Universidad Politécnica de Madrid

Escuela Técnica Superior de Ingenieros de Telecomunicación

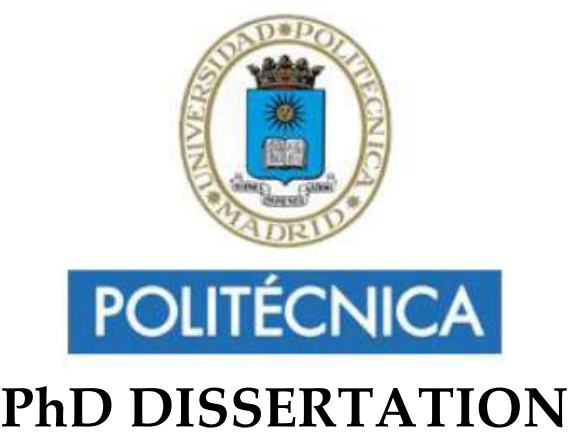

\title{
CONTRIBUTION TO HETEROGENEOUS SERVICE PROVISION AND FLEXIBLE PROCESS EXECUTION IN HUMANIZED CYBER-PHYSICAL SYSTEMS
}

\author{
Author \\ Borja Bordel Sánchez \\ MSc, Telecommunications Engineer \\ Advisors \\ Tomás Robles Valladares \\ Ramón Alcarria Garrido \\ Professors at Technical University of Madrid \\ September 2018
}







\section{(6-1) \\ POLITÉCNICA}

Título de la Tesis: CONTRIBUTION TO HETEROGENEOUS SERVICE PROVISION AND FLEXIBLE PROCESS EXECUTION IN HUMANIZED CYBER-PHYSICAL SYSTEMS

Departamento: Departamento de Ingeniería de Sistemas Telemáticos

Autor: Borja Bordel Sánchez

Directores: Tomás Enrique Robles Valladares

Ramón Pablo Alcarria Garrido

Tribunal nombrado por el Excmo. y Magfco. Sr. Rector de la Universidad Politécnica de Madrid, el día de Septiembre de 2018

\section{Presidente:}

Vocal:

Vocal:

Vocal:

Secretario:

Suplente:

Suplente:

Realizado el acto de defensa y lectura de Tesis el día de Septiembre de 2018 en la E.T.S. de Ingenieros de Telecomunicación de Madrid.

\section{Calificación:}

EL PRESIDENTE

LOS VOCALES

EL SECRETARIO 


\section{Keywords}

humanized cyber-physical systems, flexible process execution, service provision, heterogeneous services, human provided services, security, mobility management, self-configuration 


\section{Abstract}

This PhD Thesis work is developed in the context of Humanized Cyber-Physical Systems and contributes to the heterogeneous service provision and flexible process execution over such infrastructures. Humanized Cyber-Physical Systems are characterized by embedding in a transparent way both electronic devices and software components into any type of daily-living object and/or social environment (domestic, work, etc.); so by means of implicit mechanisms and interfaces the system can understand, evolve and interact with the physical world and its inhabitants.

The overall goal of this PhD Thesis work is to design, implement and validate a Humanized Cyber-Physical System, where both humans and hardware devices are involved in the task accomplishment and process execution. The system will be able to provide, in a transparent way, complex services through the coordination of entities characterized by a heterogeneous behavior and capabilities. Moreover, the system will enable the flexible process execution, considering the dynamical evolution and particular characteristics of both humans and devices.

The design and implementation of Humanized Cyber-Physical Systems is nowadays a global trend, especially in the context of the future industry, where CPS are the basic technology for the fourth industrial revolution solutions (or Industry 4.0). Nevertheless, at this moment, it cannot be found any proposal enough flexible to be applied transversally to every type of scenario, including the manufacturing industry where human intervention is crucial and essential. In this $\mathrm{PhD}$ Thesis work it is performed a deep study with details about all proposals and standardization initiatives related to Cyber-Physical Systems. And obtained results are employed to define and validate a solution that enables the flexible process execution, the human integration in such CPS, and the accomplishment of tasks over any type of physical infrastructure through the provision of heterogeneous services.

Proposed Cyber-Physical Systems are, at the end, a new generation (much more flexible and autonomous) of the already known process execution systems. Therefore, this PhD Thesis work starts investigating the different process types that could be found in the different application scenarios, the roles that people could take on when integrated into the proposed systems and, finally, the architectures, technologies and components that could allow the execution of the previously identified process types doing use of the described entities.

This $\mathrm{PhD}$ Thesis contributes to the self-configuration and self-adaptation mechanisms, applied to Humanized Cyber-Physical Systems, with a special interest on solutions based on entities (including hardware devices, virtual elements and people) with a heterogonous behavior and capabilities.

People special characteristics make impossible the use of traditional management techniques in Humanized Cyber-Physical Systems. Thus, management is addressed 
though the definition of a new type of process (name "soft process") and the use of specific algorithms for the evaluation of the human motivational state.

Any case, in those scenarios where humans do not intervene, it is also necessary to redefine the management mechanisms, to adapt them to Cyber-Physical Systems that present a very high device density, and require great reliability and availability rates.

In order to implement the described systems, enabling communication technologies are required, which are also investigated in this $\mathrm{PhD}$ Thesis work, with a special interest for future wireless technologies.

Most innovative technologies, to be applied in critical environments such as industrial scenarios, require high performance security technologies. Then, contributions in this research area, including encryption schemes and proposals based on the concept of "trust", are also done.

Each included contribution has been validated experimentally, using different techniques and application scenarios, so its viability, and the obtained improvements in respect to the state-of-the-art, are evaluated and proved. Obtained results are reported to the scientific community through different relevant international journal papers and international conferences. 


\section{Resumen}

Este trabajo de Tesis se desarrolla en el marco de los entornos ciberfísicos con intervención humana y contribuye a la provisión de servicios heterogéneos y la ejecución flexible de procesos sobre dichas infraestructuras. Los entornos ciberfísicos con intervención humana se caracterizan por integrar de forma transparente dispositivos electrónicos y componentes software en todo tipo de objetos comunes y/o entornos sociales (laboral, doméstico etc.); para mediante mecanismos e interfaces implícitas comprender, evolucionar y relacionarse con el mundo físico y sus habitantes.

El objetivo general de este trabajo de Tesis es diseñar, implementar y validar un entorno ciberfísico con intervención humana, en el que tanto dispositivos como personas participan en la realización de tareas y la ejecución de procesos. El entorno será capaz de ofrecer de forma transparente servicios complejos mediante la coordinación de entidades de comportamiento y capacidades heterogéneos. Además, permitirá la ejecución de procesos de forma flexible, en función de la evolución dinámica y las características particulares de personas y dispositivos.

El diseño e implementación de entornos ciberfísicos centrados en la ejecución de tareas es hoy en día una tendencia global, especialmente en el contexto de la industria del futuro donde son la base de los sistemas de cuarta revolución industrial o Industria 4.0. Sin embargo, no se ha propuesto un sistema suficientemente flexible como para ser aplicado de forma transversal a todo tipo de escenarios, incluyendo la industria manufacturera donde la intervención humana es crucial e imprescindible. Este Trabajo de tesis realiza un estudio detallado de todas las propuestas e iniciativas de estandarización relacionadas con los entornos ciberfísicos, y utiliza los resultados obtenidos para definir y validar una solución que permita la ejecución flexible de procesos, la integración de personas en los citados entornos y la realización de tareas sobre todo tipo de infraestructuras mediante la provisión de servicios heterogéneos.

Los entornos ciberfísicos propuestos son, en el fondo, una nueva generación (mucho más flexible y autónoma) de los ya conocidos sistemas de ejecución de procesos. Por ello, este trabajo de Tesis comienza investigando los diferentes tipos de procesos que podrían encontrarse según diversos escenarios de interés, los roles que pueden asumir las personas integradas en estos sistemas y, finalmente, las arquitecturas, tecnologías y componentes que podrían permitir la ejecución de los procesos identificados haciendo uso de las distintas entidades descritas.

Se contribuye a los mecanismos de autoconfiguración y autoadaptación aplicados a los entornos ciberfísicos, con especial interés por los sistemas basados en entidades (ya sean dispositivos hardware, elementos virtuales o personas) de comportamiento y capacidades heterogéneos.

Las especiales características de las personas, que hacen inviable el uso de técnicas de gestión tradicionales en los entornos ciberfíscios con intervención humana, se 
abordan mediante la definición de un nuevo tipo de proceso (llamado proceso "blando") y el uso de algoritmos específicos para la evaluación del estado motivacional de las personas.

En cualquier caso, en aquellos escenarios en los que las personas no participen, también es preciso redefinir los mecanismos de gestión, para adaptarlos a sistemas con una altísima densidad de dispositivos, y que precisan de una fiabilidad y disponibilidad muy elevada.

Para lograr implementar los sistemas descritos se hacen necesarias tecnologías habilitadoras de comunicaciones, que también se investigan, con especial interés por las comunicaciones inalámbricas del futuro.

Las tecnologías más innovadoras, para ser aplicadas en entonos críticos como los escenarios industriales, requieren de seguridad, por lo que también se contribuye en esta área con técnicas basadas en cifrado y el concepto de "confianza".

Cada contribución realizada ha sido validada de forma experimental, haciendo uso de diferentes técnicas y escenarios relevantes, de forma que se compruebe su viabilidad y la mejora que éstas producen con respecto al conocimiento previo. Los resultados obtenidos se han publicado en diferentes revistas internacionales $\mathrm{y}$ conferencias de prestigio. 


\section{Contents}

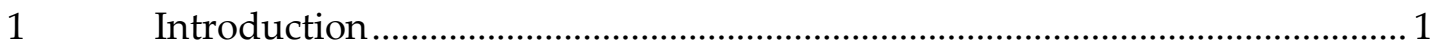

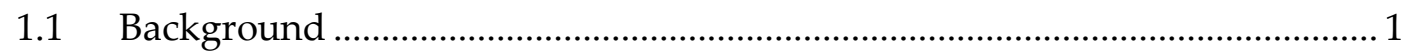

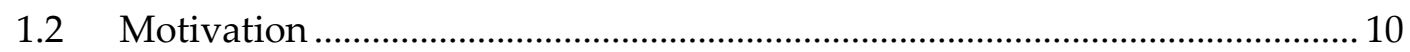

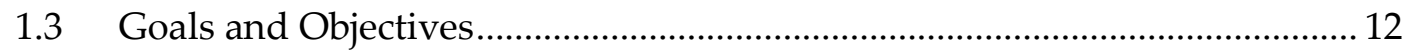

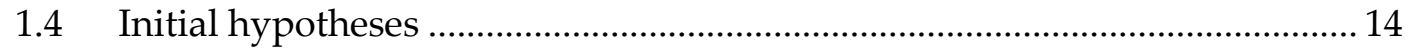

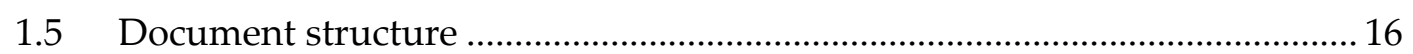

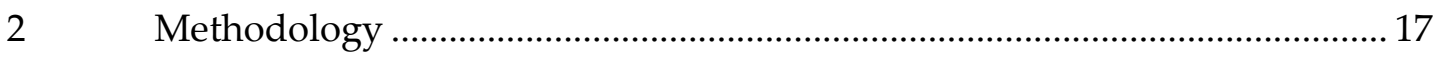

2.1 Formal aspects of the investigation in the PhD Thesis work ......................... 17

2.2 Typical scientific methodologies in engineering research............................... 19

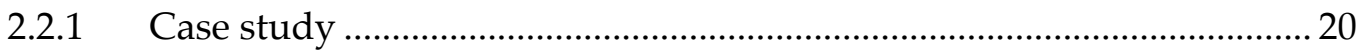

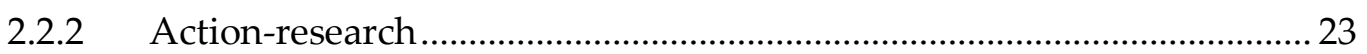

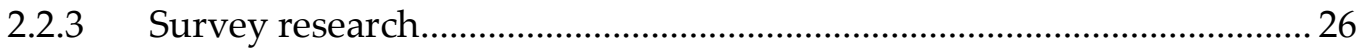

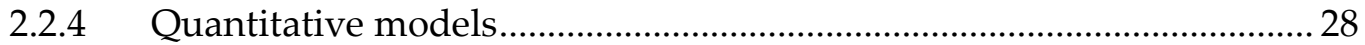

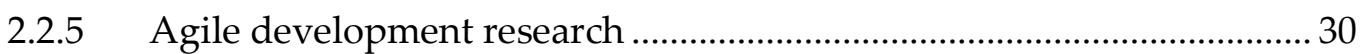

2.3 Scientific methodology in the PhD Thesis work ............................................ 31

2.3.1 General steps in the proposed research methodology............................ 31

2.3.2 Experimental methodology: relevant cases .............................................. 33

2.3.3 Particular phases in the proposed methodology ....................................... 35

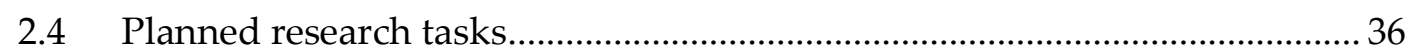

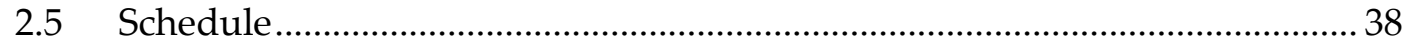

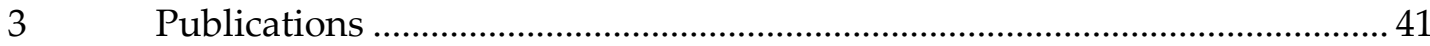

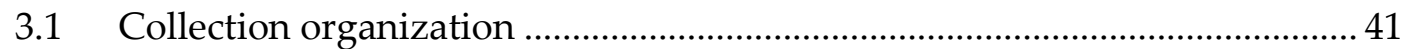

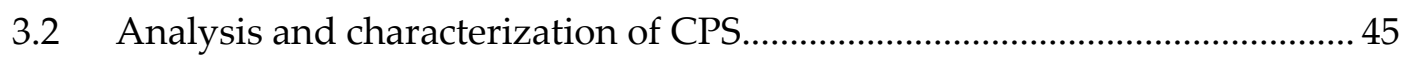

3.3 Proposed architectures: reference architecture, functional architecture and implementation architecture .................................................................... 46

3.4 Process management in Humanized Cyber-Physical Systems ...................... 49

3.5 Service self-configuration and self-adaptation in Humanized CyberPhysical Systems ........................................................................................ 52

3.6 Humanized processes. Human integration in Cyber-Physical Systems ...... 56

3.7 Device management in Humanized Cyber-Physical Systems ......................... 60

3.8 Communication models and networks in Humanized Cyber-Physical Systems. 


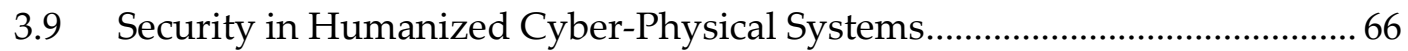

$4 \quad$ Discussion, Conclusions and Future Works ..................................................... 563

4.1 General overview of the proposed solution .....................................................56

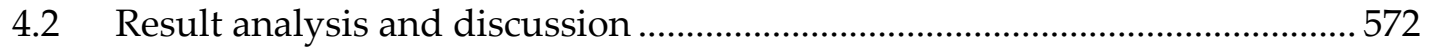

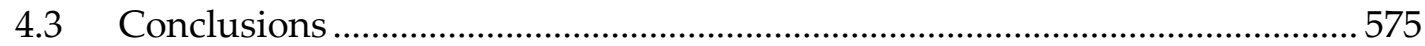

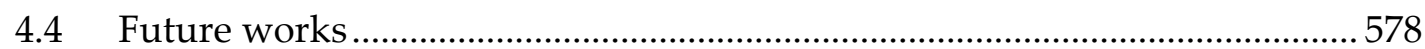

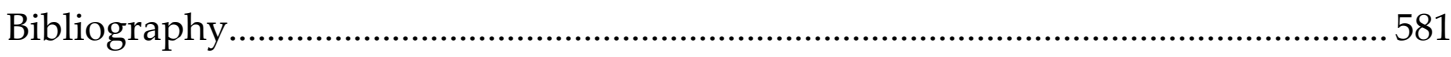

Annex A. Complete collection of publications. Additional results .............................. 585

A.1 Publications directly related to the research objectives ...................................... 585

Publications in international journals listed in JCR ranking ................................ 585

Publications in international journals listed in SJR ranking ................................ 586

Other publications in international journals ………….......................................... 586

Publications in international conferences listed in the CORE ranking ............... 587

Publications in other international conferences.....................................................58

A.2 Other publications by the author, indirectly related to this $\mathrm{PhD}$ Thesis.......... 588

Publications in international journals listed in JCR ranking.................................58 58

Publications in international journals listed in SJR ranking ................................ 588

Other publications in international journals ........................................................ 589

Publications in international conferences listed in the CORE ranking ................589

Publications in other international conferences .................................................. 590 


\section{List of figures}

Figure 1. NIST reference architecture for CPS …..................................................... 8

Figure 2. Structure of the proposed research and methodology .................................. 39

Figure 3. Proposed chronogram................................................................................. 40

\section{Tables}

Table 1. Composition of CPS in the different technological domains. ........................... 7

Table 2. Overview of the proposed solution .................................................................. 78 


\section{Acronyms}

Acronyms in italic correspond to concepts presented in this PhD Thesis work.

\begin{tabular}{|ll|}
\hline CPS & Cyber-Physical Systems \\
\hline SE & Smart Environments \\
\hline CPSS & Cyber-Physical Social Sensing \\
\hline CPO & Cyber-Physical Operation \\
\hline M2M & Machine to Machine \\
\hline WSN & Wireless Sensor Networks \\
\hline NSF & National Science Foundation \\
\hline AmI & Ambient Intelligence \\
\hline NIST & National Institute of Science and Technology \\
\hline RFC & Request For Comments \\
\hline SCADA & Supervisory Control And Data Acquisition \\
\hline QoS & Quality of Service \\
\hline KPI & Key Performance Indicator \\
\hline
\end{tabular}




\section{Introduction}

In this section, most relevant aspects in the background and the state-of-the-art are reviewed. Besides, it is described the motivation of the PhD Thesis and the goals to achieve. A first global goal is presented, which is later detailed into particular objectives, also identified in this section and achieved by the contributions developed in this PhD Thesis work.

\subsection{Background}

Humanizing technology (i.e. integrating the human actions and behavior into technological systems) is an intrinsic challenge to engineering development. The creation of interfaces and other similar mechanisms to enable a fluid interaction among people and devices is basic to ensure the success of any technology. In general, besides, the ease with which a technology penetrates society depends strongly on how similar to a human communication is to the interaction with the new system.

Classic computer theory talks about a Central Process Unit connected to a set of peripheral devices through which users can "ask for" actions that should be performed by the processor (Cohen, 1991). In that way, it could be said that computers and people stablish a dialog at a certain abstraction level. This traditional manner to interact with computers is named "explicit interactions", as (at every moment) users are aware of the expected behavior from the computer when they explicitly trigger a task or an action execution. The computer behavior, moreover, must be independent from the environment or the time evolution, to result in a useful solution.

Explicit interactions are adequate for personal devices which are action-oriented (i.e. they execute certain actions at user requests); however, most computing systems (such as industrial solutions, eHealth applications, etc.) are process-oriented, and users define their behavior as a flow before they start operating. Humanizing this second group of computing technologies is more complicated, and involves two different research lines: a new human-computer interaction paradigm; and solutions to enable 
users to define processes in an easy manner being lately executed in a flexible way depending on the computing system situation.

Furthermore, paradigms based on explicit interactions, although are useful in some applications, are unable to employ other important information sources such as information related to people desires deducted from their actions (if someone approaches a window, he probably wants to open it). Thus, in 2000, Albrecht Schmidt proposed a new paradigm called "implicit human-computer interactions" (Schmidt, 2000). It is considered as an implicit interaction every user action that is not primary focused on obtaining a response from computers, but to which processing devices response as they are programmed to understand that stimulus.

In that way, Schmidt proposed that processing devices should be aware about the environment evolution and its inhabitants, collecting information about them though sensors and actuators, and obtaining some understanding about events in the physical world. Thus, during the same year (2000) it is proposed a new and revolutionary concept: The Smart Space (Nixon, 2000).

A Smart Space is a region of the physical world where a great number of sensors, actuators and processing devices are deployed to collect and process information about the environment, being able to interact with people thanks to implicit interactions. At this point, paradigms such as "ubiquitous computing", that was defined in 1991 (Weiser, 1991), are also integrated with implicit interactions and smart spaces, in order to address problems associated to the coordination of thousands of hardware devices and the distributed process execution.

With this approach, however, Smart Spaces were only partially humanized; as people could only take a passive role (they are sensed to collect some information), and no solution to enable a flexible (distributed) process execution was provided. These technologies are critical for two reasons: first because users define the system behavior only once before the system operation and it is essential to adapt this fixed definition to the dynamic system state as time evolves; and second because final users usually do not have programming skills so they define processes using domain-specific languages, and flexible mechanisms to transform these languages into executable code are, thus, also essential.

On the other hand, Smart Spaces were still based on pre-configured rigid decision tables, so information processing was mainly based on classification algorithms. This approach was proved to be not enough and present a low humanization level, so different researches tried to improve the way in which these systems worked. Furthermore, the initial Schmidt's ideas were generalized. As defined in Schmidt's papers, implicit interactions were a catalogue of fixed stimulus-response pairs, previously configured at low-level (almost indicating the expected outputs from sensors). However, in 2002, a new idea was proposed based on pervasive systems and a new understanding of implicit interactions: Smart Environments (Das and Cook, 2005). 
Smart environments (SE) were defined, in general, as previous Smart spaces, but with an important difference (Das and Cook, 2005). Smart environments are able to capture, create, infer and apply knowledge about people and the physical world in an automatic way, adapting to the inhabitants' preferences so their user experience is highly improved. For first time, responses of engineered systems varied dynamically with time, human state, physical variables etc. and were based on the decisions made by the knowledge extraction technologies that every SE must include (contrary to decision tables employed in Smart Spaces). Although this approach brought technology to human behavior and, then, SE were a more humanized solution, people in SE took still exclusively a passive role.

Despite these improvements, besides, these systems (SE) required an intense configuration and deployment process, as their structure was much more complex than any previous proposal and employed communication and management technologies were based on traditional non-autonomous solutions. In this sense, Smart Environments were still low humanized and technologies to enable a flexible (distributed) process execution were still necessary.

On the other hand, since 1995, technologies to configure and manage networks and communicate devices in an automatic way were studied. Solutions for geographically sparse systems, deployments with a high density of computing nodes, or networks made of mobile components without any communication infrastructure were analyzed and standardized under the name of Machine-to-Machine (M2M) communications. Thanks to these new techniques, in 2001, it is defined the idea of Wireless Sensor Networks (WSN) (Akyildiz, 2002).

Wireless Sensor Networks refer an infrastructure made of a collection of processing and sensitization nodes (sometimes actuators as well) which stablish ad hoc networks in an automatic way, usually with a low power consumption, and capture information about the environment and its inhabitants. This information is shared and distributed among nodes using M2M techniques, until it is sent to the cloud through some kind of gateway. Even, some proposal for industrial scenarios where legacy systems are integrated into WSN appeared during these years.

In that way, during the first five years of the $21^{\text {st }}$ century, in the context of WSN, several problems associated to the automatic network creation, the establishment of unmanaged communication links and the autonomous sending of data and metainformation between a hardware infrastructure and a central server were solved. Using these new technologies, and despite of the fact that SE were defined in 2002, in 2005 Smart Environments were re-defined to be considered as "a part of the physical world totally interconnected through a continuous, pervasive and invisible network with sensors, actuators and processing components which are seamless embedded into daily living objects" (Cook and Das, 2004). This new generation of SE was totally autonomous, unmanaged, deployed in an ad-hoc manner, being able to capture information, send it to a central server and extract knowledge from it to make decisions 
and relate with the environment and its inhabitants in a dynamic way. This revolutionary, and much more efficient, new approach was extensively accepted and developed by several different research centers (such as the Korea Institute of Industrial Technology -KITECH- that proposed the first prototypes for industrial and domestic environments, (Baeg et al., 2007)). Although this new approach solved several lowest level problems associated to the flexible process execution, its humanization level was still inadequate, as no challenged related to the human integration was explicitly addressed.

With this new definition of SE, moreover, the number of voices claiming that old computing foundations were deprecated increased. They argued that it was not possible to develop computing systems in the same manner as in the past when important and essential paradigms such as the explicit interactions, the time independence of algorithms, the location independence of systems, etc. had been redefined or removed. These ideas were formalized one year later, in 2006, during a conference at the National Science Foundation (NSF).

Edward A. Lee, professor at Berkley University, during the referred conferred at the NSF, proposed a position paper entitled "Cyber-Physical Systems - Are Computing Foundations Adequate?" (Lee, 2006). In this paper, E. Lee defined a new technology known as "Cyber-Physical Systems", which basically was a formalization for future computing systems. In fact, WSN, Smart Spaces, Smart Environments, and many other similar proposals were, from this moment, understood as particular implementations of a new design paradigm for computing systems, which was much more revolutionary and involving much deeper changes than any proposal made up to that moment.

Cyber-Physical Systems (CPS) are defined as "integrations of computation with physical processes; where embedded computers and networks monitor and control the physical processes, usually with feedback loops where physical processes affect computations and vice versa" (Lee, 2006). In that way, learning algorithms, intelligent solutions, implicit interactions, data gathering, etc. are only particular examples of feedback control loops that characterize CPS.

Furthermore, the construction of CPS, instead of being based on the traditional computer theory, is based on control theory (and, therefore, CPS are process-oriented computing system instead of action-oriented solutions as personal devices). Thus, the objective of these systems is to execute computational processes that do not only consist of data transformations (as traditionally), but also of far richer operations that enable computing systems behave as "living creatures" which receive stimuli from the environment, interpret information from multiple sources, make a decision and return a reaction to the physical world. From the computational point of view, important topics such as the OS/programming language split, hardware/software split, understandable concurrency or networks with timing were included in the research agenda for CPS experts. 
At this moment (2006) Cyber-Physical Systems were envisioned to simplify complex distributed computing schemes, such as process execution systems, as well as task execution systems, were control loops are present intrinsically. Nevertheless, real practical characteristics that CPS have or should have were not clear. For example, some proposals consider Cyber-Physical Systems as intelligent solutions by default, but other authors argue this is only an option. In some papers CPS are defined as embedded devices which may be networked to address complex tasks in a collaborative manner; but, on the other hand, some works present CPS as systems including a pervasive platform of networked devices which solve complex problems in a choreographic manner. Then, different theoretical proposals appeared during these first moments: networked CPS, smart CPS, real-time CPS, etc. Any case all these contributions showed that CPS imply a new design procedure where computational aspects are considered together with environment's physical characteristics, so the number of synergies is maximized, the system complexity minimized, architectures simplified, efficiency increased, and cost reduced as much as possible.

In this initial understanding, besides, CPS was a very poor technology in terms of humanization. The creation of a theoretical framework for process-oriented computing solutions, actually, did not contribute to the flexible execution of processes or the integration of people in computing systems at first; as CPS were defined more like enhanced autonomous control robots of informatics systems than like a new generation of distributed control systems for future applications.

This climate of theoretical discussions was maintained until, approximately, the year 2010, when first initial prototypes related to CPS were reported (La et al., 2010). In these initial works, practical implementations of CPS were proved to require a vertical integration of several components at different abstraction layers, including a hardware platform with thousands of networked devices (from resource constraint elements to legacy systems). Deployed CPS included central management modules, prosumer interfaces, technological expert graphical interfaces, task delegation algorithms, orchestration schemes for computing solutions, etc. (Wu et al., 2011). In general, every technology previously defined in the context of WSN, Smart Environments, etc. was adapted to CPS.

Although in these initial Cyber-Physical deployments people still took exclusively a passive role and processes executed in a kind of flexible way were very few; the CPS humanization level started increasing. The integration of people was each time more important, and partial solutions to define processes using domain-specific languages, to enable users without programming skills to participate or simple process transformation techniques were reported.

During this period (2010-2014, approximately), CPS became an extremely popular paradigm, and very different domain experts tried to adapt the CPS principles to their research field. The generalization that started with the introduction of communication elements and data analytics and business components into CPS in the first reported 
prototypes intensifies with the appearance of new architectures and definitions for CPS, overall in the areas of control theory and telecommunication engineering.

In respect to control theory, as Cyber-Physical Systems paradigm is based on principles from this knowledge area, it is not strange that experts in this field employed these new computer theory technologies to design control applications and solutions much more efficient and adapted to future industry. Works belonging to control theory and related to CPS are specially focused on the characteristics of the physical environment, the hardware components and control elements.

On the other hand, once accepted that Cyber-Physical Systems are essentially ubiquitous platforms composed of thousands of networked devices, communicating with other remote components (hardware or software) through a specific technology, telecommunication experts decided to work on this new paradigm, considering CyberPhysical Systems as a new scenario of future communication networks and services. This research line became so popular that, very early, the first questions about the differences between CPS and the other great revolution of $21^{\text {st }}$ century (the Internet-ofThings) appeared.

The Internet-of-Things (IoT) appeared for first time in 1990, with the creation of a first prototype based on a kind of (smart) toaster; however, it was not formalized until 1999 (Ma, 2011). The IoT was defined as a system focused on connecting the physical and the cyber world, although during the initial moments this technology was directly related to the employment of RFID tags to associate unique identifiers to regular and daily living objects, so they are provided with a digital identity to be connected to a global network.

The Internet of Things is characterized by four basic components (Wan et al., 2013): (i) sensitization, (ii) heterogeneous access, (iii) information processing and (iv) applications and services; although additional transversal topics such as privacy or security are also usually cited. Considering this view, both WSN and CPS, as well as Machine to Machine communications, could be considered as particular realizations of application scenarios of the Internet of Things. It is important to note that, WSN could be also understood as a specific solution based on CPS principles, what is totally coherent with this approach. Any case, the difference among all these technologies is the relative weight of each functionality (or characteristic) in the global solution.

In that way, WSN are specialized on data acquisition (i.e. sensitization), including topics such as data models, the efficient use of energy, etc. On the other hand, M2M communications are focused on providing the heterogeneous access of devices, so it is enable the interconnection of a great variety of sensors and general devices being able to self-organize and operate in an autonomous way. Finally, Cyber-Physical Systems (as in the most modern approach, they are understood as a vertical integration of all layer that compose the ICT market) are usually designed as instrument to process information and provide applications and services. 
In the same way that, in general, today it is clear that Cyber-Physical Systems are a particular (and greatly relevant) scenario of the Internet of Things where the different elements that compose the IoT are mixed and configured in a specific manner, the different approaches to the paradigm of CPS that appeared in the period 2010-2014 and may be found in control theory, telecommunication engineering and computer sciences may be also understood as combinations of a same set of elements.

A deep analysis of all possible elements which may be part of CPS should be based on the study of all proposed application scenarios in research literature. However, in 2014, the Eindhoven Institute for Research on ICT (EIRCT) identified the six components which, occasionally, have been used to make up CPS (Lukkien, 2016): physical world, transducers, control components, data analytics elements, computation elements and communication components.

Thus, the simplest architecture for CPS belongs to control theory. In this area, CPS are understood as a composition of the physical world, transducers and control components (Tarraf, 2013). In some specific applications simple data analytics elements are also considered, although it is not the regular situation. As previously said, the oldest proposal on CPS is due to computer sciences researchers. In this area CPS are understood as a composition of the physical world, transducers, control components and computer sciences elements (Lee, 2015). Specialists on hybrid systems, new computational models, real-time applications and predictive models (among others) support this vision; which is, besides, the only one where humans are explicitly considered. Finally, the probably least developed approach is due to telecommunication engineering. This view is focused on hardware, services and embedded devices, so CPS are understood as a combination of transducers, control components, some data analytics elements, computer science elements and communication components.

Table 1. Composition of CPS in the different technological domains.

\begin{tabular}{|c|c|c|c|}
\hline & Control theory & Computer sciences & $\begin{array}{c}\text { Telecommunication } \\
\text { engineering }\end{array}$ \\
\hline Physical world & $\mathrm{x}$ & $\mathrm{x}$ & $\mathrm{x}$ \\
\hline Transducers & $\mathrm{x}$ & $\mathrm{x}$ & $\mathrm{x}$ \\
\hline $\begin{array}{c}\text { Control components } \\
\text { Data analytics } \\
\text { elements }\end{array}$ & Sometimes & Sometimes & Sometimes \\
\hline $\begin{array}{c}\text { Computation elements } \\
\text { Communications } \\
\text { components }\end{array}$ & & $\mathrm{x}$ & $\mathrm{x}$ \\
\hline
\end{tabular}


Table 1 summarizes the composition of CPS depending on the technological domain considered. As can be seen the underlying pervasive sensing infrastructure and the control components are the common elements to all proposals.

During this period, 2010-2014, it is made evident that Cyber-Physical Systems are an important technology to support new services and markets, to improve the efficiency of industrial systems, to enable innovative applications such as Ambient Intelligence (AmI) or the pervasive sensing platform for Smart Cities (Ramos et al. 2008). However, CPS, in 2014, it is still a research paradigm which has not totally solved the problem of humanization or process execution.

This situation is maintained until in 2014, during the Hannover Fair about industrial solution in Germany, it is defined the idea of Industry 4.0 (Jazdi, 2014). Industry 4.0 refers to a fourth industrial revolution where robotics and automatic systems, that are typical from the third one, are replaced by CPS. Therefore, in general, it is said that the fourth industrial revolution will be the era of CPS.

When the CPS paradigm receives the interest of the industrial and corporative world, other instructions get attracted towards this concept. Thus, organizations such as the standardization agencies and, in particular, the National Institute of Science and Technology (NIST) decided to create working groups focused on normalize and standardize Cyber-Physical Systems (NIST, 2014).

The main NIST's objective, which created several secondary working groups and committees, is the standardization of a CPS model enabling manufacturers to product interconnectable and interoperable equipment, as now occurs with Internet equipment regulated by RFC (Request For Comments) documents.

The NIST's vision about CPS is integrator, in order to include all different proposals about CPS that appeared over time. Cyber-Physical Systems in the NIST's approach considers all elements and components that may be part of these systems (along with any reported definition of research work). In that way, in June 2015, it is published the first reference architecture for CPS by NIST (see Figure 1).

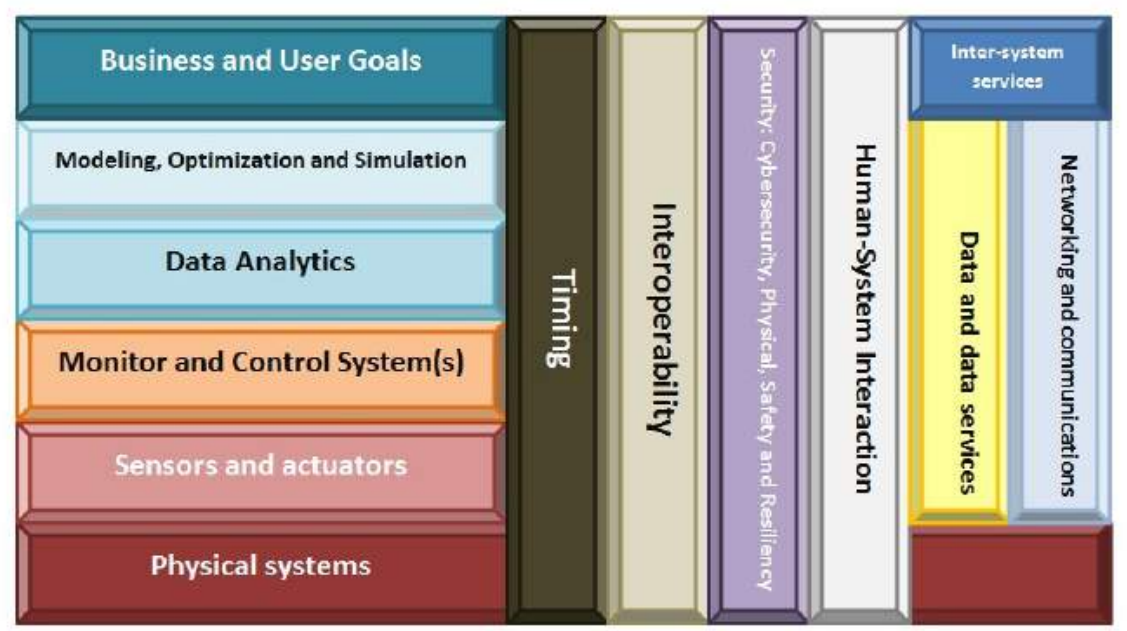

Figure 1. NIST reference architecture for CPS 
The definition of Industry 4.0 or future industry caused the interest of many different markets for Cyber-Physical Systems: automatic and massive production lines, small manufacturing industries, etc. In fact, some of the currently proposed solutions based on CPS match perfectly with some of these scenarios, especially with those based on automatic production systems, chain production companies, traditional control systems (such as SCADA (Daneels and Salter, 1999), which is closer to traditional informatics systems than to future control applications).

Nevertheless, there are many other scenarios that have not been investigated yet. In these scenarios, although the initial reference architecture proposed by NIST is available, it is not clear what components or hardware devices and equipment are required or it is necessary to design and construct; in order to enable the provision of the desired services. Specifically, as it is one of the key characteristics of CPS, it is still necessary to investigate technologies to integrate in an interoperable manner the domain-specific devices and components of these scenarios, which (in general) will be very heterogeneous and should actuate in a flexible way (according to the defined processes at each moment and the physical environment state).

A set of application scenarios which are very relevant and interesting, but they have not been considered until now, are those in which people must participle and intervene necessarily and inevitably.

Actually, in regular CPS, people are passive agents, being part of the physical environment and being sensed (and suffering the system actuations) as any other physical component, creature or agent. The final objective of these systems is to improve the user experience or life quality of people. There are, however, other scenarios where people are active agents, i.e. where services to be integrated and provided by means of Cyber-Physical Systems are finally supported and execute by people not by hardware components. The most typical example of these scenarios is manufacturing companies (where hand-made products are elaborated).

In these scenarios where humans must intervene as active agents (or service providers), it is essential to consider technologies, components and tools which allow integrating people into the system taking this role. Moreover, it is needed to consider the special characteristics of people, in respect to their behavior, work cycle, capabilities, response time, interaction with computing systems, etc.

Definitely, it became essential to solve the humanization problem that was surrounded and only partially addressed since 1990. In particular, people must be integrated not only as passive agents (as done in previous SE, Smart Spaces, first CPS, etc.) but also as active participants. Besides, since humans are now active agents, the entire system must be adapted to this new situation and, in particular, processes cannot executed using hard execution schemes (typical from systems where only intervene administrator with technological skills), but flexible procedures. 
Actually, future industry will be characterized by the great automatization of processes and the high specialization reached by Cyber-Physical Systems. Nonetheless, it must be also able to maintain those actives which must be executed by human inevitably, improving the efficiency and people wellness as much as possible.

In order to promote the development of Cyber-Physical Systems and Industry 4.0, functional components and solutions employed in both traditional scenarios (where humans are passive agents) and scenarios where people act as service providers, should be part of the same functional architecture, which will be valid for all applications.

The contributions and advances described in this PhD Thesis work address these aspects from the previous knowledge, in order to define and enable a new type of Cyber-Physical System named as "Humanized Cyber-Physical Systems", being able to integrate both people and hardware devices, all of them with heterogeneous behavior and capabilities. These systems will be also able to execute in a flexible manner all process types that may be found in industrial scenarios, AmI applications, Smart Cities, and (in general) in any future scenario of the forth industrial revolution.

\subsection{Motivation}

This PhD Thesis work is motivated by the need of defining and implementing new architectures and solutions that enable the execution of processes describing and controlling the behavior of the future engineered systems in an automatic and autonomous way, with an especial interest on scenarios where people intervene actively, and applications related to the era of Cyber-Physical Systems, also named as Industry 4.0 .

In this $\mathrm{PhD}$ Thesis work it is provided a functional architecture, together with a design and a detailed description of all components that made up it, being able to consider all possible roles that humans may take in the context of Cyber-Physical Systems; and allowing the flexible execution of all different processes that can or could be present in future fourth industrial revolution scenarios, including those with the intervention of people.

The process execution by Cyber-Physical System in scenarios where the final execution of activities and operations is in charge of platforms composed by devices and agents with capabilities and behavior very heterogeneous, including those where humans intervene, it is not solved nowadays by means of the existing execution engines and models.

Actually, to the best of my knowledge, it has been reported no model, architecture or solution based on CPS and being able to execute processes defined by domain experts at high-level, processes executed freely devices or people according to a set of rules, and/or processes defined by a sequence of phases, states or activities (a kind of balance among processes defined at high-level and processes executed freely by 
devices or people). Moreover, no CPS solution with a high humanization level, being supported at low-level by a platform of interconnected devices and active people which relate in such a way that they may coordinate in autonomous way (or through an orchestration module) to execute delegated tasks and operations.

In particular, the least studied topic is the adaptation of all exiting solutions to processes which are finally executed by humans acting as service providers; which impose very special requirements and characteristics that must be investigated.

The execution of processes by means of CPS increases the efficiency of executions, whether services are provided by devices or by humans, so motives to address the cyber-physical execution of processes are both economic and technique, and caused, in general, by the distributed and specialized execution of task according to the capabilities of the underlying physical platform.

This approach presents various advantages. For example, domain-experts are enabled to define the behavior of technological systems without needing specific knowledge (an approach known as "prosumer"), and thanks to the different transformations that process models suffer from its definition to its execution at lowlevel.

From the economic point of view, these systems allow externalizing the execution of certain activities or tasks in a dynamic way, and according to the existing capabilities in the different productions systems that are interconnected. Besides, these systems are very interesting for companies as they enable to exploit at maximum the deployed and existing productive capacity. This objective is reached by the execution of internal tasks, and the execution of activities externalized from other productions systems, agents or companies which are interconnected in a global production network. This dynamic use of resources is enabled thanks to cyber-physical technologies and usually receives the name of "Smart Manufacturing".

As most production schemes also include tasks that must be executed by people inevitably, there is an important motivation to investigate techniques that allow CPS (which will govern the future production systems) to support this type of activities. Therefore, the definition of new process models, self-configuration solutions and frameworks to integrate people into CPS present a great interest nowadays.

These new architectures will allow mixing the advantages of processes defined at high-level and advantages of systems built in a bottom-up manner (i.e. with devices that execute operations in an independent manner at low-level). Future engineered systems should allow the execution of both types of processes in a flexible way.

The referred processes, besides, will be supported by services at low, medium and high level which come from the combination and coordination of devices capabilities available in the physical platform (which also include people depending on the case). Thus, these services will have a heterogeneous character in both their composition and their comparison with other services. With this service-oriented approach, besides, 
surplus capabilities will be able to be offered to other companies through cloud services, virtual entities, etc.

In that way, there is a great motivation to investigate new device management schemes, as well as new wireless channel models and communication schemes that allow the definition, maintaining and actualization of these heterogeneous services on which process execution is supported.

On the other hand, and finally, in a future scenario where production systems and, in general, any infrastructure (include those with a critical character) will be governed and managed by means of Cyber-Physical Systems, it is essential to these systems to include security mechanisms for both protecting them against cyberattacks and making them fault resistant (against both internal and external failures). Moreover, new attacks and risk associated to these new technologies must be also investigated.

In order to reach these objectives, it is necessary to do an investigation about the types of attacks these systems could suffer, the failures could happen (among other important topics) and propose specific solutions adapted to the paradigms of future industry and Cyber-Physical Systems. Nowadays, there is a very high interest in topics related to this area (cybersecurity or cyber-physical security according to the most modern definition).

\subsection{Goals and Objectives}

The Goal of this PhD Thesis is to design, implement and validate a humanized cyberphysical system, where both humans and physical devices are involved in the task execution. The system will be able to provide, in a transparent way, complex services by means of the coordination of entities characterized by a heterogeneous behavior and capabilities. Moreover, the system will enable the flexible process execution, considering the dynamic evolution and particular characteristics of both humans and devices.

The humanized cyber-physical systems described in this $\mathrm{PhD}$ Thesis are intended to enable the integration of humans in the future feedback control systems, not only as passive inhabitants of the deployment scenarios, but also as service providers. Besides, the system will allow the implication of prosumers in the process definition, creation and execution. To do that, the humanized cyber-physical systems are defined from various perspectives, and different architectures are proposed and characterized. To develop the different definitions, models and architectures the following specific objectives are identified.

Objective\#1: Definition and characterization of the different possible process types to be execute using humanized cyber-physical systems, as well as the different entity types that may be part of these systems. Definition and characterization of the different possible roles of humans in the context of humanized cyber-physical systems, 
considering the impact of each possible role in the service provision and process execution.

Objective\#2: Proposal of a reference architecture, as well as an implementation architecture and a functional architecture, for humanized cyber-physical systems according to the roles defined in Objective\#1.

Objective\#3: Creation of a model for humanized cyber-physical systems including the required components for heterogeneous service provision and flexible process execution according to the roles, processes and entities defined in Objective $\# \mathbf{1}$ and the architectures defined in Objective\#2.

Objective \#4: Define algorithms and procedures for process definition, creation and distributed execution, which allow (at the end) the invocation of the services provided by the humanized cyber-physical system.

To achieve a useful service provision mechanism is necessary to implement selfconfiguration and self-adaptation techniques for automatic service discovery, identifying the following objectives:

Objective\#5: Define the self-configuration and self-adaptation procedures and algorithms for allowing humans to provide services (possibly with a certain quality-ofservice) which could be invoked from the final applications.

Objective \#6: Establish mechanism for self-discovery of hardware capabilities in the underlying physical platform of the humanized cyber-physical systems. Propose a selfconfiguration and self-adaptation algorithm for heterogeneous service provision, based on the coordination of various entities, possibly with a certain quality-of-service, and facilitating the invocation of services by prosumers.

To enable the human integration as service providers in the humanized cyberphysical systems, various monitoring devices and specialized algorithms and procedures are defined. The execution of processes by humans requires a much more complex mechanism of monitoring and verification. Human behavior is variable, and people may execute the same task in several different correct ways. Thus, humanized processes require specific instruments which allow a flexible process definition and verification according to the human behavior. For that, the following objectives are identified:

Objective\#7: Design and implement the devices and algorithms which enable the human monitoring, integration and interaction in the context of the humanized cyberphysical systems, especially when humans act as service providers.

Objective \#8: Create a mechanism for process definition, execution and verification, tolerant to the variable human behavior, which allows a flexible treatment of processes executed by humans. Provide an algorithm for pattern recognition and information extraction, which allows inferring the activities being performed by humans and which enables process verification in the context of flexible process execution by humans. 
Process execution in humanized cyber-physical systems need to be tolerant to changes in the hardware devices, taking into account the information provided automatically and periodically by means of the contributions described in Objective\#5. Hardware lifecycle is supported by specific management solutions and policies. The following objective is identified:

Objective\#9: Define a procedure for managing the hardware lifecycle, including both predictive and reactive techniques, in order to support flexible process execution by the physical devices. Provide an algorithm to enable hardware devices to selfmanage their resources and execute tasks in a choreographed manner (tolerant to changes in the other hardware devices).

Future humanized cyber-physical systems will be supported by pervasive hardware platforms where devices must be networked using unobtrusive (wireless) communication technologies. New solutions and network architectures, adapted to the requirements of these new engineered systems and the heterogeneous entities that form them, are required. For that, the following objective is identified:

Objective\#10: Create a communication model for cyber-physical devices communicating through a radio channel using any of the available wireless technologies. Define network architecture for future communication infrastructures being able to support the operation of humanized cyber-physical systems.

Humanized cyber-physical systems are envisioned to be employed in several different scenarios, including critical applications and solutions where private information is handled. Security policies and mechanisms are needed to guarantee the correct operation of the system and the users' privacy.

Objective\#11: Create a security solution to protect humanized cyber-physical systems against cyberattacks, unauthorized accesses, malicious components, and any other mechanism whose objective is modify the system operation.

To evaluate the applicability of the solutions developed in this PhD Thesis and to validate the usefulness of the models, policies, mechanisms and algorithms, it is necessary to evaluate the proposed solutions in different scenarios and domains.

Objective\#12: Implement applications, prototypes and proofs of concept and validate the contributions in different domains related to humanized Cyber-Physical Systems.

\subsection{Initial hypotheses}

As previously said, there are works that prove the improvement in the flexibility and humanization of production systems and other fourth industrial revolution solutions, when they are based on Cyber-Physical Systems.

The elements that motivate this PhD Thesis work, as also already said, are the needs of extending the currently existing works towards more realistic scenarios where 
people participate as active agents, and where physical platforms are composed of devices with a heterogeneous behavior and capabilities.

Considering this previous knowledge, and in order to fulfill the research objectives described in the previous sub-section, various initial hypotheses are defined, which are validated and checked in this PhD Thesis work.

Thus, the first and main hypothesis of this PhD Thesis work is:

Hyphotesis\#1: “Process execution systems based on Cyber-Physical Systems present a higher humanization and flexibility than traditional solutions, for all application scenarios, all process types and independently from the fact that final operations are executed by devices, humans or a combination of both agents".

Higher humanization and flexibility levels in process execution are evaluated considering the experience of final users and domain experts (which valuate those characteristics using some relevant KPIs), the possibility of people providing services to correct errors and modify their behavior according to tasks delegated from the management components; and the global success rate in process execution in dynamic (flexible) scenarios with human intervention.

Some previous works (especially those related to computer theory) have proved the validity of this hypothesis in the most basic scenarios, where CPS execute computational processes defined at high-level, and systems are composed of homogeneous physical platform where only hardware devices execute services and people are passive agents.

Considering the different communication technologies and solutions that have recently appeared, including those which are automatic (M2M technologies), it seems reasonable to think that the employed communication model and/or the selected network architecture influences the performance of the enabling technologies that support the humanization and flexibility of CPS. Therefore, a second hypothesis is stablished:

Hyphotesis\#2: “Communication technologies, network architectures and management solutions which support the connections among the different components in a service oriented Cyber-Physical System focused on the flexible execution of processes, have a diminishing effect in the humanization and flexibility of CPS compared to traditional systems".

In some previous works and scenarios it has been proved that there are limits in the flexibility level (and sometime also in the humanization level) that may be obtained in future computing systems depending on the selected communication scheme: selected protocols, network architectures, stability of connections, fragmentation policies, etc.

Finally, due to the need of Cyber-Physical System to be in charge of critical activities in the future, it is necessary to consider in this PhD Thesis work some factors related to security. In particular, a third and last hypothesis is proposed: 
Hyphotesis\#3: "Security technologies protect Cyber-Physical Systems against cyberattacks, unauthorized accesses, failures and other problems associated to security, but do not significantly decrease the benefit in humanization and flexibility CPS provide over traditional systems".

\subsection{Document structure}

This dissertation is structured as follows:

Section 2, Methodology, describes the employed scientific methodology to develop the research associated to this PhD Thesis work.

Section 3, Publications, presents a collection of selected papers where the most important results associated to this $\mathrm{PhD}$ Thesis work are reported to the scientist community. They contain information about all research objectives and allow proving the validity of the proposed hypotheses.

Section 4, Discussion, Conclusions and Future Works, summarizes the general solution described in the previous section. Results are analyzed, research objectives are evaluated and related to the proposed contributions, and some relevant conclusions and pending challenges for future works are reported.

Finally, Annex A, Complete collection of publications. Additional results, includes all results and research related to the PhD Thesis work, including those contributions that are secondary. 


\section{Methodology}

This section describes the scientific methodology followed to develop this $\mathrm{PhD}$ Thesis work. They are included a formal description of the investigation, a general review of the most typical methodologies in engineering research, a detailed description of the proposed methodology for this work and a list of the performed tasks together with the planned schedule.

\subsection{Formal aspects of the investigation in the PhD Thesis work}

Although there is not a universal agreement about the different research types that may be found, it is possible to find in the specialized literature several generally accepted criteria to do a formal classification of investigations.

As the objective of the proposed research is the resolution of a practical problem, including the design of new components, procedures and methods, and being, besides, the humanization and flexibility of the proposed solutions the main valuation indicators; it is possible to say that the performed investigation is technological (according to its final objective).

As technological research, this PhD Thesis work it is not only based on well-known scientific principles (as applied or theoretical sciences), but also on the experience, knowledge and professional abilities of researchers, i.e. their "expertise". In the paper "A Methodology for the Design of Application-Specific Cyber-Physical Social Sensing Co-Simulators", by Bordel et al. included in Section 3.7 and where Objetive\#9 is addressed, the importance of these factors is clearly shown. On the other hand, this PhD Thesis work has a "multiplier effect" (typical of technological investigations), as the proposed designs and solutions could be employed to enable new scenarios or as reference for new future systems. The paper "Using 5G technologies in the Internet of things handovers, problems and challenges" by Bordel et al. included in Section 3.8 and where Objetive\#10 is addressed, analyzes and proves this multiplier effect for contributions related to communication models and architectures. 
Classifying this research in terms of the type of data to be analyzed is more complicated. Two main research types are distinguished according to this criterion: qualitative and quantitative. This difficulty is caused by the fact that this $\mathrm{PhD}$ Thesis work considers both devices and people, mixed in heterogeneous scenarios; so, depending on the phenomena, variable or effect to be studied a different research type will be employed.

Globally, the research in this PhD Thesis work is quantitative: a set of hypotheses is proposed, a plan to prove their validity is designed and relevant variables in these hypotheses are measured to later analyze the acquired data by means of different techniques. If through the employed statistical or numerical methods it is deducted that it is possible to extend the obtained results (in a collection of particular scenarios) to a larger universe, then the proposed hypotheses will be considered valid.

In this case, as said in the previous section, the most relevant variables for the investigation are flexibility and humanization in CPS. Considering these variables the experimental evaluations will be performed. The paper "TF4SM: a framework for developing traceability solutions in small manufacturing companies" by Bordel et al. included in Section 3.4 and where Objetive\#4, Objetive\#7 and Objetive\#8 are addressed, proposes an experimental evaluation showing the flexibility and humanization of the designed and deployed process execution systems.

However, and despite the previous discussion, this PhD Thesis work is focused on those scenarios where humans behave as active agents into Cyber-Physical Systems. In these cases, the objective is not to obtain results to be generalized in universal laws, so a qualitative approach will be employed. In fact, investigations related to humans and CPS are focused on reconstructing the reality as could be seen from an external and neutral point of view, trying to understand the human reality: how people behave, how they live, how they feel, etc. In these cases there are no variables under study which are manipulated or controlled during the experiment, but research only aims to understand the social ecosystem that people create in Cyber-Physical Systems. The objective is to evaluate if human behavior is integrated into the system, if interactions between people and devices are correct, if people present the highest possible wellness, etc. In the paper "Assessment of human motivation through analysis of physiological and emotional signals in Industry 4.0 scenarios" by Bordel and Alcarria included in Section 3.6 and where Objetive\#7 and Objetive\#8 are addressed is included a qualitative experiment about Humanized Cyber-Physical Systems.

Any case, and independently from the data type to be analyzed, the proposed research in this PhD Thesis work is experimental.

In order to validate the proposed hypotheses sometimes it is applied an intentional manipulation of independent variables, overall they are controlled or embedded into the proposed algorithms, components or designs. In general, because of the technological nature of the proposed research, intentional variations in the intensity are applied; i.e. different values are selected for the independent variable to test the 
dependency function among the independent and dependent variables. In other circumstances, nevertheless, as for example when evaluating the second hypothesis, it is selected a measurement scheme of the effect of the independent variable. In this case, it is only evaluated if the study variable affects the interest indicators (in this case the humanization and flexibility level in the process execution).

Finally, overall in experiments where people are involved, we employed experiments with an internal control of the experimental situation. In that way, it is ensured that variations in the dependent variable are only due to variations in the independent variable. The objective is to evaluate (for example, see paper cited above in Section 3.6) if systems to represent the human state are improved if using cyberphysical techniques, guarantying that no exogenous variable is the cause of this improvement.

In summary, in this $\mathrm{PhD}$ Thesis work it is developed a technological research, quantitative (although some qualitative experiments are included) and experimental.

In respect to the hypotheses proposed in Section 1, all of them are causal, i.e. the hypotheses relate two or more variables so a causal effect or direct relation may be deducted among them. No statistical hypotheses are proposed, as the State-of-the-art does not allow imagining realistic quantitative values for humanization and flexibility for scenarios with humanized Cyber-Physical Systems and supported by heterogeneous services. Considering the results of this PhD Thesis work, in future work, hypothesis could be redefined in a statistical manner in order to maximize the humanization and flexibility.

\subsection{Typical scientific methodologies in engineering research}

The description of the scientific method proposed by Descartes in 1637 has a positivist approach, i.e. the typical approach of theoretical sciences like physics; which were the most interesting areas in that period. The problem associated to this view is the assumption of a set of repeatable and artificially controllable phenomena which are the elements under study.

However, during the $19^{\text {th }}$ century, it became clear that some investigations or fields in science do not match this scheme, as they are focused on effects that are essentially unrepeatable; like for example history research. Modern authors, like Popper, Huxley or Ackoff interpret the scientific method as a "qualified application of the common sense"(Popper, 1959; Ackoff, 1962); so nowadays there is not only one "scientific methods" but several "methodologies".

Although different methods have been proposed, most procedures focused on evaluating and correcting the scientific theories follow the same scheme: (i) observation, (ii) generalization, (iii) experimentation and (iv) validation (Rivet, 1972).

The technological research, and particularly the engineering research, although it has a special character, it shares some common aspect with the theoretical and applied 
scientific research and the positivist sciences. In particular, technological proposals must be controllable and repeatable to be useful and practical. Thus, typical technological research methods are based on this pattern (observation - generalization experimentation - validation).

Besides, engineering research presents an inductive character.

As in engineering, research starts with particular experiences and particular scenarios, reasoning must be mainly inductive, creating conclusions more abstract and general from the particular experiences. The logical reasoning is, then, ascendant in al technological research. Nevertheless, conclusions in engineering do not have a universal character, but they are very probable conclusions (it is only necessary to find a unique counterexample to reject the conclusions).

In engineering research, therefore, it is critical to develop an experimentation with enough strong and extended impact to be relevant, and make highly probable the obtained conclusions. In this case, even if a counterexample is found, its probability will be very low and maybe without any practical use, so extracted conclusions could be considered still valid in general.

Technological research, in consequence, is focused on developing experimental methods that guarantee the validity of the extracted conclusions through induction techniques. There are both, very general and very specific proposals to perform experiments in engineering; however in this section we are only reviewing those with interest for this PhD Thesis work.

\subsubsection{Case study}

Case study is a research method that allows generating new scientific theories through an induction process based on several different data sources, being of various types: qualitative, quantitative, surveys, direct observation, etc. Formally (Yin, 2017), the case study is defined as an empirical inquiry that:

- Investigates a contemporary phenomenon within its real life; when

- The boundaries between the phenomenon and context are not clearly evident; and in which

- Multiple sources of evidence are used.

Thus, the case study is an experimental methodology which employs real data in order to analyze a phenomenon and its context; as borders between the process under study and its environment are diffuse. The case study is one of the most employed methodologies in sociology, psychology, social sciences and (in general) in experiments where humans are involved (as environmental variables that effect people are very numerous and usually unknown). Case study is, actually, very useful when investigation complex phenomena where several variables affect the experiment in a non-defined manner (De Brito, 2004). The case study allows researchers to extract common, significant and relevant characteristics about the experiments varying the context which influences the phenomenon. 
The case study is a methodology based on the "ground theory"(Glasser \& Strauss, 2017), an approach which develops conclusions "grounded in data systematically gathered and analyzed"; i.e. a method focused on constructing theories from the analysis of heterogeneous data.

In this methodology conclusions are developed to explain data, so divergences never appear between the proposed theories and the real data collected. However, in order to accept that inferred theories are universally valid, it must be guaranteed that data are coherent are represent a global reality. Thus, different works investigates strategies to increase the coherence, validity and reliability of data (Yin, 2017). Data generalization must be also analytic and logic, based on representations, diagrams and graphics where the relations and deep structures among the different data sources get exposed (Miles \& Huberman, 1984).

Case study is very useful to answer questions including the tags "how" or "why", i.e. when trying to understand the underlying causes in a quite unknown phenomenon or reality. In general, the case study offers a holistic perspective (not reductionist) of a process or event.

Although case study is a valid methodology to develop new theories, it is also possible to apply this method as an experimental mechanism to prove existing hypotheses. In particular, by deepening in the causal relations of a real complex phenomenon five different objectives could be fulfilled (Voss et al., 2002):

- Exploration: During the initial moments of any research project, it is necessary to analyze the field where the work is going to be developed to stablish a set of research questions or hypotheses.

- Description: The case study may be also used to create a literal description of a phenomenon and its context.

- Theory generation: This is one of the main strengths of case study. The analysis of data allows generating new theories by induction, in a pretty simple manner.

- Theory testing: Although it is not a statistical method, case study also allows validating a set previously formulated theories or hypotheses. In this case, the case study is usually combined with other methodologies to obtain a better perspective. With this approximation, different methods and tools are employed to approach to the same reality from different points of view and avoid the research methodology weaknesses.

- Theory extension: Many times, case study is employed to slightly modify some existing theories to make them more coherent with the collected data.

Four different implementations of case study methodology may be found. On the one hand, depending on the number of considered cases it can be distinguished between single-case studies (if only one case is investigated) and multiple-case studies (if several different cases are evaluated). On the other hand, and depending on the 
manner in which each case is analyzed (as a unique entity or disagreeing the case in smaller scenarios), the methodology may be embedded or holistic.

In general, to be useful, relevant and coherent, a multiple-case study must be performed, although in some special circumstances single-case studies are also valid (Gross et al., 1971). For example, if an extreme or critical case is studied to reject an existing theory a single-case methodology may be employed. In investigations including unusual cases (such as extreme rare diseases), pilot experiences or "revelation cases" (those which are observed for first time in research) the single-case method is also accepted. In that way, if none of these especial cases is being evaluated, a multiple-case approach is required.

In order to select the cases to be studied in a multiple-case methodology, it must not be developed a statistical sampling; but a selection based on the relevance of cases. A typical error that invalids a research based on the case study methodology is selecting a high number of cases trying to improve the reliability of precision of the results (as in statistical methodologies where a large sampling space improves the precision). However, in case study it is preferred to select only cases that are critical, relevant or represent an important situation.

In general, it is accepted that a good case study approach includes between six and ten different cases (Yin, 2017). In that way, globally, at least three different relevant behaviors will be observed. It is needed, any case, a systematic approach to select cases where these relevant behaviors appear. It is important to take into account that, in case study, theories are not accepted to be valid for a enough large set of samples, but to be valid for the most relevant cases that appear in the real world.

An experiment based on a case study includes eight different phases (Eisenhardt, 1989):

- Experiment start: In this phase, the relevant behaviors to be observed are identified. Important research question focused on validating the hypotheses are proposed. In case study, however, these initial ideas are only tentative, and researches must be willing to change them if first obtained results shows it is required.

- Selection of cases: It is important to select cases corresponding to extreme, critical or rare circumstances, so the desired behavior can be observed clearly.

- Design of tools and protocols to analyze cases: The type of mechanisms to be employed during the cases analysis depends strongly on the type of data source to be obtained (surveys, documentation, devices, etc.). However, in general, the "data triangulation" paradigm is accepted. Thus, if a hypothesis is corroborated by three different independent sources, it is considered the hypothesis to be validated, as biases due to researcher are negligible. Thus, data triangulation encourages the use of different approach or tools to analyze a same reality. Data 
triangulation also encourages the participation of at least three researchers to remove biases.

- Data collection: As the amount data collected may be huge, usually data collection and data analysis are overlapped. Besides, in that way modification in the experiment can be applied dynamically, if results do not show the expected behavior. Mostly, case study is focused on qualitative data (or soft research), but also quantitative data (or hard research) or mixed sources are usually employed.

- Data analysis: Obtained data are studied in two manners, using within-case analyses and using cross-case analyses. The first step enables researcher to extract the particular characteristics of each case; and the second step is employed to compare different cases, triangle data and extract general conclusions.

- Hypotheses validation: Cases are compared two-by-two or three-by-three in order to detect hypotheses that are probably valid and those which are definitely not true.

- Explanation of divergences between results and the existing literature: Once hypotheses have been validated, divergences between the obtained results and previously reported experiences have to be detected and explained. In that way, the coherence of the proposed theory increases.

- Experiment end: Once no new relevant behaviors are observed in the selected cases, or no new cases are proposed, the experiment ends.

The huge amount of data to be analyzed, and the difficulties to extrapolate the obtained result beyond the studied cases are the main weaknesses of this methodology.

\subsubsection{Action-research}

In 1946 Kurt Lewin proposed the action-research methodology, as a new approach to develop investigation related to humans. In particular, he defined action-research as (Lewin, 1946) "a comparative research on the conditions and effects of various forms of social action and research leading to social action, being composed by a spiral of steps, each of which is composed of a circle of planning, action and fact-finding about the results of the action".

In other words, research-action is a method to investigate that mixes interventions on the reality under study with the extraction of conclusions, creating a cyclic process or "spiral of steps". Basically, Lewis argues that every research about the human factor presents a set of very special characteristics which make it to need a different a new methodology to be investigated. In particular, in his approach, experiments and phenomena to be analyzed (actions) are a unique entity.

In research-action methods, researchers perform an introspective evaluation about the situations or systems they desire to improve (how they felt, why something occurred, etc.), and of those they have been participants. This methodology is very 
employed when performing research about people in industrial or organizational scenarios (Emery et al., 1976).

The action-research methodology aims to investigate about a social system or human ecosystem, at the same time that particular exploratory actions are performed on this ecosystem (for example, investigating about solidarity at work at the same time that actions to promote this behavior are taken in a real company).

Results of these investigations must consider both, the performed actions and the observed reactions. In general action-research experiments aims to really transform a social system (not only observe the consequences of a certain action).

Some special characteristics of this methodology must be considered:

- It generates an investigation into the action, not about the action: Taken actions are part of the context where the investigation is developed. The focus is not to learn about the impact of a certain action, but learn about a social system which is being transformed by means of this action.

- Participative: Researchers must participate as active agents, not as neutral observers. Researches make things to occur as they desire, not only observe the consequence of their actions.

- Simultaneity with the action: Research is performed at real-time. As data are generated, conclusions are extracted and (if needed) modifications in the actions are taken to reach the proposed social objective. Due to this fact, quality indicators in action-research methodologies are not general, and are designed specifically for each experiment (Susman and Evered, 1978). In general, nevertheless, an experiment is valid or useful if the social objective is finally reached.

- Focused on problem solving: Action-research methods are focused on resolving a social problem or improving the general people wellness (as well as contributing to the scientific knowledge by reporting the observed behaviors). Actions must be destined to reach that objective. This type of investigations usually is about environments in change and their behavior (the actions that are causing those changes are not a research object).

Action-research experiments must follow a very strict ethical code, as people usually get manipulated during the experiments, and their situation and life may be totally changed as consequence of the taken actions.

In other methodologies the objective is to generalize the obtained results, but in action-research data collected is totally dependent on the location, moment and context of the proposed investigation, so no general theories or knowledge can be usually extracted. In this context, one of the most important risks in this methodology is the researcher not to be objective. As researcher must be participants in the experiment, they must find a balance: enough involved to be a participant, but enough neutral to describe reality objectively. In general, results based on collected data must be 
distinguished from opinions and personal observations in order to avoid these risks (Coughlan \& Coughlan, 2002).

In general, the action-research methodology is recommended to investigate three types of events (Coughlan \& Coughlan, 2002):

- Hypotheses are related to the description or behavior of a social group

- Research is focused on understanding how and why some actions improve the system

- The investigation aims to learn about the change processes

In any of these cases, the action-research methodology is composed by the same eight steps: one preliminary phase, six main phases (data collection, feedback and analysis and planning, implementation and evaluation of the action), and the final control phase.

During the preliminary phase some ethical questions must be answered. Specifically, about the adequacy of interfering in the social system, or the reasons and global benefit of performing the experiment, the interest of the hypotheses or research questions, etc.

The first main phase (data collection) is similar to the equivalent phase on other methodologies, existing both hard and soft research depending on the acquired data. As in action-research the data sources are highly influenced by the context, in the second main phase (feedback) it is evaluated the coherence and validity of the collected data before going to the next step. If no valid data are is obtained, the second phase must be repeated with the appropriate modifications.

Data analysis (the third main phase) is performed in a participative and collaborative way, among all participants with the orchestration of the researchers. A critical analysis should be performed to design the most adequate actions.

Using the conclusions extracted from the analysis of data, actions will be planned in the fourth main phase of the methodology. The selected actions must answerer a set on relevant questions to be useful and coherent enough to prove the validity of the research hypotheses. These questions usually are: what is necessary to change? what type of change is required? or what resistances are appearing?

In the fifth step, actions are implemented and in the sixth phase (evaluation) the effects of the actions are measured and participant discuss about the desired and nondesired consequences of the implemented actions. These phases are cyclical, and they will be repeated considering the new generated knowledge indefinitely and independently if actions are successful or not.

The final phase is employed to finish the experiments when participants are satisfied with the obtained results and generated changes.

Finally, in order to report an experiment performed according to the action-research method seven points must be described in order to completely characterize the 
experience: (i) Purpose, (ii) Context, (iii) Information collection methods, (iv) Description of the events, (v) Personal discussion of the researchers, (vi) Final and objective discussion and (vii) Global conclusions.

\subsubsection{Survey research}

The survey research methodology consists of selecting a population sample, representative and enough large, collect information from people by means of surveys and process information using statistical procedures to extract knowledge about a particular area. The use of surveys implies that if necessary to contact with people personally and create a collection of questions about them to obtain information about the behavior being studied.

There are three different approaches to perform a survey research (Forza, 2010):

- Exploratory: The objective of this type of surveys is to reach a better understanding about reality, especially during the first steps of an investigation. Exploratory surveys are employed to find which concepts or variables are interesting to be analyzed in detail.

- Confirmatory: These surveys are employed to prove the validity of theories or hypotheses. This approach is useful when there is a clear framework for the research or when the interest variables are already clearly identified.

- Descriptive: The objective of this surveys $s$ to obtain information to describe a certain phenomenon in the study population.

Six different phases are identified in an experiment based on survey research. First, surveys are related to the hypotheses or theoretical framework (depending on the case), later it is designed the experiment through surveys and (third) it is developed a pilot survey. If this survey is not satisfactory, the second step is repeated in cycles. Once the pilot survey is adequate, in the fourth step, it is employed to collect data. Later, and finally, data are analyzed. Eventually (in the sixth step) results are published and reported.

In survey research, it is assumed there is a previously defined set of hypotheses to be validated, together with the context where these theories should be proved. If necessary this framework may be described by means of a diagram.

Once hypotheses have been stablished, it is necessary to create a practical formulation of the previous framework. Numerical measures and empiric realities have to be introduced in order to turn the proposed statements evaluable. This step is the first phase of the survey research.

The designing of the experiment includes all tasks focused on enabling a coherent a relevant data collection: the selection of the population sample, the sampling method, the survey creation process, etc. In this phase, four types of errors may be introduced, but should be minimized (Malhorta et al., 1998). The sampling error is produced by the use of non-representative populations to create general or global results. The 
measurement error is due to divergences between the behavior to be evaluated and the selected scales to inquiry people about it in the surveys. Other factors that can cause this error are a bad writing or confusing instructions. The error known as "erroneous statistical conclusion" is due to the exiting probability to accept false hypotheses when introducing statistical instruments. Finally, the internal validity error is due to errors in the definition of the first theoretical framework or hypotheses.

In order to minimize the sampling error, the population to perform the survey research must be selected following a systematic procedure. Two variables must be carefully controlled: the size of the population (number of people that participate in the research) and the randomness of this population. In general, as the size of population increases the relevance of the obtained results goes up. On the other hand, basically, there are two methods to define the study population, probabilistic and nonprobabilistic.

Probabilistic sampling is the most adequate, as generates a population which guarantees to be representative of the general world. These methods, besides, allow generalization the obtained results. The simple random sampling, the systematic sampling or the stratified sampling (where the general word is divided into smaller sub-systems) are examples of this approach. However, in this case, it must be known the statistical composition of the considered original general world.

If secondary factors such as the cost or the required time must be considered, then, non-probabilistic sampling methods could be employed (such as the intentional sampling), although obtained results cannot be directly generalized, as the sampling error would be great.

In order to control the measurement error, one of the things to be controlled is the data acquisition. Survey research may be composed of personal interviews, telephonic questions or questionnaires by mail (both traditional mail and email) among other possibilities. In personal interviews the cost is higher, but it is possible to create a structure dialog between the participant and the researchers, so a higher amount of valuable information is usually obtained. On the other hand, the use of modern computer instruments and questionnaires by email allows automating the process of data acquisition and analysis.

Nevertheless, other factors must be considered in order to control de measurement error. In particular, the writing of the surveys is very important. It is important to guarantee that the language and words employed in the surveys are adequate for the academic level of participants. If it is ensured that participants totally understand the questions, obtained results will be useless. Furthermore, questions may be open or close. Open questions enable participants to answer questions freely, but close questions make easier to process the acquired data. However, it is important to guarantee that options in close questions are exhaustive and exclusive, to avoid doubts in participants. Finally, if options are based on scales, it is very important to select the most appropriate scale for the phenomenon to be observed. Nominal scales show 
clearly differences between participants. Ordinal scales represent order and intervals distances. Finally ratios allow analyzing relative distances.

Three last activities must be considered when defining a survey research.

First, the development of the pilot survey (Forza, 2010), in order to ensure its adequacy, should be based on three iterative cycles. These cycles must include three different groups of reviewers: academic experts, industrial experts and some participants. Academic experts should confirm the validity of the designed experiment. Industrial experts should adapt the writing to the application scenarios; and participants will correct the potential mistakes in the responses. In general, some aspects to be carefully reviewed are the clarity of instructions, the clarity of questions, the adequacy of questions and the adequacy of scales (among other issues).

Second, survey researches are very sensitive to the lack of responses or participants. If the rate of responses is very low the credibility and reliability of the proposed investigation decreases (Frohlich, 2002). Thus, techniques to increase the response rate must be deployed. For example, sending periodic reminders or trying to stablish a personal communication. If some surveys are not totally answered, the data omitted could be estimated (as surveys are usually redundant), or the survey rejected.

Finally, data analysis and the validation of hypotheses must be based on statistical mechanisms. There are two types of validation techniques: parametric and nonparametric. Parametric methods include the Pearson's correlation, Students's T distribution or ANOVA (ANalysis Of VAriance). Non-parametric methods include the Pearson's $\chi^{2}$ function and the Kolmogorov-Smirnov evaluation.

\subsubsection{Quantitative models}

"Quantitative models" is an experimental methodology with an analytic character, where reality is represented by means of ideal functions in order to predict or explain the phenomena being observed (Ackoff \& Sasieni, 1968). Employed functions may produce results with a high precision, but also through calculations much simpler and more controllable than reality.

The main advantage of quantitative models is their simplicity. If the complexity of models were as high as reality, no benefit will be obtained. Despite this simplicity, models might produce results with a high precision. The reason is that many an unknown variable configures reality, but only a set of them are responsible of controlling the most part of the phenomena being study. The key, thus, is to locate and identify these variables.

First models were based on unidimensional o two-dimensional functions, very simple, employed to show and experiment with the most important effects in a certain phenomenon. These functions, besides, should be derivable, to make the model mathematically adequate. These models are known as "ideal problems". A se con generation of models was based on statistical techniques to predict the near future by means of the analysis of the recent historical data; although these predictive models 
were unable to predict with a high precision the medium or far future. Currently, complex programs and simulators have embedded new models where several variables are considered, and whose values are based on the experience or measures.

Depending on the objective, two different types of models may be found: normative and descriptive. In normative model, it is introduced the desired results, some restrictions to the solutions, and the conditions, actions or situations that have to generated to obtain those results under the proposed restrictions are then calculated. On the other hand, descriptive models operate in reverse. It is introduced the initial situations or actions, the restriction imposed by the environment, and then the expected results for these configurations are calculated.

Besides, depending on the idealization level employed to create models, three different scientific approaches are distinguished: iconic models, analog models and symbolic models.

Iconic models represent the same proprieties of objects being studied, but usually with a change in the magnitudes or scales. Thus, iconic models can be understood as pretty deformed pictures of the reality. Iconic models tend to be very specific and difficult to manipulate during experiments.

Analog models employ some properties of the model to represents an equivalent set of properties of the object in reality, as both present the same mathematical expression. Nowadays, these models are totally mathematical (numerical) and usually are introduced in computing systems in the form of simulators. These models can be manipulated in an easier way, although they are more generalist.

Finally, symbolic models employ numbers and letter to represent equations and relations among phenomena in the real world. Mathematical relations in these models represent reality. These models are difficult to construct but are very easy to manipulate.

Iconic and analog models are usually employed in the first phases of research, and (as more information is available) symbolic models are constructed and used. Nevertheless, sometimes, the impossibility of measuring a certain variable or the bad quality of obtained data (among other cases) make necessary to employ a certain model or to reduce the precision (number of considered variables).

Research based on quantitative models consists of finding the solutions of the proposed models for a certain input configuration and using some mathematical or numerical methods (Churchman, 1971). Using the obtained results, actions on reality can be taken, although it is important to discuss about the validity and coherence of the obtained results. In general, total confidence in results obtained by models should not be maintained. If required, the initial model should be modified according to control laws, if relevant divergences between reality and predicted behavior by models are observed. 


\subsubsection{Agile development research}

Agile methodology is an approach which helps researchers to work under unpredictability through incremental, iterative work cadences, usually named as "sprints". Traditional methodologies assume that the objective, hypotheses, methods, variables to be considered, etc. can be identified before the investigation starts. However, research that implies the development of new solutions, components, algorithms, etc., such as engineering or technological investigations, deals with a huge amount of uncertainty in terms of requirement and results. It might be the case that hypotheses are not validated because of a wrong design or solution development, but with the appropriate technology, hypothesis become true.

Agile methodology provides the opportunity to assess the direction of a research through the definition of a lifecycle. This type of research does it through the creation of an interactive cycle, including a partial hypothesis validation followed by an assessment by the researchers and users until all them are satisfied with the results. In that way, by employing repeated and abbreviated research cycles, new functionalities and components can reach their optimal design in order to validate the proposed hypotheses.

Agile methodologies should not be confused with spiral approaches, where the entire research methodology is designed since the beginning. Using agile methods, it is possible to define a partial experiment considering the available information at each moment, and leave for the next cycle design decisions which cannot be taken with the available information.

Usually up to eleven different phases are identified in agile development research.

- Define the initial variables, hypotheses and objectives: The method starts with the definition of some relevant parameters. It is not necessary to define all elements at first, but some information (at least approximate) is necessary to perform the initial cycle.

- Prepare the study cases: Based on the previous information, the most relevant cases where the new functionalities have to be evaluated in order to prove the validity of hypotheses are selected.

- Prepare the global plan: Considering the selected cases, it is defined a global plan considering the cycles are going to be performed, their duration in time, the experiments they include, etc.

- Begin iteration: The research team start designing the new components, functionalities and elements to be built in this cycle.

- Build the new components, functionalities, etc.: New technologies to be employed in the experiments, which are essential and condition the hypothesis validation, are developed considering the planned designs. 
- Partial experiment: An experiment designed to evaluate the performance of the proposed new functionalities, components, etc. in their current state; and the hypothesis validity is developed.

- Evaluation: Considering the results obtained during experiments, it is evaluated the validity of hypotheses and the performance of the proposed components.

- Are results satisfactory?: This step implies a decision about if the experimental validations must continue, then new developments and experiments are required, or if the research is closed.

- Refine study cases, designs, variables, hypotheses and objectives: If new experiments are required, input values are redefined in order to increase the precision of experiments, developments and results in the next cycle.

- Final experiment and evaluation: When results, components and experiments have been concluded to be satisfactory, a final experiment and evaluation is performed.

- Final results and components or functionalities: From the final experiment it is collected information to construct the final result report and conclude the development of the new components, functionalities and technologies.

Agile development researches may be created ad hoc from existing agile project management methodologies. Depending on the specific discipline within engineering which is investigated, eXtreme, Scrum, Agile Modeling, Lean or FDD (Feature Driven Development) methodologies (among other possibilities) may be employed.

Using an agile development research in engineering investigations presents various advantages. Mainly, the quality of the obtained results and the strength of the validated hypotheses increase. Also, the cost (in time, money, etc.) of the investigation decreases. On the contrary, it is difficult to plan the end of an experiment because of the smaller temporal horizon. Besides, and finally, it is difficult to document the experimental process.

\subsection{Scientific methodology in the PhD Thesis work}

Traditional methodologies, as previously said, are specifically designed to be useful for positivist sciences. However, the proposed investigation in this $\mathrm{PhD}$ Thesis work is classified as technological, so standard methodologies must be adapted considering some engineering aspects such as the relation with the final users, the requirement capture from domain experts, etc. In this section the particular methodology designed and selected for this PhD Thesis work is presented.

\subsubsection{General steps in the proposed research methodology}

As in this PhD Thesis work it is developed an experimental research, in this work it is followed a baconian hypothetico-deductive method. This methodology includes six different steps: (i) observation, (ii) induction, (iii) hypotheses, (iv) experimentation, (v) demonstration, and (vi) theory. 
Observation consists of studying the different manifestations of a same phenomenon, as it occurs in nature. During induction, common characteristics are extracted in order to generalize the observations and define the hypotheses (the third step). Experimentation, demonstration and theory is the core of the scientific method where hypotheses are proved to be true or not.

However, in this particular case, this general approach has being adapted to engineering research by combining it with agile methodology principles. In that way, the previously described steps are linked to create fast and continuous feedback cycles. Nevertheless, in order to improve the quality of the result descriptions, it is presented the experimental validation in an aggregated manner and following the traditional scheme. First they are described the hypotheses, and later they are performed the corresponding experiments and provided the required results to support the final demonstrations.

Results of the first step (Observation) are described in Section 1.1 and Section 1.2. Results of second step (Induction) are described in Section 1.2 and Section 1.3; and hypotheses (third step) are described in Section 1.4. Experimentation, demonstration and theory are developed in papers contained in Section 3.

Observation has been performed through the reading of the previous research works in the area of Cyber-Physical Systems. As proves about the humanization and flexibility in the process execution when using Cyber-Physical Systems are systematically reported, the proposed hypotheses stablish that these characteristics may be general to all future systems, scenarios and applications. Results of this phase will be described with details in a methodological and ordered manner in the paper "Cyber-physical systems: Extending pervasive sensing from control theory to the Internet of Things" by Bordel et al., contained in Section 3.2. This paper, in that way, fulfills Objective $\# \mathbf{1}$ of this PhD Thesis work.

The induction process (Section 1.2 and Section 1.3) is straightforward since, as previously described, proves of the humanization and flexibility during the process execution in systems based on CPS are systematically reported by researchers. Thus, it is evident to induct that these characteristics may be general and common to all future scenarios, independently from the application scenario or the inclusion of people (as active agents) or devices. The referred paper, in Section 3.2, also includes arguments about this induction process and cites that supported the proposed observations and ideas.

The third phase of this method (the definition of hypotheses) has been also described in Section 1.4, considering the observations and the performed induction, also briefly presented in Section 1.2 and Section 1.3.

The core of this PhD Thesis work is focused, therefore, on the experimentation and demonstration of proposed hypotheses; a process which must enable us, moreover, to fulfill the research objectives. 


\subsubsection{Experimental methodology: relevant cases}

As can be seen in Hyphotesis\#1 (Section 1.4), the objective is to prove that the humanization and flexibility in process execution systems based on humanized CPS is common to all scenarios, all process types and independently from if tasks are finally executed by people or devices. Moreover, Hyphotesis \#2 and Hyphotesis\#3 also refers that the relation between the variable they theorize (between communication systems and humanization and flexibility; and security and humanization and flexibility respectively) is independent from the applications scenario, the composition of the physical platform, etc. In that way, only considering the writing of these hypotheses it is clear the most adequate experimental methodology to validate them is the case study.

In fact, Hyphotesis\# $\mathbf{1}$ aims to discover how the use of Cyber-Physical Systems affects the flexibility and humanization in process execution systems. Hyphotesis 2 looks for the impact of communication networks and models in the flexibility and humanization of CPS. And, finally, Hyphotesis \#3 aims to evaluate how the use and deployment of security mechanism can modify the flexibility and humanization of process execution systems. This fact, together with the fact that the proposed approach is very innovative and no research on this specific topic has been reported (as well as the fact that researcher have no control on the system behavior once operating), finally determines the case study methodology as the most appropriate experimental method in this case. This methodology, as an engineering research is conducted, is combined with agile method principles in order to reach the optimum technological designs that satisfy the proposed research objectives (see Section 1.3), and can be employed to verify the initial hypotheses.

This $\mathrm{PhD}$ Thesis work is based on a multiple-case approach, considering those scenarios and cases which are most representative of the fourth industrial revolution applications and other future engineered systems where Cyber-Physical systems will be employed. In particular, we have selected the following cases to be studied:

- Production systems and assembling lines

- Manufacturing companies

- Industry 4.0

- IoT systems and Smart Cities

- Smart Environments

- Smart agriculture

- Ambient Intelligence deployments

For each one of these cases, a holistic or embedded view will be employed, depending on the variables and behaviors to be studied in each case. In some of this cases, however, it is not employed a traditional case study methodology, but this method will be mixed with other methodologies; so the finally followed method is hybrid. 
In particular, in those experiments, as (for example) in cases related to Industry 4.0, where people act as active agents providing services an action-research methodology is employed (being researchers also participants). In that way, the increase in the workers wellness and the industry humanization, due to the use of CPS, will be evaluated in a more appropriate way. The paper "Assessment of human motivation through analysis of physiological and emotional signals in Industry 4.0 scenarios" by Bordel et al. (Section 3.6) reports an experiment following this approach.

In the same manner, in those cases were humans are only considered as passive entities (being sensed together with the physical world), a survey research is selected to evaluate the user experience or the increase in the perceived wellness. See paper "Selfconfiguration in humanized cyber-physical systems" by Bordel et al. (Section 3.5).

Finally, those scenarios where elements that belong to the future or that is impossible to implement nowadays are involved; and then it is impossible to design a controllable experiment, it is employed the quantitative models method. These models are implemented in simulators, so first evidences about the validity of the proposed hypotheses might be obtained.

Thus, the following cases and studies compose the proposed methodology for this PhD Thesis work.

Cases related to scenarios about production systems and assembling lines and manufacturing companies will be employed to perform holistic studies. The objective, then, is evaluating the humanization and flexibility in the process execution when using Cyber-Physical Systems. Hypothesis\#1 is thus evaluated and Objetive\#1, Objetive $\# 2$ and Objetive $\# 3$ addressed.

Cases based on IoT systems and Smart Cities, as they are mature technologies in many senses and there is a wide knowledge about them, will be employed to perform experiments based on quantitative models. Using the existing knowledge, it is possible to construct models with which evaluate the most innovative technical proposals. These proposals include the communication models and architectures referred in Objective\#10, which are employed to verify Hypothesis\#2. Moreover, new and genuine security solutions will be validated using quantitative models in the context of IoT system, being able to verify Hypothesis \#3 and fulfilling Objective\#11.

Within cases focused on both Smart Environments and Smart Agriculture, embedded studies are performed to partially evaluate the flexibility and humanization due to some particular functional components, algorithms or procedures. Proposals such as the self-configuration and self-adaptation mechanisms to automatically define heterogeneous services in Cyber-Physical systems (Objective\#5 and Objective\#6) or the self-management protocols to execute tasks in a choreographed manner (Objective $\#$ 9) are validated using these cases.

Finally, scenarios related to Ambient Intelligence and Industry 4.0 are employed to perform embedded studies where people represent an important element. In cases 
based on AmI deployments people will be considered passive agents to be sensed and, as said, proposals related to human monitoring will be validated. On the other hand, in cases based on Industry 4.0, humans are considered active agents and proposals related to activity recognition techniques and human integration in CPS will be validated. Objective\#7 is addressed with the first group of cases and Objective\#8 is addressed with the second group.

With the proposed experiments, it is measured, overall, the internal validity of the defined hypotheses. i.e. it is evaluated that, in fact, the improvement in the humanization and flexibility levels during the processes execution is caused by the use of Cyber-Physical Systems and not by other unknown and uncontrolled variables.

External validity of the proposed experiment, i.e. the possibility of results to be generalized, is guaranteed by a good construction of the case study methodology, as well as a good statistical configuration of the action-research, survey research and qualitative models methodologies.

\subsubsection{Particular phases in the proposed methodology}

As some of the proposed cases imply the design, construction and validation of new and innovative systems and solutions, the baconian scientific methodology must be adapted to consider these activities by combing the traditional baconian method with principles from agile methodologies. As a result, the specific scientific methodology ad hoc designed and employed in this PhD Thesis work is organized into four different phases.

The first phase (Phase\#1) includes the analysis of the state-of-the-art, the obtaining of all necessary information to define the initial hypotheses and to perform the first three steps in the baconian scientific method and the definition of the relevant cases for the case study methodology. Particular experimental methodologies for each case will be also developed in this phase. Objetive $\$ \mathbf{1}$ and Objetive $\$ \mathbf{2}$ will be addressed during this phase. "Cyber-physical systems: Extending pervasive sensing from control theory to the Internet of Things" by Bordel et al., contained in Section 3.2 describes the most part of the obtained results during this phase.

The second phase (Phase\#2) includes all technical works focused on the design and implementation of those cyber-physical components that do not exist nowadays; so its construction is required to carry out the proposed experiment in each relevant case. All objectives related to the creation, definition or implementation of algorithms, protocols, systems or solutions are addressed during this phase.

The third phase (Phase\#3) includes those experiments and studies within the case study methodology that are embedded. i.e. in this phase are carried out all partial validations in limited and controllable scenarios where only some components are considered, not the entire and global system. To this phase belong all partial evaluation performed through the action-research, survey research or quantitative models and 
methodologies. During this phase Hypothesis\#2 and Hypothesis\#3 are validated; and Objective \#10 and Objective\#11 addressed.

The fourth and final phase (Phase\#4) follows a totally holistic approach. During this phase, global scenarios will be deployed and cases including all components (already validated during the previous phase) will be studied. If required, people will be considered, and the global humanization and flexibility obtained during process execution due to the use of Cyber-Physical Systems is evaluated. During this phase Hypothesis $\mathbb{1}$ is validated.

The report of results to the scientific world takes place during the third and fourth phase, although some works may be published during the second phase, as first experimental results are obtained.

\subsection{Planned research tasks}

Considering the described methodology, the proposed phases and the research objectives described in Section 1, in this section it is proposed a collection of the most important research task developed during this $\mathrm{PhD}$ Thesis work which make up the proposed methodology.

Task\#1: Observation and analysis of the state-of-the-art. In this task the different ideas about CPS that have been reported nowadays are analyzed in order to extract some common characteristics, propose relevant applications, identify the stakeholders, select the research objectives, questions and challenges, etc.

Task\#2: Identification of relevant scenarios and hypotheses. This task includes discussions to selects, among all research opportunities related to humanized CyberPhysical Systems, the topics that are being addressed in this PhD Thesis work. As result, the initial hypotheses of this investigation are stablished.

Task\#3: Definition of architectures for CPS and process types to be considered. As hypotheses refer the independency of the results from the application scenario and the process type being executed, this task aims to identify the processes to be considered. On the other hand, with this information must be proposed an architecture for humanized CPS fulfilling those requirements.

Task\#4: Develop process models and flexible and humanized process execution algorithms for humanized CPS. Solutions, procedures, models and algorithms to enable the humanized and flexible execution of processes in humanized CyberPhysical Systems with a service-oriented configuration are developed in this task.

Task\#5: Develop the service self-configuration mechanisms and procedures. Mechanism to self-configure and maintain in an automatic way a catalogue or heterogonous services based on the coordination of devise and people with very different capabilities are developed in this task.

Task\#6: Create instruments to monitor people and integrate humans into CPS. This task contains all works focused on construct the monitoring solutions and pattern 
recognition techniques that enable people to be integrated into CPS as both, passive agents and service providers.

Task\#7: Implement self-management solutions to devices in CPS. Different solutions to allow devices to self-manage their resources and coordinate with other devices to execute activities in a choreographed manner with other components are investigated in this task.

Task\#8: Develop innovative communication models and architectures for CPS. This task includes all research initiative focused on defining and developing new communication models and network architectures, adequate to support the operations of humanized CPS.

Task\#9: Define and implement new security solutions for humanized CPS. Research works related to the creation of new security solutions to protect humanized CPS from the present and future attacks, unauthorized accessed and potential failures are addressed in this task.

Task\#10: Implement initial prototypes of components in humanized CPS. Considering the results of all previous tasks, during this activity, new components (hardware and software) as initial prototypes are constructed. These components are based on the created and defined mechanisms, protocols, models, etc. during task from task\#4 to task\#8.

Task\#11: Deployment and validation of initial prototypes in embedded cases. Considering the relevant cases identified in Task\#2, in this task developed prototypes are deployed in the interest scenarios and embedded experiments are performed according to the defined methodologies in Task\#1 and Task \#2.

Task\#12: Collaboration with international experts. During, at least, one term it is developed a research visitation to a prestigious international research institution to discuss about the obtained results, include new technologies or scenario not considered and enrich the final activities of this PhD Thesis work.

Task\#13: Integration. In order to enable the performance of more complex experiments with a holistic view, which enable the validation of the proposed hypotheses, during this task defined prototypes, components and technologies are integrated into a unique humanized CPS.

Task\#14: Deployment of the entire humanized CPS. The integrated system created during the previous task is deployed in some relevant scenarios; in order to study ate least two critical cases with a holistic approach.

Task\#15: Validation of the proposed humanized CPS with a holistic view. Considering the relevant cases identified in Task\#2, discussion developed in Task\#11, results of Task\#10 and system deployed in Task\#13, in this task holistic experiments are performed according to the defined methodologies in Task $\# \mathbf{1}$ and Task\#2. 
Task\#16: Report the obtained results to the scientific community. This task contains all works and efforts focused on publishing the obtained results and report the performed experiments to the scientific community. If adequate, some design may be protected though patents or other proper mechanisms.

Tasks from Task\#1 to Task\#3 are performed within the first phase (Phase $\# \mathbf{1}$ ) of the proposed methodology. Objective\#1 and Objective $\# \mathbf{2}$ are also addressed. Tasks from Task\#4 to Task\#9 are executed during the second phase (Phase\#2), and (basically) research objectives from Objective\#3 to Objective\#11 are fulfilled. Tasks from Task\#10 to Task\#12 belong to the third phase, when Objective\#12 is covered. Finally, tasks from Task\#13 to Task\#15 are included in the fourth phase and Objective\#12 is addressed and Hypothesis $\# \mathbf{1}$ validated.

Task\#16 is distributed in time, as explained previously, and covers Objective\#12.

Figure 2 shows graphically the entire structure of the proposed research and methodology.

\subsection{Schedule}

Considering the described methodology and the planned research activities, the total required time to develop this $\mathrm{PhD}$ Thesis work is four years.

Although tasks and phases are overlapped, in general words, during the first six months it is developed the first phase of the proposed methodology. During the next eighteen months phase two is carry out, prototypes are developed and technical works performed. Within the next eighteen months tasks related to the third phase are executed. Finally the last six months are destined to execute the fourth phase in the methodology.

Figure 3 shows the proposed schedule (or chronogram). 


\begin{tabular}{|c|c|c|c|c|c|c|c|c|c|c|c|c|}
\hline \multirow[b]{2}{*}{ Task } & \multicolumn{12}{|c|}{ Objectives } \\
\hline & Objective\#1 & Objective\#2 & Objective\#3 & Objective\#4 & Objective\#5 & Objective\#6 & Objective\#7 & Objective\#8 & Objective\#9 & Objective\#10 & Objective\#11 & Objective\#12 \\
\hline \multicolumn{13}{|l|}{ Task\#1 } \\
\hline \multicolumn{13}{|l|}{ Task\#2 } \\
\hline \multicolumn{13}{|l|}{ Task\#3 } \\
\hline \multicolumn{13}{|l|}{ Task\#4 } \\
\hline \multicolumn{13}{|l|}{ Task\#5 } \\
\hline \multicolumn{13}{|l|}{ Task\#6 } \\
\hline \multicolumn{13}{|l|}{ Task\#7 } \\
\hline \multicolumn{13}{|l|}{ Task\#8 } \\
\hline \multicolumn{13}{|l|}{ Task\#9 } \\
\hline \multicolumn{13}{|l|}{ Task\#10 } \\
\hline \multicolumn{13}{|l|}{ Task\#11 } \\
\hline \multicolumn{13}{|l|}{ Task\#12 } \\
\hline \multicolumn{13}{|l|}{ Task\#13 } \\
\hline \multicolumn{13}{|l|}{ Task\#14 } \\
\hline \multicolumn{13}{|l|}{ Task\#15 } \\
\hline Task\#16 & & & & & & & & & & & & \\
\hline
\end{tabular}




\begin{tabular}{|c|c|c|c|c|c|c|c|c|}
\hline \multirow{2}{*}{ Task } & \multicolumn{2}{|c|}{ Year 1} & \multicolumn{2}{|c|}{ Year 2} & \multicolumn{2}{|c|}{ Year 3} & \multicolumn{2}{|c|}{ Year 4} \\
\hline & Semester 1 & Semester 2 & Semester 1 & Semester 2 & Semester 1 & Semester 2 & Semester 1 & Semester 2 \\
\hline \multicolumn{9}{|l|}{ Task\#1 } \\
\hline \multicolumn{9}{|l|}{ Task\#2 } \\
\hline \multicolumn{9}{|l|}{ Task\#3 } \\
\hline \multicolumn{9}{|l|}{ Task\#4 } \\
\hline \multicolumn{9}{|l|}{ Task\#5 } \\
\hline \multicolumn{9}{|l|}{ Task\#6 } \\
\hline \multicolumn{9}{|l|}{ Task\#7 } \\
\hline \multicolumn{9}{|l|}{ Task\#8 } \\
\hline \multicolumn{9}{|l|}{ Task\#9 } \\
\hline \multicolumn{9}{|l|}{ Task\#10 } \\
\hline \multicolumn{9}{|l|}{ Task\#11 } \\
\hline \multicolumn{9}{|l|}{ Task\#12 } \\
\hline \multicolumn{9}{|l|}{ Task\#13 } \\
\hline \multicolumn{9}{|l|}{ Task\#14 } \\
\hline \multicolumn{9}{|l|}{ Task\#15 } \\
\hline Task\#16 & & & & & & & & \\
\hline
\end{tabular}

Figure 3. Proposed chronogram 


\section{Publications}

This section presents a collection of selected publications where the most relevant results related to this $\mathrm{PhD}$ Thesis work are reported to the scientific community. Papers are organized in eight thematic sections, according to the research objectives that are addressed in each paper. Moreover, the first subsection describes the general organization of the proposed collection, and the manner in which the included papers address the proposed research objectives and hypotheses. The relation between the selected papers the research methodology and research tasks is also described.

For each paper, it is included the final manuscript accepted for publication. Before the manuscript, it is also included an extended summary including all information about the publishing journal or conference.

In the next section, it is provided a general and global description of the proposed solutions, a detailed discussion and analysis of the obtained results and some relevant conclusions and ideas for future works.

\subsection{Collection organization}

The proposed collection is organized in eight thematic units or subsections.

In the first subsection "Analysis and characterization of CPS" (Section 3.2) the paradigm of Cyber-Physical Systems is analyzed in detail. From the analysis of components that may be part of a Cyber-Physical system, it is identified the different types of CPS that have been reported in the scientific literature, including the different definitions and architectures that have been proposed for each type. The most important research lines are reviewed, together with the main reported results, so a set of observations and common characteristic can be obtained to start the PhD Thesis work. In particular, the process types that might be executed in Cyber-Physical systems are analyzed, as well as the roles people can take in the context in these systems, with a special attention to works where humans act as service providers. Papers in this first thematic unit correspond with the results of Task\#1 and Task\#2. In that way, results in this subsection address Objective\#1. 
In the second subsection "Proposed architectures: reference architecture, functional architecture and implementation architecture" (Section 3.3), it is defined the idea of Humanized Cyber-Physical systems, taken as input the existing definition, especially architectures proposed by standardization organizations. The motivation of this second subsection is to generate an architecture applicable to all types of industrial scenarios (or of any other nature), in the context of the fourth industrial revolution, and including those where people intervene actively providing different services. Architectures should be able to execute, in a flexible manner, processes identified in the works presented in Section 3.2. In this subsection, it is described the design of a functional and an implementation architecture, so Objective $\# \mathbf{2}$ is covered.

Works includes in this section also describe a detailed models for humanized CyberPhysical Systems that enable the systematic description and simulation of these systems. Thus, Objective \#3 is addressed. Results included in this second thematic subsection are obtained during the execution of Task $\# 3$.

The third thematic subsection (Section 3.4, "Process management in Humanized Cyber-Physical Systems") is focused on process management in the context of humanized Cyber-Physical Systems. Considering the process types identified in the previous subsection, which mainly distinguish between processes defined at high-level and processes managed at low-level (a group within processes executed by people, devices, explicitly notified, etc. may be found), different implementations for the proposed general architectures in the previous section are proposed. It is identified the process model to be employed in each case, as well as the components to be included and elements to be configured to be able to execute processes in a flexible manner in each scenario. Finally, a detailed composition and transformation procedure, for processes to be executed in Cyber-Physical systems is described. Algorithms for the different levels in the process execution systems and mechanism to enable an humanized and flexible process management and execution are also reported. Works presented in this section address Objective $\# 4$ and validate most cases for Hypothesis\#1. Results obtained from Task \#4 and Task\#15 are reported in this third subsection.

In the fourth thematic unit (Section 3.5, "Service self-configuration and selfadaptation in Humanized Cyber-Physical Systems") it is described the selfconfiguration and dynamic self-adaptation procedures that allow generating and maintaining updated the service collection which are employed to support the execution of processes in humanized CPS. It is also described mechanism and algorithms to enable the composition of heterogeneous services by means of the coordination of entities (both people and devices) with very different behaviors and capabilities. In this thematic unit are also included algorithms that allow people to provide services in the context of humanized CPS, probably with a certain Quality of Service (QoS). In the same way, mechanisms for the self-discovery of hardware capacities as well as procedures to coordinate people and hardware elements are 
included. Objetive $\$ \mathbf{5}$ and Objective $\# \mathbf{6}$ are addressed in this subsection, which is the result of Task\#5 and Task\#11.

In the fifth thematic unit (Section 3.6, "Humanized processes. Human integration in Cyber-Physical Systems"), the required mechanisms to integrate people into CyberPhysical systems are addressed. The objective is to allow people to maintain a good wellness state, so they offer the maximum productivity in the provision of services which are executed in the system. In this subsection, Objective\#7 and Objective\#8 are covered, so mechanisms and algorithms to monitor the state of people and calculate the QoS of human provided services are investigated. Besides, pattern recognition and activity recognition techniques are developed to infer activities being performed by people and follow and control the processes executed by humans. Results of Task \#6 and Task\#11 are reported in the papers of this subsection.

The sixth thematic subsection (Section 3.7, "Device management in Humanized Cyber-Physical Systems") is focused on the device management. Techniques to manage devices in Cyber-Physical systems and their lifecycle (both predictive and reactive) are also investigated; so hardware elements can perform a flexible execution of processes depending on the dynamic situation of the physical platform. Besides, activities that cannot be executed in the underlying local platform could be resolved using virtual entities or cloud services. It is also proposed a hardware-supported algorithm that enables devices to self-manage and self-organize to take advantage of their resources and execute low-level activities in an autonomous and choreographed way. Works in this subsection address Objective $\$ \mathbf{9}$ and are develop using results of Task $\# \mathbf{7}$ and Task\#11.

The seventh subsection (Section 3.8, "Communication models and networks in Humanized Cyber-Physical Systems") is dedicated to evaluate the communication systems that support communications in humanized Cyber-Physical Systems, both among devices in a CPS and among different networked humanized CPS. In particular, in this section it is proposed a (wireless) communication channel model to be evaluated in different relevant scenarios related to future industry and similar applications. Moreover, a network architecture for future wireless communications based on 5G mobile technologies is also proposed. Objective $\# \mathbf{1 0}$ is covered by papers in this subsection which are created from results of Task $\# \mathbf{8}$ and Task\#11.

Finally, in the eighth and last thematic subsection, "Security in Humanized CyberPhysical Systems" (Section 3.9), aspects related to security in Cyber-Physical systems are evaluated. It is a transversal topic with a critical importance for all researchers, and included in the reference architecture proposed by NIST in 2015. Objective\#11 in this PhD Thesis work is covered by these works. Cryptographic solutions based on the ideas of trust and reputation, as well as intelligent security schemes that allow protecting humanized CPS against traditional and new cyberattacks are proposed. Techniques against unauthorized accesses and other innovative attacks (such as cyberphysical attacks) are also investigated. In particular, cryptographic solutions being able 
to be executed in resource constraint devices, symmetric key schemes and solutions to detect malicious components using the ideas of trust and reputation are constructed. Protocols to distribute keys and sign messages in future scenarios and CPS are also investigated. Results of Task $\# \mathbf{9}$ and Task $\# \mathbf{1 1}$ are employed to prepare the papers in this subsection.

Full-text of the select papers are provided in Section 3.10, and may be downloaded and accessed online using the information provided about each one in the corresponding section. 


\subsection{Analysis and characterization of CPS}

In this subsection they are included those works that satisfy Objective\# 1 focused on analyzing CPS and the process types that may be executed in these systems. In this section are included the following papers:

\begin{tabular}{|c|c|}
\hline Title & $\begin{array}{l}\text { Cyber-physical systems: Extending pervasive sensing from control } \\
\text { theory to the Internet of Things }\end{array}$ \\
\hline Authors & Bordel, B., Alcarria, R., Robles, T., \& Martín, D. \\
\hline Journal & Pervasive and Mobile Computing \\
\hline ISSN & $1574-1192$ \\
\hline $\begin{array}{l}\text { Impact factor } \\
\text { (JCR) }\end{array}$ & 2,349 (Q2) [JCR 2016] \\
\hline Publisher & Elsevier \\
\hline Volume & 40 \\
\hline Pages & $156-184$ \\
\hline Year & 2017 \\
\hline DOI & doi.org/10.1016/j.pmcj.2017.06.011 \\
\hline Online & https://www.sciencedirect.com/science/paper/pii/S1574119217303127 \\
\hline Abstract & $\begin{array}{l}\text { Essentially, the emerging term "Cyber-Physical Systems (CPS)" is an } \\
\text { architectural paradigm in which the pervasive sensing technologies } \\
\text { represent a fundamental part. Originally defined in the computer } \\
\text { sciences domain, the term Cyber-Physical Systems has been adapted } \\
\text { to very different domains such as the control theory or electronic } \\
\text { engineering. Even, some authors understand CPS as a particular } \\
\text { scenario of the Internet of Things (IoT) based on pervasive sensing. } \\
\text { Furthermore, recently, some works propose a definition for CPS } \\
\text { including all the features described in the different domains. In this } \\
\text { paper we provide a comprehensive analysis of the nature and } \\
\text { characteristics of the different proposals, discuss the recent attempts } \\
\text { to standardize CPS, and review the state-of-the-art on CPS for each } \\
\text { technological domain. We compare those different proposals on } \\
\text { CPS, discuss about some related terms and technologies and } \\
\text { conclude by describing the main research challenges in the area. }\end{array}$ \\
\hline
\end{tabular}




\subsection{Proposed architectures: reference architecture, functional architecture and implementation architecture}

This subsection includes Works focused on defining a set of valid architecture for humanized CPS, applicable to all possible future scenarios. Besides a model that allows the design and analysis of these new systems is also described. The following papers are included:

\begin{tabular}{|c|c|}
\hline Title & $\begin{array}{l}\text { Building smart adaptable Cyber-Physical Systems: definitions, } \\
\text { classification and elements }\end{array}$ \\
\hline Authors & $\begin{array}{l}\text { Bordel, B., Alcarria, R., Pérez-Jiménez, M., Robles, T., Martín, D., } \\
\text { \& de Rivera, D. S. }\end{array}$ \\
\hline Conference & $\begin{array}{l}\text { International Conference on Ubiquitous Computing and } \\
\text { Ambient Intelligence (UCAmI) }\end{array}$ \\
\hline CORE ranking & No \\
\hline Proceedings title & $\begin{array}{l}\text { Ubiquitous Computing and Ambient Intelligence. Sensing, } \\
\text { Processing, and Using Environmental Information }\end{array}$ \\
\hline Editors & García-Chamizo J., Fortino G., Ochoa S. \\
\hline Publisher & Springer, Cham \\
\hline ISBN & $978-3-319-26401-1$ \\
\hline Pages & $144-149$ \\
\hline Year & 2015 \\
\hline DOI & doi.org/10.1007/978-3-319-26401-1_14 \\
\hline Online & https://link.springer.com/chapter/10.1007/978-3-319-26401-1 14 \\
\hline Abstract & $\begin{array}{l}\text { The provision of systems that join the information technologies } \\
\text { with the physical world has been one of the most popular issues } \\
\text { in research in the last fifteen years. Nevertheless, the complexity } \\
\text { associated with these systems prevented many authors from } \\
\text { providing a theoretical formalization. Even it is difficult to find a } \\
\text { consensus name or a definition for this new type of systems. } \\
\text { Therefore, in this work we propose a theoretical and technical } \\
\text { formalization for these solutions, which includes a name at the } \\
\text { forefront of research: smart adaptable cyber-physical systems } \\
\text { (SACPS). We also present a complete definition for the SACPS, } \\
\text { and explain the elements and subsystem interaction. }\end{array}$ \\
\hline
\end{tabular}




\begin{tabular}{|c|c|}
\hline Title & $\begin{array}{l}\text { Physical processes control in Industry 4.0-based systems: a focus } \\
\text { on Cyber-Physical Systems }\end{array}$ \\
\hline Authors & Sánchez, B. B., de Rivera, D. S., Sánchez-Picot, Á., \& Robles, T. \\
\hline Conference & $\begin{array}{l}\text { International Conference on Ubiquitous Computing and } \\
\text { Ambient Intelligence (UCAmI) }\end{array}$ \\
\hline CORE ranking & No \\
\hline Proceedings title & $\begin{array}{l}\text { UCAmI 2016, IWAAL 2016, AmIHEALTH 2016: Ubiquitous } \\
\text { Computing and Ambient Intelligence }\end{array}$ \\
\hline Editors & García C., Caballero-Gil P., Burmester M., Quesada-Arencibia A. \\
\hline Publisher & Springer, Cham \\
\hline ISBN & $978-3-319-48799-1$ \\
\hline Pages & $257-262$ \\
\hline Year & 2016 \\
\hline DOI & doi.org/10.1007/978-3-319-48799-1_30 \\
\hline Online & https://link.springer.com/chapter/10.1007/978-3-319-48799-1_30 \\
\hline Abstract & $\begin{array}{l}\text { Industry } 4.0 \text { or cyber-industry may employ Cyber-Physical } \\
\text { Systems (CPS) profusely to organize production media in a new } \\
\text { and more efficient way. Many processes might be defined in CPS } \\
\text { and Industry } 4.0 \text {, however, physical processes are probably the } \\
\text { least studied. Therefore, in this paper we propose an architecture } \\
\text { for Industry } 4.0 \text {-based systems focused on the control of physical } \\
\text { processes. The architecture follows the CPS paradigm. } \\
\text { Moreover, a first evaluation of the performance of the proposed } \\
\text { solution, using simulation tools, is provided. The results proved } \\
\text { the proposed architecture is a valid solution for physical control } \\
\text { processes. }\end{array}$ \\
\hline
\end{tabular}




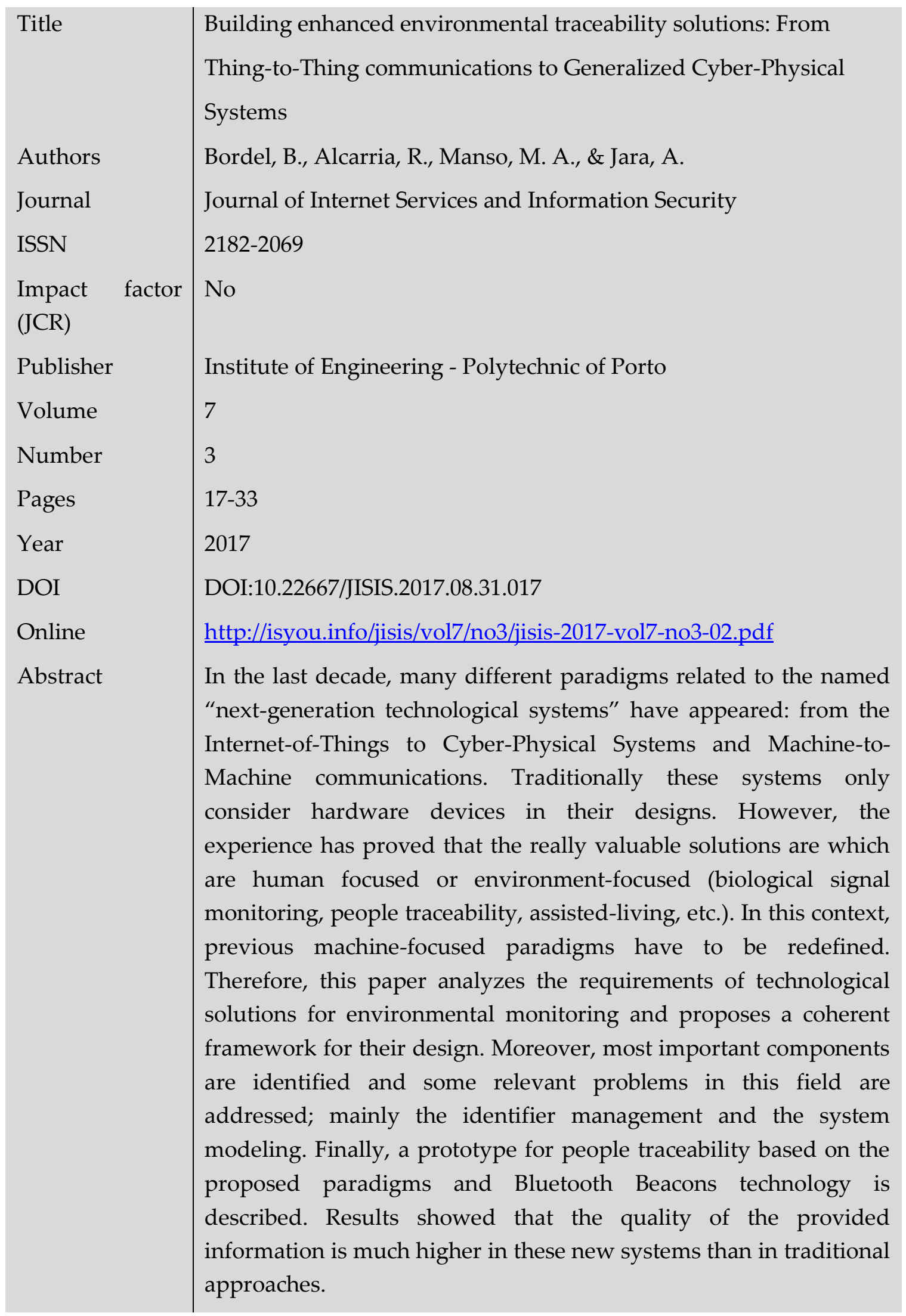




\subsection{Process management in Humanized Cyber-Physical Systems}

In this subsection the mechanisms, algorithms and specific functional architectures employed to execute processes in the context of humanized CPS are described. These processes will be executed in a flexible manner depending on the dynamic situation of the system and the catalog of heterogeneous services that support the execution. The following papers are included:

\begin{tabular}{|c|c|}
\hline Title & $\begin{array}{l}\text { Process execution in Cyber-Physical Systems using cloud and Cyber- } \\
\text { Physical Internet services }\end{array}$ \\
\hline Authors & Bordel, B., Alcarria, R., de Rivera, D. S., \& Robles, T. \\
\hline Journal & The Journal of Supercomputing \\
\hline ISSN & 1573-0484 \\
\hline $\begin{array}{l}\text { Impact factor } \\
\text { (JCR) }\end{array}$ & 1,326 (Q2) [JCR 2016] \\
\hline Publisher & Springer US \\
\hline Pages & $1-43$ \\
\hline Year & 2018 \\
\hline DOI & doi.org/10.1007/s11227-018-2416-4 \\
\hline Online & https://link.springer.com/paper/10.1007/s11227-018-2416-4 \\
\hline Abstract & $\begin{array}{l}\text { "Cyber-Physical Systems" (CPS) have emerged as the next } \\
\text { technological revolution. These new systems are commonly } \\
\text { supported by a collection of ad hoc connected devices which } \\
\text { typically collaborate in order to control some physical processes. } \\
\text { However, in recent years, many other applications based on the CPS } \\
\text { paradigm have been reported. In particular, executing user-defined } \\
\text { processes over a cyber-physical infrastructure is a very promising } \\
\text { technology for the future. Therefore, in this paper, we propose a } \\
\text { scheme which allows the creation of user-defined processes, their } \\
\text { decomposition and translation into executable orders or code, and } \\
\text { their execution using the locally available cyber-physical } \\
\text { infrastructure, cloud services and/or other services offered by remote } \\
\text { CPS through the Cyber-Physical Internet. The proposed solution also } \\
\text { enables the execution of processes with a guaranteed QoS. Moreover, } \\
\text { an experimental validation is provided in order to evaluate the } \\
\text { proposed technology performance. In particular, it is proved that } \\
\text { more than } 95 \% \text { of processes are correctly executed, and only in a } 2 \% \\
\text { of cases the minimum cost execution is not selected. }\end{array}$ \\
\hline
\end{tabular}




\begin{tabular}{|c|c|}
\hline Title & $\begin{array}{l}\text { TF4SM: a framework for developing traceability solutions in small } \\
\text { manufacturing companies }\end{array}$ \\
\hline Authors & Bordel Sánchez, B., Alcarria, R., Martín, D., \& Robles, T. \\
\hline Journal & Sensors \\
\hline ISSN & $1424-8220$ \\
\hline $\begin{array}{l}\text { Impact factor } \\
\text { (JCR) }\end{array}$ & 2,033 (Q1) [JCR 2015] \\
\hline Publisher & MDPI \\
\hline Volume & 15 \\
\hline Number & 11 \\
\hline Pages & 29478-29510 \\
\hline Year & 2015 \\
\hline DOI & doi:10.3390/s151129478 \\
\hline Online & http://www.mdpi.com/1424-8220/15/11/29478/htm \\
\hline Abstract & $\begin{array}{l}\text { Nowadays, manufacturing processes have become highly complex. } \\
\text { Besides, more and more, governmental institutions require } \\
\text { companies to implement systems to trace a product's life (especially } \\
\text { for foods, clinical materials or similar items). In this paper, we } \\
\text { propose a new framework, based on cyber-physical systems, for } \\
\text { developing traceability systems in small manufacturing companies } \\
\text { (which because of their size cannot implement other commercial } \\
\text { products). We propose a general theoretical framework, study the } \\
\text { requirements of these companies in relation to traceability systems, } \\
\text { propose a reference architecture based on both previous elements } \\
\text { and build the first minimum functional prototype, to compare our } \\
\text { solution to a traditional tag-based traceability system. Results show } \\
\text { that our system reduces the number of inefficiencies and reaction } \\
\text { time. }\end{array}$ \\
\hline
\end{tabular}




\begin{tabular}{|c|c|}
\hline Title & $\begin{array}{l}\text { Enhancing Process Control in Industry } 4.0 \text { Scenarios using Cyber- } \\
\text { Physical Systems }\end{array}$ \\
\hline Authors & Sánchez, B. B., Alcarria, R., de Rivera, D. S., \& Sánchez-Picot, Á. \\
\hline Journal & $\begin{array}{l}\text { Journal of Wireless Mobile Networks, Ubiquitous Computing, and } \\
\text { Dependable Applications }\end{array}$ \\
\hline ISSN & 2093-5374 \\
\hline $\begin{array}{l}\text { Impact factor } \\
\text { (SJR) }\end{array}$ & 0,35 (Q2) [SJR 2016] \\
\hline Publisher & Innovative Information Science \& Technology Research Group \\
\hline Volume & 7 \\
\hline Number & 4 \\
\hline Pages & $41-64$ \\
\hline Year & 2016 \\
\hline DOI & DOI:10.22667/JOWUA.2016.12.31.041 \\
\hline Online & http://isyou.info/jowua/papers/jowua-v7n4-3.pdf \\
\hline Abstract & $\begin{array}{l}\text { One of the most interesting applications of Industry } 4.0 \text { paradigm is } \\
\text { enhanced process control. Traditionally, process control solutions } \\
\text { based on Cyber-Physical Systems (CPS) consider a top-down view } \\
\text { where processes are represented as executable high-level } \\
\text { descriptions. However, most times industrial processes follow a } \\
\text { bottom-up model where processes are executed by low-level devices } \\
\text { which are hard-programmed with the process to be executed. Thus, } \\
\text { high-level components only may supervise the process execution as } \\
\text { devices cannot modify dynamically their behavior. Therefore, in this } \\
\text { paper we propose a vertical CPS-based solution (including a } \\
\text { reference and a functional architecture) adequate to perform } \\
\text { enhanced process control in Industry } 4.0 \text { scenarios with a bottom-up } \\
\text { view. The proposed solution employs an event-driven service-based } \\
\text { architecture where control is performed by means of finite state } \\
\text { machines. Furthermore, an experimental validation is provided } \\
\text { proving that in more than } 97 \% \text { of cases the proposed solution allows } \\
\text { a stable and effective control. }\end{array}$ \\
\hline
\end{tabular}




\subsection{Service self-configuration and self-adaptation in Humanized Cyber-Physical Systems}

This section includes works related to solutions and mechanism to allow the selfconfiguration, self-discovery, composition and coordination of capabilities, both hardware and human, with the objective of create a service catalog at all required levels to support the process execution in humanized CPS. The following papers are included:

\begin{tabular}{|c|c|}
\hline Title & Low-Level Service Management in Cyber-Physical Systems \\
\hline Authors & Bordel, B., Alcarria, R., Sánchez-de-Rivera, D., \& Jara, A. \\
\hline Conference & $\begin{array}{l}\text { International Conference on Innovative Mobile and Internet } \\
\text { Services in Ubiquitous Computing }\end{array}$ \\
\hline CORE ranking & No \\
\hline Proceedings title & $\begin{array}{l}\text { IMIS 2017: Innovative Mobile and Internet Services in Ubiquitous } \\
\text { Computing }\end{array}$ \\
\hline Editors & Barolli L., Enokido T. \\
\hline Publisher & Springer, Cham. \\
\hline ISBN & $978-3-319-61542-4$ \\
\hline Pages & $860-872$ \\
\hline Year & 2017 \\
\hline DOI & doi.org/10.1007/978-3-319-61542-4_88 \\
\hline Online & https://link.springer.com/chapter/10.1007/978-3-319-61542-4 88 \\
\hline Abstract & $\begin{array}{l}\text { First standardization initiatives of the Cyber-Physical Systems } \\
\text { (CPS) paradigm face a type of solutions with a top-down } \\
\text { approach. In this view, user services and applications are } \\
\text { transformed, decomposed and delegated until they are finally } \\
\text { executed by hardware devices. However, most works do not } \\
\text { describe the final execution phase, when a certain device is } \\
\text { selected to perform an action. Therefore, in this paper we describe } \\
\text { a management solution to coordinate the execution of low-level } \\
\text { services in CPS. The solution employs a probabilistic selection } \\
\text { technique based on the concept of Cost and Quality-of-Service, } \\
\text { and includes both an orchestration algorithm and a choreography } \\
\text { procedure. The proposal includes, moreover, a general framework } \\
\text { explaining all the management levels and an experimental } \\
\text { validation which evaluates the performance of the proposed } \\
\text { technology. }\end{array}$ \\
\hline
\end{tabular}




\begin{tabular}{|c|c|}
\hline Title & Self-configuration in humanized cyber-physical systems \\
\hline Authors & Bordel, B., Alcarria, R., Martín, D., Robles, T., \& de Rivera, D. S. \\
\hline Journal & Journal of Ambient Intelligence and Humanized Computing \\
\hline ISSN & $1868-5145$ \\
\hline $\begin{array}{l}\text { Impact factor } \\
\text { (JCR) }\end{array}$ & 1,588 (Q3) [JCR 2016] \\
\hline Publisher & Springer Berlin Heidelberg \\
\hline Volume & 8 \\
\hline Number & 4 \\
\hline Pages & $485-496$ \\
\hline Year & 2017 \\
\hline DOI & doi.org/10.1007/s12652-016-0410-3 \\
\hline Online & https://link.springer.com/paper/10.1007/s12652-016-0410-3 \\
\hline Abstract & $\begin{array}{l}\text { Most works on Cyber-Physical Systems (CPS) are based on classic } \\
\text { hardware infrastructures made of sensors, actuators and processing } \\
\text { devices. Usual self-configuration technologies, then, do not allow } \\
\text { humans to be integrated in CPS as service providers. Therefore, in } \\
\text { this work we propose a new self-configuration technology for } \\
\text { humanized CPS. The proposed technology uses simple binary and } \\
\text { mathematical operations in order to reduce the convergence time, } \\
\text { improve the scalability and address the dynamism introduced by } \\
\text { humans into CPS. Besides, a human-oriented quality-of-service } \\
\text { algorithm based on the Maslow pyramid is also introduced. } \\
\text { Moreover, an experimental validation is conducted in order to } \\
\text { validate the proposed solution as a useful and scalable self- } \\
\text { configuration technology for humanized Cyber-Physical Systems. }\end{array}$ \\
\hline
\end{tabular}




\begin{tabular}{|c|c|}
\hline Title & $\begin{array}{l}\text { Fast self-configuration in service-oriented Smart Environments for } \\
\text { real-time applications }\end{array}$ \\
\hline Authors & Bordel, B., Alcarria, R., Sánchez de Rivera, D., Martín, D., \& Robles, T. \\
\hline Journal & Journal of Ambient Intelligence and Smart Environments \\
\hline ISSN & $1876-1364$ \\
\hline $\begin{array}{l}\text { Impact factor } \\
\text { (JCR) }\end{array}$ & 0,809 (Q4) [JCR 2016] \\
\hline Publisher & IOS Press \\
\hline Volume & 10 \\
\hline Number & 2 \\
\hline Pages & $143-167$ \\
\hline Year & 2018 \\
\hline DOI & 10.3233/AIS-180479 \\
\hline Online & $\begin{array}{l}\text { https://content.iospress.com/papers/journal-of-ambient-intelligence- } \\
\text { and-smart-environments/ais479 }\end{array}$ \\
\hline Abstract & $\begin{array}{l}\text { Smart Environments (SE) aim to satisfy the experience of individuals by } \\
\text { the provision of systems, services and devices. Although many } \\
\text { approaches may be followed in order to build a SE, a service-based } \\
\text { design is the most promising one nowadays. In these architectures, both } \\
\text { physical devices and even humans may provide low-level services } \\
\text { based on their capabilities. These low-level services are composed and } \\
\text { connected in order to create high-level services, employed by managers } \\
\text { to describe the behavior of the SE. This composition process } \\
\text { traditionally requires the human intervention, so changes in services are } \\
\text { not automatic. However SE should operate at real-time, thus the } \\
\text { collection of available services must be always updated. In the state of } \\
\text { the art this challenge has been only partially covered, and proposed } \\
\text { solutions are still heavy and manual. Therefore, in this work we propose } \\
\text { a new fast semi-automatic self-configuration (and automatic self- } \\
\text { adaptation) technology for service-oriented SE. The proposed } \\
\text { technology is based on the definition of templates at different levels } \\
\text { which are related by means of semantic information. Moreover, simple } \\
\text { binary mathematical operations are included in order to reduce the } \\
\text { convergence time and improve the scalability. A first reduced extension } \\
\text { for hybrid approaches (including both humans and physical devices) is } \\
\text { also provided. Finally, an experimental validation is conducted in order } \\
\text { to evaluate the convergence time of the proposed solution in different } \\
\text { scenarios, and demonstrate it is scalable towards future deployments } \\
\text { involving thousands of devices and (possibly) humans. }\end{array}$ \\
\hline
\end{tabular}




\begin{tabular}{|c|c|}
\hline Title & $\begin{array}{l}\text { Plug-and-play transducers in cyber-physical systems for device- } \\
\text { driven applications. }\end{array}$ \\
\hline Authors & Bordel, B., de Rivera, D. S., \& Alcarria, R. \\
\hline Conference & $\begin{array}{l}\text { International Conference on Innovative Mobile and Internet } \\
\text { Services in Ubiquitous Computing }\end{array}$ \\
\hline CORE ranking & No \\
\hline Proceedings title & $\begin{array}{l}\text { Innovative Mobile and Internet Services in Ubiquitous Computing } \\
\text { (IMIS), } 2016 \text { 10th International Conference on }\end{array}$ \\
\hline Publisher & IEEE \\
\hline ISBN & $978-1-5090-0984-8$ \\
\hline Pages & $316-321$ \\
\hline Year & 2016 \\
\hline DOI & 10.1109/IMIS.2016.68 \\
\hline Online & https://ieeexplore.ieee.org/abstract/document/7794384/ \\
\hline Abstract & $\begin{array}{l}\text { One of the most popular IoT-based technologies nowadays are } \\
\text { Cyber-Physical Systems (CPSs). In its lowest level, CPSs include a } \\
\text { set of transducers which must be automatically connected and } \\
\text { configured to achieve a seamless union with the environment. } \\
\text { This problem, however, is usually addressed supposing devices } \\
\text { with large processing capabilities, instead of small sensors and } \\
\text { actuators seamless integrated in the physical world. Thus, in this } \\
\text { paper we propose a new plug-and-play technology specially } \\
\text { designed for being applied in reduced capacities devices } \\
\text { integrated in CPSs. Moreover, an experimental validation is } \\
\text { conducted in order to validate the proposal and evaluate its } \\
\text { performance. }\end{array}$ \\
\hline
\end{tabular}




\subsection{Humanized processes. Human integration in Cyber-Physical Systems}

This section includes Works focused on the design and evaluation of devices and algorithms to monitor people and enable their integration into humanized CPS. These works also include process models adequate to be executed by humans, as well as pattern recognition technologies to infer activities being performed by people. The following papers are included:

\begin{tabular}{|c|c|}
\hline Title & $\begin{array}{l}\text { Process execution in humanized Cyber-physical systems: soft } \\
\text { processes }\end{array}$ \\
\hline Authors & Bordel, B., Alcarria, R., \& Jara, A. \\
\hline Conference & Information Systems and Technologies (CISTI) \\
\hline CORE ranking & C - Regional \\
\hline Proceedings title & $\begin{array}{l}\text { Information Systems and Technologies (CISTI), } 2017 \text { 12th Iberian } \\
\text { Conference on }\end{array}$ \\
\hline Publisher & IEEE \\
\hline ISBN & 978-9-8998-4347-9 \\
\hline Pages & $1-7$ \\
\hline Year & 2017 \\
\hline DOI & 10.23919/CISTI.2017.7975901 \\
\hline Online & https://ieeexplore.ieee.org/abstract/document/7975901/ \\
\hline Abstract & $\begin{array}{l}\text { One of the most common applications for Cyber-Physical Systems } \\
\text { (CPS) is process execution. Nowadays, different description } \\
\text { languages are employed in order to define, command and control } \\
\text { the execution of processes using the elements making up a CPS. } \\
\text { However, this approach creates rigid (or hard) processes, which } \\
\text { are only valid in certain scenarios. The inclusion of legacy } \\
\text { systems, new Industry } 4.0 \text { devices and, overall, humans as part of } \\
\text { CPS, has caused the appearance of new paradigms which cannot } \\
\text { be addressed with traditional execution techniques. Therefore, in } \\
\text { this paper a new type of (soft) processes, designed to be employed } \\
\text { in humanized CPS, is presented. The proposal is based on the } \\
\text { concept of "strain", which is used to determine if a deformable } \\
\text { process has been correctly executed. Finally an experimental } \\
\text { validation is provided in order to evaluate the performance of the } \\
\text { proposed technology. }\end{array}$ \\
\hline
\end{tabular}




\begin{tabular}{|c|c|}
\hline Title & $\begin{array}{l}\text { Assessment of human motivation through analysis of physiological } \\
\text { and emotional signals in Industry } 4.0 \text { scenarios }\end{array}$ \\
\hline Authors & Bordel, B., \& Alcarria, R. \\
\hline Journal & Journal of Ambient Intelligence and Humanized Computing \\
\hline ISSN & $1868-5145$ \\
\hline $\begin{array}{l}\text { Impact factor } \\
\text { (JCR) }\end{array}$ & 1,588 (Q3) [JCR 2016] \\
\hline Publisher & Springer Berlin Heidelberg \\
\hline Pages & $1-21$ \\
\hline Year & 2017 \\
\hline DOI & doi.org/10.1007/s12652-017-0664-4 \\
\hline Online & https://link.springer.com/paper/10.1007/s12652-017-0664-4 \\
\hline Abstract & $\begin{array}{l}\text { Traditional methods to evaluate the human motivation in companies } \\
\text { include surveys, statistical techniques and psychological analysis. } \\
\text { However, generating the required information using these methods } \\
\text { is very costly and time demanding. As a solution, Industry } 4.0 \\
\text { paradigm allows integrating Ambient Intelligence systems into the } \\
\text { daily industrial operations in order to digitalize those activities. This } \\
\text { paper proposes a solution to automatically assess the human } \\
\text { motivation in Industry } 4.0 \text { scenarios with Ambient Intelligence } \\
\text { infrastructure. The estimation is based on both physiological and } \\
\text { emotional signals which are acquired (through on-body and } \\
\text { environmental sensors) and processed in real-time using web } \\
\text { services. The final representation of the human motivation is based } \\
\text { on the extended Maslow's hierarchy of needs. Moreover, an } \\
\text { experimental validation is provided, in order to evaluate the } \\
\text { performance of the proposed solution. }\end{array}$ \\
\hline
\end{tabular}




\begin{tabular}{|c|c|}
\hline Title & Recognition of activities of daily living in Enhanced Living \\
\hline & Environments \\
\hline Authors & Bordel, B., Pérez-Jiménez, M., \& Sánchez-de-Rivera, D \\
\hline Journal & IT CoNvergence PRActice (INPRA) \\
\hline ISSN & $2288-0860$ \\
\hline $\begin{array}{l}\text { Impact factor } \\
\text { (JCR) }\end{array}$ & No \\
\hline Publisher & Innovative Information Science \& Technology Research Group \\
\hline Volume & 4 \\
\hline Number & 4 \\
\hline Pages & $18-31$ \\
\hline Year & 2016 \\
\hline Online & http://isyou.info/inpra/papers/inpra-v4n4-03.pdf \\
\hline Abstract & $\begin{array}{l}\text { Enhanced Living Environments consider the recognition of the } \\
\text { Activities of Daily Living (ADLs) being performed by users as first } \\
\text { step in the aid plans. Some works proved the information about the } \\
\text { objects with which a person interacts robustly characterizes the } \\
\text { ADL's identity. However, designing aid plans based on these raw } \\
\text { data is a very complicated task, as an expert in both technology and } \\
\text { occupational sciences is required. In addition, the plans produced by } \\
\text { these experts are not platform-independent, to be closely linked to } \\
\text { the hardware characteristics. Therefore, the aim of this paper is to } \\
\text { design a two-phase solution capable of acquiring data from users, } \\
\text { and of extracting information about the ADL being performed to } \\
\text { trigger the execution of the proper aid plans. This solution } \\
\text { disengages the technological and occupational domains, so that aid } \\
\text { plans could be applied to any environment. Moreover, an } \\
\text { experimental validation is conducted in order to validate the } \\
\text { proposed technology as a valid solution for ADL recognition. }\end{array}$ \\
\hline
\end{tabular}




\begin{tabular}{|c|c|}
\hline Title & $\begin{array}{l}\text { T4AI: A system for monitoring people based on improved wearable } \\
\text { devices }\end{array}$ \\
\hline Authors & Pérez-Jiménez, M., Sánchez, B. B., \& Alcarria, R. \\
\hline Journal & $\begin{array}{l}\text { Research Briefs on Information \& Communication Technology } \\
\text { Evolution }\end{array}$ \\
\hline ISSN & 2383-9201 \\
\hline $\begin{array}{l}\text { Impact factor } \\
\text { (JCR) }\end{array}$ & No \\
\hline Publisher & Innovative Information Science \& Technology Research Group \\
\hline Volume & 2 \\
\hline Pages & $1-16$ \\
\hline Year & 2016 \\
\hline DOI & DOI:10.22667/ReBiCTE.2016.03.31.001 \\
\hline Online & http://rbisyou.wixsite.com/rebicte/volume-2-2016 \\
\hline Abstract & $\begin{array}{l}\text { Monitoring people is central to many applications. Some works from } \\
\text { the literature prove that information about that the sequence of } \\
\text { objects a person uses while performing an activity robustly } \\
\text { characterizes both, the activity and the quality of its execution. In this } \\
\text { paper we present a novel system called "Toolkit for activities } \\
\text { Inference" (T4AI) for inferring the activities executed by people from } \\
\text { the interactions with objects. The system includes as main element a } \\
\text { cybernetic glove based on wearable RFID readers and sensors. Our } \\
\text { proposal includes an improved RFID technology being able to be } \\
\text { used in metallic environments (such as industry scenarios). } \\
\text { Moreover, an experimental validation was conducted in order to } \\
\text { determine the performance of the proposed system. }\end{array}$ \\
\hline
\end{tabular}




\subsection{Device management in Humanized Cyber-Physical Systems}

Mechanisms that enable the hardware device management and its lifecycle in the context of humanized CPS, as well solutions to execute tasks in a choreographed, autonomous and self-managed manner are reported in this section. The following papers are included:

\begin{tabular}{|c|c|}
\hline Title & $\begin{array}{l}\text { Predictive algorithms for mobility and device lifecycle management } \\
\text { in Cyber-Physical Systems }\end{array}$ \\
\hline Authors & Sánchez, B. B., Alcarria, R., De Rivera, D. S., \& Sánchez-Picot, A. \\
\hline Journal & EURASIP Journal on Wireless Communications and Networking \\
\hline ISSN & $1687-1499$ \\
\hline $\begin{array}{l}\text { Impact factor } \\
\text { (JCR) }\end{array}$ & 1,529 (Q3) [JCR 2016] \\
\hline Publisher & SpringerOpen \\
\hline Volume & 2016 \\
\hline Number & 228 \\
\hline Year & 2016 \\
\hline DOI & doi.org/10.1186/s13638-016-0731-0 \\
\hline Online & $\begin{array}{l}\text { https://jwcn- } \\
\text { eurasipjournals.springeropen.com/papers/10.1186/s13638-016-0731-0 }\end{array}$ \\
\hline Abstract & $\begin{array}{l}\text { Cyber-Physical Systems (CPS) are often composed of a great number } \\
\text { of mobile, wireless networked devices. In order to guarantee the } \\
\text { system performing, management policies focused on becoming } \\
\text { transparent to high-level applications, the changes in the hardware } \\
\text { platform have to be implemented. However, traditional reactive } \\
\text { methodologies and basic proposed predictive solutions are not valid } \\
\text { either due to the extremely dynamical behavior of CPS or because } \\
\text { the high number of involved devices prevents fulfill the timing } \\
\text { requirements. Therefore, in this paper, we present an advance } \\
\text { predictive solution for managing the mobility and device lifecycle, } \\
\text { being able to meet all requirements of CPS. The solution is based on } \\
\text { an infinite loop, which calculates, in each iteration, a sequence of } \\
\text { future system states using a CPS simulator and interpolation } \\
\text { algorithms. Furthermore, an experimental validation is provided in } \\
\text { order to determine the performing of the proposed solution. }\end{array}$ \\
\hline
\end{tabular}




\begin{tabular}{|c|c|}
\hline Title & $\begin{array}{l}\text { A Methodology for the Design of Application-Specific Cyber- } \\
\text { Physical Social Sensing Co-Simulators }\end{array}$ \\
\hline Authors & $\begin{array}{l}\text { Sánchez, B. B., Alcarria, R., Sánchez-Picot, Á., \& Sánchez-de-Rivera, } \\
\text { D. }\end{array}$ \\
\hline Journal & Sensors \\
\hline ISSN & $1424-8220$ \\
\hline $\begin{array}{l}\text { Impact factor } \\
\text { (JCR) }\end{array}$ & 2,677 (Q1) [JCR 2016] \\
\hline Publisher & MDPI \\
\hline Volume & 17 \\
\hline Number & 10 \\
\hline Pages & 2177 \\
\hline Year & 2017 \\
\hline DOI & doi.org/10.3390/s17102177 \\
\hline Online & http://www.mdpi.com/1424-8220/17/10/2177 \\
\hline Abstract & $\begin{array}{l}\text { Cyber-Physical Social Sensing (CPSS) is a new trend in the context of } \\
\text { pervasive sensing. In these new systems, various domains coexist in } \\
\text { time, evolve together and influence each other. Thus, application- } \\
\text { specific tools are necessary for specifying and validating designs and } \\
\text { simulating systems. However, nowadays, different tools are } \\
\text { employed to simulate each domain independently. Mainly, the cause } \\
\text { of the lack of co-simulation instruments to simulate all domains } \\
\text { together is the extreme difficulty of combining and synchronizing } \\
\text { various tools. In order to reduce that difficulty, an adequate } \\
\text { architecture for the final co-simulator must be selected. Therefore, in } \\
\text { this paper the authors investigate and propose a methodology for the } \\
\text { design of CPSS co-simulation tools. The paper describes the four } \\
\text { steps that software architects should follow in order to design the } \\
\text { most adequate co-simulator for a certain application, considering the } \\
\text { final users' needs and requirements and various additional factors } \\
\text { such as the development team's experience. Moreover, the first } \\
\text { practical use case of the proposed methodology is provided. An } \\
\text { experimental validation is also included in order to evaluate the } \\
\text { performing of the proposed co-simulator and to determine the } \\
\text { correctness of the proposal. }\end{array}$ \\
\hline
\end{tabular}




\begin{tabular}{|c|c|}
\hline Title & $\begin{array}{l}\text { A Hardware-Supported Algorithm for Self-Managed and } \\
\text { Choreographed Task Execution in Sensor Networks }\end{array}$ \\
\hline Authors & Bordel, B., Miguel, C., Alcarria, R., \& Robles, T. \\
\hline Journal & Sensors \\
\hline ISSN & $1424-8220$ \\
\hline $\begin{array}{l}\text { Impact factor } \\
\text { (JCR) }\end{array}$ & 2,677 (Q1) [JCR 2016] \\
\hline Publisher & MDPI \\
\hline Volume & 18 \\
\hline Number & 3 \\
\hline Pages & 812 \\
\hline Year & 2018 \\
\hline DOI & doi.org/10.3390/s18030812 \\
\hline Online & http://www.mdpi.com/1424-8220/18/3/812 \\
\hline Abstract & $\begin{array}{l}\text { Nowadays, sensor networks are composed of a great number of tiny } \\
\text { resource-constraint nodes, whose management is increasingly more } \\
\text { complex. In fact, although collaborative or choreographic task execution } \\
\text { schemes are which fit in the most perfect way with the nature of sensor } \\
\text { networks, they are rarely implemented because of the high resource } \\
\text { consumption of these algorithms (especially if networks include many } \\
\text { resource-constrained devices). On the contrary, hierarchical networks } \\
\text { are usually designed, in whose cusp it is included a heavy orchestrator } \\
\text { with a remarkable processing power, being able to implement any } \\
\text { necessary management solution. However, although this orchestration } \\
\text { approach solves most practical management problems of sensor } \\
\text { networks, a great amount of the operation time is wasted while nodes } \\
\text { request the orchestrator to address a conflict and they obtain the } \\
\text { required instructions to operate. Therefore, in this paper it is proposed a } \\
\text { new mechanism for self-managed and choreographed task execution in } \\
\text { sensor networks. The proposed solution considers only a lightweight } \\
\text { gateway instead of traditional heavy orchestrators and a hardware- } \\
\text { supported algorithm, which consume a negligible amount of resources } \\
\text { in sensor nodes. The gateway avoids the congestion of the entire sensor } \\
\text { network and the hardware-supported algorithm enables a } \\
\text { choreographed task execution scheme, so no particular node is } \\
\text { overloaded. The performance of the proposed solution is evaluated } \\
\text { through numerical and electronic ModelSim-based simulations. }\end{array}$ \\
\hline
\end{tabular}




\subsection{Communication models and networks in Humanized Cyber- Physical Systems}

This section works relative to communication systems and humanized CPS are collected. In particular, a new wireless communication channel model is proposed and a new network architecture for future systems is designed. The following papers are included:

\begin{tabular}{|c|c|}
\hline Title & $\begin{array}{l}\text { Mobile Wireless Sensor Networks: Modeling and Analysis of Three- } \\
\text { Dimensional Scenarios and Neighbor Discovery in Mobile Data } \\
\text { Collection }\end{array}$ \\
\hline Authors & Robles, T., Bordel, B., Alcarria, R., \& Martin, D. \\
\hline Journal & Adhoc \& Sensor Wireless Networks \\
\hline ISSN & $1551-9899$ \\
\hline $\begin{array}{l}\text { Impact factor } \\
\text { (JCR) }\end{array}$ & 1,034 (Q4) [JCR 2016] \\
\hline Publisher & Old City Publishing \\
\hline Volume & 35 \\
\hline Pages & $67-104$ \\
\hline Year & 2017 \\
\hline Online & $\begin{array}{l}\text { http://www.oldcitypublishing.com/journals/ahswn-home/ahswn- } \\
\text { issue-contents/ahswn-volume-35-number-1-2-2017/ }\end{array}$ \\
\hline Abstract & $\begin{array}{l}\text { WSNs, as important part of the Internet of Things, became an essential } \\
\text { held of study in recent years. However, most works use simplified } \\
\text { scenarios for its analysis, disregarding variables that affect the data } \\
\text { collection process. In this paper, we present a new model for data } \\
\text { collection, considering the connection time among the nodes as a } \\
\text { work's main part. In addition, a real three-dimensional model is } \\
\text { described. The analysis of this new model will allow us to discover } \\
\text { new problems for the WSN operation. We start describing both two- } \\
\text { dimensional and three-dimensional scenarios, and continue } \\
\text { comparing the operation of different types of mobile nodes (such as } \\
\text { walking people or drones).Later, we analyze the connection time, the } \\
\text { rate and the range of the most used Wireless communication } \\
\text { technologies on WSN (like Bluetooth or } 802.15 .4 \text { ). Joining both results, } \\
\text { we created a model for data collection and analyzed how to build } \\
\text { more efficient WSNs. }\end{array}$ \\
\hline
\end{tabular}




\begin{tabular}{|c|c|}
\hline Title & $\begin{array}{l}\text { Using } 5 G \text { technologies in the Internet of things handovers, } \\
\text { problems and challenges }\end{array}$ \\
\hline Authors & Sánchez, B. B., Sánchez-Picot, Á., \& De Rivera, D. S \\
\hline Conference & $\begin{array}{l}\text { International Conference on Innovative Mobile and Internet } \\
\text { Services in Ubiquitous Computing }\end{array}$ \\
\hline CORE ranking & No \\
\hline Proceedings title & $\begin{array}{l}\text { Innovative Mobile and Internet Services in Ubiquitous Computing } \\
\text { (IMIS), } 2015 \text { 9th International Conference on }\end{array}$ \\
\hline Publisher & IEEE \\
\hline ISBN & $978-1-4799-8873-0$ \\
\hline Pages & $364-369$ \\
\hline Year & 2015 \\
\hline DOI & 10.1109/IMIS.2015.56 \\
\hline Online & https://ieeexplore.ieee.org/abstract/document/7284976/ \\
\hline Abstract & $\begin{array}{l}\text { The junction of both revolutions, the Internet of Things and } \\
\text { mobile technologies, seems to be the key of the future. However, } \\
\text { most works about this solution use a really simplified scenario, } \\
\text { where the application of the mobile technologies to the IoT is } \\
\text { problems-free. In this paper, we present a study considering the } \\
\text { problems of the handovers in LTE and } 5 \mathrm{G} \text { networks. This view } \\
\text { allows us to discover new challenges and limitations to the 5G- } \\
\text { based IoT operation. }\end{array}$ \\
\hline
\end{tabular}




\begin{tabular}{|c|c|}
\hline Title & $\begin{array}{l}\text { Virtualization-Based Techniques for the Design, Management } \\
\text { and Implementation of Future } 5 \mathrm{G} \text { Systems with Network Slicing }\end{array}$ \\
\hline Authors & Bordel, B., de Rivera, D. S., \& Alcarria, R. \\
\hline Conference & World Conference on Information Systems and Technologies \\
\hline CORE ranking & C \\
\hline Proceedings title & $\begin{array}{l}\text { WorldCIST'18 2018: Trends and Advances in Information } \\
\text { Systems and Technologies }\end{array}$ \\
\hline Editors & Rocha Á., Adeli H., Reis L., Costanzo S. \\
\hline Publisher & Springer, Cham. \\
\hline ISBN & $978-3-319-77712-2$ \\
\hline Pages & $133-143$ \\
\hline Year & 2018 \\
\hline DOI & doi.org/10.1007/978-3-319-77712-2_13 \\
\hline Online & https://link.springer.com/chapter/10.1007/978-3-319-77712-2 13 \\
\hline Abstract & $\begin{array}{l}\text { Emerging } 5 \mathrm{G} \text { communications aim to simplify the current } \\
\text { inefficient and heterogeneous collection of wireless solutions for } \\
\text { future systems. However, contrary to traditional mobile } \\
\text { networks, } 5 \mathrm{G} \text { networks must consider many different } \\
\text { application scenarios (Internet-of-Things, wearable devices, etc.). } \\
\text { In this context it is defined the concept of network slicing, a } \\
\text { technique where network resources are packaged and assigned } \\
\text { in an isolated manner to set of users according to their specific } \\
\text { requirements. The use of Virtual Network Functions and other } \\
\text { similar technologies is a first step in this challenge, but deeper } \\
\text { changes are required. Therefore, in this paper we present a } \\
\text { virtualization-based technique for the design, management and } \\
\text { implementation of future } 5 \mathrm{G} \text { systems with network slicing. The } \\
\text { proposed technique employs extensively current virtualization } \\
\text { technologies such as Docker or Kubernetes in order to create, } \\
\text { coordinate and manage slices, services and functional } \\
\text { components in future } 5 \mathrm{G} \text { networks. A simulation scenario } \\
\text { describing these future mobile networks is also provided, in } \\
\text { order to obtain first evidences of their predicted performance. }\end{array}$ \\
\hline
\end{tabular}




\subsection{Security in Humanized Cyber-Physical Systems}

This last subsection finally collects papers related to security aspects in humanized CPS. It is included cryptographic solutions and methods to detect malicious components based on the ideas or reputation and trust among other technologies. The following papers are included:

\begin{tabular}{|c|c|}
\hline Title & $\begin{array}{l}\text { Detecting Malicious Components in Large-Scale Internet-of- } \\
\text { Things Systems and Architectures }\end{array}$ \\
\hline Authors & Bordel, B., Alcarria, R., \& Sánchez-de-Rivera, D. \\
\hline Conference & World Conference on Information Systems and Technologies \\
\hline CORE ranking & $\mathrm{C}$ \\
\hline Proceedings title & $\begin{array}{l}\text { WorldCIST 2017: Recent Advances in Information Systems and } \\
\text { Technologies }\end{array}$ \\
\hline Editors & Rocha Á., Correia A., Adeli H., Reis L., Costanzo S. \\
\hline Publisher & Springer, Cham. \\
\hline ISBN & $978-3-319-56535-4$ \\
\hline Pages & $155-165$ \\
\hline Year & 2017 \\
\hline DOI & doi.org/10.1007/978-3-319-56535-4_16 \\
\hline Online & https://link.springer.com/chapter/10.1007/978-3-319-56535-4 16 \\
\hline Abstract & $\begin{array}{l}\text { Current large-scale Internet-of-Things systems and architectures } \\
\text { incorporate many components, such as devices or services, } \\
\text { geographic and conceptually very sparse. Thus, for final } \\
\text { applications, it is very complicated to deeply know, manage or } \\
\text { control the underlying components, which, at the end, generate } \\
\text { and process the data they employ. Therefore, new tools to avoid } \\
\text { or remove malicious components based only on the available } \\
\text { information at high level are required. In this paper we describe } \\
\text { a statistical framework for knowledge discovery in order to } \\
\text { estimate the uncertainty level associated with the received data } \\
\text { by a certain application. Moreover, these results are used as } \\
\text { input in a reputation model focused on locating the malicious } \\
\text { components. Finally, an experimental validation is provided in } \\
\text { order to evaluate the performance of the proposed solution. }\end{array}$ \\
\hline
\end{tabular}




\begin{tabular}{|c|c|}
\hline Title & $\begin{array}{l}\text { Protecting Industry } 4.0 \text { Systems Against the Malicious Effects of } \\
\text { Cyber-Physical Attacks }\end{array}$ \\
\hline Authors & Bordel, B., Alcarria, R., Sánchez-de-Rivera, D., \& Robles, T. \\
\hline Conference & $\begin{array}{l}\text { International Conference on Ubiquitous Computing and } \\
\text { Ambient Intelligence (UCAmI) }\end{array}$ \\
\hline CORE ranking & No \\
\hline Proceedings title & UCAmI 2017: Ubiquitous Computing and Ambient Intelligence \\
\hline Editors & Ochoa S., Singh P., Bravo J. \\
\hline Publisher & Springer, Cham \\
\hline ISBN & $978-3-319-67585-5$ \\
\hline Pages & $161-171$ \\
\hline Year & 2017 \\
\hline DOI & doi.org/10.1007/978-3-319-67585-5_17 \\
\hline Online & https://link.springer.com/chapter/10.1007/978-3-319-67585-5 17 \\
\hline Abstract & $\begin{array}{l}\text { Industry } 4.0 \text { refers a new industrial paradigm based on Cyber- } \\
\text { Physical Systems principles. In these new, complex and highly } \\
\text { interdependent systems, the traditional definition of "cyber- } \\
\text { attack" is not enough to represent all the situations may occur. } \\
\text { Furthermore, traditional security policies and defense strategies } \\
\text { are not designed to be effective in scenarios mixing cyber and } \\
\text { physical elements. In this context, this work presents a new idea } \\
\text { about what cyber-physical attacks are, and a technological } \\
\text { solution to protect and compensate the malicious effects of these } \\
\text { attacks in Industry } 4.0 \text { systems. The proposal is based on a } \\
\text { specific description language (CP-ADL) for cyber-physical } \\
\text { attacks, and a mathematical framework allowing a decision } \\
\text { making about the most adequate defense strategy. Finally, and } \\
\text { experimental validation is provided, showing with our proposal } \\
\text { the impact of cyber-physical attacks is highly reduced. }\end{array}$ \\
\hline
\end{tabular}




\begin{tabular}{|c|c|}
\hline Title & $\begin{array}{l}\text { An intra-slice security solution for emerging } 5 \mathrm{G} \text { networks based on } \\
\text { pseudo-random number generators }\end{array}$ \\
\hline Authors & Bordel, B., Orúe, A. B., Alcarria, R., \& Sánchez-de-Rivera, D. \\
\hline Journal & IEEE Access \\
\hline ISSN & 2169-3536 \\
\hline $\begin{array}{l}\text { Impact factor } \\
(\mathrm{JCR})\end{array}$ & 3,240 (Q1) [JCR 2016] \\
\hline Publisher & IEEE \\
\hline Volume & 6 \\
\hline Pages & $16149-16164$ \\
\hline Year & 2018 \\
\hline DOI & 10.1109/ACCESS.2018.2815567 \\
\hline Online & https://ieeexplore.ieee.org/document/8315003/ \\
\hline Abstract & $\begin{array}{l}\text { Future } 5 G \text { networks must provide communication services to a great } \\
\text { and heterogeneous collection of scenarios: from traditional mobile } \\
\text { communications to emerging applications such as Industry } 4.0 \text { or the } \\
\text { Internet of Things (IoT). In this context, the network slicing technique } \\
\text { is defined, where network resources are packaged and assigned in an } \\
\text { isolated manner to the sets of users according to their specific } \\
\text { requirements. Two different domains are, thus, defined: the intra- } \\
\text { slice domain (where dedicated and specific solutions have to be } \\
\text { deployed) and the inter-slice domain (including transversal } \\
\text { solutions). One of the key topics which should be redefined } \\
\text { following this approach is security. Traditionally, some solutions } \\
\text { (such as stream ciphers) were not considered in mobile networks. } \\
\text { However, } 5 \mathrm{G} \text { systems will be extensively employed in other new and } \\
\text { very distinct scenarios, where requirements are different. For } \\
\text { example, the use of resource constrained devices with little mobility } \\
\text { and real-time data streaming in certain IoT applications suggests the } \\
\text { use of stream ciphers (and other similar techniques) as the main } \\
\text { security solutions. Therefore, in this paper, we investigate and } \\
\text { propose a new security solution for emerging } 5 \mathrm{G} \text { networks, to be } \\
\text { applied in the intra-slice domain. The proposed solutions employ } \\
\text { lightweight pseudo-random number generators in order to provide } \\
\text { the keystream used in stream ciphers which protect the private } \\
\text { information and hide the communication signals in the frequency } \\
\text { spectrum using spread spectrum techniques. We also describe and } \\
\text { evaluate a first implementation of the proposed solution, using both, } \\
\text { a simulation scenario and a real deployment. }\end{array}$ \\
\hline
\end{tabular}




\subsection{Selected full-texts}

In this subsection full-texts of the selected papers are provided. 


\title{
Cyber-Physical Systems: Extending pervasive sensing from control theory to the Internet of Things
}

\author{
Borja Bordel ${ }^{1, *}$, Ramón Alcarria ${ }^{2}$, Tomás Robles ${ }^{1}$, Diego Martín ${ }^{1}$
}

1 Department of Telematics Systems Engineering. Universidad Politécnica de Madrid. Avenida Complutense no 30. 28040 - Madrid (España); E-Mails: bbordel@dit.upm.es; diego.martin.de.andres@upm.es; tomas.robles@upm.es;

2 Department of Topographic Engineering and Cartography. Universidad Politécnica de Madrid. Campus Sur, 28031 Madrid, Spain; E-Mail: ramon.alcarria@upm.es

* Author to whom correspondence should be addressed; E-Mail: bbordel@dit.upm.es; Tel. 915495700 ext. 3035

\begin{abstract}
Essentially, the emerging term "Cyber-Physical Systems (CPS)" is an architectural paradigm in which the pervasive sensing technologies represent a fundamental part. Originally defined in the computer sciences domain, the term Cyber-Physical Systems has been adapted to very different domains such as the control theory or electronic engineering. Even, some authors understand CPS as a particular scenario of the Internet of Things (IoT) based on pervasive sensing. Furthermore, recently, some works propose a definition for CPS including all the features described in the different domains. In this paper we provide a comprehensive analysis of the nature and characteristics of the different proposals, discuss the recent attempts to standardize CPS, and review the state-of-the-art on CPS for each technological domain. We compare those different proposals on CPS, discuss about some related terms and technologies and conclude by describing the main research challenges in the area.
\end{abstract}

\section{KEYWORDS}

Cyber-Physical Systems; Pervasive Sensing; Machine-to-Machine; Wireless Sensor Networks; Hybrid systems; Internet-of-Things; Data services; Pervasive computing 


\section{INTRODUCTION}

Essentially, the emerging Cyber-Physical Systems (CPS) are feedback control systems based on pervasive sensing [1]. Thus, CPS embody a vision of physical devices (sensors, actuators and sensor-enable mobile devices) performing feedback control loops, where devices provide and receive information from a control system which executes a certain application. In general, it assumes physical devices are seamless integrated into daily living objects, making up the called embedded systems or embedded devices.

The presence of feedback loops supported by a pervasive sensing infrastructure is the common characteristic to all proposals on CPS. From this common core, and depending on the considered technological domain, CPS may be focused on very different issues such as improving the integration level in embedded devices, building real-time applications or providing customized services in the context of the Internet of Things (IoT). Then, around each vision, authors have identified different lines in CPS research, have proposed specific architectures and have described particular solutions addressing the concrete problems of CPS in their area.

Furthermore, very recently (final months of 2013), the main standards organizations (such as the International Organization for Standardization -ISO- and the European Telecommunications Standards Institute -ETSI-) have created public working groups in order to become CPS into a standard technology. The pioneer in these efforts was the National Institute of Standards and Technology -NIST-, which proposed in its first conclusions (published in September 2015) [2] a horizontal architecture for CPS including all previous proposals. As a result, very different works can be found under the name of Cyber-Physical systems.

Moreover, CPS are currently maturing from its origin as research paradigm to a variety of commercial products $[3,4]$. CPS have many potential applications from smart infrastructure (grid, water, gas, etc.) and smart health care to smart manufacturing and smart transportation, where pervasive sensing is indispensable [5]. These applications have an important potential to benefic companies, societies and, in general, human lives.

Due to the relevance of CPS, different review papers about this topic have appeared in the literature during the last ten years. However, because of the extended use (and certain abuse) of the paradigm "Cyber-Physical Systems" nowadays, it is necessary a new effort to collect and organize the proposed contributions, considering the special circumstances that surrounds the term (lack of agreement about its definition, confusion with similar concepts and previous terms, the interest of the technological researchers on adjusting their ideas to this new paradigm, etc.). In fact, some of previous review works [88][113] propose or defend a particular definition of CPS which is employed as a framework to select the contributions to be referred. As a consequence, they offer a partial vision of this new paradigm. On the other hand, different review works about special topics related to CPS have been reported: self-adaptation [299], security [175], modeling [113], etc. These papers use to be exhaustive and very useful, but they do not offer a global context (it would be interesting, for example, to know if all reviewed contributions understood CPS in the same way or if all authors envision the same type of systems). A third group of surveys [44][114] is focused on future challenges and current trends (or similar issues). The objective of these papers, nevertheless, is not to offer a review about the state-of-the-art but to detect tendencies and problems to be solved. Finally, a great number of survey works [13][66][189][6][5] indicates the existence of different definitions and visions for 
CPS, and sometimes even the different defended architectures are analyzed, but, due to the enormous number of different proposals and the difficulties to classify them, reviewed works are usually organized depending on the application scenario (vehicular systems, medical environments, etc.), mixing works based on different concepts of CPS (which, as we are seeing, even include different elements and components). Therefore, in this paper a new comprehensive review of Cyber-Physical Systems is provided, considering all proposed definitions and visions. Reviewed works include not only which are directly referred as belonging to CPS, but also proposals about topics whose relation with CPS is not clear (Machine-to-Machine, Wireless Sensor Networks...) and other works which fit the different requirements to be considered as CPS. A first explanation of the global context is provided, identifying the elements which may compose CPS and proposing a classification for contributions related to CPS based on the ideas of the Eindhoven Institute for Research on ICT (so proposals inspired by a common definition of CPS are presented together). Finally, as a novelty, not only research works are reviewed but also standardization initiates, which (possibly) will determine the future of CPS.

The rest of the paper is organized as follows. Section 2 introduces the main components of CPS, their features and their relation with the different technological domains. Most important aspects on CPS, which are going to be reviewed from different approaches, are also presented here. Section 3 reviews CPS as defined in control theory, and analyzes the state-of-the-art on the main research challenges. Section 4 presents CPS as understood in computer sciences. Section 5 is focused on communication engineering and its proposals, works, and solutions on CPS. Finally, Section 6 presents the vision of the standards organizations on CPS, as well as the published preliminary conclusions. Finally, Section 7 presents the main future challenges in CPS research and Section 8 concludes the paper.

\section{OVERVIEW OF CYBER-PHYSICAL SYSTEMS}

Works on CPS have helped to the definition of new and extremely interesting emerging technologies in pervasive sensing. However, in the last months Cyber-Physical systems has become into an extremely popular term [6]: the original definition has been extended [7], new concepts and elements (such as virtual sensors) have been added [8], etc. In that way, learning about the composition of CPS might be a complicated task, as many different proposals can be found.

Therefore, in this section we list and describe all the elements which could be part of CyberPhysical Systems, and show how combining these elements in different proportions it is possible to obtain the main approaches identified in CPS sciences. Moreover, most important aspects on CPS are identified, and a brief summary about how each topic is addressed from each one of the listed approaches is provided.

\subsection{ELEMENTS IN CYBER-PHYSICAL SYSTEMS}

A deep analysis of all possible elements which may be part of CPS should be based on the study of all proposed application scenarios in research literature. However, in 2014, the Eindhoven Institute for Research on ICT (EIRCT) identified the six components which, occasionally, have been used to make up CPS [9]: physical world, transducers, control components, data analytics 
elements, computation elements and communication components. Although this proposal has not been communicated (until now) in a regular scientific journal, (as we are seeing) it is a powerful instrument to categorize in a coherent way all the existing definitions and works about CPS. Using the ideas of the EIRCT, a genuine review methodology and a survey with a totally new approach to all exiting works may be provided (as we are able to study all the contributions supported by a common understating of CPS together).

The physical world includes all the elements with which the other components in the system cannot communicate explicitly (i.e. the elements with which cyber components cannot stablish a dialog by means of engineered methods and solutions). As it is one of the main characteristics of CPS, cyber elements must interact with the physical world. However, while cyber components may communicate directly among them through common protocols, interfaces or access technologies (a paradigm known as "explicit interactions"), interactions among cyber components and elements in the physical world have to be performed through sensors and actuators, whose outcomes are processed (using, for example, learning techniques, probably one of the most important aspects of CPS that are being studied) in order to infer the state of the physical world and make decisions (a paradigm known as "implicit interactions"). More formally, in explicit interactions, each one of the agents to be communicated "informs" in a certain level of abstraction what it expects the others to do (by means of textual messages, using a GUI, speech input, etc.) [10]. On the contrary, implicit interactions are based on actions performed by the agents that are not primarily aimed to interact with the others, but they understand as input $[10,11]$. Then, elements in the physical world interact with the other components in the system by means of sensors and actuators which sense and influence them and analyze their behavior [11].

Depending on the application domain, the physical world may be limited to some specific elements or not. In the most general case, the physical world includes two parts: the physical system and the physical environment [12]. The physical system includes all the engineered devices (such as production systems or programmable controllers) which ideally are codesigned with the rest of the CPS, but also might be an existing legacy system. The physical environment refers to physical phenomena relevant to the system (raining, temperature in a room, etc.) [13]. People are a particularly important element in the physical world. Thus, systems that consider humans as part of the physical world are known as Human-in-the-loop systems [14]. Basically human-in-the-loop systems can be classified in three categories depending on the role occupied by humans [14]: (i) systems where humans may control the operation [15] (in that point CPS connect to software-defined networking -SDN- [16]), (ii) systems where humans are only passively monitored [17] and (iii) hybrid systems of the previous [18]. In contrast to the physical world, the rest of the components in a CPS it is said belong to the cyber world or cybernetics.

Transducers refer to the pervasive sensing platform. It includes sensors, actuators and sensorenable mobile devices organized in one or several networks [13]. Transducers usually are organized in nodes which may include sensors, actuators, processors, communication capabilities and batteries [19]. These nodes are seamless integrated into daily-living objects creating the next generation of embedded devices [13]. At logical level, it is common to suppose that sensors and actuators are grouped in two different networks (see Figure 1). Pervasive sensing platform is responsible for acquiring data from the physical world, and transmit that information to the control components. Besides it receives data from the control components and must act on the physical world in the adequate way. In general transducers are organized as 
a wireless network, although wired solutions are also possible. Moreover, transducers may be intelligent, virtual and/or distributed. Nowadays, however, intelligent transducers are much common than virtual or distributed nodes. In particular, intelligent transducers (more commonly named as smart transducers) [249] are seamless integration between sensors (or actuators) and processors, so a new device being able to self-adapt to the environment and perform some initial processing actions is obtained. IEEE 1451 standard defines and proposes different solutions to interact with this type of transducers, such as the Transducer Electronic Data Sheets (TEDS) [250], which allow the self-description of transducers and their plug-and-play connection.

Control components group all the hardware and software elements focused on managing the pervasive sensing platform. These components acquire data from sensors, perform local processing and control the actuators in order to reach the wanted state in the physical world [12]. Control tools typically present a continuous dynamic, although depending on the application in CPS they also may present a discrete behavior inherited from computing systems [19]. In general, these tools must make possible to control "by design" the behavior of the pervasive sensing platform [20]. Clearly, due to the distributed architecture of pervasive sensing platforms, control components used to be decentralized too (although that is not mandatory) [21].

Data analytics elements include all software programs focused on processing, filtering, and storing the information from the different control components. In the past, these elements used to be considered part of control components and/or computation elements. However, the increased integration between the cyber and physical world presents important challenges which deserve to be treated independently [21]. Besides, most of these challenges (such as the huge amount of information produced and the heterogeneity of the generated data) are addressed by specific technologies like Big Data. For all that, important institutions as the EIRCT are considering data analytics elements as an independent component of CPS [9]. These elements perform pattern recognition, decision-making, predictive analysis and machine-learning, among other capabilities. If necessary, they also include visual analytics for controllers, managers or general users [12].

Computation elements include a heterogeneous group of elements belonging to computer sciences. Technologies such as computational models; action-oriented, realistic, timely goals [12]; rigorous timing models for microprocessors [22, 23], real-time computing [24] and virtualization [9] are part of this group. These components are usually employed to adapt the system to internal and external changes through "switching" between different operation "modes". As a result, CPS might be considered as a hybrid system [19]. Finally, this group also includes security techniques and policies, from secure hardware $[25,26]$ to pure software solutions [27] and hybrid approaches [28].

Communication components refer, basically, a group of technologies focused on Internet services. Some examples of the elements included are cloud-computing [29], service composition [30], future wired and wireless technologies for supporting the connectivity [31], energy efficient protocols [32], machine-to-machine communications (M2M) [33] and intelligent mobility management [34].

Figure 1 shows a generic holistic view for CPS where all the previous components are considered. As we are seeing later simpler views are possible depending on the considered technological domain. 


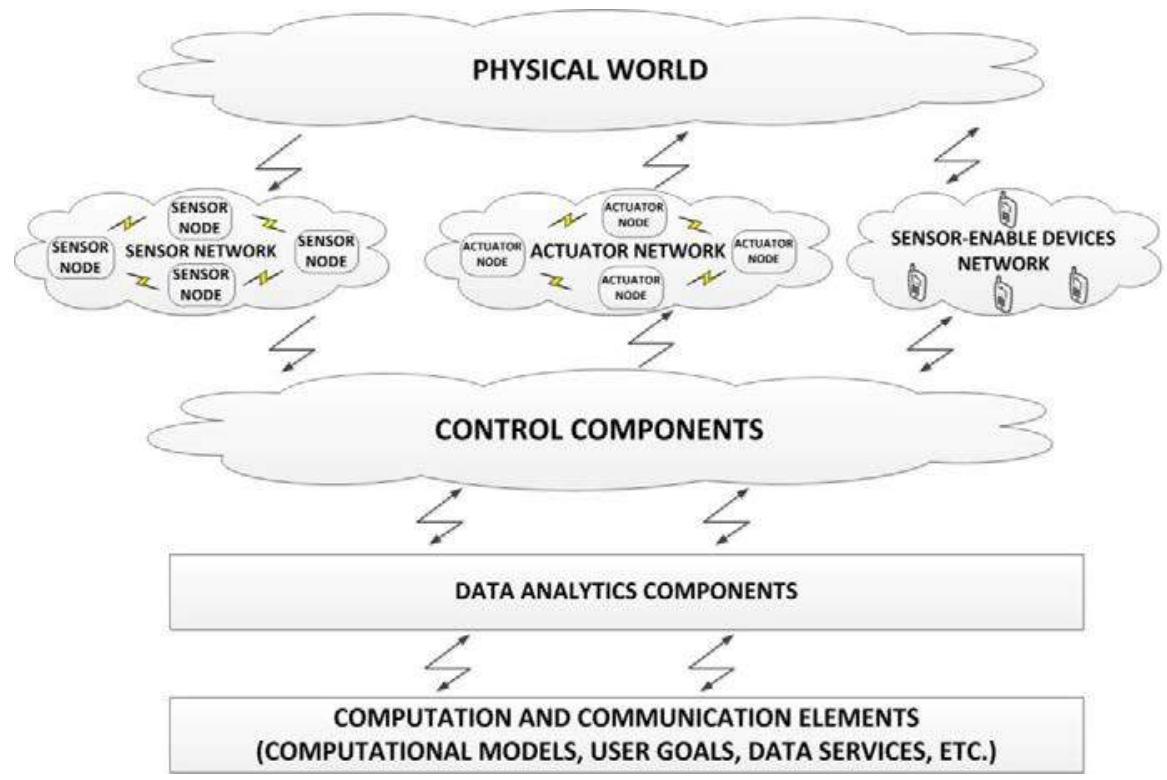

Figure 1: Holistic view of CPS

\subsection{MAIN APPROACHES IN CPS SCIENCES}

CPS has been addressed from different views by the scientific community [13]. There exist several proposals and definitions, some of them extremely specific and focused on a particular scenario as [35]. However, among all these proposals it may be also found approaches with a great impact and to which several authors have contributed. Without being exhaustive, it is noteworthy the proposals of (i) Lee [24] which describes CPS as "integrations of computation and physical processes"; (ii) Raijkumar [36] which proposes CPS as "physical and engineered systems whose operations are monitored, coordinated and integrated by a computing core"; (iii) Marwedel [37] which describes them as "embedded systems together with their physical environment" and (iv) Kim [19] which says "CPS are the next generation of engineered systems in which computing, communication and control technologies are tightly integrated".

As can be seen, proposal (i) is focused on the processes, while the others are focused on the systems. Moreover, proposal (iv) does not consider the physical world in the definition, while in the others is one of the main parts. On the other hand, proposals (iii) and (iv) highlight the improvement of the integration level while (i) and (ii) are focused on the synchronization of cyber and physical world.

As a popular term CPS are understood in different ways by different authors, therefore trying to fix a uniform definition is useless [38]. Nevertheless, the EIRCT proposes that all approaches in CPS (from more famous to which are only developed by the authors who proposed them) can be classified into four main groups depending on the elements which the authors consider as part of CPS [9]. In this work, we identify these four groups with the main research areas in telecommunication engineering: control theory, computer sciences, communication engineering, and vertical systems.

The simplest architecture for CPS belongs to control theory. In this area, CPS are understood as a composition of the physical world, transducers and control components. In some specific applications simple data analytics elements are also considered [39, 40], although it is not the 
regular situation. The importance of CPS in control theory has been showed in many studies such as [41, 42, 43, 44] and others such as the above-mentioned Marwedel's [37], Sztipanovits' and Gill's [45], which understands CPS as "physical, biological and engineered systems whose operations are integrated, monitored, and controlled by a computational core".

The oldest proposal on CPS is due to computer sciences researchers. In fact, the term CPS was coined around 2006 at the National Sciences Foundation (NSF) together with the Berkeley University [1]. It is probably the view which has had the greatest impact, with several related projects running since 2008 [3, 46, 47]. In this area CPS are understood as a composition of the physical world, transducers, control components and computer sciences elements. Specialists on hybrid systems, new computational models, real-time applications and predictive models (among others) support this vision. The definition proposed by E. Lee in his famous position paper "Cyber-physical systems; are computing foundations adequate?" [24] is the base of contributions in this technological domain.

The probably least developed approach is due to communication engineering. This view is focused on hardware, services and embedded devices, so CPS are understood as a combination of transducers, control components, some data analytics elements, computer science elements and communication components. In this approach, the physical world is considered to be outside the system's limits (i.e. it is not considered a part of CPS, but an environment that surrounds them and whose changes they are aware of). Approaches such as proposed by Kim [19] belong to this technological domain. Researches in this area tend not to include the word "Cyber-Physical Systems" in their works, although the proposed technologies could be considered a contribution in this field (as Wireless Sensor Networks [48], M2M communications [33], etc.). Thus, the number of works about CPS in communication engineering is much fewer than in other areas.

Finally, vertical approach for CPS considers the entire stack, from end-user customized services and computational models, to the pervasive sensing infrastructure and the physical world. Then, in this area, CPS are understood as a combination of all the components mentioned in Section 2.1. This theory of CPS is mainly supported by the standard organizations (such as the NIST) [12] and some research institutes as the EIRCT [12]. This perspective for CPS is the newest, proposed around 2013, but due to its great potential has been rapidly spread [38].

Table 1: Composition of CPS in the different technological domains

\begin{tabular}{|c|c|c|c|c|}
\hline & Control theory & $\begin{array}{c}\text { Computer } \\
\text { sciences }\end{array}$ & $\begin{array}{c}\text { Communication } \\
\text { engineering }\end{array}$ & Vertical system \\
\hline Physical world & $\checkmark$ & $\checkmark$ & & $\checkmark$ \\
\hline Transducers & $\checkmark$ & $\checkmark$ & $\checkmark$ & $\checkmark$ \\
\hline Control components & $\checkmark$ & $\checkmark$ & $\checkmark$ & $\checkmark$ \\
\hline Data analytics elements & Sometimes & Sometimes & Sometimes & $\checkmark$ \\
\hline Computation elements & & $\checkmark$ & $\checkmark$ & $\checkmark$ \\
\hline $\begin{array}{c}\text { Communication } \\
\text { components }\end{array}$ & & & $\checkmark$ & $\checkmark$ \\
\hline
\end{tabular}

Table 1 summarizes the composition of CPS depending on the technological domain considered. As can be seen the underlying pervasive sensing infrastructure and the control components are the common elements to all proposals. Any case, it is clear that CPS are something more than just control tools [9], traditional embedded systems, real-time applications 
and/or desktop applications [49], and represent a new type of systems which are not available yet but which will have a great impact on the world $[9,36]$.

In this context, CPS are characterized by some common aspects to all approaches, which makes them very different from any other previous engineered system. In particular, CPS involve some of the most modern techniques and popular research topics: learning solutions (especially those related to pattern recognition), context-aware systems, self-configuration and self-adaptation techniques, mobile solutions and fault-tolerant implementations (among other). However, although important aspects are common to all approaches, each technological domain addresses them from a different point of view. Below, a brief analysis of this situation for each aspect is provided.

First, context-awareness is defined as the ability of systems (specifically mobile systems) to link their operation with changes in the environment [261]. Consequently, it is an intrinsic characteristic to CPS. However, while telecommunication researchers try to define system architectures being able to manage the devices' context [29]; control theory authors look for an optimum stable control algorithm (or function) including as much context information as possible [265].

Secondly, machine learning is a pretty old concept referring the ability of computers to learn without being explicitly programmed [262]. Nowadays, is a specific field belonging to computer sciences, so these authors contributed the most to this area with respect to CPS (defining adaptive physical models [88] and hybrid automatons [266] among other works). Control theory researchers are also interested in the topic, specifically if humans are monitored (a type of human-in-the-loop systems [17]).

Self-configuration and self-adaptation are properties which allow devices to automatically perform the installation procedures to get the basic operational parameters and to obtain the necessary information for operation; modifying its configuration in an autonomous way without restarting or stopping the system operation when an unexpected change occurs [269] [270]. Although autonomic computing studies (computer sciences) consist of bringing to a given system the self-* properties [268], in the particular case of CPS, communication engineering authors are the most prolific on the topic (usually, moreover, in applications related to industrial scenarios [54]).

Fault-tolerance is the ability of systems to operate regularly in case of a failure event [263]. This property is very important in critical systems (such as energy control infrastructures), but it is rarely required to CPS (although some CPS applications are also critical [267]). In this context, computer sciences authors are almost the only ones which have developed proposals in this topic (and, most of them, related to security [275]).

Finally, mobility is a very famous property of systems being able to modify its position (logical or geographical) without having to modify or interrupt its regular operation [272]. Although it is not a mandatory requirement, devices integrated into CPS are usually considered mobile. Thus, most authors working on CPS address problems associated with mobility. Control theory researches (for example) investigate the impact of fluctuations due to mobility in the control loop stability [273]. Computer sciences authors enhance traditional solutions (such as authentication) to be applied to mobile CPS [274]. And communication engineers propose different mechanisms (e.g. protocols) to support mobility in CPS [271]. 
Many concepts and technologies are relatively similar to CPS: embedded systems [50], wearable technologies [51], legacy systems [52] and Industry 4.0 [53, 54, 55], pervasive sensing [56], machine-to-machine communications [57], etc. The relation between CPS and some of them is often confusing. Some other terms are often associated to CPS, such as cloud computing [58], pervasive computing [59] or system-of-systems [60, 61], while "Internet of Things" shares very similar characteristics to CPS.

The term "Internet-of-Things" (IoT) represents the idea of several objects which, through unique addressing policies, are able to interact with each other and cooperate with their neighbors to reach some goals [62]. As we are seeing, this definition is similar in some aspects to CPS (networked devices, common goals, etc.); however there is not any official correspondence between CPS and IoT, so various theories about the relation between both terms may be found.

In [63], Koubâa identified that the frontier between CPS and the IoT is not clearly defined since both concepts have been driven in parallel from two independent communities, although they have always been closely related. In general, four main theories are discussed nowadays.

First, some authors propose CPS and IoT are independent concepts [50], which refer different realities. Thus, in [64], Chen identifies four topic which become different CPS and IoT: (i) CPS work in a close-loop manner, (ii) the network scale of CPSs is not as large as IoT, (iii) in CPS it is necessary a deep integration between the cyber and physical world, and (iv) CPS may not necessarily be connected to Internet (although this last point depends on the considered technological domain, as we are seeing).

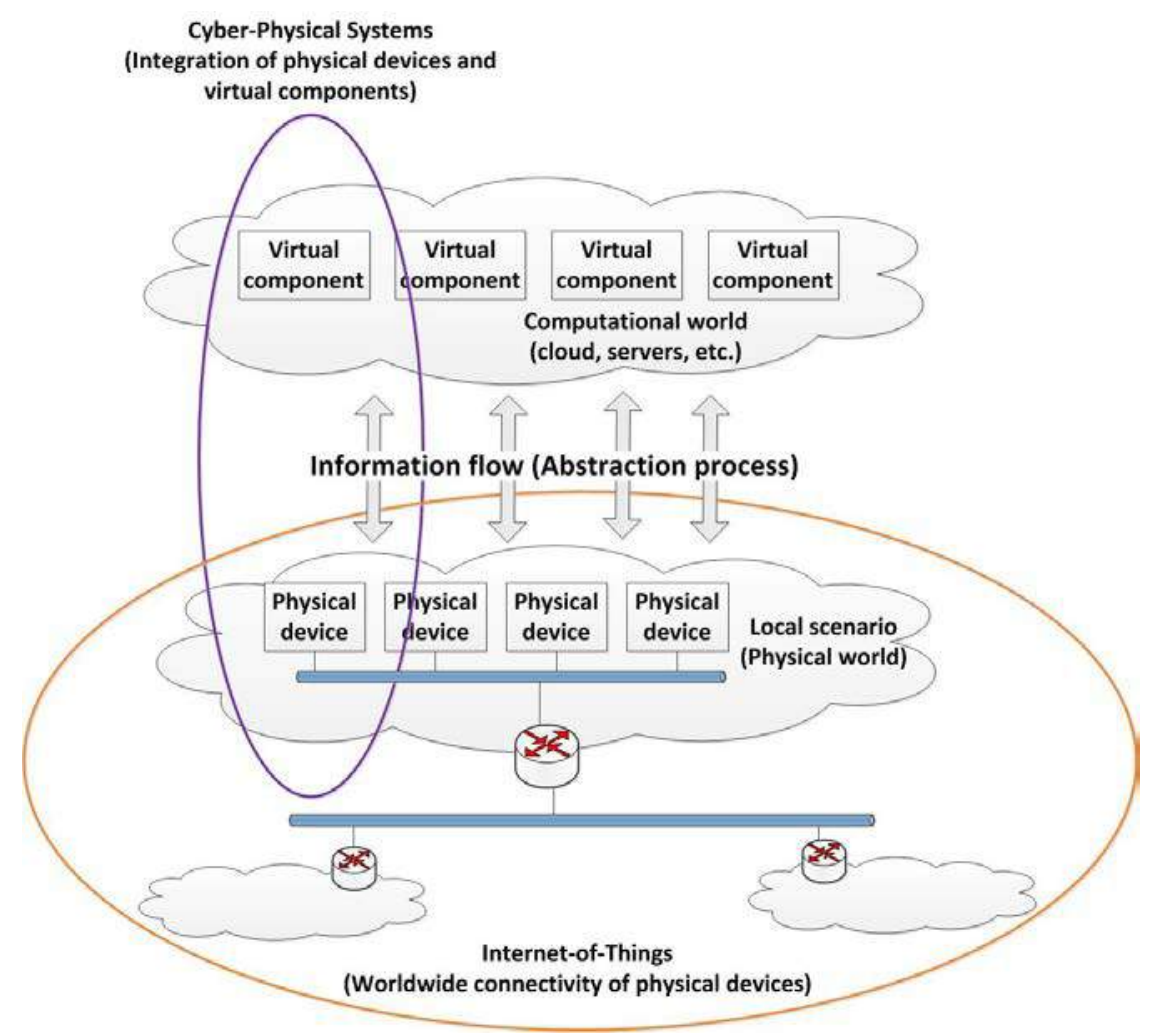

Figure 2: Relation between CPS and IoT 
In this group, other authors defend both terms are related to the same general scenario, but represent different views. For them, IoT has a horizontal view (i.e. IoT scenarios only consider hardware components and the technologies to communicate them) focused on creating a global network of daily living objects with worldwide connectivity. On the other hand, CPS has a vertical approach including from networked physical devices to computational models, control policies or data services [50] (see Figure 2).

Other authors propose that IoT and CPS refer the same reality. In this group all opinions are not equal. Some authors defend that both terms are interchangeable because they always were synonyms. Others think they are synonyms because IoT is being smoothly extended from RFID technologies to something more general [65]. Finally, some authors think both refer to the same but IoT is more supported by European and Chinese researchers and CPS by American organizations [50].

A third group of authors, thinks that IoT, CPS, and the rest of similar terms are only "buzzwords" for referring the future totally connected world [65]. Finally, other researchers defend CPS belongs to IoT, so CPS is only a new pattern or particular scenario of IoT [66].

\section{CYBER-PHYSICAL SYSTEMS IN CONTROL THEORY}

Control theory is the branch of engineering that deals with the methodologies and tools for modifying the behavior of dynamic systems as desired. One of the most extended ways of applying control is by means of feedback loops, whose inputs are provided by a pervasive sensing platform [67]. This particular area of control theory is known as feedback control theory.

As we said in the introduction, CPS are, basically, feedback control systems based on pervasive sensing. Therefore, researchers on feedback control theory rapidly adopted the CPS paradigm in order to design the next generation of control systems.

In this Section we review the basis of feedback control theory, describe the main research lines on CPS from the point of view of control experts, and present the most important contributions made in the cited lines.

\subsection{PROBLEM DESCRIPTION}

In the most general (and realistic) case, the problem of designing a controller is to correctly model the response of the system under control when its inputs are modified, as well as the inputs which must be applied to the system to obtain a certain response. Generally speaking, the objective of a controller is to make the temporal evolution of some process or system, $x(t)$, behaves in a desired way by manipulating in time some input, $s(t)$. For example, in the pasteurization process the liquid has to be heated and cooled various times following a specific pattern. The temporal evolution of temperature $T(t)$, then, has to be controlled to follow this pattern by varying the intensity level $I(t)$ of the refrigeration system.

Depending on the definition of $x(t)$ and $s(t)$ the objective might be to keep $x(t)$ close to some equilibrium point (a regulator problem) or to keep $x(t)-r(t)$ close to some equilibrium point, 
with $r(t)$ a reference temporal evolution (a servo problem) [68]. Thus, the formula modeling the temporal evolution of a controller is as follows [67] (1).

$$
x(t)=(F(t)+\Delta(t))(s(t))+n(t)
$$

In that expression (1), $n(t)$ is an unknown noise or disturbance, $F(t)$ the transference function and $\Delta(t)$ represents unknown perturbations in the transference function. Figure 3 shows a block diagram for a general controller. As can be seen, systems in control theory (including the proposed CPS) present a continuous dynamics, as the physical time (which is continuous) governs these systems.

In a really common practical problem not only $n(t)$ and $\Delta(t)$ are unknown, but also the transference function $F(t)(\cdot)$ [69]. In many cases, the temporal evolution $x(t)$ cannot be expressed explicitly as in expression (1), as it is the solution of a differential problem without analytical solution. In these cases, the mathematical formulation of the control problem used to be as seen in expression (2).

$$
x \dot{x(t)}=F_{t}(x(t))+u(t)
$$

Where $x(t)$ is the output temporal evolution and $u(t)$ is the controller signal. The unknown disturbances are considered null. This situation is typical of feedback systems where the appearance of $x(t)$ is only due to the mathematical model as it has not a real existence in the physical world [70].

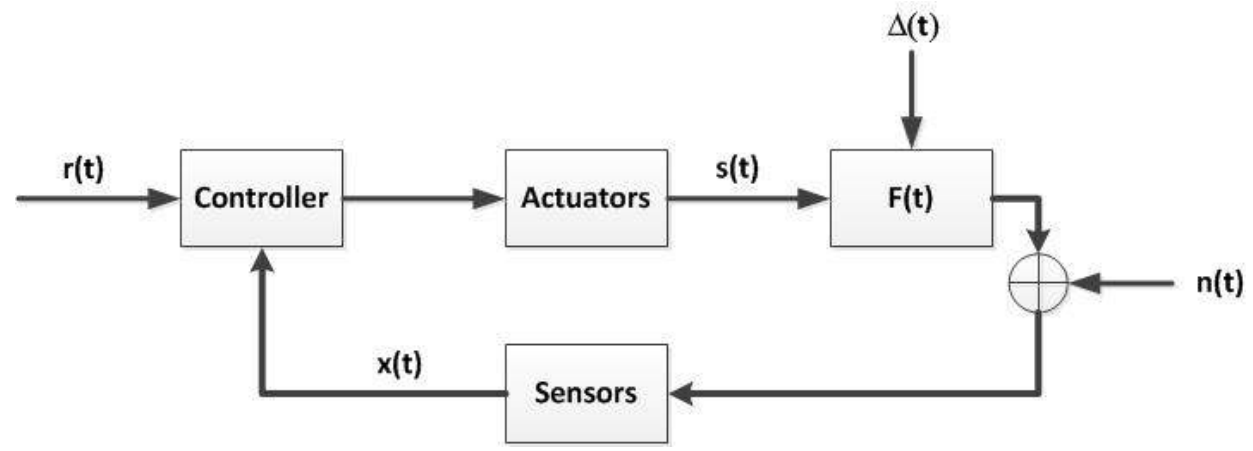

Figure 3: Block diagram for a general controller

Systems as described in Figure 3 are usually based on Programmable Logic Controllers (PLC), a type of microcontroller specifically designed to be integrated in feedback control systems. These devices can be networked, but as they are factory-centered (geographically dispersed locations cannot be connected), networks present a high rate and delays are negligible. Then, design techniques may be the same if controllers are networked or not. This type of networked PCL is usually known as Distributed Control Systems (DCS) [71].

Sometimes, however, feedback loops are closed through a shared network, creating the called Networked Control Systems (NCS) [72], which can be deployed in geographically dispersed locations. PLC, DCS and NCS are not new concepts, and usually are considered as legacy control systems [13].

Nevertheless, the named above legacy systems face some challenges, such as the impact of the network-induced delays in the stability of NCS, which may be addressed using the CPS 
paradigm. Moreover, traditional legacy systems and new CPS share several characteristics, although CPS entail requirements far beyond the expectation of legacy control systems [13, 45]. Therefore, in several scenarios CPS can improve the performance of traditional systems, integrate existing legacy systems in a uniform infrastructure or directly replace the traditional feedback control systems $[73,74]$.

\subsection{CPS IN CONTROL THEORY: THE BASIS}

As can be seen in Figure 3, feedback control systems are made of controllers and sensors and actuators (the pervasive sensing platform), so the proposed architectures for CPS in control theory usually follow this scheme (see Figure 4). Thus, the main definitions for CPS in control theory use to remark aspects such as the control processes, sensing capabilities or the physical world. Apart from the proposals cited in Section 2.2 [37, 45], it must be mentioned the definition proposed by Sztipanovits [75] which describes CPS as "a computer system that processes and reacts to data from external stimuli from physical world and make decision that also impact the physical world". This definition is important because it considers, besides control components and the pervasive sensing platform, data analytics elements, so more complicated control systems can be developed using the CPS paradigm (such as some alternatives to SCADA systems [76]).

The definition of a reference architecture for CPS is a pending challenge (see Section 7). However, some authors have tried to address this issue. In the case of CPS in control theory, the proposed architectures are simple. In Figure 4 can be seen the architecture proposed by Sztipanovits [77], which is one of the most used in CPS for control theory [78, 79, 80]. It consists of three layers: the physical layer corresponds to the physical world, and groups a collection of objects whose behavior is determined by physical laws; the second layer (called "platform") comprises the hardware elements (the pervasive sensing platform); and the third layer (the software layer) involves all the control algorithms, methodologies, etc. Similar architectures are described in other works such as [81].
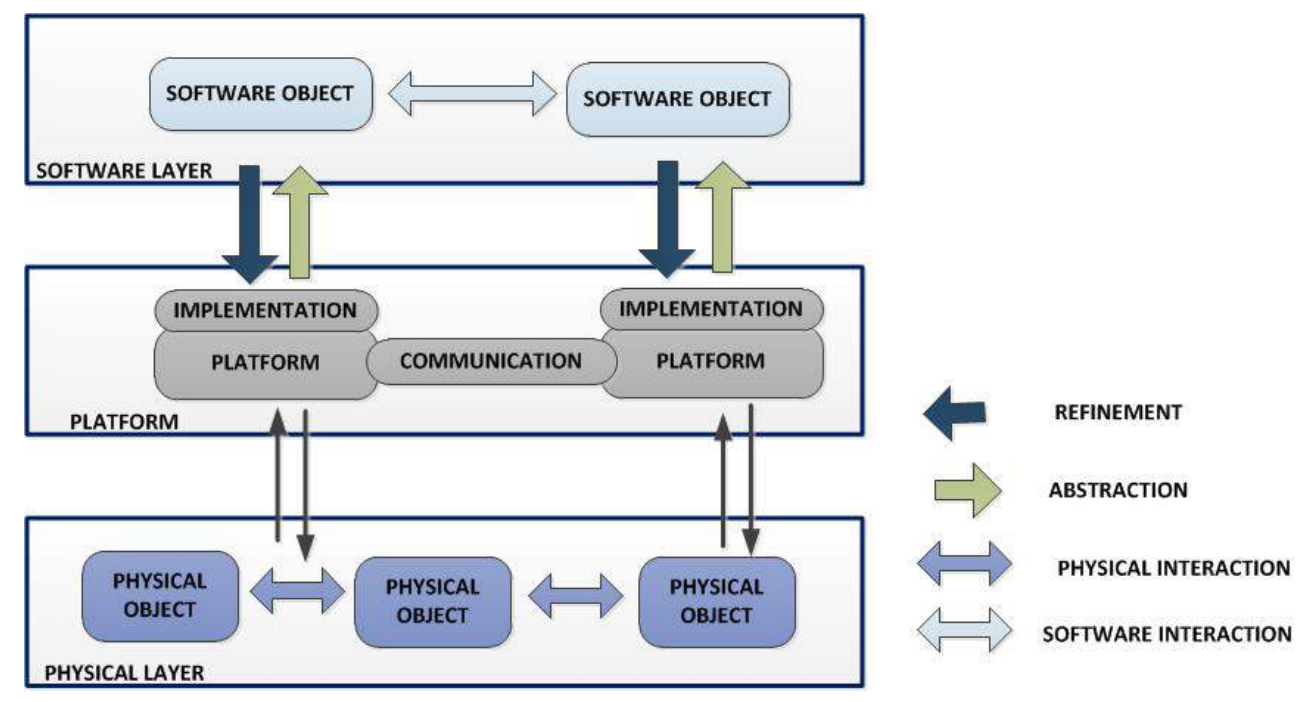

Figure 4: Layers in CPS along [77] 
CPS in control theory present a collection of particular characteristics which define the system operation. Some of these characteristics are common, as we are seeing, to all CPS (independently of the technological domain or application); others, however, are particular of the control theory domain. In $[49,81,82]$ authors identified the following six characteristics for CPS in control theory:

- Cyber capabilities are deployed in the every element in the platform: the software is embedded in the hardware.

- Components in CPS are closely integrated

- CPS are networked

- CPS show dynamically reorganizing/reconfiguring: CPS, as very complicated systems, must have adaptive capabilities.

- Control loops in CPS must be closed. In CPS the most advanced feedback control technologies have to be widely applied.

- Operation must be dependable, and certified in some cases

\subsection{RESEARCH TOPICS ON CPS AND CONTROL THEORY}

Reviewing the works on CPS in control theory $[71,73,74]$ two main research lines can be identified [9]. The first tries to obtain high-performance control system; the second tries to reach a deep penetration of sensing and actuation into the physical world. In the first line, the challenges which are usually addressed are: the obtaining of a much tighter integration among the different components (see Figure 5) and the creation of robust systems being able to work under uncertainty and complexity. In the second line, the definition of new miniaturizing techniques and the improvement in the processing capabilities integrated in sensors and actuators are the most common topics ${ }^{1}$.

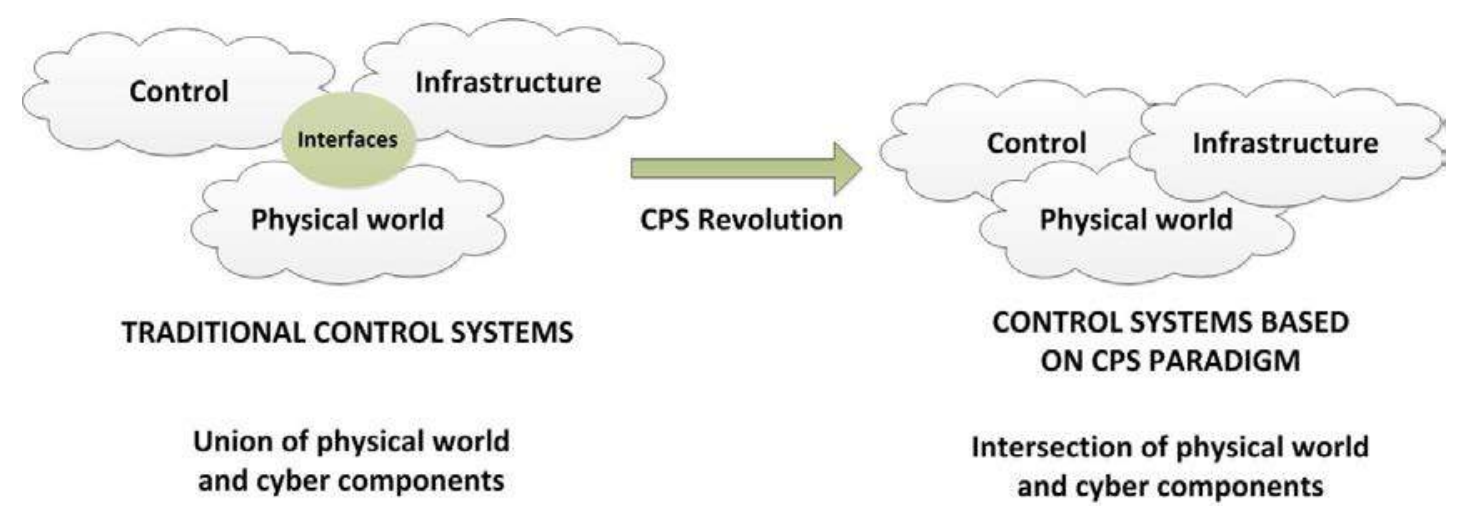

Figure 5: Evolution from legacy control systems to CPS

Some authors have proved that the Moore's law practically resolves the challenges related with the second research line $[9,83]$. Therefore, most works on CPS are focused on obtaining highperformance control system.

The creation of high-performance systems through the increment of the integration level among the different components in CPS is usually addressed by selecting only two components and trying to embed one of them in the other.

\footnotetext{
${ }^{1}$ Boldface words are used to emphasize the main research topic in each technological domain
} 
The most common topic is trying to integrate control policies in the pervasive sensing platform. Traditionally, control policies are completely independent from the infrastructure, and they are programmed after deploying the system [84]. This approach, however, is not valid to meet the characteristics expected from CPS, because of their intrinsic complexity and dynamical behavior. To meet those characteristics and being able of applying complex control policies, the control processes, the physical world and the pervasive sensing infrastructure must be modeled together. In this context, the most challenging situation in CPS modeling is to select a set of consistent models for physical environment, software, hardware, etc. In this way, one very interesting topic on CPS research is model definition, creation and integration. In [85], for example, it is proposed a really successful new framework for CPS modeling called "CPS modeling integration hubs". The work describes a new methodology and modeling environment for CPS, including a core composed of architecture, verification, analysis, hardware and software models (together with a requirement repository) which may be complemented with new modules if required. The selected modeling language is based on ModelicaML [248]. It is a remarkable work, as authors have tested the proposal with good results by modeling power grids, micro-robotics or vehicle management $[86,87]$.

Other important topic is network integration in control systems. CPS propose a scheme where networks are part of the control components, and latency requirements and storage restrictions are not only addressed with network engineering, but also using knowledge about the physical processes [9]. However, compared with legacy control systems, CPS for control theory are still at an elementary stage [88]. Thus, related to the inclusion of networks in the control components, usually only one topic is addressed: the sample scheme [19]. The traditional approach consists of sampling the physical world periodically or at predetermined instants. However, CPS allow implementing "control-on-demand" policies [9] which enable an important saving of resources as energy. For example, in the called event-based control systems, sampling is only triggered when a certain event occurs (formally that is named as Riemann or Lebesgue sampling [89]) and the called self-triggered control systems can decide the intervals when the physical signals may be unsampled and the appropriate instant to sample it again [90, 91, 92].

The creation of robust systems being able to work under uncertainty and complexity is basically focused on the impact of networks uncertainties (noise, delays, packet losses, etc.) in the studies about CPS stability (extremely important in feedback systems). In order to include the network-induced delays in traditional stability models, several proposal have appeared: some of them based on lossless channels in which the packet priority is dynamically modified [93, 94] or the maximum transmission interval is limited [95]. Others propose mathematical analysis such as Lyapunov exponents [96], hybrid systems [97] (see Section 4) or asynchronous dynamical systems (ADS) [98]. Finally, the most realistic proposals consider channels with losses, modeled as Markov chains [99] or channels with additive gaussian noise [100, 101, 102, 103].

Finally, apart from the main research lines, two additional groups of contributions may be found: on the one hand, contributions on the main CPS aspects and, on the other hand, miscellaneous topics of which we are reviewing the most usual ones: decentralized systems and control techniques for CPS.

With respect to the key aspects identified in Section 2.2, context-awareness is maybe the most studied topic in control theory. In general, works on this topic deal with the problem of making 
a control decision considering (at least) all the relevant information about the context [276]. In order to create a stable control loop, between the information acquisition and the actuation it must pass a limited time, so different proposals in order to reach these objectives may be found. Some works propose a predictive scheme [276], while others (focused on human environments) deal with fuzzy control techniques (the human activities are considered context information) [265]. Furthermore, proposals about human environments are also the basic scenario for machine learning works in control theory. In fact, one of the most challenging scenarios for control theory is human control, as human behavior is quite random and complex. Thus, in works about the human-in-the-loop problem, pattern recognition techniques for human activity inferring (a type of machine learning solutions) are usually included [277]. In general, it seems that Bayesian networks are the most adequate technique, so control application for humans tend to consider them [17][278]. Recent works about self-adaptation in relation to CPS in control theory are sparse. In this context, self-adaptation is usually named as "self-tuning", understood as the ability of control systems to modify its internal parameters in order to optimize an objective function [279]. Typical works on this topic are not specifically designed to CPS although they may be applied to this field. Mathematical analysis about the undelaying dynamical problems (stability, fixed points, etc.) are the main contribution in papers on selftuning systems [280][281]. On the contrary, fault-tolerant control is a very popular topic, although most contributions are pretty similar. The basic idea is to construct a control parametric function, being able to recover the stability (partially or totally) if any malfunction event appears [282][283][284]. Finally, different proposals on control with CPS including mobile nodes have been reported. First works on the topic tried to control mobile robots by means of parametric functions [291] (recently similar papers have been also published [290]). Nowadays however, the interest is generally focused on Wireless Sensor Networks (WSN) with mobile nodes, and the fluctuations due to mobility [285][286]. In [273], for example, it is proposed a new control loop scheme for mobile nodes, where the WSN is connected to both, the input and the output of the controller (traditionally it is only connected to the input).

With respect to the miscellaneous topics, in fact, decentralized (distributed) control systems are also usually threated in works on CPS [104]. Sometimes, these works are specifically focused on large-scale systems [105], or on optimal control [106] or real-time applications [107]. On the other hand, specific control techniques for CPS have been also proposed: new design methodologies based on achieving the balances among robustness, schedulability and power consumption [108] have appeared; particular controllers involving resilient power junction have been designed [109] and specific problems and application have been investigated [110, 111].

As CPS for control theory present the simplest architecture, and control systems are interesting for industrial companies, this technological domain is the one with the greatest real application for the CPS nowadays. One of the most promising fields is Smart Manufacturing [13]. CPS in the future industry (now being developed, for example, in Germany) will help to improve efficiency, productivity and safety and to create new forms of flexible work and collaboration [112].

\section{CYBER-PHYSICAL SYSTEMS IN COMPUTER SCIENCES}

The term "Cyber-Physical Systems" emerged around 2006, when it was coined by Helen Gill at the NSF [113]. Very soon, in the domain of computer sciences, E. Lee [24] was the first who 
wondered about the validity of traditional assumptions in computer sciences for the next technological revolution. As result, computer sciences are the domain where most contribution can be found to CPS (from theoretical results to industrial projects).

In this section we review the origin of CPS, the most remarkable theoretical results on CPS in this technological domain and the main research lines for CPS in computer sciences.

\subsection{THE ORIGIN OF CPS}

In the physical world, the time passes inexorably and it is intrinsically concurrent. However, nowadays, abstractions in computer sciences do not reflect these properties. Lee argued that technical progress is impossible while abstractions in computer sciences and properties of the physical world do not match [114]. CPS is the proposed solution to bridge that abstraction gap.

CPS are usually related to "cyberspace" (used by William Gibson in the novel Neuromancer), however, the roots of the term CPS are older and deeper [1]. CPS comes from the word "cybernetics," which was coined by Norbert Wiener [115]. Wiener derived the term from the

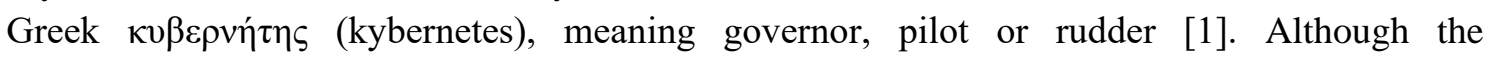
mechanisms he used did not involve digital computers, some authors claim [1][113] that the basis of his work are similar to those used today in a huge variety of computer-based systems.

Unlike in control theory, where sensing technologies have been traditionally employed, in computer sciences pervasive sensing was introduced by CPS. Actually, the Wiener's notion of cybernetics was deeply rooted in closed-loop feedback, so CPS moved to computer sciences the traditional pervasive sensing and actuation technologies from feedback control.

Although all approaches for CPS show a technology that deeply connects the physical world with the cyber world [115], computer sciences experts claim their approach for CPS is more foundational and durable than the others, because it does not refer to either implementation approaches (as in communication engineering) nor particular applications (as in control theory).

Most experts on CPS in computer sciences agree with the original definition [116, 117, 118, 119]. So, "a cyber-physical system is an integration of computation with physical processes. Embedded computers monitor and control the physical processes, usually with feedback loops where physical processes affect computations and vice versa". For these authors, CPS is about the intersection, not the union, of the physical world and the cyber components.

\subsection{THEORETICAL RESULTS}

As we said, most authors in computer sciences agree with the definition proposed by E. Lee for CPS. However, other interpretations, with much less impact, have been propounded. In [36], for example, "CPS are physical and engineered systems whose operations are monitored, coordinated, controlled and integrated by a computing core". Despite these differences, the way in which authors view CPS in computer sciences is similar. Actually, integration of electronic components in physical objects is not new. Embedded devices [120] have been successfully employed in many sectors such as automotive, aircraft control, games or home appliances. Nevertheless, these devices used to be "closed boxes" that do not offer any service to the 
outside. The radical transformation authors on CPS from computer sciences envision comes from connecting several of these devices, over which complex software applications are deployed [121].

Architectures for CPS in the computer sciences domain are more complex than the ones presented in the control theory domain; mainly because they used to include computation elements such as semantic technologies or context-aware applications. Figure 6 shows the prototype architecture for CPS described in [122], which is one of the most cited proposals $[123,124,125]$. In control theory architectures are usually designed following implementation criteria (external systems in one layer, hardware in other one and software in the third one). However, in computer sciences all layers include hardware and software elements, and architectures used to be designed depending on the abstraction level.

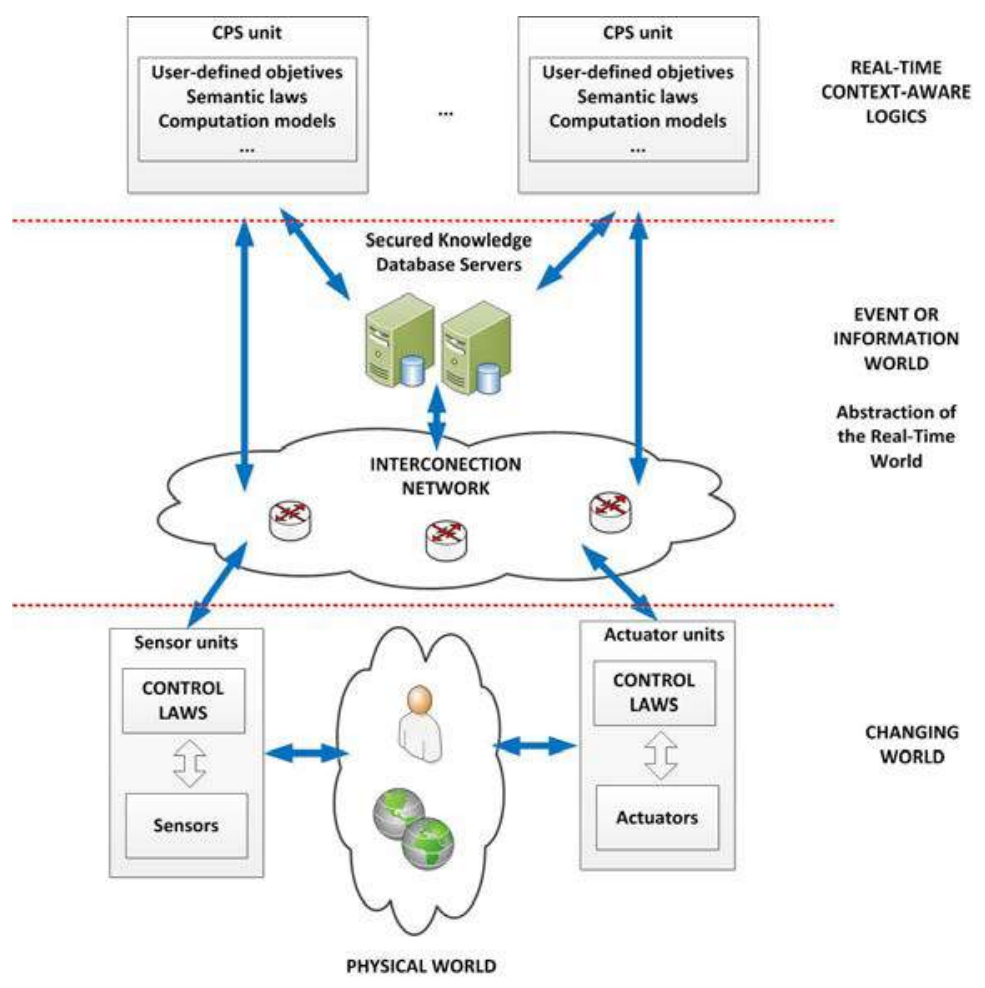

Figure 6: Layers in CPS along [122]

As it can be seen in Figure 6, the proposed architecture consists of three different layers. The first layer (called "changing world") includes all elements with continuous dynamics and which behavior is controlled by physical laws, i.e. the physical world, transducers and control components (embedded in the sensing platform). The second layer (the information world) contains some simple data analytics elements which translate from "raw facts" (events) produced by sensors and actuator to abstract information used in computing elements. Finally the third layer (real-time context-aware logics) models at high-level the changing-world and determines at real-time the system's behavior. In the third layer computational algorithms would run in parallel with processes in the physical world, so CPS must coordinate two "types of time" [126] (contrary to what happens in control theory, where only the physical time is considered).

The characteristics that must show a system to truly be a CPS have been also deeply investigated. Although many proposals may be found, there exists a certain agreement about the necessity a system to show the following four characteristics to be a CPS [127]: 
- Heterogeneity: CPS may include several types of devices. The underlying pervasive sensing platform must be able to incorporate and configure sensor nodes (with a certain amount of memory), mobile devices (such as smartphone) and networked servers.

- Unreliable networking: Devices in the pervasive sensing platform are usually connected through low-power wireless communication technologies such as 802.15.4 [128] and Nordic [129]. These technologies show a high rate of packet loss and CPS may be able to work under these circumstances.

- Mobility: In CPS mobility must be supported. In general devices in CPS interact opportunistically, as the movement may be determined by unpredictable factors such as the human behavior.

- Tight environmental coupling between the system and the external world. In CPS the system border tends to be unclear [9].

The creation of the "Cyber-Physical Systems Virtual Organization" (CPS-VO) in the U.S. [130] has allowed deepening in the conceptual issues on CPS following the computer sciences approach. In particular, we must remark the concept map proposed by the Berkley University which summarizes the characteristics, requirements an application of CPS [131]. In Figure 7 we include a graphic presentation of that concept map.

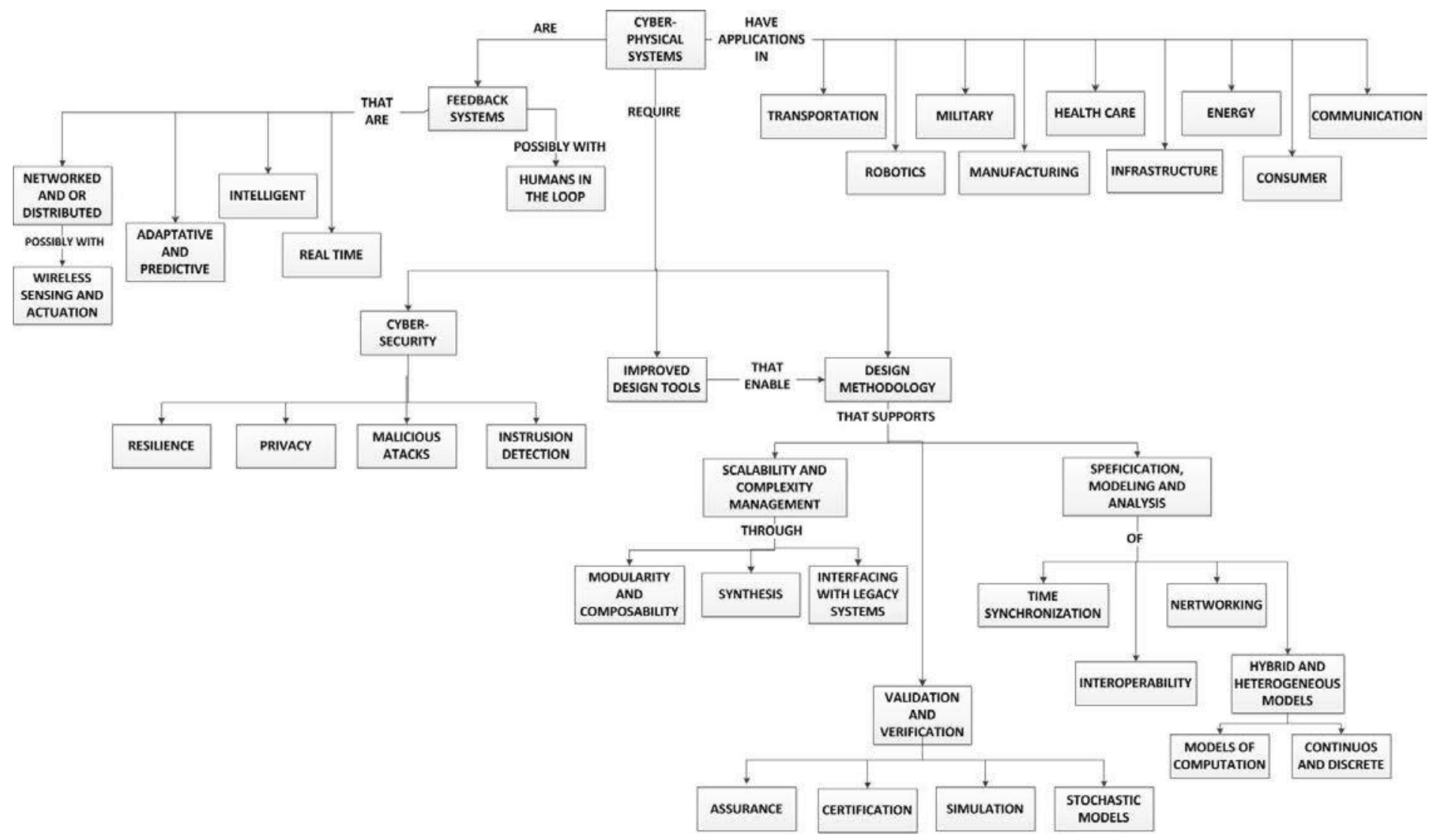

Figure 7: CPS: the concept map. Source: Berkley University

Figure 7 contains two important characteristics about CPS in computer sciences, not showed before, which must be remarked. First, nowadays, as we said in the introduction, CPS is a particular application of pervasive sensing. However, although all current works on CPS include 
a sensing platform $[132,133]$, computer sciences experts also envision other types of interaction between the physical and the cyber world (unknown today), then CPS in Figure 7 are "system possibly with wireless sensing and actuation". And, second, traditional feedback systems (including CPS for control theory) are reactive: the system receives a stimulus and it reacts [37]. However, CPS in computer sciences may be also predictive: the system reacts to a future stimulus in order to avoid, modify or cause it [134]. This new philosophy requires technologies to analyze all data available (from the past and present) and predict the future. In this point, CPS joins with Big Data and similar solutions [9].

\subsection{RESEARCH TOPICS ON CPS AND COMPUTER SCIENCES}

Research lines on CPS in the computer sciences domain are much more numerous than in control theory. In general terms, each box in Figure 7 represent a research topic which involves various specific technical challenges. For example, three issues arise with the pervasive sensing platform (named as "wireless sensing and actuation" in Figure 7): data models for distributed sensor data [135], location of sensors and actuators, and time synchronization [131].

However, some works have identified the main research lines in CPS in the computer science domain. Namely [19]: hybrid systems, distributed systems, real-time applications and security. Other topics, such as certification, have been scarcely investigated, and only preliminary reports on the state of the art have appeared [121].

A hybrid system is one system which mixes continuous dynamics and discrete dynamics. As CPS in computer sciences include both the physical world (continuous dynamics) and computing elements (discrete dynamics), usually they are designed, analyzed and validated as hybrid systems. The most simple hybrid systems employed in the CPS sciences are switched system. These systems switch among various continuous operation modes, along a control signal [136]. Overall, with these systems the stability is studied [137]. A more general framework for CPS are hybrid automaton (HA) which allows designing complex dynamics. Several mathematical formalisms for HA and CPS have appeared: from works about general issues [138], to studies for CPS specification [139] and validation [140]. Particularly important are frameworks for algorithm verification, which allows validating the behavior of software to CPS "in theory" [141]. Furthermore, well-known results bout HA complexity, such as the concepts of simulation and bisimulation [142, 143], are being used in order to reduce the intrinsic complexity to CPS when analyzing them. Finally, HA also allow automatic synthesizing of CPS given a specification and using supervisory control [144], game theory [145], piecewise-affine hybrid systems [146] or discrete-time linear systems [147]. Based on hybrid systems and HA have appeared for algorithm validation [148] and system synthesis [149].

CPS are intrinsically distributed (see Figure 7). Therefore, one of the most important issues on CPS is verifying the behavior in the global system. However, due to the complexity of the problem, no general solution has been proposed. In particular studies about the correctness in distributed CPS are mainly focused in automobile traffic control systems [150, 151]. Besides, works about the design of distributed CPS have appeared, for example for automated traffic intersection [152]. All these works, however, use pencil-paper techniques [153, 154], and automated methods would be valuable. 
CPS executes real time applications. In order to enable real-time working, CPS must include three technologies [19]: real-time scheduling, real-time systems and real-time networking. In all these areas there exist classical results which are applicable to CPS, and new results specific of CPS.

In real-time scheduling many results are classical proposal, now applied to CPS [155, 156, 157]. However, with the objective of supporting temporally predictable execution of the computing tasks in CPS, some authors have proposed intelligent algorithms. These algorithms must order adequately all the computing tasks in a CPS, so that all temporal constraints of the task are fulfilled. Among other proposals, it may be found real-time queuing techniques [158], real-time scheduling for distributed systems [159, 160], techniques of resource reservation for real-time applications [161] and real-time scheduling algorithms for embedded systems [162].

In respect of real-time systems, CPS face integrating different types of applications (real-time and not real-time) over the same pervasive sensing platform [9], moving from a federate approach to an integrated approach (see Figure 8). To overcome such issue, middleware based on the proper abstractions from the system complexities are used $[19,151]$.

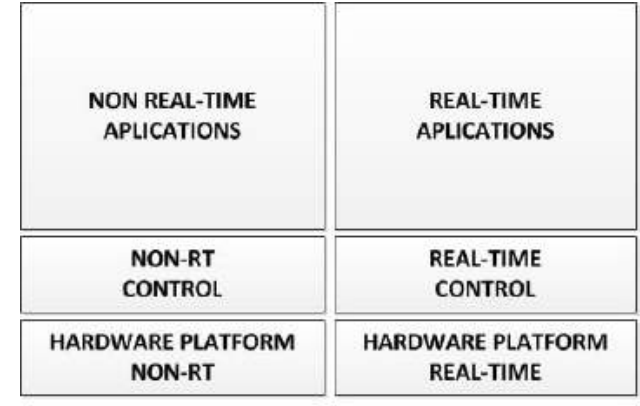

FEDERATED

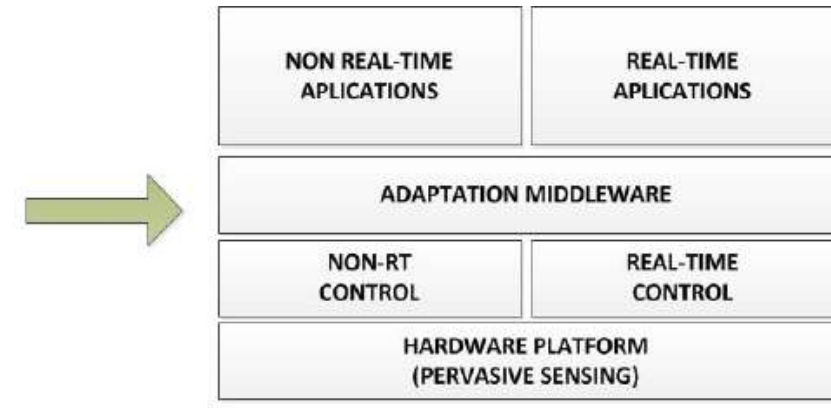

INTEGRATED

Figure 8: Evolution from traditional federated systems to integrated CPS

One of the best known examples of these adaptation middleware is the Common Object Request Broker Architecture (CORBA) [163]. It allows the interoperability among software objects running in different machines in a heterogeneous distributed environment. There exist other proposal, such as real-time CORBA [164], which includes real-time scheduling. Etherware [150] is a middleware to be deployed in large-scale networked feedback systems. OSA+ [165] is another middleware for distributed real-time embedded systems. Finally, some authors have proposed programming languages for real-time systems such as the successful Signal [166] and Esterel [167].

The last element related to real-time application in CPS is real-time networking. This issue has been widely studied, and most results employed in CPS are solutions of the state-of-the-art $[168,169]$. However, some specific proposals such as routing protocols for real-time pervasive sensing platforms $[170,171]$ are sometimes investigated.

Security is basic for systems which pretend to control critical infrastructures such as transport, health care or military equipment $[172,173]$. Thus, many works on particularities $[174,175]$ of security in CPS and its vulnerabilities have appeared [176, 177]. Mainly, CPS tend to be distributed and involve thousands of embedded system which, moreover, may operate at realtime, so intelligent analysis cannot be easily made. Some generic proposals are about including 
security in the physical world [178], and other maintain the traditional scheme of detecting attacks $[179,180]$. However, most works on safe CPS are focused on specific implementation for Electric Power Grid [181, 182, 183].

A special case of security solutions for CPS are which are specifically designed to CPS including mobile nodes. At this point, one of the most important identified aspects of CPS is addressed. In general, in computer sciences works, mobility is considered as any other characteristic to be included in the models [289]. However, in the case of security, mobility has a special recognition. In particular different soft signature technologies (typically certificateless techniques) have been defined [274][287] [288]. Following this line, we are reviewing the stateof-the-art in the other key aspects of CPS in relation to computer sciences. Context-awareness is not the most common topic in works about CPS in computer sciences. However, some contributions have been reported. In particular ontologies describing the different information fields that describe a context have been proposed [293][294]. Additionally, logical rules, (semantic) algorithms and procedures in order to process the context information (adding variables, comparing contexts, etc.) have been described [292]. Machine learning, on the contrary, is a very popular issue. Three main types of contributions may be found. First (and probably the most common approach) different machine learning techniques are proposed with the objective of detecting cyber-attacks and intruders in CPS. Pattern recognition technologies based on Markov's chains [296] or Bayesian inference [297] are common, but other proposals such as neural networks may be also found [298]. The second topic related to machine learning is physical model creation [88]. As describing systems as complicated as CPS may be a difficult task, different instruments to assist CPS designers to perform this activity have been proposed, several of them based on machine learning techniques [295]. The third and last topic is research on hybrid automatons. Hybrid automatons combine traditional finite state machines with logical rules and machine learning techniques in order to reason about the state of the physical world [266]. It is a common approach for CPS applied to natural and social sciences (biology, education, etc.). With respect to fault-tolerant systems, the most interesting works about cyberphysical applications belong to the computer sciences domain. In particular, advanced solutions (usually mathematical models) to keep CPS operating under the planned constraints by means of an external control (even in case of a failure event) have been reported [275]. As closure, self-configuration and self-adaptation policies are not usually addressed in a separated way as, in computer sciences, CPS are adaptive by default. However, in 2016 a team of Swedish computer science researchers proposed a systematic methodology and algorithm to study the state-of-the-art on the self-adaptation technologies for CPS [299]. Only a small number of the reviewed references are provided, but conclusions are remarkable. For example, they found that self-adaptation is understood in very different ways (as reconfigurability, reliability or interoperability among other possibilities); and that most self-adaptation technologies for CPS are integrated into high-level applications and are based on mapping functions.

Finally, other interesting topics addressed in the research literature are CPS simulation [184], where even a simulator called CyPhySim $[185,186]$ has been developed, and modeling [113, 187]. 


\section{CYBER-PHYSICAL SYSTEMS IN COMMUNICATION ENGINEERING}

The use of the term CPS in communications engineering is very recent and low. Experts on communication engineering use to employ older terms such as smart environments, Internet-ofThings (IoT) or Sensor Networks which may all fall under the CPS design umbrella [188]. However nowadays it is not clear the relation between these terms and CPS (see Section 2.3). In general, communication engineering experts understand CPS as a particular scenario of IoT where the underlying hardware is a pervasive sensing platform and where Internet services allow creating feedback control loops [66].

In this section we briefly review the characteristics of CPS in communication engineering, as well as the main research topics.

\subsection{CHARACTERISTICS OF CPS IN COMMUNICATION ENGINEERING}

Definitions of CPS in communication engineering are not very common. In general, however, they are focused on the Internet Services and the underlying devices (the traditional embedded devices). One example of these definition is proposed by Kim [19] which says "CPS are the next generation of engineered systems in which computing, communication and control technologies are tightly integrated".

Table 2: Characteristics of CPS in communication engineering

\begin{tabular}{|c|c|}
\hline Non-particular characteristics & Specific characteristics \\
\hline $\begin{array}{l}\text { Components in CPS include various } \\
\text { encapsulations, materials, software types, } \\
\text { dynamics, etc. }\end{array}$ & $\begin{array}{l}\text { Components in CPS may present both pre-defined } \\
\text { and ad-hoc connections }\end{array}$ \\
\hline $\begin{array}{l}\text { CPS must join the a digital part and an analog part } \\
\text { CPS are heterogeneous systems }\end{array}$ & $\begin{array}{l}\text { CPS should implement various problem-solving } \\
\text { strategies }\end{array}$ \\
\hline $\begin{array}{l}\text { CPS should present some level of intelligence } \\
\text { CPS may change their behavior dynamically }\end{array}$ & $\begin{array}{l}\text { CPS must build knowledge from both built-in } \\
\text { formal knowledge (obtained from the pervasive } \\
\text { sensing platform) and knowledge generated by } \\
\text { artificial intelligence }\end{array}$ \\
\hline Components in CPS are open systems & CPS include distributed decision-making \\
\hline $\begin{array}{l}\text { Components in CPS have to reorganize themselves } \\
\text { depending on the circumstances }\end{array}$ & CPS should support distributed problem solving \\
\hline $\begin{array}{l}\text { CPS must be able to learn from history and be } \\
\text { unsupervised }\end{array}$ & $\begin{array}{l}\text { CPS should be context-depending } \\
\text { CPS present different spatial scales and temporal }\end{array}$ \\
\hline $\begin{array}{l}\text { Different strategies to maintain the integrity, } \\
\text { security and reliability of CPS should be } \\
\text { implemented }\end{array}$ & ranges \\
\hline
\end{tabular}

Works about the characteristics of CPS are works much more exhaustive. In [189], for example, they are identified sixteen basic characteristics a system much show to be considered as CPS. Some of these characteristics are common to other approaches, but others are particular of the communication engineering domain (although they optionally could be also present in other 
domains). Table 2 classifies the cited characteristics as they appear or not in other domains. Similar characteristics have been proposed in other works such as [190].

Architectures for CPS in communication engineering may be presented following a networklike scheme or a logical stack. In Figure 9 it is presented a network-like architecture described originally in [191].

As can be seen, the architecture is similar to other proposals for generic Internet services [192], including three entities (hardware platform, control and end-user services) connected through the Internet. In some cases, each entity is, at same the time, described as a collection of sub-nets or networked servers, creating a hierarchic architecture [178].

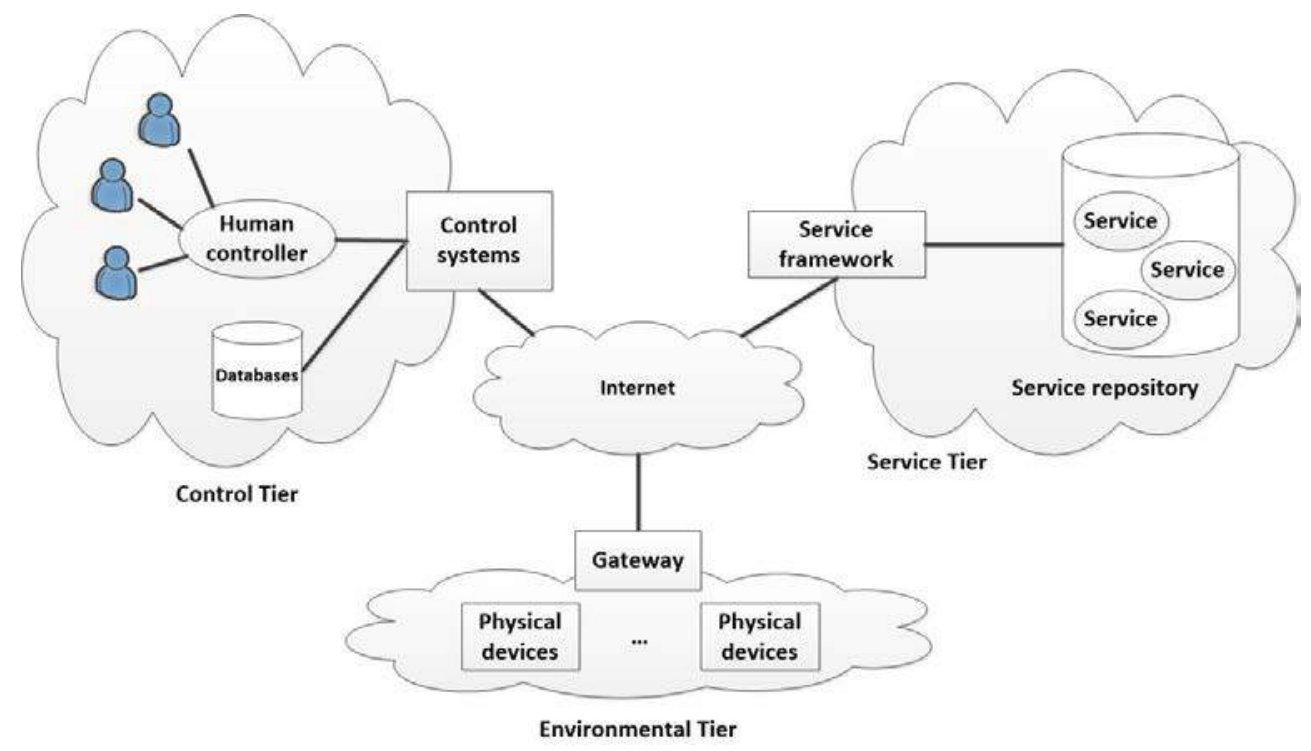

Figure 9: Architectures for CPS in a network-like scheme [191]

On the other hand, Figure 10 shows a proposed architecture for CPS [193] with a great impact (described as a logical stack including very common and famous technological such as OSGi [194] and JNI [195]). In this case, the architecture also presents three layers: the physical layer includes the hardware devices, the second provides control and the third is dedicated to service provision. All layers (as well as the humans or machine which uses the services) are communicated through the Internet (or local-area network, depending on the case). In this approach the physical world are not considered part of the system. 


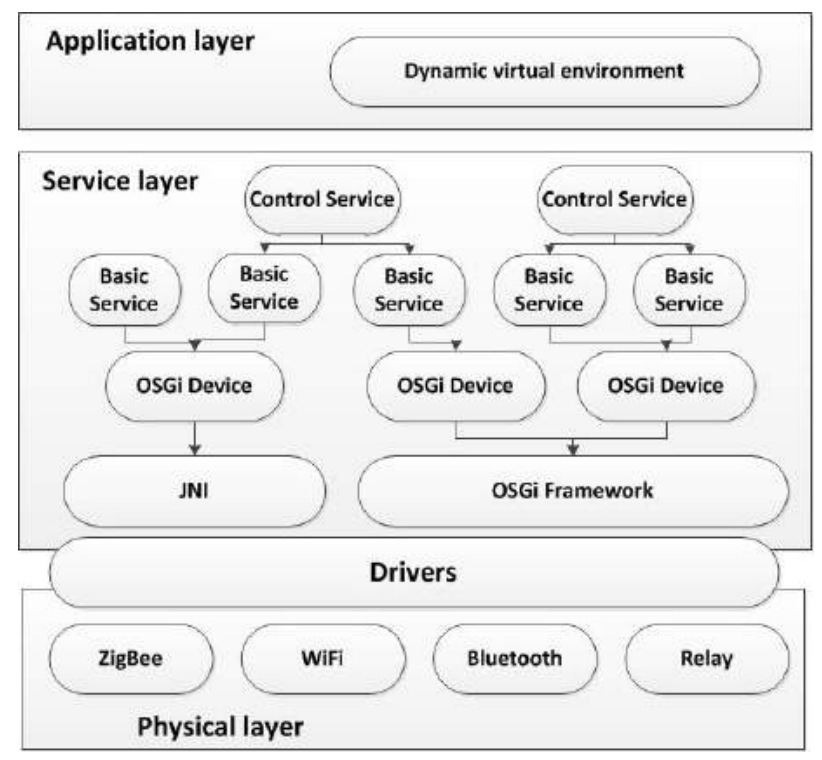

Figure 10: Architectures for CPS in a logical stack [193]

Other architectures for CPS in communication engineering, similar to which showed in Figure 9 and Figure 10, may be found in [190] and [66].

\subsection{RESEARCH TOPICS ON CPS AND COMMUNICATION ENGINEERING}

Research topics on CPS in communication engineering include all the issues traditionally related to IoT, (Mobile) Wireless Sensor Networks (WSN) or Machine-to-Machine Communications. Nevertheless, as the relation among these concepts and CPS is not clear, usually they are treated independently [196]. Any case, many works have tried to compile the state-of-the-art in these topics [197, 198, 199, 200, 201].

CPS and IoT share many research topics and usually almost any solution produced for one scenario is directly applicable to the other. In this field, most complicated issues, such as the self-configuration of heterogeneous hardware platforms are not usually addressed (in this case a possible solution might be found in [64], where network configuration is based on the concept of "reputation" of a node). The most common topics, then, are related to data services. Particularly important are the topics of service composition, service management and service access [62]. In CPS, the most investigated solution for composed services is expressing them as Business Process Execution Language (BPEL) executable files, where each task calls a service (composed -as workflows could be nested- or simple) [202]. Service management in CPS is usually based on semantic technologies and the definition of different concepts of Quality-ofService [203, 204]. Service access in CPS is especially challenging due to the different interfaces offered by end-devices. Two basic solutions are usually proposed: embedded TCP/IP stacks in all devices [205] and translation layers which adapt a generic web services language (offered to the applications) to the specific interfaces of the different devices [206].

Related to (Mobile) Wireless Sensor Networks, the most popular topic is the definition of energy-efficient routing protocols [207]. Some proposals are based on specifically adapted traditional unicast protocols [208]. Others propose new approaches, being especially important GPS-based approaches [209]. Another important problem in WSN is modeling the coverage 
area of sensors. In order to completely cover a certain extension, sensors must be properly placed. The problems associated with this issue have been deeply investigated [210], and some mathematical approaches proposed [211]. The problem of replacing batteries [212], techniques to sleep sensors [213] and technologies to filter automatically the relevant information while routing packets are also usual topics [214].

Research on device-to-device communications (D2D) is also a very important topic, as it is one of the most promising fields on communications engineering (it is applied to CPS, IoT and mobile networks, among other areas). Based on this technology, mobile nodes in a CPS may be networked and coordinate to better understand the environment and relate to the physical world in a more efficient way. Historically, D2D communications were defined to enable multi-hop relays in cellular networks [251]. However, nowadays, several different proposals may be found. In relation to CPS, enhanced studies about peer-to-peer communications based either on mobile communications (typically LTE) [252] or on wide-area radio access technologies [253][254] are the most interesting works. Usually, power consumption [255], traffic offload [256], or the number of concurrent D2D links [257] are the most interesting variables to enhance. Nevertheless, other topics are also relevant. For example, the inclusion of QoS or power constraints in session negotiation [258], as well as the use of new generation multicast technologies [259][260], are promising proposals.

Finally, in M2M communications most popular research topics are the analysis of new radioaccess technologies (RAT) for low-power, low-rate communications and/or massive access communications. Some proposals are based on mobile communications [215], but most of them are based on Home-area networks (HAN) such as Bluetooth [216] and, mainly, ZigBee or IEEE 802.15.4 [217, 218].

Although, as we said above, self-configuration of heterogeneous hardware platforms is not usually addressed, other types of self-configuration and self-adaption are successfully investigated. In particular, different works about how to address self-* properties in CPS for industrial applications may be found [54][300][301]. Typically, these proposals obtain a pyramid-like architecture where self-adaptation is placed in the top level. Works about selfadaptable (sometimes understood as self-aware) hardware architecture for cyber-physical devices have been also reported [301][302]. The last common topic about CPS self-adaption in communication engineering is the creation of middleware layers to support the self-* properties [303]. In order to finish this section we are reviewing the existing literature on the other key aspects of CPS. The definition of architectures and functional implementations of CPS is also common in relation to context-awareness. Architectures being able to store and manage context information [29] and/or provide context-dependent services (in medical environments, for example) [304][305] are common. Moreover, very recently (2017), context-aware sensing and actuation based on CPS (a technology which allows systems to require, obtain and use in an intelligent way context information) has also received attention [306] (applied, for example, to vehicular systems [307]). Fault-tolerance is rarely required to CPS, but it has been exhaustively investigated in WSN. About this topic, most works propose different fault-tolerant routing or topology-management protocols [309][310][311], but fault-tolerant WSN applications (specially for critical scenarios such as medical environments) may be also found [308]. With respect to mobility, communication engineers defined in 2013 the concept of "mobile CPS" [271]. In the same paper, the authors propose different protocols to support the mobility of cyber-physical nodes. Originally identified with crowdsensing systems, mobile CPS are now a well-known term, and many applications and works about "CPS and mobile computing" have 
appeared [312][313][314] (most of them focused on protocols and solutions to integrate both functions). Finally, machine learning and CPS is not a primary research topic for communication engineers. In general, works including learning techniques use them as instruments to build a bigger system (which is the research target). In fact, learning techniques are commonly employed in smart (or cyber) manufacturing systems [316] or in the called Cyber-Physical Production Systems (CPPS) [315]

\section{STANDARDIZING CYBER-PHYSICAL SYSTEMS}

CPS paradigm has received a great support from academia, institutions, governments and industries since the beginning. In Section 4 we cited some organizations such as the NSF or the CPS-VO (both from the U.S.), although the European Union through the Advanced Research and Technology for Embedded Intelligence Systems (ARTEMIS) project [219] and the European Commission through the Horizon2020 program [220] have also greatly supported the CPS revolution. As a result of this governmental support, the main standards organizations have included the CPS (one way or another) in their agendas. In this Section we are reviewing the different attempts to standardize CPS and we compare all approaches reviewed in this work.

\subsection{OVERVIEW OF CPS AND STANDARDS ORGANIZATIONS}

Basically, three standards organizations have addressed the challenge to normalize the CPS: the International Organization for Standardization (ISO), the European Telecommunications Standards Institute (ETSI) and the National Institute of Standards and Technology (NIST). Other important institutions such as the German Academy of Science and Engineering are also working on CPS [221], but its results do not have the consideration of standards.

The ISO, in collaboration with the International Electrotechnical Commission (IEC), created in 2012 a standardization special working group (SWG) [222] to develop a standard on the Internet-of-Things (IoT). Strictly, the ISO is not working on CPS, however, as we saw in Section 2.3, more and more authors show that IoT and CPS are different perspectives of the same concept. One of the purposes of the ISO is, precisely, to determine how much similar IoT and CPS are.

The SWG for IoT, named first as ISO/IEC JTC 1/SWG 5 and later as ISO/IEC JTC 1/SWG 10, was established in 2012 at the 27th plenary meeting of ISO/IEC JTC 1[223] in China. ISO's vision of CPS is similar to the approach of communication engineering, so the collaboration between the SW10 and the SW7 (dedicated to Sensor Networks) is official [224]. Nowadays, two important documents have been publicly distributed: an IoT mind map [225] and a report about differences of IoT, M2M, and CPS [226] (both published in the final months of 2014). However, no result about architectures, applications, etc. is public yet.

The ETSI is totally focused on communications and networking in the context of IoT and CPS. In particular, this organization is responsible of the standards about Machine-to-Machine (M2M) communications [227]. This technology (among others) is widely employed in CPS, however, the ETSI, nowadays, is not working in standardizing CPS as a whole. 
Finally, the NIST, is totally focused on standardizing CPS as a particular technology, different from any other proposal [2]. Its vision of CPS is different from all the previous, and includes all the components cited in Section 2, so all the different approaches for CPS are included in the NIST's proposals. The CPS Public Working Group was established in 2014 and in September 2015 it generated its first public results. The NIST has proposed a notational architecture for CPS, has tried to fix the vocabulary and is trying to develop the key problems of CPS.

Table 3 compares the action of the different standards organizations.

Table 3: Comparison among the action for standardizing CPS in different organizations

\begin{tabular}{|c|c|c|c|}
\hline Topic & ISO/IET & ETSI & NIST \\
\hline $\begin{array}{c}\text { Element } \\
\text { standardized }\end{array}$ & Internet-of-Things & $\begin{array}{l}\text { Machine-to-Machine } \\
\text { communications }\end{array}$ & Cyber-Physical Systems \\
\hline Start date & 2012 & 2009 & 2014 \\
\hline Objetive(s) & $\begin{array}{l}\text { Developing definitions for IoT } \\
\text { and vocabulary } \\
\text { Developing a reference } \\
\text { architecture for IoT } \\
\text { Developing other IoT relevant } \\
\text { standards such as IoT use- } \\
\text { cases, network level } \\
\text { technologies, or } \\
\text { interoperability }\end{array}$ & $\begin{array}{l}\text { Developing and maintaining } \\
\text { standards about: } \\
\text { Requirements (ETSI TS } 102 \\
689 \text { ) } \\
\text { Functional architecture (ETSI } \\
\text { TS } 102690 \text { ) } \\
\text { Interface descriptions (ETSI TS } \\
102921 \text { ) }\end{array}$ & $\begin{array}{l}\text { Developing a consensus } \\
\text { definition, reference } \\
\text { architecture, and a common } \\
\text { lexicon and taxonomy } \\
\text { Ensuring that timing, } \\
\text { dependability, and security } \\
\text { are considered as first order } \\
\text { design principles }\end{array}$ \\
\hline Current state & $\begin{array}{l}\text { Active, last publication on } \\
\text { January } 2015 . \\
\text { Next standards announced: } \\
\text { IoT - Definition and } \\
\text { Vocabulary ISO/IEC } \\
\text { (ISO/IEC NP 20924) } \\
\text { IoT - Reference Architecture } \\
\text { (AWI 30141) }\end{array}$ & $\begin{array}{l}\text { Active, last publication on } \\
\text { March } 2016\end{array}$ & $\begin{array}{l}\text { Active, last publication on } \\
\text { September } 2015\end{array}$ \\
\hline $\begin{array}{l}\text { Published } \\
\text { documents }\end{array}$ & Preliminary report [226] & $\begin{array}{l}\text { Technical Specification (TS) } \\
\text { and Technical Report (TR) } \\
\text { published for all topics (not } \\
\text { European standard) } \\
\text { [227] }\end{array}$ & $\begin{array}{l}\text { Draft for the CPS } \\
\text { framework [230] }\end{array}$ \\
\hline
\end{tabular}

\subsection{THE CASE OF THE NIST}

The NIST define "Cyber-Physical Systems or "smart" systems as co-engineered interacting networks of physical and computational components" [228]. In that way, the NIST sees CPS as a vertical system which may include, eventually, any other implementation belonging to any other technological domain. Moreover, the NIST is leading a wide program to advance on CPS, including the Cyber-Physical Systems Public Working Group (CPS PWG), formed by NIST in 2014. This group has identified five key areas in CPS [2]:

- Vocabulary and Reference Architecture

- Use Cases

- Timing

- Cybersecurity and Privacy

- Data Interoperability 
Only contributions in two areas have been published. First it has been published a report about timing [229] where different technologies such as IEEE 1588 Precision-Time Protocol (PTP), general concepts such as the phase delay and standard as the ITU T Recommendation G.8264 are reviewed. Second a report about a general framework for CPS has been distributed [178]. In this report, the NIST shows the idea of an "Internet of CPS" where systems deployed in different part of the world may interact, offer services to the others, etc. (see Figure 11). Besides, a functional decomposition of these systems is also proposed [230], and a template to describe CPS is also included.

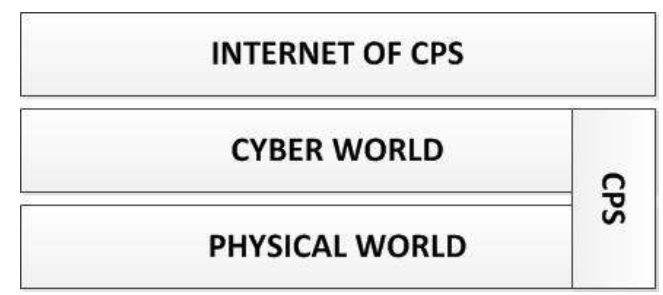

Figure 11: "Internet of CPS" NIST vision

Moreover, the NIST has proposed a notational architecture for CPS (see Figure 12) and now is investigating the different characteristics which present CPS. The proposed architecture consists of six layers and six transversals capabilities which must be included in each layer. The first four layers in the stack match with four of the explained components in Section 2. The fifth layer (Modeling, optimization and simulation) develops and maintain the dynamical computational models. The sixth layer (Business and user goals) refers to the measurable goals defined by users which must be reached. With respect to the transversals capabilities, all of them are well known, except the "Internet-system services", with this name, the NIST refers the connection between the local CPS and the Internet of CPS it envisions.

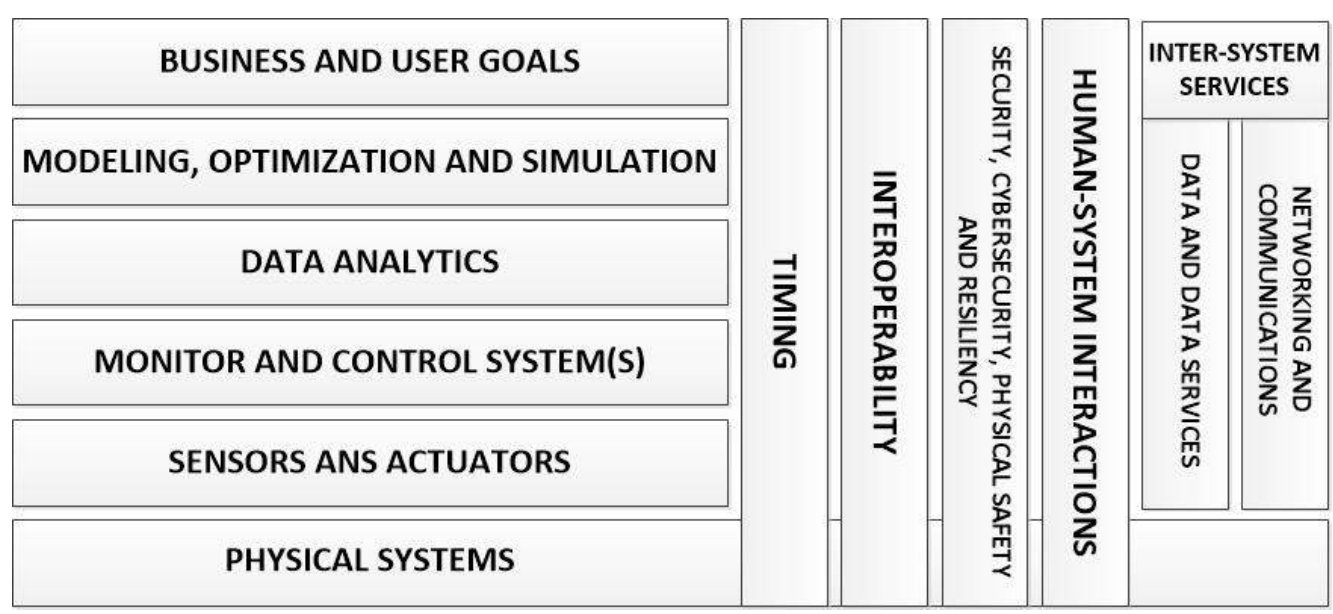

Figure 12: NIST's notational architecture for CPS

The proposals and documents of the NIST are extremely new, so few works considering those results have been published [38]. 
Once reviewed the main four approaches for CPS, in Table 4 we compare the principal characteristics of CPS in each one.

Table 4: Comparison among the different approaches for CPS

\begin{tabular}{|c|c|c|c|c|}
\hline Element & Control theory & Computer sciences & $\begin{array}{c}\text { Communication } \\
\text { engineering }\end{array}$ & NIST \\
\hline $\begin{array}{l}\text { Type of the } \\
\text { dynamics }\end{array}$ & Continuous & Hybrid & Discrete & Hybrid \\
\hline $\begin{array}{l}\text { CPS are } \\
\text { understood as... }\end{array}$ & $\begin{array}{l}\text { Improved } \\
\text { networked control } \\
\text { systems }\end{array}$ & $\begin{array}{l}\text { Integration of computational } \\
\text { and physical process }\end{array}$ & $\begin{array}{l}\text { Framework for } \\
\text { advanced Internet- } \\
\text { services }\end{array}$ & $\begin{array}{l}\text { Networked smart } \\
\text { systems }\end{array}$ \\
\hline $\begin{array}{l}\text { Number of } \\
\text { layers in } \\
\text { reference } \\
\text { architectures }\end{array}$ & 3 & 3 & 3 & 6 \\
\hline $\begin{array}{l}\text { Layers in } \\
\text { reference } \\
\text { architectures }\end{array}$ & $\begin{array}{l}\text { Physical world } \\
\text { Hardware } \\
\text { Software }\end{array}$ & $\begin{array}{l}\text { (Different abstraction levels) } \\
\text { Physical world } \\
\text { Information } \\
\text { Logics }\end{array}$ & $\begin{array}{l}\text { Physical platform } \\
\text { Network and } \\
\text { control } \\
\text { Services }\end{array}$ & $\begin{array}{l}\text { Different } \\
\text { capabilities) } \\
\text { Physical world } \\
\text { Sensors } \\
\text { Control } \\
\text { Data analytics } \\
\text { Models } \\
\text { User goals } \\
\end{array}$ \\
\hline Research topics & $\begin{array}{l}\text { Incrementing the } \\
\text { integration level } \\
\text { (new models, and } \\
\text { new sample } \\
\text { schemes) } \\
\text { Building robust } \\
\text { systems } \\
\text { New control } \\
\text { techniques }\end{array}$ & $\begin{array}{l}\text { Hybrid systems } \\
\text { Distributed CPS } \\
\text { Real-time applications (RT } \\
\text { scheduling, RT systems and } \\
\text { RT networking) } \\
\text { Security } \\
\text { Simulation } \\
\text { Modeling } \\
\text { Improvements in the } \\
\text { pervasive sensing platform } \\
\text { (models for distributed data, } \\
\text { time synchronization and } \\
\text { sensors location) }\end{array}$ & $\begin{array}{l}\text { Wireless Sensor } \\
\text { Networks (energy- } \\
\text { efficient protocols, } \\
\text { coverage models, } \\
\text { batteries } \\
\text { replacement, etc.) } \\
\text { M2M and D2D } \\
\text { communications } \\
\text { (new RAT) } \\
\text { Data services } \\
\text { (service access, } \\
\text { composition and } \\
\text { management) }\end{array}$ & $\begin{array}{l}\text { CPS Framework } \\
\text { Reference } \\
\text { architecture for } \\
\text { CPS } \\
\text { Timing } \\
\text { Cybersecurity } \\
\text { Data } \\
\text { interoperability } \\
\text { Use cases }\end{array}$ \\
\hline
\end{tabular}

Besides, once reviewed the most relevant works on the different key aspects of CPS identified in Section 2.2 for each one of the technological domain, Table 5 presents a comparative study as a summary.

Table 5: Comparison among the typical works on the key aspect for the technological domains

\begin{tabular}{|l|l|l|l|}
\hline \multicolumn{1}{|c|}{ Topic } & \multicolumn{1}{|c|}{ Control theory } & \multicolumn{1}{c|}{ Computer sciences } & \multicolumn{1}{c|}{$\begin{array}{c}\text { Communication } \\
\text { engineering }\end{array}$} \\
\hline $\begin{array}{l}\text { Context- } \\
\text { awareness }\end{array}$ & $\begin{array}{l}\text { Stable control algorithms (or } \\
\text { functions) considering context } \\
\text { information (using predictive } \\
\text { techniques or fuzzy control) }\end{array}$ & $\begin{array}{l}\text { Ontologies for context } \\
\text { information and algorithms to } \\
\text { process and compare that } \\
\text { information. }\end{array}$ & $\begin{array}{l}\text { Context-aware sensing } \\
\text { Architecture for context- } \\
\text { ware CPS } \\
\text { Context-aware service } \\
\text { provision }\end{array}$ \\
\hline $\begin{array}{l}\text { Machine } \\
\text { learning }\end{array}$ & $\begin{array}{l}\text { Pattern recognition for human } \\
\text { activity detection }\end{array}$ & $\begin{array}{l}\text { Cyber-attacks detection by } \\
\text { means of pattern recognition } \\
\text { Instruments based on learning } \\
\text { for physical model creation } \\
\text { Hybrid automatons }\end{array}$ & $\begin{array}{l}\text { Smart manufacturing } \\
\text { Cyber-Physical Production } \\
\text { Systems } \\
\text { (employed as technology } \\
\text { belonging to large }\end{array}$ \\
\hline
\end{tabular}




\begin{tabular}{|l|l|l|l|}
\hline & & systems) \\
\hline $\begin{array}{l}\text { Self-adaptation } \\
\text { and self- } \\
\text { configuration }\end{array}$ & $\begin{array}{l}\text { Mathematical analysis of } \\
\text { dynamics describing "self- } \\
\text { tuning" systems }\end{array}$ & $\begin{array}{l}\text { Adaptive models to support } \\
\text { self-* properties } \\
\text { Systematic methodology and } \\
\text { algorithm to study the state-of- } \\
\text { the-art }\end{array}$ & $\begin{array}{l}\text { Middleware creation } \\
\text { supporting self-* } \\
\text { properties } \\
\text { Self-adaptable architecture } \\
\text { definition } \\
\text { Self-adaptable hardware } \\
\text { platforms }\end{array}$ \\
\hline Fault-tolerance & $\begin{array}{l}\text { Description of parametric } \\
\text { control functions being able to } \\
\text { correct the effect of failures }\end{array}$ & $\begin{array}{l}\text { Mathematical analysis of } \\
\text { solutions to keep CPS under } \\
\text { the regular operation } \\
\text { constraints }\end{array}$ & $\begin{array}{l}\text { Fault-tolerant routing } \\
\text { protocols for WSN } \\
\text { Fault-tolerant WSN for } \\
\text { critical applications }\end{array}$ \\
\hline Mobility & $\begin{array}{l}\text { Control systems for mobile } \\
\text { robots } \\
\text { Enhanced feedback control } \\
\text { loops for mobile WSN }\end{array}$ & $\begin{array}{l}\text { Regular models for CPS } \\
\text { including mobility } \\
\text { considerations } \\
\text { Special security solutions for } \\
\text { mobile nodes in CPS }\end{array}$ & $\begin{array}{l}\text { Definition of "mobile } \\
\text { CPS" } \\
\text { Crowdsensing } \\
\text { Integration of CPS and } \\
\text { mobile computing }\end{array}$ \\
\hline
\end{tabular}

\section{FUTURE RESEARCH CHALLENGES}

Although architectures, applications and research lines for CPS depend on the considered technological domain, most challenges in CPS sciences are common for all approaches. As Internet has changed the way in which we communicate with others, CPS will change the way in which we deal with the physical world [19]. The challenges which must be addressed to support that revolution range from fundamental scientific and engineering issues to institutional and societal topics [232].

Many reports have identified research opportunities [232], scientific challenges [232, 233], technological barriers [233] and business opportunities [234] for CPS. In this Section we compile and describe some of the most remarkable challenges identified, and propose new promising areas for research.

\subsection{SCIENTIFIC AND ENGINEERING CHALLENGES}

The creation of CPS as a new type of engineered systems needs to address various scientific and technical challenges:

- Technical solutions for obtaining integrated heterogeneous systems are needed [232]. CPS contains many heterogeneous components, some of them distributed, which have to work together to create the expected performance. Mainly, modeling languages and a rigorous semantics are needed for describing the interactions (physical, computational and communications) among heterogeneous devices. Another approach consists of constructing systems with components designed in different domains and sectors without the advantage of any common standard. This second vision, however, is much more challenging and nowadays is far beyond the state-of-the-art [74].

- New pervasive sensing architectures being able of providing services (for top-down applications) and data (for traditional bottom-up applications) are required, in order to support the enormous variety of application in CPS 
- Methodologies to address the complexity intrinsic to CPS have to be developed [19]. Languages for modeling the system characteristics and behavior at different abstraction levels and independently of the application domain are needed. Besides automatic tools to transform models, compose application, build and deploy the components and incorporate verification and validation capabilities are required [235, 236].

- Deep and extensive theoretical works addressing the dynamics of CPS are required [19]. The complex dynamics generated by the tight relation among cyber and physical worlds are unexplored. Significant theoretical results are necessary to allow understanding the behavior of communications, computation, control and applications when working unified [237].

- Models representing the different types of devices are also needed (remarking, overall, the interfaces and the interaction capabilities).

- Advance towards human-focused applications over CPS, facing three main challenges [14]. First a comprehensive understanding of the complete list of human-in-the-loop applications is needed. As we said in Section 2.1 all human-in-the-loop application can be classified in three types, however, due to the complicated human behavior and its great dependency of the external conditions a richer taxonomy [238, 239] is needed. Second, automatic tools to create models of human behaviors are also necessary. Applying existing toolboxes for computer networks to systems involving humans is essential to deploy CPS in the real world [240, 241]. Finally, the traditional feedback control scheme has to be modified properly to incorporate humans. The human models could be placed in several locations (outside the loop, inside the controller, inside the transducers, in various locations, etc.), and theoretical and practical results about the different options must be generated [242].

- New scheduling politics for pervasive sensing platforms are necessary, allowing devices to discover their capabilities and self-manage their resources [243].

- Appropriate software platforms, APIs and Integrated Development Environments (IDEs) are required. The development of reliable, scalable and evolvable CPS needs tools at various levels of abstraction in order to hide the underlying complexity (distributed components, reconfigurations, etc.) to high-level developers [244].

- Methodologies for measuring the system performance have to be developed [232]. Verifying the performance, security and other requirements block the development of CPS. Nowadays, validating CPS capabilities is extremely costing, so new approaches and tools are necessary [43].

- A new generation of pervasive sensing technologies has to be developed [188]. Integrating pervasive sensing platforms in CPS involves two main challenges. First a specific model for the sensors and actuators life-cycle has to be defined [245, 246], specially indicating the consequences in higher layers of the variations at low-level. And, second timing in CPS is one of the most important topics, synchronizing the 
pervasive sensing infrastructure, and the other components in CPS, is a challenging situation [247].

Finally, in Table 6 we describe some additional challenges which depend on the particular application of CPS considered: aviation, defense, healthcare, critical infrastructure, environment monitoring and automotive [127].

Table 6: CPS challenges along the application

\begin{tabular}{|l|l|}
\hline \multicolumn{1}{|c|}{ Application } & \multicolumn{1}{c|}{ Challenges } \\
\hline Aviation and defense & $\begin{array}{l}\text { Mainly, new security protocols have to be designed. } \\
\text { Extremely precise control components are also necessary. } \\
\text { Developing a technology for high power technology will be important. }\end{array}$ \\
\hline Healthcare & $\begin{array}{l}\text { A new generation of analysis, synthesis and integration technologies are } \\
\text { required. } \\
\text { Interoperability algorithms will be also basic in the future. }\end{array}$ \\
\hline Critical infrastructure & $\begin{array}{l}\text { Mainly, extremely precise control components are necessary. } \\
\text { New developing methodologies in order to guarantee the quality of the } \\
\text { software will be also desirable. }\end{array}$ \\
\hline Environment monitoring & $\begin{array}{l}\text { Obtaining improved pervasive sensing technologies is the main challenge: } \\
\text { reaching low-power consumption, the creation of disregarded systems and } \\
\text { very precise in time platforms are the most important issues. }\end{array}$ \\
\hline Automotive & $\begin{array}{l}\text { Developing a technology for high power technology will be, in practice, the } \\
\text { only challenge for future. }\end{array}$ \\
\hline
\end{tabular}

\subsection{INSTITUTIONAL AND SOCIETAL CHALLENGES}

The revolution of CPS is not only technological, but also social and economic, as they will change the way in which people relate with the environment. Thus, the public institutions and the general society must address various challenges [232]:

- The trust of society on CPS performance, opportunities and value must be strengthened. Actions to assure that CPS are trustworthy and secure, as well as properly information campaigns to integrate the general society in the CPS revolution are pending.

- An effective model of governance must be agreed. CPS may be large-scale systems, including international applications controlling critical infrastructures such as transport, energy, health, etc. Organizations to provide both local and global regulations are necessary. These institutions will publish standards, will manage future issues on interest-conflicts and will enhance the development of CPS from the public sector with the appropriate economic incentives.

- New business models have to be investigated. The Internet revolution has changed, in many ways, the economy. CPS will transform the economy deeper than any previous technology. Those new business models, however, are not well-defined yet. This lack of proved business model seems to be blocking investments in new deployments and CPS research. 
- Professionals on CPS are necessary. CPS have a cross-disciplinary nature. Then, professional knowing about mathematics, statics, engineering, computer sciences, sociology, etc. are essential to start the CPS revolution. Nowadays, there is a lack of concentrated and multi-disciplinary educational programs oriented to develop CPS. In the past, the academic world has faced similar challenges, as with the integration of Internet sciences or, more recently with bio-engineering.

\section{CONCLUSIONS}

Cyber-Physical Systems are a particular application of pervasive sensing technologies, where feedback control loops are deployed as end-user application. However, as a popular word, CPS are understood in different ways in different technological domains. Thus, understanding the characteristics and impact of CPS is a very complicated task.

In this work we have reviewed the elements which may be part of CPS, and, after describing the origin of CPS and its relation with Internet-of-Things, we have introduced the four main approaches for CPS existing today: control theory, computer sciences, communication engineering and vertical systems. For each domain we have explained the reference architectures with more impact, described the characteristics of CPS and review the state-of-theart in the main research lines. Besides, we have reviewed the recent attempts to standardize the CPS. Finally, we also have discussed the main future unsolved challenges.

Originally defined in the computer sciences domain, the term CPS rapidly evolved towards other areas, increasing the dispersion in the architectures, characteristics, use-cases and, even, future challenges described for CPS. This phenomenon has been enhanced by the appearance of other terms (such as Industry 4.0, Internet-of-Things or Wireless Sensor Networks) not clearly separated from CPS, and used sometimes as synonyms. The interest of the standards organizations in normalizing the CPS will help in the future to reduce the ambiguity which surrounds that term. In particular, the efforts of the NIST in fixing a common architecture, vocabulary and reference architecture are helping companies and researchers to clearly shaping CPS technologies.

In conclusion, nowadays many equally important proposals for CPS are found. However, in the next future, the use of CPS paradigm in practical technologies and the creation of new standards related to CPS will help to narrow the scope and boundaries of these systems.

\section{ACKNOWLEDGMENTS}

The research leading to these results has received funding from the Ministry of Economy and Competitiveness through SEMOLA project (TEC2015-68284-R) and from the Autonomous Region of Madrid through MOSI-AGIL-CM project (grant P2013/ICE-3019, co-funded by EU Structural Funds FSE and FEDER). Borja Bordel has received funding from the Ministry of Education through the FPU program (grant number FPU15/03977). 


\section{CONFLICTS OF INTEREST}

The authors declare that there is no conflict of interest regarding the publication of this paper.

\section{REFERENCES}

[1] Lee, E. A., \& Seshia, S. A. (2011). Introduction to embedded systems: A cyber-physical systems approach. Lee \& Seshia.

[2] National Institute of Standards and Technology. CPS Public Working Group. pages.nist.gov/cpspwg (accessed on 16 May 16, 2016)

[3] Industrial Cyber-Physical Systems Center. www.icyphy.org/ (accessed on 16 May 16, 2016)

[4] Fujitsu's Smart Glove. www.fujitsu.com/global/about/resources/news/pressreleases/2014/0218-01.html (accessed on 16 May 16, 2016)

[5] Sanislav, T., \& Miclea, L. (2012). Cyber-physical systems-concept, challenges and research areas. Journal of Control Engineering and Applied Informatics, 14(2), 28-33.

[6] Krämer, B. J. (2014). Evolution of Cyber-Physical Systems: A Brief Review. In Applied Cyber-Physical Systems (pp. 1-3). Springer New York.

[7] Bordel, B., Alcarria, R., Pérez-Jiménez, M., Robles, T., Martín, D., \& de Rivera, D. S. (2015). Building Smart Adaptable Cyber-Physical Systems: Definitions, Classification and Elements. In Ubiquitous Computing and Ambient Intelligence. Sensing, Processing, and Using Environmental Information (pp. 144-149). Springer International Publishing.

[8] Talcott, C. (2008). Cyber-physical systems and events. In Software-Intensive Systems and New Computing Paradigms (pp. 101-115). Springer Berlin Heidelberg.

[9] J. Lukkien. Cyber-Physical Systems: a perspective. http://www.win.tue.nl/ johanl/docs/CPS\%20v2\%20presented.pdf (accessed on 16 May 16, 2016)

[10] Schmidt, A. (2000). Implicit human computer interaction through context. Personal technologies, 4(2-3), 191-199.

[11] Schmidt, A., Gellersen, H. W., \& Merz, C. (2000, October). Enabling implicit human computer interaction: a wearable RFID-tag reader. In Wearable Computers, The Fourth International Symposium on (pp. 193-194). IEEE.

[12] National Institute of Standards and Technology. CPS Public Working Group Presentation. http://www.nist.gov/el/upload/CPS-PWG-Kickoff-Webinar-Presentation-FINAL. pdf (accessed on 16 May 16, 2016)

[13] Gunes, V., Peter, S., Givargis, T., \& Vahid, F. (2014). A Survey on Concepts, Applications, and Challenges in Cyber-Physical Systems. Transactions on internet and information systems, 8(12), 4242-4268.

[14] Munir, S., Stankovic, J. A., Liang, C. J. M., \& Lin, S. (2013). Cyber Physical System Challenges for Human-in-the-Loop Control. In Feedback Computing.

[15] Tsui, K. M., Kim, D. J., Behal, A., Kontak, D., \& Yanco, H. A. (2011). "I want that": Human-in-the-loop control of a wheelchair-mounted robotic arm. Applied Bionics and Biomechanics, 8(1), 127-147.

[16] Kirkpatrick, K. (2013). Software-defined networking. Communications of the ACM, 56(9), 16-19.

[17] Kay, M., Choe, E. K., Shepherd, J., Greenstein, B., Watson, N., Consolvo, S., \& Kientz, J. A. (2012, September). Lullaby: a capture \& access system for understanding the sleep 
environment. In Proceedings of the 2012 ACM Conference on Ubiquitous Computing (pp. 226234). ACM.

[18] Zeiler, W., HOUTEN, R., Boxem, G., Vissers, D., \& Maaijen, R. (2011). Indoor air quality and thermal comfort strategies: the human-in-the-loop approach. In Proc. Int. Conf. Enhanced Building Oper.

[19] Kim, K. D., \& Kumar, P. R. (2012). Cyber-physical systems: A perspective at the centennial. Proceedings of the IEEE, 100(Special Centennial Issue), 1287-1308.

[20] Zambonelli, F. (2006). Self-management and the many facets of nonself. IEEE Intelligent Systems, 21(2), 50-58.

[21] Conti, M., Das, S. K., Bisdikian, C., Kumar, M., Ni, L. M., Passarella, A., ... \& Zambonelli, F. (2012). Looking ahead in pervasive computing: Challenges and opportunities in the era of cyber-physical convergence. Pervasive and Mobile Computing, 8(1), 2-21.

[22] Edwards, S. A., \& Lee, E. A. (2007, June). The case for the precision timed (PRET) machine. In Proceedings of the 44th annual Design Automation Conference (pp. 264-265). ACM.

[23] Edwards, S. A., Kim, S., Lee, E. A., Liu, I., Patel, H. D., \& Schoeberl, M. (2009). A disruptive computer design idea: Architectures with repeatable timing. In 2009 IEEE International Conference on Computer Design (pp. 54-59). IEEE Service Center.

[24] Lee, E. A. (2006, October). Cyber-physical systems-are computing foundations adequate. In Position Paper for NSF Workshop On Cyber-Physical Systems: Research Motivation, Techniques and Roadmap (Vol. 2).

[25] Trusted Computing Group, Specifications, www.trustedcomputinggroup.org. (accessed on 16 May 16, 2016)

[26] Sailer, R., Zhang, X., Jaeger, T., \& Van Doorn, L. (2004, August). Design and Implementation of a TCG-based Integrity Measurement Architecture. In USENIX Security Symposium (Vol. 13, pp. 223-238).

[27] Castelluccia, C., Francillon, A., Perito, D., \& Soriente, C. (2009, November). On the difficulty of software-based attestation of embedded devices. In Proceedings of the 16th ACM conference on Computer and communications security (pp. 400-409). ACM.

[28] Perito, D., \& Tsudik, G. (2010, September). Secure Code Update for Embedded Devices via Proofs of Secure Erasure. In ESORICS (Vol. 6345, pp. 643-662).

[29] Wan, J., Zhang, D., Zhao, S., Yang, L., \& Lloret, J. (2014). Context-aware vehicular cyberphysical systems with cloud support: architecture, challenges, and solutions. Communications Magazine, IEEE, 52(8), 106-113.

[30] Rao, J., \& Su, X. (2004). A survey of automated web service composition methods. In Semantic Web Services and Web Process Composition (pp. 43-54). Springer Berlin Heidelberg. [31] Golatowski, F., Blumenthal, J., Handy, M., Haase, M., Burchardt, H., \& Timmermann, D. (2003, June). Service-oriented software architecture for sensor networks. In International Workshop on Mobile Computing, IMC (Vol. 3).

[32] Yao, Y., Cao, Q., \& Vasilakos, A. V. (2015). EDAL: An energy-efficient, delay-aware, and lifetime-balancing data collection protocol for heterogeneous wireless sensor networks. Networking, IEEE/ACM Transactions on, 23(3), 810-823.

[33] Aijaz, A., \& Aghvami, A. H. (2015). Cognitive machine-to-machine communications for internet-of-things: a protocol stack perspective. Internet of Things Journal, IEEE, 2(2), 103-112. [34] Chai, H. S., Choi, J. Y., \& Jeong, J. (2015). An Enhanced Secure Mobility Management Scheme for Building IoT Applications. Procedia Computer Science, 56, 586-591.

[35] Sridhar, S., Hahn, A., \& Govindarasu, M. (2012). Cyber-physical system security for the electric power grid. Proceedings of the IEEE, 100(1), 210-224. 
[36] Rajkumar, R. R., Lee, I., Sha, L., \& Stankovic, J. (2010, June). Cyber-physical systems: the next computing revolution. In Proceedings of the 47th Design Automation Conference (pp. 731736). ACM.

[37] Marwedel, P. (2010). Embedded system design: Embedded systems foundations of cyberphysical systems. Springer Science \& Business Media.

[38] Bordel Sánchez, B., Alcarria, R., Martín, D., \& Robles, T. (2015). TF4SM: A Framework for Developing Traceability Solutions in Small Manufacturing Companies. Sensors, 15(11), 29478-29510.

[39] Kaisler, S. (2012). Advanced Analytics. CATALYST Technical Report, i_SW Corporation, Arlington, VA.

[40] Kaisler, S., Armour, F., Espinosa, J. A., \& Money, W. (2013, January). Big data: Issues and challenges moving forward. In System Sciences (HICSS), 2013 46th Hawaii International Conference on (pp. 995-1004). IEEE.

[41] Tarraf, D. C. (2013). Control of Cyber-Physical Systems. Proc. of Lecture Notes in Control and Information Sciences, 449.

[42] Poovendran, R., \& Sampigethaya, K. (2009). A Community Report of the 2008 HighConfidence Transportation CPS Workshop.

[43] Baheti, R., \& Gill, H. (2011). Cyber-physical systems. The impact of control technology, 12, 161-166.

[44] Cyber Physical Systems: Situation Analysis of Current Trends, Technologies, and Challenges, in Proceeding of NIST CPS Workshop, 2012. events.energetics.com/NISTCPSWorkshop/pdfs/CPS_Situation_Analysis.pdf (accessed on 16 May 16, 2016)

[45] Gill, H. (2010, November). Cyber-Physical Systems: Beyond ES, SNs, and SCADA. In Presentation in the Trusted Computing in Embedded Systems (TCES) Workshop.

[46] CPS Steering Group, Cyber-Physical Systems Executive Summary, (2008). iccps.acm.org/2011/_doc/CPS-Executive-Summary.pdf (accessed on 16 May 16, 2016)

[47] President's Council of Advisors on Science and Technology (PCAST), Leadership Under Change: Information Technology $R \& D$ in a Competitive World, (2007). https://www.nsf.gov/geo/geo-data-policies/pcast-nit-final.pdf (accessed on 16 May 16, 2016) [48] Bordel Sanchez, B., Sánchez-Picot, Á., \& Sanchez De Rivera, D. (2015, July). Using 5G Technologies in the Internet of Things Handovers, Problems and Challenges. In Innovative Mobile and Internet Services in Ubiquitous Computing (IMIS), 2015 9th International Conference on (pp. 364-369). IEEE.

[49] Huang, B. X. (2008). Cyber physical systems: a survey. Presentation Report

[50] Guihai Chen. Internet of Things towards

Ubiquitous and Mobile Computing. research.microsoft.com/enus/UM/redmond/events/asiafacsum2010/presentations/Guihai-Chen_Oct19.pdf (accessed on 16 May 16, 2016)

[51] Latré, B., Braem, B., Moerman, I., Blondia, C., \& Demeester, P. (2011). A survey on wireless body area networks. Wireless Networks, 17(1), 1-18.

[52] Bennett, K. (1995). Legacy systems: Coping with success. Software, IEEE, 12(1), 19-23.

[53] Lasi, H., Fettke, P., Kemper, H. G., Feld, T., \& Hoffmann, M. (2014). Industry 4.0. Business \& Information Systems Engineering, 6(4), 239.

[54] Lee, J., Bagheri, B., \& Kao, H. A. (2015). A cyber-physical systems architecture for industry 4.0-based manufacturing systems. Manufacturing Letters, 3, 18-23.

[55] Jazdi, N. (2014, May). Cyber physical systems in the context of Industry 4.0. In Automation, Quality and Testing, Robotics, 2014 IEEE International Conference on (pp. 1-4). IEEE. 
[56] Park, J. H., Chao, H. C., Hussain, S., \& Yen, N. Y. (2014). Pervasive sensing technologies and emerging trends. International Journal of Distributed Sensor Networks.

[57] Gilani, S. (2009). The promise of M2M: how pervasive connected machines are fueling the next wireless revolution. Embedded Systems White Paper.

[58] Peter Mell \& Timothy Grance. The NIST Definition of Cloud Computing faculty.winthrop.edu/domanm/csci411/Handouts/NIST.pdf (accessed on 16 May 16, 2016)

[59] Satyanarayanan, M. (2001). Pervasive computing: Vision and challenges. Personal Communications, IEEE, 8(4), 10-17.

[60] Adaptive Cooperative Control in urban (sub) systems. Project web page. projectaccus.eu/ (accessed on 16 May 16, 2016)

[61] Maier, M. W. (1996, July). Architecting principles for systems-of-systems. In INCOSE International Symposium (Vol. 6, No. 1, pp. 565-573).

[62] Atzori, L., Iera, A., \& Morabito, G. (2010). The internet of things: A survey. Computer networks, 54(15), 2787-2805.

[63] Koubâa, A., \& Andersson, B. (2009). A vision of cyber-physical internet. In 8th International Workshop on Real-Time Networks (RTN'09) (pp. 1-6). Instituto Politécnico do Porto. Instituto Superior de Engenharia do Porto.

[64] Chen, D., Chang, G., Sun, D., Jia, J., \& Wang, X. (2012). Modeling access control for cyber-physical systems using reputation. Computers \& Electrical Engineering, 38(5), 10881101.

[65] Sabina Jeschke. Cyber-Physical Systems - A matter of multidisciplinarity www.ima-zlwifu.rwth-

aachen.de/fileadmin/user_upload/INSTITUTSCLUSTER/Publikation_Medien/Vortraege/downl oad//CPS_23Nov2012.pdf (accessed on 16 May 16, 2016)

[66] Wan, J., Chen, M., Xia, F., Di, L., \& Zhou, K. (2013). From machine-to-machine communications towards cyber-physical systems. Computer Science and Information Systems, 10(3), 1105-1128.

[67] Doyle, J. C., Francis, B. A., \& Tannenbaum, A. R. (2013). Feedback control theory. Courier Corporation.

[68] Skogestad, S., \& Postlethwaite, I. (2007). Multivariable feedback control: analysis and design (Vol. 2). New York: Wiley.

[69] Dorf, R. C., \& Bishop, R. H. (1998). Modern control systems.

[70] Franklin, G. F., Powell, J. D., \& Emami-Naeini, A. (1994). Feedback control of dynamics systems. Addison-Wesley, Reading, MA.

[71] Butkovski1̌, A. G. (1969). Distributed control systems. Elsevier Publishing Company.

[72] Gupta, R. A., \& Chow, M. Y. (2010). Networked control system: overview and research trends. Industrial Electronics, IEEE Transactions on, 57(7), 2527-2535.

[73] The U.S. Department of Energy, The Smart Grid: An Introduction energy.gov/sites/prod/files/oeprod/DocumentsandMedia/DOE_SG_Book_Single_Pages(1).pdf (accessed on 16 May 16, 2016)

[74] High Confidence Software and Systems Coordinating Group. High-confidence medical devices: Cyber-physical systems for 21st century health care, A Research and Development Needs Report, NCO/NITRD, (2009). http://www.whitehouse.gov/files/documents/cyber/NITRD - High-Confidence Medical Devices.pdf (accessed on 16 May 16, 2016)

[75] Sztipanovits, J. (2007, March). Composition of cyber-physical systems. In Engineering of Computer-Based Systems, IEEE International Conference on the (pp. 3-6). IEEE. 
[76] Supervisory Control and Data Acquisition (SCADA) systems, Technical Information Bulletin 04-1, (2004). http://scadahacker.com/library/Documents/ICS_Basics/SCADA Basics NCS TIB 04-1.pdf (accessed on 16 May 16, 2016)

[77] Sztipanovits, J., Koutsoukos, X., Karsai, G., Kottenstette, N., Antsaklis, P., Gupta, V., \& Wang, S. (2012). Toward a science of cyber-physical system integration. Proceedings of the IEEE, 100(1), 29-44.

[78] Khaitan, S. K., \& McCalley, J. D. (2015). Design techniques and applications of cyberphysical systems: A survey. Systems Journal, IEEE, 9(2), 350-365.

[79] Fitzgerald, J., Larsen, P. G., \& Verhoef, M. (2014). Collaborative Design for Embedded Systems. Springer.

[80] Lattmann, Z., Nagel, A., Scott, J., Smyth, K., Porter, J., Neema, S., \& Mavris, D. (2012, August). Towards automated evaluation of vehicle dynamics in system-level designs. In ASME 2012 International Design Engineering Technical Conferences and Computers and Information in Engineering Conference (pp. 1131-1141). American Society of Mechanical Engineers.

[81] Miclea, L., \& Sanislav, T. (2011, September). About dependability in cyber-physical systems. In Design \& Test Symposium (EWDTS), 2011 9th East-West (pp. 17-21). IEEE.

[82] Gupta, A., Kumar, M., Hansel, S., \& Saini, A. K. (2013). Future of all technologies-The Cloud and Cyber Physical Systems. Future, 2(2).

[83] Tummala, R. R. (2006). Moore's law meets its match (system-on-package). Spectrum, IEEE, 43(6), 44-49.

[84] Erdem, E. Y., Chen, Y. M., Mohebbi, M., Suh, J. W., Kovacs, G. T., Darling, R. B., \& Böhringer, K. F. (2010). Thermally actuated omnidirectional walking microrobot. Microelectromechanical Systems, Journal of, 19(3), 433-442.

[85] Zhou, Y., \& Baras, J. S. (2013). CPS modeling integration hub and design space exploration with application to microrobotics. In Control of Cyber-Physical Systems (pp. 2342). Springer International Publishing.

[86] Wang, B., \& Baras, J. S. (2012, June). Integrated modeling and simulation framework for wireless sensor networks. In Enabling Technologies: Infrastructure for Collaborative Enterprises (WETICE), 2012 IEEE 21st International Workshop on (pp. 268-273). IEEE.

[87] Spyropoulos, D., \& Baras, J. S. (2013). Extending Design Capabilities of SysML with Trade-off Analysis: Electrical Microgrid Case Study. Procedia Computer Science, 16, 108-117.

[88] Shi, J., Wan, J., Yan, H., \& Suo, H. (2011, November). A survey of cyber-physical systems. In Wireless Communications and Signal Processing (WCSP), 2011 International Conference on (pp. 1-6). IEEE.

[89] Åström, K. J., \& Bernhardsson, B. (2002). Comparison of Riemann and Lebesque sampling for first order stochastic systems. In Proceedings of the 41st IEEE Conference on Decision and Control, 2002 (Vol. 2, pp. 2011-2016). IEEE.

[90] Velasco, M., Fuertes, J., \& Marti, P. (2003, December). The self triggered task model for real-time control systems. In Work-in-Progress Session of the 24th IEEE Real-Time Systems Symposium (RTSS03) (Vol. 384).

[91] Michael, D., \& Wang, X. (2009). Self-Triggered Feedback Control Systems With FiniteGain L2 Stability. IEEE Transactions on Automatic Control, vol. 54, no. 3, pp. 452-467, 2009.

[92] Anta, A., \& Tabuada, P. (2010). To sample or not to sample: Self-triggered control for nonlinear systems. Automatic Control, IEEE Transactions on, 55(9), 2030-2042.

[93] Walsh, G. C., \& Ye, H. (2001). Scheduling of networked control systems. Control Systems, IEEE, 21(1), 57-65.

[94] Walsh, G. C., Ye, H., \& Bushnell, L. G. (2002). Stability analysis of networked control systems. Control Systems Technology, IEEE Transactions on, 10(3), 438-446. 
[95] Branicky, M. S., Phillips, S. M., \& Zhang, W. (2000). Stability of networked control systems: Explicit analysis of delay. In American Control Conference, 2000. Proceedings of the 2000 (Vol. 4, pp. 2352-2357). IEEE.

[96] Nešić, D., \& Teel, A. R. (2004). Input-output stability properties of networked control systems. Automatic Control, IEEE Transactions on, 49(10), 1650-1667.

[97] Montestruque, L. A., \& Antsaklis, P. J. (2003). On the model-based control of networked systems. Automatica, 39(10), 1837-1843.

[98] Zhang, W., Branicky, M. S., \& Phillips, S. M. (2001). Stability of networked control systems. Control Systems, IEEE, 21(1), 84-99.

[99] You, K., \& Xie, L. (2011). Minimum data rate for mean square stabilizability of linear systems with Markovian packet losses. Automatic Control, IEEE Transactions on, 56(4), 772785 .

[100] Schulman, L. J. (1996). Coding for interactive communication. Information Theory, IEEE Transactions on, 42(6), 1745-1756.

[101] Simsek, T., Jain, R., \& Varaiya, P. (2004). Scalar estimation and control with noisy binary observations. Automatic Control, IEEE Transactions on, 49(9), 1598-1603.

[102] Ostrovsky, R., Rabani, Y., \& Schulman, L. J. (2009). Error-correcting codes for automatic control. Information Theory, IEEE Transactions on, 55(7), 2931-2941.

[103] Sukhavasi, R. T., \& Hassibi, B. (2011, December). Anytime reliable codes for stabilizing plants over erasure channels. In Decision and Control and European Control Conference (CDCECC), 2011 50th IEEE Conference on (pp. 5254-5259). IEEE.

[104] Espinosa, M. M. (2010). Contributions to the control of networked cyber-physical systems (Doctoral dissertation, University of California Los Angeles).

[105] Sandell Jr, N. R., Varaiya, P., Athans, M., \& Safonov, M. G. (1978). Survey of decentralized control methods for large scale systems. Automatic Control, IEEE Transactions on, 23(2), 108-128.

[106] Rotkowitz, M., Cogill, R., \& Lall, S. (2010). Convexity of optimal control over networks with delays and arbitrary topology. International Journal of Systems, Control and Communications, 2(1-3), 30-54.

[107] Baillieul, J., \& Antsaklis, P. J. (2007). Control and communication challenges in networked real-time systems. Proceedings of the IEEE, 95(1), 9-28.

[108] Zhang, F., Szwaykowska, K., Wolf, W., \& Mooney, V. (2008, November). Task scheduling for control oriented requirements for cyber-physical systems. In Real-Time Systems Symposium, 2008 (pp. 47-56). IEEE.

[109] Kottenstette, N., Karsai, G., \& Sztipanovits, J. (2009, August). A passivity-based framework for resilient cyber physical systems. In Resilient Control Systems, 2009. ISRCS'09. 2nd International Symposium on (pp. 43-50). IEEE.

[110] Huang, H., Sun, Y. L., Yang, Q., Zhang, F., Zhang, X., Liu, Y., \& Sierra, F. (2010, April). Integrating neuromuscular and cyber systems for neural control of artificial legs. In Proceedings of the 1st ACM/IEEE International Conference on Cyber-Physical Systems (pp. 129-138). ACM.

[111] Ny, J. L., \& Pappas, G. J. (2010, April). Robustness analysis for the certification of digital controller implementations. In Proceedings of the 1st ACM/IEEE International Conference on Cyber-Physical Systems (pp. 99-108). ACM.

[112] Germany Trade\&Invest, Industrie 4.0 - Smart Manufacturing for the Future, (2013). www.its-owl.de/fileadmin/PDF/News/2014-01-14-Industrie_4.0-

Smart_Manufacturing_for_the_Future_German_Trade_Invest.pdf (accessed on 16 May 16, 2016) 
[113] Lee, E. A. (2015). The past, present and future of cyber-physical systems: A focus on models. Sensors, 15(3), 4837-4869.

[114] Lee, E. A. (2008, May). Cyber physical systems: Design challenges. In Object Oriented Real-Time Distributed Computing (ISORC), 2008 11th IEEE International Symposium on (pp. 363-369). IEEE.

[115] Wiener, N. (1961). Cybernetics or Control and Communication in the Animal and the Machine (Vol. 25). MIT press.

[116] Sha, L., Gopalakrishnan, S., Liu, X., \& Wang, Q. (2009). Cyber-physical systems: A new frontier. In Machine Learning in Cyber Trust (pp. 3-13). Springer US.

[117] Jensen, J. C., Chang, D. H., \& Lee, E. A. (2011, July). A model-based design methodology for cyber-physical systems. In Wireless Communications and Mobile Computing Conference (IWCMC), 2011 7th International (pp. 1666-1671). IEEE.

[118] Tabuada, P. (2006, October). Cyber-physical systems: Position paper. In NSF Workshop on Cyber-Physical Systems.

[119] Wan, J., Suo, H., Yan, H., \& Liu, J. (2011). A general test platform for cyber-physical systems: unmanned vehicle with wireless sensor network navigation. Procedia Engineering, 24, 123-127.

[120] Jiménez, M., Palomera, R., \& Couvertier, I. (2013). Introduction to Embedded Systems. Springer.

[121] Lee, E. A. (2007). Computing foundations and practice for cyber-physical systems: A preliminary report. University of California, Berkeley, Tech. Rep. UCB/EECS-2007-72.

[122] Tan, Y., Goddard, S., \& Perez, L. C. (2008). A prototype architecture for cyber-physical systems. ACM Sigbed Review, 5(1), 26.

[123] Lim, S., Chung, L., Han, O., \& Kim, J. H. (2011, February). An interactive cyber-physical system (CPS) for people with disability and frail elderly people. In Proceedings of the 5th international conference on ubiquitous information management and communication (p. 113). ACM.

[124] Xiao-Le, W., Hong-Bin, H., Su, D., \& Li-Na, C. (2012). A service-oriented architecture framework for cyber-physical systems. In Recent Advances in Computer Science and Information Engineering (pp. 671-676). Springer Berlin Heidelberg.

[125] Li, Q., Qin, W., Han, B., Wang, R., \& Sun, L. (2011). A case study on REST-style architecture for cyber-physical systems: Restful smart gateway. Computer Science and Information Systems, 8(4), 1317-1329.

[126] Bonakdarpour, B. (2008). Challenges in transformation of existing real-time embedded systems to cyber-physical systems. ACM SIGBED Review, 5(1), 11.

[127] Wan, K., Man, K. L., \& Hughes, D. (2010). Specification, analyzing challenges and approaches for cyber-physical systems (CPS). Engineering Letters, 18(3), 308.

[128] IEEE Standard 802.15.4d-2009, Low-power Wireless Network technology, IEEE Computer Society, April 17, 2009.

[129] Nordic, Low-power Wireless Network Technology, www.nordicsemi.com/index.cfm?obj=product\&act=display\&pro=97 (accessed on 16 May 16, 2016)

[130] Cyber-Physical Systems Virtual Organization (CPS-VO). Webpage cps-vo.org/ (accessed on 16 May 16, 2016)

[131] Berkeley University. Cyber-Physical Systems webpage. cyberphysicalsystems.org/ (accessed on 16 May 16, 2016) 
[132] Ilic, M. D., Xie, L., Khan, U. A., \& Moura, J. M. (2010). Modeling of future cyberphysical energy systems for distributed sensing and control. IEEE Transactions on Systems, Man, and Cybernetics-Part A: Systems and Humans, 4(40), 825-838.

[133] Tang, L. A., Yu, X., Kim, S., Han, J., Hung, C. C., \& Peng, W. C. (2010, December). Trualarm: Trustworthiness analysis of sensor networks in cyber-physical systems. In Data Mining (ICDM), 2010 IEEE 10th International Conference on (pp. 1079-1084). IEEE.

[134] Lee, J., Lapira, E., Bagheri, B., \& Kao, H. A. (2013). Recent advances and trends in predictive manufacturing systems in big data environment. Manufacturing Letters, 1(1), 38-41.

[135] Kang, W., Kapitanova, K., \& Son, S. H. (2012). RDDS: A real-time data distribution service for cyber-physical systems. Industrial Informatics, IEEE Transactions on, 8(2), 393-405. [136] Liberson, D. (2003). Switching in systems and control, Systems \& Control: Foundation \& Applications.

[137] Lin, H., \& Antsaklis, P. J. (2009). Stability and stabilizability of switched linear systems: a survey of recent results. Automatic control, IEEE Transactions on, 54(2), 308-322.

[138] Platzer, A. (2010). Logical analysis of hybrid systems: proving theorems for complex dynamics. Springer Science \& Business Media.

[139] Alur, R., Courcoubetis, C., Henzinger, T. A., \& Ho, P. H. (1993). Hybrid automata: An algorithmic approach to the specification and verification of hybrid systems (pp. 209-229). Springer Berlin Heidelberg.

[140] Kaynar, D. K., Lynch, N., Segala, R., \& Vaandrager, F. (2010). The theory of timed I/O automata. Synthesis Lectures on Distributed Computing Theory, 1(1), 1-137.

[141] Henzinger, T. A. (2000). The theory of hybrid automata (pp. 265-292). Springer Berlin Heidelberg.

[142] Park, D. (1981). Concurrency and automata on infinite sequences (pp. 167-183). Springer Berlin Heidelberg.

[143] Milner, R. (1989). Communication and concurrency (Vol. 84). New York etc.: Prentice hall.

[144] Tabuada, P. (2008). An approximate simulation approach to symbolic control. Automatic Control, IEEE Transactions on, 53(6), 1406-1418.

[145] Pola, G., Girard, A., \& Tabuada, P. (2008). Approximately bisimilar symbolic models for nonlinear control systems. Automatica, 44(10), 2508-2516.

[146] Belta, C., \& Habets, L. C. (2006). Controlling a class of nonlinear systems on rectangles. Automatic Control, IEEE Transactions on, 51(11), 1749-1759.

[147] Tabuada, P., \& Pappas, G. J. (2006). Linear time logic control of discrete-time linear systems. Automatic Control, IEEE Transactions on, 51(12), 1862-1877.

[148] Frehse, G., Le Guernic, C., Donzé, A., Cotton, S., Ray, R., Lebeltel, O., ... \& Maler, O. (2011, July). SpaceEx: Scalable verification of hybrid systems. In Computer Aided Verification (pp. 379-395). Springer Berlin Heidelberg.

[149] Mazo Jr, M., Davitian, A., \& Tabuada, P. (2010, July). Pessoa: A tool for embedded controller synthesis. In Computer Aided Verification (pp. 566-569). Springer Berlin Heidelberg. [150] Baliga, G. B. (2005). A middleware framework for networked control systems.

[151] Graham, S., Baliga, G., \& Kumar, P. R. (2009). Abstractions, architecture, mechanisms, and a middleware for networked control. Automatic Control, IEEE Transactions on, 54(7), 1490-1503.

[152] Kowshik, H., Caveney, D., \& Kumar, P. R. (2011). Provable systemwide safety in intelligent intersections. Vehicular Technology, IEEE Transactions on, 60(3), 804-818. 
[153] Loos, S. M., Platzer, A., \& Nistor, L. (2011). Adaptive cruise control: Hybrid, distributed, and now formally verified. In FM 2011: Formal Methods (pp. 42-56). Springer Berlin Heidelberg.

[154] Platzer, A. (2011, April). Quantified differential invariants. In Proceedings of the 14th international conference on Hybrid systems: computation and control (pp. 63-72). ACM.

[155] Buttazzo, G. (2011). Hard real-time computing systems: predictable scheduling algorithms and applications (Vol. 24). Springer Science \& Business Media.

[156] Baruah, S. K., Rosier, L. E., \& Howell, R. R. (1990). Algorithms and complexity concerning the preemptive scheduling of periodic, real-time tasks on one processor. Real-time systems, 2(4), 301-324.

[157] Abdelzaher, T. F., Sharma, V., \& Lu, C. (2004). A utilization bound for aperiodic tasks and priority driven scheduling. Computers, IEEE Transactions on, 53(3), 334-350.

[158] Doytchinov, B., Lehoczky, J., \& Shreve, S. (2001). Real-time queues in heavy traffic with earliest-deadline-first queue discipline. Annals of Applied Probability, 332-378.

[159] Mok, A. K., Feng, X., \& Chen, D. (2001). Resource partition for real-time systems. In Real-Time Technology and Applications Symposium, 2001. Proceedings. Seventh IEEE (pp. 75-84). IEEE.

[160] Shin, I., \& Lee, I. (2008). Compositional real-time scheduling framework with periodic model. ACM Transactions on Embedded Computing Systems (TECS), 7(3), 30.

[161] Rajkumar, R., Juvva, K., Molano, A., \& Oikawa, S. (1997, December). Resource kernels: A resource-centric approach to real-time and multimedia systems. In Photonics West'98 Electronic Imaging (pp. 150-164). International Society for Optics and Photonics.

[162] Pillai, P., \& Shin, K. G. (2001, October). Real-time dynamic voltage scaling for lowpower embedded operating systems. In ACM SIGOPS Operating Systems Review (Vol. 35, No. 5, pp. 89-102). ACM.

[163] The Object Management Group (OMG). CORBA. www.corba.org/ (accessed on 16 May $16,2016)$

[164] Schmidt, D. C., Levine, D. L., \& Mungee, S. (1998). The design of the TAO real-time object request broker. Computer Communications, 21(4), 294-324.

[165] Brinkschulte, U., Bechina, A., Picioroaga, F., Schneider, E., Ungerer, T., Kreuzinger, J., \& Pfeffer, M. (2001). A microkernel middleware architecture for distributed embedded realtime systems. In Reliable Distributed Systems, 2001. Proceedings. 20th IEEE Symposium on (pp. 218-226). IEEE.

[166] LeGuernic, P., Gautier, T., Le Borgne, M., \& Le Maire, C. (1991). Programming realtime applications with SIGNAL. Proceedings of the IEEE, 79(9), 1321-1336.

[167] Berry, G., \& Gonthier, G. (1992). The Esterel synchronous programming language: Design, semantics, implementation. Science of computer programming, 19(2), 87-152.

[168] Seto, D., Lehoczky, J. P., Sha, L., \& Shin, K. G. (1996, December). On task schedulability in real-time control systems. In Real-Time Systems Symposium, 1996., 17th IEEE (pp. 13-21). IEEE.

[169] Kelly, F. P., Maulloo, A. K., \& Tan, D. K. (1998). Rate control for communication networks: shadow prices, proportional fairness and stability. Journal of the Operational Research society, 237-252.

[170] Li, Y., Chen, C. S., Song, Y. Q., \& Wang, Z. (2007). Real-time QoS support in wireless sensor networks: a survey. In 7th IFAC International Conference on Fieldbuses \& Networks in Industrial \& Embedded Systems-FeT'2007. 
[171] He, T., Stankovic, J. A., Lu, C., \& Abdelzaher, T. (2003, May). SPEED: A stateless protocol for real-time communication in sensor networks. In Distributed Computing Systems, 2003. Proceedings. 23rd International Conference on (pp. 46-55). IEEE.

[172] J. Leyden. (2008) Polish teen derails tram after hacking train network. The Register. www.theregister.co.uk/2008/01/11/tram_hack/ (accessed on 16 May 16, 2016)

[173] R. Esposito. (2006) Hackers penetrate water system computers. ABC News. blogs.abcnews.com/theblotter/2006/10/hackers_penetra.html (accessed on 16 May 16, 2016)

[174] Peisert, S., Margulies, J., Nicol, D. M., Khurana, H., \& Sawall, C. (2014). Designed-in Security for Cyber-Physical Systems. Security \& Privacy, IEEE, 12(5), 9-12.

[175] Moholkar, A. V. Security for Cyber-Physical Systems.

[176] Gollmann, D. (2012). Security for Cyber-Physical Systems. In Mathematical and Engineering Methods in Computer Science (pp. 12-14). Springer Berlin Heidelberg.

[177] Cardenas, A. A., Amin, S., \& Sastry, S. (2008, June). Secure control: Towards survivable cyber-physical systems. In The 28th International Conference on Distributed Computing Systems Workshops (pp. 495-500). IEEE.

[178] Cárdenas, A. A., Amin, S., Lin, Z. S., Huang, Y. L., Huang, C. Y., \& Sastry, S. (2011, March). Attacks against process control systems: risk assessment, detection, and response. In Proceedings of the 6th ACM symposium on information, computer and communications security (pp. 355-366). ACM.

[179] Pasqualetti, F., Dörfler, F., \& Bullo, F. (2011, December). Cyber-physical attacks in power networks: Models, fundamental limitations and monitor design. In Decision and Control and European Control Conference (CDC-ECC), 2011 50th IEEE Conference on (pp. 21952201). IEEE.

[180] Fawzi, H., Tabuada, P., \& Diggavi, S. (2011, September). Secure state-estimation for dynamical systems under active adversaries. In Communication, Control, and Computing (Allerton), 2011 49th Annual Allerton Conference on (pp. 337-344). IEEE.

[181] Sridhar, S., Hahn, A., \& Govindarasu, M. (2012). Cyber-physical system security for the electric power grid. Proceedings of the IEEE, 100(1), 210-224.

[182] Mo, Y., Kim, T. H. J., Brancik, K., Dickinson, D., Lee, H., Perrig, A., \& Sinopoli, B. (2012). Cyber-physical security of a smart grid infrastructure. Proceedings of the IEEE, 100(1), 195-209.

[183] Hahn, A., Ashok, A., Sridhar, S., \& Govindarasu, M. (2013). Cyber-physical security testbeds: Architecture, application, and evaluation for smart grid. Smart Grid, IEEE Transactions on, 4(2), 847-855.

[184] Broman, D., Greenberg, L., Lee, E. A., Masin, M., Tripakis, S., \& Wetter, M. (2014). Requirements for Hybrid Cosimulation (No. UCB/EECS-2014-157). California Univ. Berkeley Dept. of electrical engineering and computer sciences.

[185] Lee, E. A., Niknami, M., Nouidui, T. S., \& Wetter, M. (2015, October). Modeling and simulating cyber-physical systems using CyPhySim. In Proceedings of the 12th International Conference on Embedded Software (pp. 115-124). IEEE Press.

[186] Brooks, C., Lee, E. A., Lorenzetti, D., Nouidui, T. S., \& Wetter, M. (2015, April). CyPhySim: a cyber-physical systems simulator. In Proceedings of the 18th International Conference on Hybrid Systems: Computation and Control (pp. 301-302). ACM.

[187] Al Faruque, M. A. EDA for Cyber-Physical Energy Systems Design.

[188] NIWeek 2015 Keynote Presentations (2015) www.ni.com/niweek/keynote-videos/ (accessed on 16 May 16, 2016)

[189] Horvath, I., \& Gerritsen, B. H. (2012). Cyber-physical systems: Concepts, technologies and implementation principles. In Proceedings of TMCE (Vol. 1, pp. 7-11). 
[190] Wu, F. J., Kao, Y. F., \& Tseng, Y. C. (2011). From wireless sensor networks towards cyber physical systems. Pervasive and Mobile Computing, 7(4), 397-413.

[191] La, H. J., \& Kim, S. D. (2010, August). A service-based approach to designing cyber physical systems. In Computer and Information Science (ICIS), 2010 IEEE/ACIS 9th International Conference on (pp. 895-900). IEEE.

[192] Da Xu, L., He, W., \& Li, S. (2014). Internet of things in industries: a survey. Industrial Informatics, IEEE Transactions on, 10(4), 2233-2243.

[193] Lai, C. F., Ma, Y. W., Chang, S. Y., Chao, H. C., \& Huang, Y. M. (2011). OSGi-based services architecture for cyber-physical home control systems. Computer Communications, 34(2), 184-191.

[194] Alliance, O. (2003). Osgi service platform, release 3. IOS Press, Inc.

[195] Gordon, R., \& Essential, J. N. I. (1998). Java Native Interface. Prentince Hall PTR.

[196] Sheng, Z., Yang, S., Yu, Y., Vasilakos, A., Mccann, J., \& Leung, K. (2013). A survey on the ietf protocol suite for the internet of things: Standards, challenges, and opportunities. Wireless Communications, IEEE, 20(6), 91-98.

[197] Whitmore, A., Agarwal, A., \& Da Xu, L. (2015). The Internet of Things-A survey of topics and trends. Information Systems Frontiers, 17(2), 261-274.

[198] Li, S., Da Xu, L., \& Zhao, S. (2015). The internet of things: a survey. Information Systems Frontiers, 17(2), 243-259.

[199] Yick, J., Mukherjee, B., \& Ghosal, D. (2008). Wireless sensor network survey. Computer networks, 52(12), 2292-2330.

[200] Akyildiz, I. F., Su, W., Sankarasubramaniam, Y., \& Cayirci, E. (2002). Wireless sensor networks: a survey. Computer networks, 38(4), 393-422.

[201] Chen, K. C., \& Lien, S. Y. (2014). Machine-to-machine communications: Technologies and challenges. Ad Hoc Networks, 18, 3-23.

[202] Spiess, P., Karnouskos, S., Guinard, D., Savio, D., Baecker, O., Souza, L. M. S. D., \& Trifa, V. (2009, July). SOA-based integration of the internet of things in enterprise services. In Web Services, 2009. ICWS 2009. IEEE International Conference on (pp. 968-975). IEEE.

[203] Hydra Middleware Project, FP6 European Project, www.hydramiddleware.eu (accessed on 16 May 16, 2016)

[204] Xia, F., Ma, L., Dong, J., \& Sun, Y. (2008, July). Network QoS management in cyberphysical systems. In Embedded Software and Systems Symposia, 2008. ICESS Symposia'08. International Conference on (pp. 302-307). IEEE.

[205] Duquennoy, S., Grimaud, G., \& Vandewalle, J. J. (2009, May). The Web of Things: interconnecting devices with high usability and performance. In Embedded Software and Systems, 2009. ICESS'09. International Conference on (pp. 323-330). IEEE.

[206] Buckl, C., Sommer, S., Scholz, A., Knoll, A., Kemper, A., Heuer, J., \& Schmitt, A. (2009, May). Services to the field: An approach for resource constrained sensor/actor networks. In Advanced Information Networking and Applications Workshops, 2009. WAINA'09. International Conference on (pp. 476-481). IEEE.

[207] Pantazis, N., Nikolidakis, S. A., \& Vergados, D. D. (2013). Energy-efficient routing protocols in wireless sensor networks: A survey. Communications Surveys \& Tutorials, IEEE, 15(2), 551-591.

[208] Liu, J., \& Hong, X. (2009). An online energy-efficient routing protocol with traffic load prospects in wireless sensor networks. International Journal of Sensor Networks, 5(3), 185-197. [209] Chen, D., \& Varshney, P. K. (2007). On-demand geographic forwarding for data delivery in wireless sensor networks. Computer Communications, 30(14), 2954-2967. 
[210] Younis, O., Krunz, M., \& Ramasubramanian, S. (2006). Node clustering in wireless sensor networks: recent developments and deployment challenges. Network, IEEE, 20(3), 2025.

[211] Booth, L., Bruck, J., Franceschetti, M., \& Meester, R. (2003). Covering algorithms, continuum percolation and the geometry of wireless networks. Annals of Applied Probability, $722-741$.

[212] He, T., Krishnamurthy, S., Luo, L., Yan, T., Gu, L., Stoleru, R., ... \& Abdelzaher, T. F. (2006). VigilNet: An integrated sensor network system for energy-efficient surveillance. ACM Transactions on Sensor Networks (TOSN), 2(1), 1-38.

[213] Ye, W., Heidemann, J., \& Estrin, D. (2002). An energy-efficient MAC protocol for wireless sensor networks. In INFOCOM 2002. Twenty-First Annual Joint Conference of the IEEE Computer and Communications Societies. Proceedings. IEEE (Vol. 3, pp. 1567-1576). IEEE.

[214] Zhang, H., Arora, A., Choi, Y. R., \& Gouda, M. G. (2007). Reliable bursty convergecast in wireless sensor networks. Computer Communications, 30(13), 2560-2576.

[215] Lien, S. Y., Chen, K. C., \& Lin, Y. (2011). Toward ubiquitous massive accesses in 3GPP machine-to-machine communications. Communications Magazine, IEEE, 49(4), 66-74.

[216] Stojmenovic, I. (2014). Machine-to-machine communications with in-network data aggregation, processing, and actuation for large-scale cyber-physical systems. Internet of Things Journal, IEEE, 1(2), 122-128.

[217] Fadlullah, Z. M., Fouda, M. M., Kato, N., Takeuchi, A., Iwasaki, N., \& Nozaki, Y. (2011). Toward intelligent machine-to-machine communications in smart grid. Communications Magazine, IEEE, 49(4), 60-65.

[218] Xia, F., Vinel, A., Gao, R., Wang, L., \& Qiu, T. (2011). Evaluating IEEE 802.15. 4 for cyber-physical systems. EURASIP Journal on Wireless Communications and Networking, 2011(1), 1-14.

[219] The Artemis Embedded Computing Systems Initiative. www.artemis-ju.eu/home_page (accessed on 16 May 16, 2016)

[220] The EU Framework Program for Research and Innovation (Horizon2020) ec.europa.eu/programmes/horizon2020/ (accessed on 16 May 16, 2016)

[221] Acatech - National Academy of Science and Engineering (December 2011), CyberPhysical Systems Driving force for innovation in mobility, health, energy and production, Position Paper http://www.acatech.de/fileadmin/user_upload/Baumstruktur_nach_Website/Acatech/root/de/Pu blikationen/Stellungnahmen/acatech_POSITION_CPS_Englisch_WEB.pdf (accessed on 16 May 16, 2016)

[222] Sangkeun Yoo. ISO/IEC JTC 1/WG 10Working Group on Internet of Things iotweek.eu/wp-content/uploads/2015/06/07-JTC-1-WG-10-Introduction.pdf (accessed on 16 May $16,2016)$

[223] ISO/IEC JTC 1 Information technology www.iso.org/iso/iso_technical_committee?commid=45020 (accessed on 16 May 16, 2016)

[224] ISO/IEC JTC 1 Internet of Things Preliminary Report 2014. (2014) www.iso.org/iso/internet_of_things_report-jtc1.pdf (accessed on 16 May 16, 2016)

[225] Report of the 1st Meeting of ISO/IEC JTC 1/SWG 5/Ad Hoc Group 1 standards.incits.org/apps/group_public/download.php/41360/ISO-IECJTC1-

SWG5_N0037_Report_of_the_1st_Meeting_of_ISO_IEC_.pdf (accessed on 16 May 16, 2016) [226] Moayeri, Nader (2013). A Collection of Definitions for the IoT, M2M, MTC, and CPS. 
[227] ETSI. Internet of Things Webpage www.etsi.org/technologiesclusters/technologies/internet-of-things (accessed on 16 May 16, 2016)

[228] NIST Webpage for CPS www.nist.gov/cps/ (accessed on 16 May 16, 2016)

[229] DRAFT Timing Framework for Cyber-Physical Systems https://s3.amazonaws.com/nistsgcps/cpspwg/pwgglobal/CPS_PWG_Timing_Annex_for_Draft_Framework_for_Cyber-

Physical_Systems_Release_0.8_September_2015.pdf (accessed on 16 May 16, 2016)

[230] DRAFT Framework for Cyber-Physical Systems https://s3.amazonaws.com/nistsgcps/cpspwg/pwgglobal/CPS_PWG_Draft_Framework_for_Cyber-

Physical_Systems_Release_0_8_September_2015.pdf (accessed on 16 May 16, 2016)

[231] David Wollman. Cyber-Physical Systems Public Working Group (CPS PWG): Draft CPS Framework Overview Webinar pages.nist.gov/cpspwg/library/ (accessed on 16 May 16, 2016)

[232] Strategic R\&D Opportunities for $21^{\text {st }}$ Century Cyber-Physical Systems. (2013) http://www.nist.gov/el/upload/12-Cyber-Physical-Systems020113_final.pdf (accessed on 16 May 16, 2016)

[233] Foundations for Innovation in Cyber-Physical Systems (2013) http://www.nist.gov/el/upload/CPS-WorkshopReport-1-30-13-Final.pdf (accessed on 16 May $16,2016)$

[234] Strategic vision and business drivers for $21^{\text {st }}$ Century Cyber-Physical Systems (2013) http://www.nist.gov/el/upload/Exec-Roundtable-SumReport-Final-1-30-13.pdf (accessed on 16 May 16, 2016)

[235] Denker, G., Dutt, N., Mehrotra, S., Stehr, M. O., Talcott, C., \& Venkatasubramanian, N. (2012). Resilient dependable cyber-physical systems: a middleware perspective. Journal of Internet Services and Applications, 3(1), 41-49.

[236] Kai Höfig (2014) A vehicle control platform as safety element out of context. HiPEAC Computing Systems Week, Barcelona, Spain, rts.eit.uni-kl.de/hipeac-ws0514/Presentations/KaiHoefig.pdf (accessed on 16 May 16, 2016)

[237] Jeremiah Gertler, (2012) U.S. Unmanned Aerial Systems fas.org/sgp/crs/natsec/R42136.pdf (accessed on 16 May 16, 2016)

[238] Wood, A. D., Stankovic, J. A., Virone, G., Selavo, L., He, Z., Cao, Q., ... \& Stoleru, R. (2008). Context-aware wireless sensor networks for assisted living and residential monitoring. Network, IEEE, 22(4), 26-33.

[239] Liu, A., \& Salvucci, D. (2001, August). Modeling and prediction of human driver behavior. In Intl. Conference on HCI.

[240] Dickerson, R. F., Gorlin, E. I., \& Stankovic, J. A. (2011, October). Empath: a continuous remote emotional health monitoring system for depressive illness. In Proceedings of the 2nd Conference on Wireless Health (p. 5). ACM.

[241] Mohammed, S., Fraisse, P., Guiraud, D., Poignet, P., \& Makssoud, H. E. (2005, December). Towards a co-contraction muscle control strategy for paraplegics. In Decision and Control, 2005 and 2005 European Control Conference. CDC-ECC'05. 44th IEEE Conference on (pp. 7428-7433). IEEE.

[242] Aguiar, Y., Vieira, M., Galy, E., Mercantini, J. M., \& Santoni, C. (2011). Refining a user behaviour model based on the observation of emotional states. Proc. COGNITIVE, 36-40.

[243] Dunbar, M. (2001). Plug-and-play sensors in wireless networks. Instrumentation \& Measurement Magazine, IEEE, 4(1), 19-23.

[244] Colombo, A. W., Karnouskos, S., \& Bangemann, T. (2014). Towards the Next Generation of Industrial Cyber-Physical Systems. In Industrial cloud-based cyber-physical systems (pp. 122). Springer International Publishing. 
[245] Hansmann, U., Merk, L., Nicklous, M. S., \& Stober, T. (2013). Pervasive computing handbook. Springer Science \& Business Media.

[246] Bröring, A., Maué, P., Janowicz, K., Nüst, D., \& Malewski, C. (2011). Semanticallyenabled sensor plug \& play for the sensor web. Sensors, 11(8), 7568-7605.

[247] Gievska, S., \& Sibert, J. (2005, October). Using task context variables for selecting the best timing for interrupting users. In Proceedings of the 2005 joint conference on Smart objects and ambient intelligence: innovative context-aware services: usages and technologies (pp. 171176). ACM.

[248] Schamai, W., Fritzson, P., Paredis, C., \& Pop, A. (2009, December). Towards unified system modeling and simulation with ModelicaML: modeling of executable behavior using graphical notations. In Proceedings of the 7th International Modelica Conference; Como; Italy; 20-22 September 2009 (No. 043, pp. 612-621). Linköping University Electronic Press.

[249] Song, E. Y., \& Lee, K. (2008). Understanding IEEE 1451-Networked smart transducer interface standard-What is a smart transducer?. IEEE Instrumentation \& Measurement Magazine, 11(2).

[250] Dunbar, M. (2001). Plug-and-play sensors in wireless networks. IEEE Instrumentation \& Measurement Magazine, 4(1), 19-23.

[251] Lin, Y. D., \& Hsu, Y. C. (2000, March). Multihop cellular: A new architecture for wireless communications. In INFOCOM 2000. Nineteenth Annual Joint Conference of the IEEE Computer and Communications Societies. Proceedings. IEEE (Vol. 3, pp. 1273-1282). IEEE.

[252] Lei, L., Zhong, Z., Lin, C., \& Shen, X. (2012). Operator controlled device-to-device communications in LTE-advanced networks. IEEE Wireless Communications, 19(3).

[253] Alliance, W. (2010). Wi-Fi peer-to-peer (P2P) specification v1. 1. Wi-Fi Alliance Specification, 1, 1-159.

[254] Camps-Mur, D., Garcia-Saavedra, A., \& Serrano, P. (2013). Device-to-device communications with Wi-Fi Direct: overview and experimentation. IEEE wireless communications, 20(3), 96-104.

[255] Xiao, X., Tao, X., \& Lu, J. (2011, September). A QoS-aware power optimization scheme in OFDMA systems with integrated device-to-device (D2D) communications. In Vehicular Technology Conference (VTC Fall), 2011 IEEE (pp. 1-5). IEEE.

[256] Bao, X., Lee, U., Rimac, I., \& Choudhury, R. R. (2010). DataSpotting: offloading cellular traffic via managed device-to-device data transfer at data spots. ACM SIGMOBILE Mobile Computing and Communications Review, 14(3), 37-39.

[257] Han, T., Yin, R., Xu, Y., \& Yu, G. (2012, September). Uplink channel reusing selection optimization for device-to-device communication underlaying cellular networks. In Personal Indoor and Mobile Radio Communications (PIMRC), 2012 IEEE 23rd International Symposium on (pp. 559-564). IEEE.

[258] Su, L., Ji, Y., Wang, P., \& Liu, F. (2013, April). Resource allocation using particle swarm optimization for D2D communication underlay of cellular networks. In Wireless Communications and Networking Conference (WCNC), 2013 IEEE (pp. 129-133). IEEE.

[259] Du, J., Zhu, W., Xu, J., Li, Z., \& Wang, H. (2012, September). A compressed HARQ feedback for device-to-device multicast communications. In Vehicular Technology Conference (VTC Fall), 2012 IEEE (pp. 1-5). IEEE.

[260] Seppälä, J., Koskela, T., Chen, T., \& Hakola, S. (2011, March). Network controlled device-to-device (D2D) and cluster multicast concept for LTE and LTE-A networks. In Wireless Communications and Networking Conference (WCNC), 2011 IEEE (pp. 986-991). IEEE. 
[261] Rosemann, M., \& Recker, J. C. (2006). Context-aware process design: Exploring the extrinsic drivers for process flexibility. In The 18th International Conference on Advanced Information Systems Engineering. Proceedings of Workshops and Doctoral Consortium (pp. 149-158). Namur University Press.

[262] Munoz, A. (2014). Machine Learning and Optimization. Available online: https://www. cims. nyu. edu/ munoz/files/ml_optimization. pdf (accessed on 16 May 16, 2016)

[263] González, O., Shrikumar, H., Stankovic, J. A., \& Ramamritham, K. (1997, December). Adaptive fault tolerance and graceful degradation under dynamic hard real-time scheduling. In Real-Time Systems Symposium, 1997. Proceedings., The 18th IEEE (pp. 79-89). IEEE.

[265] Vainio, A. M., Valtonen, M., \& Vanhala, J. (2008). Proactive fuzzy control and adaptation methods for smart homes. IEEE Intelligent Systems, 23(2).

[266] Wing, J. M. (2006). Computational thinking. Communications of the ACM, 49(3), 33-35. [267] Lee, I., \& Sokolsky, O. (2010, June). Medical cyber physical systems. In Design Automation Conference (DAC), 2010 47th ACM/IEEE (pp. 743-748). IEEE.

[268] Autonomic Computing: IBM's perspective on the state of Information Technology. 2001. Available online: http://www.research.ibm.com/autonomic/manifesto/autonomic_computi ng.pdf (accessed on 16 May 16, 2016)

[269] Hu, H., Zhang, J., Zheng, X., Yang, Y., \& Wu, P. (2010). Self-configuration and selfoptimization for LTE networks. IEEE Communications Magazine, 48(2).

[270] Erradi, A., Maheshwari, P., \& Tosic, V. (2006, November). Policy-driven middleware for self-adaptation of web services compositions. In ACM/IFIP/USENIX International Conference on Distributed Systems Platforms and Open Distributed Processing (pp. 62-80). Springer Berlin Heidelberg.

[271] Hu, X., Chu, T. H., Chan, H. C., \& Leung, V. C. (2013). Vita: A crowdsensing-oriented mobile cyber-physical system. IEEE Transactions on Emerging Topics in Computing, 1(1), 148-165.

[272] Valkó, A. G. (1999). Cellular IP: A new approach to Internet host mobility. ACM SIGCOMM computer communication review, 29(1), 50-65.

[273] Xia, F., Tian, Y. C., Li, Y., \& Sung, Y. (2007). Wireless sensor/actuator network design for mobile control applications. Sensors, 7(10), 2157-2173.

[274] Xu, Z., Liu, X., Zhang, G., He, W., Dai, G., \& Shu, W. (2008, June). A certificateless signature scheme for mobile wireless cyber-physical systems. In Distributed Computing Systems Workshops, 2008. ICDCS'08. 28th International Conference on (pp. 489-494). IEEE.

[275] Wang, X., Hovakimyan, N., \& Sha, L. (2013, April). L1Simplex: fault-tolerant control of cyber-physical systems. In Proceedings of the ACM/IEEE 4th International Conference on Cyber-Physical Systems (pp. 41-50). ACM.

[276] Kyselova, A. N. N. A. (2013). Context Data Analysis For Microgrid Control System. Visnyk NTUU" KPI": Informatics, operation and computer science, 2013(58).

[277] Philipose, M., Fishkin, K. P., Perkowitz, M., Patterson, D. J., Fox, D., Kautz, H., \& Hahnel, D. (2004). Inferring activities from interactions with objects. IEEE pervasive computing, 3(4), 50-57.

[278] Schirner, G., Erdogmus, D., Chowdhury, K., \& Padir, T. (2013). The future of human-inthe-loop cyber-physical systems. Computer, 46(1), 36-45.

[279] Clarke, D. W., \& Gawthrop, P. J. (1979, June). Self-tuning control. In Proceedings of the Institution of Electrical Engineers (Vol. 126, No. 6, pp. 633-640). IET Digital Library.

[280] Marzbanrad, J., \& Tahbaz-zadeh Moghaddam, I. (2016). Self-tuning control algorithm design for vehicle adaptive cruise control system through real-time estimation of vehicle parameters and road grade. Vehicle System Dynamics, 54(9), 1291-1316. 
[281] Noack, R., Jeinsch, T., Sari, A. H. A., \& Weinhold, N. (2014, September). Data-driven self-tuning control by iterative learning control with application to optimize the control parameter of turbocharged engines. In Methods and Models in Automation and Robotics (MMAR), 2014 19th International Conference On (pp. 839-844). IEEE.

[282] Li, H., Gao, H., Shi, P., \& Zhao, X. (2014). Fault-tolerant control of Markovian jump stochastic systems via the augmented sliding mode observer approach. Automatica, 50(7), 1825-1834.

[283] Yin, S., Luo, H., \& Ding, S. X. (2014). Real-time implementation of fault-tolerant control systems with performance optimization. IEEE Transactions on Industrial Electronics, 61(5), 2402-2411.

[284] Chamseddine, A., Theilliol, D., Zhang, Y. M., Join, C., \& Rabbath, C. A. (2015). Active fault-tolerant control system design with trajectory re-planning against actuator faults and saturation: Application to a quadrotor unmanned aerial vehicle. International Journal of Adaptive Control and Signal Processing, 29(1), 1-23.

[285] Kumar, R., Kansal, A., \& Srivastava, M. (2004). Distributed Control over Ad-hoc Sensor Actuator Networks. NESL Technical Report NESL-TR-Jan-2004.

[286] Li, S. F. (2006, January). Wireless sensor actuator network for light monitoring and control application. In Consumer Communications and Networking Conference, 2006. CCNC 2006. 3rd IEEE (Vol. 2, pp. 974-978). IEEE.

[287] Zhang, Y., Xu, C., Yu, S., Li, H., \& Zhang, X. (2015). SCLPV: Secure Certificateless Public Verification for Cloud-Based Cyber-Physical-Social Systems Against Malicious Auditors. IEEE Transactions on Computational Social Systems, 2(4), 159-170.

[288] Sharma, G., Bala, S., \& Verma, A. K. (2016). An Improved RSA-based Certificateless Signature Scheme for Wireless Sensor Networks. IJ Network Security, 18(1), 82-89.

[289] Kim, J. E., \& Mosse, D. (2008). Generic framework for design, modeling and simulation of cyber physical systems. ACM SIGBED Review, 5(1), 1.

[290] Fink, J., Ribeiro, A., \& Kumar, V. (2012). Robust control for mobility and wireless communication in cyber-physical systems with application to robot teams. Proceedings of the IEEE, 100(1), 164-178.

[291] Kanayama, Y., Kimura, Y., Miyazaki, F., \& Noguchi, T. (1990, May). A stable tracking control method for an autonomous mobile robot. In Robotics and Automation, 1990. Proceedings., 1990 IEEE International Conference on (pp. 384-389). IEEE.

[292] Zhuikov, V., \& Kyselova, A. (2013, April). Integration of context-aware control system in microgrid. In Electronics and Nanotechnology (ELNANO), 2013 IEEE XXXIII International Scientific Conference (pp. 386-390). IEEE.

[293] Anderson, C., Suarez, I., Xu, Y., \& David, K. (2015, November). An Ontology-Based Reasoning Framework for Context-Aware Applications. In International and Interdisciplinary Conference on Modeling and Using Context (pp. 471-476). Springer International Publishing.

[294] Babu, A. K. A., \& Sivakumar, R. (2015). Development of type 2 fuzzy rough ontologybased middleware for context processing in ambient smart environment. In Intelligent Computing and Applications (pp. 137-143). Springer India.

[295] Jha, S., Gulwani, S., Seshia, S. A., \& Tiwari, A. (2010, April). Synthesizing switching logic for safety and dwell-time requirements. In Proceedings of the 1st ACM/IEEE International Conference on Cyber-Physical Systems (pp. 22-31). ACM.

[296] Mitchell, R., \& Chen, R. (2015). Behavior rule specification-based intrusion detection for safety critical medical cyber physical systems. IEEE Transactions on Dependable and Secure Computing, 12(1), 16-30. 
[297] Mitchell, R., \& Chen, R. (2013). Effect of intrusion detection and response on reliability of cyber physical systems. IEEE Transactions on Reliability, 62(1), 199-210.

[298] Mitchell, R., \& Chen, I. R. (2014). A survey of intrusion detection techniques for cyberphysical systems. ACM Computing Surveys (CSUR), 46(4), 55.

[299] Muccini, H., Sharaf, M., \& Weyns, D. (2016, May). Self-adaptation for cyber-physical systems: a systematic literature review. In Proceedings of the 11th International Symposium on Software Engineering for Adaptive and Self-Managing Systems (pp. 75-81). ACM.

[300] Bagheri, B., Yang, S., Kao, H. A., \& Lee, J. (2015). Cyber-physical Systems Architecture for Self-Aware Machines in Industry 4.0 Environment. IFAC-PapersOnLine, 48(3), 1622-1627. [301] Dutt, N., Jantsch, A., \& Sarma, S. (2015, November). Self-aware cyber-physical systemson-chip. In Proceedings of the IEEE/ACM International Conference on Computer-Aided Design (pp. 46-50). IEEE Press.

[302] Sarma, S., Dutt, N., Gupta, P., Venkatasubramanian, N., \& Nicolau, A. (2015, March). Cyberphysical-system-on-chip (cpsoc): A self-aware mpsoc paradigm with cross-layer virtual sensing and actuation. In Design, Automation \& Test in Europe Conference \& Exhibition (DATE), 2015 (pp. 625-628). IEEE.

[303] Gurgen, L., Gunalp, O., Benazzouz, Y., \& Gallissot, M. (2013, March). Self-aware cyberphysical systems and applications in smart buildings and cities. In Proceedings of the Conference on Design, Automation and Test in Europe (pp. 1149-1154). EDA Consortium.

[304] Li, T., Cao, J., Liang, J., \& Zheng, J. (2015). Towards context-aware medical cyberphysical systems: design methodology and a case study. Cyber-Physical Systems, 1(1), 5-23.

[305] Li, Y., Horváth, I., Rusák, Z., van der Vegte, W., \& Zhang, G. (2016). Conceptualization of a real-time information processing platform for context-aware informing Cyber-Physical Systems. Proceedings of ASME IDETC CIE.

[306] Malekian, R., Wu, K., Reali, G., Ye, N., \& Curran, K. (2017). Cyber-physical systems and context-aware sensing and computing.

[307] Reliable vehicle type recognition based on information fusion in multiple sensor networks Fuliang Lib, , Zhihan Lva, ,

[308] Bhuiyan, M. Z. A., Wang, G., Cao, J., \& Wu, J. (2015). Deploying wireless sensor networks with fault-tolerance for structural health monitoring. IEEE Transactions on Computers, 64(2), 382-395.

[309] Azharuddin, M., Kuila, P., \& Jana, P. K. (2015). Energy efficient fault tolerant clustering and routing algorithms for wireless sensor networks. Computers \& Electrical Engineering, 41, 177-190.

[310] Azharuddin, M., \& Jana, P. K. (2015). A distributed algorithm for energy efficient and fault tolerant routing in wireless sensor networks. Wireless Networks, 21(1), 251-267.

[311] Bagci, H., Korpeoglu, I., \& Yazıc1, A. (2015). A distributed fault-tolerant topology control algorithm for heterogeneous wireless sensor networks. IEEE transactions on parallel and distributed systems, 26(4), 914-923.

[312] Wolf, M. (2014). High-performance embedded computing: applications in cyber-physical systems and mobile computing. Newnes.

[313] Wan, J., Zhang, D., Sun, Y., Lin, K., Zou, C., \& Cai, H. (2014). VCMIA: a novel architecture for integrating vehicular cyber-physical systems and mobile cloud computing. Mobile Networks and Applications, 19(2), 153-160.

[314] Nakauchi, K., Bronzino, F., Shoji, Y., Seskar, I., \& Raychaudhuri, D. (2016, July). vmen: Virtual mobile cloud network for realizing scalable, real-time cyber physical systems. In Proceedings of the Workshop on Distributed Cloud Computing (DCC). 
[315] Monostori, L. (2014). Cyber-physical production systems: Roots, expectations and R\&D challenges. Procedia CIRP, 17, 9-13.

[316] Lee, J., Bagheri, B., \& Jin, C. (2016). Introduction to cyber manufacturing. Manufacturing Letters, 8, 11-15. 


\title{
Building Smart Adaptable Cyber-physical Systems: Definitions, classification and elements
}

\author{
Borja Bordel, Ramón Alcarria, Marina Pérez-Jiménez, Tomás Robles, Diego Martín, \\ Diego Sánchez de Rivera \\ Technical University of Madrid \\ bbordel@dit.upm.es, ramon.alcarria@upm.es, marina.perez@isom.upm.es, \\ tomas.robles@upm.es, diego.martin.de.andres@upm.es, \\ diego.sanchezderiveracordoba@gmail.com
}

\begin{abstract}
The provision of systems that join the information technologies with the physical world has been one of the most popular issues in research in the last fifteen years. Nevertheless, the complexity associated with these systems prevented many authors from providing a theoretical formalization. Even it is difficult to find a consensus name or a definition for this new type of systems. Therefore, in this work we propose a theoretical and technical formalization for these solutions, which includes a name at the forefront of research: smart adaptable cyber-physical systems (SACPS). We also present a complete definition for the SACPS, and explain the elements and subsystem interaction.
\end{abstract}

Keywords: smart adaptable cyber-physical systems, SACPS, RFID, wearable technologies, Wireless HAN, cybernetic devices

\section{Introduction}

Making a research in the smart environment field is complicated. The causes are, overall, the term's ambiguity and the lack of a bibliographic base (extended and coherent) which fixes the limits, elements, technologies, etc. in this kind of systems.

Therefore, the main objective of this work is presenting a new concept, with technical character and without ambiguity, which delimits the application area of the (until now) so-called "smart environment". We are talking about Smart Adaptable Cyber-Physical Systems (SACPS).

The rest of the paper is organized as follows: In section two we review the previous efforts of some authors for defining this type of systems and try to extend them with the now-a-days knowledge. Section three presents a classification for SACPS. Finally, section four is dedicated to the elements which make up a SACPS and to their functions. 


\section{History, definition and related work}

The first efforts for integrating information technologies in the physical world were based on electronics. Thus, from 2000, several terms have appeared in the literature and, overall, have been proposed in conferences, to refer to smart distributed electronic systems (more or less embedded in the physical world): Smart Home [1] [2], Smart Office [3], Intelligent Home [4], Smart Environments [5], Adaptive Versatile Environments [6], Interactive Spaces [7], Problem-Solving Environments [8], etc.

In a parallel way, for these terms some definitions appeared. Thereby, in 2000, [9] said that "A smart space is a region of the real world that is extensively equipped with sensors, actuators and computing components". The problem of this definition, viewed today, lies in the fact that it is incomplete, because the "intelligent factor" disappears, as the definition only considers hardware components. Due to that, in 2002, other authors decide to propose their own definition. [1] defines a Smart Environment as "a system that is able to autonomously acquire and apply knowledge about the environment and adapt to its inhabitants' preferences and requirements in order to improve their experience". This second option, however, is unspecific. Although it explains the expected operation way (from a high level point of view), including the human factor and not including any reference to hardware or software elements, makes difficult to determine when a system agrees with the definition and when not.

So much so that, in 2005 , the same authors in [5] proposed a new definition: "A smart environment is a physical world that is interconnected through a continuous network abundantly and invisibly with sensors, actuators and computational units, embedded seamlessly in the everyday objects of our lives". This last definition, much more complex, was endorsed by other authors, such as the famous KITECH (Korea Institute of Industrial Technology), nevertheless, as in the first case; this definition does not include any reference to the "intelligent part" of the system.

From 2010, more or less, the interest on these systems decrease, and researches abandon attempts to formalize the theoretical framework. At this moment, a new concept captures all interest: the Cyber-Physical Systems (CPS). The most popular definition, presented in [10], defines the CPS as "integrations of computation and physical processes. Embedded computers and networks monitor and control the physical processes, usually with feedback loops where physical processes affect computations and vice versa."

In 2010, hardware infrastructure for ubiquitous systems has started maturing, what allows defining CPS from a more behavioral and specific point of view. However, although any future attempt must apply CPS's principles, this name also groups systems that are "not enough smart" (for example, as we said, in 2002 it became clear that smart systems have to "adapt to its inhabitants' preferences and requirements").

In order to join the CPS's principles with all previous knowledge, we define the Smart Adaptable Cyber-Physical Systems (SACPS).

A Smart Adaptable Cyber-Physical Systems (SACPS) is a physical world that is interconnected through a continuous network abundantly and invisibly with sensors, actuators and computational units, embedded seamlessly in the everyday objects and/or clothes. The resulting system must be monitored from a control process man- 
agement entity, and must define feedback loops where information and collected data from sensors affect physical processes. The system must be adaptable to different domains and be oriented to prosumer user, for which must implement selfconfiguration and dynamic self-adaptation capabilities.

In this definition, as we can see, all aspects previously seen are present:

The first definition's part refers the communications physical infrastructure and its relation with the external world. As can be seen, it is really similar to the definition proposed in [5] for the "Smart Environments", to which a reference to the modern wearable technologies has been added.

The second part, in relation with process control and feedback loops, is directly related with the work philosophy in CPS (orient to process, Big Data, etc.).

The third and last part (relative to system self-configuration and its adaptation to prosumer user) gathers the necessity (showed in [1]) to make the system autonomous and adaptable to the users' preferences and requirements.

\section{Elements in a SACPS. Layered model.}

All SACPS are parts of the physical world, which connects with the rest of the reality through a permeable interface that allows the transference of materials, objects, energy, user, etc. (see Figure 1(a)).

However, despite they can be considered as a unit, inside each SACPS various subsystems can be distinguished. Each subsystem will encapsulate some elements and functions from the complete systems. Namely:

Physical subsystem: It includes all the elements from the ordinary world that are part of the SACPS, but which they have not been modified to include any electronic instrument, communication device or process element. This subsystem also includes the power supply, the physical space occupied by the system and all the information stored in several variables, own the physical world (temperature, blood pressure...). Here are also integrated all users that take advantage of the SACPS's services, but which are not experts neither in the system's technology base nor in its specific application field. In what follows, we will name as "habitants" to this kind of users.

Cybernetic subsystem: It includes all the elements from the ordinary world that have been modified in some way, in order to integrate (seamless) electronic instrumental, communication devices and/or process elements. Smart furniture, wearables or any other similar device (we will call "cybernetic devices" to them) belong to this subsystem. This subsystem is, besides, in charge of monitoring the physical subsystem and acting over it. Cybernetic devices, at the same time, can be made of up to three different modules: sensorisation module, process and execution module, and communications module. Sensorisation module includes all electronic instrumentation and the device's actuators, such as displays, LED, etc. Process and execution module includes, if there was one, the device's microcontroller unit and all its peripherals. This part is in charge of executing the task ordered from the smart subsystem, controlling sensors and actuators, executing micro-services, etc. Finally, communications module includes all the infrastructure and software, necessary to allow the communi- 
cation among the cybernetic devices, and between the device and the smart. Depending on how many modules implements a cybernetic device; we can distinct three classes of devices:

- Tagged cybernetic device: We use this name with devices that only implement the communications module. Furthermore, that module can only communicate with other cybernetic devices with which link connection will be available (never with the smart subsystem). The amount of information which can communicate is, besides, limited. The employed terminology comes from RFID technology, where this kind of modules is called "tag RFID".

- Peripheral cybernetic device: These devices implement the sensorisation module and a reduce functions communications module. Because these devices do not have a complete communications module, they only can communicate with other cybernetic devices with which link connection exits. This time, however, there are not limitations in the amount of transmittable data.

- Full cybernetic device: Finally, devices of this group implement the three possible modules (with full functions). These devices, typically, will receive information from their sensors and from close peripheral devices and tagged objects. With this information it will execute the delegated task, and will inform to the smart subsystem if it were necessary.
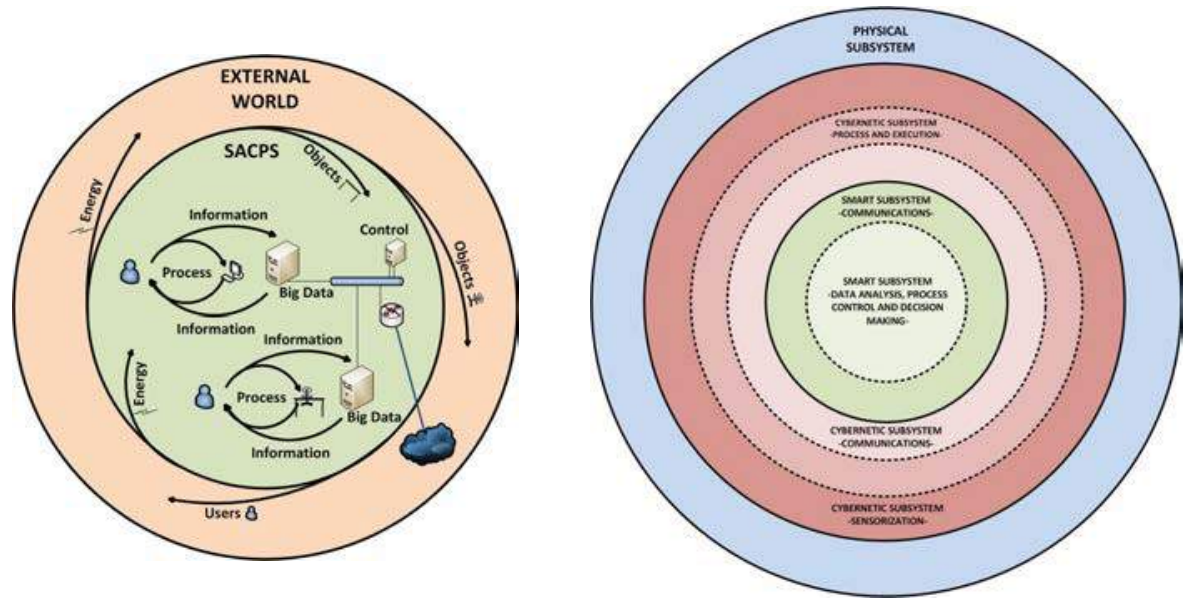

Fig. 1(a). Relation between the external world and a SACPS.

(b) Layered model of a generic SACPS

Smart subsystem: It includes all the infrastructure, applications, software, and management systems which controls and monitorizes the other subsystems, processes received data and feedbacks future process with the information extracted from these data. It can be divided in other two subsystems: communications subsystem (that makes possible the information flow with the cybernetic subsystem) and data analysis, process control and decision making subsystem. Here, besides, it will be implemented the politics that will allow the self-configuration and dynamic self-adaptation of the SACPS. This subsystem, moreover, includes those users which are not experts 
in the SACPS's base technology, but they are experts in the system's application field (prosumer users). These users, as can act directly over the smart subsystem, so that they can adjust the global behavior of the SACPS to their requirements.

It is usual in this kind of technologies (see [10]) representing the system as a set of concentric layers, where as you progress inward, you get way from the physical world. Then, the layered model for a generic SACPS can be expressed as in Figure $1(\mathrm{~b})$.

\section{Classification of SACPS}

Within the set of SACPS several classifications can be made: depending on the application field, depending on the number of elements, etc. The nature of the main data source considered differentiates all SACPS in these three categories:

Actor-focused systems: These systems are totally oriented to obtain data from SACPS's habitants. The interest is focused on how the users behave and what they feel. Due to the nature of these systems, it is indispensable that data sampling, processing, and decision making to be at real-time and execute in high-speed (with the purpose of supplying feedback as soon as possible). Of course, this does not impede, in addition, other systems for data analysis to be included. From an electronic point of view, these systems are extensively equipped with biometrical sensors and wearable devices (see Figure 2); all of them powered in an independent and continuous way, in order to guarantee a permanent access to users' data. Systems thought for helping in some types of rehabilitation, or labor stress detection, are some of the applications of the SACPS of this category.

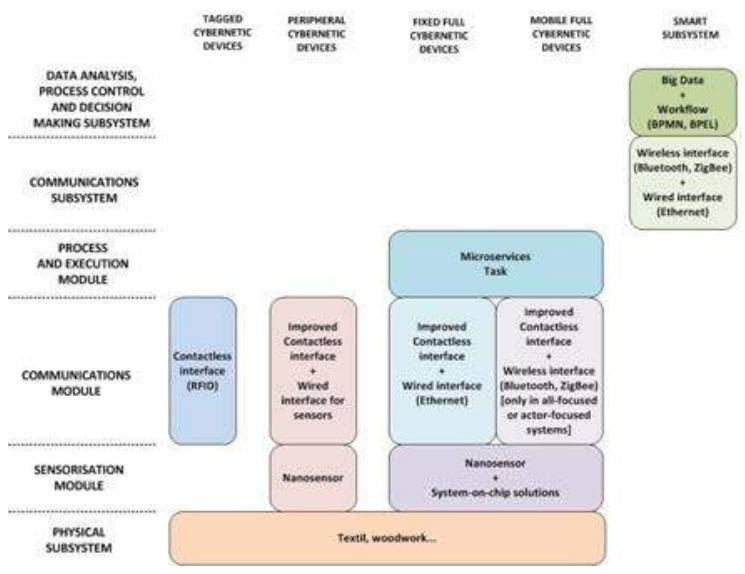

Fig. 2. Base technologies employed in SACPSs

Object-focused systems: In these systems the interest is focused on the interactions among the devices which make up the SACPS. In these systems, moreover, habitants become elements with the same importance, and at the same level, than furniture, objects. Applications deployed in this type of systems do not demand, in general, a 
continuous data supply, so data sampling can be based on collection-stored-delivery schemes. Furthermore, at hardware level, a more relaxed scheme of data sampling allows the extensive use of communication systems where one of the extremes is passive (see Figure 2). Traceability systems, control process and stock management, are typical application examples that belong to this group of SACPS.

All-focused systems: These systems are really complex, as they integrate both visions from the previous groups. On the one hand, they implement applications typical to the object-focused systems, where habitants and object are treated in the same way. On the other hand, the information provided by the actor-focused systems is also necessary here, so user monitoring infrastructure must be included. At electronic level, they are systems really similar to actor-focused ones, although, at application level, they implement (besides) functionalities typical from object-focused systems. It is a really useful point of view in systems destined to critical or dangerous resources management such as explosives or toxic gases. In these situations, knowing infrastructure information (stock, etc.) is as important as knowing actor information (i.e. the biological state of worker that manipulate the material).

\section{References}

1. Das S.K., Cook D. J.: The Role of Prediction Algorithms in the MavHome Smart Home Architecture. In IEEE Wireless Communications, 9 (6), 77-84, Dec 2002.

2. Das S.K., Cook D. J.: Agent Based Health Monitoring in Smart Homes. In Proceedings of the International. Conference on Smart Homes and Health Telematics (ICOST), Singapore, Sept 2004, (Keynote Talk).

3. Le Gal C., Martin J., Lux A., Crowley, J. L.: Smart Office: Design of an Intelligent Environment on IEEE Intelligent Systems, 16 (4), July-Aug 2001.

4. Lesser E.: The Intelligent Home Testbed. On Proceedings Of Autonomy Control Software Workshop, Jan 1999

5. Das S.K., Cook D. J.: Smart Environments: Technology, Protocols, and Applications. On Wiley, 2005.

6. Youngblood M., Cook D. J., Holder L. B.: Managing Adaptive Versatile Environments. Proceedings of IEEE International Conference on Pervasive Computing and Communications, 351-360, 2005.

7. Fox A., Johanson B., Hanrahan P., Winograd T.: Integrating Information Appliances into an Interactive Space. On IEEE Computer Graphics and Applications, 20 (3), 54-65, 2000.

8. Srivastava M. B.: Smart Kindergarten: Sensor-Based Wireless Networks for Smart Problem-Solving Environments. On Proceedings ACM Conference on Mobile Computing and Networking, 2001.

9. Nixon P., Dobson S., Lacey G.: Managing Smart Environments. In Proceedings of the Workshop on Software Engineering for Wearable and Pervasive Computing. June, 2000

10. Lee E.: Cyber physical systems: Design challenges. On Proceedings on 11th IEEE International Symposium on Object Oriented Real-Time Distributed Computing (ISORC), 2008. 363-369 


\title{
Physical processes control in Industry 4.0-based systems: a focus on Cyber-Physical Systems
}

\author{
Borja Bordel, Diego Sánchez de Rivera, Álvaro Sánchez-Picot, Tomás Robles \\ Technical University of Madrid \\ bbordel@dit.upm.es, diego.sanchezderiveracordoba@gmail.com, alvaro.spicot@gmail.com, \\ tomas.robles@upm.es
}

\begin{abstract}
Industry 4.0 or cyber-industry may employ Cyber-Physical Systems (CPS) profusely to organize production media in a new and more efficient way. Many processes might be defined in CPS and Industry 4.0, however, physical processes are probably the least studied. Therefore, in this paper we propose an architecture for Industry 4.0-based systems focused on the control of physical processes. The architecture follows the CPS paradigm. Moreover, a first evaluation of the performance of the proposed solution, using simulation tools, is provided. The results proved the proposed architecture is a valid solution for physical control processes.
\end{abstract}

Keywords: Cyber-Physical Systems, Industry 4.0, functional architectures, physical processes

\section{Introduction}

Most authors agree Industry 4.0 is any solution for industry where information technologies belonging to the next technical revolution are used [1]. In particular, a new type of integrated systems has become really popular: the Cyber-Physical Systems (CPS). CPS are integrations of physical and computational processes [2].

The use of CPS technologies in order to create the Industry 4.0 has been analyzed in various works [3]. However, most works are focused in the deployment of CPS in manufacturing scenarios, where processes have the form of business processes (a collection of activities of tasks, making a workflow). Then, companies which work with physical processes (as pasteurization or chemical reactions) are not usually considered.

\section{$2 \quad$ Processes in Industry 4.0 and CPS}

Many types of process can be defined in CPS. In Figure 1 we present a taxonomy for processes in CPS. Computational processes are described as a collection of activities, related by means of some transitions. Business processes may be orchestrated if a high-level entity controls the workflow and orders the execution of each task; or they may be choreographed if a collection of devices operating independently makes up 
the final high-level process. Finally, depending on the way in which the system is managed (if devices explicitly notify the changes or a supervisory module is deployed) the process might be event-driven, device-driven or human-driven. Physical processes (see Figure 2) and processes mixing activities and physical processes (hybrid processes) are also present in CPS.

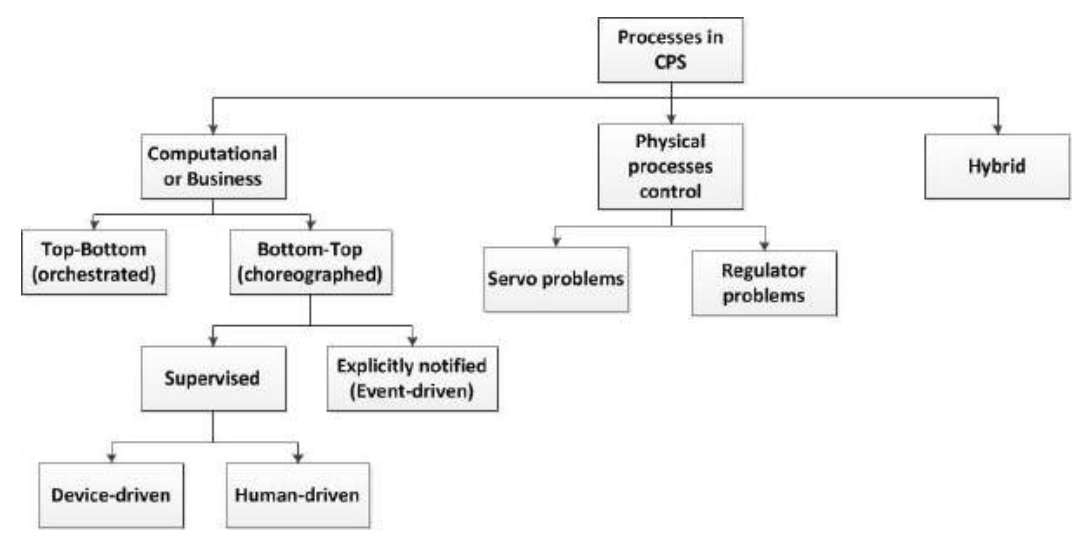

Fig. 1. Taxonomy of processes in CPS

SERVO PROBLEM

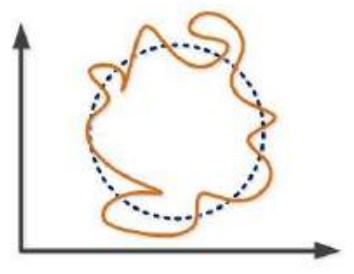

The system is controlled

to follow a certain flow

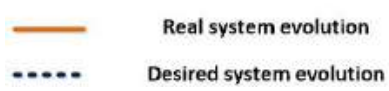

REGULATOR PROBLEM

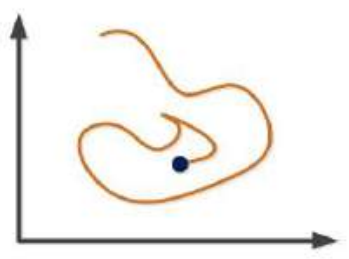

The system is controlled to reach an equilibrium point

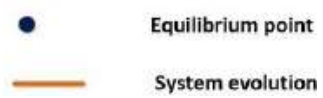

Fig. 2. Different physical processes in the state space

\section{Requirements analysis. Proposed architectures}

In this Section we are analyzing the specific requirements of CPS in industrial scenarios, as well as the needs of systems to perform physical processes control.

First, some specific requirements have to be considered in a CPS architecture for Industry 4.0. 
- REQ\#1: the architecture should support designed control systems for both, computational (including human-driven) and physical processes

- REQ\#2: high-level applications should be independent of the hardware platform, which may be heterogeneous.

- REQ\#3: the architecture should include elements to evaluate the system performing and determine if any result does not correspond to the expected one and/or some defined rules or tasks are not satisfied

- REQ\#4: methods for informing domain experts and modules to allow them to define the processes, activities and/or rules to be executed in the system should be considered in the architecture

- REQ\#5: the architecture should consider tools for self-management and automatic control of the hardware platform

- REQ\#6: procedures to extract valuable information from the data generated by the hardware platform should be considered

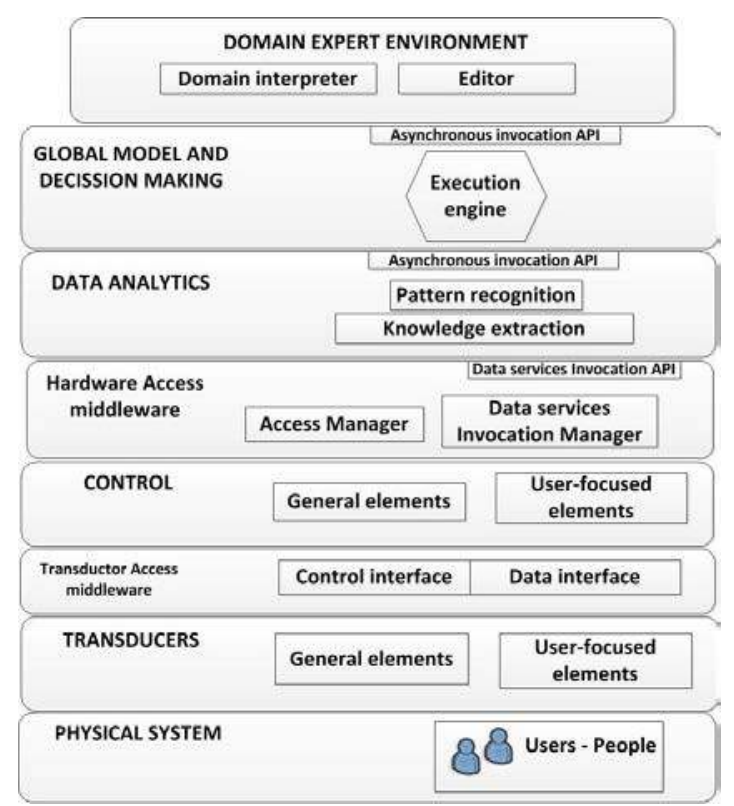

Fig. 3. Proposed architecture for CPS in Industry 4.0 scenarios

In the research literature, various architectures for CPS may be found [4]. Among all these proposals, two are especially interesting for this work. Namely: the $5 \mathrm{C}$ architecture [5] and the architecture created by National Institute of Standards and Technology (NIST) [6]. Taking into account these previous proposals, we present in Figure 3 a general functional architecture for CPS in Industry 4.0 scenarios. Then, considering the general functional architecture for CPS presented in Figure 3, and the requirements cited above, the detailed functional architecture showed in Figure 4 for physical processes control may be deducted. 


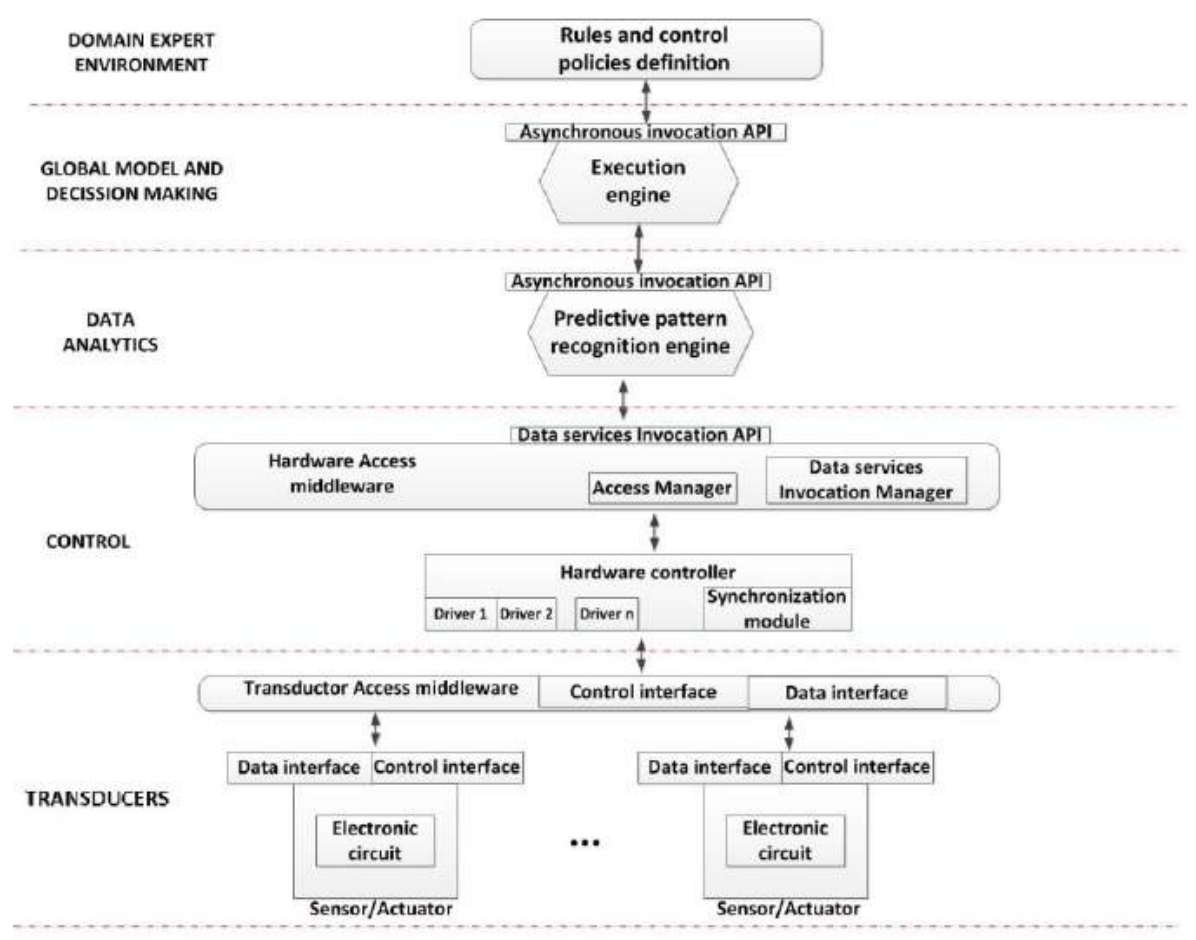

PHYSICAL SYSTEM

Fig. 4. Proposed architecture for CPS-based physical processes control systems

The proposed architecture includes sensors and actuators used for controlling the physical processes. A hardware controller is also considered to manage data from transducers. It also includes all the needed drivers to operate the sensors and actuators, and a synchronization module being able of link the CPS evolution to the temporal evolution of the physical process. A middleware is included between transducers and the hardware controller. The data services invocation manager transforms raw data in the hardware platform into structure data being able of feed some remote data services. In the data analytics layer a predictive pattern recognition engine is implemented. This engine offers different services which may be invoke from the data services invocation manager. These services are responsible of identifying the state of the physical world and/or predict the future states. With this information the predictive pattern recognition engine may invoke high-level data services in order to decide the system's reaction.

Finally, in an execution engine in the global model and decision making layer the information from the predictive pattern recognition engine is evaluated along the rules and control policies defined by the users. The reaction of the systems is immediately obtained and transmitted towards the transducers layer. At the same time, all the information about the physical world state and the system's reaction is showed in the 
domain expert environment (by means of which users can also define the rule or control policies to be applied).

\section{Experimental validation: system simulation}

In this Section we are simulating the performing of our proposal when used to control the cooling process in a steel factory.

The physical process of a piece of steel getting cooled was simulated using the three-dimensional heat equation. Actuators, represented by the additional term in the equation, along with a set of temperature sensors, made up the hardware platform of the transducers layer. The data is transmitted to a hardware controller in the control layer (Figure 4), where a software phase-locked loop synchronizes the system behavior with the temporal evolution of the heat equation. The hardware controller sends data to the data services invocation manager, which invokes a REST service which represents the predictive pattern recognition engine at the data analytics layer. Data analytics elements process the information using a simplified model of the heat equation's solution to calculate the next states of the physical processes. In the global model and decision making layer an execution engine is deployed. Information about the current process state and the calculated future steps are sent to the execution engine, where the next value for the actuators in the transducers layer (represented by the control term in the heat equation) is calculated. In the domain expert environment the objective of the control system was to force the cooled process to follow a predefined temporal evolution, by default.
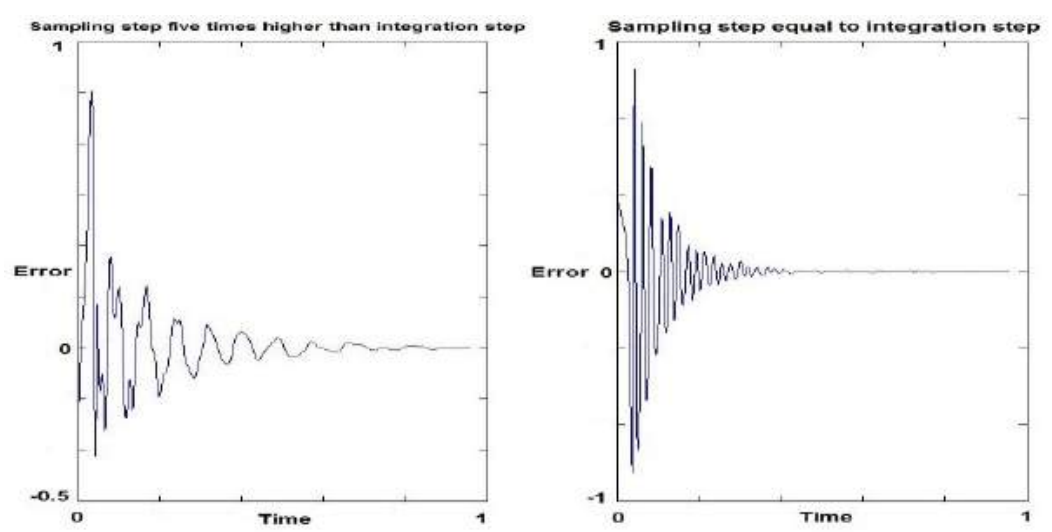

Fig. 5. Spatial media of the instantaneous error

\section{$5 \quad$ Results}

The main problem in the proposed architecture is guaranteeing the stability of the control loop so that the physical process behaves as desired. Figure 5 shows the spa- 
tial media of the instantaneous error in the control loop in two different cases: (i) the sampling step is equal to the integration step and (ii) the sampling step is five times higher than the integration step. The media is presented normalized.

As can be seen, in both cases the loop is stable and, finally the physical process behaves as desired (the error tends to zero). However, the time needed to reach the equilibrium goes up when the sampling period increases. Particularly, when the sampling step is five times higher than the integration step, the double time is necessary to reach the equilibrium. Moreover, the error varies faster when the sampling period is smaller, as can be seen in Figure 5. Any case, Figure 5 proves the proposed architecture is a valid solution for physical control processes.

\section{Conclusions}

In this paper we have investigated the use of CPS to control physical processes in the context of Industry 4.0. We have proposed a general architecture for CPS in Industry 4.0, and used that architecture to design a control system for physical processes. Moreover, we have proposed an experimental validation performed on a simulation environment. The results proved the proposed architecture is a valid solution for physical control processes. Besides, we discovered the importance of the sampling scheme in the system performance; thus, the use of advanced sampling schemes (such as on-demand sampling) will be another key to the success of CPS and Industry 4.0.

\section{Acknowledgments}

The research leading to these results has received funding from the Ministry of Economy and Competitiveness through SEMOLA project (TEC2015-68284-R)

\section{References}

1. Rüßmann, M., Lorenz, M., Gerbert, P., Waldner, M., Justus, J., Engel, P., \& Harnisch, M. (2015). Industry 4.0: The Future of Productivity and Growth in Manufacturing Industries. Boston Consulting Group.

2. Lee, E. A. (2006, October). Cyber-physical systems-are computing foundations adequate. In Position Paper for NSF Workshop On Cyber-Physical Systems: Research Motivation, Techniques and Roadmap (Vol. 2).

3. Jazdi, N. (2014, May). Cyber physical systems in the context of Industry 4.0. In Automation, Quality and Testing, Robotics, 2014 IEEE International Conference on (pp. 1-4)

4. Tan, Y., Goddard, S., \& Perez, L. C. (2008). A prototype architecture for cyber-physical systems. ACM Sigbed Review, 5(1), 26

5. Lee, J., Bagheri, B., \& Kao, H. A. (2015). A cyber-physical systems architecture for industry 4.0-based manufacturing systems. Manufacturing Letters, 3, 18-23.

6. Bordel Sánchez, B., Alcarria, R., Martín, D., \& Robles, T. (2015). TF4SM: A Framework for Developing Traceability Solutions in Small Manufacturing Companies. Sensors, 15(11), 29478-29510 


\title{
Building enhanced environmental traceability solutions: From Thing-to-Thing communications to Generalized Cyber-Physical Systems
}

\author{
Borja Bordel $^{1 *}$, Ramón Alcarria ${ }^{2}$, Miguel Ángel Manso ${ }^{2}$, and Antonio Jara ${ }^{3}$ \\ ${ }^{1}$ Department of Telematics Systems Engineering, Universidad Politécnica de Madrid \\ Calle Ramiro de Maeztu, 7, 28040 Madrid, Spain \\ bbordel@dit.upm.es \\ ${ }^{2}$ Department of Topographic Engineering and Cartography, Universidad Politécnica de Madrid \\ Calle Ramiro de Maeztu, 7, 28040 Madrid, Spain \\ \{ramon.alcarria,m.manso\}@upm.es \\ ${ }^{3}$ Institute of Information System,. University of Applied Sciences Western Switzerland \\ Route de Moutier 14, 2800 Delémont, Switzerland \\ jara@ieee.org
}

\begin{abstract}
In the last decade, many different paradigms related to the named "next-generation technological systems" have appeared: from the Internet-of-Things to Cyber-Physical Systems and Machine-toMachine communications. Traditionally these systems only consider hardware devices in their designs. However, the experience has proved that the really valuable solutions are which are humanfocused or environment-focused (biological signal monitoring, people traceability, assisted-living, etc.). In this context, previous machine-focused paradigms have to be redefined. Therefore, this paper analyzes the requirements of technological solutions for environmental monitoring and proposes a coherent framework for their design. Moreover, most important components are identified and some relevant problems in this field are addressed; mainly the identifier management and the system modeling. Finally, a prototype for people traceability based on the proposed paradigms and Bluetooth Beacons technology is described. Results showed that the quality of the provided information is much higher in these new systems than in traditional approaches.
\end{abstract}

Keywords: Thing-to-Thing communications, Generalized Cyber-Physical Systems, people traceability, ubiquitous computing, pervasive sensing, environmental monitoring

\section{Introduction}

Cyber-Physical Systems (CPS) are integrations of physical and computational processes [15]. Although there is not a unified definition, this paradigm refers to a new generation of embedded devices, being able to interact in an enhanced way with the physical world [9]. In this way, communications between elements in a CPS must be based on machine-to-machine (M2M) techniques (those totally and automatically managed by devices [16]). Other similar systems, such as the Internet-of-Things (IoT) or the Wireless Sensor Networks (WSN), also belong to this new generation of engineered solutions and are usually analyzed together. A typical architecture for any of these systems (see Figure 1) does not include humans or any other living creature or environmental element (total automatization is the final objective). However, in the last years, it has been proved that the really interesting applications in this new era

\footnotetext{
Journal of Internet Services and Information Security (JISIS), volume: 7, number: 3 (August 2017), pp. 17 33

${ }^{*}$ Corresponding author: E.T.S.I. Telecomunicación. Universidad Politécnica de Madrid. Avenida Complutense $n^{0} 30$. 28040 - Madrid (Spain), Tel: (+34) 915495700 (Ext. 3035)
} 
are which have humans and their environment as central actor. Many works related to biological signal monitoring [27], human traceability [9], human activity analysis [20], assisted living [29], etc. have been reported; and several successful commercial applications in this field have been placed into the market [21]. In this context, some authors have identified a new problem named as "the human-in-the-loop problem" [35]. It refers to the introduction of humans in the new technological systems, primary designed to operate without considering any human factor. Different roles have been identified for humans in these new solutions, although it is not clear how supporting the human intervention.

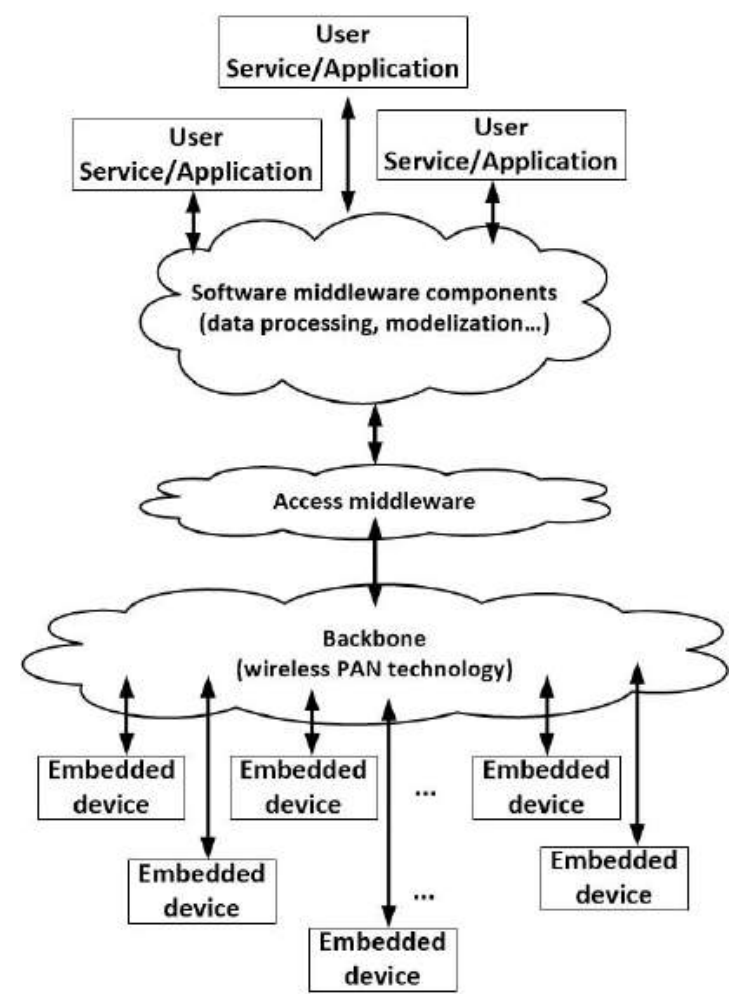

Figure 1: General architecture for a next-generation technological system

Some new paradigms, such as the humanized Cyber-Physical Systems [8], have been proposed, but they only cover some particular aspects of the problem (for example, the integration of humans as service providers). Many important issues, then, are not addressed (such as the way in which animals and other living creatures may be integrated into the system). Moreover, environmental monitoring or traceability solutions require specific instruments not usually included in traditional technological frameworks. In particular, it is required a representation and modeling instrument, and every element (or thing) in the environment must be provided with a hardware device (which cannot be seamless integrated because of obvious reasons) and an identifier.

Therefore, in this paper it is proposed a new concept named as Generalized Cyber-Physical Systems (GCPS) specially designed to support environmental traceability solutions using the CPS principles and other technological paradigms of the new era. In this context, Machine-to-Machine communications turn into Thing-to-Thing communications, as important information comes from living creatures and other physical elements, not from embedded machines (as occurs in WSN or IoT scenarios). Besides, the proposed framework and architecture consider different layers focused on representing the state of the environment being monitored (a UML model for this type of solutions is described).

The rest of the paper is organized as follows: Section 2 introduces the state of the art in studies about 
the integration of humans and other living creatures in technological systems. Section 3 proposes the concept of GCPS and a framework to design environmental control solutions, and analyzes the transition from the Machine-to-Machine (M2M) paradigm to the Thing-to-Thing (T2T) approach. Besides, the problem of the identifier management in these solutions is described and discussed. Section 4 provides an experimental validation based on a system designed following the described principles. Finally, Sections 5 and 6 describe some experimental results and some conclusions of our work.

\section{State of the Art}

Applications of CPS to traceability are numerous. Most of them are based on RFID (Radio Frequency Identification) solutions [36], where regular Things are provided with a miniaturized RFID chip [9]. However, recently, other proposals based on Wireless Sensor Networks [42] or Smart Transducers have been reported [8]. In general, nevertheless, all these papers consider CPS as a collection of physical objects which are operated by users, who are external participants. This vision is currently changing and each time more proposals of CPS including humans and other living creatures are reported.

Different proposals about human and other living creatures into CPS, WSN or IoT scenarios have been proposed.

As said in the Introduction, the "human-in-the-loop" systems are a special type of CPS which include humans in different roles. Basically, three different types of systems including people have been identified [28]: (i) systems directly controlled by humans, (ii) systems monitoring humans which take appropriate actions, and (iii) a combination of (i) and (ii). However, despite this exhaustive analysis, most works related to human-in-the-loop systems and applications are focused on monitoring humans [35, 17, 17], as it is the most challenging and revolutionary proposal. Any case, apart from the challenges identified for human integration into CPS, other aspects (such as animal traceability) are not addressed. Besides, in many applications humans are considered as a part of the environment instead of a new element in the CPS.

Some remarkable works in the field of human-in-the-loop systems are which are focused on univocally assigning identifiers to people [40]. These works may be used as the first step in traceability solutions, as they allow managers to change dynamically the identification of humans.

Other important proposals describe the called "Humanized Cyber-Physical Systems" [8]. This concept refers to CPS where humans are provided with the required instruments to be considered service providers, in the same way as sensors, actuators and the rest of hardware devices. Different proposals on the self-configuration of these systems [8], their design [44, 45] or about other similar ideas such as the "humanized cyber-physical services" [1] may be found.

Some relevant works about CPS consider animals as an additional regular actor by default [5] . Animals are provided with sensing instruments [5] or are monitored using enhanced pattern recognition techniques [39] in the same way than humans. A similar research line is focused on animal control, using cyber-physical technologies. The basic idea of these proposals is to seamless integrate electronic devices (in particular, smart sensors and actuators) into animals with the objective of controlling their behavior [41] [43].

In respect to the inclusion of other living creatures in the system, different proposal may be found. Some of them are focused on the integration of miniaturized electronic devices into animals or plants [30]. However, these proposals are complicated to apply to uncontrolled or ad hoc scenarios. Most remarkable works analyzed natural emergency situation (for example, a forest fire [30]). In these works mathematical model to understand the living creature behavior are executed using cyber-physical instruments. These ideas are very valuable, as may support advanced services in traceability solutions. Finally, in the last group of works, animals are passive agents, whose lives are improved by means of specific 
services supported by a Cyber-Physical infrastructure [3, 13] (for example, they might be moved during grazing to enhance the nutrients in the field).

In comparison with all these previous proposals, our work proposes a coherent general framework, being able of considering both humans and other living creatures (in any of the possible roles). The basic idea is to monitor the environment and the activities of the creatures and humans; as well as to trace the aspect related to them which are considered relevant.

Considering these different approaches, different models and various modeling techniques for CPS have been described. Models based on semantic agents [25] are the most recent proposal. However, models based on matrix algebra [19] for pervasive sensing platforms based on CPS paradigm, and traditional solutions based on hybrid techniques [14] may be also found. In general, nevertheless, these proposals do not cover the entire stack of layers described for CP in the most common CPS reference architectures [9][7]. In this work, therefore, it is proposed a UML model covering all the relevant aspects of CPS [6].

\section{A New Cyber-Physical Framework for Traceability}

In this Section it is proposed a general framework for traceability, based on the integration of humans and other living creatures into CPS. This new type of systems is called Generalized Cyber-Physical Systems, and presents a specific architecture which is analyzed and described in the first subsection. In the second subsection it is presented the concept of Thing-to-Thing communications and discussed the identifier management. In the third subsection the system modeling is addressed.

\subsection{General Framework: Analysis and Proposal}

A generic traceability system is basically composed of two different sub-systems: the data acquisition and the information representation (see Figure 2 (a)). This division is also valid for Cyber-Physical Systems (see Figure 2 (b)) where the "Modeling" layer is specifically focused on information representation and "Sensors and actuators" on information acquisition.

As main difference, traditional traceability solutions consider elements to be monitored as external entities to the system, but CPS include all the creature or devices with interact with other components in the system. In this context, traceability solution in the new era (the Industry 4.0, as called as the era of the CPS [23]) must follow the principles of the Cyber-Physical infrastructures. In that way, traditional CPS are insufficient as they are only focused on environmental monitoring (temperature, humidity, etc.). Furthermore, other definition such as Humanized CPS [8], Industrial CPS [11], etc. present the same problem, as they only consider a certain part of the physical world.

In order to overcome these problems, a new definition is proposed: the Generalized Cyber-Physical Systems (GCPS). A GCPS is a CPS which integrates industrial production systems, humans, other living creatures, and any other desired element into its physical platform in the same way as embedded devices, sensors and actuators. These new elements must be able to provide services, execute processes, and perform any other activity which previously was supported only by hardware devices.

GCPS require including new components in the systems, specifically focused on adapting the new elements in the physical platform to the interfaces in the high-level layers. In that way, as User-focused components were defined in Humanized CPS; in GCPS we define the "Dedicated components" to fulfill these requirements (see Figure 3 (a)). Considering these definition, richer reference architecture for GCPS may be proposed (see Figure 3 (b)).

Basically, five different new layers have been included in the "Modeling level". These new layers represent a virtual instance of the system (or system daemon, using the proposed terminology) which 

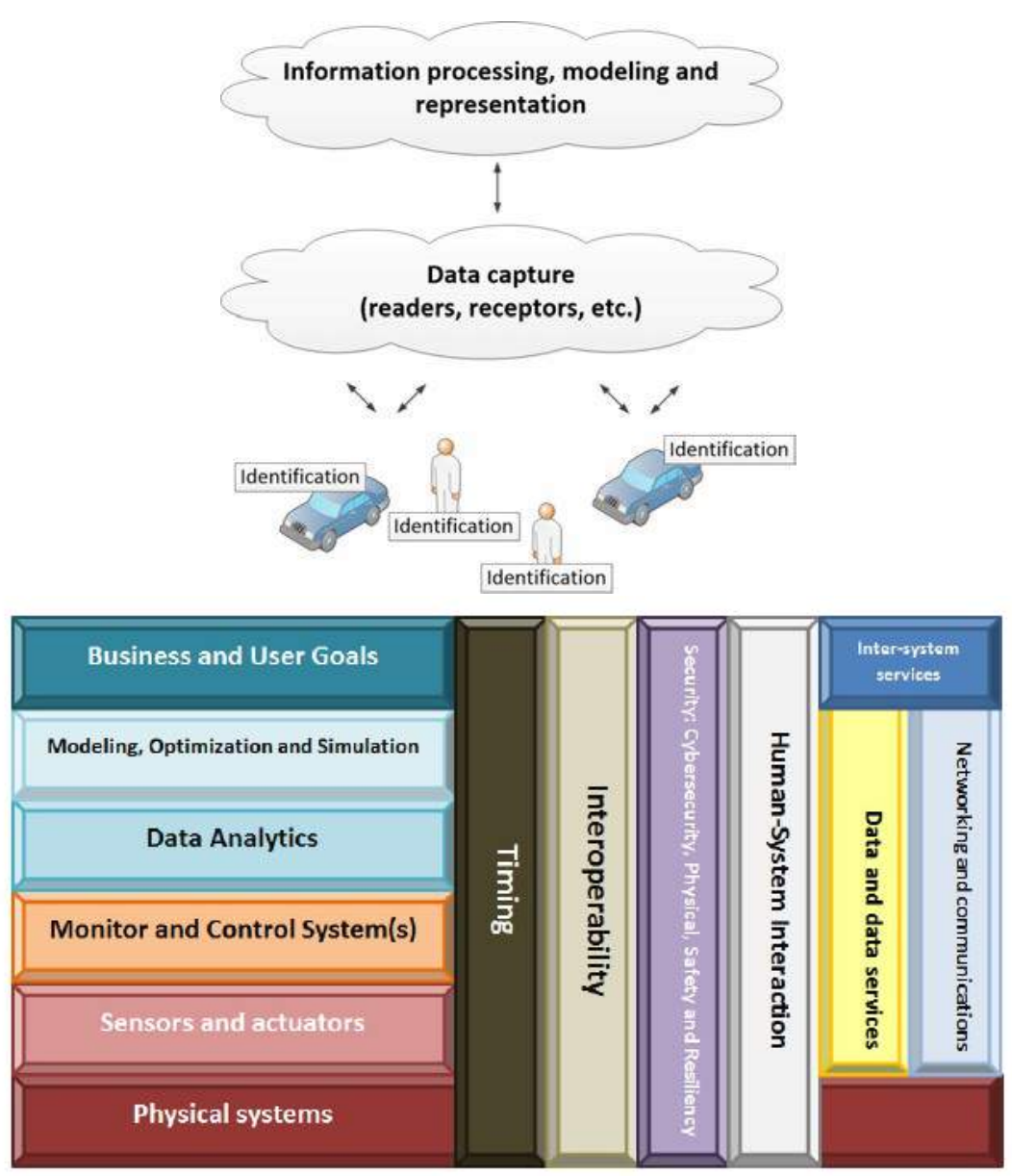

Figure 2: Basic architecture (a) traditional traceability systems (b) Cyber-Physical System

describe the state of the physical platform and the environment. Using this daemon the geographically sparse architecture of a GCPS (especially if the environment to be monitored occupies a large area) may be managed in an easier way. Besides, other functionalities such automation, intelligence, etc. and other cognitive capabilities are supported in a centralized manner. Finally, this daemon allows user to obtain information about the current and past state of the system in a very fast way and, if required, future predictions about the CPS could be also calculated. Below, the five different new layers are analyzed [34]:

- Virtualization interface: This layer offers an interface to invoke virtualization functionalities (such as create a new daemon, halt an instance, etc.). This interface offers the possibility of automating the management of the system daemons (for example, to create dynamically one when required).

- Virtual services layer (VS layer): A virtual service (VS) is an abstract representation of real service offered at medium-level (usually named as "production level" [31]). This layer allows managing the lifecycle of services, and (in our case) it contains the monitoring and traceability (and possibly prediction [10]) services employed in traceability solutions.

- Composite virtual devices layer (CVD layer): A composite virtual device is a virtual instance representing a group of virtual devices which work together in order to reach a common objective (for example, execute a service). These instances are managed at the appropriate layer. Examples 


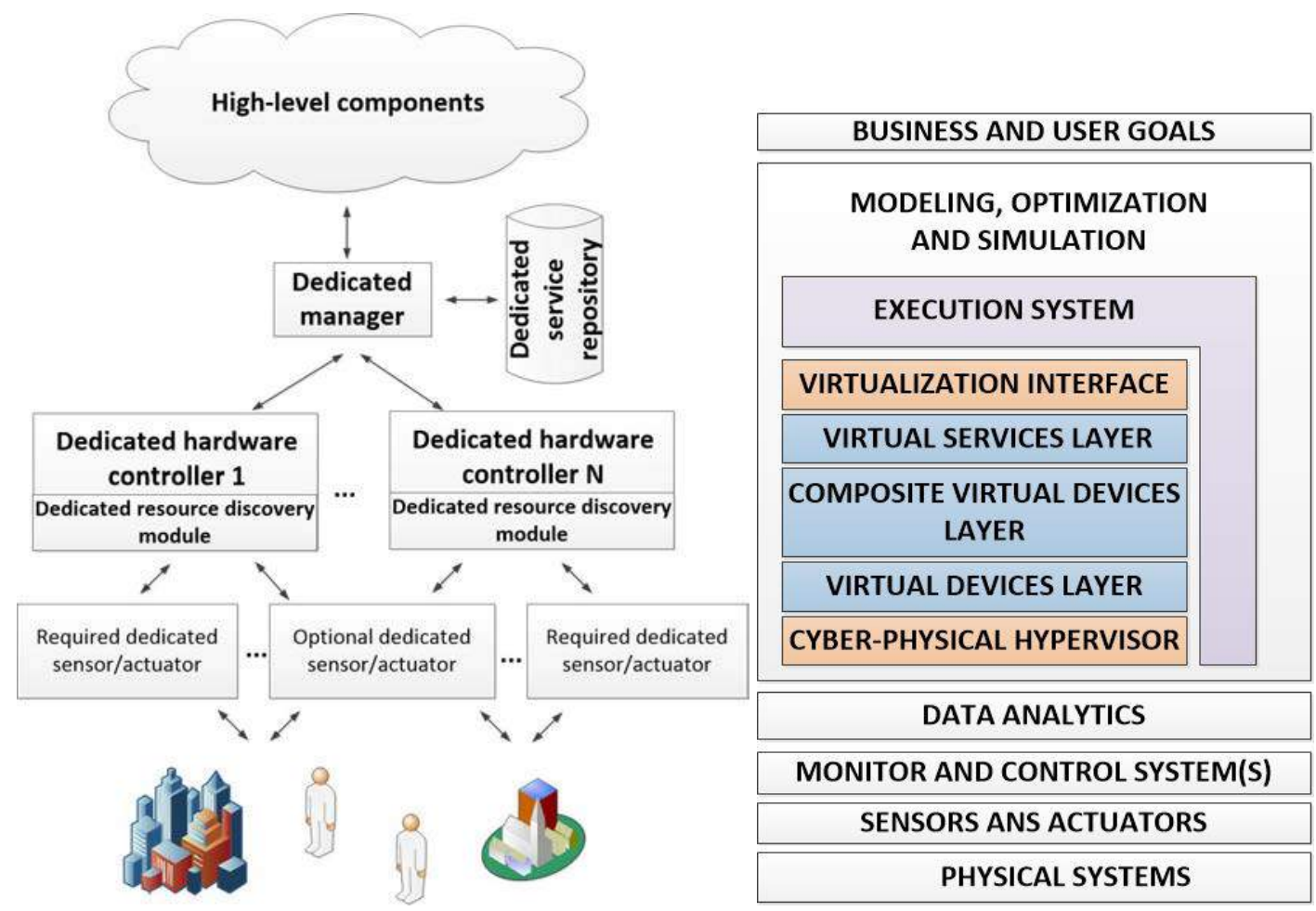

Figure 3: Architecture of a GCPS (a) functional (b) reference

of CVD are a set of sensors connected to a broker, or a subsystem including different embedded devices.

- Virtual devices layer (VD layer): Virtual devices refer to a representation of the embedded devices in the system (usually sensors and actuators, but also the called "self-managed devices" -see Section 3.3.). This functionality is useful in scenarios considering a geographically sparse infrastructure (for example, a company with different locations which desirers a complete description about the state of the entire company in the headquarters, such as it is offered in SCADA systems).

- Cyber-Physical hypervisor: This layer represents a specific hypervisor technology, focused on turning independent the real infrastructure from the virtual instance. Different virtualization techniques for CPS have been reported [18, 4].

- Apart from the previously cited layers, an execution system has to be also considered, in order to execute the user applications and services, described at the highest level. This system has been presented in previous works [31].

Finally, in order to create the daemon and the contained VD and CVD, it is necessary to acquire the required information to create a successful virtual representation of the system. Moreover, it is needed a model to represent all devise and elements in the system, used as base for the creation of the system daemon.

In the next subsections, both solutions are described: data acquisition and system modeling.

\subsection{Data Acquisition: T2T Communications}

In traditional traceability systems, every element to be monitored is provided with an electronic device containing a unique identifier which describes the identity of the element. In this context, the same 
identifier (for example when using RFID tags) allows the element to be identified, to be identified the embedded electronic device, to manage communications, etc. In that way, communications are (at the end) described as Machine-to-Machine communications (M2M) as the objective is to communicate the embedded device with a remote machine in an automatic and self-managed way. Thus, information is provided by the embedded device, not by the element to be monitored.

When using GCPS, this vision changes. Now, not every object, human or element (hereinafter "Thing" in a global way) is provided with an embedded device, as "Things" may be monitored using many other types of techniques and data analysis algorithms (such as implicit interactions [22]). Nevertheless, each Thing has to be provided, in the same way, with a unique identifier (which is part of its identity). In this new paradigm M2M communications are not valid, as information is directly provided by the Thing to be monitored. Then, we use the new concept of Thing-to-Thing communications (T2T).

T2T communications [37] is an emerging term, typical from Industry 4.0, next-generation IoT systems and CPS applications, describing a scenario where a physical Thing (including humans and any other living creature) communicates with some remote physical Things (in an automatic, implicit and self-managed way) through a technological infrastructure (typically a communication network).

In the analyzed traceability systems, T2T communications are used by environmental things to inform the central system about their position, presence, state, etc. Then, the provided information is collected and employed to instantiate the system daemon.

In this new context, however, identity management is a very complicated task. As not every Thing is provided with an embedded device containing a unique identifier, the identity of a monitored Thing is composed (at least and necessarily) of four different numbers (identifiers):

- Monitored Thing ID (MT_ID): This identifier it is associated with the Thing to be monitored and characterizes it. It is created, defined and assigned by the system managers. In general no global or international organism is in charge of the management of these identifiers.

- Associated device ID (ADID): In order to be able to communicate with other remote elements, each Thing has to be associated a device providing this functionality. This device has to be also provided with an identifier. A same device may be associated with different Things at different moments. In computer sciences, these identifiers are called OUI (Organizationally Unique Identifier). They are composed by 24 bits and are managed by the IEEE (such as MAC addresses, which could be also employed as AD_ID).

- Communication management ID (CM ID): This identifier depends on the network where the associated device and/or the monitored Thing are integrated. In global communication networks (such as Internet) they are manage by international organisms, although in local deployment (Bluetooth networks) might be assigned by system owners. IP addresses are the most common example.

- Platform management ID (PM_ID): Finally, a provisional identifier is required to communicate and manage the associated device and the monitored Thing before to be integrated in a communication network. This identifier may be a specific address (or set of addresses) specifically booked for this purpose (as in the Internet) or a new and provisional identifier as in mobile networks.

The total identity of a monitored Thing, which changes dynamically, depends on the four previously named numbers. It is not clear how the four identifiers have to be composed and related (or even if they must be related in some way) in order to create the global Thing's identity. Proposals from the computer networks world usually deal with hierarchical constructions (in a similar way as in the IPv6 standard some identifiers are calculated from others) or the creation of Internet-like directions (for example, MT_ID@CM_ID/AD_ID). On the other hand, mobile communication experts usually prefer to employ the four identifiers in an independent way, considering four different registers and application scopes. In our case (see Section 4) we are using four independent numbers. 


\subsection{Modeling a Generalized Cyber-Physical System}

In order to represent in a correct way the system state using the functionalities of the system daemon, it is required a model for GCPS including all the possible elements in those system. Figure 4, Figure 5, and Figure 6represent a UML model describing an entire GCPS. In order to propose and validate the describe model, it has been taken into account different sources: the Berkeley's conceptual map about CPS [38], relevant works about CPS modeling [14, 24, 26], and previous practical experiences on next-generation technological systems simulation [2, 32]. Below the most important aspects are explained.

First, as can be seen, GCPS are made of two different types of elements: cyber elements and physical elements (but both belong to the system). Some examples of physical elements are provided (some of the most important) but others could be considered. On the other hand, cyber elements correspond to the layer in the reference architecture showed on Figure 2

Sometimes transducers are implemented in a same physical device together with control components, creating a self-managed device. If a self-managed device, besides, is able to execute scripts and processes described at prosumer level, then it is said the device is a sub-system. Sub-systems are very typical in humanized systems, as people are able to understand prosumer language without problems (for example, if natural language is considered as prosumer description language). In other cases, sensors and actuators could be connected among them through a wireless broker, acting as control component.

In respect to processes, their classification, descriptions and decomposition have been analyzed in detail in the state of the art [31]. Same considerations may be done in respect to physical object and variables [33].

Considering the proposed UML diagram, and the previously described architecture, the activity diagram of the proposed tool is presented on Figure 7. As can be seen, the described UML model is instantiated in two steps. During the first phase, physical constraints are generated: the number of involved people, the physical configuration of the scenario, etc. In order to create the corresponding simulation script, information from users and the real deployment is collected. All interaction between the real deployment and the virtualized platform are performed trough the cyber-physical hypervisor, which provides the adequate interface. During second phase, cyber elements are instantiated (as well as services) and system operation starts. The system state is constantly updated considering the events coming from the real deployment. When timer expires, for example at end of a working day, statistical results are showed to managers.

\section{Experimental Validation}

An experiment was designed in order to validate the proposed solution as a valid technology for creating enhanced traceability solutions. In order to perform the experiment a traceability systems based on Bluetooth Beacons was constructed.

Fourteen (14) people were provided with an independent hardware device storing a 16-bits number acting as humans' identity. Each device, besides, was provided with a MAC address by the manufacturing company (Samsung, in this case), so this identifier was employed as Associated device ID.

As hardware platform, it was selected the Artik 020 architecture, consisting on C-programmable microprocessor and a Bluetooth Low-Energy communication module. In that way, communications management ID was selected by experts among all the available addressed (in this case it was selected a broadcast address, so the same identifiers could be used as CM_ID and PM_ID). Devices were configured to act as Beacons (see Figure 8).

The system was deployed in an open laboratory of the Technical University of Madrid, where people provided with electronic devices were asked to perform a certain itinerary. A one square kilometer area was employed to perform the experiments. Different obstacles were deployed in the area, so it 

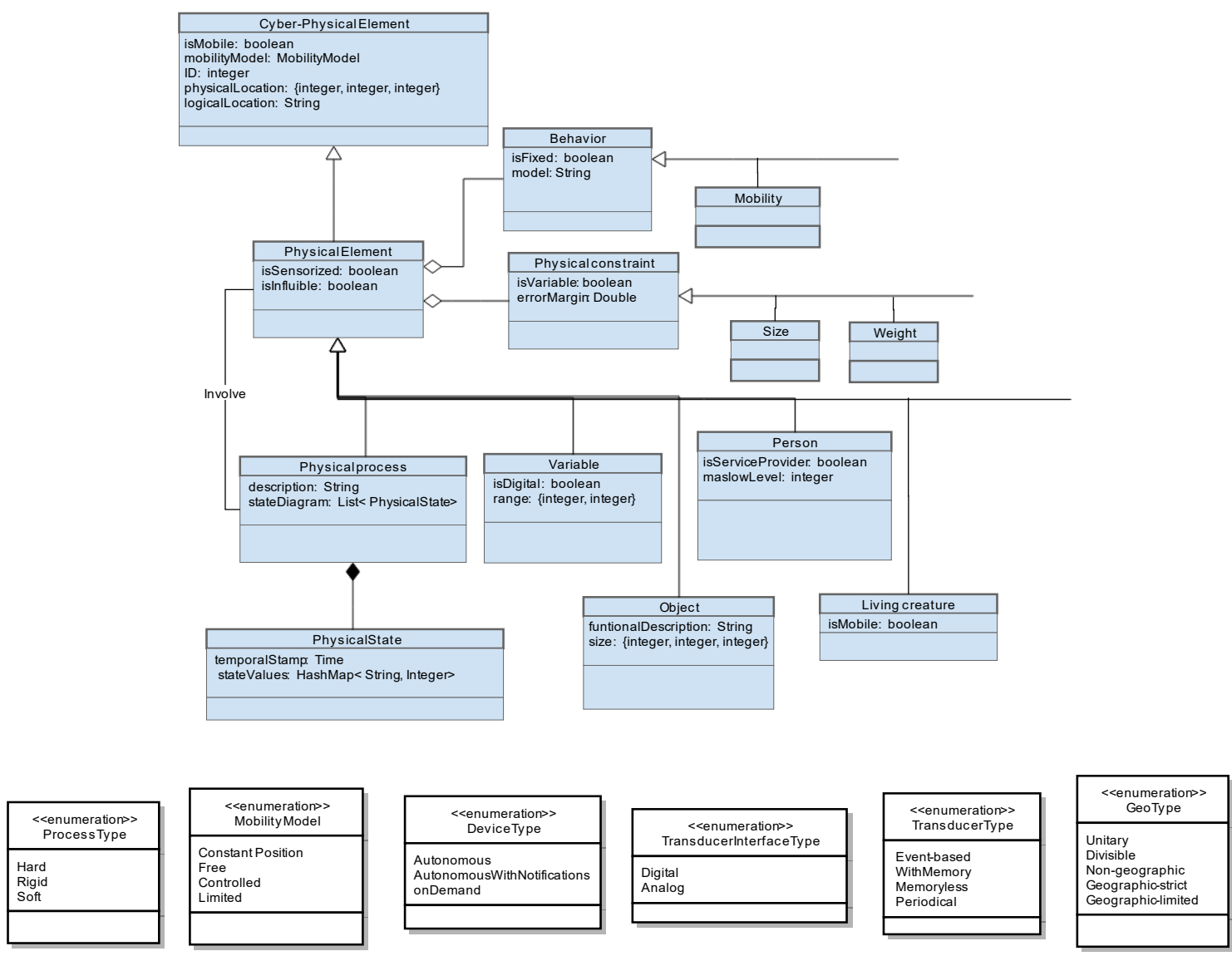

Figure 4: UML description of a GCPS (part 1)

was monitored if people were able to cross them, the time they employed, etc. In order to monitor the people actuation, different Artik 530 platform were deployed around the environment. These machines, in addition to Bluetooth communications, they are able to use WiFi technology. Using this interface, data about people were sent to a remote host, were a Java application was running. That application was described in different previous works about traditional traceability systems [9]. At the same time, data were introduced in a system daemon, previously configured using the Libvirt library (as Samsung Artk 530 platform is based on Linux machines).

Selected traditional traceability systems were exhaustively described in the state of the art [9]. It consists of a system involving some RFID-powered elements such as gloves and tables. These devices are connected with a central server where information from hardware devices is collected and processes in order to track the itinerary of the object under study. A second system was also deployed, based on a pre-CPS view [12]. In particular, in this system, regular workers are in charge of evaluating the quality and evolution of the product, employing different media to notify changes to the control system.

Records created using both methods (Java application as in traditional traceability systems and a virtual instance of the system as proposed in GCPS) were stored. At the end of the experiment, people were asked to describe their itinerary as much detailed as possible. The three information sources were compared.

Simulation description language was based on a objet-orient programming language, such as $\mathrm{C}++$ (employed in other popular simulator such as NS3). Figure 9 shows a fragment of the proposed sce- 


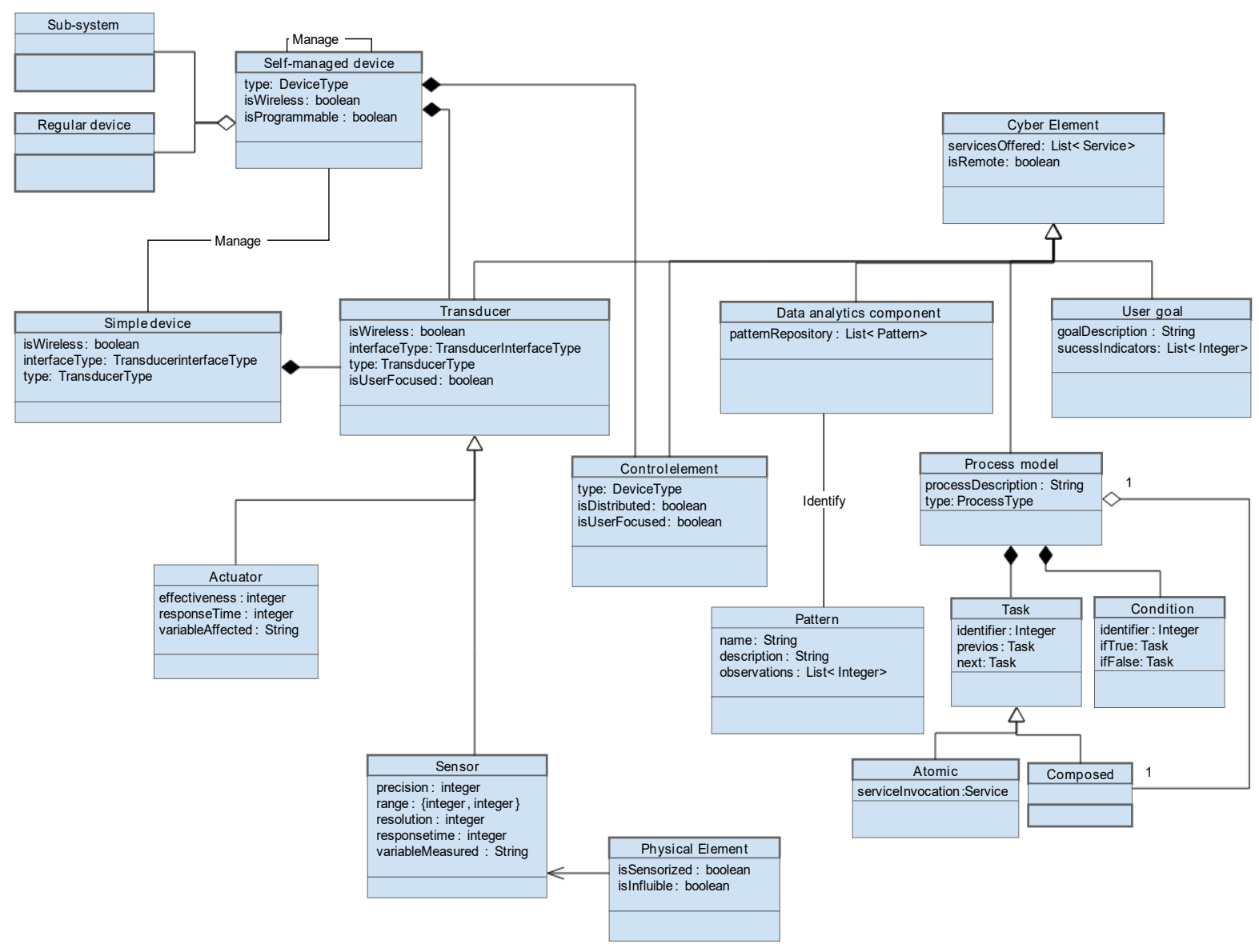

Figure 5: UML description of a GCPS (part 2)

nario. Programming tasks were performed using a regular Integrated Development Environment (IDE) specifically designed for the selected language.

\section{Results and Discussion}

In this Section a comparison about the three different obtained records of information is performed. Figure 10 shows a comparison between the number of errors found the record of the traditional traceability systems compared to the number of errors when using the proposed GCPS.

The difference is small between systems based on CPS but two factors may be considered as the cause of the seen improvement in the case of GCPS. First, errors in the management of information are more common in traditional systems. As traditional systems consist of a database containing the state of the different monitored people, errors occurring when accessing or writing into the database turn into traceability errors. Nevertheless, GCPS are supported by a virtualized platform, so the data structure as much more complex and secure. Second, traditional traceability systems do not include consistency checks (for example, a person cannot walk 3 kilometers in one minute). Thus, some errors which are detected by the consistency control policies in the system daemon are accepted by traditional traceability systems (which, for example, cannot ask for confirmation to the hardware platform when receiving incoherent data).

If systems which are not based on CPS paradigm are considered, differences are much greater. 


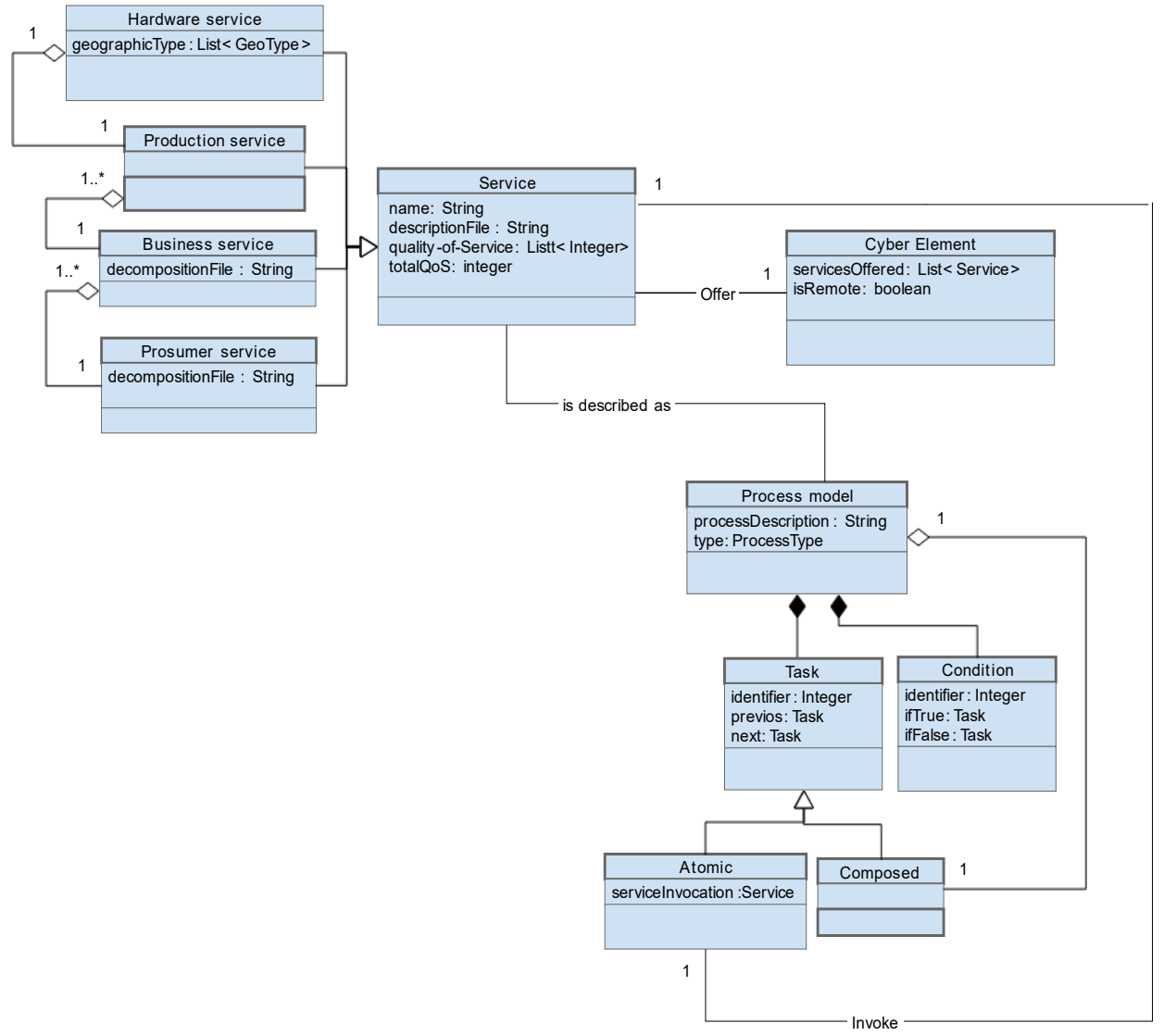

Figure 6: UML description of a GCPS (part 3)

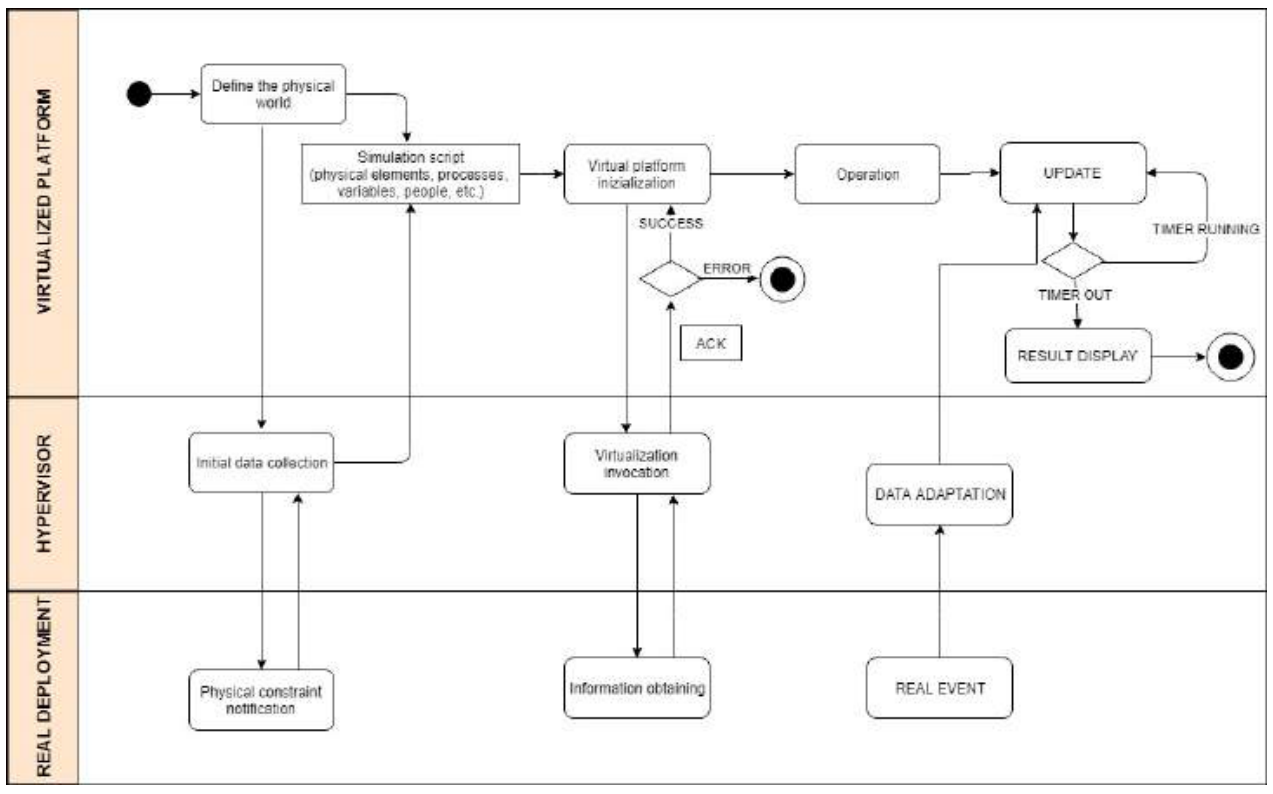

Figure 7: Activity diagram of the proposed tool 


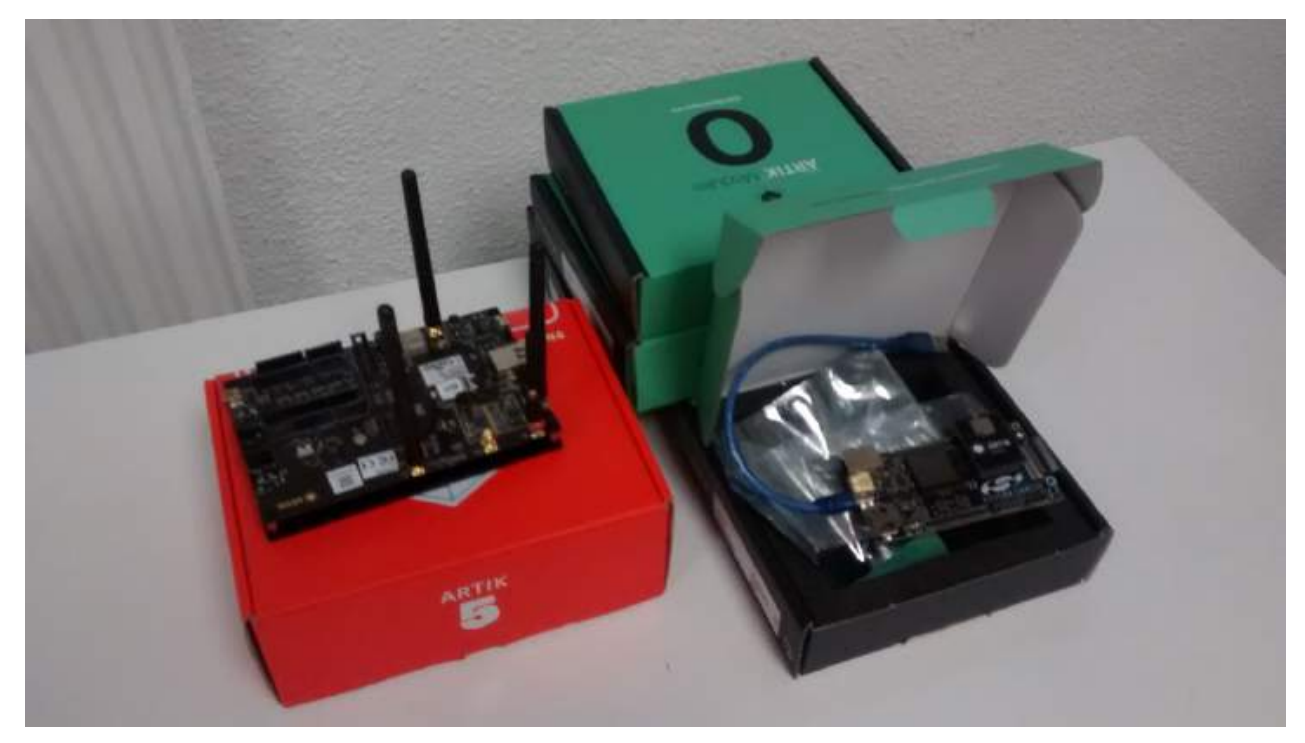

Figure 8: Deployed system

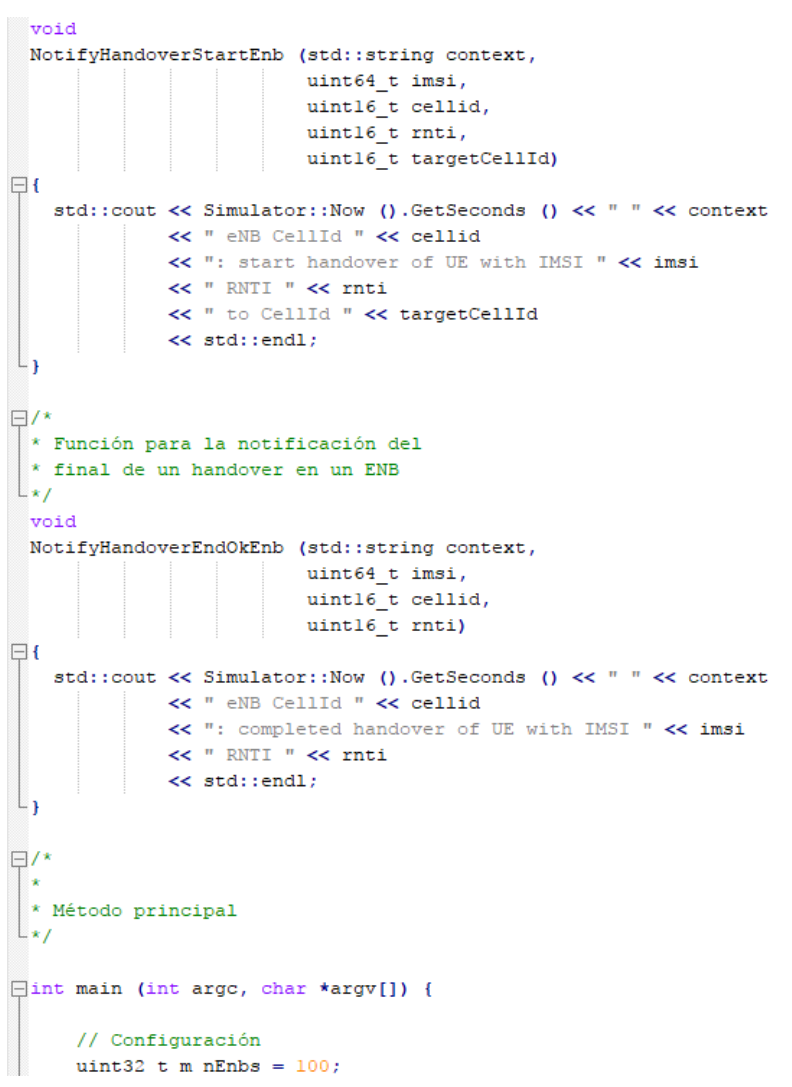

Figure 9: Screenshot of the employed IDE

Causes for this increase have been analyzed previously in other works [9], and in general are related to the possibility of obtaining real-time information from the system, as well as the possibility of implementing predictive techniques in order to obtain some information about the future.

Figure 11 compares the number of parameters maintained for each human in the traceability system, 


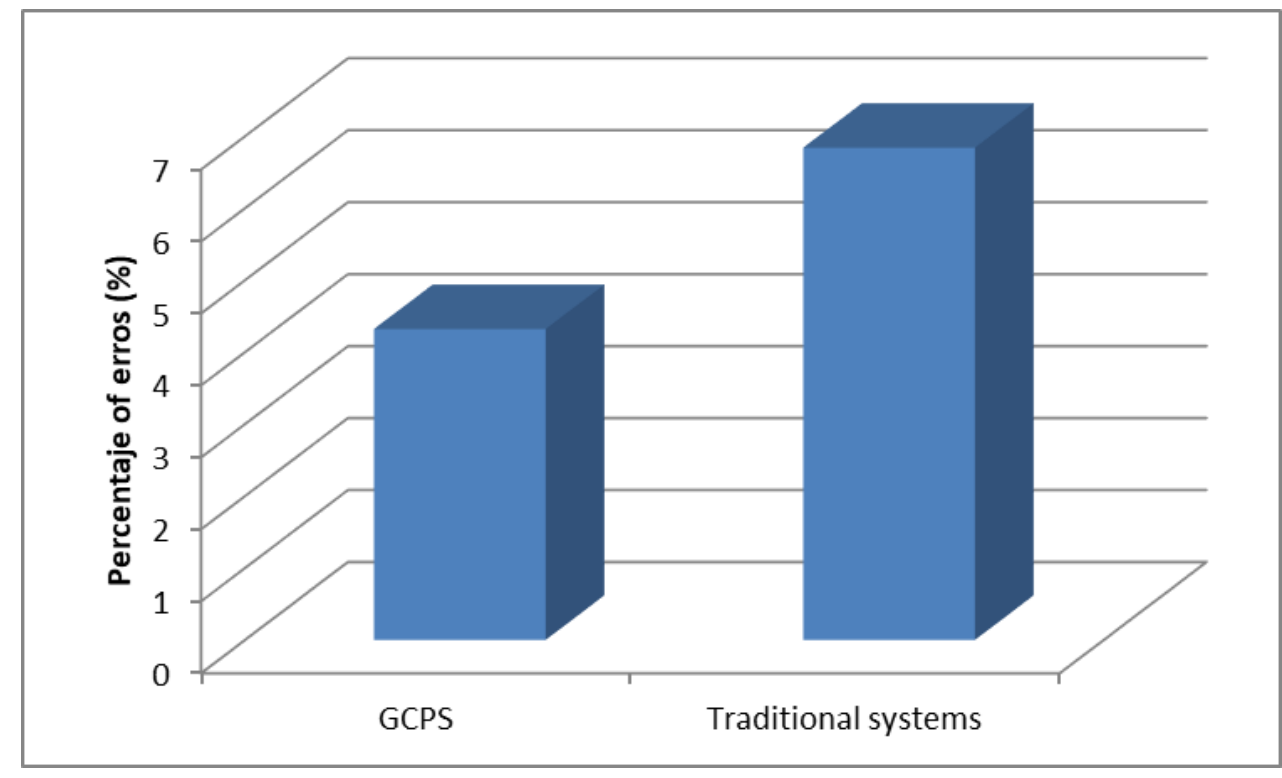

Figure 10: Comparison in the number of traceability errors

in both, traditional solutions and GCPS. As can be seen, in this case, the number of parameters is much greater (a $40 \%$ higher in the case of GCPS). That is because of the capability of the system daemon to extract new information from received one from the physical platform (functionality not included in traditional solutions). System which are not based on CPS paradigm require a high level of human intervention, and (usually) controlled parameters per item are much lower (in order to do profitable the system deployment).

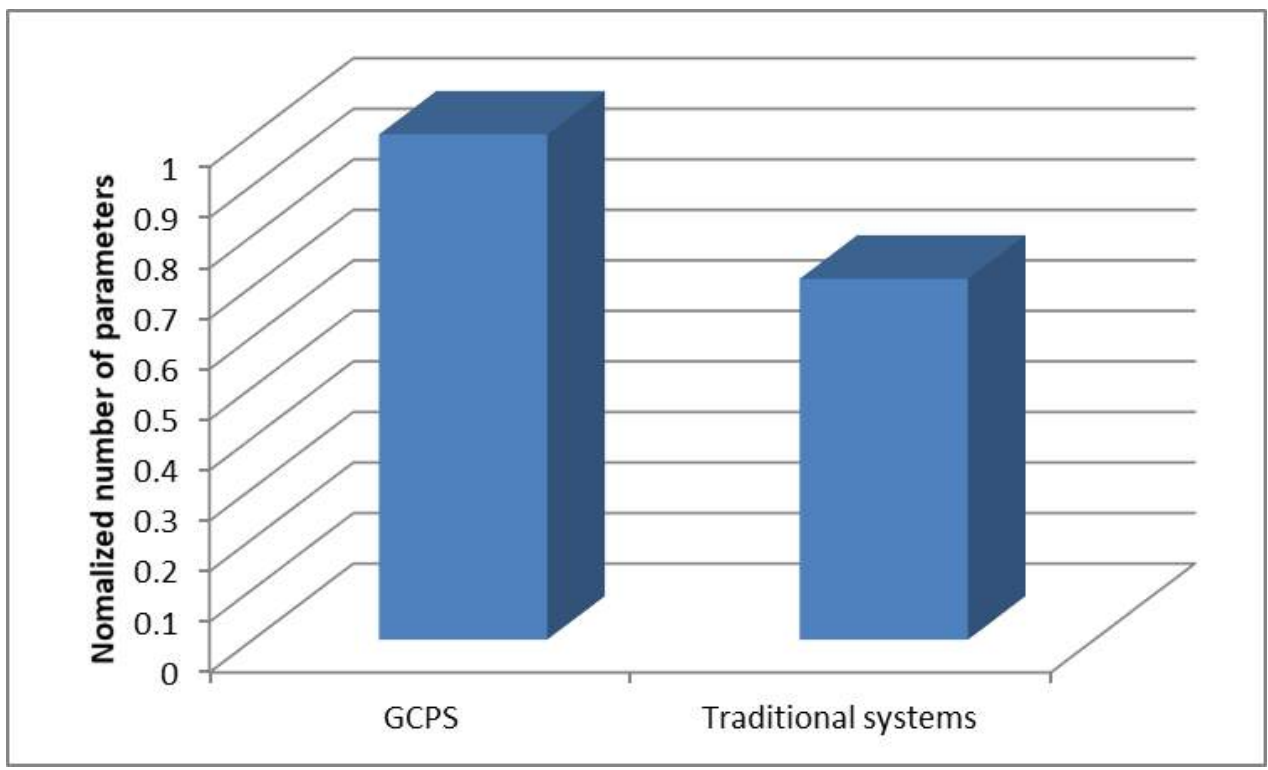

Figure 11: Comparison in the number of parameters per person

Finally, in order to show a more detailed comparison, Table 1 shows the values of some remarkable parameters. 


\begin{tabular}{cccc}
\hline Parameter & GCPS & $\begin{array}{c}\text { Traditional systems } \\
\text { (CPS) }\end{array}$ & $\begin{array}{c}\text { Traditional systems } \\
\text { (no CPS) }\end{array}$ \\
\hline $\begin{array}{c}\text { Maximum number of } \\
\text { parameters per entity }\end{array}$ & 154 & 107 & 5 (estimated) \\
\hline $\begin{array}{c}\text { Maximum number of } \\
\text { entities in the system }\end{array}$ & Undefined & 1024 & 200 (estimated) \\
\hline Mean calculation delay (s) & 17 & 34 & 52 \\
\hline Mean initialization period (s) & 72 & 24 & Not applicable \\
\hline $\begin{array}{c}\text { Integration with } \\
\text { supervisory control } \\
\text { systems }\end{array}$ & Yes & Yes & No \\
\hline
\end{tabular}

Table 1: Parameter comparison

\section{Conclusions}

Enhanced traceability solutions belong to the new era, usually named as the Industry 4.0 or the CyberPhysical Systems era. Traditionally, technological systems look for the total automation, maintaining humans and other living creatures outside the system. This situation is incompatible with the creation of next-generation traceability solution following the CPS principles. Therefore, in this paper it is proposed a new concept named as Generalized Cyber-Physical Systems, being able of including not only embedded devices, but also any other important or relevant element (including, humans, animals, etc.). Moreover, it is described the evolution of Machine-to-Machine communications to Thing-to-Thing communications, due to the integration of new elements into CPS. Finally, as GCPS are based on a virtualized instance of the system, it is proposed a simulation model. The experimental validation showed that the quality of the provided information is much higher in these new systems than in traditional approaches.

\section{Acknowledgments}

The research leading to these results has received funding from the Ministry of Economy and Competitiveness through SEMOLA project (TEC2015-68284-R) and by the Centre for the Development of Industrial Technology (CDTI) through PERIMETER SECURITY project (ITC-20161228). Borja Bordel has received funding from the Ministry of Education through the FPU program (grant number FPU15/03977). Ramon Alcarria thanks the Ministry of Education, Culture and Sport for the professor's mobility program.

\section{References}

[1] A. Ahmad, A. Paul, M. M. Rathore, and H. Chang. Smart cyber society: Integration of capillary devices with high usability based on Cyber-Physical System. Future Generation Computer Systems, 56:493-503, March 2016.

[2] A. T. Al-Hammouri. A comprehensive co-simulation platform for cyber-physical systems. Computer Communications, 36(1):8-19, December 2012.

[3] R. Arghandeh, A. von Meier, L. Mehrmanesh, and L. Mili. On the definition of cyber-physical resilience in power systems. Renewable and Sustainable Energy Reviews, 58:1060-1069, May 2016.

[4] R. F. Babiceanu and R. Seker. Big Data and virtualization for manufacturing cyber-physical systems: A survey of the current status and future outlook. Computers in Industry, 81:128-137, September 2016. 
[5] R. Baheti and G. H. Cyber-physical systems: The Impact of Control Technology, 2011. http://www. ieeecss.org/general/impact-control-technology, [Online; Accessed on August 1, 2017].

[6] B. Bordel, R. Alcarria, D. Martín, T. Robles, and D. S. de Rivera. Self-configuration in humanized CyberPhysical Systems. Journal of Ambient Intelligence and Humanized Computing, 8(4):485-496, September 2017.

[7] B. Bordel, R. Alcarria, M. Pérez-Jiménez, T. Robles, D. Martín, and D. S. de Rivera. Building Smart Adaptable Cyber-Physical Systems: Definitions, Classification and Elements. In Proc. of the 9th International Conference on Ubiquitous Computing and Ambient Intelligence (UCAml'15), Puerto Varas, Chile, volume 9454 of Lecture Notes in Computer Science, pages 144-149. Springer, Cham, December 2015.

[8] B. Bordel, D. S. de Rivera, and R. Alcarria. Plug-and-Play Transducers in Cyber-Physical Systems for Device-Driven Applications. In Proc. of the 10th International Conference on Innovative Mobile and Internet Services in Ubiquitous Computing (IMIS'16), Fukuoka, Japan, pages 316-321. IEEE, July 2016.

[9] B. Bordel Sánchez, R. Alcarria, D. Martín, and T. Robles. TF4SM: A Framework for Developing Traceability Solutions in Small Manufacturing Companies. Sensors, 15(11):29478-29510, November 2015.

[10] B. Bordel Sánchez, R. Alcarria, D. Sánchez de Rivera, and A. Sánchez-Picot. Predictive algorithms for mobility and device lifecycle management in Cyber-Physical Systems. EURASIP Journal on Wireless Communications and Networking, 2016(1):228:1-228:13, December 2016.

[11] A. W. Colombo. Industrial cloud-based cyber-physical systems : the IMC-AESOP approach. Springer International Publishing, 2014.

[12] I. E. Commission. International standard iec 62264-1: Enterprise-control system integration part 1: Models and terminology. IEC: Geneva, Switzerland, 2003. https://www.iso.org/standard/57308.html. [Online; Accessed on August 1, 2017].

[13] C. Coopmans, B. Stark, A. Jensen, Y. Q. Chen, and M. McKee. Cyber-Physical Systems Enabled by Small Unmanned Aerial Vehicles. In K. P. Valavanis and G. J. Vachtsevanos, editors, Handbook of Unmanned Aerial Vehicles, pages 2835-2860. Springer Netherlands, 2015.

[14] P. Derler, E. A. Lee, and A. S. Vincentelli. Modeling Cyber-Physical Systems. Proc. of the IEEE, 100(1):1328, January 2012.

[15] Edward A. Lee. Cyber-Physical Systems - Are Computing Foundations Adequate? Position Paper for NSF Workshop On Cyber-Physical Systems: Research Motivation, Techniques and Roadmap, Austin, Texas, USA, October 2006.

[16] Geng Wu, S. Talwar, K. Johnsson, N. Himayat, and K. D. Johnson. M2M: From mobile to embedded internet. IEEE Communications Magazine, 49(4):36-43, April 2011.

[17] M. Gillham, G. Howells, and S. Kelly. Assistive Trajectories for Human-in-the-Loop Mobile Robotic Platforms. In Proc. of the 6th International Conference on Emerging Security Technologies (EST'15), Braunschweig, Germany, pages 56-61. IEEE, September 2015.

[18] J. He, Y. Geng, Y. Wan, S. Li, and K. Pahlavan. A Cyber Physical Test-Bed for Virtualization of RF Access Environment for Body Sensor Network. IEEE Sensors Journal, 13(10):3826-3836, October 2013.

[19] M. D. Ilic, L. Xie, U. A. Khan, and J. M. F. Moura. Modeling of Future Cyber-Physical Energy Systems for Distributed Sensing and Control. IEEE Transactions on Systems, Man, and Cybernetics - Part A: Systems and Humans, 40(4):825-838, July 2010.

[20] M. P. Jimenez, B. B. Sánchez, and R. P. A. Garrido. T4ai: A system for monitoring people based on improved wearable devices. Research Briefs on Information \& Communication Technology Evolution (ReBICTE), 2(1):1:1-1:16, June 2016.

[21] Jody Ranck. Gigaom - The wearable-computing market: a global analysis. Technical report, Gigaom, 2012.

[22] W. Ju and L. Leifer. The Design of Implicit Interactions: Making Interactive Systems Less Obnoxious. Design Issues, 24(3):72-84, July 2008.

[23] H. Lasi, P. Fettke, T. Feld, and M. Hoffmann. Industry 4.0. Business \& Information Systems Engineering, 6(4):239-242, 2014.

[24] E. A. Lee. Cyber Physical Systems: Design Challenges. In Proc. of the 11th IEEE International Symposium on Object and Component-Oriented Real-Time Distributed Computing (ISORC'08), Orlando, Florida, USA, pages 363-369. IEEE, May 2008. 
[25] J. Lin, S. Sedigh, and A. Miller. Modeling Cyber-Physical Systems with Semantic Agents. In Proc. of the IEEE 34th Annual Computer Software and Applications Conference Workshops (COMPSACW'10), Seoul, South Korea, pages 13-18. IEEE, July 2010.

[26] P. Marwedel. Embedded System Design. Springer, Dordrecht, 2011.

[27] I. Mohino-Herranz, R. Gil-Pita, J. Ferreira, M. Rosa-Zurera, and F. Seoane. Assessment of Mental, Emotional and Physical Stress through Analysis of Physiological Signals Using Smartphones. Sensors, 15(10):2560725627, October 2015.

[28] S. Munir, J. A. Stankovic, C.-J. M. Liang, and S. Lin. Cyber physical system challenges for human-in-theloop control. In Proc. of the 8th International Workshop on Feedback Computing, San Jose, California, USA. USENIX, June 2013.

[29] P. Rashidi and A. Mihailidis. A Survey on Ambient-Assisted Living Tools for Older Adults. IEEE Journal of Biomedical and Health Informatics, 17(3):579-590, May 2013.

[30] V. Ruchkin, V. Fulin, B. Kostrov, A. Taganov, and A. Kolesenkov. Forest fire monitoring by means of cyberphysical system. In Proc. of the 5th Mediterranean Conference on Embedded Computing (MECO'16), Bar, Montenegro, pages 30-34. IEEE, June 2016.

[31] B. Sánchez, R. Alcarria, D. Sańchez-De-Rivera, and Á. Sańchez-Picot. Enhancing process control in industry 4.0 scenarios using Cyber-Physical systems. Journal of Wireless Mobile Networks, Ubiquitous Computing, and Dependable Applications, 7(4):41-64, 2016.

[32] B. B. Sanchez, A. Sanchez-Picot, and D. S. D. Rivera. Using 5G Technologies in the Internet of Things Handovers, Problems and Challenges. In Proc. of the 9th International Conference on Innovative Mobile and Internet Services in Ubiquitous Computing (IMIS'15), Blumenau, Brazil, pages 364-369. IEEE, July 2015.

[33] A. Sanchez-Picot, D. Martin, D. S. de Rivera, B. Bordel, and T. Robles. Modeling and Simulation of Interactions Among People and Devices in Ambient Intelligence Environments. In Proc. of the 30th International Conference on Advanced Information Networking and Applications Workshops (WAINA'16), CransMontana, Switzerland, pages 784-789. IEEE, March 2016.

[34] C. Sarkar. Virtualizing the internet of things. http://www.es.ewi.tudelft.nl/phd-theses/ 2016-Sarkar.pdf, [Online; Accessed on August 1, 2017].

[35] G. Schirner, D. Erdogmus, K. Chowdhury, and T. Padir. The Future of Human-in-the-Loop Cyber-Physical Systems. Computer, 46(1):36-45, January 2013.

[36] H. Song, D. B. Rawat, S. Jeschke, and C. Brecher. Cyber-physical systems : foundations, principles and applications. Academic Press, 2017.

[37] T. to Thing Research Group. Thing-to-thing. charter-irtf-t2trg-01, December 2015. https:// datatracker. ietf .org/rg/t2trg/about/, [Online; Accessed on August 1, 2017].

[38] B. University. Cps concept map. http://cyberphysicalsystems.org. [Online; Accessed on August 1, 2017].

[39] K. Wan, D. Hughes, K. L. Man, and T. Krilavicius. Composition challenges and approaches for cyber physical systems. In Proc. of the IEEE International Conference on Networked Embedded Systems for Enterprise Applications (NESEA'10), Suzhou, China, pages 1-7. IEEE, November 2010.

[40] H. Wang, S. Gong, X. Zhu, and T. Xiang. Human-In-The-Loop Person Re-Identification. Computing Research Repository, abs/1612.01345, December 2016.

[41] T. Wark, K. Prayaga, J. O’Grady, M. Reed, A. Fisher, C. Crossman, W. Hu, Y. Guo, P. Valencia, P. Sikka, P. Corke, C. Lee, and J. Henshall. The design and evaluation of a mobile sensor/actuator network for autonomous animal control. In Proc. of the 6th international conference on Information processing in sensor networks (IPSN'07), Cambridge, Massachusetts, USA, pages 206-2015. ACM, April 2007.

[42] F.-J. Wu, Y.-F. Kao, and Y.-C. Tseng. From wireless sensor networks towards cyber physical systems. Pervasive and Mobile Computing, 7(4):397-413, August 2011.

[43] F. Xia and Feng. QoS Challenges and Opportunities in Wireless Sensor/Actuator Networks. Sensors, 8(2):1099-1110, February 2008.

[44] X. Yue, H. Cai, H. Yan, C. Zou, and K. Zhou. Cloud-assisted industrial cyber-physical systems: An insight. Microprocessors and Microsystems, 39(8):1262-1270, November 2015. 
[45] Z. Zhang, X. Li, X. Wang, and H. Cheng. Decentralized Cyber-Physical Systems: A Paradigm for CloudBased Smart Factory of Industry 4.0. In L. Thames and D. Schaefer, editors, Cybersecurity for Industry 4.0, pages 127-171. Springer International Publishing, 2017.

\section{Author Biography}

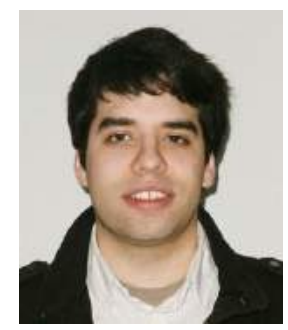

Borja Bordel received the B.S. degree in telecommunication engineering in 2012 and the M.S. telecommunication engineering in 2014, both from Technical University of Madrid. He is currently pursuing the Ph.D. degree in telematics engineering at Telecommunication Engineering School, UPM. His research interests include CyberPhysical Systems, Wireless Sensor Networks, Radio Access Technologies, Communication Protocols and Complex Systems.

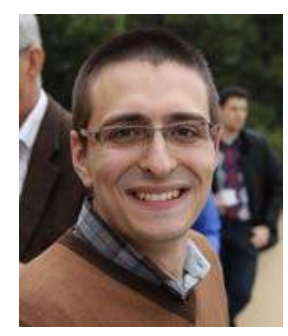

Ramón Alcarria received his M.S. and Ph.D. degrees in Telecommunication Engineering from the Technical University of Madrid in 2008 and 2013 respectively. Currently, he is an assistant professor at the E.T.S.I Topography of the Technical University of Madrid. He has been involved in several R\&D European and National projects related to Future Internet, Internet of Things and Service Composition. His research interests are Service Architectures, Sensor Networks, Human-computer interaction and Prosumer Environments.

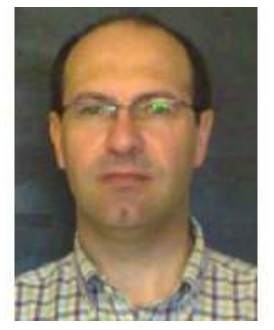

Miguel Manso received a M.S. in Telecommunication Engineering and Ph.D. in Geographic Engineering degrees from Technical University of Madrid in 2003 and 2009 respectively. He is an Associate Professor on Geomatics Engineering at the Technical University of Madrid since 1992. He has participated in some national and international projects related to Geospatial Data and Cartography. His research areas are Sensor Networks and Web Enablement, Spatial Data Infrastructure and Spatial Databases.

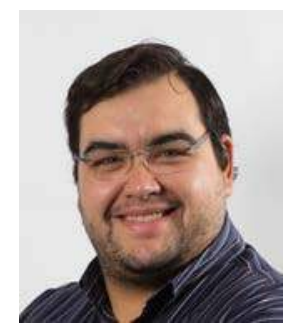

Antonio Jara received his Ph.D. at the Intelligent Systems and Telematics Research Group of the University of Murcia (UMU) from Spain. He is the Vice-chair of the IEEE Communications Society Internet of Things Technical Committee, CTO and co-founder of the Smart Cities company viBrain Solutions, Assistant Prof. PostDoc at University of Applied Sciences Western Switzerland (HES-SO). His research interests are WSNs (6LoWPAN and ZigBee) and RFID applications in building automation and healthcare. 


\title{
Process execution in Cyber-Physical Systems using Cloud and Cyber- Physical Internet services
}

\author{
Borja Bordel $^{1}{ }^{1 *}$, Ramón Alcarria ${ }^{2}$, Diego Sánchez de Rivera ${ }^{1}$, Tomás Robles ${ }^{1}$
}

1 Department of Telematics Systems Engineering. Universidad Politécnica de Madrid. Avenida Complutense no 30. 28040 - Madrid (España); E-Mails: bbordel@dit.upm.es; diego.sanchezderiveracordoba@gmail.com; tomas.robles@upm.es;

2 Department of Topographic Engineering and Cartography. Universidad Politécnica de Madrid. Campus Sur, 28031 Madrid, Spain; E-Mail: ramon.alcarria@upm.es

* Author to whom correspondence should be addressed; E-Mail: bbordel@dit.upm.es; Tel. 915495700 ext. 3035

\begin{abstract}
"Cyber-Physical Systems" (CPS) have emerged as the next technological revolution. These new systems are commonly supported by a collection of ad hoc connected devices which typically collaborate in order to control some physical processes. However, in recent years, many other applications based on the CPS paradigm have been reported. In particular, executing user-defined processes over a cyber-physical infrastructure is a very promising technology for the future. Therefore, in this paper, we propose a scheme which allows the creation of userdefined processes, their decomposition and translation into executable orders or code, and their execution using the locally available cyber-physical infrastructure, cloud services and/or other services offered by remote CPS through the Cyber-Physical Internet. The proposed solution also enables the execution of processes with a guaranteed QoS. Moreover, an experimental validation is provided in order to evaluate the proposed technology performance. In particular, it is proved that more than $95 \%$ of processes are correctly executed, and only in a $2 \%$ of cases the minimum cost execution is not selected.
\end{abstract}

\section{KEYWORDS}

Cyber-Physical Systems; Workflow execution; Cyber-physical process modeling; CyberPhysical internet; Cloud services; Process execution; YAWL;

\section{FUNDING}

The research leading to these results has received funding from the Ministry of Economy and Competitiveness through SEMOLA project (TEC2015-68284-R) and from the Autonomous Region of Madrid through MOSI-AGIL-CM project (grant P2013/ICE-3019, co-funded by EU Structural Funds FSE and FEDER). Borja Bordel has received funding from the Ministry of Education through the FPU program (grant number FPU15/03977) 


\section{INTRODUCTION}

The emerging term "Cyber-Physical Systems" refers to the integration of computational and physical capabilities using ad hoc sensor and actuator networks which, typically, are seamlessly integrated into daily living objects [25]. However, in recent years (since 2014, approximately), works about CPS also consider other devices such as microcontrollers [18], legacy systems [14] or even humans [44], which greatly extend the system's capabilities. Based on these improved CPS, new applications, architectures and functionalities have been reported [51][48]. Moreover, these new paradigm has been also employed to develop solutions in traditional fields such as the ad hoc and wireless sensor networks, Internet of Things (IoT) or pervasive computing [12].

In this context, one of the most promising proposals is the so-called service-oriented CPS [24]. In this kind of CPS, the physical infrastructure offers a collection of services by means of some software elements (such as service composition engines [2]). These services may be used to perform a certain fixed application (deployed by technological experts), or might be included in a prosumer [37] environment where non-expert users can design, build, deploy and remove their own applications in a dynamic way.

In a particular realization of the second case, services are employed to execute user-defined processes. In these processes, services are invoked and linked with each other in a certain way, in order to perform a collection of tasks or activities. Processes may describe business tasks, control activities or assisted living policies among other possibilities. Besides, each process can be executed once, various times, when a certain event happens, periodically, etc. Furthermore, most times, processes have to include services which are not supported by the underlying physical infrastructure. Sometimes these services are provided by external companies (such as the bank services), other times services are provided from a remote location (for example, if the company manages various production plants) and other services are supported by a virtual infrastructure. In the first and third case cloud services are employed [53]; in the second case, services provided by remote CPS might be used if the systems are connected to the so-called Cyber-Physical Internet [32][21] -CPI-. CPI is a network of CPS which publicly offers services that can be invoked remotely.

In order to enable non-expert users to define their processes, services must be described in a high level of abstraction. Then, instruments to decompose and translate users' designs into executable code and actions are required, as well as tools for controlling the execution and the temporization. However, current proposals only cover this objective partially. Thus, the main contribution of this work is to define a scheme being able to perform these tasks using new and emerging technologies such as the Cyber-Physical Systems paradigm.

Therefore, the objective of this paper is to describe a technical solution, including a functional architecture and the required algorithms, which enables users to define their own processes, which locates the selected services locally, in the cloud or in the CPI (selecting the minimum cost location if various are available), and being able to decompose users' designs into executable code and control the execution and the temporization. Additionally, the proposed solution allows users to perform executions with a guaranteed Quality-of-Service (QoS).

The rest of the paper is organized as follows. Section 2 analyzes the state of the art on task and process execution, delegation and transformation in CPS. Section 3 presents the proposal, including the reference architecture, the functional architecture and the required algorithms and 
mathematical formalization. Section 4 explains the experimental validation and Section 5 presents the results. Finally, Section 6 concludes the paper.

\section{STATE OF THE ART ON PROCESS AND TASK EXECUTION, DELEGATION AND TRANSFORMATION IN CPS}

Traditional implementations of CPS try to relate physical and computational processes [23][9]. Thus, in these systems, computational processes are defined as state machines where transitions between states depend on the physical processes [15]. The system behavior in each state, as well as the transitions, is defined at very low level (fixing the actions performed for each device) so, typically, these systems act as closed systems only manipulated by technological experts. A possible generalization of this solution [7] not only considers physical processes, but also any other event which can trigger a transition (for example, an alarm). Even more general proposals, such as the named Elastic Systems [28], report the integration of people in those processes. In this solution, however, domain experts continue having problems to define their own processes as it is mandatory to know how the system works at very low level.

In order to allow domain experts to define their own processes for CPS, more modern proposals have included the concept of "task" [34] instead of the state machines. A task represents a collection of actions to be performed in order to obtain a certain output. Relating the outputs and the inputs of the different tasks, a global process may be defined. A task can be described in many different abstraction levels, from human language to binary code. Thus, some proposals [42] describe a scheme where various translation (or transformation) engines (typically three cascaded elements) turn a high-level description of a process into a machine-level description to be executed by the underlying devices.

Most usually employed transformation techniques nowadays are based on a set of transformation rules which are applied by a software agent [13]. In many cases, this agent takes the form of a compiler [3], sometimes implementing advanced solutions such as predictive compilation [10]. In the last years, besides, semantic solutions have been proposed and successfully deployed [43]. Finally, specific solutions are also available. For example, it may be found transformation engines for embedded microcontrollers [6].

Previously described proposals, however, present a limited usefulness, mainly because of two facts: first, metadata cannot be easily specified and, second, standard description languages are not developed enough. In order to address the lack of adequate process description languages (especially medium-level languages) several authors have tried to extend existing standard solutions such as BPMN [13][41] or BPEL [3][45]. Nevertheless, these proposals do not solve the problem, as no execution, transformation engine, or any other tool, is adapted or extended to these proposals. In respect to the metadata specification, temporization is probably the most important issue. In all previously mentioned works, temporization must be included (when possible) using complicated notations as it is not natively supported. Then, papers proposing various schedulers for CPS temporization control have been proposed [56][19]. However, the integration of these schedulers with the other elements is not clear at all.

In order to address this problem, researchers on ad hoc wireless sensor networks (and on CPS) have proposed to describe tasks not as a collection of actions, but as a sequence of services which must be invoked [24][22]. Then, an orchestrator (or central execution engine) manages 
the synchronization among the different services in order to obtain a coherent result [38]. Some proposals even describe management algorithms which adapt the evolution of the orchestration policies depending on the variable external physical processes [27]. These solutions allow specifying temporization conditions in a very easy way in the orchestrator, although other problems (such as the need of knowing the system behavior at very low level) remain.

On the other hand, various works on CPS consider the idea of including not only services offered from the local physical platform, but also from the cloud [1] or from remote installation through the Internet [49] (named as Cyber-Physical Internet or Internet of CPS when connects various CPS). In all these works, however, the location of every service is stablished by default, and for every service only one location is available.

In any case, if various locations where tasks might be executed are available, a task delegation algorithm must be implemented (in order to transfer tasks to the system in charge of executing them). In the simplest task delegation algorithm, the root agent turns on a certain secondary node when a task (or various tasks) is being delegated [11]. In this solution, each node works independently. The root node suppresses the task from its execution schedule when the task is delegated to his secondary node, which is programmed to perform this task. This solution is the simplest and easiest to implement and deploy; however, is a very rigid scheme which hardly adapts to changes in the platform. In a more advanced technique, nodes are software agents. These agents offer public methods to be invoked by other agents paying back the agreed "price" [31]. Using this platform, agents may delegate parts of the execution in a very efficient way. Nevertheless, problems such as object serialization or method publication have not been totally resolved. Finally, service-oriented solutions have been proposed. In these works, various platforms share information to discover the list of available services, and to order the platform to execute a certain task or collection of them [35]. In this case all the available technologies for distributed systems and cloud-computing may be used (serialization, coherence, etc.). To improve the dynamism of the service-oriented solutions they might be complemented with an algorithm to determine the most adequate location to execute the tasks (if various available) [16]. These algorithms are based on cost functions, whose parameters may vary dynamically.

The objective of this paper is to go beyond the current technological state, proposing the first unified service-oriented process execution scheme, based on the emerging CPS. Our proposal considers a service-oriented solution, where various transformation engines allow users to describe processes in a very high abstraction level and, later, translate these designs into executable code. In order to employ standard modules and techniques, a well-known standard process description language such as YAWL [47] is used in our proposal. As a novelty, two different execution engines are considered, in order to interact with the cloud and the emerging Cyber-Physical Internet and, at the same time, wean the particularities of the physical devices and the high-level services. In our solution, a service may be offered in different locations and the most convenient location to execute it is selected dynamically. The task, then, could be delegated. Moreover, also as a novelty, together with every process description file, a metadata description file can be uploaded to the execution engines, contributing to control the temporization, the QoS and other parameters. 


\section{PROPOSED SOLUTION}

In this Section the technical solution is detailed. In the first subsection the reference and a general description of the solution are presented. And, in the second subsection, the technical proposal is described in detail.

\subsection{ARCHITECTURES, PREVIOUS CONFIGURATIONS, OVERVIEW OF THE PROPOSAL}

The main problem to address when designing a solution for process execution in CPS is the need of allowing users to describe processes in a high level of abstraction and, later, translate them into instructions compatible with devices.

In order to address this problem, we are basing our solution in a reference architecture (see Figure 1) proposed by the National Institute of Standards and Technology (NIST) which represents a CPS as a stack of six abstraction levels [7].

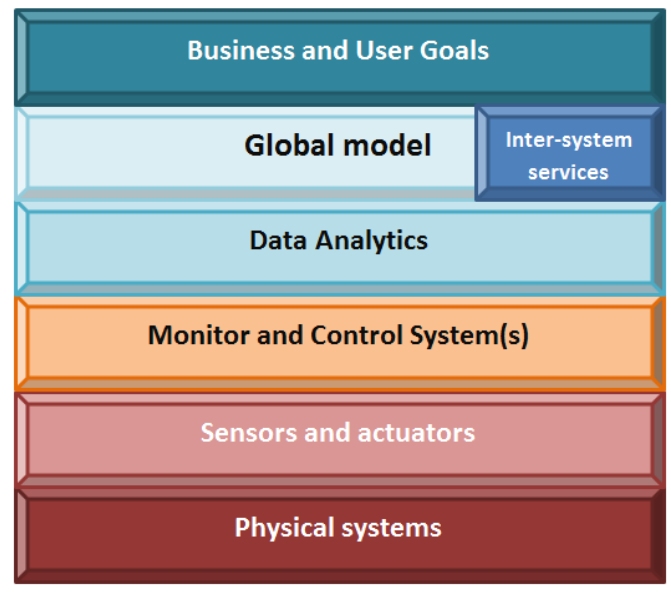

Figure 1: Reference architecture for CPS

In Figure 1, the first layer is dedicated to Business and user goals. That includes the process definition in a human understandable language and the definition of the objectives of the defined processes. In Global model, a global first executable representation of the processes must be obtained. In this layer each service must be located locally, in the cloud or in other Internet locations (the last two elements are known as Inter-system services), selecting the most convenient if available in various locations. Data analytics layer weans the higher layers from the physical devices' low-level details. For that it implements technologies such as pattern recognition or knowledge extraction. Finally, Monitor and control systems layer is dedicated to manage the hardware devices represented by the Sensors and actuators layer. Physical systems layer refers to the environment which surrounds the CPS.

Considering the division of the functionalities in a CPS proposed by the NIST (see Figure 1), a unified process execution scheme for CPS may be described as a sequence of five steps: process design, business execution, probabilistic execution, hardware access and hardware execution. Figure 2 presents a graphic representation for this "lifecycle", identifying each step with the reference layer (Figure 1) which contains the functionalities employed to perform the actions included in the step. 
The first step, process design, includes all actions to obtain executable process description. In this step prosumer users define their own processes in any domain language, including natural English and graphic methods. Besides, specialized instruments translate the description provided by prosumer users into a standard workflow description language, such as YAWL [56]. Finally, files containing metadata and an executable description of the process at business level (disaggregating the prosumer services into collections of business services) are generated.

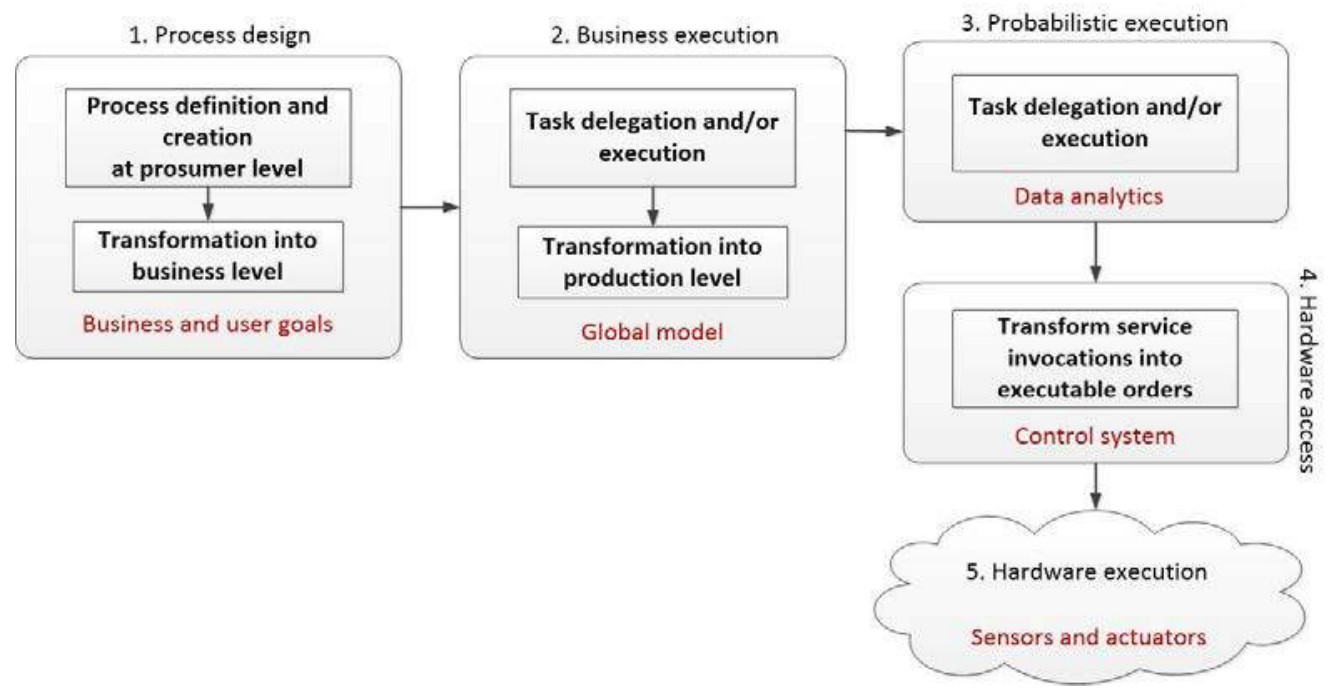

Figure 2: Lifecycle of a process in the proposed solution

In the second step, business execution, most important actions in the system are performed. At this step the process execution is initiated and orchestrated. For each service it is calculated where to execute each service task, considering the available locations for each service, the cost, quality, and the user's restrictions for each location (described in the metadata file). Tasks to be delegated are transmitted to the appropriate remote system using the delegation services. The description files of the tasks to be executed locally are translated into a production level description, considering the underlying hardware platform. Metadata are also transformed in the appropriate way.

The third step, probabilistic execution, encapsulates all actions intended (1) to execute and orchestrate the processes at production level; and (2) to wean the hard definition of computational processes from the aleatory behavior of hardware. For example, sensors present an intrinsic error which must be taken into account; various measurements made in a row offer different values, etc.

In the fourth step, hardware access, generic service invocations done by the execution engines are translated into the execution orders required by the underlying ad hoc network. In order to allow the inclusion of virtual devices [26] in the ad hoc network, at this step services may also be redirected to the cloud, if the service is not supported by real devices but virtual.

Finally, in the fifth step, hardware execution, the execution actions are processed by underlying network of physical and virtual devices which are ad hoc connected [50]. Reports about the results are also generated. 


\subsection{DETAILED EXPLANATION OF THE PROPOSAL}

In this Section we are describing in detail the proposed solution, algorithms and data format employed in the first four steps showed in Figure 2: process design, business execution, probabilistic execution and hardware access. The last step (hardware execution) depends on the underlying ad hoc network, and contributing to this process is not the objective of this paper (Section 3.2.4 describes some details).

\subsubsection{Process design}

Figure 3 presents a detailed description of the functional components involved in the process design. At this step, processes and tasks are based on prosumer services. These services are obtained as a composition of business services (see Section 3.2.2), cloud services and Internet services (which are business services provided by remote CPS). Prosumer services are described in a high level and are human-understandable, in order to allow prosumer users to define their own processes. The catalogue of available prosumer services is maintained in the prosumer services repository. Apart from the list of services no additional information is required to be included in this repository. Methods to calculate and maintain this catalogue of available services are very varied [30]: manual, automatic [8], based on sematic technologies, on messaging, etc. Any of them is valid and applicable to our proposal, which is independent from the system configuration.

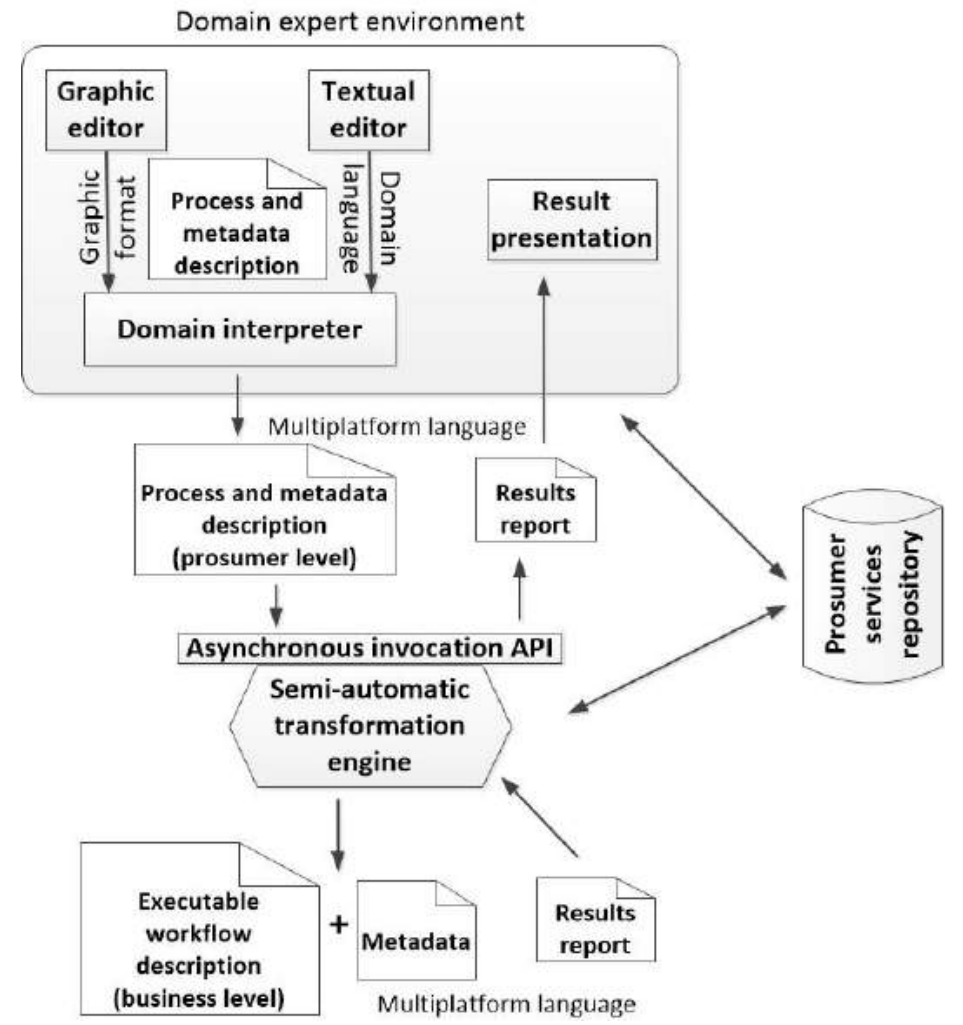

Figure 3: Detailed functional architecture for process design

Once, information about the prosumer services is available in the corresponding repository, and using the tools provided in the domain expert environment, prosumer users may design their own processes to be executed in the CPS as a logical sequence (or workflow) of prosumer services. 
information for these topics is included, some information is aggregated by default (see Table $1)$.

Although textual editor enables users to employ their domain language, prosumer environments are commonly based on graphic design. Using the graphic editor, users may create processes based on the available prosumer services without knowing any domain description language. Technologies such as YAWL (which we are employing) or BPMN can be employed at this point. Figure 4(b) presents the output of the graphic editor for the same process described in Figure 4(a). In this case, metadata are introduced in the system using the same graphic interface, although this information is not presented in the process representation.

In the process descriptions provided by users, tasks are completely executed by invoking only one prosumer service for each task. Thus, using the YAWL terminology, in those description files tasks are considered as atomic, as no secondary decomposition (labeled with the tag NetFactsType in YAWL) for the tasks is explicitly included in the file, see Figure 4, although prosumer services could be decomposed into various business services. Graphically, that is indicated in Figure 4(b) by employing a simple square for representing tasks.

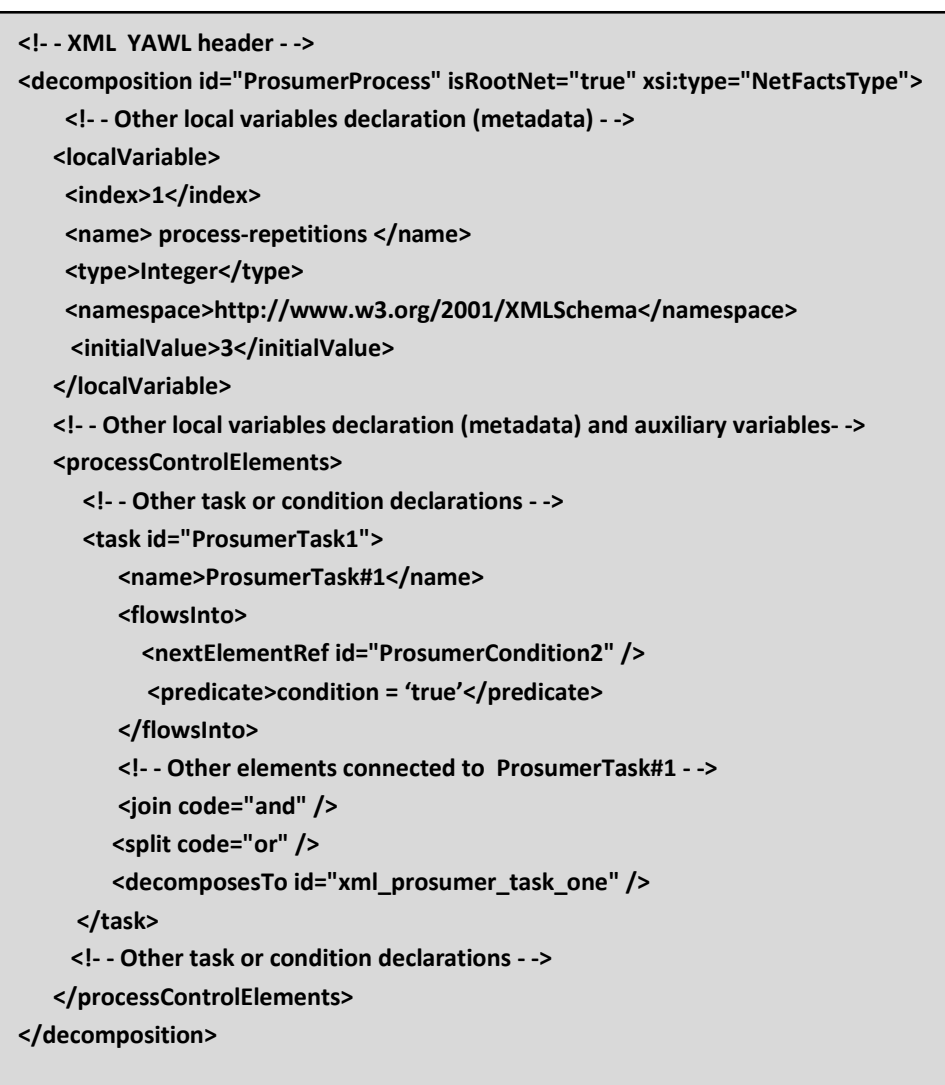

Figure 5: Prosumer process description

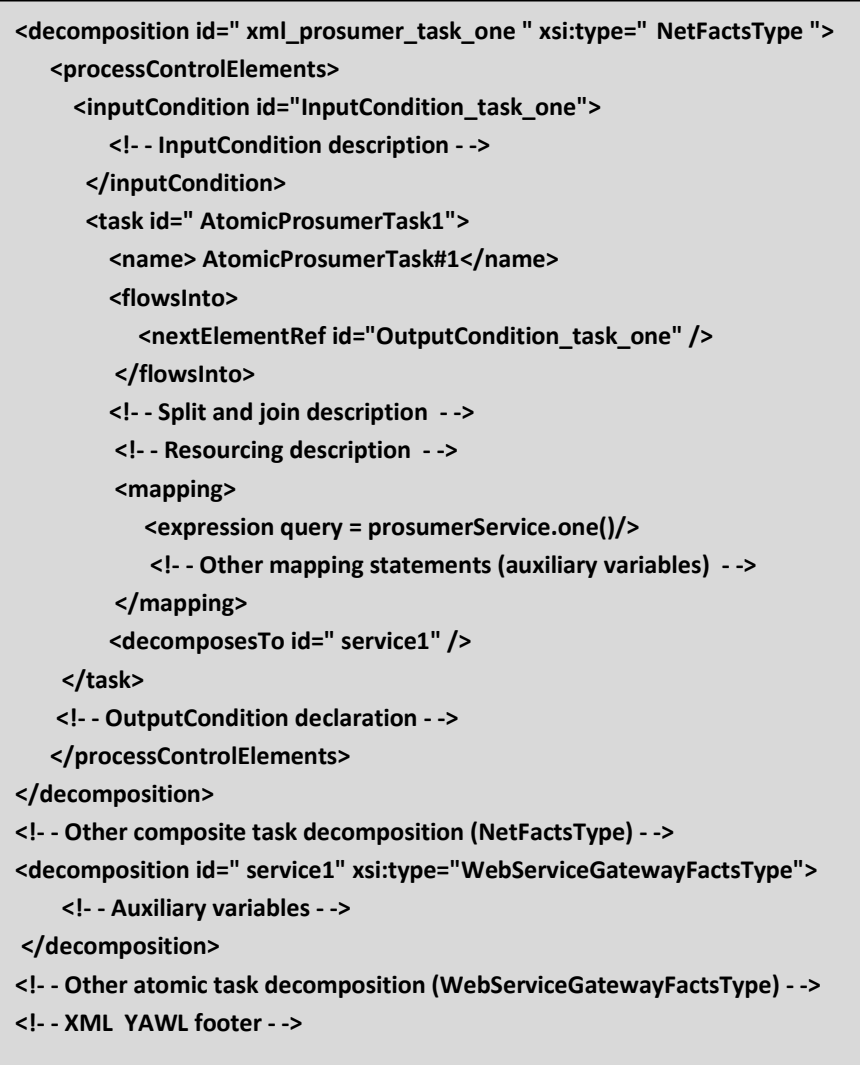

Once the process is completely described, it is translated into a new workflow description language by a domain interpreter. The domain interpreter generates a process description at prosumer level (including metadata) which depends neither on the technological domain nor on the underlying hardware platform. At this level, process is still described using prosumer services. Any executable language such as BPEL or IPM-PDL [58] can be used. In our case we are employing the XML-based version of YAWL. Figure 5 presents the output of the domain interpreter. 
As can be seen in Figure 5, the obtained process description from the domain interpreter includes a secondary decomposition for each task in the process, then in those description files tasks are described as composite tasks (in YAWL terminology). However, in practice, these tasks remain atomic because, as can be seen, secondary decompositions only include one task, which is completely executed invoking one prosumer service. This "artificial" way of representing tasks is intended to simplify following steps in the process execution. In fact, in business execution, general tasks are not truly executed but delegated. Moreover, most delegation services in CPS cannot receive or execute a description file containing an isolated task, and it must be included in a complete workflow. As a solution, domain interpreter generates a workflow of composite tasks, where each task is composed by a workflow with only one task (see Figure 5 and Figure 6). Thus, in order to delegate a task, it is only necessary to send the corresponding decomposition (together with the metadata) to the target CPS.

In Figure 5, additionally, decompositions with the label WebServiceGatewayFactsType may be found. These statements are mandatory in YAWL in the case of invoking external services to the YAWL execution environment in order to execute tasks. In fact, in our case services are placed in the cloud, Internet or provided from an ad hoc network. They contain information about the input and output variables involved in the service execution. Nevertheless, this type of decompositions is not considered when analyzing whether tasks are atomic or composite in a certain description file.

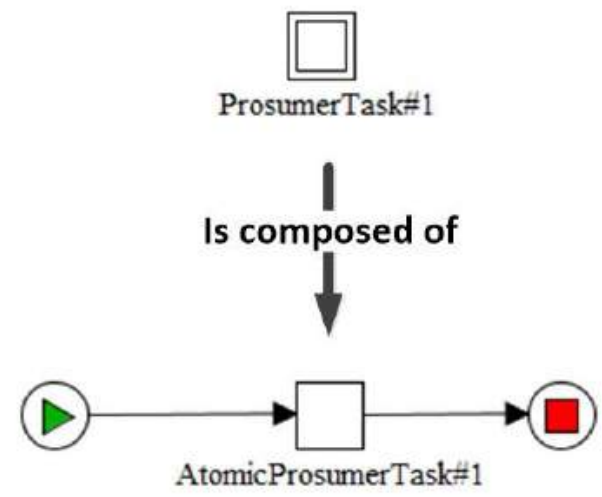

Figure 6: Prosumer process description

The file generated by the domain interpreter is stored in the domain expert environment until users decide to upload it to the execution system. The first element in the execution system is the first semi-automatic transformation engine which offers an asynchronous invocation API to the domain expert environment. Although many invocation APIs could be used (SOAP, for example), in this case we have selected a REST-based interface. Thus, users may perform four different actions. Table 2 describes those four possibilities.

Generally, the first semi-automatic transformation engine transforms the prosumer platformindependent process description generated by the domain interpreter into two different files: a business platform-independent process description and a metadata description file at business level. Process description language employed at business level may be the same employed at prosumer level or different. In our case we are maintaining YAWL as description language. At this point, processes are described using business services instead of prosumer services. 
Table 2: Operation of the REST interface in the first transformation engine

\begin{tabular}{|c|c|c|c|}
\hline $\begin{array}{c}\text { Action } \\
\text { (HTTP operation) }\end{array}$ & Required Input & Expected Output & Explanation \\
\hline PUT & $\begin{array}{l}\text { Process and } \\
\text { metadata } \\
\text { description } \\
\text { document }\end{array}$ & $\begin{array}{l}\text { Unique } \\
\text { identification of } \\
\text { the process }\end{array}$ & $\begin{array}{l}\text { The process is uploaded to the } \\
\text { execution system (transformed and } \\
\text { stored in the business execution). It } \\
\text { will be never executed if users do not } \\
\text { activate the process. }\end{array}$ \\
\hline POST & $\begin{array}{l}\text { Process } \\
\text { metadata } \\
\text { description } \\
\text { document }\end{array}$ & $\begin{array}{l}\text { Unique } \\
\text { identification of } \\
\text { the process and } \\
\text { XML report about } \\
\text { the execution } \\
\text { results }\end{array}$ & $\begin{array}{l}\text { The process is uploaded to the } \\
\text { execution system (transformed and } \\
\text { stored in the business execution) and } \\
\text { executed (at the moment indicated in } \\
\text { the metadata) }\end{array}$ \\
\hline GET & $\begin{array}{l}\text { Unique } \\
\text { identification of the } \\
\text { process }\end{array}$ & $\begin{array}{l}\text { XML report about } \\
\text { the execution } \\
\text { results }\end{array}$ & $\begin{array}{l}\text { The process corresponding to the } \\
\text { provided identification is executed at } \\
\text { the moment indicated in the metadata. }\end{array}$ \\
\hline REMOVE & $\begin{array}{l}\text { Unique } \\
\text { identification of the } \\
\text { process }\end{array}$ & Acknowledgement & $\begin{array}{l}\text { The process corresponding to the } \\
\text { provided identification is removed } \\
\text { from the execution system (business } \\
\text { engine) }\end{array}$ \\
\hline
\end{tabular}

In the literature, many different technologies to implement transformation engines may be found. Holistic methodologies such as the "Business Implementation Methodology" (BIM) [59] have been reported to be efficient and successful. The implementation of process model transformation engines is not the objective of this paper, but a brief description is provided. Detailed descriptions about these solutions are available in the state-of-the-art [60]. In fact, as part of the transformation engines, in order to translate processes from a very high abstraction level into executable code we provide a mapping between processes and software components. Based on MDA (Model Driven Architecture) principles we establish correlations between states in initial business process definition language and the states of the PIM (Platform Independent Model) and the PSM (Platform Specific Model) defined in MDA. A business process model at the PIM level, ready to be transformed into lower level process description language, is then established. The transformation process is completed by mapping platform-independent elements of the business process model into platform specific ones.

In respect to metadata, any of the available description languages may be used (EML, XML, ISO19506, etc.). However, two facts should be considered. First, prosumer users can include new and unforeseen metadata and, second, at business execution some tasks (and the corresponding metadata) might be delegated to external services (such as bank services) which have not agreed on a common document structure for describing metadata. Thus, in this proposal, we used the Resource Description Framework (RDF) in order to describe metadata. Figure 7 presents both description files for the process presented previously in Figure 4 and Figure 5.

As can be seen, the ProsumerTask\#1 and ProsumerTask\#3 tasks have been disaggregated into two workflows involving various additional tasks (i.e. additional services) in order to create the process description at business level. Besides, metadata information has been removed from the process description document. However, in general the structure of the description document is 
the same. Tasks continue being described as composite tasks (indicated by the double square, see Figure 7(c)), and the only difference is the use of business services instead of the prosumer services. Differences could be more if different languages were employed.

Much more interesting, however, is the metadata RDF description document. As can be seen, firstly, the metadata related to the entire process are listed: start time, global quality or the number of times the process must be executed. Later, for each task at business level, some additional metadata are also included. In general, for example, quality parameters present (at this level) the structure cited in Section 3.1. In that way, task delegation becomes easier: it is only necessary to transmit to the target CPS the decomposition and metadata associated with the task.
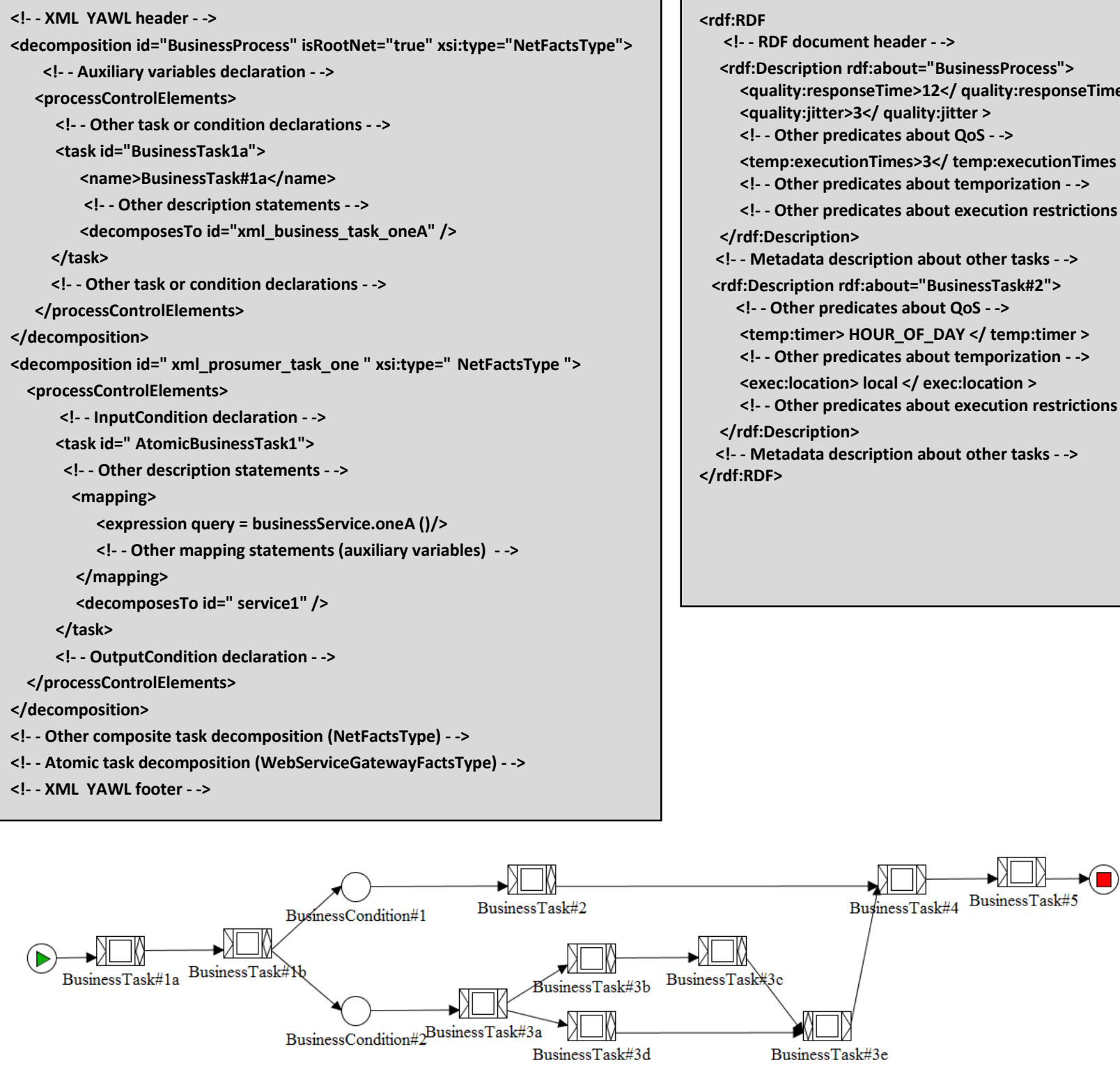

Figure 7: (a) XML business process description (b) Business metadata description (c) Graphic representation of the business process description 
Although the first semi-automatic transformation engine offers four different services to prosumer users (see Table 2), it does not store, manage or execute any process. In fact, most of these functionalities are provided by the business execution engine. Table 3 describes the behavior of the transformation engine.

Table 3: Transformation engine behavior

\begin{tabular}{|c|l|}
\hline Action (HTTP operation) & \multicolumn{1}{c|}{ Explanation } \\
\hline PUT & $\begin{array}{l}\text { The received process description is translated and } \\
\text { sent to the business execution engine to be stored. }\end{array}$ \\
\hline POST & $\begin{array}{l}\text { The received process description is translated and } \\
\text { sent to the business execution engine to be stored } \\
\text { and executed if possible. }\end{array}$ \\
\hline GET & $\begin{array}{l}\text { Redirects the information to the business engine in } \\
\text { order to initiate the execution of the corresponding } \\
\text { process }\end{array}$ \\
\hline REMOVE & $\begin{array}{l}\text { Redirects the information to the business engine in } \\
\text { order to remove the corresponding process }\end{array}$ \\
\hline
\end{tabular}

The transformation engine also generates the prosumer results reports (in this case using XML language) which are showed to users in the result presentation module. These reports are obtained by composing the business results reports by means of a kind of the "inverse algorithm" of the transformation algorithm. Figure 8 shows a possible results report about the process in Figure 7.

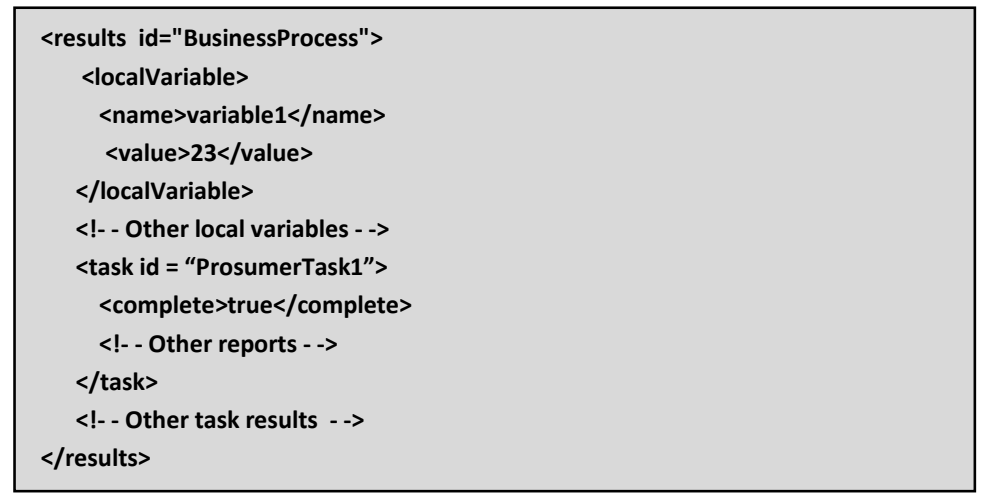

Figure 8: Results reports at prosumer level

As final idea, many technologies can be used for implementing the transformation engine. Compilers technologies [5], sematic solutions [43] or specific technologies for descriptions refactoring [55] are valid.

\subsubsection{Business execution}

This second step concentrates most of the contributions of this work. Figure 9 shows a detailed functional architecture including the components involved in the business execution. In this step, business services are considered. These medium-level services are compositions of production services (see Section 3.2.3), focused on describing functionalities in a platform- 
independent way, which helps tasks and processes to be delegated to external CPS. The corresponding repository, the business services repository, stores the catalogue of available services in the platform. It is the most important repository, and (besides the basic information about services) it must include (for each service) additional information about the execution cost and the guaranteed QoS. In particular, we are assuming this information is provided in the most typical way in CPS [33]: the cost is expressed as an integer, and the QoS is represented by a vector of integer values [52]defining each value a quality parameter (availability, jitter, etc.). Finally, two more remote repositories (not included in Figure 9) must be available: the repository of cloud services and the repository of Internet Services. These two repositories must present the same information of the business repository, but are managed by third-party entities, so they are not part of the proposed system. These repositories could be consulted through the inter-system services API.

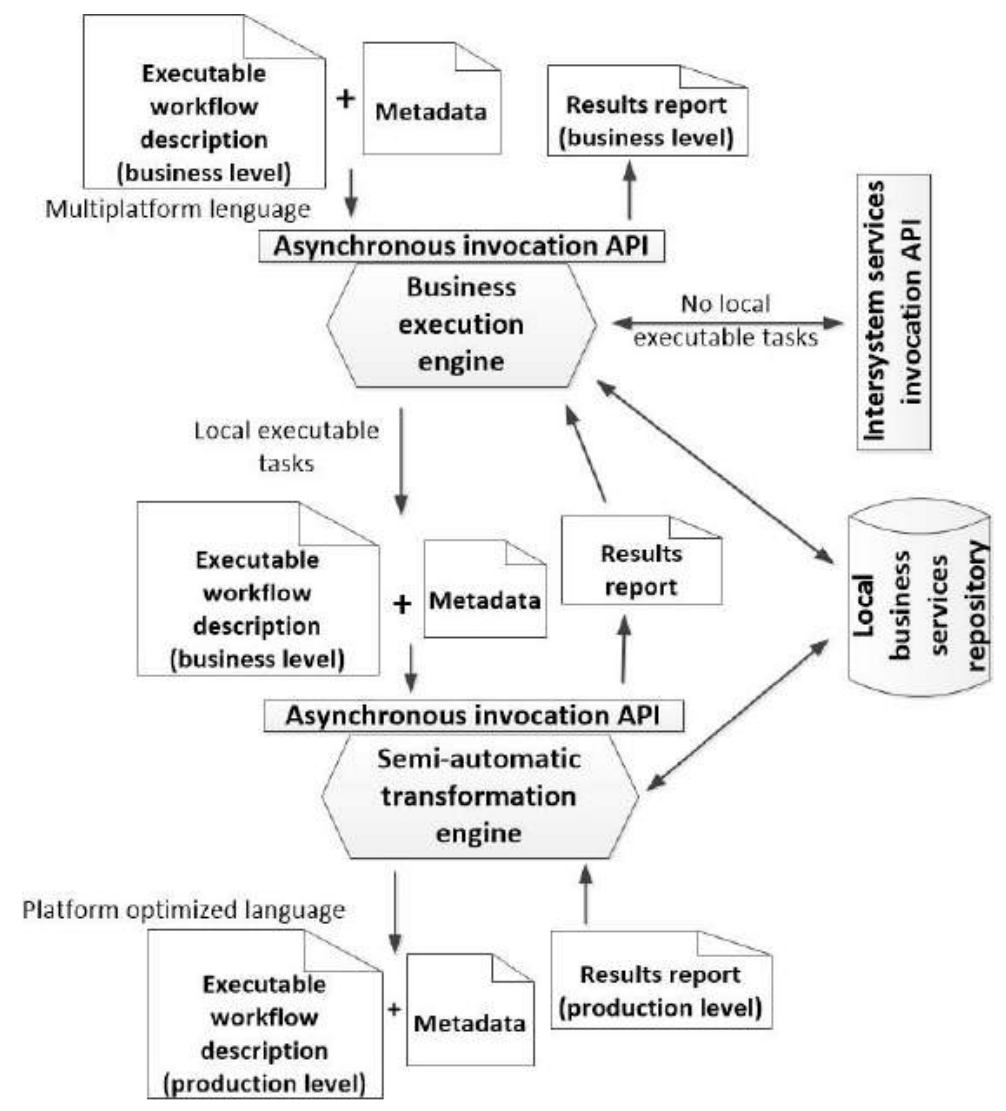

Figure 9: Detailed functional architecture for business execution

As we explained in Table 3, it is the business execution engine the entity which actually maintains and executes processes. This engine receives the output from the first semi-automatic transformation engine (the YAWL description at business level and the RDF document) and acts depending on the invoked HTTP operation and the information provided in the metadata. This engine presents towards the first transformation engine an asynchronous invocation API (REST-based in this case) whose behavior is exactly described in Table 2. In this case, all the described actions are in fact performed by the engine.

In order to perform all those functions, business execution engine is made up of various functional components. Figure 10 presents that structure. 


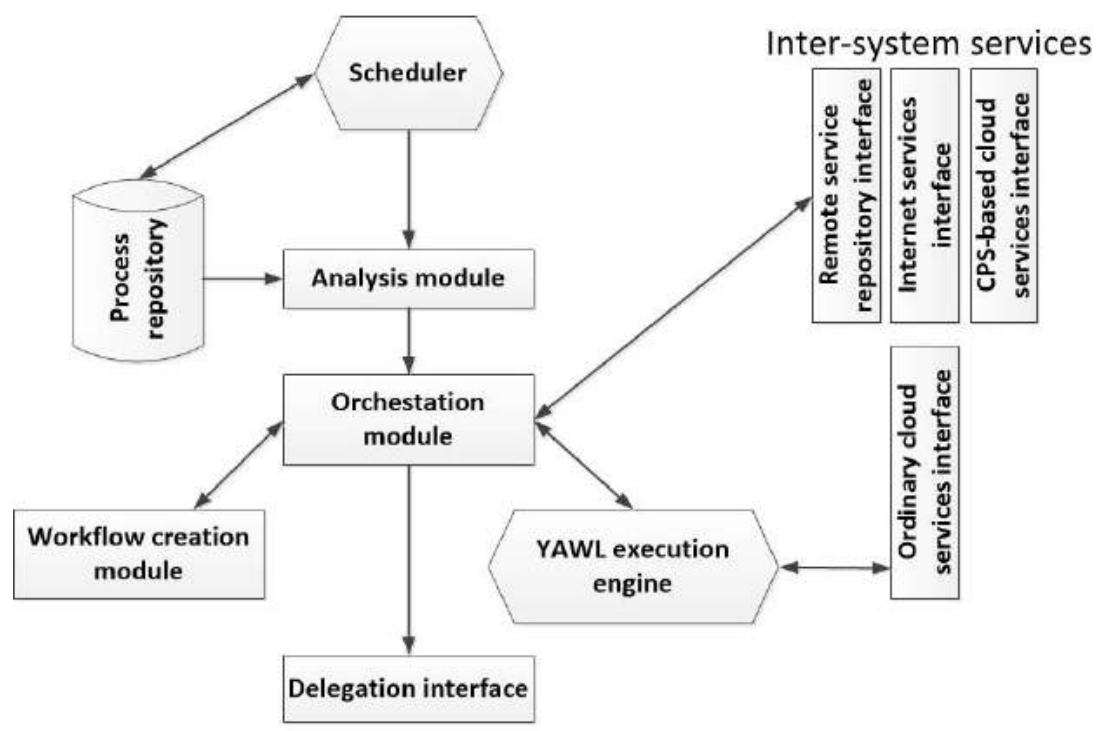

Business execution engine

Figure 10: Functional architecture for the business execution engine

Operations invoked by users are received by the scheduler, which assigns the unique identification to the process and stores both documents (process and metadata description) in the process repository. If a GET or REMOVE operation is ordered, the corresponding actions are also performed. If a certain process gets active (because a GET or POST operation is executed), the scheduler loads the temporization metadata about the whole workflow. Then, when the start time conditions are reached the scheduler loads the process and metadata description documents into the analysis module.

The analysis module parses the process description document and locates the first-order business synchronization points. First-order business synchronization points are placed on the workflow knots (points where more than two elements are connected). The InputCondition and the OutputCondition are always also first-order business synchronization points. The sequence of elements (tasks and conditions) which connect two knots is a branch. One element is never shared by various branches. Figure 11 represents the branches and the first-order business synchronization points of process on Figure 7(c).

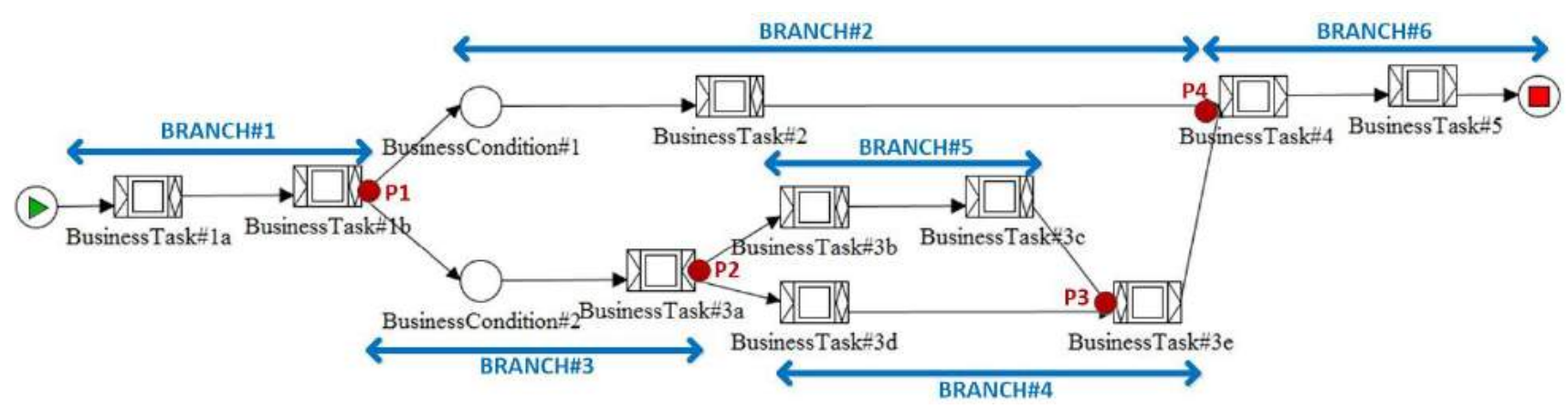

Figure 11: First-order business synchronization points and branches in a workflow 
Considering the document structure showed in Figure 7(a), Algorithm 1 presents a solution to calculate the first-order business synchronization points of a workflow. At these points, at least, the execution will be stopped in order to evaluate the execution evolution. Moreover, in knots where various branches finish, the engine also synchronizes the different flows; waiting for some or all of them in OR-joins and AND-joins, or discarding all of them except the first in XOR-joins. It is important to note that Algorithm 1 includes references to XML fields that are only employed in YAWL workflow descriptions (see Figure 7(a)). Therefore, if other description language is employed, the names of these field should be replaced to which are used in the corresponding language.

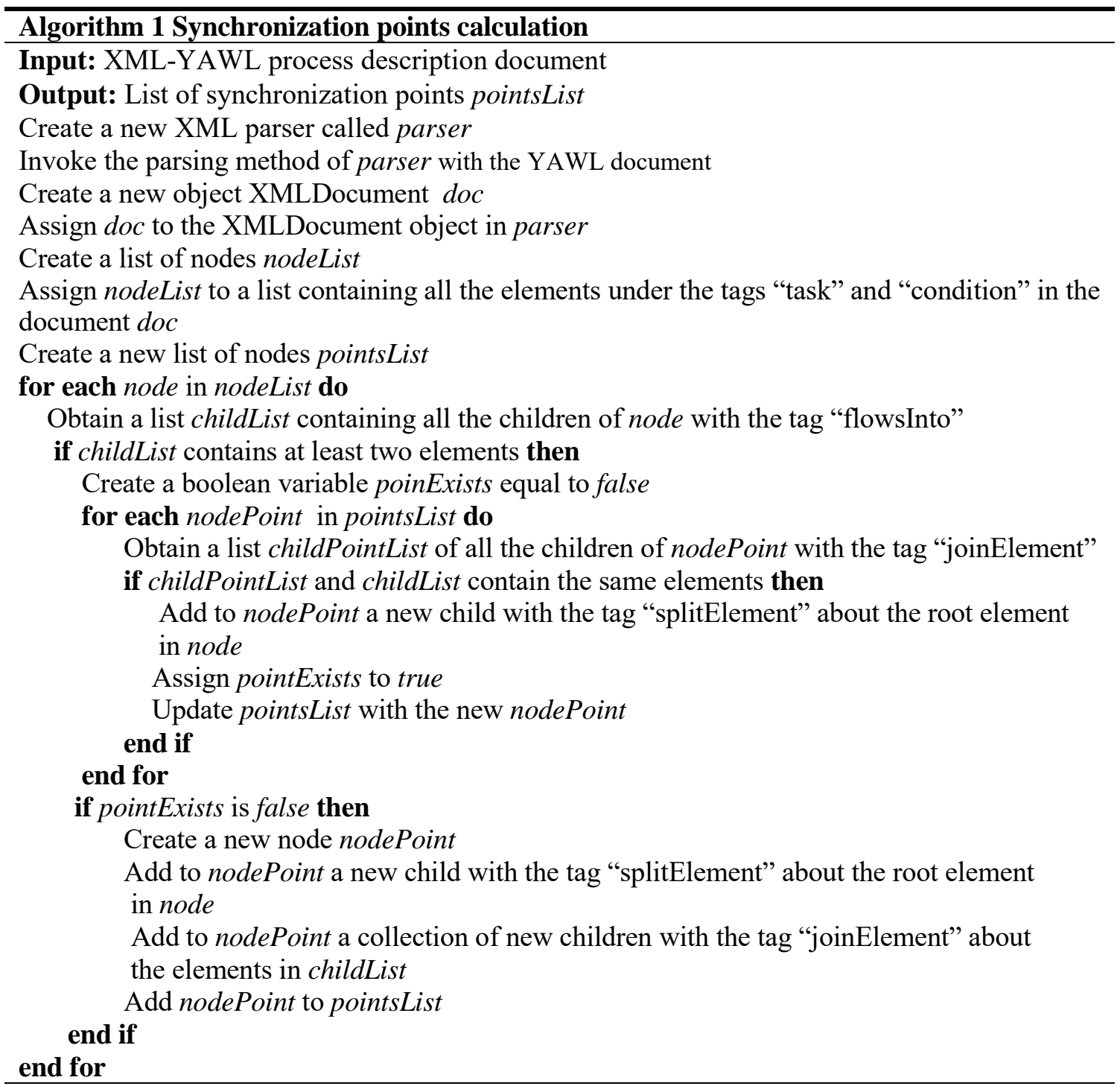

When all first-order business synchronization points have been calculated, the workflow is divided into branches. Algorithm 2 describes a method to perform this action.

Once all first-order business synchronization points and branches on the workflow have been located, the analysis results and the process and metadata descriptions are transmitted to the orchestration module. The orchestration module executes the process branch-by-branch. However, if in some synchronization point various branches are born (typically in AND-splits or OR-splits where various conditions become true), then these branches are executed in 
parallel. For example, in Figure 11 branches BRANCH\#2 and BRANCH\#3 (probably together with BRANCH\#4 and BRANCH\#5) will be executed in parallel.

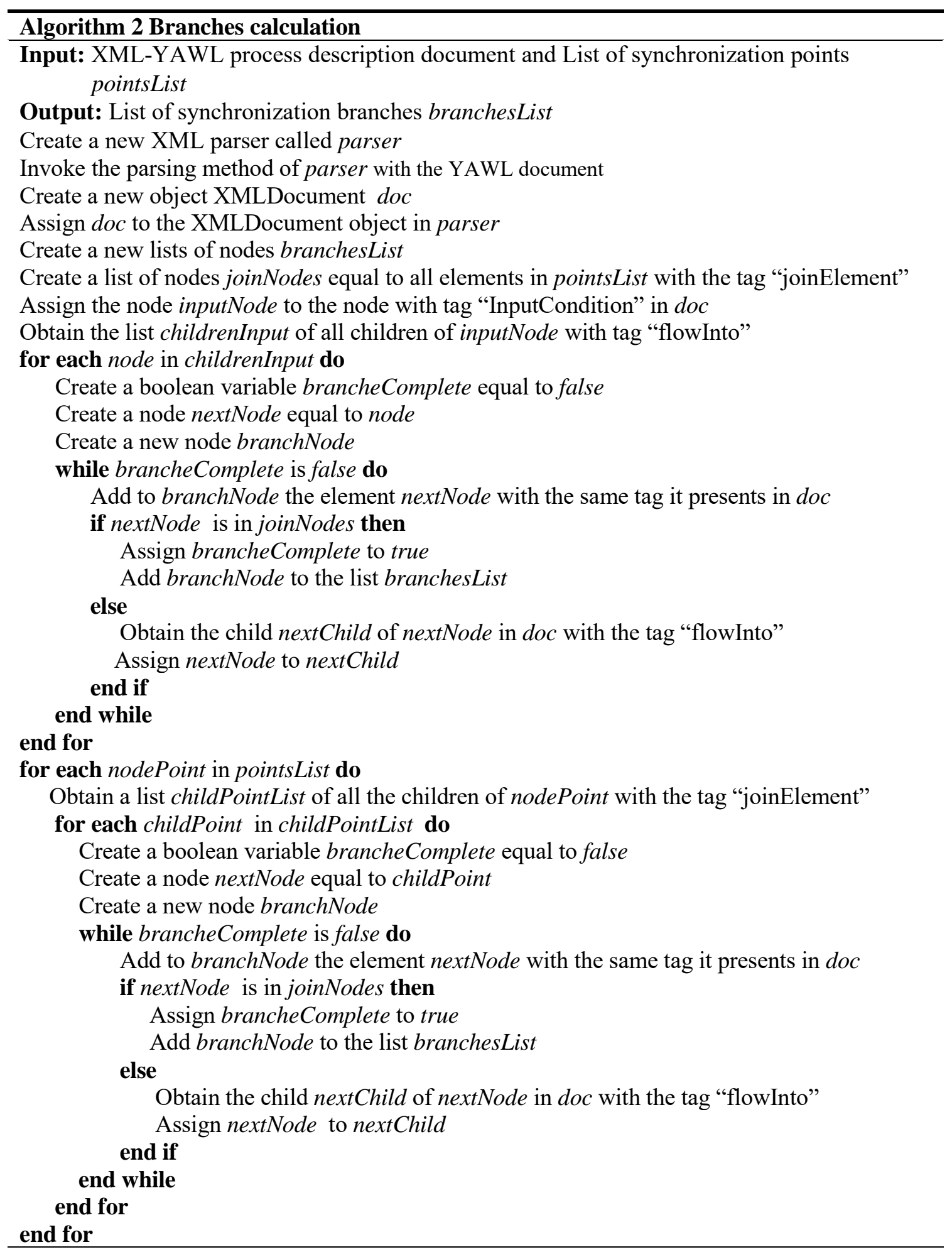

The orchestration module may delegate the entire branch, execute it in the cloud or execute it locally. Additionally, second-order business synchronization points could be added and, then, each task or collection or tasks would be executed in a different location. In general, several of these options would be available. Then, a decision algorithm is required. This algorithm is based 
on mandatory metadata (QoS and execution restrictions) about the tasks which compose the branch and information about the cost of executing the services in each available location. In particular, a hierarchy of requirements is proposed:

1. Execution restrictions indicated by users: If any task must be executed or cannot be executed in a certain location, all the possibilities including the forbidden options will be discarded.

2. Quality-of-Service: For each task (i.e. business service) only the locations which guarantee a higher quality than the one demanded by users will be considered.

3. Execution cost: Finally, if various possibilities remain adequate, the one which presents the lowest execution cost will be selected.

Execution restrictions indicated by users may be directly used as a first filter. For example, in Figure 7(b) it is indicated that BusinessTask\#2 must be executed on the local ad hoc network. However, both other two criteria (QoS and execution cost) require a study about how to perform the selection.

As we said, information about the QoS is stored for each business service in the appropriate repository (local business service repository or remote repositories for cloud or Internet services). This information is presented as an $n_{k}$-dimensional vector of integer values (1), indicating the value of a collection of quality parameters (availability, response time, etc.).

$$
Q o S_{o f f e r}=\left(\alpha_{1}, \alpha_{2}, \ldots, \alpha_{n_{k}}\right)
$$

It must be noted that $k$ parameter is an index to indicate the particular business service we are referring to. In general, the value of $n$ parameter depends on the considered service (thus, for a particular one the value would be $n_{k}$ ). Moreover, the particular parameters selected for indicating the guaranteed QoS also depends on the service, location, etc.

On the other hand, from the metadata description document (RDF document) it is possible to deduct also a collection of quality parameters for each task (i.e. business service), as said in (2).

$$
Q o S_{\text {request }}=\left(\beta_{1}, \beta_{2}, \ldots, \beta_{m_{k}}\right)
$$

As in the previous case, for each task the indicated quality parameters may not be the same, and the dimension of the vector $Q o S_{\text {request }}$ can also vary (thus, for a particular one the value would be $m_{k}$ ).

In those conditions, a location $L$ offers for a certain service $k$ a guaranteed QoS higher than which required by users if the relation (3) is verified.

$$
\sum_{i=1}^{m_{k}} \rho_{i} \beta_{i}<\sum_{j=1}^{n_{k}} \mu_{j}^{L} \alpha_{j}^{L}
$$

Where $\rho_{i}$ is the relative weight associated with the i-th quality parameter $\beta_{i}$ included in the RDF document in relation to the service $k$. Besides, $\mu_{j}^{L}$ is the relative weight associated with the $\mathrm{j}$-th quality parameter $\alpha_{j}^{L}$, associated to the service $k$ when executed in the location $L$. Relative weights are very important as they allow giving more relevance to some parameters than others. For example, to ensure that no service with a certain QoS parameter $\alpha_{j}^{L}$ below a hard limit 
$\beta_{i}$ indicated in the RFD document is invoked, the weight of this parameter must be more than $50 \%$ of the total combined QoS.

As an additional requirement, in order to obtain comparable results, it must be fulfilled that $\sum_{i=1}^{m_{k}} \rho_{i}=\sum_{j=1}^{n_{k}} \mu_{j}^{L}$.

Once all the available and adequate locations for each task have been obtained, various options in order to execute the branch are, in general, possible. It must be taken into account that for a branch including a total amount of $e$ elements, the total possible execution schemes is $3^{e}$ (as three different locations are possible). Then, it is selected the execution scheme with the lowest cost.

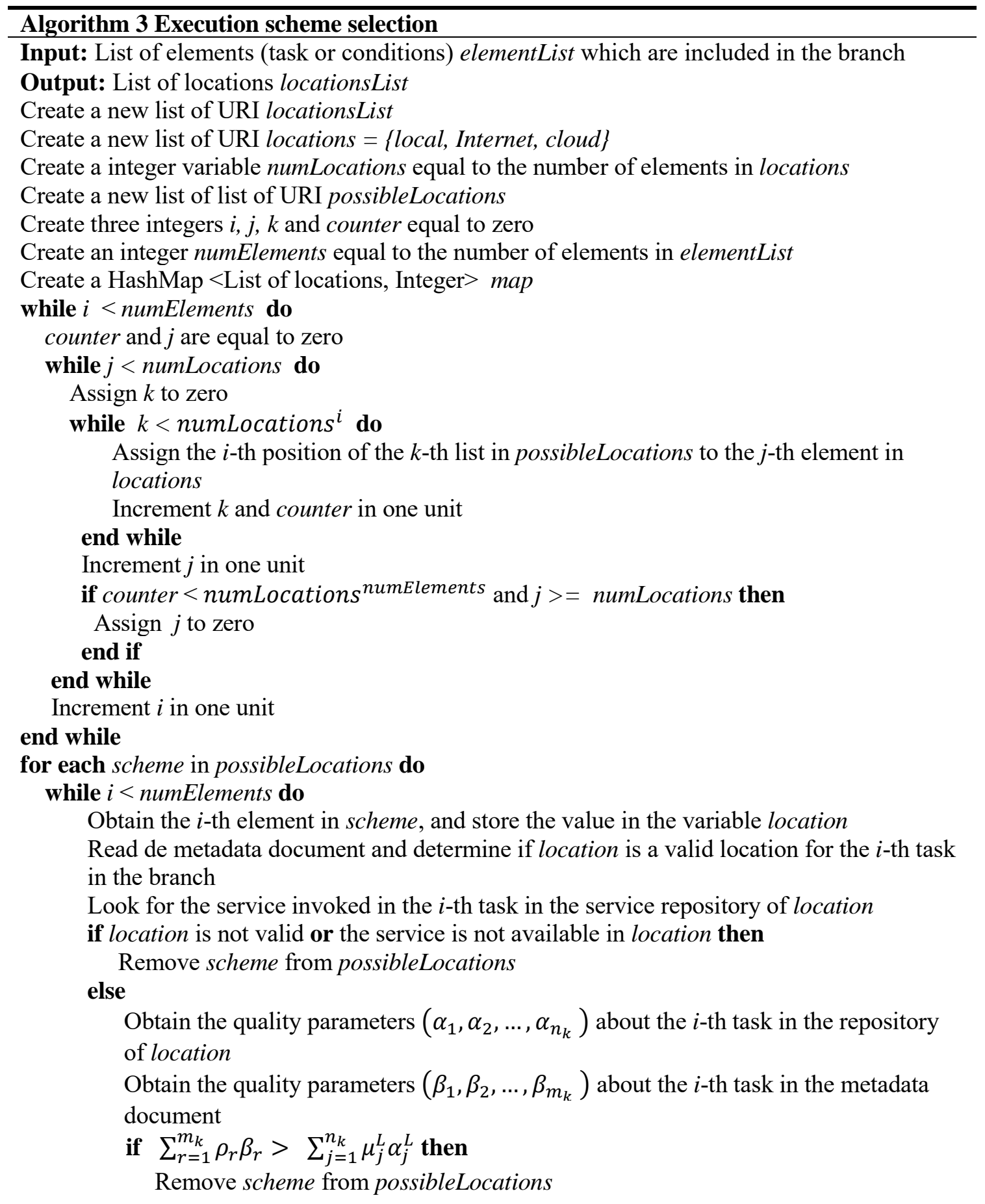




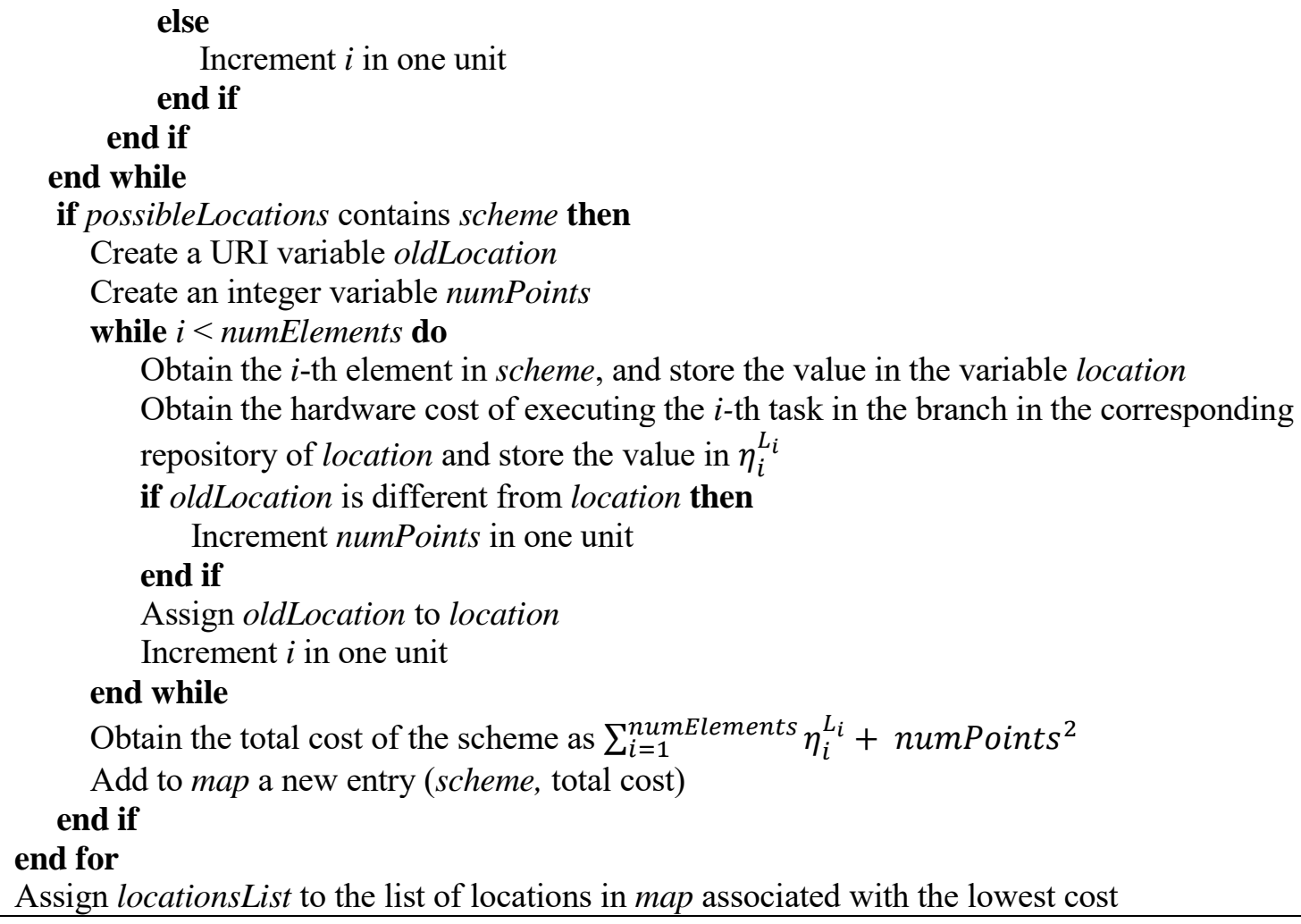

In general, the execution cost is obtained as the addition of two quantities. First, the hardware cost of executing a certain service $k$ in a certain location $L, \eta_{k}^{L}$. And, second, the cost of the orchestration actions $\delta$. This second quantity depends on the number of second-order synchronization points introduced, i.e. the number of fragments into which the entire branch is divided. Thus, the total execution cost for a certain execution scheme $s$ is obtained as (4). Briefly, this expression adds the hardware cost of executing each service in the selected location $L_{i}$, up to a total of $\tau$ services that compose the branch under study, and later adds the orchestration cost, which is a function of the selected scheme.

$$
c_{s}=\sum_{i=1}^{\tau} \eta_{i}^{L_{i}}+\delta_{s}=\sum_{i=1}^{\tau} \eta_{i}^{L_{i}}+f(s)
$$

The values of $\eta_{i}^{L_{i}}$ are provided in the service repositories together with the quality information, and function $f(s)$ may be defined as desired. In our case, we are proposing a quadratic cost function (5). Where $p_{s}$ is the number of second-order business synchronization points in the scheme $s$. As execution costs $\eta_{i}^{L_{i}}$, which are dimensionless values, usually normalized, $f(s)$ does not have any unit. It is only an indicator of the orchestration cost. Typical orchestration algorithms present a complexity of $n^{2}$ with the number of elements to manage. In our scenario, elements to manage are the parts in which a branch is divided, an amount that corresponds to the number of orchestration points. Therefore a quadratic function may be used to represent the computational cost of orchestrating a branch with $p_{s}$ orchestration points.

$$
f(s)=p_{s}^{2}
$$

Finally, Algorithm 3 describes the decision algorithm which allows selecting the most adequate execution scheme. This algorithm calculates all the possible execution schemes, and, later, 
discards every scheme which is forbidden by users, does not fulfill the quality requirements, or involves executing a service in a non-available location. In the last step, the scheme with the lowest execution cost is selected.

Mathematically, Algorithm 3 solves a graph problem (see Figure 12). Figure 12 represents the equivalent graph problem of calculating the execution scheme for a branch with three elements (tasks or conditions). The problem consists of finding the path between the initial node and the final node with the lowest cost. In expression (4), the total cost of moving among the nodes for the lowest cost path has been expressed as the cost function $f(s)$, however, if desired, it is also possible to associate a different cost for each path between each couple of nodes (see Figure 12) and substitute the cost function for the expression (6).

$$
\sum_{i=1}^{\tau} \Psi_{i}^{L_{i}-L_{i+1}}
$$

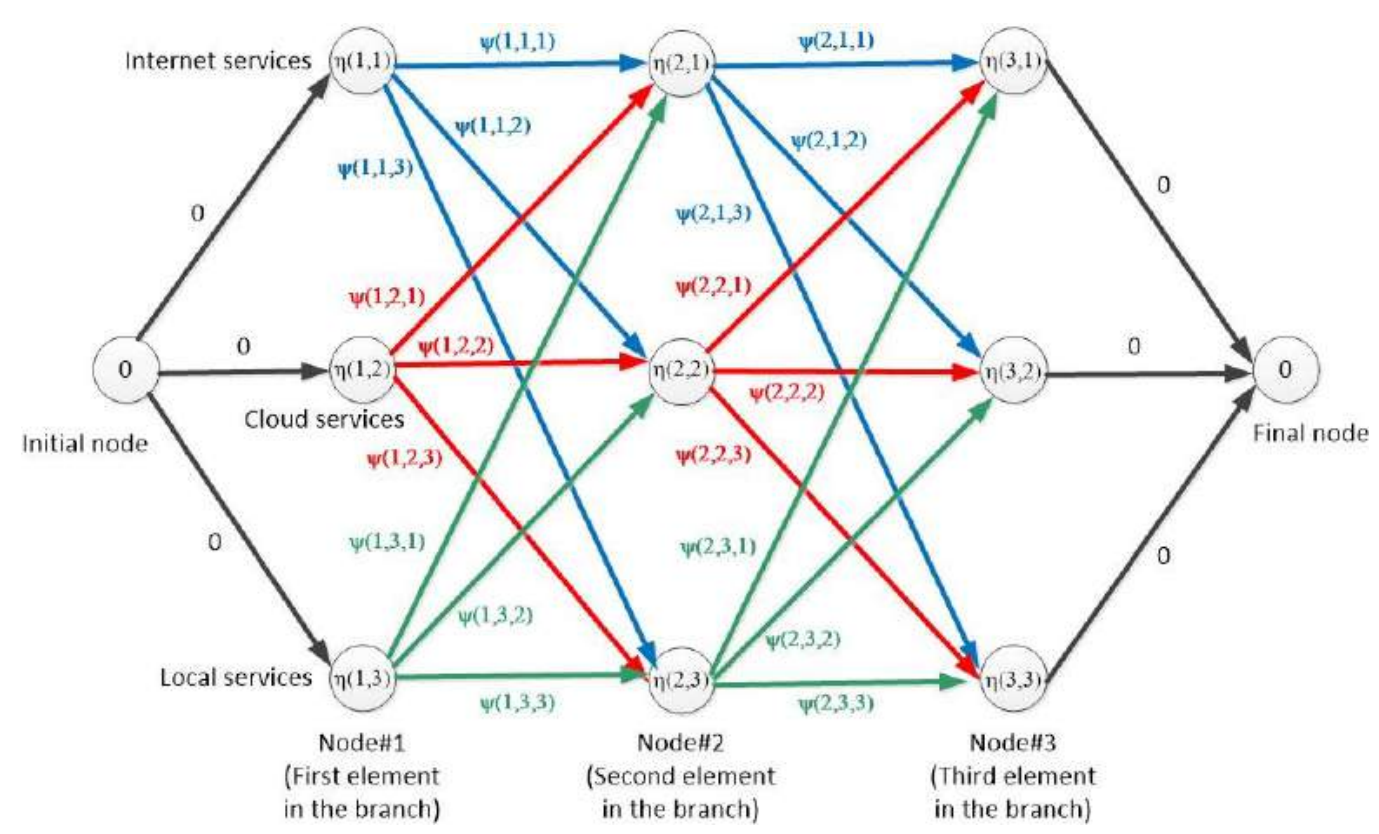

Figure 12: Equivalent graph problem for a branch of three elements. For representation purposes, subscripts have been reordered, consider that $\eta_{i}^{L_{i}}=\eta\left(i, L_{i}\right)$

When the most adequate execution scheme for each branch is selected, then, information about the elements (tasks and conditions) which compose the branch is extracted from the process description document and from the metadata description document. At this point, RDF technology and the use of composite tasks in the YAWL description simplifies the processing activities. 

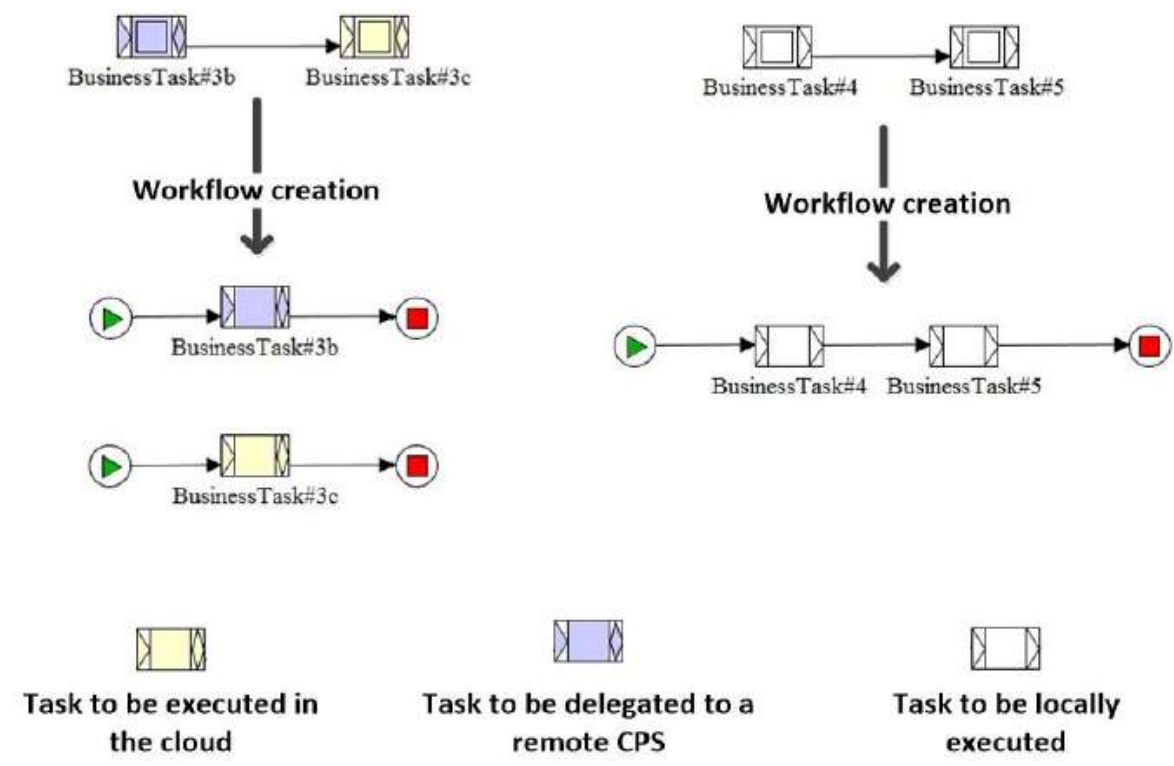

Figure 13: Workflow creation process in the business engine

This information is transmitted to the workflow creation module, which elaborates a new (or various) complete process description and metadata description documents related to the branch to be executed. In general, it creates a new workflow at business level with the tasks between every couple of second-order business synchronization points (see Figure 13). The output of this module is a collection of YAWL descriptions (using now atomic tasks) and a collection of metadata descriptions which can be finally delegated or executed (see Figure 14). As a novelty, the module could add some additional metadata, for example, about the expected precision in the probabilistic execution. Moreover, information about QoS and execution restrictions can be removed.
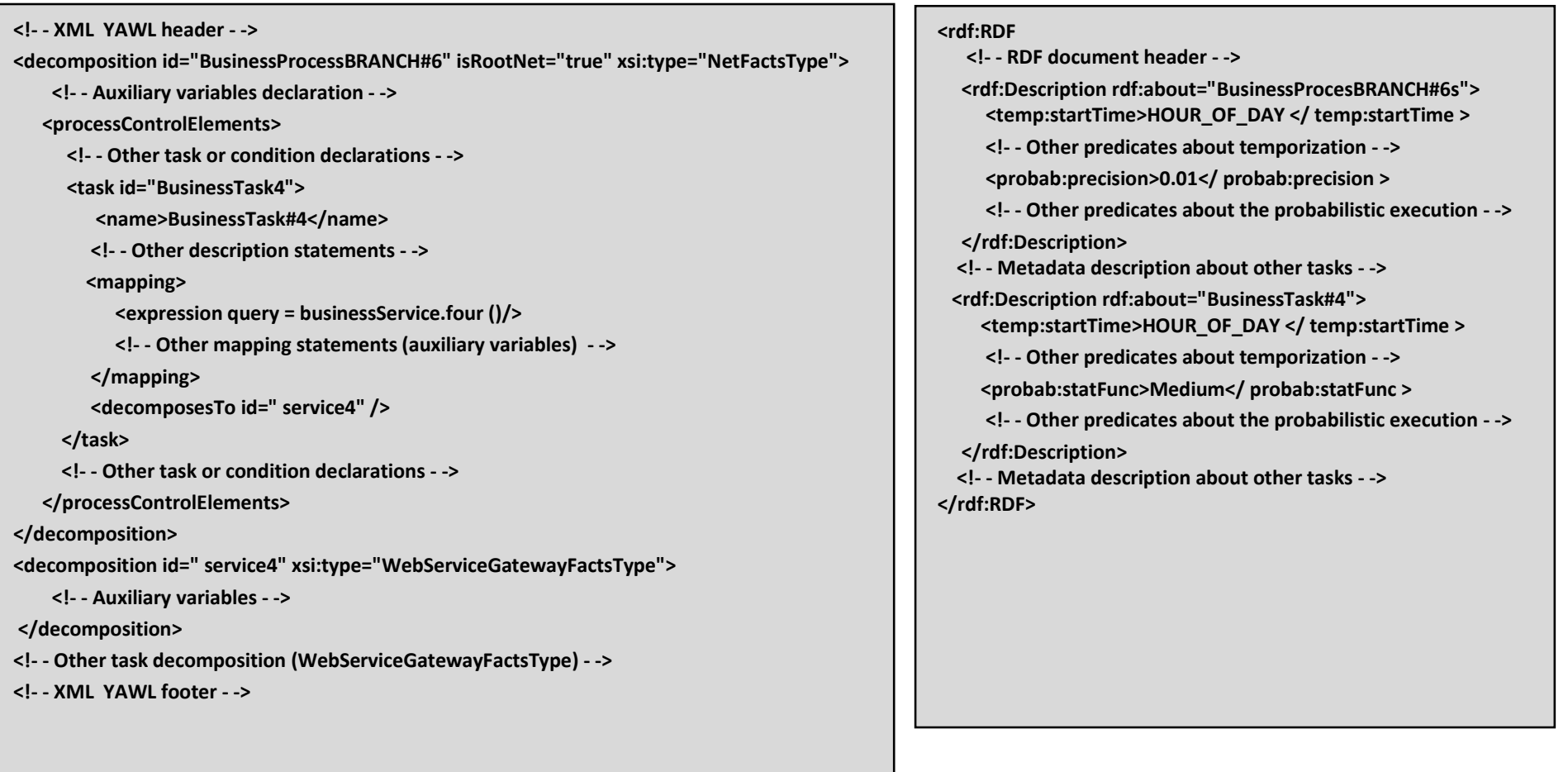
Four different actions can be taken by the business engine at this point. If a workflow must be executed locally, the corresponding process and metadata description is sent through the delegation interface. In another case, the workflow must be delegated to a remote CPS (using the appropriate interface). More complex is the execution of services in the cloud. Sometimes, cloud services are CPS-oriented, and then the process description and metadata description may be sent through the appropriate interface as the previous cases. However, other times, cloud services are not CPS-oriented, and the process and metadata description cannot be directly sent. In those cases, process description is sent to an internal YAWL engine (connected to the cloud) which executes the process at business level and requests the cloud services using ordinary techniques [46].

Tasks which are delegated to cloud or Internet services will be executed in those systems and only results report will return. In the case of tasks to be executed locally, the execution continues.

As showed in Figure 9, process descriptions at business level for tasks to be executed locally are translated into platform-dependent language by a second semi-automatic transformation engine. This engine presents a behavior identical to the first transformation engine, including the same interface explained in Table 2 and Table 3 . In this transformation process, platform-independent descriptions are transformed in platform-optimized descriptions (see Figure 15). Basically, although a second language could be used if necessary, business services are replaced by production services. In our case, we are maintaining YAWL as description language.

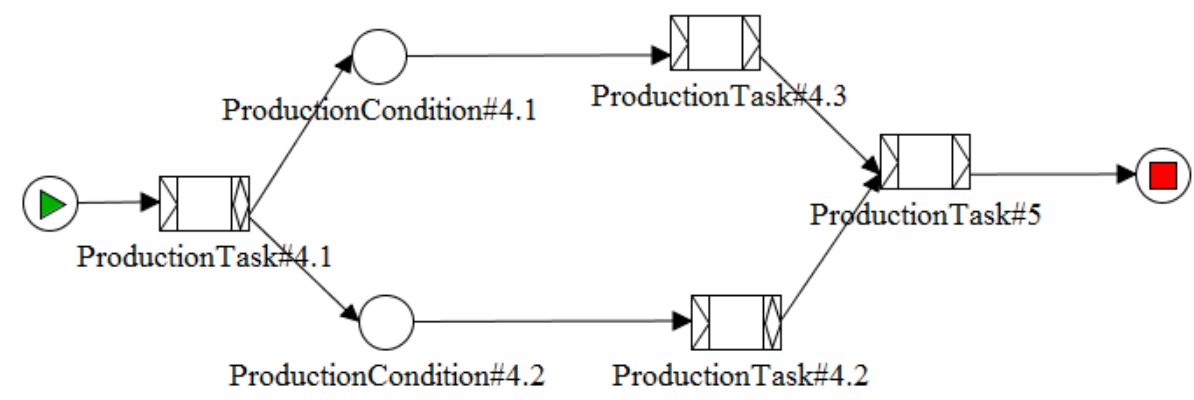

Figure 15: Workflow at production level for the BRANCH\#6 on Figure 11

In respect to metadata, they are also transformed into production level. At this level, all metadata must be referred to a particular characteristic of the underlying ad hoc network. For example, if the ad hoc network contains temperature sensors, the maximum admissible error in temperature should be added as metadata. This kind of information will be very important for the probabilistic execution.

In this first work, three metadata about probabilistic execution are considered. First, the maximum admitted error in measures. Second, the number of realizations required for each measure. And third, the statistic function that is applied to the realizations in order to obtain the final value (medium, median, etc.). Figure 16 represents the process description and the metadata document obtained as result of this second transformation process. 


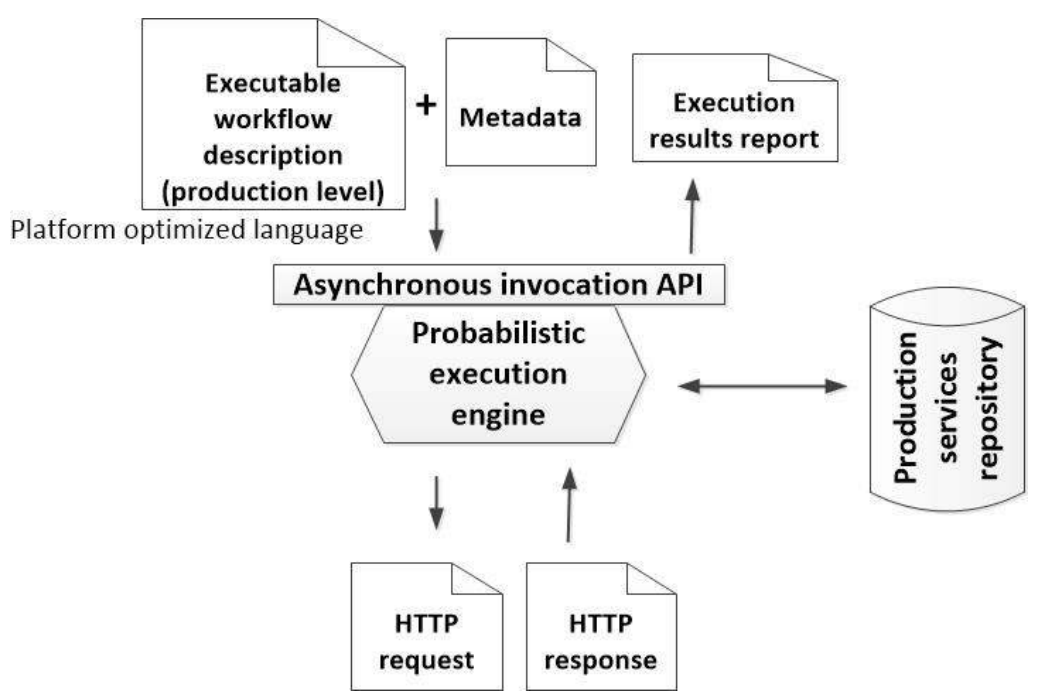

Figure 17: Functional architecture for the probabilistic execution

The probabilistic engine, basically, receives the process description document at production level and the metadata document and executes every task or condition, considering the provided metadata. Executing a task or condition implies the invocation of a certain service, which in our case, is offered by a hardware access infrastructure. These services are REST-based services, so the probabilistic engine must generate the corresponding HTTP requests (in general GET operations) and, as a result, will receive the resulting HTTP response (see Figure 18)

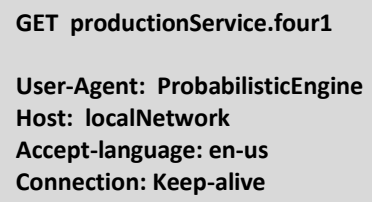

HTTP/1.1 200 OK

Date: Mon, 12 Jul 2012 12:42:33 GMT

Server: localNetwork

Content-Length: $\mathbf{8 8}$

Content-Type: text/xml

Connection: Closed

$<\mathrm{xml}>$

$<$ result $>32.5</$ result $>$

$</ \mathbf{x m l}>$

Figure 18: Example of the generated HTTP request and response by the probabilistic engine for the productionTask\#4.1

Some additional components can be distinguished as part of the probabilistic engine (see Figure 19). 


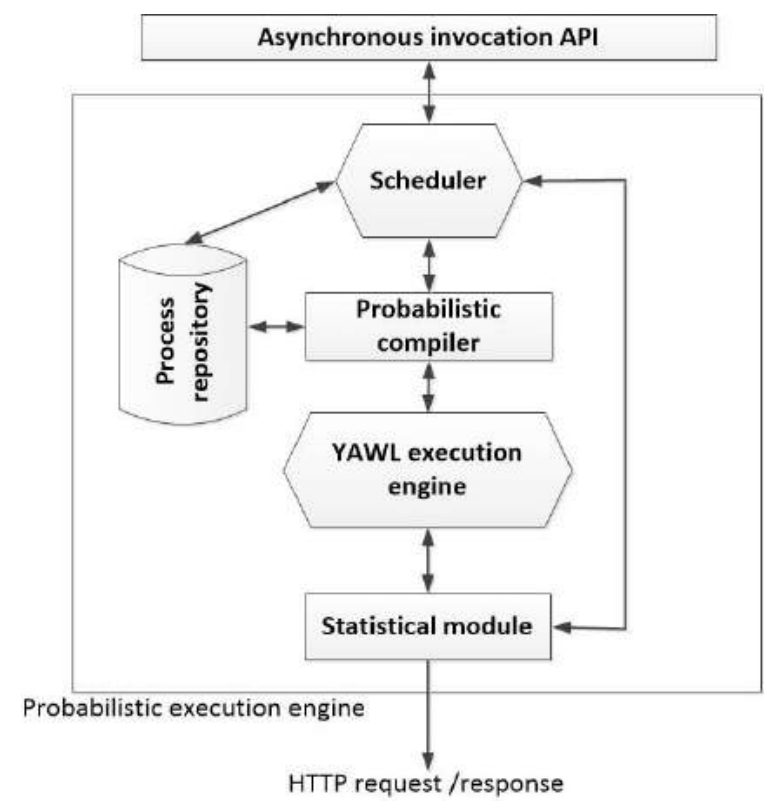

Figure 19: Functional architecture of the probabilistic execution engine

As seen in Figure 19, and similar to the case of the business engine, the probabilistic engine also implements a scheduler which receives REST operations. This scheduler assigns a unique identification to any delegated process and stores it in the process repository. The assigned identifications are sent back to the orchestration module in the business engine which maintains them until a user invokes a REMOVE operation at prosumer level. When a process gets active (because a GET or POST operation is received), the scheduler loads the metadata description document and evaluates the temporization conditions. When these conditions are fulfilled, it reads the process description document from the repository and sends it to a probabilistic compiler. That compiler also receives from the scheduler the metadata information about the probabilistic execution. In particular, it receives information about the maximum admissible error in the variables' value.

Table 4: Relation between hard and probabilistic control flow conditions

\begin{tabular}{|c|c|}
\hline Maximum admissible error: $\boldsymbol{\varepsilon}_{\text {max }}$ \\
\hline Hard control flow condition & Probabilistic control flow condition \\
\hline $\operatorname{var}>\alpha$ & $\operatorname{var}>\alpha+\varepsilon_{\max }$ \\
\hline $\operatorname{var}<\alpha$ & $\operatorname{var}<\alpha-\varepsilon_{\max }$ \\
\hline $\operatorname{var}=\alpha$ & $\operatorname{var} \geq \alpha-\varepsilon_{\max } \& \&$ var $\leq \alpha+\varepsilon_{\text {max }}$ \\
\hline $\operatorname{var} \geq \alpha$ & $\operatorname{var} \geq \alpha+\varepsilon_{\max }$ \\
\hline $\operatorname{var} \leq \alpha$ & $\operatorname{var} \leq \alpha-\varepsilon_{\max }$ \\
\hline
\end{tabular}


This compiler transforms the hard control flow conditions (i.e. the relations of equality or order which involves variables whose value depends on a service execution), into new conditions considering the maximum admissible error indicated in the RDF document. Table 4 presents the main hard control conditions which are present in a process description and the resulting probabilistic control conditions obtained from the probabilistic compiler for each case.

The obtained process description from the probabilistic compiler is sent to an internal YAWL engine which executes the process. The YAWL engine executes the workflow, and invokes the services composing every task and condition. The behavior of this engine is the standard [46].

At the same time, the scheduler reads the metadata document and extracts the remaining information about the probabilistic execution. This information is loaded in a statistical module which intercepts the service invocations (HTTP requests) done by the YAWL engine. This interception scheme allows employing standard stable implementations for the YAWL execution engine, which reduces the costs and complexity of the system. Two different types of services may be invoked: data collection services and actuation services. Depending on the type of service involved in the intercepted HTTP request, the statistical module runs different algorithms. In order to distinguish the service type, it may be indicated in the metadata (although it is not mandatory in our proposal) or may be deducted from the number and type of output and input variables. Typically, data collection services ask for different output variables (integer, string, etc.), and action services provide input variables, although sometimes boolean output variables are also considered.

Data collection services are intended to obtain certain data from the ad hoc network. If one of these services is invoked, the statistical module analyzes the corresponding metadata and creates a probabilistic study based on each intercepted HTTP request and the metadata information. This study basically consists of repeating the operation the number of times indicated in the metadata (or the required number until the hardware error is below the maximum indicated in the metadata), and later obtaining from the results the final value applying the indicated statistical function. For example, considering the information provided in Figure 15, when the YAWL engine executes the task productionTask\#4.1 and invokes the service productionService.four1, the statistical module intercepts the HTTP request and performs a probabilistic study based on five different evaluations of the service productionService.four1. The medium of the obtained values will be returned as final result to the YAWL engine in the HTTP response by the statistical module. These probabilistic studies pretend to wean the computational processes from the aleatory facts typical of hardware devices and physical world. Algorithm 4 describes the behavior of this statistical module. 


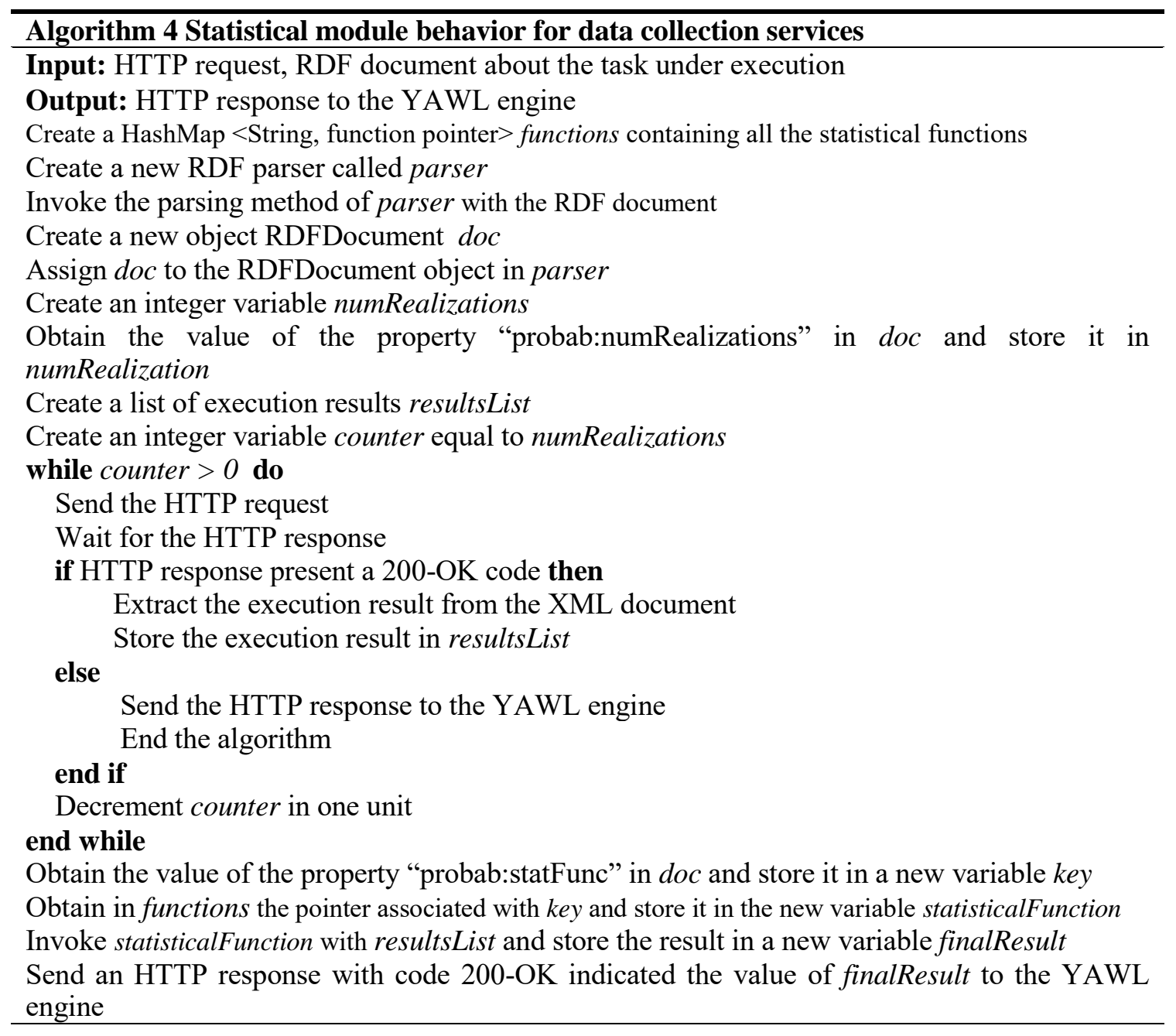

On the other hand, actuation services are focused on performing a certain action,e.g. moving robotic arm, turning on the heater, etc. In this case the statistical module only performs two actions. First, input variables are rounded in order to adjust the precision of these values to which provided by the ad hoc network and to which applied by users in the metadata. Second, if finally any input variable presents a value smaller than the global precision, then this variable is removed from the HTTP request before being sent. If it is the only variable in the request, the corresponding HTTP request is not transmitted and the statistical module directly creates a 200OK response to be sent to the execution engine. For example, considering a robotic arm which its movement precision was one degree, every robotic arm's input variable would be rounded to an integer value. Moreover, HTTP requests applying for movements smaller than one degree would be not transmitted.

Additionally, the statistical module may generate a report about the "quality" of the execution; i.e. about the final error committed $\varepsilon_{t}$ which depends on the hardware error $\varepsilon_{h}$, experimental error $\varepsilon_{e}$ and mathematical error $\varepsilon_{m}$ in the ad hoc network and the precision applied by users $\varepsilon_{u}$, as well as the statistical function applied $f_{s}$. See expression (7). In some occasions, the precision applied by users $\varepsilon_{u}$ is given in the form of the number of realizations which must be performed $n$.

$$
\varepsilon_{t}=F\left(\varepsilon_{h}, \varepsilon_{e}, \varepsilon_{m}, \stackrel{\varepsilon_{u}}{n}, f_{s}[\cdot]\right)
$$


These reports are directly transmitted to the scheduler, which can include this information in the production results reports. Then, this information would be transmitted to the business and the prosumer level in order to inform users about the obtained final precision.

\subsubsection{Hardware access}

In the last step, service invocations (HTTP requests) from the probabilistic execution engine (particularly from the statistical module) must be mapped into execution orders adequate for the underlying ad hoc network. Moreover, virtual devices can be included, so this mapping process must consider also this possibility. Figure 20 shows a functional architecture of the proposed solution for this final step.

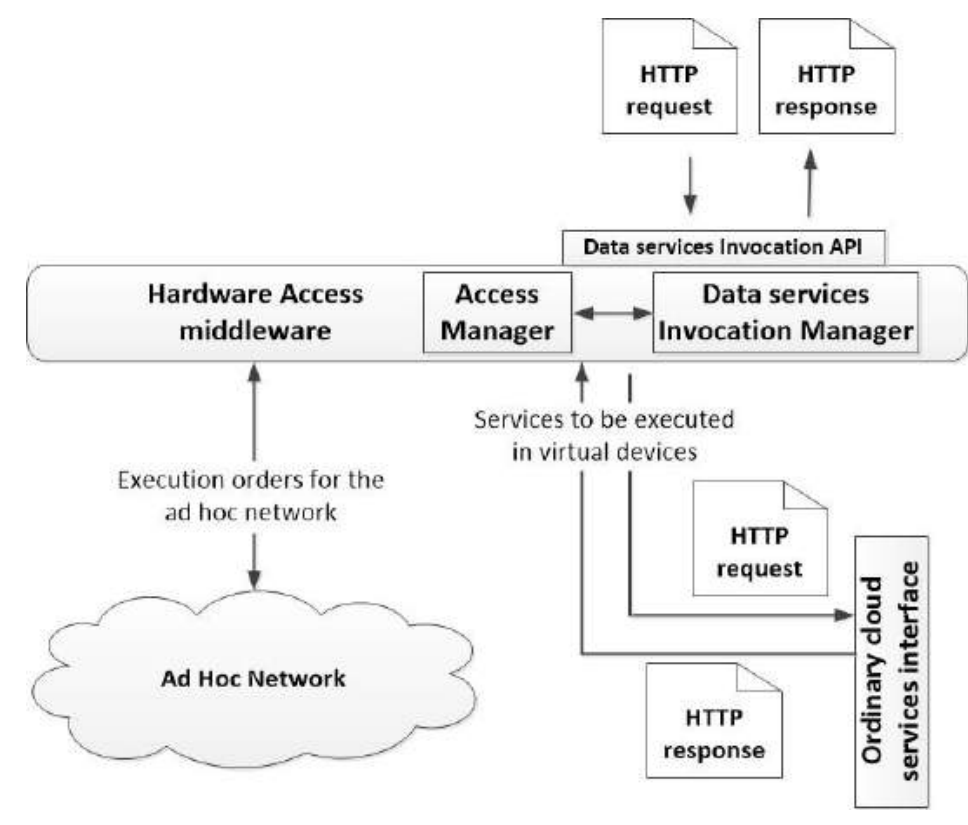

Figure 20: Functional architecture for the hardware access

As can be seen, the proposed solution is based on a middleware which connects the probabilistic execution engine with the ad hoc network and, if necessary, the cloud. The middleware offers an interface to the probabilistic engine based on REST services (as in the other cases). Once a HTTP request is received, the Access manager decides if that service is provided by the underlying ad hoc network or if it is supported by virtual devices and, then, it must be redirected to the cloud.

If the invoked service is supported by virtual devices, then the Access manager sends the original HTTP request to the Data Services invocation manager, which creates a new HTTP request indicating the web location and the name of the cloud service implementing the virtual device. This request is sent through the appropriate interface. When the HTTP request is received, the Data Services invocation manager builds a new response adequate for the original request which is sent to the probabilistic engine by the Access Manager. 
On the other hand, if the invoked service is provided by the local ad hoc network, the Access manager only indicates to the Data Services invocation manager the service to be executed (the original HTTP request is not sent). Then, the Data Services invocation manager creates the appropriate execution orders and asks the ad hoc network to perform those actions. The result is sent back to the Access manager which creates the HTTP response.

Execution order may follow many formats, including Publication/Subscription messages [7], radio signals which are collaboratively addressed [57] or frames from the mobile technologies [54].

\subsection{OTHER IMPORTANT ASPECTS: SECURITY, SCALABILITY AND FAULT RESILIENCE}

The proposed solution and framework presents clear advantages for future industrial companies (those will appear after the fourth industrial evolutions), new smart buildings, and modern intelligent management solutions for critical infrastructures (among other applications). The described technology allows sharing a common production infrastructure among different companies or users, so costs are drastically reduced. New paradigms such as Manufacturing as a Service (MaaS) [61], Drone as a Service (DaaS) [62] or Sensing as a Service (SaaS) [63] could be easily supported by means of the proposed techniques.

However, the intrinsic complexity of CPS, together with difficulties associated to process management and transformation, cause the proposal to present some problems. First, to be effective, many heavy software components must be deployed and connected. Transforming and executing processes are costly operation, so the proposed solution is not valid for constraintresources systems (for example, isolated low-power wireless sensor networks). Besides, although it is not an essential requirement, a high-quality communication infrastructure is needed. The efficiency of the proposed scheme depends strongly on the capacity of components to interact pretty fluently. Opportunistic communications and other similar technologies reduce in a very dramatic manner the quality of services supported by our proposal. Furthermore, as many interconnected services are required to maintain the global system available, these systems are vulnerable to Denial of Service (DoS) attacks.

Any case, different characteristics of the system guarantee its security and protection and its fault resilience. In respect to the first topic (security), REST interfaces may be easily extended to implement secure protocols, usually based on Public Key Infrastructures and certificates. In particular, HTTP protocol can be replaced by HTTPS (Secure HTTP) mechanisms. Besides, in order to avoid illegal accesses, a token-based solution can be deployed in combination to REST services. Most recent engineered REST services, such social networks or Openstack [64], employ this technology [65]. In relation to the second topic (fault resilience), the proposed scheme tolerates a huge amount of types of faults. Each REST request may be answered either with a success message or with a fault notification. Task, activity, branch or process may be paused by the execution engine in the higher level, and be resumed later. If the fault remains, and depending on the process definition and the selected configuration, the execution can continue or the user can be notified using the domain expert environment.

On the other hand, because of the complexity of the proposed architecture, scalability is probable one of the key issues to be faced. In fact, some important considerations must be done in relation to the maximum number of processes being executed at the same time. The 
maximum number of processes to be executed at the same time depends strongly on the selected implementation of the YAWL execution engine and the underlying hardware platform. No studies have been reported about the scalability of most common implementation, based on Java technologies. Nevertheless various scalable implementations of YAWL engines have been proposed [66][67]. Furthermore, the use of REST technologies, instead of SOAP for example, guarantees that scalability is only conditioned by execution engines and not by the communication components.

Finally, it is important to note that users may configure the system to accept different definitions of costs, metrics, parameters... The domain expert environment (using the domain interpreter) and the first transformation engine should only include the homogenization laws to transform user definitions into "user restrictions" according to the defined data formats. In that way, parameters can be defined in a heterogeneous manner, according to user abilities or knowledge.

\section{EXPERIMENTAL VALIDATION}

An experimental validation was carried out in order to evaluate the performance of the proposed solution. 


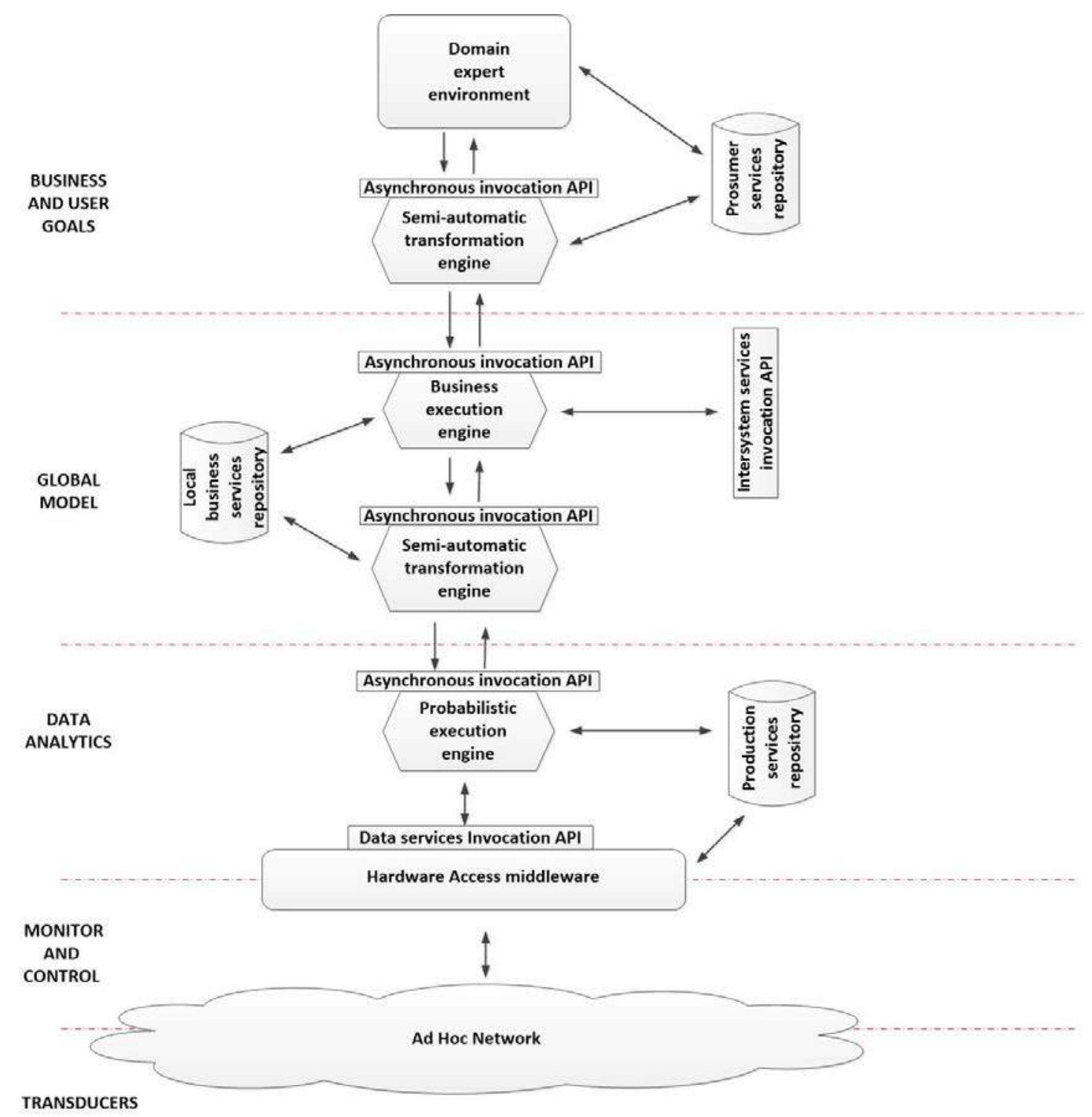

Figure 21: Complete functional architecture for the experimental validation

The validation consisted of one experiment where several processes tried to be executed. The validation was performed using a real deployment of the proposed solution, where simulated components and real software components were connected with real ad hoc network. Every component in the CPS created a log file describing its operation during the experiment. Finally, from the analysis of this log files, various results and conclusions were extracted.

Functional architectures represented in Figure 3, Figure 9, Figure 10, Figure 19 and Figure 20 were connected in order to create the complete process execution system for CPS (see Figure 21). All these components were implemented using Java technologies in a Linux server. In particular, databases were implemented using HSQLDB solutions [40], interfaces were based on RESTful library [36] and YAWL engines were implemented using the public distribution in Java [46]. Additionally, all described algorithms were also implemented using the Java programming language.

On the other hand, a real ad hoc network including sensor and actuator nodes was deployed. Each sensor node in the ad hoc network included a temperature sensor, a humidity sensor, a presence sensor and a light intensity sensor. Each actuator node included a buzzer and a light 
bulb. The complete network consisted of two actuator nodes and five sensor nodes. Nodes in the ad hoc network communicated over ZigBee [39] wireless technology. Over this radio communications technology, a MQTT publication/subscription $(\mathrm{P} / \mathrm{S})$ system was deployed in order to transmit the execution orders to the nodes (and the results from the nodes to the $\mathrm{P} / \mathrm{S}$ broker). Some previous works have showed the details of this configuration [29].

In order to connect the ad hoc network with the Linux server where software components were deployed, the server was provided with a ZigBee antenna. Besides, the P/S broker was also deployed in the Linux server and communicated through an Internet socket with the Data services invocation manager. In that way, the ad hoc network gets connected to the process execution system (in order to obtain the complete CPS), by means of a Hardware Access Middleware based on the P/S paradigm. Figure 22 shows all components involved in the described implementation.

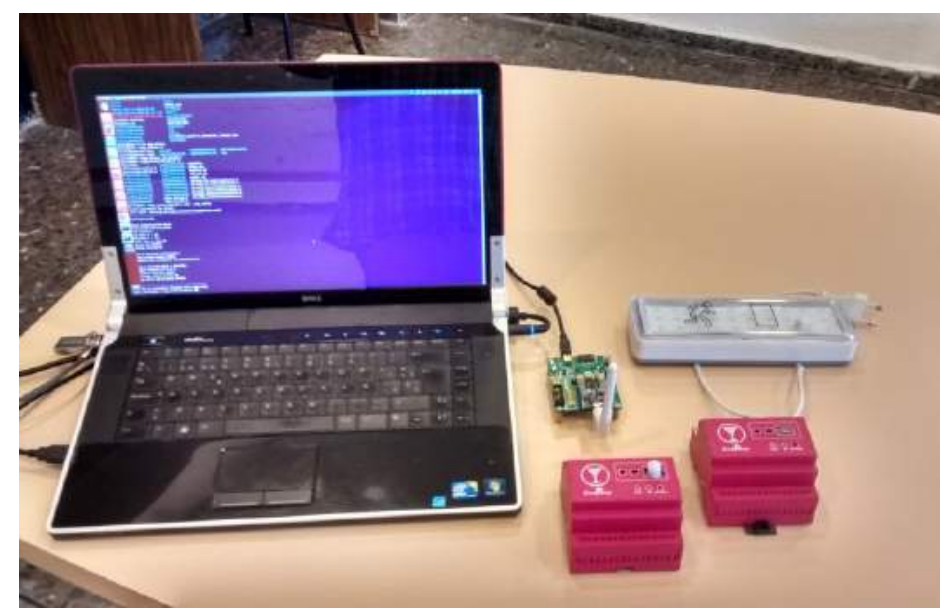

Figure 22: Deployment for the experimental validation

The described system was deployed at the Technical University of Madrid and represents a basic CPS-supported Smart Home, one of the most relevant applications for CPS and process execution systems where users do not have technological skills [4].

The proposed CPS acted as local system. In order to validate all functionalities of the proposed solution, this system should be connected to other CPS through the Cyber-Physical Internet, and be connected to the cloud. However, in order to simplify the deployment, both locations were simulated using Linux virtual machines hosted in the Linux server. In total, four virtual machines were implemented: three of them ran software agents simulating remote CPS and the fourth machine ran a virtual cloud. All machines were created using Kernel-based Virtual Machines (KVM) [20] by means of a python script and the Libvirt API [17]. The connection among the virtual machines and the Linux server was made through the public Internet using public IP addresses. 
Table 5: Available services in the local CPS for the experimental validation

\begin{tabular}{|c|c|c|c|}
\hline Level & Service ID & Name & Description \\
\hline \multirow{6}{*}{ Production } & Production\#1 & Production.getTemp & $\begin{array}{l}\text { It returns the value of the last } \\
\text { temperature measurement done by } \\
\text { the specified node. }\end{array}$ \\
\hline & Production\#2 & Production.getHum & $\begin{array}{l}\text { It returns the value of the last } \\
\text { humidity measurement done by the } \\
\text { specified node. }\end{array}$ \\
\hline & Production\#3 & Production.getLight & $\begin{array}{l}\text { It returns the value of the last } \\
\text { measurement of the light intensity } \\
\text { done by the specified node. }\end{array}$ \\
\hline & Production\#4 & Production.getPresen & $\begin{array}{l}\text { It returns a boolean indicating if the } \\
\text { presence sensor is activated or not }\end{array}$ \\
\hline & Production\#5 & Production.setLED & $\begin{array}{l}\text { It changes the state of the light bulb } \\
\text { in the specified actuator node }\end{array}$ \\
\hline & Production\#6 & Production.setAlarm & $\begin{array}{l}\text { It changes the state of the buzzer in } \\
\text { the specified actuator node }\end{array}$ \\
\hline \multirow{4}{*}{ Business } & Business\#1 & Business.getFireInf & $\begin{array}{l}\text { It obtains the data related to the } \\
\text { possible presence of a fire from the } \\
\text { node or nodes placed in an specified } \\
\text { area }\end{array}$ \\
\hline & Business\#2 & Business.getPeopleInf & $\begin{array}{l}\text { It obtains the data related to the } \\
\text { people state from the node or nodes } \\
\text { in an specified area }\end{array}$ \\
\hline & Business\#3 & Business.alarm & $\begin{array}{l}\text { It activates an alarm indicating a } \\
\text { dangerous situation }\end{array}$ \\
\hline & Business\#4 & Business.systemReset & $\begin{array}{l}\text { It returns all the system to the initial } \\
\text { state (no alarm) }\end{array}$ \\
\hline \multirow{4}{*}{ Prosumer } & Prosumer\#1 & Prosumer.isFire & $\begin{array}{l}\text { It returns a boolean indicating if there } \\
\text { is a fire in the indicated area }\end{array}$ \\
\hline & Prosumer\#2 & Prosumer.getDangerLevel & $\begin{array}{l}\text { It returns an integer indicating the } \\
\text { danger level in the indicated area }\end{array}$ \\
\hline & Prosumer\#3 & Prosumer.activateEvac & $\begin{array}{l}\text { It activates the execution of the } \\
\text { evacuation plan }\end{array}$ \\
\hline & Prosumer\#4 & Prosumer.reset & $\begin{array}{l}\text { It returns the system to the initial } \\
\text { state (no evacuation) }\end{array}$ \\
\hline
\end{tabular}

In order to reduce the influence of external variables in the results, the system was manually configured. In particular, service descriptions in the service repositories were stablished manually and in a fixed way for all the experiment's duration. Table 5 describes the considered services for this validation. As can be seen, services are typical of an emergency alert system. Information about the execution costs and the guaranteed QoS was randomly configured.

Additionally, remote CPS and the cloud also offer some services at business level. Table 6 describes these services. 
Table 6: Available services in the remote locations for the experimental validation

\begin{tabular}{|l|l|l|l|}
\hline \multicolumn{1}{|c|}{ Location } & \multicolumn{1}{|c|}{ Service ID } & \multicolumn{1}{c|}{ Name } & \multicolumn{1}{c|}{ Description } \\
\hline Cloud & Cloud\#1 & Cloud.calDangerLevel & $\begin{array}{l}\text { It determines the danger level in } \\
\text { an area whose ambient parameters } \\
\text { are sent }\end{array}$ \\
\hline Cloud & Cloud\#2 & Could.calFire & $\begin{array}{l}\text { It determines if a fire is active in } \\
\text { an area whose ambient parameters } \\
\text { are sent }\end{array}$ \\
\hline CP Internet & Internet\#1 & Internet.setSprinkler & $\begin{array}{l}\text { It activates the sprinkler system of } \\
\text { the building }\end{array}$ \\
\hline CP Internet & Internet\#2 & Internet.closeDoor & $\begin{array}{l}\text { It activates the automatic door } \\
\text { closure system of the building }\end{array}$ \\
\hline CP Internet & Internet\#3 & Internet.setOutAlarm & $\begin{array}{l}\text { It activates the exterior horn } \\
\text { associated with the building }\end{array}$ \\
\hline
\end{tabular}

As we said in Section 3, prosumer services are compositions of business services; and business services are compositions of production services. Then, services in Table 5 and Table 6 are interrelated. These decompositions are also manually stablished in a fixed way. Thus, no process composition technology must be deployed or included in the experimental validation (which reduces the variables to be taken into account). Table 7 shows these decompositions.

Considering the previously described infrastructure, the following experiment was performed. Every component in the local execution system was programmed to create a log file. Besides, a script to randomly generate processes at prosumer level was created. Sixty (60) different processes were obtained. During the experiment each one of these processes was executed four times. In total two hundred and forty (240) registries were obtained.

At the end of the experiment, all log files were collected and analyzed. In order to validate the proposed solution a process is considered to be correctly executed if various conditions are verified:

1. The ad hoc network executes the desired actions indicated by users at prosumer level, and follows the desired parameters (QoS, maximum admissible error, etc.)

2. The algorithm based on studying the process at business level branch by branch finally obtains the lowest cost execution scheme.

3. Experimental errors do not cause the appearance of false results.

If any of the three cited conditions is not verified, the execution process is considered incorrect, although a valid result is obtained. 
Table 7: Service decompositions for the experimental validation

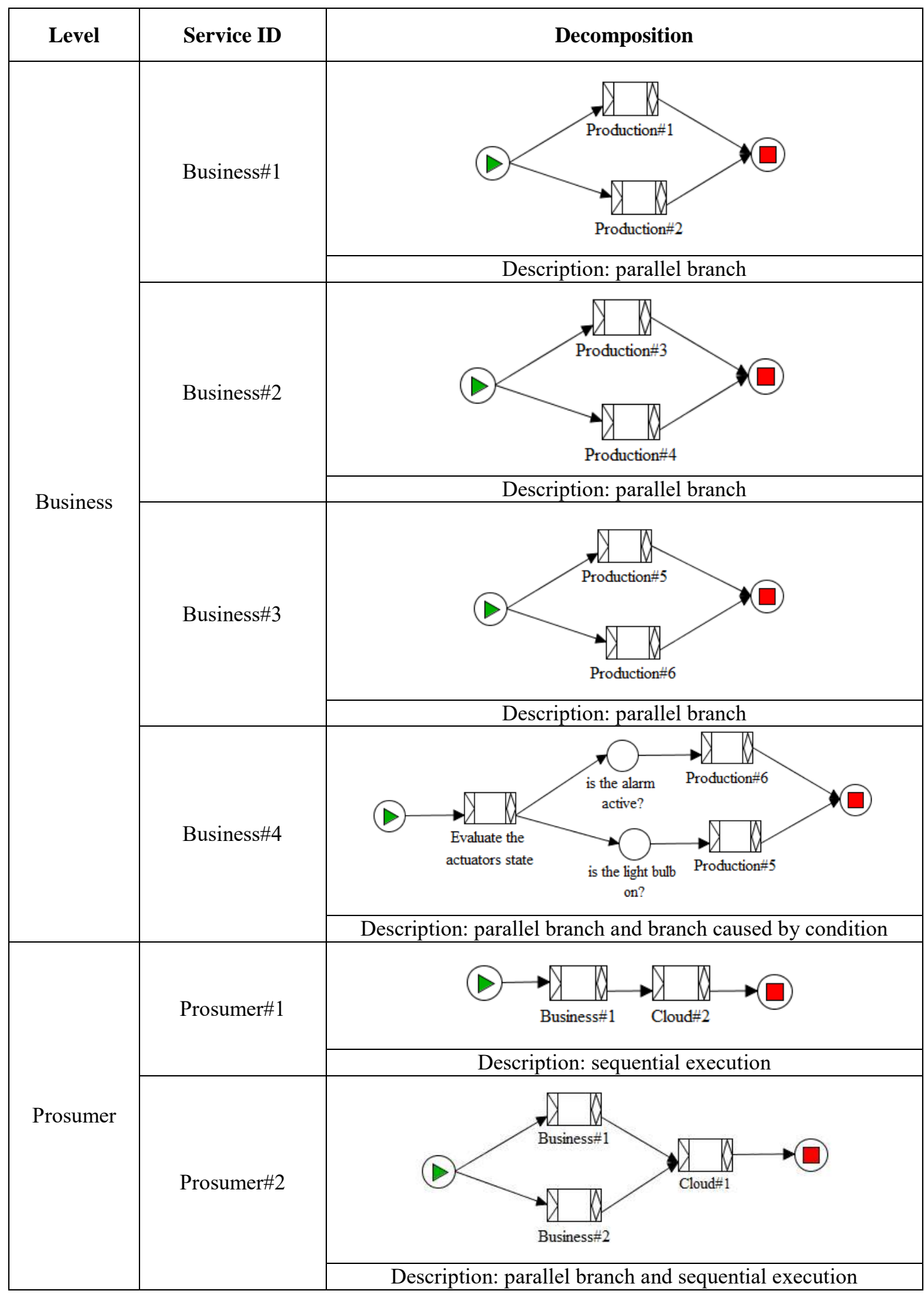




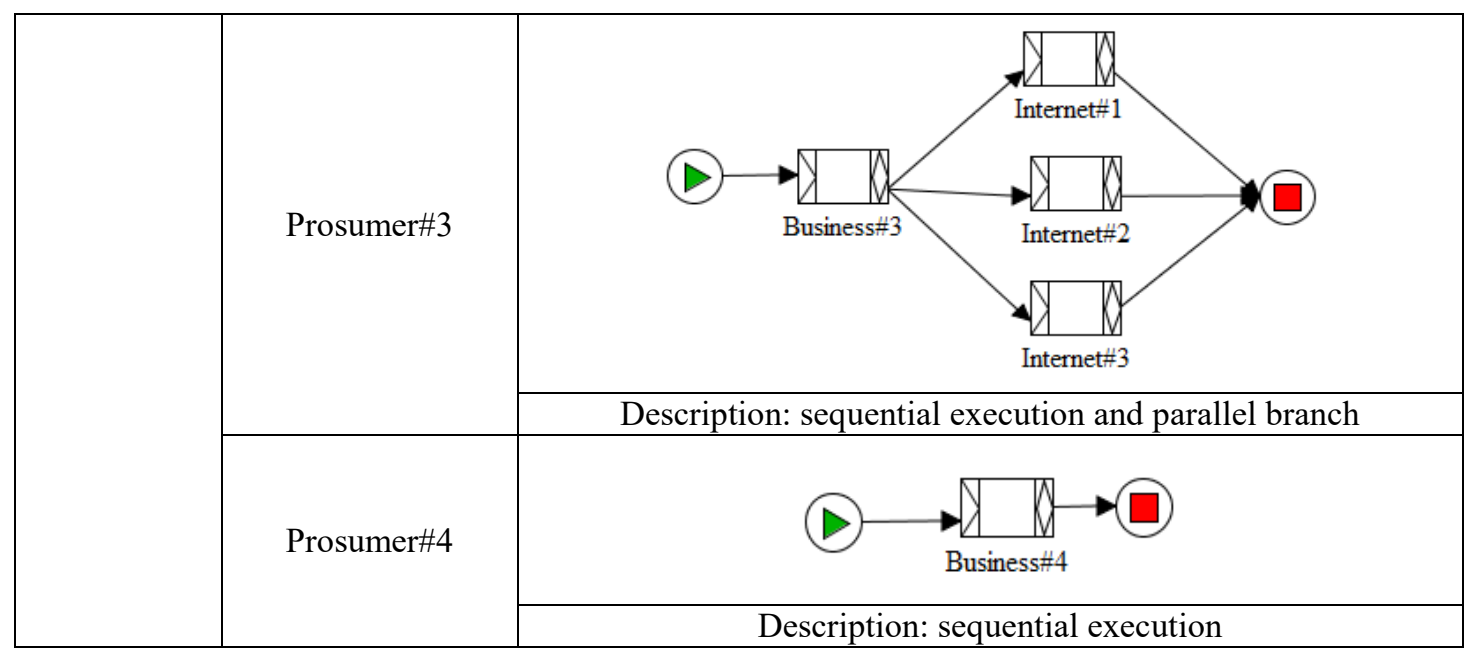

\section{RESULTS}

From the analysis of the log files generated by every component in the deployed CPS, obtained during the described experiment (see Section 4), various results can be extracted.

First Figure 23 shows the percentage of processes successfully executed. As can be seen $95.4 \%$ of the processes were successfully executed (the three proposed conditions were fulfilled). Additionally, around in a $4.6 \%$ of cases the system was not able to correctly execute the process designed by users. The causes of these fails are studied below.

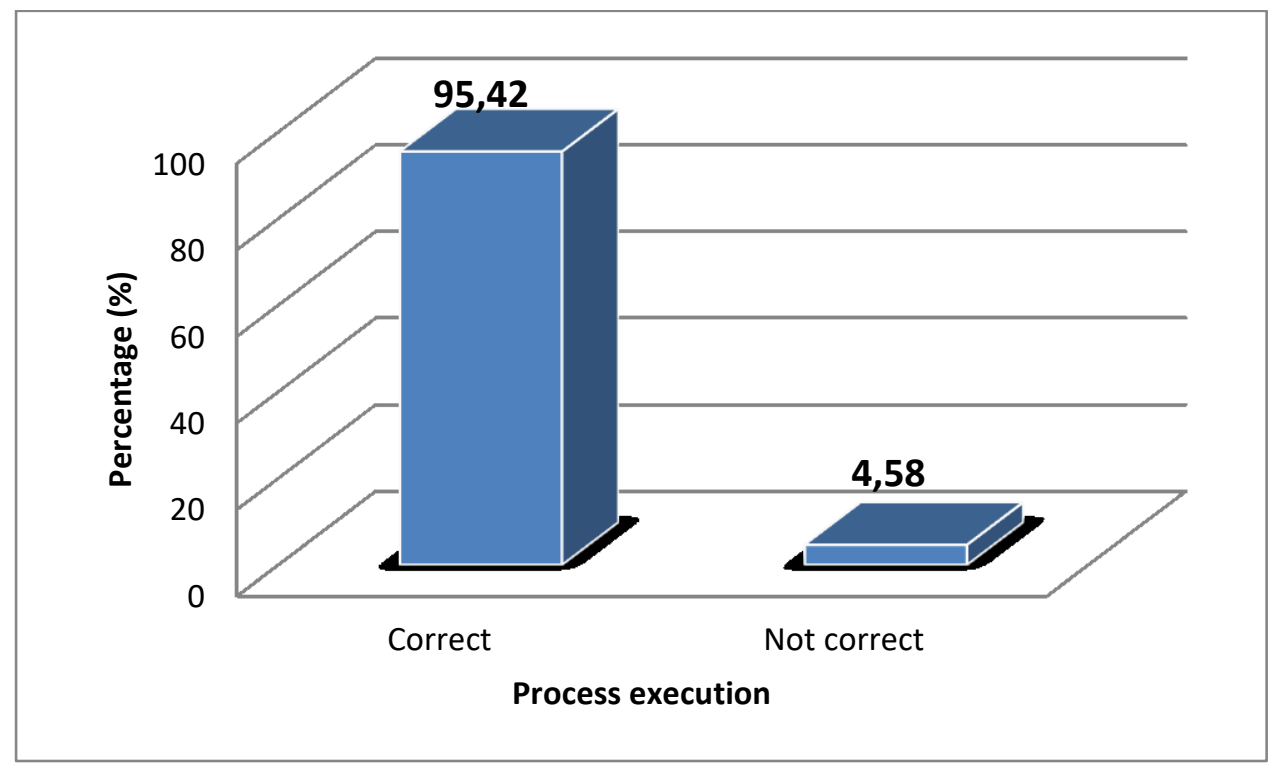

Figure 23: Global results of the experimental validation

Figure 24 shows the wrong process executions divided into three groups depending on the condition which did not get fulfilled. In some cases, more than one condition is broken, so the same execution can be counted various times. 


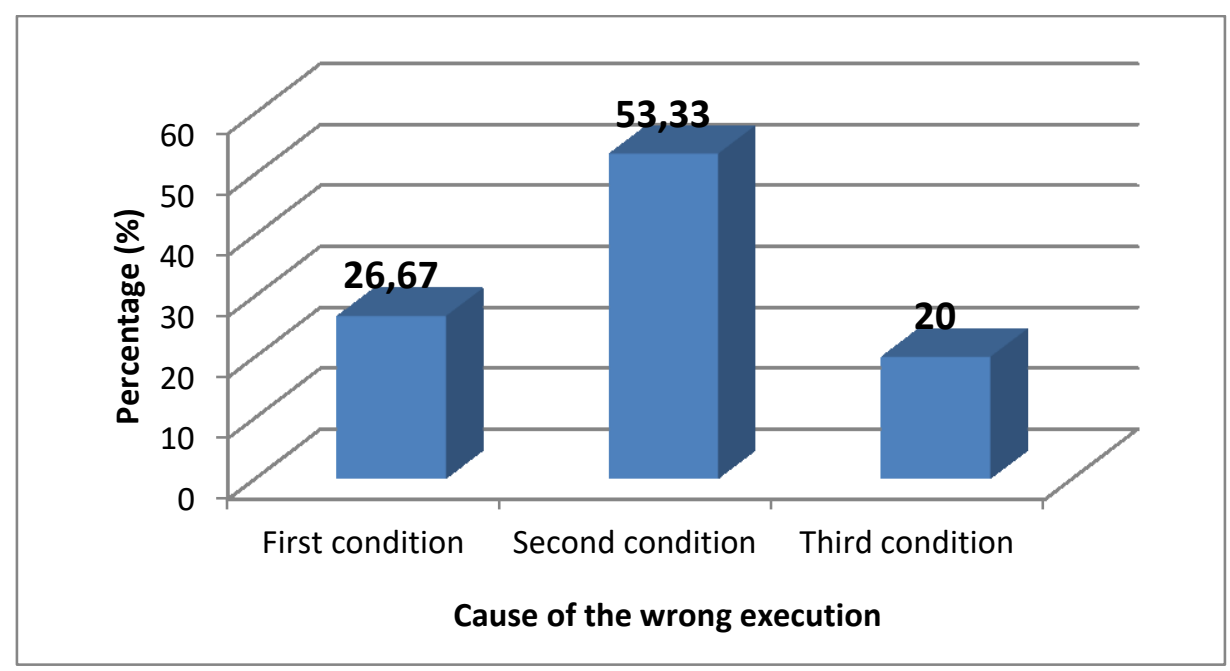

Figure 24: Causes of the wrong executions

As can be seen, more than $50 \%$ of wrong executions are due to inefficacies in the proposed study of the process description (branch-by-branch) at business level (algorithm 3). In fact, in synchronization points where only XOR-splits and XOR-joins are involved, synchronization activities are not necessary. As at these points only one output branch and one input branch are considered, no synchronization activity is required (i.e. it is not necessary to wait for other branches being executed in parallel or running various branches at the same time) and the synchronization points only exist virtually (in practice only two flows collide at the same point). Thus, these synchronization points are in fact second order points which introduce an orchestration cost which is not considered in the algorithm. In that way, the synergies of executing an entire branch in a unique location are ignored. If the branch which is going to get active was known previously, then the best execution scheme could be calculated. However, this selection is done dynamically, and the proposed algorithm cannot evaluate these situations. Nevertheless, in absolute terms, these processes represent only a $2.38 \%$ of cases (the $53 \%$ of the wrong execution -a $4.5 \%$ of the total cases-).

Moreover, in $20 \%$ of cases, wrong executions are due to problems at the probabilistic execution step. In the total amount of cases, wrong executions appear when data collection services were involved. These problems are generated by experimental errors which influence the system during a time longer than the time employed by the probabilistic execution engine in executing the corresponding task. Thus, despite doing a statistical study (including various realizations and statistical functions), the final results are not correct. Advanced techniques could be necessary in order to address this challenge.

Finally, in $26.7 \%$ of cases the actions finally executed by the underlying ad hoc network were not desired by users, or the parameters they indicated were not respected. Causes of these wrong executions are varied. In Figure 25 these causes are showed. 


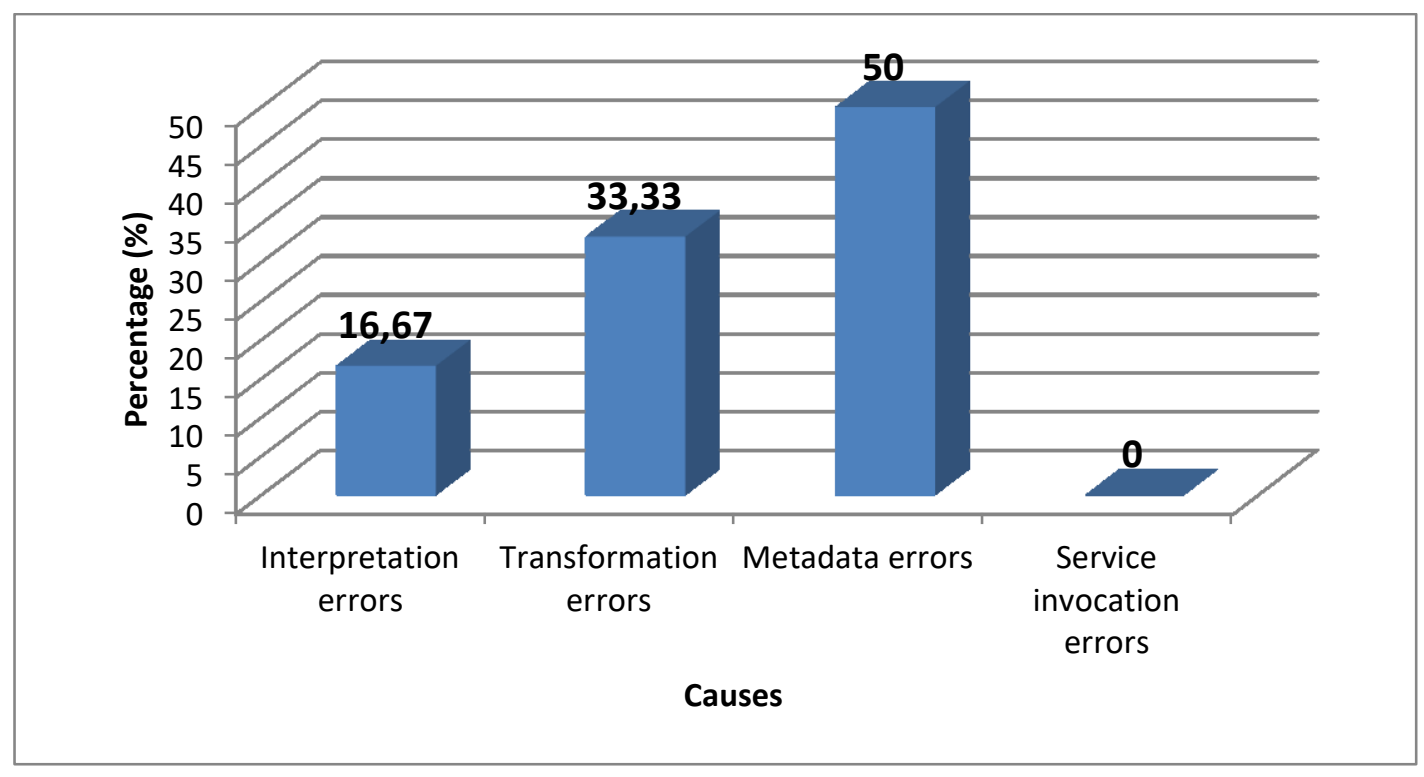

Figure 25: Causes of wrong executions (first condition)

In general, the final cause of the violation of the first condition for a successful execution is the proposed scheme based on multiple successive code and data transformations. Although, in absolute terms, this problem only appears in $1 \%$ of cases, if any little error in any of the transformation phases appears (in the metadata or in the process description), the successive transformation stages increase the error, so the final executed actions are not related to the service desired by users. As can be seen in Figure 25 this problem is especially critical in the case of metadata. Parameter transformation (describing the QoS, the statistical execution, etc.) is a complicated and delicate activity, and any minimal a variation in their value represents a great difference at runtime. Recursive feedback transformation algorithms could be necessary in order to avoid these problems.

\section{CONCLUSIONS AND FUTURE WORKS}

Cyber-Physical Systems have evolved from their origin as advanced embedded devices to a paradigm for the next generation of technological systems. In particular, systems to execute user-defined processes based on service-oriented CPS have emerged as a promising field. Many proposals related to this topic may be found, although none of them truly allow users to define processes in a high-level of abstraction which, later, may be executed in a traditional ad hoc network or pervasive computing infrastructure.

This work proposes a complete scheme (based on CPS paradigm) which covers this gap. The proposal unifies previous works (such as papers related to code transformation and service provision in CPS), and describes new solutions. Contributions include an algorithm for process execution orchestration, execution algorithms based on the concept of guaranteed QoS and probabilistic techniques to wean the random behavior of physical devices from the hard definition of computational processes. 
An experimental validation was performed in order to evaluate the proposal. Results show a $95 \%$ of user-defined processes (at prosumer level) are correctly executed. Additionally, orchestration algorithms only fail in a $2 \%$ of cases, due to the use of complex XOR-splits and XOR-joins. On the other hand, advanced statistical techniques should be implemented in order to avoid the effect of long-duration experimental errors. Moreover, corrective algorithms in the transformation stages should be also considered (especially when transforming metadata). The two last problems only affect the $1 \%$ of process executions.

Future works should address the described challenges. Besides, as the proposed experimental validation only considers a first basic implementation of CPS (in a scenario similar to a smart building), future works should also evaluate the performance of the proposal in applications such as traffic flow management (smart cities), electric power generation management and personalized health care devices. Finally, advanced studies about the performance of the proposed framework should also be carried out: scalability of fault resiliency are parameters to be investigated.

\section{REFERENCES}

[1] Abid, H., Phuong, L. T. T., Wang, J., Lee, S., \& Qaisar, S. (2011, October). V-Cloud: vehicular cyber-physical systems and cloud computing. In Proceedings of the 4th International Symposium on Applied Sciences in Biomedical and Communication Technologies (p. 165). ACM.

[2] Al Ridhawi, Y., \& Karmouch, A. (2015). Decentralized plan-free semantic-based service composition in mobile networks. IEEE Transactions on Services Computing, 8(1), 17-31.

[3] Appel, S., Frischbier, S., Freudenreich, T., \& Buchmann, A. (2013). Event stream processing units in business processes. In Business Process Management (pp. 187-202). Springer Berlin Heidelberg.

[4] Bakakeu, J., Schäfer, F., Bauer, J., Michl, M., \& Franke, J. (2017). Building Cyber-Physical Systems-A Smart Building Use Case. Smart Cities: Foundations, Principles, and Applications, 605-639.

[5] Boekhold, M., Karkowski, I., Corporaal, H., \& Cilio, A. (1999, March). A programmable ANSI C transformation engine. In International Conference on Compiler Construction (pp. 292-295). Springer Berlin Heidelberg.

[6] Bonzini, P., \& Pozzi, L. (2006, October). Code transformation strategies for extensible embedded processors. In Proceedings of the 2006 international conference on Compilers, architecture and synthesis for embedded systems (pp. 242-252). ACM.

[7] Bordel Sánchez, B., Alcarria, R., Martín, D., \& Robles, T. (2015). TF4SM: A Framework for Developing Traceability Solutions in Small Manufacturing Companies. Sensors, 15(11), 29478-29510.

[8] Bordel, B., Alcarria, R., Martín, D., Robles, T., \& de Rivera, D. S. (2016). Selfconfiguration in humanized Cyber-Physical Systems. Journal of Ambient Intelligence and Humanized Computing, 1-12

[9] Bordel, B., Alcarria, R., Robles, T., \& Martín, D. (2017). Cyber-physical systems: Extending pervasive sensing from control theory to the Internet of Things. Pervasive and Mobile Computing, 40, 156-184. 
[10] Childers, B., Davidson, J. W., \& Soffa, M. L. (2003, April). Continuous compilation: A new approach to aggressive and adaptive code transformation. In Parallel and Distributed Processing Symposium, 2003. Proceedings. International (pp. 10-pp). IEEE

[11] Clauß, S., \& Schulte, A. (2014, October). Task delegation in an agent supervisory control relationship capability awareness in a cognitive agent. In 2014 IEEE International Conference on Systems, Man, and Cybernetics (SMC) (pp. 825-830). IEEE.

[12] Conti, M., Das, S. K., Bisdikian, C., Kumar, M., Ni, L. M., Passarella, A., ... \& Zambonelli, F. (2012). Looking ahead in pervasive computing: Challenges and opportunities in the era of cyber-physical convergence. Pervasive and Mobile Computing, 8(1), 2-21.

[13] Czarnecki, K., \& Helsen, S. (2003, October). Classification of model transformation approaches. In Proceedings of the 2nd OOPSLA Workshop on Generative Techniques in the Context of the Model Driven Architecture (Vol. 45, No. 3, pp. 1-17).

[14] Delsing, J., Carlsson, O., Arrigucci, F., Bangemann, T., Hübner, C., Colombo, A. W., ... \& Kyusakov, R. (2014). Migration of SCADA/DCS Systems to the SOA Cloud. In Industrial Cloud-Based Cyber-Physical Systems (pp. 111-135). Springer International Publishing.

[15] Derler, P., Lee, E. A., Tripakis, S., \& Törngren, M. (2013, April). Cyber-physical system design contracts. In Proceedings of the ACM/IEEE 4th International Conference on CyberPhysical Systems (pp. 109-118). ACM.

[16] Griffiths, N. (2005, July). Task delegation using experience-based multi-dimensional trust. In Proceedings of the fourth international joint conference on Autonomous agents and multiagent systems (pp. 489-496). ACM.

[17] Hat, R. (2012). libvirt: The virtualization API. Available online: http://libvirt. org.

[18] Kane, M., Zhu, D., Hirose, M., Dong, X., Winter, B., Häckell, M., ... \& Swartz, A. (2014, April). Development of an extensible dual-core wireless sensing node for cyber-physical systems. In SPIE Smart Structures and Materials+ Nondestructive Evaluation and Health Monitoring (pp. 90611U-90611U). International Society for Optics and Photonics.

[19] Kim, J., Lakshmanan, K., \& Rajkumar, R. R. (2012, April). Rhythmic tasks: A new task model with continually varying periods for cyber-physical systems. In Proceedings of the 2012 IEEE/ACM Third International Conference on Cyber-Physical Systems (pp. 55-64). IEEE Computer Society.

[20] Kivity, A., Kamay, Y., Laor, D., Lublin, U., \& Liguori, A. (2007, July). kvm: the Linux virtual machine monitor. In Proceedings of the Linux symposium (Vol. 1, pp. 225-230).

[21] Koubaa, A., Andersson, B.: A vision of cyber-physical internet. In: Proceedings of the Workshop of Real-Time Networks (RTN 2009), Satellite Workshop to (ECRTS 2009)

[22] Kyusakov, R., Eliasson, J., Delsing, J., van Deventer, J., \& Gustafsson, J. (2013). Integration of wireless sensor and actuator nodes with IT infrastructure using serviceoriented architecture. IEEE Transactions on industrial informatics, 9(1), 43-51.

[23] Lee, E. A. (2006, October). Cyber-physical systems-are computing foundations adequate. In Position Paper for NSF Workshop On Cyber-Physical Systems: Research Motivation, Techniques and Roadmap (Vol. 2).

[24] Lin, K. J., \& Panahi, M. (2010, July). A real-time service-oriented framework to support sustainable cyber-physical systems. In 2010 8th IEEE International Conference on Industrial Informatics (pp. 15-21). IEEE.

[25]Liu, L., Zhao, S., Yu, Z., \& Dai, H. (2015). A big data inspired chaotic solution for fuzzy feedback linearization model in cyber-physical systems. Ad Hoc Networks, 35, 97-104.

[26]Maier, S., Herrscher, D., \& Rothermel, K. (2007). Experiences with node virtualization for scalable network emulation. Computer Communications, 30(5), 943-956. 
[27] Marrella, A., \& Mecella, M. (2017). Adaptive Process Management in Cyber-Physical Domains. In Advances in Intelligent Process-Aware Information Systems (pp. 15-48). Springer International Publishing.

[28] Moldovan, D., Copil, G., \& Dustdar, S. (2017). Elastic systems: Towards cyber-physical ecosystems of people, processes, and things. Computer Standards \& Interfaces.

[29] Morales, A., Alcarria, R., Martin, D., \& Robles, T. (2014). Enhancing evacuation plans with a situation awareness system based on end-user knowledge provision. Sensors, 14(6), 11153-11178.

[30]Muccini, H., Sharaf, M., \& Weyns, D. (2016, May). Self-adaptation for cyber-physical systems: a systematic literature review. In Proceedings of the 11th International Workshop on Software Engineering for Adaptive and Self-Managing Systems (pp. 75-81). ACM.

[31]Mueller, M. (2015). Reducing Hazards in Multiagent Task Delegation. In Wirtschaftsinformatik (pp. 1679-1693).

[32] National institute of Standards and Technology (NIST). (June 2014). DRAFT Framework for Cyber-Physical Systems https://s3.amazonaws.com/nistsgcps/cpspwg/pwgglobal/CPS_PWG_Draft_Framework_for_CyberPhysical_Systems_Release_0_8_September_2015.pdf (accessed on 19 August, 2016)

[33] Qiao, L., Kao, S., \& Zhang, Y. (2011). Manufacturing process modelling using process specification language. The International Journal of Advanced Manufacturing Technology, 55(5-8), 549-563.

[34] Rajkumar, R. R., Lee, I., Sha, L., \& Stankovic, J. (2010, June). Cyber-physical systems: the next computing revolution. In Proceedings of the 47th Design Automation Conference (pp. 731-736). ACM.

[35] Reuillon, R., Chuffart, F., Leclaire, M., Faure, T., Dumoulin, N., \& Hill, D. (2010, June). Declarative task delegation in OpenMOLE. In High performance computing and simulation (hpcs), 2010 international conference on (pp. 55-62). IEEE.

[36] Richardson, L., \& Ruby, S. (2008). RESTful web services. " O'Reilly Media, Inc.".

[37] Robles, T. S., Alcarria, R., Morales, A., \& Martín, D. (2015). Supporting variability dependencies for rule-based service compositions in prosumer environments. International Journal of Web and Grid Services, 11(1), 57-77.

[38] Seiger, R., Huber, S., \& Schlegel, T. (2016). Toward an execution system for self-healing workflows in cyber-physical systems. Software \& Systems Modeling, 1-22.

[39] Sikora, A. (2005). IEEE802. 15.4 and ZigBe for Sensor and Actuator Networks. In Embedded World Conference (pp. 189-197).

[40] Simpson, B., \& Toussi, F. (2004). Hsqldb User Guide.

[41]Sungur, C. T., Spiess, P., Oertel, N., \& Kopp, O. (2013, July). Extending bpmn for wireless sensor networks. In 2013 IEEE 15th Conference on Business Informatics (pp. 109-116). IEEE.

[42] Sztipanovits, J., Koutsoukos, X., Karsai, G., Kottenstette, N., Antsaklis, P., Gupta, V., ... \& Wang, S. (2012). Toward a science of cyber-physical system integration. Proceedings of the IEEE, 100(1), 29-44.

[43] Tamarit, S., Marino, J., Vigueras, G., \& Carro, M. (2016, March). Towards a SemanticsAware Code Transformation Toolchain for Heterogeneous Systems. In First Workshop on Program Transformation for Programmability in Heterogeneous Architectures (PROHA'16).

[44] Tan, Y., Goddard, S., \& Perez, L. C. (2008). A prototype architecture for cyber-physical systems. ACM Sigbed Review, 5(1), 26. 
[45] Tranquillini, S., Spieß, P., Daniel, F., Karnouskos, S., Casati, F., Oertel, N., ... \& Voigt, T. (2012, September). Process-based design and integration of wireless sensor network applications. In International Conference on Business Process Management (pp. 134-149). Springer Berlin Heidelberg.

[46] Van Der Aalst, W. M., Aldred, L., Dumas, M., \& ter Hofstede, A. H. (2004, June). Design and implementation of the YAWL system. In International Conference on Advanced Information Systems Engineering (pp. 142-159). Springer Berlin Heidelberg.

[47] Van Der Aalst, W. M., \& Ter Hofstede, A. H. (2005). YAWL: yet another workflow language. Information systems, 30(4), 245-275.

[48] Wan, J., Chen, M., Xia, F., Di, L., \& Zhou, K. (2013). From machine-to-machine communications towards cyber-physical systems. Computer Science and Information Systems, 10(3), 1105-1128

[49] Wan, J., Zhang, D., Zhao, S., Yang, L. T., \& Lloret, J. (2014). Context-aware vehicular cyber-physical systems with cloud support: architecture, challenges, and solutions. IEEE Communications Magazine, 52(8), 106-113.

[50] Wu, J., \& Stojmenovic, I. (2004). Ad hoc networks. COMPUTER-IEEE COMPUTER SOCIETY-, 37(2), 29-31.

[51] Wu, F. J., Kao, Y. F., \& Tseng, Y. C. (2011). From wireless sensor networks towards cyber physical systems. Pervasive and Mobile Computing, 7(4), 397-413.

[52] Xia, F., Ma, L., Dong, J., \& Sun, Y. (2008, July). Network QoS management in cyberphysical systems. In Embedded Software and Systems Symposia, 2008. ICESS Symposia'08. International Conference on (pp. 302-307). IEEE.

[53] Yue, X., Cai, H., Yan, H., Zou, C., \& Zhou, K. (2015). Cloud-assisted industrial cyberphysical systems: an insight. Microprocessors and Microsystems, 39(8), 1262-1270.

[54] Zeng, Y., Li, D., \& Vasilakos, A. V. (2013). Real-time data report and task execution in wireless sensor and actuator networks using self-aware mobile actuators. Computer Communications, 36(9), 988-997.

[55] Zhang, J., Lin, Y., \& Gray, J. (2005). Generic and domain-specific model refactoring using a model transformation engine. In Model-driven Software Development (pp. 199-217). Springer Berlin Heidelberg.

[56] Zhang, F., Szwaykowska, K., Wolf, W., \& Mooney, V. (2008, November). Task scheduling for control oriented requirements for cyber-physical systems. In Real-Time Systems Symposium, 2008 (pp. 47-56). IEEE.

[57] Zhang, W., Wen, Y., \& Wu, D. O. (2015). Collaborative task execution in mobile cloud computing under a stochastic wireless channel. IEEE Transactions on Wireless Communications, 14(1), 81-93.

[58] Choi, I., Song, M., Park, C., \& Park, N. (2003). An XML-based process definition language for integrated process management. Computers in Industry, 50(1), 85-102.

[59] Duarte, F. J., Machado, R. J., \& Fernandes, J. M. (2012, January). BIM: a methodology to transform business processes into software systems. In International Conference on Software Quality (pp. 39-58). Springer, Berlin, Heidelberg.

[60] Santos, N., Duarte, F. J., Machado, R. J., \& Fernandes, J. M. (2013, January). A transformation of business process models into software-executable models using mda. In International Conference on Software Quality (pp. 147-167). Springer, Berlin, Heidelberg.

[61] Tao, F., Cheng, Y., Zhang, L., \& Nee, A. Y. (2017). Advanced manufacturing systems: socialization characteristics and trends. Journal of Intelligent Manufacturing, 28(5), 10791094. 
[62] Alcarria, R., Bordel, B., Manso, M. Á., Iturrioz, T., \& Pérez, M. (2018, January). Analyzing UAV-Based Remote Sensing and WSN Support for Data Fusion. In International Conference on Information Theoretic Security (pp. 756-766). Springer, Cham.

[63] Perera, C., Zaslavsky, A., Christen, P., \& Georgakopoulos, D. (2014). Sensing as a service model for smart cities supported by internet of things. Transactions on Emerging Telecommunications Technologies, 25(1), 81-93.

[64] Sefraoui, O., Aissaoui, M., \& Eleuldj, M. (2012). OpenStack: toward an open-source solution for cloud computing. International Journal of Computer Applications, 55(3).

[65] Cirani, S., Picone, M., Gonizzi, P., Veltri, L., \& Ferrari, G. (2015). Iot-oas: An oauth-based authorization service architecture for secure services in iot scenarios. IEEE sensors journal, 15(2), 1224-1234.

[66] Kukla, T., Kiss, T., Terstyanszky, G., \& Kacsuk, P. (2008, November). A general and scalable solution for heterogeneous workflow invocation and nesting. In Workflows in Support of Large-Scale Science, 2008. WORKS 2008. Third Workshop on (pp. 1-8). IEEE.

[67] Schunselaar, D. M., Verbeek, H. M. W., Reijers, H. A., \& van der Aalst, W. M. (2014, September). YAWL in the cloud: supporting process sharing and variability. In International Conference on Business Process Management (pp. 367-379). Springer, Cham. 
Article

\title{
TF4SM: A Framework for Developing Traceability Solutions in Small Manufacturing Companies
}

\author{
Borja Bordel Sánchez ${ }^{1, *}$, Ramón Alcarria ${ }^{2}$, Diego Martín ${ }^{1}$ and Tomás Robles ${ }^{1}$ \\ 1 Departamento de Ingeniería de Sistemas Telemáticos, Universidad Politécnica de Madrid, \\ Avenida Complutense n³0, Madrid 28040, Spain; E-Mails: diego.martin.de.andres@ upm.es (D.M.); \\ tomas.robles@upm.es (T.R.) \\ 2 Departamento de Ingeniería Topográfica y Cartografía, Universidad Politécnica de Madrid, \\ UPM Campus Sur, Km 7.5 de la Autovía de Valencia, Madrid 28031, Spain; \\ E-Mail: ramon.alcarria@upm.es \\ * Author to whom correspondence should be addressed; E-Mail: bbordel@ dit.upm.es; \\ Tel.:+34-91-549-57-00 (ext. 3035).
}

Academic Editor: Albert M. K. Cheng

Received: 20 July 2015 / Accepted: 18 November 2015 / Published: 20 November 2015

\begin{abstract}
Nowadays, manufacturing processes have become highly complex. Besides, more and more, governmental institutions require companies to implement systems to trace a product's life (especially for foods, clinical materials or similar items). In this paper, we propose a new framework, based on cyber-physical systems, for developing traceability systems in small manufacturing companies (which because of their size cannot implement other commercial products). We propose a general theoretical framework, study the requirements of these companies in relation to traceability systems, propose a reference architecture based on both previous elements and build the first minimum functional prototype, to compare our solution to a traditional tag-based traceability system. Results show that our system reduces the number of inefficiencies and reaction time.
\end{abstract}

Keywords: traceability; real-time manufacturing; manufacturing execution system; cybernetic devices; cybernetic glove; cybernetic table; prosumer; RFID; Bluetooth 


\section{Introduction}

In recent years, companies, government institutions, researchers and traders, among others, have stressed the importance of manufacturing process monitoring systems as a primary source of information. Projects such as [1] or works such as [2,3] demonstrate the importance of the ability of systems to extract valuable data from these processes. However, as the complexity of manufacturing processes increases, monitoring (especially if involving human operators) becomes very costly and inefficient [4]. From the beginning, solutions based on information technologies were adopted to reduce the complexity associated with traceability by operators. Relevant works, such as [5-7], have developed successfully traceability solutions categorized as "Smart Environments" or "Wireless Sensor Networks". Moreover, approximately between 2000 and 2010, a number of important manufacturing companies (specially leading vehicle manufacturers) started to employ radio frequency identification (RFID) to facilitate traceability in manufacturing processes. First, Volvo Trucks established a RFID system to achieve continuous production [8,9]. Later, Toyota implemented information systems based on RFID to track auto parts production [10,11]. Most recently, in 2012, the Chinese manufacturer Guangdong Greatoo Molds Inc. deployed a RFID-enabled manufacturing execution system as a case study [12].

However, the previous examples relate to large-sized companies, while most manufacturers are actually small and medium-sized companies, which must face financial and technological challenges when they attempt to implement RFID-based traceability systems. Currently, most of the RFID systems are provided by important information technology companies, such as IBM or Ubisense. The kind of solutions developed by these Information Technologies (IT) providers makes the cost and technical requirements of adopting their RFID solutions too high for small and medium-sized manufacturing companies [13,14]. When a small manufacturing company considers applying an automated traceability system (particularly a RFID-based system) three basic questions must be confronted [12]. The first is how to deploy RFID devices to collect real-time data in a cost-effective way. The second is how to translate the real-time data into meaningful information to enable the field operator's convenient operations (e.g., inefficiency detection and correction). The third is how to integrate the RFID system into the company's current production processes.

We argue herein that a framework strongly based on Cyber-Physical Systems (CPS) can successfully address all the previous questions. As described in the relevant NSF reports [15] CPS are integrations of computation and physical processes. Embedded computers and networks monitor and control the physical processes, usually with feedback loops where physical processes affect computations and vice versa. However, this initial definition has been modified and expanded by many authors. If in the first instance CPS referred to a kind of networked embedded systems running real-time applications, nowadays CPS (as described by the National Institute of Standards and Technology [16]) have a more vertical approach, including from the user goals to the underlying physical system. In addition, in this second focus, issues related to traceability systems, such as the Human-System Interaction, are a main part of the architecture [17].

Notwithstanding this great interest, the heterogeneity in CPS has revealed several problems in industrial applications that cannot be easily solved through current control, communications, and software theory [18]. Consequently, some industrial researchers have recently returned to the simplicity and uniformity of Wireless Sensor Networks (WSNs) [19] to integrate them with traditional intelligent 
industrial systems. Thus, new definitions, such as Industrial Wireless Sensor Networks [20] have appeared. Nonetheless, the knowledge created around Cyber-Physical Systems could provide many advantages over existing industrial applications [18].

In this paper we propose a framework based on CPS to reduce the complexity associated with its application in manufacturing scenarios, including other aspects originally derived from traditional intelligent industrial solutions. The objective of this paper is to describe this framework, and to validate its usability as a traceability solution for reducing the number of inefficiencies and to improve their time response in companies. Thus, the contribution of this paper is the proposal of a CPS-based traceability framework, merging both CPS technologies and traceability capabilities. Our work also includes a validation of how a proof of concept of this framework can reduce inefficiencies and improve time response for industrial processes in companies.

In order to validate the Traceability Framework for Small Manufacturers (TF4SM) as a useful framework to develop traceability systems and applications, the authors conducted an experimental validation to address the following two research questions:

1. Would the time response to inefficiencies improve by deploying a system based on our TF4SM in companies?

2. Is it possible to reduce the number of inefficiencies in productive processes using a TF4SM-based system?

The experimental validation consisted in two typical kinds of manufacturing processes: logistical shortages detection process in warehouses and productive activities that lead to a quality system checkpoint. A statistical analysis of the results showed that a system based on our TF4SM provides a significant improvement in time response to inefficiencies in companies and a remarkable reduction in the number of inefficiencies due to procedural errors.

The rest of the paper is organized as follows: Section 2 introduces the state of the art in traceability systems and automated industrial processes, as well as different theories about networked embedded systems and related commercial products. Section 3 analyzes the requirements of small manufacturers, presents the reference architecture of our new framework and the design of the first minimum functional prototype based on it. Section 4 describes our proposal hardware implementation and our proposed process model. Section 5 provides an experimental validation of this approach. Finally, Sections 6 and 7 explain some results of this experimental validation and the conclusions of our work.

\section{State of the Art in Traceability Systems, Different Theories of Networked Embedded Systems, and RFID-Based Products}

During the last fifteen years, many concepts referring to networked embedded systems have appeared. As mentioned in the Introduction, some of them have already been used as a framework for building traceability solutions. However, we argue that the CPS paradigm fits better with traceability solutions than any other previous definition.

In this Section we review the state of the art in traceability solutions and automated industrial processes. Later, we analyze different definitions of networked embedded systems to find the one which best fits with traceability systems. Finally, a review of RFID integration in daily living objects is provided. 


\subsection{Automated Industrial Processes and Traceability Systems: State of the Art}

The application of software engineering to industrial automation spans from manufacturing automation to process control systems and energy automation systems. There is ample evidence of the extensive use of automated systems in industrial environments. For example, the German Engineering Federation has calculated [21] the ratio of control software to the cost of machinery where it is used has doubled in value from $20 \%$ to $40 \%$ in one decade.

The Automation Research Corporation (ARC) advisory group [22] and the International Electrotechnical Commission (IEC) [23] distinguish various kinds of automation products for industrial processes, among which we find the high and low power AC drivers, human machine interfaces (HMIs) and programmable logic controllers (PLC). In most solutions, several of these products must be integrated creating libraries for the interconnection which, in most cases, are proprietary technologies [24]. Thus, typically, there is not a homogeneous solution that could be standardized (except for certain cases, such as Object Linking and Embedding - OLE—-for Process Control, usually called OPC [25]). Therefore, our approach should be a complete integrated solution, which would include all the necessary functions for automated industrial processes. This approach would allow a reduction in the system's complexity, control the investment needed, remove technical positions and, ultimately, reduce the cost of deploying and operating systems [26]. In that way, we could address the first basic question presented in the Introduction ("how to deploy RFID devices to collect real-time data in a cost-effective way?").

Later, nowadays, most components automated industrial process solutions virtualized, however, the structure is similar to that of the traditional hardware architecture [27]. In these systems, there are four types of devices communicating via networks: an engineering and simulation station (including a database and a programming software), a human-machine interface (HMI), at least one programmable logic controller (PLC) with control software and, at least, one driver controlling machines. It would be a good practice to adapt the TF4SM-based system to this generic structure (already tested in industry).

On the other hand, traditional automated industrial systems require workers to train in both software engineering and industrial processes (as PLC must be programmed in proprietary low-level languages [28,29]). In order to improve the cost efficiency (so important in small companies) a prosumer-oriented [30] framework would be desirable. Thus, only industrial domain experts would be necessary.

Furthermore, the embedded devices usually considered in automated industrial processes (including traceability solutions), implement a fixed network architecture model, making it impossible to monitor workers, replace the devices easily or rearrange the machine distribution [31,32]. This increases the cost of system updates and tracking of manual processes. Then, adaptable policies should be included in traceability systems for small companies, so workers can be monitored without affecting their movement and machines could be replaced, rearranged or removed without modifying the system configuration.

Specifying now the debate on existing traceability systems for small-sized companies, five different technological states coexist in small companies nowadays, namely:

1. There are some manufacturers that do not apply any traceability system, such as small handicraft companies where production processes do not generate intermediate products [33].

2. Some companies implement systems based on invoices and control record sheets (commonly known as "system based on pen and paper" [34]). In these systems, each product carries a label 
with a serial number and a description. An operator is responsible for, at each stage of the production process, writing both data items in the appropriate form. Although these systems work fine, the use of IT enables a larger amount of data to be handled, and thereby it becomes realistic to develop traceability systems with very detailed information about both the product and its processing history $[35,36]$.

3. We find companies which implement systems based on Personal Digital Assistants (PDAs) [37]. In these systems, each product still carries a label with a serial number and a description; however, in this case the responsible operator, instead of using control record sheets, uses a PDA to transmit data in real-time. Although these systems reduce the time requirements related to traceability processes, they still are extremely sensitive to human errors.

4. Around ten years ago, in order to reduce human errors in traceability systems, labels with the serial number and the description of each product were replaced by codes (e.g., QR or barcodes) storing both data [38]. Then, equipping PDAs with the appropriate reader is enough to avoid operators having to manually input any data.

5. More recently, the old stickers with printed codes have been replaced by RFID labels [39]. This is basically due to two factors [40]: RFID tags can store kilobytes of information, and they also can include security and privacy policies (what it is especially important in confidential products). These systems have been widely applied in the manufacturing sector.

Our framework intends to improve current traceability systems in such a way that new systems would not require performing traceability-specific tasks; instead, traceability information can be deduced from the usual activities of operators.

\subsection{Networked Embedded Systems and Traceability Systems}

As stated in the previous section, we need a complete integrated solution to collect data in real-time in the most cost-effective way. Moreover, the framework must include adaptation capabilities and allow the development of system architectures similar to the generic structure of automated industrial processes. Finally, if product life cycle has to be deduced from the usual activities of operators, RFID readers (we selected RFID as the base technology for its successful results in previous deployments) must be seamlessly integrated into daily objects.

Given these premises, it is necessary to first select a basic theory that will help us develop our framework. It is clear that this basis must be related to networked embedded systems. The first efforts for integrating information technologies in the physical world and supporting different applications (including traceability systems), occurred in the electronics world. Since 2000, several terms have appeared in the literature and have been proposed in conferences to describe smart distributed electronic systems (more or less embedded in the physical world): Smart Home [41,42], Smart Office [43], Intelligent Home [44], and Smart Environments [5,45,46], among others.

In parallel, some of the existing definitions delimited what kind of infrastructure could be considered one of these new systems. In 2000, [7] stated that: "a smart space is a region of the real world that is extensively equipped with sensors, actuators and computing components". In 2002, some authors decided to propose their own definitions. [47] defines a Smart Environment as: "a system that is able to autonomously acquire and apply knowledge about the environment and adapt to its inhabitants' 
preferences and requirements in order to improve their experience". In 2005, the same authors proposed in [5] a new definition: "a smart environment is a physical world that is interconnected through a continuous network abundantly and invisibly with sensors, actuators and computational units, embedded seamlessly in the everyday objects of our lives".

Around 2010 interest in these systems waned, and researchers abandoned attempts to formalize the theoretical framework. At that time, a new concept had captured everyone's attention: cyber-physical systems (CPS) [48]. The term "cyber-physical systems" emerged around 2006, when it was coined by Gill at the National Science Foundation in the United States. Later, Lee developed the term in a relevant NSF report [15]. However, in this work CPS are described as networked entities for real-time processing. Thus, research challenges are related to computational problems such as the creation of real-time applications or operating systems [49]. Meanwhile the interest in CPS has changed to more vertical issues such as networking, communications, data services, decision making and pattern recognition.

Extremely important is the reference architecture (see Figure 1) proposed in June 2015 by the National Institute of Standards and Technology (NIST). The CPS public working group proposes that: "cyber-physical Systems or "smart" systems are co-engineered interacting networks of physical and computational components". Besides being more general than the original, this new definition and reference architecture present the advantage of being the standard on which commercial products of CPS will be based.

Table 1 compares the most important theories about networked embedded devices in relation to the classical features of RFID-based traceability systems (as described in [49]). Besides, the requirements deducted in the previous sections related to the three basic questions about traceability systems in small manufacturers (see the Introduction) are also included. As can be seen, CPS (as defined by the NIST) are aligned to the requirements of traceability systems, so we selected its reference architecture as the basis for our framework.

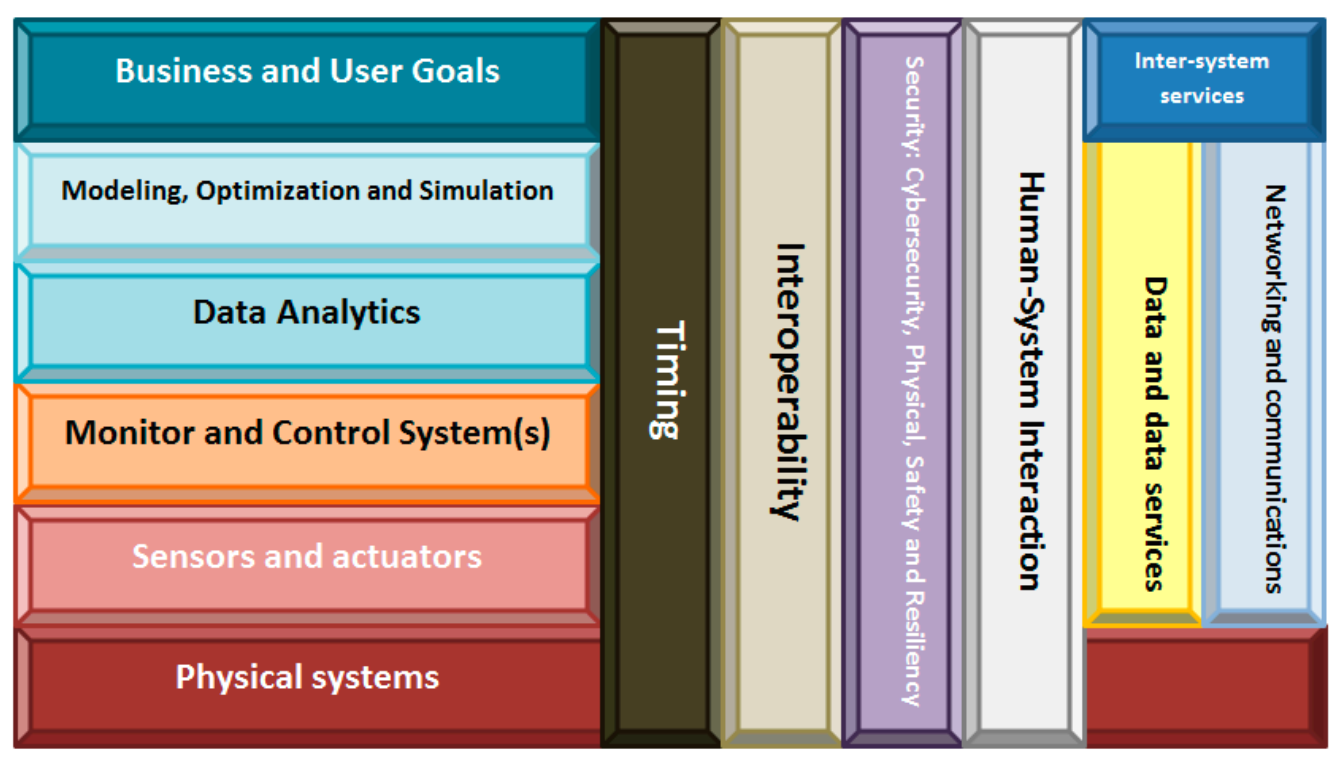

Figure 1. Reference CPS architecture (NIST). 
Table 1. Comparison among different theories about networked embedded systems in relation to traceability systems.

\begin{tabular}{|c|c|c|c|c|c|}
\hline Feature and Requirements & $\begin{array}{c}\text { Smart Space } \\
\text { Along [7] } \\
\end{array}$ & $\begin{array}{c}\text { Smart Environments } \\
\text { Along [1] }\end{array}$ & $\begin{array}{c}\text { Smart Environments } \\
\text { Along [5] }\end{array}$ & $\begin{array}{c}\text { CPS } \\
\text { From [15] } \\
\end{array}$ & $\begin{array}{c}\text { CPS From } \\
\text { NIST }\end{array}$ \\
\hline Object identification capability & $\checkmark / x$ & $x$ & $x$ & $\checkmark$ & $\checkmark / x$ \\
\hline Real-time data capture & $\checkmark$ & $\checkmark$ & $\checkmark$ & $\checkmark$ & $\checkmark$ \\
\hline Execution engine & $x$ & $x$ & $x$ & $x$ & $\checkmark / x$ \\
\hline Decision capability & $x$ & $x$ & $x$ & $\checkmark / x$ & $\checkmark$ \\
\hline Learning capability & $x$ & $\checkmark$ & $x$ & $\checkmark / x$ & $\checkmark$ \\
\hline Prosumer focused & $x$ & $x$ & $x$ & $x$ & $\checkmark / x$ \\
\hline Dynamical adaptation & $\checkmark / x$ & $\checkmark / x$ & $\checkmark / x$ & $\checkmark / x$ & $\checkmark$ \\
\hline Vertical approach & $x$ & $\checkmark$ & $x$ & $x$ & $\checkmark$ \\
\hline $\begin{array}{c}\text { Can offer meaningful } \\
\text { human-understandable information }\end{array}$ & $x$ & $\checkmark$ & $\checkmark / x$ & $\checkmark / x$ & $\checkmark$ \\
\hline
\end{tabular}

$\checkmark$ : Included in the definition; $\mathbf{x}$ : Not considered in the definition; $\checkmark / \mathbf{x}$ : Available in some works.

In the last three years, various works relating to CPS and process monitoring and traceability systems have appeared. In [50], the future demands on automation systems (based on CPS) are shown, and new strategies for software deployment of automation applications and communication systems are discussed; more specifically, the system presented in [51] describes an industrial cloud-based CPS.

Some works, such as [52], focused on designing communication models for monitoring systems and processes using CPS while others provide simulation environments for CPS-based industrial systems ([53] describes a CPS research project to support virtual design and verification of industrial processes).

However, as we mentioned in the Introduction and can be seen in [18], the actual deployment of CPS in manufacturing environments has several drawbacks that are not easily resolved. We argue the new reference architecture proposed by the NIST will allow us to develop a framework being able to address these issues.

\subsection{RFID-Based Products}

Compared to other research on automated industrial processes and traceability systems (usually focused on simulation environments [54], software compatibility [55,56], wireless sensor networks [20], or safety in automation systems [57]), our framework will not only consider high-capacity generic computing platforms, but also both software and hardware components. Thus, all knowledge of low-energy computing platforms, seamless integration of electronic systems and real-time communications (originating from the world of CPS) can be applied to the manufacturing environment.

In this section, we focus on hardware components and review the state of the art in relation to the integration of RFID tags and readers in different everyday objects. In that way the third basic question about traceability systems in small companies (how to integrate RFID systems into the company's current production processes?) is answered: by means of embedded tags and readers in daily objects.

Due to the business interest in control and traceability systems, over the years, some products, articles, conferences and so on have been about issues related to our aim (directly or indirectly). 
On the one hand, as we have mentioned, since 2000, various research proposals for integrating electronic systems at home or in the workplace emerged: [41] present the concept of "Smart Home", in [43] a "Smart Office" is designed and an "Intelligent Home" is planned in [44].

Later, between 2003 and 2004, the concept of "smart furniture" became very popular in research. Various research papers on this subject, such as the general framework presented in [58,59], appeared. Furthermore, some patents, such as [60], in which a RFID smart chair is described, were also given.

Finally, related to wearable technologies, several papers describe their use in traceability systems, especially cybernetic gloves. Thus, in 2005 a first prototype of a cybernetic glove appeared [61], and recently, in February 2014, Fujitsu presented its own design for industrials environments [62]. Independently, textile industry advances in the seamless integration between electronics and textiles, will enable a qualitative leap in wearable products [63,64].

On the other hand, although they are not considered integrated systems, several commercial "smart products" for stock control, environment adaptation to user and traceability have been developed recently.

In 2011, a smart poker table was presented in Italy by GTI Gaming [65]. Based on RFID technology, this table implements a real-time technology capable of calculating the amount of pot, and rake, reporting defects, identifying the dealer, establishing a network of tables and so on.

Later, between December 2013 and February 2015, up to three smart clinical medicine dispensers were presented in Europe. The first, in Portugal [66], was developed with Fujitsu's collaboration. The others [67,68], developed in Spain in June 2015, are still undergoing tests.

Nevertheless, the most successful product in the market is SATO's VINICITY technology [69]. This technology is able to read various RFID tags at once, so it has been employed in several "smart products" such as trays, tables and medicine dispensers.

\section{Analysis of Manufacturing Scenarios. TF4SM: First Prototype Design}

Manufacturing processes require precise control over each one of the phases comprising a process. Therefore, traceability becomes an increasingly important functionality to be implemented in process control systems. This section analyzes manufacturing scenarios in small companies (the motivation scenario of our work), and the requirements to be met to enable precise monitoring and traceability information acquisition. After describing the requirements of small manufacturing companies, we present the new traceability framework and the design of a first minimum functional prototype is proposed.

\subsection{Analysis of Manufacturing Scenarios: Motivation Scenario}

Manufacturing companies, in their day to day operation, perform various kinds of processes, such as logistic processes (including the receiving process and the inventory process) and production processes. The receiving process models the arrival of some units that must be tagged with RFID and reported in the corresponding receipt notice (which it was traditionally printed and, nowadays, it is digitalized). The received units may optionally aggregate into larger logistics units. If some units are not included in a purchase order, typically they must be discarded. After that, the received logistics units are moved to a warehouse. The execution results are reported in the corresponding transfer notice (which, one more time, was traditionally printed and, nowadays, it is digitalized). An inventory process is executed in 
parallel, and applies to all stored items that are cyclically counted. It would be a desirable feature that the system will issue alerts in cases of shortage or inconsistency of inventoried goods.

During the production process, warehouse operators select and supply the required quantities of raw materials that are specified in the production task instructions and attach the RFID labels of their containers onto the electronic reader. The production operator attaches his personal identification card to start a production task. Once the production process is finished the operator prints new RFID and/or barcode labels (depending on the system implemented) for the remaining materials that are scheduled for return to the warehouse and the products (ready or semi-finished) that are processed during each production task. Traceability information is used to execute consistency checks to determine quantities of raw materials consumed, the spoilage, the utilization of tools and labor, as well as the machine operation and stop times. It would be a desirable feature that the system will issue alarms in case of discrepancy (i.e., illegal operator or equipment), excessive consumption, and/or low production rate.

Taking into account the previous description, some additional requirements (introduced by the specific needs of small-sized manufacturing companies) have been considered in the framework development (apart from the ones presented in the previous sections):

- REQ\#1: the event monitoring function and production task traceability must be independent of the location of the information source and the hardware used. Traditional control systems were limited to the capability of the controller residing inside.

- REQ\#2: it should define a flexible and extensible architecture for the integration of various systems, wireless sensor networks (WSN), actuators, execution engines, among other things.

- REQ\#3: it should be capable of suspending current operations when the data obtained is corrupt, the task performed does not correspond to the expected one and/or some defined rules for the tasks are not satisfied.

- REQ\#4: the system must be easily configurable to allow communication with new elements and seamless interchange of new events. Traditional tag-based systems required early binding, that is, a configuration stage to link data sources and consumers of data.

- REQ\#5: the infrastructure must be capable of identifying each user or user role to determine the task to be performed. User roles are also considered for the cases when regulation does not allow identification of employees.

- REQ\#6: the system must assign tasks to different users who collaborate to complete the process.

- REQ\#7: ability to reconstruct every production step. This is required both for regulatory compliance and as an important basis for production environments.

\subsection{TF4SM: A New Traceability Framework for Small Manufacturing Companies}

As we have seen, improving traceability systems in small-sized manufacturing companies inevitably requires using information technologies. Among all the available technologies, we selected cyber-physical systems (and specifically, CPS according to the NIST definition) as the most appropriate. Therefore, we took the reference architecture showed in Figure 1 as our basis. Over it, we added or changed the elements necessary to fully check the requirements described in the previous sections. Our proposed Traceability Framework for Small Manufacturers (TF4SM) is the result of this process (Figure 2). 


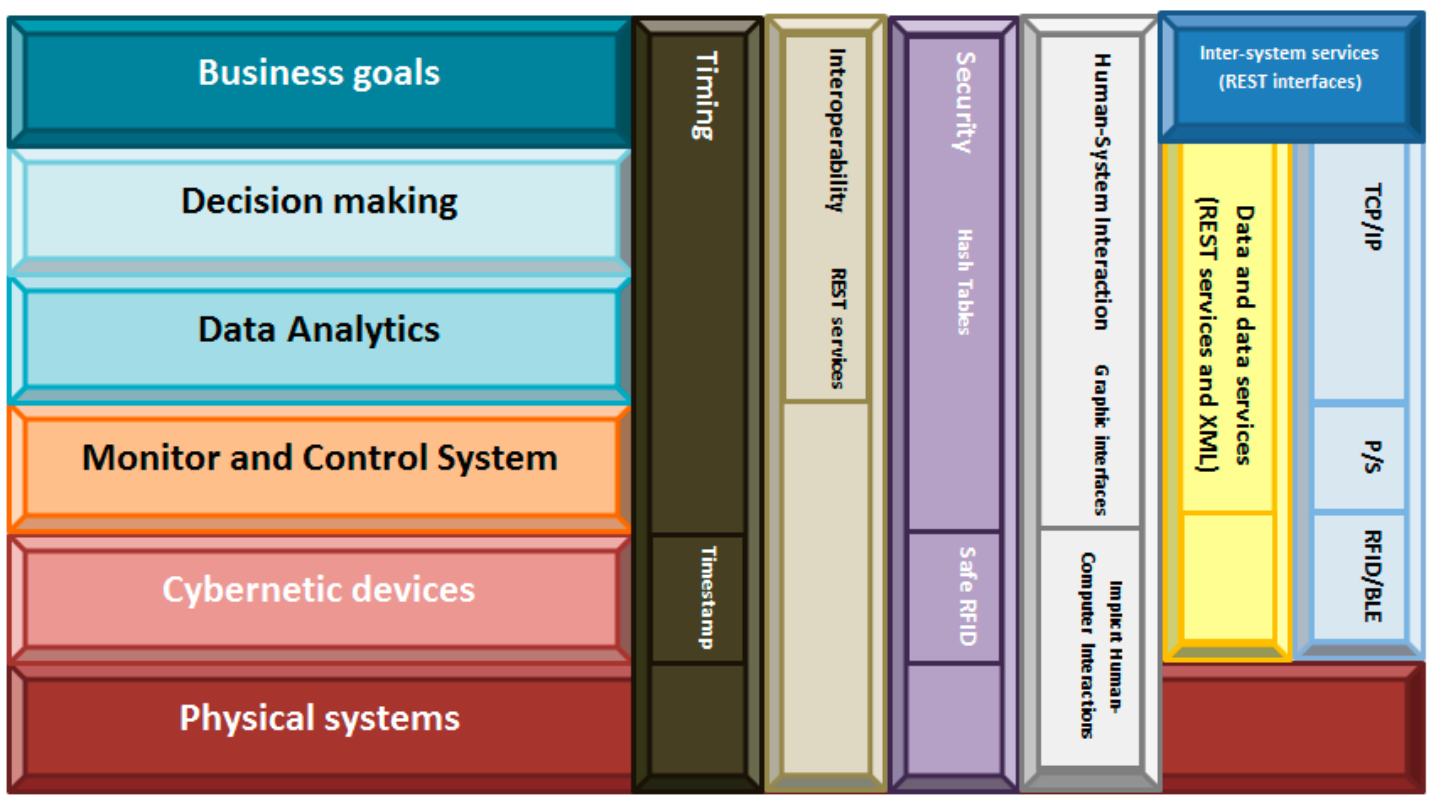

Figure 2. TF4SM architecture.

Next, we are briefly describing each part of the framework, and how they cover the previously presented requirements. The TF4SM framework consists of six levels of abstraction:

- The physical systems include all instruments, industrial systems and manufacturing elements and products present in the company's facilities.

- Cybernetic devices layer refers to the embedded devices which contains RFID readers and/or RFID tags, as well as to all elements which make up the subjacent pervasive computing system [70]. The inclusion of mobile computing in the framework (we describe the communications plane later) partially covers REQ\#1 (the system must show correct performance, independently of the information source's location). In addition, working with embedded devices allows us to answer the third basic question about traceability systems: how to integrate RFID system into the company's production processes. Although this paper refers to cybernetic devices, this kind of technologies has been commonly studied as "smart objects". For example, in [71] the relevance of smart objects is highlighted in several application scenarios.

- The Monitor and Control System refer to the interlayer which adapts cybernetic devices to high level computing schemes. It contains, mainly, data acquisition from RFID readers and data pre-processing. The inclusion of this layer allows creating hardware independent traceability systems. Embedded devices are placed below this layer and knowledge feedback loops are defined on top. This breakdown between knowledge management processes and the hardware which supports the system agree fully with the second part of REQ\#1.

- The Data Analytics layer, as in NIST reference architecture, processes data from different devices for pattern recognition and extracts data using machine learning and data mining. It also includes visual analytics oriented to the operator. Moreover, pattern recognition capability fulfills REQ\#7 (which describes the ability to reconstruct every production step), as it allows inferring workers' activities from data collected by RFID readers. The algorithms included in this layer also answer the last basic question which remains unresolved (how to translate the real-time data into 
meaningful information for the field operator?). Decision making uses results from Data Analytics as input for determining the next action which must be executed (issue an alert, suspend an operation, etc.). Thanks to the support of Data Analytics layer corrupt data can be detected, and this layer can order an operation to be suspended. In that way, REQ\#3 is covered, and some desirable features are included.

- Finally, in the Business Goals layer, rules about alerts, user's roles, minimum stock, automatic orders or emergency situations can be defined. The interface offered by the system at this level will be specific of the application domain (depend on the product manufactured in the company), so any technological expert must control the traceability system (what was desirable). As this layer is placed at human abstraction level, identities, workflows, tasks or collaborative work can be defined. Thus, REQ\#5 and REQ\#6 get fulfilled.

- Apart the previously described layers, the framework architecture includes six planes which cross vertically all the layers:

- Timing: In traceability systems, time issues are not critical. Therefore, in our framework timing has been resolved including a timestamp in each data produced by the cybernetic devices. Any other solution is considered as this datum is enough for tracing the products' life;

- Interoperability: Traceability systems are conceived as corporative infrastructure, so typically any external access will be admitted. In the case of joining various systems, communication will be performed at high level through Representational State Transfer (REST) interfaces, much more flexible than other technologies;

- Security: Security systems native from RFID-based traceability systems (based on safe RFID tags [72] and hash tables) have been widely proved as enough safe, so we maintain the same technologies in our framework;

- Human-System interaction: Two different levels are distinguished. At cybernetic devices layer, any direct interface toward the physical world is described, so the communication will be based on Implicit Human-Computer Interaction (IHCI) [73]. In higher layers, specific graphical interfaces will be defined to communicate operators with the system;

- Data and data services: The same philosophy applied in the case of "Interoperability" is valid here;

- Networking and communications: Communications must support a flexible architecture [74,75]. In order to achieve this objective four technologies coexist in our proposed framework. First, cybernetic devices must implement RFID technology (to trace products' life) and Bluetooth Low-Energy (BLE) standard (to transmit the readings toward the monitor and control system). The use of wireless technologies, in addition, supports the creation of a mobile computing scheme. Second, over BLE, a Publish/Subscribe protocol will be deployed. Actually, the use of the Publish/Subscribe communication paradigm facilitates binding between data sources and consumers of data [76], as the intermediate entity called "broker" manages registration and decouples entities in time and space. This improves system architecture flexibility (which deals with REQ\#4 and REQ\#2), as data sources do not have to be aware of the existence or number of data consumers and vice versa. Furthermore, some Publish/Subscribe communication protocols, such as the Message Queuing Telemetry Transport (MQTT), support reliable communication that includes retransmission of lost frames and acknowledge 
events (which guaranteed the delivery of information). Finally, the communication among the monitor and control system and the higher abstraction layers will be performed over standard network technologies such as TCP/IP (which allows using the existent communications infrastructure in the company).

- Inter-systems services: One more time, the same philosophy applied in the case of "Interoperability" is valid here.

\subsection{First Prototype Design}

Once presented the reference architecture for our framework, we design and build the first minimum functional prototype based on it. In this section, we first evaluate the minimum features we must include in the prototype for being able to validate its usability. Later, we present the list of elements which have to be built and the physical architecture considered.

In manufacturing companies three basic elements are involved: workers, products and the workplace (usually a bench of some type). In some traceability solutions, such as fully automated solutions, only products and the workplace are taking into account. However, these kinds of solutions are commonly designed for heavy industry companies (such as automakers), where companies have very extensive work areas, lots of investment and production processes use high-volume products. In these scenarios, high-tech expensive deployments such as robotics (which may be part of both the traceability system and the production system), or high arches for automated reading of low frequency RFID tags (which can be used without risk of reading tags of wrong areas thanks to the breadth of the production area) are feasible solutions. In contrast, in small manufacturing companies products are made with small supplies, which does not require manipulation by robots (that sometimes cannot even be done), so that investment in traceability systems based on robotics is not justified (especially considering the resources these companies usually have). Furthermore, deployment of fully automated systems often requires large spaces, not always available in small companies (sometimes made up of less than five workers), so alternatives must be evaluated. In works on this issue $[9,12]$, it is clear that the key to overcome this difficulty passes to monitor actions performed by workers without replacing them with robots, including heavy infrastructure in the work area or assigning additional tasks to operators which decline their productivity. Therefore, our prototype must include human interaction, and workers have to be incorporated into the system in the most appropriate way.

Products, as considered in the TF4SM framework, will be provided with a NFC tag which identifies each one unequivocally. Later, this NFC tag must be detected when products are placed on the work table and when they are being manipulated by workers, so both elements (workers and work areas) have to be provided with NFC reader capability. For the operators, we designed a cybernetic glove with only NFC reader capability as the objective was to create a very light wearable device, which does not impede work, and for which "pay attention once placed" is not necessary. For the workspace, NFC reader capability was not enough (we designed a cybernetic surface with four different reader zones), so four types of LED are included to notify the user of the progress in the process.

Previously described devices complete the cybernetic devices layer. Although in the general case layers can be implemented in different systems, in this first prototype we implement them into the execution engine to orchestrate the whole system. In order to enable human-system interactions at the 
top level, a visual application running in a visualization platform (or human-machine interface) is also provided. Through this platform, the state of the production process can be monitored and workflows and tasks can be defined (being also able to consult their compliance). Then, the final prototype's physical architecture can be seen in Figure 3a.

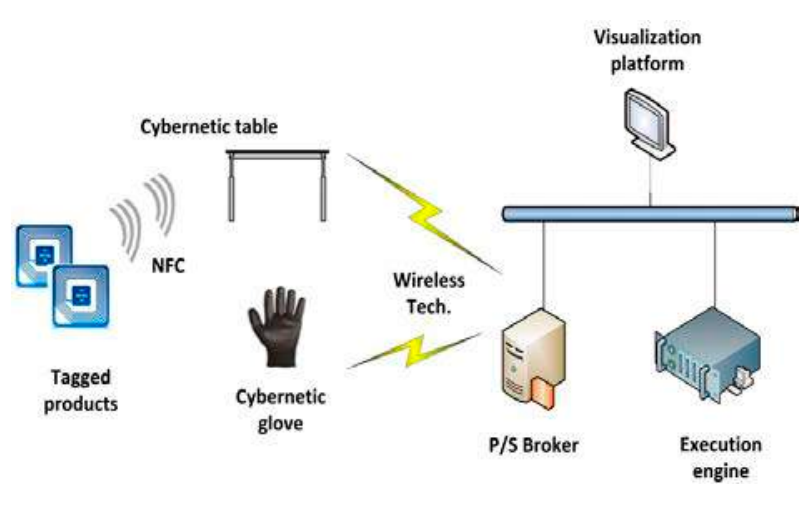

(a)

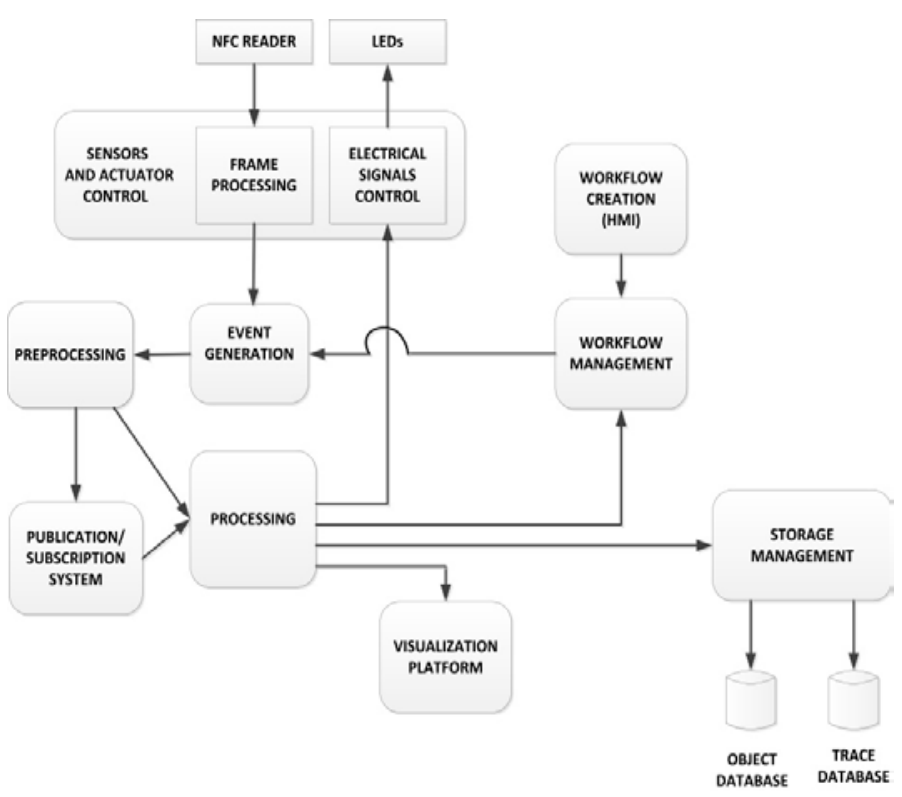

(b)

Figure 3. (a) Designed TF4SM physical architecture (b) Designed TF4SM functional architecture.

As Figure 3a shows, another element has been included: a Publish/Subscribe broker. This element allows the spatial and temporal decoupling between publishers and subscribers and, in our system; it avoids having to reconfigure the rest of devices each time a device goes on or off. However, as this element belongs to the networking and communications plane and was not the focus of this work, it was omitted (an in-depth discussion about this subject can be found in articles, such as [77]). A functional architecture can be also considered (Figure 3b). First, we developed the NFC reader capability and the LED handling capability. These modules must be controlled from a component capable of extracting useful information from the NFC frames and capable of generating the electrical signal needed for turning on (or off) the LEDs. The cybernetic table and the cybernetic glove both support these capabilities. The event generation capability was later distributed among all the cybernetic devices and the execution engine. Depending on the system's state at a particular moment any of these components can generate an event (see Section 4.2). Once an event has been generated, an evaluation step (executed in the same device where the event was produced) decides if the event must be published (using the Publish/Subscribe system) or any action must be directly executed (both options may be necessary). All the components in the prototype (cybernetic devices, execution engine and visualization platform) have processing capability (so they form a pervasive computing scheme). The processing step can cause a change in visualization platform, in LED state, a workflow update and/or an operation over the databases (in Section 4.1 the storage capacity of the system is described in detail). Both workflow management and storage management capabilities belong to the execution engine. Finally, depending 
on the workflow defined in the human-machine interface (HMI, also implemented in the execution engine), a workflow update can generate a new event, which performs the same cycle described.

\section{System Construction}

This section explains the implementation of the solution that was designed following the TF4SM framework, and its deployment in the application scenario.

\subsection{System Implementation}

As we have said in Section 3.3, the system must be made up of the following four elements: a cybernetic glove, a cybernetic table, an execution engine which communicates with cybernetic devices by means of a byte-oriented protocol, and a visualization platform displaying the status of the monitoring system and process execution.

One of the most important components in the designed system is the cybernetic glove. Even workers monitoring the system should increase process efficiency. However, human behavior is complex and sometimes random. Because of this, false measurements, erroneous events and similar mistakes can appear in the system if a cybernetic glove alone is considered (for example, as we will see later, if in the production process the glove passes near a tag it might be read, even when the object has not been picked up or used). In order to eliminate these measurement errors, more cybernetic devices are necessary (in this case we have selected a table because of the type of process considered). With the additional information provided by these additional devices, and using a proper algorithm (running in the execution engine), a more accurate picture of the current situation can be obtained.

Figure 4 shows our prototype for a cybernetic glove. The cybernetic glove consists of a plastic package placed on the front side of the wrist (so as not to impede movement), a galvanized copper coil to allow NFC communication with tagged cybernetic devices, and a synthetic textile support, whose properties do not significantly affect the magnetic field generated by the NFC module.

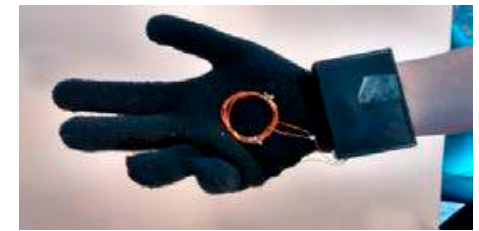

(a)

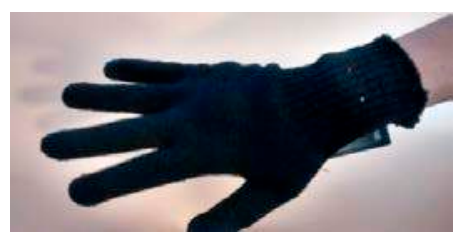

(b)

Figure 4. Example of hand movement with our cybernetic glove, (a) palm and (b) back sides.

In Figure 5a we present the electronic scheme of the cybernetic glove. As can be seen, five elements compose the glove: a LiPO battery, a galvanized copper coil as an inductive antenna, an RDM8800 NFC chip [78], an Arduino Nano platform, and an HC-06 Bluetooth slave module. The cybernetic table is also provided with four different types of LED.

A brief explanation of the function of each element follows:

- The LiPO battery has a capacity of $850 \mathrm{mAh}$ and $3.7 \mathrm{~V}$. As the total current consumption of the glove is $140 \mathrm{~mA}$, the glove has the capacity to operate for about $6 \mathrm{~h}$;

- The galvanized copper coil acts as an inductive antenna, resonant at NFC frequency (13.56 MHz); 
- The RDM8800 NFC chip receives physical signals from the antenna, and produces a data frame encapsulating, among other fields, the identifier of the tagged cybernetic device that is in contact with the glove. The output interface is UART-serial type (at 9600 bauds);

- The Arduino Nano platform receives data from RDM8800 by UART and extracts the tagged cybernetic device's identifier. Finally, it encapsulates the identifier in an application protocol message and transmits the message by a second UART at 19,200 bauds;

- The HC-06 Bluetooth slave module receives data by UART at 19,200 bauds and transmits them through a Bluetooth 3.0 interface;

- The table's LEDs are used as actuators to notify the user of different types of events.

Thanks to NFC technology, and controlling some of the antenna configurations, we can greatly reduce the distance at which the glove detects the presence of a tagged cybernetic object (see Figure 6). Thus, we can say that the glove performs readings by contact.

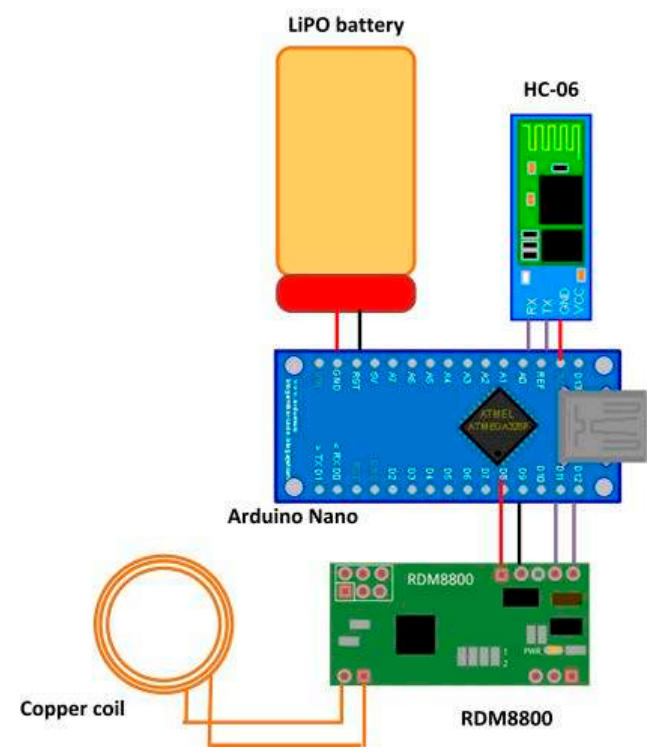

(a)

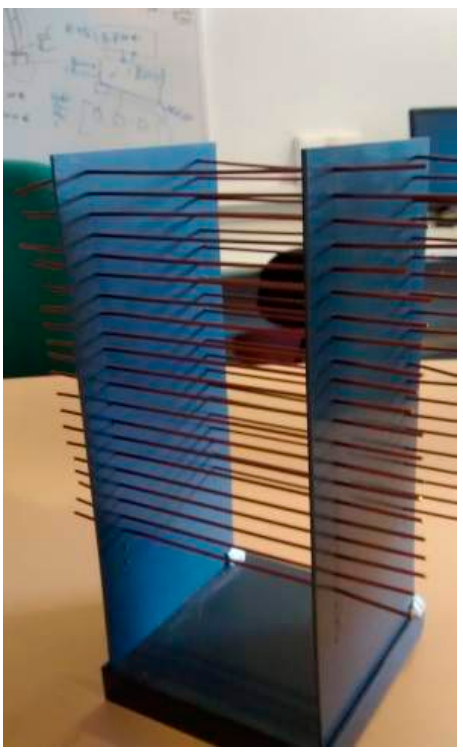

(b)

Figure 5. (a) Electronic scheme of the cybernetic glove; (b) Plastic holder graduated in height for measuring glove behavior.

Despite this, it is necessary to consider some limitations. On the one hand, metallic objects cannot be considered due to their capacity to affect magnetic fields. On the other, as the magnetic field generated by the NFC reaches a maximum distance of $1.5 \mathrm{~cm}$ and only one device can be detected at once by the glove, we must guarantee that only one tagged cybernetic device is less than $1.5 \mathrm{~cm}$ from the glove.

In order to measure the data, from which we prepared Figure 6, we used a plastic holder with several grids, separated (from each other) vertically by $0.5 \mathrm{~cm}$ (see Figure $5 \mathrm{~b}$ ). The experimental procedure was conducted as follows:

- Five points were selected in height: $0 \mathrm{~cm}, 1 \mathrm{~cm}, 1.5 \mathrm{~cm}, 1.8 \mathrm{~cm}$ and $2 \mathrm{~cm}$;

- For each point in height, a set of 10 measurements were made. The probability of correct reading for each point was calculated by means of Laplace definition Equation (1): 


$$
P(\text { correct reading })=\frac{\text { number correct readings }}{\text { number tries }}
$$

- The second step was repeated ten times, obtaining ten different values of the correct reading probability for each point in height. Figure 5 shows the clouds of points obtained, along with a piecewise linear fit (blue line) obtained by minimizing the mean square error. The bubble size indicates the number of times a given value has occurred.

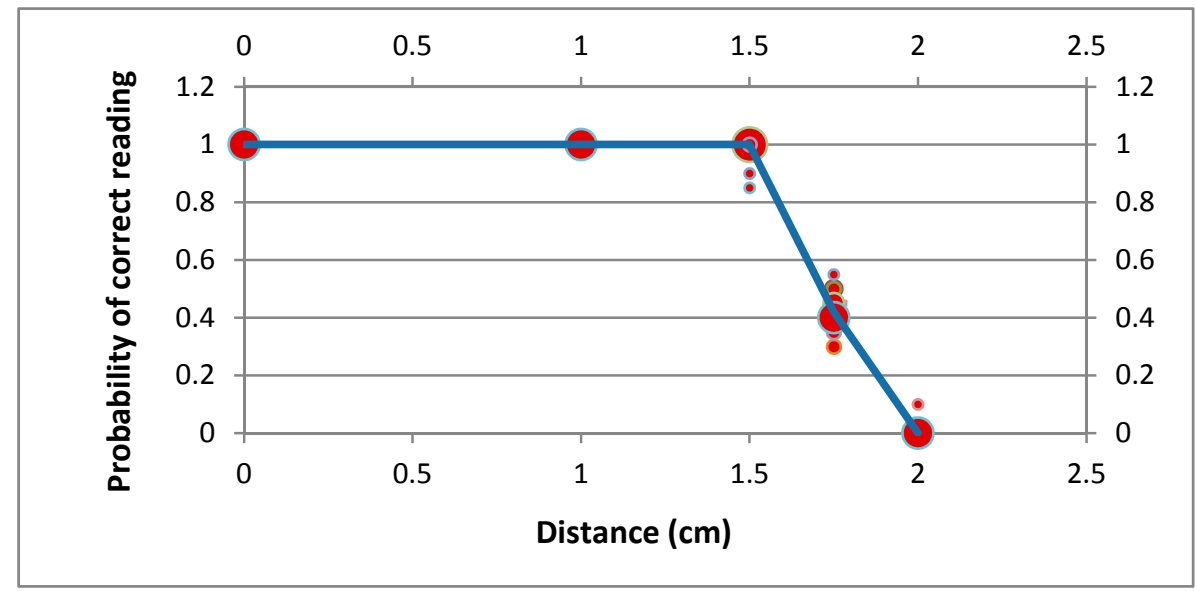

Figure 6. Evolution of the detection probability based on distance between the glove and the tagged device.

In Figure 7a we present our prototype for a cybernetic table. It is made of a plastic material (which does not interfere with electromagnetic fields) and on whose surface we have defined four detector areas.

In Figure $7 \mathrm{~b}$ an electronic scheme of the table is presented. As can be seen, there are four dedicated microcontrollers (one for each detector area), and one manager microcontroller. When a dedicated microcontroller has data to transmit, it requests permission from the manager, and transfers the message using a physical protocol. This physical protocol consists of two lines: the permission line serves as output for manager and input for dedicated microcontrollers, and the solicitation line serves as input for manager microcontroller and output for dedicated units.

The protocol is defined below and is also presented in Figure 8:

- When a dedicated microcontroller wants to transmit data using the Bluetooth module connected to the manager unit, it sets the solicitation line to high value;

- The manager unit recognizes this signal change and checks whether or not the Bluetooth module is in use. If it is not, it clears the Bluetooth buffers and deletes old transitions. It also sets the permission line to high value to indicate that it is expecting Bluetooth connections;

- When the dedicated microcontroller detects a high level in the permission line, it starts the data transmission. At the end of the transmission, it also sets the solicitation line to low level;

- Finally, when the solicitation line changes to low level, the manager microcontroller also sets the permission line to low level again.

Additionally, the maximum distance at which a tagged device is detected by the table is much shorter than the corresponding distance in the glove (see Figure 9). As can be seen, this maximum distance is, 
in this case, $2.5 \mathrm{~mm}$. To obtain this result, the same procedure explained for Figure 6 was followed (although, in this case, only four points in height were considered).

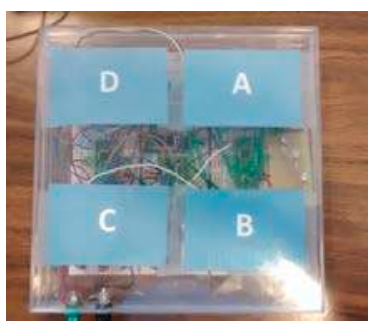

(a)

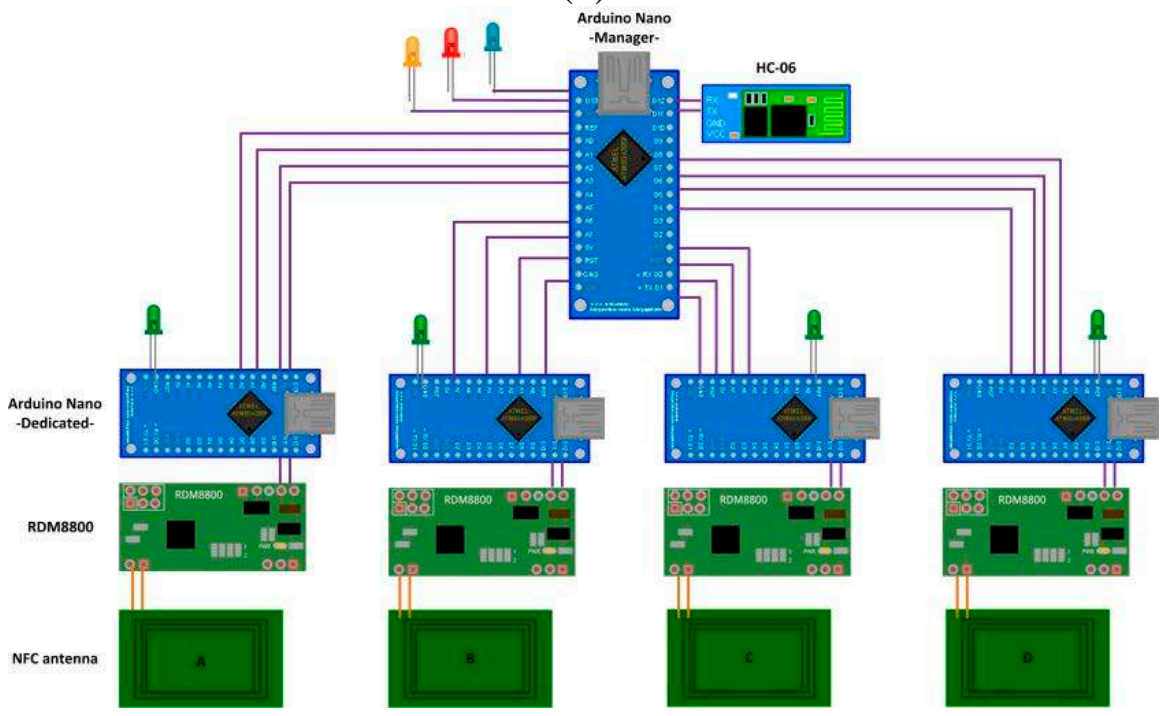

(b)

Figure 7. (a) Prototype of a cybernetic table; (b) Electronic scheme of the cybernetic table.

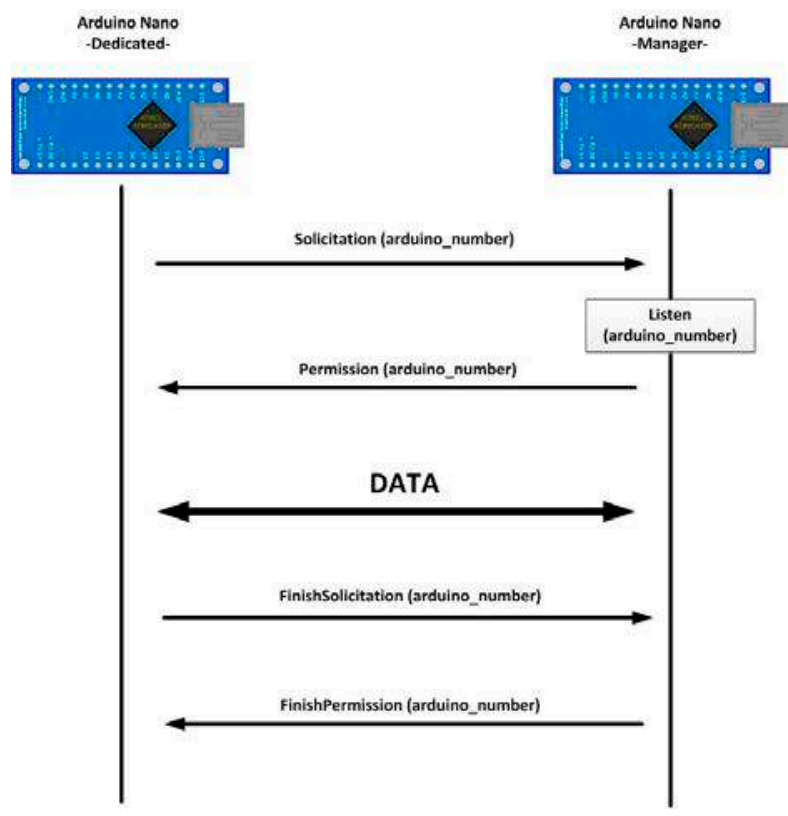

(a)

Figure 8. Cont. 


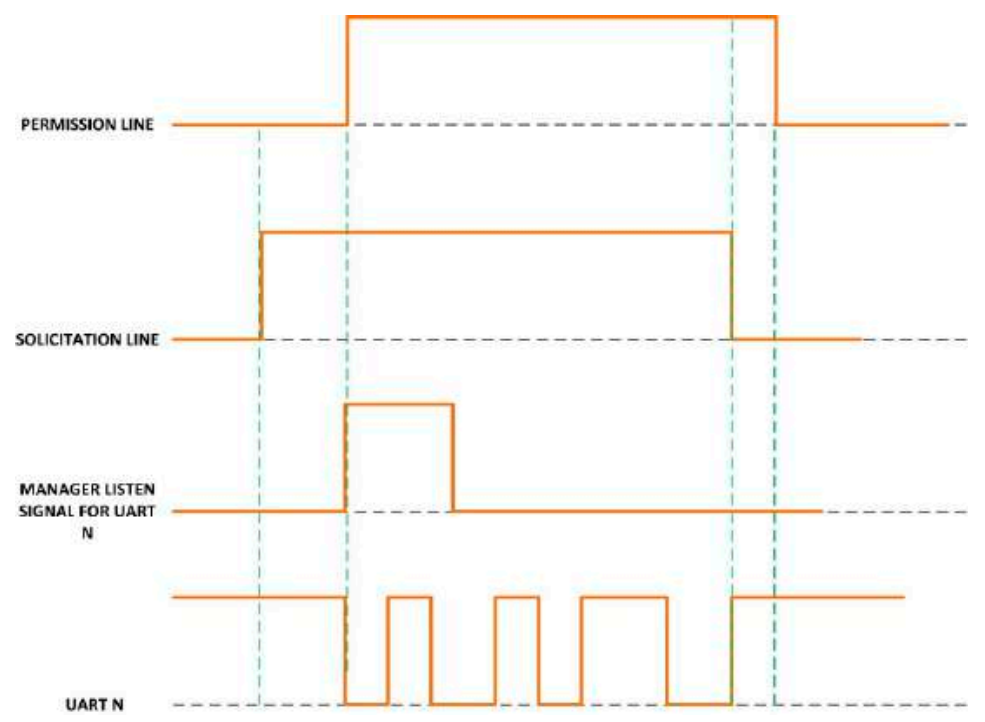

(b)

Figure 8. Physical protocol to allow communication between the manager Arduino and dedicated Arduinos, (a) Sequence diagram; (b) Chronogram.

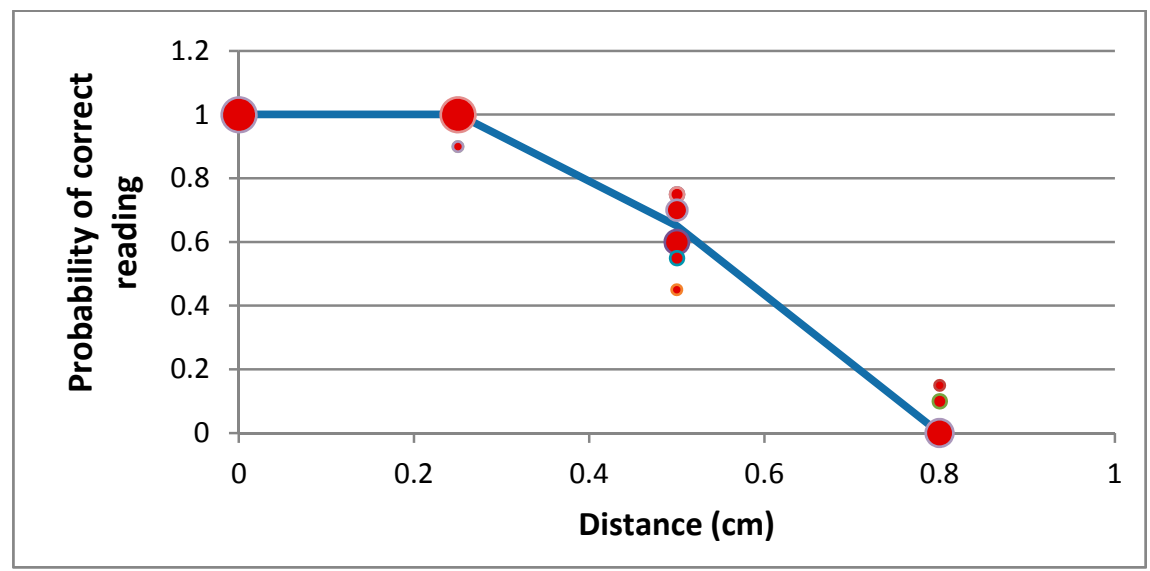

Figure 9. Evolution of the detection probability based on the distance between the table and the tagged device.

Figure 10a shows the functional decomposition of the execution engine. As can be seen, four elements can be characterized (see Section 3.3 and Figure 3b). First, there is an HMI through which users can define the system's behavior and workflows. Second, it includes a processor where workflow management, storage management, event processing and generation and system coordination are executed. Finally, two databases for information storing are also included. The first one stores the traces generated by the system in order to be able to recover past executions. The second one stores all the information about cybernetic devices, users and tagged objects (such as ID, logical name and actions allowed). All the elements that make up the execution engine are built using Java technologies, such as JavaX Bluetooth libraries and hsqlDB manager.

As we have explained in the previous sections, the execution engine has workflow and storage management capabilities. Thus, it will usually be informed about all the events in the system. However, as we have said in Section 3 and will see in Tables 2 and 3, our TF4SM framework (and consequently 
this first prototype) allows distributed event processing. Each event may be processed on the same device where it is generated (totally or partially), or it can be transmitted toward remote components. It is also possible to perform both actions.

Other capabilities, such as signal power or signal quality measurements, are considered to detect when a device is entering or leaving the system (this way the system will adapt automatically).

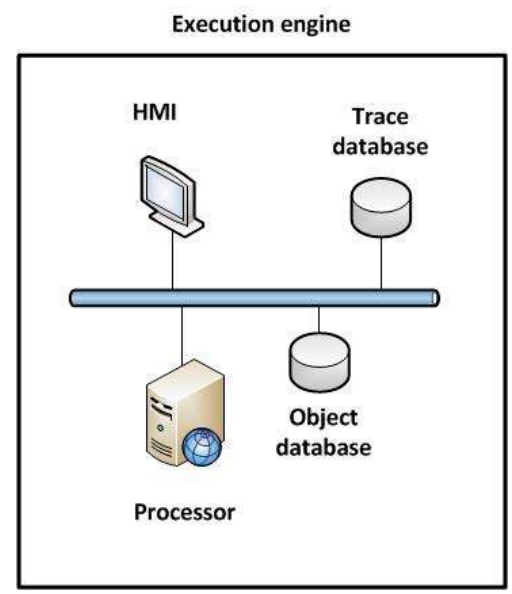

(a)

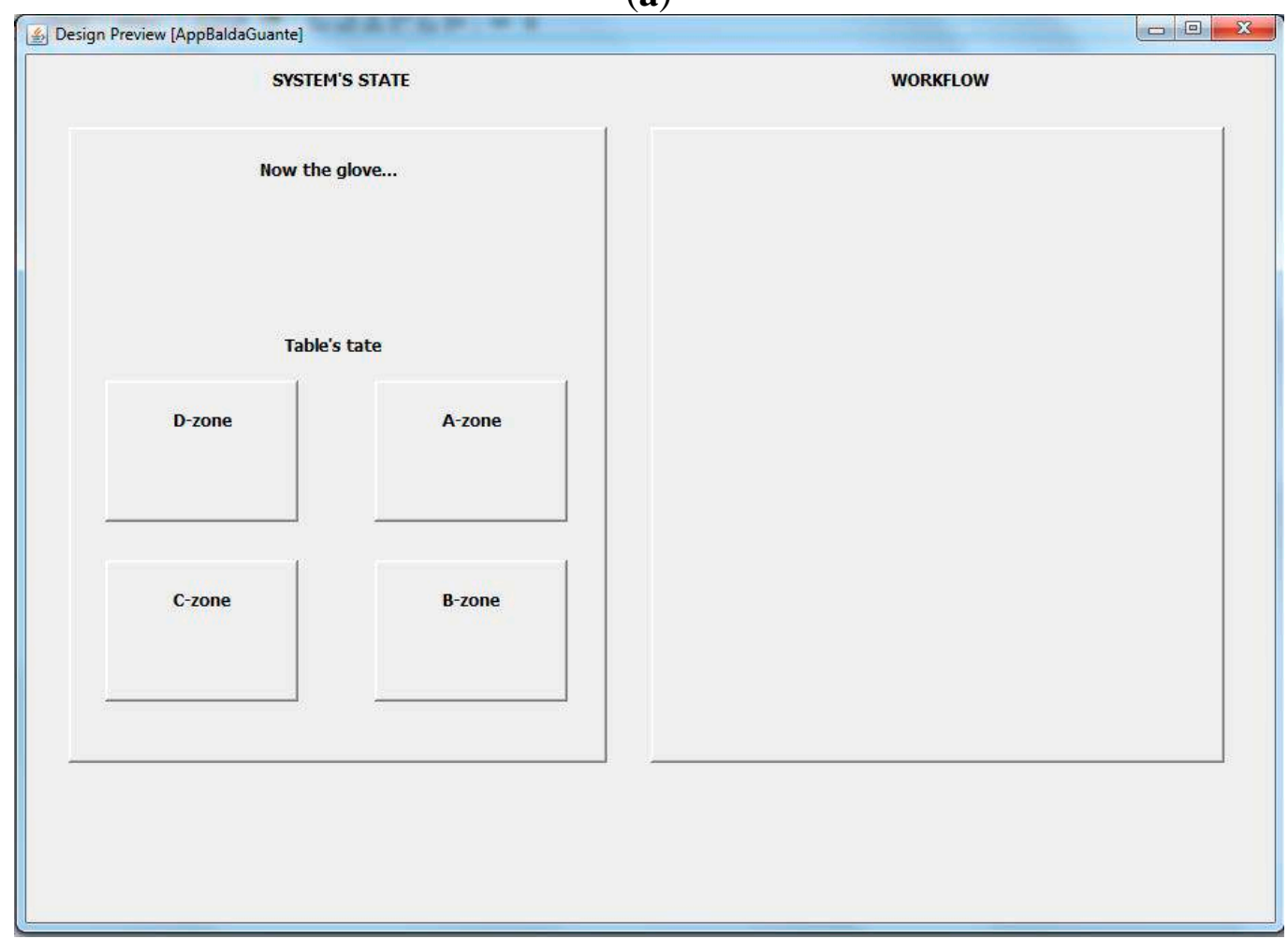

(b)

Figure 10. (a) Functional decomposition of the execution engine; (b) Visualization platform.

The visualization platform consists of a Java graphical application on which the system state and workflows advances are constantly shown (Figure 10b). Finally, we must describe the byte-oriented application protocol that allows communication among the different cybernetic devices and the execution engine. A byte-oriented (also called character-oriented or text-oriented) protocol is a communications 
protocol in which full bytes are used as control codes. The main advantage of these protocols is that they can be directly understood by humans.

In many CPS scenarios [79,80], other technologies such as XML or JSON are preferred, as they are also efficient, human readable and can also be transmitted via HTTP. However, this is not possible with the selected hardware (which only has $2 \mathrm{~KB}$ of SRAM and $30 \mathrm{~KB}$ available in EEPROM). First, specific and heavy libraries are necessary (because Arduino is not designed for parsing XML or JSON documents). Nevertheless, the EEPROM space is almost full with Arduino libraries (which includes String library), Publish/Subscribe protocol code and NFC processing code, so it would be quite impossible to add any additional library. Second, the control characters needed in XML or JSON are more than the ones used in a specific protocol, so communication slows down in these cases (We used virtual communication infrastructures and an $8 \mathrm{MHz}$ processor). And, finally, a rigid message structure allows processing the messages while they are being received, so not all the characters must be stored, and the available SRAM needed is smaller. Therefore, we have designed a specific byte-oriented application protocol for this scenario.

In this case, only two different messages are considered: DEVICE ADVERTISEMENT message and EVENT message (Figure 11).

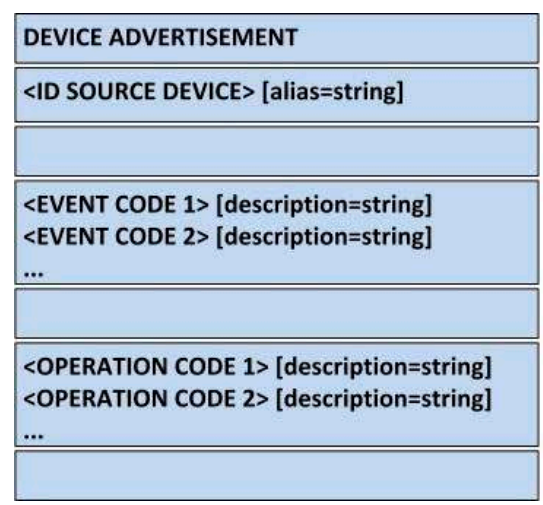

(a)

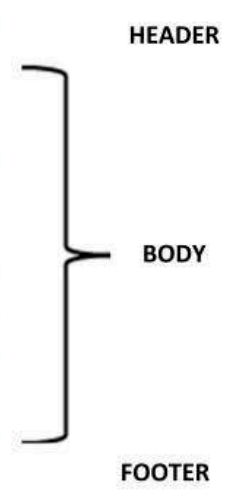

FOOTER

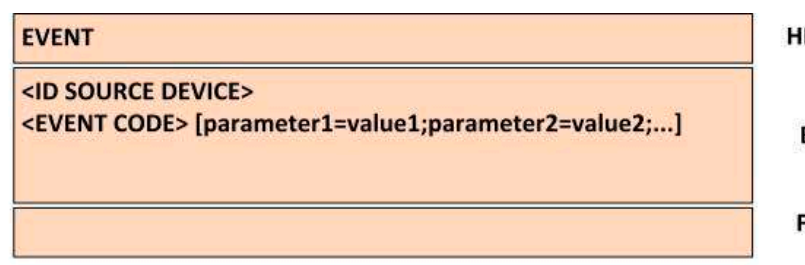

HEAD

FOO

Figure 11. Scheme of protocol messages; (a) DEVICE ADVERTISEMENT message; (b) EVENT message.

When a new cybernetic device (uniquely identified throughout the world by its Bluetooth MAC address) is connected to the prototype, the first thing it must do is to inform about the events it can generate (for example, new tagged-object detected) and the operations it can execute (such as blinking a LED). This way, the execution engine (which will be subscribed to the appropriate topic) can present that information on the HMI, and users are able to use them in their workflows and processes. This way the proposed prototype is adapted to the arrival of new devices and is adaptable to use new capabilities provided by the cybernetic device. This information is sent via a DEVICE ADVERTISEMENT message. The message structure is as follows:

- In the header line, we must indicate the message type: Device Advertisement;

- In the first line of the body, we must place the identification of the new device, followed by a blank space and (optionally) an alias which will appear on the HMI; 
- Then, an empty line indicates the beginning of the list of events the device can generate. In each line, first the event code must be placed (see Section 4.2). Later, optionally and separated by a blank space, a description of the event including its parameters, meaning or any other information can be incorporated;

- A new empty line indicates the end of the list of events, and the beginning of the list of actions the device can execute. The list format is the same as in the previous case;

- The message ends with an empty line.

Being strict, as our prototype is only going to have two types of devices (cybernetic gloves and cybernetic tables), a manual configuration of these elements could be done, and the ADVERTISEMENT message is dispensable. However, in order to verify the full performance of the TF4SM framework (including the initial configuration) we have chosen to consider this transaction. An EVENT message is sent each time a cybernetic device generates an event, and this event must be notified to another device. The message structure is as follows:

- In the header line, we must indicate the message type: Event;

- In the first line of the body, we must place the identification of the device which notifies the event;

- In the second line the event code (see Section 4.2) must be placed first. Then, after a blank space, it is possible to place as many parameters as required in the event, following the format "parameter = value" and separated from each other by semicolons. The carriage return marks the end of the parameter list;

- The message ends with an empty line.

\subsection{Process Model}

Having described the physical implementation, in this section we now present how workflow definition and verification work in our prototype and, in general, how a TF4SM-based system operates.

In our prototype, we consider a workflow as a collection of states related to each other by transitions, which are executed when one or various events occur (as in a finite-state machine). Examples of workflows are the inventory and the manufacturing processes explained in the motivation scenario. Additionally, workflows are considered logical objects, so they encapsulate their own attributes and methods (such as the owner or the date of the last execution).

In each state two activities are executed, one when entering the state (entry activity) and another when exiting (exit activity). The problem is that the activities that are usually described in a workflow consist of several actions that cannot be run directly on cybernetic devices, but should be modeled using the operations they offer. An example of activity is the production task explained in the motivation scenario. Activities encapsulate their own attributes and methods (such as the activity number).

We define an activity as an ordered collection of atomic operations. One operation can only be executed if all the previous operations have been successfully completed. As activities are the smallest logical object, if one atomic operation fails, the whole activity fails. Finally, we call atomic operations those operations which can be executed only with one interaction in the prototype (for example, picking up a tagged-object, turning on a LED, etc.). Atomic operations match operations announced by 
cybernetic devices in their DEVICE ADVERTISEMENT message. Table 2 presents the atomic operations currently supported in our system.

Table 2. Atomic operations currently supported in the TF4SM.

\begin{tabular}{|c|c|c|}
\hline Operation & Source & Description \\
\hline LED_ON & Cybernetic Table & $\begin{array}{l}\text { Turns on the LED specified in the identification parameter. If durationTime value is not } \\
\text { included, LED remains on indefinitely. Otherwise, LED is on during durationTime seconds. } \\
\text { If the LED is already on, nothing happens. }\end{array}$ \\
\hline LED_OFF & Cybernetic Table & $\begin{array}{l}\text { Turns off the LED specified in the identification parameter. If durationTime value is not } \\
\text { included, LED remains off indefinitely. Otherwise, LED is off during durationTime seconds. } \\
\text { If the LED is already off, nothing happens. }\end{array}$ \\
\hline TAG_PUSH_GLOVE & Cybernetic Glove & $\begin{array}{l}\text { Turns on the glove's NFC interface. If any parameter is included, the operation finishes when } \\
\text { any NFC tag is read. If durationTime value is specified NFC interface stays on during } \\
\text { durationTime seconds. If identification parameter is included, NFC interface stays on until } \\
\text { the tag with the specified ID is read. It is possible to combine durationTime and identification } \\
\text { value to obtain more complex behaviors. }\end{array}$ \\
\hline TAG_PUSH_TABLE & Cybernetic Table & $\begin{array}{l}\text { Turns on the table's NFC interface associated with activeZone area. If any parameter is } \\
\text { included, operation finishes when any NFC tag is read. If durationTime value is specified } \\
\text { NFC interface stays on during durationTime seconds. If identification parameter is included, } \\
\text { NFC interface stays on until the tag with the specified ID is read. It is possible to combine } \\
\text { durationTime and identification value to obtain more complex behaviors. }\end{array}$ \\
\hline TAG_POP_GLOVE & Cybernetic Glove & $\begin{array}{l}\text { Turns off the glove's NFC interface. If any parameter is included, operation finishes when the } \\
\text { NFC tag that is being read is withdrawn or if there is none, immediately. } \\
\text { If durationTime value is specified NFC interface stays on during durationTime seconds and } \\
\text { then turned off. }\end{array}$ \\
\hline TAG_POP_TABLE & Cybernetic Table & $\begin{array}{l}\text { Turns off the table's NFC interface associated with activeZone area. If any parameter is } \\
\text { included, operation finishes when the NFC tag which is being read is withdrawn, or, if there } \\
\text { is none, immediately. } \\
\text { If durationTime value is specified NFC interface stays on during durationTime seconds and } \\
\text { then turned off. }\end{array}$ \\
\hline SHOW_MESSAGE & Visualization platform & Shows on visualization platform a pop-up window, where the content of message value is printed. \\
\hline
\end{tabular}

Besides the atomic operations, as we mentioned in Section 3.3, cybernetic devices can also generate, process and receive different types of events. In an evaluation step, it is decided whether or not one event is processed in the same device where it is generated, or transmitted by means of an EVENT message to other devices (to execution engine, for example, in order to update workflow state). Table 3 presents the events that are currently supported in our system.

Considering the motivation scenario (see Section 3.1), although various workflows can be executed in parallel (receiving process and inventory process), activities in each workflow such as the production task can always be executed sequentially. We simplify our process design and support only linear workflows. In this scheme, only one set of events allows advancing to the next state, and any other set causes the workflow to fail (see Figure 12).

Finally, three states are mandatory in all the workflows defined in this first prototype. First, in all workflows the fail state must be included. When a WORKFLOW_FAIL event is triggered, workflow transits toward fail state from any other state. Second, the final state is also mandatory. 
Table 3. Events currently supported in the TF4SM.

\begin{tabular}{|c|c|c|}
\hline Event & Source & Description \\
\hline TAG_PUSH_GLOVE & Cybernetic Glove & $\begin{array}{l}\text { This event is triggered when an NFC tag is detected by the cybernetic glove. In identification } \\
\text { parameter, the read tag's ID must be indicated. }\end{array}$ \\
\hline TAG_PUSH_TABLE & Cybernetic Table & $\begin{array}{l}\text { This event is triggered when one NFC tag is detected by the cybernetic table. The activeZone value } \\
\text { includes the reader area where the tag has been detected and in identification parameter, the tag's ID } \\
\text { must be indicated. }\end{array}$ \\
\hline TAG_POP_GLOVE & Cybernetic Glove & $\begin{array}{l}\text { This event is triggered when an NFC tag previously detected by the cybernetic glove is withdrawn. } \\
\text { In identification parameter, the withdrawn tag's ID must be indicated. }\end{array}$ \\
\hline TAG_POP_TABLE & Cybernetic Table & $\begin{array}{l}\text { This event is triggered when one NFC tag previously detected by the cybernetic table is withdrawn. } \\
\text { The activeZone value includes the reader area where the tag has been withdrawn and in } \\
\text { identification parameter, the withdrawn tag's ID must be indicated. }\end{array}$ \\
\hline WORKFLOW_START & Execution engine & $\begin{array}{l}\text { This event is triggered when a workflow has been recovered by a user and must start. In identification } \\
\text { parameter the workflow ID is indicated. }\end{array}$ \\
\hline WORKFLOW_END & Execution engine & $\begin{array}{l}\text { This event is triggered when a workflow has finished successfully. In identification parameter the } \\
\text { workflow ID is indicated. }\end{array}$ \\
\hline ACTIVITY_COMPLETE & Execution engine & $\begin{array}{l}\text { This event is triggered when an activity has finished successfully. In identification parameter the } \\
\text { activity ID is indicated and, in parentIdentification, the workflow's ID to which this activity belongs } \\
\text { is included. This event will allow not only store traces of atomic operations and activities. }\end{array}$ \\
\hline ACTIVITY_FAIL & Execution engine & $\begin{array}{l}\text { This event is triggered when an activity has failed. In identification parameter the activity ID is } \\
\text { indicated and, in parentIdentification, the workflow's ID to which this activity belongs is included. }\end{array}$ \\
\hline WORKFLOW_FAIL & Execution engine & $\begin{array}{l}\text { This event is triggered when a workflow has failed. In identification parameter the workflow } \\
\text { ID is indicated. }\end{array}$ \\
\hline WORKFLOW_NEW & Execution engine & $\begin{array}{l}\text { This event is triggered when a new workflow is created in the execution engine. In the workflow } \\
\text { parameter, the new workflow is codified using bytes. }\end{array}$ \\
\hline
\end{tabular}

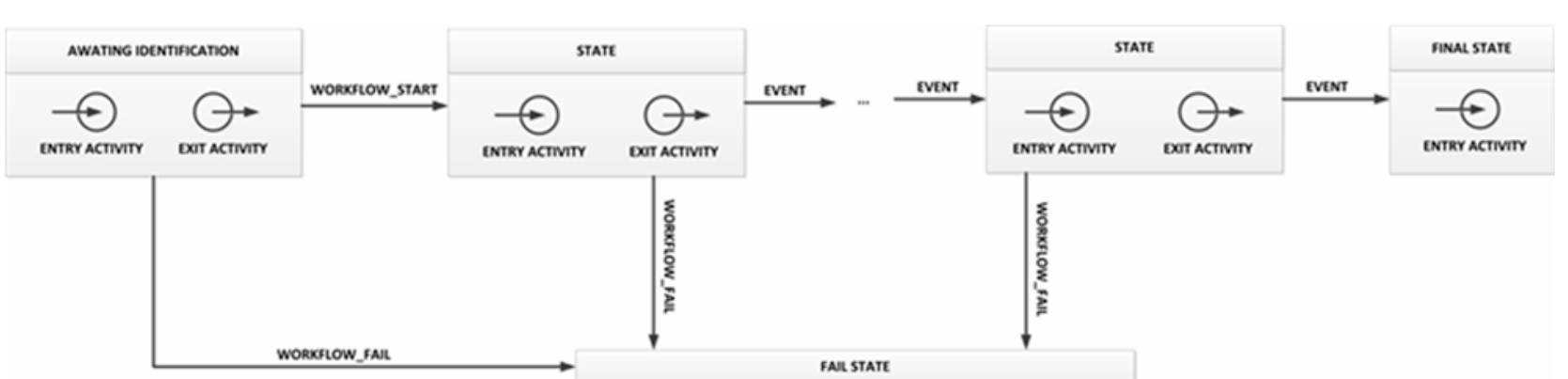

Figure 12. General workflow format in the TF4SM-based prototype.

This state represents the completion of the state machine and does not have exit activity. Third, the awaiting identification state must be considered an initial state. This state represents the workflow's starting point, and always has to be defined as follows:

- Entry activity: first a SHOW_MESSAGE operation must be executed in order to ask users to identify themselves. Then, a TAG_PUSH_GLOVE operation turns on the glove's NFC interface, which is not deactivated until the users again uses his ID to close the state; 
- As the execution engine subscribes to the corresponding topic (see Section 4.3), it will receive information on the user's ID. The execution engine checks whether the workflow owner user is the same user that was identified. If so, a WORKFLOW_START event is generated;

- Transition: the workflow will execute the transition toward the next state when a WORKFLOW_START event is received;

- Exit activity: this activity is made up of only one SHOW_MESSAGE operation, to indicate to users that the workflow verification has started.

Although the process model described is a simplification, it matches the model used in a large number of manufacturing companies. For example in [81] the cigarette manufacturing process is described by workflows consisting on sequences of activities beginning at an initial activity and ending at a completion activity. Transitions between activities also are run when the associated trigger event occurs. For all this, the model can be considered valid to verify the usability of our prototype.

\subsection{System Deployment}

Our first TF4SM-based prototype was deployed as a laboratory prototype at the Technical University of Madrid. The scenario was built simulating the scenario we can find in a real small-sized manufacturing company.

In the object database we have registered 56 different objects and 36 different user profiles. In the execution engine we have developed only one workflow, made up of 10 different states (13, if we take into account the mandatory states describe above). Finally, in this prototype, devices have been configured as Table 4 shows.

Table 4. Devices' configuration.

\begin{tabular}{ll}
\hline Device & Configuration \\
\hline Cybernetic glove & All the events generated in the glove are directly published without been processed. \\
\hline & The TAG_PUSH_TABLE event turns on one green LED. The TAG_POP_TABLE event turns off the same \\
& green LED. The WORKFLOW_START event turns on an orange LED. \\
Cybernetic table & $\begin{array}{l}\text { Both TAG_PUSH_TABLE and TAG_POP_TABLE events are also published. The WORKFLOW_END } \\
\text { event turns on a blue LED for 10 seconds, and the WORKFLOW_FAIL does the same with a red LED. }\end{array}$ \\
\hline Visualization platform & It is subscribed to all the topics that are used to update the platform. \\
\hline Execution engine & It is subscribed to all the topics that are used to update the workflow, and also to publish all the events it generates. \\
\hline
\end{tabular}

\section{Experimental Validation}

This research paper attempts to answer the following research questions:

1. Would the time response to inefficiencies improve by deploying a system based on our TF4SM in companies?

2. Is it possible to reduce the number of inefficiencies in productive processes using a TF4SM-based system?

An experimental validation was carried out in order to address these research questions. In this experimental validation two different experiments were executed. In the first one, the response time to inefficiencies (specifically, product shortages) is qualitatively compared in our proposed protoype with 
a traditional tag-based traceability system (similar to the one described in [23]). In the second one, we compare a traditional tag-based traceability system with our proposed TF4SM-base prototype, where notifications to users about process execution are activated for the number of inefficiencies by worker.

The total response time to inefficiencies may be grouped into three different times (Figure 13). The time-to-alert time starts when the inefficiency occurs and finishes when an alert is generated in the process monitoring system. Secondly, the time-to-reaction time starts when an alert is generated in the process monitoring system and finishes when the person in charge responds to the alert and identifies the solution. Finally, the time-to-solution time involves all the time needed to solve the problem previously identified.

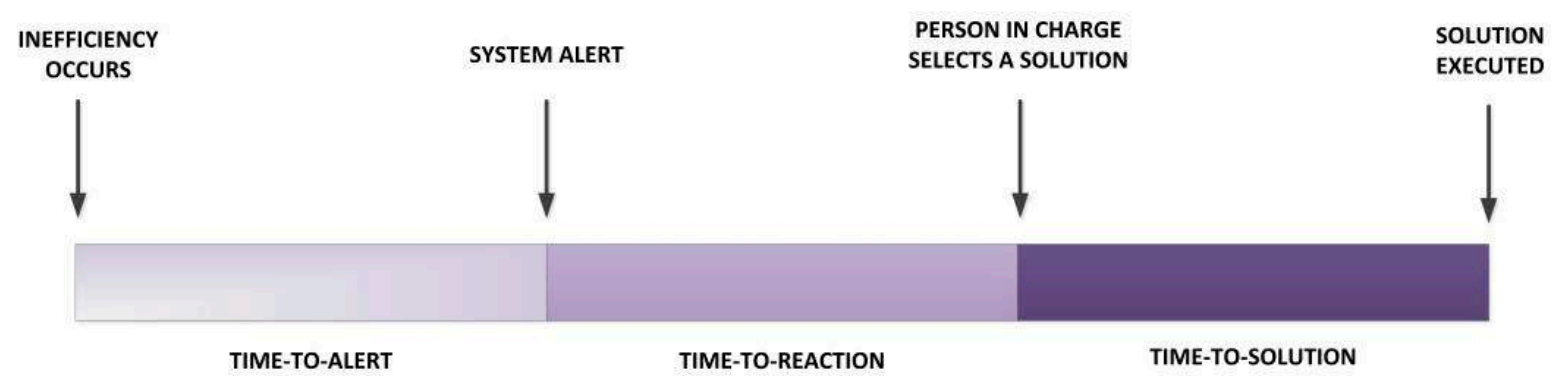

Figure 13. Response time to inefficiencies decomposition.

One of the most important inefficiency types, in which response time is critical, is product shortages. If the amount of remaining product is not accurately controlled, it might not only stop production (such as when other types of inefficiencies occur), but also all the processes in which the product is needed (which can be most processes if it is a basic product).

In product shortages, the time-to-solution is independent of the monitoring process implemented as it is more related to the presence or not of automatic order systems. Consequently, we conducted an experiment to qualitatively compare a traditional tag-based traceability system with our prototype for the time-to-alert and time-to-reaction times in the case of product shortage.

Thirty six (36) people were involved in this first experiment. In our manufacturing scenario, these people were divided into two equal groups called the TF4SM and TAG groups. Both groups were isolated from each other. Within each group four different productive processes were assigned at random among the participants, and one additional person was in charge of solving the possible product shortages. 56 different products were located in the warehouse, but the amount of some of them was insufficient to finalize all the processes in execution.

In the TF4SM group all the products were on a cybernetic table that monitored the amount of remaining product. Each worker also wore our cybernetic glove. The person in charge was also provided with a mobile visualization platform for controlling the warehouse state.

In the $T A G$ group, each worker had a tag-reader, which he had to pass over the product's tag before removing one unit from the warehouse. There was also a control point where commercial retail control software runs, and where the person in charge could verify the products available in the warehouse.

Finally, an external observatory was placed in both groups to qualitatively evaluate the time-to-alert and time-to-reaction times. 
In the second experiment, the number of inefficiencies was evaluated. In general, inefficiencies were due to three types of errors. Firstly, there were inefficiencies due to procedural errors. In these cases, an activity was not executed in the correct order, unfinished or some step had not been executed. Secondly, there were inefficiencies due to execution errors. In these cases, all steps of the activity were executed, but (at least) one of them had not been developed as expected (a badly tightened screw or badly applied paint were examples of such errors). Finally, there were inefficiencies due to other errors, such as power outages and shortages.

In traditional tag-based systems, all errors are considered equal, and were evaluated at the workflow's end (in the so-called quality control point). However, our TF4SM-base prototype allows to provide users with real-time information on process execution and, therefore, it enables the possibility of notifying procedure errors as they occur. Thus, workers can correct their last action, thereby reducing the number of errors when the product is delivered for quality control.

To compare the inefficiencies in a manufacturing company where a traditional tag-based traceability system was deployed with the inefficiencies in a manufacturing company where our proposed TF4SM-base prototype was available, we conducted the following validation.

Thirty six (36) people were involved in this second experiment. In our warehouse scenario, these people were divided into two separate groups. TF4SM was made up of 20 people and non-TF4SM the remaining 16.

The TF4SM group went one by one to a cybernetic table where they had to run a 10-step workflow. Each user had 16 kinds of products, of which only nine had to be processed, and they wore our cybernetic glove. During the execution of the workflow, our prototype monitored the process in real-time and alerted workers by means of a red LED whether they had performed an action that was not provided. At any time workers could undo any action. At the end of the workflow, an expert validated the final product and, in case of inefficiency, he specified the number of errors and type.

The non-TF4SM group worked in a similar way, except that in this case; the prototype was turned off, leaving only as checkpoint the final quality control (as in most manufacturing processes).

\section{Results}

This section presents and discusses the results obtained in the experimental validation. Results are shown following the research questions defined in this paper. Table 5 summarizes the magnitude order of the time-to-alert and time-to-reaction times for the first experiment.

Table 5. Devices' configuration.

\begin{tabular}{ccc}
\hline System Employed & Time-to-Alert Time & Time-to-Reaction Time \\
\hline Tag-based system & Less than $10 \mathrm{~s}$ & Between 1 and $3 \mathrm{~min}$ \\
TF4SM & Immediate & Less than $30 \mathrm{~s}$ \\
\hline
\end{tabular}

Some considerations should be given to Table 5. First, we verified that time response to inefficiencies improved by deploying our prototype. As the system required no consumables, the only expenditure was the initial investment, so we can say that we have achieved this improvement. Second, we can explore some reasons for this improvement. In respect to time-to-alert time, the need for human intervention in traditional tag-based systems causes, inevitably, higher delays. Operations, such as finding the tag or 
preparing the reader, can be made really fast, but automatic systems (such as the one TF4SM framework proposes) will always be much faster than humans. With respect to time-to-reaction time, the event monitoring independence from hardware (achieved in TF4SM framework) allows to develop mobile platforms that are permanently connected to the person in charge (who can respond to the emergency very quickly). In traditional tag-based systems, software commercial platforms require hard configurations and, in most cases, specific hardware to be deployed. Therefore, as in our experiment, in most companies there is a fixed control point that is periodically reviewed by managers. Depending on the review period, events may be processed faster or slower.

Considering the second experiment, Figure 14 shows two boxplot measuring the number of errors committed by the TF4SM group (median = 3.5, $\mathrm{SD}=1.76$ ) and the non-TF4SM group (median =6, $\mathrm{SD}=2.28$ ).

As both boxplots have an overlapping area, a Mann-Whitney U test was conducted to confirm whether the use of our proposed TF4SM framework reduces the number of errors. The Mann-Whitney U test is a nonparametric test of the null hypothesis that two samples come from the same population against an alternative hypothesis, comparing the mean values of the two samples. It is used to evaluate if two different data populations are similar or different (higher or lower). The $p$-value indicates the significance level of Mann-Whitney $U$ test. The results are positive and support the previous assertion within the expected significance, $p<0.005$ (U-values omitted as the comparison results have relatively little importance).

As can be seen, our proposed TF4SM framework does not eliminate the inefficiencies, but it allows workers to make some mistakes (specifically, procedure errors) and gives them the opportunity to correct a production task, which, in the end, reduces the number of inefficiencies (answering in this way our second research question).

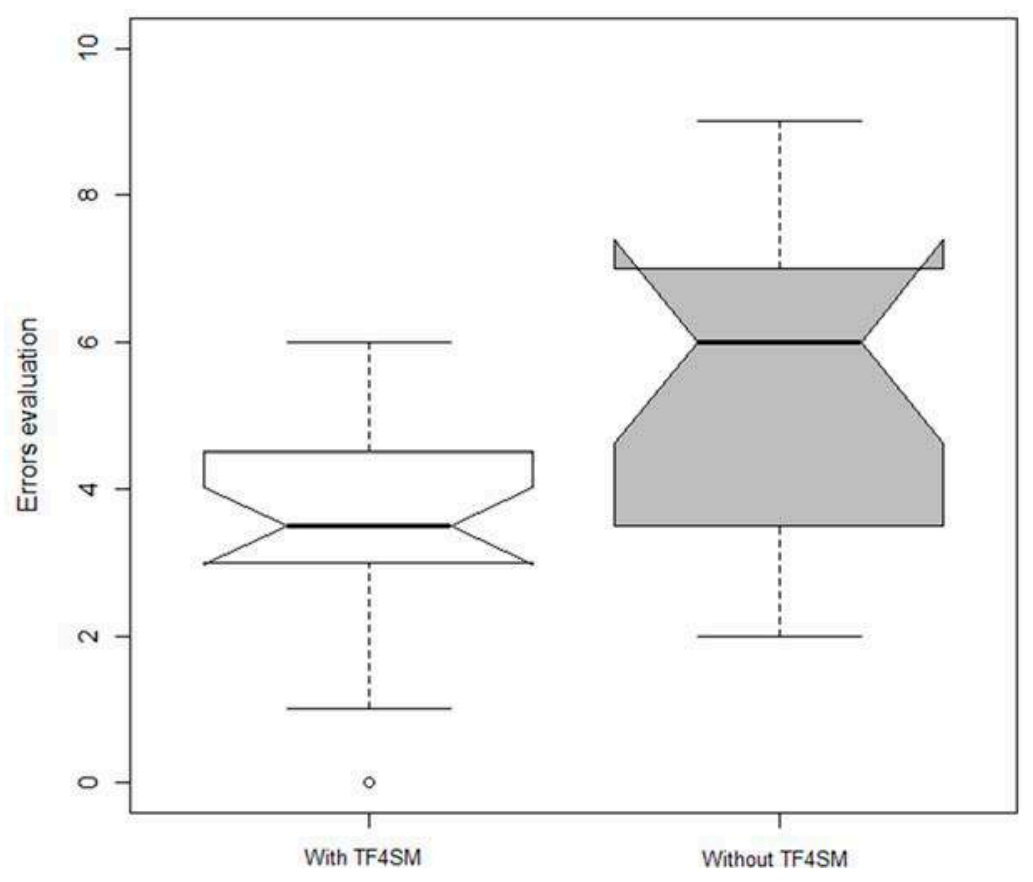

Figure 14. Response time to inefficiencies decomposition. 


\section{Conclusions}

Traceability systems are rapidly becoming very important in manufacturing sector. Governments, costumers, workers among others ask companies for tighter controls in production processes. However, the complexity associated with manufacturing processes nowadays makes this task greatly complicated, especially for small companies. On the one hand, traditional traceability systems increase costs when logistics increases in complexity, and the necessary investments are not always acceptable for small companies. On the other hand, any type of completely automatic process monitoring or traceability system is widely used in industry, so companies are reluctant to implement such solutions (except in the case of proprietary solutions, something unattainable for small companies). Our Traceability Framework For Small Companies (TF4SM) fills this gap, by allowing real-time traceability and process monitoring through a flexible, open architecture (based on CPS NIST definition) capable of adapting to all types of manufacturing companies.

With TF4SM-based systems small manufacturing companies can monitor both workers' actions and movement of the products at any given moment. This, together with the ability to use mobile platforms for system control, allows a significant reduction in the response time to inefficiencies obtained in our systems, compared to traditional tag-based traceability systems.

Moreover, the use of real-time monitoring enables the possibility of notifying users of their errors, allowing the workers to correct them (when it is possible). Thus, the number of inefficiencies in companies that implement TF4SM-based traceability systems is lower than in companies that use traditional traceability systems.

\section{Author Contributions}

The contributions described in this work are distributed among the authors in the way that follows: All the authors conceived and designed the experiments; Borja Bordel built the prototypes; Ramón Alcarria contributed to process modeling and design; Diego Martín performed the experiments; Diego Martín and Tomás Robles analyzed the data; Borja Bordel and Ramón Alcarria wrote the paper.

\section{Conflicts of Interest}

The authors declare no conflict of interest.

\section{References}

1. European Project: Real-time Monitoring and Optimization of Resource Efficiency in Integrated Processing Plants (FP7-NMP-2013-SMALL-7), Supported by the European Commission. Available online: http://cordis.europa.eu/project/rcn/110822_en.html (accessed on 26 August 2015).

2. Woodall, W.H.; Montgomery, D.C. Some current directions in the theory and application of statistical process monitoring. J. Qual. Technol. 2014, 46, 78-94.

3. Weske, M. Business Process Management: Concepts, Languages, Architectures; Springer Science \& Business Media: Berlin, Germany, 2012.

4. Condea, C.; Thiesse, F.; Fleisch, E. RFID-enabled shelf replenishment with backroom monitoring in retail stores. Decis. Support Syst. 2012, 52, 839-849. 
5. Cook, D.; Das, S. Smart Environments: Technology, Protocols and Applications; John Wiley \& Sons: Hoboken, NJ, USA, 2004.

6. Gungor, V.C.; Hancke, G.P. Industrial wireless sensor networks: Challenges, design principles, and technical approaches. IEEE Trans. Ind. Electron. 2009, 56, 4258-4265.

7. Al Agha, K. Which wireless technology for industrial wireless sensor networks? The development of OCARI technology. IEEE Trans. Ind. Electron. 2009, 56, 4266-4278.

8. Cao, H.; Folan, P.; Mascolo, J.; Browne, J. RFID in product lifecycle management: A case in the automotive industry. Int. J. Comput. Integr. Manuf. 2009, 22, 616-637.

9. Dai, Q.Y.; Zhong, R.Y.; Zhou, K.; Jiang, Z.Y. A RFID-enabled Real-Time Manufacturing Hardware Platform for Discrete Industry. In Proceeding of the 6th CIRP-Sponsored International Conference on Digital Enterprise Technology, Hong Kong, China, 14-16 December 2010; Springer-Verlag: Berlin, Heidelberg, 2010; Volume 66, pp. 1743-1750.

10. Banks, J.; Pachano, M.A.; Thompson, L.G.; Hanny, D. RFID Applied; John Wiley \& Sons: Hoboken, NJ, USA, 2007.

11. Ilie-Zudor, E.; Kemény, Z.; van Blommestein, F.; Monostori, L.; van der Meulen, A. A survey of applications and requirements of unique identification systems and RFID techniques. Comput. Ind. 2010, 62, 227-252.

12. Dai, Q.; Zhong, R.; Huang, G.Q.; Qu, T.; Zhang, T.; Luo, T.Y. Radio frequency identification-enabled real-time manufacturing execution system: A case study in an automotive part manufacturer. Int. J. Comput. Integr. Manuf. 2012, 25, 51-65.

13. Hur, S.M.; Jeong, S.; Suh, S.H. An experimental approach to RFID system performance prediction model. Int. J. Comput. Integr. Manuf. 2009, 22, 686-697.

14. Bendavid, Y.; Cassivi, L. Bridging the gap between RFID/EPC concepts, technological requirements and supply chain e-business processes. J. Theor. Appl. Electron. Commer. Res. 2010, 5, 1-16.

15. Lee, E.A. Cyber-physical systems-Are computing foundations adequate? In NSF Workshop on Cyber-Physical Systems: Research Motivation, Techniques and Roadmap, Austin, TX, USA, 16-17 October 2006.

16. National Institute of Standards and Technology. Cyber-Physical Systems Homepage. Available online: http://www.nist.gov/cps/ (accessed on 19 November 2015).

17. National Institute of Standards and Technology. CPS Public Working Group Presentation. Available online: http://www.nist.gov/el/upload/CPS-PWG-Kickoff-Webinar-Presentation-FINAL.PDF (accessed on 26 October 2015).

18. Wang, Y.; Vuran, M.C.; Goddard, S. Cyber-physical systems in industrial process control. ACM SIGBED Rev. 2008, 5, doi:10.1145/1366283.1366295.

19. Wu, F.; Kao, Y.; Tseng, Y. From wireless sensor networks towards cyber physical systems. Pervasive Mob. Comput. 2011, 7, 397-413.

20. Li, S. Industrial wireless sensor networks. Int. J. Distrib. Sensor Netw. 2014, 2014, 1-2.

21. Software Im Maschinenbau-Lästiges Anhängsel Oder Chance Zur Marktführerschaft? Available online: http://www.software-kompetenz.de/servlet/is/21700/StetterSW_im_Maschinenbau.pdf?command =downloadContent\&filename=Stetter-SW_im_Maschinenbau.pdf (accessed on 26 October 2015).

22. Automation Expenditures for Discrete Industries. Available online: http://www.arcweb.com/ market-studies/pages/automation-systems-for-discrete-industries.aspx (accessed on 26 October 2015). 
23. International Electrotechnical Commission. International Standard IEC 62264-1: Enterprise-Control System Integration Part 1: Models and Terminology; IEC: Geneva, Switzerland, 2003.

24. Hussain, T.; Frey, G. Migration of a PLC controller to an IEC 61499 compliant distributed control system: Hands-on experiences. In Proceedings of the IEEE International Conference on Robotics and Automation, Barcelona, Spain, 18-22 April 2005; pp. 3984-3989.

25. OPC 2.1 Standard. Available online: https://opcfoundation.org/ (accessed on 26 October 2015).

26. Davenport, T.H. Process Innovation: Reengineering Work through Information Technology; Harvard Business Press: Boston, MA, USA, 2013.

27. Vyatkin, V. Software engineering in industrial automation: State-of-the-art review. IEEE Trans. Ind. Inform. 2013, 9, 1234-1249.

28. Thramboulidis, K.; Zoupas, A. Real-time Java in control and automation: A model driven development approach. In Proceedings of the 10th IEEE Conference on Emerging Technologies and Factory Automation, Catania, Italy, 19-22 September 2005; pp. 8-46.

29. Jimenez, M.; Rosique, F.; Sanchez, P.; Alvarez, B.; Iborra, A. Habitation: A domain-specific language for home automation. IEEE Softw. 2009, 26, 30-38.

30. Alcarria, R.; Robles, T.; Morales, A.; López-de-Ipiña, D.; Aguilera, U. Enabling flexible and continuous capability invocation in mobile prosumer environments. Sensors 2012, 12, 8930-8954.

31. Erl, T. Service-Oriented Architecture: Concepts, Technology, and Design; Prentice Hall PTR: Upper Saddle River, NJ, USA, 2005.

32. Jammes, F.; Smit, H. Service-oriented paradigms in industrial automation. IEEE Trans. Ind. Inform. 2005, 1, 62-70.

33. Kirova, V.; Kirby, N.; Kothari, D.; Childress, G. Effective requirements traceability: Models, tools, and practices. Bell Labs Tech. J. 2008, 12, 143-157.

34. Moe, T. Perspectives on traceability in food manufacture. Trend Food Sci. Technol. 1998, 9, 211-214.

35. Muldoon, M. Integrating information systems to improve competitiveness. Brew. Distill. Int. 1992, $23,13-14$.

36. Wall, B. Golden gains from weightwatching. Potato Bus. World 1995, 3, 34-37.

37. Li, M.; Qian, J.P.; Yang, X.T.; Sun, C.H.; Ji, Z.T. A PDA-based record-keeping and decision-support system for traceability in cucumber production. Comput. Electron. Agric. 2010, 70, 69-77.

38. Seine, K.; Kuwabara, S.; Mikami, S.; Takahashi, Y.; Yoshikawa, M.; Narumi, H.; Koganezaki, K.; Wakabayashi, T.; Nagano, A. development of the traceability system which secures the safety of fishery products using the QR code and a digital signature. In Proceedings of the OCEANS'04. MTTS/IEEE TECHNO-OCEAN '04, Kobe, Japan, 9-12 November 2004; pp. 476-481.

39. Hsu, Y.-C.; Chen, A.-P.; Wang, C.-H. A RFID-enabled traceability system for the supply chain of live fish. In Proceedings of the IEEE International Conference on Automation and Logistics, Qingdao, China, 1-3 September 2008; pp. 81-86.

40. Gandino, F.; Montrucchio, B.; Rebaudengo, M.; Sanchez, E.R. On improving automation by integrating RFID in the traceability management of the Agri-food sector. IEEE Trans. Ind. Electron. 2009, 56, 2357-2365.

41. Das, S.K.; Cook, D.J. Agent based health monitoring in smart homes. In Proceedings of the International Conference on Smart Homes and Health Telematics (ICOST), Singapore, 15-17 September 2004; pp. 3-14. 
42. Das, S.K.; Cook, D.J.; Battacharya, A.; Heierman, E.O., III; Lin, T-Y. The role of prediction algorithms in the MavHome smart home architecture. IEEE Wirel. Commun. 2002, 9, 77-84.

43. Le Gal, C.; Martin, J.; Lux, A.; Crowley, J.L. Smart office: Design of an intelligent environment. IEEE Intell. Syst. 2001, 4, 60-66.

44. Lesser, V.; Atighetchi, M.; Benyo, B.; Horling, B.; Raja, A.; Wagner, T.; Xuan, P.; Zhang, S. The intelligent home testbed. In Proceedings of the Autonomy Control Software Workshop, Seattle, WA, USA, 29 January 1999.

45. Misra, A.; Das, S.K. Location estimation (determination and prediction) techniques in smart environments. In Smart Environments: Technology, Protocols and Applications; Cook, D., Das, S., Eds.; John Wiley \& Sons: Hoboken, NJ, USA, 2005; pp. 193-228.

46. Nixon, P.; Lacey, G.; Dobson, S. (Eds.) Managing Interactions in Smart Environments: 1st International Workshop on Managing Interactions in Smart Environments (MANSE'99), Dublin, December 1999; Springer-Verlag: London, UK, 2000.

47. Derler, P.; Lee, E.A.; Vincentelli, A.S. Modeling cyber-physical systems. Proc. IEEE 2012, 100, 13-28.

48. Lee, E.A. The past, present and future of cyber-physical systems: A focus on models. Sensors $\mathbf{2 0 1 5}$, 15, 4837-4869.

49. Kelepouris, T.; Pramatari, K.; Doukidis, G. RFID-enabled traceability in the food supply chain. Ind. Manag. Data Syst. 2007, 107, 183-200.

50. Riedl, M.; Zipper, H.; Meier, M.; Diedrich, C. Cyber-physical systems alter automation architectures. Annu. Rev. Control 2014, 38, 123-133.

51. Colombo, A.; Karnouskos, S.; Bangemann, T. Towards the Next Generation of Industrial Cyber-Physical Systems. In Industrial Cloud-based Cyber-physical Systems; Colombo, A., Bangemann, T., Karnouskos, S., Delsing, J., Stluka, P., Harrison, R., Jammes, F., Lastra, J.L., Eds.; Springer International Publishing: Geneva, Switzerland, 2014; pp. 1-22.

52. Al-Anbagi, I.; Erol-Kantarci, M.; Mouftah, H.T. A reliable IEEE 802.15. 4 model for cyber physical power grid monitoring systems. IEEE Trans. Emerg. Top. Comput. 2013, 1, 258-272.

53. Blackburn, M.; Denno, P. Virtual design and verification of cyber-physical systems: Industrial process plant design. Proced. Comput. Sci. 2014, 28, 883-890.

54. Gupta, I.; Kaur R. Realization of automated industrial pollution control system using LabVIEW. Int. J. Res. 2014, 1, 834-838.

55. Suender, C.; Wenger, M.; Hanni, C.; Gosetti, I.; Steininger, H.; Fritsche, J. Transformation of existing IEC 61131-3 automation projects into control logic according to IEC 61499. In Proceedings of the IEEE International Conference on Emerging Technologies and Factory Automation, Hamburg, Germany, 15-18 September 2008; pp. 369-376.

56. Wenger, M.; Zoitl, A.; Sunder, C.; Steininger, H. Transformation of IEC 61131-3 to IEC 61499 based on a model driven development approach. In Proceedings of the 7th IEEE International Conference on Industrial Informatics, Cardiff, UK, 23-26 June 2009; Volume 1, pp. 715-720.

57. Von Krosigk, H. Functional safety in the field of industrial automation. The influence of IEC 61508 on the improvement of safety-related control systems. Comput. Control Eng. J. 2000, 11, 13-18.

58. Ito, M. Smart Furniture: Improvising ubiquitous hot-spot environment. In Proceedings of the 23rd International Conference on Distributed Computing Systems Workshops, Providence, RI, USA, 19-22 May 2003; pp. 248-253. 
59. Tokuda, H.; Takashio, K.; Nakazawa, J.; Matsumiya, K.; Ito, M.; Saito, M. SF2: Smart furniture for creating ubiquitous applications. In Proceedings of the International Symposium on Applications and the Internet, Tokyo, Japan, 26-30 January 2004; pp. 423-429.

60. Hagale, A.R.; Kelley, J.E.; Rozich, R. RFID Smart Office Chair. U.S. Patent 6,964,370, 15 November 2005.

61. Fishkin, K.P.; Philipose, M.; Rea, A. Hands-on RFID: Wireless wearables for detecting use of objects. In Proceedings of the 9th International Symposium on Wearable Computers, Osaka, Japan, 18-21 October 2005; pp. 38-43.

62. Fujitsu's Smart Glove. Available online: http://www.qore.com/articulos/17335/Fujitsu-desarrolla-unguante-de-realidad-aumentada (accessed on 26 October 2015).

63. Kirstein, T.; Cottet, D.; Grzyb, J.; Tröster, G. Textiles for signal transmission in wearables. In Proceedings of the ACM of First Workshop on Electronic Textiles (MAMSET 2002), San Jose, CA, USA, 4 October 2002.

64. Marculescu, D. Electronic textiles: A platform for pervasive computing. IEEE Proc. 2003, 91, 1995-2018.

65. Giochi SmartPoker. Available online: http://www.gtigaming.com/en/product-service/smartpoker-2/ (accessed on 18 July 2015).

66. Argos Smart Clinical Cupboard. Available online: http://sicolareshigia.com/armario-de-control-destock/ (accessed on 18 July 2015).

67. Palex Smart Clinical Cupboard. Available online: http://www.europapress.es/extremadura/salud00924/noticia-infanta-cristina-badajoz-acogera-forma-piloto-armario-inteligente-suministro-materialsanitario-20150225143205.html (accessed on 26 October 2015).

68. Izco Smart Clinical Cupboard. Available online: http://www.farodevigo.es/economia/2014/09/28/ viguesa-izco-lanza-fabricacion-armarios/1102202.html (accessed on 26 October 2015).

69. SATO's VINICITY Technology. Available online: http://www.satovicinity.com/sp/ products_magellan_pjm_rfid_smart_readers.asp (accessed on 26 October 2015).

70. Wolf, M. High-Performance Embedded Computing: Applications in Cyber-Physical Systems and Mobile Computing, 2nd ed.; Morgan Kaufmann: Burlington, MA, USA, 2014.

71. Kortuem, G.; Kawsar, F.; Fitton, D.; Sundramoorthy, V. Smart objects as building blocks for the internet of things. IEEE Internet Comput. 2010, 14, 44-51.

72. Osaka, K.; Takagi, T.; Yamazaki, K.; Takahashi, O. An efficient and secure RFID security method with ownership transfer. In Proceedings of the International Conference on Computational Intelligence and Security, Guangzhou, China, 3-6 November 2006; Volume 2, pp. 1090-1095.

73. Schmidt, A. Implicit human computer interaction through context. Pers. Technol. 2000, 4, 191-199.

74. Fouquet, F. A dynamic component model for cyber physical systems. In Proceedings of the 15th ACM SIGSOFT Symposium on Component Based Software Engineering, Bertinoro, Italy, 25-28 June 2012; pp. 135-144.

75. Kim, K.; Panganamala, R. Cyber-physical systems: A perspective at the centennial. IEEE Proc. 2012, 100, 1287-1308.

76. Morales, A.; Robles, T.; Alcarria, R.; Cedeño, E. On the support of scientific workflows over Pub/Sub brokers. Sensors 2013, 13, 10954-10980. 
77. Fortino, G.; Guerrieri, A.; Russo, W. Agent-oriented smart objects development. In Proceedings of the IEEE 16th International Conference on Computer Supported Cooperative Work in Design, Wuhan, China, 23-25 May 2012; pp. 907-912.

78. RDM 8800 Datasheet. Available online: ftp://imall.iteadstudio.com/Modules/IM131218001/ DS_IM131218001.pdf (accessed on 26 October 2015).

79. Tang, L. Trustworthiness analysis of sensor data in cyber-physical systems. J. Comput. Syst. Sci. 2013, 79, 383-401.

80. Schmitt, C. TinyIPFIX: An efficient application protocol for data exchange in cyber physical systems. Comput. Commun. 2014, doi:10.1016/j.comcom.2014.05.012.

81. Zuo, X.; Tan, W.; Lin, H. Cigarette production scheduling by combining workflow model and immune algorithm. IEEE Trans. Autom. Sci. Eng. 2014, 11, 251-264.

(C) 2015 by the authors; licensee MDPI, Basel, Switzerland. This article is an open access article distributed under the terms and conditions of the Creative Commons Attribution license (http://creativecommons.org/licenses/by/4.0/). 


\title{
Enhancing Process Control in Industry 4.0 Scenarios using Cyber-Physical Systems
}

\author{
Borja Bordel Sánchez ${ }^{1 *}$, Ramón Alcarria ${ }^{2}$, Diego Sánchez-de-Rivera ${ }^{1}$, and Álvaro Sánchez-Picot ${ }^{1}$ \\ ${ }^{1}$ Department of Telematics Systems Engineering. Universidad Politécnica de Madrid \\ \{bbordel, diegosanchez, asanchez\}@dit.upm.es \\ ${ }^{2}$ Department of Topographic Engineering and Cartography. Universidad Politécnica de Madrid \\ ramon.alcarria@upm.es
}

\begin{abstract}
One of the most interesting applications of Industry 4.0 paradigm is enhanced process control. Traditionally, process control solutions based on Cyber-Physical Systems (CPS) consider a top-down view where processes are represented as executable high-level descriptions. However, most times industrial processes follow a bottom-up model where processes are executed by low-level devices which are hard-programmed with the process to be executed. Thus, high-level components only may supervise the process execution as devices cannot modify dynamically their behavior. Therefore, in this paper we propose a vertical CPS-based solution (including a reference and a functional architecture) adequate to perform enhanced process control in Industry 4.0 scenarios with a bottom-up view. The proposed solution employs an event-driven service-based architecture where control is performed by means of finite state machines. Furthermore, an experimental validation is provided proving that in more than $97 \%$ of cases the proposed solution allows a stable and effective control.
\end{abstract}

Keywords: Cyber-Physical Systems, Industry 4.0, Process Control, State Machine, Legacy Systems

\section{Introduction}

In the last ten years many solutions based on Cyber-Physical Systems (CPS) [1] have been proposed: from Ambient Intelligence (AmI) and healthcare systems [2] to traceability solutions [3]. However, the best opportunity to develop relevant products based on CPS was born in 2014 with the Industry 4.0 paradigm [4]. Industry 4.0 refers to the deep integration of next generation information technologies (such as CPS or AmI) into industrial scenarios, solutions and procedures. In that way, innovations about CPS finally could be applied to a particular application scenario, and specific and tangible contributions would be expectable.

As CPS are usually defined as integrations of computation with physical processes [5], the most direct application of CPS in Industry 4.0 scenarios is enhanced process control. In fact, traditional process control is based on closed feedback loops [6] where (i) data about the process are collected using sensors, (ii) the acquired information is processed and the actions to be taken are calculated and (iii) actuators change their state along the calculated decisions. On the contrary, CPS may enhance traditional process control as they refer a class of devices being able to get synchronized with physical processes [7]. In that way, it is not necessary to define additional feedback loops, as CPS integrates all the mechanisms to reach and maintain a synchronized state.

Depending on the defined architecture, data flow in CPS may be vertical, horizontal or even transversal. Any case, most proposals on CPS-based process control describe systems where processes are

\footnotetext{
Journal of Wireless Mobile Networks, Ubiquitous Computing, and Dependable Applications, 7:4 (December 2016), pp. 41.64

${ }^{*}$ Corresponding author: E.T.S.I. Telecomunicación. Universidad Politécnica de Madrid. Avenida Complutense $\mathrm{n}^{\mathrm{0}} 30$. 28040 - Madrid (España), Tel. 915495700 ext. 3035
} 
defined by users using high-level languages, which are later translated into executable code [8]. This code is, finally, executed by hardware devices. In general, this approach is known as top-down view [9]. However, in real industrial scenarios, processes used to be hard-programmed into low-level devices, and no interface to modify that programming is publicly offered. Devices only notify important events or changes by activating a flag which may be monitored using technologies such as OPC [10]. This situation is the final cause of the implementation of supervisory control systems such as SCADA [11] which may integrate all the flags from devices and translate them into human-readable information. This approach is usually known as bottom-up view. The authors argue that CPS-based process control systems for Industry 4.0 have to be designed to replace the old supervisory control systems, integrating hardware devices into new and enhanced process control solutions. New CPS architectures and solutions following a bottom-up approach are, then, required.

Therefore, in this paper, a new solution for bottom-up process control systems based on CPS is described. The presented solution includes a reference architecture which is defined from the numerous proposals which have been described in the research literature and from the particular requirements of Industry 4.0 scenarios. A detailed functional architecture is also provided, whose behavior is based on the sending of events among the different components which made up the system. The entire solution is based on the concept of service, which is complemented with finite state machine technologies such as Amazon States Language (ASL). Furthermore, in order to guarantee a stable control, the described solution supports executions with a guarantee Quality-of-Service (QoS).

The rest of the paper is organized as follows: Section 2 presents the state of the art in CPS architectures and process control systems. Section 3 presents the contributions of the article. Section 4 describes the experimental validation. Finally, Section 5 and 6 explain some results of this experimental validation and the conclusions of our work.

\section{State of the art}

Ten relevant CPS reference architectures have been described in research literature or in different drafts by standardization organizations [12]. Namely (Figure11 represents all of them):

- ARCH\#1. Service-based CPS architecture. Proposed by La et al. [13], it is the architecture most similar to the traditional process control systems. Basically, it consists on a physical platform, a control system and, as a novelty; a service framework which allows performing control tasks by means of Internet services (which provides a higher flexibility). Its main disadvantage is its low integration level, which should be improved in order to advance towards more efficient solutions as required by Industry 4.0. Moreover, deploying advanced functionalities (as Industry 4.0 requires) may be complicated if tiers are not explicitly independent and adapted. Thus, additional modules should be added in order to improve its usability.

- ARCH\#2. Basic prototype architecture for CPS. Proposed by Tan et al. [14] it improves various aspect $s$ of ARCH\#1. First, it proposes an event-focused architecture (including a publication/subscription network) which allows implementing both policies of hard and soft control. Additionally, a knowledge extraction module is considered, so hardware-dependent information is independent from the high-level events (although the second ones are calculated from the first ones). A virtual representation of the low-level devices is also considered, where each virtual instance has its own state which may change depending on the received events. As main disadvantage, using this architecture is very difficult to control a process including several devices, as no centralized control unit is deployed (control laws are embedded in hardware devices). Moreover, as the system description is distributed, a heavy context must be maintained (including all the virtual instances and their 
Enhancing Process Control in Industry 4.0 Scenarios using Cyber-Physical Systems
B. Bordel, R. Alcarria, D. Sánchez, A. Sánchez
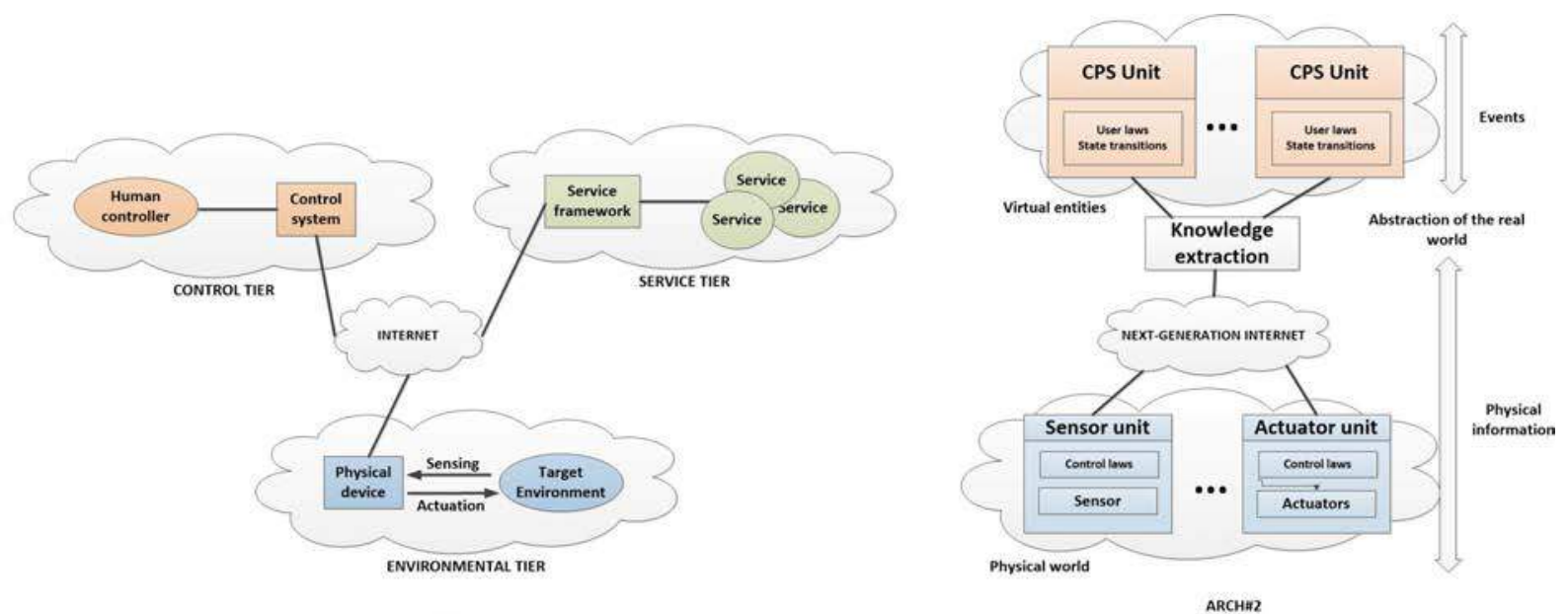

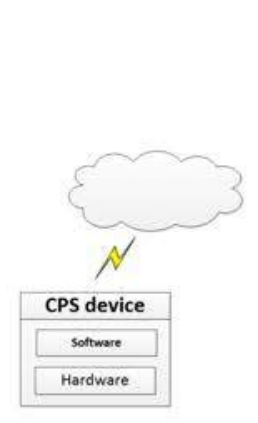

ARCH\#3

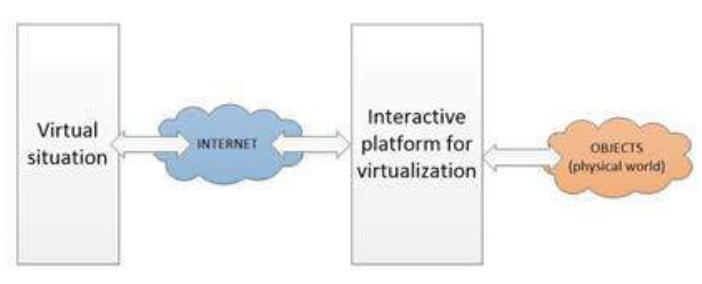

ARCH=6
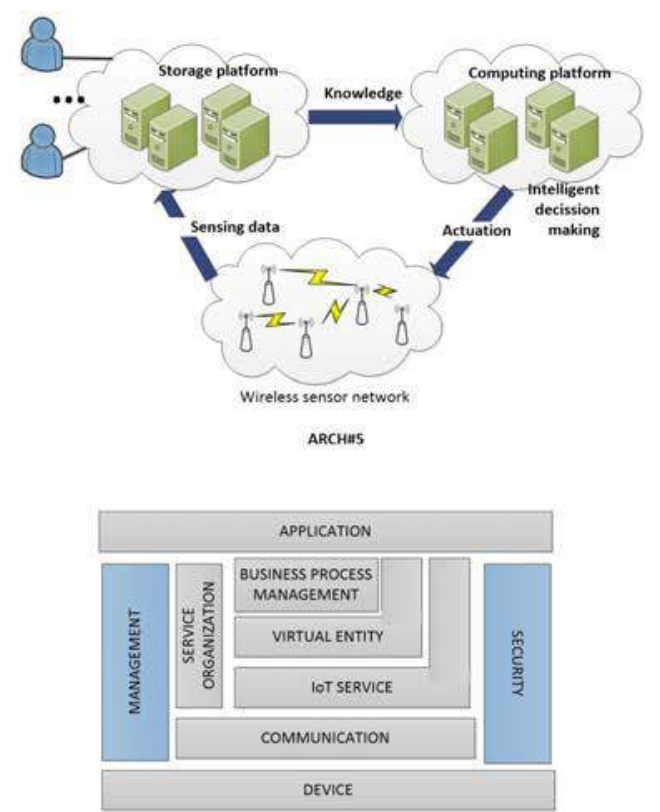

ARCH\#7

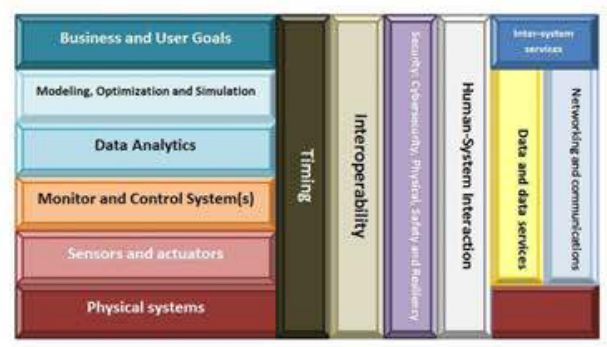

ARCHW8

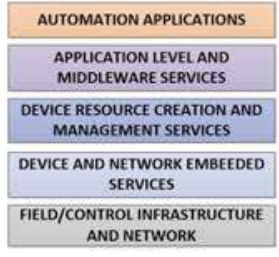

ARCHA4

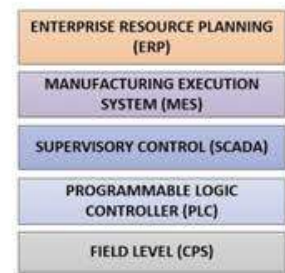

ARCHA9

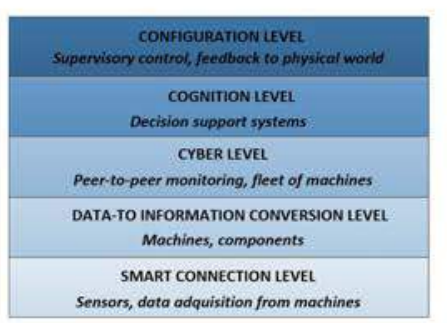

ARCH $\# 10$

Figure 1: Most important proposed architectures on CPS

states) which difficult real-time control (many calculation could be required in order to evaluate the control actions to be taken). 
- ARCH\#3. EuroCPS project architecture. This architecture was proposed as a result of the European project EuroCPS [15]. Basically it represents CPS as a networked collection of embedded devices. It is a very flexible proposal; as almost any current system may be understand as a collection of networked devices. However, it is too much general to meet the specific requirements of Industry 4.0 scenarios (for example, it is not clear how to deploy an enhanced control system).

- ARCH\#4. IoT@Work project architecture. Proposed as a result of the IoT@Work project [16], it was not proposed as a CPS architecture (originally was designed to Internet-of-Things systems). However, some authors have employed it in CPS applications, so should be considered in this group. It is a very conceptual architecture made of five different layers. It is a service-based proposal which addresses problems such as the inter-layer operation. However, it was designed with a top-down view which does not fill at all with Industry 4.0 scenarios. Moreover, it is focused on the deployment of automation applications, which is only a particular case in the Industry 4.0 environment. It is a valuable approach, although, in general, it should be more specific (considering some Industry 4.0 problems such as the geographically spared production locations).

- ARCH\#5. CPS architecture model. Proposed by Wan et al. [17], this architecture is very focused on hardware components. Three different subsystems are distinguished: wireless sensors network, storage and applications platform and a computing system. This structure is very similar to ARCH\#1 and to the traditional process control systems. Its integration level should be improved and connections with other technological systems (such as cloud computing infrastructures) are not clearly indicated as required by Industry 4.0 paradigm. As important characteristic, for first time, an intelligent decision making system (as well as humans) is considered in order to obtain the actuator responses.

- ARCH\#6. Networked CPS architecture. This architecture was proposed by Lai [18] using very traditional concepts about ordinary distributed systems. It is pretty similar to ARCH\#2, and shows the same difficulties to be applied to Industry 4.0 scenarios. As a difference, it explicitly considers an adaptation layer between the virtual entities and the physical world (an important characteristic which should be considered in later proposals).

- ARCH\#7. ISO-IEC architecture. Proposed by the ISO organization [19], it was designed as architecture for the Internet-of-Things and, later, it was applied to CPS. As ARCH\#4 it is a very conceptual proposal, although it is much more exhaustive. As a novelty, it mixes both concepts: virtualization and a service-based approach. As a main disadvantage, it does not consider an information extraction module or any similar instrument to become independent the low-level information from the high-level data. Additionally, this architecture is designed following a top-down view, which does not meet the requirement of Industry 4.0 paradigm. However, it is important to remark the inclusion of a layer dedicated to process management at business level.

- ARCH\#8. NIST architecture. This is the most recent proposal. The National Institute of Standards and Technology proposed in 2014 a reference architecture for CPS focused on being used as based for industrial product [20]. It fact, it considers all the elements necessary to meet the Industry 4.0 (although this architecture is previous to such paradigm): a low-level control layer and a business process management layer, both independent by means of a data analytics layer. It shows, moreover, a clear endpoint to connect with external services (such as cloud computing). Only small additions should be added in order to develop a bottom-up process control system using this architecture. 
- ARCH\#9. AIOTI (EC) architecture. The European Alliance for Internet of Things Innovation (AIOTI) has recently proposed an alternative architecture for CPS [21]. This proposal is also focused on industrial solutions, although it is much more conservative that which proposed by NIST. In particular, it maintains the same structure of traditional process control systems (including the SCADA systems), changing the ordinary sensors and actuators for some embedded devices they called Cyber-Physical Systems. It is very complicated to fit Industry 4.0 new designs to AIOTI architecture, so it has not been almost employed in third-party proposals.

- ARCH\#10. 5C architecture. Proposed by Lee et al. it is the first architecture proposed for Industry 4.0 systems. It is a very remarkable proposal, although it is complicated to be used as base for real developments. It describes five kinds of functions and/or modules which should be present in every CPS in order to fulfill Industry 4.0 requirements. However, capital issues about information technologies (communications, security, data format, etc.) are not addressed, so this proposal used to be employed to check that a certain system meets the Industry 4.0 requirements instead of as a reference architecture.

Considering all the previous discussions, our proposal will be based on NIST architecture, to which some slightly modifications will be added (see Section 3.1). Moreover, it will be checked considering all the functions and requirements showed by $5 \mathrm{C}$ architecture.

Apart from general CPS architectures which could be employed to develop Industry 4.0 systems, some specific works on process control systems based on CPS may be found [22]. These proposals are not very numerous and mostly focused on Smart Grids. These works are usually previous to the CPS definition, but in some occasions the authors employ this paradigm to develop the control subsystem [23] [24]. On the other hand, proposals on pure process control systems based on CPS are rare. In general, they are focused on general aspects about industrial systems [25] or propose process control solutions based on simple networked sensors and actuators (as in ARCH\#3) [26].

Our work, then, fulfills a gap in research, proposing a fully functional process control system, considering all the requirements of Industry 4.0 [27]. In order to reach this objective, the previously cited architectures and their main characteristics are taken into account. Thus, an event-driven service-based solution is designed, considering the NIST reference architecture and the concepts of "state" and "state transition".

\section{Proposed solution}

In this Section the technical solution is described. In the first subsection the reference architecture, as well as a general view of the solution performance, are presented. And, in the second subsection, the technical proposal is presented in detail by means of a specific functional architecture.

\subsection{Architectures and overview of the proposal}

Figure 2 represents the reference architecture considered as base for our proposal. As said before, basically it corresponds with the NIST CPS architecture, where three small modifications have been included. First, two middleware layers have been included in order to show explicitly their existence. Second, the name of the top layer has been modified to clearly show their functions. And, finally, some important components have been remarked inside each layer.

A brief explanation about the proposed architecture (layer by layer) is provided below:

1. Physical system. It represents all the elements which are part of the process and which should be monitored. Most production systems and legacy infrastructures belong to this layer. 


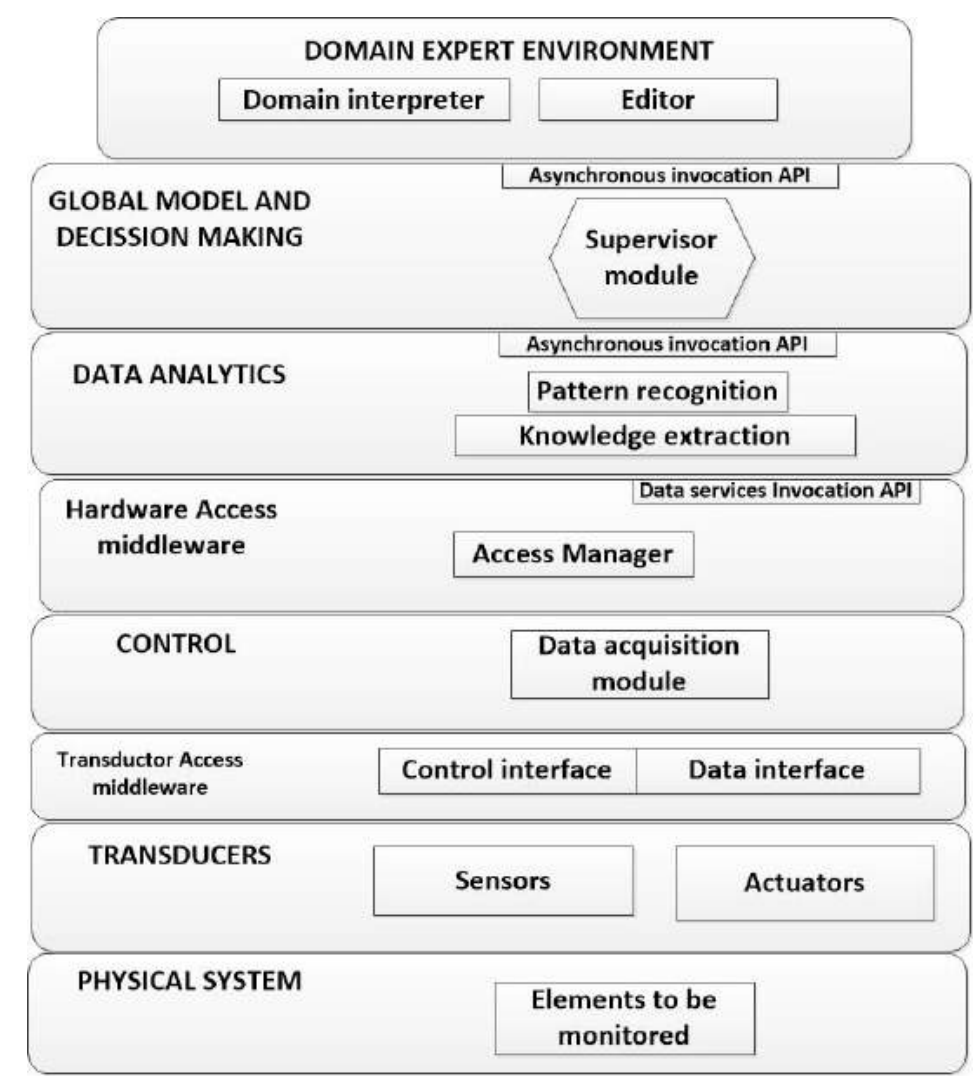

Figure 2: Reference architecture

2. Transducers. It includes both, sensor and actuators. It usually are embedded into the physical system as are included in the same case that the production systems. It refers the modules in charge of monitoring the system activity and activate (or not) a certain flag in the system's hardware interface. This module also may monitor certain input flags in order to trigger a new function or stop the system operation.

3. Transductor access middleware. It refers the protocol employed to transport the information provided by transducers towards the control layer, where a data message codifying the obtained information will be constructed. In most industrial scenarios it is based on the OPC protocol. Although previously only a data interface has been described, in some occasions a control interface could be also available. The system behavior would be the same in both cases, so in this work we are only considering a data interface.

4. Control. It manages the data acquisition by means of the transductor access middleware and elaborates the data format to be transmitted to the higher layers. On the other hand, it interpreters the messages form the high-level components and orders the appropriate changes in the actuators using the cited middleware. In some cases, some of these functionalities are directly embedded in production systems (when next generation infrastructures are considered), so all the previous layers are totally integrated making up a self-managed device.

5. Hardware access middleware. In general, any type of middleware will be adequate. However, in this proposal we are employing an event-based approach (which perfectly fits with the behavior of low-level layers), including publication/subscription technologies (as in ARCH\#1, see Section 2). 
Thus, in this case we are deploying a publication/subscription (P/S) middleware (particularly a MQTT middleware [26]) through which information (expressed as events) is transmitted to processing and decision making modules. An access manager acting as MQTT broker is also considered. Finally, this layer includes a Data service Invocation API, employed to subscribe (or unsubscribe) to a certain type of events.

6. Data analytics. This layer receives the events from the control layer, processes them and extracts the important information. Depending on the sequence of received events, it also may detect a known pattern. With this information, this layer transmits a new high-level (business) event to the supervisor module. In this case, as only two elements are related in the event transmission no P/S technology is required (moreover, its application would be highly inefficient). Instead of that, an asynchronous invocation API (based, for example, on REST services) is included.

7. Global model and decision making. This layer finally receives the high-level events previously generated and updates the current state. When a certain state is left an output activity may be trigger and, besides, when a new state is reached an input activity might be also executed. These activities, in fact, will be used to robustly control the underlying process being executed by the monitored production infrastructure. At this stage, additionally, connections with other systems would be available if needed. Finally, a new event at prosumer (human-understandable) level is generated in order to update the interface for managers or controllers.

8. Domain expert environment. Using this environment, controllers and/or managers may model the sequence of states a certain process may follow. It is important to note that, in general, a detailed description of the process may be not provided as the process evolution depends on the production system programming (usually provided with a PLC -Programmable Logic Controller-) which is unknown (it uses to be protected proprietary technology). However, the sequence of states (which might be as detailed as possible) is well-known as usually it is defined by the type of industry or process.

With this structure, as said before, the proposed architecture fulfills the requirements of Industry 4.0 systems [28]. In particular, (1) self-configure for resilience, (2) self-adjust for variation and (3) self-optimize for disturbance is provided by an adequate control using the input and output activities described above. A (4) remote visualization for humans is provided with the domain expert environment and (5) decision making is considered in the data analytics layer. Other secondary requirements are supported by the default by the physical system or transducers (such as plug\&play).

Table 1 compares the ten described architectures in Section 2 and the solution proposed above. In particular, several important requirements are evaluated, proving our proposal is the unique solution which meets all the described needs for Industry 4.0 scenarios.

Considering the previously proposed architecture, a general overview about how our proposal works is provided on Figure 3. As can be seen, firstly, domain experts should model the process as a fine state machine using graphic domain-specific tools. An interpreter, then, transforms the graphic representation into computer code (such as XML). The generated code is put on memory by the supervisor module in the form of a state transition table. If necessary, the module subscribes to the adequate kinds of events (business events, which are independent from the underlying infrastructure) and updates the system state when necessary. It also orders the execution of the input and output activities and generates the prosumer events (expressed in domain specific languages and easily human-readable format) employed to inform users about the global situation. At this point connections with cloud and other control system could be available. 
Enhancing Process Control in Industry 4.0 Scenarios using Cyber-Physical Systems

B. Bordel, R. Alcarria, D. Sánchez, A. Sánchez

\begin{tabular}{|c|c|c|c|c|c|c|c|c|c|c|c|}
\hline \multirow{2}{*}{$\begin{array}{l}\text { Requirements / } \\
\text { Functionalities }\end{array}$} & \multicolumn{11}{|c|}{ Architectures for Industry 4.0} \\
\hline & $\# 1$ & $\# 2$ & $\# 3$ & $\# 4$ & $\# 5$ & \#6 & \#7 & $\# 8$ & $\# 9$ & \#10 & Our proposal \\
\hline High integration level & & $\checkmark$ & $\checkmark$ & $\checkmark$ & & & $\checkmark$ & $\checkmark$ & $\checkmark$ & $\checkmark$ & $\checkmark$ \\
\hline Independent subsystems & & $\checkmark$ & $\checkmark$ & $\checkmark$ & & $\checkmark$ & $\checkmark$ & $\checkmark$ & $\checkmark$ & $\checkmark$ & $\checkmark$ \\
\hline Central control unit & $\checkmark$ & & & & $\checkmark$ & & & $\checkmark$ & $\checkmark$ & $\checkmark$ & $\checkmark$ \\
\hline $\begin{array}{l}\text { Knowledge extraction } \\
\text { module }\end{array}$ & & $\checkmark$ & & & & $\checkmark$ & & $\checkmark$ & $\checkmark$ & $\checkmark$ & $\checkmark$ \\
\hline $\begin{array}{l}\text { Specific problems of } \\
\text { Industry } 4.0 \text { considered }\end{array}$ & & $\checkmark$ & & $\checkmark$ & & $\checkmark$ & & $\checkmark$ & & $\checkmark$ & $\checkmark$ \\
\hline Bottom-up approach & $\checkmark$ & $\checkmark$ & $\checkmark$ & $\checkmark$ & $\checkmark$ & $\checkmark$ & & $\checkmark$ & $\checkmark$ & $\checkmark$ & $\checkmark$ \\
\hline $\begin{array}{l}\text { Connection with other } \\
\text { systems (cloud...) }\end{array}$ & $\checkmark$ & $\checkmark$ & $\checkmark$ & & $\checkmark$ & $\checkmark$ & $\checkmark$ & $\checkmark$ & & $\checkmark$ & $\checkmark$ \\
\hline $\begin{array}{l}\text { Adaptation layers } \\
\text { are considered }\end{array}$ & & & & $\checkmark$ & & $\checkmark$ & & & $\checkmark$ & $\checkmark$ & $\checkmark$ \\
\hline $\begin{array}{l}\text { Interface for } \\
\text { prosumers included }\end{array}$ & $\checkmark$ & & & & $\checkmark$ & & & $\checkmark$ & & & $\checkmark$ \\
\hline $\begin{array}{l}\text { New CPS era tools } \\
\text { are considered }\end{array}$ & $\checkmark$ & $\checkmark$ & $\checkmark$ & & $\checkmark$ & & $\checkmark$ & $\checkmark$ & & $\checkmark$ & $\checkmark$ \\
\hline $\begin{array}{l}\text { Easy to design a system } \\
\text { using the architecture }\end{array}$ & & $\checkmark$ & & & & $\checkmark$ & $\checkmark$ & $\checkmark$ & $\checkmark$ & & $\checkmark$ \\
\hline
\end{tabular}

Table 1: Comparison among the mentioned architectures

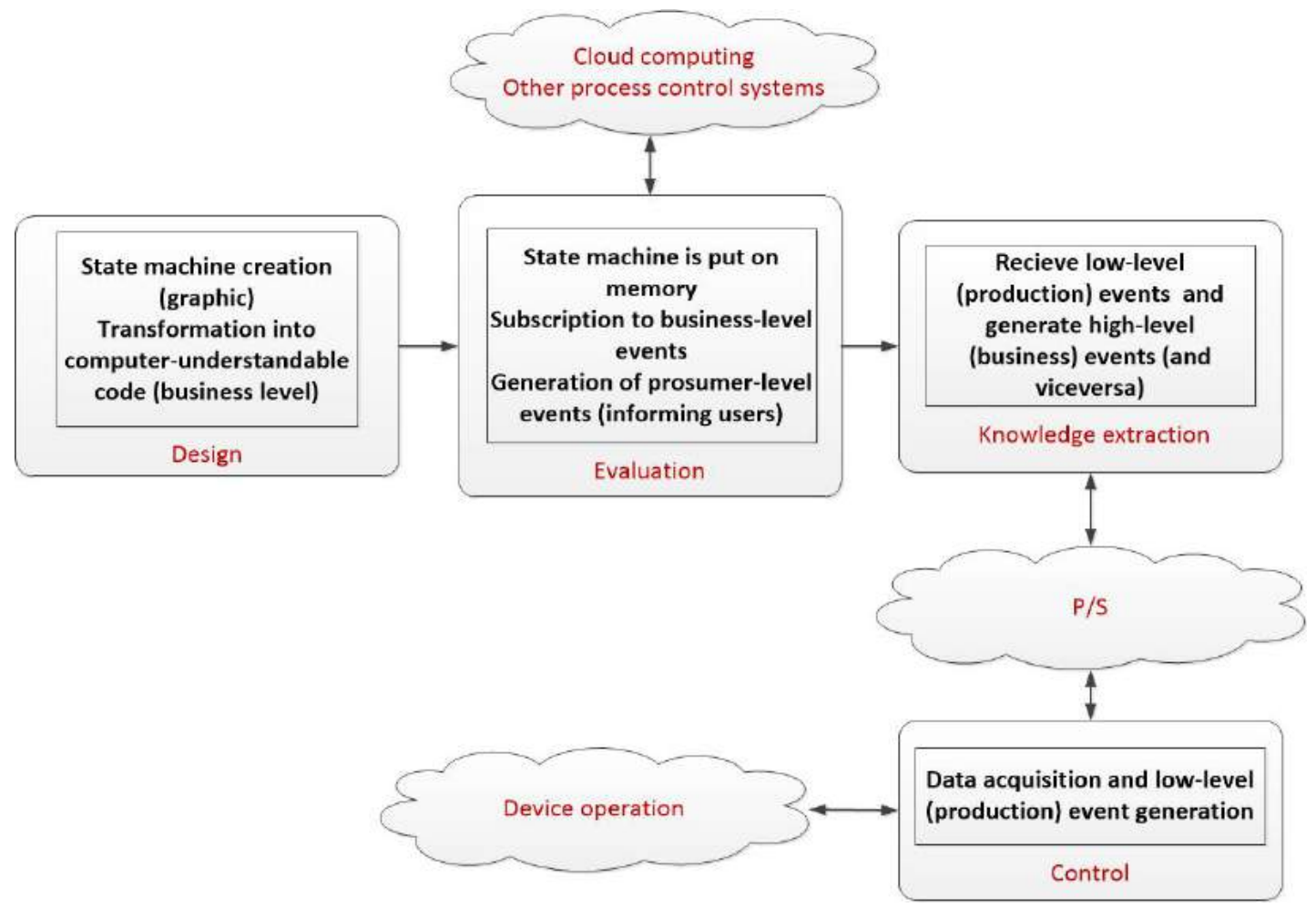

Figure 3: Proposal overview 
The necessary business-level events to update the state in the supervisor module are provided by the knowledge extraction component, which obtains that information from the processing of the production (low-level) events received from the hardware devices. As many hardware devices could be deployed, a $\mathrm{P} / \mathrm{S}$ network is the most adequate mechanism to transport the produced events. To this network, control components will be connected in order to generate and receive the production events. Finally, by means of an industrial protocol (such as OPC) and sensors and actuators, control components will obtain and provide the necessary information from/to hardware devices and production systems.

\subsection{Detailed explanation}

Figure 4 presents the detailed functional architecture of our proposal, and Figure 5 shows a UML diagram describing in a formal way the solution's behavior.

In order to perform control policies over a certain process, the first step is to design the finite state machine (FSM) which models the process, including the input (or entry) and output (or exit) activities for each state. This first model is constructed using a domain specific language, such as graphic DOT language [29] (employed in many contexts such as manufacturing companies) or the UML state charts [30]. Domain experts, thus, may employ the most adequate language for each scenario and programming skills are not required. Figure 6 6 represents a generic process modeled as a FSM.

Additionally, the entry and exit activities must be described. As usually said in the literature [3] activities are ordered collections of atomic operations (or service invocations in service-based architectures). In the prosumer interface, as it is focused on domain experts, all these elements are described in a high abstraction level which we named as prosumer level. At this level, information is expressed in a human-understandable format which, moreover, is independent from the underlying infrastructure. As in the case of the FSM, in order to describe the entry and exit activities a domain language will be employed. Many languages are available depending on the particular type of industry considered. For example, the Process Specification Language (PSL) [31] may be employed in manufacturing scenarios.

In general, descriptions obtained from prosumer interface will depend on the specific application considered and, furthermore, in some cases various languages could be supported at the same time. Then, in order to homogenize the system operation a domain interpreter is added. This interpreter transforms domain-specific descriptions into generic descriptions, employing common languages such as XML. However, the resulting FSMs and activities are still described at prosumer level. Therefore, it is not required that the obtained code from the interpreter to be executable. Languages such as the XML language for UML state charts [32] or the W3C State Chart XML language [33] could be employed in the FSM descriptions. Finally, activities are modeled using a workflow description XML-based language such as BPMN-XML [34], although is our proposal we are employing YAWL [35]. Figure 7]represents the obtained description from the interpreter using as input the description on Figure 6 and the W3C State Chart XML language.

At this step, the designed FSM must be verified in order to perform the control activities. In order to do that, the FSM description (as well as the entry and exit activity descriptions) is uploaded to the verification system, which presents an asynchronous invocation API to communicate with the prosumer interface. This interface may be based on any technology, although in our proposal we are employing REST interfaces [36]. When the REST process verification service is invocated, the FSM description is uploaded and a bidirectional communication based on web sockets is stablished between the user interface and the transformation module (through an unused transport port, in our case port 35357). These open sockets are used to transmit the prosumer events to the prosumer interface in order to inform users about the process evolution. In later occasions, the transmission of events will be based on P/S networks. However, in this case, only two entities are sharing information so P/S solutions are very inefficient; peer-to-peer communications are preferable. 
Enhancing Process Control in Industry 4.0 Scenarios using Cyber-Physical Systems

B. Bordel, R. Alcarria, D. Sánchez, A. Sánchez

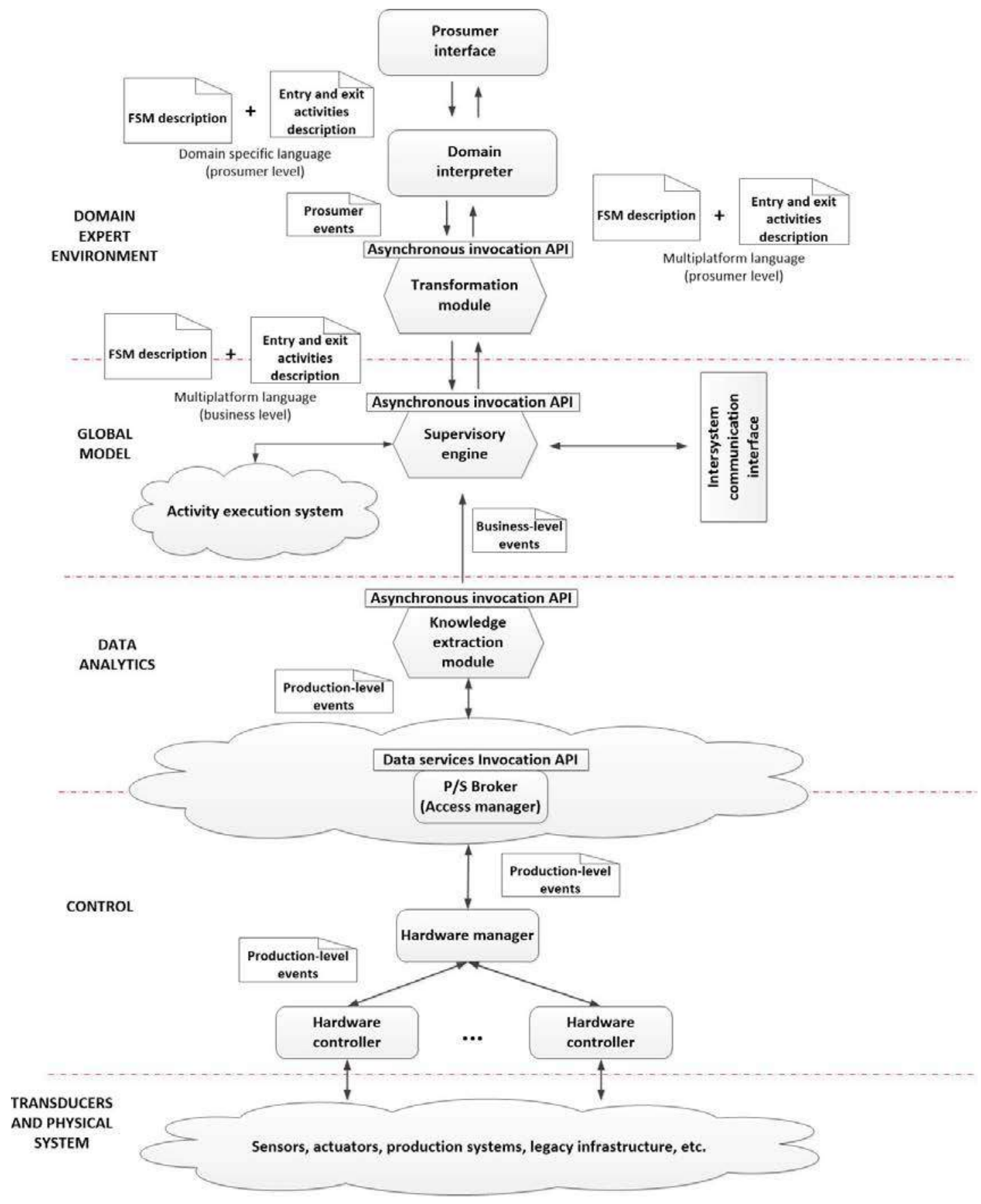

Figure 4: Detailed functional architecture

The first module in the verification system still belongs to the domain expert environment. It is a transformation module whose function is to transform the high abstraction level (prosumer) descriptions obtained from the domain interpreter into executable machine-understandable code which present a lower level of abstraction that we have named as "business level". Business-level descriptions are also independent of the underlying hardware platform. Languages employed at this point to describe FSMs may be the same as employed by the interpreter (for example the W3C State Chart XML language) or a new language can be used. Executable description languages such as which described by Bowne [37], 
Enhancing Process Control in Industry 4.0 Scenarios using Cyber-Physical Systems

B. Bordel, R. Alcarria, D. Sánchez, A. Sánchez

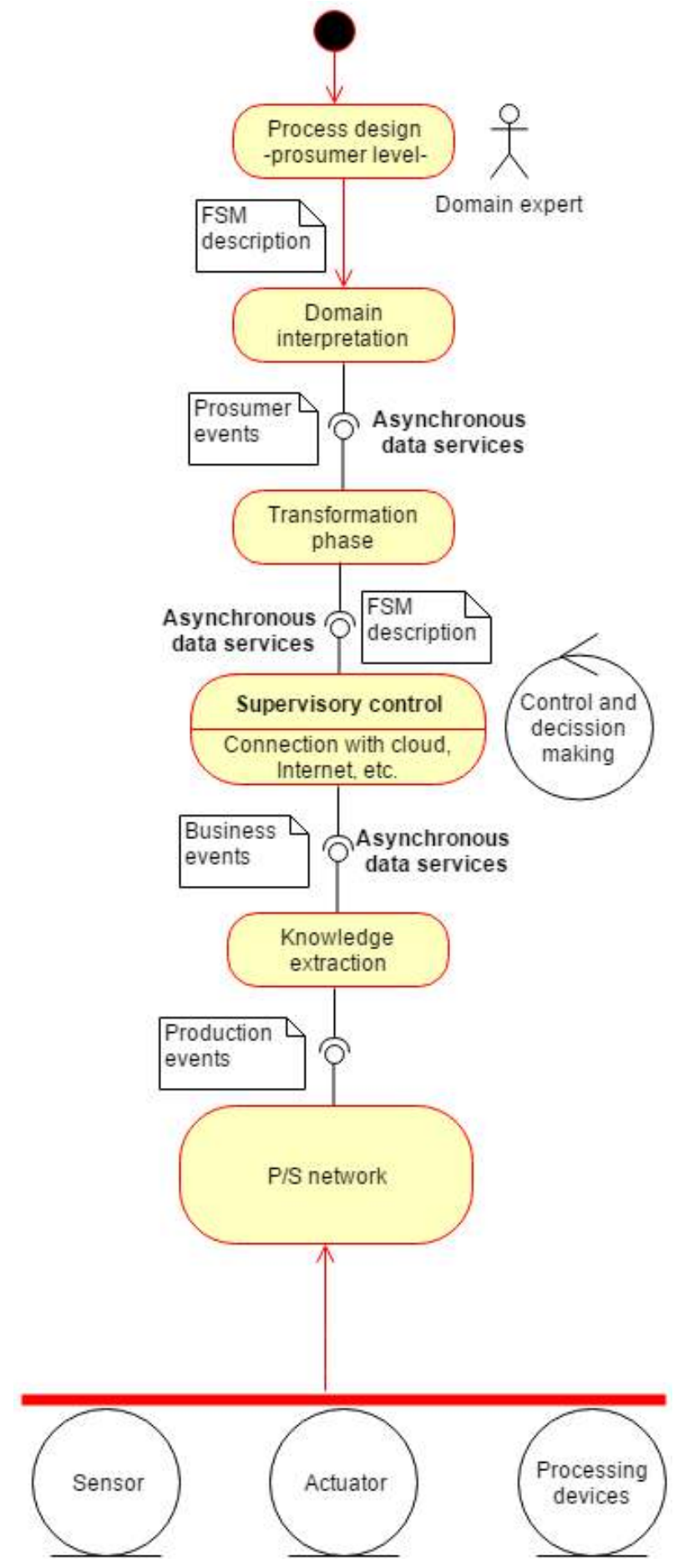

Figure 5: UML description of system behavior

the XASM language (An Extensible, Component-Based Abstract State Machines Language) [38] or the .NET framework for State Machine Workflows [39] may be very useful at this point. Figure 8 presents the output of the transformation module when description on Figure 7 is the input (when using the W3C State Chart XML language). 


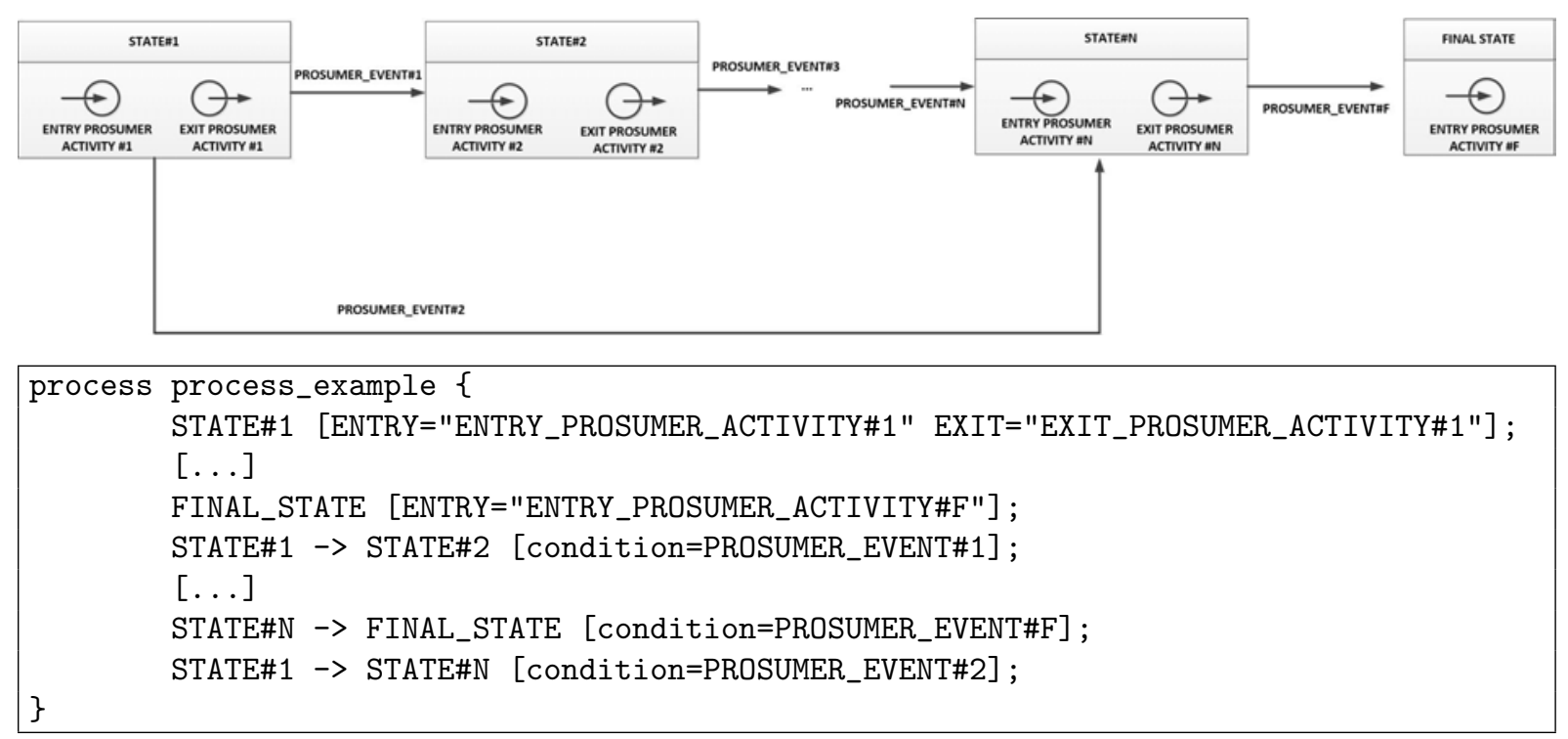

Figure 6: Generic process at prosumer level (prosumer interface). (a) Graphic representation. (b) DOT description

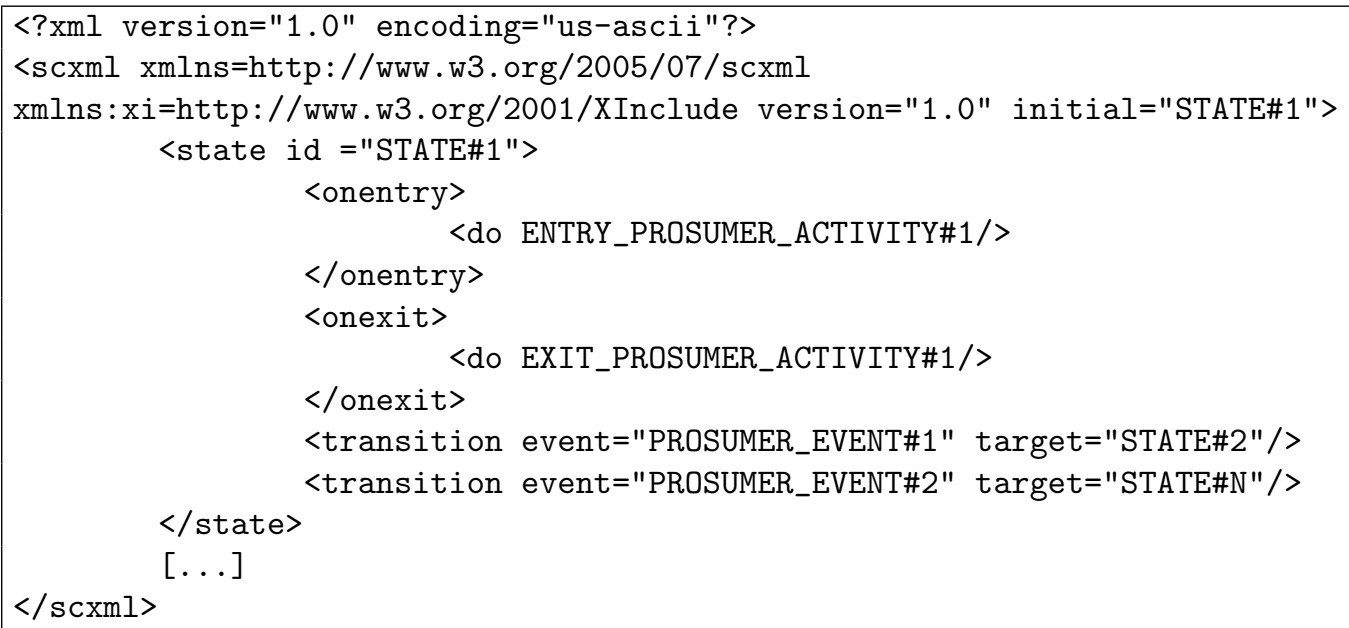

Figure 7: FSM description generated by the domain interpreter

Using the same procedure as described above, this new description is uploaded to a supervisory engine using REST services. In this engine, the adequate execution environment is provided, depending on the language employed in the business-level description. For example, if .NET technologies are employed, a C++ engine is included in this module. If, as in the proposed solution, XML technologies are employed, the uploaded description is transformed into a state transition table (see Figure 9).

State transition tables are maintained in memory in the supervisory engine, so the process state is continuously updated depending on the received business events. A new event is generated and sent to the transformation module each time the FSM is updated. Additionally, each time a state is reached or left, the corresponding entry or exit activity is executed by means of the activity execution system. This subsystem must be able to execute processes described at business level following a certain XML. Resolving this new problem is not the focus of our work, but many systems like this have been described in the literature [40, 41] which may be applied here. 


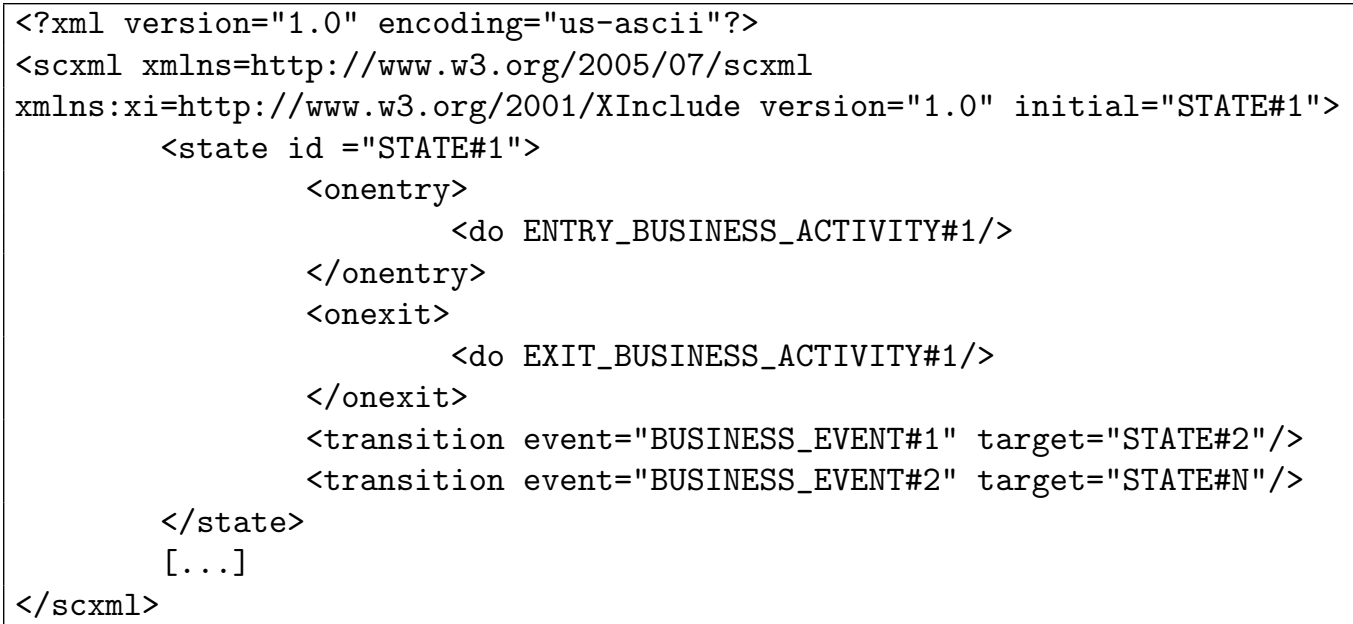

Figure 8: FSM description generated by the transformation module

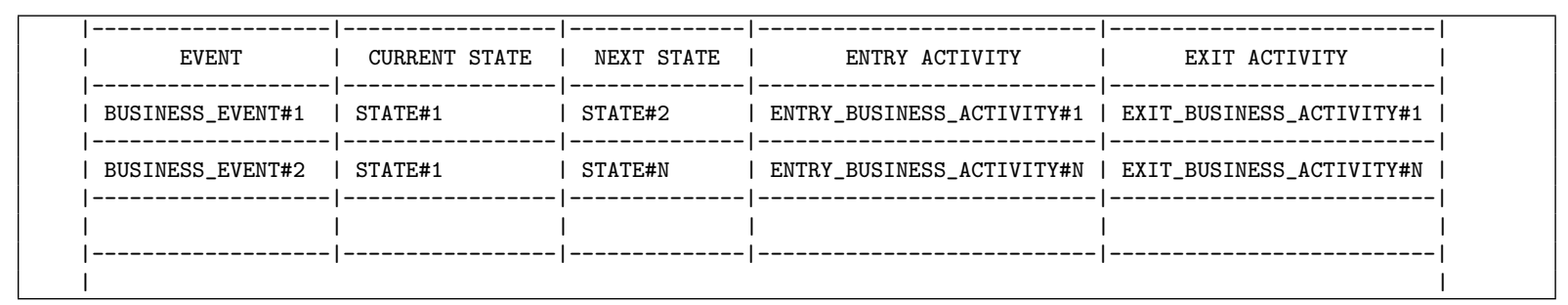

Figure 9: State transition table in the supervisory engine

Finally, the supervisory module may also receive FSM descriptions through the Intersystem communication interface. These descriptions are remotely generated, but they must be verified in the local system. Languages employed to describe these remote FSMs may be varied, although JSON-based technologies are the most adequate. In our particular solution, the Amazon States Language is employed [42].

The cited business-level events come from a knowledge extraction module, being able of detecting patterns and other platform-independent information by the analysis of the received production events. This module may implement different technologies [43, 44], depending on the application scenario. This module participates in a P/S network which transports production events from the underlying platform (which executes the process) to the verification system. Production events are expressed in a low abstraction level and they are platform-dependent (dynamic ranges, error messages, etc. follow a specific format of the installed devices). Almost any P/S technology may be applied, although in this case we have selected MQTT middleware.

MQTT is agnostic in respect to the transmitted messages, so production events may follow the desired format: XML, JSON or a proprietary design [3]. Subscriptions are managed by a broker who acts as access manager.

Production events are generated and published by hardware controllers which are devices in charge of monitoring sensors and changing the state of actuators by means of a standard industrial protocol such as OPC. These sensors and actuators (usually embedded in the production systems and legacy infrastructures) allow, finally, controlling the process which is being executed by hardware devices. Several hardware controllers may be deployed in an industrial scenario depending on the number of devices to be controlled. In order to coordinate the operation of the controllers, a hardware manager is also included. 
Nevertheless, in our solution, it is only a transparent proxy (usually it analyzes the generated event looking for remarkable information, but in this first work we are not considering these functionalities).

\section{Experimental validation}

In order to validate the proposed solution as a valid system for process control in Industry 4.0 scenarios, a first real implementation of our proposal was developed. The system was deployed in a laboratory of the Technical University of Madrid, where various emulated production systems were implemented. Three different Linux machines, implementing each one four virtual computers based on Ubuntu 16.04 operating system were started up. Each virtual machine implemented an emulation program representing a different production system provided with an OPC interface. Emulation programs were developed using MATLAB/Simulink tools.

Different electronic device production systems were emulated, in particular three different types were considered: manufacture of diodes, manufacture of capacitors and manufacture of logic gates. Four identical system of each type were implemented. Only one process was programmed in each type of production system. In total, then, three different processes were considered.

The host where each group of four virtual machines was executed acted as hardware controller. A forth computer connected with the other three by means of an Ethernet network acted as hardware manager. In this same fourth computer, the P/S broker was implemented.

The rest of the components in the functional architecture showed in Figure 4 were implemented in the cloud. Specifically, the Amazon Web Services (AWS) were employed (see Figure 10)
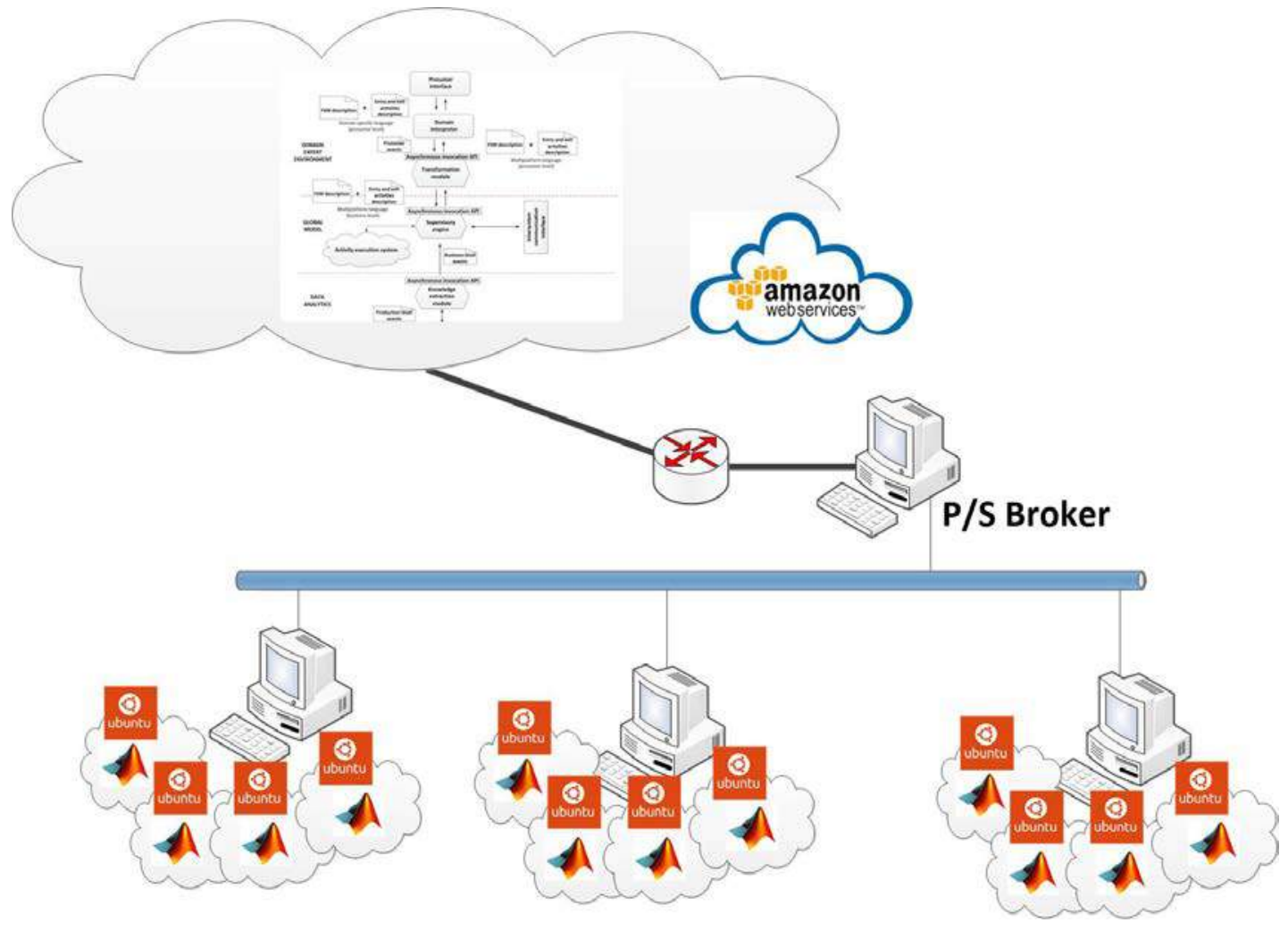

Figure 10: Scheme of the deployed infrastructure

The prosumer interface was based on a simple web page where the FSMs corresponding with each 
process could be programmed using DOT language. Entry and exit activity description were written using the YAWL designer and were uploaded using the provided form in the same prosumer interface (see Figure 11).

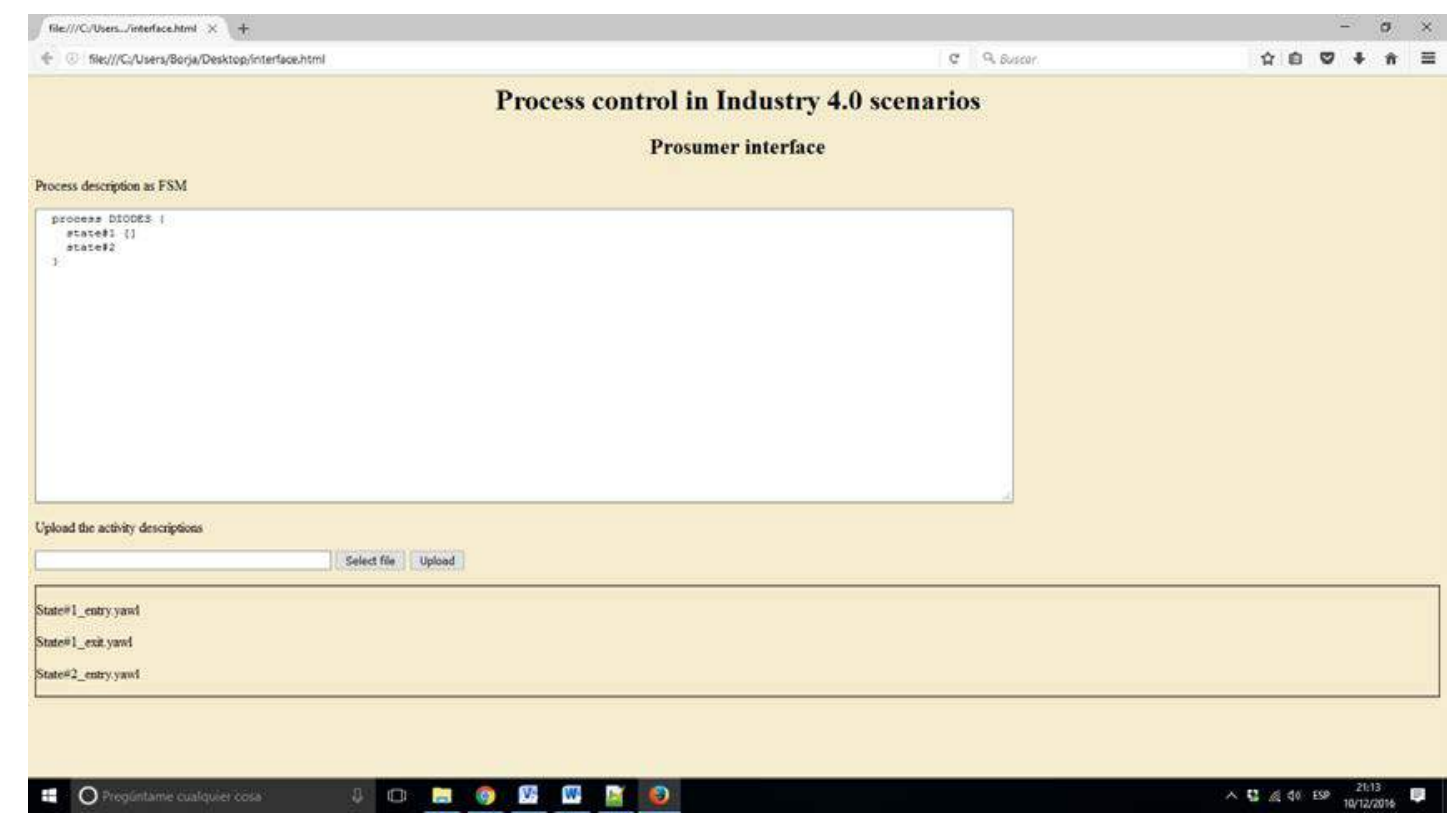

Figure 11: Prosumer interface

Three different experiments were planned in order to validate the proposed solution using the described implementation.

During the first planned experiment, emulation programs were designed to, randomly, cause malfunctions which the proposed process control solution should address and solve. Malfunctions presented different levels of severity and requirements in the response time. The experiment was repeated fifteen (15) times. At the end of the experiment, the proposed solution controlled a total of 180 processes. Data about the process evolution were collected for each of the monitored processes. From the analysis of this information we evaluated the performance of the proposed technology.

In order to value and contextualize the obtained results from the first experiment, a second infrastructure was deployed. It consisted of a traditional supervisory control system. In this new design, the described virtualized production systems were connected to a SCADA system through Programmable Logic Controllers (PLCs). The selected communication protocol was OPC. The SCADA system was developed using the openSCADA [45] project and was hosted in an Amazon Web Services Account (see Figure 12).

The second experiment is focused on the response time of the proposed solution. In fact, in order to provide a stable and robust control the process control system must react before the process state change significantly. This second experiment presents two parts.

In the first part, a fixed number of processes being executed was considered. In this case, ten (10) different processes were included. Then, the number of events per minute generated by the virtual production systems and the virtual sensors was increased. The increasing rate was $r=10 \frac{\text { events }}{\min ^{2}}$. The response time was measured in each case. The experiment finished when the response time prevented the system to provide a stable process control.

In the second part, the number of processes being controlled was increased periodically in one unit. The number of generated events per minute was fixed. For each number of processes being controlled, 


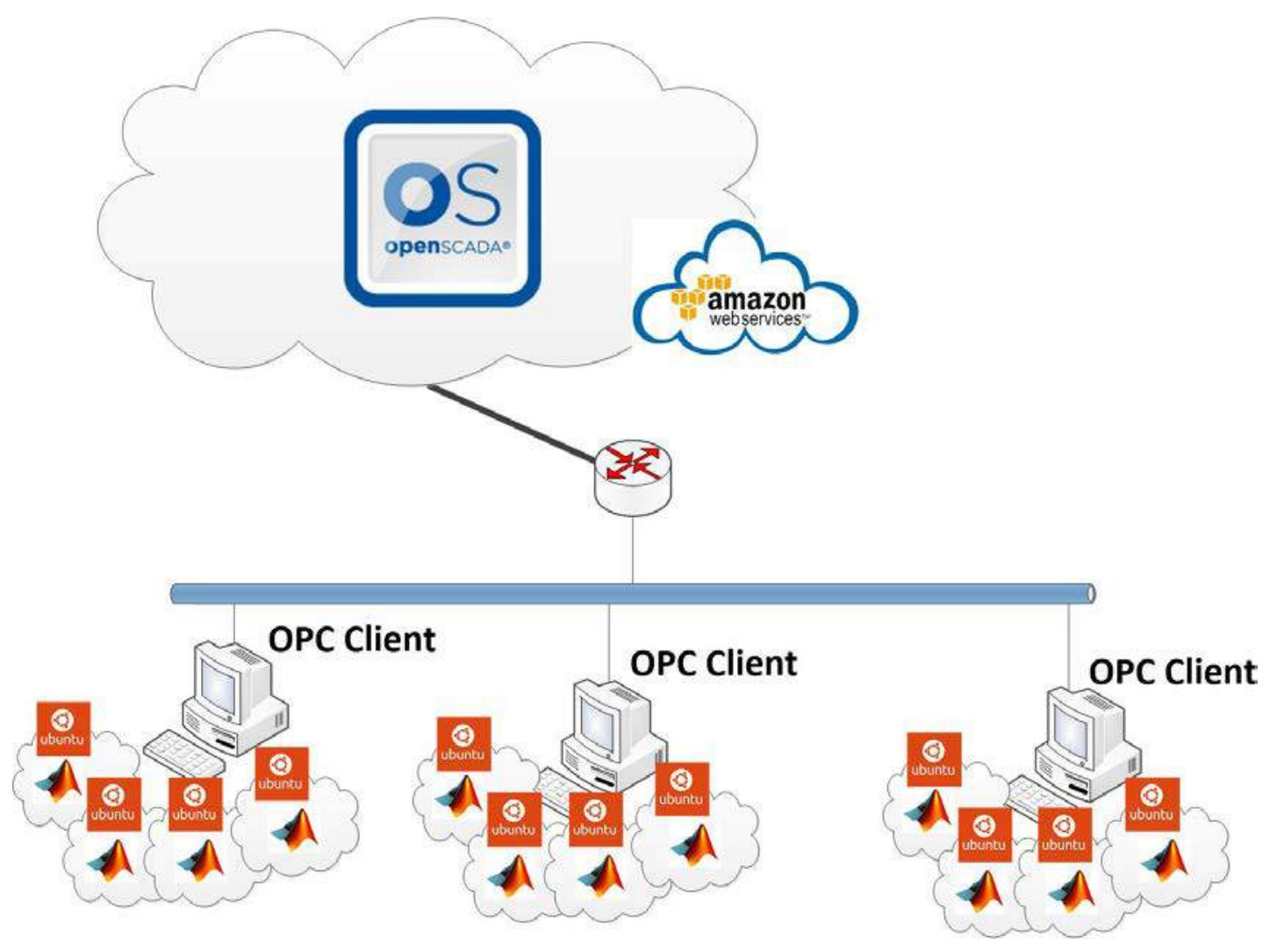

Figure 12: Traditional supervisory control system

the response time was measured. The experiments finished when the control system could not provide a stable and robust control.

Finally, the third experiment measured the user satisfaction and usability. Some experts on industrial systems (professors and post-graduate students in the Technical University of Madrid) were asked to define some control policies at prosumer level using the interface described in Figure 11. Moreover, they executed the designed control policies using the described infrastructure in the first experiment. Later, they answered a survey in order to evaluate their satisfaction with the system performance and its usability.

\section{Results}

Figure 13 shows the aggregated results of the experiment described in Section 4 . As can be seen in $97.22 \%$ of cases, the malfunctions were satisfactory corrected by means of the applied control policies. Only in $2.8 \%$ of cases the applied control was unsatisfactory and the process get uncontrolled and, finally, the entire system get blocked. These values are similar, and even slightly better, than showed on other proposals about industrial process control using the CPS paradigm [3].

As said previously, malfunctions in the production systems presented different levels of severity and requirements in the response time, so it is interesting to evaluate the causes of the failures in the proposed process control solution. Figure 14 shows a classification.

As can be seen, most failures are due to a response time too large. In this cases, malfunctions were resoluble, designed control policies were the correct, but the time required to communicate the events to 


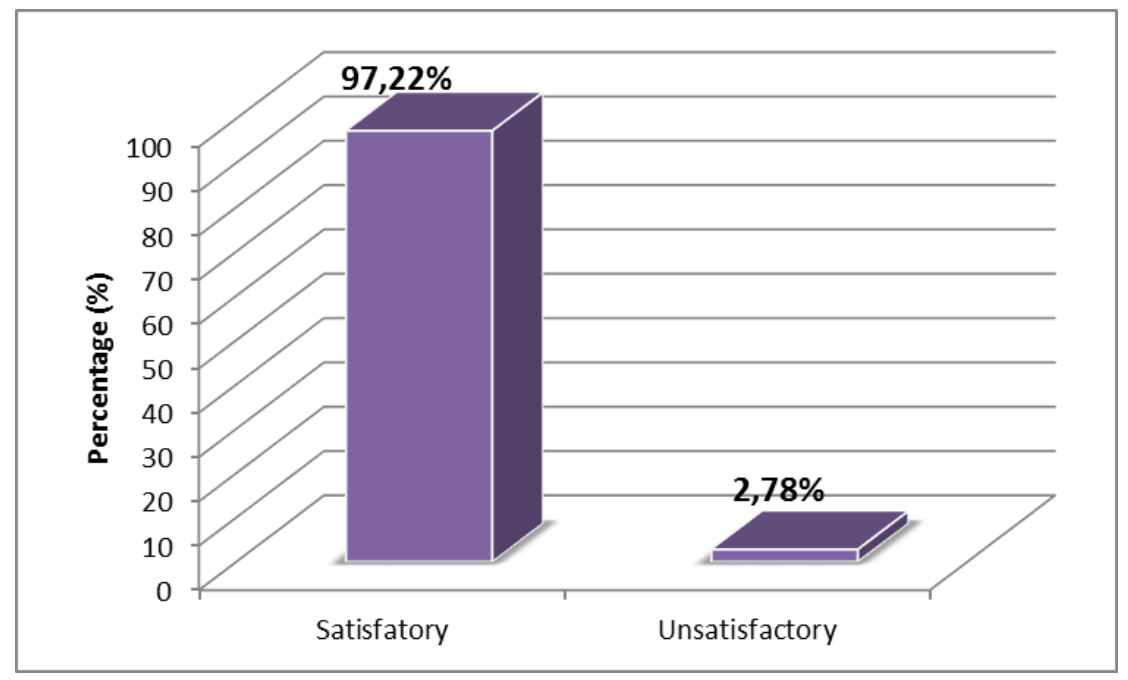

Figure 13: Aggregated results

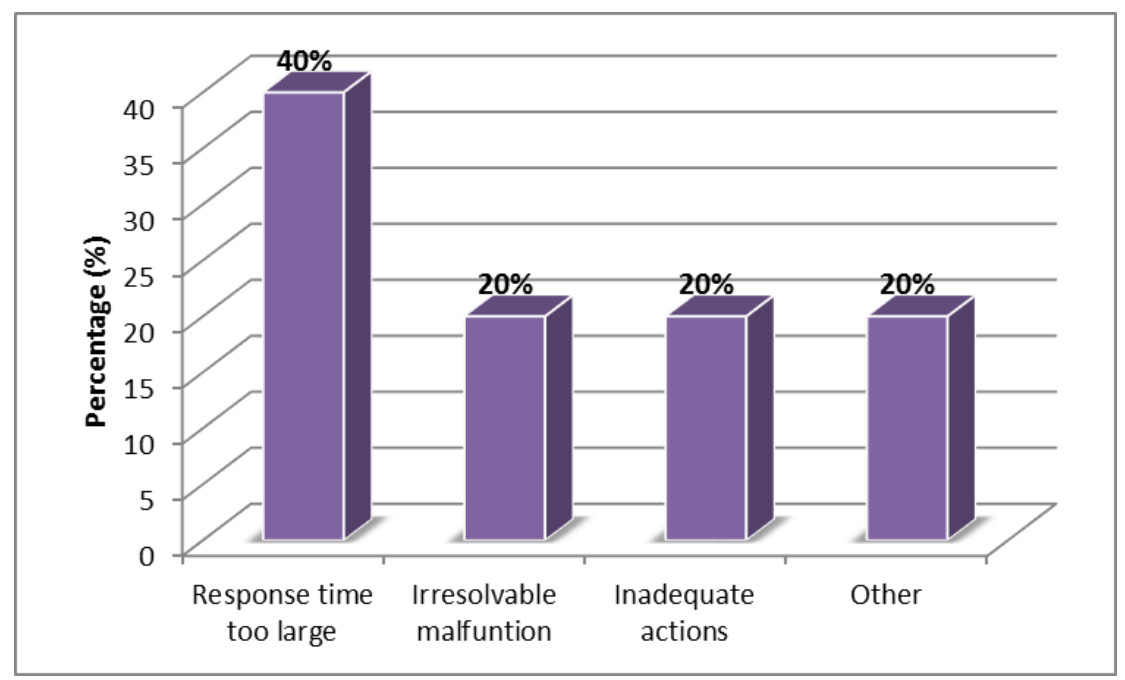

Figure 14: Causes of solution failures

the supervisory engine and, later, to send the taken decision to the emulated systems was too large, so, when applied, control policies were not enough to correct the process evolution. In order to solve this problem, the hardware manager could implement small low-level control rules in order to address in a minimum time critical situations.

Another important case to be taken into account is that, sometimes, malfunctions cannot be resolved. In every industrial scenario there exist some malfunctions that cannot be addressed by means of control policies (mechanical failures, supply failures, etc.), so our proposal should be complemented with other types of systems (such as quality assurance or maintenance chains).

As also said previously, in order to contextualize these results, they were compared with the ones obtained from a traditional supervisory process control system (see Figure 15). The obtained results proved that, although our proposal is slightly better than other solution for process control in Industry 4.0, traditional systems (based on SCADA) present a lightly better behavior. In fact, the deployed traditional system is capable of a successful control of $98.9 \%$ of processes, around $1.5 \%$ more than the results 
obtained using our technology.

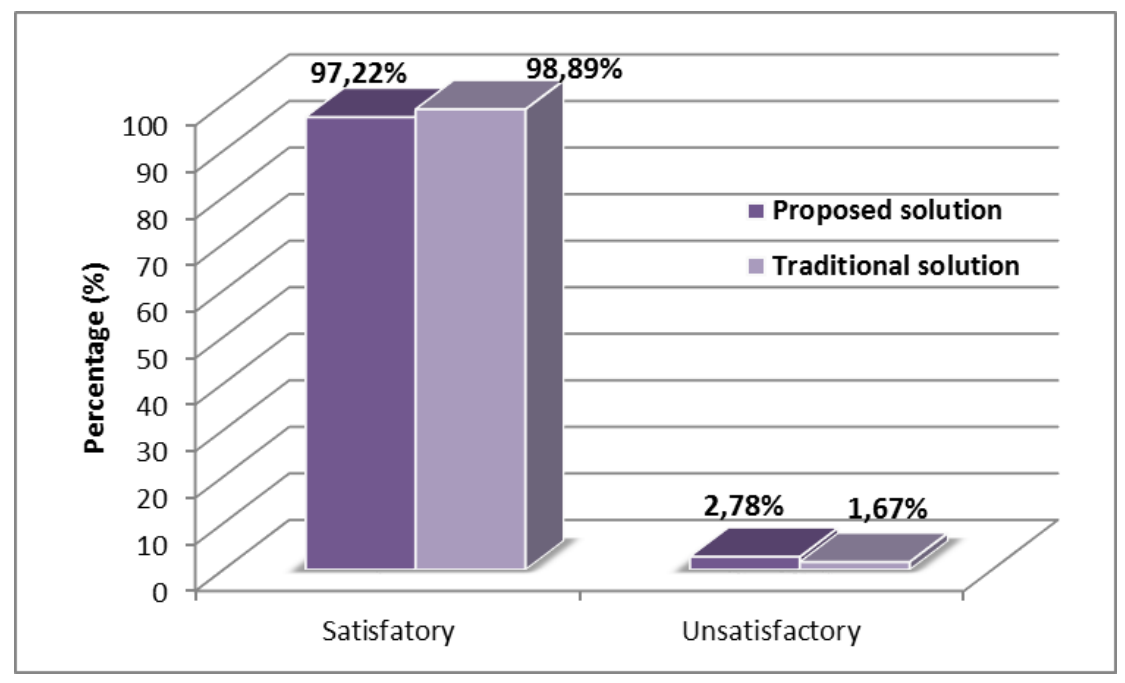

Figure 15: Comparison with traditional systems

This difference, however, is not especially remarkable and may be associated with the years of experience and the hundreds of different and new versions of SCADA published in the last 25 years.

Figure 16 shows the results for the first part of the second experiment, were the response time of the system is evaluated varying the events per minute generated by the virtualized production systems and sensors. As the response time is a stochastic process, the values showed on Figure 16 represent the temporal mean of those processes.

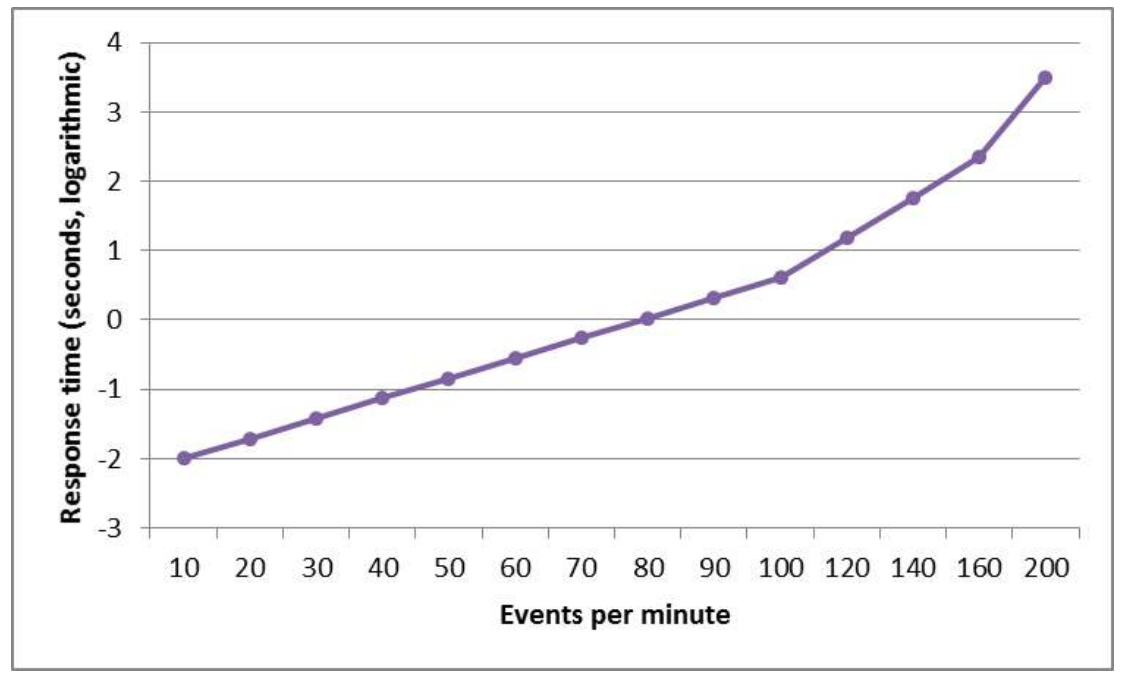

Figure 16: Response time depending on the number of events per minute

As can be seen, the response time follows an exponential function on the number of generated events per minute (linear in the logarithmic scale). Approximately (see Figure 17) every event is successfully processed until 100 events per minute are generated, which produces a response time about 100 seconds. If response time grows up to one minute, then, the system gets blocked and control policies cannot be correctly applied. More or less, this situation is reached for an event generation speed of $r=140$ events $/ \mathrm{min}$ 
The exposed values are much higher than the expected ciphers in a real industrial scenario, so our proposal is perfectly applicable. It must be noted that, if required, the number of hardware managers may be increased in order to create a distributed infrastructure (being able to admit more events per minute).

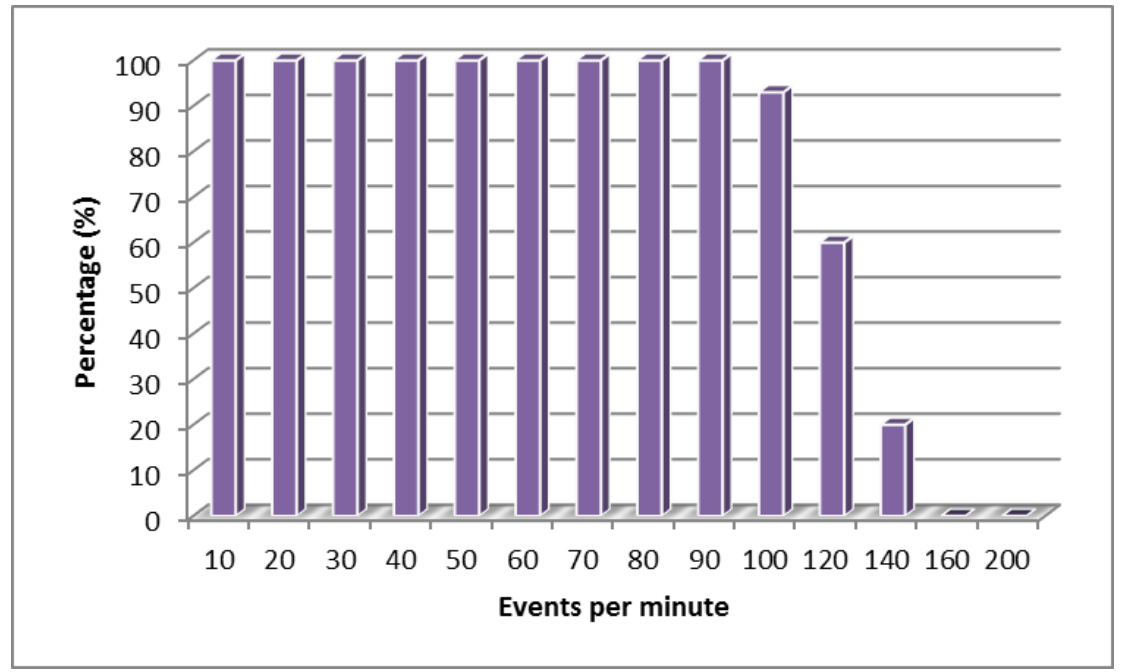

Figure 17: Percentage of events successfully processed

Results of the second part of the second experiment are showed on Figure 18 .

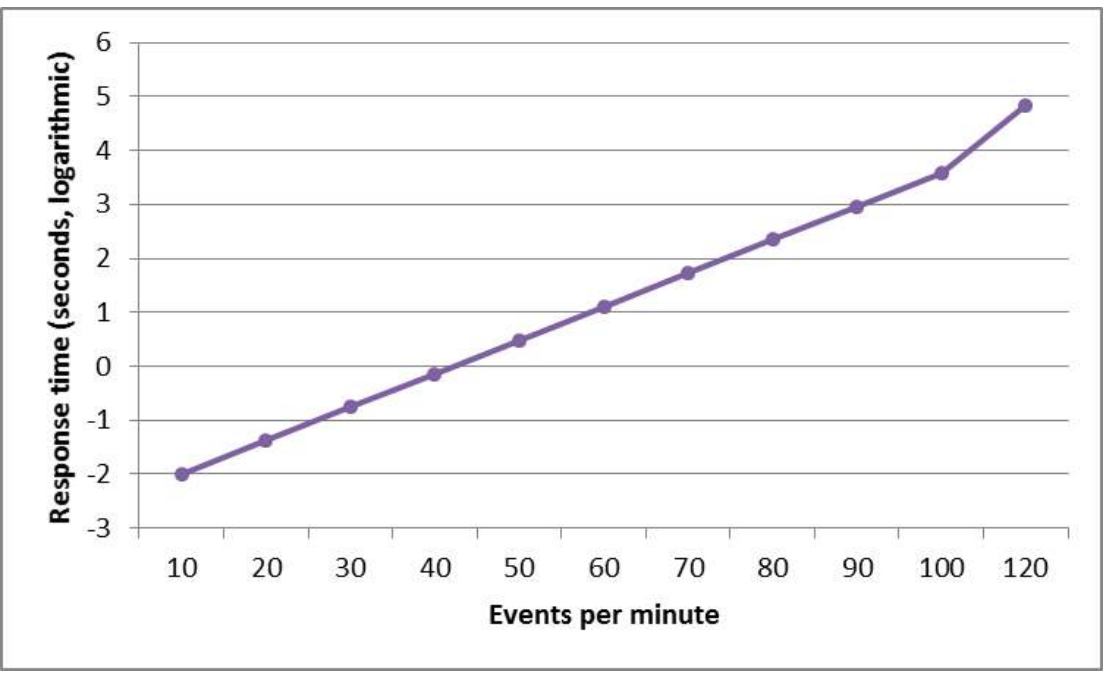

Figure 18: Response time depending on the number of processes being executed

As can be seen, the tendency in the response time depending on the number of processes being executed is very similar to which observed on Figure 16 . It presents an exponential evolution where problems appear when more than 50 processes must be controlled at the same time. If the number of processes goes up to 80, the system cannot operate (see Figure 19).

As said previously, the supervisory module may be replicated if more processes should be considered, although, in general, this number is much higher than the medium needs of industry.

Finally, Table 2 shows the results of the surveys answered by prosumer users. They valued with numbers from 1 to 9 different aspects of the proposed technology and the traditional supervisory control 


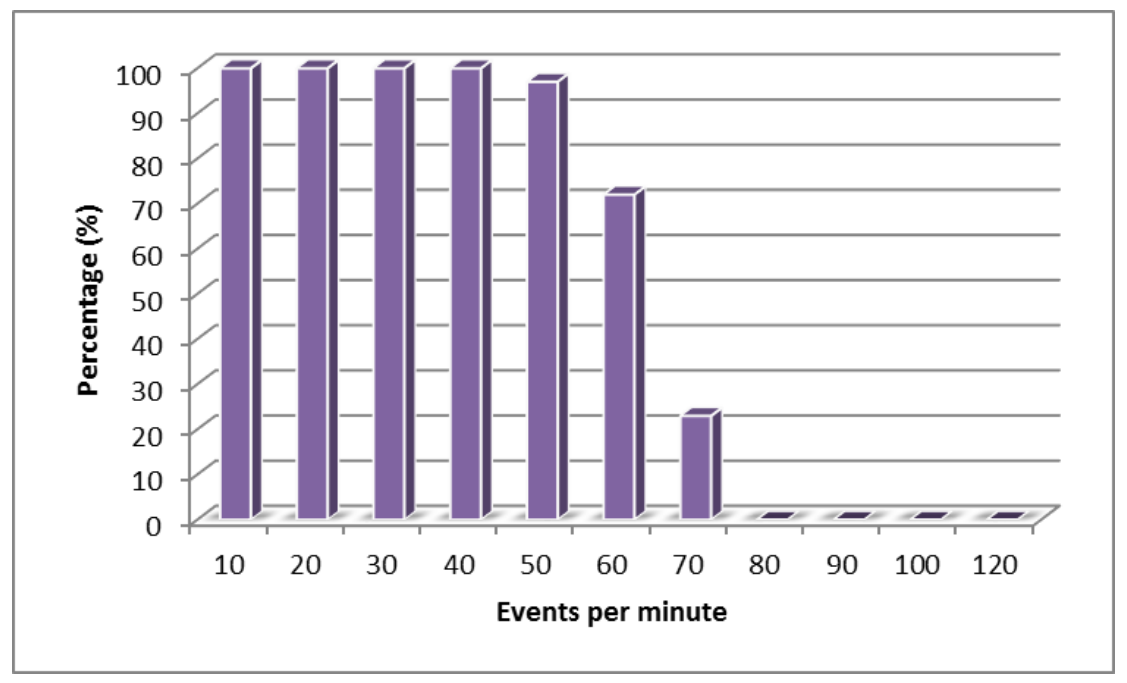

Figure 19: Percentage of events successfully processed

\begin{tabular}{ccc}
\hline \multirow{2}{*}{ Aspects } & \multicolumn{2}{c}{ Type of system } \\
\cline { 2 - 3 } & Proposed solution & Traditional solutions \\
\hline $\begin{array}{c}\text { Ease to create } \\
\text { control policies }\end{array}$ & 8.83 & 6.83 \\
\hline $\begin{array}{c}\text { Quality } \\
\text { of information } \\
\text { in the user interface }\end{array}$ & 4.33 & 7.13 \\
\hline $\begin{array}{c}\text { Flexibility to model } \\
\text { particular processes }\end{array}$ & 8.19 & 6.59 \\
\hline $\begin{array}{c}\text { Correct application } \\
\text { of control policies }\end{array}$ & 9.00 & 8.91 \\
\hline $\begin{array}{c}\text { Detection of } \\
\text { serious errors }\end{array}$ & 7.79 & 7.65 \\
\hline
\end{tabular}

Table 2: Results of the third experiment

systems. Number 1 implies users found totally inadequate a certain aspect, and number 9 that they found it totally adequate. Number 5 represents indifference. Table 2 shows the medium of all answers.

As can be seen, in general, the proposed solution is better valued by experts than traditional systems. This is especially true when talking about the creation of models and control policies. As the proposed technology allows the definition of policies and models at prosumer level (in a domain expert language), experts feel much more comfortable using the system. However, the proposed interface was only a first prototype, so it was the worst valued aspect in our proposal. Future versions should improve the provided interface, integrating all required tools in a unique window.

\section{Conclusions}

In this paper a solution for process control in Industry 4.0 scenarios is described. The proposed solution is based on the Cyber-Physical Systems paradigm, and focused on processes being executed by lowlevel hardware devices which should be supervised by high-level components (a view usually known as 
bottom-up). The proposed technology is based on the NIST reference architecture for CPS, which has been slightly modified. The entire solution is designed to meet the requirements of Industry 4.0 scenarios. Basically, our proposal consists on an interface where domain experts may model the processes to be controlled as finite state machines. The created model is transformed twice in order to obtain an executable business-level description of the FSM, including two activities per state (the entry and the exit activities) which are employed to apply the control policies. The experimental validation showed that the proposed system presents a performance comparable to the existing control systems, but it also shows all the advantages of the CPS and Industry 4.0 paradigms. Moreover, the described performance may be easily increased if low-level simple control rules are also considered, for which the described solution also contains a module in order to support these functionalities in the future.

\section{Acknowledgments}

The research leading to these results has received funding from the Ministry of Economy and Competitiveness through SEMOLA project (TEC2015-68284-R) and from the Autonomous Region of Madrid through MOSI-AGIL-CM project (grant P2013/ICE-3019, co-funded by EU Structural Funds FSE and FEDER). Borja Bordel has received funding from the Ministry of Education through the FPU program (grant number FPU15/03977)

\section{References}

[1] R. R. Rajkumar, I. Lee, L. Sha, and J. Stankovic, "Cyber-Physical Systems," in Proc. of the 47th Design Automation Conference (DAC'10), Anaheim, California, USA. ACM, June 2010, pp. 731-736.

[2] S. A. Haque, S. M. Aziz, and M. Rahman, "Review of Cyber-Physical System in Healthcare," International Journal of Distributed Sensor Networks, vol. 2014, no. 4, pp. 1-20, April 2014.

[3] B. Bordel Sánchez, R. Alcarria, D. Martín, and T. Robles, "TF4SM: A Framework for Developing Traceability Solutions in Small Manufacturing Companies," Sensors, vol. 15, no. 11, pp. 29478-29510, November 2015.

[4] H. Lasi, P. Fettke, T. Feld, and M. Hoffmann, "Industry 4.0," Business \& Information Systems Engineering, vol. 6, no. 4, October 2014.

[5] Edward A. Lee, "Cyber-Physical Systems - Are Computing Foundations Adequate?" in Position Paper for NSF Workshop On Cyber-Physical Systems: Research Motivation, Techniques and Roadmap, vol. 2. UC Berkeley, October 2006.

[6] J. C. Doyle, B. A. Francis, and A. R. Tannenbaum, Feedback Control Theory. Dover Publications, 2009.

[7] Y. Yalei and Z. Xingshe, "Cyber-Physical Systems Modeling Based on Extended Hybrid Automata," in Proc. of the 5th International Conference on Computational and Information Sciences (ICCIS'13), Hubai, China. IEEE, June 2013, pp. 1871-1874.

[8] H. Fawzi, P. Tabuada, and S. Diggavi, "Secure Estimation and Control for Cyber-Physical Systems Under Adversarial Attacks," IEEE Transactions on Automatic Control, vol. 59, no. 6, pp. 1454-1467, Junuary 2014.

[9] M. Conti, S. K. Das, C. Bisdikian, M. Kumar, L. M. Ni, A. Passarella, G. Roussos, G. Tröster, G. Tsudik, and F. Zambonelli, "Looking Ahead in Pervasive Computing: Challenges and Opportunities in the Era of Cyber-Physical Convergence," Pervasive and Mobile Computing, vol. 8, no. 1, pp. 2-21, February 2012.

[10] J.-P. Chou, L.-C. Chen, Y. Zhang, and L.-H. Pan, "OPC Unified Architecture for Industrial Demand Response," International Journal of Security and Its Applications, vol. 6, no. 2, pp. 275-280, April 2012.

[11] S. A. Boyer, Scada: Supervisory Control And Data Acquisition 4th edition. International Society of Automation, 2009.

[12] B. Bordel, R. Alcarria, M. Pérez-Jiménez, T. Robles, D. Martín, and D. S. de Rivera, "Building Smart Adaptable Cyber-Physical Systems: Definitions, Classification and Elements," in Proc. of the 9th International 
Conference on Ubiquitous Computing and Ambient Intelligence. Sensing, Processing, and Using Environmental Information (UCAml'15), Puerto Varas, Chile, ser. Lecture Notes in Computer Science, vol. 9454. Springer International Publishing, December 2015, pp. 144-149.

[13] H. J. La and S. D. Kim, “A Service-Based Approach to Designing Cyber Physical Systems," in Proc. of the 9th International Conference on Computer and Information Science (ICIS'10), Kaminoyama, Japan. IEEE, August 2010, pp. 895-900.

[14] Y. Tan, S. Goddard, and L. C. Pérez, "A Prototype Architecture for Cyber-Physical Systems," ACM SIGBED Review, vol. 5, no. 1, pp. 1-2, January 2008.

[15] EuroCPS project, https://www.eurocps.org/[Online; Accessed on December 10, 2016].

[16] A. M. Houyou, "Internet of things at work: Architecture approach to interoperability," Presentation slide at IoT Interoperability Workshop Paris, March 2012, http://www.probe-it.eu/wp-content/uploads/2012/04/ 11-IoT@Work_Interoperability-Architecture.pdf [Online; Accessed on December 10, 2016].

[17] J. Wan, M. Chen, F. Xia, L. Di, and K. Zhou, "From Machine-to-Machine Communications towards CyberPhysical Systems," Computer Science and Information Systems, vol. 10, no. 3, pp. 1105-1128, June 2013.

[18] C.-F. Lai, Y.-W. Ma, S.-Y. Chang, H.-C. Chao, and Y.-M. Huang, "OSGi-based services architecture for Cyber-Physical Home Control Systems," Computer Communications, vol. 34, no. 2, pp. 184-191, February 2011.

[19] “ISO Study Report on IoT Reference Architectures/Frameworks," August 2014, http://www.sis.se/PageFiles/ 15118/Study\%20Report\%20on\%20IoT\%20Reference\%20Architectures\%20and\%20Frameworks.pdf [Online; Accessed on December 10, 2016].

[20] NIST Cyber-Physical Systems, http://www.nist.gov/cps/[ [Online; Accessed on December 10, 2016].

[21] "Alliance for internet of things innovation report," Report: AIOTI WG11 - Smart manufacturing, http: //www.aioti.org/wp-content/uploads/2016/10/AIOTIWG11Report2015.pdf] [Online; Accessed on December 10, 2016].

[22] S. Wang, J. Wan, D. Li, and C. Zhang, "Implementing Smart Factory of Industrie 4.0: An Outlook," International Journal of Distributed Sensor Networks, vol. 12, pp. 1-10, January 2016.

[23] S. Sridhar, A. Hahn, and M. Govindarasu, "Cyber-Physical System Security for the Electric Power Grid," Proc. of the IEEE, vol. 100, no. 1, pp. 210-224, January 2012.

[24] A. W. Colombo, Industrial Cloud-Based Cyber-Physical Systems. Springer International Publishing, 2014.

[25] Y. Wang, M. C. Vuran, and S. Goddard, "Cyber-Physical Systems in Industrial Process Control," ACM SIGBED Review, vol. 5, no. 1, pp. 1-2, January 2008.

[26] R. Alcarria, T. Robles, A. Morales, and E. Cedeño, "Resolving Coordination Challenges in Distributed Mobile Service Executions," International Journal of Web and Grid Services, vol. 10, no. 2/3, pp. 168-191, April 2014.

[27] B. Bordel, D. Sánchez-de Rivera, A. Sánchez-Picot, and T. Robles, "Physical Processes Control in Industry 4.0-Based Systems: A Focus on Cyber-Physical Systems," in Proc. of the 10th International Conference on Ubiquitous Computing and Ambient Intelligence (UCAml'16), San Bartolomé de Tirajana, Gran Canaria, Spain. Springer International Publishing, November 2016, pp. 257-262.

[28] J. Lee, B. Bagheri, and H.-A. Kao, "A Cyber-Physical Systems architecture for Industry 4.0-based manufacturing systems," Manufacturing Letters, vol. 3, pp. 18-23, January 2015.

[29] E. Gansner, E. Koutsofios, and S. North, "Drawing graphs with dot," AT\&T Bell Laboratories, Tech. Rep. 910904-59113-08TM, 2006.

[30] J. Lilius and I. P. Paltor, "Formalising UML State Machines for Model Checking," in Proc. of the Unified Modeling Language: Beyond the Standard Second International Conference Fort Collins (UML99'), Colorado, USA, ser. Lecture Notes in Computer Science, vol. 1723. Springer Berlin Heidelberg, October 1999, pp. 430-444.

[31] L. Qiao, S. Kao, and Y. Zhang, "Manufacturing Process Modelling using Process Specification Language," The International Journal of Advanced Manufacturing Technology, vol. 55, no. 5, pp. 549-563, July 2011.

[32] J. L. Risco-Martín, S. Mittal, B. P. Zeigler, and J. M. De La Cruz, "From UML State Charts to DEVS State Machines using XML," in Proc. of the ACM/IEEE 10th International Conference on Model-Driven 
Enhancing Process Control in Industry 4.0 Scenarios

using Cyber-Physical Systems

B. Bordel, R. Alcarria, D. Sánchez, A. Sánchez

Engineering Lanuages and Systems (MODELS'07), Nashville, Tennessee, USA, January 2007, pp. 35-48.

[33] "State Chart XML (SCXML): State Machine Notation for Control Abstraction," W3C Recommendation, September 2015, https://www.w3.org/TR/scxml/ [Online; Accessed on December 10, 2016].

[34] B. Silver, Bpmn Method and Style, 2nd Edition, with Bpmn Implementer's Guide. Cody-Cassidy Press, 2011.

[35] W. van der Aalst and A. ter Hofstede, "YAWL: Yet Another Workflow Language," Information Systems, vol. 30, no. 4, pp. 245-275, June 2005.

[36] M. zur Muehlen, J. V. Nickerson, and K. D. Swenson, "Developing Web Services Choreography Standards - the Case of REST vs. SOAP," Decision Support Systems, vol. 40, no. 1, pp. 9-29, July 2005.

[37] M. C. Browne and E. M. Clarke, "SML-a high level language for the design and verification of finite state machines," Technical Report. Carnegie Mellon University, Tech. Rep. 1565, 1985.

[38] M. Anlauff and M. Anlauff, "Xasm - An Extensible, Component-Based Abstract State Machines Language," in Proc. of the International Workshop on Abstract State Machines - Theory and Applications (ASM'OO), Monte Verità, Switzerland, ser. Lecture Notes in Computer Science, vol. 1912. Springer Berlin Heidelberg, March 2000, pp. 69-90.

[39] “.NET framework for State Machine Workflows," https://msdn.microsoft.com/en-us/library/ee264171(v=vs. 110).aspx [Online; Accessed on December 10, 2016].

[40] W. Du, M.-C. Shan, and A. Elmagarmid, "Pre-locking scheme for allowing consistent and concurrent workflow process execution in a workflow management system," US Patent: US6078982 A, June 1998, http://www.google.tl/patents/US6078982.

[41] I. Altintas, C. Berkley, E. Jaeger, M. Jones, B. Ludascher, and S. Mock, "Kepler: An Extensible System for Design and Execution of Scientific Workflows," in Proc. of the 16th International Conference on Scientific and Statistical Database Management (SSDBM'04), Santorini Island, Greece. IEEE, June 2004, pp. 423424.

[42] “Amazon States Language specification,” https://states-language.net/spec.html] [Online; Accessed on December 10, 2016].

[43] C. H. Chen, L. F. Pau, and P. S. P. Wang, Handbook of Pattern Recognition and Computer Vision. World Scientific, 1993.

[44] O. Maimon and L. Rokach, Data Mining and Knowledge Discovery Handbook. Springer US, 2010.

[45] OpenSCADA, http://openscada.org [Online; Accessed on December 10, 2016]. 


\section{Author Biography}

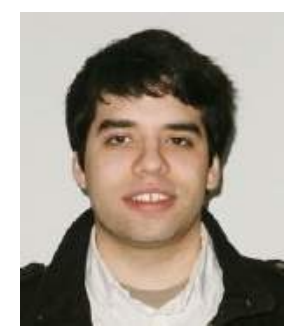

Borja Bordel received the B.S. degree in telecommunication engineering in 2012 and the M.S. telecommunication engineering in 2014, both from Technical University of Madrid. He is currently pursuing the Ph.D. degree in telematics engineering at Telecommunication Engineering School, UPM. His research interests include CyberPhysical Systems, Wireless Sensor Networks, Radio Access Technologies, Communication Protocols and Complex Systems.

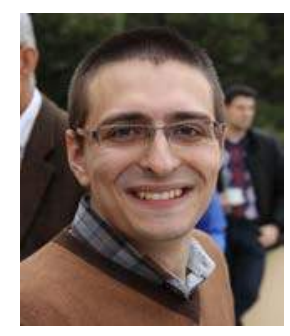

Ramón Alcarria received his M.S. and Ph.D. degrees in Telecommunication Engineering from the Technical University of Madrid in 2008 and 2013 respectively. Currently, he is an assistant professor at the E.T.S.I Topography of the Technical University of Madrid. He has been involved in several R\&D European and National projects related to Future Internet, Internet of Things and Service Composition. His research interests are Service Architectures, Sensor Networks, Human-computer interaction and Prosumer Environments.

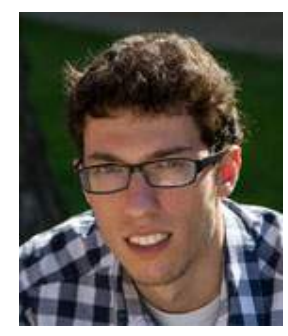

Diego Sánchez de Rivera holds a B.S. and a M.S. degree in Telecommunication Engineering from Technical University of Madrid. Currently he is a Ph.D. student and works as a research engineer in the Department of Telematics Systems at the E.T.S.I. Telecommunications of the Technical University of Madrid. His research interests are Internet of Things, Sensor Networks, Cyber-physical systems, Electronic displays and Web Development.

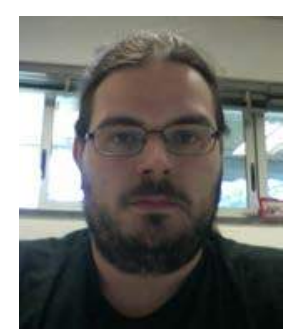

Álvaro Sanchez Picot received his M.S. degree in Telecommunication Engineering from Technical University of Madrid in 2014. Currently he is a Ph.D. student at the E.T.S.I. Telecommunications in the Department of Telematics Systems Engineering. His research interest is focused on Sensor Networks, Simulation of Network Communications, Wireless Communications and Web development. His thesis title is "Contribution to the integration of network simulators and social simulation environments for the modeling of environments and smart devices". 


\title{
Low-level service management in Cyber-Physical Systems
}

\author{
Borja Bordel $^{1,2}$, Ramón Alcarria ${ }^{1,2}$, Diego Sánchez-de-Rivera ${ }^{2}$, Antonio Jara $^{1}$ \\ ${ }^{1}$ Institute of Information Systems. University of Applied Sciences Western Switzerland \\ ${ }^{2}$ On leave from: Universidad Politécnica de Madrid, Madrid, España \\ bbordel@dit.upm.es; ramon.alcarria@upm.es; diegosanchez@dit.upm.es; jara@ieee.org
}

\begin{abstract}
First standardization initiatives of the Cyber-Physical Systems (CPS) paradigm face a type of solutions with a top-down approach. In this view, user services and applications are transformed, decomposed and delegated until they are finally executed by hardware devices. However, most works do not describe the final execution phase, when a certain device is selected to perform an action. Therefore, in this paper we describe a management solution to coordinate the execution of low-level services in CPS. The solution employs a probabilistic selection technique based on the concept of Cost and Quality-ofService, and includes both an orchestration algorithm and a choreography procedure. The proposal includes, moreover, a general framework explaining all the management levels and an experimental validation which evaluates the performance of the proposed technology.
\end{abstract}

Keywords: Cyber-Physical Systems, low-level services, service management, hardware devices

\section{Introduction}

Cyber-Physical Systems (CPS) are integrations of physical and computational processes [1]. This general definition, however, does not allow describing a uniform architecture or implementation, so several proposals may be found [2][3]. In this context, various authors and standard organizations are trying to fix the CPS paradigm defining different reference architectures. One of the most important it is the NIST architecture [4]. On the other hand, all these proposals follow a top-down approach. In these systems, firstly, user applications and services are defined at high-level and, later, they are transformed, decomposed and delegated in order to request hardware devices to perform the corresponding actions [5].

Most steps in this execution process have been discussed previously in many articles. Several contributions about task delegation [6], workflow decomposition [7] or service matchmaking [8] may be found. However, all these proposals are, at the end, based on a virtualized hardware platform which is implemented, traditionally, in a gateway or controller [5]. This component receives service invocations through a uniform interface and resolves the execution using the virtual hardware. This solution, nevertheless, does not meet the requirements of a real scenario, where real hardware devices with very heterogeneous and (most time) proprietary interfaces are included. Moreover, current systems tend to be ubiquitous, so hardware platforms present many redundancies (devices with the same functions). In this context, a solution to manage 
the service execution at low-level it is needed. The proposed solution should be able to collect information about hardware devices and, using it, decide the particular element which must execute each action (sending the corresponding order to it, using the adequate message and/or interface).

Therefore, in this paper a technology to manage the low-level service execution in CPS is proposed. The solution considers a first data collection process employed to determine the execution cost and the Quality-of-Service (QoS) offered by each device. Then, using a probabilistic selection procedure it is dynamically selected the device in charge of executing each action. Depending on the implementation, selected devices may delegate the execution (a paradigm usually named as choreography) or the hardware manager (see Figure 2) must coordinate the entire process (orchestration).

The rest of the paper is organized as follows: Section 2 describes the technical proposal including a first general framework and a mathematical formalization. Section 3 includes the experimental validation of the proposed technology, and Section 4 shows the obtained results. Finally, Section 5 presents some conclusions.

\section{Formalization of the proposed solution}

In the most general scenario, Cyber-Physical Systems implement the architecture showed on Figure 1(a). Each CPS acts as a networked autonomous system, connected with other CPS by means of the called Cyber-Physical Internet (CPI). Basically, the CPI includes a central service management infrastructure containing a service repository and a service manager. These components maintain the list of available services, publicly offered by CPS to be invoked from other CPS. The manager must (among other functionalities) determine (using, for example, semantic annotations [10]) if different services are really the same and response the queries about the stored services in the repository. The CPI, moreover, represents the backbone (usually the public Internet) which connects and communicates the different CPS among them and with the CPI infrastructure.

Then, each individual CPS relates with the CPI through an Intersystem communication interface which feeds the Execution system, together with the local Domain expert environment. Finally, a physical platform is in charge of the final execution. This layer may include different types of hardware devices (sensors, actuators, legacy systems, etc.) and different controllers. In this context, a service management solution for CPS should be composed of three different layers (see Figure 1(b)). The Connection level represents all the required procedures to integrate the peripherals with the central microcontroller of each device (physical connection) [9]; and the solution which connects each device with the other cyber-physical devices and with the hardware manager (interoperability). Interoperability mechanisms may be implemented in the microcontroller of each device or in an external broker (or hardware controller). The second level (Logical level) includes the coordination technologies employed by the hardware manager (see Figure 2) to execute low-level services using the underlying hardware platform (or virtual components if also considered). Logical solutions include device selection, low-level service delegation, etc. 


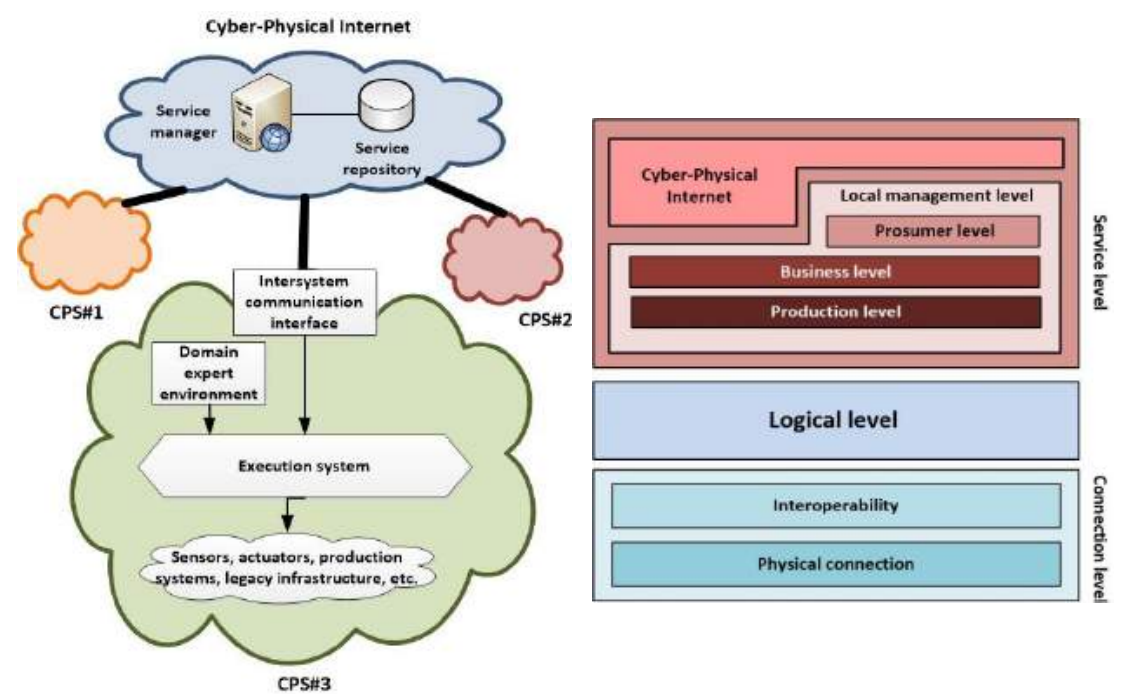

Fig. 1. (a) Typical CPS architecture (b) Architecture of a service management solution in CPS

The proposed technology in this paper belongs to this level. Finally, service level integrates all the service-focused technologies which transform low-level services (sometimes called hardware services) into more abstracted entities nearest human comprehension (Local management level) and share resources publicly with other CPS through the CPI (Cyber-Physical Internet). Typically, three different service abstraction layers are considered: production level (which integrates services with a direct correspondence with hardware services), business level (representing services expressed into any technical executable language) and prosumer level (where services are described using any domain description language). As said above, mapping the previously described works (see the Introduction) into the proposed architecture for service management in CPS, it may be proved that the cited gap in CPS research corresponds exactly with the Logical level. Therefore, this paper describes a logical level technology. This technology includes three different procedures: hardware monitoring, service execution through individual management and service execution through group management.

\subsection{Hardware monitoring}

The proposed technology follows the best effort paradigm, as it is considered that services do not include any indication about the QoS. However, in order to optimize the resource consumption, the proposed device selection for service execution it is not random, but it considers the current state of the hardware platform. Then a hardware monitoring process must be considered. Two types of information are included: the execution cost Q and a collection of hardware operation quality indicators $\Sigma$ (such as availability). Figure 2 presents a functional architecture of the application scenario.

Information about hardware is provided by hardware controllers (the components which implement the interoperability functions), so costs are different for each 
service and location (i.e. hardware controller). Mathematically, then, costs are a set of sets (1)

$$
Q=\left\{q^{1}, q^{2}, \ldots, q^{N}\right\}=\left\{\left\{q_{1}^{1}, q_{2}^{1}, \ldots, q_{M_{1}}^{1}\right\}, \ldots,\left\{q_{1}^{N}, q_{2}^{N}, \ldots, q_{M_{N}}^{1}\right\}\right\}
$$

Where $N$ is the number of hardware controllers and $M_{i}$ the number of services in the $\mathrm{i}$-th hardware controller. Thus $q_{j}^{i}$ is the execution cost of the $\mathrm{j}$-th service in the $\mathrm{i}$-th location.

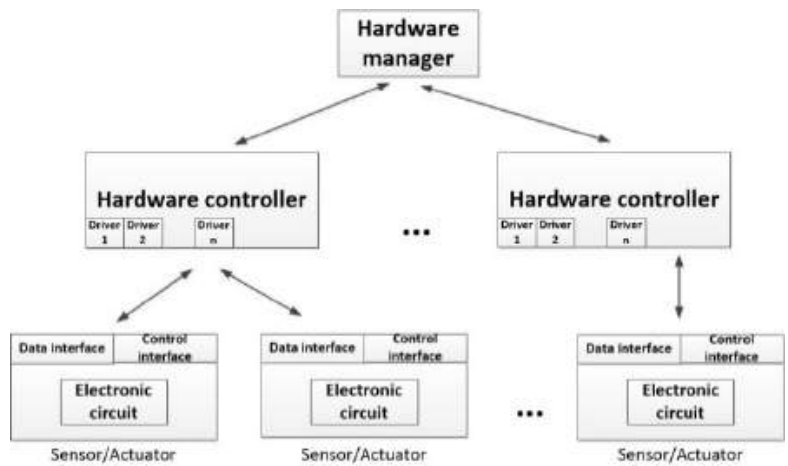

Fig. 2. Functional architecture of the application scenario

On the other hand, for each location and service, a set of values is employed as hardware operation quality indicators (2). Thus, mathematically, a triple nested list represents the quality indicators (3)

$$
\begin{gathered}
\Sigma_{j}^{i}=\left\{\sigma_{1}^{i, j}, \sigma_{2}^{i, j}, \ldots, \sigma_{R}^{i, j}\right\} \\
\Sigma=\left\{\Sigma^{1}, \Sigma^{2}, \ldots, \Sigma^{N}\right\}=\left\{\left\{\Sigma_{1}^{1}, \Sigma_{2}^{1}, \ldots, \Sigma_{M_{1}}^{1}\right\}, \ldots,\left\{\Sigma_{1}^{N}, \Sigma_{2}^{N}, \ldots, \Sigma_{M_{N}}^{1}\right\}\right\}
\end{gathered}
$$

Where $R$ is the number of considered indicators. Quality indicators (such as response time or availability) may be directly measured, and/or present very wellknown expressions [11]. However, the execution cost must be obtained through a proprietary procedure (4).

$$
q_{j}^{i}=f_{\text {cost }}\left(q_{j_{\text {user }}}^{i}, q_{j_{\text {hardware }}}^{i}\right)
$$

The cost of the $j$-th service in the $\mathrm{i}$-th location is obtained through a cost function which considers two contributions: a cost specified by the administrator $q_{j}^{i}{ }_{\text {user }}$ (employed to penalize certain equipment, do load balancing, etc.) and a hardware cost representing the resource consumption $q_{j}^{i}$ hardware . Depending if both contributions are independent or if they can compensate each other, the cost function may be a weighted arithmetic mean (5) or a geometric mean (6).

$$
\begin{gathered}
f_{\text {cost }}(\because)=\lambda_{1} q_{j_{\text {user }}}^{i}+\lambda_{2} q_{j_{\text {hardware }}^{i}} \\
f_{\text {cost }}(\because)=\sqrt{q_{j_{\text {user }}}^{i} \cdot q_{j_{\text {hardware }}^{i}}}
\end{gathered}
$$

Finally, the hardware cost is obtained as a weighted mean of a collection of resource consumption indicators $\psi_{k}$ (such as the battery consumption or the required 
processing time). These indicators must be defined to take values in the interval $[0,1]$, being 1 the value indication a higher cost (7).

$$
q_{j_{\text {hardware }}}^{i}=\sum_{k} \beta_{k} \cdot \psi_{k}
$$

In Section 3 we propose some particular examples of the named resource consumption indicators.

\subsection{Service execution through individual management}

Low-level services may be classified into various different classes attending to it geographical requirements (see Figure 3).

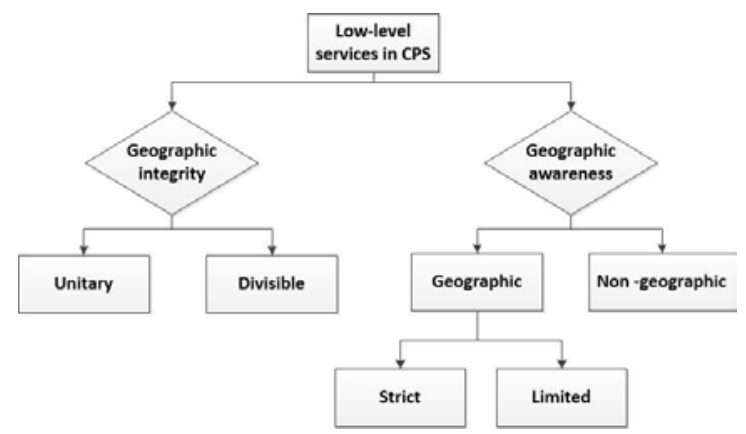

Fig. 3. Service classification depending on the geographic requirements

Unitary services are those which must be executed entirely in the same location (i.e. by the same hardware controller). Services which may be decomposed in parts to be executed in different controllers are divisible. On the other hand, geographic services must be executed in one particular location (geographically strict services) or in any of the location belonging to a certain group (geographically limited services). Geographic services must be complemented with metadata about the geographic restrictions. Non-geographic services may be executed in any location or hardware controller.

When a service invocation arrives to the hardware manager, it determines the group of locations $\mathcal{L}$ which may execute the service (depending on the geographic restrictions and the capabilities of each controller). Then, the set of locations $\mathcal{L}$ is considered as the sample space of a multidimensional random variable $\mathcal{X}$. Each one of the dimensions in this random variable corresponds with one quality indicator (see subsection A) or with the execution cost. Then the variable $\mathcal{X}$ presents $R+1$ dimensions. As physically all dimensions are independent, they are also statistically independent, and (then) the probability density function for the i-th service may be easily calculated (8).

$$
f_{x}\left(\ell_{j}\right)=\frac{q_{i}^{j}}{\sum_{j} q_{i}^{j}} \cdot \prod_{k=1}^{R} \frac{\sigma_{k}^{i, j}}{\sum_{j} \sigma_{k}^{i, j}}
$$


In order to make all quantities comparable, all indicators and cost should be mapped into the same interval. Once built the probability function, different strategies may be followed to execute the service. In this section we are considering the hardware manager interacts individually with the hardware controllers.

Figure 4 represents various message sequence charts describing the whole procedure. We are explaining briefly each step:

1. The hardware manager selects the execution location (i.e. the hardware controller) following the probability function previously calculated.

2. The hardware manager sends the proper execution order to the selected hardware controller using the adequate data format and communication technology. Typically, a byte-oriented proprietary protocol [12] and low-energy communications (such as 802.15.4 [13]) will be employed.

3. If the selected hardware controller can execute the requested service, then the execution is successful in one step (Case A, see Figure 4). It performs the proper actions and returns the result to the hardware manager.

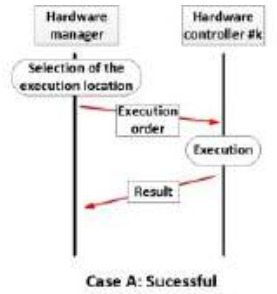

execution in one step

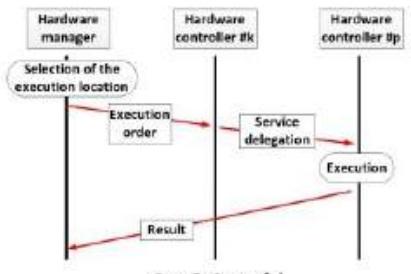

Case B: Sucessful execution in two steps
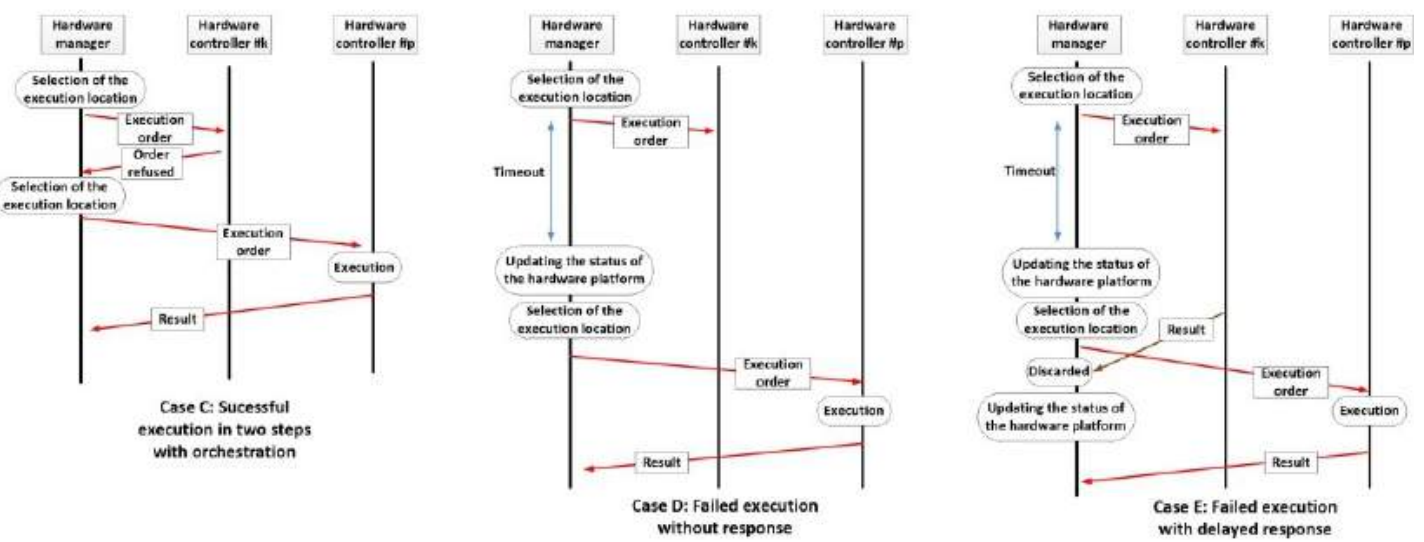

Fig. 4. Message sequence charts (service execution through individual management)

4. If the execution order cannot be executed, but the controller has resources to manage the petition, two different possibilities appear. If, at the interoperability level, a choreography algorithm is implemented, then the hardware controller may delegate the service execution to other location (if exists). This secondary hardware controller (HC) will execute the service and will send the result to the hardware manager (case B). If choreography algorithms are not available, the selected hardware controller (hardware controller \#k on Figure 4) should send a rejection message to the hardware manager (HM). At this point, the HM acts as orchestrator 
and removes this $\mathrm{HC}$ from the list of possible execution locations $\mathcal{L}$, recalculates the probability function and performs a new selection (case $\mathrm{C}$ ). This process may be repeated as many times as needed.

5. Sometimes, HCs may get congested and execution orders cannot be neither performed nor managed. No response to the hardware manager is then sent. In order to resolve these situations, each time an execution order is sent, the HM activates a timer. If at the timeout no response is received (case D), the state of the hardware platform is updated (services offered by that $\mathrm{HC}$ are declared unavailable, the timeout is increased, etc.); and a new selection (removing the unavailable location) is performed.

6. Finally, in some occasions, the $\mathrm{HC}$ may send a result to the hardware manager after the timeout (case E). At the reception, this results is discarded (a new execution order was sent), but the hardware platform state in updated once more (declaring available the services of this $\mathrm{HC}$ but with a greater response time).

As main advantage of this solution, the execution effort is very balanced among all the available devices, and the execution time of a low-level service may be reduced to a minimum (for a successful execution in one step). However, this execution time is very variable, and grows much if it is necessary to order the same execution several times. Eq (9) represents a general expression for the mean execution time when using this first proposal.

$\overline{T_{\text {exe }}}=(1-p)\left(2 T_{\text {com }}+T_{\text {proc }}\right)+\sum_{k=1}^{n-1}(1-p) p^{k}\left(2 T_{\text {com }}+T_{\text {proc }}+k \overline{T_{\text {fal }}}\right)+p^{n} n \overline{T_{\text {fall }}} \quad \forall n \geq 2$
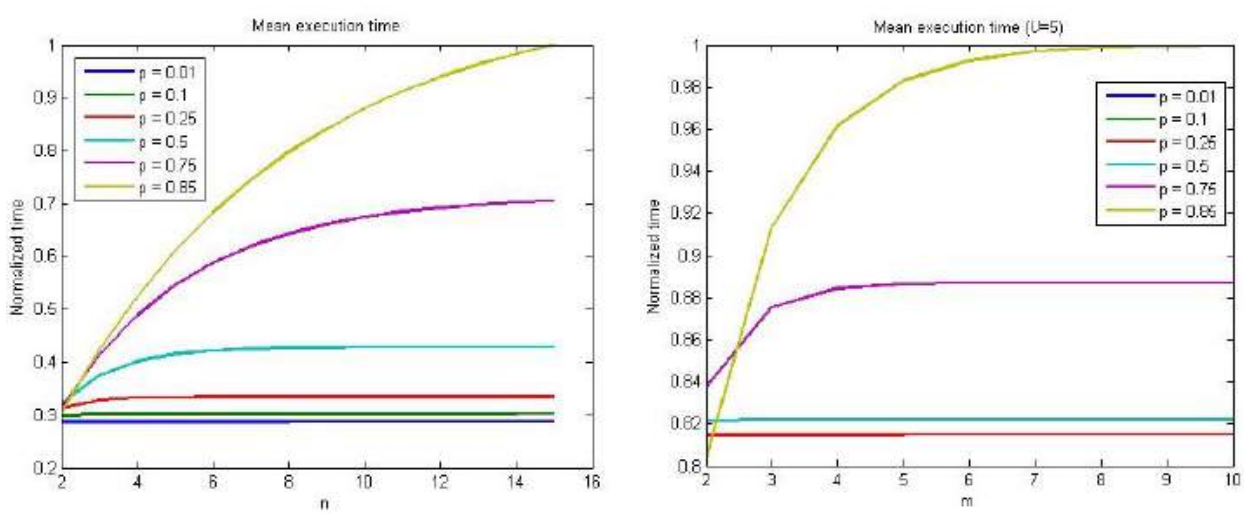

Fig. 5. (a) Graphical representation of the execution time: Eq (9)

(b) Graphical representation of the execution time: Eq (10-12)

Where $p$ is the probability that a HC refuses or that it does not answer to an execution order. $T_{\text {com }}$ is the required time to communicate a $\mathrm{HC}$ and the HM. $T_{\text {proc }}$ is the needed time by a $\mathrm{HC}$ to perform the actions related to a certain service. And $\overline{T_{\text {fall }}}$ is the mean time needed by the HM to notice a $\mathrm{HC}$ is not going to execute a service (for example, $T_{\text {fail }}=2 T_{\text {com }}$ if a rejection message is sent). Finally, $n$ is the number of HC which may execute the low-level service. Figure 5(a) represents graphically Eq. (9) for different values of $n$ and $p$. As can be seen, the execution time may grow up a $230 \%(p=0.85)$. Some applications, however, do not tolerate these variations in the 
execution time, and a more stable solution is required. This problem is addressed with the second proposed algorithm.

\subsection{Service execution through group management}

The basic idea of this second procedure is to introduce a first additional phase, where the hardware manager asks various hardware controllers if they are able to execute a certain low-level service.

When a service invocation arrives to the HM, it selects a group of $U$ hardware controller which may execute this service (following the probability function described in the previous section). If the total amount of HC which may execute the service in the system is $V$, then a total of $\left(\begin{array}{l}U \\ V\end{array}\right)$ groups can be defined. Then (see Figure 6 ) an execution petition is sent to all these HC. The hardware manager waits for the responses (a timer is launched in order to avoid blocking situations) which may accept the petition (offering some additional information, the expected processing time, for example) or reject the offer. Depending in the responses, the hardware platform state may be updated.

Finally, considering the $\mathrm{HC}$ which accept the execution petition it is constructed (in the HM) a new probability function in order to determine the device which must execute the service. From this point, the procedure continues as described in the case of a service execution through individual management

The additional initial phase makes the mean execution time greater, but much more stable (10-12). Figure 5(b) represents graphically the evolution of the mean execution time for different values of $m$ and $p$. As can be seen, in this case, there is only a $25 \%$ variation between the best and the worst situation (in the previous procedure the equivalent value was around a $250 \%$ ), although times are higher.

$$
\begin{gathered}
\overline{T_{\text {exe }}}=(1-q) T_{\text {success }}+\sum_{k=1}^{m-1}(1-q) q^{k}\left(T_{\text {success }}+k \overline{T_{\text {fall }}}\right)+m \overline{T_{\text {fall }}} \forall m \geq 2 \\
T_{\text {success }}=\left(2 T_{\text {com }}+T_{\text {proc }}\right)+2 T_{\text {com }}+T_{\text {dec }} \\
q=\prod_{k=1}^{U} p
\end{gathered}
$$

Where $T_{\text {dec }}$ is the time employed by the HM to construct the additional probability functions, and $m$ is the number of groups which may be created with the $U$ HCs which can execute a service. Considering any of the proposed management procedures, if no hardware controller may execute the service there are two alternatives. If the service is unitary, then the invocation is refused and an error is returned to the higher level. However, if the service is divisible, then, the HM may decompose the service and employ any of the previously described procedures which its parts. Any case, if any of the parts cannot be executed, the entire invocation is refused and an error is returned by the HM. 


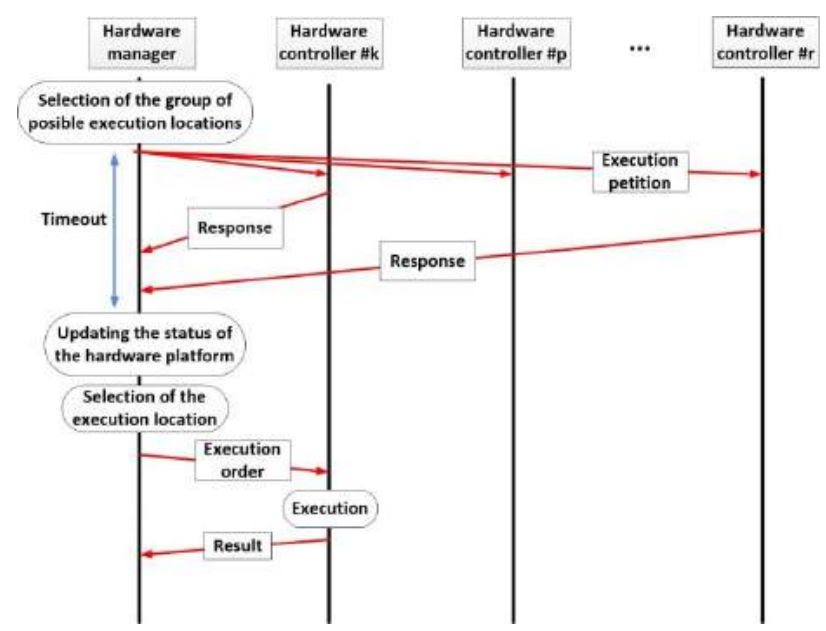

Fig. 6. Message sequence chart (service execution through group management)

\section{Experimental validation}

An experimental validation was designed in order to validate the proposal and analyze the performance of the proposed technology. The experimental validation consisted of a simulated scenario based on the NS3 simulator. The deployed architecture contained one hardware manager, fifteen hardware controllers and one hundred and ten (110) sensors and actuators (homogeneously distributed among the hardware controllers).

In order to implement the described procedures in the components of the simulated scenario, each node in the NS3 simulator was provided with a tap bridge (or ghost node). These bridges are able to connect an internal NS3 component with an external entity, which allows implementing in a very easy way complex algorithms and other proprietary solutions for which there are no libraries. In particular, the hardware manager and the hardware controller were connected with virtual Linux machines based on the KVM technology. Sensors and actuators were created by means of Simulink software and deployed in a new virtual Linux machine connected with the NS3 nodes.

Hardware controllers were connected with hardware manager by means of a Bluetooth backbone. An XML-based solution was employed as data format for this communications (HM-HC). The connection among sensors and hardware controller was resolved using the IEEE 1451 technology [14]. Figure 7 represents the planned scenario and the simulator configuration. All costs defined by users were fixed to the value of the unit $q_{u s e r}=1$. Contributions in the cost calculation were considered independent, so the cost function $f_{\text {cost }}(\because)$ ) was defined as the arithmetic mean. 


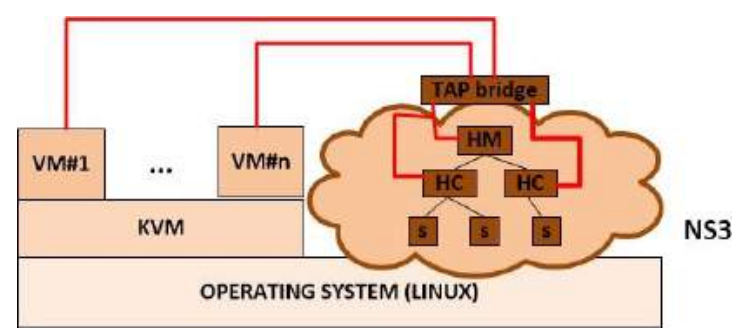

Fig. 7. Simulation scenario

The cost due to hardware considerations was understood as the arithmetic mean of two indicators: the battery consumption $\psi_{\text {bat }}$ and the occupied memory $\psi_{\text {mem }}(13-14)$. All services in the system were defined as unitary and non-geographic.

$$
\begin{gathered}
\psi_{\text {bat }}=\frac{\text { reduction in the battery charge }}{\text { total battery capacity }} \\
\psi_{\text {mem }}=\frac{\text { occupied memory }}{\text { total installed memory }}
\end{gathered}
$$

Finally, two different experiments were performed. During the first experiment connections among components were considered permanent, and losses in the Bluetooth backbone were increased progressively. The execution time was monitored when employing an individual management approach. During the second experiment, connections were considered ephemeral. The number of successful communications and the service execution time were monitored when employing both described procedures. Results were compared.

\section{Results}

In this section we present the results obtained in the experimental validation. Figure 8(a) represents the results of the first experiment. As can be seen, the execution time grows monotonously. As the losses rate goes up, the number of necessary iterations to perform a successful execution grows, so the execution time increases. The variation is, as indicated in theory, near two magnitude orders. The evolution law is almost linear. On the other hand, in regular situations (networks without congestion) the execution time is very low (around 0.1 normalized temporal units).

In Figure 8(b), the results of the second experiment compare the evolution in the execution time obtained for the first procedure (individual management) and for the second one (group management). Groups of hardware controllers are made of five elements. As seen in theory, the execution time is higher when considering group management (in the best situation the execution time grows seven times). However, as showed on Figure 8(b), the evolution law is almost constant, so almost no variations in the execution time are suffered. 

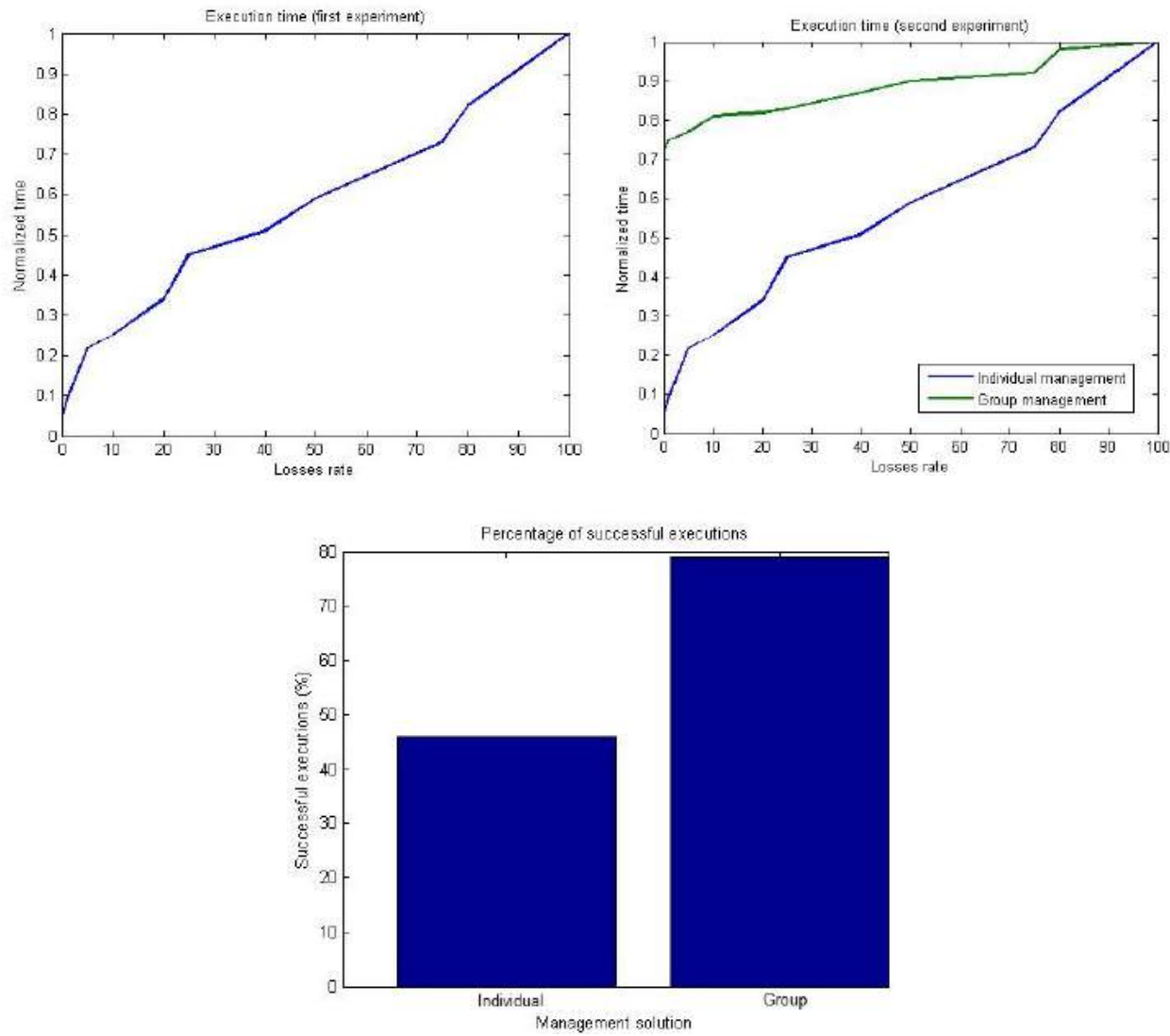

Fig. 8. (a) Results of the first experiment (b) Results of the second experiment (execution time)

(c) Results of the second experiment (successful executions)

This approach is very useful in scenarios involving ephemeral connections (as in mobile wireless sensor networks). These connections may tolerate a higher execution time, but variations are badly supported. Figure 8(c) shows the improvement in the number of successful communications (around a $50 \%$ increase).

\section{Conclusions}

Cyber-physical Systems require a solution for low-level service management. This solution must address the required procedures to finally associate a service execution with a hardware device.

In this paper we have proposed a low-level service management technology based on three different procedures. This solution belongs to the level named as "logic" in the proposed general framework. The first procedure considers a hardware monitoring process in order to evaluate the hardware platform state. The second one describes a 
service management and execution method based on individual management. Finally, a solution for service execution based on group managements is also proposed.

The experimental validation showed that individual management provides a very low execution time in the best case, but this time grows very fast if the situation of the hardware platform gets worse. In this cases a group management generates a higher execution times but much more stable.

Acknowledgments. Borja Bordel has received funding from the Ministry of Education through the FPU program (grant number FPU15/03977). Additionally, the research leading to these results has received funding from the Ministry of Economy and Competitiveness through SEMOLA project (TEC2015-68284-R) and from the Autonomous Region of Madrid through MOSI-AGIL-CM project (grant P2013/ICE3019, co-funded by EU Structural Funds FSE and FEDER).

\section{References}

1. 1. Lee, E.A. Cyber-physical systems - Are computing foundations adequate? In NSF Workshop on Cyber-Physical Systems: Research Motivation, Techniques and Roadmap, Austin, TX, USA, 16-17 October 2006.

2. Rajkumar, R. R., Lee, I., Sha, L., \& Stankovic, J. (2010, June). Cyber-physical systems: the next computing revolution. In Proceedings of the 47th Design Automation Conference (pp. 731-736).

3. Sha, L., Gopalakrishnan, S., Liu, X., \& Wang, Q. (2009). Cyber-physical systems: A new frontier. In Machine Learning in Cyber Trust (pp. 3-13). Springer US.

4. National Institute of Standards and Technology. Cyber-Physical Systems Homepage. Available online: http://www.nist.gov/cps/ (accessed on 17 March 2017).

5. Bordel Sánchez, B. , Alcarria, R., Sánchez-de-Rivera, D., \& Sánchez-Picot, A. Enhancing Process Control in Industry 4.0 Scenarios using Cyber-Physical Systems. Journal of Wireless Mobile Networks, Ubiquitous Computing, and Dependable Applications, 7:4 (December 2016), pp. 41-64

6. Horváth, K. A., \& Telek, M. (2015, May). Task Delegation in a Peer-to-Peer Volunteer omputing Platform. In International Conference on Analytical and Stochastic Modeling Techniques and Applications (pp. 115-129). Springer International Publishing.

7. Jaradat, W., Dearle, A., \& Barker, A. (2014, December). Workflow partitioning and deployment on the cloud using orchestra. In Proceedings of the 2014 IEEE/ACM 7th International Conference on Utility and Cloud Computing (pp. 251-260). IEEE Computer Society.

8. Kritikos, K., \& Plexousakis, D. (2014). Novel optimal and scalable nonfunctional service matchmaking techniques. IEEE Transactions on Services Computing, 7(4), 614-627.

9. Bordel, B., de Rivera, D. S., \& Alcarria, R. (2016, July). Plug-and-play transducers in Cyber-Physical Systems for device-driven applications. In Innovative Mobile and Internet Services in Ubiquitous Computing (IMIS), 2016 10th International Conference on (pp. 316-321). IEEE.

10. Talantikite, H. N., Aissani, D., \& Boudjlida, N. (2009). Semantic annotations for web services discovery and composition. Computer Standards \& Interfaces, 31(6), 1108-1117.

11. Shenker, S. (1997). Specification of guaranteed quality of service.

12. Bordel Sánchez, B., Alcarria, R., Martín, D., \& Robles, T. (2015). TF4SM: a framework for developing traceability solutions in small manufacturing companies. Sensors, 15(11), 29478-29510.

13. Callaway, E., Gorday, P., Hester, L., Gutierrez, J. A., Naeve, M., Heile, B., \& Bahl, V. (2002). Home networking with IEEE 802.15. 4: a developing standard for low-rate wireless personal area networks. IEEE Communications magazine, 40(8), 70-77.

14. Lee, K. (2000). IEEE 1451: A standard in support of smart transducer networking. In Instrumentation and Measurement Technology Conference, 2000. IMTC 2000. Proceedings of the 17th IEEE (Vol. 2, pp. 525-528). IEEE. 


\title{
Self-configuration in humanized Cyber-Physical Systems
}

\author{
Borja Bordel $^{1}{ }^{1}$, Ramón Alcarria ${ }^{2}$, Diego Martín ${ }^{1}$, Tomás Robles ${ }^{1}$, Diego Sánchez de Rivera ${ }^{1}$ \\ 1 Department of Telematics Systems Engineering. Universidad Politécnica de Madrid. Avenida \\ Complutense $\quad \mathrm{n}^{\mathrm{o}} \quad$ 30. $28040 \quad$ - Madrid (España); E-Mails: bbordel@dit.upm.es; \\ diego.martin.de.andres@upm.es; tomas.robles@upm.es; diego.sanchezderiveracordoba@gmail.com \\ 2 Department of Topographic Engineering and Cartography. Universidad Politécnica de Madrid. \\ Campus Sur, 28031 Madrid, Spain; E-Mail: ramon.alcarria@upm.es
}

* Author to whom correspondence should be addressed; E-Mail: bbordel@dit.upm.es; Tel. 915495700 ext. 3035

\begin{abstract}
Most works on Cyber-Physical Systems (CPS) are based on classic hardware infrastructures made of sensors, actuators and processing devices. Usual self-configuration technologies, then, do not allow humans to be integrated in CPS as service providers. Therefore, in this work we propose a new selfconfiguration technology for humanized CPS. The proposed technology uses simple binary and mathematical operations in order to reduce the convergence time, improve the scalability and address the dynamism introduced by humans into CPS. Besides, a human-oriented quality-of-service algorithm based on the Maslow pyramid is also introduced. Moreover, an experimental validation is conducted in order to validate the proposed solution as a useful and scalable self-configuration technology for humanized Cyber-Physical Systems.
\end{abstract}

\section{KEYWORDS}

Cyber-Physical Systems; HCI; Humanized computing; Self-configuration; Humanized CPS; Maslow pyramid

\section{ACKNOWLEDGMENTS}

The research leading to these results has received funding from the Ministry of Economy and Competitiveness through SEMOLA project (TEC2015-68284-R) and from the Autonomous Region of Madrid through MOSI-AGIL-CM project (grant P2013/ICE-3019, co-funded by EU Structural Funds FSE and FEDER).

\section{CONFLICTS OF INTEREST}

The authors declare that there is no conflict of interest regarding the publication of this paper. 


\section{INTRODUCTION}

Traditional Cyber-Physical Systems (CPS) are defined (Lee 2006) as integrations of computation with physical processes. Embedded computers and networks monitor and control the physical processes, usually with feedback loops where physical processes affect computations and vice versa. Nevertheless, CPS paradigm is increasingly applied to scenarios where physical processes are executed by humans, and physical devices are only a medium through which order the execution of tasks and monitor their performance. In (Bordel Sánchez et al 2015), for example, a scenario is presented where CPS paradigm is applied to manufacturing companies.

Despite these antecedents, most practical implementations of CPS usually do not include humans as service providers (Cardenas et al 2008) or they treat them as an ordinary component (Schirner et al 2008) (usually identified with the monitoring infrastructure), so the resulting system is not in fact humanized.

In order to properly integrate humans in CPS as service providers, in (Bordel et al 2015) the authors proposed a particular implementation of CPS by adding some additional requirements. Basically, we included prosumer (an acronym formed by the words producer and consumer) user's requirements saying that "the system must be adaptable to different domains and be oriented to prosumer user, for which must implement self-configuration capabilities". In this context, configuration is understood as the process which allows discovering the capabilities of humans and physical devices present in the system, and propagating them to the cybernetic world wrapped as services. This process runs in the system boot, and gets updated periodically. These new requirements enable the integration of humans as service providers (producers) in humanized CPS, in the same way as traditional CPS include sensors, actuators or processing devices. Figure 1 shows the comparison between traditional CPS and humanized CPS.

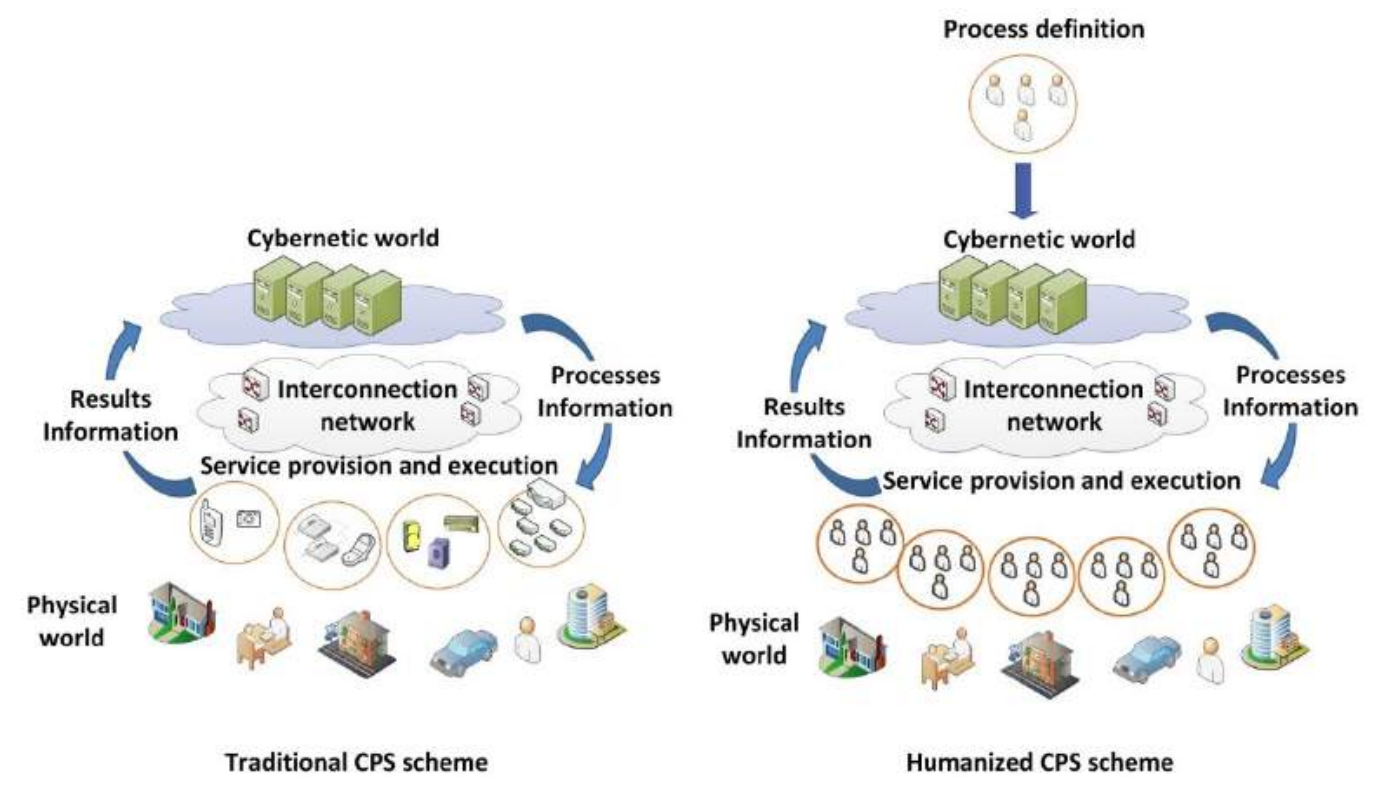

Figure 1. Comparison between the architecture of traditional CPS and the humanized CPS

Although in (Bordel et al. 2015) an implementation architecture for humanized CPS is described, and general technologies are associated to each module, it remains undefined the particular algorithms, functional components, etc. which implement the cited self-configuration capabilities and become a CPS into a humanized system. Therefore, the objective of this paper is to extend the definition presented in (Bordel et al. 2015) describing the particular self-configuration technologies implemented in CPS to support human integration. We implement the proposed solutions and carry out an experimental validation in order to validate the performance of the proposed technology, and to compare the obtained results against previous proposals. 
The rest of the paper is organized as follows: Section 2 introduces the state of the art in self-configuration solutions for Cyber-Physical Systems. Section 3 presents the proposed technology. Section 4 provides an experimental validation of the proposal. Finally, Sections 5 and 6 explain some results of this experimental validation and the conclusions of our work.

\section{STATE OF THE ART}

Adequate self-configuration policies are basic for human integration in CPS (Kao et al 2015; Park et al 2015) Nowadays, no special self-configuration technology has been described for including humans in CPS as service providers. Moreover, most works on CPS which consider humans represent them as the monitoring infrastructure which controls their performance. So, the usual self-configuration technologies for CPS only operate with physical devices.

Some of these proposals are based on the maintenance of a centralized heavy context describing the system state. In (Dillion, Potdar et al.2011) and (Dillion, Zhuge et al 2011) self-configuration functionalities are supported by an intelligent scheduler installed in each device, communicating with a central core where decisions are taken. In SOCRADES project (Colombo et al 2010) a self-configuration technology based on petri nets is proposed. However, every reconfiguration results in a manual effort. In the same way, in (Hoang et al 2012), a special middleware for self-configuration where virtual components represent the real physical devices is proposed. However, it also needs manual effort from the system administrator. Finally, AutoPnP (Keddis et al 2013) project deals with automatically adding and configuring new components using artificial intelligence algorithms.

Other solutions are based on the exchange of large amounts of data describing in a comprehensive way the system state. In (De Lemos et al 2013), a solution for self-configuration based on control loops is proposed. In (Mönks et al 2014) a distributed technology for self-configuration is described. Each device generates a description file when a change occurs in the system and it is processed by the rest of the components in order to modify the system's configuration. Finally in (Wang et al 2014) it is proposed a service-oriented self-configuration policy, based on semantic technologies. Each device generates a description file when a change occurs in the system, which is processed in order to decide if a service has to be added, deleted or modified.

All these proposals, however, cannot be easily extended in order to represent real humans. The first proposals require the execution of complex algorithms to modify the stored heavy context. Then, the reconfiguration process tends to be slow. Such solutions, therefore, are adequate for systems made of physical devices where the number and variety of changes is low (typically adding and removing components). However, in systems including humans as service providers, the configuration varies in a very fast and dynamic way, and the use of the previous algorithms may cause the system never reaches a stable state. The problem of the second type of proposals is the great amount of generated signaling. In some cases, the signaling load on the system is close to $50 \%$, which is very difficult to maintain and scale when the system's configuration varies in a very fast and dynamic way as in humanized CPS.

Therefore, basically, it is necessary to design a self-configuration technology being able to support the fast and dynamic way in which the humans' state changes. The solution should generate a minimal load of signaling and employ simple algorithms being able to update the system state as fast as possible. For that, we propose a service-oriented solution based on simple binary and mathematical operations, mixed with a human-oriented quality-of-service algorithm based on the Maslow pyramid (Maslow 1943). 


\section{PROPOSAL: A SELF-CONFIGURATION SOLUTION FOR HUMANIZED SYSTEMS}

This section analyzes the humanized CPS and the previous considerations which have to be taken into account in the design of the proposed self-configuration technology. Moreover, the functional architecture which supports the proposed self-configuration solution, its theoretical formalization and the practical algorithms implemented are described.

\subsection{PREVIOUS CONSIDERATIONS}

In Figure 1 we showed the basic scheme for humanized CPS. As can be seen, humans are included as service providers. However, humans are much more complex than physical devices. Thus, a deep analysis of all possible scenarios relating humanized CPS should be based on the definition and characterization of humans in several different situations. But in our case, we are going to work over some common characteristics for all cases, which are:

1- All involved humans in the system have the same academic and professional qualification.

2- It is only considered people without mental illness or special needs.

3- Services provided by humans could be characterized just with the number of people involved in the execution.

4- The way in which humans perform a service depends on their motivation

5- Humans' motivation can be characterized by means of the usual Maslow's hierarchy of needs (Maslow 1943)

The first and second points guarantee a certain level of homogeneity in humans. In general, the services that a person can execute depend on their education, experience, physical and psychological skills, etc. However, treating each user in a particular way requires performing an evaluation and cataloging process first. Since the taxonomy of users (Cotterman et al 1989) is not the objective of this work, in this proposal we have considered that all users have the same profile.

The third point enables to consider services involving various people. In almost any scenario, there exist tasks which have to be executed by means of the collaboration of various workers (like carrying a heavy object or doing a warehouse inventory). Our self-configuration technology allows this type of services.

Finally, fourth and fifth points explain how to evaluate the quality-of-service (QoS) provided by humans depending on their physiological and psychological state. In that way, our self-configuration technology not only considers the presence or absence of users, but also their emotional capacity to execute a particular service.

\subsection{FUNCTIONAL ARCHITECTURE AND MATHEMATICAL FORMALIZATION}

The proposed self-configuration technology is service-oriented: various software components analyze the humans present in the system at each moment, and compose the services which could be executed in such humanized CPS in that particular moment. The services, as well as some information about the offered QoS, are registered in a local and/or remote repository to be invoked in the end user's applications.

The proposed functional architecture for supporting the described technology can be seen in Figure 2. Various components can be distinguished.

User-focused sensors and actuators: They include the entire dedicated hardware infrastructure to the interaction with humans. The capabilities of these devices are not propagated to the higher layers and offered to the end-users wrapped as services. Such capabilities are only accessed by the user-focused hardware controller in order to monitor humans and act over them. We distinguish two different types of devices. On the one hand the required devices, which are those with which humans must interact to be integrated in the CPS (if a person is outside the coverage area of any of them, he is not considered as an available service provider). On the other hand, the optional devices, which provide additional information 
about humans (which could be very important) but they do not influence the decision of considering a person as an available service provider or not.

User-focused hardware controller: This component is responsible for gathering data from the userfocused sensors and actuators, and translating this information into valid indicators about the humans' state. Each controller includes a User discovery module which determines the number of humans acting as available service providers in the system.

User manager: It coordinates the operation of the different user-focused hardware controllers. It decides at each moment the controller to which the execution of a specific atomic service (those which may be directly matched with the humans' abilities) is delegated.

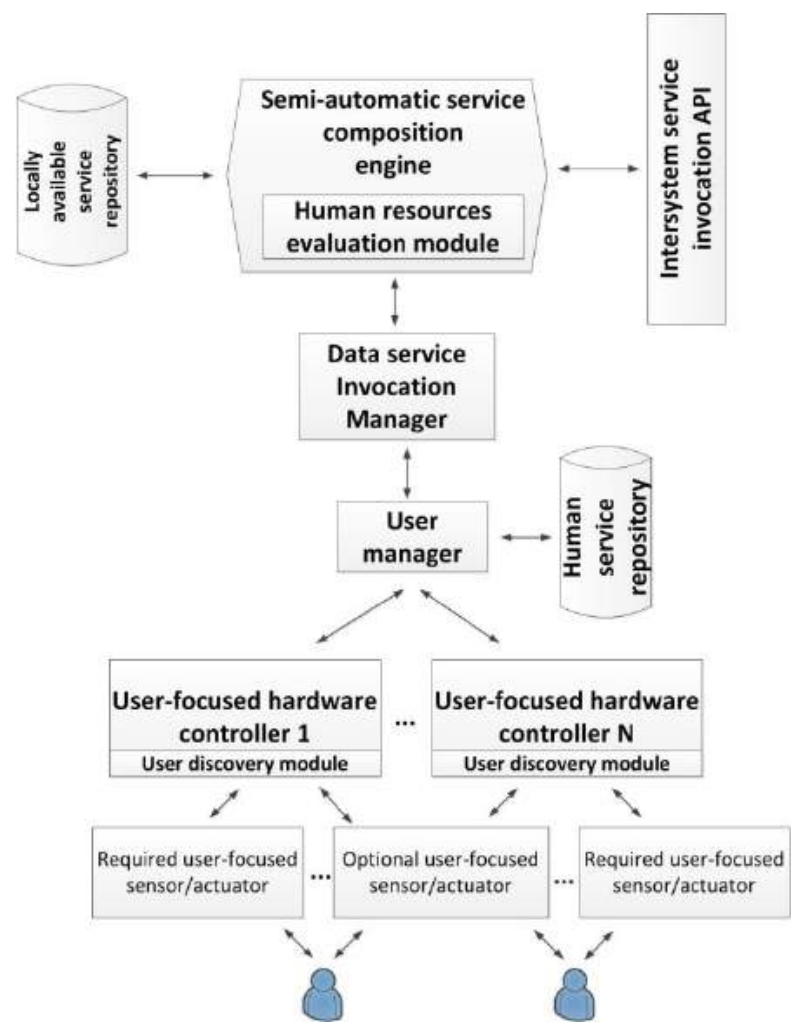

Figure 2. Functional architecture

Human service repository: It contains all the necessary information about the atomic services provided by humans.

Data service invocation manager: It becomes independent the physical platform (at low level) and the services domain (at high level). It translates calls to high-level services into invocations to the User managers. It also adapts the responses of the manager to the callback format described in the high-level service.

Semi-automatic service composition engine: This component composes high-level services using semiautomatic techniques and the information about the atomic services from the Data Services Invocation Manager. In this paper we are not discussing the particular technology employed in the process of service composition. Any of the proposed solutions in the research literature, such as (Domingues et al 2011) or Hydra Project (European commission 2005) will be valid. This component is also responsible for calculating the QoS offered, for which it includes special modules such as the Human resources evaluation module (which executes an algorithm based on the Maslow pyramid). Finally, once determined the composed services, this module registers the obtained services in a Locally available 
service repository so they can be called from local applications, and/or in a public remote repository through the Intersystem service invocation API, so they can be called from applications in the cloud.

Over this architecture, the following self-configuration process is executed in order to determine the available services in the system.

First, each Users-focused hardware controller discovers the sensors and actuators which it controls. Then, each controller gathers a first amount of data from these devices and creates a descriptor $u d$. The descriptor of the i-th User-focused hardware controller can be denoted as (1).

$$
u d_{i}=\left\{n_{i}, q_{i}, y_{i}\right\}
$$

The descriptor includes three elements. $n_{i}$ indicates the number of humans recognized as available service providers. $q_{i}$ is a cost vector where each position indicates the cost of accessing to the user-focused hardware to perform a certain action. Finally, $y_{i}$ is a collection of indicators about the humans' state obtained from the processing of the gathered data from the user-focused sensors.

In the third step all users-focused hardware controllers send their descriptors to the Users manager. Then, in the Users manager four collections may be described: $U D$ the collection of descriptors, $Q$ the collection of cost vectors, $N U$ the collection of the numbers of users and $Y$ the collection of indicators.

$$
\begin{aligned}
& U D=\left\{u d_{i}, i=1 \ldots M\right\} \\
& Y=\left\{y_{i}, i=1 \ldots M\right\} \\
& Q=\left\{q_{i}, i=1 \ldots M\right\} \\
& N U=\left\{n_{i}, i=1 \ldots M\right\}
\end{aligned}
$$

Where $M$ is the number of User-focused hardware controllers in the system.

Using (2), (4) and (5) the Users manager generates a partitioned matrix $U M$ describing the atomic services provided by humans, as well as the QoS offered for each service due to the hardware platform.

$$
U M=\left(\sigma_{h}\left|H Q_{g}\right| H Q_{c}\right)
$$

As can be seen in (6), $U M$ is composed of three submatrices. $\sigma_{h}$ is a binary column matrix where each row indicates if a certain atomic service is available. $H Q_{g}$ is a matrix describing the quality due to hardware (users-focused sensors and actuators) for each atomic service in one particular moment. Each row in $H Q_{g}$ contains the quality parameters of the atomic service to which it is referred the same row in $\sigma_{h} . H Q_{c}$ is a matrix describing the context-depending QoS (no guaranteed) due to user-focused hardware. Sometimes, the system presents surplus resources (such as optional user-focused sensors) that could be assigned to the existing services in order to improve their quality of execution. These resources, however, cannot be guaranteed, and their availability depends on the system's context. The relation between the collections $U D, Y$ and $Q$ and the matrix $U M$ is denoted as a vector function $G$ (7).

$$
\left(\sigma_{h}, H Q_{g}, H Q_{c}\right)=\vec{G}(N, Q)=\left(g_{1}(N U), g_{2}(Q, \Sigma), g_{3}(Q, \Sigma)\right)
$$

Where $\Sigma$ is a collection of results about measurements on the users-focused hardware to determine the quality of service execution. Humans are not included in these measurements because their state will be considered later through an algorithm based on the Maslow's pyramid. Both $\Sigma$ and $Y$ are periodically updated so the system's configuration is refreshed each time step.

Once the $U M$ matrix, the collection of human indicators $Y$ and the collection $N U$ have been constructed, these three elements are transmitted to the Data services invocation manager. 
In the Data service invocation manager, $U M$ matrix is mapped over a description file (typically an XML file) where atomic services are described as high-level services. For example, in the case of using Simple Object Access Protocol (SOAP) technologies, we would use a Web Services Description Language (WSDL) file. For its part, collections $Y$ and $N U$ will be also mapped on a description file, which might be also based on XML.

Then, both description files are sent to the Semi-automatic service composition engine. In this component, first it is run an algorithm based on the usual Maslow's hierarchy of needs (8).

$$
m l=\operatorname{maslow}\left(Y, m t, m l_{-h}\right)
$$

The function maslow takes the collection of indicators $Y$ and produces a vector $m l$ where each position represents the motivation level on the Maslow's pyramid (see Figure 3) which a certain person occupies. $m t$ is meta-information about the indicators such as the maximum possible value. And $m l_{-h}$ is the history of the motivation level occupied by humans in the past.

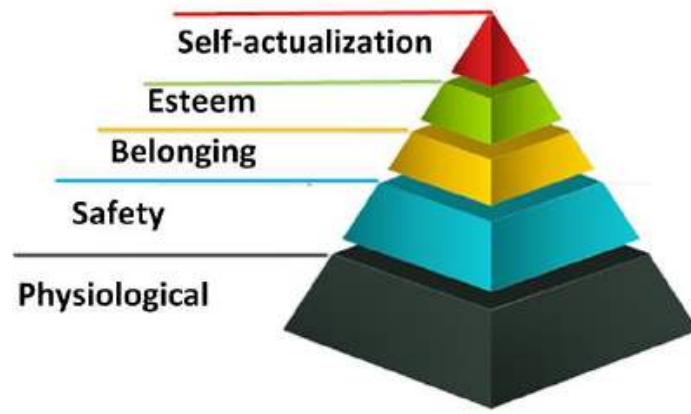

Figure 3. Maslow's hierarchy of needs or Maslow's pyramid

Then, the engine composes a collection of high-level services $S$ using the atomic services provided by and humans.

$$
S=\left\{s_{j}, j=1, \ldots, L\right\}
$$

Where $L$ is the number of composed services created in the semi-automatic engine.

The $\mathrm{j}$-th service of the collection can be denoted as (10).

$$
s_{j}=\left\{X M L, Q o S_{g}, Q o S_{c}, B C\right\}
$$

Where $X M L$ describes the service's input, output and its decomposition in atomic services. $Q o S_{g}$ represents the final guaranteed $\mathrm{QoS}$ for the composed service (expressed as a numerical vector where each position represents the value of one quality parameter). $Q o S_{c}$ expresses the context-depending QoS for the final composed service. And $B C$ represents the behavior constraints for the composed service (such as time or range constraints for service availability).

The $X M L$ and $B C$ elements are generated during the service composition process. $Q o S_{g}$ and $Q o S_{c}$ are calculated from quality parameters of all atomic services which compose a composed service.

$$
\begin{aligned}
& Q o S_{g}=q u a l \_g\left(H Q_{g}^{1}, \ldots, H Q_{g}^{R_{2}}, m l, N U\right) \\
& Q o S_{c}=q u a l_{-} c\left(H Q_{c}^{1}, \ldots, H Q_{c}^{R_{2}}, m l, N U\right)
\end{aligned}
$$

Where $H Q_{g}^{r}$ are the guaranteed quality parameters due to the user-focused hardware of the r-th atomic service which is part of the composite service. The $H Q_{c}^{r}$ parameters have the same interpretation as the 
above, but applied to context-depending quality parameters. And $R_{2}$ is the number of atomic services which are part of the composite service.

The functions qual_c and qual_g mix the quality parameters due to the user-focused hardware platform with the quality parameters due to humans. In Figure 4 it is presented a scheme about the QoS estimation in the proposed technology.

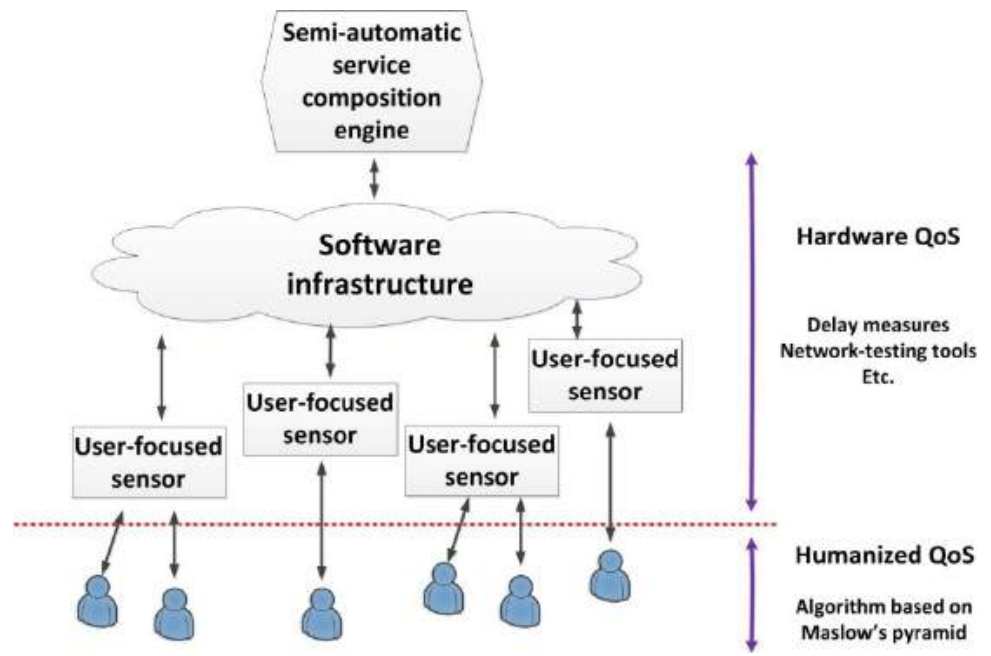

Figure 4. Estimation of the QoS in humanized CPS

As can be seen in Figure 4, traditional definitions, methodologies and procedures (such as the iperf network-testing tool) are used to evaluate the QoS provided by the user-focused hardware platform. As these technologies are not easily applicable to humans, in order to include the humans' performance into the quality parameters, a specific algorithm based on the Maslow's hierarchy of needs is implemented for estimating the "human QoS". Functions qual_c and qual_g join both values.

Finally, each composited service may present three different states: available, unavailable and deleted. Table 1 describes the conditions which must fit a service to present each state.

Table 1. Services' states in humanized CPS

\begin{tabular}{|c|l|}
\hline State & \multicolumn{1}{|c|}{ Description } \\
\hline Available & $\begin{array}{l}\text { A service is available when all the atomic services which compose it are } \\
\text { available, and the guaranteed QoS of the composite service surpasses the } \\
\text { established QoS thresholds. }\end{array}$ \\
\hline Unavailable & $\begin{array}{l}\text { A service is unavailable either when one of the atomic services which } \\
\text { compose it becomes unavailable, or when the guaranteed QoS of the } \\
\text { composite service falls below the established QoS thresholds. } \\
\text { A service which is considered unavailable may return to the available } \\
\text { state without refreshing the service composition process. }\end{array}$ \\
\hline Deleted & $\begin{array}{l}\text { A service is considered as deleted when gets unavailable for a time which } \\
\text { surpasses a certain limit. } \\
\text { A service which is considered deleted only may return to the available } \\
\text { state after refreshing the service composition process in the semi- } \\
\text { automatic engine. }\end{array}$ \\
\hline
\end{tabular}


As can be seen, in Table 1 it is referred some QoS thresholds which all services must surpass to be considered available. These thresholds are applied in a two-phase process.

In the first phase each quality parameter in $Q o S_{g}$ must surpass a limit $k_{i}(13)$.

$$
Q o S_{g}^{i}>k_{i} \quad i=1, \ldots, P
$$

Where $P$ is the number of considered quality parameters in the system (i.e. the number of position in $Q o S_{g}$ ). In order to abbreviate the notation we also can denote (14)

$$
Q o S_{g}>K
$$

Where $K=\left\{k_{i} \quad i=1, \ldots, P\right\}$

If the first phase is verified, then it is evaluated a second phase. In this new phase the total guaranteed QoS $Q o S_{t o}$ must surpass a limit $Q o S_{t h}(15)$.

$$
Q o S_{t o}>Q o S_{t h}
$$

The expression which allows obtaining the total guaranteed QoS from the vector $Q o S_{g}$ is as follows (16).

$$
Q o S_{t o}=\sum_{i=1}^{P} \omega_{i} \frac{Q o S_{g}^{i}}{\lambda_{i}}
$$

Where $\lambda_{i}$ is the standard value of the i-th quality parameter and $\omega_{i}$ is the relative weight of the i-th parameter in total estimation of the offered QoS.

\subsection{IMPLEMENTATION}

In the previous Section the mathematical formalization of the proposed self-configuration mechanism was explained. In this Section a particular implementation is proposed and, according to this implementation, the functions, data format, vector, etc. are fixed.

The proposed deployment was based on a manufacturing scenario, where each worker acts as service providers for which all of them are provided with separated NFC-enabled gloves such as described in (Bordel Sánchez et al 2015). Besides, in order to collect data about humans' state a Bitalino board (Bitalino 2014) was carried out for every worker. Figure 5 shows a prototype of the mentioned glove.

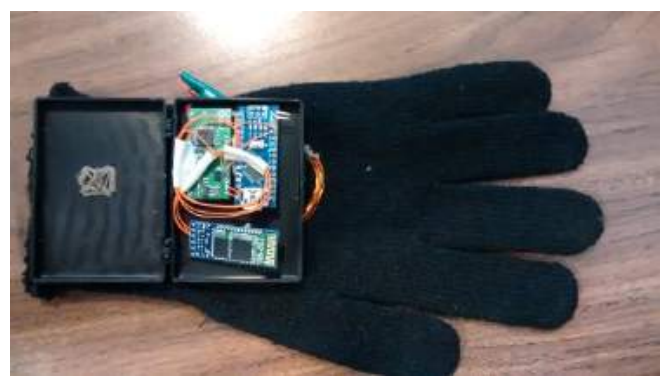

Figure 5. Prototype of the proposed NFC-enabled glove

Moreover, the manufacturing scenarios included two smart worktable (provided with various NFC readers and LEDs) being able to detect the number of objects on their surface and inform the users. The electronic scheme and validation of this smart worktable may be also found in (Bordel Sánchez et al 2015). Figure 6 shows a prototype of the cited worktable. 


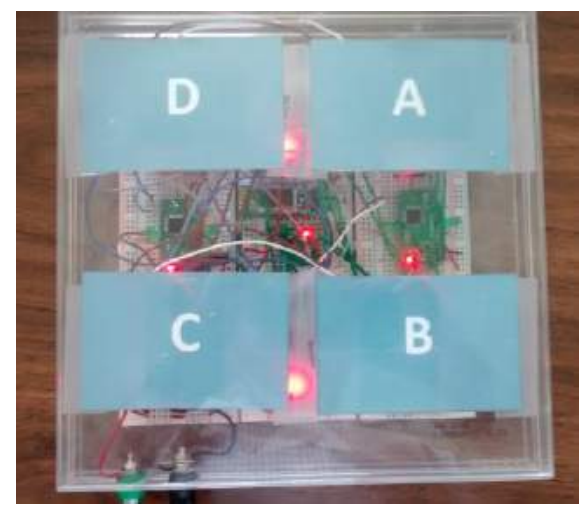

Figure 6. Prototype of the proposed smart worktable

Programming in the previously cited hardware components was completely redefined for this work. In particular, it was designed to include two functional components from functional architecture in Figure 2: user-focused sensors and the user-focused hardware controllers (including the appropriated interfaces, protocols and other characteristics, necessary to run the proposed self-configuration algorithm). The rest of the components in the functional architecture described in Figure 2 were implemented in a central server using Java technologies. Hardware components were connected through an ad-hoc Bluetooth LowEnergy network and software elements were communicated using Internet sockets.

Considering the details cited above about the particular implementation proposed (and specially the information about the hardware devices involved), it is possible to fix the vector and matrix format for all the data elements described in the previous sections. Table 2 shows the proposal.

Table 2. Data format in self-configuration process for humanized CPS

\begin{tabular}{|c|c|}
\hline Element & Pattern \\
\hline$\sigma_{h}$ & (Carrying object $\quad$ Remove object off table Place object on table) \\
\hline$H Q_{g}$ and $H Q_{c}$ & $\left(\begin{array}{ccc}\text { Cost }(\text { element_1) } & \text { Response time }(\text { element_1) } & \text { Availability }(\text { element_1) } \\
\text { Cost }(\text { element_2 }) & \text { Response time }(\text { element_2) } & \text { Availability }(\text { element_2 }) \\
\ldots & \ldots \\
\text { Cost }(\text { element_N }) & \text { Response time }(\text { element_N }) & \text { Availability }(\text { element_N } N)\end{array}\right)$ \\
\hline$y_{i}$ & (Stress level) \\
\hline$\Sigma$ & (Response time Availability) \\
\hline $\begin{array}{c}Q o S_{g} \text { and } \\
Q o S_{c}\end{array}$ & (Cost Response time Availability) \\
\hline
\end{tabular}

For simplicity, we have implemented reduced-dimensions vectors and matrix in order to clarify the proposed technology, though in practice they may be as big as needed.

Once described the data format, we are specifying the practical functions implementation (also considering the information previously provided).

Function $g_{1}$ is implemented by means of a hash table which relates a key (the value $N U$ ) with a value (the vector $\sigma_{h}$ ). This table must be preconfigured depending on the deployment scenario. Basically, function $g_{1}$ obtains the available atomic services from the number of humans present in the system. Later, functions $g_{2}$ and $g_{3}$ are presented below (17) (18). Function $g_{2}$ calculates the guarantee QoS, which 
matches with the maximum cost offered by the user-focused hardware controllers for a certain atomic service. Function $g_{3}$ obtains the context-depending QoS, which corresponds with the minimum cost offered by the user-focused hardware controllers for a certain atomic service, adequately modified if additional capabilities are available in the system.

$$
\begin{gathered}
H Q_{g}^{i}=g_{2}(Q, \Sigma)=\left(\max \left(\left\{\sum q_{j} c_{i} \quad j=1, \ldots, Q_{1}\right\}\right) \quad \Sigma^{1} \quad \Sigma^{2}\right) i=1, \ldots, Q_{2} \\
H Q_{c}^{i}=g_{3}(Q, \Sigma)=\left(\min \left(\left\{\sum q_{j} c_{i}+\sum \mu_{i} q_{j} v u_{j} \quad j=1, \ldots, Q_{1}\right\}\right) \quad \Sigma^{1} \quad \Sigma^{2}\right) i=1, \ldots, Q_{2}
\end{gathered}
$$

Where $c_{i}$ in a binary vector where each position indicates the need to include or not a certain capability in order to provide a certain atomic service. $Q_{1}$ is the number of user-focused hardware controllers (locations) where the atomic service is available and $Q_{2}$ is the number of atomic services defined in the system. Finally, vector $\mu_{i}$ is a numerical vector where each position indicates the modification in the QoS of the $\mathrm{i}$-th atomic service due to the existence of additional available capabilities. And $v u_{j}$ is a binary vector indicating the user-focused hardware capabilities available in the $j$-th user-focused hardware controller.

The maslow function is defined to be independent of the number and type of selected indicators to represent the users' state. In our case we have selected as indicator the stress level. In (Cohen et al 1983) a graduated scale from 0 to 56 is used to represent the stress in humans. Algorithm 1 presents the described function.

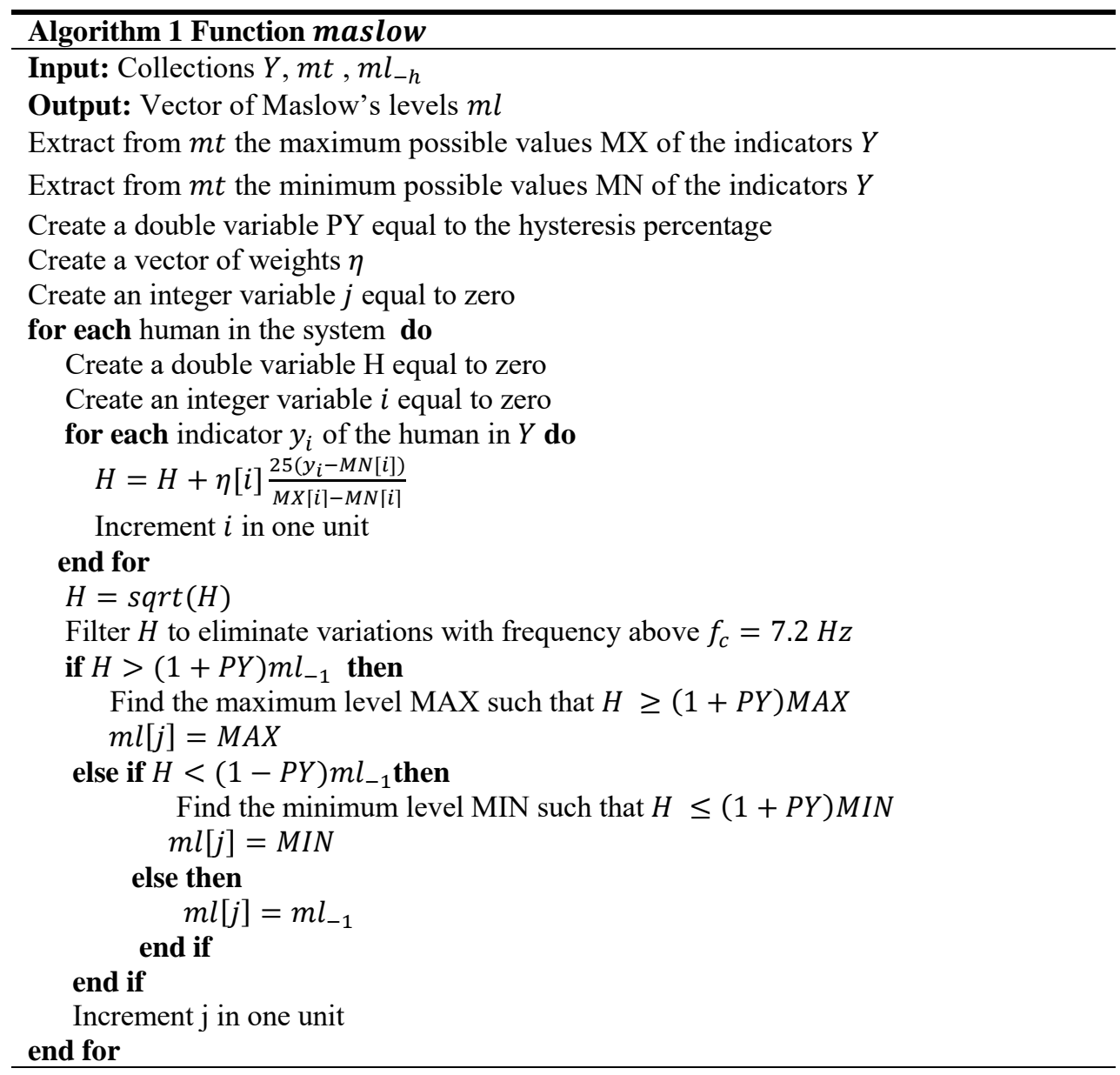

Algorithm 1, in summary, executes the following steps. First, all indicators are mapped on the interval $[0,25]$ in order to get the algorithm independent of the indicators' scale. In the case there were various indicators, each one would be weighted depending on its relative importance. Later, it is applied the 
square root function in order to obtain a value inside the interval $[0,5]$. The use of a square root function (and not a linear function) represents the increasing difficulty of reaching the levels of Maslow's hierarchy of needs (see Figure 7(a)).

Later, the obtained sample is filtered. In (Millot et al 2002) and (JaSkowski et al 1995) is showed that the reaction time in humans varies between $400 \mathrm{~ms}$ and $140 \mathrm{~ms}$, depending on the stimulus, the context, etc. Then, all variations with frequency greater than $f_{c}=\frac{1}{140 \mathrm{~ms}} \approx 7.2 \mathrm{~Hz}$ must be removed, as they are due to numerical noise, devices' precision, etc. not to changes in the humans' state.

Finally the resulting sample is mapped on the Maslow's pyramid to calculate the level where each person is. The selected map function is a multilevel hysteresis cycle (see Figure 7(b)). This function, which has a certain amount of memory, avoids changes in the humans' state due to parasite causes such as the limited precision of the algorithm. Moreover, the cycle's width grows up when increasing the Maslow's level, reflecting the crescent resistance of people to change their state.
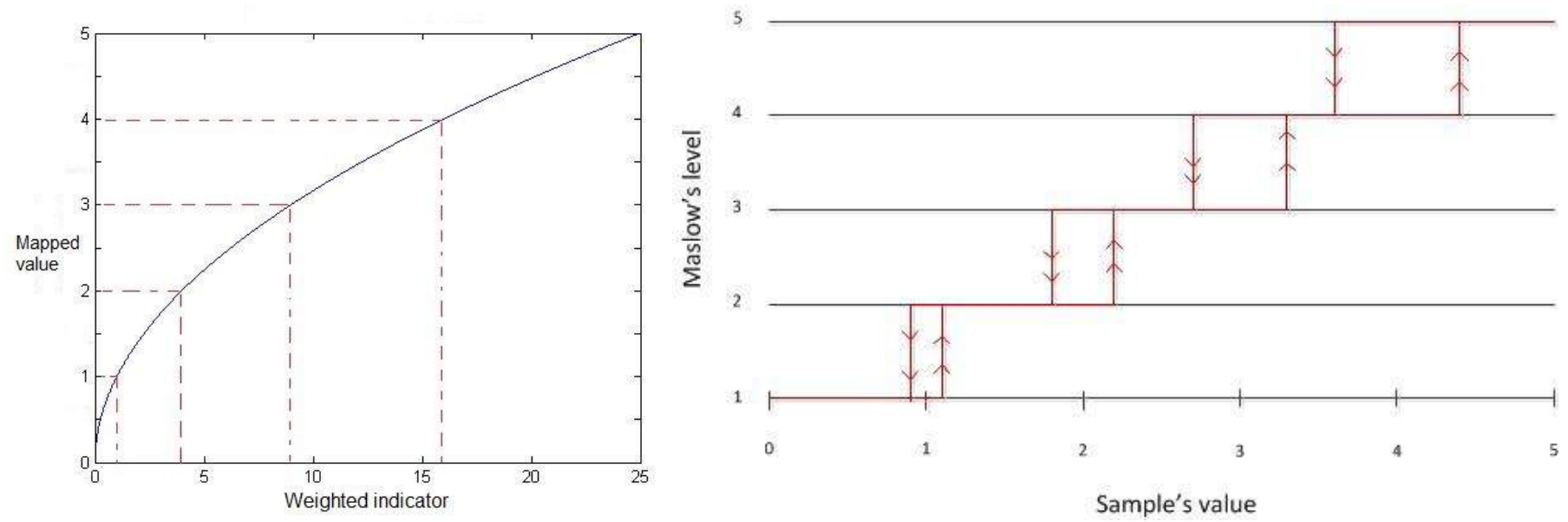

Figure 7. (a)Square root interval mapping function (b) Multilevel hysteresis cycle map function

Finally, expressions (27) and (28) show the particular implementation of qual_g and qual_c functions.

$$
\begin{aligned}
Q o S_{g} & =\sum_{i=1}^{R_{2}} \gamma_{i}\left(\delta_{1}^{i} H Q_{g}^{i}+\delta_{2}^{i} Q_{r e f}^{i}\left(1+m l_{r e f}^{i}-\min \{m l\}\right)\right) \\
Q o S_{c} & =\sum_{i=1}^{R_{2}} \gamma_{i}\left(\delta_{1}^{i} H Q_{c}^{i}+\delta_{2}^{i} Q_{r e f}^{i}\left(1+m l_{r e f}^{i}-\max \{m l\}\right)\right)
\end{aligned}
$$

Where $\gamma_{i}$ is the relative weight of the QoS due to the $\mathrm{i}$-th atomic service in the total QoS. $\delta_{1}^{i}$ represents the relative weight of the QoS due to user-focused hardware in the total QoS of the i-th atomic service, and $\delta_{2}^{i}$ represents the relative weight of the QoS due humans in the total QoS of the $\mathrm{i}$-th atomic service. Finally, $m l_{r e f}^{i}$ is the reference level in the Maslow's pyramid for the person who provides the i-th atomic service; and $Q_{r e f}^{i}$ is the reference QoS provided by the person who executes the i-th atomic service when he is located in the $m l_{\text {ref }}^{i}$ level in the Maslow's pyramid.

In the following sections, for simplicity, all the parameters describing weights are fixed following a uniform distribution (i.e. all the parameters have the same value, $\omega=\frac{1}{N}$ where $N$ is the number of considered elements). 


\section{EXPERIMENTAL VALIDATION}

Two experiments were designed in order to analyze the performance of the proposed self-configuration technology. In the first one, the evolution of the guaranteed QoS when the humans' state varies is compared in our solution with a traditional service-oriented self-configuration technology for CPS (such as described in (Wang et al 2014)). In the second one, we study the maximum number of humans our solution can consider at the same time.

Sixteen (16) people were involved in this first experiment. As we said, we deployed a manufacturing scenario (similar to described in (Bordel Sánchez et al 2015)) in two research laboratories where we placed a temperature control system. The scenario included various cybernetic gloves (one for each worker included in our system -eight in total as we are seeing later-) and two smart worktables, and, additionally, a central server as previously described. Additionally, people were monitored using an electrocardiogram (ECG) sensor, included in a Bitalino board (Bitalino 2014). The involved people were divided into two equal groups. Both groups were isolated from each other. In one of the laboratories, the proposed architecture, algorithms and functions for self-configuration were implemented in the described infrastructure. In the other, the same infrastructure was deployed, but in the central server only one virtual Java machine was implemented. In that virtual machine the technology proposed in (Wang et al 2014) was executed, being programmed also using Java technology. The QoS offered by each system was monitored. Every 5 minutes, the temperature rose in laboratories $3^{\circ} \mathrm{C}$. Thus, the comfort of people progressively went down. The impact of this deterioration in the offered QoS by each system was monitored.

In the second experiment, only one laboratory was used. In this place, the same manufacturing scenario was deployed, where a virtual machine in the central server cited in Section 3.3 executed the proposed self-configuration technology. In this experiment, the maximum number of humans which our solution can consider at the same time was evaluated. First, only one human was included in the system. Then, the temperature in the laboratory raised $10{ }^{\circ} \mathrm{C}$ during 20 seconds. The person's state changed various times and we measured the medium convergence time of the self-configuration technology. We repeated the same experiment increasing the number of involved humans until the proposed technology is not capable of reaching a stable state. Then, the maximum number of humans is obtained.

The first experiment is designed to force people to descend to the first level in the Maslow's pyramid. The top levels in the Maslow's hierarchy of needs include elements which difficultly may be controlled in a laboratory: feelings, self-esteem, job security, etc. However, in the first level (and, in some cases, in the second one) elements about the physical environment are present, which can be manipulated in a laboratory. We have selected to manipulate the temperature as it impacts strongly in the people physiological state, but it does not jeopardize health (within limits that are controlled very strictly). Therefore, when people start descending in the Maslow's pyramid, changes in the QoS will be observed.

The second experiment evaluates the relation between the time a person or group of people requires to change the state in the Malow's pyramid, and the time the proposed algorithm needs to detect the change. When both values become equal no more people could be considered in systems which implement our self-configuration technology.

In general, people involved in both experiments are always considered as service providers. Nevertheless, people may perform different roles and, even, the same person can be considered as service provider or consumer depending on the point of view, the service or the temporal instant. Basically, a person is a service provider of a certain service in a certain moment if he is performing any action which is part of the tasks associated to the service. A person is a service consumer if he has applied for a service (whether then he should execute some actions related to the service provision). Table 3 summarizes this explanation and shows some examples. 
Table 3. Explanation about the human roles in Humanized CPS

\begin{tabular}{|c|l|l|}
\hline & \multicolumn{1}{|c|}{ Service provider } & \multicolumn{1}{c|}{ Service consumer } \\
\hline A person occupies the role... & $\begin{array}{l}\text { If he participates in the tasks } \\
\text { associated with the service } \\
\text { execution in a certain moment }\end{array}$ & $\begin{array}{l}\text { If he has applied for the service } \\
\text { execution }\end{array}$ \\
\hline Example & $\begin{array}{l}\text { The person who searches in the } \\
\text { warehouse for a certain material } \\
\text { unit and places it on the } \\
\text { worktable }\end{array}$ & $\begin{array}{l}\text { A person asks the warehouse for } \\
\text { a certainaterial unit }\end{array}$ \\
\hline
\end{tabular}

\section{RESULTS AND DISCUSSION}

This section presents and discusses the results obtained in the experimental validation.

The results of the first experiment can be seen in Figure 8. Various regions may be distinguished in Figure 8. In the first region, humans occupy a very low level in the Maslow's pyramid due to the elevated temperature, so the total guaranteed QoS gets really reduced. Nevertheless, hardware components continue running without problems, so traditional self-configuration technologies offers a QoS much higher than the available. In the second region, the temperature is reduced and humans rise in the Maslow's pyramid until occupy the reference level. In that situation, the QoS offered by the hardware platform and the sum of the human QoS and user-focused hardware QoS differ by less than 1\%. That is the design situation of the traditional self-configuration technologies. Finally, in the third region, humans occupy a level above the reference, so they can offer a QoS higher than expected. Then the total QoS gets increased as can be seen in Figure 8 .

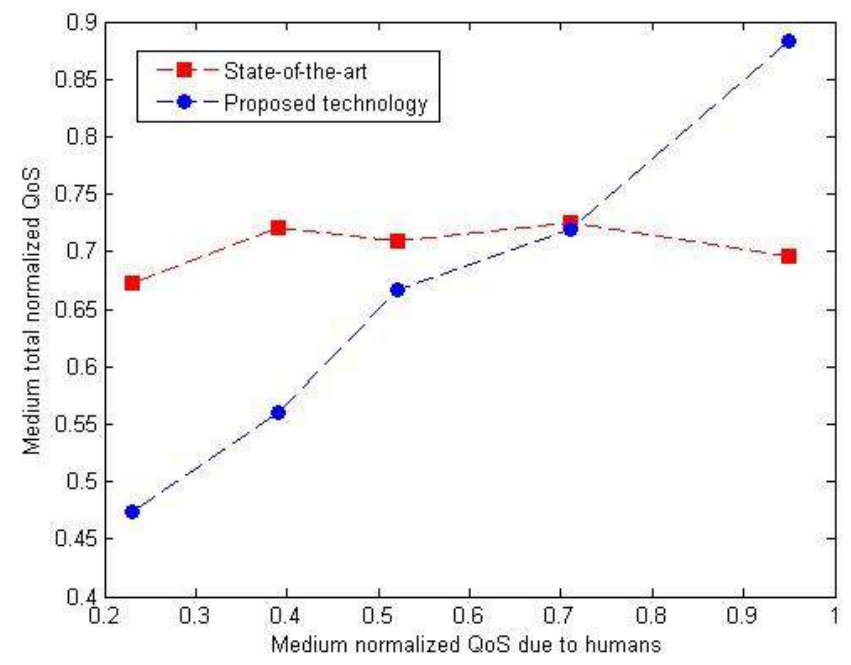

Figure 8. QoS comparison between our proposal and results obtained in the state-of-the-art

It is important to note that the slope of the quality evolution in the proposed technology depends on the selected scale for the Maslow's pyramid. This dependency is not very strong as the medium normalized value is considered in the results, which greatly reduces the influence of factors such as the selected scales. However, it can be easily proved that, due to the definition of functions qual_g and qual_c, a variation about a $50 \%$ in the final total quality (before considering the medium normalized value) is produced if human service providers go up or down one level in the Maslow's hierarchy of needs. Any case, the three previously identified areas remain, independently of the particular numerical values of the $\mathrm{Y}$-axis. 
In conclusion, Figure 8 shows how important human intervention is in the provision of the technological services. In works relating ambient intelligence solutions, where humans and Quality-of-Experience occupy a central role, most times only hardware devices are considered in the quality calculation. However, as it can be seen, human factors may reduce the final obtained quality in up to $40 \%$ (in the worst case) so, in order to approximate research results to the real performing, humans' state should be also considered (employing emotions detection, biometry, etc.).

On the other hand, an improvement close to $20 \%$ in the obtained final quality is generated when humans occupy the top level in the Maslow's pyramid. Thus, humans not only may reduce the final quality but also become an added value which can cover the hardware deficiencies and improve its performing.

Figure 9 represents the results of the second experiment. As can be seen, when the convergence time reaches the value of, approximately, 22 seconds, the system cannot get configured properly. In that moment, we had integrated 38 humans in the system. Although modifying some considerations about the validation scenario, results could be subtly different; this second experiment allows us to evaluate the growth rate of convergence time with the number of humans. A simple mathematical study proves that the convergence time grows with a rate of $n \log (n)$, being $n$ the number of integrated humans. This rate improves the complexity associated with precedents such as the Dijkstra algorithm and the exhaustive analysis of the atomic services (which grow with a rhythm of $n^{2}$ ). Besides, the maximum convergence time is also improved with respect to the precedents (where, depending on the case, the convergence time reaches a maximum of 70 seconds, approximately (Wang et al 2014)). However, a maximum of 22 seconds is high enough to prevent the execution of services with strong requirements about latency, so this fact must be considered if necessary.

In future works, the growing rate of the convergence time should be approximated to a function of order $n$. In general, Cyber-Physical Systems are large-scale deployments which should include as many people as required, so a function of order $n$ generates better results (as $n<n \cdot \log (n)$ when $n \rightarrow \infty)$.

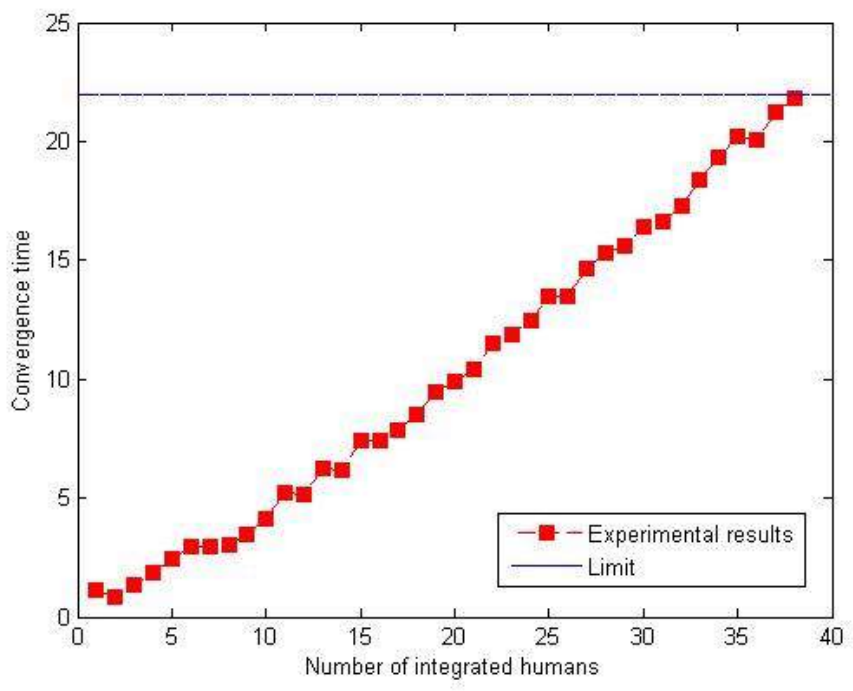

Figure 9. Evolution of the convergence time along the number of integrated humans

\section{CONCLUSIONS}

In this paper a new self-configuration technology for humanized Cyber-Physical Systems (CPS) is proposed. Humanized CPS include humans as service providers, in the same way as traditional CPS include sensors and actuators. The proposed technology is based on simple binary, logical and mathematical operations in order to reduce the convergence time and improve the scalability. As humans 
are much more complex than physical devices, the proposed solution includes an algorithm based on the Maslow's hierarchy of needs with which an estimate Quality-of-Service is calculated.

The proposed self-configuration technology implies the transmission of a very small amount of signalization, as all the process is based on binary or numerical vectors which may be transmitted in a very efficient way. Moreover, the described functions are made of simple logical or mathematical operations (additions and multiplications) which can be executed really quickly in any processor. Therefore, the proposed algorithms allow configuring the system in a much lower time than the previous proposals. Furthermore, the concept of Quality-of-Service allows addressing the dynamism introduced by humans in CPS. In that way, when the psychological or physiological state of humans changes, it is not necessary to execute the complete process of self-configuration, and only the value of the QoS is updated (which is much faster).

\section{REFERENCES}

Bitalino (2014). Project Bitalino [online] http://www.bitalino.com/ Accessed 26 March 2016

Bordel Sánchez, B., Alcarria, R., Martín, D., \& Robles, T. (2015). TF4SM: A Framework for Developing Traceability Solutions in Small Manufacturing Companies. Sensors, 15(11), 29478-29510.

Bordel, B., Alcarria, R., Pérez-Jiménez, M., Robles, T., Martín, D., \& de Rivera, D. S. (2015). Building Smart Adaptable Cyber-Physical Systems: Definitions, Classification and Elements. In Ubiquitous Computing and Ambient Intelligence. Sensing, Processing, and Using Environmental Information (pp. 144-149). Springer International Publishing.

Cardenas, A. A., Amin, S., \& Sastry, S. (2008, June). Secure control: Towards survivable cyber-physical systems. In The 28th International Conference on Distributed Computing Systems Workshops (pp. 495-500). IEEE.

Cohen, S., Kamarck, T., \& Mermelstein, R. (1983). A global measure of perceived stress. Journal of health and social behavior, 385-396.

Colombo, A. W., Karnouskos, S., \& Mendes, J. M. (2010). Factory of the future: A service-oriented system of modular, dynamic reconfigurable and collaborative systems. In Artificial intelligence techniques for networked manufacturing enterprises management (pp. 459-481). Springer London.

Cotterman, W. W., \& Kumar, K. (1989). User cube: a taxonomy of end users. Communications of the ACM, 32(11), 1313-1320.

De Lemos, R., Giese, H., Müller, H. A., Shaw, M., Andersson, J., Litoiu, M., \& Weyns, D. (2013). Software engineering for self-adaptive systems: A second research roadmap (pp. 1-32). Springer Berlin Heidelberg.

Dillon, T., Potdar, V., Singh, J., \& Talevski, A. (2011, May). Cyber-physical systems: Providing Quality of Service (QoS) in a heterogeneous systems-of-systems environment. In Digital Ecosystems and Technologies Conference (DEST), 2011 Proceedings of the 5th IEEE International Conference on (pp. 330-335). IEEE.

Dillon, T. S., Zhuge, H., Wu, C., Singh, J., \& Chang, E. (2011). Web-of-things framework for cyberphysical systems. Concurrency and Computation: Practice and Experience, 23(9), 905-923. 
Domingues, J., Damaso, A., Nascimento, R., \& Rosa, N. (2011). An energy-aware middleware for integrating wireless sensor networks and the internet. International Journal of Distributed Sensor Networks, 2011.

European commission (2005). Proyect Hydra. [online] http://www.hydramiddleware.eu/articles.php?article id=68 Accessed 26 March 2016

Hoang, D. D., Paik, H. Y., \& Kim, C. K. (2012). Service-oriented middleware architectures for cyberphysical systems. International Journal of Computer Science and Network Security, 12(1), 79-87.

JaSkowski, P., Rybarczyk, K., Jaroszyk, F., \& Lemanski, D. (1995). The effect of stimulus intensity on force output in simple reaction time task in humans. Acta neurobiologiae experimentalis, 55, 57-57.

Kao, H. A., Jin, W., Siegel, D., \& Lee, J. (2015). A Cyber Physical Interface for Automation SystemsMethodology and Examples. Machines, 3(2), 93-106.

Keddis, N., Kainz, G., Buckl, C., \& Knoll, A. (2013, February). Towards adaptable manufacturing systems. In Industrial Technology (ICIT), 2013 IEEE International Conference on (pp. 1410-1415). IEEE.

Lee, E. A. (2006, October). Cyber-physical systems-are computing foundations adequate. In Position Paper for NSF Workshop On Cyber-Physical Systems: Research Motivation, Techniques and Roadmap (Vol. 2).

Maslow, A. H. (1943). A theory of human motivation. Psychological review, 50(4), 370.

Millot, J. L., Brand, G., \& Morand, N. (2002). Effects of ambient odors on reaction time in humans. Neuroscience letters, 322(2), 79-82.

Mönks, U., Trsek, H., Dürkop, L., Geneiß, V., \& Lohweg, V. (2014). Assisting the design of sensor and information fusion systems. Procedia Technology, 15, 35-45.

Park, J., Lee, S., \& Yoon, T. (2015). Designing Goal Model for Autonomic Control Point of CyberPhysical Systems (CPS). Indian Journal of Science and Technology, 8(19).

Schirner, G., Erdogmus, D., Chowdhury, K., \& Padir, T. (2013). The future of human-in-the-loop cyberphysical systems. Computer, (1), 36-45.

Wang, T., Niu, C., \& Cheng, L. (2014, December). A Two-Phase Context-Sensitive Service Composition Method with the Workflow Model in Cyber-Physical Systems. In Computational Science and Engineering (CSE), 2014 IEEE 17th International Conference on (pp. 1475-1482). IEEE. 


\title{
Fast self-configuration in service-oriented Smart Environments for real-time applications
}

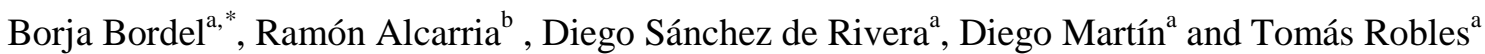 \\ ${ }^{a}$ Department of Telematics Systems Engineering. Universidad Politécnica de Madrid. Avenida Complutense $n^{o} 30$. \\ 28040 - Madrid (España); E-Mails: bbordel@dit.upm.es; diego.sanchezderiveracordoba@gmail.com; \\ diego.martin.de.andres@upm.es; tomas.robles@upm.es \\ ${ }^{\mathrm{b}}$ Department of Topographic Engineering and Cartography. Universidad Politécnica de Madrid. Campus Sur, \\ 28031 Madrid, Spain; E-Mail: ramon.alcarria@upm.es
}

\begin{abstract}
Smart Environments (SE) aim to satisfy the experience of individuals by the provision of systems, services and devices. Although many approaches may be followed in order to build a SE, a service-based design is the most promising one nowadays. In these architectures, both physical devices and even humans may provide low-level services based on their capabilities. These low-level services are composed and connected in order to create high-level services, employed by managers to describe the behavior of the SE. This composition process traditionally requires the human intervention, so changes in services are not automatic. However SE should operate at real-time, thus the collection of available services must be always updated. In the state of the art this challenge has been only partially covered, and proposed solutions are still heavy and manual. Therefore, in this work we propose a new fast semi-automatic self-configuration (and automatic self-adaptation) technology for serviceoriented SE. The proposed technology is based on the definition of templates at different levels which are related by means of semantic information. Moreover, simple binary mathematical operations are included in order to reduce the convergence time and improve the scalability. A first reduced extension for hybrid approaches (including both humans and physical devices) is also provided. Finally, an experimental validation is conducted in order to evaluate the convergence time of the proposed solution in different scenarios, and demonstrate it is scalable towards future deployments involving thousands of devices and (possibly) humans.
\end{abstract}

Keywords: Smart environments; Self-configuration; Services composition; Quality of service; Real-time applications

*Corresponding author. E-mail: bbordel@ dit.upm.es 


\section{Introduction}

Cook and Das [1] define a Smart Environment (SE) as one that is able to acquire and apply knowledge about the environment and its inhabitants in order to improve their experience in that environment.

Traditionally, SE can be understood as monolithic systems where the different existing layers are strongly integrated. Thus, in these architectures, the operation of SE is described as ad hoc feedback loops of (i) perceiving the state of the environment, (ii) reasoning about the sensed state together with some goals defined by users and (iii) acting over the environment to change its state. Thus, perception of the environment is a bottom-up process [2]. With this view, every component in the system depends on each other (e.g. the users' goals should be measurable employing the underlying hardware devices), and various experts must be involved not only in the system deployment but also during the operation [3].

With the objective of getting independent the different elements in a SE and, overall, of enabling domain experts with no programming or technological skills (such as health care professionals) to operate SE without technical assistance, newer serviceoriented architectures are being applied to SE. Service-based designs are currently very promising [4][5], as they define different levels of abstraction which are independent and might be employed in very different contexts (services are encapsulated and can be easily transferred to new systems and applications). Thus, economies of scale are enhanced, and the deployment of SE is promoted.

The introduction of service-based approaches causes the traditional SE operation to change [6]. Particularly, a new first discovery process must be considered, where low-level services (those which are directly supported by the hardware capabilities) are listed and transformed into medium-level and high-level services. In that way, the SE operation should be described as, (i) the physical layer publishes the available services in a central server by means of a transformation process, (ii) then, the user's applications look in the central server for the required services to improve the inhabitants experience following the rules indicated by system managers and (iii) using the selected services the applications monitor the state of the environment, reason about that state and act to change the situation. This new approach maintains the bottom-up perception of Smart Environments, but turns independent all the different layers without including heavy software elements such as specialized operating systems [2].

Another advantage of applying a service-based approach is the possibility of including humans as service providers [7]. In this context, a "service provided by humans" is any work performed by people at the request of the SE, with the aim of improving the overall experience of the inhabitants. For example, in a certain SE the system may ask a person (by means of a mobile application, or any other mechanism) to open a window if the ambient temperature goes above the specified range. Then, it is said the service "open a window" is provided by humans. Traditionally, studies on the relation between humans and SE are focused on behavior monitoring [8][9] or activities recognition [10][11]. In that way, humans are usually considered as "consumers" (i.e. applicants for system actions to improve their experience). However, projects such as the MavHome [12] (Managing an Adaptive Versatile Home) have introduced humans in SE in various roles (a separated problem known as "humans-in-the-loop" [13]). Figure 1 shows the resulting scheme for service-based Smart Environments. As can be seen, humans appear as services consumers (in the top part of the architecture) and as services providers (in the physical layer, together with hardware components).

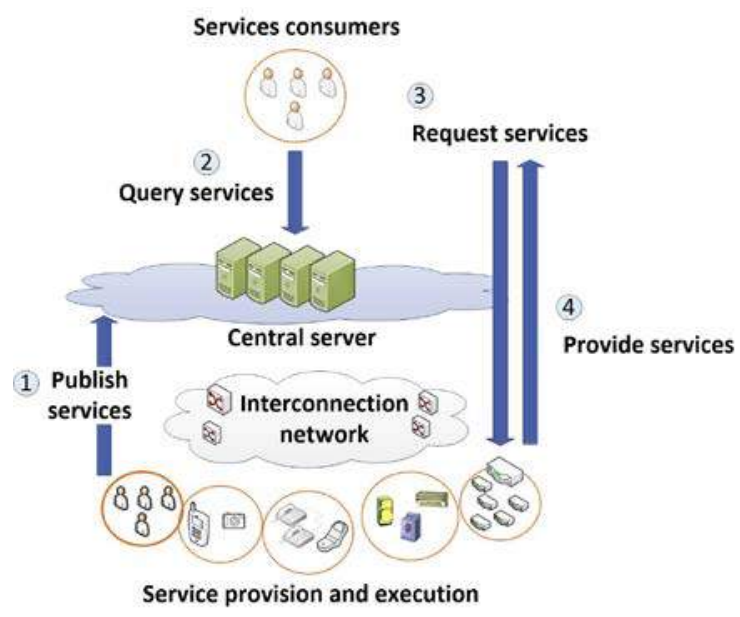

Service-oriented Smart

Environments scheme

Fig. 1. Service-oriented Smart Environment scheme

Nevertheless, service-based systems introduce new challenges and problems. In traditional SE based on feedback loops, the system is designed to react to the stimuli before the state in the physical world changes 
(the stability criterion). Service-oriented solutions should maintain these temporal requirements as SE are much more valuable if real-time operation is supported [14]. However, in these systems, the necessary abstraction process to transform low-level services into high-level services is often performed by people, as it is required to understand the "meaning" (i.e. the semantic) of every service to perform it [15]. Thus, the cited abstraction process cannot be executed during the operation time as it prevents the fulfillment of the real-time requirements (because it is a manual process and, then, very time consuming). Thus, the definition of services at the different levels is performed during the configuration time, and the created service collections remain immutable until an expert updates them manually. Nonetheless, changes in the physical platform occur in the same way that in the traditional SE, so commonly there are services configured by experts which are not executable during a certain time due to variations in the hardware components, or even in humans (life cycle, interferences, temperature...) [16].

In order to address these inconsistencies, two types of solutions have been proposed. First, some solutions try to find out if a certain service is available before invoking it [17]. In order to do that, information about the hardware devices is provided to high-level components, a solution which breaks the independency among abstraction layers typical of service-based solutions. Moreover, transferring exhaustive descriptions about low-level components to high-level layers (and later processing them) is a very costly process, so real-time operation in the SE is usually compromised [18].

On the other hand, some solutions include new functionalities in hardware components so that they can refuse non-executable service invocations [19]. If a certain invocation is refused, the high-level layers must look for another equivalent service and repeat the call [20]. This iterative process may be really complex and time consuming, so many times realtime operation is not supported [17].

Therefore, the objective of this paper is to address these problems, proposing a semi-automatic selfconfiguration (and automatic self-adaptation) technology for service-oriented Smart Environments, compatible with real-time applications, and being able to maintain the independency among the different abstraction levels. In order to do that, we define various types of templates at different levels; we represent information by means of binary vectors which may be processed using simple operations and include semantic and Quality-of-Service (QoS) infor- mation in order to maintain updated at every time the list of available services.

The authors, besides, implemented the proposed solutions and carried out an experimental validation in order to validate its performance (especially, its convergence time and scalability).

The rest of the paper is organized as follows: Section 2 introduces the state of the art in the different technologies involved in the proposed problem and solution. Section 3 presents the proposed technology. Section 4 provides an experimental validation of the proposal. Finally, Sections 5 and 6 explain some results of this experimental validation and the conclusions of our work.

\section{State of the art}

A complete solution for service-based SE requires implementing two different procedures: configuration mechanisms and adaptation mechanisms.

A configuration mechanism is the process through which all components in a system perform the installation procedures to get the basic operational parameters and to obtain the necessary initial information for operation. The configuration process takes place at the pre-operational phase [21]. Thus, automatic solutions are not needed, as (in theory) the preoperational time may be as large as required. However, in practice, it is impossible to manually configure systems including a great number of devices, so (at least) semi-automatic solutions are needed in order to perform a first automatic phase which reduces the information volume until it is manageable by people. As, partially, the system may be configured by itself, these technologies are usually known as semiautomatic self-configuration solutions.

On the other hand, an adaptation mechanism is focused on dynamically modifying the system configuration without restarting or stopping the system operation. Although various types of adaptation may be defined, in this context, adaptation represents the system reaction when an unexpected change occurs in any of its components. The basic adaptation actions include removal, addition, and replacement of hardware components [22]. As the adaptation process occurs at operation time, and (while being executed) the correct system behavior is not guaranteed, adaptation algorithms (in theory) should be fast and are always automatic (so normally they are named as self-adaptation policies) 
Self-adaptation mechanisms, moreover, usually depend on the selected configuration solution. In general, if the implemented configuration mechanism is a self-configuration one, no specific self-adaptation solution is defined: it is enough (as occurs in the Internet and the OSPF protocol) to dynamically refresh the configuration process during the operation, and the system gets automatically adapted to the new circumstances. In any case, if a different solution for self-adaptation is required, it must be specifically designed to update the configuration of the system under study.

In our proposal, two different mechanisms are defined. On the one hand, a semi-automatic selfconfiguration solution, based on the definition (by domain experts) of service semantic templates which are instantiated automatically using the information provided by the hardware platform is proposed. Hardware information is also automatically collected using low-level templates and binary operations. Mechanisms to turn independent both levels are also included.

On the other hand, as the instantiation of semantic templates is a very complex process [23], a lighter self-adaptation algorithm is defined. In particular, during the configuration process, QoS data are also collected, which are employed to define services as available, unavailable or deleted. Using this solution, the system may be updated in a very fast way and, any case, if required, it is also possible to perform a hard refresh of the configuration process.

Moreover, as we are seeing, the proposed technologies may be extended to hybrid systems including devices and humans.

Then, in this section we analyze the state of the art on various issues: from previous self-configuration and self-adaptation solutions, to technologies related to the semantic service composition.

Previous configuration solutions for serviceoriented Smart Environments are divided, basically, into two different groups.

In the first group, solutions based on the virtualization of the hardware platform in a central computing core can be found. The idea of these proposals is to create a virtual model for each one of the physical components in the system and, then, obtain the list of available services and configure the system implementing the configuration policies into the virtualized hardware platform and defining mapping techniques. Some of these works propose a specialized middleware layer which maintains the virtualized model [24][25]. Others create an agent-based system representation where every device is represented by an agent in an agent directory [3] (agent-based solutions present the advantage of being able to be extended to hybrid systems). This approach, however, present two main disadvantages. First composed services are manually defined by experts during the system configuration and human intervention is required to apply any update to the service collections (for example, in [24] composed service "healthcare virtual sensor" it is created by joining three types of sensor services). And, second, services in the different abstraction layers are not independent, as (in order to design service composition) experts must know both levels: the underlying hardware platform and the high-level services to be offered (as indicated in the previous example).

Solutions based on the use of semantic technologies together with the virtual hardware platform are a special case in this first group [26]. Although these technologies could be employed to make independent the different abstraction layers in the architecture, these works usually create a unique medium-level layer where semantic is employed to define ontologies with the objective of detecting services with "the same meaning" and avoid replicated services in the service catalogue. In that way, problems previously cited usually appears too in this type of proposals.

In comparison to these previous works, our solution employs light semantic technologies to reach a semi-automatic solution where abstraction levels are independent. In particular, experts must only create service semantic templates in any domain language (so programming skills are not required). These templates are hardware independent, so they may be employed in any context, system or application (which makes easy to share knowledge and reduce costs). In a second phase, these templates are automatically instantiated by a composition engine using mediumlevel information from the hardware devices. This additional medium-level layer decouples the highlevel services from hardware platform, so no knowledge about the technological basis is required to operate a SE (which meets the needs of experts such as health care professionals).

As a result, the proposed technology includes three different independent abstraction layers. In the second group of previously existing configuration technologies, solutions are based on the exchange of large amounts of data (among the different components) describing in a comprehensive way the system state. Then, devices independently calculate their own system state. In general, these solutions are automatic: the system runs a configuration procedure during which the operation is stopped and, once fin- 
ished, the operation is started. For example, in [27] a distributed technology for self-configuration is described (each device creates a description file which is processed by the rest of the components). As automatic solutions, these configuration policies do not require to be complemented with adaptation mechanisms (which is an important advantage). Nevertheless, generating, exchanging and processing exhaustive descriptions about the system requires an important effort from the devices, especially if reduced capabilities devices are deployed and we consider a large-scale system [27]. Additionally, this approach does not allow turning independent the different abstraction layers in the system, so managers must have technological skills and economy of scale is not promoted (knowledge and experience cannot be easily shared as every deployment is an ad hoc design).

In order to reduce this effort and, then, accelerate the configuration process we have designed a mechanism through which devices may share information about them using binary vectors (which might be transmitted very quickly and processed with simple logical operations). At low-level, archetype-based templates are defined. These templates are instantiated using binary information (yes/no answers) which may be understood as simple binary vectors. The vectors may be aggregated using logical operations. This information is collected by a medium-level data service manager which translates the aggregated binary descriptions into a traditional services description document (such as an XML).

Automatic self-configuration policies may be used as self-adaptation policies if, when a change occurs, the system is entirely re-configured. However, some specific adaptation technologies have been also described.

Systems configured by means of mechanisms based on the virtualization of the hardware platform (see above) tend to implement (over the same virtual infrastructure) complex intelligent algorithms to get adapted to the changes. For example, in [28] and [29] self-adaptation functionalities are supported by an intelligent scheduler installed in each device, communicating with a central core where decisions are taken. Similar to those proposals, in [30] selfadaptation process is based on software agents (called Multilevel self-configuration acting units). When a change occurs in the system, agents execute a recurrent algorithm of protocols negotiation and session establishment. In SOCRADES project [31] a self-adaptation technology based on petri nets is proposed. However, every reconfiguration results in a manual effort. Finally, AutoPnP [32] project deals with automatically adding new components (one of the most common adaptation cases, see above) using artificial intelligence algorithms.

In all those proposals, due to the need of executing complex algorithms both locally and remotely, modifying the virtualized hardware platform (which may include several entities is large-scale SE) and renegotiating the session parameters, the adaptation process tends to be slow, which makes difficult its execution under real-time constraints.

In our proposal, the most complex step is service composition. In order to avoid having to refresh this process when any change occurs in the SE, abstraction layers in the architecture are totally independent. In general, hardware devices present redundancies, backup solutions, etc. which cause that many times changes in the physical platform do not affect the provided services. Our proposal considers this fact and provides mechanisms to detect if a certain change in the hardware platform must be translated into a new service composition process. Thus, it is only necessary to refresh the service composition phase if new services must be added.

On the other hand, self-adaptation solutions based on the exchange of information among the different devices making up the SE have been described. The operating principle is similar to the automatic configuration solutions described above. However, while configuration policies only can restart the system operation (see previous description), self-adaptation mechanisms consider the past system configurations and update the system state dynamically by taking into account the new information without stopping the operation. In [33], a solution for self-adaptation based on control loops is proposed. A decision making unit receives a continuous data flow from every device describing its state, analyzes together all received data and acts over the system. Additionally, semantic solutions are really popular. Thus, in [34], [35] and [36] semantic technologies are used to process the information about the system and decide if a service has to be added, deleted or modified (later we are describing this topic in more detail). Finally, solutions based on the generation and transmission of event notifications have been also proposed [37].

As main advantage, with this second type of solutions, the adaptation process is much faster than which obtained when using genetic and other types of complex algorithms. However, these solutions present the same disadvantages as studied configuration solutions (a great effort from the system is required and abstraction layers are not independent). 
A binary data format has been included in our solution in order to address these problems. Nevertheless, this information may not be enough in some occasions, especially when a certain service is present but its performance it is not adequate (if, for example, degradation due to interferences wants to be considered). Thus, QoS data are also included. These data do not affect the service composition process, so all the previously cited benefits are not penalized, but are employed to dynamically decide if a high-level service is available or not. In this way, the system may get adapted much more dynamically to changes and several different types of situations.

As final detail, the key element in our proposal is the semantic service composition based on templates (although many other proposals have been included in order to design a vertical solution).

Traditionally, service composition has been a manual task. However, in the last decade various works on semi-automatic service composition have appeared, most of them following the named process-driven paradigm [20]. Basically, in this type of solutions, low-level or atomic services are automatically collected and users must create composed services by connecting several of these atomic services [38][39]. Problems associated with these solutions have been described above.

In order to reduce the human intervention in the service composition process, semantic technologies have been applied. In general, these proposals are designed to build the future Web 3.0 [40], so web services are employed. The general idea is to include a semantic reasoner being able to decompose a highlevel service into low-level services depending on their meaning [41][42], user preferences [20] or a set of constraints [43]. At the end, all these works try to execute the service with the most similar meaning to that desired by the users [20]. In this approach, however, the reasoning phase occurs each time a service is invoked during the operation time. Thus, in scenarios involving a great number of services and/or devices, the required time to resolve this process prevents the use of these solutions in real-time applications [20]. Moreover, the reasoning algorithms are typically platform dependent, so they cannot be freely applied to new scenarios or applications [20].

Our solution, on the contrary, performs the service composition during the pre-operational time (in the configuration procedure), so system operation is not affected. We are, moreover, applying the technology of Semantic Annotations [44] in order to include semantic in the lightest way. Additionally, in order to promote the collaboration among users and the econ- omy of scale (typical of the SE revolution) we are basing the proposed semantic service composition on hardware independent templates which may be shared and employed in very different scenarios or applications.

Finally service-oriented designs present the advantage of being able to be applied to hybrid systems (including devices and humans). In fact, our proposal may be extended to this type of SE, and we present (see Section 3) a first possible way to design that extension. Nowadays, no configuration solutions for Smart Environments involving humans and physical devices at the same time have been proposed. Furthermore, proposals on self-configuration for the problem of humans-in-the-loop are based on previous works about self-organizing systems (different from Smart Environments, as self-organizing systems are more focused on the ability of reacting to stimuli -in any way-, than on improving the individuals experience). Among all the existing technologies, Collective Intelligence (COIN) has been the most studied [45] [46]. This technology is particularly useful as it allows incorporating user satisfaction functions in the configuration algorithm executed in the system manager. Other technologies such as ant-based models [47] or Multi-agent coordination [48] have been also proposed, although they are not focused on SE.

\section{Proposal: a fast self-configuration solution for Smart Environments}

This section analyzes the functional architecture which supports the proposed self-configuration and self-adaptation solutions, describes their theoretical formalization and presents their operation.

\subsection{Solution overview. Functional architecture}

Our solution is based on three different independent abstraction layers. Figure 2 shows the general proposed scheme (reference architecture). 


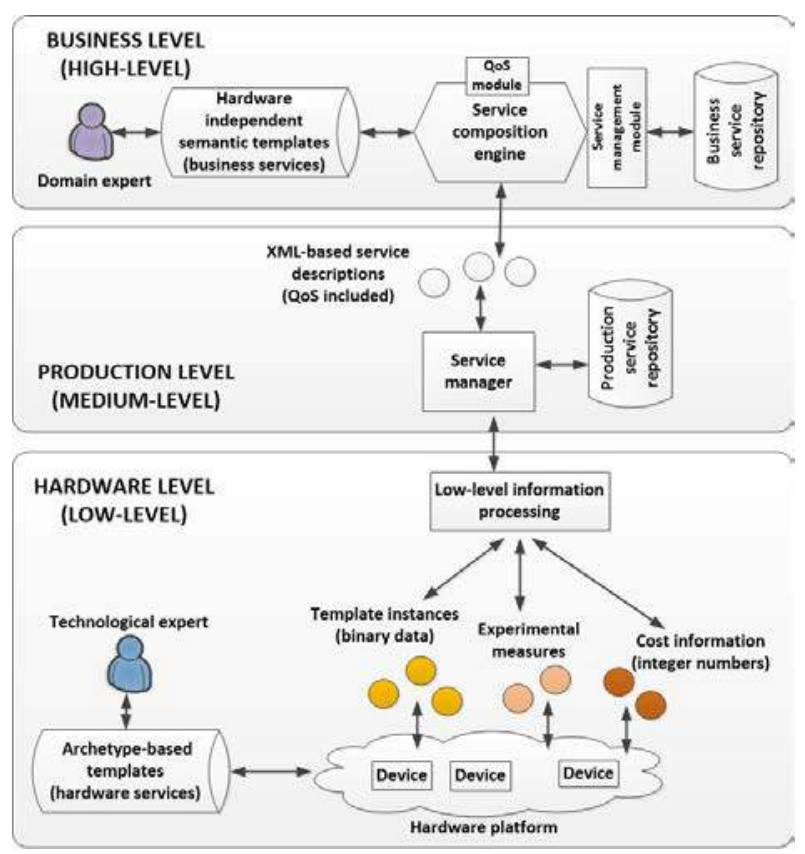

Fig. 2. Reference architecture

In the lowest level (called hardware level) a first expert appears. A technological expert (who is required to deeply know the base technology but which is not required to have knowledge about the application scenario) must indicate the hardware services provided by the infrastructure (currently or, if desired, including future extensions). These services must be embedded into hardware and depends on the devices to have certain capabilities. Then, every hardware service may be expressed as the collection of capabilities which together support the service. A certain capability (such as measuring temperature or adding integer numbers) may be present in several devices, so it can be understood as an archetype. An archetype is the original pattern or model of which all things of the same type are representations or copies [49]. As a certain capability either is present in a device or is not (there are no more options) the instances of these archetypes are binary (yes/no). In conclusion, every hardware service may be represented as a template listing all the archetypes (or capabilities) involved in its execution. The technological expert must create these templates (one for each service) which will be automatically instantiated by hardware devices (using binary data) during configuration process.

During the configuration procedure, binary information from the cited instances may be processed at hardware level in order to reduce the amount of data to be transmitted. The obtained instances, besides, must include a first simple semantic characterization also provided by the technological expert with the low-level templates. The result of this processing is sent to the next level.

At medium-level layer (called production level) services are abstracted from the underlying devices. In particular, obtained instances are translated into a generic service description language (such as XML) where semantic information is automatically completed and configured as Semantic Annotations. Moreover, proprietary APIs and interfaces (typical of hardware devices) are substituted by a uniform and generic API (using REST technologies, for example). The obtained description is totally agnostic in respect to the underlying infrastructure. A first service repository may be created at this level.

The generated generic service description at medium level, however, still contains services which have no value for final users in a SE. In order to create this value, it is added a final high-level layer (called business level). At business level a domain expert must indicate the relevant services for his application, including their most significant parts and their meanings. This information (provided using a domain description language) makes up a collection of semantic templates. These templates are introduced in a composition engine together with the description obtained from the production level. Then, the engine finally creates and defines the collection of services which are in theory available in the SE (by means of semantic matching).

The process previously described, however, does not take into account factors such as the minimum expected performance by SE users from a service. Therefore, in our solution, basic information about available services is complemented with additional data about Quality-of-Service. Moreover, these QoS information is employed in a self-adaptation mechanism which is triggered once finalized the configuration process (see Section 3.3).

In this work we call "QoS information" any representation of the system performance pertaining a certain service. This representation can be numerical (quantitative), but it might consist of only one value or of a collection of them. QoS may be represented by means of technical measurable parameters (jitter, delay, packet losses rate, etc.) or through dimensionless values obtained from the application of functions to the cited parameters in order to obtain representative ratios (availability, invocation cost, etc.). QoS is, at the end, employed to indicate the current value of certain important network parameters. Besides, QoS is used to evaluate if network parameters are compat- 
ible with the expectances of users (which should also indicate the desired QoS, usually through representative ratios as employing network parameters requires technical knowledge). Other definitions of quality (not based on technical parameters and/or including the user perception) are not considered in this work.

Two different information sources are considered into the QoS calculation.

On the one hand, periodical experimental measures about some remarkable network parameters are done (delay, jitter, transmission rate, etc.). These measures are performed by the low-level processing module. The results depend on the device positions (if mobile), time, interferences, etc. As these measures may change very dynamically, the evaluation period will be a critical parameter in our solution (during that period service catalogue will not be updated).

On the other hand, information about the cost of executing a certain service in the hardware platform is collected. This information is provided by devices to the low-level processing module in an autonomous way (without a previous request). Costs are integer numbers representing the expense in battery charge, RAM memory, CPU and processing time (among other factors) which the execution of a certain service (or capability) causes in each device. The algorithm used to obtain these costs is not the focus of this article (various proposals may be found in the literature). Cost information, in general, changes in a very slow way (it depends on hardware and embedded software which is not variable), so devices simply notify variations to the processing module when occurring.

The low-level information processing module analyzes the received information (costs and experimental measures) and obtains hardware-independent remarkable ratios representing the offered QoS (both the maximum QoS and the guaranteed QoS). In that way, abstraction layers continue being independent as low-level information is not sent to the production level. Moreover, the entire system does not have to be dimensioned to accept a data flow which grows as the hardware-platform scales. It is enough to adequately design the infrastructure.

At production level, the QoS information is integrated into the same XML service descriptions previously described. Finally, this information, at business level, is employed to calculate a weighted quality value which is compared with some threshold defined in the system. The service management module, then, depending on this comparison decides if a service is available, unavailable or deleted.
It is important to note that recalculating the service composition is not necessary in order to update a service state, as it depends only on QoS information. In that way, service catalogue is updated in a very fast way, as the most time consuming step (the service composition process, as evaluated in some papers [50][51]) is avoided. As we are seeing later, with this self-adaptation mechanism (as can be seen is totally automatic) the service composition process must be executed again when a new service has to be added.

The technologies described above must be supported by various functional components. Several functional architectures for service-oriented Smart Environments have been proposed [52] [53]. Any of them may be complemented with the proposed technology, if adequate components are added. In that way, the proposed functional architecture for supporting our proposal can be seen in Figure 3. Various components can be distinguished.

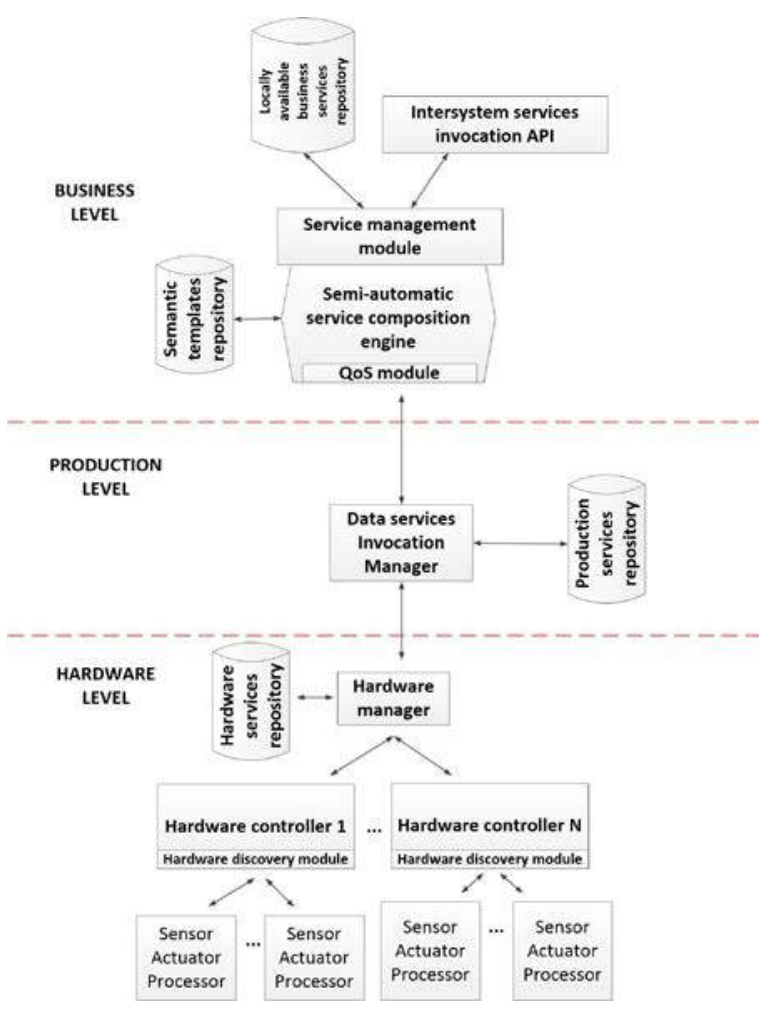

Fig. 3. Functional architecture

- Sensors, actuators and processors: They include all the traditional devices present in the hardware infrastructure of Smart Environments and ubiquitous computing systems. From the sim- 
plest sensors to the called Smart Objects [54] are included.

- Hardware controller: This component is responsible for discovering, setting up and managing the hardware devices. Its main utility in our solution is to obtain and instantiate the low-level archetype-based templates (from Hardware manager). The architecture includes as many controllers as required by the hardware platform. Typically, each smart device [55] implements its own hardware controller and traditional devices are connected to a different hardware controller acting as broker. Each hardware controller includes a Hardware discovery module which obtains the necessary information to instantiate the cited archetype-based templates (using any of the existing technologies [56])

- Hardware manager: It coordinates the operation of the different hardware controllers. It manages the archetype-based templates and the instances generated by the underlying hardware controllers. Basically, it processes all the archetype-based templates describing every hardware service, in order to create a unique template (called master template) containing all the relevant capabilities (i.e. capabilities which are named at least one time in the service templates). This master template is sent to the hardware controllers which have to instantiate it. Moreover, it processes the low-level data to be submitted to medium-level components. It also maintains information about the different locations (devices) where a certain capability is available (although this problem is more related to the SE operation and it is not analyzed in this work).

- Hardware services repository: It contains all the necessary information about the hardware services (sometimes also named as atomic services) It stores the archetype-based templates, as well as the simple semantic information associated with each one. It also contains the master template and the generated instance. The hardware manager uses that information to decide which atomic services are available.

- Data services invocation manager: This production level component makes independent the hardware platform (at low level) and the service domain (at high level). It translates the archetype-based template instances into a generic service description document and offers to the business level components a service interface based on generic technologies (such as REST) which hides the proprietary and heterogeneous APIs employed by hardware devices. Thus, the resulting description is totally agnostic from the underlying devices.

- Production services repository: This is a secondary component which stores the list of production services described by the data services invocation manager in the corresponding document. It also stores the relation between the proprietary hardware interfaces and the generic one (based on REST technologies) offered to business level components.

- Semi-automatic service composition engine: This component composes high-level services using semi-automatic techniques and the information provided by the Data Services Invocation Manager. Semi-automatic techniques require some human intervention, but in a time previous to the composition process. In our case, domain experts must create a collection of semantic templates describing high-level services (business services). Following a matching mechanism, the semantic content of the medium-level service description is employed to instantiate the semantic templates. As a result, a traditional executable XML service description will be obtained. In this paper we are not discussing the particular matching algorithm employed in the process of services composition. Any of the proposed solutions in the research literature, such as [57] or Hydra Project [58] will be valid.

This component, besides, include two important modules. The QoS module transforms the production level data about quality into a unique parameter (for each business service) through a weighting and aggregation procedure. The result is sent to the service management module which compares it to the minimum thresholds indicated by users and decides if a service is available, unavailable or deleted

Finally, once determined the available composed services, they may be registered in a Locally available business services repository so they can be called from local applications, and/or in a public remote repository through the Intersystem services invocation API, so they can be called from applications in the cloud. 


\subsection{Self-configuration solution: mathematical formalization}

Using the architecture proposed on Figure 3, the following self-configuration process may be executed in order to determine the available services in a Smart Environment.

First, each Hardware controller discovers the sensors, actuators and processors which it controls, as well as the capabilities of these devices. This process might be performed by means of any of the existing technologies such as IEEE 1451 [59] or the Composite Capability/Preference Profiles (CC/PP) W3C recommendation [60].

In parallel, the Hardware manager processes the defined archetype-based templates describing the hardware services. These templates may be described in various languages, although the most appropriate is ADL2 (Archetype Description Language, version 2.0) [61]. From the processing of all templates a master template will be obtained, containing all the capabilities employed in, at least, one service (see Figure 4). Notes describing the basic semantic of services and capabilities are also included.

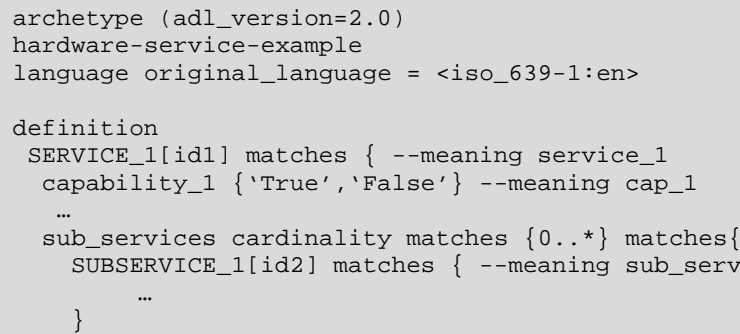

Fig. 4. Example of an archetype-based template (top) and of a possible master template (bottom) using ADL2

An additional advantage of employing a serviceoriented solution is that it is not necessary to model the universe of all possible devices [62]. It is enough to model the hardware capabilities and hardware services of interest for the particular application scenar- io. The list or the ontology of relevant capabilities may be based on an ad hoc definition or on any of the exiting generic proposals [63][64].

It is important to note that devices may present other capabilities not included in the archetype-based templates. Even, new devices with new capabilities could be added. However, in our proposal (in order to speed up the configuration process) only the required capabilities to execute hardware services are considered. The others, as no service employs them (probably they are not of interest in the application scenario), they will never be invoked, and it has no sense to include them in the configuration process. On the other hand, it must be said that SE are planned systems, not ad hoc deployments. Thus, new devices are always added by experts and (in that case) they also could include the corresponding archetype-based template (if necessary). In future work, besides, this procedure will be investigated to be automated.

Then, each Hardware controller obtains the master template and instantiates it. In general, instances of archetype-based templates are XML documents. However, in this case, all the fields in the template are Boolean variables, so the instance may be understood as a binary vector. Thus, after the instantiation process each Hardware controller creates a descriptor $h d$. The descriptor of the i-th Hardware controller can be denoted as indicated in Eq. (1).

$$
h d_{i}(t)=\left\{v_{i}(t), q_{i}(t)\right\}
$$

The descriptor includes two elements. $v_{i}$ is a binary vector where each bit determines the presence or absence of a certain capability (the instance of the master template). $q_{i}$ is a cost vector where each position indicates the cost of accessing a certain capability (if available) in the i-th hardware controller. As previously said, this cost includes the expense in battery charge, RAM memory, CPU and processing time (among other factors representing the hardware and software configuration). Both elements depend on time, as new sensors could be added, more efficient procedures could be programmed, etc.

In the third step, all hardware controllers send their descriptors to the Hardware manager. Then, in the Hardware manager three collections may be described: $H D$ the collection of descriptors, $V$ the collection of binary capabilities vectors and $Q$ the collection of cost vectors.

$$
\begin{aligned}
H D(t) & =\left\{h d_{i}(t), i=1, \ldots, N(t)\right\} \\
V(t) & =\left\{v_{i}(t), i=1, \ldots, N(t)\right\} \\
Q(t) & =\left\{q_{i}(t), i=1, \ldots, N(t)\right\}
\end{aligned}
$$

Where $N(t)$ is the number of Hardware controllers in the Smart Environment. All the variables are 
time dependent, as changes in the hardware platform may cause changes in these values.

Strictly speaking, the collection $H D(t)$ already contains $V(t)$ and $Q(t)$. However, for more clarity, three different collections have been defined. This is because the purpose of the sets is very different. While $H D(t)$ is destined to be stored into the hardware services repository (in order to allow the future execution of atomic services), $V(t)$ and $Q(t)$ are going to be employed, independently, in the next steps of the configuration process (so two separated collection are needed).

Using Equations (2), (3) and (4) the Hardware manager generates a partitioned matrix $D M(t)$ describing the available atomic services in the hardware platform, as well as the QoS offered for each service.

$$
D M(t)=\left(\sigma_{d}(t)\left|Q_{g}(t)\right| Q_{c}(t)\right)
$$

As can be seen in Eq. (5), DM(t) is composed of three submatrices.

$\sigma_{d}(t)$ is a binary column matrix where each row indicates if a certain atomic service is available (each hardware service, when defined through an archetype-based template is provided with a unique numerical integer identifier, which may be employed to determine the position in this column which is referred to it). $Q_{g}$ is a matrix describing the guaranteed QoS for each atomic service in one particular moment. Each row in $Q_{g}$ contains the quality parameters of the atomic service to which it is referred the same row in $\sigma_{d}$. Finally $Q_{c}$ is a matrix describing the context-depending QoS (no guaranteed) for each atomic service in one particular moment. Sometimes, the system presents surplus resources that could be assigned to the existing services in order to improve their quality of execution. These resources, however, cannot be guaranteed, and their availability depends on the system's context.

The relation between the collections $H D(t), V(t)$ and $Q(t)$ and the matrix $D M(t)$ is denoted as a vector function $F$, represented in Eq. (6).

$$
\begin{gathered}
\left(\sigma_{d}, Q_{g}, Q_{c}\right)=\vec{F}(V, Q)= \\
=\left(f_{1}(V), f_{2}(Q, \Sigma), f_{3}(V, Q, \Sigma)\right)
\end{gathered}
$$

As can be seen, this vector function has three components.

Function $f_{1}$ employs the collection of instances of the master template (bit vectors) produced by hardware controllers to determine if each one of the described hardware services in the corresponding repository is supported by the infrastructure or not. In a traditional approach, an instance transformation would be applied (using matching techniques) in or- der to obtain the instances of all archetype-based templates (one per hardware service, equal for all hardware controllers) from the instances of the master template (one per hardware controller, equal for all hardware services). However, this process is complex and heavy [65], and it may not fit teal-time constraints. This problem may be solved if master template instances are understood as bit vectors, as, then, $f_{1}$ might be described as a sequence of logical (binary) operations which are executed in a very dynamic and fast way.

For that, when creating the master template, Hardware manager should define a new collection of binary templates $C(t)$, identifying for each hardware service the capabilities involved in its execution (7).

$$
C(t)=\left\{c_{i}, \quad i=1, \ldots, M(t)\right\}
$$

Being $M(t)$ the number of available hardware services in the infrastructure in a certain moment.

The format of these binary templates must be the same as the master template instances: each bit determines the presence or absence of a certain capability in a hardware service. Then, function $f_{1}$ may be described as in Algorithm 1.

Algorithm 1. Implementation of function $f_{1}$ using pseudocode

\begin{tabular}{c|c}
\hline $\mathbf{N}$ & Algorithm $\mathbf{1}$ Function $\boldsymbol{f}_{\mathbf{1}}$ \\
\hline 1 & Input: Collection $V$ \\
2 & Output: Column matrix $\sigma_{d}$ \\
3 & Generate a binary vector of zeros $d s$ \\
4 & Generate an integer $i$ equal to one $(i=1)$ \\
5 & for each element $v_{i}$ in $V$ do \\
6 & $d s$ is equal to $v_{i}$ bitor $d s$ \\
7 & end for \\
8 & $d s$ is equal to compl $d s$ \\
9 & for each binary template $c_{i}$ in $C$ do \\
10 & VM = $\left(c_{i}\right.$ bitand $\left.d s\right)$ \\
11 & if VM != 0 then \\
12 & BR is equal to zero \\
13 & else then \\
14 & BR is equal to one \\
15 & end if \\
17 & $\sigma_{d}^{i}$ is equal to BR \\
18 & Increment $i$ in one unit \\
19 & end for \\
\hline
\end{tabular}

In summary, Algorithm 1 condenses in a binary vector $d s$ (which is initialized in line 3 ) all the existing capabilities in the hardware controllers. For that, an OR operation is applied to all master template instances expressed as bit vectors $v_{i}$ (lines 5 to 7 in Algorithm 1). Later, for each atomic service it is ap- 
plied (using an AND operation) a mask $c_{i}$ (the corresponding binary template described above) to the binary vector $d s$ in order to identify if it is required any capacity not available on the hardware platform (line 10). The result (VM) is a vector of zeros if all required capabilities are present in the hardware platform or a vector with non-null positions if any capability is not present. Then, the value of the VM parameter may be used to decide if a certain hardware service is available $(\mathrm{VM}=0)$ or not, and, in that way, fixing the corresponding value (the $\mathrm{i}$-th position of the $\sigma_{d}$ column) in consequence. Then, in order to advance to the next position in the $\sigma_{d}$ column, an integer named as $i$ (initialized with value one in line 4 ) is updated (line 18).

Functions $f_{2}$ and $f_{3}$, which together the described function $f_{1}$ make up the vector function $F$, are presented in Equations (8) and (9).

$$
\begin{gathered}
Q_{g}^{i}(t)=f_{2}(Q(t), \Sigma(\mathrm{t}))= \\
\left(\begin{array}{c}
\max \left(\left\{q_{j} c_{i} j=1, \ldots, L(t)\right\}\right)+q_{c o}^{i} \\
R_{1}(M o(\Sigma)) \\
\ldots \\
R_{S}(M o(\Sigma)) \\
i=1, \ldots, M(t)
\end{array}\right)^{T} \\
\left(\begin{array}{c}
\min \left(\left\{q_{c}^{i}(t)=f_{3}(V(t), Q(t), \Sigma(\mathrm{t}))=\right.\right. \\
\left.\left.\sum \mu_{i} q_{j} v_{j} j=1, \ldots, L(t)\right\}\right)+q_{c o}^{i} \\
R_{1}\left(P_{90}(\Sigma)\right) \\
R_{S}\left(P_{90}(\Sigma)\right) \\
i=1, \ldots, M(t)
\end{array}\right)^{T}
\end{gathered}
$$

Briefly, function $f_{2}$ creates a vector describing the guaranteed global cost of executing a certain hardware service (the i-th service) and the guaranteed quality parameters associated. In particular, the first component, it adds the cost of accessing to all the capabilities which make up an atomic service, considering the maximum value among all the possible location where the service is available (being $L(t)$ the number of locations where it is available at a certain moment). In fact, in general, the guaranteed cost is the worst case (although certain services have reserved resources, but this is not the focus of this paper). The cost $q_{c o}^{i}$ is a correction factor applied if the ithe hardware service is provided by the coordination of various devices and there is a cost of coordination.
The rest of the components are relevant ratios $R_{1}, \ldots, R_{S}$ (defined by technological experts according to users' needs and the application scenario) calculated from the experimental measures $\Sigma(\mathrm{t})(10)$.

$$
\Sigma(\mathrm{t})=\left\{\sigma_{i}(t-p T) i=1, \ldots, K p=1, \ldots, P\right\}
$$

$\Sigma(\mathrm{t})$ is a collection of $K$ experimental measures about QoS on the system $\sigma_{i}$ (for example, its response time, packet loss rate, etc.). These measurements are periodically repeated and refreshed with a period of $T$. As we design a solution to maintain updated the system at real time, the value of this period will be critical in our proposal. The collection of experimental measures considers the $P$ past evaluations of the experimental measures.

Examples of relevant ratios are availability, blocking probability, etc. In order to obtain the guaranteed value of these ratios, in their calculation it is employed the most probable value (mode) of the experimental measures, $M o(\Sigma)$.

On the other hand, the function $f_{3}$ creates a vector describing the context-depending costs and QoS (i.e. values for the cost and QoS parameters which are not guaranteed and may be reached or not depending on the system context). The first component, the execution cost, is calculated in the same way than in function $f_{2}$. However, in this case, more than one device may be used at the same time to support a certain capability. Then, a correction factor is applied. In particular, $\mu_{i}$ is a numerical vector where each position indicates the modification in the QoS due to the existence of additional available capabilities.

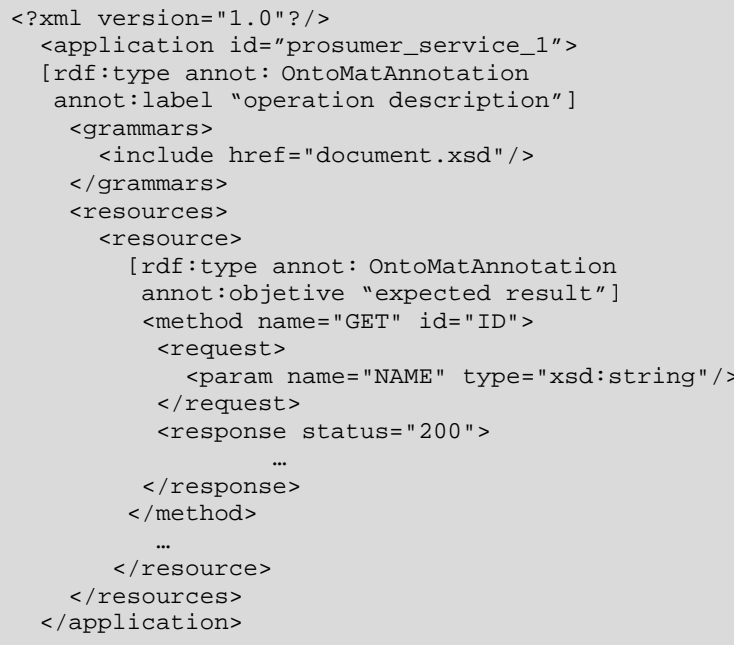

Fig. 5. Example of a WADL document with Semantic Annotations

In the same line, hardware independent relevant ratios employed to inform about QoS to production 
level components are the same than in function $f_{2}$. In this case, nevertheless, in their calculation it is employed the 90-th percentile $P_{90}$ (i.e. the value such $90 \%$ of measures are equal or worse than it) instead of the mode of measures.

Once $D M(t)$ matrix has been constructed, it is transmitted to the Data services invocation manager (production level). In the Data services invocation manager, matrices are mapped over a description file (typically an XML file) where atomic services are described as production-level services. For example, using REST services we would use a Web Application Description Language (WADL) file. Moreover, the short notes about semantics included in archetype-based templates are processed and condensed in various Semantic Annotations [36] (for that the master template is also sent to the production level). Figure 5 presents an example of this document.

As can be seen on Figure 5, Semantic Annotations are included in square brackets, describing the "meaning" of each application (prosumer service), request, etc. Once created, XML files are sent to the Semi-automatic service composition engine.

Then, the engine composes a collection of highlevel (or business level) services $S(t)$ using the production services described in the WADL document, as expressed in Eq. (11). The collection of composed services is time dependent as it may be change depending on the hardware platform configuration.

$$
S(t)=\left\{s_{j}, \quad j=1, \ldots, I\right\}
$$

Where $I(t)$ is the number of composed services created in the semi-automatic engine.

In order to create this collection of business services, the composition engine must apply a semantic matching technology to the production service description file, together with the semantic templates for business services defined by domain experts. These templates describe the parts (invocations) a service should have, as well as their meaning. Comparing these templates with Semantic Annotations in XML description documents the collection of service is created (applications and requests with the same meaning than desired by users are connected in order to build the high-level services). Business services (as well as semantic templates) are described, then, using any of the existing executable workflow description language (YAWL, for example [66]), probably complemented with Semantic Annotations. In respect to the semantic matching technology, any of the techniques described in the literature may be employed [67][68].
In that way, the $\mathrm{j}$-th service of the collection can be denoted as in Equation (12).

$$
s_{j}=\left\{X M L, Q o S_{g}(t), Q o S_{c}(t), B C(t)\right\}
$$

Where $X M L$ describes the service's input, output and its decomposition in prosumer services. $Q o S_{g}$ represents the final guaranteed QoS for the composed service (expressed as a numerical vector where each position represents the value of one quality parameter). $Q o S_{c}$ expresses the context-depending QoS for the final composed service. And $B C$ represents the behavior constraints for the composed service (such as time or range constraints for service availability).

The $X M L$ and $B C$ elements are generated during the service composition process, by means of the cited semantic matching technique. However, $Q o S_{g}$ and $Q o S_{c}$ are calculated from quality parameters of all production services (see Eq. (8) and Eq. (9)) which make up a composed service, using Equations (13) and (14) in the QoS module

$$
\begin{aligned}
& Q o S_{g}=\text { qual_g }\left(Q_{g}^{1}, \ldots, Q_{g}^{R_{1}}\right) \\
& Q o S_{c}=\text { qual_c }\left(Q_{c}^{1}, \ldots, Q_{c}^{R_{1}}\right)
\end{aligned}
$$

Where $Q_{g}^{r}$ are the guaranteed quality parameters of the r-th production service provided by physical devices which is part of the composite service. The $Q_{c}^{r}$ parameters have the same interpretation as the above, but applied to context-depending quality parameters. Finally, $R_{1}$ is the number of production services provided by physical devices which are part of the $j$-th composite service.

Several implementations for the functions qual_g and qual_c may be proposed. In this paper, we define them as indicated in Eq. (15) and Eq. (16). In that way, $Q o S_{g}$ and $Q o S_{c}$ are two real vectors, although in other implementation could be Bdimensional complex or integer vectors.

$$
\begin{gathered}
Q o S_{g}=\sum_{i=1}^{R_{1}} \beta_{i} Q_{g}^{i} \\
Q o S_{c}=\sum_{i=1}^{R_{1}} \beta_{i} Q_{c}^{i}
\end{gathered}
$$

Where $\beta_{i}$ is the relative weight of the QoS due to the $\mathrm{i}$-th production service provided by physical devices in the total QoS.

Once obtained the entire collection of composed services by the Semi-automatic service composition engine (and the corresponding QoS), they are registered in the Locally available services repository. Through this repository, composed services can be 
called from local applications, and/or from remote applications through the Intersystem services invocation API.

\subsection{System self-adaptation: mathematical formalization}

The described procedure in the previous section generates a fixed list of services, associated each one with two parameters representing the aggregated quality. However, the situation of the hardware platform may change, and the provided services should get affected in consequence.

Among all the described steps, the most time consuming is service composition in the Semi-automatic service composition engine (as indicated by various authors [51][65]). The rest of operations are simple matrix calculations which might be executed at realtime. The objective, then, is to define a selfadaptation policy which does not need to repeat the entire services composition process.

Table 1. Services' states in Smart Environments

\begin{tabular}{|c|l|}
\hline State & \multicolumn{1}{c|}{ Description } \\
\hline \multirow{5}{*}{ Available } & $\begin{array}{l}\text { A service is available when all the } \\
\text { atomic services which compose it are } \\
\text { available, and the guaranteed QoS of } \\
\text { the composite service surpasses the } \\
\text { established QoS thresholds. }\end{array}$ \\
\hline \multirow{5}{*}{ Unavailable } & $\begin{array}{l}\text { A service is unavailable either } \\
\text { when one of the atomic services } \\
\text { which compose it becomes unavaila- } \\
\text { ble, or when the guaranteed QoS of } \\
\text { the composite service falls below the } \\
\text { established QoS thresholds. } \\
\text { A service which is considered una- } \\
\text { vailable may return to the available } \\
\text { state without refreshing the service } \\
\text { composition process. }\end{array}$ \\
\hline \multirow{7}{*}{ Deleted } & $\begin{array}{l}\text { A service is considered as deleted } \\
\text { when gets unavailable for a time } \\
\text { which passes a certain limit. } \\
\text { A service which is considered de- } \\
\text { leted only may return to the available } \\
\text { state after refreshing the service com- } \\
\text { position process in the semi- } \\
\text { automatic engine. }\end{array}$ \\
\hline
\end{tabular}

In order to reach this objective, we associate to each composed service a service state. Thus, each composited service may present three different states: available, unavailable and deleted. Table 1 describes the conditions which must fit a service to present each state.

As can be seen, in Table 1 it is referred some QoS thresholds which all services must pass to be considered available. These thresholds are applied in a twophase process in the Service management module.

In the first phase each quality parameter in $Q o S_{g}$ must pass a limit $k_{i}$ as expressed in Eq. (17).

$$
Q o S_{g}^{i}>k_{i} \quad i=1, \ldots, B
$$

Where $B$ is the number of considered quality parameters in the system (i.e. the number of elements in $\left.Q o S_{g}\right)$. Considering the functions proposed in Eq. (15) and Eq. (16), $B$ is equal to the unit (in other implementation it could present greater values). In order to abbreviate the notation we also can denote Eq. (17) as indicated in Eq. (18).

$$
Q o S_{g}>K
$$

Where $K=\left\{k_{i} \quad i=1, \ldots, B\right\}$

If the first phase is verified, then it is evaluated a second phase. In this new phase the total guaranteed QoS $Q o S_{t o}$ must pass a limit $Q o S_{t h}$ as indicated in Equation (19).

$$
Q o S_{\text {to }}>Q o S_{t h}
$$

The expression which allows obtaining the total guaranteed QoS from the vector $Q o S_{g}$ is as expressed in Equation (20).

$$
Q o S_{t o}=\sum_{i=1}^{P} \omega_{i} \frac{Q o S_{g}^{i}}{\lambda_{i}}
$$

Where $\lambda_{i}$ is the standard value of the $i$-th quality parameter and $\omega_{i}$ is the relative weight of the i-th parameter in total estimation of the offered QoS.

Using our proposal typical fluctuations in the state of hardware devices do not causes the execution of a new service composition process. This process only has to be initiated in two situations:

- When a previously deleted composed service is available again

- When a new hardware service is discovered by the Hardware manager

In the first case, even when the service is considered deleted, both the Semi-automatic service composition engine and the Locally available services repository maintains in memory the service information. Thus, the complete composition process does not have to be executed, and only a new collection 
modifying the affected service has to be generated in the engine.

In the second case, the complete composition process has to be repeated. New composed services could appear and they must be calculated and incorporated in the repository. Nevertheless, if the employed service composition technique allows conditional composition [50], this process could be simplified by restricting it to an expansion of the composed services due to the appearance of a new atomic service. If this solution is available, the Data services invocation manager would create a special XML description file indicating to the Semi-automatic service composition engine the new physical platform situation and the condition on which the new composition process should be based.

It must be noted that any change in the physical layer which does not cause a modification in the atomic services is not propagated towards the Data services invocation manager. As the proposed functional architecture is made of independent abstraction layers, changes in the hardware level which do not affect the hardware services are directly managed by the hardware controllers or the Hardware manager. This approach allows an important reduction in the average needed time to update the system state.

\subsection{Extension to hybrid systems}

Service-oriented architectures for SE present the possibility of including humans as service providers. However, most configuration and adaptation solutions for SE do not consider this situation (see Section 2). On the contrary, our proposal may be easily extended to hybrid systems (those which include both humans and devices in their low-level infrastructure).

Previous works [69] have proved that the number of humans in a Cyber-Physical System, together with their motivation state (which represents the execution quality), may characterize the available human provided services in the system. Therefore, in order to configure human provided services, our solution must collect and include this information in the previously described configuration and adaptation processes (Section 3.2 and 3.3).

Then, in the case of existing humans in a SE, sensors focused on acquiring information about people should be included into the hardware platform (called user-focused devices). These devices are controlled by user-focused controllers, where data analysis algorithms are included (in order to deduct the number of people and their motivation state from sensor in- formation). With this information, a description matrix $U M(t)$ should be defined (21) (equivalent to the matrix $D M(t)$, employed to describe the low-level services provided by hardware devices).

$$
U M(t)=\left(\sigma_{h}(t)\left|H Q_{g}(t)\right| H Q_{c}(t)\right)
$$

As matrix $D M(t), U M(t)$ it is made of three submatrices. $\sigma_{h}$ is a binary column matrix where each row indicates if a certain low-level service provided by humans is available. $H Q_{g}$ is a matrix describing the guaranteed quality and $H Q_{c}$ is a matrix describing the context-depending QoS (no guaranteed). Both matrices are calculated from the users' motivation state, which may be completed with other estimations such as the stress level.

Algorithms and functions employed to construct the matrix $U M$ from sensor information have been described in previous works [69]. Thus, in this proposal we are focusing on the configuration process more than in the data analytics. Moreover, for human provided services, archetype-based templates are not required; only one "master pseudo-template" must be built. The objective of this pseudo-template is to register the services a certain quantity of people may provide in the application scenario (never to be instantiated as regular templates). Then, this pseudotemplate lists these services (categorized depending on the number of people) with their meanings (see Figure 6).

Once matrix $U M$ is constructed, the appropriate module (usually called "user manager" [69], see Figure 8) sends it to the production level (to the Data services invocation manager).

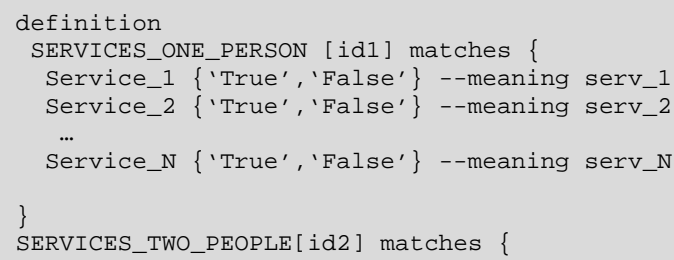

Fig. 6. Master pseudo-template for human provided services

At production level, the Data services invocation manager includes human provided services in the created XML service description document, together with services provided by hardware devices. This module makes no difference between both types of services. If required by business level components, it 
may include a new label indicating if a service is provided by devices or by humans but it is not mandatory.

From this point, the configuration process could be performed in the same way as if only devices were included into the low-level infrastructure. However, the calculation of the total QoS in Eq. (15) and Eq. (16) affects the self-adaptation solution, which should consider the dynamical behavior of humans, in order to avoid unnecessary calculations. Thus, in case of existing humans as service providers, we propose to modify these equations along expressions (22) and (23).

$$
\begin{aligned}
& Q o S_{g}=\alpha_{1}\left(\sum_{i=1}^{R_{1}} \beta_{i} Q_{g}^{i}\right)+\alpha_{2}\left(\sum_{i=1}^{R_{2}} \gamma_{i}\left(\delta_{1}^{i} \mathcal{L} \mathcal{P}\left[H Q_{g}^{i}\right]\right)\right) \\
& Q o S_{c}=\alpha_{1}\left(\sum_{i=1}^{R_{1}} \beta_{i} Q_{c}^{i}\right)+\alpha_{2}\left(\sum_{i=1}^{R_{2}} \gamma_{i}\left(\delta_{1}^{i} \mathcal{L} \mathcal{P}\left[H Q_{c}^{i}\right]\right)\right)
\end{aligned}
$$

Where $\alpha_{1}$ is the relative weight of the QoS due to the physical devices in the total QoS, and $\alpha_{2}$ is the relative weight of the QoS due to production services provided by humans in the total QoS. $\beta_{i}$ is the relative weight of the QoS due to the $\mathrm{i}$-th production level service provided by physical devices in the total QoS due to hardware devices. $\gamma_{i}$ has the same meaning of $\beta_{i}$ but applied to services provided by humans. Finally, $\mathcal{L P}[\cdot]$ represents a low-pass filter, focused on removing the very high-frequency variations in the quality parameters due to humans, as their behavior tends to be very dynamic, but QoS is not really affected for these modifications [69]. The particular configuration of this filter will depend on the application scenario.

No more modifications are required.

\subsection{Implementation}

Once described the mathematical formalization, in this subsection we present a completely useful specific implementation of the proposed self-configuration technology (others could be proposed and developed).

For this first implementation we have selected as deployment scenario a manufacturing scenario where a Smart Environment for product control, traceability and workers performance monitoring has been deployed. In this scenario several types of sensors, actuators and physical devices could be included. However, in this first evaluation only three instruments (smart objects) were considered: a RFIDenabled glove [70], a worktable provided with NFC readers and LEDs for user notification [70], and an autonomous wireless device with e-paper display and low-energy communication for information presentation [71].

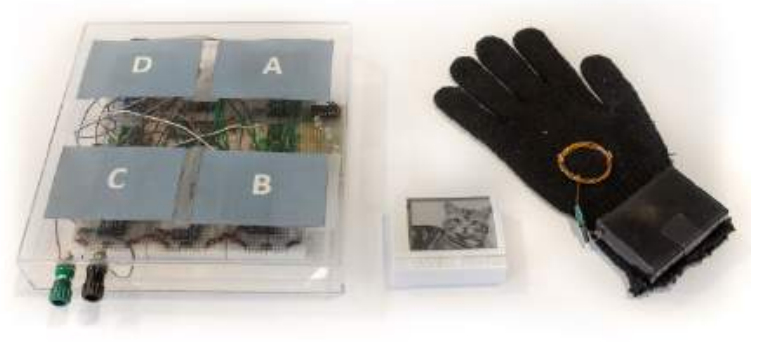

Fig. 7. Prototypes implementation

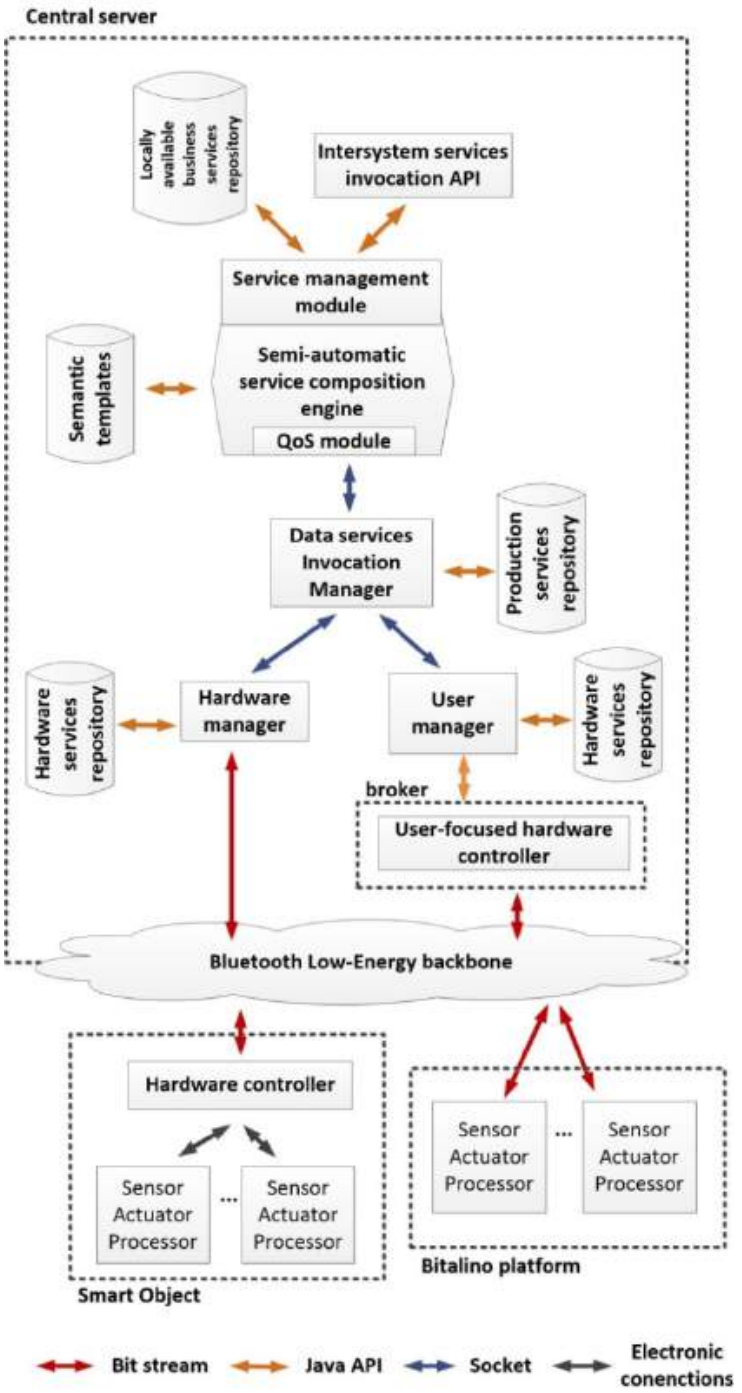

Fig. 8. Implementation architecture 
All these devices were based on commercial technologies. Figure 7 shows a prototype of each one of the described devices. The final deployment scenario would include as much devices as necessary, so various copies of each one of the presented prototypes in Figure 7 could be considered.

On the other hand, human service providers were monitored by means of a Bitalino [72] platform (the user-focused device), whose generated data about the humans' state (stress level, heart rate, etc.) were used as input to determine the people's motivation state using any of the proposed algorithms in the existing literature [69].

The software components (such as the Semiautomatic service composition engine) were implemented in Java technology. These components were deployed in a central server. All the physical components were connected through a Bluetooth LowEnergy backbone. This technology (due to its low power consumption) fits perfectly the requirement of the proposed scenario, where devices are provided with a small battery as power supply.

Figure 8 shows a detailed implementation architecture where all the defined components in the functional architecture (Figure 3) are mapped. It is important to note that components dedicated to control humans acting as service providers (the called userfocused components) have also been included. In particular, as can be seen, the prototypes showed on Figure 7 implement an embedded Hardware controller but Bitalino platform requires a broker acting as User-focused hardware controller which is implemented using Java technologies in the central server.

Data transmission among the components implemented using Java technologies is based on the common Java serialization solutions (such as the $\mathrm{Ob}$ jectOutputStream class). The transmission of the data generated by the Bitalino platform and the userfocused hardware controllers deployed as brokers in the central server employs the proprietary solutions provided by Bitalino software.

Finally, the transmission of the descriptors $h d_{i}$ from the hardware controllers (deployed in the smart objects) to the Hardware manager (running in the central server) is based on a Bluetooth binary stream. Descriptors $h d_{i}$ may be easily serialized sending at first the binary vector $v_{i}$ and later the cost vector $q_{i}$. In the same way, binary stream is employed to transmit the master archetype-based template from hardware manager to hardware controllers.

Now, considering the proposed implementation, the structure of the different vectors, matrixes and collection described in Section 3.2 may be fixed. Moreover, the selected semantic service composition technology must be also presented.

Table 2 presents the vector and matrix format for all the data elements described in the previous subsection (3.2). As can be seen, for simplicity (templates are much more easily created), in this case we have selected the hardware services coincident with some hardware capabilities, although that is not mandatory (in general, an atomic service uses various hardware capabilities). This option, nevertheless, does not affect the number of mathematical operations performed during the self-configuration process, so results about the convergence time and scalability are valid. Moreover, reduced-dimensions vectors and matrix have been selected in order to clarify the proposed technology, though in practice they may be as big as needed.

On the other hand, matrices $H Q_{g}$ and $H Q_{c}$ are not included in Table 2, as they are usually made of dimensionless parameters which do not match with any common definition. In this work, we are assuming these matrices are numerical values (real numbers) obtained as indicated in any of the previous works on this topic [69].

In general, the particular choices described in Table 2 are stablished with the objective of obtaining results which could be easily interpreted in Section 5 . Matrices $v_{i}, q_{i}$ and $\sigma_{d}$ are directly deducted from the capabilities of hardware prototypes showed on Figure 7. Human provided services in matrix $\sigma_{h}$ are selected to be simple activities, so no specific formation or great (physical or mental) efforts are required from people (what makes simple the development of the experimental validation). In respect to QoS measures and matrices (i.e. matrices $\Sigma, Q_{g}, Q_{c} Q o S_{g}$ and $Q o S_{c}$ ), parameters are selected as they are the most adequate to represent the proposed production and business services. Other typical parameters such as bandwidth, reliability of data precision have no sense in a scenarios based on RFID readers (as they do not affect in a determinant way that technology). Moreover, the selected parameters are easily understandable which is helping us to understand the results obtained in Section 5. In any case, the two parameters chosen represent two basic dimensions of a quality service: its delay and its reliability.

In respect to the semantic service composition, a technology focused on web service matching is implemented [43]. Finally, in the next sections, for simplicity in the experiment design, all relative weights have been fixed following a uniform distribution. 
Table 2. Data format in fast self-configuration process for Smart Environments

\begin{tabular}{|c|c|}
\hline Element & Pattern \\
\hline$v_{i}$ and $q_{i}$ & (NFC reading Power LEDS Signal processing Measure temperature Show a picture) \\
\hline$\sigma_{d}$ & ( $N F C$ reading Power LEDS Measure temperature Show a picture) \\
\hline$\sigma_{h}$ & (Carrying object Remove object off table Place object on table) \\
\hline$\Sigma$ & (Response time Availability) \\
\hline$Q_{g}, Q_{c}$ & $\left(\begin{array}{ccc}\text { Cost }(\text { element_1) } & \text { Response time }(\text { element_1) } & \text { Availability(element_1) } \\
\operatorname{Cost}(\text { element_2) } & \text { Response time }(\text { element_2) } & \text { Availability(element_2) } \\
\ldots & \ldots & \ldots \\
\text { Cost }(\text { element_N }) & \text { Response time }(\text { element_N }) & \text { Availability }(\text { element_N })\end{array}\right)$ \\
\hline$Q o S_{g}$ and $Q o S_{c}$ & (Cost Response time Availability) \\
\hline
\end{tabular}

\section{Experimental validation}

The proposed solution presents the advantage over previous proposals to be able to be applied in realtime applications and scenarios. In order to reach this objective three different designs have been included:

- The semantic service composition is not performed at operation time but during the configuration phase. As this time consuming process does not have to be executed each time a service is called, the service invocations are highly accelerated.

- A self-adaptation policy based on the concept of QoS is deployed. As service composition is performed before the system operation it is required a solution to update the list of available services at real-time. Our proposal classifies services as available, deleted or unavailable using simple mathematical operations for QoS calculation.

- Information from the low-level platform in order to calculate the QoS is collected using binary data formats and simple logical operations. In that way, adaptations may be performed with a much reduced time consume, as almost no information must be transmitted (so system resources are not consumed).

In this section, the proposed technology is evaluated as a valid solution for real-time operation, considering the three mentioned designs. Additional advantages such as the possibility of including humans as service providers, and the independence among the different abstraction layer in the architecture are not evaluated as they are directly deducted from the previous descriptions.

The proposed experimental validation mixes two different techniques. On the one hand, a real deployment was performed in the first experiment; but, on the other hand, a simulation environment was employed in the second, third and fourth experiments.

In the first experiment the required convergence time for the initial system configuration and the medium convergence time needed by the system to react to changes in the physical level are evaluated. For that, a Smart Environment as described in Section 3.5 employing the proposed self-configuration technology was deployed at the Technical University of Madrid. Three different scenarios were evaluated:

- In the first scenario fifteen smart objects made up the hardware platform: five cyber gloves, five worktables and five electronic ink displays.

- In the second scenario only seven smart objects were included: three electronic ink displays, two cyber gloves and two worktables. Additionally, eight humans acting as service providers are also part of the environment.

- Finally, in the third scenario, exclusively fifteen humans acting as service providers made up the Smart Environment.

- For each scenario, the experiment consisted of two phases.

- In the first phase the system was powered on and the time employed in the initial selfconfiguration measured. Then, the system was powered off again. This routine was repeated twenty-four times four each scenario. 
- In the second phase, for each scenario, the system was powered on, and it was kept operating for three hours. Data about the different system adaptations performed, and the convergence time employed in each one of these adaptations were collected. As said in Section 3.2, the refreshing period $T$ of the experimental measures $\Sigma(\mathrm{t})$ employed to calculate the QoS and update the list of available services is critical. Different values for this parameter were also considered in each in of the proposed scenarios during the second phase. Table 3 shows the number of registers obtained for each scenario and value for the period $T$ in this second phase.

Table 3. Number of registers in the second phase of the first experiment

\begin{tabular}{|c|c|c|c|c|}
\hline \multirow{2}{*}{ Scenario } & \multicolumn{4}{|c|}{ Refreshing period (s) } \\
\cline { 2 - 5 } & $\mathbf{5}$ & $\mathbf{1 0}$ & $\mathbf{3 0}$ & $\mathbf{6 0}$ \\
\hline $\begin{array}{c}\text { First scenario } \\
\text { (fifteen devices) }\end{array}$ & 142 & 106 & 97 & 89 \\
\hline $\begin{array}{c}\text { Second scenario } \\
\text { (seven devices and } \\
\text { eight humans) }\end{array}$ & 248 & 173 & 145 & 109 \\
\hline $\begin{array}{c}\text { Third scenario } \\
\text { (fifteen humans) }\end{array}$ & 346 & 263 & 207 & 192 \\
\hline
\end{tabular}

In the second experiment, the evolution of the convergence time of the proposed self-configuration technology when increasing the number of elements in the physical platform (both, humans and devices) is evaluated. In this experiment a simulated deployment was employed (mainly, due to the difficult of performing experiments involving greats amount of people).

The simulation was performed using the NS3 simulator [73]. In that simulator every element was represented by an agent, which implements the proposed self-configuration technology. Data gathered in the first experiment were used to model the behavior of the different devices and humans (then the obtained results present a good quality). Twelve different simulated scenarios were evaluated. In the first scenario only one element was included in the physical platform; in the last scenario 10000 (ten thousands) elements were considered. For each one of these twelve scenarios, three simulations were performed. Each simulation represented three hours of system operation. Data about the self-adaptations performed were gathered (specially the notifications about the start and the end of the self-configuration process).
The third experiment was designed to evaluate the evolution of the resource consumption (rate) in the system due to the proposed technology when increasing the number of elements in the physical platform (both, humans and devices). The experiment was really similar to the one performed in the second case. However, some modifications in the simulated scenarios were developed; basically various sinks in order to control the signalization load in the system must be added.

With this new simulation scenario, the same procedure as described for the second experiment was followed (twelve different scenarios including from one physical element to ten thousands, and three simulation performed for each scenario). Then, data about the signalization in the system was collected.

Finally, in the fourth experiment, previous results are compared to traditional configuration and adaptation solution for service-oriented SE. In particular, a traditional smart configuration solution based on semantic service compositions and the transmission of description files among components [36][20] was selected.

\section{Results}

In this Section, results of the proposed experiment in Section 4 are presented and analyzed.

Figure 9 presents the results of the first phase of the first experiment. Temporal values in the $\mathrm{Y}$-axis were normalized by the maximum value, as the important information is the relation among the results obtained for each scenario.

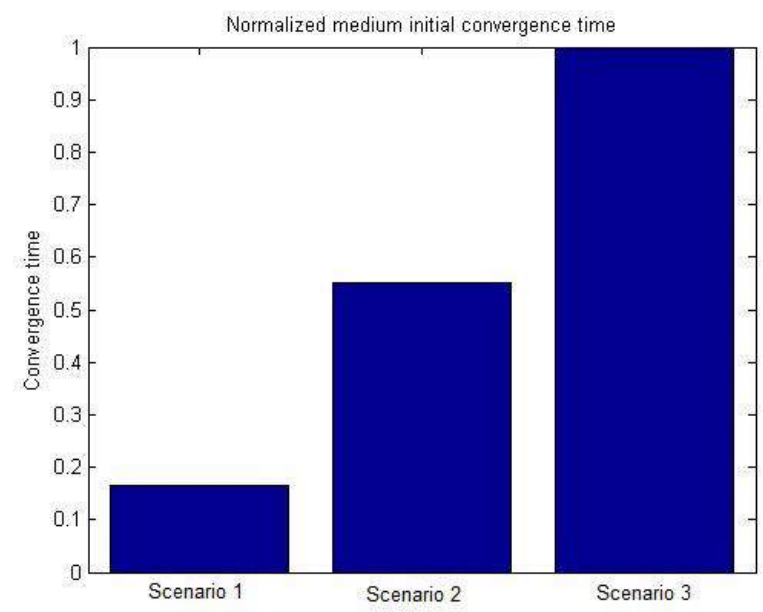

Fig. 9. Normalized medium initial convergence time 
Figure 9 represents the medium time obtained from the twenty-four realizations developed. As can be seen, the maximum time is required in the third scenario, when fifteen humans are included in the Smart Environments. This time is $45 \%$ less (approximately) if only eight humans are considered (as in the second scenario). If only fifteen hardware devices are included, the required time is a $15 \%$ of the required time when fifteen humans were considered (third scenario), and a $65 \%$ less than the required time when eight humans and seven devices were included (second scenario).

As main conclusion, it is clear that Smart Environments including humans require more time to configure than system only made of hardware devices. This fact may be caused by two factors.

First, determining the humans' state requires more resources. Hardware components only must transmit two vectors to the Hardware manager; however, user-focused hardware manager needs to collect various frames of data and apply a data processing algorithm before determining the stress level in humans.

And, second, as the user-focused hardware controller is implemented in a software broker running in the central server (instead of in the same hardware device as in the case of smart objects), communications between sensors and controllers tend to be slower and need more time.

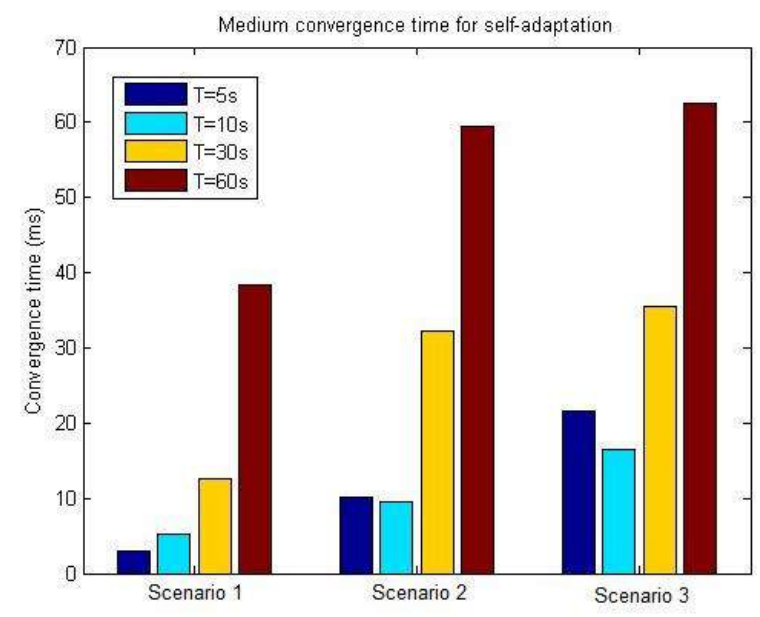

Fig. 10. Medium convergence time for self-adaptation

In respect to the second phase of the first experiment, first we are analyzing data on Table 3. As can be seen, as the refreshing period goes down, the number of adaptation processes performed grows. These additional records correspond to temporal fluc- tuations which are addressed if the refreshing time is enough short, but which are not detected if not (and then during a certain time the list of available services is not updated).

Figure 10 shows the results of the second phase of the first experiment. Figure 10 represents the medium time required for system adaptation in each one of the proposed scenarios, for the different proposed refreshing periods.

As can be seen in Figure 10, in general, the required time for self-adaptation continues being higher when humans are considered. In particular, the medium time when considering fifteen humans in the Smart Environment is up to three times higher than which obtained in the case of only existing hardware devices. Moreover, in the case of the second scenario (eight humans and seven hardware devices) the convergence time is around a $40 \%$ fewer than the value obtained for the third scenario.

Apart from these tendencies, two important facts about the refreshing period must be considered. First, very small values for the refreshing period do not guarantee a shorter convergence time. As can be seen on the results of the third scenario, the convergence time for $T=5 s$ is higher than which obtained for $T=10 s$. That is due to the fact that, for $T=5 s$, the system invests a lot of processing time on doing measures, and algorithm for motivation state calculation (then) cannot take profit from all resources in the user-focused controller.

And, second, if the refreshing period is too high, the convergence time is practically equal to it. In practice, as the required calculation time to perform an adaptation is relatively small, when the refreshing period goes up a certain threshold (around $T=20 \mathrm{~s}$ ), the convergence time does not depend on the processing time, but on the waiting time to the next experimental measure.

Nevertheless, despite these observations, the obtained convergence time is very low. In comparison, for example, similar experiments for LTE networks [44] showed that the required time for a handover is around $20 \mathrm{~ms}$ (and network reconfiguration is perfectly executed at real-time in most cases).

From these results, it is possible to guarantee the proposed solution as useful in real-time applications.

Figure 11 shows the results of the second experiment, where the evolution of the convergence time of the proposed self-configuration technology when increasing the number of elements in the physical platform is evaluated. Results are normalized to the 
maximum value as the interesting information is the tendency, not the particular temporal values.

As can be seen, the convergence time is almost invariant with respect to the number of elements in the physical platform. The medium normalized convergence time presents only around a $7 \%$ of variation when the number of elements in the physical platform increases in four magnitude orders (from 1 to 10000).

This result, actually, greatly improves the precedents. For example, some previous proposals [27] reported variations of a $600 \%$, approximately, when the number of elements in the physical layer is multiplied by three.

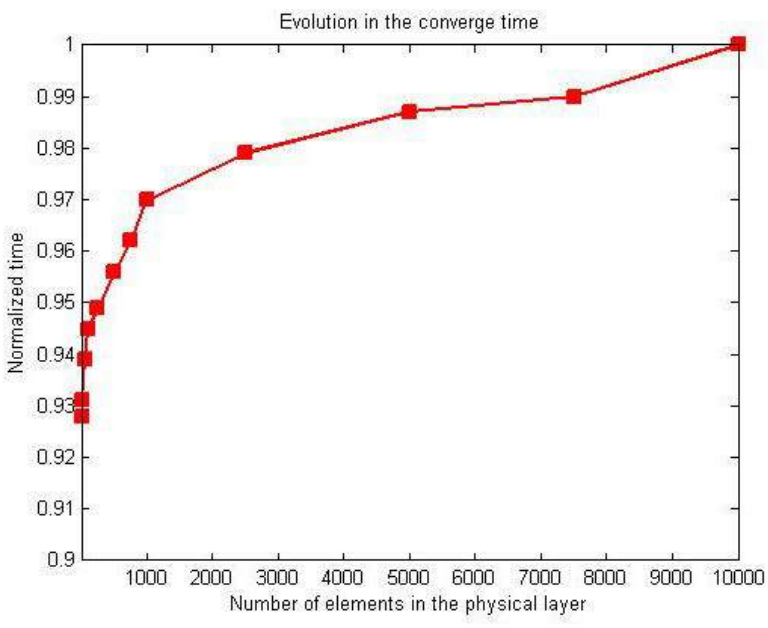

Fig. 11. Evolution in the convergence time vs. number of elements in the physical platform

The explanation of this improvement is a mix of various causes. In the proposed solution, the described functional architecture (Figure 3) presents a tree topology. In that way, information may be compiled in the low layers (i.e. hardware controller), and in the top layers (i.e. hardware manager, service composition engine, etc.) algorithms are independent of the number of elements included in the physical platform. Moreover, in the hardware controllers information about the system's state is codified using binary vectors and algorithms are based on logical operations. Thus, the convergence time increases lightly when increasing the number of elements in the physical platform, but the impact is very limited as showed in Figure 11.

Therefore, it is possible to say the proposed technology guarantees the scalability of the Smart Environments, at least considering the required convergence time.
Figure 12 shows the results of the third and last experiment. In this Figure the evolution of the resource consumption in the system is represented versus the number of elements included in the physical layer. The resource consumption seen in one simulation (as percentage) is calculated using the expression of Equation (24).

$\frac{\text { Bytes transmitted dedicated to conf or adapt }}{\text { Total of bytes transmited }} \cdot 100$

As can be seen, the resource consumption increases linearly when increasing the number of elements in the physical layer.

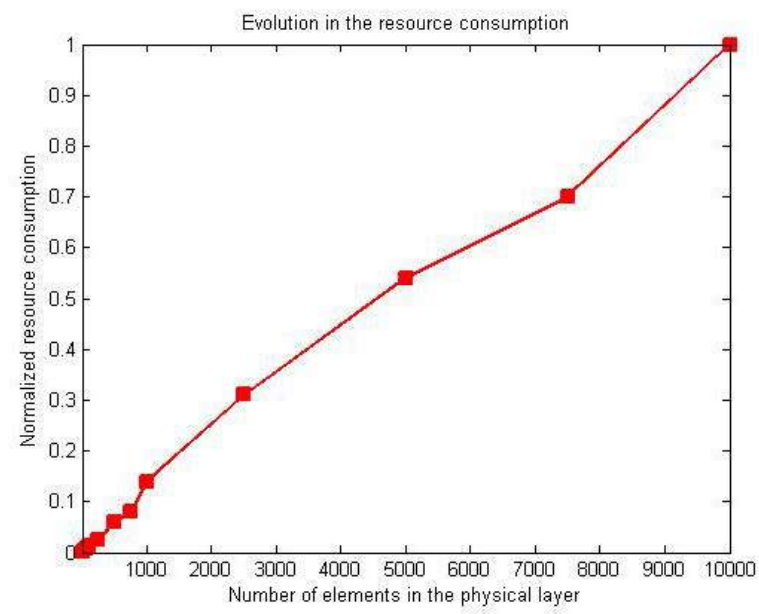

Fig. 12. Evolution in the resource consumption vs. number of elements in the physical platform

This result is coherent with the proposed scenario, where every additional smart object incorporates one hardware controller which transmits a fixed amount of bytes of signalization. The same reasoning is valid in the case of humans.

A linear increase improves the behavior of the previous proposals [19], which traditionally present an increase of $n^{2}$ order. However, it is a limiting characteristic of the proposed technology which must be taken into account (in particular when designing the Bluetooth backbone or when selecting the appropriate central server for the Smart Environment).

Finally, Figure 13 shows the results of the fourth experiment. In this figure, the required calculation time to perform the system configuration in our proposal is compared with the results obtained using previous technologies. 
As can be seen, previous solutions require much more calculation time to perform the system configuration than the proposed technology (up to an order of magnitude higher). The evolution in both cases (the configuration process of the proposed technology and previous proposals) present an exponential evolution created by the service composition engine (whose reasoning time usually grows following this law). However, in the analyzed previous technology the increase is much faster as to the reasoning time of the composition engine it must be added the required time to collect the information from the hardware platform and processing it (which also evolves with and exponential law). On the other hand, the proposed processing algorithm based on simple logical operations, if programmed adequately, requires a constant calculation time, independent from the number of offered hardware services. Thus, the calculation time is only due to the service composition technology selected.

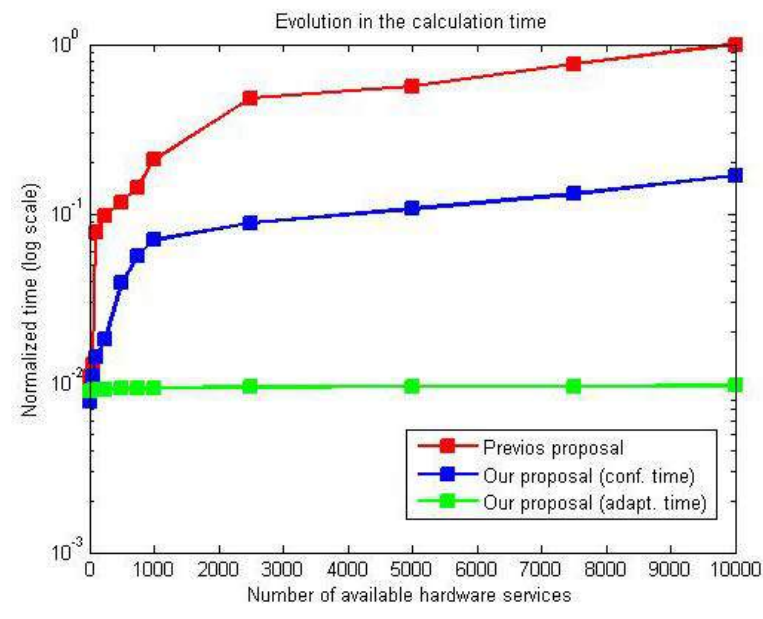

Fig. 13. Evolution in calculation time vs. number of available hardware services in the physical platform

Furthermore, there is a basic difference. The showed calculation time for previous proposals (red line) must be assumed each time a service is invoked. On the contrary, the configuration delay in our solution is only present at the pre-operational time and if a hard refresh is required. In any other case, the selfadaptation solution maintains updated the list of available services (green line). In that case, as seen previously, the calculation time remains almost constant and it is much lower than in the other cases (as the service composition process is not performed).
In conclusion, the proposed technology fulfills the objective of improving the existing technologies in order to be able to be applied to real-time scenarios.

\section{Conclusions}

Smart Environments are greatly extended, for example, as support for Ambient Intelligence applications. In order to become a real useful technology, Smart Environment must operate at real-time and support automatic self-configuration and selfadaptation.

The purpose of the current paper is to describe a new fast self-configuration technology to be applied in Smart Environment for real-time applications. Additionally, as a novelty, the proposed technology is able to include both service provided by hardware devices and services provided by humans.

The proposed solution is based on a functional architecture made of various independent components which, using low-level templates, simple binary and numeric vectors and matrices, transmit the state of the physical layer to a service composition engine. The engine, then, calculates the available services in the system using sematic templates. The solution is service-oriented and employs the concept of qualityof-service in order to reduce the time required in the self-adaptation process. For that, the quality-ofservice is defined as a vector where various parameters are represented (humans' physiological state, available processing slots, availability, etc.). Then, a service calculated by the composition engine is available if each one of the parameters is higher than a certain limit and, besides, the global quality (calculated using a cost function and the quality-of-service vector) is also higher than a threshold. Otherwise, the service is unavailable or deleted.

In this work, an experimental validation consisting of three experiments about the scalability and the convergence time of the proposed solution was also performed. Then, the following conclusions can be drawn from the present study.

First, the proposed solution requires a convergence time which allows its application to real-time scenarios. In particular, the achieved value for the convergence time is lower than obtained for other technologies such as Long-Term Evolution (LTE) networks.

Second, the described solution includes both humans and hardware devices. However, performance is better in scenarios where only hardware compo- 
nents are considered. Mainly, this fact is due to the higher resources needed to determine the humans' state; and because an external broker is required as user-focused hardware controller. In future works, mechanisms to obtain the same behavior independently of the physical layer composition should be developed.

Third, in terms of the convergence time, the scalability of the proposed solution is guaranteed. The impact of the number of elements of the physical layer in the convergence time is extremely limited, so large scale deployments may be considered. This is very important, considering concepts such as the Internet of Things and Pervasive Sensing, based on increasing the density of hardware devices in daily scenarios.

And, finally, the limiting characteristic of the proposed solution is the resource consumption. The proposed technology highly improves the performance of the previous proposals, but the resource consumption continues depending linearly on the number of elements in the physical platform. Future works should improve this aspect.

\section{Acknowledgements}

The research leading to these results has received funding from the Ministry of Economy and Competitiveness through SEMOLA project (TEC201568284-R) and from the Autonomous Region of Madrid through MOSI-AGIL-CM project (grant P2013/ICE-3019, co-funded by EU Structural Funds FSE and FEDER). Borja Bordel has received funding from the Ministry of Education through the FPU program (grant number FPU15/03977).

\section{References}

[1] D.J. Cook, Youngblood, L.B. Holder and E.O. Heierman, Automation intelligence for the smart environment, in: Proceedings of the International Joint Conference on Artificial Intelligence, 2005.

[2] D. J Cook, S. K. Das, How smart are our environments? An updated look at the state of the art. Pervasive and mobile computing, 3(2) (2007), 53-73.

[3] R. Alcarria, T. Robles, A. Morales, S. González-Miranda, New service development method for prosumer environments. In: Proceedings of the 6th International Conference on Digital Society , pp. 86-91, 2012

[4] A. W. Colombo, T. Bangemann, S. Karnouskos, J. Delsing, P. Stluka, R. Harrison, R., J. L. Lastra. Industrial cloud- based cyber-physical systems. The IMC-AESOP Approach. 2014

[5] F. Jammes, S. Karnouskos, B. Bony, P. Nappey, A.W. Colombo, J. Delsing, T. Bangemann, Promising technologies for soa-based industrial automation systems. In: Industrial CloudBased Cyber-Physical Systems, pp. 89-109. Springer International Publishing. 2014

[6] C. L. Wu, C. F. Liao, L. C. Fu, Service-oriented smart-home architecture based on OSGi and mobile-agent technology. IEEE Transactions on Systems, Man, and Cybernetics, Part C (Applications and Reviews), 37(2) (2007), , 193-205.

[7] D. Cook, D. Das. Smart environments: Technology, protocols and applications (Vol. 43). (2004). John Wiley \& Sons.

[8] A. Helal, D. J. Cook M. Schmalz. Smart home-based health platform for behavioral monitoring and alteration of diabetes patients. Journal of diabetes science and technology, $\mathbf{3}(\mathbf{1})$ (2009), 141-148.

[9] N. K. Suryadevara, S. C. Mukhopadhyay, R. Wang, R. K. Rayudu. Forecasting the behavior of an elderly using wireless sensors data in a smart home. Engineering Applications of Artificial Intelligence, 26(10), (2013), 2641-2652.

[10]D. J. Cook, N. C. Krishnan, P. Rashidi. Activity discovery and activity recognition: A new partnership. IEEE transactions on cybernetics, 43(3), (2013), 820-828.

[11]N. Roy, A. Misra, D. Cook. Ambient and smartphone sensor assisted ADL recognition in multi-inhabitant smart environments. Journal of ambient intelligence and humanized computing, 7(1), (2016), 1-19.

[12] G.M. Youngblood. MavHome: Managing an Adaptive Versatile Home, Oct. 2003.Website: mavhome.uta.edu [accessed: 02/2017].

[13]S. Munir, J. A. Stankovic, C. J. M. Liang, \& S. Lin. Cyber physical system challenges for human-in-the-loop control. In: Presented as part of the 8th International Workshop on Feedback Computing. (2013).

[14]D. De, W. Z. Song, M. Xu, C. L. Wang, D. Cook, \& X. Huo. Findinghumo: Real-time tracking of motion trajectories from anonymous binary sensing in smart environments. In: Distributed Computing Systems (ICDCS), (2012) IEEE 32nd International Conference on (pp. 163-172). IEEE.

[15] V. X. Tran, H. Tsuji. Owl-t: A task ontology language for automatic service composition. In: Proceedings of IEEE International Conference on Web Services (2007), pp. 1164-1167. IEEE.

[16]D. D. Hoang, H. Paik, \& C. K. Kim. Service-oriented middleware architectures for cyber-physical systems. International Journal of Computer Science and Network Security, 12(1) (2012), 79-87.

[17]R. Alcarria, T. Robles, A. Morales, D. López-de-Ipiña, U. Aguilera. Enabling flexible and continuous capability invocation in mobile prosumer environments. Sensors, 12(7) (2012), 8930-8954.

[18]F. Zeshan, R. Mohamad. Semantic web service composition approaches: overview and limitations. International Journal of New Computer Architectures and their Applications 1(3), (2011), 640-651.

[19]J. Wang, Q. Zhu, Y. Ma. An agent-based hybrid service delivery for coordinating internet of things and 3rd party service providers. Journal of Network and Computer Applications, 36(6) (2013), 1684-1695

[20] S. Mayer, R. Verborgh, M. Kovatsch, F. Mattern, Smart Configuration of Smart Environments. IEEE Transactions on Automation Science and Engineering, 13(3), (2014) 12471255 
[21]H. Hu, J. Zhang, X. Zheng, Y. Yang, P. Wu, Selfconfiguration and self-optimization for LTE networks. IEEE Communications Magazine, 48(2), (2010), 94-100.

[22] A. Erradi, P. Maheshwari, V. Tosic, Policy-driven middleware for self-adaptation of web services compositions. In: proceedings of International Conference on Distributed Systems Platforms and Open Distributed Processing, 2006, (pp. 62-80). Springer Berlin Heidelberg.

[23] Y. Charif, N. Sabouret, An overview of semantic web services composition approaches. Electronic Notes in Theoretical Computer Science, 146(1), (2006), 33-41.

[24] M. S. Familiar, J. F. Martínez, I. Corredor, \& C. García-Rubio. Building service-oriented smart infrastructures over wireless ad hoc sensor networks: A middleware perspective. Computer Networks, 56(4), 1303-1328. (2012).

[25]M. S. Familiar, J. F. Martínez \& L. López. Pervasive smar spaces and environments: a service-oriented middleware architecture for wireless ad hoc and sensor networks. International Journal of Distributed Sensor Networks, (2012).

[26]C. Gouin-Vallerand, B. Abdulrazak, S. Giroux, \& A. K. Dey, A context-aware service provision system for smart environments based on the user interaction modalities. Journal of Ambient Intelligence and Smart Environments, 5(1), (2013), 47-64.

[27]U. Mönks, H. Trsek, L. Dürkop, V. Geneiß, \& V. Lohweg,.. Assisting the design of sensor and information fusion systems. Procedia Technology, 15, (2014), 35-45.

[28]T. Dillon, V. Potdar, J. Singh, \& A. Talevski. Cyber-physical systems: Providing Quality of Service $(Q o S)$ in a heterogeneous systems-of-systems environment. In: Proceedings of the 5th IEEE International Conference on Digital Ecosystems and Technologies Conference (DEST), (2011) (pp. 330-335). IEEE.

[29]T. S. Dillon, H. Zhuge, C. Wu, J. Singh, \& E. Chang. Web of - things framework for cyber-physical systems. Concurrency and Computation: Practice and Experience, 23(9), (2011), 905-923.

[30]A. Smirnov, A. Kashevnik, N. Shilov, A. Makklya, \& O Gusikhin. Context-aware service composition in cyber physical human system for transportation safety. In: 13th International Conference on ITS Telecommunications (ITST), (2013) (pp. 139-144). IEEE.

[31] A. W. Colombo, S. Karnouskos, J. M. Mendes. Factory of the Future: A Service-oriented System of Modular, Dynamic Reconfigurable and Collaborative Systems. In: Artificial Intelligence Techniques for Networked Manuf Enterprises Management, pp. 459-481. Springer, 2010.

[32] AutoPNP consortium. AutoPNP - Plug and Play for Automation Systems 2014 Available from: http://www.autopnp.com/ (accessed on $6^{\text {th }}$ July 2016).

[33]H. C. Cheng, R. de Lemos, H.Giese, P. Inverardi, J. Magee. Software Engineering for Self-Adaptive Systems: A Research Roadmap Self-Adaptive Systems, Springer. Berlin Heidelberg (2009), pp. 1-26, 2009.

[34] T. Wang, C. Niu, \& L. Cheng, A Two-Phase Context-Sensitive Service Composition Method with the Workflow Model in Cyber-Physical Systems. In: Computational Science and Engineering (CSE), (2014) IEEE 17th International Conference on (pp. 1475-1482). IEEE.

[35]L. Baresi, S. Guinea, \& A. Shahzada. SeSaMe: towards a semantic self adaptive middleware for smart spaces. In: International Workshop on Engineering Multi-Agent Systems (pp 1-18). (2013, May). Springer Berlin Heidelberg.

[36]S. Mayer, N. Inhelder, R. Verborgh, R. Van de Walle \& F. Mattern, Configuration of smart environments made simple: Combining visual modeling with semantic metadata and rea- soning. In: International Conference on the Internet of Things (IOT), (2014) (pp. 61-66). IEEE.

[37]L. Baresi, \& S. Guinea, Event-Based Monitoring of ServiceOriented Smart Spaces. In: IEEE 8th International Conference on Service-Oriented Computing and Applications (SOCA) (pp 123-130). (2015) IEEE

[38]K. Baina, B. Benatallah, F. Casati, F. Toumani, F. Modeldriven web service development. In: Proceedings of International Conference on Advanced Information Systems Engineering. 2004. (pp. 290-306). Springer Berlin Heidelberg.

[39]M. Rietzler, J. Greim, M. Walch, F. Schaub, B. Wiedersheim, M. Weber, M. Homeblox: Introducing processdriven home automation. In: Proceedings of the 2013 ACM conference on Pervasive and ubiquitous computing adjunct publication, 2013, (pp. 801-808). ACM.

[40] J. Hendler, Web 3.0 Emerging. Computer, 42(1), (2013), 111 113.

[41]H. N. Talantikite, D. Aissani, N. Boudjlida. Semantic annotations for web services discovery and composition. Computer Standards \& Interfaces, 31(6), (2009) 1108-1117.

[42] Y. Charif, N. Sabouret. An overview of semantic web services composition approaches. Electronic Notes in Theoretical Computer Science, 146(1), (2006), 33-41.

[43]E. Karakoc, P. Senkul, Composing semantic Web services under constraints. Expert Systems with Applications, 36(8), (2009), 11021-11029.

[44]E. Oren, K. Möller, S. Scerri, S. Handschuh, M. Sintek, What are semantic annotations. Relatório técnico. DERI Galway, 9(2006), pp.62.

[45]D. H. Wolpert, K. R. Wheeler, \& K. Tumer. Collective intelligence for control of distributed dynamical systems. EPL: $E u$ rophysics Letters, 49(6), (2000). 708.

[46] G. D. M. Serugendo, A. Karageorgos, O. F. Rana, \& F. Zambonelli. Engineering self-organising systems: nature-inspired approaches to software engineering (Vol. 2977). (2004). Springer.

[47]G. Di Caro, M. Dorigo. Ant colonies for adaptive routing in packet-switched communications networks. In: Proceedings of PPSN V - Fifth International Conference on Parallel Problem Solving from Nature, volume 1498 of LNCS. SpringerVerlag, (1998).

[48]N. Jennings, K. Sycara, M. Wooldridge. A Roadmap of Agent Research and Development. Autonomous Agents and MultiAgent Systems, 1(1) (1998) pp. 7-38

[49] V. Bicer, O. Kilic, A. Dogac, G. B. Laleci. Archetype-based semantic interoperability of web service messages in the health care domain. International Journal on Semantic Web and Information Systems 1(4), (2005), 1-23.

[50]E. Sirin, B. Parsia, D. Wu, J. Hendler, D. Nau. HTN planning for web service composition using SHOP2. Web Semantics: Science, Services and Agents on the World Wide Web, 1(4), (2004), 377-396.

[51]L. Zeng, B. Benatallah, M. Dumas, J. Kalagnanam, Q. Z Sheng. Quality driven web services composition. In: Proceedings of the 12th international conference on World Wide Web, 2003. (pp. 411-421). ACM.

[52]V. Degeler, L. I. L. Gonzalez, M. Leva, P. Shrubsole, S Bonomi, O. Amft, \& A. Lazovik. Service-oriented architecture for smart environments (short paper). In: IEEE 6th International Conference on Service-Oriented Computing and Applications (pp. 99-104). (2013) IEEE.

[53]F. Furfari, M. Girolami, S. Lenzi, \& S. Chessa. A serviceoriented zigbee gateway for smart environments. Journal of Ambient Intelligence and Smart Environments, 6(6), (2014), 691-705. 
[54]G. Kortuem, F. Kawsar, D. Fitton, V. Sundramoorthy. Smart objects as building blocks for the internet of things. IEEE Internet Computing. 14, 2010, 44-51.

[55] J. W. Gardner, V. K. Varadan, \& O. O. Awadelkarim. Mi crosensors, MEMS, and smart devices (Vol. 1). (2001). Chichester: Wiley.

[56] B. Bordel, D. Sánchez de Rivera, R. Alcarria. Plug-and-Play Transducers in Cyber-Physical Systems for Device-Driven Applications. In: proceedings of 10th International Conference on Innovative Mobile and Internet Services in Ubiquitous Computing (IMIS), 2016 (pp. 316-321). IEEE.

[57] J. Domingues, A. Damaso, R. Nascimento, N. Rosa, An Energy-Aware Middleware for Integrating Wireless Sensor Networks and the Internet, International Journal of Distributed Sensor Networks, (2011), pp. 1-19, 2011.

[58]Hydra $\quad$ Project $\quad$ Available http://www.hydramiddleware.eu/articles.php?article id=68. (accessed on $6^{\text {th }}$ July 2016)

[59] K. Lee. IEEE 1451: A standard in support of smart transducer networking. In: Proceedings of the 17th Conference on Instrumentation and Measurement Technology, (2000). p. 525528.

[60]Composite Capability/Preference Profiles (CC/PP) current status page Available from: https://www.w3.org/standards/techs/ccpp\#w3c_all (accessed on $6^{\text {th }}$ July 2016)

[61]T. Beale, S. Heard. Archetype Definition Language. openEHR Foundation, 2007, 47.

[62]P. Kostelnik, M. Sarnovsky, J. Hreno, M. Ahlsen, P. Rosengren, P., Kool, M. Axling. Semantic devices for ambient environment middleware. In: Proceedings of EURO TrustAMI, Internet of Things and Services Workshop. 2008

[63] M. Compton, P. Barnaghi, L. Bermudez, R. GarcíA-Castro, O. Corcho, S. Cox, V. Huang, The SSN ontology of the W3C semantic sensor network incubator group. Web semantics: science, services and agents on the World Wide Web, 17, 2012 , 25-32.

[64] A. Bandara, T. R. Payne, D. de Roure, G. Clemo An ontological framework for semantic description of devices. 2004
Available

online: http://eprints.soton.ac.uk/262689/1/DOPoster2.pdf (accessed on $6^{\text {th }}$ July 2016)

[65]N. Silva, J. Rocha. Semantic Web Complex Ontology Mapping. Web Intelligence, 2003, pp. 82-88

[66] V. M. Van Der Aalst, A. H. Ter Hofstede. YAWL: yet another workflow language. Information systems, 30(4), 2005, 245 275.

[67]F. Giunchiglia, P. Shvaiko, M. Yatskevich. Semantic matching. Encyclopedia of Database Systems, 2009, pp. 2561-2566. Springer US.

[68] M. Paolucci, T. Kawamura, T. R. Payne, K. Sycara. Semantic matching of web services capabilities. In: Proceedings of International Semantic Web Conference, 2002. (pp. 333-347). Springer Berlin Heidelberg.

[69]B. Bordel, R. Alcarria, D. Martín, T. Robles, D. Sánchez de Rivera. Self-configuration in humanized Cyber-Physical Systems. Journal of Ambient Intelligence and Humanized Computing, 1-12.

[70]B. Bordel Sánchez, R. Alcarria, D. Martín \& T. Robles. TF4SM: A Framework for Developing Traceability Solutions in Small Manufacturing Companies. Sensors, 15(11), (2015), 29478-29510.

[71]D. Sánchez de Rivera, R. Alcarria, D. Martín de Andres, B. Bordel, T. Robles, An autonomous Information Device with E-Paper Display for Personal Environments In: Proceedings of IEEE International Conference on Consumer Electronics (ICCE), (2016), Las Vegas, NV, 2016

[72] Bitalino homepage. Available from: http://www.bitalino.com/ (accessed on $6^{\text {th }}$ July 2016)

[73]Ns-3 Simulator homepage. Available from: https://www.nsnam.org/ (accessed on $6^{\text {th }}$ July 2016). 


\section{Plug-and-play transducers in Cyber-Physical Systems for device-driven applications}

\author{
Borja Bordel, Diego Sánchez de Rivera \\ Department of Telematics Systems Engineering \\ Universidad Politécnica de Madrid \\ Madrid (España) \\ bbordel@dit.upm.es, \\ diego.sanchezderiveracordoba@gmail.com
}

\author{
Ramón Alcarria \\ Department of Topographic Engineering and \\ Cartography \\ Universidad Politécnica de Madrid \\ Madrid (España) \\ ramon.alcarria@upm.es
}

\begin{abstract}
One of the most popular IoT-based technologies nowadays are Cyber-Physical Systems (CPSs). In its lowest level, CPSs include a set of transducers which must be automatically connected and configured to achieve a seamless union with the environment. This problem, however, is usually addressed supposing devices with large processing capabilities, instead of small sensors and actuators seamless integrated in the physical world. Thus, in this article we propose a new plugand-play technology specially designed for being applied in reduced capacities devices integrated in CPSs. Moreover, an experimental validation is conducted in order to validate the proposal and evaluate its performance.
\end{abstract}

Cyber-Physical Systems; Internet of Things; plug-and-play technologies; transducers;

\section{INTRODUCTION}

One of the most complex IoT-based solutions, where IoT components get complemented with additional functionalities as distributed control or intelligence management and processing, are Cyber-Physical Systems (CPSs) [1].

As described in the relevant NSF reports [2], CPSs are integrations of computation and physical processes. Embedded computers and networks monitor and control the physical processes, usually with feedback loops where physical processes affect computations and vice versa. However, this initial definition has been modified and expanded by many authors. Thus, nowadays CPSs (as described by the National Institute of Standards and Technology [3]) have a more vertical approach, including from the user goals to the underlying physical system [4].

In this new vertical architecture, feedback loops can operate in two senses: bottom-up and top-down [5]. Although both approaches are interesting, the natural way of executing the loops is bottom-up (monitoring first the physical process, deciding later the actions to be taken, and acting over the physical process in the last step) [6]. Three different types of applications can be built with this approach:

- Event-driven applications: In these applications, high-level actions are triggered by a particular event or set of events.

- Human-driven applications: In these applications, the system's behavior changes depending on the activities being performed by users.
- Device-driven applications: In these applications, hardware devices perform a certain process independently, and top layers supervise the execution by means of the data produced by the hardware platform.

Among all the previous applications, device-driven applications are the most critical. In this kind of scenarios data are not only processed locally in hardware, but also remotely by data analytics components. Thus, information about transducers must be transmitted to higher layers in the system. Furthermore, hardware controllers, to which transducers are connected, are typically reduced capabilities devices, as they must be as small as possible to be seamless integrated in daily living objects. Industrial control systems are a typical example of these scenarios. In industrial production systems, assembly lines are controlled using Programmable Logic Controllers (PLCs) running independently a certain number of tasks. Above them, a system of data acquisition and processing (SCADA, for example) monitors the entire process and undertakes supervision.

However, most employed solutions nowadays for plugand-play transducers (as IEEE 1451 standard [7]) either they require the use of high capacities components, which are not admissible in this scenario, or cannot move the information about transducers beyond the controller to which they are connected.

Therefore, in this paper we propose a new plug-and-play technology for transducers integrated in CPSs for devicedriven applications. The objective of the paper is to present the technical solution, to describe a first implementation and to validate the usability and performance of the proposed solution.

The rest of the paper is organized as follows: Section II presents the state of the art in relation to plug-and-play transducers. Section III describes the technical proposal and presents a practical implementation. Section IV includes the experimental validation of the proposed technology, and Section V shows the obtained results. Finally, Section VI presents some conclusions.

\section{STATE OF THE ART}

Various solutions have been proposed in the research literature about the problem of plug-and-play transducers in CPS. On the one hand, some works [8] explain how create a 
wireless network [9] made of several transducers. On the other hand, other articles [10] [11] are focused on connecting scattered devices through an ad-hoc network (based, for example in the IEEE 1073 medical bus and Publication/Subscription networks). These solutions, however, do not fit in our scenario; mainly, because all previous proposals seek to create a totally connected network of transducers, but our goal is to connect to the controller and configure automatically the transducers, as well as, to support the information transmission to the data analytics components.

If standardized solutions (i.e. industrial products) are considered, the standards family IEEE 1451 addresses exactly the problem of supporting a plug-and-play architecture based on transducers and microcontrollers [12] [7]. The IEEE 1451 family provides a common interface and enabling technology for the connectivity of smart transducers (a concept referring integrations between transducers and processors) to controllers and data acquisition systems. The standardized Transducer Electronic Data Sheets (TEDS) [13] specified by IEEE 1451.2 allow the self-description of transducers and the interfaces provide a standardized mechanism to facilitate the plug-and-play of transducers to hardware controllers. The inclusion of 1451.4 to the family of 1451 standards will meet the needs of the analog transducer users [14].

Nevertheless, despite being a standard solution, it has not been widely adopted. In fact, IEEE 1451 presents various problems; most of which impede the use of this solution in CPS.

First, the standard only defines 16 TEDS templates. If a transducer type different to these 16 standard templates is added, users should define a new template (for which users need technical knowledge). Thus, although IEEE 1451 is an open and standard solution (a great advantage over other proposals), it is common to include proprietary TEDS templates in practical implementations. Therefore, the solution obtained would be also proprietary and the interoperability typical of standard technologies would disappear (becoming equal this alternative to any other proposal done by a particular researcher.).

Second, IEEE 1451 is a complex standard. Algorithms based on TEDS (which are byte-oriented messages) require large amounts of memory (for both, code and data) and high computing capabilities. In our scenario, hardware controllers' size is much more important than computing capabilities; so, for being able to reach a seamless integration, miniaturized low capacities microcontrollers are usually selected as hardware controllers. Specifically, a TED message has a length of 92 bytes, which has to be maintained in SRAM memory. Taking into account this value and considering that miniaturized microcontrollers usually have around $1 \mathrm{~KB}$ of SRAM, in a hardware controller only between 5 and 10 transducers could be considered (depending on the variables and other data generated by the program). This limit is low enough for being admissible in most applications.

Finally, small controllers do not implement IEEE 1451 interface. Miniaturized processing elements only implement the basic communication interfaces (as SPI or I2C), so to support IEEE 1451 standard additional circuits should be added, which would obstruct a seamless integration.

Therefore, authors concluded that a new proposal for supporting plug-and-play transducers in CPS is needed. This solution must be independent of the transducer type, must be defined at low-level (i.e. we need a bit oriented solution), have to be based on common communication interfaces and communication messages should be short and easily processed. Thus, we have designed a plug-and-play software serial interface, capable of recognizing the transducers connected to a hardware controller.

\section{PROPOSAL}

In this section, we describe the proposed technical solution and its first particular implementation.

\section{A. Technical solution}

The plug-and-play architecture described below can be implemented in any controller or transducer, with the proviso that programming capability and a Serial Peripheral Interface (SPI) (the most common in microelectronics) must be available. SPI ports allow full-duplex communications using four independent lines:

- Master Output Slave Input (MOSI) line, to transmit data from the master device to the slave device.

- Master Input Slave Output (MISO) line, to transmit data from the slave device to the master device.

- Clock (CLK) line, for sharing the synchronization signal.

- Slave Select (SS) line, to active the slave device.

For analog sensors, an intermediate stage, including the above requirements, should be added (Figure 1).

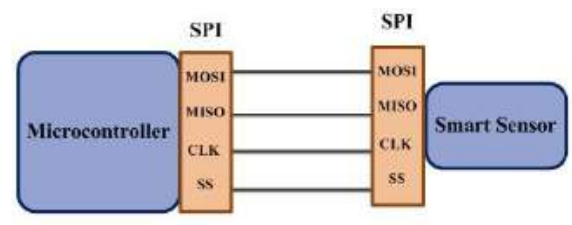

(a)

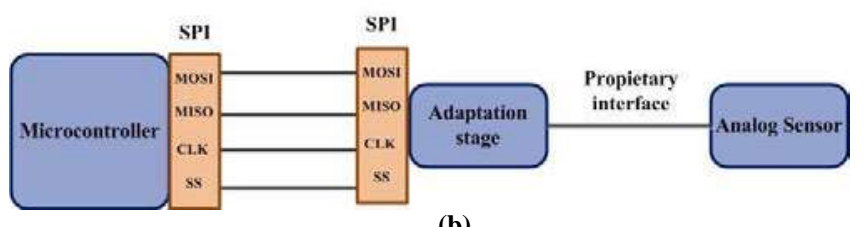

(b)

Figure 1. (a) Physical architecture for sensors with computing capabilities (b) Physical architecture for analog sensors

Then, suppose a digital transducer and a hardware controller related through a physical interface SPI. The controller acts as master and the transducer acts as slave device. Over this physical interface, two logical interfaces are defined: the control one and the data one (see Figure 2). 


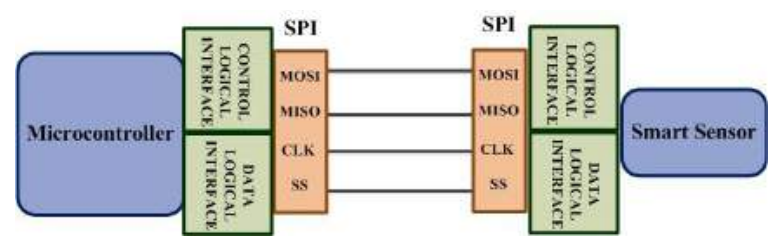

Figure 2. Logical interfaces in the plug-and-play architecture

The form of sharing the physical lines between these two logical interfaces is based on the inclusion of a header (called "Interface ID") in the frames circulating through the interface (Figure 3). In this proposal, the interface ID has one-byte length.

It must be noted that with a byte, 256 different logical interfaces could be defined. Although in this first version of the architecture only two interfaces are considered (control and data), in the future, other interfaces can be used to, for example, distinguish between continuous data output sensors (such as biometrics) and sensors that generate data slowly (such as RFID readers).

Two basic parameters characterize a digital transducer: the data length and the frequency with which the existence of new data in the sensor should be consulted.

The first parameter it is vital for system operation. In the SPI interfaces, the master device must activate the Slave Select signal (SS) during a time equal to the time required by the slave device to perform the transmission of its new data, so it is imperative that the master device previously knows such duration. Since the bit time is fixed, knowing the length (in bits) of the data produced by the sensor (the slave device) is enough to become available the transmission.

Depending on the configuration, the second parameter may be unnecessary. In some configurations, external interruptions are previously configured in the microcontroller unit (MCU), which can be triggered by transducers. Thus, when a transducer has new data, it interrupts the normal execution of the MCU and the microcontroller does not have to know the frequency with which new data is generated. However, this solution, though valid, requires the microcontroller to have an interruption pin for each transducer, which is not always guaranteed. Therefore, our architecture considers another solution, in which a software interruption for every peripheral is configured, so that the normal program execution in the MCU goes to data acquisition routine when a timer, which controls the data timing, expires. On the downside, the microcontroller must first know the time with which to set the timer.

Known these two parameters, each time the timer expires, the data acquisition routine activates the corresponding transducer (slave module) for the appropriate time. During that time, the sensor transmits a data frame whose structure is shown in Figure 3(a). In the data frame shown in Figure 3(a), various fields can be distinguished

The first byte is dedicated to the Interface ID as described above. The next two bytes are used as Sensor ID. This field could be suppressed in applications where data were locally processed into the hardware; however, as we said, in devicedriven applications processing is also done in data analytics components and the Sensor ID field is needed. In order to simplify the processing, the Sensor ID is divided into two parts: the first byte indicates the sensor type and the second byte indicates the specific sensor of that class which generated the frame (if various are included). Considering a CPS as a local system, the Sensor ID assignation process can be performed by users according to needs of their system (which may vary depending on the application). However, it must be ensure that each Sensor ID is unique within each CPS, in order to guarantee a proper system performing. Finally, the payload may be as long as needed.

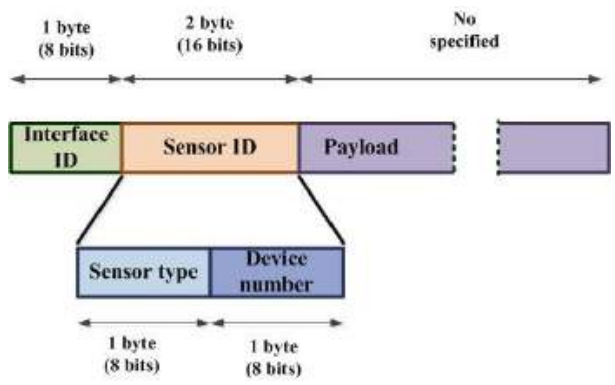

(a)

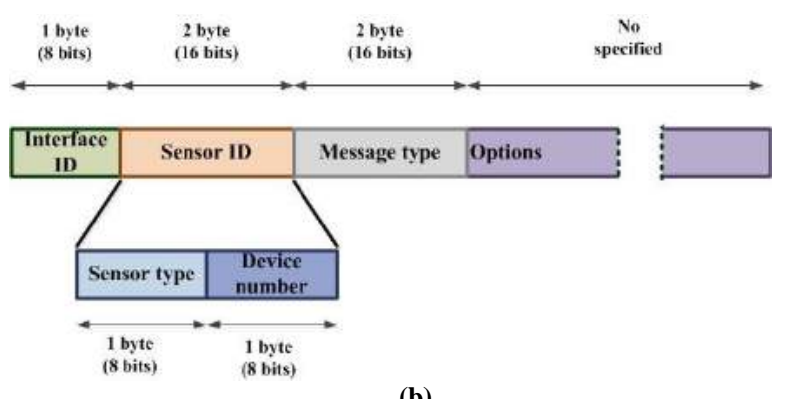

(b)

Figure 3. (a) Data frame structure (b) Control frame structure

Once described the data frame, in Figure 3(b) we present the control frame structure. As can be seen, some fields are common to the data frame (interface and sensor ID) but two new fields are considered. The third byte (message type) represents the control message that is transmitted, and the remaining bytes contain the message options (as much as needed). More than 65500 control messages can be defined (we reserved 2 bytes for that field), which exceeds the needs of the vast majority of applications.

When the system is powered, the microcontroller requests the description from all slaves. For that, it activates the appropriate "slave select" signal and sends a Description request message through the SPI interface (in the case of having only a SPI port, it can be multiplexed using any of the available techniques [15]). We must note that control messages have a specific length, so the master always knows how much time to activate the slave select signal. After requesting one slave, the master looks for the answer. If the slave sends a Description response message, the master records that SPI port as occupied and transmits a Description $A C K$ message. If no answer is received, the port is discarded. Table I details the messages cited above. 
TABLE I. CONTROL MESSAGES DESCRIPTIONS

\begin{tabular}{|l|l|}
\hline $\begin{array}{c}\text { Message } \\
\text { name }\end{array}$ & \multicolumn{1}{|c|}{ Options } \\
\hline $\begin{array}{l}\text { Description } \\
\text { request }\end{array}$ & $\begin{array}{l}\text { Only one field of 16 bits (called "clock } \\
\text { frequency"), containing de clock frequency of the } \\
\text { processor (expressed in MHz) }\end{array}$ \\
\hline $\begin{array}{l}\text { Description } \\
\text { response }\end{array}$ & $\begin{array}{l}\text { Two fields. The first one of 16 bits (called "data } \\
\text { length") containing the length of data produced by } \\
\text { the sensor (in bits). The second one of 16 bits } \\
\text { (called "division factor"), containing the integer } \\
\text { number for which the clock frequency of the } \\
\text { processor must be divided to obtain the reference } \\
\text { frequency of the sensor. }\end{array}$ \\
\hline $\begin{array}{l}\text { Description } \\
\text { ACK }\end{array}$ & Not used \\
\hline
\end{tabular}

Once all ports have been inspected, the microcontroller runs Algorithm 1. In this configuration process transducers are listed, associated with the physical port to which they are connected and grouped along their period (expressed as a multiple of the minimum period existing in the platform) in a hash table. A two-dimensional array is also generated to be used as decreasing counter in the interrupt service routine.

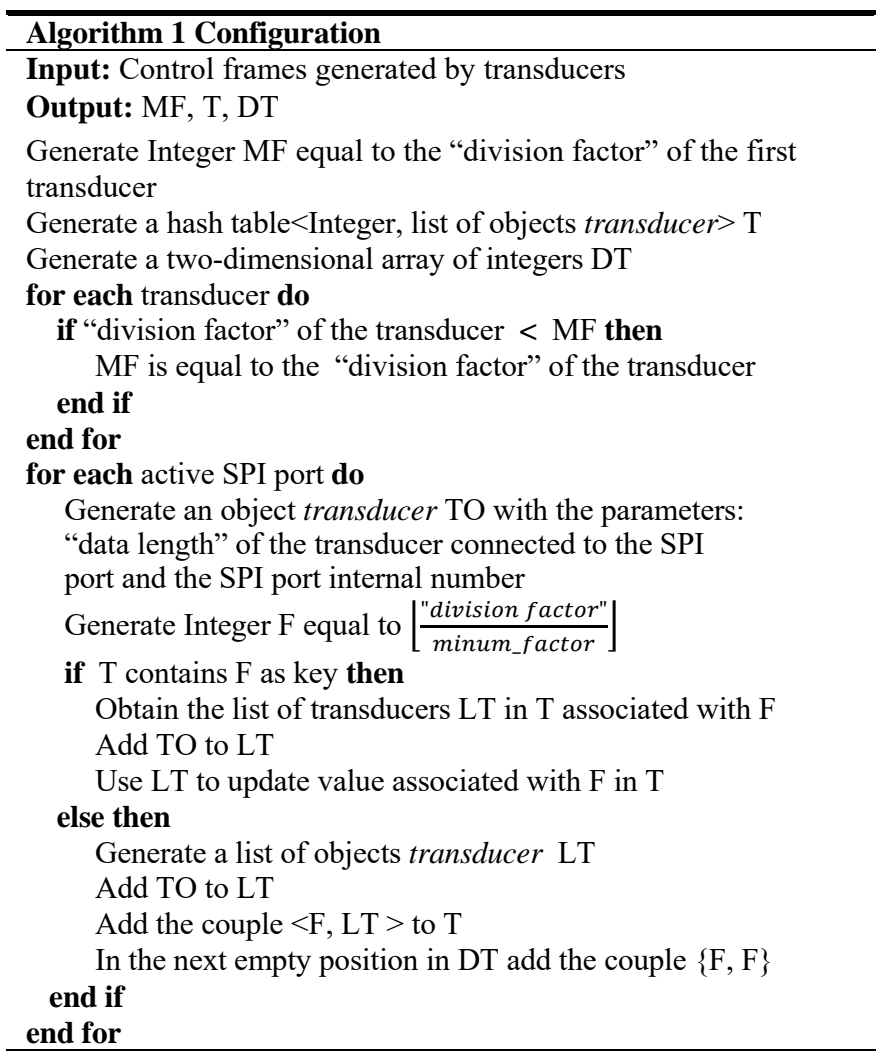

Then, a software interruption is configured to become active each " $M F$ " clock cycles. The interrupt service routine, which allows acquiring data from transducers connected to the hardware controller, is described in Algorithm 2. Briefly, each time an entry in $D T$ arrives at zero; all the transducers associated with the period stored in that entry are read.

With this architecture, great processing capabilities are not required to controllers, as all the included operations can easily be implemented in assembly code (although some structures, such as the hash tables, must be properly adapted). Moreover, there are no limits in the use of different types of sensors; and, overall, data and code storage capacities may be much more reduced. For example, maintaining all the information about 10 sensors with IEEE 1451 TEDSs requires, in average, around 900 bytes; with the described solution, in average, only 150 bytes are needed (approximately).

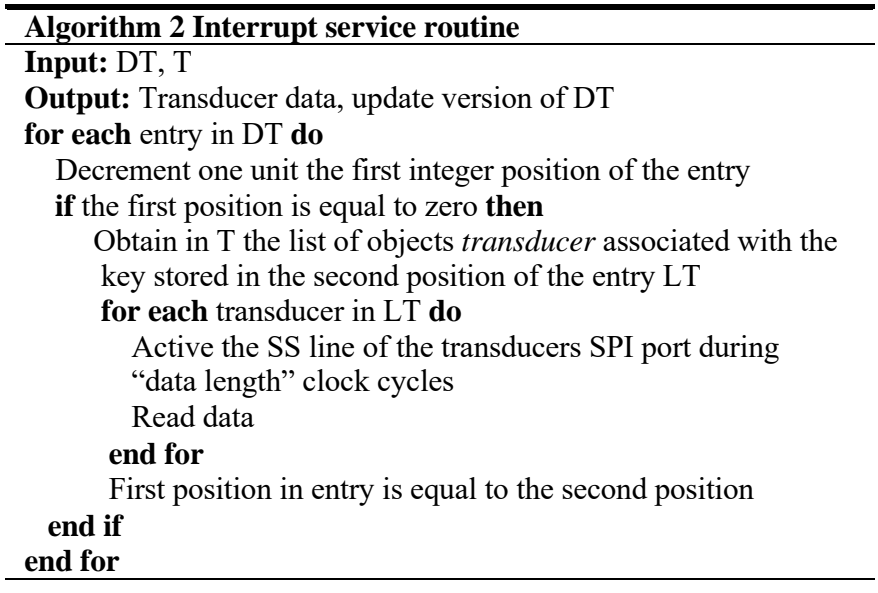

\section{B. Prototype implementation}

The proposed technology was implemented, for first time, in a collection of three sensors (two three-axial accelerometers and a RFID reader [16]) connected to a QFN microcontroller [17], acting as hardware controller. On the other hand, data analytics components were implemented in a MATLAB application, deployed in a personal computer. In this first prototype, these components collected the received data from hardware platform and stored them in a numerical matrix. Both layers (hardware and data analytics) were connected through a wireless interface (specifically a ZigBee interface [18]). The resulting hardware platform can be seen in Figure 4.

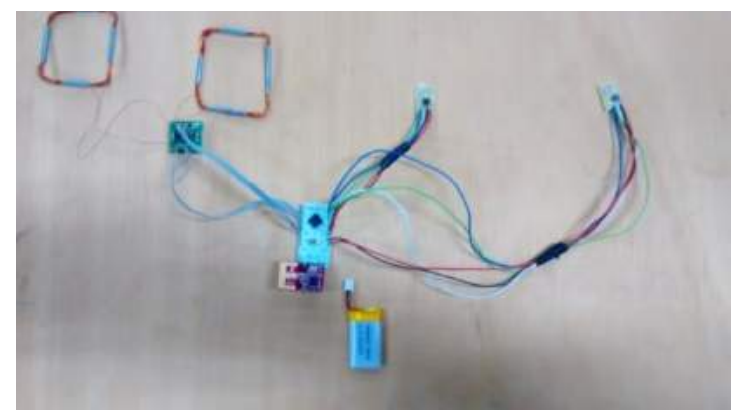

Figure 4. Hardware platform implemented

The described algorithms were codified using assembly code, where the particular values of Interface ID, Sensor ID 
and Message type employed in the implementation can be seen in Table II.

TABLE II. CONTROL MESSAGES DESCRIPTIONS

\begin{tabular}{|c|c|c|}
\hline \multirow{2}{*}{ Field } & Value \\
\hline \multirow{3}{*}{ Interface ID } & Data & 0xDD \\
\cline { 2 - 3 } & Control & 0xCC \\
\hline \multirow{3}{*}{ Sensor ID } & Accelerometers & 0x0101 \\
& RFID & 0x0102 \\
\cline { 2 - 3 } & Description request & 0x0201 \\
\cline { 2 - 3 } Message type & Description response & 0xAA \\
\cline { 2 - 3 } & Description ACK & 0xAB \\
\cline { 2 - 3 } & & $0 x A C$ \\
\hline
\end{tabular}

\section{EXPERIMENTAL VALIDATION}

An experimental validation was designed in order to validate the proposal and analyze the performance of the proposed technology. The experimental validation was divided in two different experiments.

In the first experiment, transducers were placed in a controlled situation, so we can predict their outputs [19]. A MATLAB application received data sent by the hardware controller, and it compared the expected values to which it acquired. In that way, we validated both configuration algorithm and data acquisition at hardware level. Moreover, we validated the usefulness of the frames' headers (i.e. the Sensor ID) in the processing step by the data analytics components. In total there were 144 records per sensor analyzed.

In this experiment, accelerometers were placed with the intrinsic Z-axis aligned with the vertical line. Then, the expected output (expressed as G-forces) was $\{x=0 g, y=$ $0 g, z=1 g\}$. Considering that accelerometers are a really precise sensors (sensitive, for example, to vibrations), it is probable that the real obtained output vary around the expected value with a certain tolerance. Therefore, we considered valid all data in a margin of $\pm 10 \%$ around the expected value. Over the RFID reader was placed a tag, so the expected output was the tag's ID.

In the second experiment, we turned off the hardware platform, and then we turned it on. A MATLAB application (see Figure 5) showed dynamically in a graphic interface (GUI) data received from hardware controller. In the moment of turning on the hardware platform, we ran a timer which was stopped when the first valid datum was showed in the MATLAB GUI. In that way, we obtained an estimation of the time needed to execute the initial configuration of the hardware controller. The experiment was repeated twelve times.

This experiment was performed using a special environment, where a software timer is configured in the following way: it starts counting time when a special line gets powered. This line was connected to the power supply of the hardware platform. Later, it stops counting time when the first valid datum is received and processed. In order to verify data properly the same hardware configuration done in the first experiment was maintained.

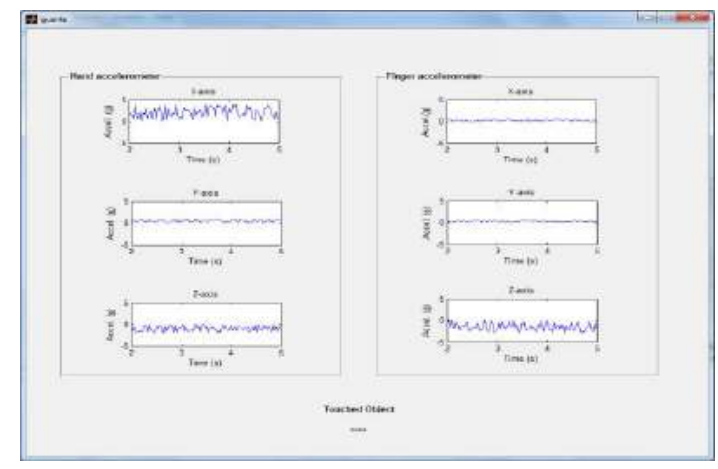

Figure 5. Capture of the MATLAB application for data processing in the second experiment

\section{RESUlts}

In this section we present the results obtained in the experimental validation, the results are presented according to the experiments proposed in the previous section.

\section{A. First experiment}

Experts analyzed every single record about the data received from the hardware controller using semi-automatic techniques. The results of this analysis are shown in Table III

TABLE III. CONTROL MESSAGES DESCRIPTIONS

\begin{tabular}{|c|c|c|c|}
\hline Sensor & Correct data & Incorrect data & Hit rate \\
\hline Accelerometer \#1 & 137 & 7 & $95 \%$ \\
\hline Accelerometer \#2 & 139 & 5 & $96.5 \%$ \\
\hline RFID reader & 142 & 2 & $98.6 \%$ \\
\hline Total & 418 & 14 & $96.8 \%$ \\
\hline
\end{tabular}

As can be seen, the consolidated hit rate is near $97 \%$, being the hit rate for all sensors above the $95 \%$. In fact, the hit rate increases when considering transducers with lower frequency (i.e. sensors which produces data in a slower rate). In that way, RFID reader presents a hit rate $4 \%$ higher than presented by the first accelerometer.

The explanation of this phenomenon is related to the transducer discovery process. Sensors with high frequencies (such as accelerometers) could be read before the discovery process is complete, so there exists a convergence period during which data received could be corrupted. This problem could be easily solved by adjusting a security temporal slot in the data analytics components.

\section{B. Second experiment}

Twelve realizations of the second experiment were done. A normalized histogram, expressing the time needed to 
receive a valid datum from the hardware controller once the hardware platform get powered (convergence time) is shown in Figure 6.

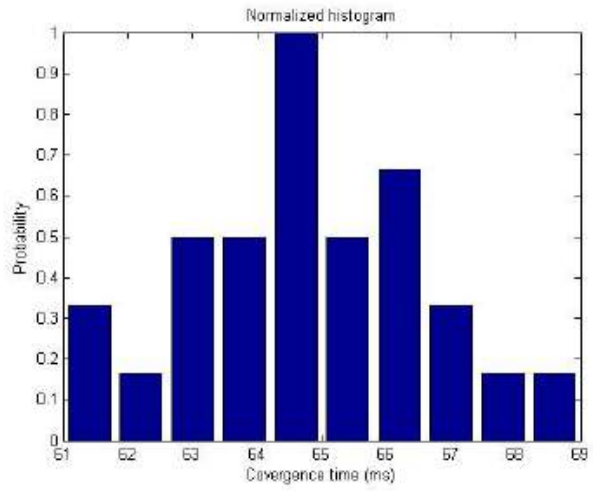

Figure 6. Normalized histogram of the convergence time

As can be seen, the dispersion is quite low (around a 5\% over the medium value), being (in medium) the convergence time around $65 \mathrm{~ms}$. This time implies that, at least, one datum from all sensors with a frequency higher than $15 \mathrm{~Hz}$ is lost. Of course, as the sensor rate increases, the number of lost data will also increase.

\section{CONCLUSIONS}

In view of the results presented in the previous section, some reflections may be drawn:

The proposed solution is valid to implement high performance plug-and-play transducers. Device-driven applications based on CPSs may implement this technology in order to improve the integration level and ease the automatic system configuration. Particularly, it must be remarked the simple algorithms needed to execute the configuration and the support provided by the Sensor ID field to data analytics components.

Moreover, the proposed plug-and-play technology is adequate to be implemented in scenarios where IT infrastructure must be seamless integrated in the environment. Specially, in those cases in which components must be miniaturized and their capacities reduced. Some important particular cases of this scenario are wearable devices and Ambient Intelligence (AmI) environments.

Besides, biomedical sensors, which produce data at frequencies around $50 \mathrm{~Hz}$, must implement a security temporal slot in order to not consider erroneous values due to the convergence process. In application of human monitoring this consideration might be important.

Finally, using the Sensor ID field, data analytics components may execute complex algorithms in an easier way. Thus, applications such as emotions detection could be implemented over hardware platforms using the proposed technology.

\section{ACKNOWLEDGMENT}

The research leading to these results has received funding from the Ministry of Economy and Competitiveness through SEMOLA project (TEC2015-68284-R) and from the
Autonomous Region of Madrid through MOSI-AGIL-CM project (grant P2013/ICE-3019, co-funded by EU Structural Funds FSE and FEDER).

\section{REFERENCES}

[1] J. Wan. "From machine-to-machine communications towards cyberphysical systems". Computer Science and Information Systems, 2013, vol. 10 , no 3 , p. 1105-1128.

[2] E.A Lee. "Cyber-physical systems-Are computing foundations adequate?" In NSF Workshop on Cyber-Physical Systems: Research Motivation, Techniques and Roadmap, Austin, TX, USA, 16-17 October 2006.

[3] National Institute of Standards and Technology. Cyber-Physical Systems Homepage. Available online: http://www.nist.gov/cps/ (accessed on 25 February 2016).

[4] B. Bordel Sánchez, R. Alcarria, D. Martín and T. Robles "TF4SM: A Framework for Developing Traceability Solutions in Small Manufacturing Companies". Sensors 2015, 15, 29478-29510.

[5] M. Conti. "Looking ahead in pervasive computing: Challenges and opportunities in the era of cyber-physical convergence". Pervasive and Mobile Computing, 2012, vol. 8, no 1, p. 2-21.

[6] B. Bordel, R. Alcarria, M. Pérez-Jiménez, T. Robles, D. Martín, and D. Sánchez de Rivera. "Building Smart Adaptable Cyber-Physical Systems: Definitions, Classification and Elements". In Ubiquitous Computing and Ambient Intelligence (pp. 144-149). Springer International Publishing.

[7] E. Y. Song and K. Lee "Understanding IEEE 1451-Networked smart transducer interface standard-What is a smart transducer?" IEEE Instrumentation \& Measurement Magazine, 2008, vol. 11, no 2, p. 1117.

[8] T. Falck "Plug'n play simplicity for wireless medical body sensors". Mobile Networks and Applications, 2007, vol. 12, no 2-3, p. 143-153.

[9] T. Robles, B. Bordel, R. Alcarria and D. Martín "Mobile wireless sensor networks: Modeling and analysis of three-dimensional scenarios and neighbor discovery in mobile data collection", Ad Hoc \& Sensor Wireless Networks 2016 (In press)

[10] J. Yao, R. Schmitz, S. A. Warren. "A wearable point-of-care system for home use that incorporates plug-and-play and wireless standards". IEEE Transactions on Information Technology in Biomedicine, 2005, vol. 9, no 3, p. 363-371.

[11] A. Bröring "Semantically-enabled sensor plug \& play for the sensor web". Sensors, 2011, vol. 11, no 8, p. 7568-7605.

[12] K. Lee. "IEEE 1451: A standard in support of smart transducer networking". In Proceedings of the 17th Conference on Instrumentation and Measurement Technology, 2000. p. 525-528.

[13] M. Dunbar. "Plug-and-play sensors in wireless networks". IEEE Instrumentation \& Measurement Magazine, 2001, vol. 4, no 1, p. 1923.

[14] D. Potter "Smart plug and play sensors". IEEE Instrumentation \& Measurement Magazine, 2002, vol. 5, no 1, p. 28-30.

[15] F. Miesterfeld. "Method for serial peripheral interface (SPI) in a serial data bus". U.S. Patent No 4,739,324, 19 Abr. 1988.

[16] RDM8800 NFC reader datasheet. Available online: http://wiki.iteadstudio.com/RDM8800 (accessed on 25 February 2016)

[17] PIC16(L)F1825/1829 Data Sheet. Available online: http://ww1.microchip.com/downloads/en/DeviceDoc/41440B.pdf (accessed on 25 February 2016)

[18] MRF24J40MA Data Sheet. Available online: http://ww1.microchip.com/downloads/en/DeviceDoc/70329b.pdf (accessed on 25 February 2016)

[19] R. Alcarria, T. Robles, A. Morales, D. López-de-Ipiña, and U. Aguilera, "Enabling flexible and continuous capability invocation in mobile prosumer environments", 2012. Sensors, 12(7), 8930-8954. 


\title{
Process execution in humanized Cyber-Physical Systems: soft processes
}

\author{
Borja Bordel $^{1,2}$, Ramón Alcarria ${ }^{1,2}$, Antonio Jara ${ }^{1}$ \\ ${ }^{1}$ Institute of Information Systems. University of Applied Sciences Western Switzerland (HES-SO) \\ Sierre, Vallais, (Switzerland) \\ ${ }^{2}$ On leave from Universidad Politécnica de Madrid (UPM). \\ Madrid (España) \\ bbordel@dit.upm.es; ramon.alcarria@upm.es; jara@ieee.org
}

\begin{abstract}
One of the most common applications for CyberPhysical Systems (CPS) is process execution. Nowadays, different description languages are employed in order to define, command and control the execution of processes using the elements making up a CPS. However, this approach creates rigid (or hard) processes, which are only valid in certain scenarios. The inclusion of legacy systems, new Industry 4.0 devices and, overall, humans as part of CPS, has caused the appearance of new paradigms which cannot be addressed with traditional execution techniques. Therefore, in this paper a new type of (soft) processes, designed to be employed in humanized CPS, is presented. The proposal is based on the concept of "strain", which is used to determine if a deformable process has been correctly executed. Finally an experimental validation is provided in order to evaluate the performance of the proposed technology.
\end{abstract}

Cyber-Physiscal Systems; soft processes; process execution; humanized computing; process strain

\section{INTRODUCTION}

Cyber-Physical Systems (CPS) are traditionally understood as integrations of computation with physical processes. Embedded computers and networks monitor and control the physical processes, usually with feedback loops where physical processes affect computations and vice versa [1]. However, recent proposals deal with integrating new elements into the CPS architecture in order to extend the range of possible applications. This is the case of Industry 4.0 systems, where a new generation of industrial embedded devices is considered [2]; the new networked control systems, which integrates legacy infrastructures [3]; and, overall, the humanized CPS, which include humans as services providers in the architecture's lowest layer, together with hardware devices [4].

In this new context, the science of CPS must take the next step. Different articles have studied the applications this new ecosystem of engineered solutions enables [5]. One of the most commonly cited is process execution. In fact, totally automated process execution systems are the basis of many of the most interesting applications today, such as Ambient Intelligence [6]. This, however, is a particularly challenging objective, as many different types of processes may be executed on CPS and, even, in a same application scenario. Therefore, most proposals on this topic are based on the simplest scenario: a CPS made of hardware devices executing a certain workflow, defined in any process description language (for example,
BPEL [7] or YAWL [8]). In order to determine if the proposed workflow has been correctly executed, a collection of user objectives or rules is usually defined. These objectives are, then, employed as strict (or hard) conditions which cannot be contradicted in any way.

In order to consider the possible variations due to the stochastic variables of the physical world, a certain tolerance margin is usually defined. However, this approach presents various conflicting situations. For example, is a workflow correctly executed if all conditions are met in the limit of the tolerance margin? In general, a global measure about the difference between the described process and the tasks finally executed is required.

Hard conditions, moreover, are inadequate when humans are considered. People, contrary to the deterministic behavior of devices, tend to be very diverse, and boolean evaluations cannot be designed to consider all possible cases. If only one general condition is defined, many wrong situations would be allowed. On the other hand, it is impossible to predict all the possible correct ways in which people perform a certain process. Then, a new way to define and understand processes in humanized CPS is required.

Therefore, the objective of this paper is to adapt a new concept of process (called soft processes) to (humanized) Cyber-Physical Systems. These processes are characterized by a strain function, which generalizes the traditional idea of tolerance, and allows determining if the execution of a collection of tasks matches a certain previously defined workflow. By means of a new representation of processes as deformable solid bodies, a strain tensor field and machine learning solutions, it is proposed a mathematical formalization which supports the presented idea. Finally, an experimental validation is conducted, in order to evaluate the improvement in the decision-making. Using our proposal, more than $93 \%$ of correct processes in a humanized CPS are correctly considered, compared to $70 \%$ obtained in traditional deployments.

The rest of the paper is organized as follows. Section 2 describes the state of the art on soft processes and process execution in CPS. Section 3 presents the mathematical formalization of the proposed technology, based on the concept of strain. Section 4 and Section 5 proposes an experimental validation and presents its results. Finally Section 6 concludes the paper. 


\section{STATE OF THE ART}

The concept of "soft process" has been used in many fields in order to represent a relaxation in the characteristics of the existing processes. For example, the term was used in manufacturing to refer the integration of statistics into production systems [9] and, in physics, to represent nonperturbative reactions [10].

In a more technological area, the concept has been employed to refer coding/decoding based on diffused information in cooperative communications [11]. Finally, the idea has been employed in automatons, in order to create workflows with adaptive timing conditions [12], by means of (for example) utility functions.

These ideas, however, have not been applied to CPS. Process execution in CPS is still based on the definition of an executable model in any workflow description language, which is accompanied by a collection of user objectives used as hard conditions of success [13]. If any of the proposed conditions is not strictly met, then the execution is considered failed.

With this approach, different process execution systems based on CPS paradigm have been proposed. Some of them consist of finite state machines controlling the process being executed by low-level devices [2]. Others try to adapt workflow description languages (such as BPEL4WS) to incorporate user objectives and be able to use them in CyberPhysical Systems [14]. Frameworks and instruments to design processes using graphic languages (UML or BPMN, for example) have been also presented [15].

Finally, some proposals deal with the idea of creating a vertical process execution system, being able to include both humans and hardware devices [16]. These proposals, however, do not address the basic problem (human behavior does not match a hard definition of processes), and usually try to develop brokers which hide the complexity of human behavior offering a hardware-like interface.

Contrary to these previous works, our technology is specifically designed to be applied in humanized CyberPhysical Systems, so it is unnecessary to adapt or develop new components in order to homogenize human and hardware behavior. Moreover, our proposal is agnostic in respect to the implementation architecture or technology, so it can be deployed in any existing system.

\section{STRAIN OF A PROCESS: SOFT AND HARD PROCESSES}

Current process descriptions, focused on CPS or not, use to include a collection of user objectives which executed tasks should met in order to be considered valid (see Figure 1). Processes which must fulfill these conditions in a hard way are called hard processes. In this type of processes, it is made a boolean decision about executions, and no variation in respect to the created model (however small) is allowed.

Rigid processes make the requirements about executions more flexible. In these processes, (small) tolerance margins are usually defined, and results are considered valid if belong to a certain interval around the real expected value. These tolerance margins are usually given as relative variations (1) or absolute normalized values (2).

$$
\begin{gathered}
e=\frac{s_{\text {max }}-s_{\text {ref }}}{s_{\text {ref }}}=\frac{\Delta S}{s_{\text {ref }}} \\
\lambda=\frac{s_{\text {max }}}{s_{\text {ref }}}=e+1
\end{gathered}
$$

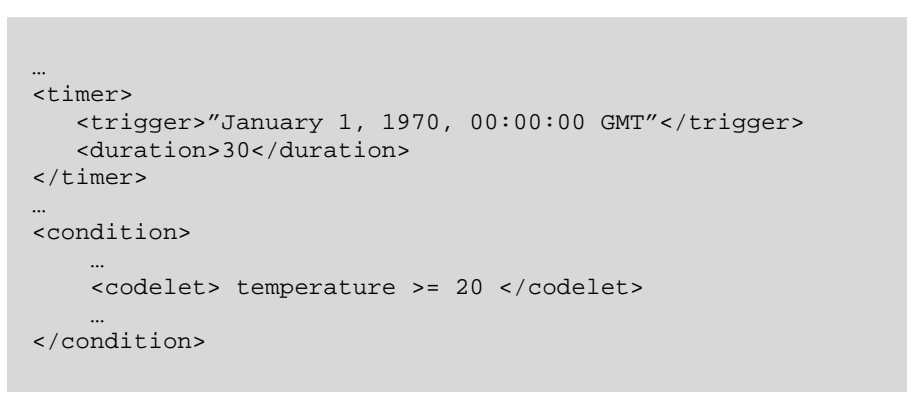

Figure 1. Fragment of a process description (YAWL code) describing user rules

Previous expressions, however, are only useful if tolerance margins are small (i.e. $|e| \ll 1 \Rightarrow|e|<0.1$ ). If bigger values want to be considered, or additional effects (not presentable with tolerances) should be admitted it is needed a new theory and new instruments. Processes considering any of these complex effects are called soft process or deformable processes (see Figure 2).

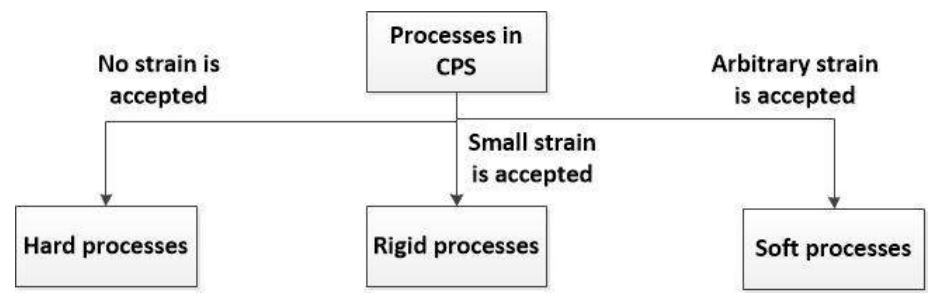

Figure 2. Classification of processes on a CPS, dependeing on the admissible strain

In order to represent complex admissible variations in the process execution in respect to the created model, it is defined the concept of strain of a process.

The strain of a process, $\varepsilon$, is the change which experiments a workflow when it is executed, in respect to the designed model. It is cause by internal efforts (i.e. it exists a modification in the task configuration). In that sense, a temporal movement of the entire workflow (for example) does not generate any strain.

In order to study the strain a process has suffered during its execution, it is useful to represents processes as deformable solid bodies in a Cartesian system. In order to do that, a certain workflow $W$ it is understood as a collection of Cartesian points, representing each one a task (3).

$$
W=\left\{t_{i}, i=1, \ldots, N\right\}
$$

Being $N$ the number of tasks in the workflow $W$. Now, each task (point) it has associated a numerical position vector where each component represents the value of one condition or restriction about this task (execution order, start time, duration, etc.). As different tasks may present different restrictions, vectors must consider a dimension for every 
possible condition, making a total of $M$ dimensions (4). If a certain condition (i.e. component of the corresponding vector) is not relevant for a certain task, it would be initialized with an arbitrary value.

$$
\vec{x}=\overrightarrow{t_{l} O}=\left(x_{1}, x_{2}, \ldots, x_{M}\right)
$$

Then, the model of a process may be drawn in a $M$ dimensional Cartesian system, as seen on Figure 3.

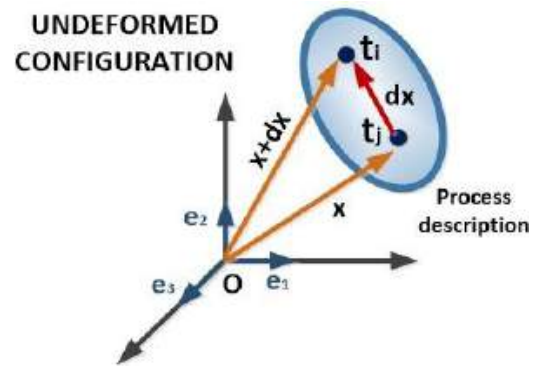

Figure 3. Undeformed configuration of a process in a M-dimensional space

In this context, the execution of a process description may be represented by a mathematical application $T_{D}(5)$, which generates a deformed process $W^{*}$ (see Figure 4).

$$
T_{D}: W \subset \mathbb{R}^{M} \rightarrow W^{*} \subset \mathbb{R}^{M}
$$

The proposed solution tries to evaluate the produced strain, and determine if it beats the maximum admissible threshold. Two different approaches are considered. In the first one, conditions associated with tasks are supposed to be independent. In the second one, a more realistic scenario is considered, and conditions show interdependencies.

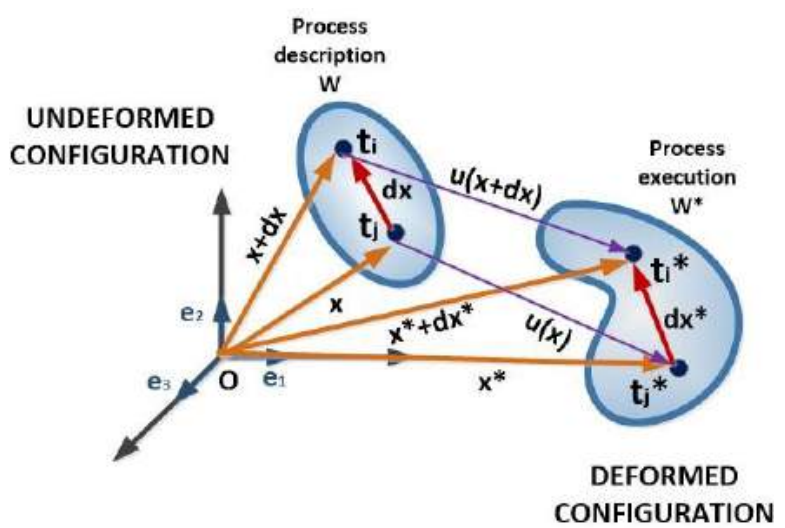

Figure 4. Deformation of a process in a M-dimensional space

\section{A. Conditions as independent variables}

In the case of existing multiple conditions, but independent (i.e. modifications in one of them do not imply modifications in the others), the study of the strain of the process gets greatly simplified (as each condition may be analyzed separately).

As analyzed in previous works about strain in mechanics [17], the expression employed to estimate the strain in any dimension of the work space (i.e. in any considered condition) depends on the magnitude order of the produced deformation. In particular, it is defined the stretching of a condition (6) as the reference magnitude to determine the most adequate expression. Table I shows the most representative strain calculation methods

$$
\lambda=\frac{S_{\text {execution }}}{S_{\text {definition }}}
$$

TABLE I. DIFFERENT STRAIN CALCULATION FUNCTIONS

\begin{tabular}{|c|l|c|}
\hline \multirow{2}{*}{ Rotation } & \multicolumn{2}{|c|}{ Strain } \\
\cline { 2 - 3 } & Large $\left(\left|\varepsilon_{U}\right|>0.1\right)$ & Small \\
\hline \multirow{2}{*}{ Large } & $\begin{array}{c}\text { Hencky strain } \\
\varepsilon_{H}=\ln (\lambda)\end{array}$ & $\begin{array}{c}\text { Green strain } \\
\varepsilon_{G}=\frac{1}{2}\left(\lambda^{2}-1\right) \\
\text { Almasi strain }\end{array}$ \\
& & $\varepsilon_{A}=\frac{1}{2}\left(1-\frac{1}{\lambda^{2}}\right)$ \\
\hline Small (or zero) & $\begin{array}{l}\text { Ad hoc logarithmic } \\
\text { definitions }\end{array}$ & $\begin{array}{c}\text { Unitary strain } \\
\varepsilon_{U}=\lambda-1\end{array}$ \\
\hline
\end{tabular}

In Table I a new concept is shown: the rotation of a process. Rotation is a property of cyclic processes, whose tasks (sometimes) maybe executed in a different order than originally planned, and the workflow execution is still valid. This phenomenon is usually not allowed in CPS, but humans continuously perform this kind of actions, so we are considering it in our proposal. However, if desired, it may be not taken into account and the rest of the proposed framework remains invariant.

Then, as can be seen, for each condition in every task, it must be evaluated the value of the unitary strain. The definition of this quantity, as can be seen on Table I and Eq. (1) matches the traditional idea of relative variation. As, commonly, processes are considered rigid $\left(\left|\varepsilon_{U}\right|<0.1\right)$ and no rotation is allowed, this definition is the most used nowadays (at this point our theory meets perfectly the existing works on this topic). If this unitary strain is small, and rotation is also small, this result is final. If not, considering the obtained value, it must be calculated the adequate expression. In the case of existing a small rotation and a large strain, ad hoc logarithmic definitions are required. Various works on mechanics have studied this point [18]. Nevertheless, in our case, it could be also employed the Hencky strain, if no more specific expression is available.

As conditions are supposed to be independent, once calculated all the strain functions, it will be obtained a collection of values $d^{t_{i}}$ for each task (7). Conditions initialized with arbitrary values do not change due to the workflow execution, so strain has a value equal to zero and it will not affect the future calculations.

$$
d^{t_{i}}=\left\{\varepsilon_{1}^{t_{i}}, \varepsilon_{2}^{t_{i}}, \ldots, \varepsilon_{M}^{t_{i}}\right\}
$$

Then, in order to evaluate the global strain $\varepsilon^{t_{i}}$ of a task $t_{i}$, all obtained partial values $\varepsilon_{i}^{t_{i}}$ must be aggregated. This action must be performed by means of a Euclidean module 
(distance), or through a simple addition among other possibilities. In this first proposal, we are considering the natural addition of all amounts (8).

$$
\varepsilon^{t_{i}}=\sum_{j=1}^{M} \varepsilon_{j}^{t_{i}}
$$

Finally, at this point, it is necessary to evaluate the global strain of the workflow by aggregating the strain of each task. The same instruments employed in the previous phase could be used in this new case (9).

$$
\varepsilon^{W^{*}}=\sum_{i} \varepsilon^{t_{i}}
$$

As a result of this method, finally, a collection of strain estimations will be obtained (related to conditions, tasks and the entire workflow). In order to decide if a certain execution is correct or not, then, it has to be evaluated if the produced deformation $\varepsilon^{W^{*}}$ beats the maximum allowed threshold $\varepsilon^{t h}$.

This approach presents various advantages. As conditions are supposed to be independent, each one may be studied separately, and strain may be easily calculated from the process model and the performed execution. However, a certain error is committed (the obtained strain is lower than which really produced). In order to correct this effect, conditions are considered to show interdependencies.

\section{B. Conditions as non- independent variables}

In general, in a workflow, any deformation produced in a certain variable or condition affects other tasks in the process. For example, if the start time suffers a delay, the measured temperature will vary (as it changes with time). In order to represent the strain of a workflow, when various components must be considered, it is necessary a tensor field instead of a numerical value.

For any arbitrary deformation, it is defined the strain tensor $F$ as the Jacobian matrix of the application $T_{D}(10)$.

$$
F=\nabla T_{D}=\left(\begin{array}{ccc}
\frac{\partial x_{1}^{*}}{\partial x_{1}} & \cdots & \frac{\partial x_{1}^{*}}{\partial x_{M}} \\
\frac{\partial x_{M}^{*}}{\partial x_{1}} & \cdots & \frac{\partial x_{M}^{*}}{\partial x_{M}}
\end{array}\right)
$$

This tensor disagrees the produced deformation of a variable in various components, depending on the cause: intrinsic causes or extrinsic factors (i.e. deformations in other variables). As Eq. (10) is a very unpractical expression, the strain tensor $F^{t_{i}}$ of the task $t_{i}$ is usually calculated with a secondary procedure (11). Vectors are referred to Fig. 4.

$$
\begin{gathered}
F^{t_{i}}=\left(\begin{array}{ccc}
\varepsilon_{11}^{t_{i}} & \ldots & \varepsilon_{1 M}^{t_{i}} \\
\varepsilon_{M 1}^{t_{i}} & \ldots & \varepsilon_{M M}^{t_{i}}
\end{array}\right) \\
\varepsilon_{i j}^{t_{i}}=\frac{1}{2}\left(\frac{\partial u_{i}}{\partial x_{j}}+\frac{\partial u_{j}}{\partial x_{i}}+\sum_{k=1}^{M} \frac{\partial u_{k}}{\partial x_{j}} \frac{\partial u_{k}}{\partial x_{i}}\right)
\end{gathered}
$$

In the case of small deformations, some approximation could be done, although (in general) all terms must be considered.

Contrary to the strain values obtained when conditions are considered independent, the strain tensor it is not able to be directly obtained from the process model and/or its executions. Therefore, it is required a machine-learning algorithm which, with time, may "memorize" the behavior of the workflow and obtain the different components in the tensor strain. For example, each component could be supposed to be a certain percentage of the total obtained deformation and employing a genetic algorithm to adapt these percentages as more information about executions is obtained. However, in this initial work we are not discussing in detail this point, as future papers will deal with this topic.

Once obtained the tensor field, all the strain tensors should be aggregated in order to finally obtain a global measure about the workflow. As in the previous case, the addition of all obtained tensors is a possible solution (12).

$$
F^{W^{*}}=\sum_{i} F^{t_{i}}
$$

As valuating the maximum admitted strain using tensors is a complicated task, usually (at the end) it is obtained a numerical estimation from the tensor, for example, considering the module (13).

$$
\varepsilon^{W^{*}}=\left\|F^{W^{*}}\right\|
$$

The same threshold defined in the previous case could be applied then here.

\section{EXPERIMENTAL VALIDATION}

In order to evaluate the proposed technology an experimental validation was planned and carried out. A process execution system based on the CPS paradigm was deployed at the Technical University of Madrid and various experiments were performed.

The deployed process execution system consisted of a wearable device (a smart glove) based on RFID technology, a worktable provided with RFID readers [19] and various sensitization nodes [20]. Components were connected with a central server, where the execution engine is hosted and data about the executions is collected, by means of Bluetooth technology. The process description is based on finite state machines and user objectives, defined as temporal linear logic rules. A detailed explanation about the deployed system, how it works and the different manners in which states are visited may be found in previous works [13].

Ten people participated in our experimentation, performing different processes about Activities of Daily Living (ADL) [21]. In particular six processes were selected to be developed (see Table II). People were divided into two different groups (two different humanized CPS were organized). The first group executed processes considering a hard definition of tasks (traditional approach) [13]. The second group performed the activities considering a soft definition of processes (our proposal). 
TABLE II. TESTED PROCESSES

\begin{tabular}{|c|l|}
\hline Activity number & \multicolumn{1}{|c|}{ Activity description } \\
\hline 1 & Use a microwave \\
\hline 2 & Adjust the thermostat \\
\hline 3 & $\begin{array}{l}\text { Turn on TV, watch it for a few } \\
\text { minutes, turn it off }\end{array}$ \\
\hline 4 & Take some pills \\
\hline 5 & Phone to get the horoscope \\
\hline 6 & Make yourself a cup of coffee \\
\hline
\end{tabular}

With this infrastructure two different experiments were carried out.

The first experiment compared the obtained results (percentage of executions correctly considered) using a traditional approach (hard definition of processes) and which obtained using our proposal. During this experiment, our solution was configured to suppose conditions are independent variables.

The second experiment evaluated the difference between considering conditions as independent variables or not (in this case, a genetic algorithm was employed to obtain the different components in the strain value). In particular it is evaluated the improvement in the obtained results, depending on the number of executions performed.

\section{RESULTS}

Figure 5 shows the results of the first experiment. In this figure the percentage of executions correctly considered when using the proposed technology and a traditional approach are compared.

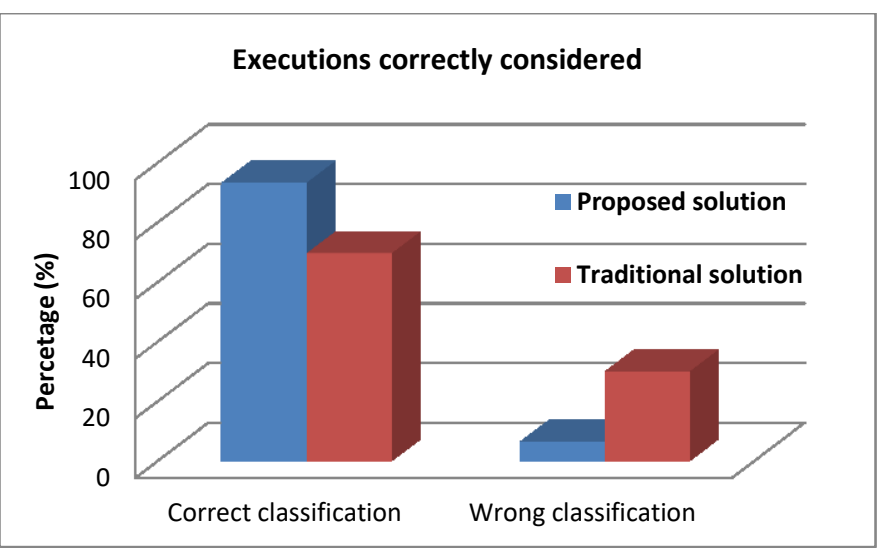

Figure 5. Executions correctly considered (first experiment)

As can be seen, our solution improves in a $30 \%$ the performing of traditional technologies, by considering the concept of strain of a process execution. In particular, around $93 \%$ of correct executions are catalogued as valid by the proposed technology, contrary to the lower $70 \%$ reached by traditional solutions. On the other hand, this result is the natural tendency when hard conditions are relaxed, as more cases than before as considered correct. It is important to note, then, that false positives are not enough numerous to compensate this improvement, so the proposed technology present a clear advantage over traditional approaches.

Nevertheless, despite these good global results, the proposed solution presents a worse behavior in certain particular aspects. Specifically, see Figure 6, it presents a worse false positive rate compared to traditional technologies.

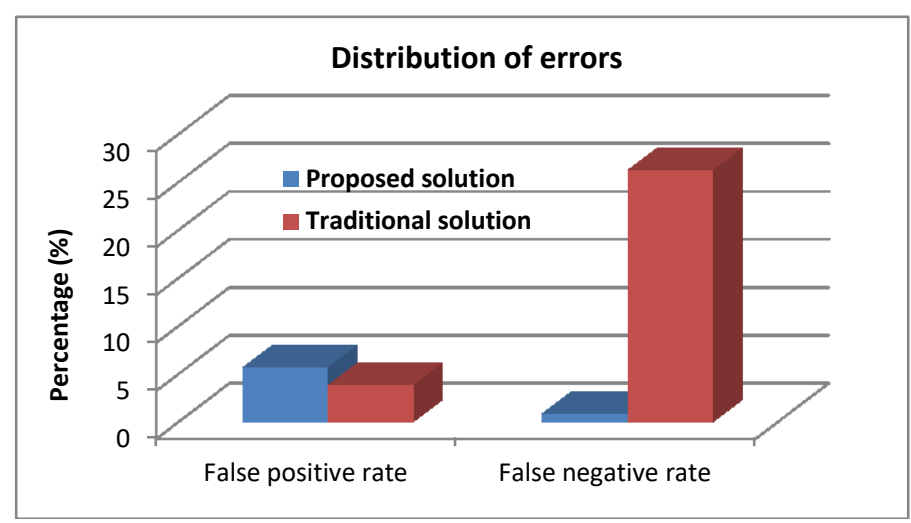

Figure 6. Distribution of errors (first experiment)

As can be seen on Figure 6, the false positive rate is almost a $50 \%$ higher in the proposed solution than in previous technologies (a fact that was expectable as rules are in general relaxed). Moreover, as said, when conditions are considered as independent variables, the obtained strain estimation is lower than real deformation, so more false positives are committed. However, the great difference in the false negative rate clearly turns the proposed solution better than the state of the art.

Figure 7, finally, compares the obtained results when conditions are considered as independent variables, and when are supposed to be non-independent variables. As a machinelearning algorithm is included, results will vary with the number of performed executions.

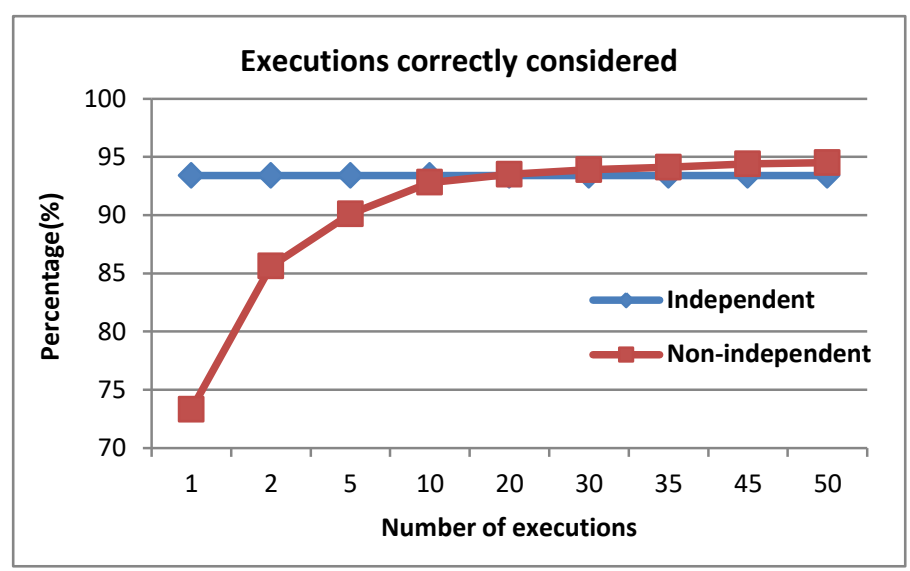

Figure 7. Executions correctly considered (second experiment)

As can be seen three different regions may be distinguished. In the first region (up to ten executions) the approach considering conditions as non-independent variables is clearly worse. During this period the machine-learning algorithm converges to a certain stable configuration. In the second region (between ten and thirty executions) both 
approaches are pretty similar (the percentage of correct executions considered valid presents a difference lower than $1 \%$ between both configurations). However, in the third region (from thirty executions), once the genetic algorithm has learn about the process being executed, it is shown a slight improvement (around 1.5\% of increasing the success rate) if conditions are considered as non-independent variables.

In general, the calculation effort is much higher if a machine-learning algorithm has to be deployed, so it the application scenarios requires this level of precision. Any case, in order to avoid the first region (where system performing is not adequate) hybrid system could be designed; mixing both approaches (conditions as independent and nonindependent variables).

\section{CONCLUSIONS}

The inclusion of humans in Cyber-Physical Systems (CPS) in order to create the new humanized CPS has caused traditional process definitions and execution solutions not to be valid. In particular, user goals, rules and conditions cannot be expressed in a hard way, as people tend to behave in an unpredictable and variable way.

In this context we proposed a new way of defining processes, based on the concept of strain. Strain it is employed as a global measure about the deformation a certain process suffers when it is executed. Processes which admit this type of approach are called soft processes. Moreover, experiments showed that soft processes greatly improve the performing of traditional hard definitions when applied to humanized CPS.

Future works should consider hybrid approaches (both, mixing hard and soft processes, and conditions as independent and non-independent variables) and developing machinelearning algorithm specially focused on the proposed scenario.

\section{ACKNOWLEDGMENT}

The research leading to these results has received funding from the Ministry of Economy and Competitiveness through SEMOLA project (TEC2015-68284-R) and from the Autonomous Region of Madrid through MOSI-AGIL-CM project (grant P2013/ICE-3019, co-funded by EU Structural Funds FSE and FEDER). Borja Bordel has received funding from the Ministry of Education through the FPU program (grant number FPU15/03977).

\section{REFERENCES}

[1] Lee, E. A. (2006, October). Cyber-physical systems-are computing foundations adequate. In Position Paper for NSF Workshop On CyberPhysical Systems: Research Motivation, Techniques and Roadmap (Vol. 2).

[2] Sánchez, B. B., Alcarria, R., Sánchez-de-Rivera, D., \& Sánchez-Picot, A. Enhancing Process Control in Industry 4.0 Scenarios using CyberPhysical Systems. Journal of Wireless Mobile Networks, Ubiquitous Computing, and Dependable Applications 7:4 (December 2016), pp. 4164

[3] Yue, D., Tian, E., \& Han, Q. L. (2013). A delay system method for designing event-triggered controllers of networked control systems. IEEE Transactions on Automatic Control, 58(2), 475-481.
[4] Bordel, B., Alcarria, R., Martín, D., Robles, T., \& de Rivera, D. S. Selfconfiguration in humanized Cyber-Physical Systems. Journal of Ambient Intelligence and Humanized Computing, 1-12.

[5] Bordel B., Sánchez de Rivera D., Sánchez-Picot Á., Robles T. (2016) Physical Processes Control in Industry 4.0-Based Systems: A Focus on Cyber-Physical Systems. In: García C., Caballero-Gil P., Burmester M., Quesada-Arencibia A. (eds) Ubiquitous Computing and Ambient Intelligence. UCAmI 2016. Lecture Notes in Computer Science, vol 10070. Springer, Cham

[6] Martín, D., Alcarria, R., Sánchez-Picot, Á., \& Robles, T. (2015). An ambient intelligence framework for end-user service provisioning in a hospital pharmacy: a case study. Journal of medical systems, 39(10), 116.

[7] Pantazoglou, M., Pogkas, I., \& Tsalgatidou, A. (2014). Decentralized enactment of BPEL processes. IEEE Transactions on Services Computing, 7(2), 184-197.

[8] Van Der Aalst, W. M., \& Ter Hofstede, A. H. (2005). YAWL: yet another workflow language. Information systems, 30(4), 245-275.

[9] Mole, K. F., Ghobadian, A., O'Regan, N., \& Liu, J. (2004). The use and deployment of soft process technologies within UK manufacturing SMEs: an empirical assessment using logit models. Journal of Small Business Management, 42(3), 303.

[10] Kolb, P. F., Heinz, U., Huovinen, P., Eskola, K. J., \& Tuominen, K. (2001). Centrality dependence of multiplicity, transverse energy, and elliptic flow from hydrodynamics. Nuclear Physics A, 696(1-2), 197215.

[11] Sneesens, H. H., \& Vandendorpe, L. (2005, December). Soft decode and forward improves cooperative communications. In Computational Advances in Multi-Sensor Adaptive Processing, 2005 1st IEEE International Workshop on (pp. 157-160). IEEE.

[12] Izosimov, V., Pop, P., Eles, P., \& Peng, Z. (2008, March). Scheduling of fault-tolerant embedded systems with soft and hard timing constraints. In Proceedings of the conference on Design, automation and test in Europe (pp. 915-920). ACM.

[13] Bordel Sánchez, B., Alcarria, R., Martín, D., \& Robles, T. (2015). TF4SM: a framework for developing traceability solutions in small manufacturing companies. Sensors, 15(11), 29478-29510.

[14] Colombo, A. W., Bangemann, T., Karnouskos, S., Delsing, J., Stluka, P., Harrison, R., ... \& Lastra, J. L. (2014). Industrial cloud-based cyberphysical systems. The IMC-AESOP Approach.

[15] Seiger, R., Keller, C., Niebling, F., \& Schlegel, T. (2015). Modelling complex and flexible processes for smart cyber-physical environments. Journal of Computational Science, 10, 137-148.

[16] Seiger, R., Huber, S., \& Schlegel, T. (2015, June). Proteus: An integrated system for process execution in cyber-physical systems. In International Conference on Enterprise, Business-Process and Information Systems Modeling (pp. 265-280). Springer International Publishing.

[17] Onaka, S. (2012). Appropriateness of the Hencky equivalent strain as the quantity to represent the degree of severe plastic deformation. Materials Transactions, 53(8), 1547-1548.

[18] Latorre, M., \& Montáns, F. J. (2014). On the interpretation of the logarithmic strain tensor in an arbitrary system of representation. International Journal of Solids and Structures, 51(7), 1507-1515.

[19] Martín, D., Bordel, B., Alcarria, R., Sánchez-Picot, Á., de Rivera, D. S., \& Robles, T. (2016). Improving Learning Tasks for Mentally Handicapped People Using AmI Environments Based on Cyber-Physical Systems. In Ubiquitous Computing and Ambient Intelligence: 10th International Conference, UCAmI 2016, San Bartolomé de Tirajana, Gran Canaria, Spain, November 29-December 2, 2016, Proceedings, Part I 10 (pp. 166-177). Springer International Publishing.

[20] Morales, A., Alcarria, R., Martin, D., \& Robles, T. (2014). Enhancing evacuation plans with a situation awareness system based on end-user knowledge provision. Sensors, 14(6), 11153-11178.

[21] Katz, S.; Ford, A. B.; Moskowitz, R. W.; Jackson, B. A.; Jaffe, M. W. Studies of illness in the aged: the index of ADL: a standardized measure of biological and psychosocial function. Jama, 1963, 185(12), 914-919. doi:10.1001/jama.1963.03060120024016 


\title{
Assessment of human motivation through analysis of physiological and emotional signals in Industry 4.0 scenarios
}

\author{
Borja Bordel $^{1, *}$, Ramón Alcarria ${ }^{2}$ \\ 1 Department of Telematics Systems Engineering. Universidad Politécnica de Madrid. Avenida \\ Complutense nº 30. 28040 - Madrid (España); E-Mails: bbordel@dit.upm.es \\ 2 Department of Topographic Engineering and Cartography. Universidad Politécnica de Madrid. \\ Campus Sur, 28031 Madrid, Spain; E-Mail: ramon.alcarria@upm.es
}

* Author to whom correspondence should be addressed; E-Mail: bbordel@dit.upm.es; Tel. 915495700 ext. 3035

\begin{abstract}
Traditional methods to evaluate the human motivation in companies include surveys, statistical techniques and psychological analysis. However, generating the required information using these methods is very costly and time demanding. As a solution, Industry 4.0 paradigm allows integrating Ambient Intelligence systems into the daily industrial operations in order to digitalize those activities. This paper proposes a solution to automatically assess the human motivation in Industry 4.0 scenarios with Ambient Intelligence infrastructure. The estimation is based on both physiological and emotional signals which are acquired (through on-body and environmental sensors) and processed in real-time using web services. The final representation of the human motivation is based on the extended Maslow's hierarchy of needs. Moreover, an experimental validation is provided, in order to evaluate the performance of the proposed solution.
\end{abstract}

\section{KEYWORDS}

Industry 4.0; Human motivation; Maslow; Emotions recognition; ECG; Ambient Intelligence; Humanized computing

\section{ACKNOWLEDGMENTS}

Borja Bordel has received funding from the Ministry of Education through the FPU program (grant number FPU15/03977). Additionally, the research leading to these results has received funding from the Ministry of Economy and Competitiveness through SEMOLA project (TEC2015-68284-R) and from the Autonomous Region of Madrid through MOSI-AGIL-CM project (grant P2013/ICE-3019, co-funded by EU Structural Funds FSE and FEDER).

\section{CONFLICTS OF INTEREST}

The authors declare that there is no conflict of interest regarding the publication of this paper. 


\section{INTRODUCTION}

Improving the efficiency and the productivity is a key element for companies. Since 1970 many works on investigating the factors which influence the efficiency and the productivity of the industrial activities have appeared (Rosenberg, et al., 1980 and Black et al. 1996). One of the most important conclusions of these papers is that human factors are the most important element to be taken into account (Nossal et al., 2010). In order to address this situation two types of solutions have been adopted. On the one hand, traditional technological solutions try to replace humans, as much as possible, for robots and automatic production systems (MacDuffie et al., 1992). On the other hand, in companies which depend on human labor (such as manufacturing companies) many times psychological departments have been created. These departments must coach workers in order to improve their efficiency (Wickens et al., 2015).

In general, it is very well known that all the employees in a company (including workers and managers) must be motivated in order to really improve the quality, efficiency and productivity of industrial processes (Datta et al., 2005). General and intermediate managers require information about the workers' motivation in order to implement the appropriate policies and organizational culture. Nowadays, traditional methods to obtain that information are based on surveys and statistical procedures, such as the Chapman's test (Chapman 2012). Nevertheless, two main problems are associated with these methods. First, results need a lot of time to be available (workers have to answer the survey; reports must be elaborated, etc.). However, in some occasions rapid (or automatic) reactions would be desirable. Second, only aggregated values can be obtained; i.e. it is evaluated the global motivation of workers (at the end of the day), however it is not detected whether some particular activity, moment or place along the day specially affect workers.

In order to address these two challenges, an infrastructure to monitor every worker at every moment should be available. Usually, this kind of systems is identified as Ambient Intelligence (AmI) systems (Martin et al, 2015). However, integrating AmI solutions with industrial scenarios is also a problematic issue. Fortunately, in 2014, a new concept emerged in order to address this challenge: Industry 4.0 (Lassi et al., 2014). Industry 4.0 paradigm provides a framework for a new type of industrial companies which deeply integrates information technologies in their daily activities. One of the most promising fields for Industry 4.0 is, precisely, integrating AmI and pervasive computing systems in companies (Schmidt et al., 2015).

Nonetheless, while Industry 4.0 has addressed the challenge of integrating AmI infrastructures into industrial companies, no proposal about how to use the produced data to assess the workers' motivation has appeared. Therefore, this paper describes a technical solution for the assessment of human motivation in Industry 4.0 scenarios. In this work, the key contribution is:

- A model, calculation algorithm and technological system to represent and obtain the motivational state of people from physiological and emotional signals.

- Other secondary contributions such as the employed methods or protocols to extract these physiological and emotional signals are also described.

The proposed solution includes two mathematical models (one simpler and other more elaborated) which relate both physiological and emotional signals obtained from users, with their motivation level. Physiological signals are obtained by means of digital signal processing from an electrocardiogram (ECG) signal acquired using on-body sensors. Emotional signals are generated using video processing. All the acquired signals are processed together, using web services, in order to obtain the final estimation of the motivation level. The motivation level representation is based on the extended Maslow's hierarchy of needs (Maslow et al., 1970). As an improvement in respect to traditional solutions, the proposed technology offers real-time information about the human motivation, and allows a continuous monitoring of workers. Demotivation situations may be located and addressed, in that way, in an extremely precise and rapid way, which helps to strongly increase the global industrial efficiency. 
The rest of the paper is organized as follows: Section 2 introduces the state of the art in human motivation theories and assessment techniques (especially in industrial scenarios). Section 3 presents the proposed technology. Section 4 provides an experimental validation of the proposal. Finally, Sections 5 and 6 explain some results of this experimental validation and the conclusions of our work.

\section{STATE OF THE ART}

In the last sixty years many works about the human motivation in the workplaces (including industrial scenarios) have been proposed. Works on this topic may be classified into two groups (Krocht, 2007): motivation models and motivation assessment solutions.

Four basic theories and models about human motivation have been proposed: the Theory $\mathrm{X} / \mathrm{Y}$, the motivator-hygiene theory, the valence model and the Maslow's hierarchy of needs.

The most recent motivation model was proposed by Douglas McGregor (McGregor 1985). In fact he proposed two different models called Theory X and Theory Y. McGregor claimed that personnel policies have strategic organizational importance and, in particular, managers must assume one of two things: (i) humans naturally want to grow, take responsibility, and care about their jobs; or (ii) humans are passive, dependent, and lazy. The first assumption is known as Theory Y, and companies adhered to this theory try to create in workers intrinsic motivation (Ryan et al., 2000) by the promotion of coaches, work teams, and policies to strengthen their own self-control (Weisbord et al., 1987). On the other hand, companies implementing the second assumption (Theory $\mathrm{X}$ ) implements externally controlled environments, with close supervision (Weisbord et al., 1987) in order to induce external motivation on workers. Motivation policies applying both theories have reported favorable results.

Some years before, Herzbeg (Herzbeg 1966) showed that some factors cause demotivation when they are not present, but if present they do not cause motivation. Other, when present, they creates satisfaction and motivation. The first group of factor is known as hygiene factors and the second group as motivational factors (Herzbeg 1986). This theory is usually named as the "motivator-hygiene theory" (Bassett-Jones et al., 2005). In the 60s, Vroom (Vroom, 1982) defined the valence as the amount of value a person places upon the probable outcome of his or her actions. Then, people are motivated to the extent that they may do something resulting in a desired outcome or reward. Porter and Lawler (Porter et al., 1968) expanded this theory by clarifying some points and introducing the concept of feedback loop (a way to provide people with information about their actions in real time, and then give them a chance to change those actions, pushing them toward better behaviors.).

However, the most referenced motivation model is the Maslow's hierarchy of needs (Maslow et al., 1970). This theory currently has much support and continues being extremely used (for example, in the sales sector (Berl et al., 1984)). Maslow claimed that people move up a pyramid of needs as they satisfy each of them. Thus, unsatisfied needs motivated workers until they are fulfilled. This model is totally compatible with other theories, such as the taxonomy of human motivation in the workplace proposed by Ryan (Ryan et al., 2000). 


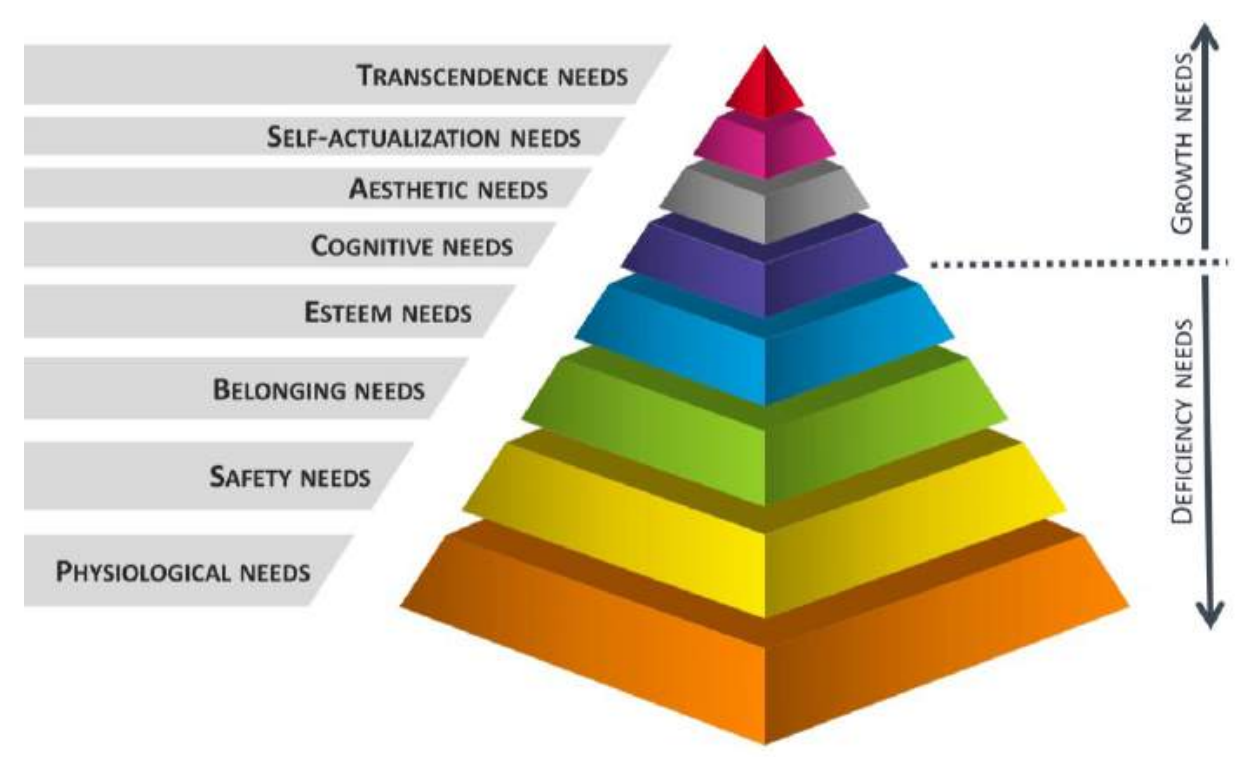

Figure 1. Maslow's hierarchy of needs. Extended model

The original model was proposed by Maslow in 1943 (Maslow, 1943) and described a hierarchy including only five levels (five basic needs): physiological needs, safety needs, belonging needs, esteem needs and self-actualization needs. This proposal corresponds with the world-famous Maslow's pyramid which has been applied successfully in many companies and research areas (Bordel et al., 2017). However, some years later, Maslow extended his proposal (Maslow et al., 1970) by adding new levels to the hierarchy and dividing needs into two different groups (deficiency needs and growth needs). This second model, known as the extended Maslow's hierarchy of needs, use to be represented as an eightlevel pyramid (see Figure 1).

Historically, Maslow's model has been very employed in order to create motivational workplaces (Mustafa 1992 and Martin et al., 2013) However, some authors have proposed alternative models (Alderfer 1969), which finally have been discarded.

Other proposals, such as the Motivation Assessment Scale (MAS) (Newton et al., 1991), focused on severe/profound mental handicap may be found, although they are not the objective of this paper. Any case, many minor models may be found (Steers et al., 2004) and applied in some specific situations.

In respect to motivation assessment solutions, information required in order to locate people in the cited model may be really varied. Traditionally, worker's satisfaction level was measured in relation to some key aspects such as money, operational autonomy, etc. (Tampoe 1993). More recently, new parameters have been defined. For example, the Operational Organization Support (Rhoades et al., 2002) (POS) indicates how much the workers feel the organization supports them. Fairness in the organization (procedural justice, distribute justice and interactional justice) (Colquitt et al., 2005), organizational commitment (Meyer at., 2002) or the organization citizenship behavior (OCB) (Podsakoff et al., 2000) are other indicator which usually are measured in order to evaluate worker' motivation. However, as can be seen, all these measures are company-centric, so they discard personal aspects. More realistic workercentric measures may help companies to obtain more valuable information.

Previously cited proposals determine the way in which humans may be motivated and how this motivation can be measured and quantified (Reeve 2014). However, methods and tools in order to acquire data relative to workers motivation and processing them are also required. 
No proposal about real-time data collection is described. Traditional solutions, which are nowadays the most extended, include from periodical surveys (Kroth et al., 2007) to productivity level measures (Ryan et al., 2000) in order to feed the motivation models and make decisions. Tools such as the Chapman's test (Chapman 2012) or the Implicit Association Test (Brunstein et al., 2004) are still extremely common.

On the other hand, more technological proposals may also be found, although they are very rare. Instruments such as virtual reality simulators to strengthen the human motivation (Green et al., 2006) are the most well-known (and almost unique) proposals.

Any case, all these instruments require much time to monitor and evaluate subjects, and strange variables (as the sincerity of the people) cannot be despised and may generate false data and results.

Our proposal considers as motivation model the extended Maslow's hierarchy of needs, as it is the most famous, employed and validated framework. In comparison with the previously cited works, our solution enables the real-time monitoring of workers so demotivation factors may be located in an extremely precise and rapid way, helping companies to improve the global industrial efficiency. Worker-centric measures are performed, so all aspects related to humans are considered. Information in order to evaluate the motivation level is taken from two different sources: a personal device for electrocardiogram (ECG) monitoring, and the company's video system (included, for example, into the security systems). Thus, the motivation assessment includes two types of information: physiological and emotional (extracted using video processing for emotion tracking).

\section{PROPOSAL: AN INFRASTRUCTURE AND ALGORITHM TO EVALUATE THE HUMAN MOTIVATION}

This section describes the motivational model taken as base in the proposed technological solution. Moreover, a mathematical model in order to relate the motivation level and the obtained physiological and emotional signals is presented. Finally, the entire technological solution (including from the personal device design to the result presentation) is described and implemented.

\subsection{MATHEMATICAL MODEL}

As can be seen on Figure 1, the extended Maslow's hierarchy of needs is an eight-level pyramid divided into two big groups. Each level represents a set of needs which may motivate humans when they are not totally $(100 \%)$ fulfilled. Different needs might be fulfilled in different percentages at the same time. However, as Maslow indicated, the satisfaction degree of a certain needs is not independent from how much fulfilled are the others (Maslow et al., 1970 and Maslow, 1943). In particular, needs in a certain level cannot be totally (100\%) fulfilled until all the previous needs in the hierarchy are totally fulfilled too.

Thus, the motivation state of humans $\mathcal{L}$ may be expressed as an eight-dimensional vector (1) indicating the satisfaction degree (percentage) of needs associated with each one of the eight levels on the Maslow's hierarchy.

$$
\mathcal{L}=\left(\ell_{1}, \ldots, \ell_{8}\right)
$$

Some needs (such as safety needs, $\ell_{2}$ ) are related to the physical integrity and the physical state in general. Others (for example, self-actualization needs, $\ell_{7}$ ) depend on the emotional state of people. Finally, some needs are depending on both facts (emotions and physical state) (Duval et al., 2006). Then, in our proposal, the motivation state is calculated as a function of two types of signals: emotional signals and physiological signals (see Figure 2). 


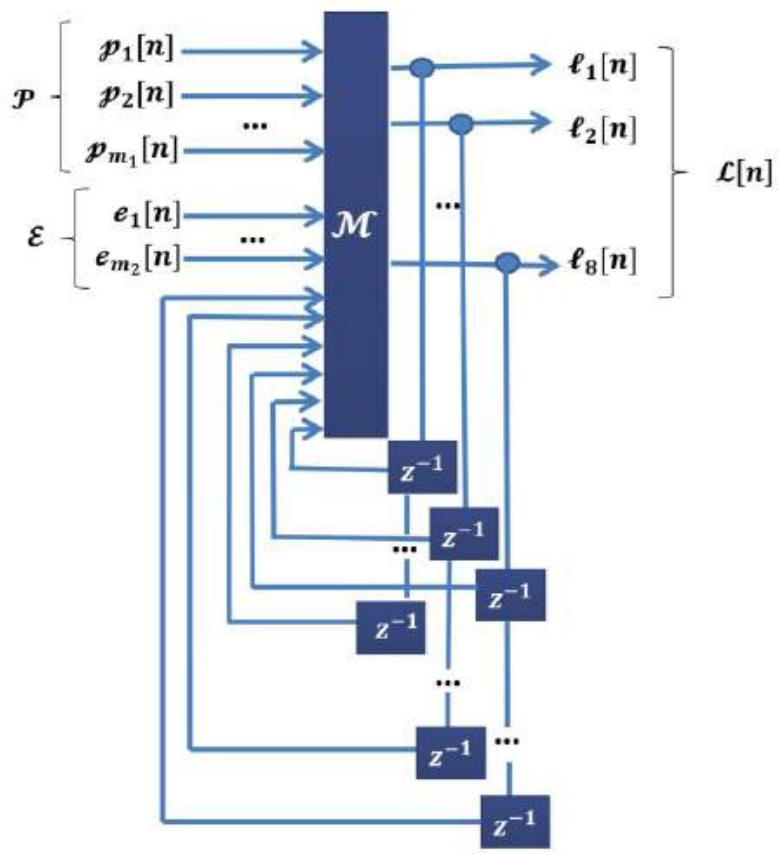

Figure 2. Motivation calculation algorithm as block diagram

As can be seen, the proposed calculation algorithm is based on digital signal processing. The current motivational state of a human $\mathcal{L}[n]=\left(\ell_{1}[n], \ldots, \ell_{8}[n]\right)$ is obtained as output of a digital system, characterized by the mathematical operator $\mathcal{M}[\cdot]$, which takes as inputs the physiological signals $\mathcal{P}=\left\{p_{1}[n], \ldots, p_{m_{1}}[n]\right\}$, the emotional signals $\mathcal{E}=\left\{e_{1}[n], \ldots, e_{m_{2}}[n]\right\}$ and the past values of the motivational state (being $m_{1}$ the considered number of physiological signal and $m_{2}$ the number of employed emotional signals). Hereinafter, in order to simplify the mathematical expressions, we are considering the input signals (both physiological and emotional) as part of a collection $\mathcal{S}=\left\{s_{1}[n], \ldots, s_{m}[n]\right\}$, where $m=m_{1}+m_{2}$. Thus, $s_{k}[n]=p_{k}[n]$ for $k=1, \ldots, m_{1}$ and $s_{k+m_{1}}[n]=$ $e_{k}[n]$ for $k=1, \ldots, m_{2}$.

$$
\left(\ell_{1}[n], \ldots, \ell_{8}[n]\right)=\mathcal{L}[n]=\mathcal{M}[\mathcal{P}, \mathcal{E}, \mathcal{L}[n-1], \ldots, \mathcal{L}[0]]
$$

The objective of this section is to define the mathematical expression of the operator $\mathcal{M}[\cdot]$, so that the obtained signal $\mathcal{L}[n]$ really represents the motivation state of the human under study. Two different possibilities are going to be considered.

In a simplified model, it is suppose the effect of every input (including all signals and past values of the motivational state) on the obtained motivation state is independent, so the final value may be calculated as the sum of all the independent effects. If a more realistic and advanced model wants to be applied, inputs are considered not totally independent and the final obtained value cannot be calculated as a simple sum, as we are explaining below.

\section{a) A simplified model}

In a first approach, we can make the assumption that every input in the digital system is independent. Thus, the evolution of the considered signals is not correlated. Then, it can be reasonably assumed that the effect of each signal on the final value of the motivation state is also independent. Therefore, the mathematical operator $\mathcal{M}[\cdot]$ may be expressed as the weighted sum of all these independent effects (2). 


$$
\ell_{i}[n]=\sum_{k=1}^{m} \alpha_{k} f_{k}\left(s_{k}[n]\right)+\sum_{j=1}^{i-1} \beta_{j} g_{j}\left(\ell_{j}[n]\right)+\sum_{r=1}^{n} \gamma_{r} h_{r}\left(\ell_{i}[n-r]\right)
$$

As can be seen the current satisfaction degree of the needs in the i-th level of the Maslow's hierarchy of needs depends on three contributions. First the current value of the emotional and physiological signals, second, the current satisfaction degree of the previous needs in the hierarchy and, third, the past values of the satisfaction degree of the needs in the i-th level of the Maslow's pyramid (considering they are causal signals).

The first and third contributions are specific proposals of our work (see Figure 2). The second contribution represents the Maslow's observations about the relation among the different needs in the hierarchy (Maslow et al., 1970 and Maslow, 1943) (see Section 2).

Expression (2) also considers three parameter families and three function families. The parameter families $\alpha_{k}, \beta_{k}$ and $\gamma_{k}$ are collections of unknown fixed weights indicating the relative influence of each input in the final result. Function families $f_{k}, g_{k}$ and $h_{k}$ are families of unknown functions which must be determined so that the final result really represents the human motivation state.

Now, we are considering the Taylor series expansion centered at the origin (or Maclaurin series expansion) for every unknown function (3).

$$
\begin{aligned}
& f_{k}(x)=\sum_{q_{1}=0}^{\infty} \frac{f_{k}^{\left(q_{1}\right)}(0)}{q_{1} !} x^{q_{1}}=\sum_{q_{1}=0}^{\infty} a_{k, q_{1}} x^{q_{1}} \\
& g_{k}(x)=\sum_{q_{2}=0}^{\infty} \frac{g_{k}^{\left(q_{2}\right)}(0)}{q_{2} !} x^{q_{2}}=\sum_{q_{2}=0}^{\infty} b_{k, q_{2}} x^{q_{2}} \\
& h_{k}(x)=\sum_{q_{3}=0}^{\infty} \frac{f_{k}^{\left(q_{3}\right)}(0)}{q_{3} !} x^{q_{3}}=\sum_{q_{3}=0}^{\infty} c_{k, q_{3}} x^{q_{3}}
\end{aligned}
$$

Then, the proposed model for human motivation state calculation may be expressed as a power sum (4).

$$
\begin{aligned}
\ell_{i}[n]=\sum_{k=1}^{m} \alpha_{k}\left(\sum_{q_{1}=0}^{\infty} a_{k, q_{1}}\left(s_{k}[n]\right)^{q_{1}}\right)+\sum_{j=1}^{i-1} \beta_{j}\left(\sum_{q_{2}=0}^{\infty} b_{j, q_{2}}\left(\ell_{j}[n]\right)^{q_{2}}\right) \\
+\sum_{r=1}^{n} \gamma_{r}\left(\sum_{q_{3}=0}^{\infty} c_{r, q_{3}}\left(\ell_{i}[n-r]\right)^{q_{3}}\right)
\end{aligned}
$$

Considering that summations are linear operators, and grouping real fixed parameters in only one quantity, expression (4) may be reduced (5).

$$
\begin{aligned}
\ell_{i}[n]=\sum_{k=1}^{m} \sum_{q_{1}=0}^{\infty} \eta_{k, q_{1}}\left(s_{k}[n]\right)^{q_{1}}+\sum_{j=1}^{i-1} \sum_{q_{2}=0}^{\infty} \lambda_{j, q_{2}}\left(\ell_{j}[n]\right)^{q_{2}} \\
+\sum_{r=1}^{n} \sum_{q_{3}=0}^{\infty} \mu_{r, q_{3}}\left(\ell_{i}[n-r]\right)^{q_{3}}
\end{aligned}
$$

Expression (5) in totally equivalent to expression (2), however, infinite summations are not practical as they cannot be programmed or calculated using digital processing. Then, infinite summations must be transformed into finite summations assuming a certain error in the model (6). Moreover, the effect of past values of the motivation state should be limited to some samples around the current state. 


$$
\begin{aligned}
\ell_{i}[n] \approx \hat{\ell}_{i}[n]= & \sum_{k=1}^{m} \sum_{q_{1}=0}^{q_{1, \max }} \eta_{k, q_{1}}\left(s_{k}[n]\right)^{q_{1}}+\sum_{j=1}^{i-1} \sum_{q_{2}=0}^{q_{2, \max }} \lambda_{j, q_{2}}\left(\ell_{j}[n]\right)^{q_{2}} \\
& +\sum_{r=1}^{r_{\max }} \sum_{q_{3}=0}^{q_{3, \max }} \mu_{r, q_{3}}\left(\ell_{i}[n-r]\right)^{q_{3}}
\end{aligned}
$$

The order of the total considered error (7) is, thus, depending on the number of elements included in each summation (as, at the end, it limits the number of terms in the Taylor series expansion).

$$
\left(\ell_{i}[n]-\hat{\ell}_{i}[n]\right)=\max \left\{\left(s_{k}[n]\right)^{q_{1, \max }} k=1, \ldots, m ;\left(\ell_{j}[n]\right)^{q_{2}, \max } j=1, \ldots, i-1 ;\left(\ell_{i}[n-r]\right)^{q_{3}, \max } \quad r=1, \ldots, r_{\max }\right\}
$$

In conclusion, in order to complete the model, it is only necessary to obtain the proper values for the parameter families $\eta_{k, q_{1}}, \lambda_{j, q_{2}}$ and $\mu_{r, q_{3}}$. Thus, in total, it is necessary to calculate $m \cdot q_{1, \max }+(i-1) q_{2, \max }+r_{\max } \cdot q_{3, \max }$ parameters, which may be understood as the unknowns in a system of linear algebraic equations. In the regular case, more equations than unknowns will be available, so the mathematical problem should be solved using the mean square errors technique.

It is important to note that, in case of considering the parameter families $\alpha_{k}, \beta_{k}$ and $\gamma_{k}$ as variable weights depending on the particular value of the emotional and physiological signals (or the previous emotional state), the final obtained expression for the digital system would be the same (6). In particular, the multiplication of two functions depending on the same variable is a third function depending on the same variable (it is enough to apply the Cauchy product with the two associated Taylor expanded series).

Additionally, if desired, the parameter families $\alpha_{k}, \beta_{k}$ and $\gamma_{k}$ may be considered time dependable. In that way, expression (6) gets lightly transformed (8). As a result, the equivalent system of linear algebraic equations should be evaluated dynamically.

$$
\hat{\ell}_{i}[n]=\sum_{k=1}^{m} \sum_{q_{1}=0}^{q_{1, \max }} \eta_{k, q_{1}}[n] \cdot\left(s_{k}[n]\right)^{q_{1}}+\sum_{j=1}^{i-1} \sum_{q_{2}=0}^{q_{2, \max }} \lambda_{j, q_{2}}[n] \cdot\left(\ell_{j}[n]\right)^{q_{2}}+\sum_{r=1}^{r_{\max } q_{3, \max }} \sum_{q_{3}=0}^{\alpha_{r, q_{3}}}[n] \cdot\left(\ell_{i}[n-r]\right)^{q_{3}}
$$

\section{b) A more realistic model}

In the real world, emotional and physiological signals are not independent, as they represent information about a same person. For example, the beats per minute and breaths per minute behave and evolve in a similar way, but they are two different physiological signals. Moreover, motivation state is not independent from emotional and physiological signals (in fact our models assumes the opposite). In those conditions, the digital system is modeled as a unique multi-dimensional function (9).

$$
\ell_{i}[n]=F\left(s_{k}[n] k=1, \ldots, m ; \ell_{j}[n] j=1, \ldots, i-1 ; \ell_{i}[n-r] r=1, \ldots, n\right)
$$

Then, using the multi-dimensional Taylor series expansion centered at the origin, the model may be expressed as (10); where Tis the total number of considered variables (i.e. $T=m+i-1+n$ ). As can be seen, the value of $T$ grows as $n$ does it, which is not a desirable characteristic for a practical model.

$$
\ell_{i}[n]=\sum_{h=0}^{\infty} \sum_{\forall h_{1}+\ldots+h_{T}=h} \sigma_{h_{1} \ldots, h_{T}}\left(\left(s_{1}[n]\right)^{h_{1}} \cdot \ldots \cdot\left(s_{m}[n]\right)^{h_{m}} \cdot\left(\ell_{1}[n]\right)^{h_{m+1}} \cdot \ldots \cdot\left(\ell_{i-1}[n]\right)^{h_{m+i-1}} \cdot\left(\ell_{i}[n-1]\right)^{\left.h_{m+i} \cdot \ldots \cdot\left(\ell_{i}[0]\right)^{h_{T}}\right)}\right.
$$

Where the values of $\sigma_{h_{1}, \ldots, h_{T}}$ parameters are formally expressed depending on a multinomial coefficient and on the value of the partial derivatives at the origin (11).

$$
\sigma_{h_{1}, \ldots, h_{T}}=\left(\begin{array}{c}
h \\
h_{1}, \ldots, h_{T}
\end{array}\right) \frac{\partial^{T} F(0, \ldots, 0)}{\partial s_{1}[n] \cdot \ldots \cdot \partial s_{m}[n] \cdot \partial \ell_{1}[n] \cdot \ldots \cdot \partial \ell_{i-1}[n] \cdot \partial \ell_{i}[n-1] \cdot \ldots \cdot \partial \ell_{i}[0]}
$$


Once more, infinite summations should be removed in order to obtain a practical model, so the final expression (12) assumes a certain error (13).

$$
\ell_{i}[n] \approx \hat{\ell}_{i}[n]=\sum_{h=0}^{h_{\max }} \sum_{\forall h_{1}+\cdots+h_{T}=h} \sigma_{h_{1}, \ldots, h_{T}}\left(\left(s_{1}[n]\right)^{h_{1}} \cdot \ldots \cdot\left(s_{m}[n]\right)^{h_{m}} \cdot\left(\ell_{1}[n]\right)^{h_{m+1}} \cdot \ldots \cdot\left(\ell_{i-1}[n]\right)^{h_{m+i-1}} \cdot\left(\ell_{i}[n-1]\right)^{\left.h_{m+i} \cdot \ldots \cdot\left(\ell_{i}[0]\right)^{h_{T}}\right)}\right.
$$

In this case, estimating the order of the committed error is more complicated than in the previous case; however it is possible to stablish a first approximation (13).

$$
\vartheta\left(\ell_{i}[n]-\hat{\ell}_{i}[n]\right)=\left(s_{k}[n]\right)^{h_{\max }} \quad k \in\{1, \ldots, m\}
$$

As in the simple model, in order to complete the definition of the digital system it is necessary to obtain the value of the $\sigma_{h_{1}, \ldots, h_{T}}$ parameters. These values may be calculated as the solutions of a system of linear algebraic equations (10) using the mean square error. As previously, $\sigma_{h_{1}, \ldots, h_{T}}$ parameters may be considered variable in time if desired, and the mathematical expression for the digital system remains as indicated (10).

This more realistic model, in fact, allows representing complex effects, so more precise results are obtained. However, it presents various disadvantages. First, in order to determine all the elements in the second summation in expression (10), it is necessary to solve a complex mathematical problem: locating all the sets of $T$ numbers whose sum is equal to $h$. In particular, this problem does not have a general solution, so it is very complicated to program an algorithm presenting this realistic model. And, second, multiplications are the dominant mathematical operation in the model instead of additions, so a higher calculation time is required in order to obtain the motivation level. Thus, it should be guaranteed, in order to preserve the real-time quality, that a calculation is finished before new information (a new sample) gets available.

Therefore, unless a higher level of precision is mandatory, it is preferable to employ the simple model as the resulting system is easier to manage.

Any case, in order to determine the value of the unknown parameters, an excitation process of the different motivation states should be performed. Some authors have proved that motivation films, horror movies and other audiovisual contents may modify the motivation and stress level of users (MohinoHerranz et al., 2015). Then, during a calibration process, users visualize these contents and unknown parameters are fixed to generate the adequate motivation level.

\subsection{PHYSIOLOGICAL SIGNAL OBTENTION}

Recently, previous works have proved that physiological signals may be used to determine the emotions that users under study are feeling (Park et al., 2013). The objective of this section is to design a system being able to extract that information and introduce it in the motivation calculation model.

As we have said, two different types of signal are employed in our model: physiological and emotional. Physiological signals are obtained from a personal device, employed to monitor humans and as personal information device. The electronic scheme of the proposed device is shown on Figure 3. 


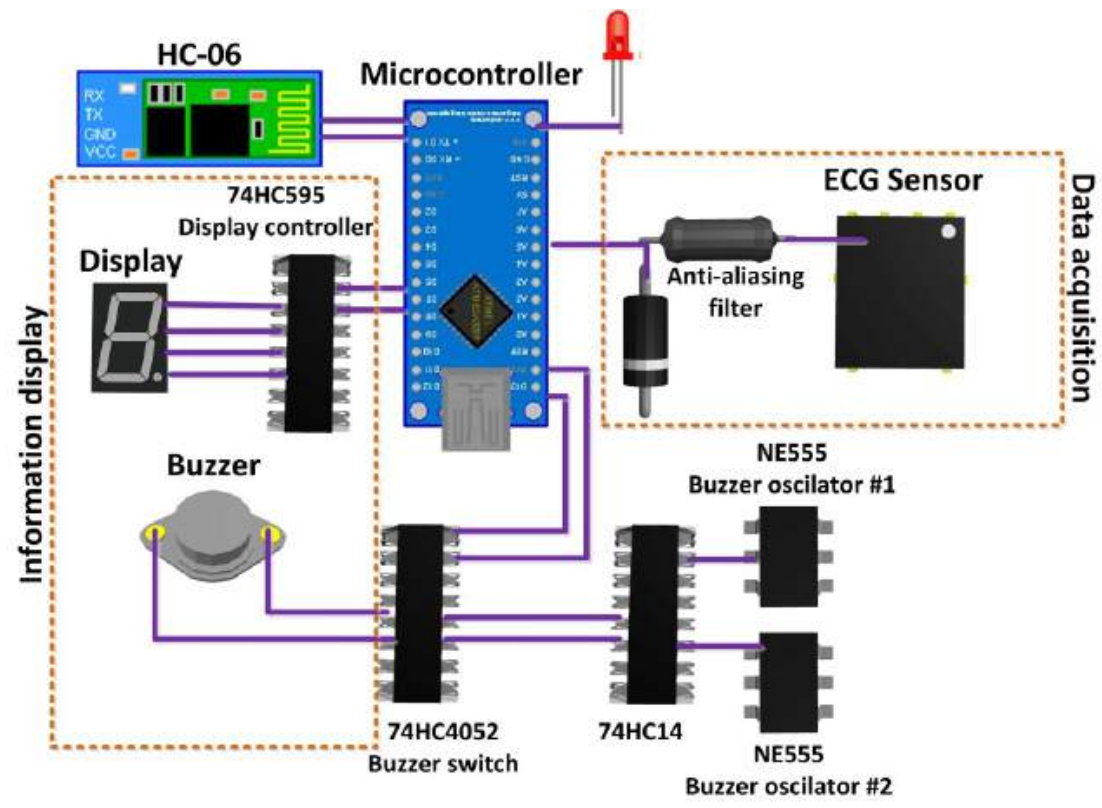

Figure 3. Electronic scheme of the personal device

As can be seen, the device may be divided into two parts. The first part is dedicated to data acquisition (includes de ECG sensor) and, the second part, is dedicated to inform users (it includes a display to display visual information and a buzzer in order to display sound information). The ECG sensor (Bitalino 2017) employs three on-body electrodes in order to capture the required information (see Figure 4(a)). The device also includes a small microcontroller in order to manage the device and the Bluetooth communications which transmits the information to a processing application and receives the information to be displayed in the device. Below a detailed description of the employed components is provided:

- A red LED indicating if the system is powered on

- An ECG sensor sensor (Bitalino 2017) from Bitalino toolkit, provided by PLUX wireless biosignals S.A. Output signal presents a bandwidth of $40 \mathrm{~Hz}$ (from $0.5 \mathrm{~Hz}$ to $40 \mathrm{~Hz}$, approximately) and a dynamical range of $3 \mathrm{~V}$ (signal ranges from $-1.5 \mathrm{~V}$ to $1.5 \mathrm{~V}$ ). This sensor employs three electrodes (in order to make differential measures).

- An anti-aliasing filter, based on a resistor-capacitor low-pass configuration. This analog filter presents a cut frequency of $40 \mathrm{~Hz}$, in order to avoid problems in the digitalization process.

- Two NE555 temporization circuits, configured as astable multi-vibrators (oscillators). The frequency of the produced signal is different for each circuit. The first one generates a signal of $f_{1}=1 \mathrm{~Hz}$ and the second employs a frequency of $f_{2}=2 \mathrm{~Hz}$. In both cases the duty-cycle is configured for a value of $\mathrm{DC}=50 \%$

- A smith-trigger integrated circuit $74 \mathrm{HC} 14$ destined to clearly define the edges of the signal from the NE555 circuits.

- A 74HC4052 multiplexer/demutiplexer circuit focused on controlling the signal delivered to the buzzer. Four possible options are considered: any of the signal produced by the NE555 circuits, a continuous $\mathrm{V} \_c c=5 \mathrm{~V}$ signal from the power source, or a continuous signal GND $=0 \mathrm{~V}$. Depending the delivered signal the sound produced by the buzzer changes.

- A buzzer as a sound information device

- A 7 segments LCD display, showing the higher level in the Maslow's hierarchy whose needs have been fulfilled at all (100\%).

- A controller circuit 74HC595 (shift register) in order to show the cited information on the display 
- A microcontroller (Arduino Nano) which manages the entire system. This controller is in charge of sampling and digitalizing the analog signal from the anti-aliasing filter. It also selects the signal delivered to the buzzer and build the messages transmitted to a processing application with the information from the ECG

- A HC-06 Bluetooth module employed to transmit and receive information from the processing application.

As can be seen, the proposed implementation of the personal devices is pretty obtrusive, and (in real scenarios) it would make difficult for workers to perform their daily activities when wearing this device. Thus, the creation of an unobtrusive personal device (for example, integrated in an intelligent watch) is a future challenge to be addressed and solved.

As said, the above-mentioned hardware is complemented with a processing application (implemented in Java) which finally generates the physiological signals employed in the model (see Section 3.1). Figure 4(b) represents the processing application. This application runs in a smart device (smartphone, tablet, etc.) provided with Bluetooth connectivity, which also controlled by the user. It receives messages from the hardware device, processes the obtained samples and obtains the physiological signals. Obtained signals are transmitted to a remote server through web sockets (see Figure 5). The remote server calculates the motivation state and returns the information to the processing application which sends the appropriate message to the personal device (in order to inform users). Section 3.4 deeply describes the global system's behavior.
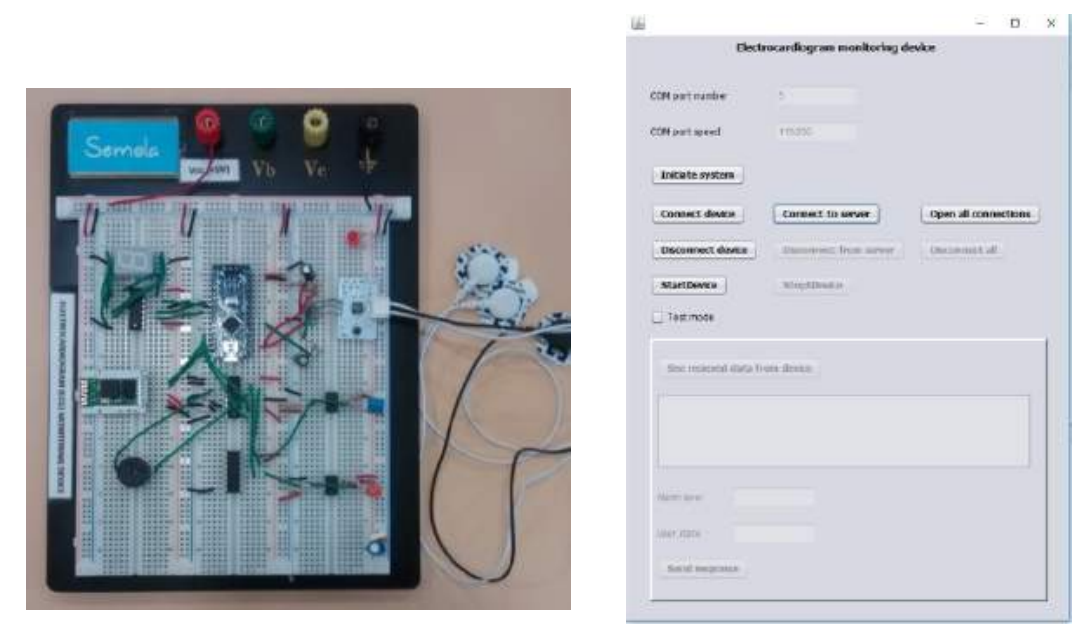

Figure 4. (a) Personal device implementation (b) Processing application interface

Seven different physiological signals are considered and calculated in the processing application (see Figure 5): (i) the raw electrocardiogram signal $\operatorname{raw}[n]$, (ii) the direct current (DC) level in the ECG $D C[n]$, (iii) the respiration frequency $R F[n]$, (iv) the low-frequency components in the ECG $L F[n]$, (v) the high-frequency components in the ECG $H F[n]$, (vi) the beats per minute $B P M[n]$ and (vii) the pulsations per minute $P P M[n]$. These signals represent the entire information that may be obtained from an ECG, so they contain enough data to obtain a valid representation of the users' state.

In some occasions, workers may oppose to be monitored for motivation and efficiency control, so in Industry 4.0 scenarios (as which we are describing), it is recommended to provide additional services (such as e-health or security) to persuade workers to use the personal devices. 

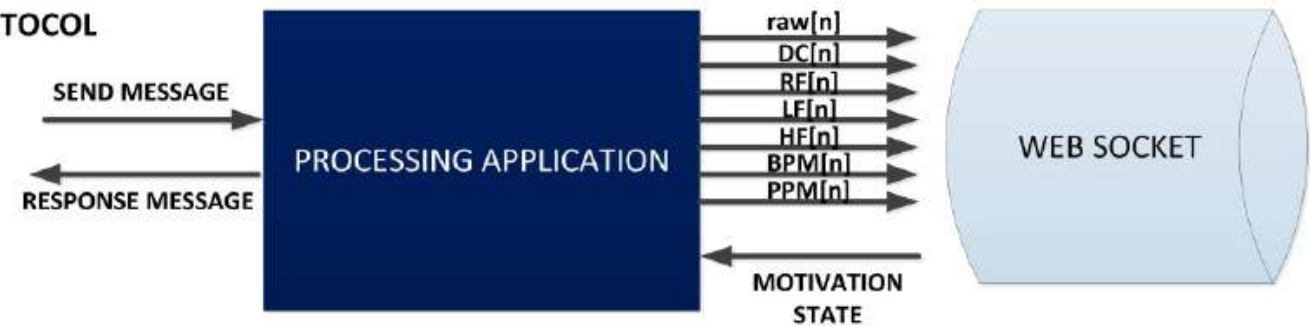

Figure 5. Processing application scheme to obtain the physiological signals (functional diagram)

In this module, real-time requirements are hard and must be specially considered. In particular, it must be guaranteed that every sample is processed before a new sample gets available. For that, it is proposed the processing scheme shown on Figure 6. A detailed explanation of the proposed scheme is provided below.

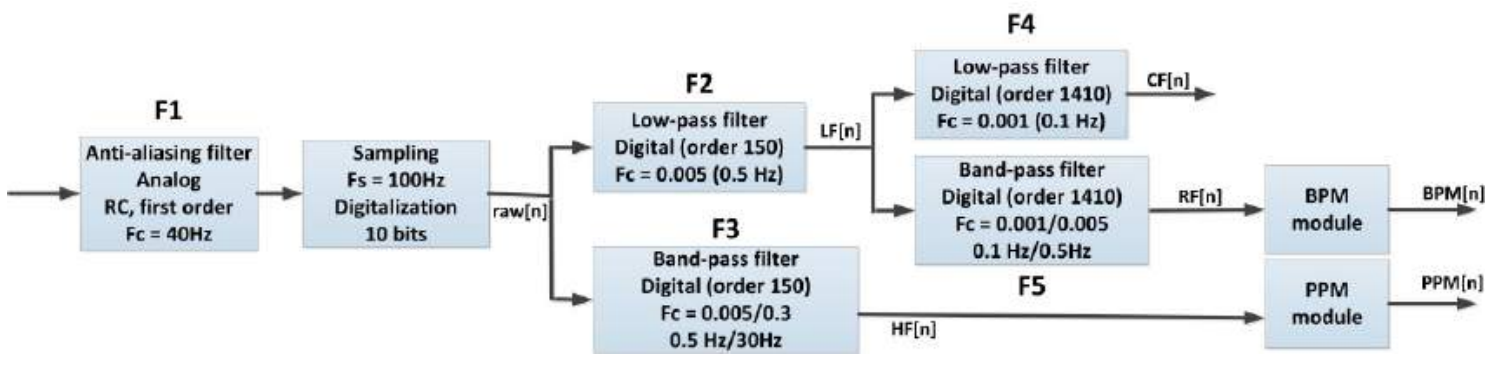

Figure 6. Processing scheme to obtain the physiological signals (filter bank)

- Filter F1 is an anti-aliasing analog filter, based on a resistor-capacitor configuration (as explained above). The cut frequency is $f_{c}=40 \mathrm{~Hz}$. As the input signal presents a bandwidth of $40 \mathrm{~Hz}$ (centered at the origin) it is enough to include a first order filter. This filter is implemented using electronic components, but in advance all filters are implemented using digital signal processing in the processing application.

The ECG sensor includes mechanisms (Bitalino, 2017) to avoid the contamination of the ECG signal with the power signal (as important information is stored in some very lo-frequency bands, see Figure 6). However, this unique analog (hardware) filter (Filter F1) may introduce some low-frequency noise if some design decisions are not properly taken. In particular, in this case, we have selected a resistor-capacitor configuration (not an active filer) which does not require to be powered. In that way, power supply is directly connected to the ECG sensor and to the microcontroller (see Figure 3), and no power signal is accidentally added to ECG signal in this filter.

- The output of the F1 filter (a signal limited in band to $40 \mathrm{~Hz}$ ) is sampled and digitalized. In order to respect the Nyquist criterion the sampling frequency is fixed to $f_{s}=100 \mathrm{~Hz}$ (one sample each $10 \mathrm{~ms}$ ). Moreover, as the dynamic range of the signal is $3 \mathrm{~V}, 10$ bits allow a resolution of $3 \mathrm{mV}$ (approximately). This value is enough for our application. The sampled and digitalized signal is the first physiological signal, $\operatorname{raw}[n]$

Using digital signal processing, the sampled signal $\operatorname{raw}[n]$ is filtered. Various works study the relevant information contained in the different frequency bands of an ECG signal users (Mohino-Herranz et al., 2015, Lee et al., 2006 and Piccirillo et al., 1996). In particular four different bands are considered in our proposal.

- $\quad \mathrm{F} 2$ is a low-pass filter with a cut normalized frequency of $f_{c}=0.005$ (around $0.5 \mathrm{~Hz}$ in natural frequency). This band contains both, information about the respiration frequency and about the DC component. The output of this filter is a new physiological signal called $L F[n]$. Considering a ripple of $\varepsilon_{F 2}=0.13 \mathrm{~V}$ in the passband and the stopband (which means, approximately, a $4 \%$ of 
the dynamic range) and a transition bandwidth of $\Delta f_{F 2}=0.002$, the filter must present an order of $N_{F 2}=150$ using Finite Impulse Response (FIR) technologies. This implies to accumulate samples during 1.5 seconds (150 samples) before being able to filter. Moreover, considering sums and multiplications, this filter should perform $O_{F 2}=2 \cdot N_{F 2} \cdot f_{s}=30000$ ops (operations per second). We consider both facts meet the real-time requirements. In particular, the transition bandwidth was selected to obtain a filter order compatible with real-time requirements (the length of an FIR filter required to meet given specifications (Kaiser 1974) is approximately (14)), respecting a good-quality filtering (the transition bandwidth is around a $40 \%$ of the cut frequency).

$$
N=\frac{-20 \cdot \log _{10}\left(\sqrt{\varepsilon_{\text {pass }} \cdot \varepsilon_{\text {stop }}}\right)-13}{14.6 \cdot \Delta f}
$$

- Filter F3 is the complementary of filter F2. It is a band-pass filter between normalized frequencies $f_{1}=0.005$ and $f_{2}=0.3$. Characteristics are selected equal to which explained for filter F2, so the same considerations are valid. This filter takes as input the raw sampled signal and extracts the high frequency components, which are a new physiological signal named as $H F[n]$.

- As said before, signal $L F[n]$ contains two types of information, so they must be isolated. Filter F4 extracts the DC signal $D C[n]$ in the ECG. For that, this filter obtains the base band with normalized cut frequency $f_{c}=0.001$ (around $0.1 \mathrm{~Hz}$ in terms of natural frequency). Considering a ripple of $\varepsilon_{F 4}=0.08 \mathrm{~V}$ in the passband and the stopband and a transition bandwidth of $\Delta f_{F 4}=$ 0.0004 (the $40 \%$ of the cut frequency) the filter (implemented using FIR technologies) should present an order of $N_{F 4}=1410$. This would imply to accumulate samples during 14.1 seconds and make $O_{F 4}=2 \cdot N_{F 4} \cdot f_{s}=282000$ ops. These conditions cannot be considered meeting the real-time requirements. Thus, in order to maintain the same filtering quality and improve the processing speed, this filter is implemented using Interpolated Finite Impulse Response (IFIR) technologies (Neuvo et al., 1984). IFIR filters are actually two cascaded filters (see Figure 7).

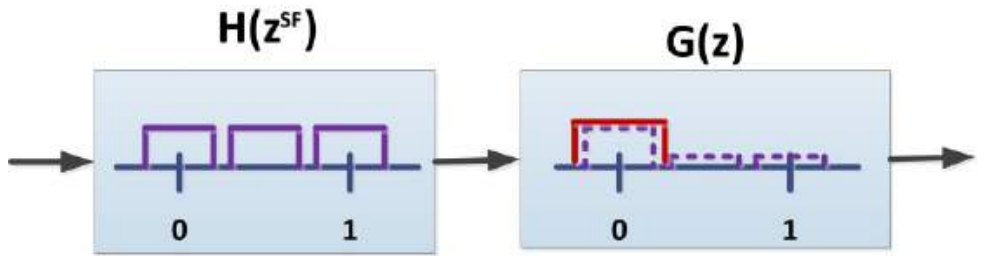

Figure 7. IFIR filter structure

The first one is a filter with the same frequency response as the equivalent FIR filer, but expanded in a factor $S F$, so it presents an order of $N_{H}=\frac{N_{F I R}}{S F}$. The obtained impulse response $h_{H}[n]$ is then expanded in a factor $S F$ (named by Neuvo (Neuvo et al., 1984) as stretch factor), so in the Fourier domain the filter get compressed in the same factor (obtaining the desired filter) and it is obtained the final filter $H\left(z^{S F}\right)$. However, the zero-insertion process also introduce replicas of the filter frequency response which must be removed with a second filter $G(z)$, acting as an interpolation filter.

In the case of the F4 filter, as the passband is very narrow, the stretch factor may be high. In particular we employed a value of $S F=30$, so $N_{H}=47$. In the same way, the interpolation filter may present a low order. In this case we selected $N_{G}=60$. In conclusion, although the IFIR filter behaves as a FIR filter with order 1410, in fact it is only necessary to accumulate $N_{H}+N_{G}=107$ samples and perform $O_{F 4}=2 \cdot\left(N_{H}+N_{G}\right) \cdot f_{S}=21400$ ops. These characteristics meet the real-time requirements.

- Finally, filter F5 extracts the respiration frequency using a passband filter between normalized frequencies $f_{1}=0.001$ and $f_{2}=0.005$. In respect to the design process, the same 
considerations made for filter F4 are valid here. As output, filter F5 generates a new physiological signal named as $R F[n]$

- Additionally, two calculation modules are included to obtain the pulsation per minute and the breaths per minute. For both modules there exist some well-known algorithms which may be directly applied. In particular, the pulsation per minute may be calculated using simple threshold based algorithms (Duval et al., 2006) or autocorrelation based analysis (Fujii et al., 2013) (among other possibilities). In this work we are considering the second case. The breaths per minutes are calculated using the calculation algorithm of beats per minute (Duval et al., 2006).

As final detail, it must be discussed the communication interfaces. As indicated in Figure 5, two different interfaces are included in the processing application.

First, as the built personal device includes a reduced size and capabilities microcontroller, typical communications tools (such as XML files or JSON objects) cannot be implemented to send information between the device and the processing application. A simple protocol, not requiring parsing libraries or a great amount of RAM memory is required. This situation has been studied previously (Bordel Sánchez et al., 2015) and a byte-oriented proprietary protocol is the best solution. Figure 8 shows the four messages composing the designed protocol.

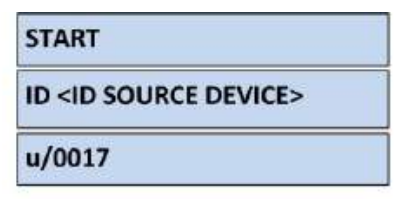

\begin{tabular}{|l|}
\hline SEND \\
\hline \hline ID <ID SOURCE DEVICE > \\
AMOUNT <PAYLOAD LENGTH > \\
MAX_VALUE <MAXIMUN VALUE IN DATA> \\
MIN_VALUE <MINIMUM VALUE IN DATA> \\
DATA \\
\hline \hline CLIST OF SAMPLES> \\
\hline \hline U/0017 \\
\hline
\end{tabular}

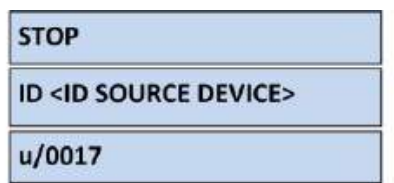

\begin{tabular}{|l|}
\hline RESPONSE \\
\hline BUZZER $<$ SIGNAL> \\
DISPLAY <NUMBER> \\
\hline \hline u/0017 \\
\hline
\end{tabular}

Figure 8. Byte-oriented protocol employed between the personal device and the processing application (message format)

The proposed protocol includes four messages. The "START" message activates a personal device from the processing application; and the "STOP" message finishes the operation. "SEND" message is employed by the hardware device to send to the processing application all the acquired ECG samples; and "RESPONSE" message is used by the processing application to inform the hardware device about the information to be displayed. All the messages finish with the "end of transaction" Unicode character. These messages are transmitted through a Bluetooth interface with a rate of $115200 \mathrm{bits} / \mathrm{s}$, communicating the personal device and the processing application. 


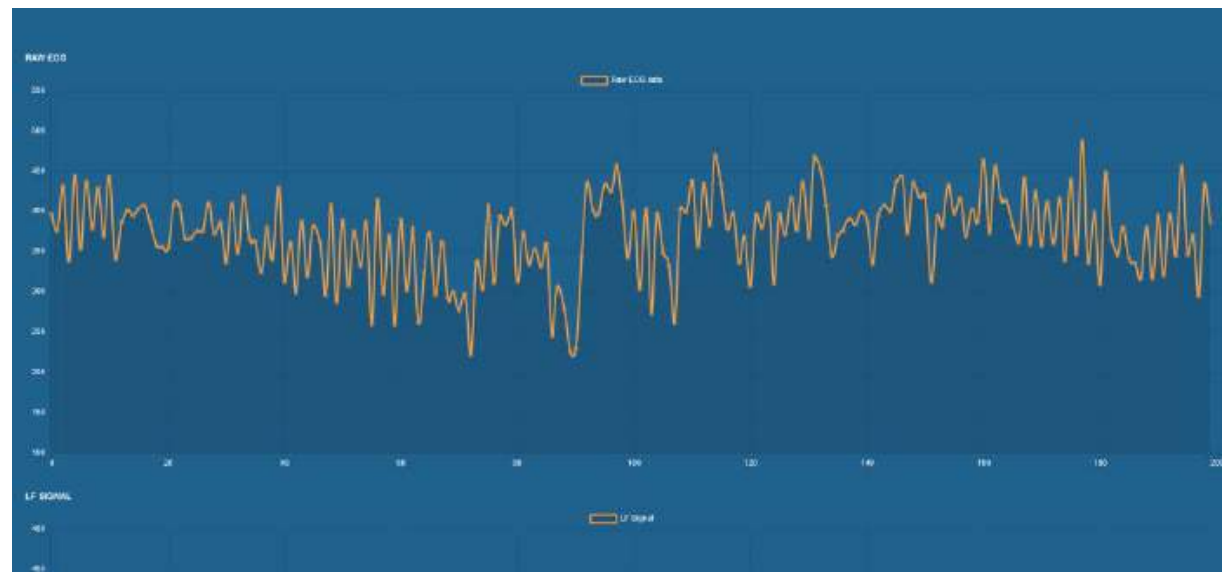

Figure 9. Web interface (management) showing some of the obtained physiological signals

Another important detail is the power consume associated with communications. To be practical, the personal device's battery should last, at least, an entire working day. In order to reach this objective, the Bluetooth module must be powered off when not used. Thus, a communication temporization is planned. The microcontroller acquires all the samples which can be transmitted in a unique SEND message whose transmission lasts, at maximum, 10ms (the time between two consecutive sampling routines). Considering the configured Bluetooth speed and the structure of the SEND messages, the maximum number of samples is around 27. During all this time the Bluetooth module remains powered off. Then, when the described SEND message is built, it is transmitted using the Bluetooth communications. When the transmission finishes, the Bluetooth module keeps powered on during a certain time, looking for the RESPONSE message. If no message is received, or the timer expires, the module is powered off another time.

\subsection{EMOTIONAL SIGNAL OBTENTION}

The process for obtaining the emotional signals is simpler than which described for the physiological ones. In particular, emotional signals are obtained by means of video processing techniques from the video stream provided by the available video systems in the company (for example, the security system).

Emotion tracking is a very complex discipline, with many authors working on it (Metallinou et al., 2011, Imbrasaite et al., 2013 and Bailenson et al., 2008). However, in this case, it is not necessary to develop a new specific technology, as many commercial or free solutions are available. Kairos (Kairos 2017), CrowdEmotion (CrowdEmotion 2017) or Affectiva (Affectiva 2017) are companies and/or research projects dedicated to emotion tracking. In particular, in this case, we are using the library provided by Affectiva.

Affectiva provides an artificial intelligence algorithm, based on the research of the Massachusetts Institute of Technology (MIT) Media Lab. The emotion analysis algorithm employs computer vision and new face and emotion classifiers, trained to cover a wide range of facial expressions. Various software development kits (SDKs) are distributed under a free license for personal or open source projects. Thus, may types of application may incorporate the emotion recognition functionalities provided by Affectiva. In this case, we are using the JavaScript SDK.

In order to obtain the emotional signals, the output of the video system is connected to a static web page including the Affectiva JS-SDK. Then, every frame is processed using the emotion recognition algorithm and several emotional signals are obtained as response (see Figure 10). Results, as well as the video stream being analyzed are displayed in a web interface, accessible by managers. 


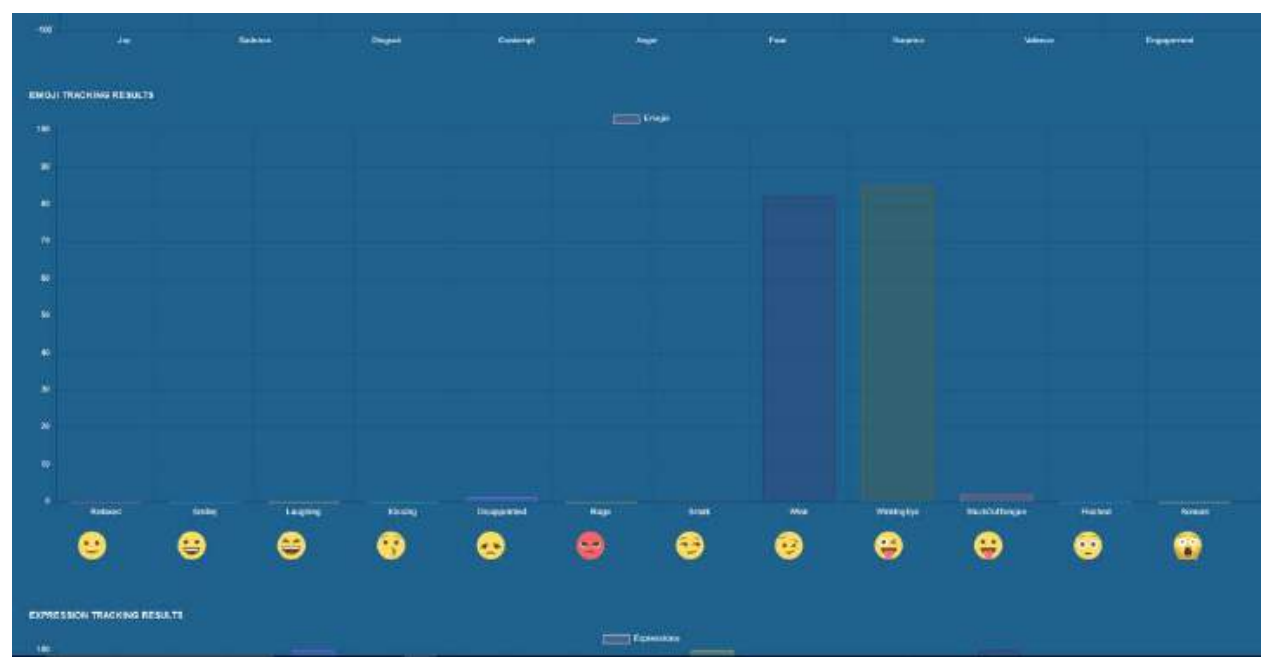

Figure 10. Web interface (management and webRTC page) showing the some emotional signals

The video stream is captured by the static web using WebRTC (Bergkvist et al., 2012) technologies, and the frames are sent to a cloud service (based on a REST interface) using AJAX invocations. In that way, moreover, results may be dynamically displayed in the web interface without refreshing the page (see Figure 11).

Forty-three different signals (belonging to three categories) are obtained from the emotion tracking system (see Table 1). In the motivation model (see Section 3.1) can be included as many signals as desired. Thus, a JSON object is sent from the emotional signals generating module to a remote server where these data are mixed with the physiological signals in order to evaluate the motivation level (see Figure 11). In particular, the JSON object must contain only the emotional signals to be included in the model. In our case, we are considering all the obtained signals.

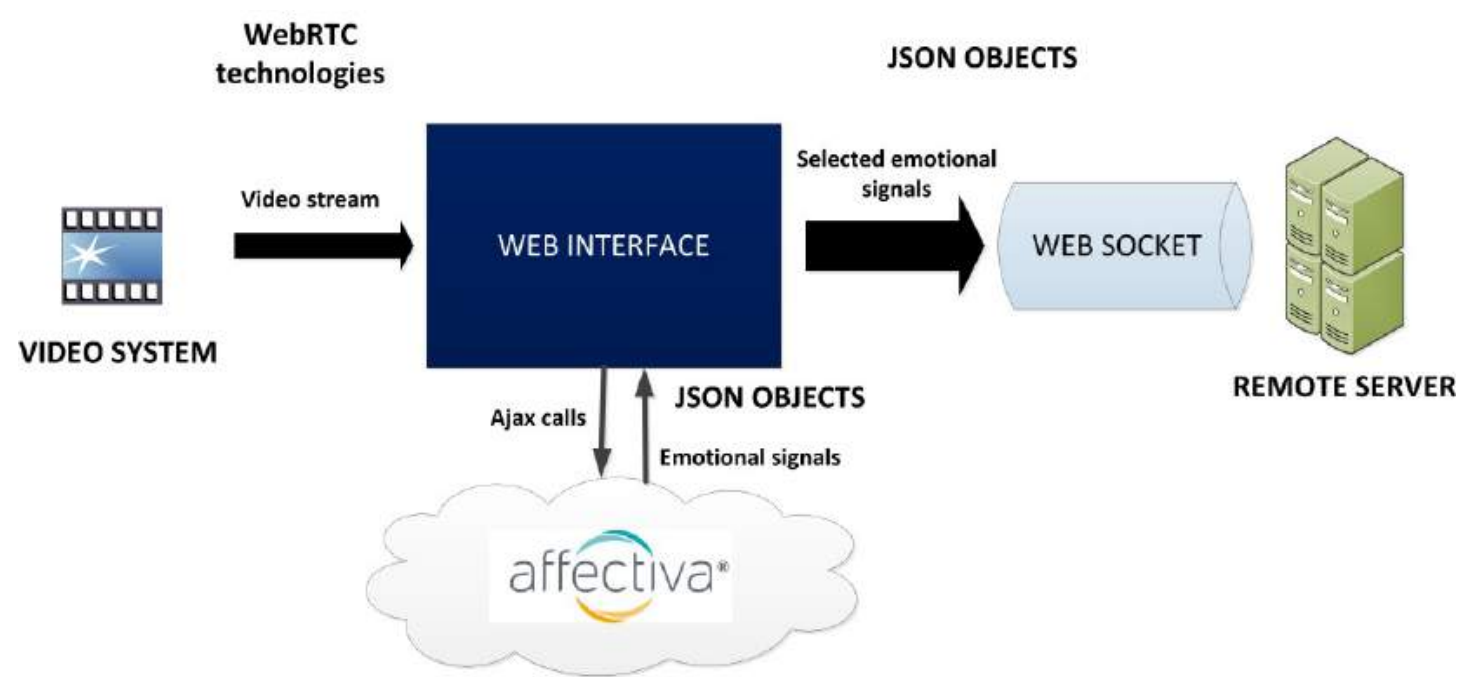

Figure 11. Scheme of the emotional signal obtaining module (functional architedture)

Additionally, the emotion tracking system offers information about the appearance of people being monitored. In particular four features are evaluated: gender, age, ethnicity and the presence of glasses. In advanced scenarios or applications, if desired, these data may be used to select different motivation models adapted to the particular characteristics of the person being monitored (so more precise results will be extracted). 
Table 1. Emotional signals obtained from the emotion tracking service

\begin{tabular}{|c|c|c|}
\hline \multicolumn{3}{|c|}{ Type of signal } \\
\hline Expressions & Emojis & Emotions \\
\hline Smile & Relaxed & Joy \\
\hline innerBrowRaise & Smiley & Sadness \\
\hline browFurrow & Laughing & Contempt \\
\hline noseWrinkle & Kissing & Anger \\
\hline UpperLipRaise & Dissapointed & Fear \\
\hline LipCornerDepressor & Rage & Surprise \\
\hline chinRaise & Smirk & Valence \\
\hline lipPucker & Wink & Engagement \\
\hline lipPress & StuckOutTongueWinkingEye & \\
\hline lipSuck & StuckOutTongue & \\
\hline mouthOpen & Flushed & \\
\hline Smirk & Scream & \\
\hline eyeClousure & DominantEmoji & \\
\hline \multicolumn{3}{|l|}{ Attention } \\
\hline \multicolumn{3}{|l|}{ lidTighten } \\
\hline \multicolumn{3}{|l|}{ jawDrop } \\
\hline \multicolumn{3}{|l|}{ Dimpler } \\
\hline \multicolumn{3}{|l|}{ eyeWiden } \\
\hline \multicolumn{3}{|l|}{ CheekRaise } \\
\hline lipStretch & & \\
\hline
\end{tabular}

\subsection{GLOBAL SYSTEM}

The global architecture of the proposed system is shown on Figure 12.

As can be seen, the global system is composed by the union of the physiological signal obtention module (see Section 3.2), the emotional signal obtention module (see Section 3.3) and a remote server where the mathematical model describing the human motivation is applied (see Section 3.1). Additionally, three new modules are added: two adaptation modules to incorporate the emotional and physiological signals to the mathematical model in a coherent way, and a web interface for managers where results are shown and from which they may control the entire system.

The new web interface consists of two static pages (see Figure 13) from which the system may be started, stopped or monitored. Moreover, in a second page, results about the motivation state are shown. 


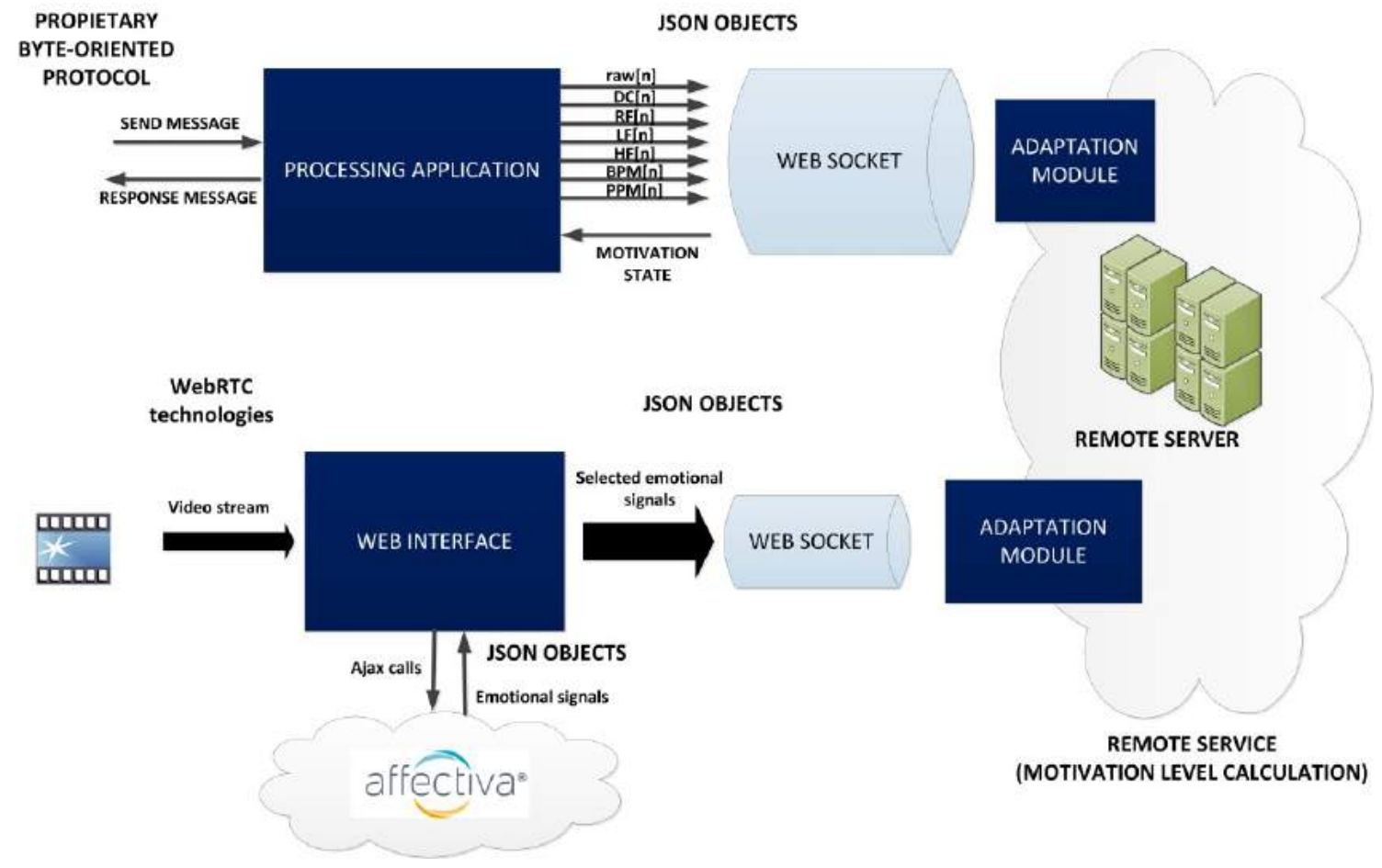

Figure 12. Global architecture (functional architecture) of the proposed solution

In respect to the adaptation modules two facts may be addressed. First, each signal presents a different dynamic range which is not recommendable in order to facilitate the motivation estimation. Second, the sample rate obtained from the physiological signals is much higher than which obtained from emotional signal. In order to obtain precise estimations both rates should be equal.

Thus, the adaptation modules present a first step where the signal's values are mapped into the range $[0,100]$, so the maximum and minimum values for every signal are now equal. Later, as we said in Section 3.2, the physiological signals (in order to respect the Nyquist criterion and truly represent the physiological information) present a rate of $\boldsymbol{R}_{\boldsymbol{p}}=\mathbf{1 0 0} \boldsymbol{s p s}$ (samples per second). However, the emotional signals (as they come from a video stream composed of $25 \mathrm{fps}$-frames per second-) present a

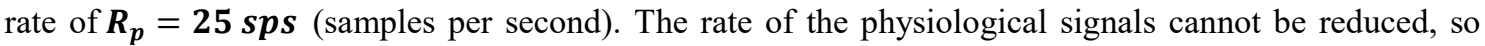
the rate of the emotional signals is increased in the corresponding adaptation module by adding an interpolation stage which expands the emotional signals by a factor four.

Once generated the adapted signals, they reach the remote server where they are introduced, sample by sample, in the human motivation mathematical model. Expression (8) should be used to obtain a faster but less precise estimation. Expression (10) generates better-quality information employing more calculation time. Any case, as result of the human motivation estimation, a collection of eight signals $\mathcal{L}$ are obtained, describing the motivation in real-time. These new signals are displayed in the web interface for result presentation.

As can be seen, models in expression (8) and expression (10) include, each one, a collection of unknown parameters. As said in Section 3.1, a calibration process should be performed in order to determine these values before. This process may be generic and, then, the same values for the unknown parameters are employed to estimate the motivation of every person. In another possibility, different values are employed to estimate the motivation of every person. This second option generates better results, but requires implementing a repository to store all the possible values and an identification service to determine the identity of the person being monitored (based, for example, on the face recognition system).

In this work, we have designed a first calibration process. The unknown parameters are considered to have the same value for all people, so the calibration process was performed only one time. As this work is not focused on optimizing or improving the calibration process, the proposed calibration is rather inefficient and it requires a remarkable amount of time (for a functional deployment this process should 
be enhanced). On the other hand, as the number of users to be monitored remains low (less than twenty users) no repository or identification service is required in this initial case.
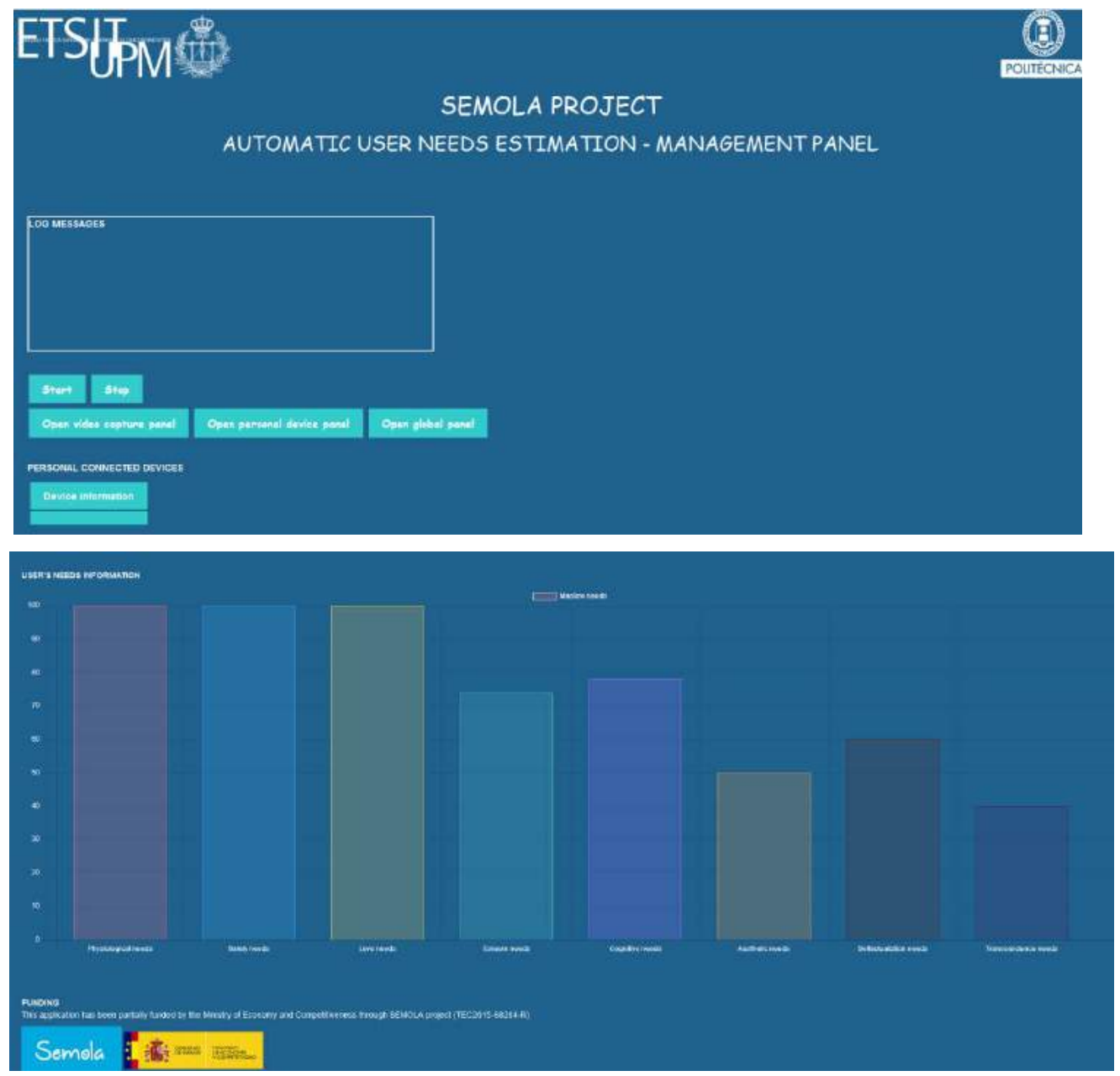

Figure 13. Web interface (management and results)

The personalized calibration process lasts around forty (40) minutes. As proposed by other authors (Duval et al., 2006), special multimedia contents will be visualized by users in order to excite a certain motivation state. Considering this previous work, it can be assumed that specific motivations can be realistically induced in subjects by exposing them to specific multimedia content. Then, measures to determine the value of the unknown parameters will be performed. Figure 14 shows the scheme of the proposed calibration process.

As can be seen, eight (8) different activities are programmed:

- At the first phase, a gallery of suggestive photos about food and water will excite the feelings of hunger and thirst in people (in a similar way as ads invoke these feelings). These physiological needs will carry users to the first motivation state, represented by the vector $\mathcal{L}=(\mathbf{5 0}, 0,0,0,0,0,0,0)$.

- During the second activity, people visualize a segment of the movie "REC" (2007) by Filmax. This movie is a psychological horror film which causes on people a sensation of unsafety and insecurity. In that way, the second motivation state $\mathcal{L}=(\mathbf{1 0 0}, \mathbf{5 0}, \mathbf{0}, \mathbf{0}, \mathbf{0}, \mathbf{0}, \mathbf{0}, \mathbf{0})$ is excited

- At the third stage, feeling about family, love and affection were come out by displaying a promotional video about soldiers coming home. The third motivation $\mathcal{L}=(\mathbf{1 0 0}, \mathbf{1 0 0}, \mathbf{5 0}, \mathbf{0}, \mathbf{0}, \mathbf{0}, \mathbf{0}, \mathbf{0})$ state is expected to appear. 


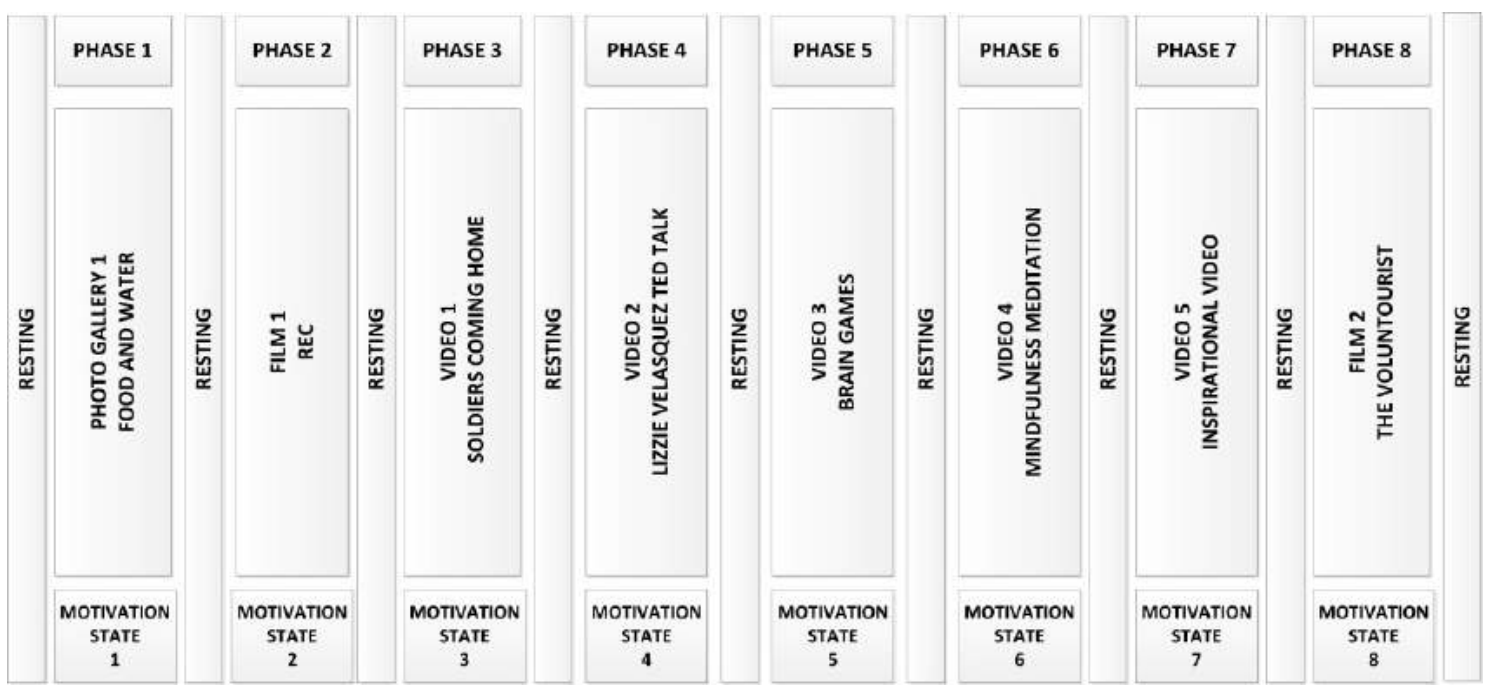

Figure 14. Temporal scheme of the calibration process. Sequence of activities

- In order to excite the fourth motivation state $\mathcal{L}=(\mathbf{1 0 0}, 100,100,50,0,0,0,0)$ the famous TED talk performed by Lizzie Velasquez about self-esteem, respect and self-confident was shown.

- A motivation state based on cognitive needs, described by the vector $\mathcal{L}=(100,100,100,100,50,0,0,0)$, was strengthen by showing a composition of short brain games (including their explanation).

- At the sixth phase, aesthetic needs must be enhanced in order to excite the sixth motivation state which is described by the vector $\mathcal{L}=(\mathbf{1 0 0}, \mathbf{1 0 0}, \mathbf{1 0 0}, \mathbf{1 0 0}, \mathbf{1 0 0}, \mathbf{5 0}, \mathbf{0}, \mathbf{0})$. A sensation of balance was caused by a video on mindfulness meditation (a segment of four minutes approximately).

- The seventh motivation state is caused by self-actualization needs such as realizing personal potential. In order to enhance these needs on people an inspirational video was displayed. A collection of eleven (11) videos were available, cheering up people to follow their objectives. The vector $\mathcal{L}=(100,100,100,100,100,100,50,0)$ describes this state.

- Finally, the eight motivation state $\mathcal{L}=(\mathbf{1 0 0}, \mathbf{1 0 0}, \mathbf{1 0 0}, \mathbf{1 0 0}, \mathbf{1 0 0}, \mathbf{1 0 0}, 100,50)$ was excited by showing a segment of the movie "The voluntourist" (2012) by The Voluntourist's team. This state was based on the need of helping the others (transcendence).

Some of these multimedia contents, in general, could not move some people to the desired motivational state. However, for this first implementation, it has been performed the calibration process choosing a person which is affected by the presented material.

Various measurements were performed during the calibration process, so the unknown parameters in the mathematical model $\left(\boldsymbol{\alpha}_{\boldsymbol{k}}, \boldsymbol{\beta}_{\boldsymbol{k}}\right.$ and $\left.\boldsymbol{\gamma}_{\boldsymbol{k}}\right)$ could be determined by means of the mean square errors technique (a set of linear equation will be obtained, whose solutions are calculated by optimizing the committed error).

In a first proof, it was observed that the attention paid by people to the audiovisual content contaminated video recognition measures. Therefore, a resting short period (around a minute) was placed before and after every activity. This period helped the system in two ways. First, the expression of attention disappeared from people's face and more realistic measures about emotion tracking could be performed. And, second, this resting period weaned the different activities.

Any case, different calibration processes could be designed depending on the type of organization, the culture, the gender, etc. 


\section{EXPERIMENTAL VALIDATION}

The validation of this paper aims to discuss whether the proposed mathematical models and the related technical solution correctly determine the motivation level of some users from the physiological and emotional signals. In order to evaluate the performance of the proposed technology, some experiments with users were proposed. In these experiments users were monitored and performed some activities. We used the proposed technical solution to measure their motivational level and compare it with an estimation extracted from traditional surveys. In this section we describe the validation process considering the context, plan and data gathered from the experiments.

\subsection{EXPERIMENT SETUP}

An experiment was conducted employing as main material the described system. The final objective of this experiment as to answer some questions regarding the effectiveness of the proposed technological solution, in terms of the validity of the obtained motivational state estimations.

Two research questions were formulated:

- Q1: The proposed solution estimates in an adequate way the people motivational state? In other words, is there any significant difference between the obtained result using the proposed technology and the obtained results using traditional evaluation techniques such as physiological surveys?

- Q2: Is the proposed processing algorithm fast enough to consider the proposed solution as a (almost) real-time technology? i.e. Does the system offer a result while the motivational state is still valid?

\subsection{METHOD AND PARTICIPANTS}

The validation described in this paper was planned, guided, monitored and evaluated by its authors (hereafter experts), who have more than five years of experience in knowledge management, Internet of Things and data analysis.

The experts chose a working environment as a scenario to carry out the experiments. This environment was recreated in a medium-size room at the Technical University of Madrid. A working environment is suitable to validate the proposed model and technical solution, as the ultimate goal of our contribution is to evaluate the people's motivation in Industry 4.0 scenarios in order to improve efficiency and productivity in companies. In this specific experiment, a software company was emulated.

Eighteen (18) people participated in the experiment, ranging from 26 to 56 years old. People had careers related to software engineering, working in ICT-related companies or research centers. All the participants were treated anonymously by the application and the experts. No personal data related to the volunteer identification were stored or diffused. All the experiments were performed under the conditions of respect for individual rights and ethical principles that govern research involving humans. The selection process performed by experts took into account different profiles, with various technical skills and experience level. The heterogeneity in the experimental groups (both, the control and the test groups) was planned so that volunteers provide different motivation-related responses in the defined activities. Groups were configured considering the principles of gender equality. It was guaranteed that all groups were composed of comparable populations. 
Three different groups of participants were defined: one control group and two test groups. Groups are employed to compare the obtained results using different estimation methods for the motivational state.

- Control group: This group performed the experiment without the monitoring of any engineered system. At the end of each activity, a regular (and universally accepted) survey (Chapman 2012) focused on estimating the motivation level using the Maslow's pyramid was performed by each participant. In general, the Maslow's hierarchy of needs may be understood as a "long term" theory (i.e. it refers people attaining/realizing something over a longer period in life). However, as indicated by the Maslow (Maslow et al., 1970) this theory is also valid to daily life and may be applicable to smaller time scales and short-term needs. Thus, specific motivations can be realistically induced in subjects by exposing them to specific multimedia content.

- Test group \#1: This group performed the planned experiment being monitored by the proposed system, where the simple calculation algorithm was implemented.

- Test group \#2: This group performed the planned experiment being monitored by the proposed system, where the complex calculation algorithm was implemented.

Participants were divided in a homogeneous way among the three defined groups. As the number of available personal biometric measuring devices was limited (as well as the number of computers) the experiment was performed in six different sessions, in three days in a row. In each session 3 users were involved. Each user performed the same experiment.

The calibration phase lasted around forty (40) minutes and was performed taking as reference one random additional person. In order to avoid distortions due to physiological and psychological fatigue caused by this large calibration phase, the additional person did not participate in the experiment (only performed the calibration process), and no more calibration tasks were developed with the other eighteen (18) participants. Besides, in order to preserve as maximum the spontaneity of the reactions, the personal that performed the calibration process was not aware of the final objective of the experiment. Any case, the calibration phase is a challenge to be solve in future works. Once the system was calibrated, each person performed a data acquisition phase (the experiment lasted around one hour for each person). In this phase, a sequence of six activities was planned:

- ACT\#1: Graphic design. In this activity people was asked to design (using a computer or manual techniques) a user interface. No requirements were established.

- $\mathrm{ACT} \# 2$ : Requirements capture. People under study in this step must capture the requirement about a certain application expressed by a couple of additional people (not under study) acting as a customers.

- ACT\#3: Programming. People were asked to program a certain algorithm (a searching algorithm in huge arrays) whose solution was provided using plane text.

- ACT\#4: Quality of assurance. An instructions brochure was provided to people, including a survey. Users had to perform the procedures indicted in the brochure and measure the required time to perform them. Information was fulfilled in the survey.

- ACT\#5: Management. Users were included in a representation of a management meeting were they should decide which people should be fired in order to not incur losses in the current year. Each member in the meeting was provided with a laptop.

- ACT\#6: Creative meetings. Finally users participated in a creative meeting were a new product was designed. Particularly, they participated in a brain storming session. As the Activity\#5, each member was provided with a laptop. 
In order to turn independent the feelings caused by each activity, a resting period was included before every of them. During this period people could not talk with the other participants.

\subsection{DATA GATHERING: TEST VARIABLES}

During the data acquisition phase three different primary information sources were considered. First, users in test groups were monitored while performing the activities described in Section 4.2 by means of a video system installed in the distributed laptops and personal computers. Second, each person was also provided with a personal biometric device (see Section 3.2) in order to capture the physiological signals (see Figure 15). These two information sources were connected to the motivation level calculator (see Figure 12) where both calculation algorithms (see Section 3.1) were running. Thus, two real-time estimations of the people motivation (made of a collection of eight signals, $\mathcal{L}$ ) were obtained. These collections were stored in a database so that statistical analysis function (normalization, interpolation, means, and median calculations) are performed. And third, and finally, a survey based on Chapman's proposals was answered by each participant in the control group. This survey was answered after each activity, during the resting period before starting the next one. The survey consisted of twenty five questions about the personal state after participating in each activity. Thus, a collection of eight values describing the motivation state at the final moment of each activity was obtained and stored in a database.
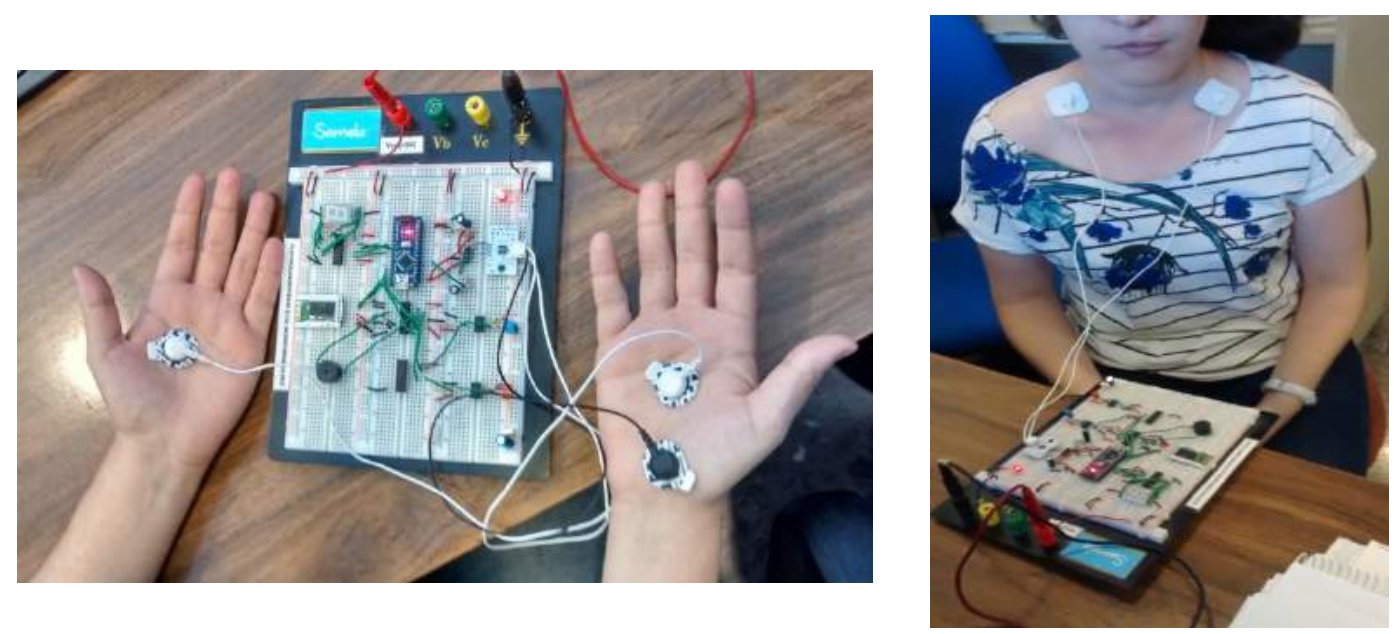

Figure 15: Data gathering during experimental validation

As instruments such as Chapman's surveys are employed nowadays in companies in order to evaluate the motivation of workers, we are considering that the information provided by this source describes the real motivation state of people. Our objective, therefore, is to evaluate how similar are the results obtained by our system to the results obtained using the survey.

Additionally, in order to evaluate the real-time operation of the system, a measure about the required time to obtain a motivational estimation using both proposed algorithms (see Section 3.1) were performed using simple timestamps programmed in the remote server.

\section{RESULTS AND DISCUSSION}

The box plot of the data shows that there are not relatively big differences between the groups (see Figure 16). It is important to note that although our proposed system records information in real-time, in order to compare the calculated results with the information provided by the surveys (which only evaluate the final state) only the information about the final moment is considered. This configuration is mandatory as no similar real-time systems are nowadays publicly available to do a more sophisticated comparison. However, it is important to remember (see Section 3.1) that the proposed algorithms consider the past 
values of the motivation state in order to calculate the following one, so that information is included in some way. Moreover, the particular moment considered to compare with the results obtained from the surveys is not relevant, as (in general) motivation state converges with time to a fixed value. Activities were planned to have a duration which allows reaching this convergence state.

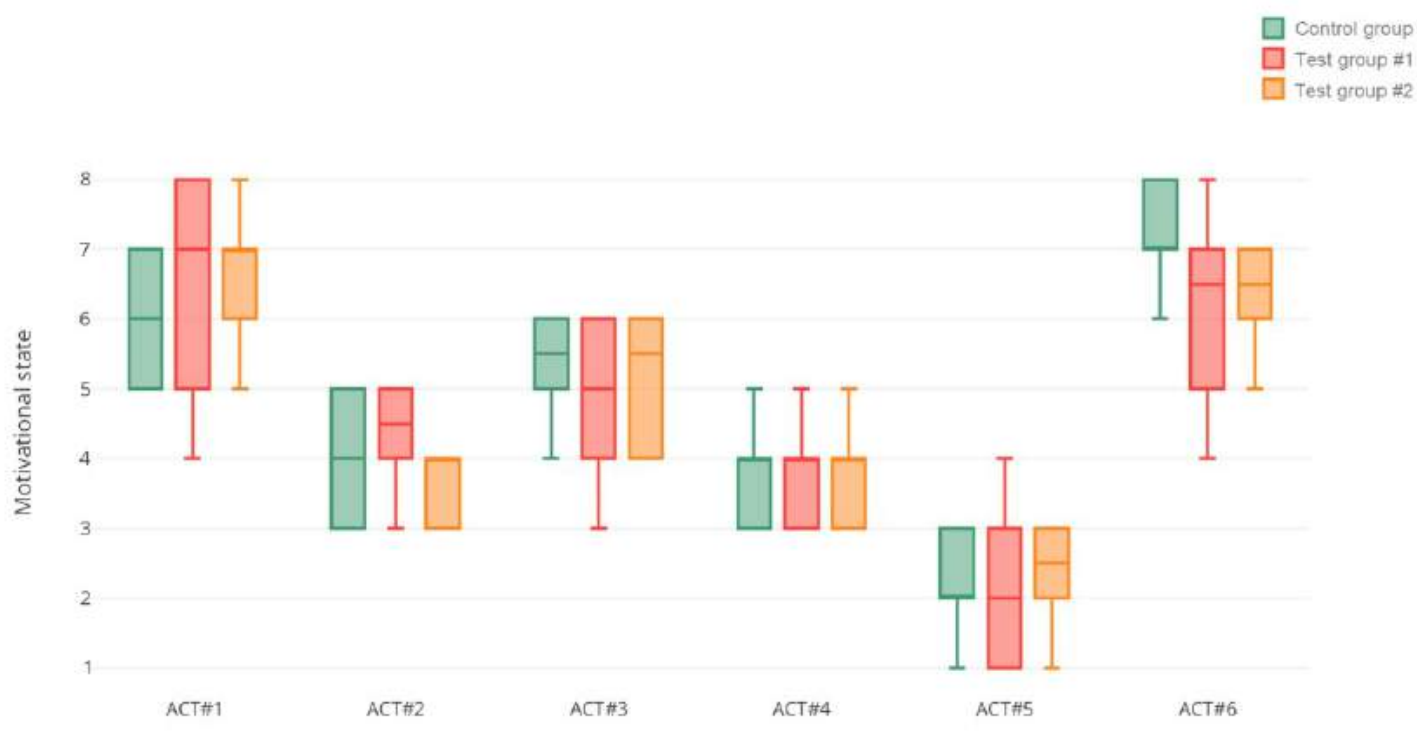

Figure 16: Obtained results. Comparison between both proposed calculation algorithms and traditional techniques

As both boxplots have an overlapping area, a Mann-Whitney U test was conducted to confirm whether the use of our proposed technology estimates the motivational state in the correct way. The MannWhitney $U$ test is a nonparametric test of the null hypothesis that two samples come from the same population against an alternative hypothesis, comparing the mean values of the two samples. It is used to evaluate if two different data populations are similar or different (higher or lower). The $\mathrm{p}$-value indicates the significance level of Mann-Whitney $U$ test. The results are positive and support the validity of the obtained results from the proposed system, considering any of the proposed algorithms (simple or realistic). The obtained value $p>0.05$ indicates there are not significant differences between any of the collected datasets (U-values omitted as the comparison results have relatively little importance). In that way, the first research question (Q1) may be answered in a positive way.

It is also important, however, to discuss the results considering each activity. See Table 2 were the significance of the obtained results (in comparison to the results generated by surveys) is evaluated. In general creative activities (such as ACT\#1 or ACT\#6) present a higher typical deviation, so it is more complicated to predict the motivation state in these cases using the proposed technique. In fact, these activities refer to motivation states such as self-actualization or transcendence, which are difficult to evaluate using physiological signals or face expressions. In order to improve this characteristic, a third information source could be added or a second-order effect (such as hysteresis functions or other type of corrections) could be considered in the model. For example, as modifying the mathematical model and calculation algorithm is a more complicate task, the communication patterns could be an interesting third information source to be added in an extension of this work. Using the previously described video infrastructure (see Section 3.3) and a new artificial vision technology, it could be inferred if workers are communicative, silent, isolated from the group, etc. These data usually are related to high-level needs, such as the need of helping the others to solve their problems. Including this information in the remote server through the appropriate adaptation module (see Figure 12), an enhanced assessment could be produced. 
Table 2. Performance of the proposed motivational state calculation algorithms depending on the activity being executed

\begin{tabular}{|c|c|c|c|c|c|c|}
\hline \multirow{2}{*}{ Algorithm } & \multicolumn{7}{|c|}{ Activity } \\
\cline { 2 - 7 } & $\mathrm{ACT} \# 1$ & $\mathrm{ACT} \# 2$ & $\mathrm{ACT} \# 3$ & $\mathrm{ACT} \# 4$ & ACT\#5 & ACT\#6 \\
\hline $\begin{array}{l}\text { Simple processing } \\
\text { algorithm }\end{array}$ & $*$ & $N S$ & $N S$ & $N S$ & $N S$ & $* *$ \\
\hline $\begin{array}{l}\text { Realistic (complex) } \\
\text { processing algorithm }\end{array}$ & $N S$ & $N S$ & $N S$ & $N S$ & $N S$ & $*$ \\
\hline
\end{tabular}

NS not significant; * significant at $p<0.05 ; * *$ significant at $p<0.005 ; * * *$ significant at $p<0.001$

Results obtained from the simple algorithm in ACT\#3 (programming) are remarkable. In general, typical deviation is similar for both algorithms. However in this activity it is much higher if the simple model is considered. A detailed study proved that as all signals are considered independent in the simple model, the emotional signal "attention" saturated the algorithm causing that an erroneous emotional state was estimated. In fact, as people remained focused on computer during this task, "attention" signal was very strong. As in the realistic model signals are considered dependent one on each other the effect of one signal in the final value is not so important, and results present a better quality.

Finally, in general, as business policies do not consider specific values, but global tendencies, an error around $15 \%$ can be perfectly tolerated (and even a $22 \%$ depending on the case). Thus, the proposed tool may be useful in Industry 4.0 scenarios.

Table 3 shows the required calculation time in each case. The average calculation time is obtained as the arithmetic average of all samples of the required time to update the motivational state in the management interface each time a change occurs in workers. The most probable calculation time is the most frequent value (those which appears most times) in the collected samples about the required time to update the motivational state (a 10\% tolerance margin is considered). Finally, maximum calculation time is the maximum value that appears in the mentioned samples.

Table 3. Evaluation of the calculation time for both proposed calculation methods

\begin{tabular}{|c|c|c|}
\hline \multirow{2}{*}{ Parameter } & \multicolumn{2}{|c|}{ Algorithm } \\
\cline { 2 - 3 } & Simple & Realistic \\
\hline $\begin{array}{c}\text { Average calculation } \\
\text { time (s) }\end{array}$ & 0.693 & 1.725 \\
\hline $\begin{array}{c}\text { Most probable } \\
\text { calculation time (s) }\end{array}$ & 0.517 & 1.421 \\
\hline $\begin{array}{c}\text { Maximum calculation } \\
\text { time (s) }\end{array}$ & 0.966 & 1.986 \\
\hline
\end{tabular}

As can be seen, the most probable calculation time in the case of using the realist algorithm is almost three times greater than the equivalent for the simple model. As the value is not so huge (in comparison to the change frequency of needs and motivation in standard humans), the realistic algorithm may be used in scenarios involving few workers, as the improvement in the precision is notable. However, in large companies, the simple algorithm could be more desirable in order to allow a truly real-time motivation station. Any case, the second research question (Q2) is also answered in a positive way. 


\section{CONCLUSIONS}

Industry 4.0 is a new paradigm which refers the inclusion of Ambient Intelligence and Cyber-Physical Systems principles into industry processes and scenarios. The final objective of this new revolution is to improve the productivity and efficiency in companies by means of the information technologies. One of the most important factors in companies is the human factor. Nowadays, human motivation is a key element in most companies, so surveys and psychological studies are usually performed.

The objective of this paper is to carry these methodologies to the next level, considering the Industry 4.0 paradigm. In particular, a system to estimate the human motivation is described. This system employs both emotional signals (obtained using video processing) and physiological signals (obtained by means of a biometric personal device also described in this work). The proposal includes, moreover, two different models (one simpler and one more realistic) in order to calculate the motivation of people in real-time.

Global results obtained from our proposal do not present significant differences in respect to which obtained from surveys. Nevertheless, second-order effect should be considered in the models in order to reduce these differences and a third information source about workers should be also included (especially when performing creative tasks). On the other hand, our proposal improves the exiting valuation techniques as it allows knowing the motivation state in real-time.

Future works, however, should address, mainly, three pending challenges: the optimization of the calibration phase, the creation of an unobtrusive ECG sensor to monito physiological signals, and the inclusion of soma correction in the proposed mathematical models in order to detect in a more precise way the growth needs.

In conclusion, the proposed system fulfills its objective, and helps companies to assess the human motivation in order to apply the most adequate business policies.

\section{REFERENCES}

Affectiva homepage http://www.affectiva.com/ (accessed on 20 August 2017)

Alderfer, C. P. (1969). An empirical test of a new theory of human needs. Organizational behavior and human performance, 4(2), 142-175.

Bailenson, J. N., Pontikakis, E. D., Mauss, I. B., Gross, J. J., Jabon, M. E., Hutcherson, C. A., ... \& John, O. (2008). Real-time classification of evoked emotions using facial feature tracking and physiological responses. International journal of human-computer studies, 66(5), 303-317.

Bassett-Jones, N., \& Lloyd, G. C. (2005). Does Herzberg's motivation theory have staying power? Journal of management development, 24(10), 929-943.

Bergkvist, A., Burnett, D. C., Jennings, C., \& Narayanan, A. (2012). Webrtc 1.0: Real-time communication between browsers. Working draft, W3C, 91 .

Berl, R. L., Williamson, N. C., \& Powell, T. (1984). Industrial salesforce motivation: A critique and test of Maslow's hierarchy of need. Journal of Personal Selling \& Sales Management, 4(1), 32-39.

BiTalino datasheet. http://bitalino.com/datasheets/ECG_Sensor_Datasheet.pdf (accessed on 20 August 2017)

Black, S. E., \& Lynch, L. M. (1996). Human-capital investments and productivity. The American economic review, 86(2), 263-267 
Bordel, B., Alcarria, R., Martín, D., Robles, T., \& de Rivera, D. S. (2017). Self-configuration in humanized cyber-physical systems. Journal of Ambient Intelligence and Humanized Computing, 8(4), 485-496.

Bordel Sánchez, B., Alcarria, R., Martín, D., \& Robles, T. (2015). TF4SM: a framework for developing traceability solutions in small manufacturing companies. Sensors, 15(11), 2947829510.

Brunstein, J. C., \& Schmitt, C. H. (2004). Assessing individual differences in achievement motivation with the Implicit Association Test. Journal of Research in Personality, 38(6), 536555.

Chapman, A. (1995 - 2012). Maslow's Hierarchy of Needs. Retrieved from http://www.businessballs.com/maslow.htm (accessed on 20 August 2017)

Colquitt, J. A., Greenberg, J., \& Zapata-Phelan, C. P. (2005). What is organizational justice? A historical overview. Handbook of organizational justice, 1, 3-58.

CrowdEmotion homepage http://www.crowdemotion.co.uk/ (accessed on 20 August 2017)

Datta, D. K., Guthrie, J. P., \& Wright, P. M. (2005). Human resource management and labor productivity: does industry matter?. Academy of management Journal, 48(1), 135-145.

Duval, S., Hashizume, H., \& Richard (2006), N. Physiological, Safety, and Belonging Needs: From Wearable Computers to Intelligent Environments.

Green, M., \& Sulbaran, T. (2006, October). Motivation assessment instrument for virtual reality scheduling simulator. In E-Learn: World Conference on E-Learning in Corporate, Government, Healthcare, and Higher Education (pp. 45-50). Association for the Advancement of Computing in Education (AACE).

Fujii, T., Nakano, M., Yamashita, K., Konishi, T., Izumi, S., Kawaguchi, H., \& Yoshimoto, M. (2013, July). Noise-tolerant instantaneous heart rate and r-peak detection using short-term autocorrelation for wearable healthcare systems. In Engineering in Medicine and Biology Society (EMBC), 2013 35th Annual International Conference of the IEEE (pp. 7330-7333). IEEE.

Herzberg, F. I. (1966). Work and the nature of man.

Herzberg, F. (1986). One more time: How do you motivate employees. New York: The Leader Manager, 433-448.

Imbrasaite, V., Baltrušaitis, T., \& Robinson, P. (2013, July). Emotion tracking in music using continuous conditional random fields and relative feature representation. In Multimedia and Expo Workshops (ICMEW), 2013 IEEE International Conference on (pp. 1-6). IEEE.

Kairos homepage https://www.kairos.com/ (accessed on 20 August 2017)

Kaiser, J. F. (1974). Nonrecursive digital filter design using the I_0-sinh window function. In Proc. IEEE Int. Symp. Circuits \& Syst., 1974.

Kroth, M., \& Kroth, M. S. (2007). The manager as motivator. Greenwood Publishing Group.

Kroth, M. Maslow-move aside! A heuristical motivation model for leaders in career and technical education (2007)

Lasi, H., Fettke, P., Kemper, H. G., Feld, T., \& Hoffmann, M. (2014). Industry 4.0. Business \& Information Systems Engineering, 6(4), 239-242.

Lee, C., Yoo, S. K., Park, Y., Kim, N., Jeong, K., \& Lee, B. (2006, January). Using neural network to recognize human emotions from heart rate variability and skin resistance. In Engineering 
in Medicine and Biology Society, 2005. IEEE-EMBS 2005. 27th Annual International Conference of the (pp. 5523-5525). IEEE.

MacDuffie, J. P., \& Krafcik, J. (1992). Integrating technology and human resources for highperformance manufacturing: Evidence from the international auto industry. Transforming organizations, 209226.

Martin, D. J., \& Loomis, K. S. (2013). Building teachers: A constructivist approach to introducing education. Cengage Learning.

Martín, D., Alcarria, R., Sánchez-Picot, Á., \& Robles, T. (2015). An ambient intelligence framework for end-user service provisioning in a hospital pharmacy: a case study. Journal of medical systems, 39(10), 116.

Maslow, A. H. (1943). A theory of human motivation. Psychological review, 50(4), 370.

Maslow, A. H., Frager, R., Cox, R. Motivation and personality (Vol. 2, pp. 1887-1904). J. Fadiman, \& C. McReynolds (Eds.). New York: Harper \& Row. 1970

McGregor, D. The human side of enterprise: 25th anniversary printing (1985). New York: McGrawHill..

Meyer, J. P., Stanley, D. J., Herscovitch, L., \& Topolnytsky, L. (2002). Affective, continuance, and normative commitment to the organization: A meta-analysis of antecedents, correlates, and consequences. Journal of vocational behavior, 61(1), 20-52.

Metallinou, A., Katsamanis, A., Wang, Y., \& Narayanan, S. (2011, May). Tracking changes in continuous emotion states using body language and prosodic cues. In Acoustics, Speech and Signal Processing (ICASSP), 2011 IEEE International Conference on (pp. 2288-2291). IEEE.

Mohino-Herranz, I., Gil-Pita, R., Ferreira, J., Rosa-Zurera, M., \& Seoane, F. (2015). Assessment of mental, emotional and physical stress through analysis of physiological signals using smartphones. Sensors, 15(10), 25607-25627.

Mustafa, H. (1992). The contributions of psychology to the study of administrative behaviour. International Journal of Public Sector Management, 5(4).

Neuvo, Y., Cheng-Yu, D., \& Mitra, S. (1984). Interpolated finite impulse response filters. IEEE Transactions on Acoustics, Speech, and Signal Processing, 32(3), 563-570.

Newton, J. T., \& Sturmey, P. (1991). The Motivation Assessment Scale: inter-rater reliability and internal consistency in a British sample. Journal of Intellectual Disability Research, 35(5), 472-474.

Nossal, K., \& Sheng, Y. (2010). Productivity growth: Trends, drivers and opportunities for broadacre and dairy industries. Australian Commodities: Forecasts and Issues, 17(1), 216.

Park, Byoung-Jun, et al. "Design of prototype-based emotion recognizer using physiological signals." ETRI Journal 35.5 (2013): 869-879.

Piccirillo, G., Vetta, F., Fimognari, F. L., Ronzoni, S., Lama, J., Cacciafesta, M., \& Marigliano, V. (1996). Power spectral analysis of heart rate variability in obese subjects: evidence of decreased cardiac sympathetic responsiveness. International journal of obesity and related metabolic disorders: journal of the International Association for the Study of Obesity, 20(9), 825-829.

Podsakoff, P. M., MacKenzie, S. B., Paine, J. B., \& Bachrach, D. G. (2000). Organizational citizenship behaviors: A critical review of the theoretical and empirical literature and suggestions for future research. Journal of management, 26(3), 513-563.

Rhoades, L., \& Eisenberger, R. (2002). Perceived organizational support: a review of the literature. 
Porter, L. W., \& Lawler, E. E. (1968). Managerial attitudes and performance.

Reeve, J. (2014). Understanding motivation and emotion. John Wiley \& Sons.

Rosenberg, R. D., \& Rosenstein, E. (1980). Participation and productivity: An empirical study. ILR Review, 33(3), 355-367.

Ryan, R. M., \& Deci, E. L. (2000). Intrinsic and extrinsic motivations: Classic definitions and new directions. Contemporary educational psychology, 25(1), 54-67.

Schmidt, R., Möhring, M., Härting, R. C., Reichstein, C., Neumaier, P., \& Jozinović, P. (2015, June). Industry 4.0-potentials for creating smart products: empirical research results. In International Conference on Business Information Systems (pp. 16-27). Springer, Cham.

Steers, R. M., Mowday, R. T., \& Shapiro, D. L. (2004). Introduction to special topic forum: The future of work motivation theory. The Academy of Management Review, 29(3), 379-387.

Tampoe, M. (1993). Motivating knowledge workers-the challenge for the 1990s. Long Range Planning, 26(3), 49-55.

Vroom, V. H. (1982). Work and motivation. Robert E. Krieger Publishing Company

Weisbord, M. R. (1987). Productive workplaces: Organizing and managing for dignity, meaning and community. Jossey-Bass.

Wickens, C. D., Hollands, J. G., Banbury, S., \& Parasuraman, R. (2015). Engineering psychology \& human performance. Psychology Press. 


\title{
Recognition of activities of daily living in Enhanced Living Environments
}

\author{
Borja Bordel $^{1 *}$, Marina Pérez-Jiménez ${ }^{2}$, and Diego Sánchez-de-Rivera ${ }^{1}$ \\ ${ }^{1}$ Department of Telematics Systems Engineering. Universidad Politécnica de Madrid, Spain \\ bbordel@dit.upm.es, diego.sanchezderiveracordoba@gmail.com \\ ${ }^{2}$ Department of Physical Electronics. Universidad Politécnica de Madrid, Spain \\ marina.perez@isom.upm.es
}

\begin{abstract}
Enhanced Living Environments consider the recognition of the Activities of Daily Living (ADLs) being performed by users as first step in the aid plans. Some works proved the information about the objects with which a person interacts robustly characterizes the ADL's identity. However, designing aid plans based on these raw data is a very complicated task, as an expert in both technology and occupational sciences is required. In addition, the plans produced by these experts are not platformindependent, to be closely linked to the hardware characteristics. Therefore, the aim of this paper is to design a two-phase solution capable of acquiring data from users, and of extracting information about the ADL being performed to trigger the execution of the proper aid plans. This solution disengages the technological and occupational domains, so that aid plans could be applied to any environment. Moreover, an experimental validation is conducted in order to validate the proposed technology as a valid solution for ADL recognition.
\end{abstract}

Keywords: HCI, ELEs, ADL, Enhanced Living Environments, Cyber-Physical Systems, Activities of Daily Living

\section{Introduction}

Helping assisted people to maintain an independent lifestyle is nowadays one of the most important research challenges. In this field, most remarkable solutions are based on seamless integration of Information and Communication Technologies (ICT) within context-aware homes with the aim of constructing Enhanced Living Environments (ELEs). Works such as [18] demonstrate the importance of these solutions.

Nevertheless, the design and implementation of these systems is a complex and multidisciplinary activity which deals with very different aspects which rarely can be addressed by only one person. Despite of this fact, most proposed ELEs are monolithic solutions where there is no division between the different scientific domains (such as the occupational sciences or electronics). Thus, works about ELEs use to show the necessity of data interpreters being specialist in both technological and occupational sciences [38]. Furthermore, such interpreters (as well as all models, patterns, etc. created by them) can only be used in some particular ELE platforms, as data format depends strongly on the underlying hardware and all the know-how created cannot be easily exported to other systems [13].

Among all the steps that compose the aid processes in ELEs, perhaps the most critical is the recognition of the activity the users are performing [28], vital for triggering the proper aid plan in each situation [18]. In context-aware homes (the base of ELEs) the most common activities are Activities of Daily

IT CoNvergence PRActice (INPRA), volume: 4, number: 4 (December 2016), pp. 18 31

${ }^{*}$ Corresponding author: E.T.S.I. Telecomunicación. Universidad Politécnica de Madrid. Avenida Complutense $n^{\circ} 30$. 28040 - Madrid (Spain), Tel. (+34) 915495700 ext. 3035 
Living (ADL) [15]. Some authors proved that the sequence of objects with which a person interacts characterizes the ADL being performed [30, 10]. Other authors, however, demonstrated implicit information (such as the hand gestures or emotions) is necessary to recognize ADL adequately [7, 9]. The utilization of computing elements to register this information is a challenge [23], as recently it has been proved that the introduction of computing elements in residences can lead stress in users and consequently modify the normal ADL execution and reduce the user quality-of-experience (QoE) [13].

Therefore, the objective of this paper is to describe a new a solution to be integrated in ELEs, being able to recognize ADLs automatically. We argue the use of Cyber-Physical Systems paradigm and reference architecture [24] allow the different abstraction levels of ELEs to become independent and, then, allow us to separate the different expert domains (becoming the aid plans platform-independent).

The authors, besides, built a prototype and carried out an experimental validation in order to validate the proposed solution as a valid technology for DL recognition. In particular, more than $85 \%$ of ADL are perfectly deducted using our proposal.

The rest of the paper is organized as follows: Section 2 introduces the state of the art in ADL recognition systems. Section 3 analyzes the requirements ELE scenarios, presents the functional architecture of the proposal and the implementation of the first prototype based on it. Section 4 provides an experimental validation of the solution. Finally, Sections 5 and 6 explain some results of this experimental validation and the conclusions of our work.

\section{State of the Art}

Activity recognition systems are made of two different parts: the monitoring subsystem and the recognition subsystem [36]. On the one hand, the monitoring subsystem acquires data from users such as users' location or the objects they touch. On the other hand, the recognition subsystem processes the acquired data and decides which is the ADL being performed.

Five main types of monitoring technologies can be identified [29]: passive infrared (PIR) motion sensors, body-worn sensors, pressure sensors, video monitoring and sound capturing. Deployments based on PIR motion sensors [25] consist of a set of sensors which detect user's position in the house. The position, as well as the time he remains in the same location, is used to recognize the ADL being executed. Body-worn sensors approach is based on different types of wearable sensors, such as accelerometers [3] or [33], with which users are provided and whose outcomes are evaluated during the activity. Other systems deploy several binary pressure sensors (they get active when touched) in home elements and places [17] and use the activation sequence of sensors to recognize the ADL being performed. Video monitoring is probably the most studied technology. With this name we refer to all technologies based on deploying cameras on the user's residence and using the recorded video to recognize the executed ADL [31, 26]. Finally, sound capturing systems consist of a set of microphones deployed in the house, allowing activity recognition by means of the audio analysis [21]. With respect to video monitoring, audio capturing presents fewer problems from the point of view of privacy.

In general, video monitoring and body-worn sensors are the most used technologies. They present less ambiguity and less noise level than the others, and often allow obtaining systems with more precision.

Very recently, activity recognition systems which do not present the monitoring subsystem have appeared [39]. However, their practical use is not clear and the obtained results are not as good as expected.

Basically, the recognition subsystem consists of a processing unit, executing an algorithm which extracts the useful information from data provided by monitoring subsystem, and decides the ADL being executed. This subsystem also stores models for all the ADL which want to be inferred. These models 
are expressed as the sequence of the data it is expected to receive from monitoring subsystem when executing a certain ADL. Thus, the process of recognition can be understood as finding the model $M=$ $\left\{m_{1}, m_{2}, \ldots, m_{n}\right\}$ that best explains the sequence of observations $O=\left\{o_{1}, o_{2}, \ldots, o_{k}\right\}$.

Some works [30] propose algorithms based on dynamic Bayesian networks which model the sequence of objects with which a person interacts. Other authors [37] propose algorithms which work with temporal probabilistic models (like naive Bayes or hidden Markov) and sensor readings. In some cases, researches try to recognize concurrent ADLs [40] and analyze the activity duration. Algorithms based on the analysis of the time a sensor is active [41] are also possible. Finally, solutions consisting of semantic technologies and ontology-based approaches have been also proposed [19].

In the ideal case, recognition subsystem would be independent of the monitoring subsystem. However, in all cases [25, 37], they are designed together as the recognition algorithm depends strongly on the data offered by the monitoring subsystem. Achieving this independence between monitoring subsystem (which belongs to the technological domain) and recognition subsystem (which belong to occupational sciences domain) is the goal of the proposed solution.

\section{Proposal: Toolkit for ADL Inference}

ELEs require precise information about the users' situation in order to execute the most appropriate aid plan in each moment. Therefore, activity recognition is an increasingly important functionality to be implemented in enhanced living systems. This section analyzes ELEs and the requirements to be met to enable precise information acquisition and ADL recognition. After describing the requirements of ELEs, we present the proposed solution and the design and implementation of a first functional prototype.

\subsection{Motivation Scenario: Requirements}

ADL recognition solutions present some important requirements which have been studied many times [14] (unobtrusiveness and flexibility are the most mentioned topics). Moreover, several prototypes and instruments in order to obtain information from users and perform ADL inference have been described [34, 4]. In this section, and taking into account these previous descriptions, some additional requirements (introduced by the specific needs of ELEs) are expressed:

- REQ\#1: ADL descriptions should be tolerant to the different possible performing ways.

- REQ\#2: The underlying hardware of ubiquitous computing system in ELEs should be tolerant to high levels of noise, light, vibration, etc.

- REQ\#3: ADL recognition system should incorporate instruments to create ADL models with minimum human effort.

- REQ\#4: Data acquisition should be transparent to users, in order to not negatively affect their daily lives.

- REQ\#5: The system should evaluate not only the identity of the ADL being performed, but also the quality of the execution. For example, in scenarios related to people with cognitive disorders in first stage aid plans depend strongly on the advance of the dementia.

\subsection{Solution Architecture}

Since 2000, several architectures have appeared in the literature to describe seamless integrations between the physical world and information technologies [6, 4]. Among all of them, cyber-physical systems (CPS) [16] are today which are receiving more attention. In that field, the reference architecture 
proposed in June 2015 by the National Institute of Standards and Technology (NIST) [24] stands out among other architectural proposals to be more general and to be considered the standard for CPS commercial products [4].

NIST's Architecture shown in Figure 1 a describes a whole system (in this case, a complete ELE). Then, for our purpose, it is possible to make a simplification (see Figure $1 \mathrm{~b}$ ). The problem of ADL recognition is basically a problem about data analytics [10, 20], thus, in general, the details about the superior and inferior levels are not important. In particular, the solutions used to control sensors and actuators do not affect the proposed system [1], so that "Sensors and actuators" and "Monitor and control system" levels can be merged into a single level ("Hardware platform"), of which it is only interesting the format and nature of the generated data. On the other hand, the subdivision of aid plans into objectives ("user goals" level) and methodologies ("Modeling, optimization and simulation" level) does not modify the following considerations, so that the two top levels can be reduced to a single level ("Occupational sciences plans") that concentrates all the elements belonging to the occupational sciences.

The resulting architecture is made of the following layers:
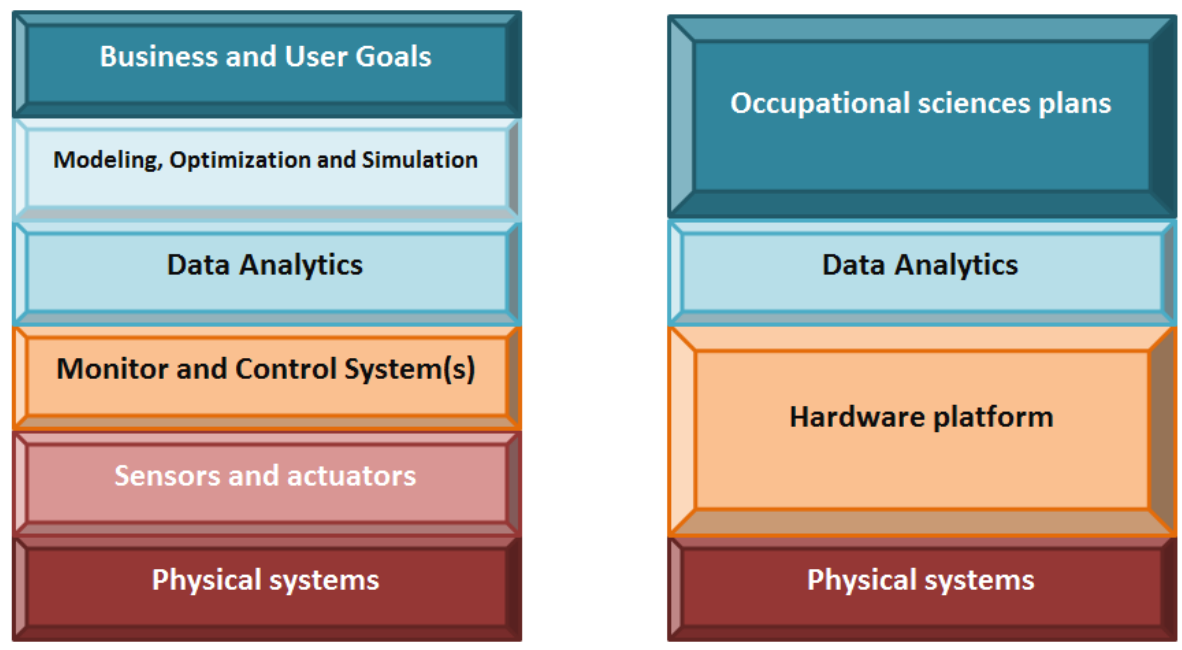

Figure 1: (a) Reference CPS architecture (NIST). (b) Reference architecture in our proposal

- Physical systems: It includes all elements in the ELE belonging to a regular residence. Furniture, kitchen and home appliances, grooming items... even the users belong to this layer.

- Hardware platform: It includes the electronic elements, processing devices and, in general, the entire ICT infrastructure deployed in the residence to monitor the users' behavior.

- Data Analytics: The inclusion of this layer is the novelty of this work. As can be seen in Figure 1 b, it uncouples the hardware layer (technological domain) and the occupational sciences models (obviously, occupational sciences domain). In this layer data from hardware platform is processed for pattern recognition and information extraction. At this level, it is evaluated the correlation between high-level models and the data generated by the hardware platform.

- Occupational sciences plans: It includes the aid plans and activities models created by the occupational sciences experts, using their own description languages, data format and, in general, their domain knowledge.

Following the reference architecture shown in Figure $1 \mathrm{~b}$, we developed our proposal. The functional architecture of the proposed technology can be seen in Figure 2. System operation is as described below. 


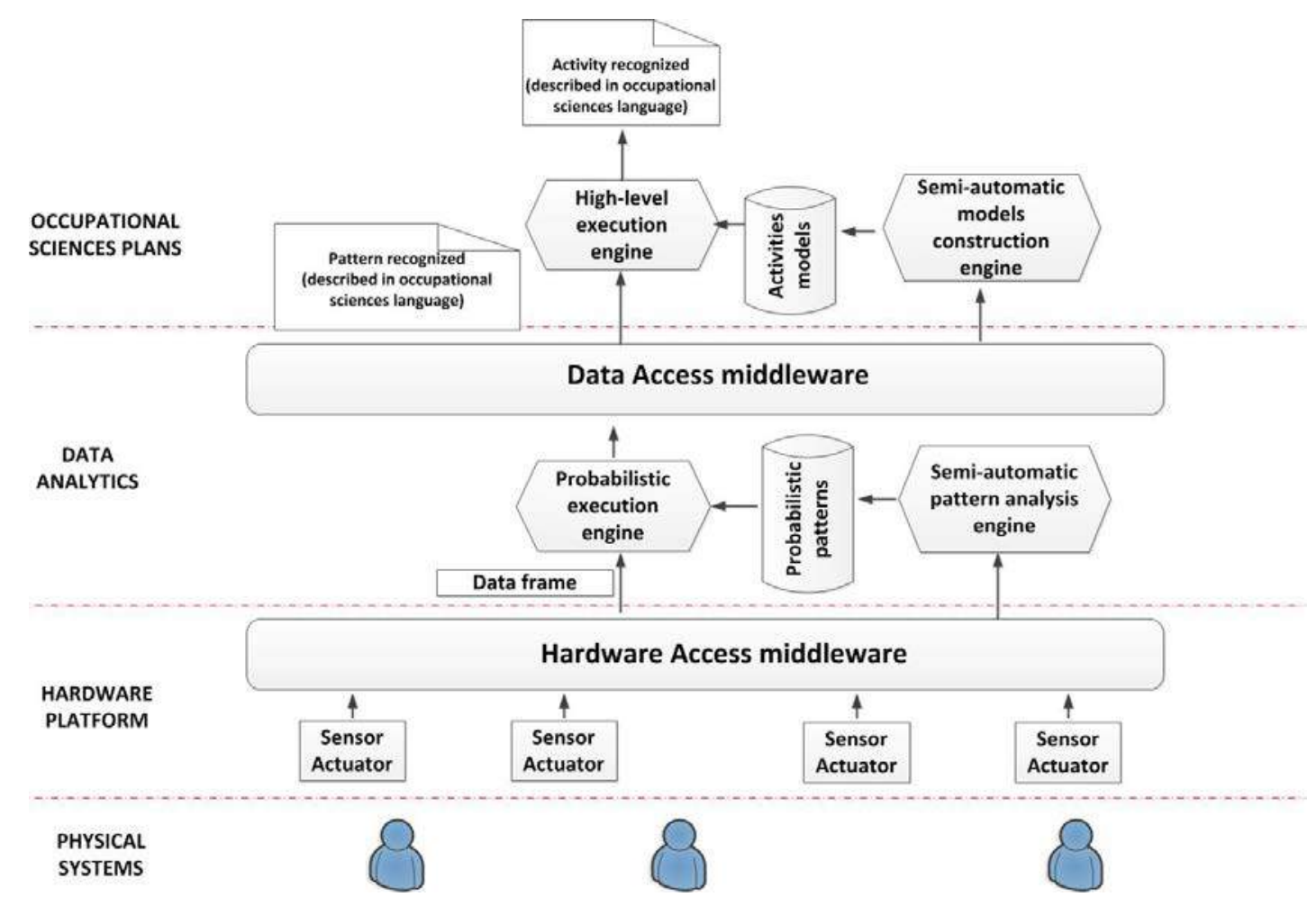

Figure 2: Functional architecture of the proposed technology

Users perform their usual activities of daily living. While executing the ADLs, they cause that sensors deployed along the residence generate data, which are transmitted in the form of frames through the appropriate hardware access middleware. Any type of middleware is valid, although considering it has to communicate two groups of devices made of various entities, Publication/Subscription paradigm [22] is probably the most adequate.

Two different operation modes can be distinguished in the proposal: training mode and recognition mode. In training mode, data frames from hardware platform are directed to a Semi-automatic pattern analysis engine, which studies the received data using machine-learning algorithms [12]. This engine will construct the data patterns associated with the atomic tasks defined in occupational sciences [2] to describe ADLs. Users are asked to execute each one of the atomic tasks, and for each one the engine will build at least one pattern which will be stored in a probabilistic pattern repository with the atomic task that represents. In recognition mode, data frames from hardware platform are directed to a probabilistic execution engine, which evaluates which of the stored patterns in the repository corresponds to the received data. In this evaluation, any of the algorithms described in Section 2 can be used. The output of this engine is a description of the recognized pattern, indicating the atomic task which has been identified using the terminology of occupational sciences [2].

The information about the recognized atomic task is transmitted through a Data access middleware, for which the same considerations made for the Hardware access middleware are valid.

In training mode, information about the recognized atomic task is directed to a Semi-automatic models construction engine, which will build the complete ADL models by means of the analysis of the information received. The ADL models (understood as a collection of atomic tasks, as in the occupational sciences [2]) will be stored in an activities models repository. Once completely trained the Data Analytics components, users are asked to execute the entire ADLs in order to calculate the ADLs models. In recognition mode, information about the recognized atomic task is directed to a high-level execution 
engine, which evaluates which of the stored models in the repository corresponds to the received data. In this evaluation, any of the algorithms described in Section 2 can be used. The output of this engine is a description of the recognized ADL, using the terminology of occupational sciences.

The use of probabilistic models and algorithms (both in Data analytics and Occupational sciences plans levels), as well as the fact that models and patterns are calculated in a training period involving users, allow us to fulfil the REQ\#1 introduced in Section 3.1. Also, this training period allows creating automatically ADL models, so REQ\#3 is also addressed.

The introduction of two recognition processes presents various advantages. First, the technological domain and the occupational sciences domain become independent. With this configuration, occupational sciences experts can design aid plans without knowing the details about the hardware platform. For example, experts can design an aid plan including an action after executing a certain atomic task, without knowing the particular datum or event which determines the end of the task.

In a more particular example, one of the simplest ADLs is "preparing a simple drink" [30]. The shortest definition of this ADL, as a collection of atomic task, could be: (1) taking the soda bottle that is on the table, (2) filling the glass which is on the table with soda and (3) leaving the bottle on the table again [27]. In scenarios involving people with dementia, it is really important to avoid accidental poisonings, so it is valuable to alert users and caregivers (if any) when they select the wrong bottle. Such notification, in our proposal, can be planned and modified by experts in occupational sciences without having to know which hardware event indicates the bottle selection at technological level. Moreover, hardware platform can be modified without having to reprogram the aid plan.

It must be noted that, as atomic tasks are indivisible in the occupational sciences theory, it has no sense to consider actions while one of them is being executed.

Second, in works which do not consider that ADLs can be described as a collection of atomic task (such as "take a glass" or "press a button"), recognition algorithms must address together all the sources of randomness in ADLs execution. Basically [3] they include sensors errors, noise and interferences in the medium and the variations in human behavior. In our solution, we separate these sources in two groups: effects due to hardware (solved in Data Analytics level) and due to the intervention of users (solved in Occupational sciences plans level). In that way, each problem's part can be addressed separately by the proper domain experts. These politics partially cover REQ\#2.

Finally, the accuracy of the resulting system may be greater than in the one from previous proposals [30]. The execution of atomic tasks has less ambiguity than the execution of complete ADLs, so the success rate of the probabilistic engine tends to be high. Later, once the atomic tasks are properly recognized, the algorithms to recognize ADLs also increase their success rate since they have more information, as they perfectly know the atomic tasks that have been executed.

\subsection{Implementation}

In this section, we design and build a first prototype in order to validate its usability as functional part of ELEs.

First, we must consider the requirements the prototype should fulfill. In the functional architecture design, we solved in a general way REQ\#1 and REQ\#3. It is also partially covered REQ\#2. However, it remains unsolved three requirements (REQ\#4, REQ\#5 and the rest of REQ\#2) which cannot be addressed in the functional architecture, as they depend on the hardware platform and the particular implementation done. Figure 3 shows the physical architecture of the proposed prototype.

Various elements can be distinguished in Figure 3 .

- Tagged products: All elements involved in the execution of ADLs (such as the kitchen appliances) get tagged with passive NFC tags. The objective is detecting when a user interacts with a particular 

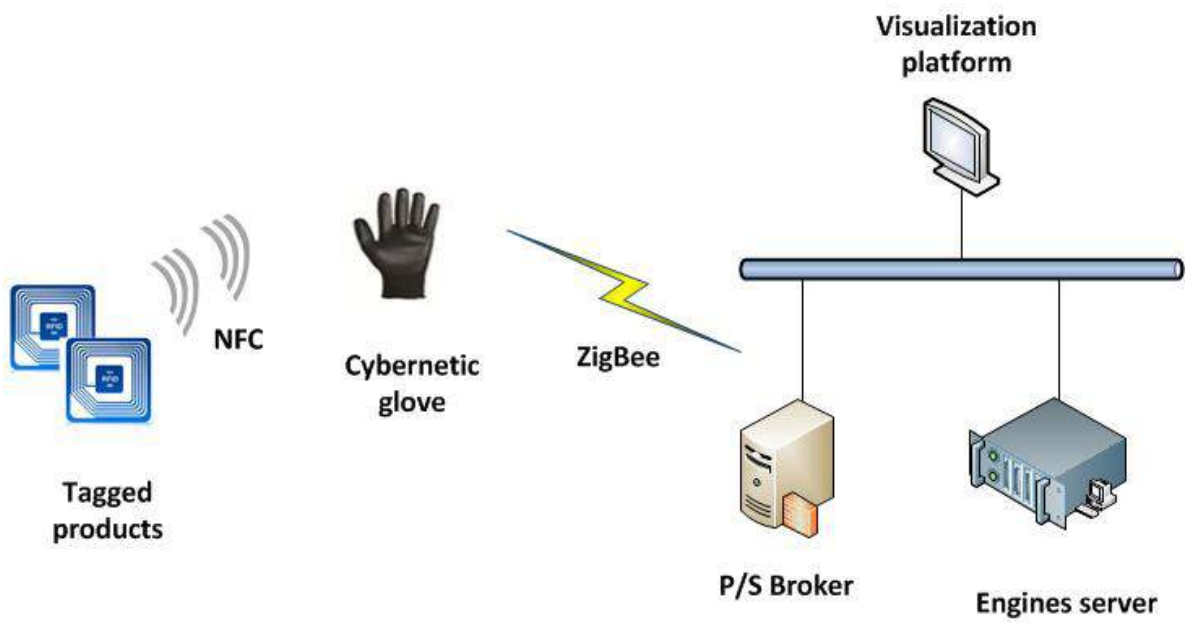

Figure 3: Proposed physical architecture

object (as described, for example in [33]). With this election, REQ\#2 is fulfilled. NFC technology is extremely tolerant to noise, vibrations, manipulation, etc. much more than any other technology (such as PIR motion sensors). Tags should be place in the proper way, in order to respect REQ\#4.

- Cybernetic glove: Users will be provided with a cybernetic glove including a NFC reader and various accelerometers. In that way, both information sources for ADL inferring are monitored: explicit (the objects with which users interact [30, 10]) and implicit (the hand gestures done during the activities execution [7, 9]). The use of accelerometers, besides, allows obtaining a measure of the quality of ADLs execution [33] (which addresses REQ\#5, although this kind of algorithms is not the objective of this article). As most involved objects in ADLs execution are hand-manipulated, creating a cybernetic glove is the most reasonable solution in order to obtain correct readings from NFC tags. However, if there were users with special needs or it was wanted to recognize other type of activities, any other NFC-based wearable device could be integrated. Finally, the glove should be designed in order to respect REQ\#4. Moreover, the glove will use a Publication/Subscription middleware based on ZigBee technology, in order to transmit the data frames [32].

- P/S Broker: This element allows the spatial and temporal decoupling between publishers and subscribers. However, as this element belongs to the networking and communications plane and was not the focus of this work, it is omitted in the rest of the article (an in-depth discussion about this subject can be found in articles, such as [11]).

- Engines server: This server executes in different processes the four engines identified in the functional architecture (see Figure 21. This server also contains the two repositories (patterns and models) described above. The communication between the different components was based on sockets.

- Visualization platform: On this platform the results of the ADL recognition process will be shown (using the terminology of occupational sciences).

Finally, we are discussing the implementation details of each component (especially about the cybernetic glove which must be designed to address REQ\#4). The cybernetic glove [34] consists of a flexible circuit placed on the wrist as a bracelet, a galvanized copper coil integrated in the fabric to allow NFC communication, two accelerometers (one placed on the index finger and the other in the center of 
the hand) and a synthetic textile support, whose properties do not significantly affect the magnetic field generated by the NFC module [34]. The integration of the electronic circuit into the glove is greatly important, as it must be designed to fulfil REQ\#4.

The engines server is composed of four different Java programs, connected among them through sockets. The Semi-automatic pattern analysis engine executes a pattern recognition algorithm called "Nonparametric clustering", which allows detecting patterns without having any previous knowledge. A complete description of the algorithm can be found in [12]. Later, considering the type of monitoring technology included in the prototype, the most appropriate algorithm to be executed in the probabilistic execution engine is a temporal probabilistic model as described in [37]. Both, accelerometers and RFID reader information is combined as explained in [35].

In occupational sciences, there is not a unique uniform description language, so one of the usually used must be chosen to be included in the prototype. Basically there are two proposals: the American one [2, 5] (made by the American Occupational Therapy Association) and the European one [8] (encouraged European network of Occupational Therapy). Because the European project normalizes terminology, not only in English but also in Spanish and German, among others languages, we decided to use their proposal [8]. So, the output of the probabilistic execution engine and the high-level execution engine will be expressed following those recommendations.

Finally, the high-level execution engine will execute an algorithm based on Bayesian networks, equal to described in [30]. In this work, it can be found also an algorithm to construct the Bayesian networks, which will be implemented in the Semi-automatic models construction engine.

The visualization platform is composed of a Java GUI where the result of the recognition process is shown.

\section{Experimental Validation}

An experiment was designed in order to validate the proposed solution as a valid technology for ADL recognition in ELEs. In particular, the experimental validation consisted of two different experiments.

In the first experiment, the rate of ADL correctly detected is evaluated. The proposed technology is deployed in a laboratory of the Technical University of Madrid, where a house-like scenario is created (see Figure 4). A traditional solution based on PIR sensors was also implemented in a similar space [25]. Eighteen people (18) were involved in the experiment. Nine people (9) performed activities in the space where our solution was available. The other people (nine) worked in the space where the PIR-based system was implemented.

A description about the activities to be performed in the different spaces was provided to participants. Table 1 shows the list of panned ADLs.

Information about the inferred activities by both deployed systems was collected and compared with the created plans. In order to do that, the Engines server was programmed to create a log file. Finally, the rate of successfully deducted ADL was calculated.

In the second experiment, the user satisfaction is evaluated by means of a survey. People using both ADL recognition systems were asked, once finished the first experiment, to fulfill a survey about their satisfaction in respect to the experience. Results are processed using statistical software in order to compare the obtained responses.

\section{Results and Discussion}

In this section we present the results obtained in the experimental validation. Figure 5 shows the aggregated results of the first experiment. As can be seen, PIR-based solutions present a rated of successfully 


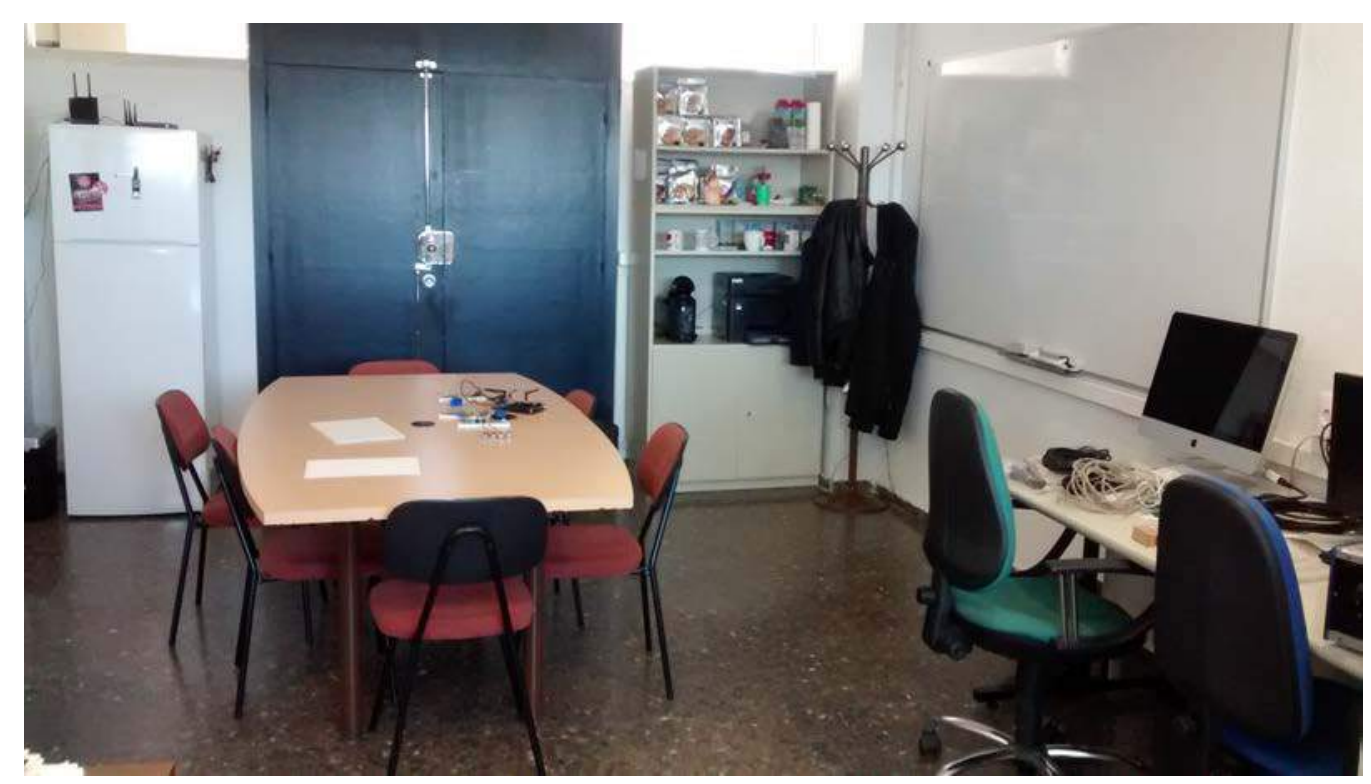

Figure 4: House-like space at the Technical University of Madrid

\begin{tabular}{cc}
\hline Activity & Evaluation form \\
\hline Transfer & Moves in and out of bed and/or in and out of chair \\
\hline Feeding & Gets food from plait or equivalent into mouth \\
\hline $\begin{array}{c}\text { Going to } \\
\text { toilet }\end{array}$ & $\begin{array}{c}\text { Gets on and off toilet } \\
\text { Arranging clothes }\end{array}$ \\
\hline Bathing & $\begin{array}{c}\text { Sponge bath } \\
\text { Tub bath } \\
\text { Shower }\end{array}$ \\
\hline Dressing & Gets clothes from closets and drawers \\
& Puts on clothes \\
\hline
\end{tabular}

Table 1: List of planned ADLs

inferred ADLs around 74\%, while in the proposed technology is around $85 \%$. This improvement is due to the proposed separation between variation due to hardware and due to humans, which allow introducing a double verification phase. Moreover, many ADLs are performed using hands, so if these extremities and their movements are controlled, more precise results are obtained (in PIR-based solutions only body movements are monitored).

However, this improvement is not homogeneous. Figure 6 presents the results of the first experiment, disaggregated per ADL.

As can be seen, activities performed, mainly, using the hands are easily detected by means of the proposed technology. Nevertheless, if the ADL implies body movements (as in transfer ADL), PIRbased technologies are more adequate and success rate is higher.

In respect to the second experiment, Figure 7 shows the results of the satisfaction survey.

A Mann-Whitney $U$ test was conducted in order to test if both surveys were statistically equal; we 


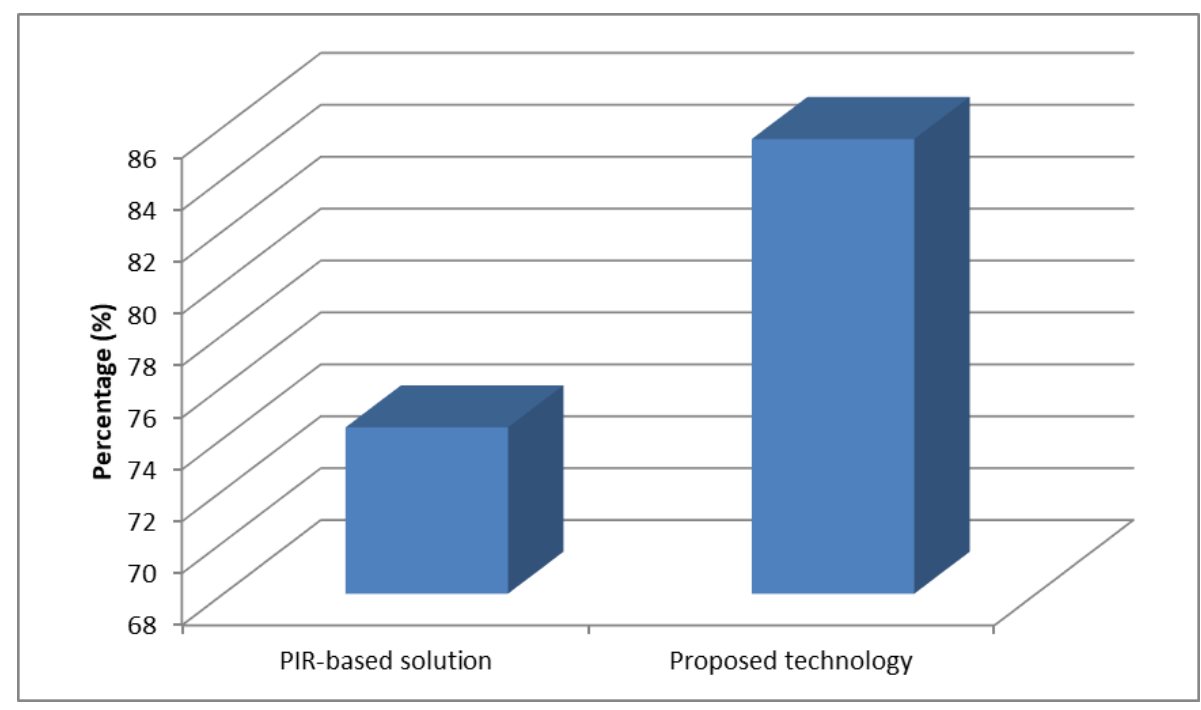

Figure 5: Aggregated results of the first experiment

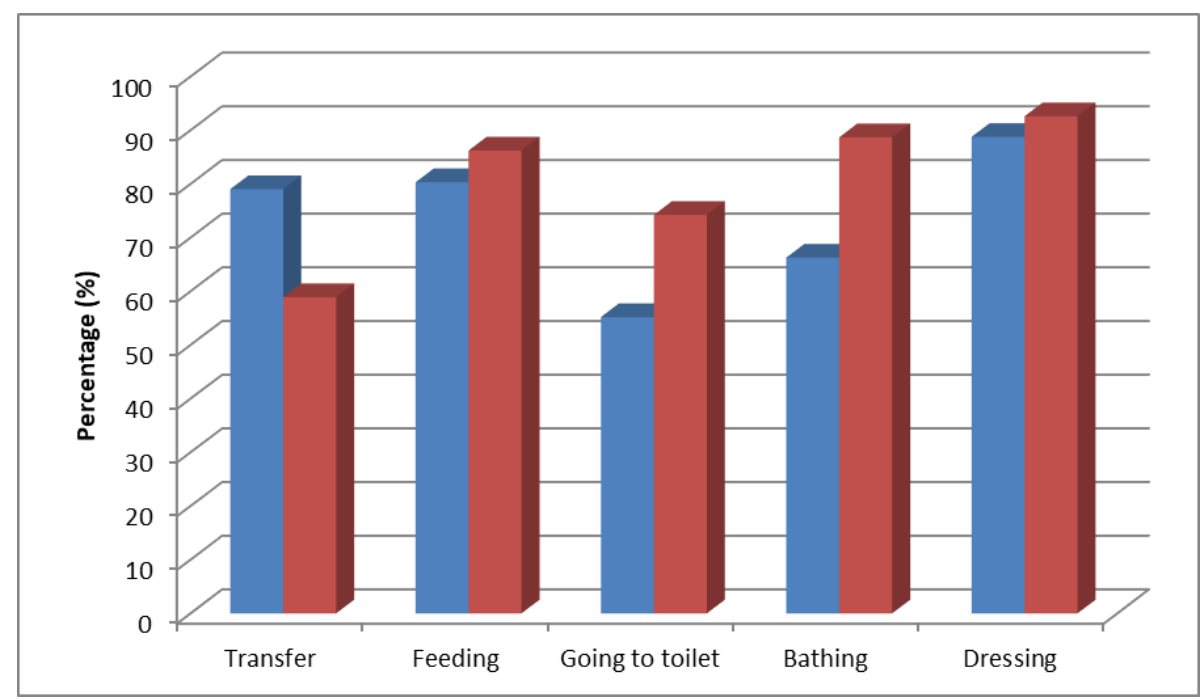

Figure 6: Disaggregated results of the first experiment
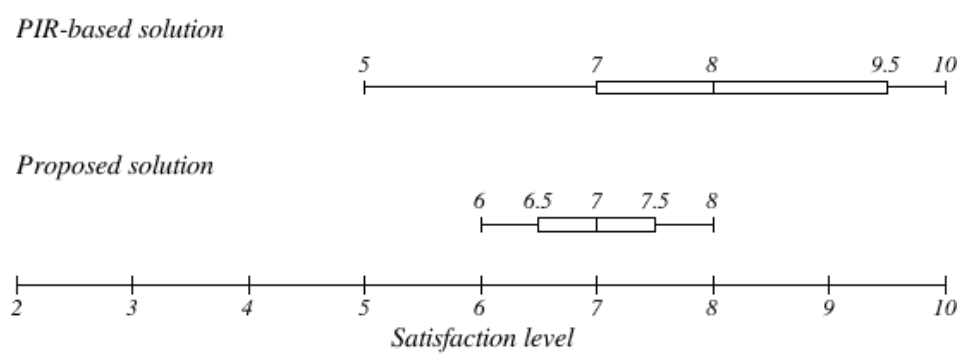

Figure 7: Results of the second experiment

could not find any statistical evidence confirming the difference between the two samples. Then, we can say both technologies provide the same degree of satisfaction. 


\section{Conclusions}

Enhanced living environments (ELEs) are rapidly becoming in one of the most profitable research fields. Seamless integrations of Information and Communication Technologies (ICT) within context-aware homes are usually the technological base over which ELEs are built. In this field, ADLs recognition is one of the most critical and widely studied activities. However, most proposed systems are monolithic solutions where there is no division between the different scientific domains, making difficult and costly their maintenance and modification. Our technology pretends to address this problem by means of a functional architecture based on Cyber-Physical paradigm and reference architecture.

In our solution, technological domain and occupational sciences domain become independent, which enables the deployment of systems only attended by expert caregivers or occupational therapists (without the intervention of hardware technicians).

We provided also two experimental validations with real users. These experiments showed that the proposed technology improves the overall rate of success; especially in ADL performed suing the hands (compared to traditional PIR-based approaches). Moreover, despite the use of a cybernetic glove, the user satisfaction level in both technologies is equivalent.

\section{Acknowledgments}

The research leading to these results has received funding from the Ministry of Economy and Competitiveness through SEMOLA project (TEC2015-68284-R) and from the Autonomous Region of Madrid through MOSI-AGIL-CM project (grant P2013/ICE-3019, co-funded by EU Structural Funds FSE and FEDER). Borja Bordel has received funding from the Ministry of Education through the FPU program (grant number FPU15/03977)

\section{References}

[1] R. Alcarria, T. Robles, A. Morales, and E. Cedeño. Resolving coordination challenges in distributed mobile service executions. International Journal of Web and Grid Services, 10(2/3):168, 2014.

[2] American Occupational Therapy Association. Occupational Therapy Practice Framework: domain and process. The American journal of occupational therapy : official publication of the American Occupational Therapy Association, 56(6):609-39, 2002.

[3] O. Banos, M. Damas, H. Pomares, A. Prieto, and I. Rojas. Daily living activity recognition based on statistical feature quality group selection. Expert Systems with Applications, 39(9):8013-8021, jul 2012.

[4] B. Bordel Sánchez, R. Alcarria, D. Martín, and T. Robles. TF4SM: A Framework for Developing Traceability Solutions in Small Manufacturing Companies. Sensors, 15(12):29478-29510, nov 2015.

[5] M. J. Borst and D. L. Nelson. Use of uniform terminology by occupational therapists. The American journal of occupational therapy : official publication of the American Occupational Therapy Association, 47(7):6118, jul 1993.

[6] D. J. Cook and S. K. Das. Smart environments : technologies, protocols, and applications. John Wiley, 2005.

[7] R. Cowie, E. Douglas-Cowie, N. Tsapatsoulis, G. Votsis, S. Kollias, W. Fellenz, and J. Taylor. Emotion recognition in human-computer interaction. IEEE Signal Processing Magazine, 18(1):32-80, 2001.

[8] ENOTHE Project (Occupational Therapy Terminology). Available online: http://enothe.eu/, last viewed May 2017.

[9] G. Fischer. User Modeling in Human-Computer Interaction. User Modeling and User-Adapted Interaction, 11(1/2):65-86, 2001.

[10] K. Fishkin, M. Philipose, and A. Rea. Hands-On RFID: Wireless Wearables for Detecting Use of Objects. In Ninth IEEE International Symposium on Wearable Computers (ISWC'05), pages 38-43. IEEE, 2005. 
[11] G. Fortino, A. Guerrieri, and W. Russo. Agent-oriented smart objects development. In Proceedings of the 2012 IEEE 16th International Conference on Computer Supported Cooperative Work in Design (CSCWD), pages 907-912. IEEE, may 2012.

[12] K. Fukunaga. Introduction to Statistical Pattern Recognition (2Nd Ed.). Academic Press Professional, Inc., San Diego, CA, USA, 1990.

[13] A. Grguric, A. M. M. Gil, D. Huljenic, Z. Car, and V. Podobnik. A Survey on User Interaction Mechanisms for Enhanced Living Environments. In ICT Innovations 2015, pages 131-141. Springer, Cham, 2016.

[14] M. P. Jimenez, B. B. Sánchez, and R. P. A. Garrido. T4ai: A system for monitoring people based on improved wearable devices. Research Briefs on Information \& Communication Technology Evolution (ReBICTE), 2:1$16,2016$.

[15] S. Katz, A. B. Ford, R. W. Moskowitz, B. A. Jackson, and M. W. Jaffe. Studies of Illness in the Aged. The Index of ADL: A Standardized Measure of Biological and Psychosocial Function. JAMA, 185:914-9, sep 1963.

[16] E. Lee and E. A. The Past, Present and Future of Cyber-Physical Systems: A Focus on Models. Sensors, 15(3):4837-4869, feb 2015.

[17] J. Lester, T. Choudhury, and G. Borriello. A Practical Approach to Recognizing Physical Activities. In Lecture Notes in Computer Science, pages 1-16. Springer, Berlin, Heidelberg, 2006.

[18] S. Loshkovska and S. Koceski. ICT innovations 2015: emerging technologies for better living. Springer International Publishing, 2015.

[19] H. Maki, H. Ogawa, S. Matsuoka, Y. Yonezawa, and W. M. Caldwell. A daily living activity remote monitoring system for solitary elderly people. In 2011 Annual International Conference of the IEEE Engineering in Medicine and Biology Society, pages 5608-5611. IEEE, aug 2011.

[20] D. Martín, J. G. Guzmán, J. Urbano, and J. Llorens. Patterns as objects to manage knowledge in software development organizations. Knowledge Management Research \& Practice, 10(3):252-274, sep 2012.

[21] D. Maunder, J. Epps, E. Ambikairajah, and B. Celler. Robust sounds of activities of daily living classification in two-channel audio-based telemonitoring. International journal of telemedicine and applications, 2013:696813, 2013.

[22] A. Morales, R. Alcarria, D. Martin, and T. Robles. Enhancing Evacuation Plans with a Situation Awareness System Based on End-User Knowledge Provision. Sensors, 14(6):11153-11178, jun 2014.

[23] L. Muguira, J. I. Vazquez, A. Arruti, J. R. de Garibay, I. Mendia, and S. Renteria. RFIDGlove: A Wearable RFID Reader. In 2009 IEEE International Conference on e-Business Engineering, pages 475-480. IEEE, 2009.

[24] National Institute of Standards and Technology. CPS Public Working Group Presentation. Available online: http://www.nist.gov/el/upload/CPS-PWG-Kickoff-Webinar-Presentation-FINAL.PDF. last viewed May 2017.

[25] T. Nef, P. Urwyler, M. Büchler, I. Tarnanas, R. Stucki, D. Cazzoli, R. Müri, and U. Mosimann. Evaluation of Three State-of-the-Art Classifiers for Recognition of Activities of Daily Living from Smart Home Ambient Data. Sensors, 15(5):11725-11740, may 2015.

[26] B. Ni, G. Wang, and P. Moulin. RGBD-HuDaAct: A Color-Depth Video Database for Human Daily Activity Recognition, pages 193-208. Springer London, London, 2013.

[27] C. D. Nugent, Xin Hong, J. Hallberg, D. Finlay, and K. Synnes. Assessing the impact of individual sensor reliability within smart living environments. In 2008 IEEE International Conference on Automation Science and Engineering, pages 685-690. IEEE, aug 2008.

[28] F. J. Ordóñez, P. de Toledo, and A. Sanchis. Activity recognition using hybrid generative/discriminative models on home environments using binary sensors. Sensors, 13(5):5460-5477, 2013.

[29] K. K. B. Peetoom, M. A. S. Lexis, M. Joore, C. D. Dirksen, and L. P. De Witte. Literature review on monitoring technologies and their outcomes in independently living elderly people. Disability and Rehabilitation: Assistive Technology, 10(4):271-294, jul 2015.

[30] M. Philipose, K. Fishkin, M. Perkowitz, D. Patterson, D. Fox, H. Kautz, and D. Hahnel. Inferring Activities from Interactions with Objects. IEEE Pervasive Computing, 3(4):50-57, oct 2004. 
[31] H. Pirsiavash and D. Ramanan. Detecting activities of daily living in first-person camera views. In Computer Vision and Pattern Recognition (CVPR), 2012 IEEE Conference on. IEEE, 2012.

[32] D. Sánchez-de Rivera, D. Martín, R. Alcarria, B. Bordel, and T. Robles. Towards a Wireless and Low-Power Infrastructure for Representing Information Based on E-Paper Displays. Sustainability, 9(1):76, jan 2017.

[33] A. Schmidt, H.-W. Gellersen, and C. Merz. Enabling implicit human computer interaction: a wearable RFIDtag reader. In Digest of Papers. Fourth International Symposium on Wearable Computers, pages 193-194. IEEE Comput. Soc, 2000.

[34] B. B. Sánchez, D. S. de Rivera, and Álvaro Sánchez-Picot. Building unobtrusive wearable devices: an ergonomic cybernetic glove. Journal of Internet Services and Information Security (JISIS), 6(2):37-52, May 2016.

[35] M. Stikic, T. Huynh, K. Van Laerhoven, and B. Schiele. ADL recognition based on the combination of RFID and accelerometer sensing. In 2008 Second International Conference on Pervasive Computing Technologies for Healthcare, pages 258-263. IEEE, jan 2008.

[36] H. Takechi, A. Kokuryu, T. Kubota, and H. Yamada. Relative Preservation of Advanced Activities in Daily Living among Patients with Mild-to-Moderate Dementia in the Community and Overview of Support Provided by Family Caregivers. International Journal of Alzheimer's Disease, 2012:1-7, 2012.

[37] T. L. M. van Kasteren, G. Englebienne, and B. J. A. Kröse. An activity monitoring system for elderly care using generative and discriminative models. Personal and Ubiquitous Computing, 14(6):489-498, sep 2010.

[38] L. Vuegen, B. Van Den Broeck, P. Karsmakers, H. Van Hamme, and B. Vanrumste. Monitoring activities of daily living using Wireless Acoustic Sensor Networks in clean and noisy conditions. In 2015 37th Annual International Conference of the IEEE Engineering in Medicine and Biology Society (EMBC), volume 2015, pages 4966-4969. IEEE, aug 2015.

[39] J. Wang, J. Bauer, M. Becker, P. Bente, L. Dasenbrock, K. Elbers, A. Hein, M. Kohlmann, G. Kolb, C. Lammel-Polchau, M. Marschollek, M. Meis, H. Remmers, H. M. zu Schwabedissen, M. Schulze, E.-E. Steen, R. Haux, and K.-H. Wolf. A novel approach for discovering human behavior patterns using unsupervised methods. Zeitschrift für Gerontologie und Geriatrie, 47(8):648-660, dec 2014.

[40] S. Zhang, S. McClean, B. Scotney, P. Chaurasia, and C. Nugent. Using duration to learn activities of daily living in a smart home environment. In Proceedings of the 4th International ICST Conference on Pervasive Computing Technologies for Healthcare. IEEE, 2010.

[41] H. Zheng, H. Wang, and N. Black. Human Activity Detection in Smart Home Environment with SelfAdaptive Neural Networks. In 2008 IEEE International Conference on Networking, Sensing and Control, pages 1505-1510. IEEE, apr 2008. 


\section{Author Biography}

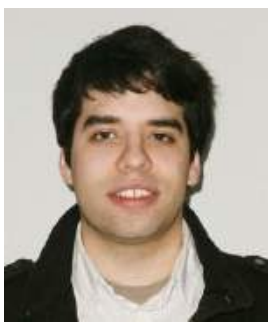

Borja Bordel received the B.S. degree in telecommunication engineering in 2012 and the M.S. telecommunication engineering in 2014, both from Technical University of Madrid. He is currently pursuing the Ph.D. degree in telematics engineering at Telecommunication Engineering School, UPM. His research interests include CyberPhysical Systems, Wireless Sensor Networks, Radio Access Technologies, Communication Protocols and Complex Systems.

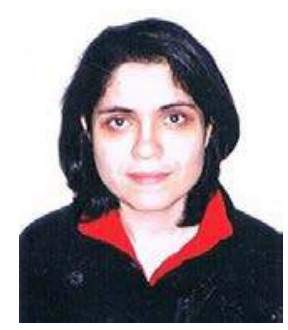

Marina Pérez-Jiménez received the B.S. degree in telecommunication engineering in 2011 and the M.S. telecommunication engineering in 2014, both from Technical University of Madrid. He is currently pursuing the Ph.D. degree in physical electronics at Telecommunication Engineering School, UPM. His research interests include magnetic sensors, microprocessors, underwater communications, aerospace technology and communications based on magnetic induction.

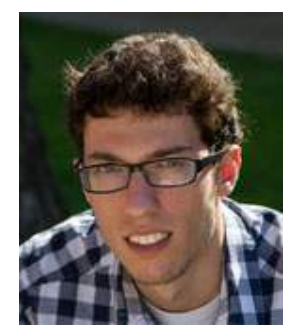

Diego Sánchez-de-Rivera holds a B.S. and a M.S. degree in Telecommunication Engineering from Technical University of Madrid. Currently he is a Ph.D. student and works as a research engineer in the Department of Telematics Systems at the E.T.S.I. Telecommunications of the Technical University of Madrid. His research interests are Internet of Things, Sensor Networks, Cyber-physical systems, Electronic displays and Web Development. 


\title{
T4AI: A system for monitoring people based on improved wearable devices
}

\author{
Marina Pérez-Jiménez ${ }^{1}$, Borja Bordel Sánchez ${ }^{2 *}$, and Ramón Alcarria ${ }^{3}$ \\ ${ }^{1}$ Department of Physical Electronics. Universidad Politécnica de Madrid \\ marina.perez@isom.upm.es \\ ${ }^{2}$ Department of Telematics Systems Engineering. Universidad Politécnica de Madrid \\ bbordel@dit.upm.es \\ ${ }^{3}$ Department of Topographic Engineering and Cartography. Universidad Politécnica de Madrid \\ ramon.alcarria@upm.es
}

\begin{abstract}
Monitoring people is central to many applications. Some works from the literature prove that information about that the sequence of objects a person uses while performing an activity robustly characterizes both, the activity and the quality of its execution. In this paper we present a novel system called "Toolkit for activities Inference" (T4AI) for inferring the activities executed by people from the interactions with objects. The system includes as main element a cybernetic glove based on wearable RFID readers and sensors. Our proposal includes an improved RFID technology being able to be used in metallic environments (such as industry scenarios). Moreover, an experimental validation was conducted in order to determine the performance of the proposed system.
\end{abstract}

Keywords: cybernetic devices, cybernetic glove, RFID, T4AI, Toolkit for activities Inference

\section{Introduction}

From the beginning, human-activity tracking techniques have focused on direct observation of people and their behavior with cameras, accelerometers, or contact switches [27]. Nevertheless, around ten years ago, a new approach was born [22] [29]. It consisted of complementing direct observation with an indirect study, deducting people's actions from their effect on the environment, especially on the objects with they interact. Thus, many works (such as [25] and [14]) have explored ways to obtain and use knowledge of person-object interactions.

Knowing which physical objects a person touches is central to many applications [27]. Logs of objects touched during a day may be, for example, the basis of "experience sampling" [7] programs whose objective is reconstructing user's behavior.

Basically, three main techniques have been applied in human-activity tracking [27]: computer vision, active sensor beacons and passive RFID. Among all these, computer vision is the least mature technology, remaining many challenges unresolved. Active sensor beacons allow us to know, very precisely, the interactions human-objects, but require the use of batteries (which hinders their long term use). On the contrary, RFID is a mature technology which provides the same accuracy as active sensor beacons, but with the advantage of not requiring the use of batteries. In return, RFID tags are unable to detect motion. Despite this inconvenience, RFID technology has been widely used for tracking human activity, especially by means of its integration in wearable devices [27] [12].

Research Briefs on Information \& Communication Technology Evolution (ReBICTE), Vol. 2, Article No. 1 (March 31, 2016)

*E.T.S.I. Telecomunicación. Universidad Politécnica de Madrid. Avenida Complutense no 30. 28040 - Madrid (España), Tel. 915495700 ext. 3035 
However, most of the proposed devices based on wearable RFID readers are really similar (they often resembles to some kind of "smart glove"), and remain in a pretty low development level.

Therefore, the objective of this paper is to design and implement a new system for human-activity tracking based on a cybernetic glove built with a new generation of wearable RFID readers and wearable sensors. We call this new system "Toolkit for activities Inference" (T4AI). In particular, the contribution of this paper includes the description of an improved RFID technology being able to be used in metallic environments.

The authors carried out an experimental validation in order to validate the presented T4AI in this paper. The statistical evidences and findings obtained during the experimental validation are very remarkable and indicate the presented system in this paper is a reliable solution for tracking the execution of the human activities.

The rest of the paper is organized as follows: Section 2 presents the state of the art in human-activities tracking systems and wearable. Section 3 presents the contributions of the article. Section 4 describes the experimental validation. Finally, Section 5 and 6 explain some results of this experimental validation and the conclusions of our work.

\section{State of the art}

RFID technology is considered in many fields (such as sales or logistic processes) as the future way in which information will be introduced in information systems (as replacement, among other solutions, of the barcode). Actually, nowadays, RFID tags are more extensively used than, for example, barcodes in many areas.

In research, since 1999, papers such as [32] have discussed how RFID tags can be used to bridge physical and virtual worlds. One year later, Albrecht Schmidt defined the "Implicit Human-Computer Interactions" as actions performed by the user that are not primarily aimed to interact with a computerized system, but which such a system understands as input [25]. Merging both ideas, concepts such as the RFID-based glove [27] or the iBracelet [12] for human monitoring were immediately developed.

The first study about an RFID-glove was [26]. This prototype is based on a low-frequency RFID technology, so the maximum range reached is quite large. However, the connection between the glove and the main host is wired, so the working distance is limited to the length of the wire. Moreover, the design of the glove it is not very ergonomic, as the hardware is not completely integrated with it.

In 2005, this research field suffered a real revolution. First a second generation of the RFID-based glove was presented in [12]. In this work, a miniaturized NFC reader was employed, instead of the low-frequency RFID reader, so the electronics integration improved substantially. Moreover, a wireless communication module was also included, removing the wire which connected the glove with the main host; thus, the working distance strongly increased. The obtained prototype (called iGlove) was also complemented with a smart bracelet (called iBracelet) for applications where it was not possible to integrate the electronics in the glove (such as in sanitary scenarios). Second, a new RFID technology including sensing capabilities was described. As we said in the introduction, around 2004 a new approach for human-activity tracking was described in [22] and [29] (among others). This technique consisted of complementing direct observation with an indirect study. However, all the described prototypes do not merge direct and indirect observation, but replace traditional instrument by RFID technology. Then, a research group from the Intel Research Seattle presented in [23] a new project (called Wireless identification and sensing platform -WISP-) merging both sensing capabilities and RFID technology. These devices (passive, programmable and provided with a microcontroller unit) interact with RFID readers and WISP readers. If a traditional RFID reader is used, only identification number is obtained. If a WISP reader is used, both the identification and data from sensors included are obtained. However, the 
WISP project presented in [23] (called alpha-WISP) is not enough, as sensing capabilities are limited to one-axis accelerometer. Thus, some months later, an improved pi-WISP project is presented in [28]. In this new article, sensing capabilities had grown for supporting three-axis sensors, so most sophisticated application can be considered. With this new pi-WISP technology available, a new iGlove and iBracelet provided with accelerometers were described in [27]. Although this was the first prototype in including both sensing and RFID capabilities, the rate reached for data collection from sensors was rather poor, so critical real-time applications were not covered in this approach.

Finally, after a period when no works about smart gloves were published, in very recent years (since 2013) various prototypes and research works have been reported [8]. In 2013, the first RFID-based glove was patented [19]. It included a RS-232 connector, a small battery and a RFID reader. In 2014, systems based on smart gloves for monitoring the children behavior were described [11]. Also in 2014, Fujitsu presented a system [1] being able to control the operators in industries, using a RFID-enabled smart glove also provided with a QR reader. In 2015 three important prototypes were implemented. First, the Italian company IDROGENET SRL deployed a system called GloReha [2], based on a computerized glove which infers the hand movements (and corrects them) in the context of neurological rehabilitation. Second, the German startup ProGlove [5] developed a wearable tool targeted at professional production processes. The wearable is basically an ergonomic smart glove being able to detect certain patterns. Finally, at the Massachusetts Institute of Technology (MIT) a smart glove based on Arduino platform and some accelerometers was successfully employed to translate the sign language into an English speech. The proposed glove, named as SignAloud [6], is based on a powerful pattern recognition engine and a voice synthesizer. Table 1 summarizes and compares the described relevant previous works.

In all cited precedents, one of the causes of the low development level of the wearable devices is the selected RFID technology. In most RFID technologies the reading range is too high [31], and it cannot be said the glove detects the touched objects when all objects in a meter radius are detected. Furthermore, the reading range in NFC (Near Field Communications, the most used solution) is between five and ten centimeters, and it may become too large depending on the application scenario. On the other hand, all the gloves cited above present a great problem: they cannot be used in metallic environments. This limitation is due to the use of passive RFID tags, which cannot obtain energy from the reader when placed on metallic surfaces (as metals "reflect" the energy). In the last five years various proposals addressing this issue have appeared [13] [10]. However, all are focused in Ultra-high frequency RFID technology, which presents a reading range around 1.5 meters [10] (a too high value) and operates in the band of $850-950 \mathrm{MHz}$ (a band legally regulated in many countries, so that the UHF RFID technology cannot be used freely, for example, in most of Europe) [31]. There also exist some commercial products for "on-metal NFC tags". Nonetheless, they are based on the creation of a gap between the surface and the tag circuit, by including several layers of different materials [4]. The result is a tag of a much larger size than usual, and that can hardly be placed on small objects which need to be precisely manipulated.

Therefore, compared with all these precedents, our proposal presents a great improvement. An improved and miniaturized RFID technology, capable of working in metallic environments, is developed. It is really important to note that many objects in all scenarios are metallic, and, as we saw, none of them could be tagged by using traditional RFID tags, as none commercial technology is totally suitable for application to a cybernetic glove. This problem, which has only been partially addressed in the literature, is resolved with the proposed technology.

\section{Toolkit for activities Inference}

We technically describe the presented contributions. In the first subsection, the application scenario is defined. In the next subsection the improved RFID technology is described. Later, in Section 3.3 the 


\begin{tabular}{|c|c|c|}
\hline Precedent & Characteristics & Problems \\
\hline First RFID-glove [26] & Very large maximum range. & $\begin{array}{l}\text { The electronic circuit is not } \\
\text { completely integrated. Wired } \\
\text { solution. }\end{array}$ \\
\hline $\begin{array}{l}\text { iGlove [12] } \\
\text { Children monitoring } \\
\text { system [11] }\end{array}$ & $\begin{array}{l}\text { RFID readers are miniaturized. } \\
\text { Wireless solution. }\end{array}$ & $\begin{array}{l}\text { Does not include any direct } \\
\text { observation technology } \\
\text { (accelerometers, sensors, etc.). }\end{array}$ \\
\hline $\begin{array}{c}\text { WISP [23] } \\
\text { Pi-WISP [28] } \\
\text { New iGlove [27] }\end{array}$ & $\begin{array}{l}\text { RFID readers are miniaturized. } \\
\text { Wireless solution, includes } \\
\text { accelerometers. }\end{array}$ & $\begin{array}{l}\text { The data rate is very poor. Critical } \\
\text { real-time applications cannot be } \\
\text { developed. }\end{array}$ \\
\hline $\begin{array}{c}\text { First patented } \\
\text { RFID-based glove } \\
{[19]}\end{array}$ & RFID readers are miniaturized. & $\begin{array}{l}\text { Does not include any direct } \\
\text { observation technology } \\
\text { (accelerometers, sensors, etc.). } \\
\text { Wired solution. }\end{array}$ \\
\hline Fujitsu smart glove [1] & $\begin{array}{l}\text { Includes accelerometers and a } \\
\text { QR reader. Wireless solution. }\end{array}$ & $\begin{array}{l}\text { The electronic circuit is not } \\
\text { completely integrated. }\end{array}$ \\
\hline GloReha [2] & $\begin{array}{l}\text { Includes accelerometers and a } \\
\text { pattern recognition system. }\end{array}$ & $\begin{array}{l}\text { Does not include RFID readers. } \\
\text { Wired solution. }\end{array}$ \\
\hline $\begin{array}{l}\text { SignAloud [6] } \\
\text { ProGlove [5] }\end{array}$ & $\begin{array}{l}\text { Includes accelerometers and a } \\
\text { pattern recognition system. } \\
\text { Wireless solution. }\end{array}$ & Does not include RFID readers. \\
\hline
\end{tabular}

Table 1: Comparison among the relevant precedents

final electronic system is presented.

\subsection{Application scenario}

From the beginning, wearable technologies and pervasive computing [20] were developed together. In fact, Mark Weiser explained in 1991 [33] [24], talking about pervasive computing, "The most profound technologies are those that disappear. They weave themselves into the fabric of everyday life until they are indistinguishable from it." Furthermore, one of the basic applications of pervasive computing is using computers and sensors to infer users' behavior in their environment. In applications performed in controlled scenarios (such as living labs or training houses), cameras and artificial vision are the proper technologies, as they are the least obtrusive and there is almost no problem associated with their deployment and operation (as conflicts with privacy or improper handlings of equipment by users). However, some applications require to evaluate the users' behavior in uncontrolled environments (such as the workplace or the user's home), which are more aggressive scenarios and deployments may not provide the precision needed by the cameras (which, for example, must be properly focused). Besides, in the second case, it is necessary to consider factors such as privacy, the space configuration (that probably will not be as open as in controlled scenarios) or the available space in the user's home. In that scenario, wearable technologies seem to be the response.

Among all the applications which fit with the second described case, the study of people with cog- 
nitive disorders in first stage is a priority (especially in an increasingly aging world) [15]. However, it remains being a difficult application area to infer whether and how people are performing activities [16]. Causes are various [22]. First, people can perform a same activity in different ways, so models adapted to these requirements are needed. Second, the aggressiveness of the uncontrolled environments causes the underlying hardware of ubiquitous computing system and wearable devices should be tolerant to high levels of noise, light, vibration, etc. And, third, the number of activities is too huge for modeling each activity separately; so automatic instruments to create these models are necessary.

In [22], researchers from Intel Research Seattle (closed in 2011) partially solved these problems. They proposed a system (called Proactive Activity Toolkit -PROACT-) which represented activities as a probabilistic sequence of objects used (which allowed addressing the first challenge in human-activities tracking). Moreover, they created probabilistic models of activity use from plain English descriptions of activities such as recipes; solving in that way the third challenge. To address the second challenge, they found RFID as a robust technology to sense the objects being used in several contexts. Nevertheless, due to the technology limitations, a huge list of objects could not be detected, such as those made of metallic materials.

Therefore, the aim of this article is building a new system (called T4AI) addressing the shortcomings of earlier prototypes. For this purpose, an improved RFID technology that detects interactions with metal objects was developed. In the background, we also worked to solve another of the basic challenges in the design of wearable devices: the communication between the glove and other hosts (for which it was made use of modern low-energy technologies).

In scenarios related to people with cognitive disorders in first stage, motion analysis is a very important complementary information source, for evaluating the quality with which activities are being performed. So, the most proper additional sensors for our application scenario are three-axial accelerometers [31].

The proposed system:

- Includes the cybernetic glove, a processing application and an analysis engine to infer the executed activities using both RFID and accelerometers data.

- Represents activities as a probabilistic sequence of objects used.

- Describes probabilistic models as TXT files, written in plain text.

With this system, authors conducted an experimental validation where various people were monitored, allowing us to determine the correct performance of the proposed solution.

\subsection{An improved RFID technology}

RFID-based wearable devices [9] have received great attention in the last 15 years (see Section 2). Furthermore, in the last five years, many miniaturized new RFID technologies for wearable devices have been proposed. However, most works [21] [17] propose including UHF RFID tags in fabrics, in order to maintain clothes "passive" (without independent power supply). The objective of these wearable devices is to monitor users' position by means of, for example, various antennas strategically placed to make a triangulation [30]. The main reason to include passive tags in fabrics, instead of active readers, is the difficulty of miniaturizing RFID readers and making them energy efficient (see Section 2). However, in our application, we are detecting objects used by the users, so passive tags must be placed in objects and the user have to be provided with and active reader (in our case, integrated in the cybernetic glove).

Two additional problems have to be taken into account in the use of traditional RFID technologies in a wearable glove. First, the minimum distance at which a tag can be read (using high frequency RFID 
solution [31]) is around 5 centimeters. This value is too high in our application, as we are only interested in the object touched by the users. In a typical daily living situation, various objects are placed together, and we need distinguish which particular object has been picked up. None other tag must be read. And, second, RFID tags cannot be placed in metallic surfaces [31]. On the one hand, traditional RFID tags cannot be read if placed in metallic surfaces, which may be found every day in our lives (in kitchen items, clothing, containers, furniture, etc.). Any case, a real monitoring of the activities of daily life cannot impose constraints on the objects used.

Therefore, to create our cybernetic glove we designed an improved RFID technology based on miniaturized active readers, being able to read tags placed on metallic surfaces and whose minimum reading distance tends to zero (direct contact). As limitation, this technology does not allow the use of commercial solutions, making it difficult to perform large deployments at low cost.

In order to be energy efficient and be able to reuse tags for different applications (which lowers system cost) the proposed technology comprises both a miniaturized, active reader with reading and writing capabilities; and rewritable passive tags. Thus, both the reader and the tags are formed by two different transponders: a transmitter module and a receiver module. The objective of this scheme is to be able to establish a full-duplex communication between reader and tags by means of the use of two different radio channels (Figure 1).

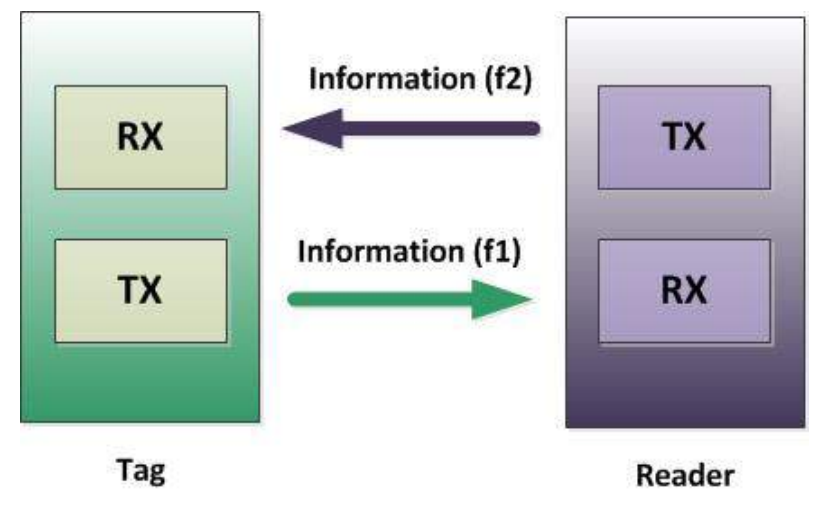

Figure 1: Communication scheme for the RFID technology

If both radio channels are sufficiently separated in the frequency domain, any relevant interference between both frequencies will occur. Over these two channels, data packets containing the identification of the tag and the reader will be sent.

The general design for the transmitter module in the reader and in the tag is the same (Figure 2(a)). The information to be transmitted modules a carrier frequency in amplitude (using ASK modulation), which is adapted to be radiated by means of an antenna tuned at the carrier frequency. The signal adapter module is an extremely narrow passband filter that only allows passage of the carrier frequency, eliminating any other component of the spectrum.

Although the general design is the same in both elements (reader and tags), the transmitter implementation is different depending on the device. The reader must be, overall, robust. It is going to be placed in a glove, so it will be subject to changes of position, shock, friction, etc., in this scenario it is important that its operation is not affected significantly in these situations. The tags, meanwhile, must be inexpensive (as it is important to have a lot to place tags in all objects of daily life). Therefore, to implement the transmitter in the reader, it has been chosen a much more complex and expensive technology, but very robust. Meanwhile, for the tag, it has been sought the most cost effective solution.

In the case of the reader, the amplitude modulation is made by means of an analog switch, selecting between the carrier signal and ground (Figure 3(a)) following a TTL signal which codifies the informa- 


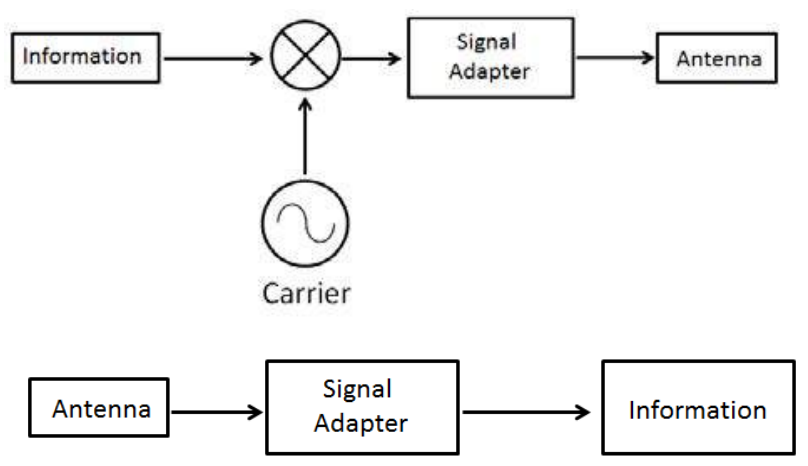

Figure 2: (a) General design for RFID transmitter (b) General design for RFID receiver

tion to be transmitted. Both, the carrier signal and the information signal are generated by the same high integrated microprocessor (to which a digital waveform generator is added). Using a software simulated oscillator reduces the size of the reader, its price, increases its robustness and allows an easy selection of the carrier frequency.
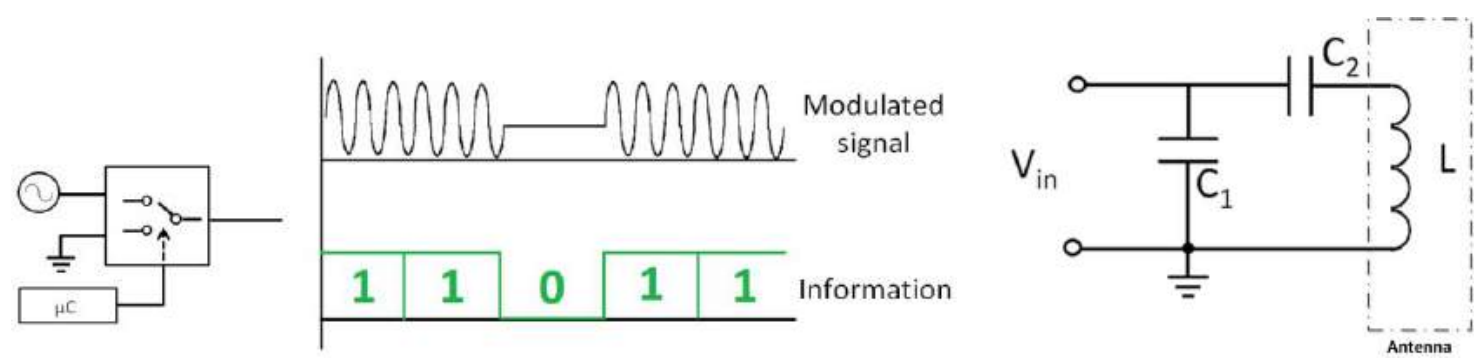

Figure 3: (a) ASK modulator in the RFID reader (b) Signal adapter circuit in the reader

This scheme for the transmitter, although it cannot generate large-signal (more than $3 \mathrm{~V}$ in amplitude) it is sufficient if the reading distance between reader and tag is very small (as in our case).

Moreover, the use of an analog switch and software oscillator (instead of the traditional analog crystal oscillators) greatly simplifies the signal adapter module. Usually [3] adaptation stages need an EMC filter and an impedance adapter circuit made of six capacitors and two inductances (which are especially problematic in high integration). In comparison, in our technology, signal adapter module only needs one passband filter made of two capacitors. To achieve this, the circuit uses the inductive impedance viewed from the end of the adaptation stage towards the antenna (when it is in transmission). Figure 6(b) presents the resultant circuit. The transference function of that circuit is similar to which showed by a second order passband filter. The advantage of that design is that, at carrier frequency, $C_{l}$ capacitor supplies current to the antenna, so the signal is amplified, and the radiated field maximum. Furthermore, at the carrier frequency and due to the fact that the circuit is resonant, the impedance of the set is minimal and negligible the energy dissipation (which greatly reduces power consumption).

In the case of the tag, the amplitude modulation is made by means of only one high integrated microprocessor. The microprocessor will generate a TTL function codifying the information to be transmitted. Then, that signal passes through the adapter signal module described above, so all components in the spectrum are cancelled, except carrier frequency, and TTL signal is "rounded" (the final result is similar to an ASK modulated carrier). This procedure is much cheaper, but is weaker and requires certain degree of stability to operate. 
The receiver module is the same in both tags and reader (a global scheme is presented in Figure 2(b)). The signal is received by means a tuned antenna, and it passes through a signal adapter module (which removes all signals in the spectrum except the modulated carrier). Later, the filtered signal passes through a demodulator, capable of extracting the information coded in the carrier.

The signal adapter module is formally the same as described above, except in this case the inductance associated with the antenna is viewed as if it were a signal source (Figure 4(a)). The received signal, therefore, is also amplified in that resonant circuit. The demodulator is made of half-wave rectifier, which transforms any period of non-zero signal at a high level of continuous voltage. Said continuous signal fed to a digital to analog converter (ADC) integrated in the microprocessor. When the ADC accumulates several values much greater than zero, it is decided, then, that has been received a ' 1 '. In opposite case, it is assumed transmitted a ' $O$ ' (Figure 4(b)). Thus, sample speed is a critical parameter of the design.
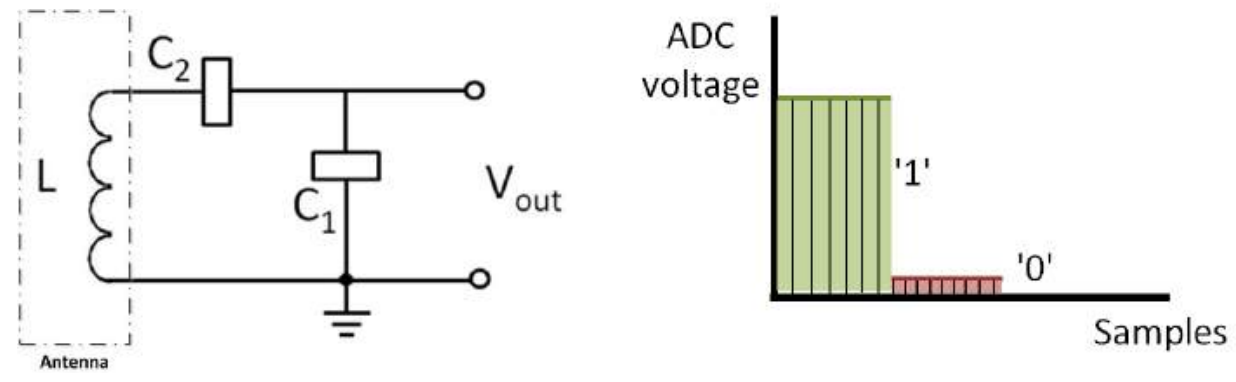

Figure 4: (a) Signal adapter circuit in the reader (b) Output of the demodulator

The physical phenomenon that allows communication between tag and reader is the mutual inductance between two coils. When by a coil of copper wire is circulating a variable current, the coil generates a magnetic field (Figure 5(a)). This field is not too intense, but at very short distance there is indeed a magnetic flux perpendicular to the coil (Figure 5(b)). When a second coil enough approaches the first, the field generated crosses the second coil and induces a current that varies as does the flow (Figure 5 (c)). This mechanism (much like how transformers work) permits the use of low power signals, and ensure that only those tags located close enough can be read (ideally those with whom the reader has contact).
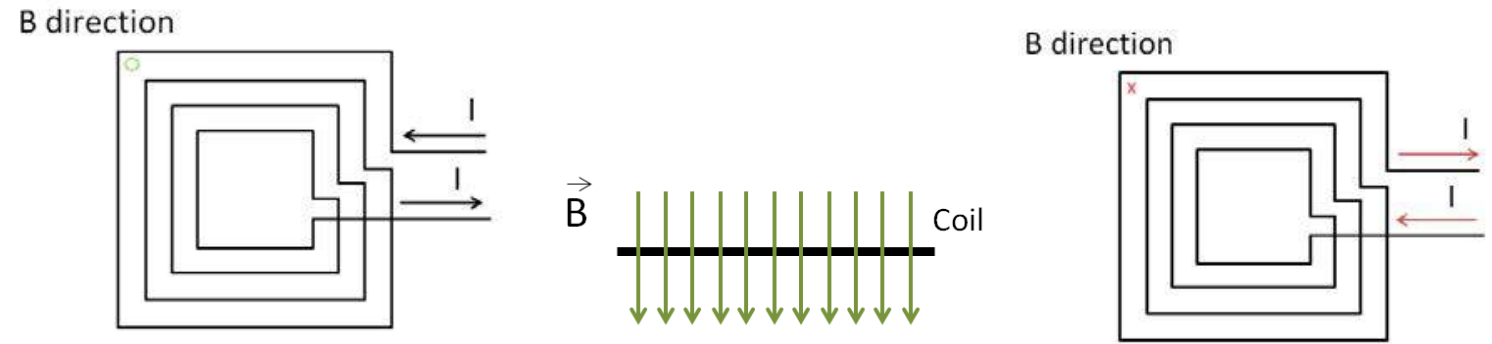

Figure 5: (a) Magnetic field generated in a coil (b) Perpendicular magnetic flux (c) Induced current in a coil by a magnetic field

In traditional RFID technologies [31] there are various ways in which the RFID reader-writer can communicate with the RFID tag. Basically, three physical processes are used: backscatter coupling, capacitive coupling and inductive coupling (which it is used in the glove). With the traditional design of RFID readers and tags, capacitive coupling are used for close range links (within 1 centimeter), inductive coupling for remote links (between $1 \mathrm{~cm}$ and 1 meter) and RFID backscatter coupling for long range 
links (more than 1 meter). However, capacitive coupling requires including rigid electrodes, acting as the plates of the capacitor, which extremely difficult to integrate into a flexible fabric. Thus, the inductive coupling is most suitable procedure (as copper coils can be very flexible), although the read range in the traditional technologies is too large. With the proposed technology, this problem disappears and close range links with inductive coupling are reachable.

Working with very low power signals and very short range links, removes all legal limitations on the use of frequency spectrum. Therefore, it has been chosen to work in the band of tens of kilohertz (up to 1000 times below the NFC band). This allows reducing the power consumption of the system for the same transmitted power, lowering the components' price and getting a greater level of integration. Furthermore, the spurious modulation effects are located far away from the passband at these frequencies, so a more robust operation is achieved.

Nevertheless, the main advantage of using inductive coupling at very short ranges is the possibility of using our technology in metallic environments. Tags can be placed over metallic surfaces, and communications may continue taking place robustly.

Finally, it is important to note that, apart from the reduction in the number of components achieved with our technology (which already greatly reduces the system size), the area occupied by the coils in our solution may be significantly less than that used in commercial solutions. In addition, for all the components mentioned above there are a highly integrated version, and even microprocessors can be developed in printed technology if the algorithm to be run in that microcontroller is prepared.

\subsection{Complete electronic system}

Once presented all the contributions in the electronic field, in this section we are describing the final electronic system on which the cybernetic glove will be based.

Figure 6 shows the global scheme of the system.

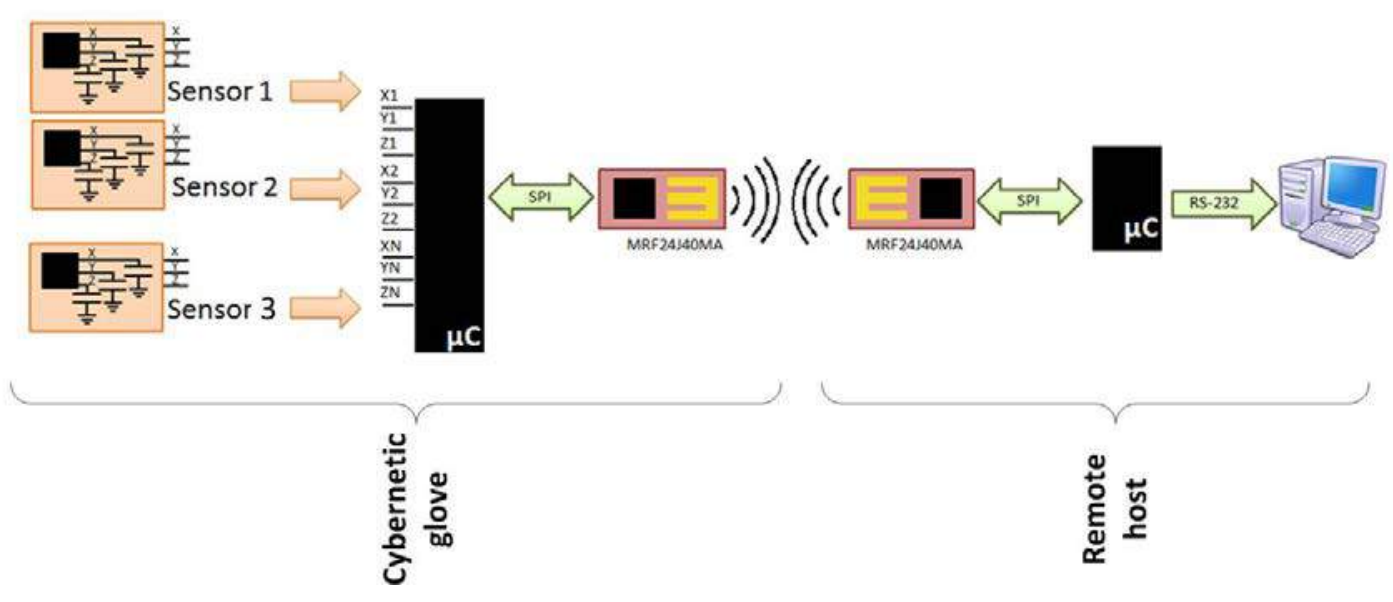

Figure 6: Global scheme of the electronic system

In the cybernetic glove, a sort of sensors is connected to a main microcontroller. Being more specific, the glove is provided with three sensors: two accelerometers and one RFID reader (see Section 3.2. The microcontroller, with the data collected, builds a data frame to be transmitted toward the remote host where processing takes place. That frame is transmitted by a traditional SPI port to a $2.4 \mathrm{GHz}$ communications module, which implements ZigBee protocol. The remote host is connected through a RS-232 port to a ZigBee receptor made of a microcontroller and a $2.4 \mathrm{GHz}$ communications module 
(equal to which is included in the glove). In that host, a MATLAB application receives, processes [18] and presents data and results.

Then, the sum of all information provided by the accelerometers and the RFID reader (transmitted by means of the processor and the communications module) enables a MATLAB application to calculate and determine the users' behavior in the remote host.

\subsection{Final prototype and system operation}

The electronic system described above was integrated in a woven glove, being able to communicate with the analysis engine and the data processing application, both implemented in MATLAB code. The resulting wearable device can be seen in Figure 7 .

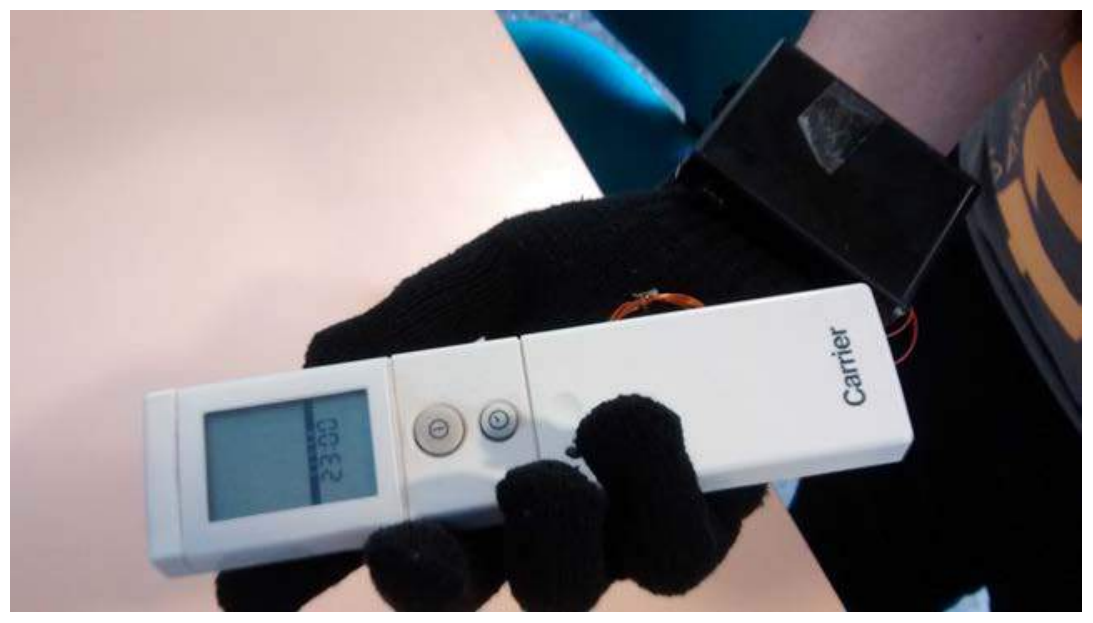

Figure 7: Final integrated smart glove

Both the analysis engine and the data processing application were implemented using MATLAB code and some of the pattern recognition functions available in that environment. In particular, the data processing application filters the received data and removes the frequency components due to electronic noise, interferences, etc. Once the important information has been extracted, it is sent to the analysis engine where a Hidden Markov Model (HMM) allows the identification of the executed activity. Figure 8 represents the graphic application used as interface between the pattern analysis engine and the users.

Finally, the system operation is described in the flowchart showed in Figure 9

\section{Experimental validation}

Table 2 shows the activities selected for this experimentation.

The experiment was split in three different phases:

- Training phase: Participants received some training about the activities they had to perform with the cybernetic glove, the activities they have to perform and how to perform it. The participants attended a lecture where they were trained and they saw the gloves for the first time, while experts presented them and performed some examples.

- Activities performing phase: Each participant was interviewed in this phase. The participant was asked to execute the 6 activities being monitored with the proposed T4AI. 


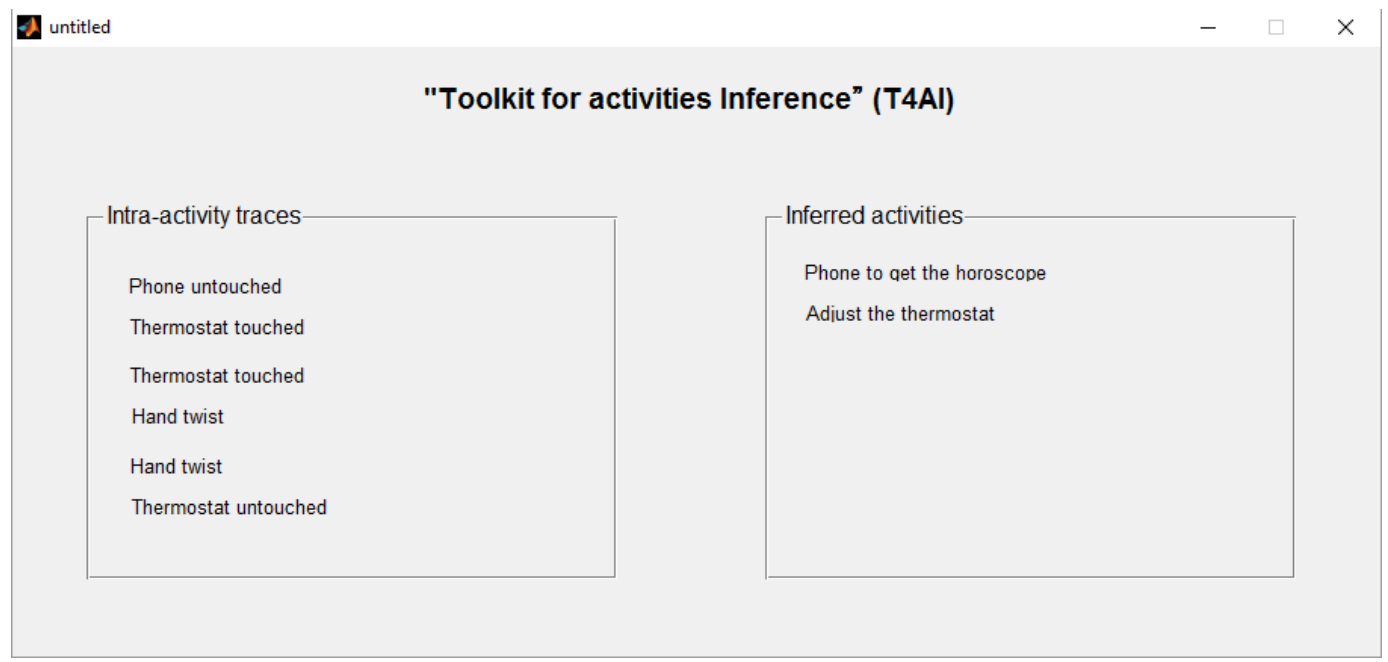

Figure 8: Graphic application in the analysis engine

\begin{tabular}{cc}
\hline $\begin{array}{c}\text { Activity } \\
\text { number }\end{array}$ & Activity description \\
\hline 1 & Use a microwave \\
\hline 2 & Adjust the thermostat \\
\hline 3 & Turn on TV, watch it for a few minutes, turn it off \\
\hline 4 & Take some pills \\
\hline 5 & Phone to get the horoscope \\
\hline 6 & Make yourself a cup of coffee \\
\hline
\end{tabular}

Table 2: Tested activities

- Evaluation phase: In this phase, experts evaluated the records obtained by the system for each participant.

The experiment was performed in a laboratory at the Technical University of Madrid (UPM). The "Toolkit for activities Inference" (T4AI) was deployed in the laboratory, in order to collect and process all data from the cybernetic glove, and for inferring the activities being performed by users. T4IA reported automatically the result of the activity at the end of each performance; experts took note about all the results. When a performance was correctly classified, it scored a true positive (TP); an incorrect it scored a false positive (FP); but when an activity occurred and was not reported by our system, it scored as a false negative $(\mathrm{FN})$.

\section{Results}

In this section we present the results obtained in the experimental validation. The analysis about the activities inferred by T4IA is shown in Table 3 . 


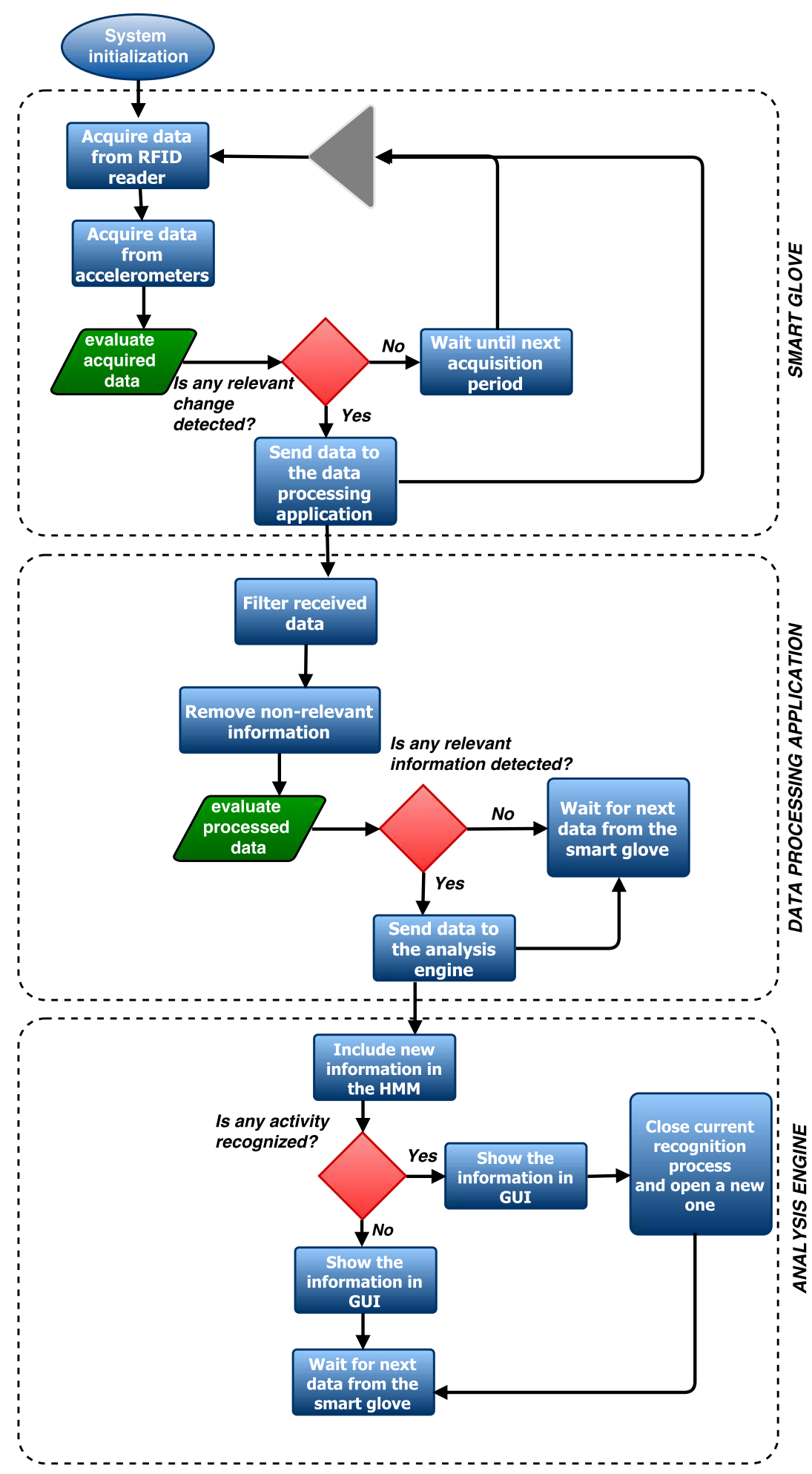

Figure 9: Flowchart of the proposed T4AI

A Mann-Whitney $U$ test was conducted in order to test if the precision and recall obtained in our experiment are significantly different from the results obtained by Philipose and Fishkin [22]; we could not find any statistical evidence confirming the difference between the two samples. 


\begin{tabular}{cccc}
\hline Activity no. & True Positives & False Positives & False Negatives \\
\hline 1 & 12 & 3 & 6 \\
\hline 2 & 15 & 0 & 3 \\
\hline 3 & 15 & 1 & 3 \\
\hline 4 & 13 & 2 & 6 \\
\hline 5 & 20 & 0 & 4 \\
\hline 6 & 10 & 3 & 5 \\
\hline \hline Total & 85 & 9 & 27 \\
\hline
\end{tabular}

Table 3: Experiment result for the RQ1 validation

Therefore, we can assert that T4IA (Toolkit for Activities Inference) used in the validation behaves the same way as system presented by Philipose and Fishkin [22]. Then, it can be said that activities performed by user can be inferred with the proposed system as with the precedent, so in the proposed T4AI no functionality is lost compared to the previous prototypes (Section 2). However, our system presents various advantages such as the possibility of considering metallic objects in the application scenarios.

\section{Conclusions}

With our proposed T4AI and its cybernetic glove, the limitations about the objects which can be manipulated by users being monitored are reduced thanks to an improved RFID technology being able to read tags placed over metallic surfaces.

We provided also an experimental validation with real users. In order to probe the correct performance of the proposed system, we investigated if the TP, FP and FN obtained using our system are significantly different from the results obtained by Philipose and Fishkin [22]. We did not find any statistical evidence confirming the difference between the two proposals.

\section{Acknowledgments}

The research leading to these results has received funding from the Ministry of Economy and Competitiveness through SEMOLA project (TEC2015-68284-R). We also thank the Autonomous Region of Madrid for the funding of MOSI-AGIL-CM project (grant P2013/ICE-3019, co-funded by EU Structural Funds FSE and FEDER).

\section{References}

[1] Fujitsu's smart glove. http://www.qore.com/articulos/17335/ Fujitsu-desarrolla-un-guante-de-realidad-aumentada [Online; Accessed on March 10, 2016] in Spanish.

[2] Gloreha system main page. http://www.gloreha.com [Online; Accessed on March 10, 2016$].$ 
[3] Measurement and tuning of a NFC and Reader IC antenna with a MiniVNA. http://www.nxp.com/ documents/application_note/AN11535.pdf [Online; Accessed on March 10, 2016].

[4] On-metal NFC technology. http://rapidnfc.com/on_metal_nfc_tags [Online; Accessed on March 10, 2016].

[5] Proglove project main page. http://www.proglove.de [Online; Accessed on March 10, 2016].

[6] Signaloud gloves web page. http://lemelson.mit.edu/winners/thomas-pryor-and-navid-azodi [Online; Accessed on March 10, 2016].

[7] L. F. Barrett and D. J. Barrett. An Introduction to Computerized Experience Sampling in Psychology. Social Science Computer Review, 19(2):175-185, May 2001.

[8] B. Bordel, R. Alcarria, M. Pérez-Jiménez, T. Robles, D. Martín, and D. S. d. Rivera. Building Smart Adaptable Cyber-Physical Systems: Definitions, Classification and Elements. In J. M. García-Chamizo, G. Fortino, and S. F. Ochoa, editors, Proc. of the 9th International Conference Ubiquitous Computing and Ambient Intelligence (UCAmI'15), Puerto Varas, Chile, volume 9454 of Lecture Notes in Computer Science, pages 144-149. Springer International Publishing, December 2015.

[9] B. Bordel Sánchez, R. Alcarria, D. Martín, and T. Robles. TF4SM: A Framework for Developing Traceability Solutions in Small Manufacturing Companies. Sensors, 15(11):29478-29510, November 2015.

[10] S. L. Chen. A Miniature RFID Tag Antenna Design for Metallic Objects Application. IEEE Antennas and Wireless Propagation Letters, 8:1043-1045, 2009.

[11] L. Escobedo, C. Ibarra, J. Hernandez, M. Alvelais, and M. Tentori. Smart objects to support the discrimination training of children with autism. Personal and Ubiquitous Computing, 18(6):1485-1497, November 2013.

[12] K. P. Fishkin, M. Philipose, and A. Rea. Hands-on RFID: wireless wearables for detecting use of objects. In Proc. of the 9th IEEE International Symposium on Wearable Computers (ISWC'05), Osaka, Japan, pages 38-41. IEEE, October 2005.

[13] B. Gao, C. H. Cheng, M. M. F. Yuen, and R. D. Murch. Low Cost Passive UHF RFID Packaging with Electromagnetic Band Gap (EBG) Substrate for Metal Objects. In 2007 Proceedings 57th Electronic Components and Technology Conference, pages 974-978, May 2007.

[14] G. R. Hayes, J. A. Kientz, K. N. Truong, D. R. White, G. D. Abowd, and T. Pering. Designing Capture Applications to Support the Education of Children with Autism. In N. Davies, E. D. Mynatt, and I. Siio, editors, Proc. of the 6th International Conference of Ubiquitous Computing (UbiComp'04), Nottingham, UK, number 3205 in Lecture Notes in Computer Science, pages 161-178. Springer Berlin Heidelberg, September 2004.

[15] Y. J. Hong, I. J. Kim, S. C. Ahn, and H. G. Kim. Activity Recognition Using Wearable Sensors for Elder Care. In Proc. of the 2nd International Conference on Future Generation Communication and Networking (FGCN'08), Hainan Island, China, volume 2, pages 302-305. IEEE, December 2008.

[16] S. Katz, A. B. Ford, R. W. Moskowitz, B. A. Jackson, and M. W. Jaffe. Studies of illness in the aged: The index of adl: a standardized measure of biological and psychosocial function. JAMA, 185(12):914-919, September 1963.

[17] K. Koski, E. Moradi, A. Vena, T. Björninen, L. Sydänheimo, L. Ukkonen, and Y. Rahmat-Samii. Characterization of electro-textiles using wireless reflectometry for optimization of wearable UHF RFID tags. In Proc. of the Progress in Electromagnetics Research Symposium, Stockholm, Sweden, Progress in Electromagnetics Research Symposium, pages 1188-1192, August 2013.

[18] N. C. Krishnan, C. Juillard, D. Colbry, and S. Panchanathan. Recognition of Hand Movements Using Wearable Accelerometers. Journal of Ambient Intelligence and Smart Environments, 1(2):143-155, April 2009.

[19] A. E. Majoros, B. C. Fredgren, P. R. Davies, and R. D. Kalinowski. Data Interface Process With RFID Data Reader Glove. Patent no. US20100097195 A1, Majoros Anthony E, USA, U.S. Classification 340/10.6; International Classification H04Q5/22; Cooperative Classification G06K7/0008; European Classification G06K7/00E, April 2010. http://www.google.com/patents/US20100097195 [Online; Accessed on March 10, 2016].

[20] A. Morales, R. Alcarria, D. Martin, and T. Robles. Enhancing Evacuation Plans with a Situation Awareness System Based on End-User Knowledge Provision. Sensors, 14(6):11153-11178, June 2014. 
[21] C. Occhiuzzi, S. Cippitelli, and G. Marrocco. Modeling, Design and Experimentation of Wearable RFID Sensor Tag. IEEE Transactions on Antennas and Propagation, 58(8):2490-2498, August 2010.

[22] M. Philipose, K. P. Fishkin, M. Perkowitz, D. J. Patterson, D. Fox, H. Kautz, and D. Hahnel. Inferring activities from interactions with objects. IEEE Pervasive Computing, 3(4):50-57, October 2004.

[23] M. Philipose, J. R. Smith, B. Jiang, A. Mamishev, S. Roy, and K. Sundara-Rajan. Battery-free wireless identification and sensing. IEEE Pervasive Computing, 4(1):37-45, January 2005.

[24] M. Satyanarayanan. Pervasive computing: vision and challenges. IEEE Personal Communications, 8(4):1017, August 2001.

[25] A. Schmidt. Implicit human computer interaction through context. Personal Technologies, 4(2-3):191-199, June 2000.

[26] A. Schmidt, H. W. Gellersen, and C. Merz. Enabling implicit human computer interaction: a wearable RFIDtag reader. In Proc. of the 4th International Symposium on Wearable Computers (ISWC'20), Atlanta, GA, USA, pages 193-194. IEEE, October 2000.

[27] J. R. Smith, K. P. Fishkin, B. Jiang, A. Mamishev, M. Philipose, A. D. Rea, S. Roy, and K. Sundara-Rajan. RFID-based Techniques for Human-activity Detection. Communications of the ACM, 48(9):39-44, September 2005 .

[28] J. R. Smith, B. Jiang, S. Roy, M. Philipose, K. Sundara-Rajan, and A. Mamishev. ID Modulation: Embedding Sensor Data in an RFID Timeseries. In M. Barni, J. Herrera-Joancomartí, S. Katzenbeisser, and F. PérezGonzález, editors, the 7th International Workshop of Information Hiding (IH'05), Barcelona, Spain, Revised Selected Papers, volume 3727 of Lecture Notes in Computer Science, pages 234-246. Springer Berlin Heidelberg, June 2005.

[29] E. M. Tapia, N. Marmasse, S. S. Intille, and K. Larson. MITes: Wireless portable sensors for studying behavior. In Proc. of the Extended Abstracts Ubicomp 2004, Nottingham, England, September 2004.

[30] R. L. S. Torres, D. C. Ranasinghe, Q. Shi, and A. P. Sample. Sensor enabled wearable RFID technology for mitigating the risk of falls near beds. In Proc. of the 2013 IEEE International Conference on RFID (RFID'13), Malaysia, pages 191-198. IEEE, April-May 2013.

[31] R. Want. An introduction to RFID technology. IEEE Pervasive Computing, 5(1):25-33, January 2006.

[32] R. Want, K. P. Fishkin, A. Gujar, and B. L. Harrison. Bridging Physical and Virtual Worlds with Electronic Tags. In Proc. of the 1999 ACM SIGCHI Conference on Human Factors in Computing Systems (CHI'99), Pittsburgh, PA, USA, pages 370-377. ACM, May 1999.

[33] M. Weiser. The Computer for the 21st Century. Mobile Computing and Communications Review, 3(3):3-11, July 1999. 


\section{Author Biography}

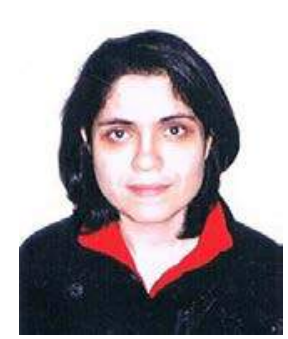

Marina Pérez received the B.S. degree in telecommunication engineering in 2011 and the M.S. telecommunication engineering in 2014, both from Technical University of Madrid. He is currently pursuing the Ph.D. degree in physical electronics at Telecommunication Engineering School, UPM. His research interests include magnetic sensors, microprocessors, underwater communications, aerospace technology and communications based on magnetic induction.

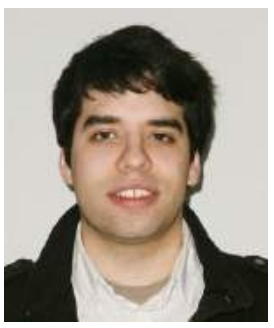

Borja Bordel received the B.S. degree in telecommunication engineering in 2012 and the M.S. telecommunication engineering in 2014, both from Technical University of Madrid. He is currently pursuing the Ph.D. degree in telematics engineering at Telecommunication Engineering School, UPM. His research interests include CyberPhysical Systems, Wireless Sensor Networks, Radio Access Technologies, Communication Protocols and Complex Systems.

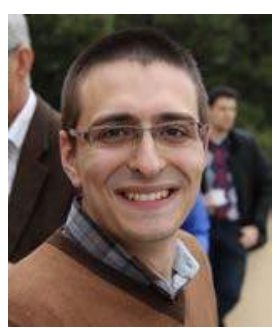

Ramón Alcarria received his M.S. and Ph.D. degrees in Telecommunication Engineering from the Technical University of Madrid in 2008 and 2013 respectively. Currently, he is an assistant professor at the E.T.S.I Topography of the Technical University of Madrid. He has been involved in several R\&D European and National projects related to Future Internet, Internet of Things and Service Composition. His research interests are Service Architectures, Sensor Networks, Human-computer interaction and Prosumer Environments. 


\title{
Predictive algorithms for mobility and device lifecycle management in Cyber- Physical Systems
}

\author{
Borja Bordel Sánchez ${ }^{1 *}$, Ramón Alcarria ${ }^{2}$, Diego Sánchez de Rivera ${ }^{1}$ and Alvaro Sánchez-Picot ${ }^{1}$
}

\begin{abstract}
Cyber-Physical Systems (CPS) are often composed of a great number of mobile, wireless networked devices. In order to guarantee the system performing, management policies focused on becoming transparent to high-level applications, the changes in the hardware platform have to be implemented. However, traditional reactive methodologies and basic proposed predictive solutions are not valid either due to the extremely dynamical behavior of CPS or because the high number of involved devices prevents fulfill the timing requirements. Therefore, in this paper, we present an advance predictive solution for managing the mobility and device lifecycle, being able to meet all requirements of CPS. The solution is based on an infinite loop, which calculates, in each iteration, a sequence of future system states using a CPS simulator and interpolation algorithms. Furthermore, an experimental validation is provided in order to determine the performing of the proposed solution.
\end{abstract}

Keywords: Cyber-Physical Systems, Mobility management, Device lifecycle, Predictive models, CPS simulation, Interpolation algorithms

\section{Introduction}

CPS are the next generation of engineered systems in which computing, communication, and control technologies are tightly integrated [1]. In theory, any type of device could be part of a Cyber-Physical System: large or miniaturized, wired or wireless, fixed or mobile, etc. However, most practical discussions about the characteristics of CPS conclude that they are composed of a great number of wireless [2], embedded [3], mobile [2] devices.

Wireless mobile devices allow creating a more flexible network architecture than other types of devices [4]. But, on the contrary, these devices tend to interact and communicate opportunistically [4], so the access to them is not guarantee, in general. Nevertheless, most CPS applications (such as energy infrastructures, transport systems, or medical instruments) require a permanent and transparent access to the hardware capabilities, so

\footnotetext{
* Correspondence: bbordel@dit.upm.es

${ }^{1}$ Department of Telematics Systems Engineering, Universidad Politécnica de Madrid, Avenida Complutense n 30, 28040 Madrid, Spain

Full list of author information is available at the end of the article
}

mobility and device lifecycle management policies are essential in CPS.

Besides, this challenging situation has turn more complex due to the appearance of concepts such as the Cyber-Physical Internet [5]. These scenarios consider the possibility of deploying various communicated CPS in the same geographical area, so mobile devices not only can move inside the coverage area of one CPS but also they can move to other CPS and later return (or not).

In traditional systems, device lifecycle and mobility are supported by means of reactive techniques, i.e., the system makes decisions or actions when a certain change occurs [6]. However, these techniques suppose the changes in the system are continuous and slow, so there is enough time to determine and execute the proper actions before a fatal error occurs. For example, in LongTerm Evolution (LTE) networks [7], a handover is executed when quality or power measures from a user equipment go below a certain limit. Then, it is suppose these measures are not going to change abruptly, so there is time to execute the handover (although it is a long and complicated process) before losing the 
connectivity definitively. However, CPS present an extremely dynamical behavior, and the system state may change rapid and randomly [8]. For example, in underwater military applications [9], the medium can degrade so fast that there is no enough time to react properly before losing access to hardware devices, since a relevant change in the signal quality is detected. Thus, reactive techniques are not valid in CPS, as the decision and actions are calculated considering situations which may change strongly during the calculation process.

On the other hand, a small number of basic predictive solutions are also available. In these proposals, the system predicts the future changes and executes the appropriate actions before they occur. However, they are based on heavy simulators which are useful in simple scenarios but which fail when used in CPS due to the high number of involved devices. In these cases, the needed time to simulate a certain future situation is higher than remaining time to really reach it. Then, the essential timing requirement of predictive solutions is not fulfilled.

Therefore, in this paper, we propose a predictive algorithm for device lifecycle and mobility management in CPS, being able to predict the future states of the system and calculate and execute the appropriate actions before future changes or fatal errors occur. The proposed algorithm is based on a CPS simulator and interpolation functions being able to calculate the future system states. These results feed a decision-making module, which is not part of the proposed predictive algorithms, but which is required to manage the mobility and the device lifecycle. The proposed solution presents a flexible definition, so it could be adapted to any application scenario of CPS (low-rate networks, intelligent devices, etc.). Moreover, an experimental validation is provided, considering a particular implementation of the proposed algorithm. The proposed experiment proves that in more than $95 \%$ of cases, the algorithm manages the system changes successfully.

The rest of the paper is organized as follows: Section 2 introduces the state of the art in mobility and device lifecycle in CPS. Section 3 presents the application scenario and proposes the predictive algorithm. Section 4 describes a particular implementation of the algorithm, used in the experimental validation. Finally, Sections 5 and 6 explain some results of this experimental validation and the conclusions of our work.

\section{State of the art}

Works on managing the device lifecycle in CPS are not common. In general, papers which treat this topic are focused on configuring the whole CPS, not only on managing the hardware platform. Then, in general, particular details about hardware issues (such as controlling the remaining battery charge in wireless devices) are not addressed.

In this area, different types of proposals are found. Some works are focused on the use of artificial intelligence algorithms being able of automatically adding, removing, and configuring components in CPS [10]. Others describe special tools (such as middleware layers) which, at the end, need the human intervention [11]. Works describing algorithms where each device generates a description file when a change occurs in the system, which is processed by the rest of the platform, may be also found [12]. Finally, papers discussing the installation of an intelligent scheduler in each device, communicating with a central core where decisions are taken are also available [13, 14].

However, as can be seen, all the previous proposals follow a reactive scheme which is not valid in our general scenario (it must be noted that, sometimes, these solutions could be valid, if some assumptions are considered).

The topic of mobility support in CPS has received more attention than device lifecycle, although it is not a main research line nowadays. In general, two types of mobility may be defined in CPS: intra-system and intersystem.

In intra-system mobility scenarios, devices move inside the coverage area of one CPS. Proposal about this topic delegate the mobility control to the underlying network [15]. In these works, devices are clients of a mobile network through which a data service (representing the CPS applications) is provided. These solutions present a good performing; however, as we said in the introduction, nowadays concepts such as the Cyber-Physical Internet have to be considered, and this scheme cannot cover the requirements of these scenarios. Then, mobility problem in CPS is focused on inter-system mobility.

In the case of inter-system mobility, works used to propose application-specific mobile frameworks (based on SOA, for example), adapted to the particular mobility requirements of their scenario $[16,17]$. Moreover, some authors incorporate GPS transponders into devices, in order to provide geographical data to a computational core. Then, the core may determine if devices are or not in the coverage area [18]. Nevertheless, all these proposals (including the cited for the intra-system case) follow a reactive scheme and are valid in very restrictive scenarios (as we said previously).

Finally, relating to inter-system mobility management, predictive schemes have been also proposed [19]. In these solutions, predictive algorithms are based on simulators which use numerical integration functions (one for each device) [20]. Then, the resulting algorithms present an order of complexity of $o\left(n^{2}\right)$ for both: the number of considered devices and the number of 
instants for which the system state is calculated. Thus, in scenarios where hundreds of devices are included, and/or where the dynamics requires to simulate the system state in several time instants, the needed time to simulate a certain future situation may be higher than remaining time to really reach it.

Therefore, basically, it is necessary to design a predictive algorithm being able to manage both the device lifecycle and the mobility and to predict the future system states before it reaches them. The solution should be as general as possible and should allow including a high number of devices and obtaining the system state in as many time instants as needed. For that, we propose an algorithm based on an infinite loop, where four steps are repeated in each iteration: (i) data about the real system state from hardware devices; (ii) a CPS simulator obtains a certain number of future system states using the data acquired; (iii) the rest of the system states are obtained using interpolation techniques; and (iv) the decisionmaking algorithms execute the adequate actions based on the predicted future system states. The use of interpolation techniques, as we are seeing, introduces a greater error in the predicted future states. However, they reduce strongly the needed time to obtain a sequence of future states. In the experimental validation section we are proving the resulting solution allow predicting and managing correctly the changes in the system, despite the additional error introduced by interpolation techniques.

\section{Proposal: a predictive algorithm based on CPS simulation}

In this section, we analyze the general characteristics of the base scenario, show the functional architecture for the proposed solution and describe the proposed predictive algorithm.

\subsection{Base scenario, functional architecture}

As we said in Section 2, we are looking for an algorithm as general as possible. However, CPS may be deployed in very different scenarios: from small-scale applications to large-scale solutions [21]. Then, a deep analysis of device lifecycle and mobility in CPS should be based on the definition and characterization of several different deployments (manufacturing [7], irrigation [22], etc.), architectures, implementations, and/ or use cases. Nevertheless, in our case, we are going to work over some general characteristics (common to most cases), which are

1. Various small-scale CPS are deployed in the same area. Besides, the high-level applications and processes associated to one CPS are not modified or affected by changes in the hardware platform.
2. The communication among the different CPS is supported by the so-called Cyber-Physical Internet [6], to which CPS are connected through an "Intersystem services interface."

3. Two types of CPS are defined: internal-CPS and border-CPS. Internal-CPS present a coverage area completely surrounded by the coverage areas of other CPS. In border-CPS, the limits of the coverage area represent the geographical limits of the CyberPhysical Internet.

4. The movement of the mobile devices can be defined as follows: (a) free, when there is not external control; (b) controlled, when it is possible to plan the and route for them and; (c) limited to one or several specific routes independently if they are free or controlled.

5. Only predictable changes are considered. In real systems, both predictable and unpredictable changes occur: devices run out of battery charge or leave a coverage area (which are predictable changes), but also they get broken down or the tasks they are executing get blocked (which are unpredictable changes). Unpredictable events have to be addressed using reactive techniques [23], which are not discussed in this work but which must complement the proposed solution in commercial deployments.

The first and second points focus the mobility problem on inter-system mobility. As we said, valid solutions for intra-system mobility may be found, and mobility problem in CPS is centered almost exclusively on intersystem mobility. The third point refers to the geographic limitation of the small-scale CPS and their underlying network which, in general, does not have worldwide coverage. Figure 1 shows graphically the resulting network architecture from the first three points.

The fourth point is directly related to the great diversity of devices which can be considered: from robots to wearable devices. Each one of these devices presents a different movement and the proposed solution should consider all of them.

Finally, the fifth point limits the scope of the proposed solution to predictable situations. These situations represent the majority of changes in the regular operation of a CPS (or, even, in traditional communication networks [24]). However, traditional reactive techniques (such as timers) may complement the proposed predictive solution, especially in commercial deployments where unpredicted system failures might occur and have to be managed.

The functional architecture for supporting the main proposal of this paper (the predictive algorithms, see Section 3.2) can be seen in Fig. 2. Various components may be distinguished. 


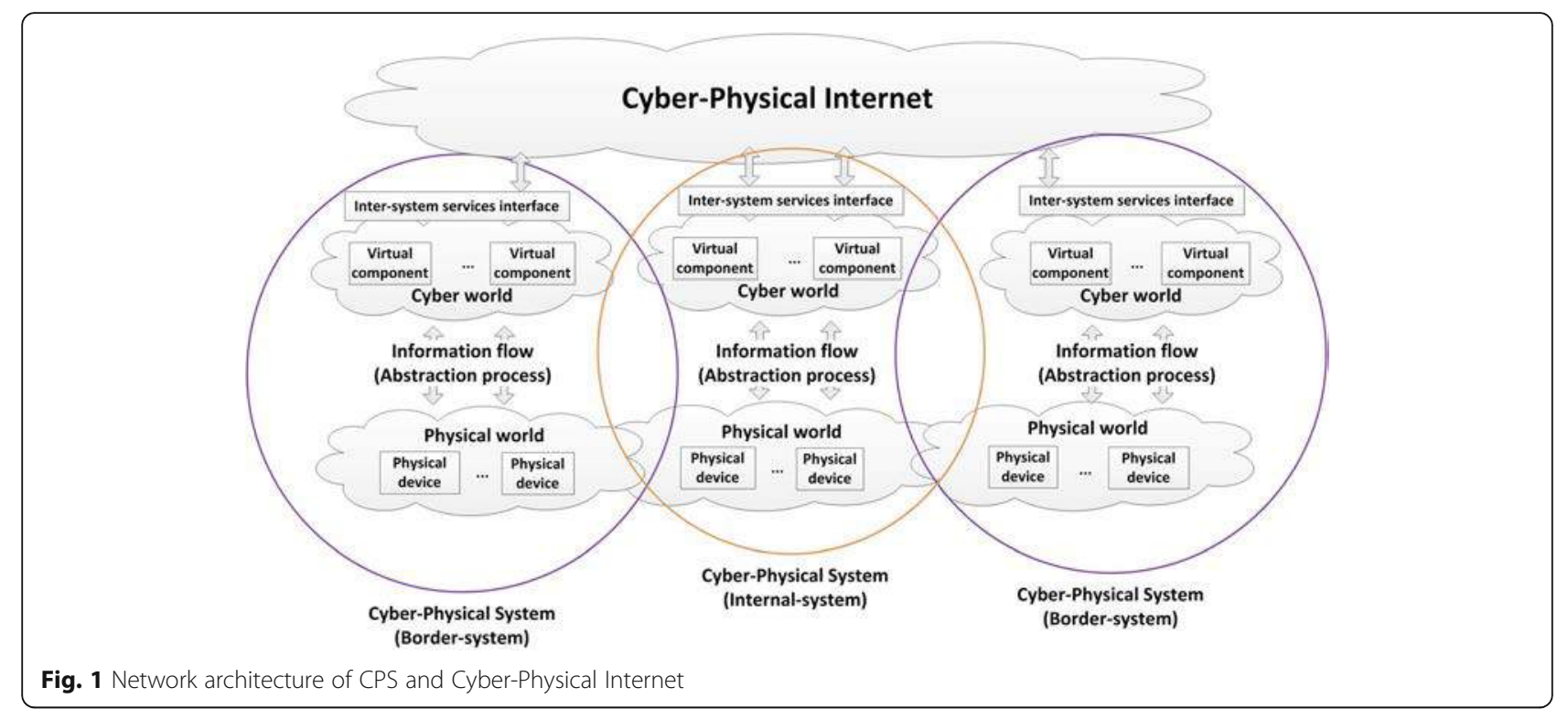

Peripherals: They include all the components which support the task execution in a CPS (sensors, actuators, processors, etc.). They communicate through an appropriate middleware (such as a serial port) with their associate controllers.

Hardware controllers: It refers to all the components which manage and control the operation of the peripherals. In particular, they include all the necessary drivers to operate the peripherals. Each hardware controller has associated a certain number of peripherals. A hardware device which implements both peripherals and, at least, one hardware controller is called self-manage device or intelligent device.
Hardware manager: The entire hardware platform is managed from this component. In particular, it includes the module which implements the proposed algorithms (the mobility and device lifecycle predictive manager). It also implements the decision-making algorithms, executes the transactions for system reconfiguration, and makes independent changes in the hardware platform from the high-level applications or processes.

\subsection{A predictive algorithm for device lifecycle and mobility management}

At the start of the system, in the mobility and device lifecycle predictive manager of the hardware manager

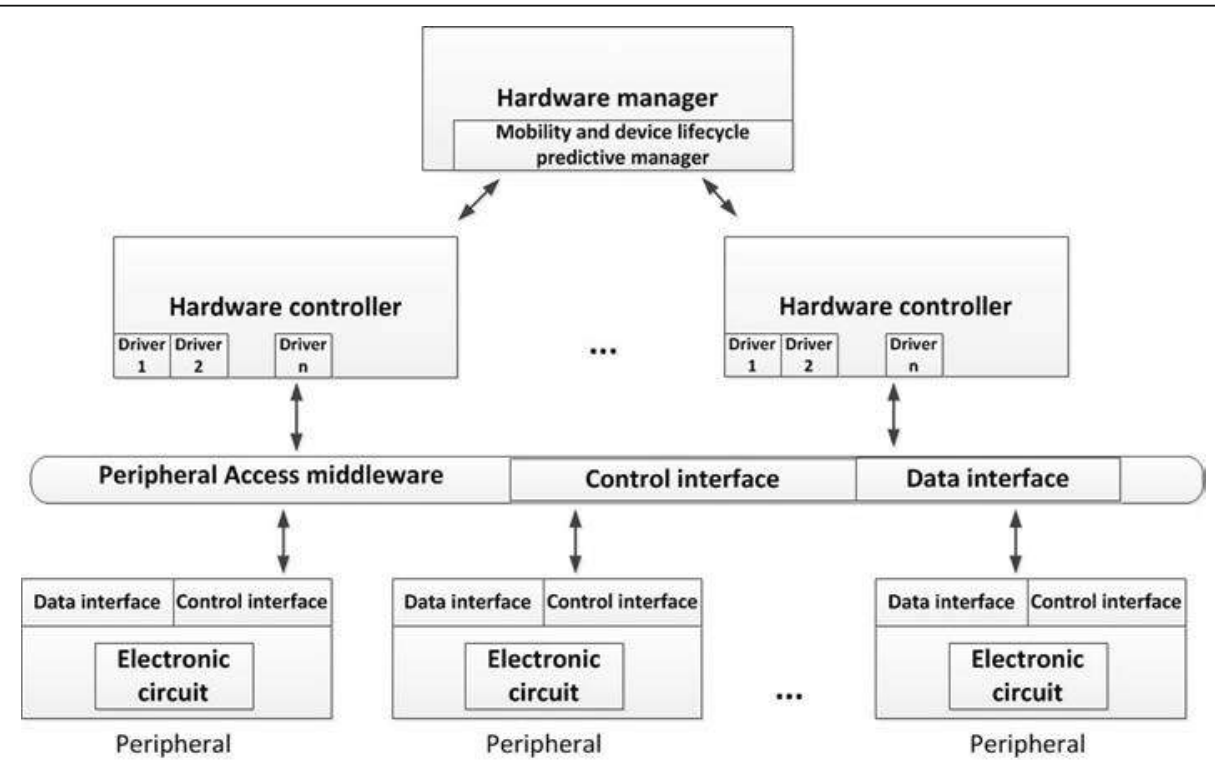

Fig. 2 Functional architecture 
showed in Fig. 2, a model $M$ of the underlying hardware platform is created. In this model, the $i$ th device is represented by a collection $m_{i}$ of $n_{i}$ relevant parameters (1).

$$
m_{i}=\left\{\operatorname{id}_{i}, \text { type }_{i}, \operatorname{param}_{1}, \ldots, \operatorname{param}_{n_{i}}\right\}
$$

The device model $m_{i}$ includes a unique identification $\operatorname{id}_{i}$ and a type identifier type ${ }_{i}$. The type identifier takes values from a set $T$, where each value represents a different class of device (2). The type identifier also indicates the pattern which must follow the collection $m_{i}$ in each case. Examples of possible relevant parameters are the geographical position or the battery charge level.

$$
T=\left\{\text { device_class }_{j} j=1, \ldots, Q\right\}
$$

Then, the entire model $M$ is a list of collections of parameters $m_{i}$ (3). In order to allow its mathematical treatment (for example, in the interpolation functions), this model can also be expressed as matrix $S(4)$. We are calling this matrix representing the value of all important parameters in the system "system state matrix" or, for simplicity, "system state."

$$
\begin{aligned}
& M=\left\{m_{1}, m_{2}, \ldots, m_{k}\right\} \\
& S=\left(\begin{array}{c}
m_{1} \\
\ldots \\
m_{k}
\end{array}\right)
\end{aligned}
$$

In (2) and (3), $k$ represents the total number of devices in the CPS. As, in general, not all device models $m_{i}$ have the same number of relevant parameters $n_{i}$. In system state, matrix dimensions are homogenized by adding as many zeros as needed (these additional zeros allow the mathematical treatment of the system state matrix but are ignored in the decision-making algorithms).

In the mobility and device lifecycle predictive manager, a simulator being able to simulate CPS scenarios is also included. This simulator will take the system state in a certain instant $t=t_{0}, S_{t_{0}}$, and will obtain the system state $h$ seconds later, $S_{t_{0}+h}$. Additional zeros added to homogenize dimensions in the system state matrix will not be taken into account.

Two additional important parameters are the time step $h$ and the total simulated time $T_{\text {sim }}$. Then, the predictive algorithm must obtain the system state each $h$ seconds, between the current time $t_{0}$ and $t_{0}+T_{\text {sim. }}$. In order to obtain useful information, the calculation should finish before the first time step passes, i.e., before $t=t_{0}+h$.

In previous proposals about predictive mobility management systems, all the needed future states are obtained by means of the CPS simulator. However, in complex systems where a great number of devices are considered, and/or where several time instants have to be obtained (i.e., the time step $h$ is very small), timing conditions cannot be fulfilled, i.e., the calculations do not finish before $t=t_{0}+h$. As a solution, we propose to generate some future states, instead of through the CPS simulator using interpolation techniques (which are much faster and lighter, although they present a greater error). Then, three different types of system states may be found:

- Intra-state: It represents the real state of the system in a certain instant $t=t_{0}$. In this state, the values of the parameters in the model are obtained from the physical devices through a data acquisition process. The cited data acquisition process could be based on a periodical transmission of information from the physical devices to the management unit; or in a solution where the management unit requests the devices for that information. In both cases, solutions based on JSON objects or XML description files are the most adequate (although any other could be used). Intra-state is the most precise way of describing the system; however, it is also the most costly (in time and processing capabilities). These states will be noted as $S^{I}$ or $I$. At least, one intra-state has to be composed at the beginning of each iteration in the infinite loop.

- Predictive state: It refers the states obtained from the simulator. To obtain a predictive state, it is necessary to dispose, first, of an intra-state. From one intra-state, we may be able to obtain as many predictive states as desired. However, the precision in the prediction goes down, as we try to predict farther temporal instant is farther than what we desire to predict. On the other hand, the time needed to calculate a predictive state is lower than necessary to acquire an intra-state. These states will be noted as $S^{P}$ or $P$.

- Interpolated state: It refers to the states obtained by interpolating two predictive states or one intra-state and one predictive state. These states are really fast to obtain but present the biggest error. This error goes up when more temporal distance exists between the states interpolated. These states will be noted as $S^{B}$ or $B$.

The proposed algorithm is based on the definition of a sequence of predictive and interpolated states, describing the system in collection of future instants $\left\{t_{0}, t_{0}+h, \ldots\right.$, $\left.t_{0}+T_{\text {sim }}\right\}$. Every sequence of states must start with an intra-state. And, later, a predictive or interpolated state is obtained for each time step, according to a predesigned pattern. The selected pattern might be very varied and should be adapted to the specific application scenario. In general, a balance between precision, calculation, speed, and the amount of data transmitted must be achieved. Figure 3 shows some possible schemes, depending on the considered scenario. 


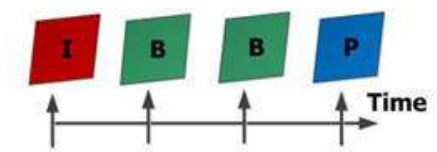

Current time

Default configuration

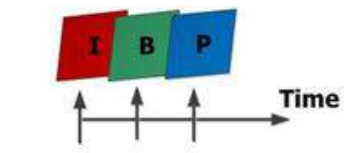

Current time

Systems evolving rapidly

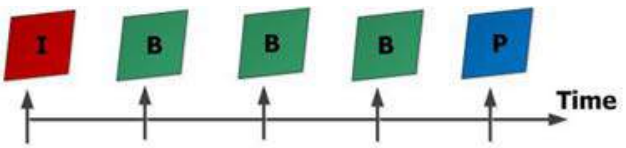

Current time

Systems evolving slowly

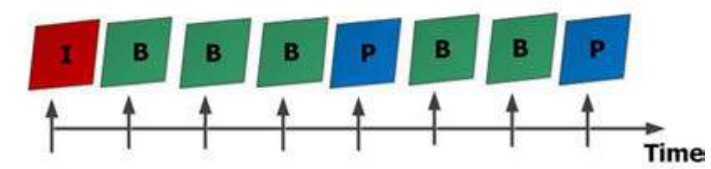

Current time

Fig. 3 Possible sequences of states

Given a certain time step $h$ and a total simulated time $T_{\text {sim }}$, the precision of the sequence of states goes up, when the number of predictive states included increases. However, the calculation time also increases and, perhaps, the timing condition cannot be met. On the contrary, if more interpolated states are considered, the calculation time will go down strongly. Nevertheless, the error in the predicted states will be greater, and some changes could not be predicted. We argue that it is possible to find a pattern adapted to the considered CPS, such that it allows fulfilling the timing condition and the additional introduced error does not affect the correct prediction of changes in the system. In Sections 4 and 5, we prove that.

On the other hand, the precision in the predicted states also goes up when the time step $h$ decreases. Nonetheless, in this case, meeting the timing condition (i.e., the calculation time must be smaller than the time step) gets more complicated. Finally, $T_{\text {sim }}$ may be used as a design parameter. In general, if the considered CPS presents a very variable behavior, the simulation time $T_{\text {sim }}$ will tend to be small (as well as the time step).

Then, in order to clarify the proposed algorithm, a numerical example is provided. In a CPS, four equal devices are available. Each device is represented by three relevant parameters. Numerically, the four models get described by

$$
\begin{array}{ll}
m_{1}=\{1,1,9,8,7\} & m_{2}=\{2,1,9,8,7\} \\
m_{3}=\{3,1,9,8,7\} & m_{4}=\{4,1,9,8,7\}
\end{array}
$$

These models are ordered as a matrix, which corresponds with the first intra-state obtained:

$$
S=\left(\begin{array}{lllll}
1 & 1 & 9 & 8 & 7 \\
2 & 1 & 9 & 8 & 7 \\
3 & 1 & 9 & 8 & 7 \\
4 & 1 & 9 & 8 & 7
\end{array}\right)=S_{t_{0}}^{I}
$$

Using a CPS simulator, a predictive stet is obtained:

$$
S_{t_{0}+T}^{P}=\left(\begin{array}{ccccc}
1 & 1 & 6 & 8 & 5 \\
2 & 1 & 1 & 0 & 0 \\
3 & 1 & 2 & 6 & 6 \\
4 & 1 & 9 & 8 & 7
\end{array}\right)
$$

And, finally, using matrix interpolation techniques, at least one interpolated state is calculated:

$$
S_{t_{0}+\frac{T}{2}}^{B}=\left(\begin{array}{ccccc}
1 & 1 & 8 & 8 & 6 \\
2 & 1 & 5 & 4 & 4 \\
3 & 1 & 6 & 7 & 6 \\
4 & 1 & 9 & 8 & 7
\end{array}\right)
$$

Then, the situation of the devices for each temporal instant is obtained by reconstructing the device models from the previous matrixes

$$
\begin{aligned}
& t=\frac{T}{2} \quad m_{1}=\{1,1,8,8,6\} \quad m_{2}=\{2,1,9,4,4\} \\
& m_{3}=\{3,1,6,7,6\} \quad m_{4}=\{4,1,9,8,7\} \\
& t=T \quad m_{1}=\{1,1,6,8,5\} \quad m_{2}=\{2,1,1,0,0\} \\
& m_{3}=\{3,1,2,6,6\} \quad m_{4}=\{4,1,9,8,7\}
\end{aligned}
$$

As we said, the proposed sequence of predictive and interpolated states must be repeated in an infinite loop, which in each iteration starts with obtaining an intrastate. This new intra-state could be obtained for an instant posterior to the calculated interpolated and predictive states. However, as we said, obtaining an intra-state is a very costly task in time. Then, a temporal gap could appear in the management activities due to the delay of the intra-state generation process. In order to avoid that, the new intra-state may be acquired for a moment before the end of the current sequence (see Fig. 4). 


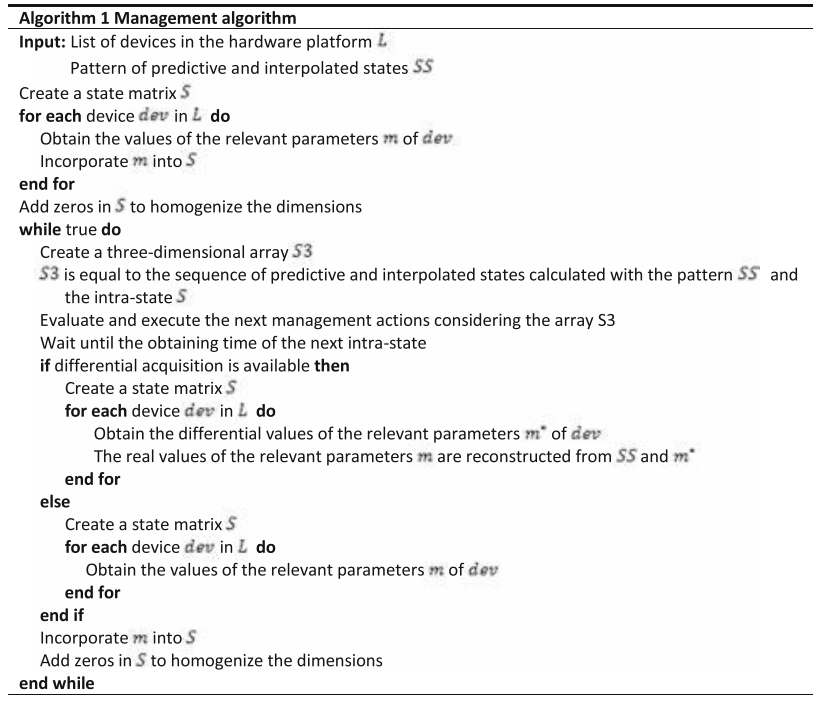

Moreover, in the second case, and if devices in the hardware platform present a certain level of intelligence, the intra-state may be generated using differential acquisition (see Fig. 4). In differential acquisition, each device is able to predict (and interpolate) its future states. Then, in order to reduce the required time to collect the information about the real device state, they only transmit to the hardware manager the differences between one of the last predictive or interpolated states in the sequence and the reality. Later, the hardware manager reconstructs the complete intrastate. This solution is in general much more efficient (especially if information compression is also enabled) and, overall, faster (which helps to meet the timing condition).

Then, considering all previous discussion, Algorithm 1 presents the general management algorithm.

In summary, Algorithm 1 creates the first intra-state and calculates the sequence of interpolated and
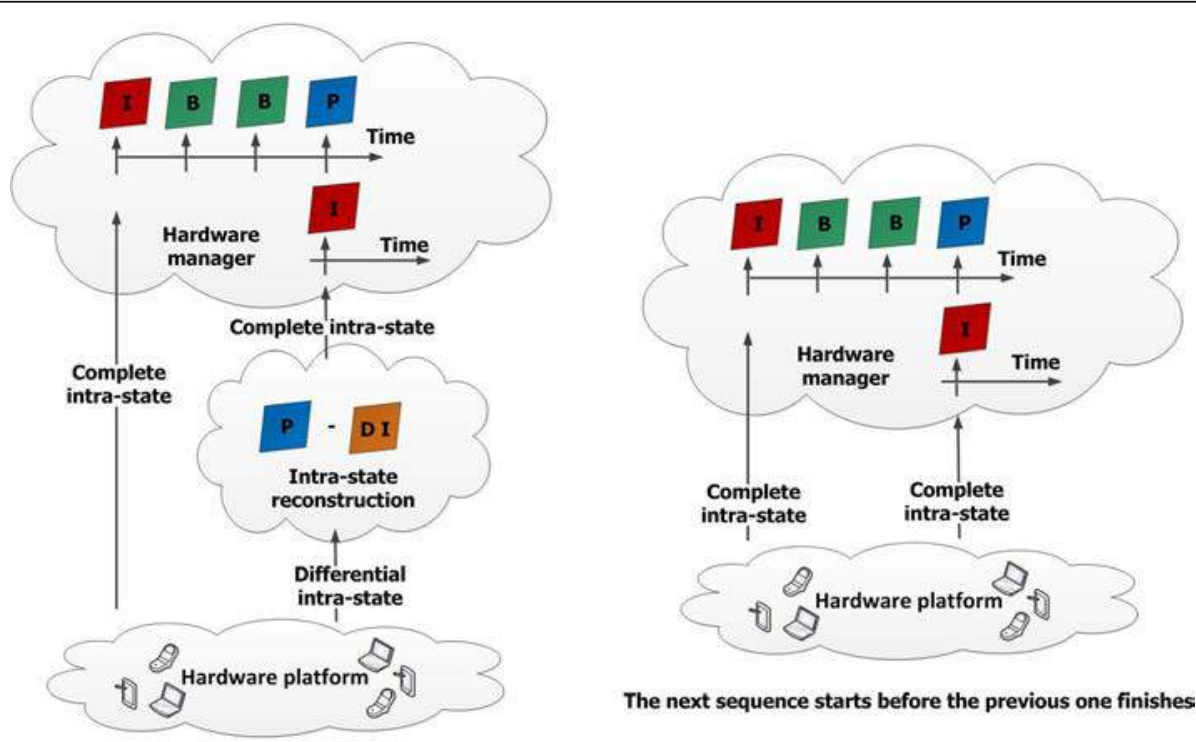

The next sequence starts before the previous one finishes

The next sequence starts before the previous one finishes Differential intra-state acquisition

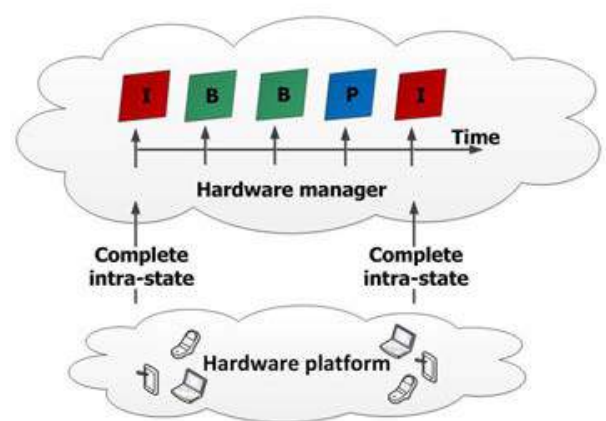

The next sequence starts when the previous one finishes

Fig. 4 Possible restarts of the sequence of states 
predictive states using the obtained intra-state and given the desired pattern. Then, it calls the decision-making process with the calculated sequence of future states and performs the appropriate actions. Finally, it waits until it is necessary to obtain the next intra-state, and then acquires it (using differential acquisition if available).

In Algorithm 1, two procedures remain undefined: the decision-making function and the calculation process of the future states (including the simulation and interpolation process). With respect to the first function (decision-making), it is totally independent of the proposed predictive algorithms (see Section 3.3). Concerning the second function (calculation of the future states), Algorithm 2 represents its implementation.

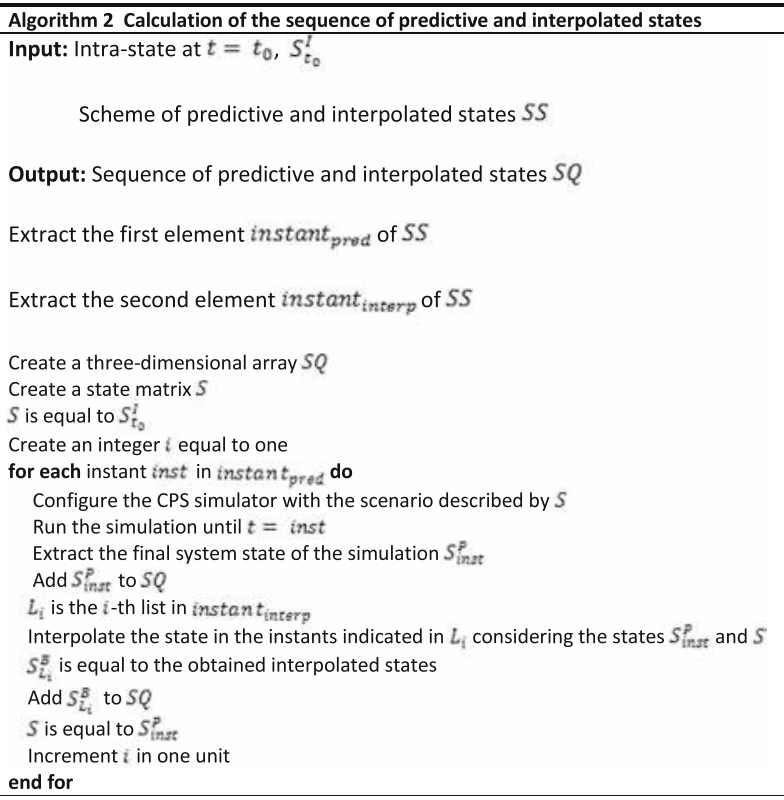

As can be seen in Algorithm 2, two parameters are taken as input: the original intra-state and the scheme of predictive and interpolated states. This scheme has the following structure (5).

$$
\mathrm{SS}=\left\{\text { instant }_{\text {pred }}, \text { instant }_{\text {interp }}\right\}
$$

SS is a list of two elements where the fist element, instant $_{\text {pred }}$, is a list of the temporal instants for which a predictive state must be calculated. The second element, instant $_{\text {interp, }}$ is a list of lists indicating the structure of the interpolated states (6).

$$
\text { instant }_{\text {interp }}=\left\{\left\{T_{1}, \ldots, T_{q}\right\}_{i}, i=1, \ldots, \text { length }\left(\text { instant }_{\text {pred }}\right)\right\}
$$

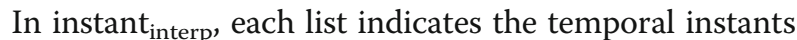
for which an interpolated state must be calculated. The first list indicates the instants between the first intrastate and the first predictive state, the second list indicates the instants between the first and the second predictive states, etc.

Considering Algorithm 2, a first numerical evaluation of the impact of using interpolated states in the calculation time may be done. As we said, simulators present an order of complexity of $o\left(n^{2}\right)$ for both, the number of considered devices and the number of instants for which the system state is calculated. On the contrary, interpolation algorithms present an order of complexity of $o(n)$ for both, the number of considered devices and the number of instants for which the system state is calculated. Then, we consider the needed time to calculate an intra-state for a certain CPS $T_{I}$, the required time to calculate one predictive state in the same CPS $T_{P}$ and, finally, the needed time to calculate one interpolated state in that CPS $T_{B}$. If we suppose the selected pattern for the sequence of future states is the default configuration (see Fig. 3), the time employed in the calculation process if interpolated states are not considered is (7). In (7) $T_{3-P}$ is the calculation time for three predictive states.

$$
T_{I}+T_{3-P}=T_{I}+3^{2} T_{P}
$$

In the same situation, if interpolated states are considered, the needed time is (8).

$$
T_{I}+T_{P}+T_{2-B}=T_{I}+T_{P}+2 T_{B}
$$

In the general case, $T_{I}>T_{P}>T_{B}$. In order to obtain a first evaluation, we imagine that $T_{I} \approx 3 T_{P}$ and $T_{P} \approx T_{B}$. In those circumstances, the use of interpolated states reduces the calculation time around $50 \%$. Then, it is clear that the use of interpolated states helps to fulfill the timing condition.

On the other hand, it is important to remark that interpolation techniques also present some disadvantages. As we said, interpolated states have a low precision, although they can be calculated in a very fast way. The second part helps to fulfill timing requirements, but the first idea refers to the need of more complex decision-making algorithms (depending on the case). If interpolation techniques are correctly designed and the CPS presents a regular behavior, then the loss of precision does not affect significantly the mobility and device lifecycle management. However, in CPS whose behavior tends to be very dynamical and changing (or if interpolation techniques are not correctly planned), the loss of precision might be remarkable and the decisionmaking modules should implement mechanism to operate in those conditions (what complicates the algorithm design and programming). 
Finally, in Algorithm 2, any of the available CPS or Internet-of-Things (IoT) simulators is valid. For example, the simulator CyPhySim [20, 25] proposed by the Berkeley University could be employed. Other options are the NS3 simulator [26], the SimpleIoTsimulator suite [27], or the junction of MATLAB and Simulink [28]. Relating to the interpolation process, also any of the available techniques may be used: from linear interpolation [29] to cubic splines [30].

\subsection{Decision-making and predictive solutions}

Once a sequence of future states is generated, the decision-making algorithms evaluate the information and decide to execute the appropriate actions. Many possible decision-making algorithms may be employed in the predictive algorithms. On the most efficient case, intelligent decision-making [31] would be used. However, in this work, we have designed a more simple strategy.

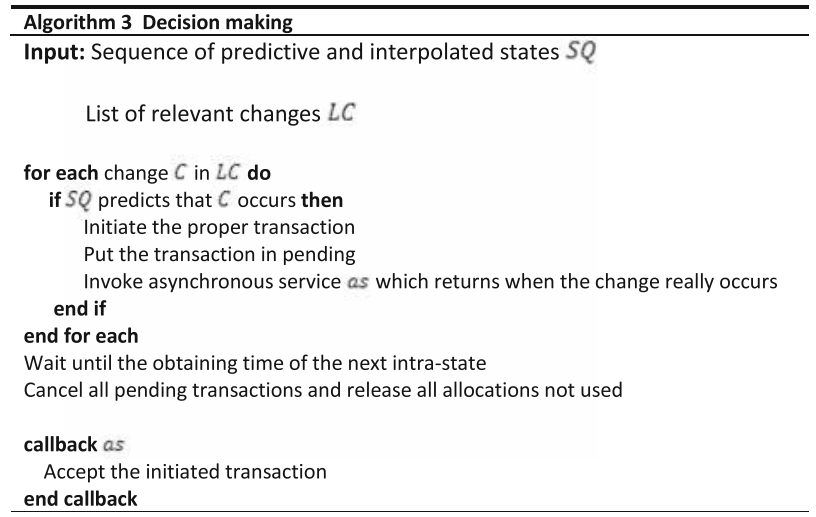

In our proposal, in each sequence of future states, the decision-making algorithm looks for the relevant changes defined in the system (such as a device running out of battery charge). Then, if any of them is found, the system will initiate the transaction for the system reconfiguration immediately. Once all the parameters have been negotiated, all data transmitted, etc., the transaction is pending. In the moment when the change really occurs, the transaction is finally accepted and the CPS is reconfigured. If, at the end, the change never happens, the transaction is canceled. Algorithm 3 presents the decisionmaking function specifically designed for this work.

On the other hand, in advanced scenarios, decisionmaking module may also change the scheme of interpolated and predictive states. As we said, CPS present a very dynamical behavior. In some circumstances, the selected scheme for the interpolated and predictive states at first might not be adequate when the CPS evolves. In those cases, the decision-making algorithms may implement mechanism to detect the increase in the number of unpredicted changes and to reconfigure the scheme of states in order to be more effective.

\section{Practical implementation and experimental validation}

For the first practical implementation of the proposed solution, we have selected as deployment scenario a laboratory at the Technical University of Madrid. The laboratory is organized in three rooms (see Fig. 5), being deployed a different CPS (i.e., a different hardware manager) in each room.

In this scenario, three predictable relevant changes can occur: (a) devices move from the coverage area of one CPS to the coverage area of another, (b) devices leave all the deployed CPS, or (c) devices run out of battery and become unavailable. In the first case, a handover must be executed. In the second and third case, if necessary, tasks being executed by the device have to be delegated or canceled. In advance scenarios, techniques for avoiding the predicted events could also be employed. For example, in applications involving humans, messages to prevent them from leaving the system could be sent. These advanced solutions, however, are not considered in this work.

In CPS, processes are not assigned to only one device. Instead, processes are divided in several tasks which are executed in the hardware platform. For this reason, if one device is transferred to other CPS, the whole context (process) cannot be transferred. Then, handovers in our CPS are managed as a case of IPv6 mobility [32] and

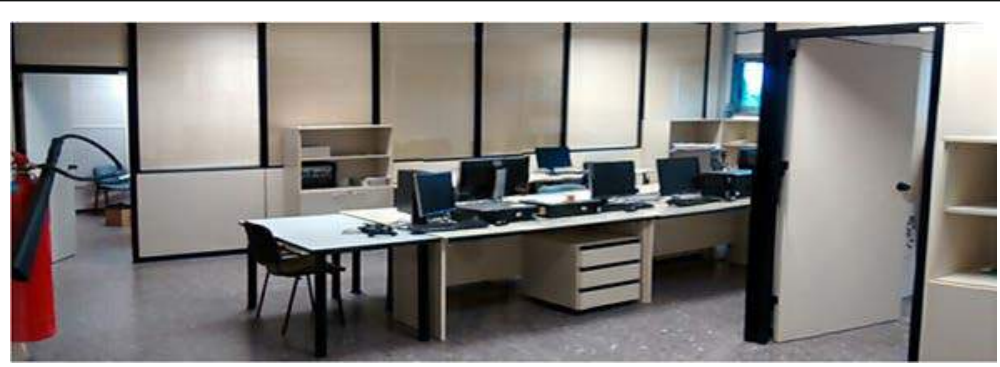

Fig. 5 Deployment scenario 


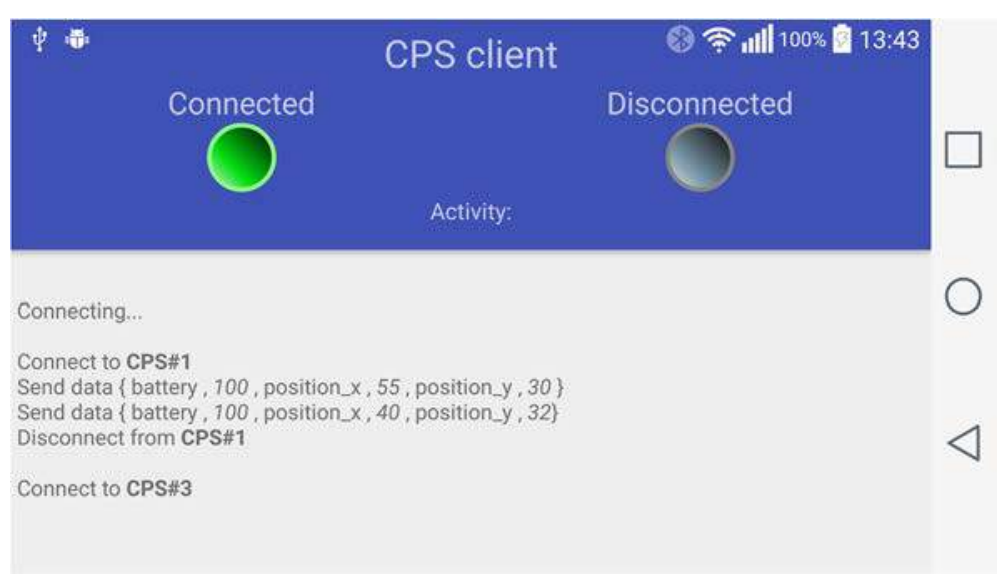

Fig. 6 CPS client application

not as traditional handovers from mobile networks. In that way, the target CPS acts only as a proxy, connecting the source CPS with the transferred device.

In the case that the device leaves all CPS or runs out of battery charge, if the estimated time to finish the assigned tasks is greater than the remaining time until the device becomes unavailable, resources are allocated in other device or devices, and the necessary data are transmitted to them. When the device becomes unavailable, the new execution is activated. If, at the end, the device remains available, the allocated resources are released.

We performed a system deployment in order to validate the proposed solution. For that, the hardware platform in the described CPS is made of various smartphones and tablets, where an application called CPS Client was installed. This application (see Fig. 6) allows the device to be part of hardware platform when pressing the connect button and releases the device by pressing the disconnect button. When acting as an element of the hardware platform, the device tries always to be connected and sends periodically the needed data to the hardware manager to create the mandatory intra-states. It also shows some information about the activity in the system.

In this scenario, differential acquisition is not available, and the employed simulator is an integration of the NS3 simulator and the suites MATLAB and Simulink [33]. The pattern of predictive and interpolated states is as follows (9) (time given in seconds). As can be seen, $h=1 \mathrm{~s}$ and $T_{\text {sim }}=3 \mathrm{~s}$. In order to select these values, we tried to not commit errors up to the maximum typical error considered in engineering. We considered people walking to have a medium speed of $1.1 \frac{\mathrm{m}}{\mathrm{s}}$ [34]. Then, in a room with $30 \mathrm{~m}$ long, in $3 \mathrm{~s}$ a person has varied its situation in more than a $10 \%$ (the typical limit to consider a value negligible in engineering).

$$
S_{t=0}^{I} \quad S_{t=1}^{B} \quad S_{t=2}^{B} \quad S_{t=3}^{P}
$$

In the proposed scheme of future system states, two interpolated states have been considered. Walking people tend to follow a very predictable path and batteries are discharged slowly, so various interpolated states may be included without committing great errors. It must be noted that a matrix cubic spline technique was implemented as interpolation technology.

In this scenario, thirty-six (36) people are provided with the CPS Client and are requested to operate in the coverage area of the CPS. They also could leave that zone. The human behavior perfectly represents the complicated dynamic of CPS. Data about the number of managed changes, the success rate and the calculation time were collected.

\section{Results and discussion}

More than 400 relevant changes were registered while performing the experimental validation. Figure 7 shows the distribution of the registered changes, depending on

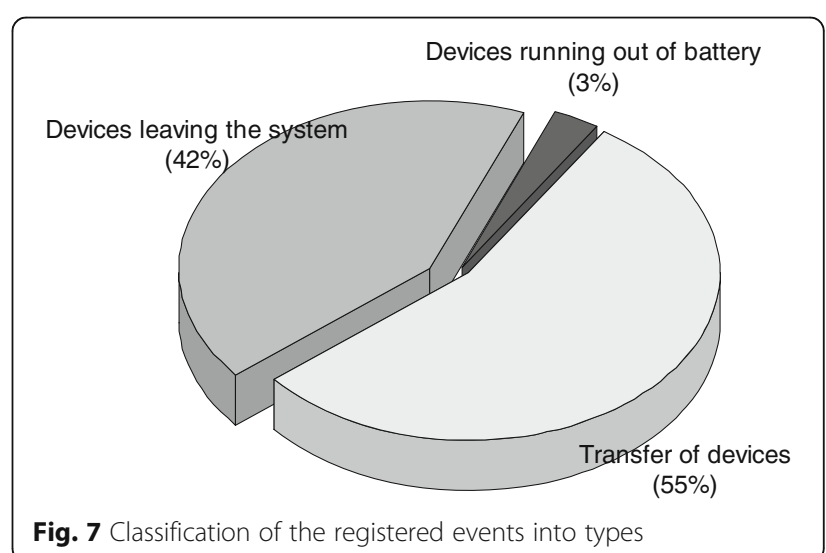


Aggregated sucess rate

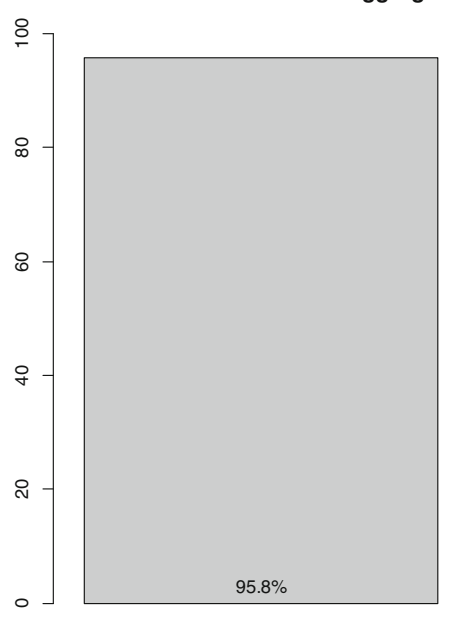

Fig. 8 Aggregated success rate

their type. As can be seen, most of them were handovers (55\%). This fact agrees with the scenario configuration, where three different CPS were deployed. On the other hand, changes about devices running out of battery charge were few (3\%), as most devices had batteries with enough capacity to be independent for dozens of hours.

Most of these changes were successfully managed, i.e., the expected actions are taken when the change occurs (and only if the change occurs). The aggregated success rate is slightly over $95 \%$ (see Fig. 8). With respect to the wrongly managed events (around a $4 \%$ ), all errors are due to changes unpredicted by the simulator, so the management algorithm failed. The underlying cause of these misinterpretations is the dynamical model employed to represent the system evolution, which for some phenomena (such as the batteries discharge process) implements laws which only are approximated mathematical functions due to the complex behavior of these phenomena. For example, typically the battery charge is overestimated, and devices may turn off before executing the adequate actions. Advanced CPS models [35] would solve this situation.

In conclusion, the proposed predictive algorithm, where patterns include interpolated states, clearly allows the correct management of changes in the hardware platform (despite the greater error included).

In fact, around the $4 \%$ of events are unpredicted by the simulator. However, some additional considerations should be given to Fig. 8. If the success rate is disaggregated into the three event types, some relevant facts might be exposed (see Fig. 9).

First, as can be seen, the success rate in the case of transferring a device to a second CPS (a handover) is higher than the aggregated rate. If we consider a device leaving the entire deployment, the success rate is, more or less, equal to the aggregated rate. These high values for the success rate may be associated to a good model for the device movement in the simulator. Then, most displacements are correctly predicted.

On the other hand, success rate in the case that a device runs out of battery charge is much fewer (around a $30 \%$ fewer) than the aggregated rate. Clearly, that is due to the complexity associated to the battery charge simulation. Generic models are employed in these simulations, but they poorly fit the real evolution of battery charge. Then, an important percentage of the events of this type are not predicted. Nevertheless, as this type of events only represents the $3 \%$ of the total amount events, the impact in the aggregated success rate is limited.

Finally, Fig. 10 represents the histogram of the calculation time employed in obtaining each one of the 250 sequences of future states generated during the experimental validation. As can be seen, the most

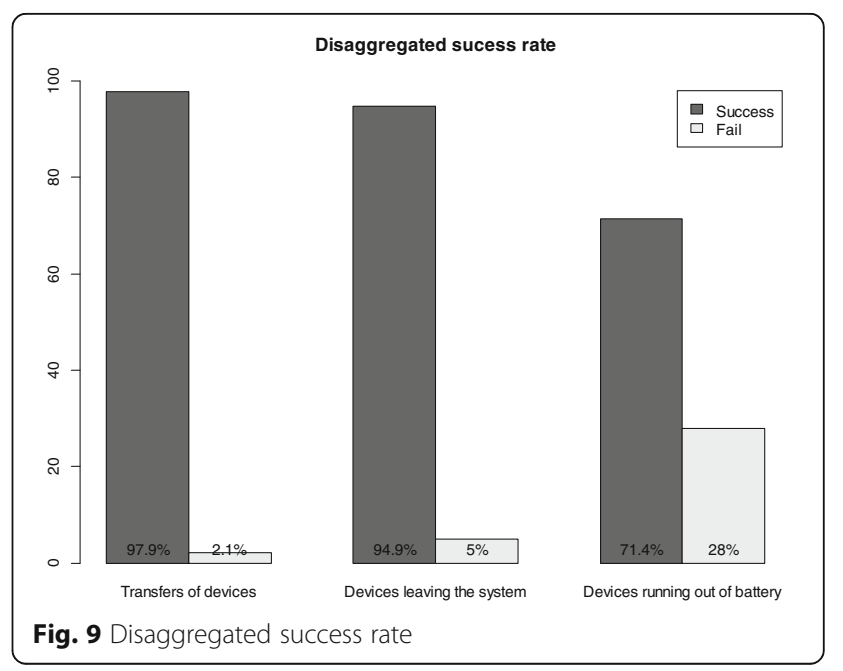




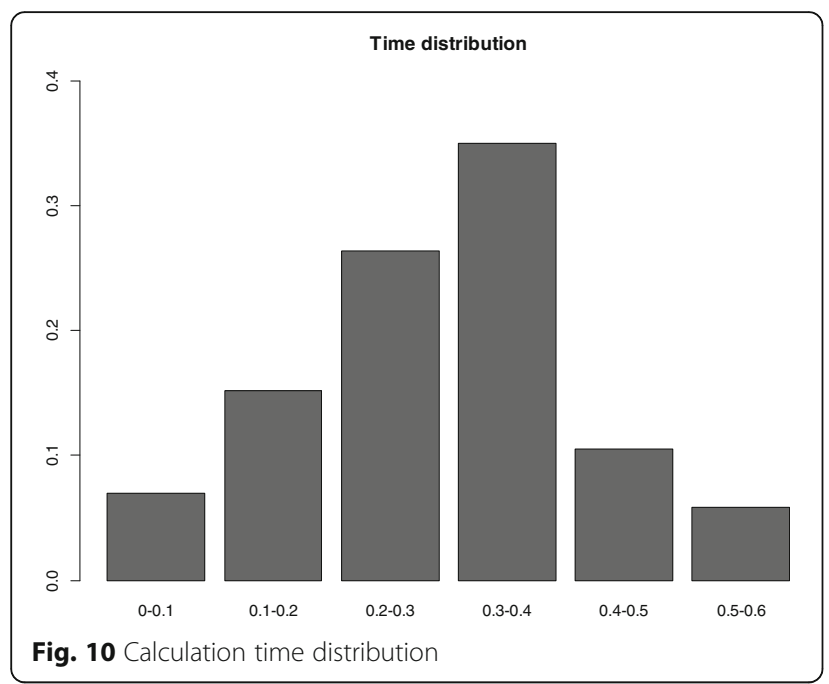

probable time is the interval of $0.3-0.4 \mathrm{~s}$, so it is clearly smaller than the time step $(h=1 s)$. Besides the maximum calculation time was $0.57 \mathrm{~s}$, which continuous being smaller than the time step. In conclusion, the proposed solution meets the timing condition.

\section{Conclusions}

Most practical discussions about the characteristics of Cyber-Physical systems (CPS) conclude that they are composed of wireless, embedded, mobile devices. Thus, techniques for mobility and device lifecycle management are necessary. Traditional reactive solutions are not valid in CPS, as they present a complex, and sometimes random, dynamic. The proposed basic predictive solution cannot be employed as they do not fulfill the timing requirements. Therefore, in this paper, we propose an advanced predictive technique for managing the mobility and device lifecycle, being able to meet all requirements of CPS. The solution is based on an infinite loop, which calculates, in each iteration, a sequence of future system states using a CPS simulator and interpolation algorithms.

As we saw, the obtained success rate is higher than the $95 \%$, so the proposed solution correctly manages the changes in the hardware platform. However, most complicated elements (such as the battery charge) present difficulties to be simulated correctly. Besides, timing requirements are comfortably met.

The proposed solution is valid for all types of CPS; however, improved simulation models are needed and tools for generating them automatically are also necessary. Obtaining these technologies will determine the future commercial success of our proposal. Moreover, reactive techniques which complement the proposed predictive solution are necessary, in order to create a really useful hardware management algorithm for CPS.

\section{Funding}

The research leading to these results has received funding from the Ministry of Economy and Competitiveness through SEMOLA project (TEC2015-68284R) and from the Autonomous Region of Madrid through MOSI-AGIL-CM project (grant P2013/ICE-3019, co-funded by EU Structural Funds FSE and FEDER).

\section{Authors' contributions}

The contributions described in this work are distributed among the authors in the way that follows: all the authors conceived and designed the solution; $\mathrm{BB}$ and RA wrote the paper; DSdeR performed the experiments; and ÁS-P programmed the simulator. All authors read and approved the final manuscript.

\section{Competing interests}

The authors declare that they have no competing interests.

\section{Author details}

${ }^{1}$ Department of Telematics Systems Engineering, Universidad Politécnica de Madrid, Avenida Complutense n³0, 28040 Madrid, Spain. ²Department of Topographic Engineering and Cartography, Universidad Politécnica de Madrid, Campus Sur, 28031 Madrid, Spain.

Received: 1 June 2016 Accepted: 15 September 2016

Published online: 22 September 2016

\section{References}

1. KD Kim, PR Kumar, Cyber-Physical Systems: a perspective at the centennial. Proc. IEEE 100(Special Centennial Issue), 1287-1308 (2012)

2. K Wan, KL Man, D Hughes, Specification, analyzing challenges and approaches for Cyber-Physical Systems (CPS). Eng. Lett. 18(3), 308 (2010)

3. M Jiménez, R Palomera, I Couvertier, Introduction to embedded systems (Springer, New York 2013). ISBN: 978-1-4614-3142-8. doi: 10.1007/978-14614-3143-5

4. S Aram, I Khosa, E Pasero. Conserving energy through neural prediction of sensed data. JoWUA. 6(1), 74-97 (2015)

5. A Koubâa, B Andersson, A vision of cyber-physical internet. In 8th International Workshop on Real-Time Networks (RTN'09) (Instituto Politécnico do Porto. Porto, 2009). http://hdl.handle.net/10400.22/3837

6. A Bindel, P Conway, L Justham, A West, New lifecycle monitoring system for electronic manufacturing with embedded wireless components. Circuit World 36(2), 33-39 (2010)

7. B. Bordel Sanchez, Á. Sánchez-Picot, \& D. Sanchez De Rivera, Using 5 G technologies in the Internet of things handovers, problems and challenges. in Innovative Mobile and Internet Services in Ubiquitous Computing (IMIS), 2015 9th International Conference on (IEEE, 2015), pp. 364-369.

8. B Bordel Sánchez, R Alcarria, D Martín, T Robles, TF4SM: a framework for developing traceability solutions in small manufacturing companies. Sensors 15(11), 29478-29510 (2015)

9. V Katiyar, N Chand, N Chauhan, Recent advances and future trends in wireless sensor networks. Int. J. Appl. Eng. Res. 1(3), 330 (2010)

10. N. Keddis, G. Kainz, C. Buckl, \& A. Knoll, Towards adaptable manufacturing systems. in Industrial Technology (ICIT), 2013 IEEE International Conference on (IEEE, 2013), pp. 1410-1415

11. DD Hoang, HY Paik, CK Kim, Service-oriented middleware architectures for Cyber-Physical Systems. Int. J. Comput. Sci. Netw. Secur 12(1), 79-87 (2012)

12. U Mönks, H Trsek, L Dürkop, V Geneiß, V Lohweg, Assisting the design of sensor and information fusion systems. Procedia Technol 15, 35-45 (2014)

13. T. Dillon, V. Potdar, J. Singh, \& A. Talevski, Cyber-Physical Systems: providing quality of service (QoS) in a heterogeneous systems-ofsystems environment. in Digital Ecosystems and Technologies Conference (DEST), 2011 Proceedings of the 5th IEEE International Conference on (IEEE, 2011), pp. 330-335.

14. TS Dillon, H Zhuge, C Wu, J Singh, E Chang, Web-of-things framework for Cyber-Physical Systems. Concurrency Comput. Pract. Exp 23(9), 905-923 (2011)

15. D. Work, A. Bayen, \& Q. Jacobson, Automotive cyber physical systems in the context of human mobility. in National Workshop on high-confidence automotive Cyber-Physical Systems (Troy, Berkeley University 2008), http:// bayen.eecs.berkeley.edu/sites/default/files/conferences/cps1.pdf 
16. V. C. M. X. Hu, W. Leung, B.C. Du, P. Seet, \& P. Nasiopoulos, A service oriented mobile social networking platform for disaster situations in Proc. 46th HICSS (2013), pp. 136-145

17. X Hu, T Chu, H Chan, V Leung, Vita: a crowdsensing-oriented mobile CyberPhysical System. Emerg. Top. Comput. IEEE Trans 1(1), 148-165 (2013)

18. C Fok, A Petz, D Stovall, N Paine, C Julien, S Vishwanath, Pharos: a testbed for mobile Cyber-Physical Systems. (The University of Texas, The Center for Advanced Research in Software Engineering, Austin, 2011). TR-ARiSE-2011-001

19. J Fink, A Ribeiro, V Kumar, Robust control for mobility and wireless communication in Cyber-Physical Systems with application to robot teams. Proc. IEEE 100(1), 164-178 (2012)

20. E.A. Lee, M. Niknami, T.S. Nouidui, \& M. Wetter, Modeling and simulating Cyber-Physical Systems using CyPhySim. in Proceedings of the 12th International Conference on Embedded Software (IEEE Press, 2015), pp. 115-124

21. R Poovendran, K Sampigethaya, SKS Gupta, I Lee, KV Prasad, D Corman, JL Paunicka, Special issue on Cyber-Physical Systems [scanning the issue]. Proc. IEEE 100(1), 6-12 (2012)

22. T Robles, R Alcarria, D Martın, M Navarro, R Calero, S Iglesias, M López, An IOT based reference architecture for smart water management processes. J. Wirel. Mob. Netw. Ubiquit. Comput. Dependable Appl 6(1), 4-23 (2015)

23. F-Y Leu, H-L Chen, C-C Cheng, Improving multi-path congestion control for event-driven wireless sensor networks by using TDMA. J. Internet Serv. Inf. Secur 5(4), 1-19 (2015)

24. P Satam, H Alipour, Y Al-Nashif, S Hariri, Anomaly behavior analysis of DNS protocol. J. Internet Serv. Inf. Secur 5(4), 85-97 (2015)

25. C. Brooks, E. A. Lee, D. Lorenzetti, T. S. Nouidui, \& M. Wetter, M. CyPhySim: a Cyber-Physical Systems simulator. in Proceedings of the 18th International Conference on Hybrid Systems: Computation and Control (ACM, 2015), pp. 301-302

26. NS3 simulator webpage. Available online: https://www.nsnam.org/ Accessed on 25 May 2016

27. SimpleloTsimulator webpage. Available online: http://www.smplsft.com/ SimpleloTSimulator.html Accessed on 25 May 2016

28. CM Ong, Dynamic simulation of electric machinery: using MATLAB/Simulink, vol. 5 (Prentice Hall PTR, Upper Saddle River, 1998)

29. G. Barequet, \& M. Sharir, Piecewise-linear interpolation between polygonal slices. in Proceedings of the tenth annual symposium on Computational geometry (ACM, 1994), pp. 93-102

30. C De Boor, A practical guide to splines, vol. 27 (Springer, New York, 1978), p. 325

31. NK Janjua, FK Hussain, OK Hussain, Semantic information and knowledge integration through argumentative reasoning to support intelligent decision making. Inf. Syst. Front. 15(2), 167-192 (2013)

32. D Johnson, C Perkins, J Arkko, Mobility support in IPv6 (No. RFC 3775), 2004

33. Dmitry Kachan. Integration of NS-3 with MATLAB/Simulink. Available online: http://epubl.Itu.se/1653-0187/2010/062/LTU-PB-EX-10062-SE.pdf. Accesed 25 May 2016

34. Y Feng, K Liu, Q Qian, F Wang, X Fu, Public-transportation-assisted data delivery scheme in vehicular delay tolerant networks. Int. J. Distrib. Sens. Netw. (2012)

35. D Martín, J García Guzmán, J Urbano, A Amescua, Modeling software development practices using reusable project patterns: a case study. J. Softw. Evol. Process 26(3), 339-349 (2014)

\section{Submit your manuscript to a SpringerOpen ${ }^{\circ}$ journal and benefit from:}

- Convenient online submission

- Rigorous peer review

- Immediate publication on acceptance

- Open access: articles freely available online

- High visibility within the field

- Retaining the copyright to your article

Submit your next manuscript at $>$ springeropen.com 


\title{
A Methodology for the Design of Application-Specific Cyber-Physical Social Sensing Co-Simulators
}

\author{
Borja Bordel Sánchez ${ }^{1, *}$, Ramón Alcarria ${ }^{2}$, Álvaro Sánchez-Picot ${ }^{1}$ and Diego Sánchez-de-Rivera ${ }^{1}$ \\ 1 Department of Telematics Systems Engineering, Universidad Politécnica de Madrid, Avenida Complutense \\ n³0, 28040 Madrid (España), Spain; alvaro.spicot@gmail.com (Á.S.-P.); diegosanchez@dit.upm.es \\ (D.S.-d.-R.) \\ 2 Department of Topographic Engineering and Cartography, Universidad Politécnica de Madrid, Campus \\ Sur, 28031 Madrid (España), Spain; ramon.alcarria@upm.es \\ * Correspondence: bbordel@dit.upm.es; Tel.: +34-91-549-57-00 (ext. 3035)
}

Received: 25 July 2017; Accepted: 19 September 2017; Published: 22 September 2017

\begin{abstract}
Cyber-Physical Social Sensing (CPSS) is a new trend in the context of pervasive sensing. In these new systems, various domains coexist in time, evolve together and influence each other. Thus, application-specific tools are necessary for specifying and validating designs and simulating systems. However, nowadays, different tools are employed to simulate each domain independently. Mainly, the cause of the lack of co-simulation instruments to simulate all domains together is the extreme difficulty of combining and synchronizing various tools. In order to reduce that difficulty, an adequate architecture for the final co-simulator must be selected. Therefore, in this paper the authors investigate and propose a methodology for the design of CPSS co-simulation tools. The paper describes the four steps that software architects should follow in order to design the most adequate co-simulator for a certain application, considering the final users' needs and requirements and various additional factors such as the development team's experience. Moreover, the first practical use case of the proposed methodology is provided. An experimental validation is also included in order to evaluate the performing of the proposed co-simulator and to determine the correctness of the proposal.
\end{abstract}

Keywords: Cyber-Physical Social Sensing; co-simulation; social simulation; networks simulators; MASON; NS3; Cyber-Physical Systems

\section{Introduction}

Cyber-Physical Social Sensing (CPSS) systems [1] are ubiquitous mobile wireless sensor networks where intelligent terminals equipped with various sensors are integrated. These terminals perceive human social information such as the environment or the social activities. CPSS include also a central server where the information is uploaded and from which users are provided with data services (see Figure 1). The final objective of CPSS is to enrich human-to-human, human-to-object, and object-to-object interactions in the physical world, human society, as well as in the virtual world [1].

As can be deducted from the previous description, CPSS are intrinsically concurrent. Various domains (such as the social, physical or cyber) coexist in time, evolve together and influence each other [2] (see Figure 1).

In CPSS, the social world includes all people sharing the same space (as large as wanted) and time, including their individual and group physical and physiological state and all human-to-human interactions among the habitants (affective or physical) [3,4]. Many specialized works on sociology and group psychology have studied these issues [5-8]. 
The physical world includes all physical phenomena and third-party devices and objects which are relevant to the CPSS operation [9]. The physical world also includes all the processes and object-to-object interactions developed by the elements which compose it. The temperature evolution in closed rooms [10], or the traditional industrial systems [11], belong to the physical world. The impact of the physical world in the social world has been exhaustively studied in the context of the so-called social environment [12-15], but always from a narrative point of view (never with practical applications).

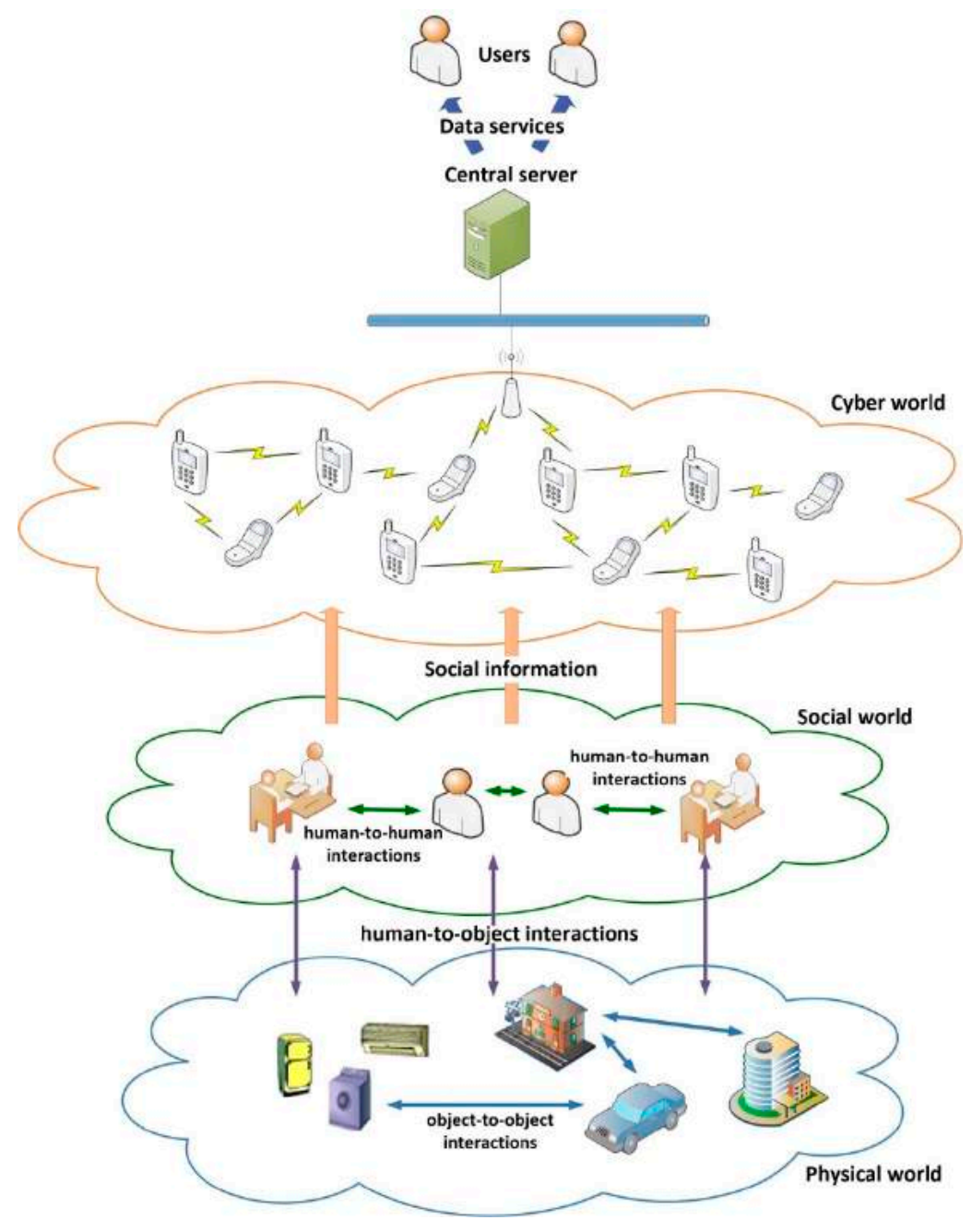

Figure 1. Cyber-Physical Social Sensing (CPSS) scheme.

Finally, the cyber world includes all the intelligent terminals [16], sensors and mobile wireless networks used to sense the social information. The relation between the cyber world and the physical world is probably the most studied. The term cyber-physical systems (CPS) [17] (which refers to the intersection of the physical and cyber worlds) is nowadays one of the most popular terms in research. On the other hand, the field of social sensing [18] (the union of the cyber and social worlds) are also turning more important day by day (with concepts such as the Social Internet-of-Things [18,19]), although it continues to be less popular than CPS.

The novelty of CPSS, then, is to consider the three subsystems (sometimes together with other domains such as the mental world [20]) evolving together, formalizing some ideas which have started to appear very recently (such as the union of CPS and social sensing) [21]. Table 1 summarizes the relations among the different subsystems as described above. 
Table 1. Different concepts about the relations among the CPSS subsystems.

\begin{tabular}{cccc}
\hline Concept & Physical World & Social World & Cyber World \\
\hline Cyber-Physical systems & $\mathrm{x}$ & & $\mathrm{x}$ \\
Social Internet-of-Things & & $\mathrm{x}$ & $\mathrm{x}$ \\
Social Environment & $\mathrm{x}$ & $\mathrm{x}$ & $\mathrm{x}$ \\
Social sensing & & $\mathrm{x}$ & $\mathrm{x}$ \\
CPSS & $\mathrm{x}$ & $\mathrm{x}$ & \\
\hline
\end{tabular}

As a current technological paradigm, CPSS present some common characteristics with other recent proposals such as the Internet-of-Things (IoT). However, while IoT solutions are focused on the global interconnection of "things" (daily living objects provided with embedded computers), CPSS are primary designed to perceive human social information including the environment, transportation and social activities [22]. In both cases, a heterogeneous hardware platform with a high density of devices is considered. Nevertheless, IoT systems consider people as users and things as the main element in the systems, while in CPSS the human factor is the basic information source and sensors (usually integrated into intelligent devices) are only the medium through which obtain that information. Technically, CPSS may be understood as an extension of IoT systems, were human factors are combined with traditional devices. As an evolution of Cyber-Physical Systems [23], CPSS use to define feedback control loops, where social information is processed and returned to social world in order to generate a certain effect. On the other hand, IoT services do not have restrictions and may be based on the simple data acquisition.

In any case, it must be noted that how the social world is integrated into CPS/IoT in order to create CPSS systems is an open issue [20]. Some works (the oldest ones) monitors social information by means of user surveys or user interfaces [24], defining kind of collaborative platforms (in order to forecast the bus arrivals [25] for example). Other define sensor networks were nodes interact with others and share information as people do in social networks [26]. Finally, and probably the most popular approach nowadays, some authors understand that CPSS should monitor people in a passive way (without human intervention, surveys or interfaces) as CPS monitors temperature or manufacturing process. In order to do that, sensors included in intelligent terminals are usually employed $[27,28]$. Using the acquired information, people behavior, movement, etc. could be tracked and the environment (public infrastructure, such as airports, for example) would react to this social information.

In this work, we are following the last approach, considering that CPSS are systems focused on monitoring people social behavior in a passive, unobtrusive and non-intrusive way.

The most important difficulty that CPSS designers face is the problem of constructing large systems, including thousands of devices, people and a great amount of information [29]. Besides, CPSS have to manage very different abstraction levels, from the high-level information typical of the social world, to the very low-level information, so common in the cyber world (especially when hardware is considered) [20]. In order to address this problem, application-specific tools are necessary for specifying and validating designs and simulating systems.

Nowadays, specific tools are employed to simulate each domain independently (a social simulator for the social world, a network simulator for the cyber world, etc.). This solution is very efficient in time, as each tool is extremely optimized to simulate a specific domain. In fact, most new tools for CPSS simulation would be less efficient and would require more computing power [30]. However, this situation also presents important disadvantages. Mainly, it firstly forces the decomposition of the CPSS scenario in different subsystems, simulating each domain in one environment and, later, relates the results obtained from each tool. All these actions must be performed by different experts, simulations becoming a very costly and complicated task. Thus, despite the probable inefficiency in time, the future is the co-simulation [31].

Nevertheless, today there is a lack of co-simulation instruments being able to simulate together all domains which made up a CPSS. The main cause of this situation is the difficulty of relating correctly the simulation of various subsystems, as well as the complexity of interaction with final 
users in order to acquire the necessary co-simulator's requirements and characteristics. In particular, integrating and/or synchronizing various simulation tools implies modifying the execution routine or the communication and graphic interfaces among other tasks. Moreover, interacting with final users in order to acquire the required system characteristics implies to coordinate a communication process among all the stakeholders. Most of these activities are very time consuming and many times researches, companies or users do not implement new co-simulation tools for not being sure whether the effort is worthwhile. In that situation, the role of software architects is vital, as they must guarantee that the selected design or solution is the most adequate for every case: depending on the application, the users' needs, the acquired requirements, the development team and the simulations to be performed (among other topics). In order to obtain a truly correct, efficient and functional co-simulator, appropriate to each specific situation, all important factors must be considered following an ordered process. However, the methodology that software architects should follow in order to acquire the users' requirements and select their own design for an application-specific co-simulator has been scarcely studied: only partial and incomplete proposals have been reported.

Therefore, in this paper a methodology for the design of CPSS co-simulation tools is proposed. The work describes the four steps that software architects should follow in order to design their own application-specific CPSS co-simulator, as well as the previous phase of requirements and characteristics capture involving both software architects and final users. The proposal includes from the characteristics fixing and the selection of the most appropriate co-simulation paradigm, to the choice of the graphic environments for results presentation. The paper provides, also, the first practical use case of the proposed methodology, implementing the obtained design using currently available technologies. Finally, results obtained from the implemented CPSS co-simulator are included. From the analysis of these experimental results, future challenges and research lines are identified.

During the last months CPSS has become a very popular research topic. Different works about the applications of the CPSS paradigm to social Big Data [32], robotics [26], people tracking [27] and wireless sensor networks (WSN) [33] have been recently reported. In most of these papers, experimental validations are based on laboratory deployments, theoretical analyses or concept proofs [24]. However, some variables (such as scalability) are not easily evaluable using these techniques and/or imply a huge cost. Thus, papers consisting of an exhaustive evaluation of algorithms for CPSS or other similar proposals usually include the design and implementation of a CPSS co-simulator [34,35]. A commercial co-simulator may require several months or years before it can be released, however, many times, CPSS researchers need to design and built and ad-hoc co-simulator in order to evaluate the performance of their proposals. The scope of these instruments is to be used as a general-purpose CPSS co-simulator, but be employed in a specific application. In that way, the objective of this paper is to guide every researcher working on CPSS (which sometimes does not have technical knowledge on programming or simulator construction) during the design process of his own application-specific co-simulator.

The rest of the paper is organized as follows. Section 2 reviews previous works about methodologies for co-simulation tools design and describes the state of the art in social, physical and cyber simulation. Section 3 describes the proposed methodology and discusses the requirements all CPSS co-simulator should fulfill. Later it deeply discusses the four steps which made up the proposed methodology, including the requirements capture and characteristic fixing, the different available co-simulation paradigms, the simulation model and simulation lifecycle, the different coordination and synchronization mechanisms, and the different options for the graphic environment for results presentation. Section 4 describes the first use case of the proposed methodology and the proposed experimental validation in order to evaluate its performance and the correctness of the proposed methodology. Finally, Section 5 shows the results obtained from the experiments. Section 6 concludes the paper and refers some future challenges. 


\section{State of the Art}

In this section, a general overview about the recent proposals on CPSS is provided. Moreover (in the second subsection), most relevant works on co-simulation are revised. In the third subsection, specific proposals about CPSS co-simulation are reviewed. Finally, current tools and proposals for simulating every domain involved in CPSS are revised (first physical processes simulators, secondly social simulators and finally network simulator).

\subsection{Recent Proposals on CPSS}

CPSS is an acronym which may refer various proposals: from Cyber-Physical Social Sensing to Cyber-Physical Social Systems [36]. Furthermore, it is not clear if all these proposals refer to the same reality or present some differences [22]. However, in both cases, CPSS represents the deep integration of traditional technological systems (such as the Internet of Things or Cyber-Physical Systems) with human and social factors, such as people behavior, social opinions or the user mental world [20].

In that way, there is not a unique manner to understand Cyber-Physical Social Sensing. Some works reviewing the different approaches may be found [37].

Some proposals understand that, in CPSS, traditional sensors (present in IoT/CPS scenarios) are substituted by user surveys or user interfaces, which are displayed on mobile smart devices [24]. In that way, people opinion is sensed, creating a kind of collaborative of participative platform for relevant data sharing, in the same way as current social networks [38]. Historically, this approach is, probably, the first one to appear. With this view, systems to predict the bus arrivals [25] or to share information among people in the context of smart cities [39] have been reported. Even, in this kind of proposals, it is very necessary to design (and have been proposed) techniques to protect user personal information (as collaborative platforms require creating a user profile) [40]. Methodologies to design CPSS in an effective way have been also reported [41]. In relation to the promising Industry 4.0 paradigm [42], proposals about new manufacturing paradigms including social networks, social businesses and other similar technologies may be found [43]

On the other hand, other works defend the proposal that CPSS are made of traditional sensors, but being able to stablish ad hoc connections in order to share relevant information with other nodes, as people do in social networks. Proposals related to social robots [26], social techniques for information dissemination in sensor networks [44], social ad hoc networks [45] and social routing protocols $[46,47]$ have been reported around this topic.

Finally, some works describe CPSS, but deal with the problem of sensing the human behavior in a passive and non-intrusive way. It is probably the most recent and popular approach nowadays. In relation to Industry 4.0, the concept of social sensor has been proposed [48]. It consists of a virtual entity acquiring information and statistics in a proactive way from the applications and sensors in the user smartphones. Moreover, works about privacy protection, in order to anonymize the acquired data from users are also common [49,50]. Sampling algorithms in order to collect information from users in an unobtrusive way have been also proposed [51]. Frameworks and architectures focused on collecting and processing information about human behavior $[27,28]$ may be also found. Service composition techniques [36], efficient wireless data transmission [52], etc., for CPSS following this last approach have been also investigated.

\subsection{State-of-the Art on Co-Simulation}

The term co-simulation may be understood in various ways. However, for this work, we are following Broman's proposal, which defines co-simulation as any simulation of a coupled system performed through the composition of various simulators [53]. However, contrary to these previous proposals focused on the coordination of tools considered as black boxes, in this work domain specific tools could be slight modified if required and possible.

Traditional works on co-simulation investigate the coordination between simulators with different time representations (discrete time and continuous time simulators, an approach usually 
named as hybrid co-simulation) [30]. Problems such as the multi-level modeling (reality may be modeled in different ways depending on the abstraction level) [54] or the stability of dynamical systems [55] have been also addressed.

In relation to the coordination of different simulation tools various standard technologies have been proposed. Basically, three of them are the most important: High-level architecture (HLA) [56], Functional Mock-up Interface (FMI) [57] and Discrete Event System Specification (DEVS) [58].

HLA standard is mainly focused on continuous time (CT) simulations. This standard defines an interface, an object model and a set of rules in order to allow simulation tools to communicate regardless the underlying platform. FMI, on the contrary, is usually employed with discrete-event oriented simulations. This standard was specifically designed to simulate Cyber-Physical Systems, and it is based on the creation of a simulation model or a software library called Functional Mock-up Unit (FMU). Finally, DEVS is also focused on discrete systems, although it is different from the previously mentioned proposal as it is a formalized rather than an available technology.

CPSS co-simulation is natively hybrid. Social and physical simulations are (in general) continuous in time, while cyber (network) simulations are based on discrete events. In that way, proposed standards are not completely useful as, as indicated by Broman [53], both standards have limitations for hybrid co-simulation. In fact, some extension of these standards for certain applications have been proposed [59,60], although these proposals do not totally resolve the problem.

\subsection{CPSS Co-Simulation}

A CPSS simulator should be able to simulate all the subsystems composing a CPSS. That implies not only representing elements belonging to each domain, but also considering specific and precise models for them. For example, as we are seeing, various social simulators allow users to include smartphones in simulations, however, no model for the cellular network, congestion, data flow, communication protocols, handovers, etc., is proposed. Physical phenomena, such as heat propagation, cannot either be represented using the appropriate dynamical model. Network and physical simulators, on the other hand, present the opposite problem. The inclusion of all these details into a unique simulation causes simulators to be heavier and slower, but results closer to reality are obtained.

In that way, as previously mentioned, works about methodologies for designing co-simulation tools are scarce. In fact, most works are focused on the implementation process, which it is a later stage to the design phase. Thus, most existing ones are focused on very particular aspects, such as the coordination of continuous and discrete-events simulators [61], the problem of mixing tools implementing time with different data structures (and accuracy) [62], or the creation of a multi-domain description language adequate for the co-simulation [63]. Works on requirements capture can be also found [64,65] and final users' needs evaluation [66,67], but they are focused on generic software, often resulting inefficient. Finally, papers addressing a complete methodology focused on the design process usually investigate procedures to create equivalent models in order to load the entire scenario (including all domains) in a unique simulator [68] (for example, representing people as mobile nodes in the network simulator). We are sure that no work proposes a complete methodology which allows software architects to design their own co-simulators.

In the next subsection, we review the state of the art on the simulation of the three main domains which compose a CPSS.

\subsection{Social, Physical and Network (Cyber) Simulation}

Although systems including five different domains in CPSS have been reported [1], typically they only include three subsystems: physical world, cyber world and social world [2]. Thus, in the context of CPSS simulation, basically three types of proposals should be taken into account: the physical processes simulators, the social simulators and the IT simulators.

The physical processes simulators are based on mathematical engines which calculate numerically the evolution of the system integrating the differential equations which describe the 
system's behavior [63]. These simulators are the oldest [69,70] as they were extremely used in manufacturing, avionics or metal factories. Nowadays, it is difficult to find research works on this topic, as a great variety of powerful commercial products may be found. Simulink [71], MapleSim [72], OpenModelica [73], Wolfram SystemModeler [74] or xcos [75] are some of the most famous and used examples. All these solutions provide a graphic interface and a programming environment which recovers the mathematical details and enable high-level developers work comfortably.

In this area, current research works try to include some aspects of the virtual world in the physical simulations. These solutions are known as mixed simulation [76]. However, most of these proposals are focused on sanitary scenarios and are difficultly extended to other applications, as specific knowledge and configurations is required.

On the other hand, first CPS (Cyber-Physical Systems [77]) simulators have been proposed [7880]. In the final design, these simulators will include the physical and the cyber world; however, nowadays they only consider the physical world and some characteristics about the cyber world. Furthermore, they cannot recover the mathematical details, so experience in numerical integration is required in order to correct manage the simulations.

In both cases, mixed and CPS simulations, the techniques used in order to include the cyber world in the physical one consist of deep modifications in the numerical algorithms and models. Moreover, in general, physical processes simulators integrate the dynamical models for total amount of simulated time and, once obtained the results (consisting of one or various discrete signals); they are showed in the graphic environment or returned in the method callback. Table 2 compares all the simulators described in respect with the most important characteristics. It is important to note that other types of simulator may be found. For example, very recently various cyber physical systems modeling frameworks aiming at modeling different physiological processes have been proposed [81,82]. Moreover, prior works in Cyber Physical systems which aimed at modeling stochastic variables with long tails which are more adequate to model human behavior have also appear [23].

Social simulation studies the interaction among social entities taking into account their psychology and their behavior, their interactions with other entities in the environment and the behavior of all entities as a group [83]. Mainly, there are two different types of social simulations. First, social-level simulations analyze situations, usually a society, as a whole, including its evolution when a change happens. Second, agent-based simulations study each person in a more detailed manner, designing a specific model for each agent (for example, the movement becomes an important factor in the simulation). In CPSS environments, agent-based simulations are preferable, as may include factors such as the movement of the individuals. On the contrary, social-level simulations are not suitable at all as important characteristics of people (movement, etc.) cannot be considered.

Table 2. Physical processes simulators.

\begin{tabular}{|c|c|c|c|c|}
\hline \multirow[b]{2}{*}{ Characteristics } & \multirow{2}{*}{$\begin{array}{c}\text { Traditional } \\
\text { Research } \\
\text { Simulators }\end{array}$} & \multirow{2}{*}{$\begin{array}{c}\text { Current } \\
\text { Commercial } \\
\text { Simulators }\end{array}$} & \multicolumn{2}{|c|}{ Current Research Simulators } \\
\hline & & & $\begin{array}{c}\text { Mixed } \\
\text { Simulators } \\
\end{array}$ & $\begin{array}{c}\text { Most Common Current } \\
\text { CPS Simulators } \\
\end{array}$ \\
\hline Include a graphic environment & No & Yes & Yes & Sometimes \\
\hline $\begin{array}{l}\text { Domain-specific knowledge is } \\
\text { required }\end{array}$ & Yes & No & Yes & Yes \\
\hline Include aspects of the cyber world & No & No & Yes & Yes \\
\hline Is a stable version & Yes & Yes & No & No \\
\hline Simulation scheme & First, all the & qulation are $\mathrm{pe}$ & formed and, la & er, results are showed \\
\hline
\end{tabular}

In agent-based simulations every element in the simulation is modeled as an agent, a unit that acts on their own, without external direction, in response to situations they encounter during the simulation [84]. An agent is updated every certain time according to the behavior defined inside its programming, what usually means some movement of the agent and maybe the influence and interaction with some other agent. Some of the agents can be very primitives and actually have 
nearly no behavior (such as a table in the middle of the room), while others (such as people) can be very complex.

In general, agent-based simulators deal with a high number of autonomous people, who enter, move around and leave a large installation, interacting with each other and with the environment (which consists of many devices with communication capabilities). Features related to autonomy, interaction, mobility [85] and openness can be achieved by employing agreement technologies [86], as well as semantic alignment [87], negotiation [88], argumentation, virtual organizations [89] and trust and reputation mechanisms [90].

Various kinds of agent-based social simulators may be found. Some of them simulate human intervention to react to events produced by sensors in the scope of Ambient Intelligence. This is the case of Ubiwise [91], TATUS [92], UbiREAL [93], and OpenSim [94]. In this sense, the DAI Virtual Lab [88] is complemented with a living lab which allows researchers to continue their work in a physical environment. These frameworks cope with the modeling of realistic environments and not only the wireless sensor networks.

Other social simulation environments can assist in the development of IoT systems by simulating artificial societies of users immersed into the intelligent environment and generating sensors events [95]. Although social simulation has been used for testing a number of closed systems, mainly in the emergency management scope, the general use of this technology for providing general AmI systems with automatic testing is still a novel field with few contributions such as UbikSim [96] and Phat [97] simulators.

Traditional works on social modeling and simulation try to obtain an analytical model to represent a certain human process [98] (such as the traffic flow [99] or crowds of pedestrians [100]).

In the last years, research works about agent-based social simulation have focused on discovering or predicting new models and behaviors, instead of on designing new simulators. Actually, there exists various professional generic agent based social simulators (apart from those which have been designed and particularized for a certain case study or application): MASON, Repast [101] and Swarm. Besides, most of these instruments provide a graphic environment, some of them based on 2D representations while others have a 3D tool [102]. Usually, in a simulator, users can run a predefined scenario by modifying some of the parameters and creating approximately the same predefined agents to see the differences between the simulations. This use of the tool requires no programming ability because the simulation can be controlled with a GUI that firstly enables the user to modify the parameters and specify how many agents are going to be simulated and, once the simulation starts, it enables pause, stop and resume the simulation while it is running. These simulators also enable some kind of batch simulation [103] where you can run some hundreds of simulations with the same or different parameters to later analyze the results generated in a log.

In order to create a different scenario and different agent models some programming is required to define the agent behavior and place the different elements in the environment. In this case, specific knowledge of the language and the behavior models are required in order to create the desired simulation despite you can create a behavior composed of several predefined behaviors

Table 3 compares all the social simulators described above.

Finally, cyber world simulators (known as IT simulators or networks simulators) include, probably, the widest catalogue of simulation solutions among all the CPSS subsystems. On the one hand, it may be found the traditional networks simulators, such as the NS3 [104]. These simulators must be configured using a generic programming environment, as they usually do not present a graphic interface. Sometimes, it is possible to show the result graphically using third-party tools. These simulators are developed professionally, so research works are not usually focused on them. There are several simulators nowadays both open-source such as NS3 or OMNet++ [105] and proprietary such as OPNET [106] or NETSIM [107]. 
Table 3. Social simulators comparison.

\begin{tabular}{ccccc}
\hline Characteristics & Social-Level & \multicolumn{3}{c}{ Agent-Based Simulators } \\
\cline { 3 - 5 } & Simulators & $\begin{array}{c}\text { Ambient } \\
\text { Intelligence }\end{array}$ & $\begin{array}{c}\text { Development of } \\
\text { IoT Systems }\end{array}$ & Generic \\
\hline Represent each people as a unit & No & Yes & Yes & Yes \\
Programming knowledge is required & No & Sometimes & Sometimes & Sometimes \\
A graphic interface is provided & Yes & Yes & Yes & Yes \\
Cyber elements may be included & No & Yes & Yes & No \\
People is the objective of the simulations & Yes & Yes & No & Yes \\
Stable tools are available & Yes & Yes & Yes & Yes \\
Simulation scheme & \multicolumn{2}{c}{ Results are showed as calculated in each time step } \\
\hline
\end{tabular}

On the other hand, new instruments for the most recent and popular concepts on IT sciences, such as the Internet-of-things (IoT), have appeared and could be used in CPSS simulations. These proposals are still been developed and many researchers are working on this topic. Some examples are the IoT simulator Cooja [108], SimpleIoTSimulator [109] or AutoSim [110]. These tools used to incorporate a graphic environment to create the scenarios. However, the customizing options are much fewer than in traditional networks simulators. Social IoT simulators are a special case among the IoT simulators [111]. In these instruments, some aspects of the social human behavior are included in the models in order to, for example, represent the human movements.

All IT simulators are event driven, "jumping" from one event to the following event in the simulation process. During the simulation, the logs generated from the processing of each event are presented (or stored) at each simulation step.

Table 4 compares all the IT simulators described above.

Table 4. Network or IT simulators comparison.

\begin{tabular}{cccc}
\hline Characteristics & $\begin{array}{c}\text { Traditional Network } \\
\text { Simulators }\end{array}$ & IoT Simulators & $\begin{array}{c}\text { Social IoT } \\
\text { Simulators }\end{array}$ \\
\hline Include a graphic environment & Sometimes & Yes & Yes \\
Programming abilities are required & Yes & No & No \\
Include social aspects & No & No & No \\
Is a stable version & Yes & Sometimes & No \\
Number of customizing options & High & Medium & Low \\
Simulation scheme & Event-driven, offering the logs in each step \\
\hline
\end{tabular}

\section{Methodology for the Implementation of Application-Specific Cyber-Physical Social Sensing Co-Simulators}

This work proposes the first methodology for the design of CPSS co-simulators. The proposed methodology supports the work of software architects, which is the first approach to the co-simulator creation. At this stage, generic discussions about the complexity of simulations to be performed are proposed; general questions about requirements, the users' profile, and the development team must be answered; and the global lines of the future implementation are stablished. In later phases (at implementation time) more specific problems (which are not the focus of this work) should be addressed. In particular, crucial aspects about the data formats and time representation should be solved.

In order to organize these activities, we propose a four-step methodology for designing a CPSS co-simulator being able of fulfilling the users' needs and requirements:

1. Selection of the co-simulation paradigm

2. Particularization of the general simulation model and simulation lifecycle

3. Selection of the appropriate coordination mechanism

4. Design of the user interface and results presentation 
Several authors have reported the interest of research on co-simulation. The possibility of simulating individual components using different simulation tools simultaneously and collaboratively enables stakeholders to evaluate deployments in a very realistic way. In order to reach this objective, individual simulation tools should be able to exchange information such as variables and their values, synchronize the time steps and, in general, orchestrate the co-operative simulation [112]. Mainly, two standard technologies have been proposed to coordinate co-simulations: the High-level architecture (HLA) [56] and the Functional Mock-up Interface (FMI) [57]. Although several tools are compatible with these standards, not all simulation tools implement the same and, even, some tools do not implement any (for example, most social simulators). Moreover, problems and restrictions in the integrated tools based on these standards have been reported [112]. And finally, some important challenges related to CPSS co-simulation are not addressed by these solutions (such as data exchange and time management in the case of FMI standard). In fact, the complexity of CPSS pushes designers to create application-specific and simple solutions, losing most of powerful advantages of standard solutions.

In that way, the proposed methodology in this paper aims to enable CPSS researchers and software architects to design the most adequate co-simulation for a given application, addressing the four most important challenges. Namely [112,113]:

- The first step addresses the "Data Flow and Concurrency". Different paradigms in order to parallelize and manage the data flow among the domain specific simulation tools are presented and different criteria to select among them depending on the situation are provided.

- Challenges related to the "Modeling language" and "System Topology representation" are addressed during the second step. A basic simulation model is proposed, and indications to adapt it to the particular scenario under study are provided. An identical process is followed with the simulation lifecycle.

- "Time management" and "System Scalability" are investigated in the third step. Different options to coordinate the different time representation and simulation speeds are studied. Besides, depending on the desired future system scalability and the scenario under study, different criteria to select the most appropriate management technique are provided.

- Finally, the fourth step is focused on "Tool heterogeneity". In order to homogenize the interaction with the proposed co-simulator as much as possible, different ideas about the possibilities for user interfaces are provided and analyzed.

As a previous phase to the application of the proposed methodology, an interaction process among software architects and final users must be performed. Our proposal also includes and regulates this process and helps both, final users and designers, to determine the requirements and characteristic applicable to the new co-simulation tool (see Figure 2). The implementation process, although it is the most time consuming task, is a post-design activity, so it is not considered in this work.

In this Section, the four steps of the proposal are explained, and the previous phase of requirements are captured to obtain from final users the characteristics which the new co-simulator should meet.

In relation to traditional software engineering methodologies, the proposed technique lacks an explicit mention to a software evaluation step. However, this phase should be considered. Once the new co-simulator is designed, its functionalities should be evaluated to prove it fulfills the user requirements. If not, a new iteration following the proposed methodology should be carried out, considering the new user inputs. 


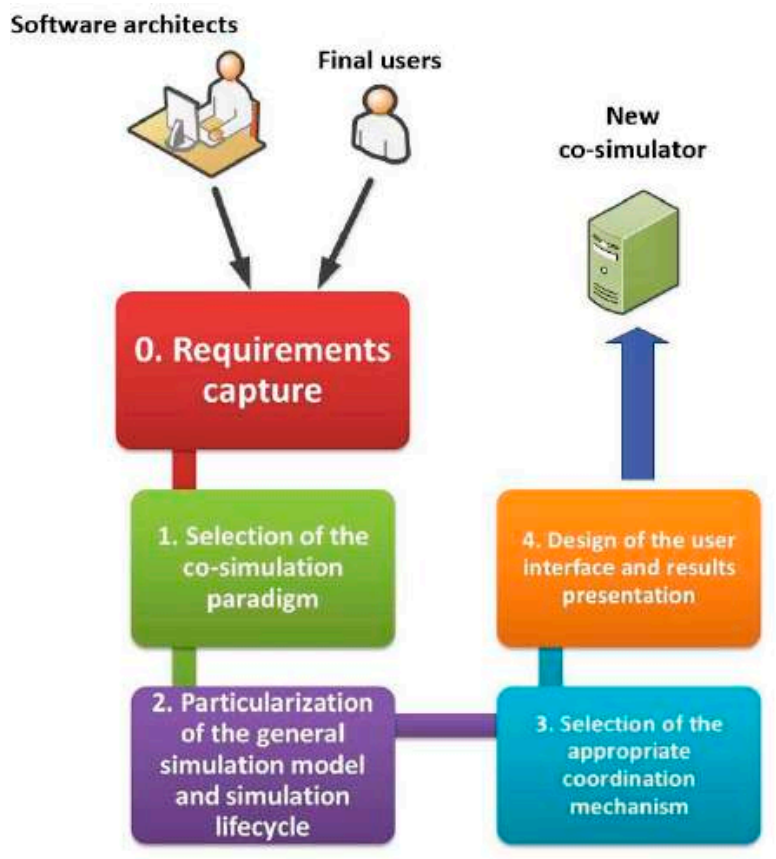

Figure 2. Scheme of the proposed methodology.

\subsection{Previous Phase: Final Users' Requirements and Characteristics Capture}

Many works have studied the requirements that co-simulation tools should fulfill in order to operate correctly and efficiently [25,30]. However, in application-specific tools, most of these generic requirements could be inapplicable and other needed characteristics may not be considered. Moreover, the interaction with final users may be complicated and very time consuming if an ordered process is not followed. Furthermore, not only traditional requirements (such as the tool's accuracy) should be considered. In particular, characteristics of the development team, final users' education and the type of simulations to be performed should be defined.

In this previous phase, software designers and final users should meet and stablish the requirements the new simulation tool should fulfill, and other important characteristics. Four important thematic blocks must be addressed: technical requirements, application requirements and characteristics, development team characteristics and final user characteristics.

In respect to technical requirements, all of them may be associated to one property among the following four [25]:

- Flexibility. The adaptation level of the proposed solution to new usages, utilization modes, technological instruments, etc. must be determined. For example, if required, the simulator should be able to be applied to new scenarios.

- Modularity. Depending on the expected use for the co-simulator, the tool should include several modules independently handled. For example, if applying many changes in the simulator structure during its operation is desirable, modules and components should be easily added and removed without affecting any other part of the tool (total modularity).

- Scalability. The upper limit for the simulation scenario complexity should be defined. For example, the maximum admissible number of agents in a particular scenario, or the level of complexity of the agent's behavior should be determined.

- Accuracy. Users should be able to select the desired accuracy level. For example, users must indicate the maximum time without updating the simulation (which allows calculating the required time step at the implementation stage, see Section 3.4)

Requirements about flexibility and modularity refer to the programming structure of the co-simulator. In particular, they indicate the implemented level of openness with the co-simulator. 
Scalability implies that the resulting co-simulator should be able to simulate future scenarios. Finally, accuracy enables users to configure the simulations according to particular configuration parameters.

In respect to application requirements, they also match the previously presented properties. Finally, characteristics related to the application scenario, the development team and final users should be considered. Relevant information about these topics should be discussed among designers and final users, in order to adapt the new simulation tool to the specific context. Table 5 presents some basic characteristics which should be considered, although others could be added.

Table 5. Relevant characteristic for a co-simulator.

\begin{tabular}{cc}
\hline Thematic Block & Relevant Characteristics \\
Application characteristics & $\begin{array}{c}\text { The main subsystem (physical, cyber or social world) in the simulation } \\
\text { Type of application (e.g., Ambient Intelligence validation or development) } \\
\text { Possible simulation scenarios considered (buildings, cities, large facilities, etc.) }\end{array}$ \\
\hline Development team characteristics & $\begin{array}{c}\text { Knowledge about simulators programming } \\
\text { Number of developers (available workforce) }\end{array}$ \\
\hline Final users characteristics & $\begin{array}{c}\text { Technical and programming skills } \\
\text { (sector professionals, researchers or students, etc.) }\end{array}$ \\
\hline
\end{tabular}

The first and third steps allow generating co-simulators which fulfil flexibility requirements. Co-simulation paradigms and coordination mechanisms are open solutions which allow easily modifying the programming of the co-simulator. Second step is designed to meet modularity and scalability requirements. The simulation model and the simulation lifecycle can be modified, extended or reduced in an independent way, and according to the users' needs. Finally, accuracy requirements are mainly supported by the fourth step. An appropriate graphic interface must enable users to configure the simulations. Relevant characteristic may affect all steps in the methodology.

The next subsections describe deeply the four main steps of the proposed methodology.

\subsection{Selection of the Co-Simulation Paradigm}

In the first step, once software designers have selected the domains involved in the simulation, requirements and characteristics have been captured and the available tools which are going to be used to compose the final co-simulator, it is necessary to select the co-simulation paradigm on which the co-simulator is being based. An explanation about all the possibilities, and the criteria for selecting the most adequate, are provided in this sub-section. It is important to note that most of these criteria are extracted from requirements captured in the previous phase (see Table 5).

Figure 3 proposes a taxonomy of the simulation paradigms, which could be applicable to the CPSS simulation.

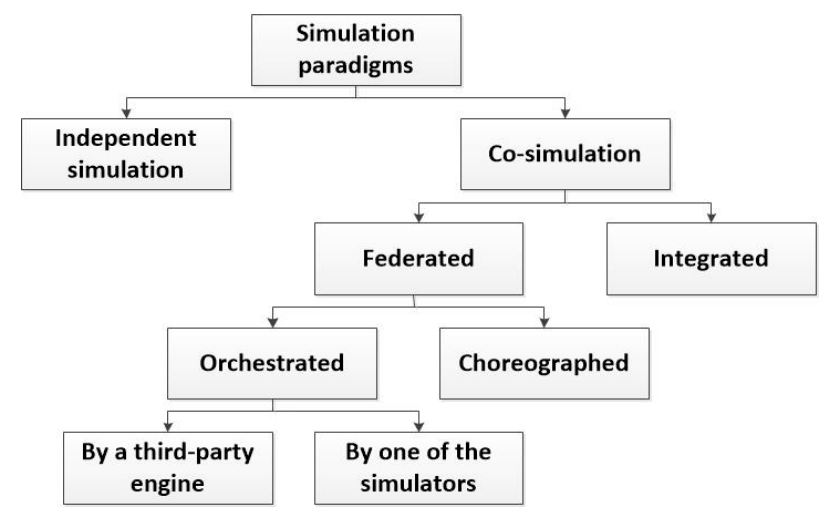

Figure 3. Taxonomy of CPSS simulation paradigms.

In general, two different types of simulation scheme may be implemented, when various domains compose a global scenario: independent simulation or co-simulation. In the independent simulation each domain is simulated in a different domain-specific simulator. In this case, once 
designed the global scenario, an expert must divide it into the different subsystems (three, in the case of CPSS - the physical, the cyber and the social worlds-) and configure each simulator separately (see Figure 4a). Finally, the results obtained from each tool must be combined with the others in order to generate the global results for the proposed scenario. As main advantage (see Section 3.4), if various work stations are available, it is possible to run all the simulations in parallel (which is very efficient in time).

On the other hand, co-simulation tools are instruments being able to process a description including all the characteristics of the global scenario (see Figure $4 \mathrm{~b}$ ). They may be implemented using different integration levels, which, at the end, causes the simulation time goes up in different quantities depending on the implementation selected. In any case, considering the same hardware platform, these solutions always require a higher simulation time.

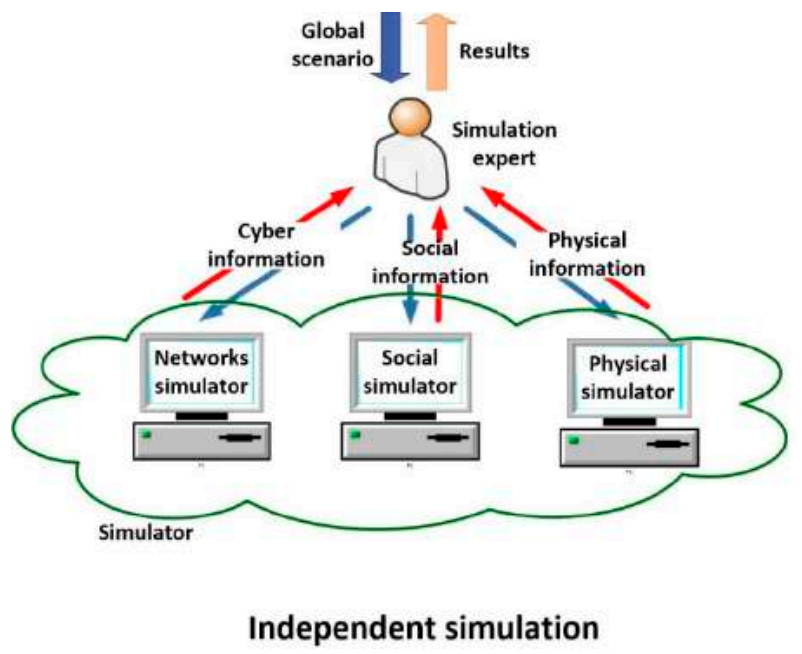

(a)

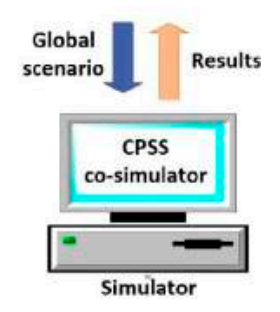

Co-simulation

(b)

Figure 4. Different simulation paradigms (a) Independent simulation (b) Co-simulation.

As we said in Section 2, recent attempts to reach a co-simulation tool for two of the CPSS subsystems are based on the deep integration of new characteristics in the existing simulator. We call this co-simulation paradigm integrated co-simulation. Integrated co-simulation tools are monolithic instruments where none separated functional part can be distinguished. In practice, they can be understood as a unique simulation algorithm. In general, this is the final objective when building co-simulation tools as, at that moment, the resulting solution is completely different from the parts. However, it is really difficult to create an integrated simulator right from the scratch. In fact, various previous works [113] have showed the complexity of combining various tools and domains in a unique simulation (even if only two different tools are considered).

In contrast, we define the federated co-simulation. In federated co-simulation various (three in CPSS-a social simulator, a network simulator and a physical process simulator-) domain-specific tools are coordinated to simulate the global scenario. Each part could be modified in different levels, but there is always a separation among the different functional parts (which maintain all their traditional characteristics). Federated co-simulation is the most profitable paradigm in the first stages for building a new simulation tool.

Now, as we said, in federated co-simulation the different parts which make up the global simulator could be modified in different level. Depending on the number of required modifications different federated co-simulation paradigm may be identified.

The paradigm which needs the highest level of development is the choreographed federated co-simulation. In this scheme, all the different domain-specific simulators have been modified to understand the global description of the simulation scenario, to select the important data for them, to configure the simulation and to share the resulting data with the other domain-specific simulator (see Figure 5a). Thus, programming is required in order to modify the simulation tools. Finally, one 
of the simulators is in charge of building the final simulation results. This federated paradigm is the closest to the integrated simulations.

In contrast to choreographed co-simulation, orchestrated federated co-simulation does not require to modify all the domain-specific tools (or the modifications are negligible), and, then, programming knowledge to modify the simulators themselves is not required in the development team. In this paradigm, an entity is in charge of receiving the global description of the scenario, processing it, and configuring the simulation in the different tools. Finally, this entity must also generate the final results using the data from the different simulators. In orchestrated by one of the simulators federated co-simulation the described entity is one on the domain-specific simulator which has been adequately modified (see Figure 5b). In orchestrated by a third-party engine federated co-simulation no modification in the domain-specific tools is required, as the described entity is a third-party program which uses the available interfaces in the simulator to interact with them (see Figure $5 \mathrm{c}$ ).

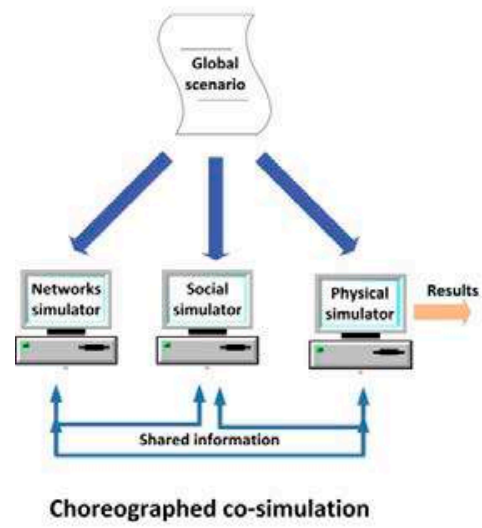

(a)

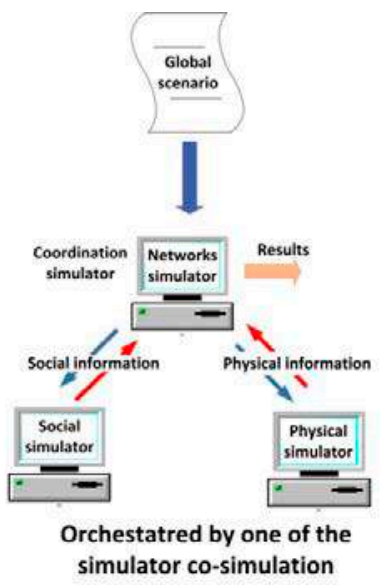

(b)

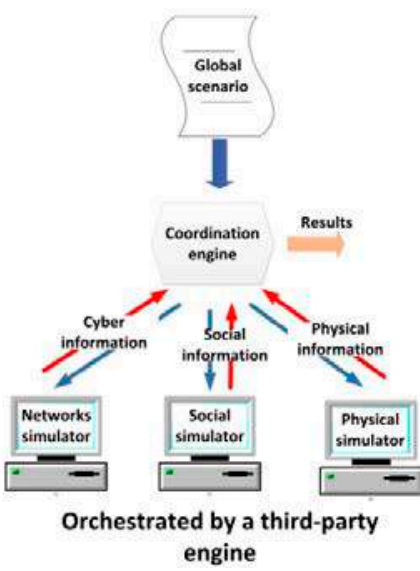

(c)

Figure 5. Different simulation paradigms (a) Choreographed (b) Orchestrated by one of the simulators (c) Orchestrated by a third-party engine.

Various proposals and commercial simulators applying different paradigms may be found. For example, in [114] it is described an orchestrated by a third-party engine federated co-simulator for CPS (based on the union of the NS3 simulator and the MASON simulator). In [115] an orchestrated by one of the simulators federated co-simulator is presented. The proposal is based on the coordination of MATLAB/Simulink (a tool which, besides, performs the coordination tasks) and the NS3 simulator. This paradigm is greatly extended (especially when MATLAB/Simulink suite is involved), for example to perform simulations about reflector antennas with the commercial software GRASP [116]. On the other hand, in [117] a Smart grid simulator based on choreographed co-simulation between the NS2 simulator and the PSLF (Positive Sequence Load Flow, software for power systems) Simulator is described; and in [118] an integrated simulator (also for Smart grids) is presented.

Selecting the appropriate co-simulation paradigm for a new simulation tools is a complicated task which depends, basically, on two factors: the technological capacity of the future developers and the future users' abilities and (mainly) the characteristics of the selected tools to make up the co-simulator. Table 6 presents and discusses the most important criteria which must be considered in the selection of a co-simulation paradigm. 
Table 6. Criteria for the selection of a co-simulation paradigm.

\begin{tabular}{|c|c|c|}
\hline Group & Criteria & Explanation \\
\hline \multirow[b]{2}{*}{$\begin{array}{l}\text { Developers' } \\
\text { knowledge and } \\
\text { users' abilities }\end{array}$} & $\begin{array}{l}\text { Technical capacity to perform } \\
\text { large developments } \\
\text { (programming to modify the } \\
\text { simulators themselves) }\end{array}$ & $\begin{array}{c}\text { Developing an integrated co-simulator requires great knowledge } \\
\text { about programming and expert people on software development. If } \\
\text { these resources are not available, federated co-simulators are } \\
\text { preferable }\end{array}$ \\
\hline & $\begin{array}{l}\text { Technical skills of users } \\
\text { (programming to implement } \\
\text { the models) }\end{array}$ & $\begin{array}{l}\text { If users have technical skills, they can perform the scenario division } \\
\text { into different domains. If that is not possible, orchestrated or } \\
\text { integrated co-simulation are the only feasible paradigms. These } \\
\text { paradigms, moreover, help to consider a high number of different } \\
\text { subsystems in the simulation without complicating the usability in } \\
\text { excess. }\end{array}$ \\
\hline \multirow{4}{*}{$\begin{array}{l}\text { Characteristic of } \\
\text { the selected } \\
\text { simulation tools }\end{array}$} & $\begin{array}{l}\text { Utilization of open } \\
\text { architecture tools }\end{array}$ & $\begin{array}{l}\text { If simulation tools present an open architecture, federated } \\
\text { co-simulation may be employed. However, if all selected simulators } \\
\text { are close architecture tools, any information could be exchanged and } \\
\text { integrated co-simulation is the solution. }\end{array}$ \\
\hline & $\begin{array}{l}\text { Availability of adequate } \\
\text { domain-specific simulator in } \\
\text { the state of the art }\end{array}$ & $\begin{array}{c}\text { If any of the available domain-specific simulators nowadays is } \\
\text { adequate to be integrated in the new co-simulator, integrated } \\
\text { co-simulator is the only valid paradigm (programming the } \\
\text { unavailable modules). }\end{array}$ \\
\hline & $\begin{array}{l}\text { Utilization of open source } \\
\text { tools }\end{array}$ & $\begin{array}{l}\text { If open architecture tools are used, and federated co-simulation is } \\
\text { going to be employed, the use of choreographed co-simulation } \\
\text { requires all simulators involved to be open source (as the code has to } \\
\text { be slightly modified). }\end{array}$ \\
\hline & $\begin{array}{l}\text { Compatibility among the } \\
\text { domain-specific tools }\end{array}$ & $\begin{array}{l}\text { If open architecture tools are used, and federated co-simulation is } \\
\text { going to be employed, the use of choreographed co-simulation } \\
\text { requires all simulators involved to be totally compatible (use the } \\
\text { same data formats, communication protocols, APIs, etc.). }\end{array}$ \\
\hline Other & $\begin{array}{l}\text { Results presentation and user } \\
\text { interaction }\end{array}$ & $\begin{array}{l}\text { If orchestrated co-simulation is being performed, and if none of the } \\
\text { modules for results presentation provided with the domain-specific } \\
\text { simulation tools meet the needs of the new co-simulator, a } \\
\text { third-party engine must be included }\end{array}$ \\
\hline
\end{tabular}

\subsection{Particularization of the General Simulation Model and Simulation Lifecycle}

Once selected the most appropriate co-simulation paradigm, the application for which the simulator is being designed must be considered. In that process, characteristics and final users' requirements captured in the previous phase (see Section 3.1) should considered as inputs. In particular, the application scenarios and the type of simulations the users are going to perform should be taking into account. The first element (application scenarios) will allow designers to describe a specific simulation model. The second one (the type of simulations) might help to propose the simulation lifecycle most adequate. The second step in the methodology is dedicated to these two tasks.

\subsubsection{CPSS Simulation Model}

The simulation model on the proposed designed co-simulator must be focused on the most relevant aspect of the reality for the particular selected application. Elements from all the subsystems may be present, but description should be much more exhaustive when representing certain parts of the world.

Figure 6 represents a basic simulation model for CPSS which can be taken as initial step in the construction of an own model. In the presented basic model four types of elements are present: in blue, general elements relating the three worlds (physic, social and cyber), in red elements from the physical world, in yellow devices belonging to the cyber world and, in green, elements in the social world.

As can be seen, general elements include the constraints and the behavior of every agent in the simulation. Social elements basically consider people, their state (particular and as a group) and the relations among them. Cyber elements refer a list of devices including sensors and actuators. The rest of agents present in the scenario belong to the physical world. 


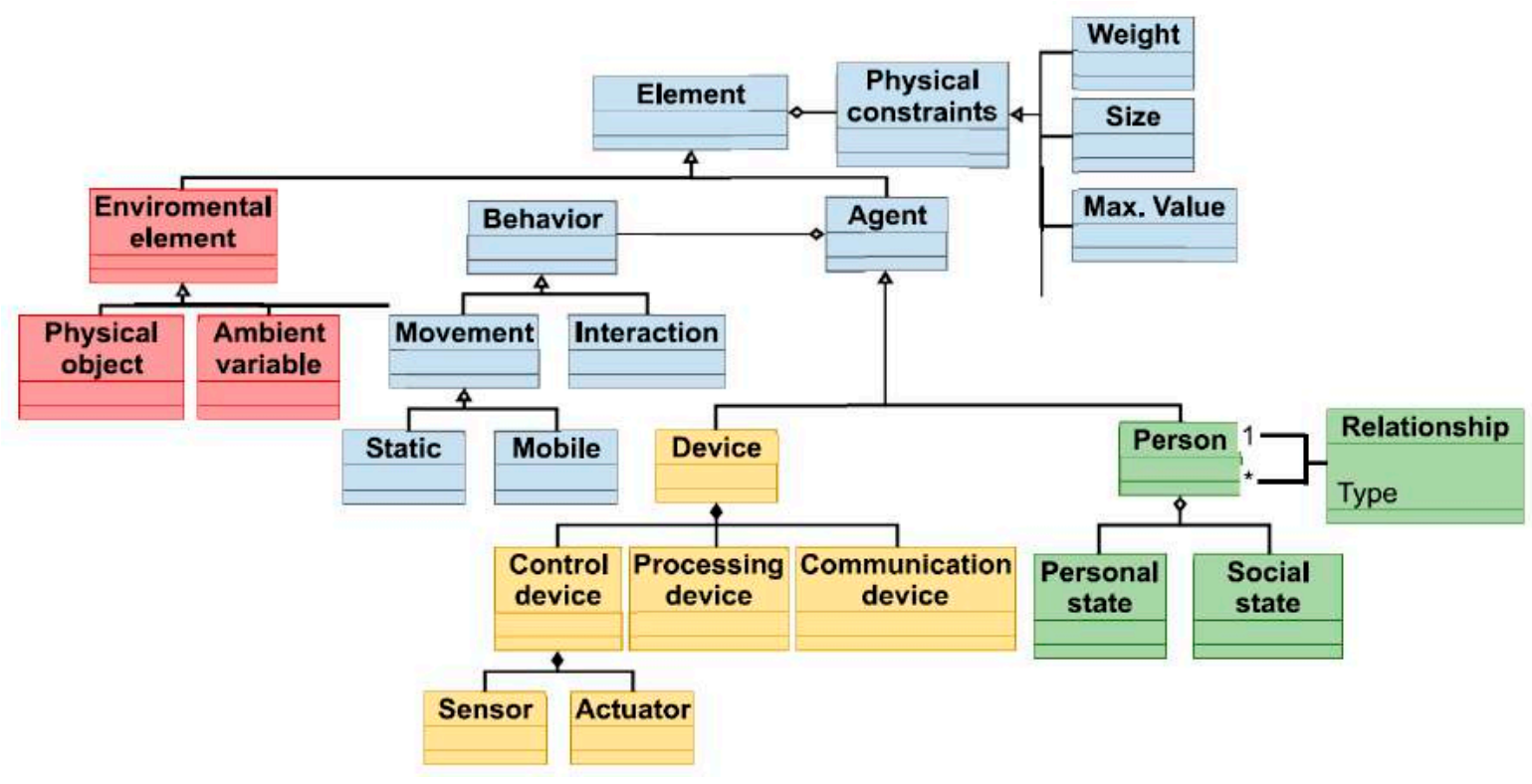

Figure 6. Base CPSS simulation model.

Using the basic model in Figure 6, the most adequate simulation model for the specific application under work must be developed. For that, some parts of the model may be reduced (for example, distinguishing between sensors and actuator could be unnecessary), and others extended (including, for example, more elements for composing a person).

Some methodologies for the creation of correct and complete simulation models have been proposed [119,120]. Basically, depending on the application and purpose of the designed co-simulator some aspect of reality could be abstracted away [121]. However, in Table 7, some general lines are given in order to build the simulation model according to the selected application, the scenarios under study and the specific problem being addressed with the co-simulator. For unreported applications nowadays, actions to be taken would be pretty similar. Thus, as main contribution to the agent-based modeling we propose a guide for software architects (as well as an initial model mixing, for first time, the three basic CPSS subsystems), in order to allow them to create the most adequate model in each case (considering current and future scenarios).

Table 7. Criteria for the creation of the simulation model.

\begin{tabular}{cc}
\hline $\begin{array}{c}\text { Application } \\
\text { Validation of ambient } \\
\begin{array}{c}\text { intelligence systems } \\
\text { [91-94,122] }\end{array}\end{array}$ & $\begin{array}{c}\text { A more specific definition of the physical world (ambient) is necessary. In } \\
\text { particular, physical laws for the evolution of relevant phenomena should be } \\
\text { modeled. Besides different types of people should be also considered } \\
\text { (depending on if they present special needs, incapacities, etc.). }\end{array}$ \\
$\begin{array}{c}\text { Development of IoT } \\
\text { systems } \\
\text { [95] }\end{array}$ & $\begin{array}{c}\text { The concept of "service" should be added in the simulation model, and a } \\
\text { more exhaustive description of the different types of devices also would be } \\
\text { desirable (in particular, a description of the interfaces is very important in IoT } \\
\text { scenarios). }\end{array}$ \\
$\begin{array}{c}\text { Social research } \\
\text { [123,124] }\end{array}$ & $\begin{array}{c}\text { Most elements in the cyber world can be removed (it is enough to include the } \\
\text { concept of "device"). On the other hand, social world must be extended, } \\
\text { including different personal and social states, different types of interrelations } \\
\text { among people and the social evolution laws. }\end{array}$ \\
$\begin{array}{c}\text { Crowd management } \\
\text { [125-127] }\end{array}$ & $\begin{array}{c}\text { A detailed model for "physical object" may be important. Models for walls, } \\
\text { doors, buildings, etc. are critical in order to manage people in the most } \\
\text { adequate way. In addition, models for the different emotions and their } \\
\text { propagation in crowds must be considered. }\end{array}$ \\
\hline $\begin{array}{c}\text { Social sensing } \\
\text { [86-90] }\end{array}$ & $\begin{array}{c}\text { Different types of sensors have to be considered, so the model must include } \\
\text { all of them. Besides, the social world requires a more exhaustive description } \\
\text { as mentioned in the case of "social research" and "crowd management". }\end{array}$ \\
\hline
\end{tabular}




\subsubsection{Simulation Lifecycle}

Depending on the type of the simulations being performed in the designed co-simulator, the simulation lifecycle may vary (more steps could be added, for example). The steps included in the simulation lifecycle are going to affect, overall, the fourth step in the methodology, dedicated to the interaction with users. For example, if all the simulations to be performed only address one goal, no step for "goal specification" is needed, and no interface dedicated to this step is required.

In general, designing the most adequate lifecycle is much easier (almost elemental) than building the simulation model. Next, a complete generic simulation lifecycle is described, from which different steps can be removed (if necessary), or where new states may be defined.

The creation of the particularized simulation lifecycle is very simple: if the tasks associated with a certain state described below do not have to be executed, this state is removed; if new tasks have to be added, new states should be defined.

Co-simulation environments are often used to test how humans relate to devices and other infrastructure objects. Simulations showing specific aspects of these relations are executed and redefined multiple times to retrieve knowledge about users and devices. The process is represented in Figure 7 and the quality assessment [128] of every step is described below.

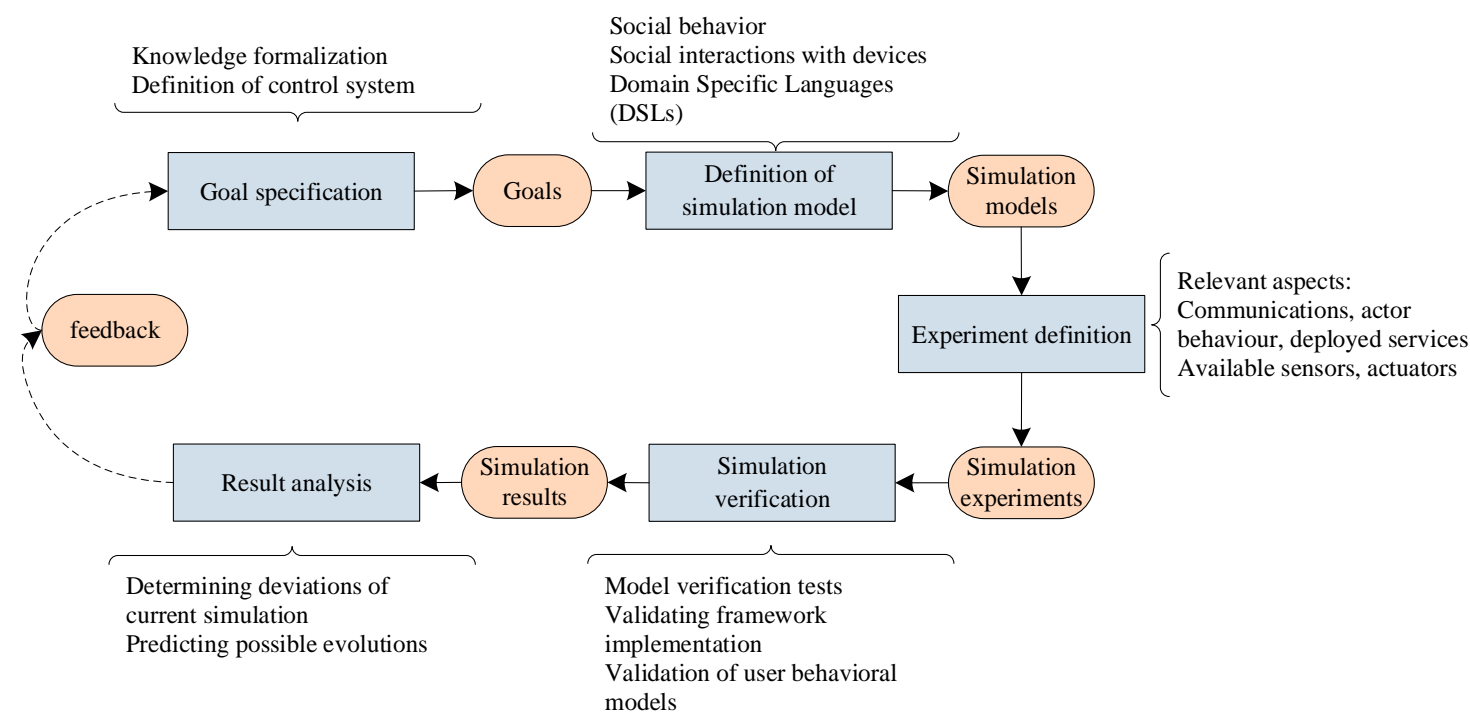

Figure 7. Basic simulation lifecycle.

Goal specification: Stakeholders meet to determine the specific problem to solve, capturing this information by field studies and/or other requirement acquisition techniques. The research problem is formally and clearly formulated. One goal commonly pursued in simulations integrating social behavior and device interaction is to check the functioning of a control system (a device or set of devices that manages and regulates the behavior of other systems in the smart environment), analyzing if this system correctly takes into account its execution context. This is a prior step to define the simulation model associated to this system, as analyzed goals can be used to model what kinds of experiments are desirable for the particular problem and the type of situations considered.

Definition of simulation model: Social behavior and social interactions with devices and the control system must be integrated into the simulation model. Some considered models (see Figure 6) are person, behavior (describe individual and collective human behavior), environmental elements or actuators, sensors (describe the physical characteristics of the AmI environment).

Domain Specific Languages (DSLs) are used to create simulation models by model-driven development techniques. Other end-user development tools present in the co-simulation framework will be used to transform these models to simulation specifications. These tools are reusable across domains and permit the developer or any other stakeholder to describe specific aspects of the problem. 
Some questions arise regarding the correct definition of the simulation model. Are there many simulation models in the literature or this is the very first of its kind? Is the underlying social theory correctly instantiated using proper methods and algorithms?

Experiment definition: Simulation experiments take into account the selected models depending on the aspects to be addressed. It is foreseen to take into account the agent behavior, and all the key elements included in the particular developed simulation model. Simulations addressing one or more of these aspects will be produced and evaluated by the developers. The required simulations will be described by the experiments model and will incorporate specific information, such as building blueprints or device limitations.

Experiment definition is done via configuration tools. Particular applications are chosen and different models are selected (users, populations, spaces, actuators, sensors), and combined, and then the different experiments that are going to be applied are prepared. Experts would facilitate end-users the task of creating those experiments by reusing existing information and allowing them to decide which information should be extracted from the simulation, for its later analysis. The result of applying these tools would be simulation specifications.

Simulation execution in co-simulation system: The problem to be simulated is parameterized, producing the problem specific components the reusable co-simulation framework requires. Actions from the experiment definition and control system behavior are applied on the simulation so as to affect the simulated actors and to product the expected effect. The system would be adjusted to deal with the different situations that might occur: necessity of repeating stimulus (without saturating the audience), unexpected problems with devices, etc. Besides, communication among devices is tested, assessing effectiveness and cost. Possible problems like bandwidth, transmission quality, etc., must be also validated.

Simulation verification: A verification step is required to ensure that simulation model and experiments were well-defined. In this step passive and active tests are conducted to verify that the model is behaving in the way it is intended to behave (internal validity). Also, some tools and techniques such as code debugging, unit testing, profiling [129] as well as test suites (histograms, spatial analysis, pattern matching, etc.) must be considered, as these techniques allow us to validate the proposed model and the implementation of the framework. Some questions arise: In terms of the definition and implementation of social behavior, is the underlying social theory correctly instantiated using proper tools or programing languages? In terms of computational efficiency, how efficient is the implementation of social behavior in terms of using computational resources? Is there any architectural implication in the publication and acquisition of user behavioral models?

Results analysis: Experiments are analyzed according domain knowledge to improve our current model and identify new needs. When the experiments are successful, an exhaustive documentation is thus available about how the system is wanted to behave. The work carried out can be used to track the behavior in the real setting.

Regarding the deployment process of services or control systems in the CPSS environment we assume that the system tested against simulations would be the same as those installed in the real environment. Using a software-in-the-loop approach, we would ensure that the conditions of these services in the simulation are the same or sufficiently similar to reality.

Once deployed, information collected during experiments can be used to determine whether current observation in the real AmI environment corresponds to a simulated scenario or whether it is necessary to launch a simulation with the current context in the real world so as to determine deviations with regards to experimentation and/or predict possible evolutions from the current situation.

Some questions arise: In terms of overall effectiveness, does the model render what is necessary for answering the initial research questions? What is the quality of the simulated infrastructure that renders the most effective simulation experience?

Finally, in order to correctly follow the defined simulation lifecycle, we need to take into account the following requirements: 
- In CPSS, we need to consider several aspects (e.g., social, physical and cyber) of the agents' behavior. For example, movement in devices can be dictated by movement of people carrying those devices. Also, in a social simulation it is useful to know if persons that walk through a corridor should have enough WiFi coverage, or would detect a Bluetooth beacon that is broadcasting a signal in one of the surrounding rooms.

- Some tools must be needed to create experiments from previous ones, by modifying functionalities or stimulus affecting simulated actors to produce expected effect. Experiment creation should be done by domain experts, who have the required experience to identify human and device behavior in knowledge bases but they also have little experience in software engineering. Thus, it is needed to provide configuration and personalization tools easy to use for domain experts and adequate to their skills.

- Tools for analyzing the results of the simulation are extremely important to deal with the amount of data that is produced by the simulation. They should be able to process and facilitate the analysis by the experts. Moreover, after real deployment of services or control systems, those tools should also be used for analyzing and comparing current data against simulations so as to identify deviations and foresee future situations. For doing that, performance data must be generated by the simulator.

\subsection{Selection of the Appropriate Coordination Mechanism}

Some of the proposed co-simulation paradigms do not require any additional decision, as the paradigm determines the entire structure of the new co-simulator. In generic designs, issues such as the data format or the communication protocols to be employed in the coordination process must be selected. If a choreographed paradigm is selected all simulators involved must be totally compatible, so no action about the data format or protocols is required (all of them should implement the same). Again, orchestrated co-simulation requires the orchestrator to implement all the protocols, interfaces, etc. employed by the rest of tools, so no action about the data format or protocols is required as well. In federated co-simulation, the key topic is the coordination of the different domain-specific tools which compose the global co-simulator. Thus, only decisions about the mechanism to synchronize the execution of the domain-specific simulators must be taken. The third step of the proposed methodology is focused on this point. In particular, two different mechanisms could be implemented (see Table 8).

Table 8. Coordination mechanisms.

\begin{tabular}{|c|c|c|}
\hline $\begin{array}{c}\text { Coordination } \\
\text { Mechanism }\end{array}$ & $\begin{array}{c}\text { Co-Simulation } \\
\text { Paradigm }\end{array}$ & Implementation \\
\hline $\begin{array}{l}\text { Parallel } \\
\text { execution }\end{array}$ & All & $\begin{array}{l}\text { Every tool executes in a separate host, processor or thread. Simulation } \\
\text { calculations are performed in parallel and results are shared with the } \\
\text { rest of simulators immediately (using the orchestrator element if } \\
\text { necessary). }\end{array}$ \\
\hline \multirow{3}{*}{$\begin{array}{l}\text { Stops and waits } \\
\text { execution }\end{array}$} & Choreographed & $\begin{array}{l}\text { In a certain order, every simulator makes its calculations. When all } \\
\text { tools have performed their execution, all of them share the results } \\
\text { with the others. }\end{array}$ \\
\hline & $\begin{array}{l}\text { Orchestrated by a } \\
\text { third-party engine }\end{array}$ & $\begin{array}{l}\text { In a certain order, the engine order every simulator to execute the } \\
\text { calculations. When each simulator finishes, it sends the results to the } \\
\text { engine and it sends the information to the other tools. }\end{array}$ \\
\hline & $\begin{array}{l}\text { Orchestrated by } \\
\text { one of the } \\
\text { simulators }\end{array}$ & $\begin{array}{l}\text { First, the orchestrator simulator performs its calculations and shares } \\
\text { the results with the other tools. Then, in a certain order, it orders } \\
\text { every simulator to execute its calculations. When each simulator } \\
\text { finishes, it sends the results to the orchestrator and it sends the } \\
\text { information to the other tools }\end{array}$ \\
\hline
\end{tabular}

The coordination mechanism is, probably, the most critical step in the proposed methodology. Although the design process can be simple (see Table 8), at implementation time, programming 
these policies forces to face various crucial problems. For instance, the event ordination if various discrete time domain-specific simulators are employed, or the definition of a common time representation when each tool employs a different data format (and accuracy). The selected solutions for these challenges depend on the chosen co-simulation paradigm at the first step, but (in general) an initial synchronization process must be performed (and configured) in order to homogenize time characteristics and variables (see Figure 8a). Then, simulation may be executed normally-evaluating the system state after each event or time step-(Figure 8b).

In order to select the most adequate coordination mechanism two factors should be considered: the difficulty of implementing the mechanism and the time required to perform a simulation when using a certain mechanism. This second criterion is directly related with the scalability requirements acquired from final users in the initial phase (Section 3.1). In general, implementing a parallel execution is much costlier than a stop-and-wait model. However, the time required to finish a certain simulation in smaller if parallel execution is considered. In order to evaluate these temporal factors, a theoretical analysis is provided below. In particular, Equation (9) provides a theoretical analysis in order to optimally select the best approach for specific situations (depending on the expected number of simulations to be performed, etc.)

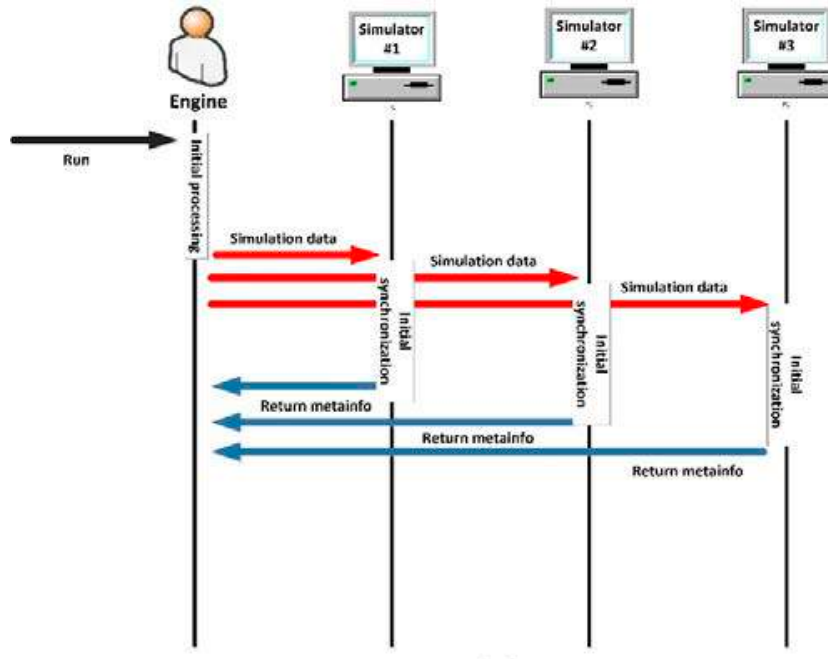

(a)

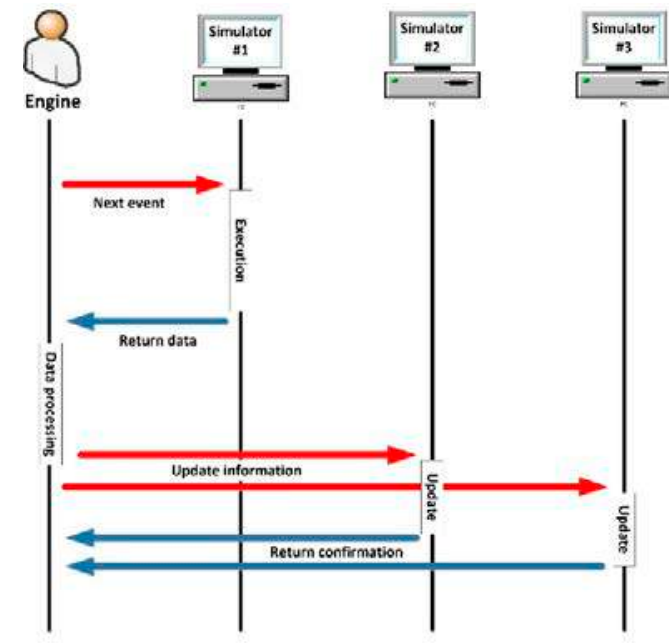

(b)

Figure 8. (a) Sequence diagram describing the initial synchronization process in a co-simulator; (b) Example of a sequence diagram about the stop-and-wait coordination mechanism when using a federated co-simulation controlled by a third-party engine.

We are supposing a simulation about a global scenario $S_{G}$ represented by means of a global model $M_{G}$ composed of information about three different domains: the physical, social and cyber world (1).

$$
M_{G}=\left\{m_{p h}, m_{s o}, m_{c y}\right\}
$$

We are calling $T_{p h}$ to the needed time to simulate the physical model $m_{p h}$ in a physical processes simulator; $T_{s o}$ to the needed time to simulate the social model $m_{s o}$ in a social simulator; and $T_{c y}$ to the needed time to simulate the cyber model $m_{c y}$ in a networks simulator. All these variables, $T_{p h}, T_{s o}$ and $T_{c y}$, represent stochastic processes which may be understood as ergodic processes whose statistical mean is referred as $E\left[T_{p h}\right]=\mu_{p h}, E\left[T_{s o}\right]=\mu_{s o}$ and $E\left[T_{c y}\right]=\mu_{c y}$. Calculation time is a stochastic process as aleatory facts (such as numerical noise) may affect the calculation speed, the time step (if it is considered as variable), etc.

As reference, in average, the total amount of time required nowadays to simulate the global model $M_{G}$ using independent simulation $\mu_{T}^{\text {indep }}$ is (2) and (3).

$$
\mu_{T-s \& w}^{\text {indep }}=\mu_{p h}+\mu_{s o}+\mu_{c y}+\mu_{\text {pro_scenario }}+\mu_{\text {pro_results }}
$$




$$
\mu_{T-\text { para }}^{\text {indep }}=\max \left\{\mu_{p h}, \mu_{s o}, \mu_{c y}\right\}+\mu_{\text {pro_scenario }}+\mu_{\text {pro_results }}
$$

In Equations (2) and (3), $\mu_{\text {pro_scenario }}$ represents the mean of a stochastic process $T_{\text {pro_scenario }}$ indicating the time an expert need to separate the global model in the different domains. In the same way, $\mu_{\text {pro_results }}$ is the statistical mean of a stochastic process $T_{\text {pro_results }}$ which indicates the required time to combine the obtained independent results. Both tasks could be performed automatically by using the adequate tools, but this implies a first initial version of a CPSS co-simulator for the application under study is available. For this work, no previous requirements are necessary, so tasks are considered to be performed manually. If the stop-and-wait coordination mechanism is implemented, the domain-specific simulations would have to be executed in a row and the total simulation time is indicated by (2). If parallel execution is available, the total simulation time is which indicated in (3). It is obvious that if parallel execution is available the mean required simulation time goes down, as $\max \left\{\mu_{p h}, \mu_{s o}, \mu_{c y}\right\} \ll\left(\mu_{p h}+\mu_{s o}+\mu_{c y}\right)$, especially when the number of included devices and people goes up (as, typically, simulation algorithms present a complexity of order $\sigma\left(n^{2}\right)$ ). Another parameter which strongly affects the simulation time is the selected value for the minimum time step (usually simulation algorithms includes variable time step solvers). In most cases, the complexity order of the simulation algorithms depending on the time step is $\sigma(n)$, so co-simulation tools usually present softer limitations related to the time step than which they present in respect to the number of considered devices. Moreover, the complexity of the simulation models and the attribute representation of agents may extend the simulation time, even in various magnitude orders (overall if non-linear models are included, which must be solved using complex numerical algorithms). In general, if more devices want to be included it is necessary to use simpler attribute representations and higher values for the time step. Obviously, as more complex models and smaller values for time step are employed, the precision in the obtained results go up. Now, in the case of an orchestrated federated co-simulation solution, a new stochastic process may be defined, representing the total simulation time $T_{T}^{\text {orches }}$ if stop-and-wait coordination mechanism is implemented (4). Moreover, another variable $T_{T-\text { para }}^{\text {orches }}$ may be defined (5) if parallel execution is available.

$$
\begin{gathered}
T_{T-s \& w}^{\text {orches }}=T_{p h}+T_{s o}+T_{c y}+T_{\text {comm }}+T_{\text {init }}+T_{\text {end }} \\
T_{T-\text { para }}^{\text {orches }}=\max \left\{T_{p h}, T_{s o}, T_{c y}\right\}+T_{\text {init }}+T_{\text {end }}
\end{gathered}
$$

In (4), $T_{\text {comm }}$ indicates the total time used in communicating the three domain-specific simulators (also a stochastic process, which includes, besides, delays due to feedback loops which are present in co-simulation tools but not on domain specific simulators). $T_{\text {init }}$ is the time used in configuring the simulation at the initial moment and $T_{\text {end }}$ the required time by the orchestrator for processing and presenting all the results by the simulators. In general, if mean values are considered, $\left(\mu_{\text {init }}+\mu_{\text {end }}\right)<\left(\mu_{\text {pro_scenario }}+\mu_{\text {pro_results }}\right)$, as the third-party engine may processes the initial configuration faster than any expert. Thus, in general, $\mu_{T}^{\text {orches }}<\mu_{T}^{\text {indep }}$.

The time expressed in (4) and (5) could be reduced if choreographed co-simulation solutions are considered. In that case, as no orchestrator is deployed, the time $T_{\text {init }}+T_{\text {end }}$ does not have to be considered. Then, the total simulation time in this case $T_{T}^{\text {choreo }}$ is represented by two new stochastic processes (6) or (7) depending on the selected coordination mechanism.

$$
\begin{gathered}
T_{T-s \& w}^{\text {choreo }}=T_{p h}+T_{s o}+T_{c y}+T_{\text {comm }} \\
T_{T-\text { para }}^{\text {choreo }}=\max \left\{T_{p h}, T_{s o}, T_{c y}\right\}
\end{gathered}
$$

Clearly, if mean values are considered, $\mu_{T}^{\text {choreo }}<\mu_{T}^{\text {orches }}$ in all cases.

Finally, as we said, in integrated solutions this step is not applicable as no coordination mechanism is needed.

As said, it is important to note that all previous temporal variables (in Equations (2)-(7)) are not fixed values. In general, the required time to perform a certain simulation is a stochastic variable which depends on many uncontrollable factors. Thus, relations among the variables may change in 
time. However, in order to support the decision making, in this section we are considering a typical case and the values can be fixed calculating the medium of various evaluations or by theoretical studies considering the algorithms complexity and the underlying hardware.

On the other hand, one additional temporal variable could be considered. Sometimes, the same event must be processed in various simulation tools, or events which are internal when using a unique domain-specific simulator must be externalize when this tool is included in a co-simulator. Then, the total time required to simulate each subsystem in a co-simulator (called, $T_{p h}^{c}, T_{s o}^{c}, T_{c y}^{c}$ ) is, in mean, greater than the required time in independent tools $\left(T_{p h}, T_{s o}, T_{c y}\right)$. In particular, this situation may be reduced to the inclusion of and additional stochastic process $T_{c o-s i m}$ representing these activities (8).

$$
T_{p h}+T_{s o}+T_{c y}<T_{p h}^{c}+T_{s o}^{c}+T_{c y}^{c}=T_{c o-s i m}+T_{p h}+T_{s o}+T_{c y}
$$

The main disadvantage of this new variable is that it depends on the particular implementation employed in the new co-simulator. Thus, discussing its possible values is outside of the scope of this paper. Nevertheless, it can be seen that (although could be partial) the previous analysis is valid as the relations among the different variables are almost independent of the additional time $T_{c o-s i m}$.

For every co-simulation paradigm, as can be seen, the difference in the mean simulation time due to the implementation of a parallel execution or a stop-and-wait mechanism is, basically, the difference between $\max \left\{\mu_{p h}, \mu_{s o}, \mu_{c y}\right\}$ and $\mu_{p h}+\mu_{s o}+\mu_{c y}$. Both values, in general, grows up with a complexity order of $\sigma\left(n^{2}\right)$ when increasing the number of agents in the scenario. However, the growth rate is higher for the expression $\mu_{p h}+\mu_{s o}+\mu_{c y}$ as can be seen in Figure 8, where a generic graphic of the evolution of both expressions is provided.

In Figure 9 two areas may be distinguished. In the first zone, both expressions are low and not very different, as the difference between $\max \left\{\mu_{p h}, \mu_{s o}, \mu_{c y}\right\}$ and $\left(\mu_{p h}+\mu_{s o}+\mu_{c y}\right)$ is not so remarkable. If simulations being performed are located in this area, stop-and-wait mechanism is preferable. Then, from a certain number of agents, the difference between $\max \left\{\mu_{p h}, \mu_{s o}, \mu_{c y}\right\}$ and $\left(\mu_{p h}+\mu_{s o}+\mu_{c y}\right)$ starts growing and, in this area, parallel execution is desirable. In order to support scalability (in the limits of a certain application) it is very important to evaluate the area where the planned co-simulator will operate.

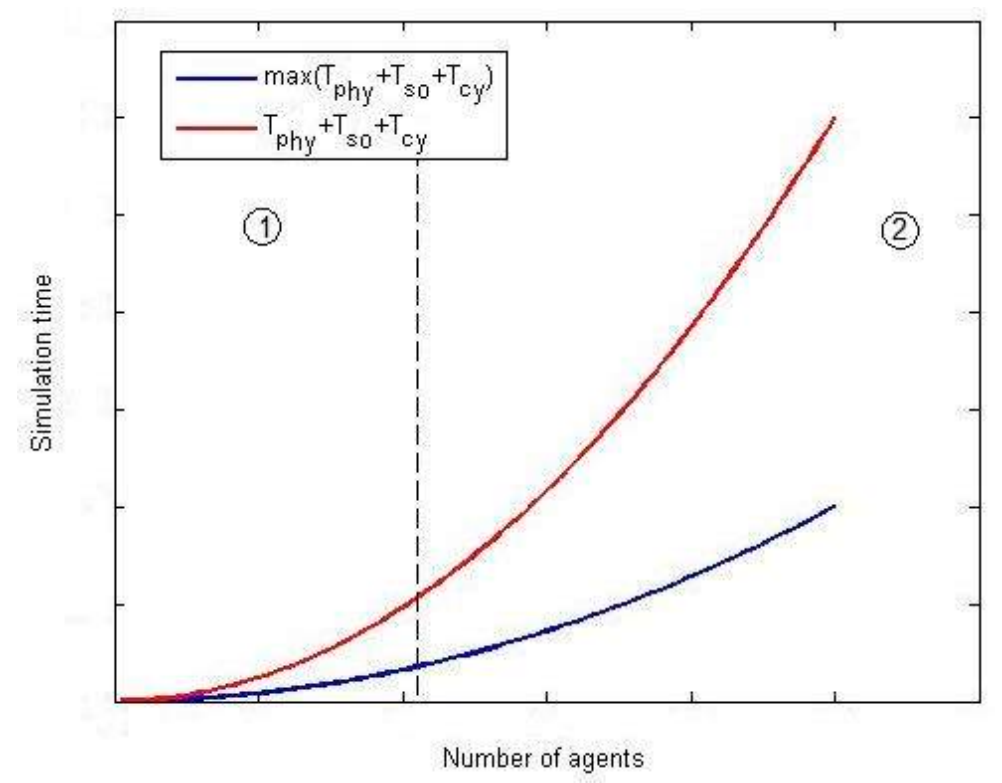

Figure 9. Generic graphic of the evolution of the simulation time.

In order to evaluate the relation between the required simulation time and the development time to be invested, one new function can be defined (9). It represents the number of simulations 
which have to be performed in order to compensate the needed additional time to implement a parallel execution coordination mechanism.

$$
S(n)=\left\lceil\frac{T_{p a r a}^{\text {deve }}-T_{s \& w}^{\text {deve }}}{T_{\text {s\&w }}^{\text {sim } n}-T_{\text {para }}^{\text {sim } n}}\right\rceil
$$

In (8), $n$ represents the number of agents in the simulation, $T_{s \& w}^{\operatorname{sim} \_n}$ the required time to perform the simulation using a stop-and-waits mechanism, $T_{\text {para }}^{\operatorname{sim}_{n} n}$ the required time to perform the simulation using parallel execution, $T_{\text {para }}^{\text {deve }}$ the needed time to develop a co-simulator using parallel execution and $T_{s \& w}^{\text {deve }}$ the needed time to develop a co-simulator using a stop-and-wait mechanism.

As $T_{\text {para }}^{\text {deve }}-T_{s \& w}^{\text {deve }}$ is a fixed number and $T_{s \& w}^{\text {sim } n}-T_{\text {para }}^{\text {sim } n}$ presents a complexity order $\sigma\left(n^{2}\right)$, then $S(n)$ presents a complexity order of $\sigma\left(\frac{1}{n^{2}}\right)$. Figure 10 represents this new function.

If simulations which are going to be performed include a great number of elements, very complex scenarios or devices extremely heterogeneous, parallel execution is the best option as $S(n)<1$. In this area, only one simulation requires an amount of time higher than which invested in implementing the parallel execution. On the contrary, if simulations to be performed are simple, the time invested in implementing a parallel solution does not make profit, as $S(n) \gg 1$. Finally, in the intermediate zone (when $S(n)>1$ but $S(n)$ is not much higher than the unit) any of the proposed mechanisms can be selected to be implemented. In that point, the utilization degree may be determinant (e.g., if many simulations are being performed).

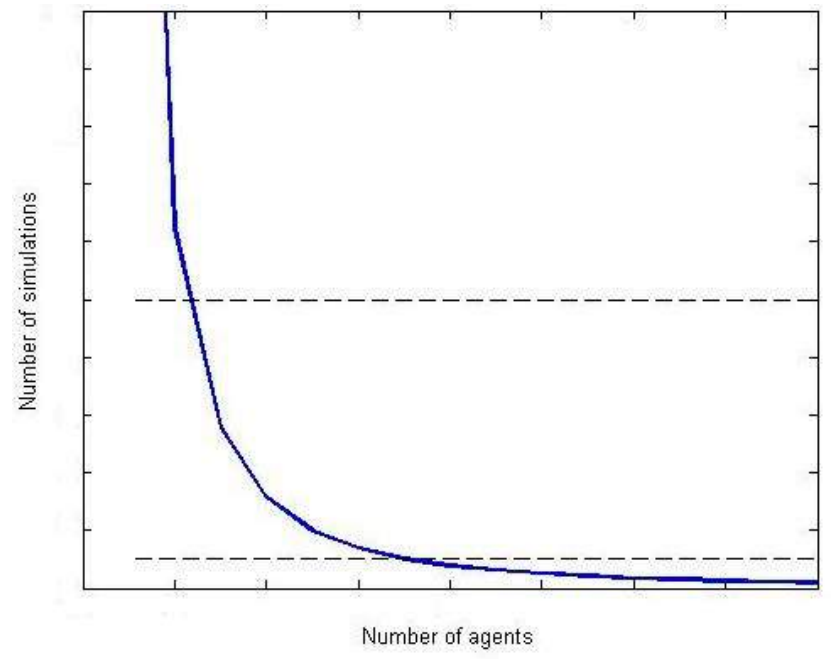

Figure 10. Function $S(n)$.

It is important to note that parallel execution does not imply to be able to parallelize various co-simulations. Domain-specific simulator may operate in parallel, but the co-simulator (as a unit) only performs one simulation each time. If parallelization capabilities are required, the adequate domain-specific simulation tools should be chosen (they must support parallel simulations) and, at implementation time, the coordination mechanism should also be able to manage various simulation at the same time.

Finally, more than the three basic subsystems of CPSS could be considered. Then, if more domain-specific simulation tools are included in the co-simulator, the expressions $\max \left\{T_{p h}, T_{s o}, T_{c y}\right\}$ and $\left(T_{p h}+T_{s o}+T_{c y}\right)$ are generalized to $\max \left\{T_{i}, i \in \operatorname{simulators}\right\}$ and $\sum_{i} T_{i}$. In this generalized expression, it can be seen that, when more domains are considered, parallel execution solutions are preferable (as the reduction in the simulation time, for any considered number of agents, is very remarkable). 


\subsection{Design of the User Interface and Results Presentation}

The final step of our methodology focuses on designing the user interface customized to the particular application, simulation model, and lifecycle. Previous steps greatly influence this final phase. For example, if the "goal specification" state is removed from the simulation lifecycle it is not necessary to develop an interface to show that information. In conclusion, depending on the previous steps, the interface will adapt their elements to show only the relevant aspects of the desired simulation. Additionally, accuracy requirements established by final users in the initial phase should be also considered, in order to create the most adequate results presentation environment. Likewise, the final users' profile should be also taken into account (see Table 5) to adequate the environment to their needs.

In every simulator, some universally required elements [130], must be always included in a user interface in order to perform a successful simulation (for example, there will be always an interface to start a simulation, stop it, or even pause it). Then, every interface for a simulator must include three tools: instruments to design a simulation, instruments to execute and control the simulation and tools to analyze the results. For each one of the three tools, some decisions must be taken (considering the most relevant aspects of the simulations to be performed with the co-simulator). We review these aspects below:

Instruments to design a simulation. User interface must permit the selection between all possible scenarios that can be found in the simulation process, this includes modeling and configuring all the elements that are present in a certain simulation. To allow this, a method to design the scenario has to be provided along with the necessary rules for a correct scheme. Basically, two options are possible: simulations are designed using predefined layouts or scenarios, or external instruments (such as programming environments) are linked to the co-simulator in order to create a new scenario with each simulation.

Instruments to execute and control the simulation. These elements are always available to interact, so the simulation process can be controlled externally. Depending on how the simulation has to be executed, the objective and if various simulations must be executed in a row, two types of tools can be used. Text interfaces and graphical interfaces are the primary options to be considered in the design of the execution and control interface. Any case, textual elements can be present in both types.

Tools to analyze the results. Results may be presented in a simulator in two manners. First there exists the "post-mortem" presentation. In this scheme, the simulation finishes creating a log file which is used later to construct and show the results (thus, graphical display needs a data source to represent the results). Secondly, in "live" presentation, results are calculated, processed and showed at the same time that the simulation advances.

Table 9 provides some criteria to select the most appropriate interface depending on the simulation that is going to be performed.

Table 9. Criteria for designing the user interface.

\begin{tabular}{cc}
\hline Tasks & Criteria \\
\hline $\begin{array}{c}\text { Design a } \\
\text { simulation }\end{array}$ & $\begin{array}{c}\text { If simulations concern only a limited collection of scenarios, predefined layouts are the } \\
\text { appropriate solution. If users must be enabled to design their own scenarios, additional external } \\
\text { instruments are required (for example 3D modeling, development environments, etc.). }\end{array}$ \\
\hline $\begin{array}{c}\text { Execute and } \\
\text { control the } \\
\text { simulation }\end{array}$ & $\begin{array}{c}\text { Text interfaces are adequate for users who performs many simulations in a row (such as in } \\
\text { Monte-Carlo simulations). In didactic applications, or if simulations are performing one-by-one, } \\
\text { graphical interfaces are desirable. }\end{array}$ \\
$\begin{array}{c}\text { Analyze the } \\
\text { results }\end{array}$ & $\begin{array}{c}\text { Simulators which generates great amounts of data require "post-mortem" tools as no enough } \\
\text { time is available to process and presents the results when performing the simulation. If the } \\
\text { monitored variables are few (such as, for example, the position of the agents), then "live" tools } \\
\text { are valid. }\end{array}$ \\
\hline
\end{tabular}

Finally, each co-simulator paradigm and even the particularization of the simulation model made on the first and second steps, require a specific set of tools to be able to configure and 
supervise the simulation. These elements can be added to the user interface in order to interact with the system involved in the simulation process. Some criteria to select the different items that a particular model's needs are defined in Table 10, where we provide some key elements to add in each co-simulation paradigm.

Table 10. User interface key elements.

\begin{tabular}{cc}
\hline Co-Simulation Paradigm & Key Elements \\
\hline Independent & $\begin{array}{c}\text { Individual controls } \\
\text { Individual results }\end{array}$ \\
\hline Choreographed & $\begin{array}{c}\text { Global scenario definition } \\
\text { Relationship conditions }\end{array}$ \\
\hline Orchestrated by a third-party engine & Third party engine connection status \\
\hline Orchestrated by one of the simulators & Main simulator selector \\
\hline
\end{tabular}

\section{Experimental Validation: Co-Simulator Development and Experiment Description}

In this Section, a practical validation for the proposed methodology is provided. We design (and implement) a particular CPSS co-simulator employing the proposed methodology, and, later, we design some experiments in order to evaluate its performance and the invested time its development; and compare those data with those obtained from other solutions. The correctness of the proposed solution will be deducted from the obtained results.

\subsection{Co-Simulator Implementation}

For this first practical use case of the proposed methodology, we decided to design a CPSS co-simulator focused on validating crowd management systems for emergencies (panic control, enhancing attention, etc.) in large facilities. These systems include the three main subsystems of CPSS: the physical world (buildings, stairs, exits, etc.), the cyber world (sensors, displays or speakers) and the social world (people, social behavior and other similar elements). General elements (see Section 3.3.1), such as time constraints, are present in all subsystems. These elements are transversal entities which condition the entire system's operations and, then, they should be considered in all subsystems and domain specific simulations.

First, we perform the requirements and characteristics capture phase. In particular, requirements about the four properties described in Section 3.1 were stablished:

- REQ\#1. Flexibility: The proposed simulation tool is focused in one particular application (crowd management), so requirements about flexibility are not imposed.

- REQ\#2. Modularity: The proposed co-simulator should allow incorporating new types of devices in the cyber world as new technologies are proposed or investigated.

- REQ\#3. Scalability: Simulations scenarios are limited to large-facilities so the maximum number of agents in a certain simulation is of various tens of thousands. As maximum, then, the co-simulator must be able to consider fifty thousands of agents. However, as we are saying later, that is not the most common case.

- REQ\#4. Accuracy: As social models present a limited accuracy (human behavior is very difficult to predict), it is not required a high level of precision in the designed tool (a medium value would be acceptable).

Moreover, simulation tools for crowd management present some specific characteristics. Namely:

- CHAR\#1. Simulations are performed by social experts, who are not programmers or technological professional. Thus, simulators cannot require technological knowledge.

- CHAR\#2. In general, particular values or states at a certain time step are not interesting. In crowd management global tendencies (e.g., is the panic growing?) are more important than particular values. 
- CHAR\#3. The most important subsystem in crowd management systems is the social world. Simulations must provide precise social information, in order to evaluate the crowd behavior. Physical and cyber worlds are secondary.

- CHAR\#4. As buildings may be complex structures, designing the models to include correctly the scenario in the simulator can be difficult in some occasions. However, although many agents might be included in one simulation, all of them present the same behavior, so the required processing capabilities to execute the simulations are limited.

- CHAR\#5. The number of agents in a certain scenario is limited. Buildings are regulated and a maximum capacity is always defined. Even when over-capacity is considered, the number of agents in a certain scenario cannot increase indefinitely.

- CHAR\#6. Results must be represented using both techniques: temporal and statistical graphics, and animations about the scenario's evolution in time.

Apart from the previously presented characteristics, other circumstances must be also considered before implementing the co-simulator (final users and development team characteristics):

- CHAR\#7. The group of future developers does not include any expert on simulators programming. Then, complicated and specific implementation cannot be addressed.

- CHAR \#8. The simulation scenarios are limited to large facilities, so user don not have to be are not enabled to design their own scenarios.

- CHAR\#9. In this case the employed domain-specific simulators were: Matlab/Simulink as physical processes simulator, NS3 as network simulator and MASON as social simulator. We chose these instruments due to their extended use in research, because they present an open architecture and, besides, MASON and NS3 are open source and, finally, due to their efficient performance.

Once the starting position has been exhaustively studied, it is possible to apply the proposed methodology in order to implement the desired co-simulator.

STEP \#1. Selection of the co-simulation paradigm

First, as said in CHAR\#7, no experts on simulators programming were available. Thus, integrated co-simulation got discarded (see Table 5).

Besides, all considered simulators present an open architecture, so federated co-simulations are enabled. Secondly, in CHAR \#9, it must be noted that MATLAB is not an open source tool, so choreographed co-simulation is not allowed. Finally, the results presentation and graphic interface provided with the selected tools do not meet the requirements of a crowd management system (in particular, any environment to visualize animations described in CHAR\#6 is available). Moreover, CHAR\#1 indicates that users are not programmers. Therefore, the proposed co-simulator must be implemented following the orchestrated by a third party engine federated co-simulation paradigm. Figure 11 presents the architecture of the proposed co-simulator. As a novelty, a database where all the logs are stored is also included (as we said, optional components could be added if necessary).

Additionally, NS3 simulator presents, by default, a modularity architecture, so REQ\#2 is fulfilled, and any type of new devices could be easily added to the resulting co-simulator. Moreover, all the domain-specific independent simulators meet scalability requirement REQ\#3. 


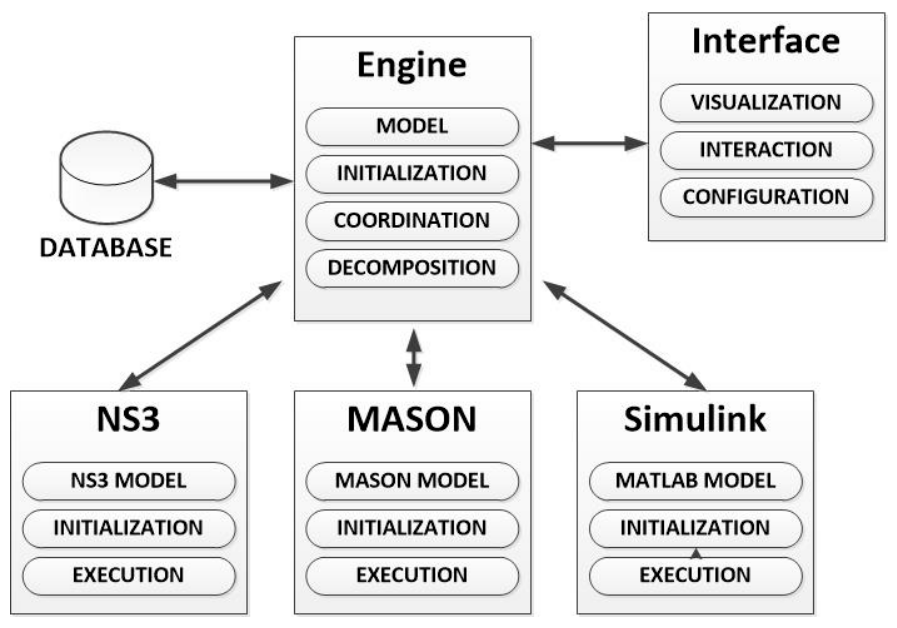

Figure 11. CPSS simulator architecture.

\section{STEP \#2. Particularization of the general simulation model and simulation lifecycle}

In this case, the simulation lifecycle is maintained as shown in Figure 6. No additional task is required and, as indicated in the characteristics cited above all the basic states are necessary.

In respect to the simulation model, $\mathrm{CHAR} \# 3$ states that the most important subsystem in crowd management systems is the social world, so this area must be expanded in the model. Besides, as indicated in Table 6, the physical world should be also slightly complemented in order to represent in the proper way the scenario. Considering this both elements, Figure 12 shows the additional part to be included in the simulation model showed in Figure 6, by extending the relations of "Personal state", "Social state" (both in green, as they are social elements) and "Physical object" (in red, as it belongs to the physical world).

In respect to devices (cyber world), which usually make up a very heterogeneous platform (the CPSS framework is known to have various types of device), the selected network simulator (NS3) has to be able to simulate all the required devices. In particular, see Figure 6, control, communication and processing devices have to be included. Different works [131] have proved that NS3 simulator may be used in that way, being possible to simulate from huge computing systems to small wireless sensor networks or cellular communications.

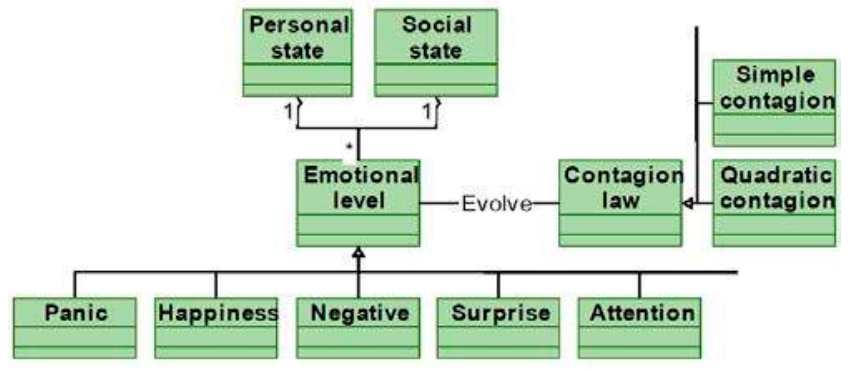

(a)

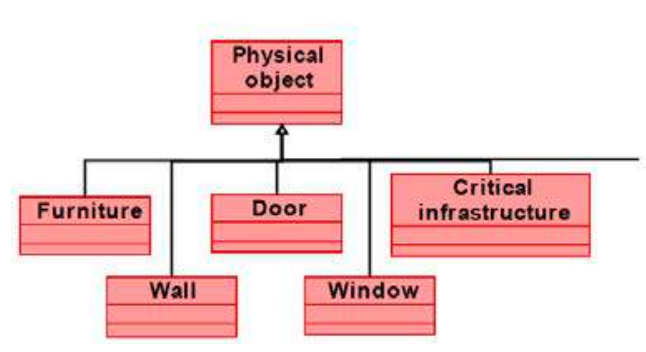

(b)

Figure 12. Extensions for the simulation model (a) Social world (b) Physical world.

\section{STEP \#3. Selection of the appropriate coordination mechanism}

In the third step, the coordination mechanism must be selected. In this case, it must be noted that three different simulation schemes are presented in the co-simulator: physical simulator makes a continuous simulation but the results are shared when the process finished; social simulator also makes a continuous simulation and shares the results each time step and, finally, network simulator makes an event-oriented simulation. However, at implementation stage, these three different schemes may be reduced to only two different schemes. In physical processes simulators, it is very common to recover the simulation routine using a program which runs the simulation each time 
step and shows the generated results. In that way, physical processes simulators (Matlab in our case) and social simulators (MSON in this works) behave in the same manner. Considering this situation, implement a parallel execution solution in the planned co-simulator would be a very difficult task.

On the other hand, as indicated in CHAR\#4, the required processing capabilities to execute the simulations are limited. Moreover, as said in CHAR\#5 the number of agents in the simulation scenarios is also limited. Thus, the simulation time tends to be small and the difference between $\max \left\{T_{p h}, T_{s o}, T_{c y}\right\}$ and $\left(T_{p h}+T_{s o}+T_{c y}\right)$ is not so remarkable. We are waiting a high value for the function $S(n)$. Therefore, stop-and-wait mechanism is preferable in our case. Next, the detailed design of the proposed coordination mechanism is explained.

When the engine receives the global model for the simulation scenario, it keeps track of the future events that are occurring in the different domain-specific simulators. The information about these events is stored in an Event Queue. Thus, the events are extracted by order, always getting the first one in time (which corresponds to the next simulation). On the one hand, a network simulator calculates the future events in a row when starting the simulation. This information is sent to the engine which stores the corresponding events in the Event Queue. On the other hand, MASON simulator (and Matlab/Simulink) updates the state each time step. The events corresponding to this time step $T_{\text {step }}$ are also stored in the Event Queue. At implementation stage, the value of $T_{\text {step }}$ in the social simulator must be fixed to meet REQ\#4 about the tool's accuracy. In our particular case, developers selected a value of $T_{\text {step }}=1 \mathrm{~s}$ as default value, however the decision making to obtain this value it not the objective of this paper.

Once the three simulators (NS3, Matlab and MASON) have been adequately configured, they halt the simulations and only update them when informed by the third-party engine. During the co-simulator operation, the engine checks the simulator to which the first event in the Event Queue belongs. Then it removes this event from the queue and informs the corresponding simulator to continue with the next step (if the corresponding simulator is Matlab or MASON) or to execute the next event (is the corresponding simulator is NS3). The proper domain-specific simulator runs the simulation and generates certain information that could be relevant to the other simulators. The engine processes this information and sends it to the other simulators which update their information and informs the engine once done.

At the end of the simulation, all generated logs are stored in the data base.

Using this approach, moreover, REQ\#3 about scalability is completely fulfilled. Simulations may take a very long time, but they admit perfectly to consider various tens of thousands of agents.

It must be noted that the previous solution it is only one of the several available possibilities. In other situations or application scenarios, the proposed scheme could not be suitable. In particular, it is also possible to create a continuous-time simulator (which updates the state each $T_{\text {step }}$ seconds) by using a different implementation of simulator class in the NS3 implementation or by recovering the entire simulator with an adequate interpolation middleware. The users' preferences or the application requirements will help developers to select the most adequate approach (see Section 3.1).

On the other, although it is not mandatory, the simplest configuration for a co-simulator which includes various continuous-time domain-specific simulators is to employ a unique $T_{\text {step }}$ for every tool. Of course, the selected value of the $T_{\text {step }}$ parameter at production time will affect the correctness and validity of the obtained results. In genera words, as $T_{\text {step }}$ gets greater, the precision of the results go down. This idea is common to all continuous-time simulators, but it is especially important in co-simulation tools as many different and independent tools are involved.

Any case, these discussions must be addressed at implementation and production time (respectively) so they are not the focus of the article.

\section{$\underline{\text { STEP \#4. Design of the user interface and results presentation }}$}

In the fourth step the user interface is designed. As said in CHAR\#8, the planned co-simulator is going to be limited to systems deployed in large facilities. Thus, users do not require additional instruments to design their own scenarios, and layouts of the main facilities (stadiums, colleges, etc.) may be provided with the simulator. 
As said in CHAR\#2, it is unnecessary to control the evolution of the simulation step by step. The interest is focused on the global tendencies, so many simulations are performed in a row in order to obtained representative statistical results. Then, a textual interface should be included for simulation control and execution. Finally, as said in CHAR\#6, different visualizations of the results have to be available. Moreover, users have to be able to calculate many important aggregated values. In conclusion, "post-mortem" results presentation is the most adequate in the proposed case. Finally, Figure 13 shows the interfaces obtained for the co-simulator.

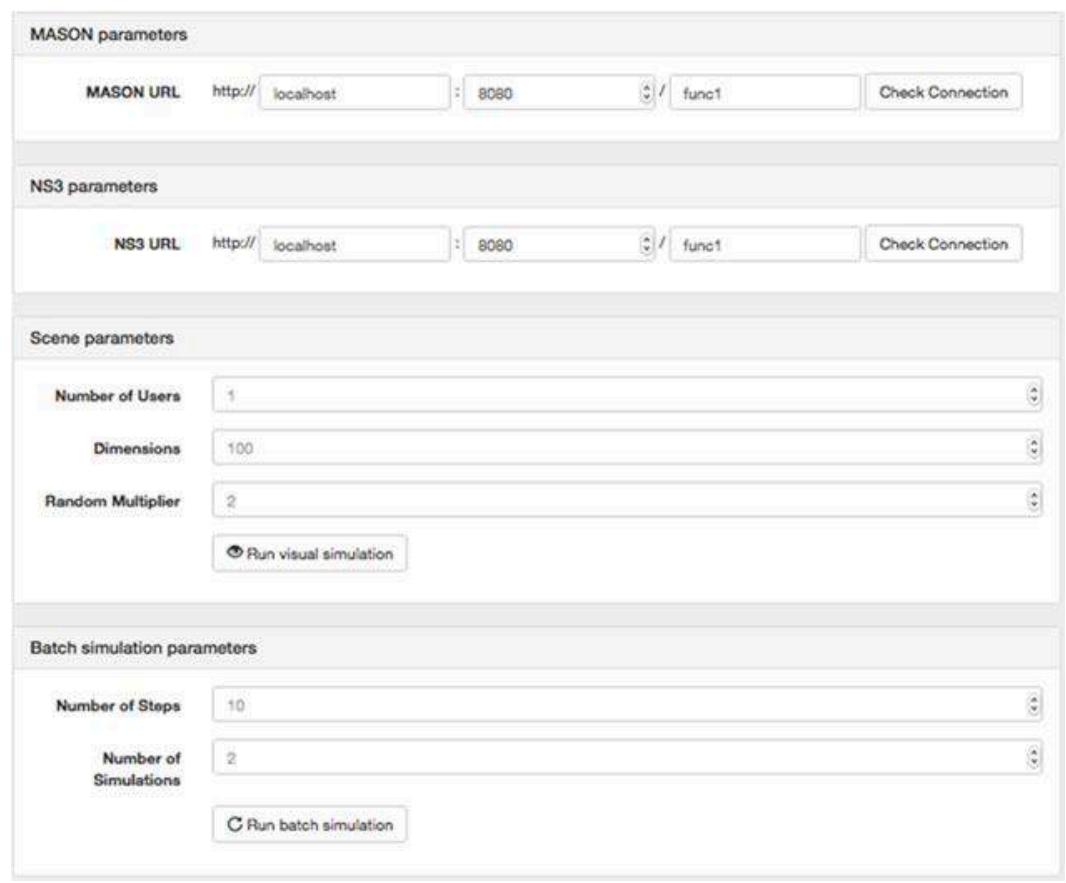

(a)

\section{HYDRA Home About Contac}

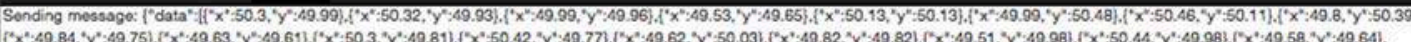

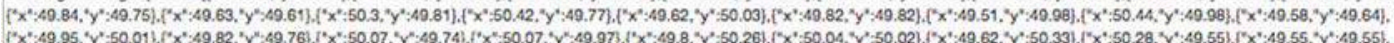

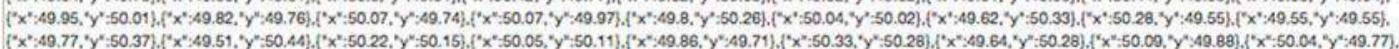

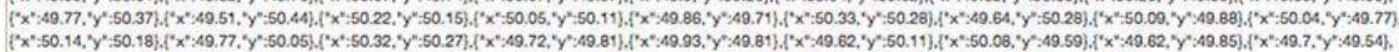

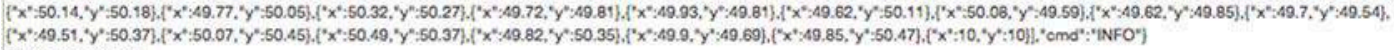

Simulation started

New message recoived:

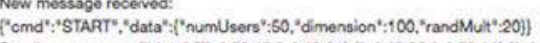

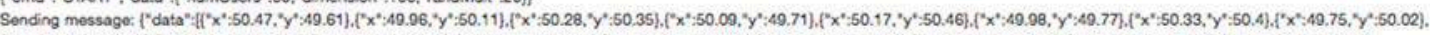

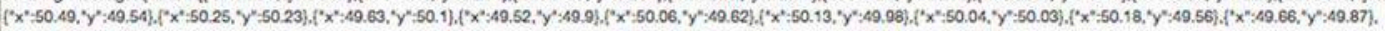

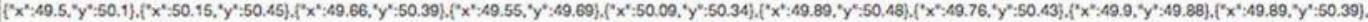

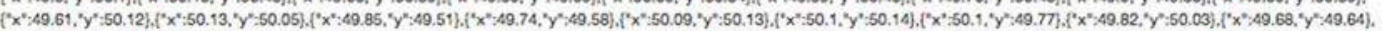

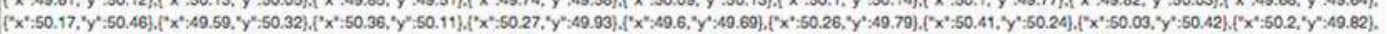

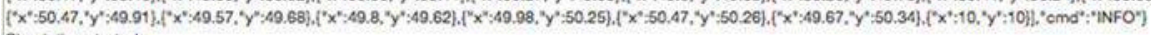
Simulasion started

New message received:

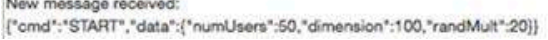

(b)

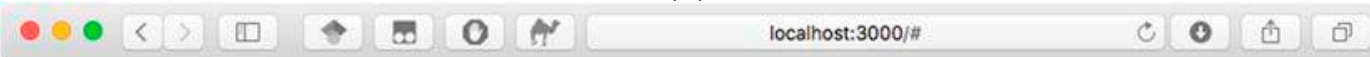

\section{HYDRA Home About Contact}

\section{Select scene}

\begin{tabular}{ll} 
Scene & Description \\
\hline Scene 0 & Several users with mobile phones move around a router, connecting and disconnecting from the WiFi. \\
\hline Scene 1 & Sensing environment \\
\hline Scene 2 & Building B at the ETSIT \\
\hline Scene 3 & People moving in a classroom building
\end{tabular}




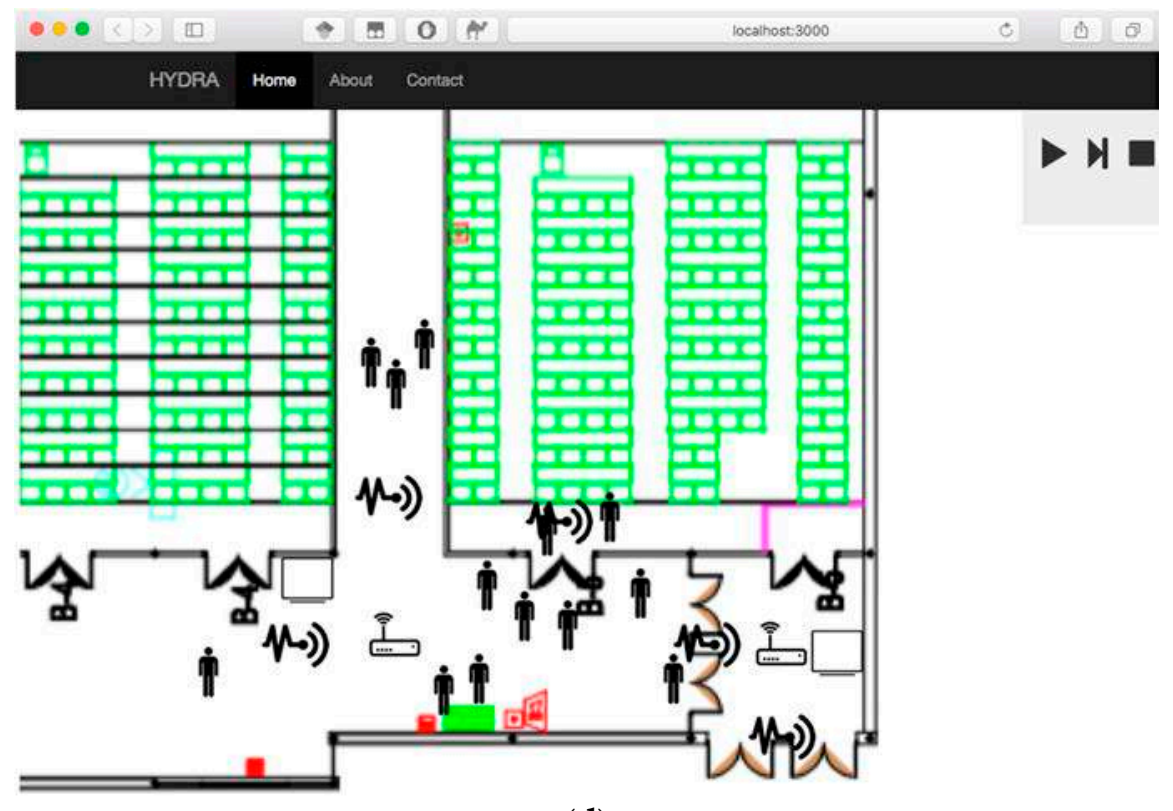

(d)

Figure 13. Simulator's user interfaces. (a) Basic configuration. (b) Simulation execution and control. (c) Simulation design. (d) Results presentation (temporal animation).

\subsection{Experiment Description}

In order to evaluate the correctness of the proposed methodology an experimental validation was designed and carried out. The objective is verifying that the proposed methodology generates the most adequate co-simulator design for a given application, as well as the operational limits of the generated tool. In order to evaluate that, the previously designed co-simulator was implemented.

The experimental validation was divided in three different experiments.

In the first experiment (named as experiment\#1) various quality parameters about the obtained co-simulator in Section 4.1 are evaluated by a crowd simulation expert. Although this first experiment pretends to be a global evaluation, the selected quality parameters are mainly focused on steps one, two and four (as well as on the final users' requirements). Thus, the second experiment (named experiment\#2) is focused on the third step. In this second experiment, temporal measurements about the required simulation time in different situations are done. Finally, in the third experiment (identified as experiment\#3), the operation limits of the generated tool are evaluated. In particular, scalability and accuracy are validated.

\subsubsection{Non-Methodological Co-Simulator Implementation}

In all the described experiments, the results are compared to the values obtained from other additional co-simulators which have been implemented without following the proposed methodology. In particular, in order to carry out the experimental validation three additional co-simulators were implemented. These new co-simulators were developed following existing proposals about this topic, instead of following the proposed methodology.

The first non-methodological co-simulator (hereinafter called "simulator\#1") it was implemented following a kind of choreographed co-simulation paradigm, where every domain-specific simulator is recovered by a middleware being able to communicate with the rest of simulators [132]. Simulation models were maintained as defined by default in the domain-specific simulators, and no additional interface was deployed. All the domain-specific simulators are running in parallel. Graphics and results were shown using the instruments provided by NS3 simulator. Figure 14a describes the architecture of this new co-simulator. The simulation update process follows an iterative paradigm, where each simulation step implies a convergence phase. Every domain-specific simulator has to calculate in an independent way a first estimation of the situation of the scenario under study in the next step, which it is shared with the rest of the tools 
later. Considering this new information, domain-specific tools modify their initial calculations and the sharing and updating process is repeated until values converge to a stable situation.

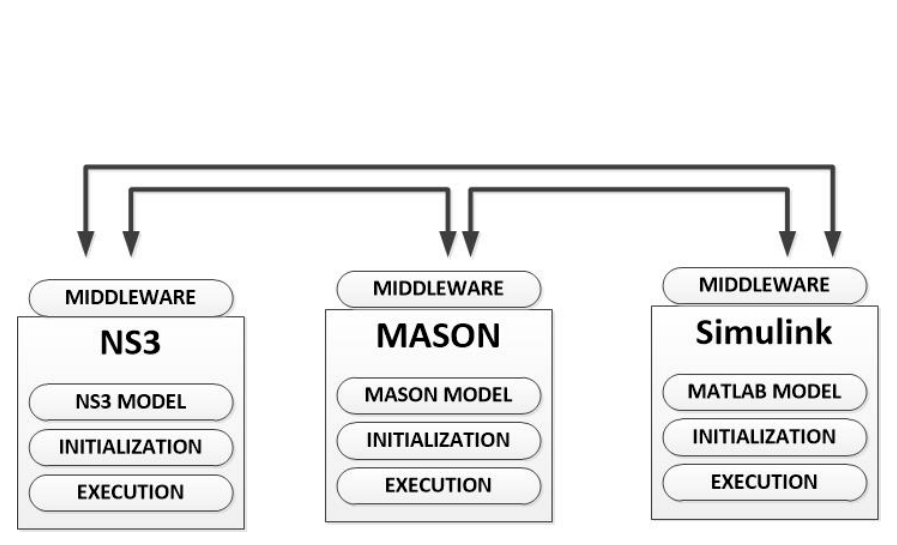

(a)

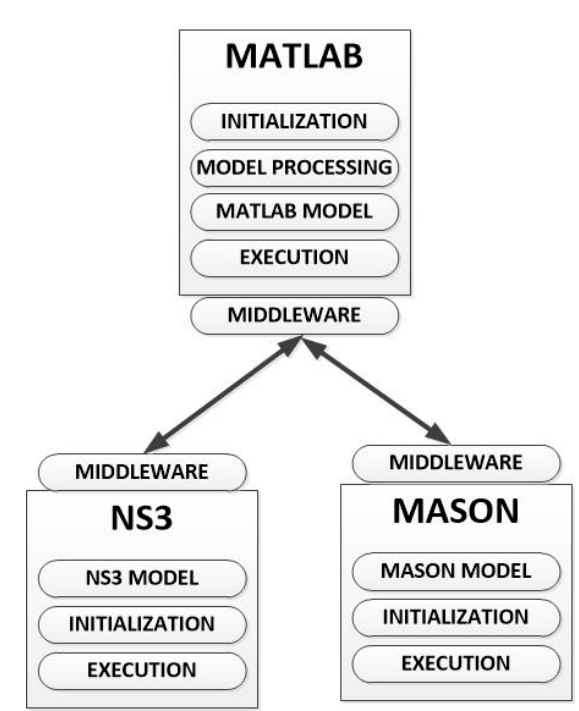

(b)

Figure 14. Secondary (auxiliary) co-simulators architecture. (a) simulator\#1. (b) simulator\#2.

The second additional co-simulator (hereinafter called "simulator\#2") was implemented following one of the most popular schemes for first concept proofs in research. It is similar to an "orchestrated by a one of the simulators" co-simulation paradigm, where the most versatile domain-specific tool controls and manage the entire simulation [115]. As in the base work, we have selected as main simulator the MATLAB suite. As in simulator\#1, simulation models were maintained as defined by default in the domain-specific simulators, and no additional interface was deployed. A "stop-and-wait" coordination mechanism was applied and graphics and results were shown using the instruments provided by MATLAB. Figure $14 \mathrm{~b}$ describes the architecture of this second new co-simulator.

Finally, a third additional non-methodological co-simulator was implemented. This final co-simulator (hereinafter called "simulator\#3"), it is identical to the methodological one. However, in this case, a parallel execution coordination mechanism is selected, instead of the stop-an-wait mechanism employed in the methodological simulator. With this structure, this co-simulator is perfect to evaluate, in a comparative way, the performing of the selected coordination mechanism (third step in the methodology).

On the other hand, in order to allow comparisons between the proposed application-specific co-simulators and state-of-the-art tools, a benchmark simulator is also considered. As said in Section 2, no simulator proposed in the state-of-the-art allows simulating CPSS considering all subsystems in the same detail level (for example, if a fire is simulated, a realistic evolution depending the scenarios has to be followed, but also people's behavior has to be adapted to this situation and the impact in communications should be also evaluated - for example interferences in radio channels due to smoke-). In that way, benchmark domain specific simulators use to present a better performance (as said previously, simulations are faster and more scalable) because their simulations are simpler (coordination delays and congestion, for example, do not appear). However, in order to stablish a reference, it has been selected a benchmark simulator focused on the simulation of the selected study scenario (see Section 4.2.3). In that way, the social simulator MASSIS [133-135] (Multi-agent system simulation of indoor scenarios) has been also deployed and employed. This simulator is based on MASON (as the proposed co-simulator), but includes new interfaces and functionalities to simulate some aspects of the cyber and physical world. 


\subsubsection{Detailed Description of Experiments}

An expert on crowd simulations was invited to evaluate the performing and adequacy of the proposed (methodological) co-simulator using a collection of quality parameters. Additionally, the same evaluation was carried out using the non-methodological co-simulators "simulator $\# 1$ " and "simulator\#2" (described above, Section 4.2.1) and MASSIS simulator. The list of quality parameters was selected to represented how much adequate is the obtained co-simulator for the application scenario. Moreover, the adaptation of the three co-simulators under study to the final users' requirements is also evaluated. These parameters were: usability by crowd management experts, facility to include new types of devices, scalability to advance scenarios, adequacy of the simulation model, accuracy of the simulations, customization and interest of the presented results.

As can be seen, experiment\#1 cannot evaluate which coordination mechanism (parallel execution or "stop-and-wait") is the most adequate for our application (mainly because that is a technical decision and depends strongly on the developers being related to the co-simulator's implementation). Then, in the second experiment, a validation was carried out in order to determine if the methodological co-simulator (Section 4.1) implements the most adequate coordination mechanism. For that, the required simulation time by the methodological co-simulator is evaluated, and compared with which required by the non-methodological co-simulator simulator\#3.

Additionally, all results are compared and valuated together with the invested development time in implementing each tool. The validation consisted of the definition of various simulations which were performed using every simulator. Data about the different simulation times were registered. Finally, as we have said in Sections 3.1 and 4.1, every designed co-simulator should fulfill the final users' requirements. However, sometimes, as time passes, the use of the designed co-simulator gets far from what originally planned. In this case, it is very interesting to know if the new tool continuous meeting the final users' requirements in that new context. Then, in the third experiment, three evaluations were carried out in order to determine the operational limits of the tools designed with our methodology. Moreover, result of the experiences described below were compared with results from all the implemented non-methodological co-simulators ("simulator\#1", "simulator\#2" and "simulator\#3").

In this third experiment, firstly, we are evaluating the scalability of the proposed simulators, depending on the number of agents per simulation. For that, the number of agents in a certain simulation is increased until the designed co-simulator cannot execute it. For every case, various attempts are programmed. Data about the success in the simulations execution are collected. Secondly, the same evaluation is repeated for a fixed number of agents but increasing the number of parameters considered for each agent. The scalability depending on the complexity of the agent model is, thus, evaluated. As previously, for every case, various attempts are programmed. And, thirdly, it is evaluated the accuracy of the resulting co-simulator. For that, the expert on crowd simulations was asked to perform a certain simulation using independent simulation techniques. The results are taken as reference. Then, various simulations modifying the temporal step when possible are performed. Differences between these simulations and the reference (the committed error) are evaluated and employed to determine the simulator's accuracy. As MASSIS describes entities with a fixed number of parameters, and time step is not easily modifiable, this benchmark simulator was only considered for the first part of the experiment. Although the obtained results are not comparable at all (as the complexity of the performed simulations is not similar), these measures may be employed as reference during the discussions.

\subsubsection{Simulation Scenario}

In order to perform the described experiments, a particular simulation scenario was defined.

One of the most interesting topics nowadays is the adequacy of public infrastructures to emergency and evacuation situations (in order to avoid human avalanches, bottlenecks at the exits, etc.). In these situations, communication networks (especially proprietary WiFi networks) support a high stress and, even and depending on the situation, they may get isolated and uncommunicated. Besides, in these situations, people many times behave in a non-standard way, so regular emergency 
systems (such as alarms, panels, etc.) are not as much effective as desired. In these scenarios, then, the physical world (where the emergency starts and evolves), the cyber world (devices and platforms employed nowadays as main information source) and social environment (people) are totally interconnected. Thus, the purpose of the planned simulations is to test the proposed methodology in the creation of a tool for a realistic CPSS application.

The simulation scenario is a representation of first floor in one building at the Technical University of Madrid. In those spaces, we consider a certain amount of people moving around with their smartphones and interacting with the rest of people. The simulation scenario includes three corridors, ten classrooms (some of which were cooled) and other minor spaces (such as a small hall). As maximum, 800 people can be at the same time in that space. Figure 15 represents that scenario.

A fire was simulated in that scenario, where and evacuation was, then, started. The differences of temperature in the building and the information provided from the emergencies system forced people to move and interact with the others. These temperature differences cause both the people movement to change and the quality of communications to go down (properties of wireless channel change). At the same time, smartphones registered the social information and transmitted the acquired data to a central server deployed in a computer center near the simulated building (where the actions to be taken in order to manage people were calculated). With this information, groups of people may be located, specific evacuation plans and information may be communicated, etc.

The simulation pretended to calculate the system evolution along the first ten minutes.

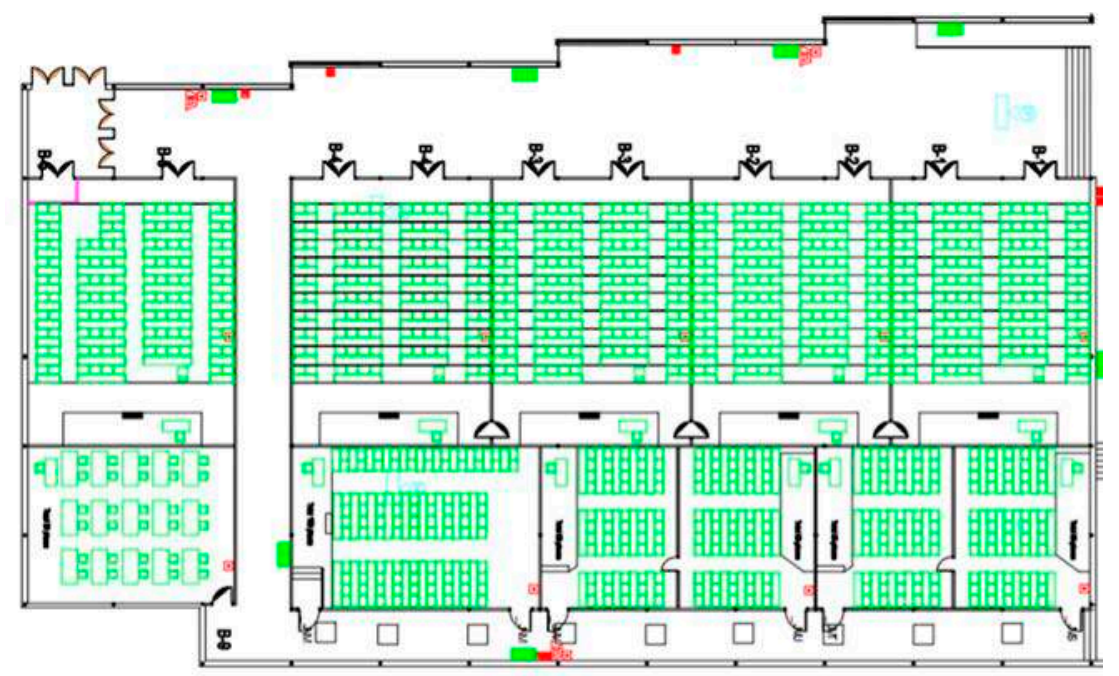

Figure 15. Simulated space.

During the first experiment (experiment\#1), the expert on crowd simulations performed various simulations modifying as much aspects as he considered adequate to evaluate the proposed quality parameters. During the second experiment (experiment\#2) the explained simulation was repeated considering different amounts of people in the building. In that way, it was possible to know if the difference in the development time between the methodological simulator and an identical simulator which implements parallel execution (simulator\#3) justifies the use of the stop-and-wait mechanism. Finally, during the third experiment (experiment\#3), simulation was repeated considering different values for the variables under study (namely, the number of agents in the scenario, the number of parameters per agent and the temporal step).

For every case, simulation was repeated fifteen (15) times and the final result was calculated as the medium value.

In order to simulate each one of the proposed sub-systems (social environment, physical world and cyber domain) libraries and state-of-the-art proposal have been employed. In particular, cellular network and WiFi elements have been simulated employing some existing NS3 libraries (WiFi and LTE modules). Social environment has been programmed using the MASON tools and proposals about emergency simulations [136]. The basic idea is to employ the inertial sensors included into 
intelligent terminals to determine the people behavior during the emergency (if panic is present, people keep calm, etc.). In these scenarios, participative sensing (as some works on CPSS describe) has no sense, and passive monitoring is the most adequate approach. Sensor outputs are emulated by the coordination of social simulation (which determines the evolution in the people behavior), network simulation (which control the behavior of smartphones) and physical simulation (which determines a realistic sensor output for each situation and agent). Physical domain is evaluated using numerical models for fire propagation [137].

\section{Experimental Validation: Results}

In this Section results of the experiments described in Section 4.2 are presented in an ordered way.

\subsection{First Experiment (Experiment\#1): Results}

Table 11 shows the conclusions of the crowd simulation expert about the quality of the presented co-simulators (the methodological one and "simulator\#1" and "simulator\#2").

As can be seen, the methodological co-simulator is globally better than the non-methodological co-simulators (implemented without following the proposed methodology), and the selected benchmark simulator, for the selected application. Thus, the methodology fulfills the objective of creating the most adequate co-simulator, given a certain application of CPSS. Next, we are reviewing in detail each one of the five quality parameters.

The expert considered all co-simulators require users to know details about the cyber world, which is not desirable at all. However, in the case of the methodological co-simulator, the third-party engine covers many of the technical details which must be controlled by user in the secondary co-simulators. In relation to this point, nevertheless, MASSIS simulator is the best as it includes a graphic interface which is very useful for crowd simulation researchers.

Similar to the previous discussion, the third-party engine offers a common and easy mechanism to extend the proposed co-simulator if new devices or domains (such as the artificial world) want to be included. On the contrary, the "simulator\#1" co-simulator requires to design a new middleware each time a new domain-specific co-simulator is included, which is not easy for non-technical users. The same problem appears on "simulator\#2" co-simulator, as every new device requires creating an equivalent description in MATLAB language, which is most times unknown by social experts. On the other hand, MASSIS simulator offers an embedded library of available devices which is very difficult to extend (it requires to initiate a new development and to have technological knowledge).

In respect to scalability, the expert found the methodological simulator may address every simulation scenario for crowd management systems verification perfectly. This characteristic is also available in the non-methodological "simulator\#2" co-simulator, as it is an intrinsic property of "stop-and-wait" coordination mechanism (as we are seeing later). On the other hand, "simulator\#1" co-simulator has many problems with certain scenarios, as it must manage a great amount of signalization (typical of choreographed schemes) which hinders the simulation performing. MASSIS simulator, however, includes a heavy 3D interface which causes large scale simulations to experiment problems when a high number of elements are simulated (see Section 5.3).

Table 11. Results about the quality parameters in the first experiment.

\begin{tabular}{ccccc}
\hline \multirow{2}{*}{ Quality Parameter } & \multicolumn{4}{c}{ Marks (0-10) } \\
\cline { 2 - 5 } & $\begin{array}{c}\text { Methodological } \\
\text { Co-Simulator }\end{array}$ & $\begin{array}{c}\text { Non-Methodological } \\
\text { simulator\#1 }\end{array}$ & $\begin{array}{c}\text { Non-Methodological } \\
\text { simulator\#2 }\end{array}$ & MASSIS \\
\hline Usability by crowd management experts & 8 & 5 & 6 & 9 \\
Facility to include new types of devices & 9 & 7 & 6 & 6 \\
Scalability to advance scenarios & 9 & 6 & 9 & 7 \\
Adequacy of the simulation model & 8 & 8 & 8 & 7 \\
Accuracy of the simulations & 7 & 8 & 8 & 8 \\
Customization & 6 & 8 & 7 & 7 \\
Interest of the presented results & 9 & 7 & 7.4 & 7.5 \\
Total & 8 & 7 & & 7 \\
\hline
\end{tabular}


The models, as the expert said, were adequate in all cases; although some improvements (such as extending the social part) could be included. Experts also indicated that simulation model included into MASSIS shows some deficiencies in respect to mobile entities (for example, they cannot correct the itinerary if a wrong path is followed).

In general, accuracy in all co-simulators is acceptable. However, non-methodological co-simulators (as well as MASSIS simulator) allow final users to control more exactly this parameter (as the third party engine hides some details), so they are more positively considered. In respect to customization, in general, the non-methodological and MASSIS co-simulators allow users to modify the models, simulation routines, etc. in an easier way than the methodological co-simulator (mainly because of the third-party engine).

Finally, the results presentation in the methodological co-simulator was very useful for crowd analyses. But the secondary co-simulators need a more extended catalogue of functionalities in this sense (for example, including advanced animations). MASSIS includes a useful graphic interface but certain representations are difficult to obtain.

In balance, as we said, the proposed methodology allowed implementing the most adequate co-simulator.

\subsection{Second Experiment (Experiment\#2): Results}

Figure 16 shows the results obtained from the second experiment in the experimental validation. The figure shows the average simulation time depending on the number of involved people and mobile devices (agents). In this figure, we compare the simulation time required by the methodological co-simulator, and by an identical simulator which implements parallel execution. Considering the maximum capacity of the scenario ( 800 people) and an over-capacity around $20 \%$, the maximum number of agents in a simulation is two thousand (one thousand people and their corresponding smartphones).

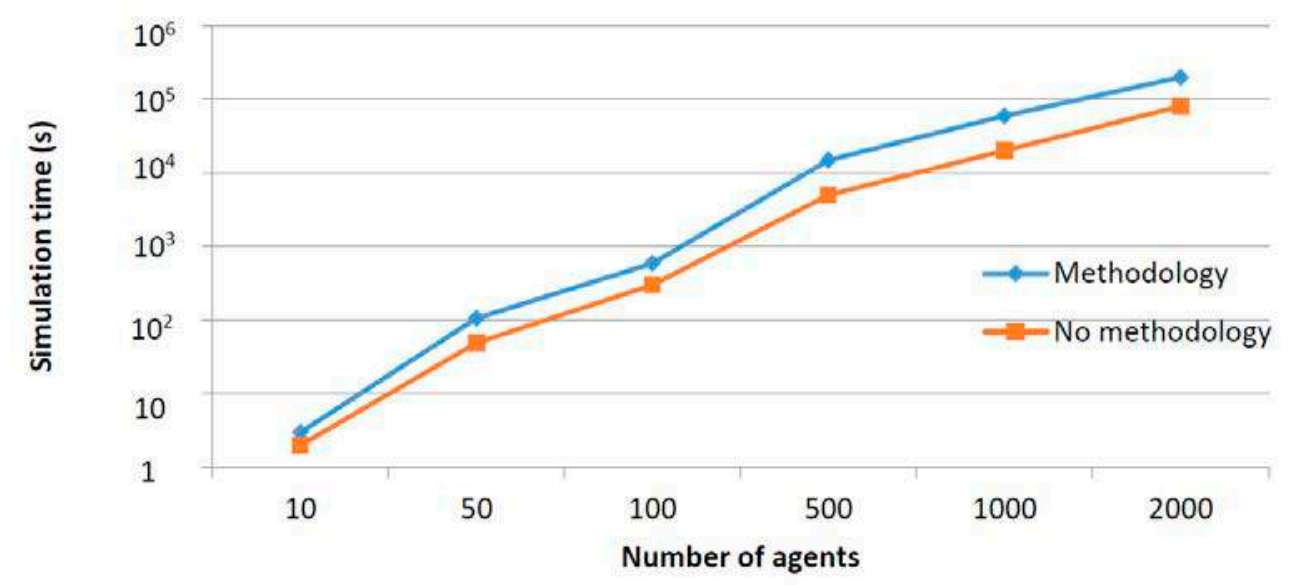

Figure 16. Simulation time along the number of involved people.

Results on Figure 16 are presented in a logarithmic scale in order to visualize correctly all the values (despite being various magnitude order different). As can be seen, the simulation time increases for both co-simulators when increasing the number of simulated people in, approximately, a complexity of order $\sigma\left(n^{2}\right)$. As it was foreseeable, the methodological co-simulator, which implements a stop-and-wait mechanism, requires more time than the co-simulator which implements parallel execution. This difference grows with the number of agents; however, it is not enough to justify the development time required to implement the parallel execution scheme.

In fact, we valued the total time required to implement the methodological co-simulator in one thousand (1000) h. In the case of the identical simulator which implements parallel execution, that time increases to five thousand (5000) h. With these values, in Figure 17 we represented the function $S(n)$, taking into account the results shown in Figure 16. 


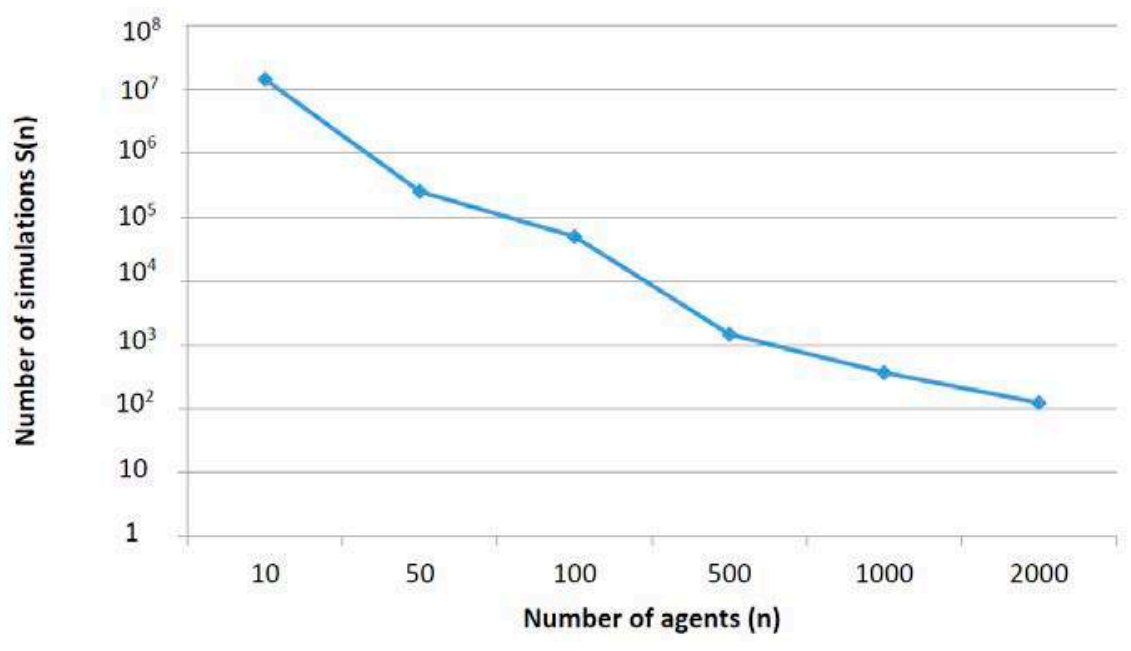

Figure 17. Function S(n).

As can be seen on Figure 17, in the worst case, more than one hundred (100) simulations must be performed to compensate the development time invested in implementing the parallel execution mechanism. This value grows up to ten million if the simplest scenario is considered. Any case, it is clear that the number of simulations is great, concluding that the most adequate mechanism, in this case, is stop-and-wait as indicated by our methodology.

\subsection{Third Experiment (Experiment\#3): Results}

Once methodology performance has been evaluated, it is important to determine the operational limits of the co-simulator.

Figure 18 shows the results about the scalability depending on the number of agents per simulation scenario. As can be seen, every simulation including 5000 agents, or fewer, is perfectly executed by the methodological co-simulator. However, as this number goes up, the simulation algorithm start being collapsed and, in some occasions, simulations cannot be performed. In particular, for a number of agents $n=50,000$ the simulation returns an execution error in $50 \%$ of times. For this work, we are considering a simulation fails (or returns an execution error) when the management entity is not able to communicate with at least one of the domain-specific simulators that make up the general co-simulator. Besides, simulations are considered blocked if the updating process in one of the domain-specific simulators does not finish before one Linux keepalive probe (employed, for example, also in TCP connections -75 s). In fact, problems in the simulation may appear due to hardware limitations, software coordination malfunctions or communication stack overflows because of the great amount of time required to perform the simulation update at each time step when the number of agents is above a certain limit. Furthermore, the maximum number of agents that may be considered without appearing problems in the simulation also depends on the machine hosting the simulation environment. In this case, we have selected a 64-bit Linux Ubuntu 16 operating system, with an Intel i5 processor and 8 GB of RAM memory. As this hardware configuration it is not specifically designed to support heavy simulations, obtained results may show a quite small maximum number of agents per simulation. However, as the objective of this experiment is to compare the performance of different co-simulator configurations, obtained measures can be considered adequate.

As can be seen in Figure 18, for $n=80,000$ or more agents the simulation algorithm never worked (in the sense expressed above). The origin of this limit is the third party engine. As this element acts as an orchestrator, all information must be processed by it. Thus, it creates a bottleneck which blocks the simulation, even when the underlying simulators may perform simulations including more agents. 
This phenomenon is common to all orchestrated schemes, including the non-methodological co-simulators "simulator\#2" and "simulator\#3". In the case of these simulators the problem aggravates. Fist, in "simulator\#2" the orchestrator is one of the domain-specific simulators (MATLAB in this case), so this element not only must perform the orchestration activities but also the simulation under evaluation. Then, the bottleneck narrows and the maximum number of agents decreases (in this case for $n=7000$ agents the simulations return an error in the $50 \%$ of cases). On the other hand, "simulator\#3" presents a parallel execution scheme. Thus, information is not generated in an ordered way (as in stop-and-wait scheme) and the third-party engine gets overloaded much sooner. This case is the most critical, as can be seen in Figure 18, and for $n=5000$ agents the simulations fail in the $60 \%$ of cases.

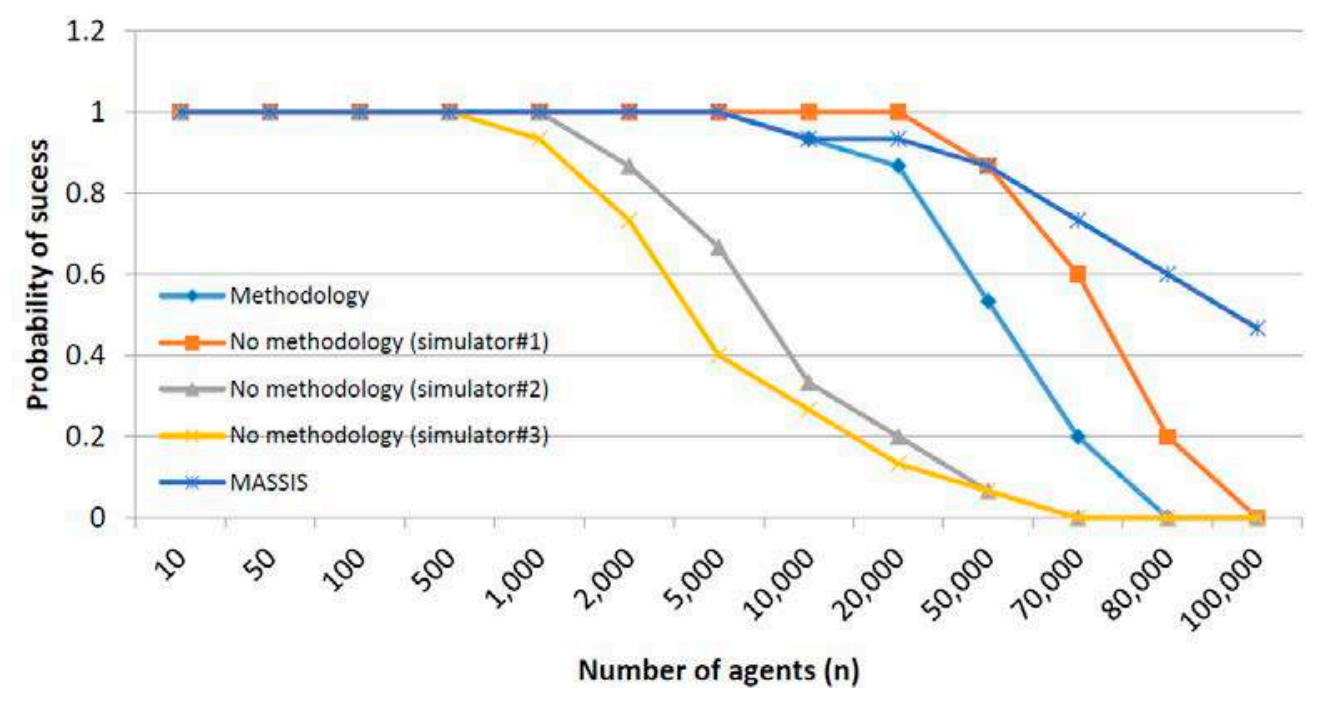

Figure 18. Scalability of the proposed co-simulators considering the number of agents.

This situation is relieved if choreographed simulations are considered, as in "simulator\#1". It presents the widest operation regime as it presents a distributed management system. In particular, this simulator gets blocked when the signaling load among the domain-specific simulators cannot be managed. This limit is reached, approximately, for $n=70,000$ agents, when simulations return an error the $40 \%$ of times.

Although these numbers may seem low, greatest public facilities may host between 100 and 200 thousand people. Furthermore, the proposed simulation scenario, as maximum, may host around three thousand people (this quantity is supported without problems by the proposed co-simulator). For large-scale simulations (including hundreds of thousands of people) the designed tool is not valid but, in this case, the proposed methodology would have produced a different co-simulator. Besides, it must be considered that the employed hardware platform is not a great computational power platform as employed in other scalability studies [138]. Thus, obtained results may be slightly worse than usual.

Finally, as can be seen, benchmark simulators, such as MASSIS, allow us to perform bigger simulations without experimental problems (even the more basic MASON simulator may be configured to include near one million of agents) [138]. However, in these cases, simulations only include social phenomena, and the proposed co-simulator also offers a perspective about the state of the physical environment and the deployed technological systems.

As said in Section 4.2, other important variable in scalability is the number of parameters considered per agent. Figure 19 shows the obtained results. As indicated in Section 4.2, the simulations were performed including 800 agents in the scenario. 


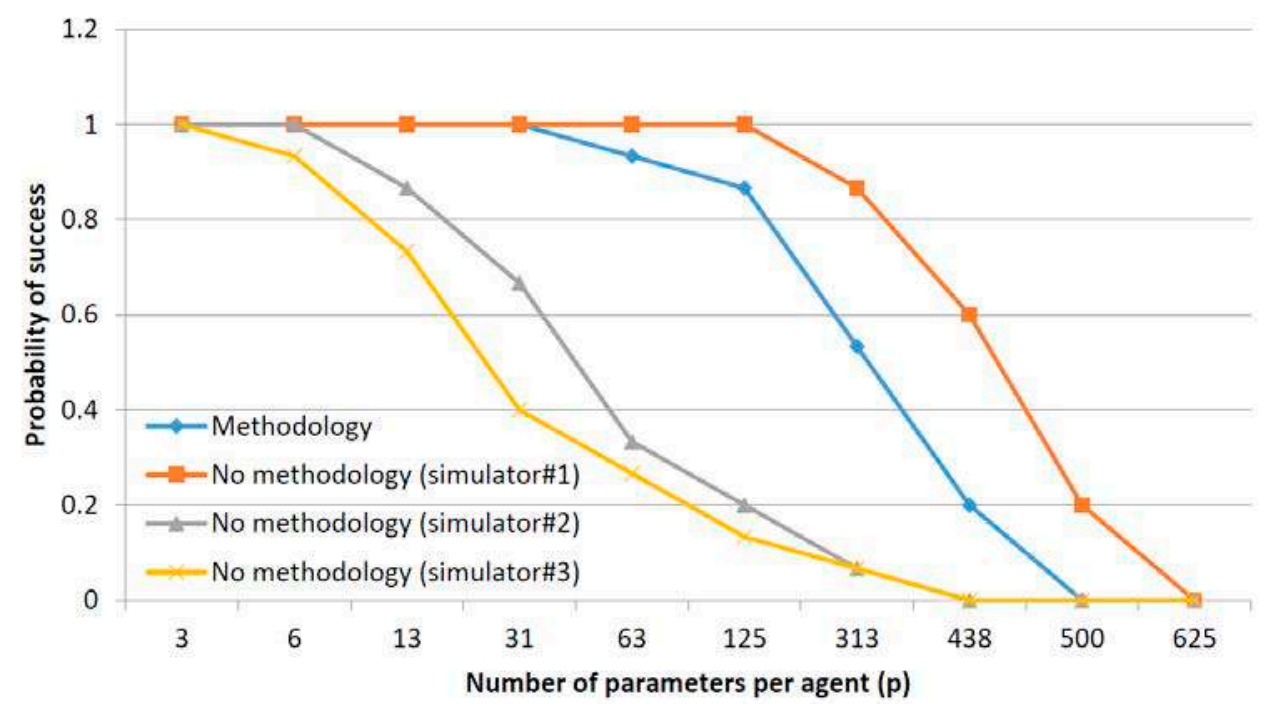

Figure 19. Scalability of the proposed co-simulators considering the number of parameters per agent.

As can be seen, tendencies in Figure 19 are very similar to those observed in Figure 18. That is coherent, as simulation algorithms and signaling load depend in the same way on the number of agents and on the number of parameters per agent. Thus, discussions presented for Figure 19 are valid for this new evaluation. In order to compare the results, Table 12 presents the most important points on Figure 19.

Table 12. Scalability of the proposed co-simulators considering the number of parameters per agent.

\begin{tabular}{ccccc}
\hline Important Points & Methodological & "simulator\#1" & "simulator\#2" & "simulator\#3" \\
\hline Maximum number of parameters without fails & 30 & 300 & 7 & 4 \\
Number of parameters 50\% of fails & 310 & 440 & 40 & 30 \\
Maximum number of parameters & 500 & 620 & 440 & 440 \\
\hline
\end{tabular}

From results on Figures 18 and 19, it is possible to say that the methodological co-simulator meets the final users' requirements. In particular, various tens of thousands (specifically between 50,000 and 80,000) may be included in a simulation scenario as indicated in REQ\#3 (which specifically indicated a limit of 50,000 agents per simulation).

Finally, Figure 20 shows the results for the accuracy evaluation. This evaluation considers a definition of global error (1) in order to evaluate the accuracy of the proposed co-simulator as the temporal step goes down (when possible to modify it). This expression represents the aggregated absolute error in the simulations, being $P\left(t_{\text {final }}\right)$ the final value (at the end of the simulation under study) of the parameter $P$ and $P_{\text {ref }}\left(t_{\text {final }}\right)$ the final value (at the end of the simulation taken as reference) of the reference parameter $P_{\text {ref }}$. Results on Figure 20 are normalized.

$$
\varepsilon=\sum_{\forall \text { agent }} \sum_{\forall \forall \text { parameter } P \text { in } A}\left|P\left(t_{\text {final }}\right)-P_{\text {ref }}\left(t_{\text {final }}\right)\right|
$$

As can be seen the committed error in the proposed methodological co-simulator is constant. That is due to the fact that the temporal step is not controllable by final users (CHAR\#2 showed that this functionality was not necessary). As explained in Section 4.1, the obtained co-simulator is event-oriented, so the value of the temporal step does not affect the final accuracy. The committed error is around $12 \%$ (Figure 20). 


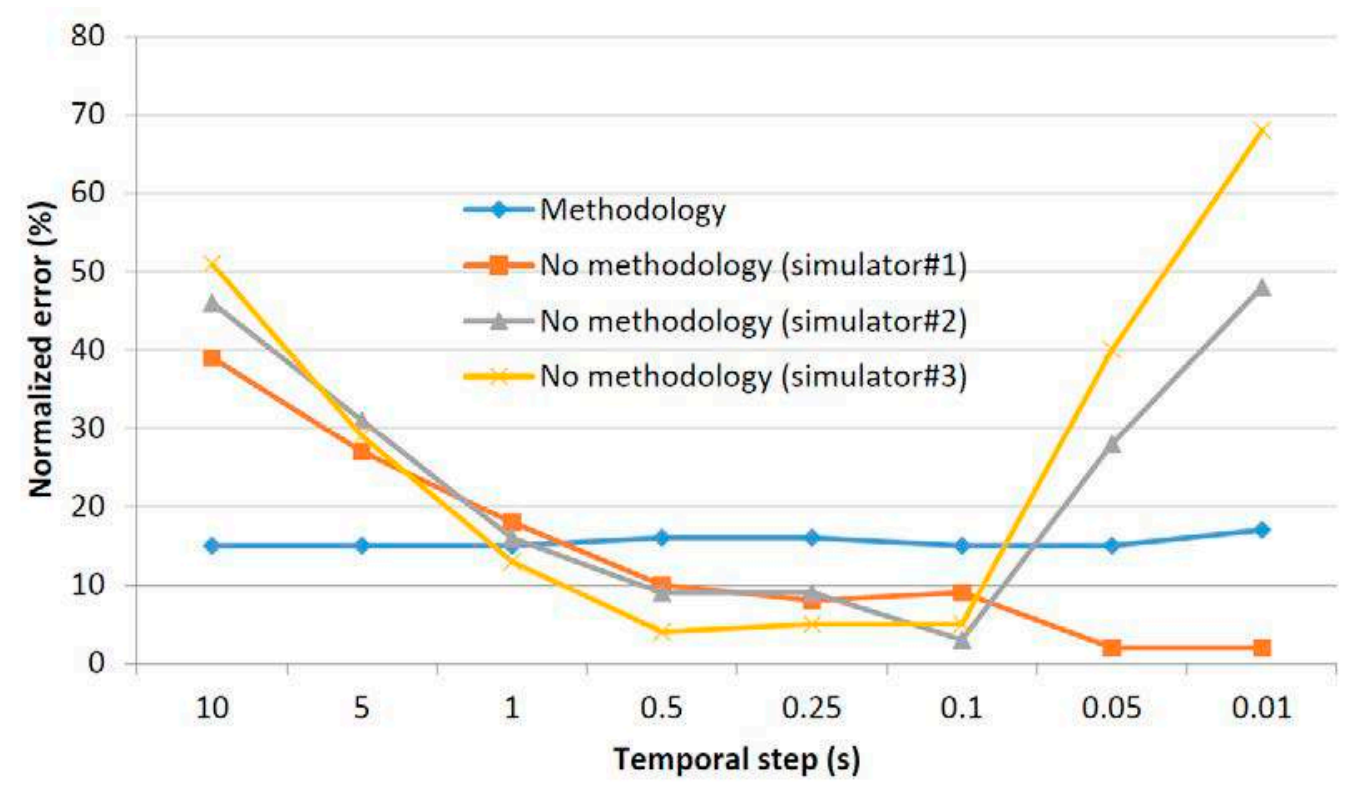

Figure 20. Accuracy of the proposed co-simulators.

In the case of the non-methodological co-simulator all of them allow to manipulate the temporal step $T_{\text {step }}$. Thus, in general, for great values of this variable the maximum error goes up (as changes in the simulation are very abrupt), and for smaller values of $T_{\text {step }}$ the error goes down (the simulation evolves in a more continuous way). However, as can be seen, "simulator\#2"and "simulator\#3" do not follow this rule at all. In these simulators, the committed error goes up again when the temporal step falls below a certain limit $\left(T_{\text {step }} \approx 0.1 \mathrm{~s}\right.$ in both cases). This evolution may be explained by the fact that, in both cases, the simulation algorithm gets blocked when the temporal step is very small. In particular, the orchestration entities cannot update the global state fast enough and some information is lost. As a consequence, the error increases.

\subsection{Discussion}

Considering the previous results, the correctness of the proposed methodology may be confirmed. The results obtained from the first experiment guarantee the selected co-simulation paradigm, the designed simulation model and lifecycle and the planned results presentation interface are the most adequate. The second experiment shows the designed simulation tool is the most adequate in terms of time (the type and number of simulation performed worth the invested time in developing the tool). In general, the correctness of the third step is much more solid, as quantitative and objective proofs have been provided (second experiment). However, the rest of steps involve the users' needs and impressions, so results are much more subjective (although a specialist has been introduced in order to reduce at minimum the human factors). For example, although scenario customization capabilities are not necessary, users prefer to have these functionalities (see Table 11).

The proposed simulator, moreover, present a wide operation range. The limiting property is the scalability, as simulations including more than 80,000 agents (or more than 500 parameters per agent) cannot be performed. Any case, final users' requirements are perfectly met.

As said, the proposed methodology is correct as it generates the most adequate tool for each application (especially in terms of costs and time). However, the possibilities of future extensions or new versions are not considered and some synergies can remain hidden when applied the proposed methodology. Any case, in these situations the most adequate simulator for a certain application it is not generated, as business or corporative arguments are the main factor to be considered.

Finally, the proposed methodology it is only the first stage in the simulator creation process, as (once defined the architecture) implementation tasks must be initiated and, finally, users should 
know how to perform valid simulations. These posterior phases introduce new variables, challenges and problems which are not the focus of the article but which influence the final obtained tool.

\section{Conclusions}

In the present paper, a new methodology to design efficient application-specific CPSS co-simulator has been described. The methodology includes four points which allow selecting the most adequate co-simulation paradigm, to particularize the general simulation model and lifecycle, to choose the better coordination mechanism and, finally, to design the proper interface to the particular application. Additionally, a previous phase for capturing the final users' requirements and characteristics is described. The obtained inputs are employed as selecting criteria in the methodology steps.

Traditional works on co-simulation are focused on very particular aspects about the implementation process, or they only consider the design of integrated co-simulators, which are not the most adequate for all applications. On the contrary, our proposal enables users to design (and implement, if desired) the co-simulator most appropriate for their applications.

First evidences that the simulators generated using the proposed methodology are the friendliest and most useful for users and experts on a certain application of CPSS are provided, as indicated for crowd experts during the proposed experiments. Moreover, the methodology also tries to be efficient in time, taking into account and balancing both elements: the required simulation time and the needed development effort. In that sense, solid evidences that our proposal helps users to select the best option among all the possibilities for a certain application, following an ordered process have been provided. Nevertheless, further analyses considering the collaboration of CPSS researchers should be performed in order to determine the final usefulness of the proposed methodology.

Future works should address the problems mentioned above, organizing deep and large-scale experimental studies involving CPSS researchers. These professionals, who should need to design a new co-simulator, would report their experience, which could be compared with data from a control group. Besides, the relation between the proposed design phase and the next stage (usually, implementation) should be analyzed in order to improve some aspects of the proposed solution (for example, enabling, in that way, the use of agile methodologies, very common in software development). Moreover, and finally, the inclusion of automatic tools in order to build valid simulation models, adequate for the designed co-simulator should be also considered.

Acknowledgments: Borja Bordel has received funding from the Ministry of Education through the FPU program (grant number FPU15/03977). Additionally, the research leading to these results has received funding from the Ministry of Economy and Competitiveness through SEMOLA project (TEC2015-68284-R) and from the Autonomous Region of Madrid through MOSI-AGIL-CM project (grant P2013/ICE-3019, co-funded by EU Structural Funds FSE and FEDER).

Author Contributions: The contributions described in this work are distributed among the authors in the way that follows: All the authors wrote the paper; Borja Bordel Sánchez and Ramón Alcarria contributed to the theoretical formalization and to the proposal of the different simulation paradigms; Diego Sánchez-de-Rivera proposed the simulation models and Álvaro Sánchez-Picot programmed the CPSS simulator.

Conflicts of Interest: The authors declare no conflict of interest.

\section{Abbreviations}

The following abbreviations are used in this manuscript:

CPSS Cyber-Physical Social Sensing

CPS Cyber-Physical Systems

\section{References}

1. Liu, Z.; Yang, D.S.; Wen, D.; Zhang, W.M.; Mao, W. Cyber-physical-social systems for command and control. IEEE Intell. Syst. 2011, 26, 92-96. 
2. Smirnov, A.; Levashova, T.; Shilov, N.; Sandkuhl, K. Ontology for cyber-physical-social systems self-organization. In Proceedings of the 16th Conference of Open Innovations Association (FRUCT16), Oulu, FI, USA, 27-31 October 2014; pp. 101-107.

3. Latour, B. Reassembling the Social-An Introduction to Actor-Network-Theory; Oxford University Press: Oxford, UK, 2005.

4. Tory Higgins, E. Social cognition: Learning about what matters in the social world. Eur. J. Soc. Psychol. 2000, 30, 3-39.

5. Forsyth, D.R. Crowds and Collective Behavior; Group Dynamics: Wadsworth, OH, USA, 2010; pp. 500-528.

6. Aguirre, B.E.; Torres, M.R.; Gill, K.B. Hotchkiss, H.L. Normative collective behavior in the Station building fire. Soc. Sci. Q. 2011, 92, 100-118.

7. Drury, J.; Novelli, D.Y.; Stott, C. Psychological disaster myths in the perception and management of mass emergencies. J. Appl. Soc. Psychol. 2013, 43, 2259-2270.

8. Huo, F.Z.; Song, W.G.; Liu, X.D.; Jiang, Z.G.; Liew, K.M. Investigation of Human Behavior in Emergent Evacuation from an Underground Retail Store. Procedia Eng. 2014, 71, 350-356.

9. National Institute of Standards and Technology. CPS Public Working Group. Available online: pages.nist.gov/cpspwg (accessed on 31 August 2016).

10. Sarbu, I.; Sebarchievici, C. Performance Evaluation of Radiator and Radiant Floor Heating Systems for an Office Room Connected to a Ground-Coupled Heat Pump. Energies 2016, 9, 228.

11. Warren, I. The Renaissance of Legacy Systems: Method Support for Software-System Evolution; Springer Science \& Business Media: New York, NY, USA, 2012.

12. Borch, C. Crowd theory and the management of crowds: A controversial relationship. Theory Cult. Soc. 2015, 32, 155-175.

13. Jiang, L.; Jingyu, L.; Shen, C.; Yang, S.; Han, Z. Obstacle Optimization for Panic Flow. Reducing the Tangential Momentum Increases the Escape Speed. PLoS ONE 2014, 9, 1-15.

14. Weidmann, U.; Kirsch, U. Pedestrian and Evacuation Dynamics; Springer: Berlin, Germany, 2014.

15. Hotchkiss, H.L.; Aguirre, B.E.; Best, E. A critique of the official report on the evacuation of the World Trade Center: Continued doubts. Disasters 2013, 37, 695-704.

16. Sánchez, B.B.; Sánchez-Picot, Á.; De Rivera, D.S. Using 5G Technologies in the Internet of Things Handovers, Problems and Challenges. In Proceedings of the 9th International Conference on Innovative Mobile and Internet Services in Ubiquitous Computing (IMIS), Blumenau, Brazil, 8-10 July 2015; pp. 364369.

17. Baheti, R.; Gill, H. Cyber-physical systems. Impact Control Technol. 2011, 12, 161-166.

18. Wang, D.; Abdelzaher, T.; Kaplan, L. Social Sensing: Building Reliable Systems on Unreliable Data; Morgan Kaufmann: Burlington, MA, USA, 2015.

19. Atzori, L.; Iera, A.; Morabito, G.; Nitti, M. The social internet of things (siot) - When social networks meet the internet of things: Concept, architecture and network characterization. Comput. Netw. 2012, 56, 35943608.

20. Wang, F.Y. The emergence of intelligent enterprises: From CPS to CPSS. IEEE Intell. Syst. 2010, 25, 85-88.

21. Social Sensing and CyberPhysical Systems. Available online: http://www3.nd.edu/ dwang5/courses/spring15/syllabus/Syllabus.pdf (accessed on 31 August 2016).

22. Zeng, J.; Yang, L.T.; Ma, J. A system-level modeling and design for cyber-physical-social systems. ACM Trans. Embed. Comput. Syst. 2016, 15, 35.

23. Bogdan, P.; Marculescu, R. Towards a science of cyber-physical systems design. In Proceedings of the IEEE/ACM International Conference on Cyber-Physical Systems (ICCPS), Chicago, IL, USA, 12-14 April 2011; pp. 99-108.

24. Xu, Y.; Luo, X.; Wang, W.; Zhao, W. Efficient DV-HOP Localization for Wireless Cyber-Physical Social Sensing System: A Correntropy-Based Neural Network Learning Scheme. Sensors 2017, 17, 135

25. Zhou, P.; Zheng, Y.; Li, M. How long to wait? Predicting bus arrival time with mobile phone based participatory sensing. In Proceedings of the 10th International Conference on Mobile Systems, Applications, and Services, Ambleside, UK, 25-29 June 2012; pp. 379-392.

26. Tang, H.; Li, L.; Xiao, N. Smooth Sensor Motion Planning for Robotic Cyber Physical Social Sensing (CPSS). Sensors 2017, 17, 393.

27. Jia, Y.; Zhou, Z.; Chen, F.; Duan, P.; Guo, Z.; Mumtaz, S. A Non-Intrusive Cyber Physical Social Sensing Solution to People Behavior Tracking: Mechanism, Prototype, and Field Experiments. Sensors 2017, $17,143$. 
28. Li, F.; Liu, G.; Liu, J.; Chen, X.; Ma, X. 3D Tracking via Shoe Sensing. Sensors 2016, 16, 1809

29. Dao, M.S.; Pongpaichet, S.; Jalali, L.; Kim, K.; Jain, R.; Zettsu, K. A real-time complex event discovery platform for cyber-physical-social systems. In Proceedings of International Conference on Multimedia Retrieval; ACM: New York, NY, USA, 2014; p. 201.

30. Broman, D.; Greenberg, L.; Lee, E.A.; Masin, M.; Tripakis, S.; Wetter, M. Requirements for Hybrid Cosimulation; No. UCB/EECS-2014-157. California University: Sacramento, CA, USA, 2014.

31. Alharthi, M.; Taha, A.E.M.; Vasilev, V.; Atkinson, K. An Acumen/NS-3 integration for modeling networked Cyber-Physical Systems. In Proceedings of the 27th Biennial Symposium on Communications (QBSC), Kingston, ON, Canada, 1-3 June 2014; pp. 189-192.

32. Guo, D.; Cao, J.; Wang, X.; Fu, Q.; Li, Q. Combating QR-Code-based compromised accounts in mobile social networks. Sensors 2016, 16, 1522.

33. Li, X.; Dai, H.N.; Wang, H.; Xiao, H. On Performance Analysis of Protective Jamming Schemes in Wireless Sensor Networks. Sensors 2016, 16, 1987.

34. Zhang, J.; Long, J.; Zhang, C.; Zhao, G. A Delay-Aware and Reliable Data Aggregation for Cyber-Physical Sensing. Sensors 2017, 17, 395.

35. Huang, C.; Marshall, J.; Wang, D.; Dong, M. Towards Reliable Social Sensing in Cyber-Physical-Social Systems. In Proceedings of the 2016 IEEE International Parallel and Distributed Processing Symposium Workshops, Chicago, IL, USA, 23-27 May 2016; pp. 1796-1802.

36. Su, Z.; Qi, Q.; Xu, Q.; Guo, S.; Wang, X. Incentive scheme for cyber physical social systems based on user behaviors. IEEE Trans. Emerg. Top. Comput. 2017, doi:10.1109/TETC.2017.2671843.

37. Sowe, S.K.; Zettsu, K. Human Factors in Cyber-Physical Social Systems: Leveraging Social Sensor Data. Inf. Model. Knowl. Bases XXVII 2016, 280, 157.

38. Ganti, R.K.; Tsai, Y.E.; Abdelzaher, T.F. Senseworld: Towards cyber-physical social networks. In Proceedings of the International Conference on Information Processing in Sensor Networks, St. Louis, MO, USA, 22-24 April 2008; pp. 563-564.

39. Cassandras, C.G. Smart cities as cyber-physical social systems. Engineering 2016, 2, 156-158.

40. Zheng, X.; Cai, Z.; Yu, J.; Wang, C.; Li, Y. Follow but no track: Privacy preserved profile publishing in cyber-physical social systems. IEEE Int. Things J. 2017, doi:10.1109/JIOT.2017.2679483.

41. Zeng, J.; Yang, L.T.; Lin, M.; Ning, H.; Ma, J. A survey: Cyber-physical-social systems and their system-level design methodology. Future Gener. Comput. Syst. 2016, doi:10.1016/j.future.2016.06.034.

42. Lasi, H.; Fettke, P.; Kemper, H.G.; Feld, T.; Hoffmann, M. Industry 4.0. Bus. Inf. Syst. Eng. 2014, 6, $239-242$.

43. Jiang, P.; Ding, K.; Leng, J. Towards a cyber-physical-social-connected and service-oriented manufacturing paradigm: Social Manufacturing. Manuf. Lett. 2016, 7, 15-21.

44. Zhang, Y.; Song, L.; Jiang, C.; Tran, N.H.; Dawy, Z.; Han, Z. A Social-Aware Framework for Efficient Information Dissemination in Wireless Ad Hoc Networks. IEEE Commun. Mag. 2017, 55, 174-179.

45. Ferrag, M.A.; Maglaras, L.; Ahmim, A. Privacy-preserving schemes for ad hoc social networks: A survey. IEEE Commun. Surv. Tutor. 2017, doi:10.1109/COMST.2017.2718178.

46. Papakipos, M.N.; Garcia, D.H.; Toksvig, M.J.M. Social ad Hoc Networking Protocol and Presentation Layer. U.S. Patent No. 9,679,336, 13 June 2017.

47. Gupta, R.; Krishnamurthi, N.; Wang, U.T.; Tamminedi, T.; Gerla, M. Routing in Mobile Ad-Hoc Networks using Social Tie Strengths and Mobility Plans. In Proceedings of the Wireless Communications and Networking Conference (WCNC), San Francisco, CA, USA, 19-22 March 2017; pp. 1-6.

48. Ding, K.; Jiang, P. Incorporating Social Sensors and CPS Nodes for Personalized Production under Social Manufacturing Environment. Procedia CIRP 2016, 56, 366-371.

49. Zhang, Y.; Cheng, Q.; Zhong, S. Privacy-preserving data aggregation in mobile phone sensing. IEEE Trans. Inf. Forensics Secur. 2016, 11, 980-992.

50. Li, Q.; Cao, G. Providing privacy-aware incentives in mobile sensing systems. IEEE Trans. Mobile Comput. 2015, 15, 1485-1498.

51. Thejaswini, M.; Rajalakshmi, P.; Desai, B. Novel sampling algorithm for human mobility-based mobile phone sensing. IEEE Int. Things J. 2014, 2, 210-220.

52. Xiao, M.; Wu, J.; Huang, L.; Liu, C. Multi-task assignment for crowdsensing in mobile social networks. In Proceedings of the 2015 IEEE Conference on Computer Communications (INFOCOM), Kowloon, China, 26 April-1 May 2015; pp. 2227-2235. 
53. Gomes, C.; Thule, C.; Broman, D.; Larsen, P.G.; Vangheluwe, H. Co-simulation: State of the art. arXiv 2017, arXiv:1702.00686.

54. Vangheluwe, H.; de Lara, J.; Mosterman, P.J. An introduction to multiparadigm modelling and simulation. In Proceedings of the AIS2002 (AI, Simulation \& Planning), Lisbon, Portugal, 7-10 April 2002.

55. Busch, M.; Schweizer, B. Numerical stability and accuracy of different cosimulation techniques: Analytical investigations based on a 2-DOF test model. In Proceedings of the 1st Joint International Conference on Multibody System Dynamics, Lappeenranta, Finland, 25-27 May 2010; Curran Associates, Inc.: Red Hook, NY, USA, 2010; pp. 25-27.

56. Kuhl, F.; Weatherly, R.; Dahmann, J. Creating Computer Simulation Systems: An Introduction to the High Level Architecture; Prentice Hall PTR: Maldon, UK, 1999.

57. Blochwitz, T. The Functional Mockup Interface for Tool independent Exchange of Simulation Models. In Proceedings of the 8th International Modelica Conference, Dresden, Germany, 20-22 March 2011; pp. 2022.

58. Molter, H.G. Discrete event system specification. In SynDEVS Co-Design Flow; Springer: Wiesbaden, Germany, 2012; pp. 9-42.

59. Bogomolov, S.; Greitschus, M.; Jensen, P.G.; Larsen, K.G.; Mikucionis, M.; Strump, T.; Tripakis, S. Co-Simulation of Hybrid Systems with SpaceEx and Uppaal. In Proceedings of the 11th International Modelica Conference (MODELICA), Versailles, France, 21-23 September 2015; Linköping University Electronic Press: Paris, France, 2015; pp. 159-169.

60. Awais, M.U.; Mueller, W.; Elsheikh, A.; Palensky, P.; Widl, E. Using the HLA for Distributed Continuous Simulations. In Proceedings of the 2013 8th EUROSIM Congress on Modelling and Simulation, Cardiff, UK, 10-13 September 2013; pp. 544-549.

61. Nicolescu, G.; Boucheneb, H.; Gheorghe, L.; Bouchhima, F. Methodology for efficient design of continuous/discrete-events co-simulation tools. In High Level Simulation Languages and Applications-HLSLA; Society for Modeling \& Simulation International: San Diego, CA, USA, 2007; pp. 172-179.

62. Falby, J.S.; Zyda, M.J.; Pratt, D.R.; Mackey, R.L. NPSNET: Hierarchical data structures for real-time three-dimensional visual simulation. Comput. Graph. 1993, 17, 65-69.

63. Lang, Y.D.; Biegler, L.T.; Grossmann, I.E. Simultaneous optimization and heat integration with process simulators. Comput. Chem. Eng. 1988, 12, 311-327.

64. Macaulay, L. Requirements capture as a cooperative activity. In Proceedings of the IEEE International Symposium on Requirements Engineering, San Diego, CA, USA, 6 January 1993; pp. 174-181.

65. Wood, D.P.; Christel, M.G.; Stevens, S.M. A multimedia approach to requirements capture and modeling. In Proceedings of the First International Conference on Colorado Springs Requirements Engineering, Colorado Springs, CO, USA, 18-22 April 1994; pp. 53-56.

66. Nunes, N.J.; Cunha, J.F. Wisdom: A software engineering method for small software development companies. IEEE Softw. 2000, 17, 113.

67. Bergman, E. Information Appliances and beyond: Interaction Design for Consumer Products; Morgan Kaufmann: Burlington, MA, USA, 2000.

68. Hessel, F.; Le Marrec, P.; Valderrama, C.A.; Romdhani, M.; Jerraya, A.A. MCI-Multilanguage distributed co-simulation tool. In Distributed and Parallel Embedded Systems; Springer: Berlin, Germany, 1999; pp. 191200.

69. Nof, S.Y.; Deisenroth, M.P.; Meier, W.L. Using physical simulators to study manufacturing systems design and control. In Proceedings of the AIIE Spring Annual Conference, Callaghan, Australia, 17-19 May 1979; pp. 219-227.

70. Mallick, S.K.; Cabezas, H.; Bare, J.C.; Sikdar, S.K. A pollution reduction methodology for chemical process simulators. Ind. Eng. Chem. Res. 1996, 35, 4128-4138.

71. Mathworks: Matlab/Simulink Homepage. Available online: http://es.mathworks.com/products/simulink/ (accessed on 31 August 2016).

72. Maple Home Page. Available online: https://www.maplesoft.com/products/maplesim/ (accessed on 31 August 2016).

73. OpenModelica Homepage. Available online: https://www.openmodelica.org/ (accessed on 31 August 2016).

74. Wolfram Homepage. Available online: http://www.wolfram.com/system-modeler/ (accessed on 31 August 2016). 
75. SciLab Homepage. Available online: https://www.scilab.org/scilab/gallery/xcos (accessed on 31 August 2016).

76. Larnpotang, S.; Lizdas, D.; Rajon, D.; Luria, I.; Gravenstein, N.; Bisht, Y.; Robinson, A. Mixed simulators: Augmented physical simulators with virtual underlays. In Proceedings of the 2013 IEEE Virtual Reality (VR), Lake Buena Vista, FL, USA, 18-20 March 2013; pp. 7-10.

77. Bordel, B.; Alcarria, R.; Pérez-Jiménez, M.; Robles, T.; Martín, D.; de Rivera, D.S. Building Smart Adaptable Cyber-Physical Systems: Definitions, Classification and Elements. In International Conference on Ubiquitous Computing and Ambient Intelligence; Springer: Berlin, Germany, 2015; pp. 144-149.

78. Brooks, C.; Lee, E.A.; Lorenzetti, D.; Nouidui, T.S.; Wetter, M. CyPhySim: A cyber-physical systems simulator. In Proceedings of the 18th International Conference on Hybrid Systems: Computation and Control, Seattle, WA, USA, 14-16 April 2015; pp. 301-302.

79. Wetter, M.; Nouidui, T.S.; Lorenzetti, D.; Lee, E.A.; Roth, A. Prototyping the next generation energyplus simulation engine. In Proceedings of the 3rd IBPSA Conference, Jeju island, South Korea, 27-29 November 2015.

80. Derler, P.; Lee, E.A.; Vincentelli, A.S. Modeling cyber-physical systems. Proc. IEEE 2012, 100, 13-28.

81. Xue, Y.; Rodriguez, S.; Bogdan, P. A spatio-temporal fractal model for a CPS approach to brain-machine-body interfaces. In Proceedings of the Design, Automation \& Test in Europe Conference \& Exhibition (DATE), Dresden, Germany, 14-18 March 2016; pp. 642-647.

82. Ghorbani, M.; Bogdan, P. A cyber-physical system approach to artificial pancreas design. In Proceedings of the Ninth IEEE/ACM/IFIP International Conference on Hardware/Software Codesign and System Synthesis, Montreal, QC, Canada, 29 September-4 October 2013; p. 17.

83. Davidsson, P. Agent Based Social Simulation: A Computer Science View. Available online: http://jasss.soc.surrey.ac.uk/5/1/7.html (accessed on 31 August 2016).

84. Allan, R.J. Survey of Agent Based Modelling and Simulation Tools; Science and Technology Facilities Council: Swindon, UK, 2010.

85. Sánchez, B.B.; Alcarria, R.; de Rivera, D.S.; Sánchez-Picot, A. Predictive algorithms for mobility and device lifecycle management in Cyber-Physical Systems. EURASIP J. Wirel. Commun. Netw. 2016, 2016, 228.

86. Ossowski, S. Agreement Technologies. In Law, Governance and Technology Series, No. 8; Springer: Heidelberg, Germany, 2013.

87. Euzenat, J.; Shvaiko, P. Ontology Matching; Springer: Heidelberg, Germany, 2007.

88. Jennings, N.R.; Faratin, P.; Lomuscio, A.R.; Parsons, S.; Wooldridge, M.J.; Sierra, C. Au-tomated negotiation: Prospects, methods and challenges. Int. J. Group Decis. Negot. 2001, 10, 199-215.

89. Ferber, J.; Gutknecht, O.; Michel, F. From Agents to Organizations: An Organizational View of MultiAgent Systems. Agent Oriented Softw. Eng. 2004, 2935, 214-230.

90. Sierra, C.; Debenham, J. Trust and honour in information based agency. In Proceedings of the 5th International Conference on Autonomous Agents and Multi Agent Systems, Hakodate, Japan, 8-12 May 2006; pp. 1225-1232.

91. Barton, J.; Vijayaraghavan, V. Ubiwise: A Simulator for Ubiquitous Computing Systems Design; HP Labs.: Palo Alto, CA, USA, 2003.

92. O'Neill, E.; Klepal, M.; Lewis, D.; O'Donnell, T.; O'Sullivan, D.; Pesch, D. A testbed for evaluating human interaction with ubiquitous computing environments. In Proceedings of the Testbeds and Research Infrastructures for the Development of Networks and Communities, Trento, Italy, 23-25 February 2005; pp. 60-69.

93. Nishikawa, H.; Yamamoto, S.; Tamai, M.; Nishigaki, K.; Kitani, T.; Shibata, N.; Yasumoto, K.; Ito, M. UbiREAL: Realistic Smartspace Simulator for Systematic Testing. Ubiquitous Comput. LNCS 2006, 4206, 459-476.

94. Tang, L.; Zhou, X.; Becker, C.; Yu, Z.; Schiele, G. Situation-based design: A rapid ap-proach for pervasive application development. In Proceedings of the 9th International Conference on Ubiquitous Intelligence \& Computing and 9th International Conference on Autonomic \& Trusted Computing (UIC/ATC), Fukuoka, Japan, 4-7 September 2012; pp. 128-135.

95. Serrano, E.; Botía, J.A. Validating ambient intelligence based ubiquitous computing systems by means of artificial societies. Inf. Sci. 2013, 222, 3-24.

96. UbikSim Website. Available online: https://github.com/emilioserra/UbikSim/wiki (accessed on 31 August 2016). 
97. Campillo-Sanchez, P.; Gómez-Sanz, J.J.; Botía, J.A. PHAT: Physical Human Activity Tester. In Hybrid Artificial Intelligent Systems; Springer: Heidelberg, Germany, 2013; Volume 8073, pp. 41-50.

98. Bogdan, P.; Marculescu, R. A fractional calculus approach to modeling fractal dynamic games. In Proceedings of the 50th IEEE Conference on Decision and Control and European Control, Orlando, FL, USA, 12-15 December 2011; pp. 255-260.

99. Helbing, D. Modeling multi-lane traffic flow with queuing effects. Phys. A Stat. Mech. Appl. 1997, 242, 175194.

100. Cao, K.C.; Chen, Y.; Stuart, D. A Fractional Micro-Macro Model for Crowds of Pedestrians based on Fractional Mean Field Games. arXiv 2016, arXiv:1602.01211.

101. Macal, C.; North, M. Introductory tutorial: Agent-based modeling and simulation. In Proceedings of the 2014 Winter Simulation Conference, Savannah, GA, USA, 7-10 December 2014; pp. 6-20.

102. Railsback, S.F.; Lytinen, S.L.; Jackson, S.K. Agent-based simulation platforms: Review and development recommendations. Simulation 2006, 82, 609-623.

103. North, M.J.; Nicholson, T.; Collier, J.R.V. Experiences creating three implementations of the repast agent modeling toolkit. ACM Trans. Model. Comput. Simul. 2006, 16, 1-25.

104. NS3 Homepage. Available online: https://www.nsnam.org/ (accessed on 31 August 2016).

105. Varga, A. The OMNeT++ discrete event simulation system. In Proceedings of the European simulation multiconference (ESM'2001), Prague, Czech Republic, 6-9 June 2001; p. 65.

106. Aneiba, A.; Chibelushi, C.C. OPNET-Based Performance Analysis of a Multi-agent Architecture for Managing the Mobile Content Delivery Process. In Information Science and Applications (ICISA); Springer: Singapore, 2016; pp. 127-137.

107. Siraj, S.; Gupta, A.; Badgujar, R. Network simulation tools survey. Int. J. Adv. Res. Comput. Commun. Eng. 2012, 1, 199-206.

108. Eriksson, J.; Österlind, F.; Finne, N.; Tsiftes, N.; Dunkels, A.; Voigt, T.; Marrón, P.J. COOJA/MSPSim: Interoperability testing for wireless sensor networks. In Proceedings of the 2nd International Conference on Simulation Tools and Techniques, Rome, Italy, 2-6 March 2009; p. 27.

109. SimpleIoTSimulator HomePage. Available online: http://www.smplsft.com/SimpleIoTSimulator.html (accessed on 31 August 2016).

110. Duarte, F.; Ferreira, A.; Fael, P. Software for Simulation of Vehicle-Road Interaction. In New Advances in Information Systems and Technologies; Springer: Berlin, Germany, 2016; pp. 681-690.

111. Nitti, M.; Atzori, L.; Cvijikj, I.P. Friendship selection in the social internet of things: Challenges and possible strategies. Int. Things J. 2015, 2, 240-247.

112. Quadri, I.; Bagnato, A.; Brosse, E.; Sadovykh, A. Modeling methodologies for Cyber-Physical Systems: Research field study on inherent and future challenges. ADA USER 2015, 36, 246.

113. Palensky, P.; Widl, E.; Elsheikh, A. Simulating cyber-physical energy systems: Challenges, tools and methods. IEEE Trans. Syst. Man Cybern. Syst. 2014, 44, 318-326.

114. Mart, D.; Bordel, B. Modeling and Simulation of Interactions among People and Devices in Ambient Intelligence Environments. Proceedings of the 30th International Conference on Advanced Information Networking and Applications Workshops (WAINA), Crans-Montana, Switzerland, 23-25 March 2016; pp. 784-789.

115. Kachan, D. Integration of NS-3 with MATLAB/Simulink; Luleå tekniska universitet: Lulea, Switzerland, 2010.

116. Grasp Homepage. Available online: http://www.ticra.com/products/software/grasp (accessed on 31 August 2016)

117. Lin, H.; Sambamoorthy, S.; Shukla, S.; Thorp, J.; Mili, L. Power system and communication network co-simulation for smart grid applications. In Proceedings of the Innovative Smart Grid Technologies (ISGT), Anaheim, CA, USA, 7-19 January 2011; pp. 1-6.

118. Karnouskos, S.; De Holanda, T.N. Simulation of a smart grid city with software agents. In Proceedings of the Third UKSim European Symposium on Computer Modeling and Simulation, Athens, Greece, 25-27 November 2009; pp. 424-429.

119. Sargent, R.G. Verification and validation of simulation models. J. Simul. 2013, 7, 12-24.

120. Jeruchim, M.C.; Balaban, P.; Shanmugan, K.S. Simulation of Communication Systems: Modeling, Methodology and Techniques; Springer Science \& Business Media: New York, NY, USA, 2006. 
121. Duarte, L.M.; Kramer, J.; Uchitel, S. Model extraction using context information. In International Conference on Model Driven Engineering Languages and Systems; Springer: Berlin/Heidelberg, Germany, 2006; pp. 380394.

122. Nieto-Hidalgo, M.; Ferrández-Pastor, F.J.; García-Chamizo, J.M.; Flórez-Revuelta, F. DAI Virtual Lab: A Virtual Laboratory for Testing Ambient Intelligence Digital Service. In Proceedings of the V Congreso Internacional de Diseño, Redes de Investigación y Tecnología para Todos, Madrid, Spain, 23-25 September 2013.

123. Polhill, J.G.; Parker, D.; Brown, D.; Grimm, V. Using the ODD protocol for describing three agent-based social simulation models of land-use change. J. Artif. Soc. Soc. Simul. 2008, 11, 3.

124. Terna, P. Simulation tools for social scientists: Building agent based models with swarm. J. Artif. Soc. Soc. Simul. 1998, 1, 1-12.

125. Duives, D.C.; Daamen, W.; Hoogendoorn, S.P. State-of-the-art crowd motion simulation models. Transp. Res. Part C Emerg. Technol. 2013, 37, 193-209.

126. Lasecki, W.S.; Murray, K.I.; White, S.; Miller, R.C.; Bigham, J.P. Real-time crowd control of existing interfaces. In Proceedings of the 24th annual ACM symposium on User interface software and technology, Barbara, CA, USA, 16-19 October 2011; pp. 23-32.

127. Perry, R.W. Incident management systems in disaster management. Disaster Prev. Manag. Int. J. 2013, 12, 405-412.

128. Cioffi-Revilla, C. On the Quality of a Social Simulation Model: A Lifecycle Framework. Adv. Soc. Simul. Adv. Intell. Syst. Comput. 2014, 229, 13-23.

129. Sargent, R.G. Verification and Validation of Simulation Models. In Proceedings of the 2007 Winter Simulation Conference, Washington, DC, USA, 9-12 December 2007.

130. Cao, H. Development of Techniques for General Purpose Simulators. Ph.D. Thesis, Stanford University, Stanford, CA, USA, 2002.

131. Alcarria, R.; Robles, T.; Morales, A.; Cedeño, E. Resolving coordination challenges in distributed mobile service executions Int. J. Web Grid Serv. 2014, 10, 168-191, doi:10.1504/IJWGS.2014.060251

132. Strasser, T.; Stifter, M.; Andrén, F.; Palensky, P. Co-simulation training platform for smart grids. IEEE Trans. Power Syst. 2014, 29, 1989-1997.

133. Pax, R.; Pavón, J. Multi-Agent System Simulation of Indoor Scenarios. In Proceedings of the 2015 Federated Conference on Computer Science and Information Systems (FedCSIS), Lodz, Poland, 13-16 September 2015; pp. 1757-1763.

134. Pax, R.; Bonett, M.C.; Gómez-Sanz, J.J.; Pavón, J. Virtual Development of a Presence Sensor Network Using 3D Simulations. In International Conference on Smart Cities; Springer: Cham, Switzerland, 2017; pp. 154-163.

135. A Framework for MultiAgent System Simulation of Indoor Scenarios. Available online: http://www.massisframework.com/ (accessed on 21 september 2017)

136. Ren, C.; Yang, C.; Jin, S. Agent-based modeling and simulation on emergency evacuation. Complex Sci. 2009, 5, 1451-1461.

137. Kodur, V.K.R.; Dwaikat, M. A numerical model for predicting the fire resistance of reinforced concrete beams. Cem. Concr. Compos. 2008, 30, 431-443.

138. Lorig, F.; Dammenhayn, N.; Müller, D.J.; Timm, I.J. Measuring and Comparing Scalability of Agent-Based Simulation Frameworks. In German Conference on Multiagent System Technologies; Springer: Cham, Switzerland, 2015; pp. 42-60.

(C) 2017 by the authors. Licensee MDPI, Basel, Switzerland. This article is an open access article distributed under the terms and conditions of the Creative Commons Attribution (CC BY) license (http://creativecommons.org/licenses/by/4.0/). 
Article

\title{
A Hardware-Supported Algorithm for Self-Managed and Choreographed Task Execution in Sensor Networks
}

\author{
Borja Bordel $^{1, *(D)}$, Carlos Miguel ${ }^{1}$ (D), Ramón Alcarria ${ }^{2}$ (iD) and Tomás Robles ${ }^{1}$ (iD \\ 1 Departamento de Ingeniería de Sistemas Telemáticos, Universidad Politécnica de Madrid, \\ Avenida Complutense No. 30, 28040 Madrid, Spain; carlos.miguel@upm.es (C.M.); \\ tomas.robles@upm.es (T.R.) \\ 2 Departamento de Ingeniería Topográfica y Cartografía, Universidad Politécnica de Madrid, \\ UPM Campus Sur, Km 7.5 de la Autovía de Valencia, 28031 Madrid, Spain; ramon.alcarria@upm.es \\ * Correspondence: bbordel@dit.upm.es; Tel.: +91-549-57-00 (ext. 3035)
}

Received: 4 February 2018; Accepted: 5 March 2018; Published: 7 March 2018

\begin{abstract}
Nowadays, sensor networks are composed of a great number of tiny resource-constraint nodes, whose management is increasingly more complex. In fact, although collaborative or choreographic task execution schemes are which fit in the most perfect way with the nature of sensor networks, they are rarely implemented because of the high resource consumption of these algorithms (especially if networks include many resource-constrained devices). On the contrary, hierarchical networks are usually designed, in whose cusp it is included a heavy orchestrator with a remarkable processing power, being able to implement any necessary management solution. However, although this orchestration approach solves most practical management problems of sensor networks, a great amount of the operation time is wasted while nodes request the orchestrator to address a conflict and they obtain the required instructions to operate. Therefore, in this paper it is proposed a new mechanism for self-managed and choreographed task execution in sensor networks. The proposed solution considers only a lightweight gateway instead of traditional heavy orchestrators and a hardware-supported algorithm, which consume a negligible amount of resources in sensor nodes. The gateway avoids the congestion of the entire sensor network and the hardware-supported algorithm enables a choreographed task execution scheme, so no particular node is overloaded. The performance of the proposed solution is evaluated through numerical and electronic ModelSim-based simulations.
\end{abstract}

Keywords: Wireless Sensor Networks; task execution; algorithms; resource management; ModelSim

\section{Introduction}

Wireless Sensor Networks (WSN) have evolved from a theoretical and research paradigm to be a real and practical technology. Actually, several new engineered systems such as Cyber-Physical Systems [1], Smart cities [2] or Industry 4.0 [3] (among other possibilities) are based on WSN. Currently, besides, WSN have been generalized [4], so they are now understood as sets of wireless smart nodes (including each one, sensors, actuators and processing devices) networked via radio links. With this new approach, WSN can not only provide information (which must be compressed and transmitted to user applications) as traditionally but also execute and get the result of tasks which are delegated by high-level applications.

First real WSN were composed of a small and homogeneous collection of nodes with a medium size [5]. Nodes in these first deployments had also a mid-range computing power, with enough resources to execute both data and control processes. However, over time, nodes became smarter 
(they implement more complex algorithms), smaller (thanks to microelectronic technology) and more resource-constraint (they are dedicated devices and have a lower cost) [6]. At the same time, the number of nodes in a WSN has gone up strongly and quickly; so WSN evolved to current pervasive sensing platforms composed of a huge number of tiny, heterogeneous, resource-constraint smart nodes [7].

In this context, collaborative or choreographed task execution schemes are which fit in the most perfect way with the nature of sensor networks, as they enable the creation of flexible ad hoc resource pools being able to execute delegated tasks [8]. The flexible and dynamic combination of nodes with different capabilities is the perfect approach to make the most of the resources of a sensor network, as resources are packed and assigned to tasks at real-time depending on the current node situation and workload. Nevertheless, although these solutions were investigated in first WSN, current software solutions for choreographed task execution and resource self-management by the smart nodes in a WSN are pretty inefficient: they are not adapted to resource constraint devices and resource consumption of these algorithms grows strongly with the number of nodes in the network.

On the contrary (see Figure 1), almost every current WSN is based on a hierarchical network design, where resource constraint nodes are grouped in clusters which are managed by more powerful computing devices usually named as "cluster heads" [9]. These cluster heads are, finally, controlled by a central orchestrator, placed in the cusp of the network hierarchy. Both, cluster heads and orchestrator present enough computing power to support any required management algorithm or solution. However, although this approach solves many management problems of large WSN with resource constraint devices, it is also very inefficient, whereas a relevant percentage of the operation time is wasted in communicating nodes with the cluster heads and the orchestrator [10]. In fact, if a conflict occurs, nodes must request cluster heads and orchestrator for a solution and wait for instructions before continuing with the operation. Next-generation WSN, applied to Industry 4.0 for example, must operate at real-time, so new and more efficient management solutions are required.

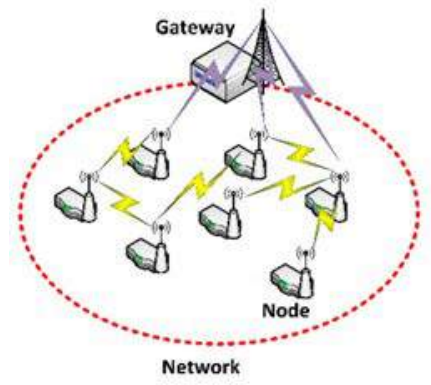

(a)

Choreographed or collaborative WSN

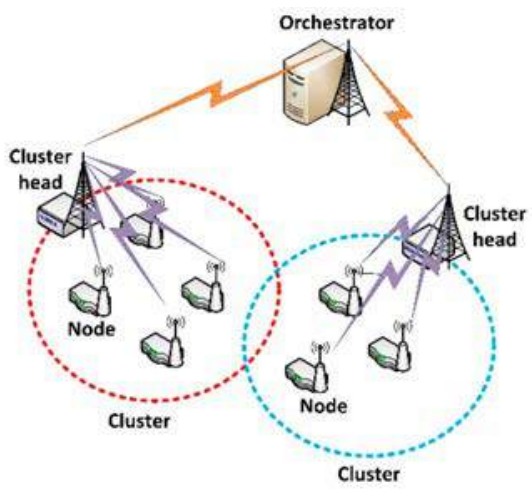

(b)

Orchestrated or

Figure 1. (a) Choreographed Wireless Sensor Network (WSN) (b) Hierarchical WSN.

Therefore, in this paper it is proposed a new solution for self-managed and choreographed task execution in sensor networks, which is adapted to resource-constraint devices and may be implemented in large WSN. This proposal is based on a horizontal network scheme where they are not required cluster heads or heavy orchestrators. Besides, the workload that sensor networks support is improved, as almost no time is wasted in communicating nodes with other components during control transactions. Thus, efficiency is also improved. These objectives are reached thanks to choreography and self-management algorithms that are implemented using hardware techniques and bit-oriented communication protocols. The use of processing resources is, in conclusion, negligible. On the other hand, to avoid the congestion of the WSN (functions that orchestrators support nowadays) our proposal is complemented with a lightweight gateway performing simple traffic engineering 
operations, which do not require bidirectional communication with nodes (so global efficiency is not affected).

The rest of the paper is organized as follows: Section 2 introduces the state of the art in management and choreography solutions for WSN in tasks execution systems. Section 3 describes the study scenario and the proposed solution including all important details. Section 4 provides an experimental validation of the contribution. Finally, Sections 5 and 6 explain some results of this experimental validation and the conclusions of our work.

\section{State of the Art}

Task execution systems are, since the beginning, one of the most important applications for WSN [11]. In these scenarios, one of the key problems is the selection of the most adequate node to execute a certain task, delegated by high-level applications. If the node, or nodes, to execute a task are selected by components in a hierarchical level superior to the nodes (such as cluster heads) it is said the network implements an orchestration management solution (see Figure 1b). On the other hand, if the nodes to execute a task are selected by means of an agreement among all nodes in the WSN (see Figure 1a) it is said the network implements a self-management solution or a choreography management solution (sometimes also named as collaborative management).

Most extended proposals about management in WSN are based on orchestration schemes [12]. In these solutions, information about node hardware capabilities is sent to the orchestrator and/or any component in charge of the network management. Then, when a task is delegated by high-level applications, a matchmaking process is performed by those components in order to find the nodes which provide the adequate functions (nodes) to execute the delegated task [13].

In general, works on orchestration schemes define a set of "node adaptors" which homogenize the control interface of nodes in order to communicate them with a middleware supported by the orchestrator [14]. This middleware is usually service-oriented and may consider artificial intelligent algorithms [14]. Some proposals include a virtual representation of the WSN in this middleware [15], so calculations and operation are performed on this virtual instance before being mapped on the real network. It is also possible to find authors describing the orchestration middleware as in mobile networks (considering a global register, a monitoring module, etc.) [16]. Finally, agent-based management architectures have been also reported. Different agent locations and manager (orchestrator) configurations were investigated [17].

As orchestration schemes need an initial configuration process (to send the information about the WSN to the manager), dynamic changes in WSN are one of the main problems in these technologies [18]. As the repetition of the initial configuration process may stop the operations in the WSN, a technique named as "context-aware management" was proposed [19]. In this technique, the orchestration middleware collects and maintains information about the entire environment and situation of nodes in the WSN. Solutions based on semantics [20] and/or pattern recognition [21] are employed to take management decision with the available information.

Any case, all previously described solutions present a common problem: they imply complex processing operations (such as analysing semantic objects, starting virtual instances, updating a huge community of agents, etc.) which make impossible to operate at real-time, except in small deployments. Thus, although managers and orchestrators have the required computational power to perform these operations, the efficiency in control transactions is very low.

In these hierarchical networks, besides, complex communication protocols and transactions are deployed and defined, as nodes in WSN must send a relevant amount of information to orchestrators. Up to ten different messages [20] may have to be exchanged by each node with the orchestrator before executing a management operation (running a delegated task, for example); and this number may grow if cluster heads or any other medium-level component is also considered [22]. These protocols and transactions, furthermore, force the nodes to maintain large state descriptions and to handle heavy data formats such as JSON (JavaScript Object Notation) [22]. 
In conclusion, an important part of the processing resources in nodes (which are resource constraint) is finally consumed by management operations, even when considering central orchestrators. This is especially critical when considering the processing time, as control transactions require large communication processes when various heavy messages must be created and interpreted.

These problems [23], nevertheless, may be solved if collaborative, choreographed or self-management solutions are implemented. In fact, although they are sparse, some works have investigated these techniques. Task allocation using choreographed technologies is usually understood as an optimization problem. The basic idea is to select the set of nodes which may execute a certain task or workflow consuming the minimum amount of resources. Various works have instigated the complexity of the underlying mathematical problem [24,25], proving that traditional task allocation techniques cannot be directly implemented in choreographed WSN due to the employment of resource-constraint devices [26].

Partial solutions to this problem have been reported optimizing the energy [27,28] or time [29] consumption for a given set of tasks. In order to solve the global multi-objective optimization, heuristic solutions [30] and stochastic heuristic methods have been investigated [31]. In particular different variations of the well-known Particle Swarm Optimization (PSO) algorithm may be found [32,33]. Other bionic intelligence methods have been also proposed to allocate tasks to be executed in an optimum way [34].

These techniques, however, are only valid if networks are static and the sequence of tasks to be executed is known. If real-time changes have to be considered, or the tasks to be executed are aleatory, either the optimization problem cannot be solved (furthermore, it has no sense), or the obtained solution is only a first approximation which could be obtained using much lighter processes.

In a more relaxed way, other proposals try to schedule the task execution, so most adequate nodes are in charge of it but no optimization or fitness function is defined. Geographical information or real-time operations [11] can be added as a novelty in this last group of solutions.

The main problem of all described choreographed techniques, nevertheless, is the same. The complexity and resource consumption of these algorithms grow with the number of nodes in the network [33]. Some of them, even, present an exponential evolution. Any case, software implementations of the described technologies have been reported to work only in WSN with a few tens of nodes. In current pervasive sensing platform, then, they cannot be employed, so orchestration techniques are usually preferred.

The proposed solution in this paper includes a hardware-supported algorithm which has negligible resource consumption and can be executed at real-time in large WSN, as its complexity does not depend on the number of considered nodes. No control transactions or communication processes are necessary to manage the infrastructure. The proposed choreography solution, besides, enables the self-management of nodes, so no network orchestrator has to be included and only a lightweight gateway must be considered.

\section{Self-Managed and Choreographed Task Execution in Sensor Networks}

This section describes the main contribution of this paper. First subsection presents the study scenario and the characteristics of the considered WSN. Second subsection presents a global overview of the proposed technique and the data format employed in task execution orders. The third subsection explains the behaviour of the proposed network gateway. And, finally, fourth subsection describes the proposed hardware-supported algorithm to be implemented in sensor nodes.

\subsection{Study Scenario}

Our scenario is designed for all types of environments where smart sensor nodes are deployed to perform cyber-physical operations, i.e., operations where either some information (such as temperature, humidity, presence, etc.) is gathered from the environment, or some effects (e.g., turning on the lights) are applied to the physical world. The node density is very high, because new deployments tend 
to be pervasive and future paradigms such as pervasive sensing platforms or smart dust present this characteristic.

Besides, hardware capabilities are redundant; so many different nodes may perform any operation within the WSN. In this initial study, we do not consider operations involving the transmission of images, video streams, sounds or other information requiring large and/or variable amount of data. Each operation consists of two messages: a bit-oriented execution order with a fixed structure and length; and a bit-oriented response including the result of the operation also with a fixed structure and length (see Section 3.2). We are also assuming the WSN performs a constant monitoring of the environment and may execute operations at any moment. However, real-time processing is not mandatory, if the application scenario does not require this characteristic (as in some agricultural systems [35]). Thus, ad hoc sensor networks and mobile nodes may be also considered. Even, other solutions, such as including the sensors in mobile nodes could be also considered if necessary.

The proposed technology belongs to the transport level in the OSI reference model [36]. As in standard Internet transactions, operation messages in WSN enable the communication of remote applications (i.e., the user application which invokes the operation and the low-level program in the smart node which finally executes it); regardless the underlying infrastructure. As in other transport level protocols, the proposed technology allows identifying the application to be invoked, considering reliable communications and generating complex transactions.

As a consequence, in our scenario, all nodes must implement a network protocol proving addressing to the entire network. No special characteristics are required from this protocol, except a broadcast and/or anycast solution [37] must be supported. If needed, a Delay-Tolerant Protocol [38] could be also included (see Figure 2a).

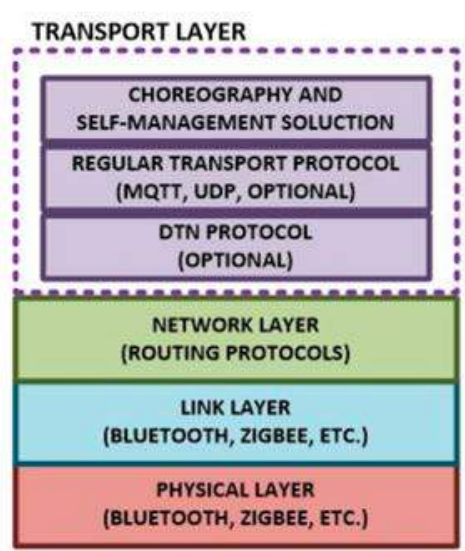

(a)

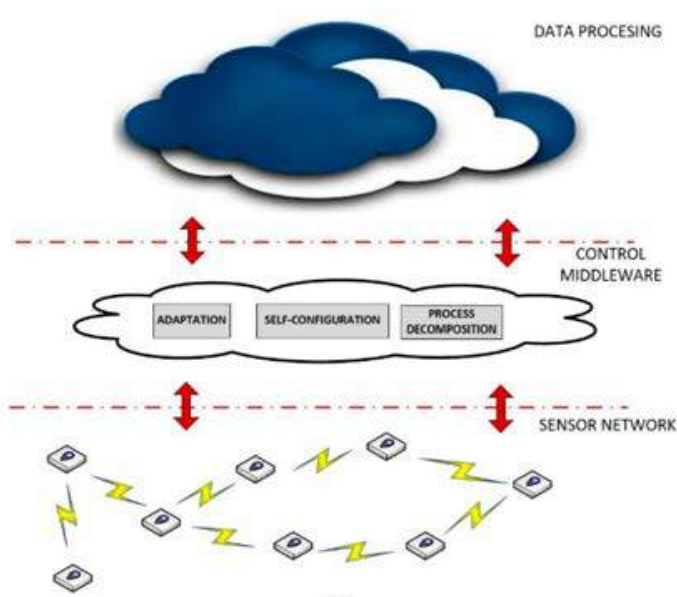

(b)

Figure 2. (a) Protocol stack in the proposed scenario (b) Scenario's logical structure: layers.

A complete list of all admissible or preferred characteristics should be based on a deep analysis and the definition and characterization of all possible application scenarios [18] but in our case, we are going to work over some common characteristics for all cases, which are:

1. Lack of infrastructure for supporting power supply or access to unlimited data storage (such as data bases).

2. Usage of specific protocols for stablishing WSN's topology in an autonomous way and for routing data from the user applications to the smart nodes which must perform the cyber-physical operations

3. Nodes may be mobile and stablish ad hoc connections. DTN technologies for communication among nodes and other components could be included.

4. The node density is very high and hardware capabilities are very redundant. 
5. The entire WSN is deployed in a unique geographical location. Thus, cyber-physical operations considering geographic information can be executed in any available node.

The first point limits the kind of technologies we can use and more specifically, the lack of power supply limits the service time of the network, which is a key element for the usability of such kind of solutions in real environments.

The second and third points explain how smart nodes create an organized network. On the one hand, as the proposed technology belong to transport level, network level solutions must be previously considered. On the other hand, as nodes may be mobile and not be available all time, Delay-Tolerant Network (DTN) technologies to allow reliable and efficient communication could be implemented.

Finally, the fourth and fifth points are directly generated by the requirements imposed by most recent and future scenarios. Industry 4.0 systems or pervasive sensing platforms, in fact, are based on very dense networks, deployed in a unique geographical location and with a very high reliability (so several redundant nodes are included).

Three layers are identified in our application scenarios: sensor network, control middleware and data processing and user applications (Figure $2 b$ ).

Sensor network refers to a set of sensorization nodes, including transducers and processing components, being able to execute cyber-physical operations (and, in consequence, tasks, see Section 3.2). In general, sensors may be distributed in a random way (if node installation is possible in all points within the deployment area) or in a planned way (if some points are preferred). Therefore, we cannot guarantee that we know every position of the fixed nodes. In this work, we are assuming we know neither the sensors' position nor network topology or the number of nodes. Moreover, in relation to the node characteristics, the proposed solution allows employing any type of device. That means we do not have to be worried about values such as the sensing range, the sensing period or the monitored variable; because our proposal is independent from sensors' features and only considers generic parameters, such as the energy consumption. In that way, these assumptions make the proposal very general and flexible.

Control middleware includes all the components employed to communicate and adapt the low-level architecture of WSN and the high-level design of user applications. Process decomposition mechanisms, self-configuration instruments and many other similar solutions are included in this layer.

Data processing layer and user applications refer to all final high-level components which receive data from WSN and control middleware, process them and (eventually) store and/or present them. This layer can be based on any standard technology like centralized services or cloud services, because in our scenarios real time operations are not mandatory if the application scenario does not require them.

\subsection{Global Overview. Protocol for Task Execution}

The proposed technology is composed of two different algorithms. The first one is implemented in the control middleware, where a lightweight gateway is included in order to avoid the congestion of the WSN (guarantying the stability of the choreography solution). The second one is implemented in all nodes of the WSN, so they can self-manage their resources and the execution of the delegated tasks.

In this context, a task is understood as the sequence of various chained cyber-physical operations (CPOs), for which it must be obtained a unique and global result by the WSN. In a more formal way, a task may be represented as a workflow of cyber-physical operations. These workflows can be described using any available description language (BPEL, BPMN, etc.), although in this article we are employing YAWL [39] because of their capability to represent almost every possible situation.

Delegated tasks can be received in the control middleware already described as workflows of cyber-physical operations or may be transformed in that middleware. Any case, this transformation process is not the objective this paper, so we are assuming we already have that workflow-like description (see Figure 3). 


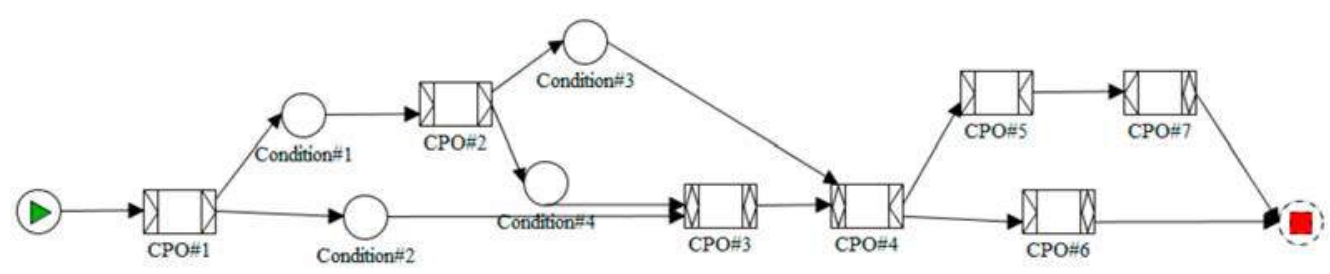

Figure 3. Task as a workflow of cyber-physical operations (CPO).

According to the Erlang's queue theory, a lightweight gateway in the control middleware evaluates if the maximum allowed load in the sensor networks has been reached (i.e., if the network is congested). If positive, the task execution is rejected. However, in order to determine the maximum allowed load in the WSN, a statistical procedure is employed, so some rejected tasks are finally executed in order to determine if the network is in fact congested or the maximum allowed load can be increased.

Accepted tasks are transformed in a set of execution orders (one per cyber-physical operation in the task workflow), which are represented using a bit-oriented format. These orders also contain information about how they are related among them in order to guarantee that inputs and outputs of the different operations are correctly linked. This characteristic enables the use of hardware-supported technologies in nodes.

Once constructed, these orders are broadcasted in the WSN. Thus, the smart nodes in the WSN include a hardware module which, once received an execution order, decides if the node can or cannot execute this operation. In this decision, the proposed algorithm considers the available resources, the interest and importance of the operation to be executed and the predicted resource consumption made when performing the operation. These resource self-management functionalities are complemented with choreography solutions, so that nodes can decide in an autonomous way which device finally executes the operation (using pseudorandom number generators); and can also compose the final result of a delegated task from the partial results of the performed cyber-physical operations (using the identifiers and the cross-references included in the execution orders).

In this proposal, these solutions are supported using only hardware technologies, so embedded software in nodes must not be modified to include coordination algorithms and critical resources (such as the operation time) are not wasted in control operations.

Figure 4 represents the global overview of the proposed technology.

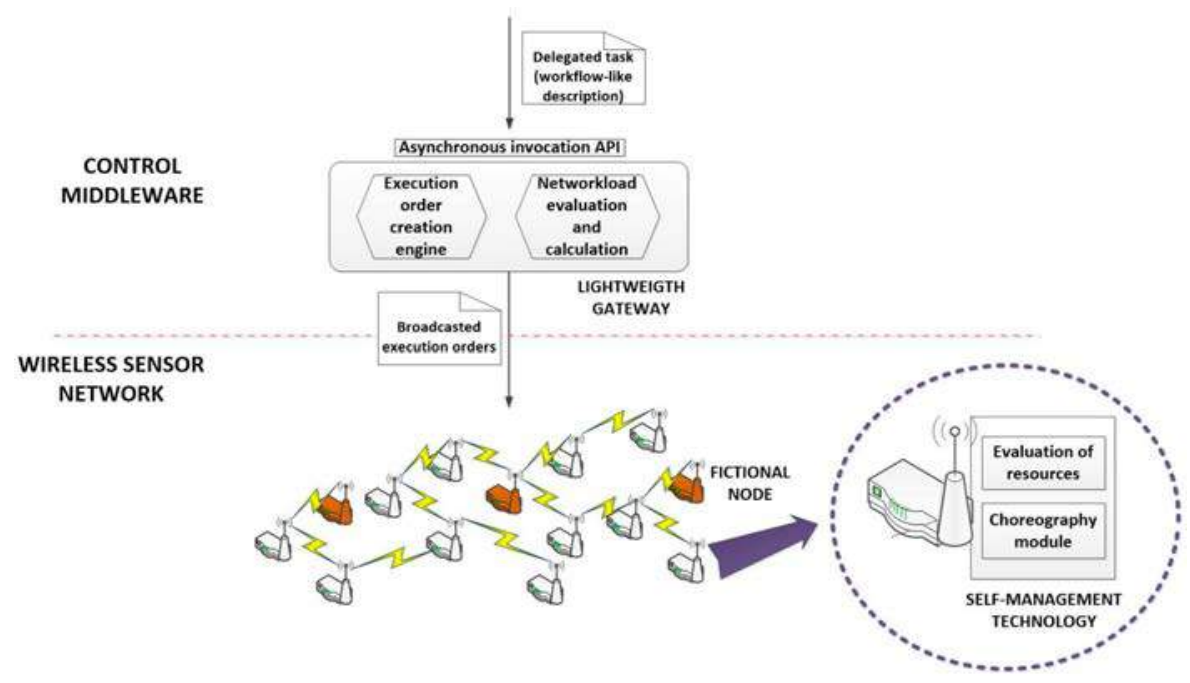

Figure 4. Global scheme of the proposed technology. 
Bit-oriented execution orders can be easily generated by the proposed lightweight gateway, or this functionality may be deployed in a second and new component. In this first work, we are assuming the gateway is in charge of this function (see Figure 4).

The proposed format for the execution orders may be seen in Figure 5. This message is needed to require the sensor network to execute a $\mathrm{CPO}$ or, at least, to evaluate the possibilities of carrying out the execution. The proposed format is byte-aligned. Eight different fields are considered in each execution order. Namely:

\section{Execution order format}

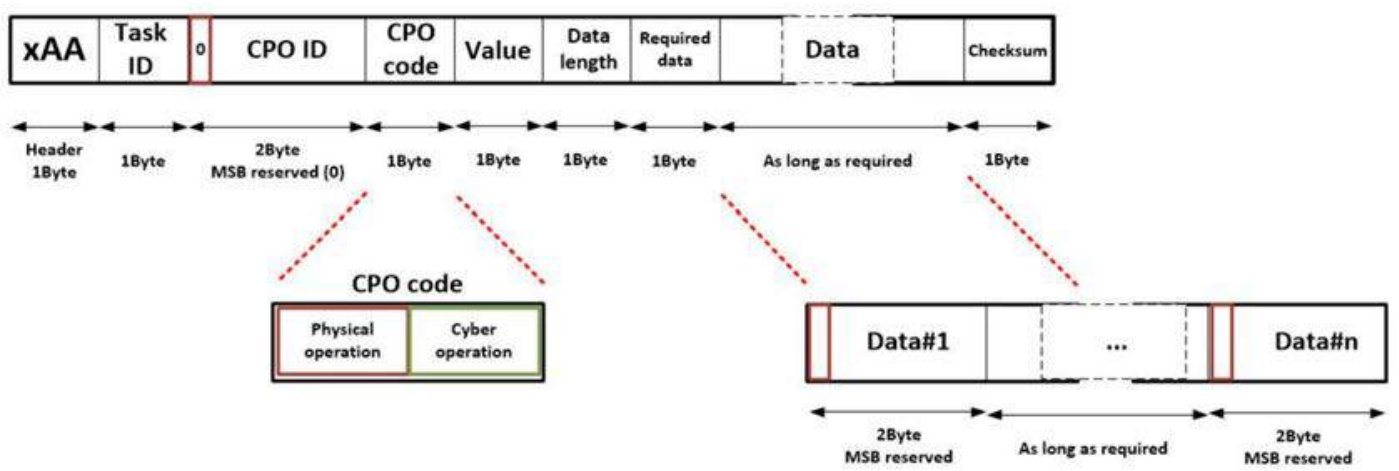

Figure 5. Bit-oriented execution order format.

- Header (1 byte). The first byte is a header, which must be completed with the hexadecimal value $x A A$, to indicate an execution order is described by this command.

- Task ID (1 byte). This field identifies the delegated task to which the CPO belongs. As maximum, 255 tasks can be executed at the same time.

- CPO ID (2 bytes). This field identifies each concurrent CPO univocally. Although 2 bytes are intended for this purpose, only fifteen bits are employed to represent the CPO identifier. The Most Significant Bit (MSB) is reserved and initialized with a binary zero. This bit will be used to link inputs and outputs of $\mathrm{CPO}$ in an easier way (see Section 3.4).

- CPO code (1 byte). This field indicated the CPO to be performed. Two hundred fifty-five (255) different operations may be considered in the WSN. In order to simplify the decision process in smart nodes about if they are able to execute an operation, the CPO is divided into two fields. The four most significant bits represent the physical operation to be performed (i.e., the transducers involved in the execution: temperature sensors, LEDs, etc.). If a node does not include that hardware no more processing is required to reject the execution. The four Least Significant Bits represent the cyber operation to be performed (i.e., obtaining an average, sending the raw collected data, etc.).

- Value (1 bytes). This field represents the value the operation has for the system. It is obtained as a composition of the importance of the delegated task for the system and the relevance of the operation within the delegated task (see Section 3.3).

- Data length (1 byte). This field indicates how many input data are required (as maximum) to execute the CPO. These data are provided in the adequate field. Each data needs 2 bytes, so the length of the data field may be calculated multiplying by two the value represented here.

- Required data (1 byte). This field indicates how many input data are required and essential to execute the CPO. This field is employed to represent the join-type of the CPO [40]. Three different join-types are possible in YAWL: AND (if an operation is not triggered until all previous operations have finished, so required data is equal to data length); XOR (if an operation is triggered once the first previous operation has finished, so required data is equal to the unit); and OR (if and operation is triggered once a certain number of previous operations -to be selected by the user- 
have finished, so required data includes a personalized value). Once all required data are available, the $\mathrm{CPO}$ is executed.

- Data (between zero and 512 bytes, as needed). In this field, all input data of the CPO are represented. Each data requires 2 bytes: the MSB is reserved (it is a flag) and the next 15 bits includes the value of the datum. If the datum is a constant, the MSB is initialized with a binary one and the next 15 bits include the value of the constant. However, on the other hand, if the datum is obtained as the output of a previous CPO, the MSB is initialized with a binary zero and the next 15 bits include the CPO ID of the operation which will produce the datum. The order in which data are listed must be fixed and established for each operation.

- Checksum (1 byte). The checksum is obtained as the XOR of all bytes in the execution order except the header. If an order is corrupted, the checksum will not be validated and the order will be discarded.

Once a CPO is performed, the corresponding node generates a message including the results of the operation, in order to allow the other nodes to execute the linked operations. This message is needed to update the state of the choreographed task execution. The format of this message is showed on Figure 6. As can be seen, fields in this new message are similar to which previously presented. In particular, six fields are considered:

Result message format

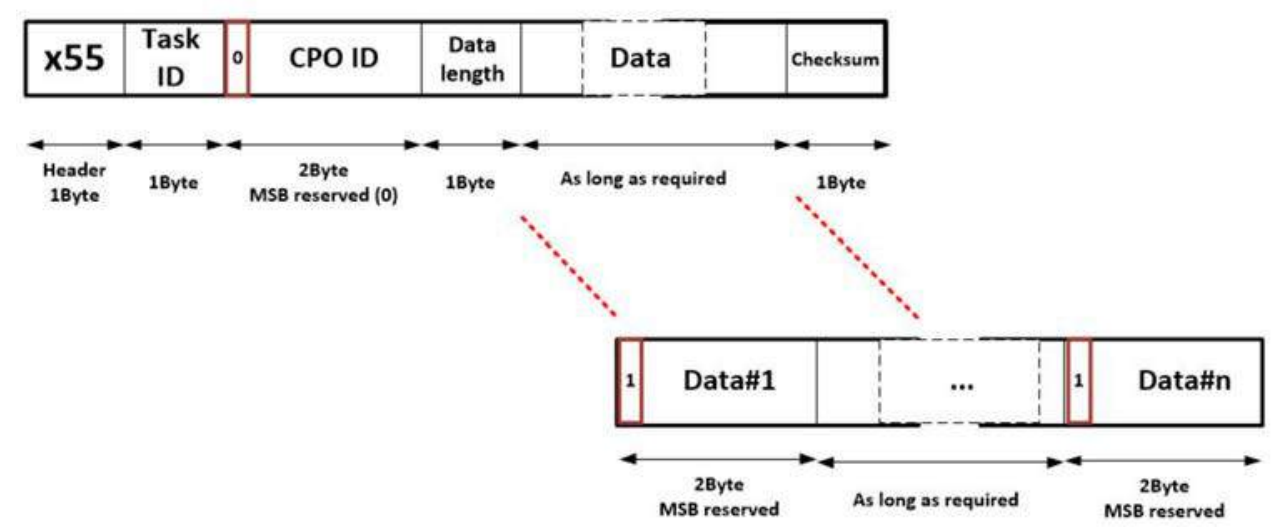

Figure 6. Bit-oriented result message format.

- Header ( 1 byte). The proposed header for result messages is $x 55$

- Task ID (1 byte). This identifier has the same meaning as explained above.

- $\quad$ CPO ID (1 byte). This identifier has the same meaning as explained above.

- Data length (1 byte). This field indicates how many output data are generated as result of the $\mathrm{CPO}$. These data are provided in the adequate field. Each data needs 2 bytes, so the length of the data field may be calculated multiplying by two the value represented here.

- Data (between zero and 512 bytes, as needed). In this field, all output data of the CPO are represented. Each data requires 2 bytes: the MSB is reserved (and initialized with a binary one) and the next 15 bits includes the particular value of the result. The order in which results are listed must be fixed and stablished for each operation.

- Checksum (1 byte). The checksum is obtained as the XOR of all bytes in the result message except the header.

As can be seen in Figure 3, sometimes workflows may include conditions, in order to select which branch (or branches) in the workflow is executed. These structures can appear depending on the split-type of operations. In YAWL, three different split-types are defined: AND, OR and XOR (with the same meaning that the join-types). Tasks of OR or XOR split-types must include conditions [40]. 
Conditions must be also executed, so they are treated as any other CPO (they have a CPO ID, they present cross-references with other operation in the data field, etc.). However, as the code to execute these conditions is not available in the nodes of the WSN (as conditions are defined at high-level by users considering the application scenarios and not the underlying network), they are not executed by hardware nodes. Execution orders of conditions are not neither broadcasted and all of them are resolved by fictional nodes (see Figure 4). Fictional nodes are virtual instances hosted in the control middleware but they behave and relate with other nodes as any other standard hardware component. In these nodes, the lightweight gateway may deploy dynamically the required code to execute the conditions in the workflow.

Some conditions will be verified, so a standard result message will be generated. However, other conditions could not be verified and nodes in charge of executing the operations in the cancelled branch (or branches) should deallocate the corresponding resources and be available for new operations. In order to enable that, a new message, the aborted operation message, was included in the proposed task execution protocol (see Figure 7). This message is much shorter and fields have the same meaning as previously described commands.

\section{Aborted operation message format}

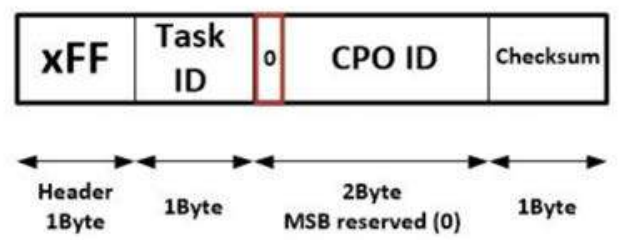

Figure 7. Bit-oriented aborted operation message format.

Finally, although at this point the necessity of including this message may not be seen clearly, it a message is needed to enable nodes to claim the right of executing a CPO ordered by the gateway. This last message is named "claim message" and its structure is very similar to the aborted message format, although the header, in this case, is initiated with the value xCC (see Figure 8). This message is totally necessary, as it is employed to ensure that (in a choreographed execution algorithm) only one node is in charge of executing each CPO. Among nodes willing to execute a CPO, a random mechanism is employed to select the final node responsible of the execution. This node must claim that execution, so the other nodes can renounce it.

\section{Claim message format}

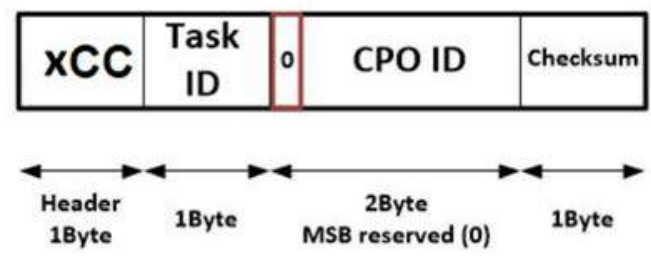

Figure 8. Claim message format.

Considering the proposed messages, smart nodes in the WSN may execute tasks in a choreographic way, as described in Section 3.4.

\subsection{Network Gateway}

The main problem of choreography solutions is that no node in the sensor network has a global understanding of the ongoing situation. In that way, networks implementing these technologies 
may get congested easily, as no node can reject the execution of a task or operation on behalf of the entire network. Timers and other similar solutions must be considered in this case in order to inform high-level applications that a task cannot be executed. However, those techniques do not enable the premature detection of congestion situations, which makes them very inefficient.

Therefore, in the proposed solution, a lightweight gateway (see Figure 4) has been included. This gateway does not need to maintain a real-time representation of the network state and only using statistical procedures can detect and prevent the congestion of the network.

A Wireless Sensor Network $\mathcal{W}$ may be seen as the union of a family of sets of servers (1). Each set of servers $\mathcal{S}_{i}$ groups the nodes being able to perform a certain CPO. As, usually, a node may execute various different operations, it may be present in several server sets.

$$
\mathcal{W}=\bigcup_{i=1}^{m} \mathcal{S}_{i}
$$

The cardinality of each set of servers (2) is usually unknown, as configuration processes are not able to collect that information in most cases. The total number of nodes in the WSN is $N_{\text {total }}$.

$$
N_{i}=\operatorname{card}\left\{\mathcal{S}_{i}\right\}
$$

In this context, delegated tasks are received by the WSN in a random manner. Nevertheless, it is reasonable to imagine that the probability distribution of task delegations (and execution of CPO) in time in WSN $f_{d}[k]$ is similar to the probability distribution of calls in time in telephony systems (3). In particular, delegations are independent among them and they are equiprobable in time; so a Poisson distribution is the most adequate.

$$
f_{d}[k]=\frac{\left(\lambda_{i} T\right)^{k}}{k !} e^{-\lambda_{i} T}
$$

Then, for each server group $\mathcal{S}_{i}$, the probability of receiving $k$ task delegations in a time period $T$ is represented by a Poisson distribution with medium $\lambda_{i} T$; where $\lambda_{i}$ is the average rate of received delegations (or ordered CPO, depending on the point of view, see Figure 9) by the group of servers $\mathcal{S}_{i}$ during the time period $T$.

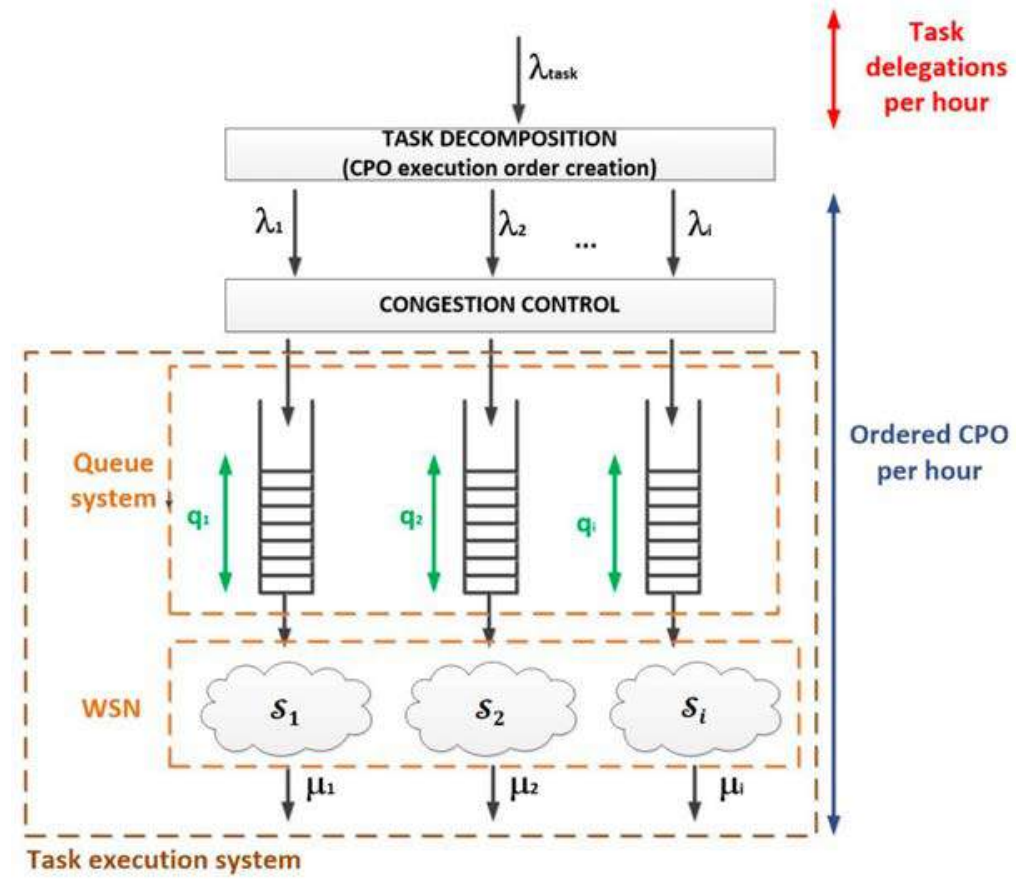

Figure 9. Queue system in the proposed solution. 
Experimentally, it is proved that the service time follows an exponential probability distribution in telephony systems. Although no similar results have been obtained for WSN, in this work we are assuming the service time in WSN follows an exponential law as well (4). Hereinafter, $\frac{1}{\mu_{i}}$ is the medium service time for the group of servers $\mathcal{S}_{i}$ (i.e., the required time to execute a certain CPO operation by a server within $\mathcal{S}_{i}$ ).

$$
f_{s t}(t)=\mu_{i} e^{-\mu_{i} t}
$$

As, in some occasions, bursts of task delegations may appear, for each group of servers it is considered a finite queue $Q_{i}$ to absorb the effect of these bursts. Each queue $Q_{i}$ has a maximum capacity of $q_{i}$ CPO execution orders. The resulting system is a hybrid queue system whose Quality-of-Service (QoS) is determined by both the waiting time in the queue and the loss probability due to the limited capacity of the queues.

Although this model considers to be defined some assumptions that are met in most cases, the traffic engineering theory guarantees that the obtained results are a good first estimation for all cases, including those which do not fulfil the initial assumptions (for example, if task delegations are periodic). General models as the proposed one by Kingman [41] could be applied for a more precise calculation.

In this scenario, it is possible to calculate the volume of CPOs $A_{i}$ supported by each group of servers $\mathcal{S}_{i}$ using the Erlang's theory (5). It is also possible to calculate the congestion factor (or utilization factor) $\rho_{i}$ which represents (if $\rho_{i}>1$ ) a permanent and structural situation of congestion in the WSN (6)

$$
\begin{gathered}
A_{i}=\frac{\lambda_{i}}{\mu_{i}} \\
\rho_{i}=\frac{A_{i}}{N_{i}}=\frac{\lambda_{i}}{N_{i} \mu_{i}}
\end{gathered}
$$

With this approach the situation of the WSN, regardless how great is the node density or the number of nodes, may be represented using only five one-dimensional arrays (with 256 values each one, one value per $\mathrm{CPO}$ ). These arrays represent the medium task delegation rate, the inverse of the medium service time (or performed-served-operation rate), the number of servers and the congestion factor (and/or the traffic volume) in the WSN (7).

$$
\begin{array}{ll}
\vec{N}=\left\{N_{i},\right. & i=0, \ldots, 255\} \\
\vec{A}=\left\{A_{i},\right. & i=0, \ldots, 255\} \\
\vec{\lambda}=\left\{\lambda_{i},\right. & i=0, \ldots, 255\} \\
\vec{\mu}=\left\{\mu_{i},\right. & i=0, \ldots, 255\} \\
\vec{\rho}=\left\{\rho_{i},\right. & i=0, \ldots, 255\}
\end{array}
$$

As in standard telephony systems, in this case we are assuming the number of users that try to use the resources of the WSN is very high. However, users have a variable behaviour in time, i.e., the situation of the WSN and the values of the mentioned arrays (7), will vary in time (although this dependency is not explicitly expressed). Because of this variability, in order to dimension systems, key indicators are evaluated during the most loaded hour (such hour when the medium task delegation rate is highest). An important topic in every WSN for task execution, then, will be to guarantee that, at no time, the hourly ordered CPO rate is above the calculated rate for the most loaded hour $\overrightarrow{\lambda_{\max }}$. The use of these "maximum" values as comparison threshold it will be very important to maintain the network in a stable situation as we are seeing.

Two relevant considerations have to be done at this moment. First, the module of vectors $\vec{A}, \vec{\rho}$, etc. may be used as a first evaluation of the situation of the network as a whole. However, the obtained result it is not precise from the traffic engineering point of view. And, second, among the presented 
arrays (7) only two of them are not modifiable as they depend on the deployed hardware devices: the number of servers $\vec{N}$ and the inverse of the service time $\vec{\mu}$. The other three parameters are dependent on each other, there is only one degree of freedom, i.e., only one parameter can be employed as independent variable. In this work, we employ $\vec{\rho}$ as such an independent variable, which is the basis of the congestion control algorithm to be deployed in the lightweight gateway (see Algorithm 1).

According to the Erlang's theory, the loss probability any of the previously described queue systems, for each group of servers, is (8). In this work, we are assuming the system must present a certain QoS level, represented by the availability of the system (expressed as probability) for each group of servers, $p_{a v}^{i}$. Then, the maximum allowed loss probability in the system $p_{\text {loss }}^{i}$ is (9). As in the previous case, one-dimensional arrays of 256 elements may be employed to represent the loss probability and availability of the entire network $\overrightarrow{p_{l o s s}}, \overrightarrow{p_{a v}}$.

$$
\begin{gathered}
p_{\text {loss }}^{i}=\rho^{q_{i}+1} \frac{1-\rho}{1-\rho^{q_{i}+2}} \\
p_{\text {loss }}^{i}=1-p_{a v}^{i}
\end{gathered}
$$

With these equations, it is possible to determine the maximum utilization factor and maximum hourly task delegation rate which can support the WSN, to guarantee the desired QoS. There is no analytic solution but it is pretty easy to obtain a numerical one (see Figure 10).

Using a statistical procedure and a sliding window filter (we have named the "Erlang filter") only task delegations compatible with the desired QoS will be accepted in the system (see Algorithm 1).

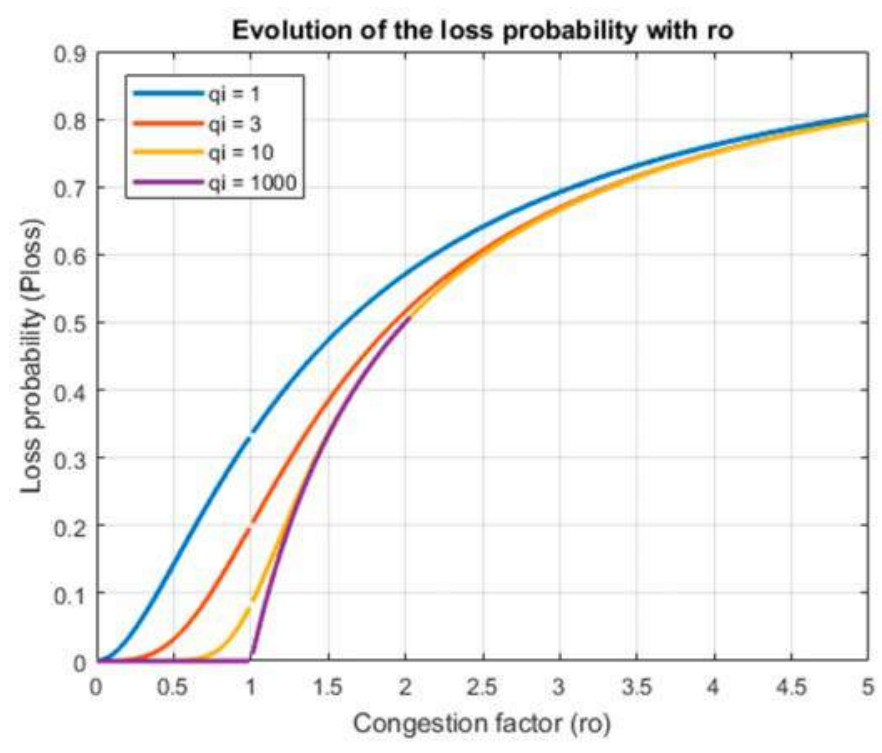

Figure 10. Evolution of the loss probability with the congestion factor.

As can be seen (Algorithm 1), the first activity to be performed when a task delegation is received is evaluating the number of $\mathrm{CPO}$ of each type which will be executed if the task is accepted. This step is very important, as tasks are delegated as a unique element but they are decomposed into CPO in the WSN. Congestion factors and the other traffic engineering parameters are, then, expressed terms of the ordered CPOs, so it is basic to know how many $\mathrm{CPO}$ will be executed per delegated task. 


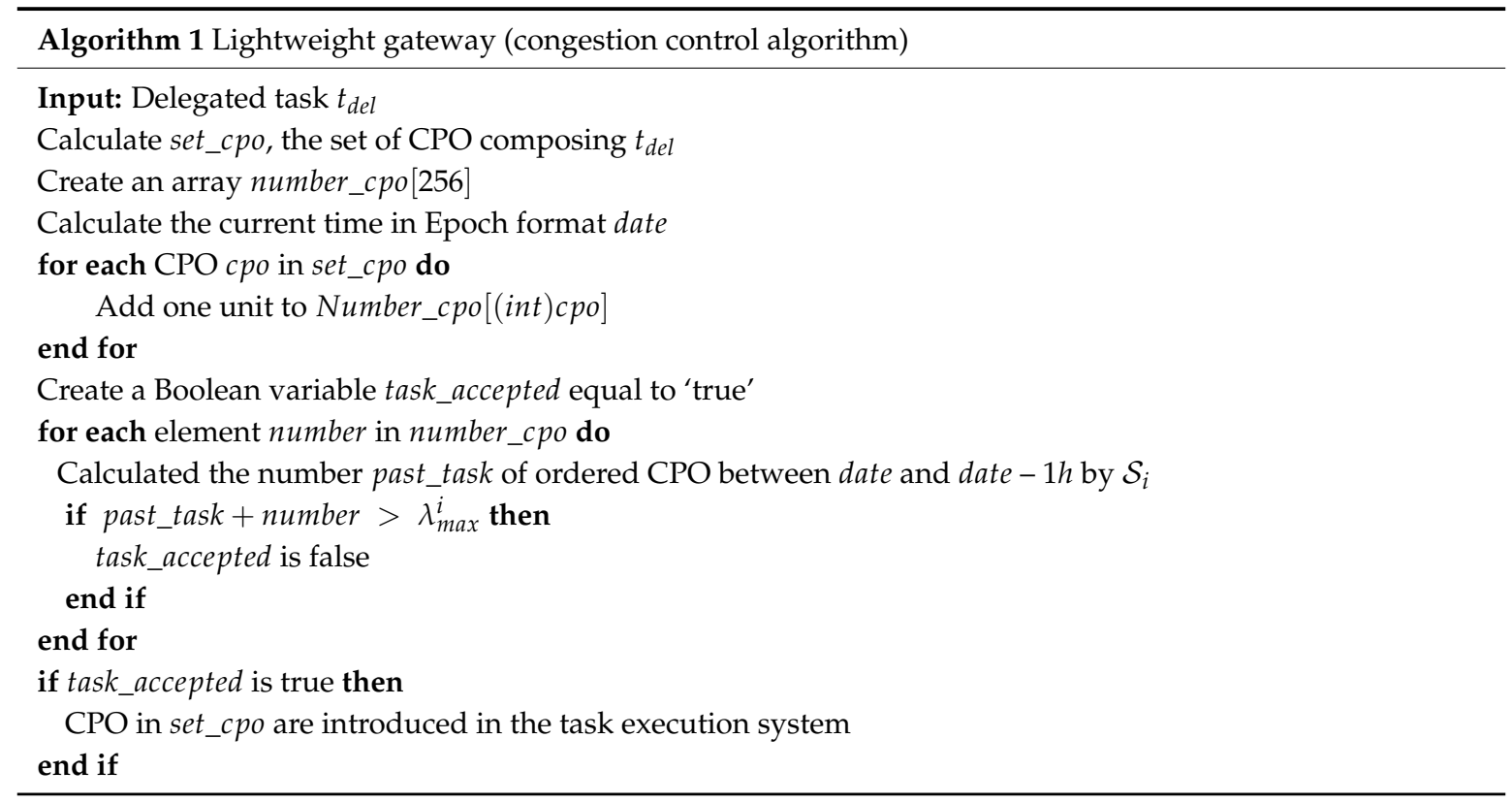

As CPO are identified by one-byte integer numbers, a simple casting of data types enables us to count the amount of CPO of each type that make up the delegated task.

The rest of the Algorithm 1 is the proposed "Erlang filter," designed to avoid the congestion in the system. Even if queues in the devices and/or the gateway could store more CPO than admitted by this filter (for example, if queues were infinite), the system becomes unstable if the utilization factor is higher than one, so this filter prevents the queue system to reach that point (see Figure 11).

The basic idea is to store a historical register of the amount of ordered CPO (not received tasks, as rejected tasks must not be included) in time. A slicing window with a width of $T$ time units (usually an hour to be coherent with the traffic engineering principled) moves through the registry and accumulates all the operations that fall within it. The value of $T$ parameter should be chosen to be large enough to remove the random fast variation in the task delegation flow; but short enough to allow observing the slow variations that occur throughout the day. Although specific values for particular applications could be calculated, the traffic engineering theory has proved that, in the general case, one hour is a good balance between both requirements. If the accumulated value (considering also the number of operations which will be introduced if the task is accepted), for any server group, is higher than the maximum allowed hourly medium CPO ordination rate, the delegated task is directly rejected, no operation is introduced in the queues.

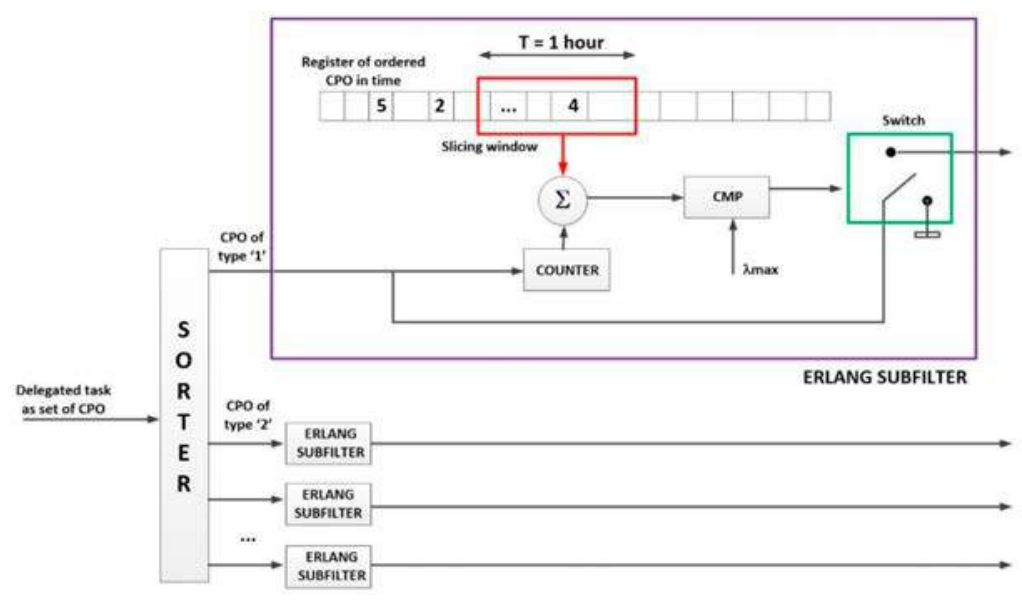

Figure 11. Structure of the proposed Erlang filter. 
Thus, a key problem is to calculate the maximum allowed hourly medium CPO ordination rate $\lambda_{\text {max }}^{i}$ for each group of servers. Considering that, as said, the maximum value of the congestion factor is obtained to fulfil the requirements of the desired QoS; and $N_{i}$ and $\mu_{i}$ are imposed by the hardware characteristics of the WSN, this maximum rate is easily calculated (10).

$$
\lambda_{\text {max }}^{i}=\rho_{i} \cdot N_{i} \cdot \mu_{i}
$$

Then, it is necessary to calculate the values of $N_{i}$ and $\mu_{i}$ of the underlaying WSN. The calculation of $\mu_{i}$ is simpler and it is showed in Algorithm 2. On the other hand, Algorithms 3 and 4 are designed to estimate the value of $N_{i}$, which is more complicated as this parameter cannot be directecly measured.
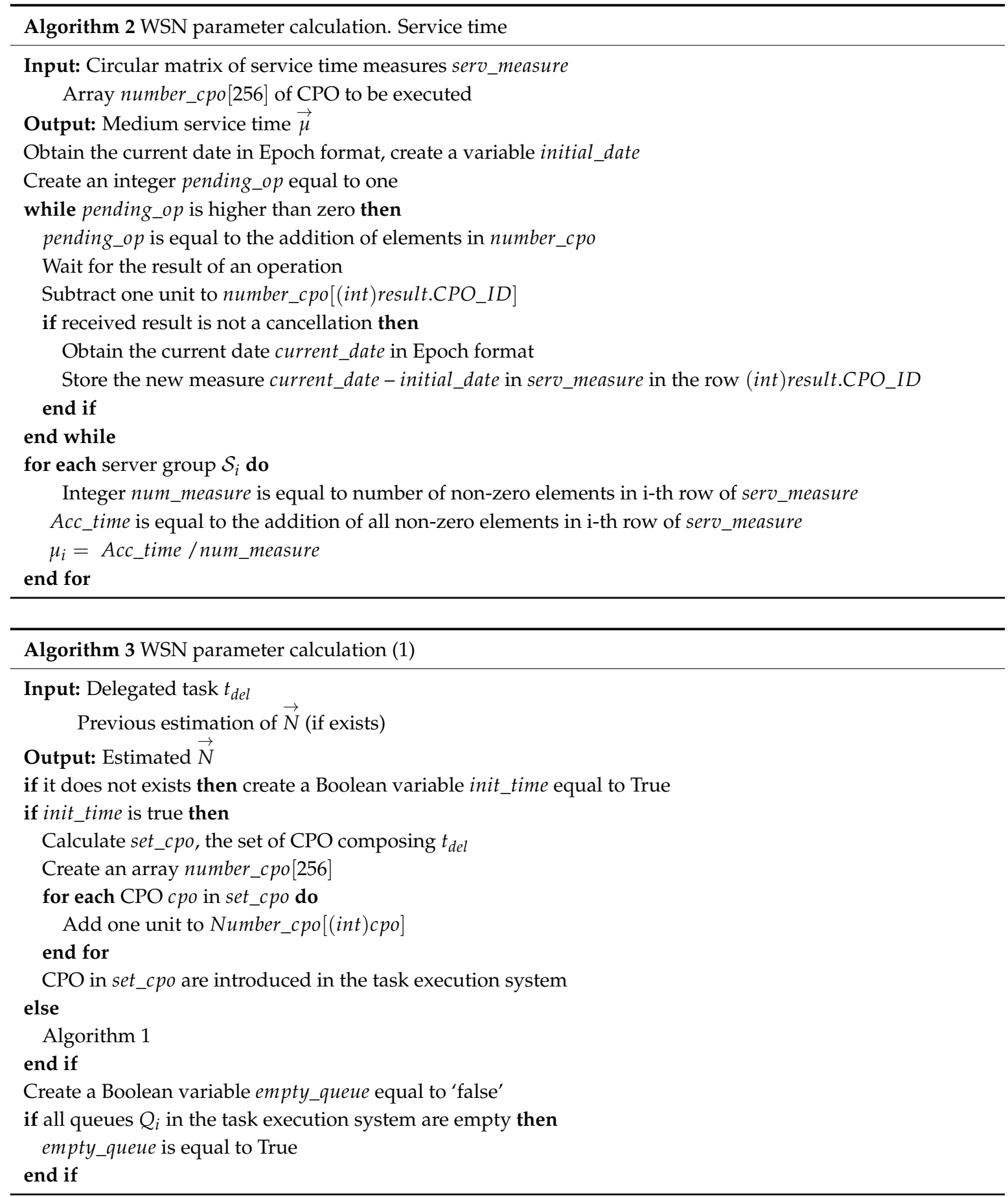

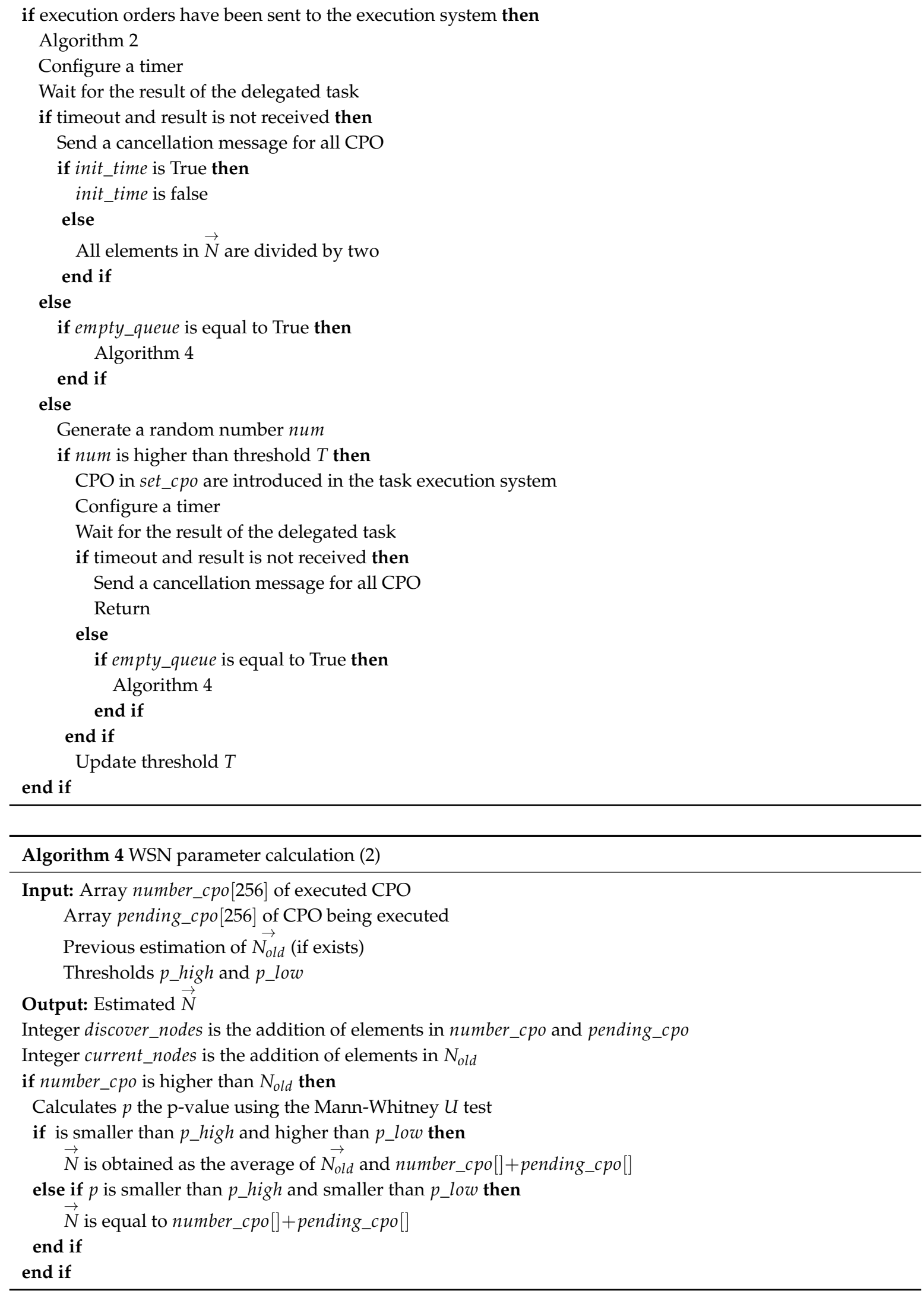

Because of the need of listening all interactions among nodes in WSN to estimate the medium service time, Algorithm 2 should be hosted in fictional nodes (see Figure 4), which are virtual entities maintained and supported by the gateway. The proposed Algorithm 3 considers a circular matrix 
where measures about the service time are stored (columns represent different time instants and rows different server groups). The number of elements per row $k$ represents the number of measures employed to calculate the medium service time.

Algorithm 2 receives information about the amount of $\mathrm{CPO}$ of each type to be performed. Then, it waits until all of them have been executed, or cancelled (in this case the new calculated medium service time will be equal to the previous one because of the algorithm design). Considering the initial time when the delegated task was triggered and the finishing time of each $\mathrm{CPO}$, a new measure of the service time is obtained (and stored in the circular matrix). It is important to remark that, as we are seeing in Section 3.4, resources of nodes get "allocated" once CPO are broadcasted and accepted. Thus, although an important time of the service time may be employed in waiting until the previous $\mathrm{CPO}$ in the task workflow are finished, it must be considered the whole time since $\mathrm{CPO}$ executions are ordered.

Once all CPO have been resolved, executed or cancelled, the medium service time is evaluated considering the new data and all previous $k-1$ samples. It is important to note that, if we assume the WSN is not congested, the service time may be considered independent from the hourly task delegation rate (and only dependent on intrinsic hardware and software factors). Thus, all measures can be employed to calculate the average, regardless the moment and external conditions when they were acquired.

Moreover, as these kind of "learning algorithms" require a certain time to converge to a stable value (while enough data are accumulated), only non-zero values in the buffer of measures will be considered to obtain the medium service time.

Algorithm 3 is the most complex algorithm to be described in this work. It makes reference to all previously described algorithms, as the evaluation of the number of nodes of each type in the WSN is a key problem without a simple solution. As we said, nodes are considered servers in our model, grouping them according to the $\mathrm{CPO}$ they can perform. As, usually, each node can perform various $\mathrm{CPO}$, the same node might be included in various server groups (see Figure 12). Nevertheless, at each time instant, a hardware smart node may only act as belonging to one group $\mathcal{S}_{i}$ (i.e., it can only perform a $\mathrm{CPO}$ at the same time, as sensor nodes usually do not allow parallel programming), although this association can change over time.

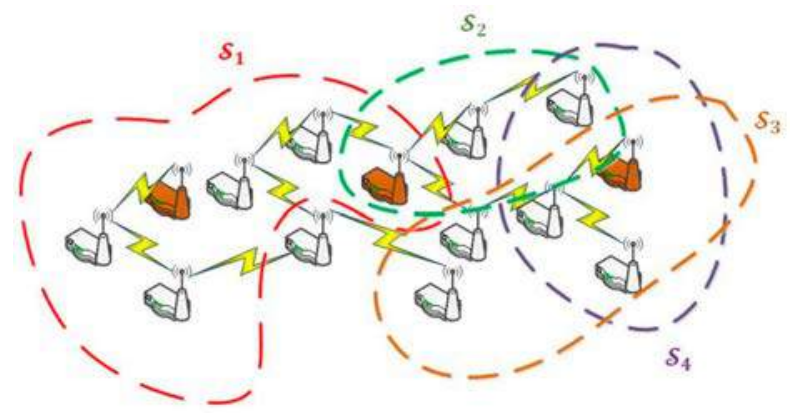

Figure 12. Distribution of nodes in the different server groups.

When estimating the number of servers per group, $N_{i}$, however, it is desirable the values to be stable over time. Oscillating variables cause the algorithms to behave in a random way and we could no guarantee the WSN is not congested. Although different policies may be employed to fix stable values for $N_{i}$ (e.g., distributing the nodes homogeneously among the shared groups), in this case these values are adjusted to the real use demand of the different server groups. Then, server groups receiving more $\mathrm{CPO}$ execution orders will include more shared nodes than less used groups.

The manner in which Algorithm 3 does this data inference is by monitoring the real capacity of WSN to execute the delegated tasks. During the initialization period, most common operations will 
need more nodes to be executed and Algorithm 3 will include these nodes in the adequate group, although they could execute other different $\mathrm{CPO}$.

Algorithm 3 considers a first initialization period, when no control about the WSN congestion is performed. Information about the underlying hardware is collected during this time, which finishes once the WSN is not able to execute a delegated task (i.e., when a previously configured timer expires and no result is received). At this moment, the number of nodes in each server group $N_{i}$ is fixed to be equal to the last value for which tasks were successfully executed.

If a congestion situation is detected (because a delegated task is not executed when the maximum allowed rate has not been exceeded) and the initial time is over, the current number of nodes in each server group $N_{i}$ is divided by two. From this point, the number of nodes in each server group should be updated until reaching its real values. This policy, in fact, must be proved to be successful in other congestion control solutions such as TCP.

Using only the previously described technique, the number of nodes will be always fixed to the value calculated during the initialization period. In order to update this value, for example if new devices are added or during the recalculation process after a congestion situation, tasks marked to be rejected can be finally processed. If these tasks are not executed by the WSN, the number of nodes is not updated. On the contrary, if these tasks are in fact executed by the WSN, that may mean more nodes than the system knows are available and vector $\vec{N}$ is updated according to this new information.

In order to select which tasks marked to be rejected are finally processed, a statistical process is developed: a random number is generated, if the number is higher than a threshold, the task is tried to be executed. The key problem in this solution is, then, the calculation of the threshold $T$. This threshold has to be smart and have memory, as depending on the result of previous discovering attempts it should be more difficult or easier to do a new attempt. Considering these ideas, the proposed function (11) to calculate the threshold $T$ includes two branches, depending on if the previous discovering attempt was successful $(s=1)$ or not $(s=0)$.

$$
T\left[n_{0}\right]= \begin{cases}\frac{P}{2}\left(1+T\left[n_{-1}\right]\right) & \text { if } s=1 \\ \frac{P}{2} T\left[n_{-1}\right] & \text { if } s=0\end{cases}
$$

The proposed function is based on the geometric series, it is recursive and evolves between zero and $P$, where $P$ is maximum number the random number generator in Algorithm 3 may deliver. In order to maintain the total randomness at the beginning, the initial value of this threshold is designed to be $T[0]=\frac{P}{2}$. The proposed function is inspired on the traditional congestion control mechanisms, that may be found (for example) in most implementations of the TCP protocol. These solutions consider exponential-like functions (like the proposed one) as they allow making the most of network resources and detect congestion situations more quickly.

The last detail to be discussed is about the updating algorithm of array $\vec{N}$. If all queues in the task execution system are empty, it is guaranteed that all pending and ordered $\mathrm{CPO}$ are been executed simultaneously. Then, the number of nodes in each server group must be, at least, equal to the number of CPO being executed at the same time.

If the number of discovered nodes is higher than the previously known amount, then, the vector $\vec{N}$ should be updated considering the new information. However, as each node can perform several different $\mathrm{CPO}$, small differences may appear but not due to the discovery of new nodes but because of movements of nodes among different server groups. Therefore, before updating the vector $\vec{N}$ it must be guarnteed that the new array presents a globally and significant improvement in the number of discovered nodes in respect to the previous values. In order to do that, Algorithm 4 considers a statistical test: the Mann-Whitney $U$ test. The Mann-Whitney $U$ test is a nonparametric test of the null hypothesis that two samples come from the same population against an alternative hypothesis, comparing the mean values of the two samples. It is used to evaluate if two different data populations 
are similar or different (higher or lower). The p-value indicates the significance level of Mann-Whitney $U$ test.

Using this test, only when a significantly better array is calculated, the values in $\vec{N}$ are updated. In practice, two different thresholds for the $\mathrm{p}$-value are considered. The first one determines if array $\vec{N}$ must be updated or not. The second inicates if the actualization must be total (the new vector replaces the old one) or partial (both arrays are combined, in this case calculating the average).

\subsection{Choreography Algorithm}

Using the previously described smart algorithms, we guarantee the WSN remains in a stable state and choreography algorithms are, then, guaranteed to be effective. Besides, as the state of the WSN may be represented using a data structure with a fixed memory consumption, independent from the number of nodes in the WSN, the gateway may be lightweight and operate at real-time. Considering these previous results, a hardware-supported algorithm is a valid solution to enable smart nodes in WSN to self-manage their resources and execute tasks in a choreographed way.

Figure 13 shows the workflow of the proposed smart self-management and choreographed task execution algorithm for WSN. The algorithm starts when the gateway introduces the corresponding $\mathrm{CPO}$ execution orders of a delegated task in the execution system. When nodes get available, those orders are extracted from the queues and broadcasted within all the WSN (using the message described in Section 3.2). When nodes listen these execution orders, each one decides randomly, $\mathrm{CPO}$ by $\mathrm{CPO}$, if they would be able to execute that operation. This decision can be made using a random number generator and a fixed threshold (if the generated random number is above the threshold the CPO is admitted). After a certain time and as node density is very high, all CPO will be tentatively accepted by several different smart nodes. At this moment, before doing any additional processing, the node verifies if it can perform the required $\mathrm{CPO}$. If the ordered $\mathrm{CPO}$ is not among the operations the node may solve, all resources are deallocated and the node returns to the initial value. On the contrary, the self-management functions are activated.

Then, if CPO is tentatively accepted, each node must evaluate two conditions in an autonomous manner. First it must be guaranteed the value of the CPO to be performed is higher than the current value of the "gas" remaining in the node. In this context, the notion of "gas" may be understood as a homogeneous variable, obtained from the aggregation of remaining resources. As the remaining amount of "gas" goes down, its value increases. Only operations whose value is higher than the value of the "gas" they are going to consume are executed. Second, apart from the value of the "gas", the node must evaluate if it has enough resources to execute the CPO. For example, although the value of the $\mathrm{CPO}$ was the highest, the operation could not be executed if it requires more than a certain time (because the node was very demanded), it would run out of battery, etc.

These two decisions represent the self-management capacity of smart nodes, as they can manage their resources in an autonomous way. Later, both processes will be explained with details.

If both previous conditions are verified, the CPO is finally accepted by the node. When an operation is accepted, the node runs a timer initialized with a random time. At timeout, the node will generate and broadcast a claim message about the accepted operation. However, if before timeout, the node receives a claim message from another node (which configured the timer with a lower value), all resources are released and CPO is not executed.

Two considerations have to be done. First, if (because of the randomness of the process) a CPO is not accepted by any node, the gateway, when no node claims the execution, will broadcast the execution order again. The entire task execution is aborted if a CPO cannot be executed after various attempts. Second, as messages need a time to be transmitted, at timeout (after broadcasting the claim message), an extra time for listening for claim messages from other nodes is considered. Claim messages received during this extra time are considered to collide. In order to resolve this situation, a new random timer is configured and the process is repeated but only by the nodes that collided. 


\section{SMART NODE}

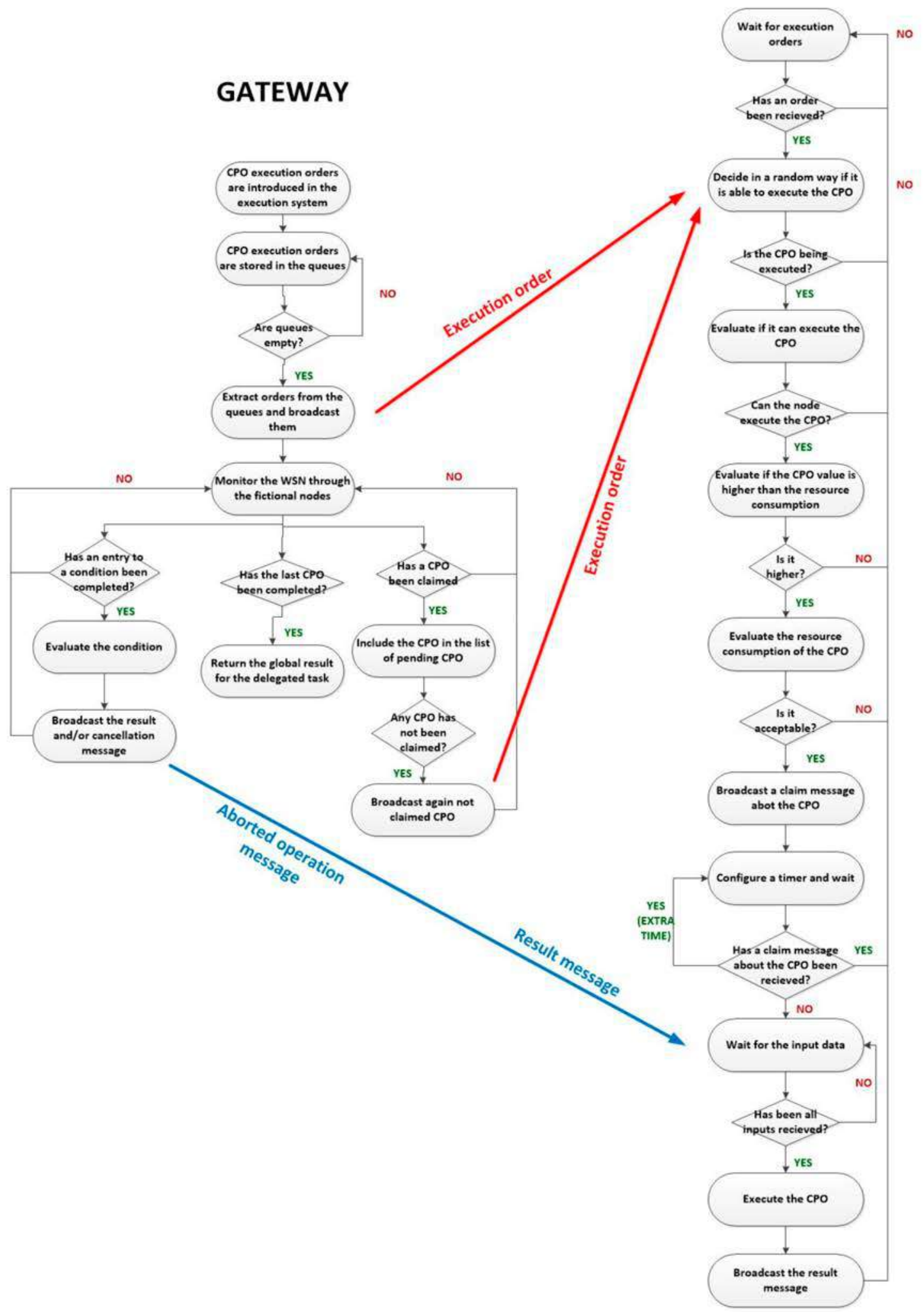

Figure 13. Workflow of the proposed smart task execution algorithm. 
The proposed mechanism to decide if a CPO is finally executed may seem complicated but once implemented using hardware technologies, the entire algorithm will be executed almost instantly.

After all this process, only one node will be in charge of executed each CPO in the delegated task. $\mathrm{CPO}$ associated to conditions in the workflow of the delegated task are directly assigned to fictional nodes, through which the gateway also monitors the behaviour of the hardware devices in the WSN.

$\mathrm{CPO}$ whose input data are only constants (the corresponding flag in those fields is fixed to a binary one) are directly executed. When a CPO is executed, the corresponding node broadcast a result message, describing its output. In that way, all nodes in charge of CPO whose input data refer to this operation will be able to replace this reference by the final constant value to be employed (the obtained result). Once the number of "required data" by any CPO is reached (because all the needed cross-references with other $\mathrm{CPO}$ have been solved) the $\mathrm{CPO}$ is directly executed and its result broadcasted.

The gateway, once received the result for the last CPO in the workflow in the delegated task, returns the global result of the execution (equal to the result of the last CPO) to the high-level applications.

The proposed algorithm solves the choreographed execution of tasks in the WSN, however, self-management functions require a more detailed discussion.

The idea of "gas" is a global representation of the available resources in a node. The initial amount of gas in a node $g_{0}$ is a parameter of the proposed algorithm. In order to prioritize the most important $\mathrm{CPO}$ at each moment, as the available resources go down, the gas is scarcer and then more valuable. Only CPO whose value $v_{c p o}$ is higher than the value of the gas $v_{g}$ it is going to consume are executed. In the proposed solution, the amount of gas each low-level instruction consumes in each node is fixed, so the amount of gas required to execute each $\mathrm{CPO}$ is also known and fixed. If the CPO is executed, the amount of consumed gas is subtracted from the remaining gas in the node. The problem, then, is evaluate the value of this gas.

As in standard economic products, the value of each unit of gas follows an exponential evolution. As gas is scarcer, its value increases in an exponential way. In order to make comparable the CPO value and the gas value, both variables take values within the interval $[0,255]$ (12). Several different works have proved that (in standard economy), as the available quantity of a product decreases, its price rises exponentially $[42,43]$. Since the resources of the sensor network are consumed, ultimately, by the users who order the task delegations, it is reasonable the cost to grow in the same way that the price of any other resource, as it gets scarcer.

$$
v_{g}=\left\lceil\left(v_{g}^{\max }-v_{0}\right)\left(1-e^{-\left(\frac{k_{\text {inf }}}{g t}\right)}\right)+v_{0}\right\rceil
$$

In the proposed assessment function, $g_{t}$ represents the amount of remaining gas at the current moment. $v_{0}$ reprsents the desired initial value for each unit of gas, as a minimum value $v_{0}=0$. $v_{g}^{\max }$ represents the desired final value for each unit of gas, as a maximum value $v_{g}^{\max }=255$. Finally, $k_{\text {inf }}$ is the inflaction constant. It indicated how fast the value of the gas increases. Approximately, for $g_{t}=\frac{k_{\text {inf }}}{5}$ the gas reaches its maximum value.

Although this expression may seem complex, in fact only one variable is present, $g_{t}$. The other parameters are design constants with a fixed value. The idea of "gas" can be employed, besides, to implement enhanced QoS techniques, even though in this paper we are not considering this option.

The implementation of exponential functions using logical gates and similar hardware solutions it is very complicated. Therefore, in order to only employ binary adders and multipliers in the proposed hardware-supported algorithm (thus, the solution can be implemented using microelectronic techniques), the Taylor's series of the proposed function is calculated and employed (13).

$$
v_{g}=\left\lceil\sum_{j=0}^{\infty} \frac{v_{g}^{(j)}\left(g_{0}\right)}{j !}\left(g_{t}-g_{0}\right)^{j}\right\rceil \approx\left\lceil v_{0}+\left(v_{g}^{\max }-v_{0}\right) \frac{k_{\text {inf }}}{g_{0}{ }^{2}}\left(g_{0}-g_{\mathrm{t}}\right)\right\rceil
$$


Regardless the value of gas, smart nodes should consider other limits: they should never spend all their resources; they should be operating during, at least, a minimum time to be profitable, etc. In order to fulfil these requirements, nodes should be able to predict the future state of their resources at each moment.

The state of the resources in a node may be represented through a column vector $\overrightarrow{r_{i}}(14)$, where $M$ is the number of resources to be considered. The resources of the entire WSN can be represented, then, by means of a matrix $\vec{r}$ where each column represents a different node (15).

$$
\begin{gathered}
r_{i}=\left[\begin{array}{c}
r_{i}^{1} \\
r_{i}^{2} \\
\ldots \\
r_{i}^{M}
\end{array}\right] \\
\vec{r}=\left[\begin{array}{ccc}
r_{i}^{1} & \ldots & r_{\mathrm{N}_{\text {total }}^{1}} \\
\ldots & \ldots & \ldots \\
r_{i}^{M} & \ldots & r_{\mathrm{N}_{\text {total }}^{M}}^{M}
\end{array}\right]
\end{gathered}
$$

In this context, the future state of the resources in the WSN, at any moment, could be evaluated through a dynamic system $\vec{F}$ depending on the current state of the resources and a stochastic process representing the task delegations. This stochastic process $\Phi$, as said, follows a Poisson's distribution (16).

$$
\vec{r}(t)=\vec{F}(\vec{r}(t), \Phi)
$$

If we particularize the model in a unique node, parameters in the dynamics might be divided into three elements (17): a column vector representing the resource self-management in the node (the future state of resources depends on the self- management policies applied by the node); a matrix $\vec{r}_{\text {cho }}(t)$, named choreography term, representing the resource state in the rest of the WSN (because of the choreography algorithm, the resource consumption in nodes depends on the behavior of the other devices); and the previously described stochastic process $\Phi$.

$$
\vec{r}_{i}(t)=\vec{F}\left(\vec{r}_{i}(t), \vec{r}_{\text {cho }}(t), \Phi\right)
$$

From the point of view of a particular node, the impact of the choreography term in the consumption of its resources is a random contribution, as it does not have information about the management the other nodes do with their resources (18). This random contribution can be understood as a stochastic process $\Phi_{\text {cho }}$ representing all CPO the other nodes have not accepted. As nodes in the WSN operate in an independent way and there are a high number of them, $\Phi_{\text {cho }}$ may be assumed to be a Possion distribution (18). Stochastic terms may be, finally, grouped (19).

$$
\begin{gathered}
\vec{r}_{i}(t)=\vec{F}\left(\vec{r}_{i}(t), \Phi_{\text {cho }}, \Phi\right) \\
\vec{r}_{i}(t)=\vec{F}\left(\vec{r}_{i}(t), \Phi_{\text {total }}\right)
\end{gathered}
$$

At this point, the proposed model using differential equations and continuous time must be transformed to a model with finite differences and discrete time (20). Besides, if we assume that all resources are independent (the memory consumption is independent from the battery discharge, for example), the dynamic model can be divided into a system of $M$ independent Equations (21).

$$
\overrightarrow{r_{i}}[n+T]=\vec{F}\left(\overrightarrow{r_{i}}[n], \Phi_{\text {total }}[T, n]\right)
$$




$$
\left\{\begin{aligned}
r_{i}^{1}[n+T] & =F_{1}\left(r_{i}^{1}[n], \Phi_{\text {total }}[T, n]\right) \\
& \ldots \\
r_{i}^{M}[n+T]= & F_{M}\left(r_{i}^{M}[n], \Phi_{\text {total }}[T, n]\right)
\end{aligned}\right.
$$

Using this model, it must be guaranteed that no resource, during the planned operation time, goes below a certain acceptable limit $r_{t h}^{j}$. These limits are calculated to gurantee the minimum survival of the node.

Each node will perform short measures (e.g., $T=10 \mathrm{~min}$ ) in order to estimate the medium value stochastic term. Using this information and the resource evaluation laws $F_{j}$, the node will calculate the resource state at the end of the planned operation time $T_{o p}$ and if it is smaller than $r_{t h}^{j}$ (for any resource) the $\mathrm{CPO}$ will be not accepted.

The final detail to be discussed are the definition of the resource evolution laws $F_{j}$. These laws must be defined before implementing the proposed algorithm using hardware technologies. They depend on the resource to be studied (for example battery charge presents an exponential evolution but memory usage follows a linear law) and should be transformed into Taylor's series if the corresponding evolution law does not present a polynomic form (so it can be implemented using binary adders and multipliers).

Considering all previous details, it is possible to propose a schematic hardware implementation. The objective of this paper is not to describe a complete low-level hardware implementation which, even, could be performed using solid state devices and microelectronic techniques in order to improve the integration level. However, some important components and signals in order to understand the behaviour of the proposed hardware-supported algorithm are identified.

The first module to be considered is the enable signal generation (see Figure 14). In this module and using the binary header of messages (se Section 3.2), the signals that activate the processing of the received message are generated. In order to do that and using XNOR logical gates, the header of the received message is compared to the four possible headers. Only the gate where both entries are equal produces a logical one at its output. Besides, if the received message is a result or a claim or cancellation message, the message must be referred to the $\mathrm{CPO}$ being currently executed. Besides, using a XNOR and a AND gate it is validated the correctness of the received message using the checksum. As a result, four enable signal are generated: "en_execution," "en_result," "en_cancel" and "en_claim." The signal associated to the received message type is set to high level.

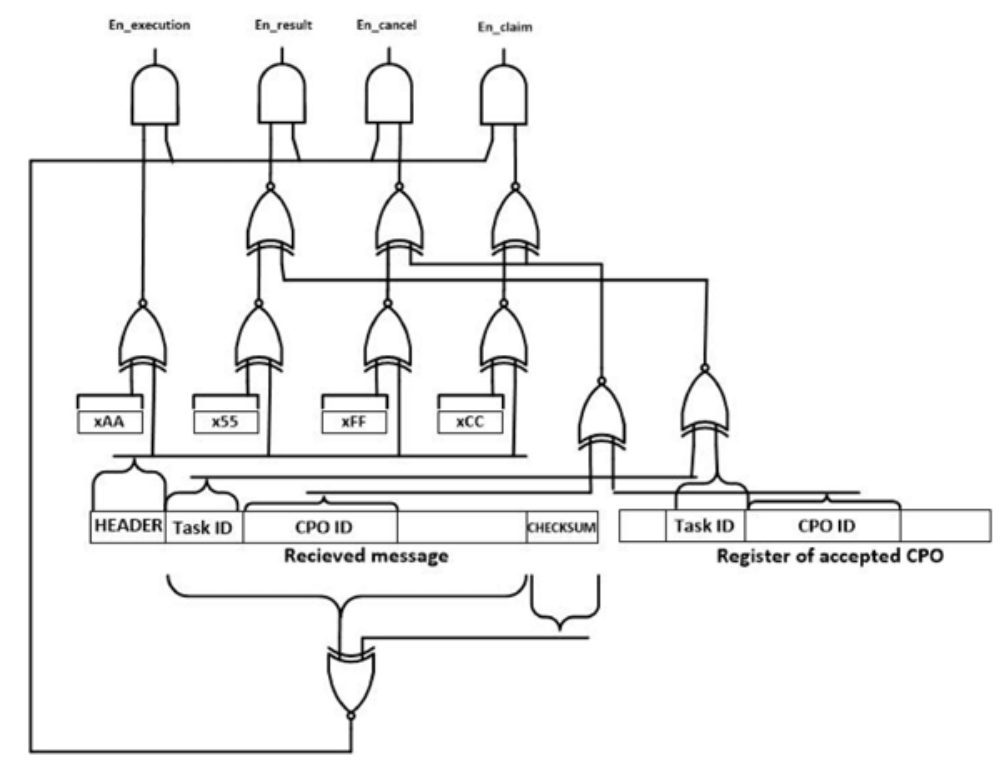

Figure 14. Enable signal generation. 
Figure 15 shows the CPO evaluation module. This circuit performs the self-management functions and the first steps in the choreographed execution of tasks. As can be seen, although in Figure 13 the proposed algorithm was showed in a sequential way, hardware technologies enable the parallel execution of the different verification phases, despite of the fact that smart nodes, usually, do not support parallel programming.

The unique important novelty in the proposed hardware implementation is the inclusion of a 256 position ROM memory. In this memory, only positions whose address corresponds with codes of CPO the node can perform have a non-empty content. The content of this memory is the amount of gas each CPO requires to be executed. In that way, doing only one query to the ROM memory two steps in the proposed algorithm are performed almost completely.

Moreover, in order to implement the entire algorithm using simple hardware technologies, Pseudorandom Number Generators (PRNG) might be constructed using XOR gates and Logical Shift Registers.

Once the "claim generation" signal is calculated, a second module starts its performance: the claim module (see Figure 16). This module generates and transmits the claim operation message (if needed) and performs the steps of the choreography algorithm that ensure that only one node in the WSN is responsible for executing a CPO.

The claim module includes a RS bistable which maintains the initial "claim generation signal" in its original value for an infinite time. This new signal (the "initial claim generation" signal) activates a counter which controls the extra waiting time for simultaneous claim messages. At the same time, a tristate buffer is enabled and the corresponding claim message is broadcasted. If during the extra time a new claim message is received, a reset signal is activated, so the last step in the CPO evaluation module is executed once more (a new random waiting time is initiated to solve the collision of claim messages). The inverse of this reset signal activates the last module of the proposed self-managed and choreographed task execution algorithm.

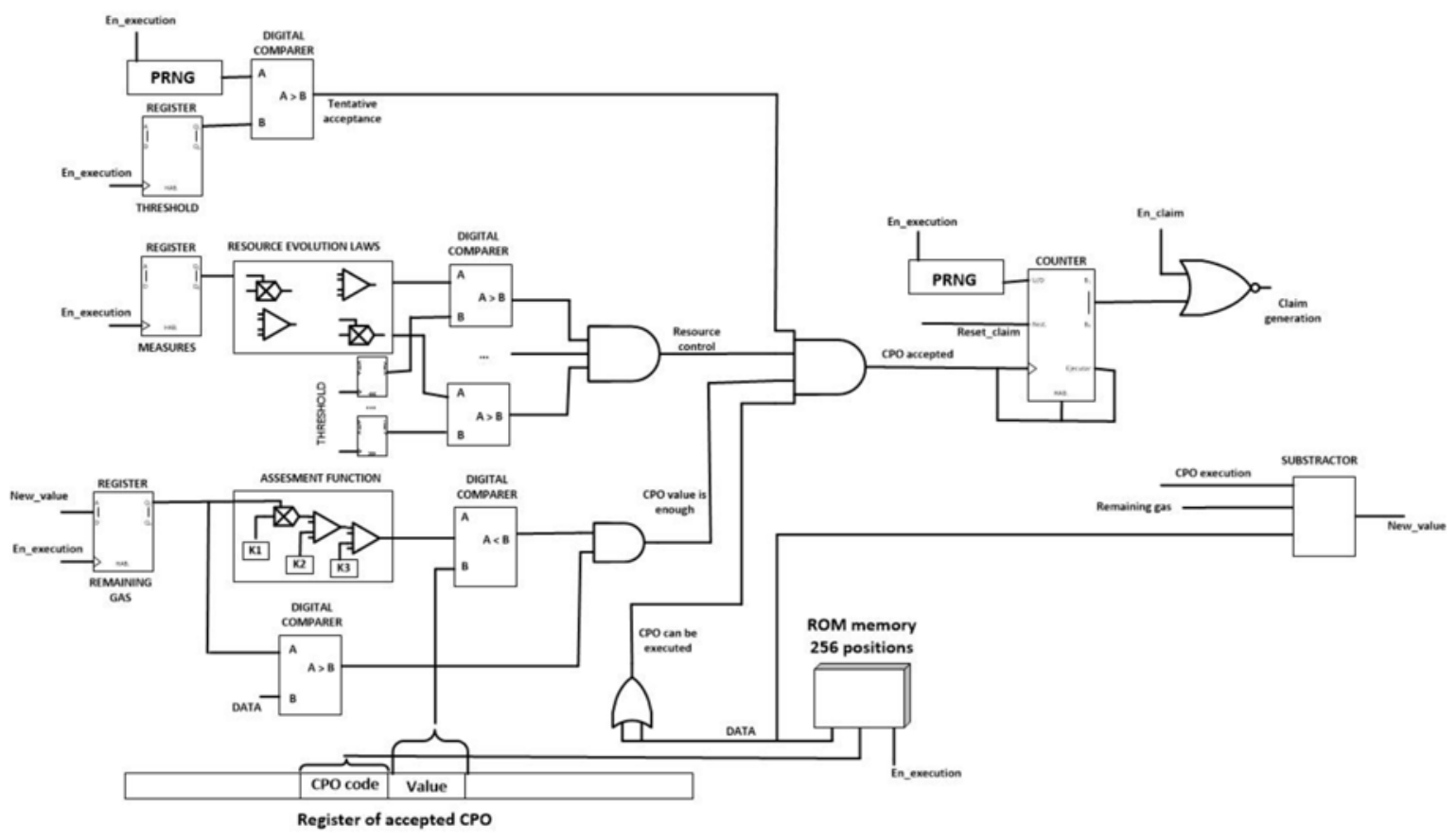

Figure 15. CPO evaluation module. 


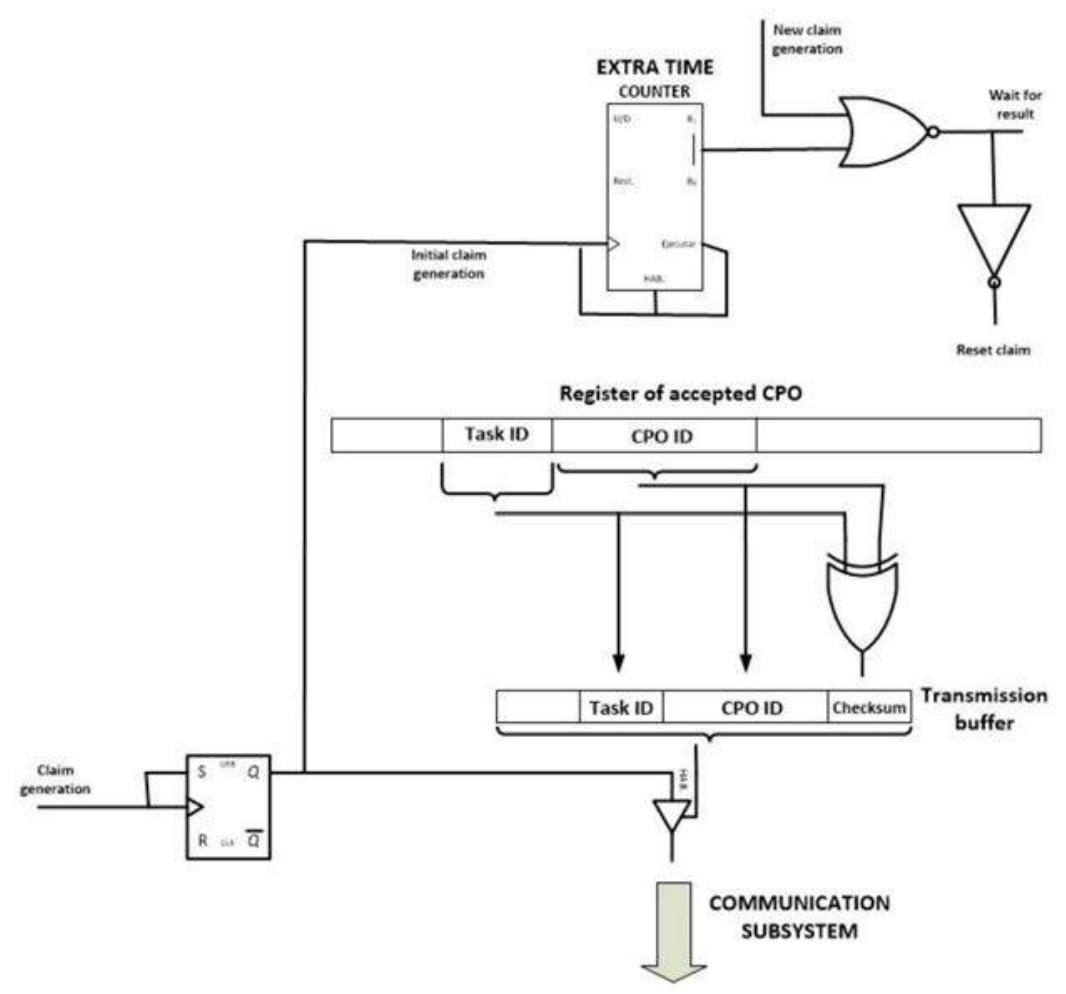

Figure 16. Claim module.

Thus and finally, the CPO execution module maintains accepted CPO in a waiting state, until the minimum number of input data are received and then the execution is finally performed (see Figure 17). In order to do that, a counter controls the number of constant data that have been received and compares the result with the required data specified in the execution order. Once both values are equal (the counter reaches the value zero), a tristate buffer sends the final CPO execution order to the application level.

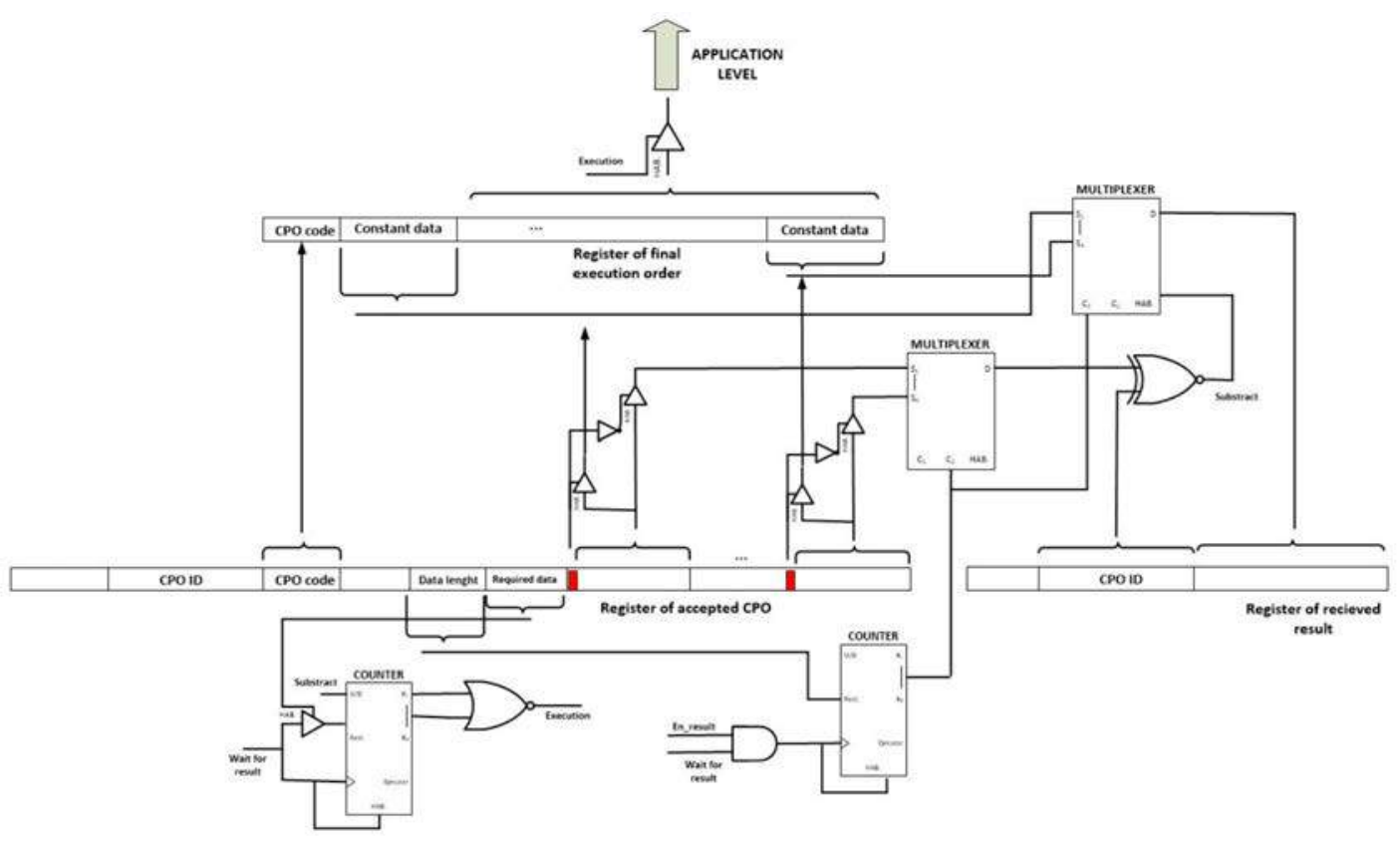

Figure 17. CPO execution module. 
Using also tristate buffers and the one bit flag that is included with each data in the CPO execution order, it is decided if the corresponding data is a constant value that can be included directly in the final execution order, or a cross-reference that must be replaced. Each time a result message is received, two multiplexers are employed to compare the CPO ID to the pending cross-references and, if success, replace the adequate reference with the received results.

Once the CPO is executed the result message can be generated and broadcasted using only one buffer. Besides, if the en_cancel signal gets activated at any time, all registers, counters and other digital circuits get reset using the appropriate pin of each element. In these both cases, no additional hardware implementation is required.

\section{Experimental Validation}

In this section, the performance of the proposed solution is evaluated. Two different types of experiments were carried out. The first type consisted of numerical simulations, employed to evaluate the correctness of the congestion control algorithm and other characteristics of the lightweight gateway behaviour. The second type was based on the ModelSim software, which enabled us to simulate the hardware implementation of the proposed self-management and choreography algorithm using the VHDL (Very High Speed Integrated Circuit Hardware Description Language) language. Using these ModelSim-based simulations we evaluated important low-level aspects of the proposed solution such as the processing delay.

In particular, four different experiments were planned and developed. The first three experiments were based on numerical simulations. The fourth one was developed using the ModelSim software.

The first experiment evaluated the performance of the proposed algorithm to obtain the value

of vector $\vec{N}$, the number of devices in each server group. Ten different $\mathrm{CPO}$ were considered during this first experiment, so ten different server groups were defined. Each node was able to perform one or two different CPO. The total number of nodes in the simulation is one hundred (100). The specific composition of the WSN is showed in Table 1. Values in Table 1 represent the total number of nodes being able to perform the $\mathrm{CPO}$ indicated in the corresponding row and column. For example, two nodes can execute both the CPO\#1 and CPO\#3; but five nodes can only execute CPO\#1.

Table 1. Composition of the WSN during the first experiment.

\begin{tabular}{|c|c|c|c|c|c|c|c|c|c|c|}
\hline & CPO\#1 & CPO\#2 & CPO\#3 & CPO\#4 & СРО\#5 & CPO\#6 & СРO\#7 & CPO\#8 & CPO\#9 & CPO\#10 \\
\hline CPO\#1 & 5 & 5 & 2 & & & & & & & \\
\hline CPO\#2 & 5 & & & & & 4 & & & & \\
\hline СРO\#3 & 2 & & & & 8 & & & & & \\
\hline СРО\#4 & & & & 10 & & & & & & \\
\hline СРО\#5 & & & 8 & & & & & 2 & & \\
\hline СРО\#6 & & 4 & & & & 4 & & & & \\
\hline СРO\#7 & & & & & & & & 5 & 5 & \\
\hline СРО\#8 & & & & & 2 & & 5 & & & 5 \\
\hline СРО\#9 & & & & & & & 5 & & & 4 \\
\hline СРО\#10 & & & & & & & & 5 & 4 & 1 \\
\hline
\end{tabular}

The convergence speed and the final value for the $\vec{N}$ depend on the delegated task configuration and workflow (as said, the algorithm was specifically designed to depend on these parameters). Therefore, we must guarantee that the obtained results are coherent (they are detected, as maximum, as much devices as included in the WSN) and that final values are not oscillating.

The second experiment was designed to investigate if the QoS parameters in the WSN are guaranteed as desired. In particular, we evaluated if the proposed algorithms maintain the congestion factor and the network load below the specified limits. For this experiment, the same WSN described for the first experiment was employed. The medium service time was simulated to be one second, so results may be analysed in an easier way. Moreover, although as described in Section 3.3, the design process should start defining the expected loss probability in the execution system, in this experiment we are selecting a value for the utilization factor $\rho_{\exp }=0.7$ regardless the associated loss probability. 
The third experiment was planned to evaluate the success rate in the execution of delegated tasks. The simulation scenario was the same of the two previous experiments but in this case, besides, workflows of $\mathrm{CPO}$ were randomly generated to be executed using the proposed solution. The success rate depending on the capacity of queues was evaluated. In this case, besides, it is interesting to repeat the experiment for different network configurations. Thus, the third experiment was repeated for a network including 100 sensors nodes, 200 sensor nodes, etc. The composition was always maintained as described in Table 1.

Finally, the fourth experiment was developed using the ModelSim software. The proposed hardware implementation was described using the VHDL language in order to evaluate the mean processing delay (as there are random components which do not present a constant delay). In this scenario, only two resources were considered relevant: the memory capacity and the battery charge. The memory capacity was modelled using a decreasing linear function with the number of performed operations. The battery charge was modelled using a decreasing exponential function, written as a Taylor's series. Additionally, the minimum value of gas was considered to be zero $v_{0}=0$ and the maximum value was defined to be $255, v_{\max }=255$. The total amount of gas at the initial time was 4096 units, $g_{0}=4096$. For this last experiment the total number of possible CPO, 256, were considered.

\section{Results}

In this Section, results of the experiments described in Section 4 are presented in an ordered way.

Figure 18 shows the results for the first experiment. Figure 18a shows the result for the total number of nodes in the WSN and Figure 18b shows the result for each one of the ten available CPO. As can be seen, the evolution of the number of discovered nodes is globally increasing. After ten complete updates (several task executions that have not forced an update of the number of nodes could be also performed during this time), the total number of nodes has reached $90 \%$ of the final value. This result, with small variations, is also valid for each individual server group.

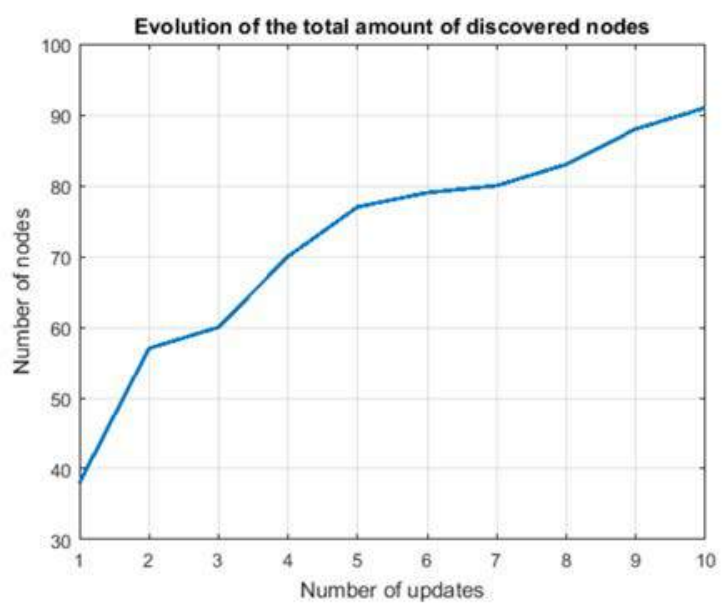

(a)

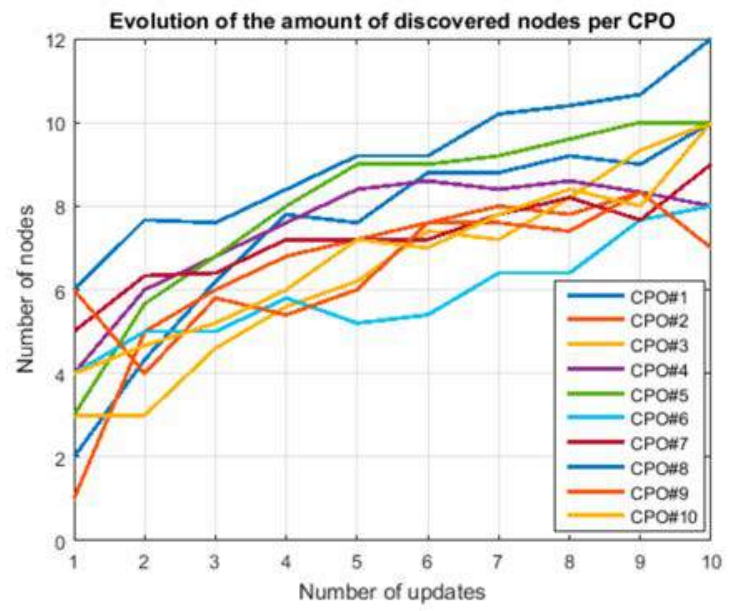

(b)

Figure 18. (a) Evolution of the total number of nodes (b) Evolution of the number of nodes per CPO.

It is important to note that the evolution of $\vec{N}$ with the number of updates is not monotonous. There are fluctuations, because of the fact that a global improvement in the value of $\vec{N}$ might imply small partial deterioration in some values. The same effect appears when the new value of $\vec{N}$ is calculated as the average value of the two previous estimations.

However, as can be seen in Figure 18, calculated estimations are not oscillating, although they can fluctuate slightly. Moreover, with time, $\vec{N}$ converges to a stable value: in this case nodes are distributed uniformly among server groups as delegated tasks are generated randomly with a uniform 
distribution of $\mathrm{CPO}$ (as said, the algorithm is designed to distribute nodes among the different groups according to the real configuration of delegated tasks).

During the second experiment, once it is proved the proposed algorithm to estimate the value of $\vec{N}$ presents the expected behaviour, we included in the simulation model the Erlang filter to evaluate the utilization factor and the network load. Figure 19 shows the obtained results. As the behaviour of the proposed filter is equal for all server groups, in this case, as can be seen, we are only studying one group. In that way, the performance of the proposed filter may be analysed in a clearer manner.

The operation of the WSN is simulated during twenty-four hours $(24 \mathrm{~h})$, considering a potential network load as showed in Figure 19b. This potential network load is directly obtained from the task delegation rate, which is configured to follow (during the day) a Gaussian-like evolution (with two peak of maximum use). This potential network load generates a potential utilization factor (see Figure 19a), where queues are supposed to be infinite.

Both presented figures are quite similar. The real utilization factor (or network load) follows the demand curve, while it allows guarantee the QoS of task executions. When demand goes above the maximum for which the WSN is designed, the Erlang filter prevents more CPOs from entering into the execution system than allowed per unit of time (exactly as planned). Small and continuous variations may appear due to the fact that the number of allowed CPO is an integer number but the network utilization factor is a real number. Besides, the effect of the slicing window included in the Erlang filter might also generate a high-frequency component.

It can be seen, also, that the execution system presents a delay in respect to the input task flow. The increasing (and decreasing) speed is lower, due to the impact of the filter that softens the variations that appear in the CPO flows.

Figure 20 shows the results of the third experiment. In this new experiment, once the WSN is guaranteed not to be congested, it is evaluated the success rate in task execution, when employing the proposed solution. In this experiment, we are only considering the CPOs (or tasks) that are accepted in the execution system. Those tasks that exceed the maximum allowed rate and were rejected in the Erlang filter are not included in the results of this experiment. All queues in the system are supposed to have the same capacity.

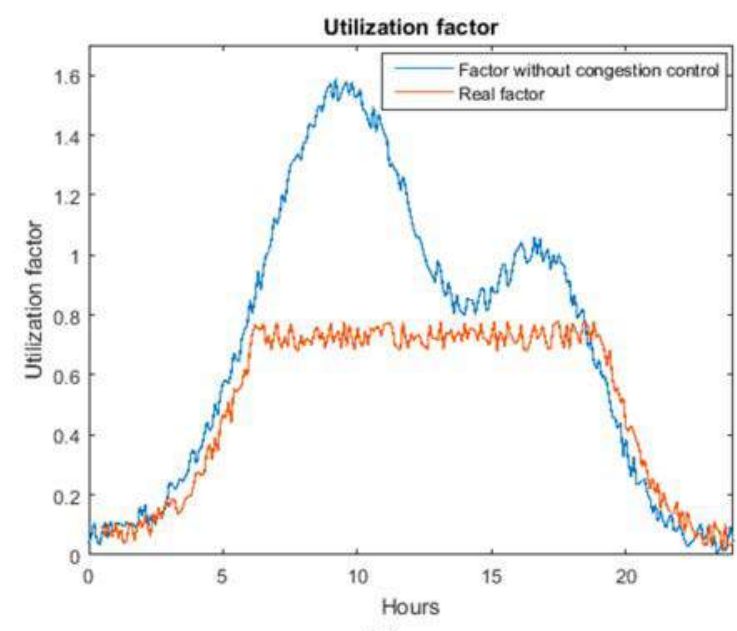

(a)

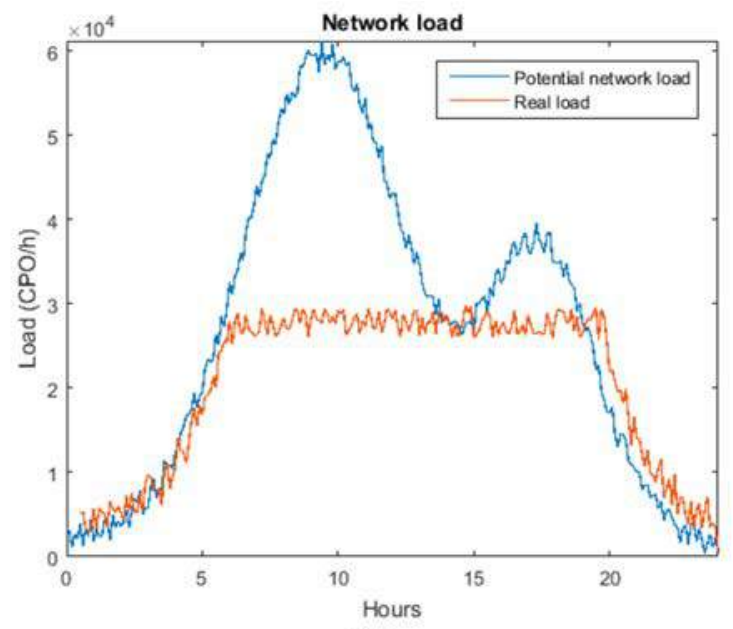

(b)

Figure 19. (a) Utilization factor (b) Network load. 


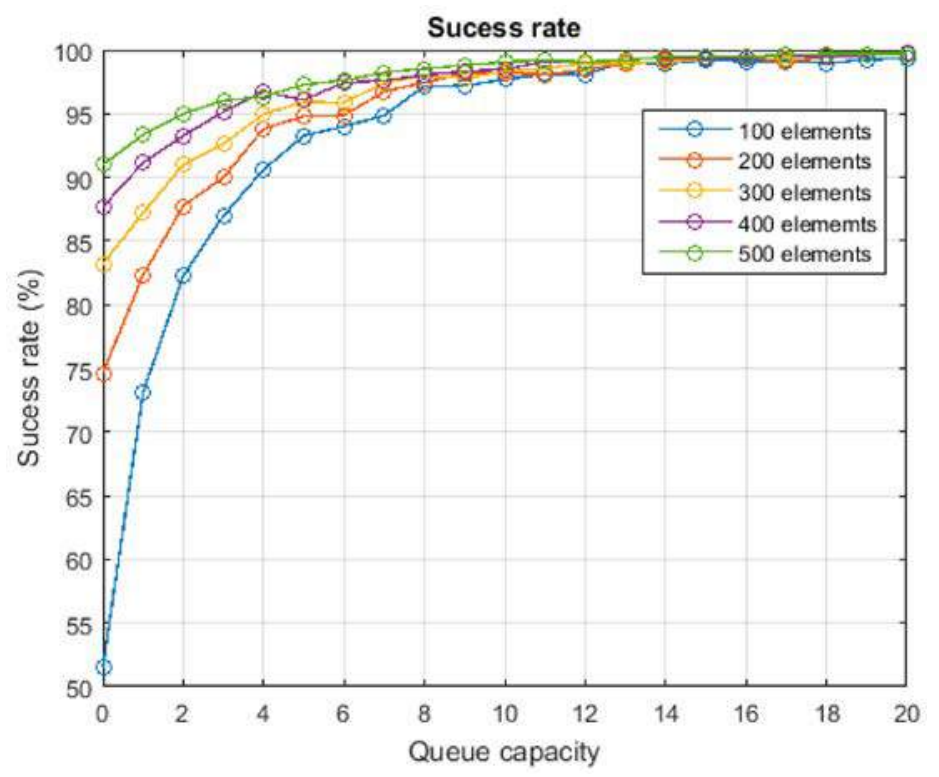

Figure 20. Evolution of the success rate.

As can be seen, if no queue is considered, the success rate is around $52 \%$ when 100 sensors are considered in the WSN. As the number of nodes in the network grows, the success rate also goes up (for 500 nodes around $92 \%$ ). For queues with a capacity of twenty (20) execution orders the success rate grows to almost 100\%, independently from the number of nodes considered in the WSN. Actually, current computing systems may easily support queues with this capacity (considering the 256 different existing server groups, a total storage capacity of 5120 orders will be required).

These results are coherent with the proposed solution. It must be noted that, after being accepted and decomposed, CPO making up a task might be introduced in a queue if all nodes being able to execute that operation are busy. If any queue is full, the corresponding CPO will be rejected and, then, the entire task will be also rejected. In fact, although the maximum hourly delegation rate is not exceeded, it may appear delegation bursts which do exceed the capacity of the sensor network. This situation is more common as the queue capacity gets lower, which reduces dramatically the success rate as seen in Figure 20.

In the context of this experiment, where all values and structures present a uniform configuration (all queues have the same capacity, tasks are composed of random CPO following a uniform distribution, etc.), it can be proved that failures are practically due in full to the loss probability of the queue system (see Figure 10). The success rate will change depending on the composition of tasks and the queue capacity of each sever group. However, these first results show that the proposed solution is a valid mechanism to enable the self-managed and choreographed task execution in sensor networks.

Finally, for the fourth experiment, a simulated hardware implementation of the proposed algorithm was developed. Using this modelling and simulation tools the mean delay when processing an execution order is evaluated. For that, the delay between the reception of the execution order and the generation of the "execution" signal (see Figure 17) was evaluated. The experiment was repeated for different values of the field "required data" (see Figure 21). 


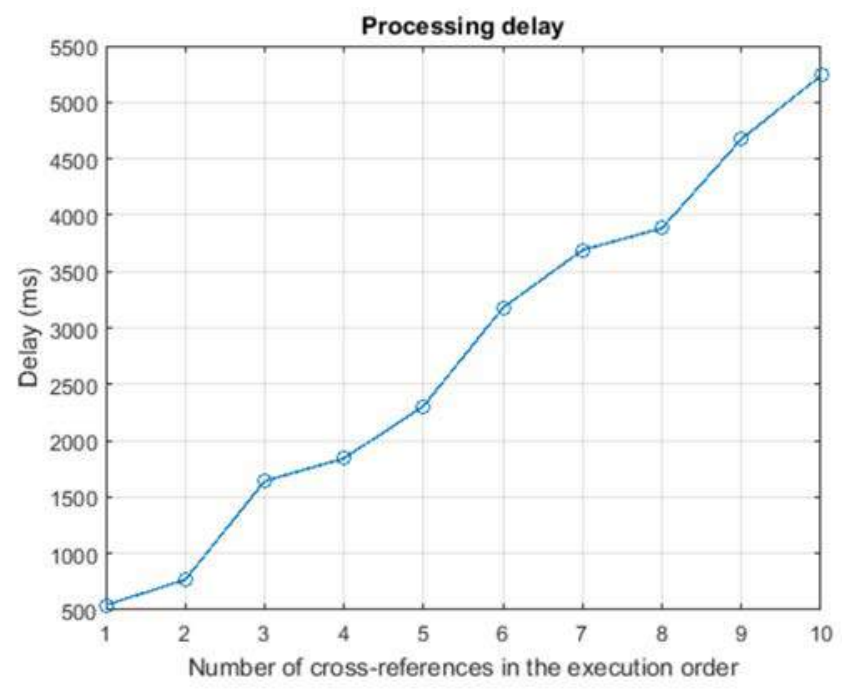

Figure 21. Processing delay.

As can be seen in Figure 21, the processing delay grows linearly with the number of cross-references. In order to guarantee this result and to avoid the WSN congestion (when exponential laws appear), the lightweight gateway must maintain the utilization factor below the unit (as proposed in this paper). In general, the minimum processing delay is slightly above $500 \mathrm{~ms}$ (including all random timers). From Figure 21, besides, it can be deducted that a workflow including ten (10) CPO requires around 6 seconds to be executed using the proposed solution.

ModelSim simulation may be also employed to obtain a footprint of the proposed solution. As the proposed algorithm is not a software solution, the footprint cannot be defined in terms of Flash Memory or SRAM. On the contrary and using the ModelSim capabilities, a good Key Performance Indicator (KPI) is the equivalent number of required logical gateways to implement the proposed hardware-supported algorithm.

Considering the maximum number of possible CPO and standard 16-bit counters, ModelSim software it is requested to generate the equivalent implementation using only logical gates. As different implementations can be selected, we obtained the mean values of all possible results. Considering all previous ideas, the calculated footprint for the proposed algorithm is 396 logical gates.

\section{Conclusions}

Wireless Sensor Networks (WSN) have evolved from a theoretical and research paradigm to be a real and practical technology. Actually, several new engineered systems such as Cyber-Physical Systems are based on WSN. Collaborative or choreographed task execution schemes fit in the most perfect way with the nature of sensor networks, as they enable the creation of flexible ad hoc resource pools being able to execute delegated tasks. Therefore, in this paper it is proposed a new solution for self-managed and choreographed task execution in sensor networks, which is adapted to resource-constraint devices and may be implemented in large WSN.

The solution includes a lightweight gateway where, using the Erlang's queue theory, it is implemented a congestion control algorithm, so it is guaranteed the underlying WSN is always below the congestion level. In this context, choreography algorithms can operate without overloading the network because of the broadcast messages. This gateway also supports virtual nodes-named fictional nodes-employed to monitor and interact with the WSN in a transparent way.

Delegated tasks are filtered in the gateway and transformed into a set of execution orders, represented using a bit-oriented protocol and broadcast messages, so nodes may use a hardware supported algorithm to execute tasks in a choreographed way. In particular, the proposed algorithm includes both, a resource self-management module (where each node may decide if it has enough 
resources to execute a $\mathrm{CPO}$ ) and a choreography module employed to decide which node (among which are able to execute the $\mathrm{CPO}$ ) is finally in charge of the execution.

Results showed that the proposed solution reaches all the proposed objectives, controlling the congestion of the WSN and showing a linear evolution of time with the number of performed CPO.

Acknowledgments: Borja Bordel has received funding from the Ministry of Education through the FPU program (grant number FPU15/03977). Additionally, the research leading to these results has received funding from the Ministry of Economy and Competitiveness through SEMOLA project (TEC2015-68284-R) and from the Autonomous Region of Madrid through MOSI-AGIL-CM project (grant P2013/ICE-3019, co-funded by EU Structural Funds FSE and FEDER).

Author Contributions: The contributions described in this work are distributed among the authors as follows: All the authors wrote the paper; Borja Bordel and Carlos Miguel Nieto contributed to the theoretical formalization and to the proposal and Tomás Robles and Ramón Alcarria proposed the simulation models and performed the experimental validation.

Conflicts of Interest: The authors declare no conflict of interest.

\section{Abbreviations}

The following abbreviations are used in this manuscript:

$\begin{array}{ll}\text { WSN } & \text { Wireless Sensor Networks } \\ \text { QoS } & \text { Quality of Service } \\ \text { CPO } & \text { Cyber-Physical Operation }\end{array}$

\section{References}

1. Bordel, B.; Alcarria, R.; Robles, T.; Martín, D. Cyber-physical systems: Extending pervasive sensing from control theory to the Internet of Things. Pervasive Mob. Comput. 2017, 40, 156-184. [CrossRef]

2. Caragliu, A.; Del Bo, C.; Nijkamp, P. Smart cities in Europe. J. Urban Technol. 2011, 18, 65-82. [CrossRef]

3. Sánchez, B.B.; Alcarria, R.; de Rivera, D.S.; Sánchez-Picot, Á. Enhancing Process Control in Industry 4.0 Scenarios using Cyber-Physical Systems. JoWUA 2016, 7, 41-64.

4. Simbeye, D.S.; Zhao, J.; Yang, S. Design and deployment of wireless sensor networks for aquaculture monitoring and control based on virtual instruments. Comput. Electron. Agric. 2014, 102, 31-42. [CrossRef]

5. Morales, A.; Alcarria, R.; Martin, D.; Robles, T. Enhancing evacuation plans with a situation awareness system based on end-user knowledge provision. Sensors 2014, 14, 11153-11178. [CrossRef] [PubMed]

6. Warneke, B.A.; Pister, K.S. An ultra-low energy microcontroller for smart dust wireless sensor networks. In Proceedings of the 2004 IEEE International Solid-State Circuits Conference, Digest of Technical Papers (ISSCC), San Francisco, CA, USA, 15-19 February 2004; pp. 316-317.

7. Mukhopadhyay, S.; Postolache, O.A. Pervasive and Mobile Sensing and Computing for Healthcare; Springer: Berlin, Germany, 2014.

8. Fortino, G.; Galzarano, S.; Gravina, R.; Li, W. A framework for collaborative computing and multi-sensor data fusion in body sensor networks. Inf. Fusion 2015, 22, 50-70. [CrossRef]

9. Jia, D.; Zhu, H.; Zou, S.; Hu, P. Dynamic cluster head selection method for wireless sensor network. IEEE Sens. J. 2016, 16, 2746-2754. [CrossRef]

10. Tolle, G.; Culler, D. Design of an application-cooperative management system for wireless sensor networks. In Proceedings of the Second European Workshop on Wireless Sensor Networks, Istanbul, Turkey, 2 February 2005; pp. 121-132.

11. Zeng, Y.; Li, D.; Vasilakos, A.V. Real-time data report and task execution in wireless sensor and actuator networks using self-aware mobile actuators. Comput. Commun. 2013, 36, 988-997. [CrossRef]

12. El Emary, I.M.; Ramakrishnan, S. (Eds.) Wireless Sensor Networks: From Theory to Applications; CRC Press: Boca Raton, FL, USA, 2013.

13. Alcarria, R.; Robles, T.; Morales, A.; Cedeño, E. Resolving coordination challenges in distributed mobile service executions. Int. J. Web Grid Serv. 2014, 10, 168-191. [CrossRef] 
14. Lan, S.; Qilong, M.; Du, J. Architecture of wireless sensor networks for environmental monitoring. In Proceedings of the 2008 International Workshop on Geoscience and Remote Sensing. ETT and GRS 2008. International Workshop on Education Technology and Training, 2008, Shanghai, China, 21-22 December 2008; Volume 1, pp. 579-582.

15. McGibney, A.; Rodríguez, A.E.; Rea, S. Managing wireless sensor networks within IoT ecosystems. In Proceedings of the 2015 IEEE 2nd World Forum on Internet of Things (WF-IoT), Milan, Italy, 14-16 December 2015; pp. 339-344.

16. Sheng, Z.; Wang, H.; Yin, C.; Hu, X.; Yang, S.; Leung, V.C. Lightweight management of resource-constrained sensor devices in internet of things. IEEE Internet Things J. 2015, 2, 402-411. [CrossRef]

17. Ruiz, L.B.; Nogueira, J.M.; Loureiro, A.A. Manna: A management architecture for wireless sensor networks. IEEE Commun. Mag. 2003, 41, 116-125. [CrossRef]

18. Robles, T.; Bordel, B.; Alcarria, R.; Martín, D. Mobile Wireless Sensor Networks: Modeling and Analysis of Three-Dimensional Scenarios and Neighbor Discovery in Mobile Data Collection. Adhoc Sens. Wirel. Netw. 2017, 35, 67-104.

19. Alcarria, R.; Bordel, B.; Jara, A. Flexible Service Provision in Context-Aware Cyber-Physical Systems. In Proceedings of the International Conference on Innovative Mobile and Internet Services in Ubiquitous Computing, Torino, Italy, 10-12 July 2017; Springer: Cham, Switzerland, 2017; pp. 873-883.

20. Ganz, F.; Barnaghi, P.; Carrez, F.; Moessner, K. Context-aware management for sensor networks. In Proceedings of the 5th International Conference on Communication System Software and Middleware, Verona, Italy, 4-5 July 2011; ACM: New York, NY, USA, 2011; p. 6.

21. Kang, S.; Lee, Y.; Min, C.; Ju, Y.; Park, T.; Lee, J.; Song, J. Orchestrator: An active resource orchestration framework for mobile context monitoring in sensor-rich mobile environments. In Proceedings of the 2010 IEEE International Conference on Pervasive Computing and Communications (PerCom), Mannheim, Germany, 29 March-2 April 2010; pp. 135-144.

22. Silva, F.A.; Ruiz, L.B.; Braga, T.R.M.; Nogueira, J.M.S.; Loureiro, A.A.F. Defining a Wireless Sensor Network Management Protocol. In Proceedings of the 4th Latin American Network Operations and Management Symposium LANOMS, Porto Alegre, Brazil, 29-31 August 2005; pp. 39-50.

23. Tsiatsis, V.; Kumar, R.; Srivastava, M.B. Computation hierarchy for in-network processing. Mob. Netw. Appl. 2005, 10, 505-518. [CrossRef]

24. Guo, W.; Xiong, N.; Chao, H.C.; Hussain, S.; Chen, G. Design and analysis of self-adapted task scheduling strategies in wireless sensor networks. Sensors 2011, 11, 6533-6554. [CrossRef] [PubMed]

25. Salman, A.; Ahmad, I.; Al-Madani, S. Particle swarm optimization for task assignment problem. Microprocess. Microsyst. 2002, 26, 363-371. [CrossRef]

26. Gerkey, B.P.; Matarić, M.J. A formal analysis and taxonomy of task allocation in multi-robot systems. Int. J. Robot. Res. 2004, 23, 939-954. [CrossRef]

27. Abdelhak, S.; Gurram, C.S.; Ghosh, S.; Bayoumi, M. Energy-balancing task allocation on wireless sensor networks for extending the lifetime. In Proceedings of the 53rd IEEE International Midwest Symposium on Circuits and Systems (MWSCAS), Seattle, WA, USA, 1-4 August 2010; pp. 781-784.

28. Chen, L.; Xue-song, Q.; Yang, Y.; Gao, Z.; Qu, Z. The contract net based task allocation algorithm for wireless sensor network. In Proceedings of the 2012 IEEE Symposium on Computers and Communications (ISCC), Cappadocia, Turkey, 1-4 July 2012; pp. 000600-000604.

29. Xiao, W.; Low, S.M.; Tham, C.K.; Das, S. Prediction based energy-efficient task allocation for delay-constrained wireless sensor networks. In Proceedings of the 6th Annual IEEE Communications Society Conference on Sensor, Mesh and Ad Hoc Communications and Networks Workshops (SECON Workshops' 09), Rome, Italy, 22-26 June 2009; pp. 1-3.

30. Yu, Y.; Prasanna, V.K. Energy-balanced task allocation for collaborative processing in wireless sensor networks. Mob. Netw. Appl. 2005, 10, 115-131. [CrossRef]

31. Jin, Y.; Jin, J.; Gluhak, A.; Moessner, K.; Palaniswami, M. An intelligent task allocation scheme for multihop wireless networks. IEEE Trans. Parallel Distrib. Syst. 2012, 23, 444-451. [CrossRef]

32. Kulkarni, R.V.; Venayagamoorthy, G.K. Particle swarm optimization in wireless-sensor networks: A brief survey. IEEE Trans. Syst. Man Cybern. Part C (Appl. Rev.) 2011, 41, 262-267. [CrossRef]

33. Yang, J.; Zhang, H.; Ling, Y.; Pan, C.; Sun, W. Task allocation for wireless sensor network using modified binary particle swarm optimization. IEEE Sens. J. 2014, 14, 882-892. [CrossRef] 
34. Elbeltagi, E.; Hegazy, T.; Grierson, D. Comparison among five evolutionary-based optimization algorithms. Adv. Eng. Inform. 2005, 19, 43-53. [CrossRef]

35. Serodio, C.; Cunha, J.B.; Morais, R.; Couto, C.; Monteiro, J. A networked platform for agricultural management systems. Comput. Electron. Agric. 2001, 31, 75-90. [CrossRef]

36. Bharti, A.M.A.T. OSI Reference Model. Int. J. 2014, 4, 55-59.

37. Metz, C. IP anycast point-to-(any) point communication. IEEE Internet Comput. 2002, 6, 94-98. [CrossRef]

38. Jain, S.; Fall, K.; Patra, R. Routing in a Delay Tolerant Network. In Proceedings of the 2004 Conference on Applications, Technologies, Architectures and Protocols for Computer Communications (SIGCOMM'04), Portland, OR, USA, 30 August-3 September 2004; ACM: New York, NY, USA, 2004; Volume 34, pp. 145-158.

39. Van Der Aalst, W.M.; Ter Hofstede, A.H. YAWL: Yet another workflow language. Inf. Syst. 2005, 30, $245-275$. [CrossRef]

40. Ter Hofstede, A.H.; van der Aalst, W.M.; Adams, M.; Russell, N. (Eds.) Modern Business Process Automation: YAWL and Its Support Environment; Springer Science \& Business Media: Berlin, Germany, 2009.

41. Kingman, J.F.C.; Atiyah, M.F. The single server queue in heavy traffic. Oper. Manag. Crit. Perspect. Bus. Manag. 2003, 57, 40. [CrossRef]

42. Karlan, D.; Zinman, J. Price and control elasticities of demand for savings. J. Dev. Econ. 2018, 130, 145-159. [CrossRef]

43. Cashin, P.; Mohaddes, K.; Raissi, M.; Raissi, M. The differential effects of oil demand and supply shocks on the global economy. Energy Econ. 2014, 44, 113-134. [CrossRef]

(C) 2018 by the authors. Licensee MDPI, Basel, Switzerland. This article is an open access article distributed under the terms and conditions of the Creative Commons Attribution (CC BY) license (http://creativecommons.org/licenses/by/4.0/). 


\title{
Mobile wireless sensor networks: Modeling and analysis of three-dimensional scenarios and neighbor discovery in mobile data collection
}

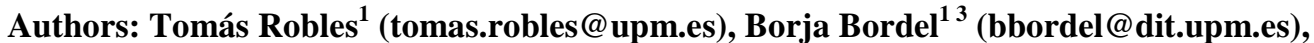 \\ Ramón Alcarria² (ramón.alcarria@upm.es), Diego Martín (diego.martin.de.andres@upm.es) \\ ${ }^{1}$ Departamento de Ingeniería de Sistemas Telemáticos. Universidad Politécnica de Madrid. Avenida Complutense $\mathrm{n}$ 은 \\ 30, "Ciudad Universitaria". 28040 - Madrid (España). Tel. 915495700 ext. 3035 \\ ${ }^{2}$ Departamento de Ingeniería Topográfica y Cartografía. Universidad Politécnica de Madrid. UPM Campus Sur, Km \\ 7.5 de la Autovía de Valencia. 28031 Madrid, Spain. Tel. 915495700 ext. 3035 \\ ${ }^{3}$ Corresponding author.
}

\section{Abbreviated title: Neighbor Discovery in three-dimensional MWSN \\ Keywords: Neighbor discovery; Mobile wireless sensor networks; Mobile data collection; Three- dimensional scenarios; Wireless technologies; Analytical model}

\begin{abstract}
:
WSNs, as important part of the Internet of Things, became an essential field of study in recent years. However, most works use simplified scenarios for its analysis, disregarding variables that affect the data collection process. In this paper, we present a new model for data collection, considering the connection time among the nodes as a work's main part. In addition, a real three-dimensional model is described. The analysis of this new model will allow us to discover new problems for the WSN operation. We start describing both two-dimensional and three-dimensional scenarios, and continue comparing the operation of different types of mobile nodes (such as walking people or drones). Later, we analyze the connection time, the rate and the range of the most used Wireless communication technologies on WSN (like Bluetooth or 802.15.4). Joining both results, we created a model for data collection and analyzed how to build more efficient WSNs.
\end{abstract}

\section{INTRODUCTION}

Data collection process from a set of fixed and distributed nodes is, nowadays, one of the most critical topics in communications research ([1], [2], [3]). Maximizing the transmission capability with the minimum consumption requires a deep study that has not been fully addressed in the literature.

With the incredible improvement of communication technologies and integration capability, new applications and network configurations have been investigated. In particular, the progress of sensor manufacturing techniques and the increase of the wireless communication efficiency enable the birth of the so called Wireless Sensor Networks (WSN) [4].

Initially, WSN were composed by a collection of fixed nodes, connected in a persistent way by means of any wireless communication technology (e.g. WiFi or Bluetooth). The problem of this configuration lies in the fact that these networks usually are isolated, that is, there is often a lack of Internet connectivity (which is required if, for example, we want to deploy services based on the cloud). To solve this situation 
most proposals are focused on adding, at least, one mobile node to WSN [5]. In that way, both elements together are named as Mobile Wireless Sensor Networks (MWSN).

Mobile nodes, as they are moving around, cannot stablish persistent connections with the fixed nodes. This fact provides a dynamic topology to the network. To face that problem, MWSN implement Delay Tolerant Networks (DTN) principles [5].

As an important component of the revolution of the Internet of Things, MWSN allow the deployment of a wide range of applications [6]: from crop control to Smart Grids environment or public transport surveillance. To make these services work properly, the fixed nodes must be capable to realize two actions while one mobile node is inside its coverage area: to stablish one temporal connection with it, and transmit as much stored data as possible.

It is clear that if the connection time (which depends on the wireless technology employed) is too high, there is no time for data collection. That is, in fact, a great problem because, at the end, it can produce the loss of most of the data. For this reason, in the last years, many schemes and algorithms for efficient data transmission (between one fixed node and one mobile node) have been presented [7].

However, all those works are focused either on increasing the transmission rate, or on optimizing the routing algorithms, assuming that, with these improvements, the connection time does not affect the data collection process (it is insignificant compared to total available time) [8]. Furthermore, due to its complexity, three-dimensional scenarios are usually reduced to two-dimensional ones, which is practical, but provides less precise results.

Thus, the novelty of this this work is the proposal and use of a new and more realistic model, for analyzing the data collection process from MWSN. Basically, we improve the model's precision by using a real three-dimensional geometrical study, and by considering the impact of the connection time between sensor network and mobile layer. Finally, a numerical study will allow us detecting new problems and limitations in the deployment and operation of MWSN.

Characterizing the time necessary to stablish the ephemeral connections among the nodes is a task depending on too many variables: the protocol stack, the communication environment, the code optimizations, etc.; for what to verify these assumptions is a really difficult topic. However, if we consider the data link and physical levels, where the number of variables is greatly reduced, it is easier to realize a comparison where clearly appears that, in real scenarios, even only with the neighbor discovery process in those levels, most of the proposed mobile nodes (specially aerial ones) remain in the coverage area too little time to be able to complete the data collection process.

With this philosophy, in this article we propose a comparison in two levels: on the one hand we will study the main mobile nodes considered nowadays (walking people, terrestrial vehicles, drones and Zeppelins). On the other hand, we will obtain the time necessary for neighbor discovery in different wireless technologies, such as Bluetooth, Bluetooth Low-Energy, 802.15.4 and WiFi (only considering the link and physical levels).

From the combination of this information, we will obtain a new model, in which connection time among the nodes does not have to be insignificant, and it is applicable directly in three-dimensional scenarios. With that, our objective is to bring out new challenges in MWSN, such as the use of low-energy technologies in three-dimensional scenarios.

As we will see, only slowest mobile nodes (e.g. walking people) or highest range radio technologies (e.g. WiFi) guarantee the transmission of great quantities of data. In other scenarios, a neighbor discovery process has to be studied because it can be decisive (especially in three-dimensional networks).

The rest of the paper is organized as follows: in Section 2 we present, classify and review the main works over MWSN and neighbor discovery in wireless technologies. Section 3 presents general model 
construction. In Section 4, the two-dimensional and the three-dimensional cinematic models are deducted, the involved variables are presented and the different mobile nodes, and their application to MWSN, are showed and studied. In that way, Section 5 presents a study of wireless communication technologies commonly used on WSN. Section 6 evaluates all the previous deductions and joins the obtained results, whereas Section 7 provides some conclusions and future work.

\section{RELATED WORKS}

In the last years, data collection from MWSN has received a lot of attention [7]. However, most works are focused on network level. For example, there is a wide range of options in relation to the routing protocols: from the ones based on TCP/IP principles [9], to the ones which employ geographical information from the scenario in which the network is placed to select the proper path [10] [11].

Regardless, some works about data collection modeling can be found [12] [13]. With respect to this previous works, we include in this article two main new contributions: on the hand, we consider physical variables such as the mobile node's speed and make our calculations over a real three-dimensional scenario; on the other hand, we take into account the time need for neighbor discovery. Moreover, related articles used to study one specific scenario, generally based on high node density environments [14] [15]. Nevertheless, in these cases, the probability of interference and/or losses is really high, therefore the time needed to stablish a connection also increases, and it may not be possible to disregard it (even if it has done for simplicity). Definitely, we need a model which considers the neighbor discovery process.

Other articles, though, are focused in empirical studies over real devices [16], introducing sometimes new variables as the power consumption [17] or the processing time [18]. In these cases, it is assumed an interference free scenario, where the node density is low enough for only trying to stablish one connection at the same time [19] (if sensors are dispersed, it is reasonable to think that only one node is in the mobile node's coverage area at the same time). Although we are going to study a similar scenario, these works do not often analyze the communication systems or processes, so they do not consider main physical variables as the mobile node speed or the quantity of data that it is possible to transmit. The objective of our work is to contribute to solve this pending challenge.

Furthermore, in the lasts months, the study of neighbor discovery process in MWSN is becoming more important. Some works, as [20], are focused on studying the connection time in MWSN from an electronic point of view (power consumption, shared resources, etc.). Others center new proposals and standard reviews (theoretical and practical), in the field of wireless communications. The objective of these reviews is always reducing the connection time, although this can be made either by adjusting the variable proposed in the standard [21] or modifying the way in which the protocol works [22]. Despite all this, any work has analyzed how these new studies or standards affect the amount of data which can be collected from a WSN. This article tries to fill that void.

Finally, with respect to three-dimensional scenarios, around seven years ago they received a lot of attention, and many articles about their connectivity and coverage problems appeared ([23], [24]). However, nowadays, most articles use projected systems which reduce one dimension of the scenario (becoming it in a two-dimensional one). In this work, we want to return to the initial approach, and we are using a real three-dimensional scenario in which, for first time, neighbor discovery process is going to be considered. Moreover, the concept of three-dimensional MWSN has been really little worked (probably due to the necessity of using physical models to study them), and most works related to three-dimensional scenarios only cover three-dimensional WSN [25] [26] (where mobile nodes are not considered). 
In conclusion, it is greatly difficult to find, in present publications, the knowledge for being able to analyze neighbor discovery in the data collection process from MWSN deployed in three-dimensionalscenarios. We assume, however, that this study is basic to discover new problems in MWSNs, and to deploy networks based on next-generation wireless technologies (as, for example, Bluetooth Low Energy). Therefore, we present a three-dimensional model for mobile data collection, which takes into account the time employed in neighbor discovery.

\section{MODEL CONSTRUCTION}

In this section, we discuss about data collection process in MWSN. We describe the environment where we considered the deployment of the WSN and propose a logical model in three layers for this scenario. Besides, we present the basic assumptions about the fixed and mobile nodes. Finally, in order to identify the variables our model has to take into account, we study the time distribution among the task carried out in the data collection process.

Our scenario is designed for natural environment where sensors are deployed for gathering information such as temperature, humidity, presence, etc. The node density is low, because the natural environment does not justify a higher number of elements. In this initial study we do not consider the transmission of images, video, sounds or other information requiring large amount of data.

We are also assuming we need a constant monitorization of the environment, but we do not need realtime processing (as, for instance, in some agricultural management systems [27]). Therefore, deploying an ad hoc sensor network and getting data collected by a mobile node (one per day, per week... depending on the sensors' memory size, the sensorized area, etc.) is the most efficient and least expensive technical solution. Other solutions, such as including the sensors in the mobile node, are more costly, even unrealizable in practice, so they will not be considered.

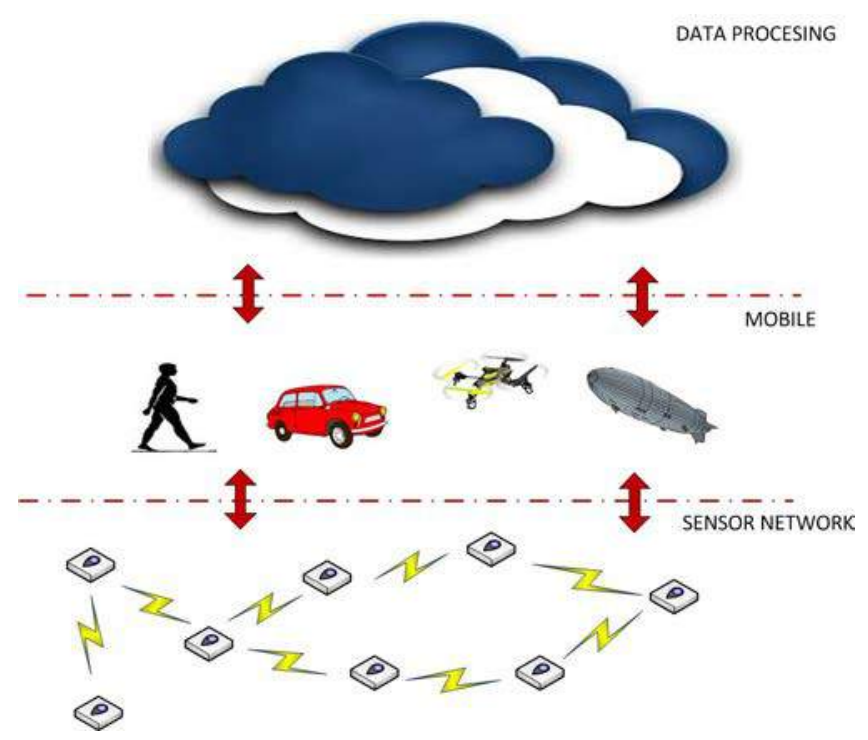

Figure 1: Scenario's logical structure: layers

Three layers are identified in this scenario: sensor network, mobile layer and data processing (Figure 1). 
Sensor network: The sensor network is distributed over a wide area, providing sensors, data gathering and support for wireless communications, but without communication infrastructure. In general, sensors may be distributed in a random way (if all geographical points are equally important) or in a planned way (if some points are more important as, for example, if we want to control the trees in a plantation). Therefore, we cannot guarantee that we know every position of the fixed nodes. In this work we are assuming we do not know neither the sensors' position nor network topology or the number of nodes.

Finally, in relation with the sensors' characteristics, our model allows employing any type of device. That means we do not have to be worried about values such as the sensing range, the sensing period or the monitored variable; because our proposal is independent from sensors' features, and only considers communication parameters.

Mobile layer: considers a mobile device (or mobile node) that is moving around a sensorized area and, using DTNs based communications, collects available sensor data. The mobile node can be single persons, cars (SUV) or air equipment such as drones or airships. This mobile equipment collects information and translates them to the data processing layer.

Due to its position, mobile layer must implement two different communication interfaces. On the one hand, it must be capable of using the sensor's communication technology (in which we focused in this work); and, on the other hand, it has to stablish a successful communication with the processing layer. In relation with this second interface, many technologies can be used, but it seems clearly enough mobile technologies are the most effective solution [28] (especially for its great communication range).

Data processing layer: this layer receives data from mobile layer, stores and processes it. This layer can be based on any standard technology like centralized services or cloud services, because in our scenarios it is not required real time communication neither feedback among sensors and data processors. A deep analysis of all possible solutions should be based on the definition and characterization of different scenarios, but in our case, we are going to work over some common characteristics for all cases, which are:

1- Lack of infrastructure for supporting communications and power supply.

2- WSN deployed for communication with sensors and actuators.

3- Usage of DTN technologies for communication between sensor layer and mobile layer.

4- Usage of specific protocols for stablishing WSN's topology in an autonomous way, and for routing data from the origin to the nodes which can transmit them to the mobile nodes.

5- Modeling of a three dimensional space: we are going to consider mobile nodes at ground level, and others that move on the air such as drones.

6- The movement of the mobile nodes can be defined as: (a) free, when there is not external control; (b) controlled, when it is possible to plan the and route for them and; (c) limited to one or several specific routes independently if they are free or controlled.

The first and second points limit the kind of technologies we can use, and more specifically the lack of power supply limits the service time of the network, which is a key element for the usability of such kind of solutions in real [29].

The third and fourth points explain how the mobile nodes can collect data from sensors. On the one hand, as mobile nodes are not available all time, we must include Delay-Tolerant Network (DTN) technologies to allow reliable and efficient communication. On the other hand, as sensors are distributed in a random way, we cannot guarantee the mobile nodes pass near all fixed nodes. That is why we need a protocol able to identify the sink nodes (those can communicate with the mobile nodes), and able to route data to them when one mobile node appears (and all this with minimal time consumption). Concepts as compression of information may be the key element [30]. 
Finally, the fifth and sixth points are directly generated by the requirements imposed by scenarios for agriculture and natural areas. There, movement of mobile nodes is not limited to the ground, and we can take advantage of drones and other aerial equipment. In addition, there is a great diversity of mobile nodes from tourists or workers performing their specific activities to specific equipment deployed and operated for performing as mobile nodes [10].

In general, any equipment with wireless communication capabilities, when none transmission is running, can behave in two different ways: or it maintains the interface powered and actively hearing for a new communication, or it turns off the communication interface (waiting for a signal which wake it up). In the first case three tasks must be executed: stablish a connection, transmit data and close the connection (see Figure $2 \mathrm{a}$ ). In the second case, to the three previous tasks, one more has to be added: the awakening process (see Figure $2 b$ ).
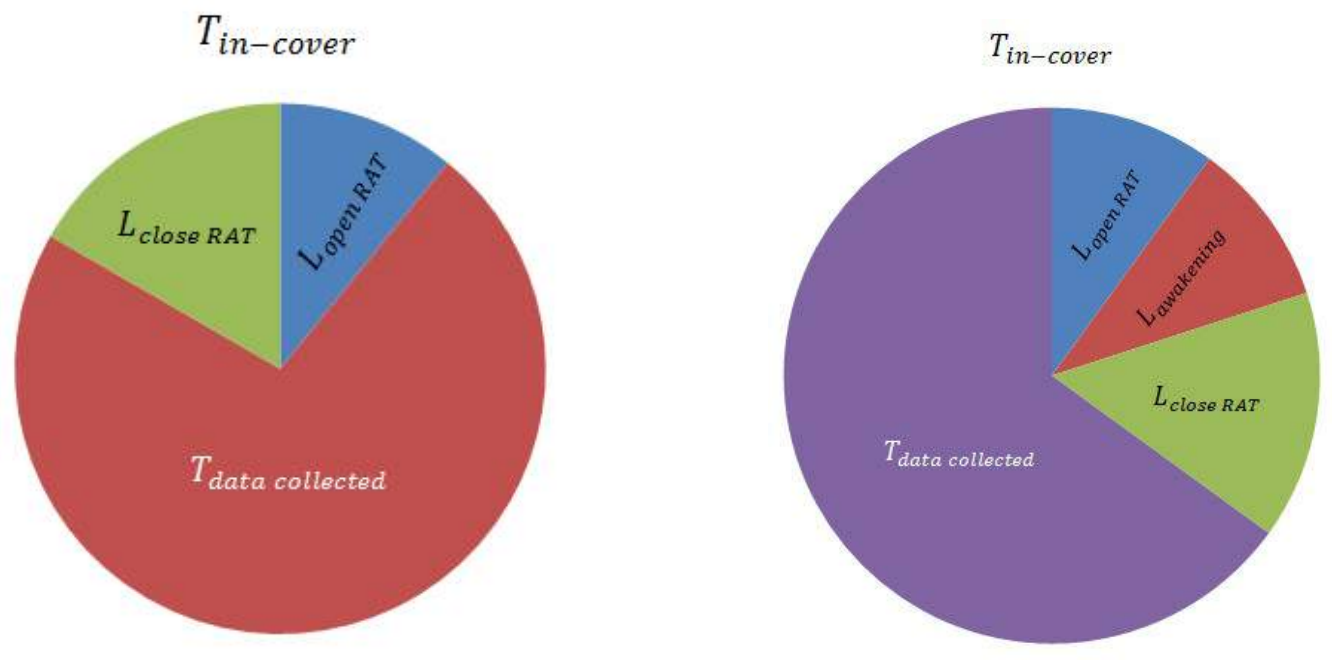

Figure 2: Distribution of the $T_{\text {in-cover }}$ time among different tasks. (a) In the case of keeping the wireless interface on all the time. (b) In the case of turning off the communication module when none transmission is running

Although turning off the communication module is the most efficient behavior in terms of power consumption, not all wireless access technologies support this possibility by default. Then, in order to obtain comparable results, we are going to consider the wireless interfaces of the fixed and mobiles nodes are always available.

The total time in which a fixed sensor and a mobile node can communicate $\left(T_{\text {in-cover }}\right)$ is function, basically, of three variables: maximum range for the Radio Access Technology (RAT) selected, called as $R$; the speed of the physical node $(v(t))$, which can vary with time, and the geometry of the scenario. In Section 4 we will discuss the analytical expression of this function.

The time needed to stablish a connection, $L_{\text {open } R A T}$, depends on the Radio Access Technology selected. Usually, the connection can be considered as established when a physical signal (beacon) is received. However, other times, it is necessary to send a MAC message.

At last, the time for a node to close a connection $\left(L_{\text {close }}\right.$ RAT $)$ depends also on Radio Access Technology selected. Nevertheless, all protocol stacks implement procedures to close a connection even when the connection is down. Therefore, in our model, we are going to suppose fixed nodes close the connection 
when the mobile node is outside their coverage area (in order to exploit the available time to transmit as much data as possible).

Then it is possible to obtain (1).

$$
T_{\text {in-cover }}=T_{\text {data collected }}+L_{\text {open } R A T}
$$

Now, considering the real rate offered, at each moment, at physical layer by the RAT $(C(t))$, we have the total amount of data collected is (2):

$$
\text { Data }_{\text {collected }}=\int_{0}^{T_{\text {data collected }}} C(t) d t
$$

Thus, finally, the expression which models the amount of data it is possible to transmit between a fixed sensor and a mobile node is (3):

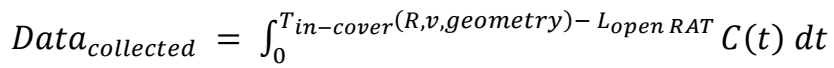

In the next section we are describing the value of $T_{\text {in-cover }}(R, v(t)$, geometry), and in Section 5 we are discussing the value of $C(t)$ and $L_{\text {open } R A T}$.

\section{CINEMATIC MODEL AND MOBILE NODES STUDY}

Once we have described the natural environment and the network logical structure, it is necessary to design a model to calculate the geometrical variables for the mobile nodes movement, and to estimate the total available time. This model includes the structure and number of nodes of the WSN, the number and location of the sensor and actuators, and the number, frequency and speed of the mobile nodes.

We base the physical model on a structure [31], where: the underlying topology on which sensors, mobile nodes and access points are placed is assumed to be a discrete and finite two-dimensional grid; only a fraction of the grid points are occupied by sensors and access points. The access points are modelled to be uniformly spaced on the grid while the sensors are randomly distributed. The mobile node motion is modelled as a simple random walk on the grid; the mobile nodes communicate with the sensors or accesspoints only when they are co-located at the grid points. Mobile nodes do not have problem of memory and we are going to assume in this first analysis that nodes have enough memory space for storing the data.

Specifications over the general initial model:

1- We assume that the mobile nodes can connect directly with the service layer by the use of suitable technology. Therefore, we assume every packet successfully transmitted to the mobile node reaches the service layer.

2- As power resources, components' size and weight and, in general, on board equipment's capabilities are higher than the sensor nodes' resources; we are assuming the uplink is the limiting.

3- We are going to use a three dimensional model as described below 
For ground mobile nodes, we use a two-dimensional model, where the sensor node is stationary and the mobile node moves around it. In the most general case (see Figure 3a), the mobile node will describe a random trajectory. However, our study has local character, and only analyzes the mobile behavior inside the sensors' coverage area. Typically, the absence of infrastructure for supporting power supply causes the coverage area of the sensor nodes is small compared with the total area occupied by the MWSN. Then, we may assume that in the vicinity of the fixed nodes, the trajectory of the mobile nodes is almost linear (see Figure 3b).
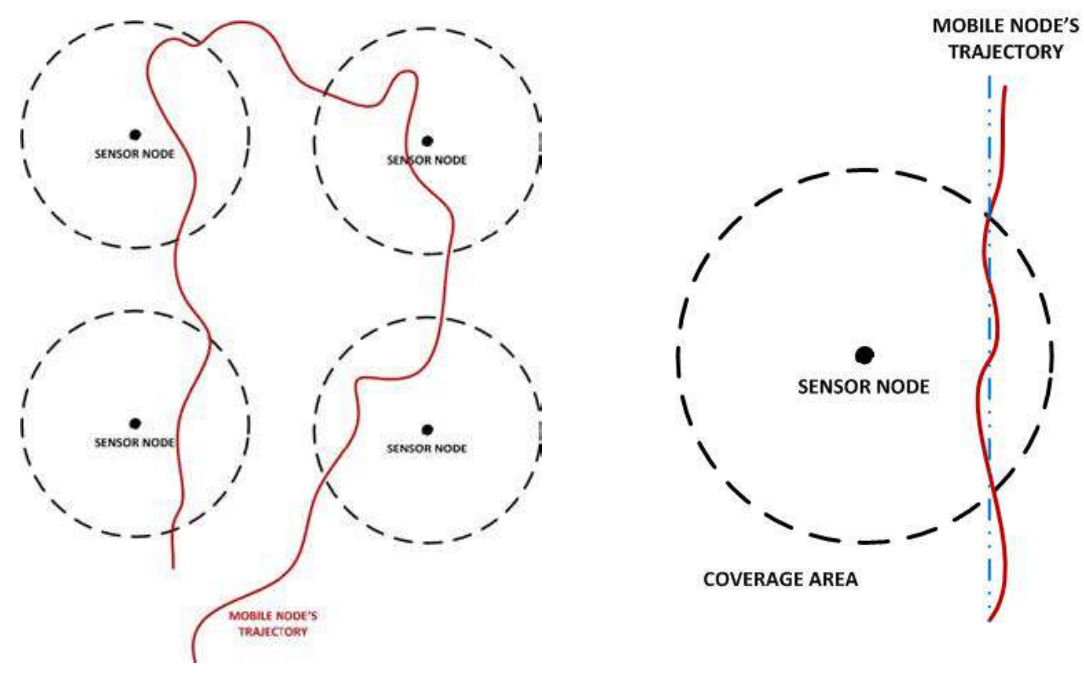

Figure 3: Mobile nodes' trajectory in a two-dimensional scenario. (a) Seeing the whole random trajectory (b) Considering only the almost linear trajectory inside each coverage area

Defining a planar Cartesian coordinate system centered on the position of the sensor (see Figure 4a), the trajectory of the mobile node inside the coverage area can be expressed as a pair of functions varying in time, as in (4).

$$
\overrightarrow{r(t)}=(x(t), y(t))
$$
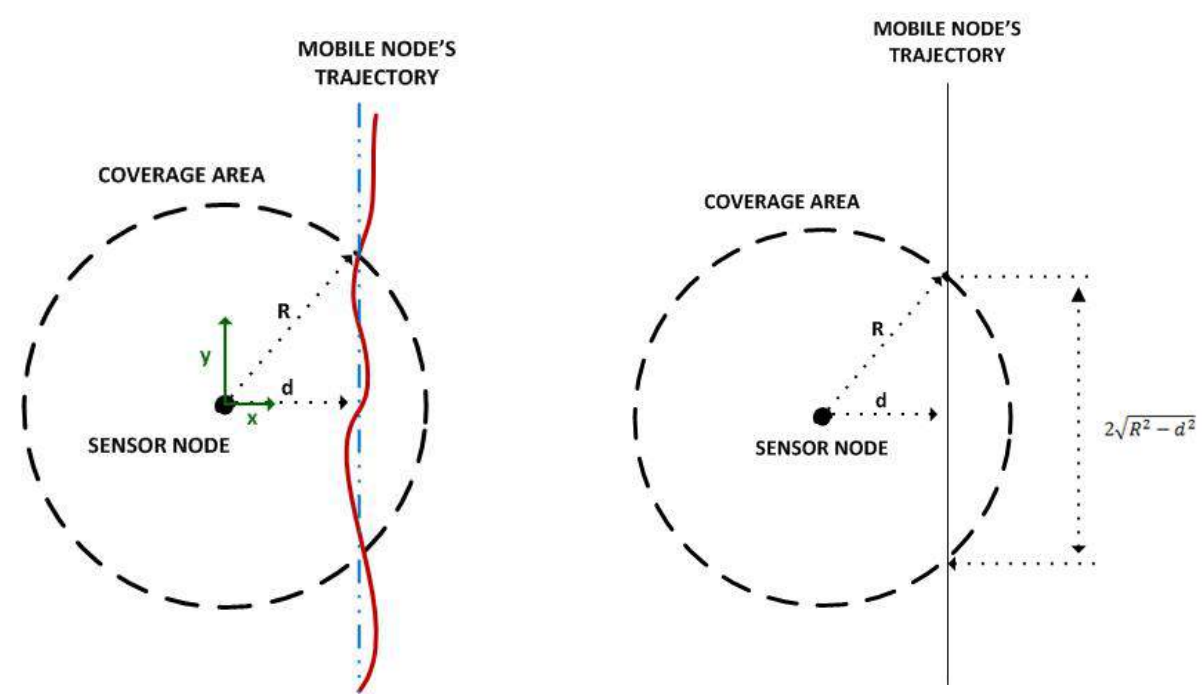

Figure 4: Geometrical scheme for two-dimensional scenarios. (a) Real case (b) Ideal case 
On the one hand, the function $y(t)$ expresses the ideal linear movement at a speed $v_{\text {linear }}(t)$. The mathematical expression of $y(t)$ is $(5)$.

$$
y(t)=\int v_{\text {linear }}(t) d t+K
$$

In the typical case, $v_{\text {linear }}(t)$ will be almost constant, and it would only be affected by small variations due to lack of speed stability of the mobile node. Then, $v_{\text {linear }}(t)$ can be understood as the addition of a constant speed $v$ and a small vibrational movement with random parameters varying in time, as can be seen in (6):

$$
v_{\text {linear }}(t)=v+B(t) \cos (\beta(t) t)
$$

Where $B(t)$ and $\beta(t)$ are stochastic processes.

$B(t)$ and $\beta(t)$ values depends on the speed stability of the mobile node, but in general they will vary smoothly and slowly (compared to the constant speed $v$ ). Therefore, in first approximation, we can consider that, within a sensor node's coverage area, they maintain a fixed value.

$$
\begin{aligned}
& B(t)=\omega_{1} \cdot \varepsilon_{1} \\
& \beta(t)=\omega_{1}
\end{aligned}
$$

The specific values of $\varepsilon_{1}$ and $\omega_{1}$ will be experimentally obtained later for each mobile node.

Then, the final expression of $y(t)$ can be seen in (10):

$$
\begin{gathered}
v_{\text {linear }}(t)=v+\omega_{1} \cdot \varepsilon_{1} \cos \left(\omega_{1} t\right) \\
y(t)=\int v_{\text {linear }}(t) d t+K=v t+\varepsilon_{1} \sin \left(\omega_{1} t\right)+K
\end{gathered}
$$

Where $K$ is an integration constant.

On the other hand, the function $x(t)$ contains the small spurious movements along the second axis (the horizontal axis in our deduction). Thus, the function $x(t)$ can be mathematically expresses as fixed distance modified by a small vibrational movement with random parameters varying in time (11).

$$
x(t)=d+A(t) \sin (\alpha(t) t)
$$

Where $A(t)$ and $\alpha(t)$ are stochastic processes.

$A(t)$ and $\alpha(t)$ values depend on the stability of the mobile node and on how rugged the ground is where the MWSN is deployed. However, as a general consideration, both parameters vary smoothly and, it is foreseeable, slowly (compared to the linear velocity of the mobile node, $v_{\text {linear }}(t)$ ). Therefore, in first approximation, we can consider that, within a sensor node's coverage area, they maintain a fixed value.

$$
\begin{aligned}
& A(t)=\varepsilon_{2} \\
& \alpha(t)=\omega_{2}
\end{aligned}
$$

The specific values of $\varepsilon_{2}$ and $\omega_{2}$ will be experimentally obtained later for each mobile node.

Now, to calculate the $T_{\text {in-cover }}$ time it is necessary to consider the mathematical expression of the coverage area. Considering it as a perfect circle we have that all the point belonging to the coverage area must verify the equation (14):

$$
x^{2}+y^{2} \leq R^{2}
$$


Seeing Figure 4a, we can see there exists two points which belong both to the node's trajectory and to the circumference that limits the coverage area. Considering the time starts running when the mobile node gets in the coverage area, the mobile node will reach these temporal points at (15) and (16):

$$
\begin{gathered}
T_{1}=0 \\
T_{2}=T_{\text {in-cover }}^{2-\text { dim }}
\end{gathered}
$$

Substituting in the expressions for $T_{1}=0$, we can calculate the value of $K$ as in (17).

$$
\begin{array}{r}
x^{2}(0)+y^{2}(0)=d^{2}+K^{2}=R^{2} \\
K=-\sqrt{R^{2}-d^{2}}
\end{array}
$$

Therefore, the trajectory of the mobile nodes, and its total speed, will be defined as in (19) and (20).

$$
\begin{gathered}
\overrightarrow{r(t)}=\left(d+\varepsilon_{2} \sin \left(\omega_{2} t\right), v t+\varepsilon_{1} \sin \left(\omega_{1} t\right)-\sqrt{R^{2}-d^{2}}\right) \\
v(t)=\left(\varepsilon_{2} \omega_{2} \cos \left(\omega_{2} t\right), v+\omega_{1} \varepsilon_{1} \cos \left(\omega_{1} t\right)\right)
\end{gathered}
$$

Finally, substituting in the expressions for $T_{2}=T_{\text {in-cover }}^{2-\text { dim }}$, we can calculate the value of $T_{\text {in-cover }}^{2-\text { dim }}$ following (21) and (22).

$$
\begin{gathered}
x^{2}\left(T_{\text {in-cover }}^{2-\text { dim }}\right)+y^{2}\left(T_{\text {in-cover }}^{2-\text { dim }}\right)=R^{2} \\
\left(d+\varepsilon_{2} \sin \left(\omega_{2} T_{\text {in-cover }}^{2-\text { dim }}\right)\right)^{2}+\left(v T_{\text {in-cover }}^{2-\text { dim }}+\varepsilon_{1} \sin \left(\omega_{1} T_{\text {in-cover }}^{2-\text { dim }}\right)-\sqrt{R^{2}-d^{2}}\right)^{2}-R^{2}=0
\end{gathered}
$$

Solving this transcendent equation in the general case is practically impossible, and numerical methods must be used. However, a simplified expression can be obtained if we consider the ideal case (Figure 4b). In that situation, all the spurious effects are cancelled, and the $T_{\text {in-cover }}^{2-d i m}$ can be directly obtained following eq. (23).

$$
\begin{gathered}
v^{2} T_{\text {in-cover,ideal }}^{2-\text { dim }}-2 v \sqrt{R^{2}-d^{2}}=0 \\
T_{\text {in-cover,ideal }}^{2-\text { dim }}=\frac{2 \sqrt{R^{2}-d^{2}}}{v}
\end{gathered}
$$

For a three dimensional model, the cinematic deduction is pretty similar to the previous one, for twodimensional scenarios. The base geometrical scheme can be seen in Figure 5.

Imaging a three-dimensional Cartesian coordinate system centered on the position of the sensor, the trajectory of the mobile node inside the coverage area can be expressed as a shortlist of functions varying in time, as in (25).

$$
\overrightarrow{r(t)}=(x(t), y(t), z(t))
$$

The expression of $x(t)$ and $y(t)$ is the same than the presented for the two-dimensional case. Then:

$$
\begin{gathered}
y(t)=\int v_{\text {linear }}(t) d t+c t e=v t+\varepsilon_{1} \sin \left(\omega_{1} t\right)+K \\
x(t)=d+\varepsilon_{2} \sin \left(\omega_{2} t\right)
\end{gathered}
$$


Where $\varepsilon_{1}$ and $\omega_{1}$ represent the speed stability of the mobile node; and $\varepsilon_{2}$ and $\omega_{2}$ reflect the horizontal stability of the aerial vehicle.

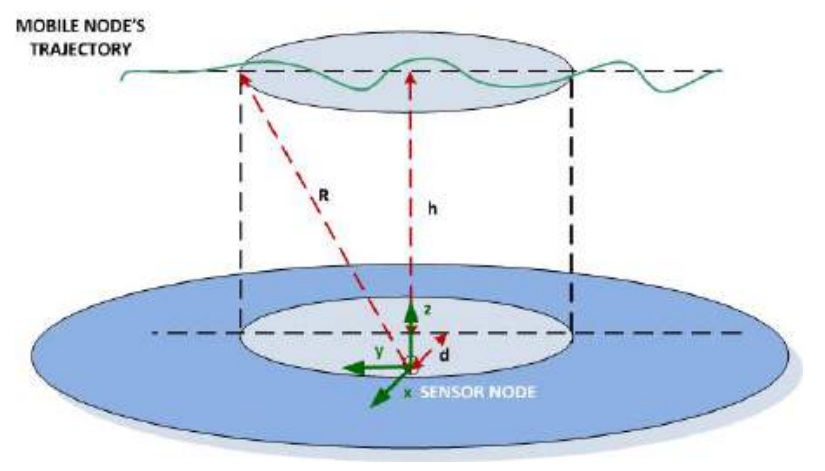

Figure 5: Geometrical scheme for three-dimensional scenarios

Now, to calculate the expression of $z(t)$, we are following a similar process to which we followed to obtain the expression of $x(t)$.

The function $z(t)$ contains the small spurious movements along the third axis (the vertical axis in our deduction). Thus, the function $z(t)$ can be mathematically expresses as fixed altitude modified by a small vibrational movement with random parameters varying in time (28).

$$
z(t)=h+C(t) \sin (\gamma(t) t)
$$

Where $C(t)$ and $\gamma(t)$ are stochastic processes.

$C(t)$ and $\gamma(t)$ values depends on the vertical stability of the aerial node. However, as in the other cases, both parameters vary smoothly and, it is foreseeable, slowly. Therefore, in first approximation, we can consider that, within a sensor node's coverage area, they maintain a fixed value.

$$
\begin{aligned}
& C(t)=\varepsilon_{3} \\
& \gamma(t)=\omega_{3}
\end{aligned}
$$

The specific values of $\varepsilon_{3}$ and $\omega_{3}$ will be experimentally obtained later for each mobile node.

Now, to calculate the $T_{\text {in-cover }}$ time it is necessary to consider the mathematical expression of the coverage area. Considering it as a perfect sphere we have that all point belonging to the coverage area must verify the equation (31).

$$
x^{2}+y^{2}+z^{2} \leq R^{2}
$$

One more time, there are two temporal points (eq. (32) y (33)) in which the trajectory crosses the limit of the coverage area.

$$
\begin{gathered}
T_{1}=0 \\
T_{2}=T_{\text {in-cover }}^{3-\text { dim }}
\end{gathered}
$$

Substituting in the expressions for $T_{1}=0$, we can calculate the value of $K$. See eq. (34)

$$
\begin{gathered}
x^{2}(0)+y^{2}(0)+z^{2}(0)=d^{2}+K^{2}+h^{2}=R^{2} \\
K=-\sqrt{R^{2}-d^{2}-h^{2}}
\end{gathered}
$$


Therefore, the trajectory of the mobile nodes, and its total speed, will be defined as in equations (36) and (37):

$$
\begin{gathered}
\overrightarrow{r(t)}=\left(d+\varepsilon_{2} \sin \left(\omega_{2} t\right), v t+\varepsilon_{1} \sin \left(\omega_{1} t\right)-\sqrt{R^{2}-d^{2}-h^{2}}, h+\varepsilon_{3} \sin \left(\omega_{3} t\right)\right) \\
v(t)=\left(\varepsilon_{2} \omega_{2} \cos \left(\omega_{2} t\right), v+\omega_{1} \varepsilon_{1} \cos \left(\omega_{1} t\right), \varepsilon_{3} \omega_{3} \cos \left(\omega_{3} t\right)\right)
\end{gathered}
$$

Finally, substituting in the expressions for $T_{2}=T_{\text {in-cover }}^{3-\text { dim }}$, we can calculate the value of $T_{\text {in-cover }}$. See (38).

$$
\begin{gathered}
x^{2}\left(T_{\text {in-cover }}^{3-\text { dim }}\right)+y^{2}\left(T_{\text {in-cover }}^{3-\text { dim }}\right)+z^{2}\left(T_{\text {in-cover }}^{3-d i m}\right)=R^{2} \\
\left(d+\varepsilon_{2} \sin \left(\omega_{2} T_{\text {in-cover }}^{3-\text { dim }}\right)\right)^{2}+\left(v T_{\text {in-cover }}^{3-\text { dim }}+\varepsilon_{1} \sin \left(\omega_{1} T_{\text {in-cover }}^{3-\text { dim }}\right)-\sqrt{R^{2}-d^{2}-h^{2}}\right)^{2}+\left(h+\varepsilon_{3} \sin \left(\omega_{3} T_{\text {in-cover }}^{3-d i m}\right)\right)^{2}-R^{2}=0
\end{gathered}
$$

As in the two-dimensional case, if we suppose the trajectory is ideal (a perfect straight line), the transcendent equation can be strongly simplified.

$$
v^{2} T_{\text {in-cover,ideal }}^{3-\text { dim }}-2 v \sqrt{R^{2}-d^{2}-h^{2}}=0
$$

And then, the solution can be expressed as in (41).

$$
T_{\text {in-cover }, \text { ideal }}^{3-\text { dim }}=\frac{2 \sqrt{R^{2}-d^{2}-h^{2}}}{v}
$$

Finally, in three-dimensional scenarios, it is also typical to eliminate the parameter representing the horizontal distance between the flying node and the fixed node $(d)$, due to difficulty of measuring this value. To do that, one value for this parameter (expressed as function of the altitude and the maximum wireless technology rage) is applied. For example, a good value to be employed is the medium value.

The maximum horizontal distance for an aerial vehicle which flies at $h$ meters, and must keep inside a sphere of radius $R$ meters can be calculated following (42).

$$
\begin{gathered}
x^{2}+y^{2}+z^{2}=d_{\max }{ }^{2}+0+h^{2}=R^{2} \\
d_{\max }=\sqrt{R^{2}-h^{2}}
\end{gathered}
$$

And the minimum is elemental to calculate.

$$
d_{\min }=0
$$

Therefore, the medium value is shown in Eq. (45):

$$
d_{\text {medium }}=\frac{\sqrt{R^{2}-h^{2}}}{2}
$$

And, finally, (46) and (47) show the practical expression obtained.

$$
\left(\frac{\sqrt{R^{2}-h^{2}}}{2}+\varepsilon_{2} \sin \left(\omega_{2} T_{\text {in-cover }}^{3-\text { dim }}\right)\right)^{2}+\left(v T_{\text {in-cover }}^{3-\text { dim }}+\varepsilon_{1} \sin \left(\omega_{1} T_{\text {in-cover }}^{3-\text { dim }}\right)-\sqrt{\frac{3}{4}\left(R^{2}-h^{2}\right)}\right)^{2}+\left(h+\varepsilon_{3} \sin \left(\omega_{3} T_{\text {in-cover }}^{3-\text { dim }}\right)\right)^{2}-R^{2}=0
$$

In the general case, or the ideal one:

$$
T_{\text {in-cover, ideal }}^{3-\text { dim }}=\frac{\sqrt{3\left(R^{2}-h^{2}\right)}}{v}
$$


Now, a final element must be considered in our cinematic model: the precision with which $T_{\text {in-cover }}^{2-d i m}$ and $T_{\text {in-cover }}^{3-d i m}$ are obtained. As $T_{\text {in-cover }}^{2-\text { dim }}$ and $T_{\text {in-cover }}^{3-\text { dim }}$ are calculated from secondary variables, it is important to express the precision in time as a function of errors in the secondary variables. This objective is practically impossible in the general case (using eqs. (22) and (46)), and numerical methods should be used to limit the error value. However, in the ideal cases, we would be able to calculate the interval in which the real result is located, from errors made by measuring the others variables (such as speed or altitude). Using error propagation theory, and considering the committed errors are small comparing with the variables' value, we can obtain eqs. (48) and (49).

$$
\begin{gathered}
\Delta T_{2-d i m}=\frac{2}{v}\left(\frac{R}{\sqrt{R^{2}-d^{2}}} \Delta R+\frac{d}{\sqrt{R^{2}-d^{2}}} \Delta d+\frac{\sqrt{R^{2}-d^{2}}}{v} \Delta v\right) \\
\Delta T_{3-\operatorname{dim}}=\frac{\sqrt{3}}{v}\left(\frac{R}{\sqrt{R^{2}-h^{2}}} \Delta R+\frac{h}{\sqrt{R^{2}-h^{2}}} \Delta h+\frac{\sqrt{R^{2}-h^{2}}}{v} \Delta v\right)
\end{gathered}
$$

In the final part of this section, we are going to discuss about the mobile node's speed and the geometrical parameters of our models, in order to evaluate $T_{\text {in-cover }}^{2-d i m}$ and $T_{\text {in-cover }}^{3-\text { dim }}$ In the three-layer model for using MWSN, we can identify different types of mobile nodes, which one can be used in various contexts and address different situations:

1- Walking people: any person carrying one communication device using the suitable technology can perform the task of mobile node. Such person can act as passive walker such as tourist in a natural area, or he/she can follow specific routes or perform controlled tasks. Typically, a walking person has a medium speed of $4 \mathrm{Km} / \mathrm{h}$ [32]. We will use this type of mobile node in home applications (as garden monitoring). Considering a typical garden, it is reasonable supposing a walking person is never further than 2 meters of a sensor.

2- Cars, off-road vehicles and in general any type of vehicle: those vehicles can move through the sensors area loaded with a node for data colleting, and as in the case of walking people, they can follow non deterministic paths or can be controlled for performing specific ways. Controlling quite big semi-urban areas (such as golf courses) is likely the most common application for these mobile nodes. $20 \mathrm{~km} / \mathrm{h}$ is a typical speed for golf carts, and, taking into account the dimensions of the touristic courses (6400 meters), we can consider 10 meters as the minimum distance between a terrestrial vehicle and a sensor.

3- Aerial vehicle and more specifically unmanaged aerial vehicles (drones) can be used for data collection. In this group, we have to distinct two types of drones: plane-type drones (which cannot be stopped on air) and helicopter-type drones (which can remain on air with zero speed). Plane-type drones are commonly used to control great natural areas (such as forest, lakes, etc.), while helicopter-type drone are employed in urban environment (as they can remain in air controlling people, cars, etc.). The speed of these mobile nodes depends on the model selected, but the most usual value for cruise speed is $60 \mathrm{Km} / \mathrm{h}$ for helicopter-type drones, and $80 \mathrm{Km} / \mathrm{h}$ for plane-type drones. Finally, drones' altitude is function of the skyline altitude [2]. Thus, in networks deployed in low-height and large-scale crops, drones can be operated at 5 meters; for monitoring dense forest or metropolitan areas drones may be fly at 10 meters (more or less); and for collecting data from the financial district (where buildings have more than 10 floors), drones must have an altitude around 100 meters.

4- Zeppelins, such type of mobile nodes can be used stay in the air for long time periods or moving over extensive areas with low energy consumption. The most common application of these 
mobile node is verifying the state of the mountain parks (like Rocky Mountain Park), where the mobile node must operate at 3000 meters or more. Their speed, however, due to the environmental risks is much lower than the aerial vehicles' one. Typically, a zeppelin does not move faster than $5 \mathrm{Km} / \mathrm{h}$.

Finally, it is necessary to evaluate the parameters related to the stability $\left(\varepsilon_{1}, \omega_{1}, \varepsilon_{2}, \omega_{2}, \varepsilon_{3}\right.$ and $\left.\omega_{3}\right)$ for each mobile node. To do that, an experimental evaluation has been conducted.

First, an electronic system to determine in a very precise way the position of each mobile node is designed. This module consisted of the following elements (Figure 6 shows an electronic diagram of the module):

- A centrimetric precision GPS module. Recently, small form factor GPS receivers have been developed. In particular, Piksi low-cost GPS receiver, from Swift Navigation [33], has a $53 \times 53 \mathrm{~mm}$ form factor and supports GPS, GLONASS and Galileo signals. By means of this sensor, latitude and longitude of the location of the mobile node can be known with centimetric precision. This device connects to the outside via serial port (UART).

- A barometric sensor. The considered GPS module calculates very precisely latitude and longitude of the location of the mobile node. However, if using aerial nodes, we wish to obtain the same precision in height. For this, a high precision barometric sensor (BMP180 in this case [34]) is included. The device's output interface is serial (I2C).

- An Arduino Nano microcontroller. It receives data from GPS module by UART and from barometric sensor by I2C. It encapsulates both data in an application message and transmits the message by a second UART at 19200 bauds.

- $\quad$ A Bluetooth module. A HC-06 Bluetooth slave module receives data by UART at 19200 bauds and transmits them through a Bluetooth 3.0 interface.

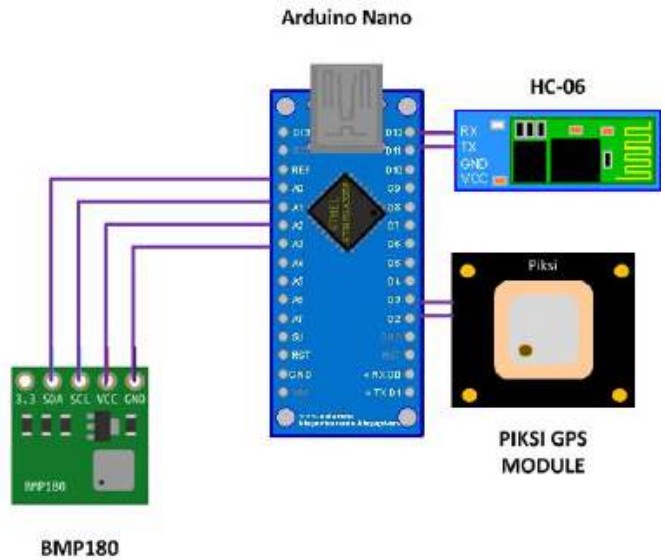

Figure 6: Electronic system to locate the position of the mobile node

Second, a Bluetooth receiver was built and connected to a general purpose computer. This receiver is made of a Bluetooth master module (HC-05 module), a microcontroller and a RS-232 adapter to connect the microcontroller with the computer. When the microcontroller receives a message from the mobile node, it sends it by RS-232 to the computer, where a MATLAB application is listening.

Finally, in the MATLAB implementation, the data are processed and stability values calculated. For that the geographic coordinates (latitude and longitude) are converted to Cartesian XY coordinates. Moreover, 
barometer data are processed to obtain the height of the node as the third Cartesian coordinate Z. Later all sequences are low pass filtered to remove high frequency numerical noise introduced by processing algorithms and sensor accuracy. Then, $\mathrm{X}$ and $\mathrm{Z}$ sample sequences are transformed by means of the FFT algorithm for expression in the frequency domain. The values of horizontal and vertical stability are given by the amplitude and pulsation of the main harmonic. The third sequence ( $\mathrm{Y}$ coordinate according to Figures 4 and 5) is numerically derived for obtaining the linear velocity on said component. Finally, the resulting sequence is transformed by means of the FFT algorithm for expression in the frequency domain. The speed stability is given by the amplitude and pulsation of the main harmonic.

For each mobile node type the experiment was performed 16 times. This number of times has been selected taking into account the experiment's restrictions. The final stability values are the median of all the particular obtained results. Finally, due to the complexity associated with zeppelins, this type of vehicle is not considered in the experiment.

The next table (Table 1) summarizes speed, distance to fixed nodes and the stability results for different mobile nodes.

Table 1: Summary of mobile nodes' characteristics

\begin{tabular}{|c|c|c|c|c|c|c|c|c|}
\hline Mobile node & $\begin{array}{c}\text { Cruise } \\
\text { speed } \\
(\mathrm{m} / \mathrm{s})\end{array}$ & $\begin{array}{l}\text { Minimum } \\
\text { distance* } \\
\quad \text { (m) }\end{array}$ & $\begin{array}{c}\varepsilon_{1} \\
(\mathbf{m})\end{array}$ & $\begin{array}{c}\omega_{1} \\
(\mathrm{rad} / \mathrm{s})\end{array}$ & $\begin{array}{c}\varepsilon_{2} \\
(\mathbf{m} / \mathbf{s})\end{array}$ & $\begin{array}{c}\omega_{2} \\
(\mathrm{rad} / \mathrm{s})\end{array}$ & $\varepsilon_{3}(\mathbf{m})$ & $\begin{array}{c}\omega_{3} \\
(\mathrm{rad} / \mathrm{s})\end{array}$ \\
\hline $\begin{array}{l}\text { Walking person } \\
\text { (WP) }\end{array}$ & 1.1 & 2 & 0.13 & 0.11 & 0.04 & 0.02 & - & - \\
\hline $\begin{array}{l}\text { Terrestrial vehicle } \\
\text { (TV) }\end{array}$ & 5.6 & 10 & 0.27 & 0.04 & 0.01 & 0.02 & - & - \\
\hline $\begin{array}{l}\text { Helicopter-type** } \\
\text { drone at } 5 \mathrm{~m} \mathrm{(H5)}\end{array}$ & \multirow{3}{*}{16.7} & 5 & \multirow{3}{*}{0.32} & \multirow{3}{*}{0.1} & \multirow{3}{*}{0.08} & \multirow{3}{*}{0.5} & \multirow{3}{*}{0.28} & \multirow{3}{*}{0.12} \\
\hline $\begin{array}{c}\text { (Helicopter-type } \\
\text { drone at } 10 \mathrm{~m}) \\
(\mathrm{H} 10)\end{array}$ & & 10 & & & & & & \\
\hline $\begin{array}{c}\text { (Helicopter-type } \\
\text { drone at } 100 \mathrm{~m} \\
(\mathrm{H} 100)\end{array}$ & & 100 & & & & & & \\
\hline $\begin{array}{c}\text { Plane-type } * * * \text { drone } \\
\text { at } 5 \mathrm{~m}(\mathrm{P} 5)\end{array}$ & \multirow{3}{*}{22.2} & 5 & \multirow{3}{*}{0.25} & \multirow{3}{*}{0.01} & \multirow{3}{*}{0.02} & \multirow{3}{*}{0.01} & \multirow{3}{*}{0.09} & \multirow{3}{*}{0.01} \\
\hline $\begin{array}{c}\text { Plane-type drone at } \\
10 \mathrm{~m}(\mathrm{P} 10)\end{array}$ & & 10 & & & & & & \\
\hline $\begin{array}{c}\text { Plane-type drone at } \\
100 \mathrm{~m} \mathrm{(P100)}\end{array}$ & & 100 & & & & & & \\
\hline Zeppelin (DIR) & 1.4 & 3000 & - & - & - & - & - & - \\
\hline
\end{tabular}

\section{WIRELESS TECHNOLOGIES STUDY}

Once we have described the characteristics of the main mobile node types, and the work scenario, in this section we are studying the wireless technologies commonly used on WSN, as well as some variants of them. Then, we will obtain the value of $L_{R A T}$ and model the data rate $C(t)$.

* Minimum distance between the fixed node and the mobile one, along the mobile node trajectory.

** Cruise speed value taken from Drone Aibot X6

*** Cruise speed value taken from E-300 Viewer 
For the scenarios described, we need the wireless technology that consumes as less power as possible. Due to the fact that the nodes lack of a permanent power connection, so we need technologies with a reduce coverage range to be capable of getting this purpose. The study will be focused in personal area networks technologies, to which the $\mathrm{WiFi}$ technology will be added with the objective of stablishing a comparison with the more extended wireless standard. The complete list of the considered technologies is: Bluetooth 3.0, Bluetooth 4.0 (particularly the Low-Energy core or BLE), 802.15.4, a variant of the 802.15.4 standard called Fast 802.15.4 proposed in [22], WiFi, considering two different protocol implementations.

In our calculations we present the particular process of neighbor discovery employed in each technology (process which determines the connection time needed).

\subsection{Neighbor discovery in Bluetooth 3.0 technology}

The neighbor discovery process of Bluetooth 3.0 is the most complex and least efficient. In this version, the neighbor discovery procedure descripted in the standard is called "inquirer process", and supposes that they exist two active devices for all time: on the one hand we find the master (which is looking for new neighbors), and on the other we find the slave (which tries to be discovered to be integrated in one network).

Periodically, the master broadcasts some special packets (named as ID packets), whose mission is to discover new devices. The packets are transmitted using 32 different frequencies, following a procedure known as Frequency Hopping (FH). The FH procedure divides the 32 available channels in two groups of 16 , called trains and labeled as A and B.

In each cycle, four sequences are broadcasted, each one made up for 256 frequency trains. In the first and third frequencies $A$ train is transmitted, and in the second and fourth $B$ train is transmitted. The time necessary to broadcast the ID packets in each channel is one slot of $625 \mu$ s [35], so 16 slots are necessary to transmit one train of frequencies (see Figure 7).

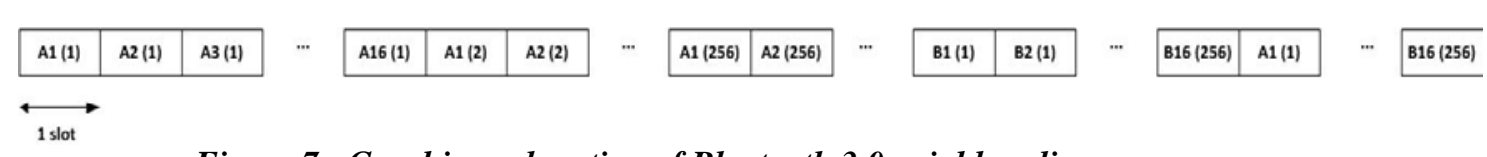

Figure 7: Graphic explanation of Bluetooth 3.0 neighbor discovery process

We suppose now a scenario where there are no interferences, thus, it's reasonable to think the discovery of one new device will be completed in one cycle. Then, if the discovery goes on in the $n$ hop, the total time spent will be (50):

$$
L_{\text {open } B T}=n \cdot t_{\text {slot }}
$$




\subsection{Neighbor discovery in Bluetooth Low-Energy technology}

As we said in Section 5.1, Bluetooth 3.0 neighbor discovery is really expensive in terms of power. Thus, when Bluetooth Special Interest Group decided to create a Low-Energy core for Bluetooth 4.0 (called BLE), they had to propose a totally renovated procedure.

In BLE, again, it exists two types of devices: scanner or initiator (which tries to discover new devices), and the advertiser (which tries to be discovered). The initiator periodically scans three special channels allocated for the advertisement broadcast (labeled as 37, 38 and 39) in a sequential way, looking for advertising packets of others. One the other hand, the advertiser transmits advertising packets in the same channels (using the FH procedure), and wait for an answer sent by the scanner. One the advertiser receives the scanner respond, it is considered the advertiser has been discovered; even the advertiser also has to respond to confirm the connection [36].

BLE standard defines two different procedures for neighbor discovery, depending on the knowledge stored in the scanner: if the scanner knows the advertiser it uses Direct advertising, if not, it uses an Indirect advertising. In our case, as the mobile node is known, we are evaluating the Indirect advertising type.

The time a device that plays the role of advertiser needs to complete an advertising event (the name used in the standard for a search cycle), is computed by (51):

$$
T_{\text {event }}=T_{\text {adver }}+\text { delay }
$$

The $T_{\text {adver }}$ is an integer multiple of $0.625 \mathrm{~ms}$, ranging from $20 \mathrm{~ms}$ to 10.24 seconds, and delay is a pseudo-random value ranging from $0 \mathrm{~ms}$ to $10 \mathrm{~ms}$ (generated by the Link Layer for each advertising event).

Inside each advertising event, for each frequency an advertising packet is sent; later, the advertiser will be listening on the same channel for a while to check if there is response coming from any initiator. According to the specification, the total time spent by channel shall be less than or equal to $10 \mathrm{~ms}$ (see figure 8).

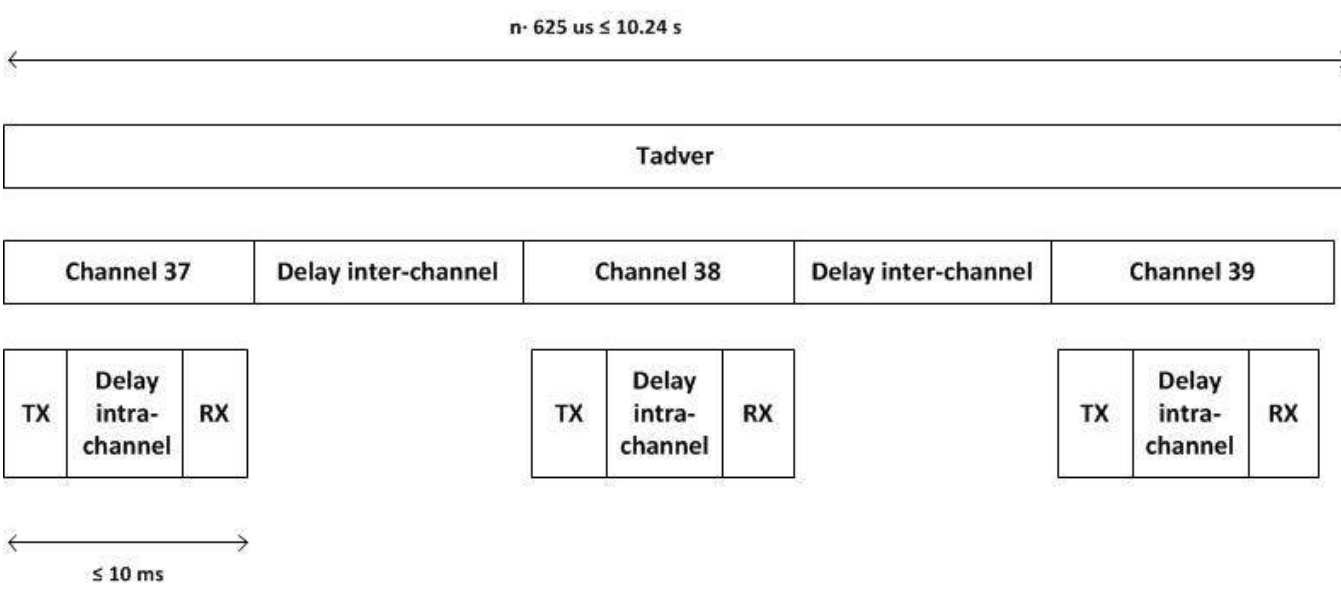

Figure 8: Graphic explanation of BLE neighbor discovery process 
In conclusion, and if we suppose that the time spent by channel is the same for the three frequencies and that there aren't any interferences, the discovery only needs one advertising event. The time necessary to complete a discovery of a device that transmits in the $n$ advertising channel is (52):

$$
L_{\text {open } B L E}=n \cdot\left(T_{t x}+T_{\text {delay intra-channel }}+T_{R X}\right)+(n-1) \cdot T_{\text {delay inter-channel }}
$$

\subsection{Neighbor discovery in 802.15 .4 technology}

Although BLE strongly reduces the power consumption (compared to Bluetooth 3.0), all Bluetooth technologies are designed for transmitting a great data flow, which not agrees at all with the assumptions exposed in Section 2. Therefore, the third technology we are considering is 802.15.4 standard, destined to support low-energy and low-rate communications.

In 802.15.4 standard [37], networks have a hierarchical structure (unlike the Bluetooth technology). Thus, in all networks must be a coordinator responsible for managing the new associations.

The new device has to send an association solicitation to the corresponding coordinator for being incorporated into a network; for which, it is necessary that the new device knows both coordinator's MAC and the frequency where the network is working (among the 16 available channels).

If we suppose the new device has no information about the network, it must scan iteratively the 16 available channels looking for the packets the coordinator broadcasted (where it is included the whole information necessary for building the association message). Once this message is sent, the device is considered to be associated to the network.

In conclusion and supposing the network operates over the $\mathrm{n}$ channel, the time spent in adding a new device to the network is (53):

$$
\begin{gathered}
L_{\text {open 802.15.4 }}=n \cdot t_{\text {scan }}+t_{\text {asso mess }}= \\
=n \cdot \text { aBaseSuperFrameDuration } \cdot\left(2^{B O}+1\right) \cdot t_{\text {sym }}+t_{\text {asso mess }}
\end{gathered}
$$

Where aBaseSuperFrameDuration and $B O$ are two integer parameters.

\subsection{Neighbor discovery in Fast 802.15 .4 technology}

As we can see in (53), connection time in 802.15.4 technology has a linear relation with the channel number where the network coordinator is transmitting. This can be very problematic if we consider a big set of channels, so in the last standard version $(802.15 .4 \mathrm{e})$ the committee has tried to optimize the scan time, although the truth is the impact of these measures is limited.

Therefore, it is so important a standard's modification proposed recently [22], called Fast 802.15.4, which reaches to take a great advantage of the switching frequency capability of 802.15.4 networks. 
They propose, then, to allocate one channel (named as Beacon Channel) to transmit over it the associating messages, and all the information needed to build this solicitation. The others channel will be used for data transmission. In that way, the time necessary to add a new device is fixed, and it is equal to (54):

$$
\begin{gathered}
L_{\text {open fast 802.15.4 }}=t_{\text {scan }}+t_{\text {asso mess }}= \\
=\text { aBaseSuperFrameDuration } \cdot\left(2^{B O}+1\right) \cdot t_{\text {sym }}+t_{\text {asso mess }}
\end{gathered}
$$

\subsection{Neighbor discovery in WiFi technology}

Finally, we are going to analyze neighbor discovery in WiFi technology. With these data, our model will also include LAN technologies, what is basic in order to obtain remarkable results in Section 6.

In 802.11 standard, neighbor discovery (known as Access Point discovery) is performed through the scanning process, in which a mobile node (called mobile station -MS- in this standard) sends a Probe Request management frame and waits for a Probe Response on each channel.

This waiting time is managed by two timers in the scanning process, namely MinChannelTime $(M i n C T)$ and MaxChannel-Time (MaxCT).

If no Probe Response is received before MinCT expires, the MS switches to the next channel and sends a new Probe Request. This may happen if no AP is operating on the channel, if the Probe Response is sent after the timer expiration, or if there is a transmission error. Otherwise, if at least one Probe Response was received, the MS waits for a longer timer (namely MaxCT). In this additional time, the MS is listening for more responses from other Access Points operating in the same channel (see Figure 9).

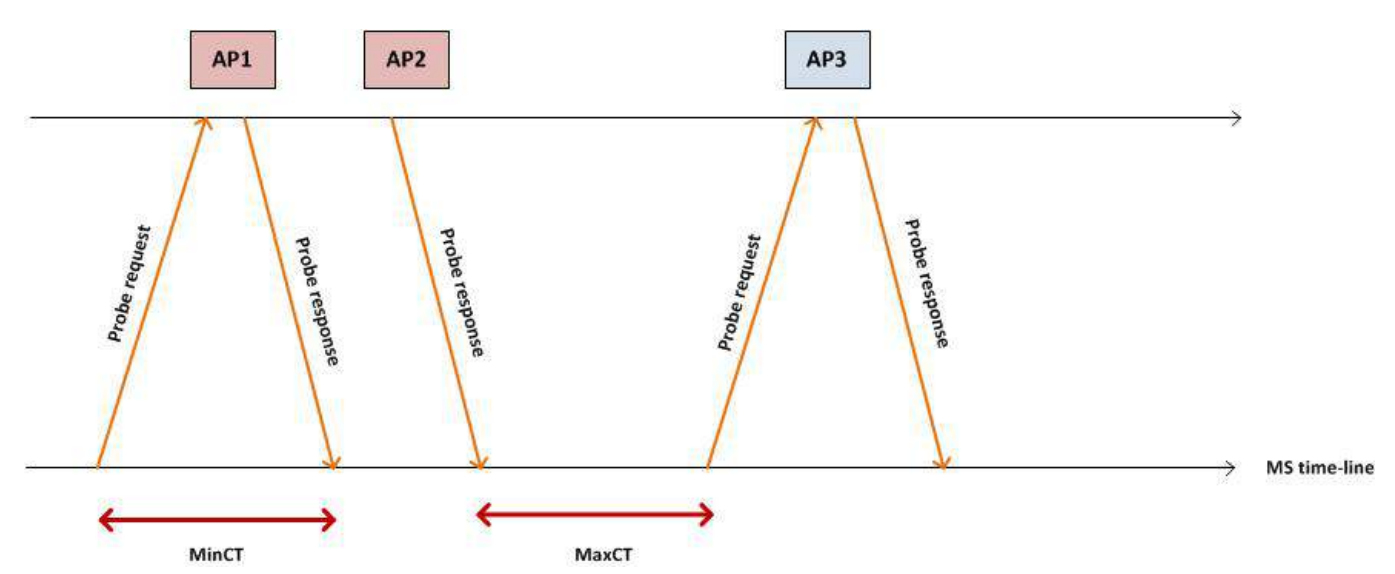

Figure 9: Graphic explanation of WiFi's Access Point Discovery

It is very important to say that although the 802.11 standard proposes 16 available channels, in Europe, only 13 channels are available [38]; so, in the following section we only consider those 13 frequencies.

Taking into account this process, the time spent in locating a new device which uses the $n$ cannel is (55):

$$
L_{\text {open } 802.11}=\operatorname{Max} C T+(n-1) \cdot \operatorname{MinCT}
$$




\subsection{Probabilistic model of $L_{\text {open } R A T}$ delay}

As we saw in Equations (50), (52), (53), (54) and (55) the $L_{\text {open } R A T}$ delay depends on $n$ (the number of the channel where the neighbor device is found). Knowing a priori this value is impossible, since it depends on the specific scenario to be considered: topography of the land, interfering systems, etc. Then, it is a random variable. Therefore, to characterize completely $L_{\text {open } R A T}$ it is not only necessary to show the mathematical expression as a function of $n$, but also the probability of each specific value (i.e. the associated probability density function).

Thus, two different probability density functions must be considered. First, one of the functions represents the probability of finding one device in a specific given channel (called $f_{n}[n]$ onwards). Second, the function that relates the value of $L_{\text {open RAT }}$ calculated for each value of $n$ with the probability of needing this time for finding a neighbor (called $f_{L}[L]$ onwards). Since the channel number is a discrete variable (ranging from $n=1$ to $n$ = number of channels), both probability density functions are also discrete.

The functions $f_{n}[n]$ and $f_{L}[L]$ are not independent, but only $f_{n}[n]$ is independent, being possible to calculate $f_{L}[L]$ from it. In protocols in which both devices which want to communicate are moving along the frequency spectrum (such as Bluetooth 3.0 and Bluetooth Low-Energy), each channel tracking is independent of the previous, so the probability of stopping the neighbor discovery process in a specific channel is equal to the probability of the device to be on that channel. Then, the relation between $f_{n}\left[n_{i}\right]$ and $f_{L}\left[L_{i}\right]$ where $L_{i}$ is the $L_{\text {open RAT }}$ delay suffered if one neighbor is found in the $n_{i}$ channel is (56).

$$
f_{L}\left[L_{i}\right]=f_{n}\left[n_{i}\right]
$$

However, in protocols in which one of the elements remains permanently transmitting in the same channel $(802.15 .4,802.11$, etc.), the $n+1$ channel is evaluated only if the device is not found in the $n$ channel (search is ordered). Thus, the relation between $f_{n}\left[n_{i}\right]$ and $f_{L}\left[L_{i}\right]$ where $L_{i}$ is the $L_{\text {open } R A T}$ delay suffered if one neighbor is found in the $n_{i}$ channel is (57).

$$
\begin{gathered}
f_{L}\left[L_{i}\right]=f_{n}\left[n_{i}\right] \quad \text { if } i=1 \\
f_{L}\left[L_{i}\right]=f_{n}\left[n_{i}\right] \prod_{k=1}^{i-1}\left(1-f_{n}\left[n_{k}\right]\right) \quad \text { if } i>1
\end{gathered}
$$

Finally, $f_{n}[n]$ must be calculated considering the characteristics of the deployment scenario, the sensor programming, etc. For example, if other radio services cover the area of the MWSN, probably it would be necessary to evaluate the interferences following the ITU-R recommendations (P.452 in this case [39]).

\subsection{Upstream radio rate $C(t)$ evaluation}

Finally, the last parameter that we must evaluate on wireless access technologies is the upstream radio rate $C(t)$. The randomness of this parameter (along with its time dependence), makes $C(t)$ in a stochastic 
process, therefore lacks deterministic mathematical expression. Thus, integrating $C(t)$ in time becomes a complicated task, but essential to our model.

In order to partially solve this problem, our model only considers the expected value of the Data $a_{\text {collected }}$ parameter. However, for calculating this value some considerations have to be taken into account. First, let's consider $C(t)$ as a stationary stochastic process, i.e. the probability distribution is the same for every instant of time. Additionally, as it is common in communications systems, we will assume that $C(t)$ has ergodic character (thus the process statistic parameters are invariant over time).

Now, we calculate the expected value of the Equation (3)

$$
\mathbb{E}\left[\text { Data }_{\text {collected }}\right]=\mathbb{E}\left[\begin{array}{ll}
\int_{0}^{T \text { in-cover }}(R, v, \text { geometry })-L_{\text {open } R A T} & C(t) d t
\end{array}\right]
$$

Considering the definition of the Riemann integral we can obtain expression (59)

$$
\begin{aligned}
& \mathbb{E}\left[\int_{0}^{T_{\text {in-cover }}(R, v, \text { geometry })-L_{\text {open }} R A T} C(t) d t\right]= \\
& =\mathbb{E}\left[\lim _{n \rightarrow \infty} \frac{T_{\text {in-cover }}(R, v, \text { geometry })-L_{\text {open } R A T}}{n} \sum_{k=1}^{n} C\left(\frac{k\left(T_{\text {in-cover }}(R, v, \text { geometry })-L_{\text {open } R A T}\right)}{n}\right)\right]
\end{aligned}
$$

Being the expected operator continuous and linear, it can be interchangeable with the limit operator. Moreover, as $L_{\text {open } R A T}$ and $C(t)$ are physical independent processes, they are also independent statistics variables. Then it is possible to obtain expression (60).

$$
\begin{gathered}
\mathbb{E}\left[\lim _{n \rightarrow \infty} \frac{T_{\text {in-cover }}(R, v, \text { geometry })-L_{\text {open } R A T}}{n} \sum_{k=1}^{n} C\left(\frac{k\left(T_{\text {in-cover }}(R, v, \text { geometry })-L_{\text {open } R A T}\right)}{n}\right)\right]= \\
=\lim _{n \rightarrow \infty} \frac{T_{\text {in-cover }}(R, v, \text { geometry })-\mathbb{E}\left[L_{\text {open } R A T}\right]}{n} \sum_{k=1}^{n} \mathbb{E}\left[C\left(\frac{k\left(T_{\text {in-cover }}(R, v, \text { geometry })-L_{\text {open } R A T}\right)}{n}\right)\right]
\end{gathered}
$$

Furthermore, as $C(t)$ is a stationary stochastic process, the expected value is constant in time. Thus, the expression (61) becomes true.

$$
\mathbb{E}\left[C\left(\frac{k\left(T_{\text {in-cover }}(R, v, \text { geometry })-L_{\text {open } R A T}\right)}{n}\right)\right]=\mathbb{E}\left[C\left(\frac{k\left(T_{\text {in-cover }}(R, v, \text { geometry })-\mathbb{E}\left[L_{\text {open } R A T}\right]\right)}{n}\right)\right]
$$

Joining expressions (58), (59) and (61) we can obtain expression (62):

$$
\begin{aligned}
& \mathbb{E}\left[\int_{0}^{T_{\text {in-cover }}(R, v, \text { geometry })-L_{\text {open } R A T}} \quad C(t) d t\right]= \\
& =\lim _{n \rightarrow \infty} \frac{T_{\text {in-cover }}(R, v, \text { geometry })-\mathbb{E}\left[L_{\text {open } R A T}\right]}{n} \sum_{k=1}^{n} \mathbb{E}\left[C\left(\frac{k\left(T_{\text {in-cover }}(R, v, \text { geometry })-\mathbb{E}\left[L_{\text {open } R A T}\right]\right)}{n}\right)\right]
\end{aligned}
$$

And considering one more time the definition of the Riemann integral (see (63))

$$
\begin{aligned}
& \mathbb{E}\left[\int_{0}^{T_{\text {in-cover }}(R, v, \text { geometry })-L_{\text {open } R A T}} C(t) d t\right]= \\
& =\int_{0}^{T_{\text {in-cover }}(R, v, \text { geometry })-\mathbb{E}\left[L_{\text {open } R A T}\right]} \mathbb{E}[C(t)] d t
\end{aligned}
$$

Integrating expression (63) and considering Equation (58), it is possible to calculate the expression (64). 


$$
\mathbb{E}\left[\text { Data }_{\text {collected }}\right]=\mathbb{E}[C(t)]\left(T_{\text {in-cover }}(R, v, \text { geometry })-\mathbb{E}\left[L_{\text {open } R A T}\right]\right)
$$

The value of $\mathbb{E}\left[L_{\text {open }} R A T\right]$ can be calculated from the discussions presented in the previous subsections, but $\mathbb{E}[C(t)]$ remains unclarified.

In general, to estimate $\mathbb{E}[C(t)]$ several realizations of $C(t)$ would be necessary, but as the process is supposed to be ergodic, the expected value can be replaced (since it coincides in value) by a time average (so that even a single embodiment can characterize the process).

To obtain the expected value of the upstream radio rate in all radio technologies described above, another experiment was conducted. Twelve fixed nodes, implementing two to two the same radio technology, were deployed in an agricultural environment (Figure 10). All nodes included only a program being able to calculate the instantaneous rate by performing a continuous data sending. So, it can be assumed that the calculated value perfectly matches the rate at radio level. Each couple of nodes was exchanged data for two hours, measuring the upstream radio rate each 500ms (in total they were taken around 14500 measurements).
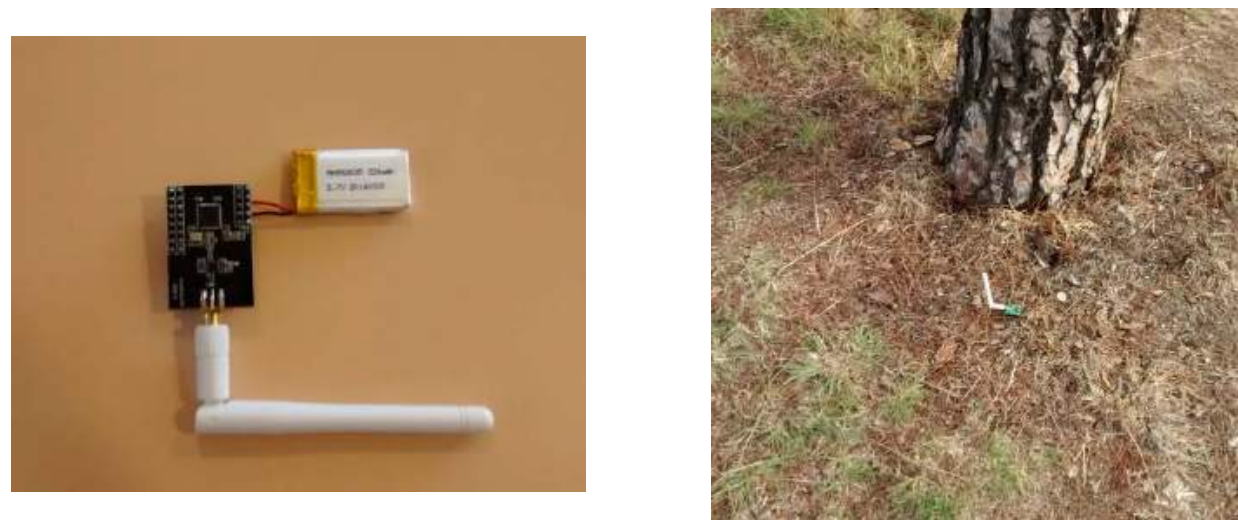

Figure 10: (a) Picture of one of the fixed node deployed. (b) Deployment scenario

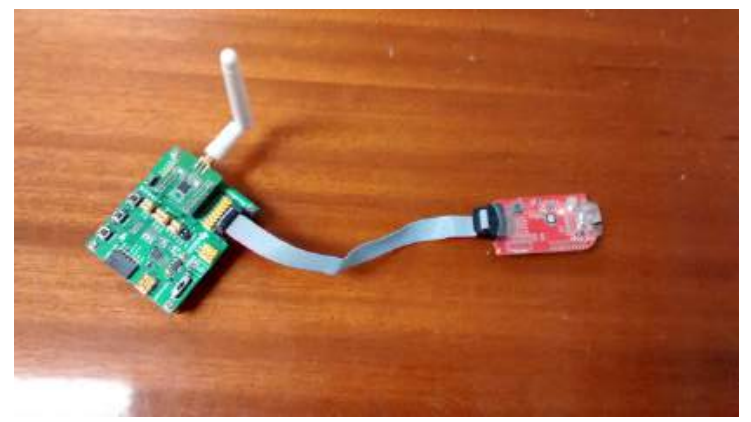

Figure 11: Data downloading by means a debugger board

At the end of the experiment, data was gathered by means a debugger board (Figure 11) and processed using numerical calculation software. Table 2 shows the expected value, the most probable value and the standard deviation obtained for each radio access technology. 
Table 2: Statistics description of the upstream radio rate for each $R A T$

\begin{tabular}{|c|c|c|c|}
\hline Radio technology & $\begin{array}{c}\text { Expected } \\
\text { value } \\
\text { (Mbps) }\end{array}$ & $\begin{array}{c}\text { Standard } \\
\text { Deviation } \\
\text { (Mbps) }\end{array}$ & $\begin{array}{c}\text { Most } \\
\text { probable } \\
\text { value } \\
\text { (Mbps) }\end{array}$ \\
\hline Bluetooth 3.0 & 0.844 & 0.03 & 0.852 \\
\hline Bluetooth Low-Energy & 0.874 & 0.04 & 0.861 \\
\hline 802.15 .4 & 0.205 & 0.01 & 0.207 \\
\hline Fast 802.15.4 & 0.204 & 0.01 & 0.204 \\
\hline WiFi (fixed temporizers) & 8.774 & 0.97 & 9.057 \\
\hline WiFi (Smart temporizers) & 8.894 & 1.11 & 8.855 \\
\hline
\end{tabular}

\section{MODEL UTILIZATION. ANALYTICAL EVALUATION}

In this section, we are going to join the results of the previous sections to create the final model. We are also describing the process which must be followed in order to obtain an estimation of the total amount of data collected. Later, we are proposing typical values for the parameters in the model to obtain some numerical results. With the study of these results new problems and limitations for the MWSNs will arise.

\subsection{Final model. Model utilization}

Considering (64), the final model can be expressed as (65).

$$
\begin{gathered}
\mathbb{E}\left[\text { Data }_{\text {collected }}\right]=\mathbb{E}[C(t)]\left(T_{\text {in-cover }}^{i-\text { dim }}-\mathbb{E}\left[L_{\text {open RAT }}\right]\right) \\
i \in\{1,2\} \\
R A T \in\{B T, B L E, 802.15 .4, \text { fast } 802.15 .4,802.11\}
\end{gathered}
$$

To obtain an estimation of the data collected by means of the expression (65), the following steps must be followed.

1- Having chosen the deployment scenario, the radio access technology for the communications and the type of mobile node which will be used as data mule, said mobile node must be characterized in relation to its horizontal stability, speed stability (and vertical stability, if an aerial node). Values such as the minimum distance at which the mule will be from the fixed sensors must be also obtained. In Section 4 we described this process (and presented some results) for some typical mule types. 
2- With the parameters calculated in the previous step, and expressions (22) and (46) (depending on if we consider a two-dimensional scenario or a three-dimensional one), the value of $T_{\text {in-cover }}^{i-\operatorname{dim}}$ may be numerically calculated.

3- Known the geometrical parameters of the deployment, a radio evaluation of the scenario must be performed. With it, and knowing the RAT employed, the probability function to find a node in a channel $\left(f_{n}[n]\right)$ may be estimated.

4- Considering the calculated $f_{n}[n]$ the probability density function $f_{L}[L]$ may be obtained. In Section 5 (expression (57)) the process is described.

5- A deep analysis of the hardware and programming employed in the fixed nodes must be conducted in order to estimate the parameters which appear in the expression of the neighbor discovery delay for the selected RAT. Taking them and $f_{L}[L]$ into account, the value of $\mathbb{E}\left[L_{\text {open } R A T}\right]$ can be calculated.

6- In order to know the value of $\mathbb{E}[C(t)]$, a temporal evaluation of the evolution of upstream radio rate should be performed. The time average of this evaluation will give us the sought value. In Section 5 we described this process (and presented some results) for the considered RAT.

7- Applying expression (65) the amount of collected data may be calculated.

\subsection{Analytical evaluation}

In this section, we are obtaining the amount of collected data using the proposed model, for all combination of the selected RAT and considered mobile nodes.

In Section 4, the stability of the considered mobile nodes was evaluated. In Section 5, the value of $\mathbb{E}[C(t)]$ for all selected RAT was also calculated. Then, to be able to evaluate the amount of data collected it is necessary, firstly, to fix the variables' values in the neighbor discovery equations (expression (50), (52), (53), (54) and (55)).

Bluetooth 3.0 technology fixes all the values of neighbor discovery process' parameters, so it is enough with the information given in Section 5.

On the contrary, in BLE technology the standard describes the neighbor discovery as a function of several variables (see expression (52)). For them, it gives only a maximum value. So, in order to fix these variables' values, we are going to employ as reference the BLE implementation used in the CC2540 device from Texas Instruments (the most common system-on-chip for this technology). The specific values are (see table 3): 
Table 3: Parameters' value for BLE

\begin{tabular}{|c|c|}
\hline Parameter & Value $(\mu \mathbf{s})$ \\
\hline$T_{t x}$ & 380 \\
\hline$T_{\text {delay intra-channel }}$ & 105 \\
\hline$T_{R X}$ & 115 \\
\hline$T_{\text {delay inter-channel }}$ & 150 \\
\hline
\end{tabular}

Considering, 802.15.4 and Fast 802.15.4 solutions, Table 4 contains all necessary data to calculate the results [13]. These values have been set comparing various implementations of 802.15.4 protocol, such as IEEE 802.15.4 from Texas Instruments, open-ZB or ZBOSS.

Table 4: Parameters' value for 802.15.4 and Fast 802.15.4

\begin{tabular}{|c|c|}
\hline Parameter & Value \\
\hline aBaseSuperFrameDuration & 960 \\
\hline BO & 3 \\
\hline Bits per symbol & 4 \\
\hline Nominal radio data rate & $250 \mathrm{Kbps}$ \\
\hline Packet size & 50 Bytes \\
\hline
\end{tabular}

Determining the temporizer values of WiFi technology, the standard does not suggest any solution so the selected policy for setting these values strongly impacts in the results. For this reason, we are studying two different solutions: the first one based on fixed values for the temporizers; and the second one based on a smart algorithm for selecting the values for each channel.

For the first implementation we have selected a value of $15 \mathrm{~ms}$ for the temporizers (most common commercial value), and for the second proposal we are going to use the algorithm described in [12].

Later, it is necessary to estimate the probability distribution function $f_{n}[n]$. In this case, due to the fact that there are no interferences in the environment, we can suppose all channel have the same probability. Therefore, $f_{n}[n]$ is a uniform probability distribution between $n=1$ and $n=N$ (both included) where $N$ is the number of the available channels. Mathematically:

$$
f_{n}[n]=\frac{1}{N}(u[n]-u[n-N-1])
$$

Being $u[n]$ is the Heaviside-step function of discrete type. 
Now, we may obtain the value of $\mathbb{E}\left[L_{\text {open } R A T}\right]$. In Table 5 , we calculate the main values that can be obtained from the random variable $L_{\text {open }} R A T$. There, we also show the expected value and nominal value of the upstream radio rate $(\mathbb{E}[C(t)])$ and the maximum range $(R)$ for every RAT. As we saw, to obtain these values we supposed a low-density natural environment and a standard transmission power (considering the nodes implement a system-on-chip, as CC25XX series from Texas Instruments).

Table 5: Numerical results of the connection time, rate and range for the considered Wireless Technologies

\begin{tabular}{|c|c|c|c|c|c|c|c|c|c|}
\hline $\begin{array}{c}\text { Wireless } \\
\text { Technology }\end{array}$ & $\begin{array}{c}\text { Minimum } \\
\text { connection } \\
\text { time }\end{array}$ & $\begin{array}{c}\text { Maximum } \\
\text { connection } \\
\text { time }\end{array}$ & $\begin{array}{c}\text { Medium } \\
\text { connection } \\
\text { time }\end{array}$ & $\begin{array}{c}\text { Most } \\
\text { probable } \\
\text { connection } \\
\text { time }\end{array}$ & Median & $\begin{array}{l}\text { Standard } \\
\text { Deviation }\end{array}$ & $\begin{array}{c}\text { Expected } \\
\text { upstream } \\
\text { Radio rate }\end{array}$ & $\begin{array}{c}\text { Nominal } \\
\text { upstream } \\
\text { Radio rate }\end{array}$ & $\begin{array}{c}\text { Maximum } \\
\text { range }(R)\end{array}$ \\
\hline Bluetooth 3.0 & $625 \mu \mathrm{s}$ & $10.24 \mathrm{~s}$ & $5.12 \mathrm{~s}$ & --- & $5.12 \mathrm{~s}$ & $3.6 \mathrm{~s}$ & $844 \mathrm{kbps}$ & $1 \mathrm{Mbps}$ & $10 \mathrm{~m}$ \\
\hline BLE & $600 \mu \mathrm{s}$ & $2.1 \mathrm{~ms}$ & $1.35 \mathrm{~ms}$ & --- & $1.35 \mathrm{~ms}$ & $612 \mu \mathrm{s}$ & $874 \mathrm{kbps}$ & $1 \mathrm{Mbps}$ & $50 \mathrm{~m}$ \\
\hline 802.15 .4 & $0.5 \mathrm{~s}$ & $2.7 \mathrm{~s}$ & $952.21 \mathrm{~ms}$ & $0.5 \mathrm{~s}$ & $1.67 \mathrm{~s}$ & $1.027 \mathrm{~s}$ & $205 \mathrm{kbps}$ & $250 \mathrm{kbps}$ & $70 \mathrm{~m}$ \\
\hline Fast 802.15.4 & $628.24 \mathrm{~ms}$ & $628.4 \mathrm{~ms}$ & $628.24 \mathrm{~ms}$ & $628.24 \mathrm{~ms}$ & $628.24 \mathrm{~ms}$ & 0 & 204 kbps & $250 \mathrm{kbps}$ & $70 \mathrm{~m}$ \\
\hline $\begin{array}{l}\text { WiFi (fixed } \\
\text { temporizers) }\end{array}$ & $15 \mathrm{~ms}$ & $210 \mathrm{~ms}$ & $62.94 \mathrm{~ms}$ & $15 \mathrm{~ms}$ & $195 \mathrm{~ms}$ & $70.14 \mathrm{~ms}$ & 8.9 Mbps & $11 \mathrm{Mbps}$ & $100 \mathrm{~m}$ \\
\hline $\begin{array}{l}\text { WiFi (smart } \\
\text { temporizers) }\end{array}$ & $15 \mathrm{~ms}$ & $121 \mathrm{~ms}$ & $6.9 \mathrm{~ms}$ & $15 \mathrm{~ms}$ & $93 \mathrm{~ms}$ & $5.49 \mathrm{~ms}$ & 8.8 Mbps & $11 \mathrm{Mbps}$ & $100 \mathrm{~m}$ \\
\hline
\end{tabular}

As we said in Section 2, it is important to highlight that, in some technologies, fixed nodes, usually spend most of the time in a sleeping mode (in order to save as much power as possible). However, in this work we are going to suppose all nodes are active all the time (so values shown in Table 5 are final).

Considering numerical data from Tables 1 and 5, the final model expressed in (65), and solving numerically the expressions (22) and (46), it is possible to calculate the amount of data collected from sensor for each technology and mobile node type. See Table 6 and Figure 12. 
Table 6: Medium time available for data collection ( $T_{\text {in-cover }}^{\text {i-dim }}-\mathbb{E}\left[L_{\text {open RAT }}\right]$ )

\begin{tabular}{|c|c|c|c|c|c|c|c|c|c|}
\hline $\begin{array}{c}\text { Medium time } \\
\text { available for data } \\
\text { collection (s) }\end{array}$ & \multicolumn{9}{|c|}{ Mobile node type } \\
\hline $\begin{array}{c}\text { Wireless } \\
\text { Technology }\end{array}$ & WP & TV & H5 & H10 & H100 & P5 & P10 & P100 & DIR \\
\hline Bluetooth 3.0 & 12.58 & 0 & 0 & 0 & 0 & 0 & 0 & 0 & 0 \\
\hline BLE & 90.90 & 17.47 & 5.15 & 5.07 & 0 & 3.8 & 3.82 & 0 & 0 \\
\hline 802.15 .4 & 126.45 & 23.75 & 6.28 & 6.25 & 0 & 4.5 & 4.45 & 0 & 0 \\
\hline Fast 802.15.4 & 126.5 & 24.08 & 6.61 & 6.55 & 0 & 4.83 & 4.78 & 0 & 0 \\
\hline $\begin{array}{c}\text { WiFi (fixed } \\
\text { temporizers) }\end{array}$ & 181.62 & 35.43 & 10.28 & 10.24 & 0 & 7.73 & 7.7 & 0 & 0 \\
\hline $\begin{array}{c}\text { WiFi (smart } \\
\text { temporizers) }\end{array}$ & 181.7 & 31.49 & 10.34 & 10.30 & 0 & 7.79 & 7.76 & 0 & 0 \\
\hline
\end{tabular}

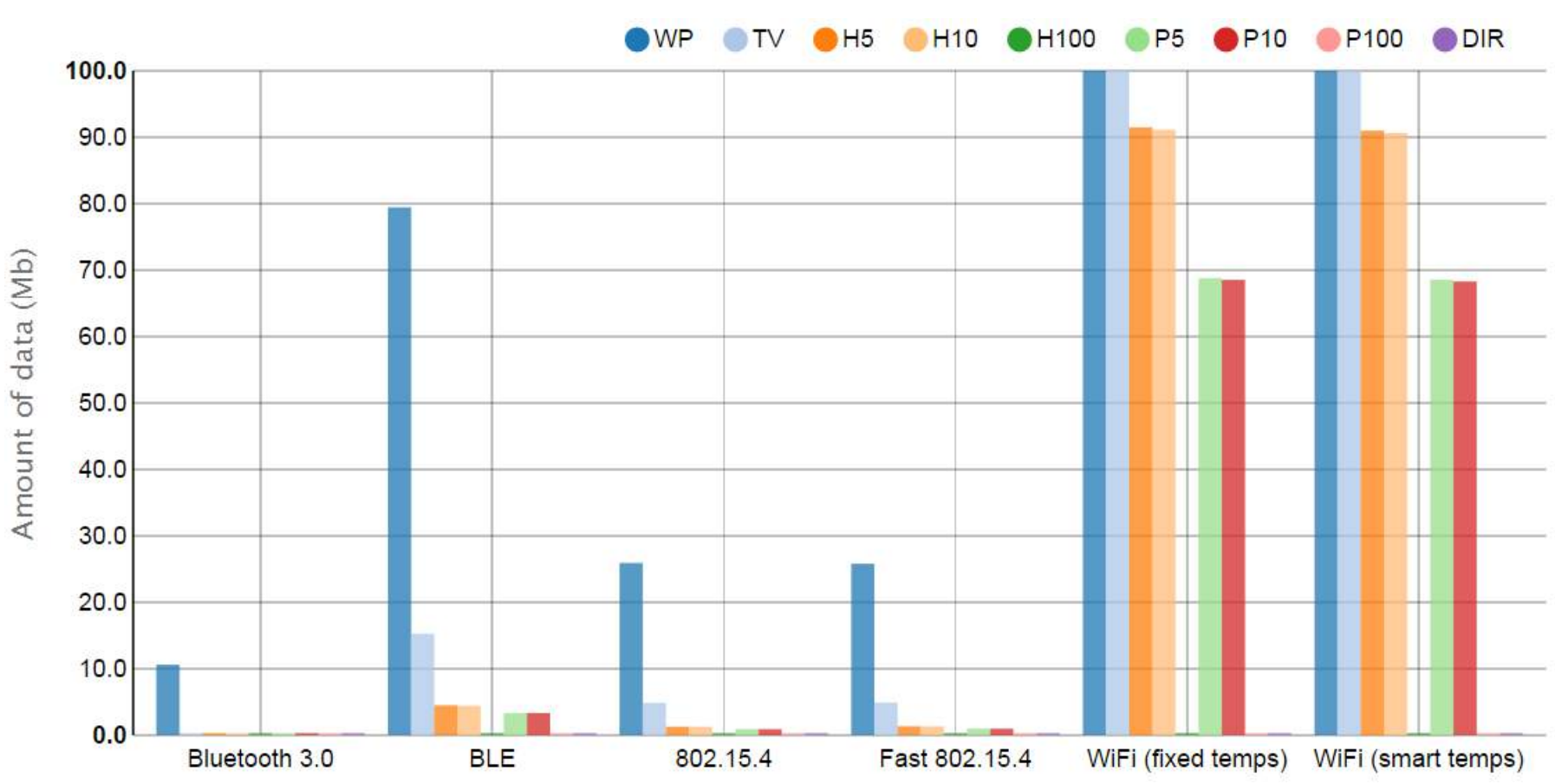

Figure 12: Transmittable data quantity during the available time (Y-limit $100 \mathrm{Mb}$ ). For WP and WiFi technologies, we found a maximum of $1.6 \mathrm{~Gb}$ and for TV and WiFi a maximum of $315 \mathrm{Mb}$.

Before extracting any conclusion, it is important to notice that the data quantity expressed in Figure 12 is calculated at radio level. The real data quantity from application level is lower. For example, in 802.15.4 standard only $2 \mathrm{kbps}$ of the $250 \mathrm{kbps}$ available at radio level can be used at application level.

Any case, a great importance result (which we should obtain) is the impact of considering real effects (such as the mobile node's stability) on the amount of data calculated. Figure 13 shows, as percentage, the 
difference between the amounts of data shown in Figure 12, and those obtained without considering stability parameters and taking, instead of the expected value of the upstream radio rate, its nominal value. As can be seen, in general, the percentage is positive, which means that the amount calculated considering "real effects" is lower than which could be obtained without considering these effects.

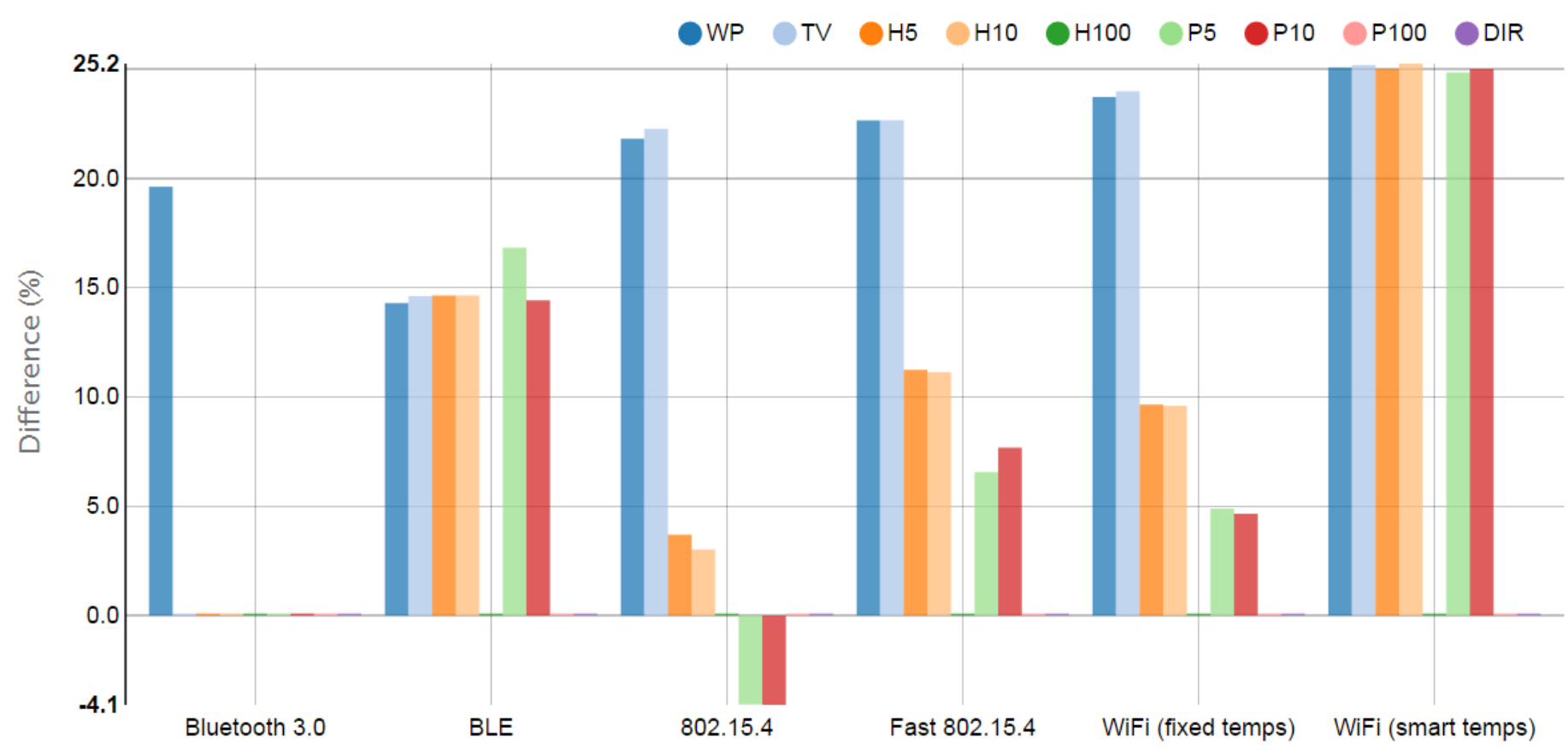

Figure 13: Difference between the amounts of data shown in Figure 12, and those obtained without considering stability parameters and considering the nominal upstream radio rate.

In Figure 13, two overlapping effects can be shown: one due to consider a more realistic mobility model and another due to consider a "real" value for the upstream radio rate. A study of both effects separately may show that the variation due to consider the stability parameters is the order of $5 \%$, being the remainder of the variation due to consider an upstream radio rate different to the nominal.

The variation in the amount of data due to consider a value different from the nominal upstream radio rate is always positive (i.e., the amount is lower because of considering a non-nominal value). However, the effect due to consider a more complex mobility model can take both signs. In particular, in the case of using a plane-type drone, the time in coverage is much higher if stability parameters are considered. Thus, in P5 and P10 scenarios, the variation is below the average; even it can be seen that the total data transmitted considering the "real" effect is greater than that if we do not consider it (in the case of using RAT 802.15.4).

\section{CONCLUSIONS AND FUTURE WORK}

Our objective in this research work is to present a new model for data collection in MWSN. A numerical analysis of this model allowed us to identify new problems in the operation of WSN, by studying the impact of the connection time and scenario's geometry in the process of data collection. The realized 
analysis includes both two-dimensional and three-dimensional scenarios, so terrestrial and flying mobile nodes can be considered.

Furthermore, the realistic parameter's values we have used allow us to extract some interesting conclusions:

In general, the connection time strongly impacts in the total available time along which the fixed and mobile nodes are in their coverage area. However, some technologies (like WiFi) are immune to the degradation due to the connection time, although these standards do not belong to the PAN set, so they are not useful in scenarios with scarce power supply.

Moreover, the values we showed in Figure 12 are calculated in a controlled scenario. In a real situation, higher layers in the protocol stack will also increase the time needed for stablishing an ephemeral connection, the interferences in the radio channel can make the devices need more than one search cycle for discover a new neighbor, the weather changes may affect the communication quality, etc. Even in this ideal scenario, the radio rate destined to data might be not enough. Considering the simplest applications, it is possible to achieve the requirements easily, but applications (and overall services) with more value added need a rate at top level that is not clear we can get.

As we can see in Figure 12, the amount of data collected is remarkable only for WiFi technology and for the slowest mobile nodes (namely, walking people and terrestrial vehicles). Nonetheless, Bluetooth 3.0 is so inefficient that only walking people can be considered as a valid mobile node. The use of drones only can be taken into account in scenarios where 802.11 technology is available, although if the application does not require a great data rate, BLE technology is also possible in low and medium altitude scenarios. Finally, it is clear that in high altitude scenarios, any PAN or LAN technology can be used. In those situations mobile technologies (such as LTE or the next 5G solutions) could be the only choice.

Therefore, in general, the main conclusion is that there are two possible scenarios: one where slow mobile nodes are employed; and other where LAN technologies (e.g. WiFi, WiMAX...) are deployed. Then, the role of distance and mobile node speed as key element is clear, so they should be the center of our future research. In that sense, it is really important to optimize the drones' trajectories as essential measure for improving the results of these systems.

Once we have described the problems associated with the necessity of stablishing an ephemeral connection, a lot of new analysis and proposals can be made. On the one hand, new standards' versions and special neighbor discovery procedures can be studied, focused on reducing the connection time. On the other hand, many extensions of this works may be made: from new analysis considering the time needed for waking up the nodes to studies based on more complicated physical scenarios, for example, one where the mobile nodes consider random paths. Furthermore, it will be very interesting a similar work that considers others wireless communication technologies or standards such as $802.11 \mathrm{v}$, LTE or WiMAX.

\section{References}

[1] Pereira, P. R., Casaca, A., Rodrigues, J. J., Soares, V. N., Triay, J., \& Cervelló-Pastor, C. (2012). From delay-tolerant networks to vehicular delay-tolerant networks. Communications Surveys \& Tutorials, IEEE, 14(4), 1166-1182.

[2] Quilter, M. C. (1997). Vegetation monitoring using low-altitude, large-scale imagery from radiocontrolled drones.

[3] Perkins, C. E. (2008). Ad hoc networking. Addison-Wesley Professional. 
[4] Feng, Y., Liu, K., Qian, Q., Wang, F., \& Fu, X. (2012). Public-transportation-assisted data delivery scheme in vehicular delay tolerant networks. International Journal of Distributed Sensor Networks, 2012.

[5] Câmara, D., Frangiadakis, N., Filali, F., \& Bonnet, C. (2011). Vehicular delay tolerant networks. Handbook of research on mobility and computing: evolving technologies and ubiquitous impacts, 356367.

[6] Ammari, H. M., Gomes, N., Jacques, M., Grosky, W., Maxim, B., Yoon, D. (2013) A Survey of Sensor Network Applications and Architectural Components. management, Adhoc \& Sensor Wireless Networks, 11,12.

[7] Yu, S., Zhang, B., Li, C., \& Mouftah, H. (2014). Routing protocols for wireless sensor networks with mobile sinks: A survey. Communications Magazine, IEEE, 52(7), 150-157.

[8] Gil-Castineira, F. (2007). Using Delay Tolerant Networks for Car2Car.

[9] Singh, H. R., Theja, N., \& Metagar, S. M. Licklider Transmission Protocol (LTP): A Protocol for Interplanetary Communication.

[10] Tseng, Y. C., Wu, F. J., \& Lai, W. T. (2013). Opportunistic data collection for disconnected wireless sensor networks by mobile mules. Ad Hoc Networks, 11(3), 1150-1164.

[11] Pásztor, B., Musolesi, M., \& Mascolo, C. (2007, October). Opportunistic mobile sensor data collection with scar. In Mobile Adhoc and Sensor Systems, 2007. MASS 2007. IEEE International Conference on (pp. 1-12). IEEE.

[12] Jain, S., Shah, R. C., Brunette, W., Borriello, G., \& Roy, S. (2006). Exploiting mobility for energy efficient data collection in wireless sensor networks. Mobile Networks and Applications, 11(3), 327-339.

[13] Zhang, X. (2009). Data collection in wireless sensor networks. University of Illinois at Chicago.

[14] Contreras, D., \& Castro, M. (2015). Experimental assessment of the adequacy of Bluetooth for opportunistic networks. Ad Hoc Networks, 25, 444-453.

[15] Franssens, A. (2010). Impact of multiple inquires on the bluetooth discovery process: and its application to localization.

[16] Liu, J., \& Chen, C. (2012). Energy analysis of neighbor discovery in Bluetooth Low Energy networks. Nokia.(nd).

[17] Aslam, S., Farooq, F., \& Sarwar, S. (2009, December). Power consumption in wireless sensor networks. In Proceedings of the 7th International Conference on Frontiers of Information Technology (p. 14). ACM.

[18] Ye, W., Heidemann, J., \& Estrin, D. (2002). An energy-efficient MAC protocol for wireless sensor networks. In INFOCOM 2002. Twenty-First Annual Joint Conference of the IEEE Computer and Communications Societies. Proceedings. IEEE (Vol. 3, pp. 1567-1576). IEEE.

[19] Intanagonwiwat, C., Estrin, D., Govindan, R., \& Heidemann, J. (2002). Impact of network density on data aggregation in wireless sensor networks. In Distributed Computing Systems, 2002. Proceedings. 22nd International Conference on (pp. 457-458). IEEE.

[20] Orlinski, M., \& Filer, N. (2015). Neighbour discovery in opportunistic networks. Ad Hoc Networks, 25, 383-392. 
[21] Montavont, N., Arcia-Moret, A., \& Castignani, G. (2013, September). On the selection of scanning parameters in IEEE 802.11 networks. In Personal Indoor and Mobile Radio Communications (PIMRC), 2013 IEEE 24th International Symposium on (pp. 2137-2141). IEEE.

[22] Sthapit, P., Choi, Y. S., Kwon, G. R., Hwang, S. S., \& Pyun, J. Y. (2013). A fast association scheme over IEEE 802.15. 4 based mobile sensor network. Proc. ICWMC.

[23] Huang, C. F., Tseng, Y. C., \& Lo, L. C. (2007). The coverage problem in three-dimensional wireless sensor networks. Journal of Interconnection Networks, 8(03), 209-227.

[24] Bai, X., Zhang, C., Xuan, D., Teng, J., \& Jia, W. (2009, May). Low-connectivity and full-coverage three dimensional wireless sensor networks. In Proceedings of the tenth ACM international symposium on Mobile ad hoc networking and computing (pp. 145-154). ACM.

[25] Ou, C. H., \& Ssu, K. F. (2008). Sensor position determination with flying anchors in threedimensional wireless sensor networks. Mobile Computing, IEEE Transactions on, 7(9), 1084-1097.

[26] Ammari, H. M., \& Das, S. K. (2009). Critical density for coverage and connectivity in threedimensional wireless sensor networks using continuum percolation. Parallel and Distributed Systems, IEEE Transactions on, 20(6), 872-885.

[27] Serodio, C., Cunha, J. B., Morais, R., Couto, C., \& Monteiro, J. (2001). A networked platform for agricultural management systems. Computers and Electronics in Agriculture, 31(1), 75-90.

[28] Munir, S. A., Ren, B., Jiao, W., Wang, B., Xie, D., \& Ma, J. (2007, May). Mobile wireless sensor network: Architecture and enabling technologies for ubiquitous computing. In Advanced Information Networking and Applications Workshops, 2007, AINAW'07. 21st International Conference on (Vol. 2, pp. 113-120). IEEE.

[29] Yick, J., Mukherjee, B., \& Ghosal, D. (2008). Wireless sensor network survey. Computer networks, 52(12), 2292-2330.

[30] Yang, H., Huang, L., \& Xu, H. (2015). Distributed Compressed Sensing in Vehicular Ad-hoc Network. Adhoc \& Sensor Wireless Networks, 25.

[31] Shah, R. C., Roy, S., Jain, S., \& Brunette, W. (2003). Data mules: Modeling and analysis of a threetier architecture for sparse sensor networks. Ad Hoc Networks, 1(2), 215-233.

[32] Bendall, M. J., Bassey, E. J., \& Pearson, M. B. (1989). Factors affecting walking speed of elderly people. Age and Ageing, 18(5), 327-332.

[33] Swift Navigation homepage, http://swiftnav.com/about.html. Visited in October 2015

[34] BMP180 Digital pressure sensor Data sheet, https://www.adafruit.com/datasheets/BST-BMP180DS000-09.pdf. Visited in October 2015

[35] Bluetooth, S. I. G. (2004). Specification of the Bluetooth System, 2004. Available via www. bluetooth. org/spec, version, 1.

[36] Bluetooth, S. I. G. (2010). Bluetooth Core Specification Version 4.0. Specification of the Bluetooth System.

[37] LAN/MAN Standards Committee. (2003). Part 15.4: wireless medium access control (MAC) and physical layer (PHY) specifications for low-rate wireless personal area networks (LR-WPANs). IEEE Computer Society. 
[38] European Table of Frequency Allocations and Applications http://www.erodocdb.dk/Docs/doc98/official/pdf/ercrep025.pdf

[39] International Telecommunication Union, Recommendation P.452, https://www.itu.int/rec/R-RECP.452/en. Visited in October 2015 


\title{
Using 5G technologies in the Internet of Things: Handovers, problems and challenges
}

\author{
Borja Bordel Sánchez, Álvaro Sánchez-Picot, Diego Sánchez De Rivera \\ Departamento de Ingeniería de Sistemas Telemáticos \\ Universidad Politécnica de Madrid \\ Madrid, Spain \\ bbordel@dit.upm.es, alvaro.spicot@gmail.com,diego.sanchezderiveracordoba@gmail.com
}

\begin{abstract}
The junction of both revolutions, the Internet of Things and mobile technologies, seems to be the key of the future. However, most works about this solution use a really simplified scenario, where the application of the mobile technologies to the IoT is problems-free. In this paper, we present a study considering the problems of the handovers in LTE and 5G networks. This view allows us to discover new challenges and limitations to the 5G-based IoT operation.
\end{abstract}

Keywords- Long-Term Evolution; 5G; Internet of Things; Handover; VWSN; Drone; NS3

\section{INTRODUCTION}

Long-Term Evolution (LTE) standards changed the traditional way in which mobile network were working [3]. Leaving circuit switched technology far behind, LTE became the first all-IP mobile network.

However, the fast increase of users in the mobile networks, and the need of extending the mobile services toward the Internet of Things (IoT) (like evacuation services [1], social aid [2], etc.), put a great pressure for going forward the fifth generation of the mobile technologies [4].

All professionals agree the next mobile generations have to be based on two basics principles [4] [5]. At physical level, new and unexploited radiochannels must be used, such as E-Band (in the EHF spectrum). And, at network level, the signalization has to be reduced (in order to avoid a network overload when the number of user goes up exponentially). While the movement of services to higher bands in frequency is a process repeated many times along the last decades, the reduction of the signalization only has been applied in some special cases; due to most services are session-focused and greatly reliable.

In general, it is supposed the signaling does not consume any significant resource from the network (in comparison with the total available) [6]. However, in IoT scenarios, where the resources are limited, the deployment of a great number of nodes may imply a high portion of the resources are used in transporting signalization. Among all control process described in mobile networks, handovers are the most significant in terms of signalization (due to its great reliability). In LTE technology, moreover, both the network and the user equipment (UE) participate in the handover, so the resources needed increase in respect to other technologies. For all that, the handovers optimization or redesign will be basic for going forward the next mobile generation.

This article aims studying the problems associated with the handovers in an IoT scenario based on LTE technology. From the results of this work, a set of conclusions will be deducted. Finally, these conclusions are used to find out the future challenges of $5 \mathrm{G}$ standards.

The rest of the paper is structured as follows. In section II, we present the work scenario, as well as the network architecture and the employed physical model. In section three, we review the LTE-handover theory and the most relevant cases for our study are analyzed. In the fourth section, we propose a simulation model for NS3 and present the results obtained of the simulator. Finally, in section V, we will discuss the results and their consequences.

\section{SCENARIO DESCRIPTION}

In this section, we present the environment where we planned the deployment of our network, describe the elements which form the topology and present a logical model in three layers for this scenario. Moreover, we explain a mathematical diagram that shows the mobile node's movement around the fixed ones.

\section{A. Base scenario description}

Our IoT scenario is based on a natural environment, where a set of fixed sensors are deployed for gathering information such as temperature, humidity, presence, etc. The node density is low, because the natural environment does not justify a greater number of elements. In this initial analysis, we do not consider images, video or other information requiring large amount of resources.

The described network is one of the called Wireless Sensor Networks (WSNs), which are composed by a collection of fixed nodes, connected in a persistent way by means of any wireless communication technology. To this WSN, at least, one vehicle node is added in order to collect data information from sensors (which form an isolated network). This entire infrastructure makes up a completed Vehicular Wireless Sensor Network (VWSN).

Three layers are defined in this scenario: sensor network, vehicular layer and data processing (Fig. 1).

- Sensor network: The sensors are distributed over a wide area providing data gathering and support for wireless communications, but without communication infrastructure. 


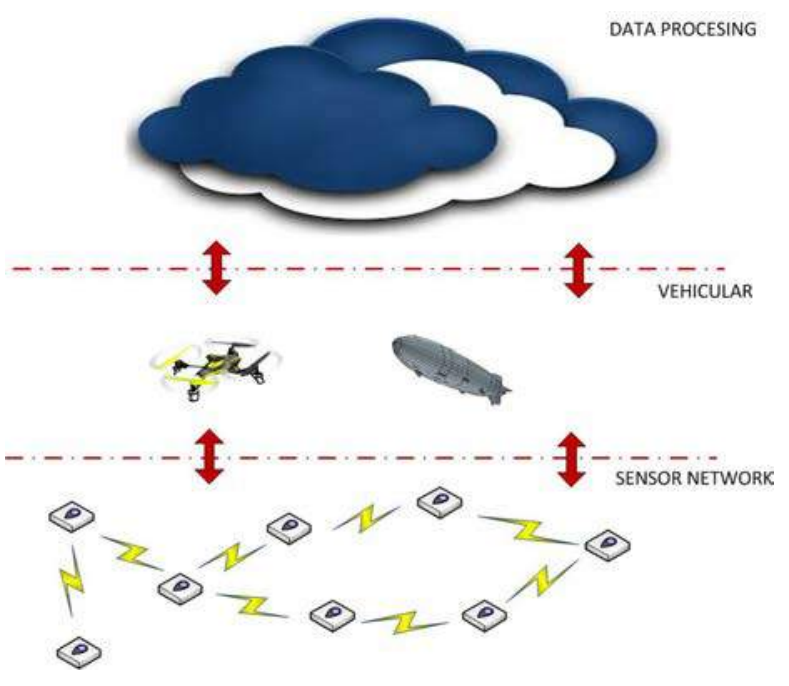

Figure 1. Scenario's logical structure:layers

- Vehicular layer: Includes a mobile device (or vehicle node) that is moving around the zone occupied by the sensors. The vehicle node uses Delay-Tolerant (DTNs) based communications for collecting available data from sensors and translates them to the data processing layer.

- Data processing layer: This layer receives data from vehicular layer, stores and processes it. This layer can be based on any standard technology like centralized services or cloud services, because in our scenario it is not required real time communication neither feedback among sensors and data processors.

Studying all possible technical solutions should be based on the definition and analysis of several different practical applications; however, in our case, we do not need be so thorough, therefore we are working over some common characteristic for all cases:

1) Lack of infrastructure for supporting communications and power supply.

2) WSN deployed for communication with sensor and actuators.

3) Usage of DTN solutions and vehicle nodes for communication.

4) Modeling of a three dimensional space: we are considering vehicles that moves on the air such as drones.

5) The movement of the aerial vehicles can be defined as: (a) free, when there is not external control; (b) controlled, when it is possible to plan the route; and (c) limited to one or several specific routes.

The first and second point limit the type of technologies we can use and more specifically the lack of power supply limits the service time of the network. The fourth and fifth points are directly generated by the requirements imposed by scenarios for natural areas. There, movement of vehicle nodes is not limited to the ground, and we can take advantage of drones and other aerial equipment.

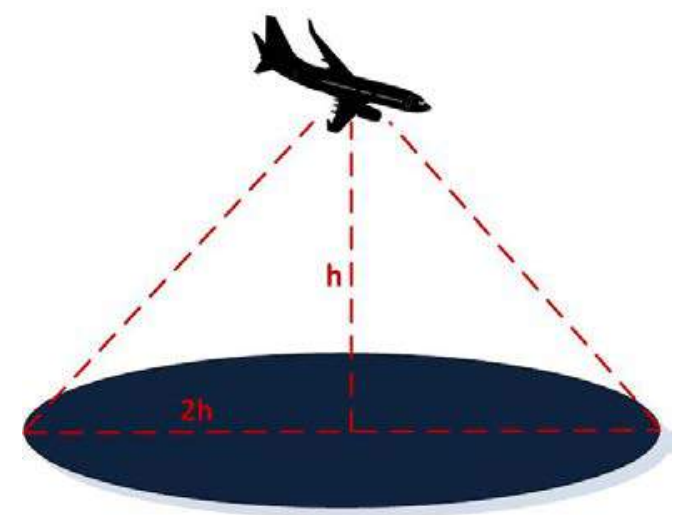

Figure 2. Geometrical scheme for three-dimensional scenarios

\section{B. Physical model}

Once we have described the natural environment and the network logical structure, it is necessary to design a model to calculate the geometrical variables for the vehicle nodes' movement. This model includes the structure and number of nodes in WSN, the number and location of the sensor and actuators, and the number, frequency and speed of the vehicle nodes.

As we said previously, we are going to focus our study in the case that the vehicle node is aerial equipment. For that, it's mandatory to use a three-dimensional model.

For a three dimensional model, which is inherently more complex than a two-dimensional, we are going to make some assumptions in order to simplify the qualitative analysis: The flying node maintains a uniform altitude over the ground $(h)$. When the mobile node is in the range of one fixed node, the transmission can be started, and when the vehicle node is beyond the range, the data transference is stopped. While $\mathrm{h}$ defined the altitude over the ground, we have to take into consideration the variations in the horizontal distance with respect to the fixed node when the vehicular node passes. In this analysis we assume that horizontal coverage of the flying node is $2 h$, so the average horizontal distance with respect to any node in the ground inside the coverage area is $h$ (see Fig. 2).

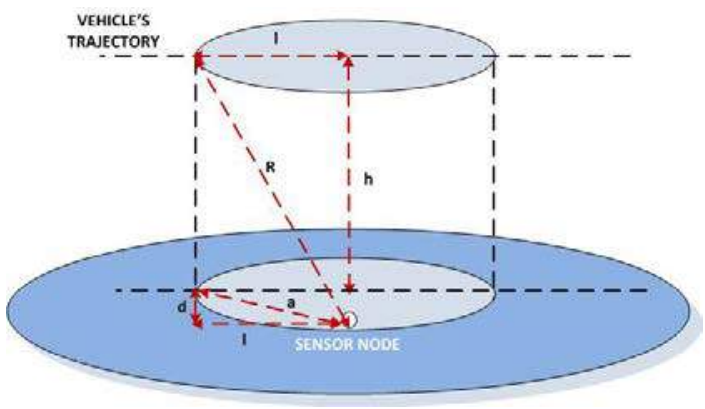

Figure 3. Detailed geometrical scheme for three-dimensional scenarios 
As seen at Fig 3, the distance when the flying node can connect with the main node is (1):

$$
l=\sqrt{R^{2}-\left(h^{2}+d^{2}\right)}
$$

The final element we need to define is the speed and altitude at which the drones operate. Next table (table I) summarizes these values for different aerial equipment.

TABLE I. AERIAL VEHICLE'S CHARACTERISTICS

\begin{tabular}{|c|c|c|}
\hline \multicolumn{1}{|c|}{ Vehicle node } & Cruise speed $(\boldsymbol{m} / \mathbf{s})$ & Altitude $(\boldsymbol{m})$ \\
\hline Helicopter-type drone $^{\mathrm{a}}$ & 66.7 & $5-100$ \\
\hline Plane-type drone $^{\text {b }}$ & 222.2 & $5-100$ \\
\hline Zeppeling & 44.4 & 3000 \\
\hline \multicolumn{2}{|c|}{$\begin{array}{c}\text { a. } \\
\text { b. } \quad \text { Cruise speed value taken from Drone Aibot X6 }\end{array}$} \\
\hline
\end{tabular}

\section{LTE HANDOVERS}

In this section we are going to discuss the different types of handover which must to be done in the described scenario. After that, we will present the process that has to be followed to execute them, when the base technology is LTE.

As can be seen in Fig. 4, the final topology of the work scenario is composed of overlapping two different networks: sensor network (isolated and low-resources); and 4G network, from which access points to cloud are part. Therefore, it is easy to see aerial vehicles will be mobile clients of both networks, so in the scenario two different types of handover will occur [7].

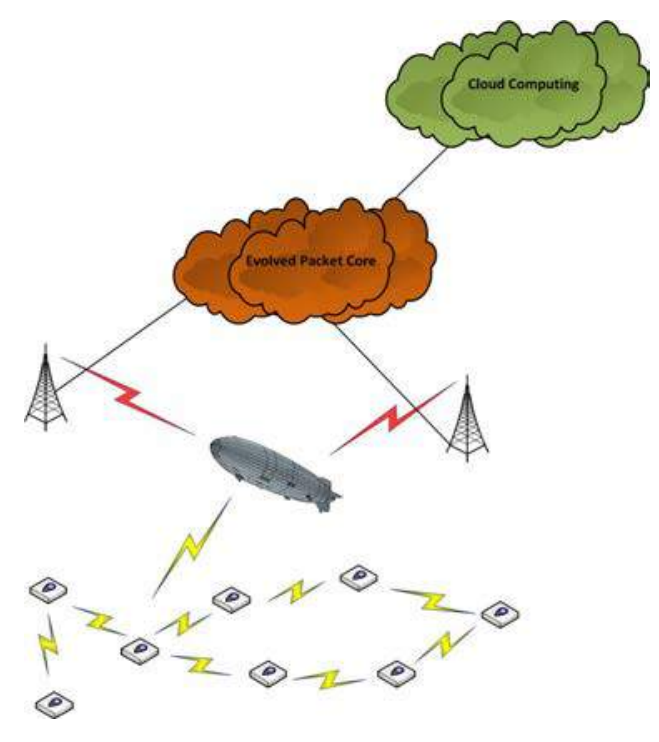

Figure 4. Scenario's netwok topology
Considering the connection of the drone with the sensor network, any factor may guarantee that the origin node (in whose coverage area the drone is) and the target node (toward the vehicle is traveling) can coordinate to execute an organized handover of the communication process. To this problem, besides, we should add the fact that the nodes lack of infrastructure for power supply, so they may not develop great coverage areas. This necessary implies a really important increase in the number of handovers, and, consequently, in the signaling load.

Ultimately, the drone-WSN communication model cannot be session-oriented, but it has to be based on the establishment of several ephemeral connections between the WSN and the aerial vehicle (in this case, an efficient routing protocol is needed to send data toward the mobile node). In that way, only LTE handovers might occur in our scenario.

Usually, there are two different types of handover approach in mobile networks [7]: Network Controlled (in this case network makes handover decisions), and Mobile Evaluated (when the UE makes handover decisions and informs network about them; although still network takes the final decision based on radio resource available in target cell). In LTE network, a hybrid approach is used. UE sends measurement information to the network, and, based on those measurements, network asks UE to move to a target cell.

Three different types of handovers are defined in LTE [3]: Intra-LTE handover (in this case source and target cells are part of the same LTE network), Inter-LTE handover (when at least one EPC component changes), and Inter-RAT (which occurs between different radio technologies).

On the one hand, in this work, it is logical supposing the mobile node is going to be client of only one LTE network, because the area where we deploy the WSN never will be larger than the area covered by the LTE network. On the other hand, although in some scenarios (for example, places where $4 \mathrm{G}$ is not deployed) might be necessary executing an Inter-RAT handover, we are not considering this case in this first analysis. Therefore, at the end, we are only considering Intra-LTE handovers. [12]:

There are, basically, two types of Intra-LTE handover

- Handover using X2 Interface: X2 is the interface between two eNodeBs (eNB). When X2 interface is present then handover is completed without Evolved Packet Core (EPC) involvement. The release of the resources at source eNodeB is triggered by target eNodeB.

- Handover using S1 Interface: S1 is the interface that connects the eNBs with the EPC. When X2 interface is not available, then handover is carried out through S1 interface, involving the EPC in the process. The EPC does not change the decisions taken by the source eNB. 


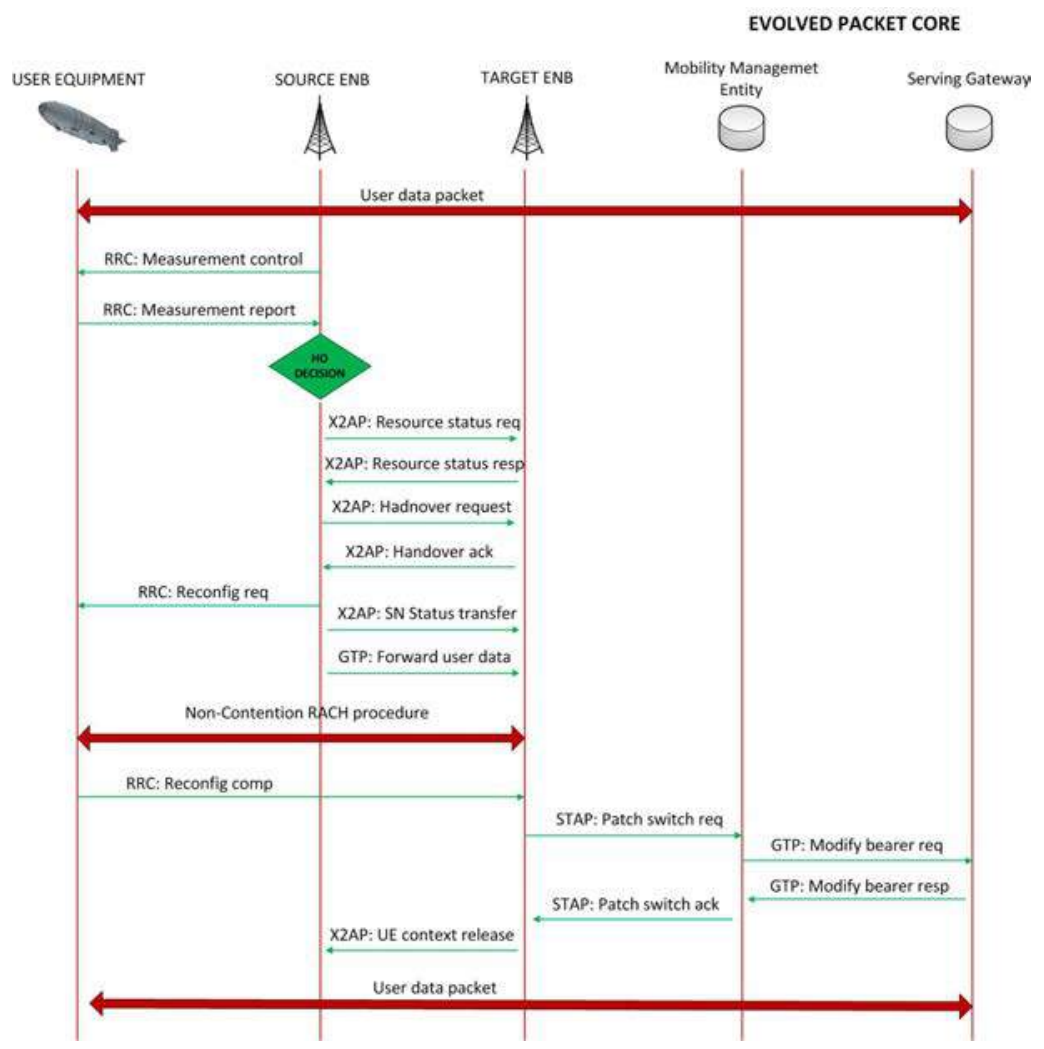

Figure 5. Message sequence of a Intra-LTE handover using X2 interface

Although both types of handover are possible, in practical cases all eNBs have activated the X2 interface. Furthermore, the differences between both types are quite small, that is why usually only handover using $\mathrm{X} 2$ interface is considered. As our objective it is not making a deep study of all 4G handover's possibilities, we are also focusing our study in handovers using X2 interface.

In Fig. 5, we present a message sequence that summarizes the handover process using X2 interface. As can be seen, the process starts with a routine evaluation of the radio link's state (first two messages). Taking into account the information sent by the UE, the source eNB decides to execute a handover. Then, source eNB and target eNB stablish a communication through X2 interface and both nodes accept the handover (messages from the third to the sixth). In the third step, UE's radio layer is updated for using the target eNB's frequencies and time slots (seventh and eleventh messages). Later, the UE's context stored in the source eNB is transferred to the target eNB (eighth, ninth and final messages). Finally, the UE stablishes a new connection with the target eNB (using a general RACH procedure) and the information stored in the EPC is modified.

\section{NS3 SIMULATION}

In this section we propose a simulation model for the NS3 simulator, with which to evaluate the probability density function of time consumption for the handover process. Later, we analyze the results of applying this model to our scenario.

The simulation model we propose it's composed of the following elements:

- 100 eNB distributed in a uniform way on the $\mathrm{X}$-axis. The lateral distance between the eNBs is one kilometer (being the first eNB placed on the origin).

- Only one EPC, to which all eNBs are connected. The throughput of the IP backbone is $100 \mathrm{Gbps}$ in all links (reachable quantity if we imagine an optical fiber infrastructure) and the MTU value is 1500 bytes.

- Only one user equipment (UE) which travels along the X-axis with a constant speed of $150 \mathrm{~m} / \mathrm{s}$.

If , now, we enable the traces of physical layer, MAC layer, RLC (Radio Link Control) layer and PDCP (Packet Data Coverage Protocol) layer, we will be able to monitor the handovers which will occur when the UE progresses along the $\mathrm{X}$-axis [10].

In order to adjust this simulation model to the characteristics of our work scenario, we are configuring the simulation in the next way: 
- Considering the WSN is deployed in an agricultural environment, it can be supposed the radiofrequency is propagating following the free space laws. Therefore, we selected the Friss model as physical model. Moreover, each eNB is transmitting a power of $+20 \mathrm{dBm}$, and gets one dedicated bearer. Finally, we are going to use a real model for RRC (Radio Resource Control) protocol (in which delays, power consumption, errors, etc. added in the radiochannel are taken into account).

- As in our scenario we have deployed DTN technologies, it is not necessary to include reliability in transport layer; so our equipment are going to implement UDP not TCP. The maximum UDP segment size is fixed in 100 bytes. With the objective of generating the realest possible scenario, each UDP entity sends a segment each $10 \mathrm{~ms}$ minimum.

- In order to guarantee the UE passes near all eNBs, the simulation's stop time will be 700 seconds.

With this model, we will do two different simulations, varying the handover algorithm used by the eNBs [13].

Firstly, we used the algorithm A2A4RsrqHandoverAlgorithm. This algorithm is based on measuring the signal quality received by the UE from each eNB. For that, the eNB uses the RSRQ (Reference Signal Received Quality) measurements defined in the LTE standards. In this case, to trigger a handover is necessary to fulfill two conditions. When the RSRQ of the serving cell is worse than the ServingCellThreshold value (first condition), the UE measures the quality of the other eNBs. Then, if the RSRQ of one of the other eNBs is, at least, equal than the serving cell's RSRQ plus the NeighbourCellOffset value (second condition) the handover will be triggered. Thus, the UE is always connected to the eNB with provides the highest C/I. In this work, the ServingCellThreshold value is fixed to 30 and the NeighbourCellOffset to one.

Secondly, we used the A3RsrpHandoverAlgorithm. Here, RSRP (Reference Signal Received Power) measurements are used as handover's trigger. In this case, it is also necessary to fulfill two conditions. In order to avoid false measurements, to trigger a handover one neighbor eNB's power must be higher than the serving cell's power in a margin of Hysteresis $\mathrm{dB}$ (first condition) during TimeToTrigger milliseconds (second condition). So, the UE is connected to the eNB from which it receives the greatest power. In this paper, Hysteresis value is fixed to $1.5 \mathrm{~dB}$ and the TimeToTrigger value to 128 milliseconds.

As can be seen in Fig. 6 and 7, the result obtained for both algorithms are really similar. It is showed, however, that in the case of using the A2A4RsrqHandoverAlgorithm the delay introduced by the handover is a little bit smaller ( 3 $\mathrm{ms}$ ). This issue may be attributed to the fact that the transmission quality is higher for the A2A4RsrqHandoverAlgorithm, so the number of errors, retransmissions, etc. is lower in this case.

Any case, in both figures exist a value much more probable than the others, so in the next steps we are using these values (table II).

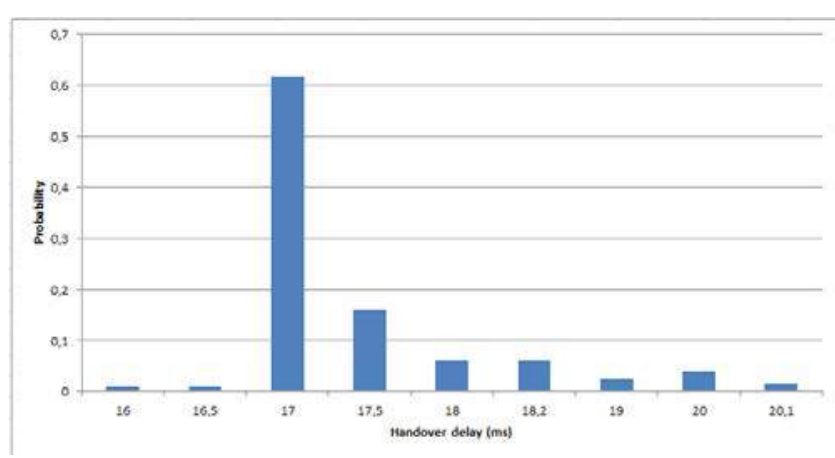

Figure 6. Densisty probability function obtained for A2A4RsrqAlgorithm

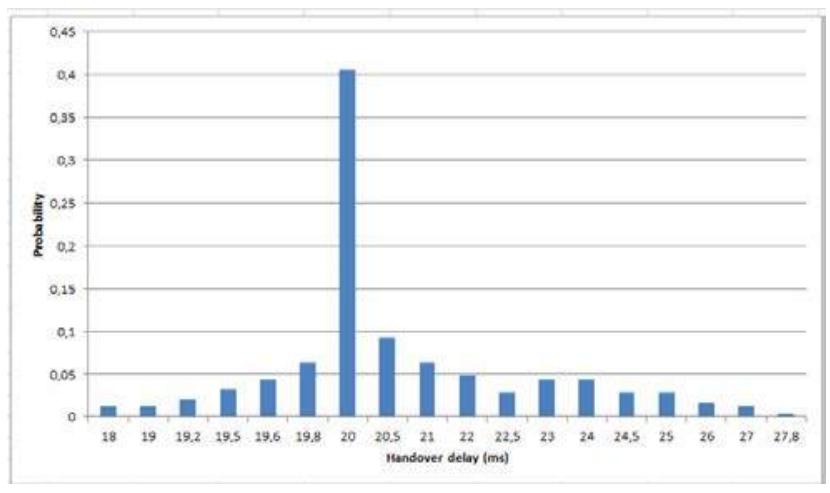

Figure 7. Densisty probability function obtained for A3RsrpAlgorithm

TABLE II. MAIN RESULTS OF NS3 SIMULATION

\begin{tabular}{|l|c|}
\hline \multicolumn{1}{|c|}{ Handover algorithm } & Most probable delay (ms) \\
\hline A2A4RsrqHandoverAlgorithm & 17 \\
\hline A3RsrpHandoverAlgorithm & 20 \\
\hline
\end{tabular}

Taking into account tables I and II, it is easy to calculate the minimum area we can plan for one cell (eq. 2). To obtain these results, we are supposing the cells are circular, and the drone flies over the diameter of the cell (see table III).

$$
\text { Area }_{\text {min }}=\pi \cdot\left(\text { speed }_{\text {vehicle }} \cdot \text { delay }_{\text {handover }}\right)^{2}
$$

TABLE III. MINIMUN POSSIBLE CELL AREA $\left(\mathrm{M}^{2}\right)$

\begin{tabular}{|c|c|c|c|}
\hline \multirow{2}{*}{ Handover algorithm } & \multicolumn{3}{|c|}{ Vehicle node } \\
\cline { 2 - 4 } & $\begin{array}{c}\text { Helicopter- } \\
\text { type drone }\end{array}$ & $\begin{array}{c}\text { Plane-type } \\
\text { drone }\end{array}$ & Zeppelin \\
\hline A2A4RsrqHandoverAlgorithm & 4 & 44.8 & 1.8 \\
\hline A3RsrpHandoverAlgorithm & 5.6 & 62 & 2.5 \\
\hline
\end{tabular}




\section{DISCUSION}

In view of the results presented in the previous section, some reflections may be drawn:

It seems to be clear that the zeppelin and the helicoptertype drone might not be problematic. Those vehicles are so slow as to allow the deployment of the Internet of Things systems based on $5 \mathrm{G}$ technologies, without redesigning the handovers. However, the time necessary to collect data from sensor is much higher than in other solutions.

In relation with the plane-type drone, as we can see, the minimum area required to directly employ the LTE handovers in systems based on $5 \mathrm{G}$ technologies it is not especially huge. Nevertheless, it is similar to the area taken up by the called femtocells [9]. So, it is a future challenge to discover if 5G-femtocells will be possible, and to develop the way in which they could be used with drones.

Furthermore, two recent events allow us to imagine how the future 5G will be; and, accordingly, to think out the challenges these standards will have to face.

On the one hand, global leaders in mobile technology (like ZTE in 2014) and wireless device testing (as Anite in November 2011) have developed tools and products for extensive LTE Multi-RAT deployments. It seems clear the 5G networks will use different physical technologies, adapted each one to the environment where it is deployed [16]. However, the handover between two radio access technologies is much more difficult and time consuming than intra-RAT handovers. So, will be possible to design multi-RAT 5G networks (which will combine the LTE cover rage and the power consuming of Bluetooth Low-Energy, for example) for our scenario? The success of the IoT in the future agriculture might depend on this.

On the other hand, in August 2013, the US Federal Communications Commission (FCC) announced a change in its rules governing the $60 \mathrm{GHz}(57-64 \mathrm{GHz})$ band [8]. Now, these frequencies are one of the key technologies for future 5G [11]. In really flats areas (as used in agriculture), where coverage area per antenna is reduced, will be possible to use these frequencies (which reduce the coverage area in factor of 60) without redefining the handovers? Several works as [14] and [15] propose a physical structure divided into two types of cells: macrocells (among which handovers can remain as in LTE technology) and microcells (among which it is not clear what kind of control communication could exist). Solving this situation will be another challenge for future $5 \mathrm{G}$ standards.

The problem of using higher frequencies will be bigger if, as it seems to be the tendency, drones become faster every year. We should notice that when the aerial vehicle's speed goes up in a $k$ factor, minimum cell area grows in a $k^{2}$ factor. The final challenge of $5 \mathrm{G}$ standard it is not to adapt the handovers to the nowadays world, but to preview the future technologies and being prepare for it.

\section{ACKNOWLEDGMENT}

This work is supported by project CALISTA TEC2012-32457.

This work has been partially supported by the Autonomous Region of Madrid through programme MOSI-AGIL-CM (grant P2013/ICE-3019, co-funded by EU Structural Funds FSE and FEDER).

\section{REFERENCES}

[1] A. Morales, R. Alcarria, D. Martin, and T. Robles, "Enhancing evacuation plans with a situation awareness system based on end-user knowledge provision". Sensors, 2014, vol. 14, no 6, p. 11153-11178.

[2] L. Atzori, A. Iera, G. Morabito. "SIoT: Giving a social structure to the internet of things". Communications Letters, IEEE, 2011, vol. 15, no 11, p. 1193-1195.

[3] S. Sesia, I. Toufik, M. Baker, "LTE: the UMTS long term evolution". New York: John Wiley \& Sons, 2009.

[4] S. Chen, J. Zhao, "The requirements, challenges, and technologies for $5 \mathrm{G}$ of terrestrial mobile telecommunication". Communications Magazine, IEEE, 2014, vol. 52, no 5, p. 36-43.

[5] P. Demestichas,. A. Georgakopoulos, D. Karvounas, K. Tsagkaris, " $5 \mathrm{G}$ on the horizon: key challenges for the radio-access network". Vehicular Technology Magazine, IEEE, 2013, vol. 8, no 3, p. 47-53.

[6] T. G. Lewis, "Network science". John Wiley \& Sons, 2009.

[7] W. C. Lee, "Mobile communications engineering". McGraw-Hill Professional, 1982.

[8] FCC resolution. "FCC modifies part 15 rules to spur the deployment of wireless services, unlicensed spectrum innovation in the 57-64 ghz band"

https://apps.fcc.gov/edocs_public/attachmatch/DOC-322747A1.pdf

[9] J. G. Andrews, H. Claussen, M. Dohler, S. Rangan., M. C. Reed. "Femtocells: Past, present, and future". Selected Areas in Communications, IEEE Journal on, 30(3), 497-508. 2012

[10] G. Piro, N. Baldo, M. Miozzo, "An LTE module for the ns-3 network simulator". Proceedings of the 4th International ICST Conference on Simulation Tools and Techniques. ICST (Institute for Computer Sciences, Social-Informatics and Telecommunications Engineering), 2011. p. 415-422.

[11] M. N. Tehrani, M. Uysal, H. Yanikomerouglu, "Device-to-device communication in $5 \mathrm{G}$ cellular networks: challenges, solutions, and future directions". Communications Magazine, IEEE, 2014, vol. 52, no 5, p. 86-92.

[12] H. Holma, A. Toskala, "LTE for UMTS-OFDMA and SC-FDMA based radio access". John Wiley \& Sons, 2009.

[13] K. Dimou, M. Wang,, Y. Yang, M. Kazmi, M. Larmo, J. Pettersson "Handover within 3GPP LTE: design principles and performance". Vehicular Technology Conference Fall (VTC 2009-Fall), 2009 IEEE 70th. IEEE, 2009. p. 1-5

[14] X. Ge., H. Cheng, M. Guizani, T. Han, "5G wireless backhaul networks: challenges and research advances". Network, IEEE, 2014, vol. 28 , no 6 , p. 6-11.

[15] N. Bhushan, J. Li, D. Mallad., R. Gilmore, D. Brenner, A. Damnjanovic, S. Geirhofer, "Network densification: the dominant theme for wireless evolution into 5G". IEEE Communications Magazine, 2014, vol. 52, no 2, p. 82-89.

[16] J. G. Andrews, S. Buzzi, W. Choi, S. Hanly, A. Lozano, A. Soong. J. C. Zhang, "What will 5G be?". 2014. 


\title{
Virtualization-based techniques for the design, management and implementation of future 5G systems with network slicing
}

\author{
Borja Bordel $^{1}$, Diego Sánchez de Rivera ${ }^{1}$, Ramón Alcarria ${ }^{1}$ \\ ${ }^{1}$ Universidad Politécnica de Madrid, \\ Madrid, España \\ bbordel@dit.upm.es, diegosanchez@dit.upm.es,ramon.alcarria@upm.es
}

\begin{abstract}
Emerging 5G communications aim to simplify the current inefficient and heterogeneous collection of wireless solutions for future systems. However, contrary to traditional mobile networks, 5G networks must consider many different application scenarios (Internet-of-Things, wearable devices, etc.). In this context it is defined the concept of network slicing, a technique where network resources are packaged and assigned in an isolated manner to set of users according to their specific requirements. The use of Virtual Network Functions and other similar technologies is a first step in this challenge, but deeper changes are required. Therefore, in this paper we present a virtualization-based technique for the design, management and implementation of future 5G systems with network slicing. The proposed technique employs extensively current virtualization technologies such as Docker or Kubernetes in order to create, coordinate and manage slices, services and functional components in future 5G networks. A simulation scenario describing these future mobile networks is also provided, in order to obtain first evidences of their predicted performance.
\end{abstract}

Keywords: 5G; network slicing; future technological systems; virtualization techniques, Docker, Kubernetes

\section{Introduction}

During the last ten years, many different communication technologies have been defined: from broadband mobile networks (such as Long-Term Evolution Advanced, LTE-A or 4G+ [1]) to Low Power Wide Area (LPWA) solutions for the Internet-ofThings (IoT) or Cyber-Physical Systems [2] (like SigFox or LoRa). Besides, users and companies have created new devices which employ standard wireless solutions (as WiFi or Bluetooth) in scenarios where they are not prepare to work efficiently [3].

In fact, the described heterogeneity and great variety of standards, proprietary solutions and ad hoc uses of traditional wireless solutions have been proved to be very inefficient. As a direct consequence, in several well-known scenarios, such as health monitoring using wearable devices, wireless sensor networks, etc. researchers are still discussing about how to provide these future systems with a global, mobile, wireless and efficient connection [4]. 
This situation, and problems such as spectrum saturation, interferences, scalability, global connectivity or hybrid scenarios where various technologies have to be mixed, has pushed the definition of a new and universal communication technology. This solution is known as $5 \mathrm{G}$ communications.

$5 \mathrm{G}$ communications are, exactly, the fifth generation of mobile networks, envisioned in such way that they may support a wide range of communication services and devices (in practice, all current and predicted future needs) [5]. Those services include IoT systems, Industry 4.0, future intelligent terminals, new ubiquitous mobile applications, etc. This service-oriented view is pretty different from traditional research topics, focused on improving and reorganizing the access and/or core infrastructure of mobile networks in order to provide users with a higher speed connection. However, 5G communications are not only about high speed communications, but also about a system being able to satisfy services with very different requirements and supported by very different devices [6].

This rethink of mobile networks naturally leads to the notion of network slicing [7], where network resources and infrastructure are divided into different vertical virtual dedicated networks, or slices, each one optimized to support a different kind of services. In order to reach this objective, flexible and programmable technologies are required, so a same physical infrastructure may provide a multitude of diverse services, independently one from the others. Software defined networks (SDN) and Virtual Network Functions (VNF) [8] are the first steps to address this challenge, but they are still (nowadays) employed with a traditional (horizontal, where all resources support all services) approach. Thus, deeper and more ambitious changes are required, specifically a more extensive and flexible use of virtualization techniques.

Therefore, the objective of this paper is to describe a new virtualization-based technique for the design, management and implementation of future 5G systems with network slicing. The proposed technique is based on the most popular virtualization technology nowadays: containers (especially Dockers). The use of these solutions, together with other coordination mechanisms for virtual entities (such as Kubernetes) fits perfectly the requirements and proposed architectures for emerging $5 \mathrm{G}$ networks. Services, slices, inter-slice coordination mechanisms and intra-slice functional components (among other minor elements) may be properly described using the cited technologies.

The rest of the paper is organized as follows: Section 2 describes the state of the art on network slicing; Section 3 describes the concept of network slicing and how virtualization techniques may be employed to design, implement and manage 5G networks considering this functionality; Section 4 presents an experimental validation using a simulation scenario in order to evaluate the predicted performance of this future networks; and Section 5 concludes the paper.

\section{State of the art on network slicing}

Network slicing (see Figure 1) aims to define a vertical dedicated network for each service type supported by $5 \mathrm{G}$ networks. This approach is very different from the traditional view where all network elements contributed to support all services, and all 
user and application were provided with connections with the same Quality-ofService (QoS) [9].

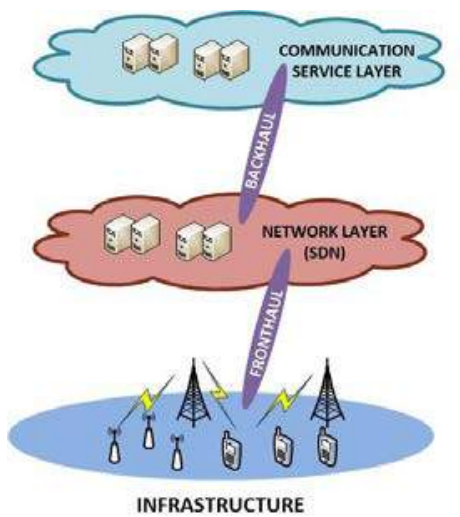

(a)

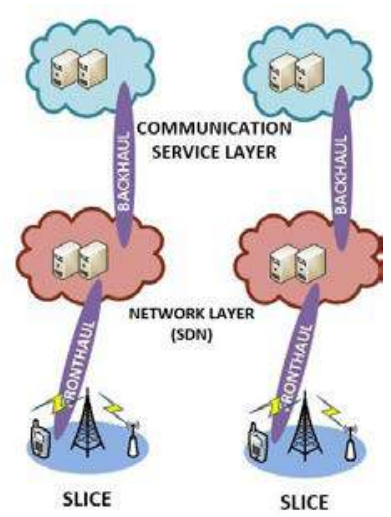

(b)

Fig. 1. Mobile network architecture (a) Traditional view (b) Network slicing

In order to reach this objective three main research areas are nowadays being worked [7]: the required infrastructure for network slicing, the network function definition and deployment and the communication service management.

Works on the required infrastructure for network slicing focus on two different subjects: the composition of this infrastructure and its virtualization. Typically, papers describing the composition of the infrastructure propose an Infrastructure as a Service (IaaS) [10] solution where generic computation elements support the entire system [11][12]. Although this solution seems to be valid of the Core Network, some authors propose also an edge computing infrastructure in order to support the Access Network and to fulfill the latency requirement of some slices [13]. Works on infrastructure virtualization are mainly focused on the Core Network, where most proposals are based on the use of Linux containers and kernel-based virtual machines [14].

Works about the network function definition and deployment are mostly related to SDN and Network Function Virtualization (NFV). Different studies about the currently available VNFs and the required new developments may be found [15]. For example, proposals about the virtualization of the LTE Enhanced Packet Core [16] have been reported.

Finally, works on communication service management are nowadays sparse. Although this area is critical for future $5 \mathrm{G}$ networks, due to the novelty of this layer in mobile communication systems and architectures, fundamental questions are still unanswered. In particular, some papers about mapping services to network components, so every service obtains the required resources to be available, may be found [17].

Our proposal is totally coherent with all these previous contributions and research, but expands the knowledge in the area by defining a technique and approach being able to address current problems in the three cited topics through a consistent and integrated technique. As main novelty, our approach simplifies and improves the slice design, management and implementation through standard technologies as Docker. 


\section{Virtualization techniques and future 5G networks}

Emerging 5G networks envision a scenario where telecommunication operators rent slices to the physical infrastructure owners, where they can deploy the communication services they offer to their clients (the final users). In that way, two different businesses are born: on the one hand, the communication service provision, and, on the other hand, the slice provision (see Figure 2).

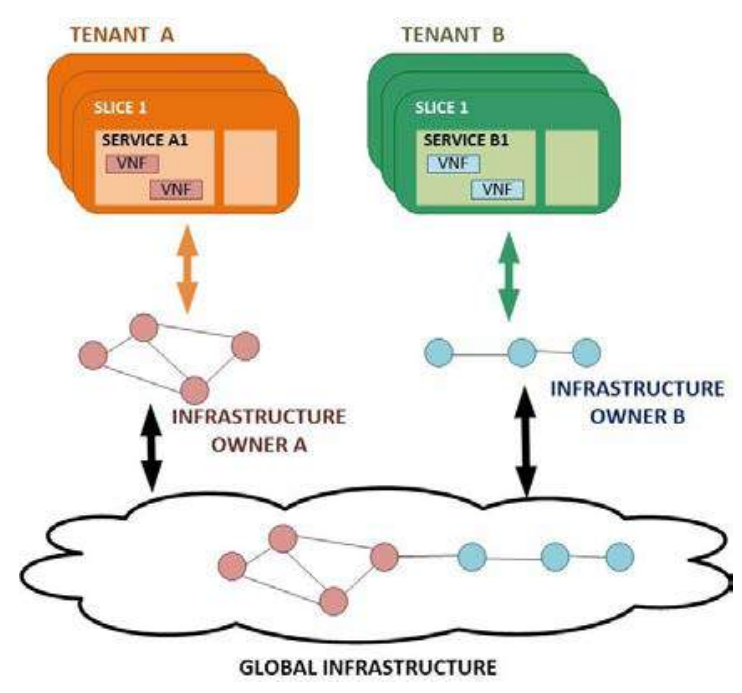

Fig. 2. Representation of future $5 \mathrm{G}$ systems with network slicing

With this approach, virtualization technologies are a key element for network slicing, as must be guaranteed the total independence, availability and reliability of slices. Besides, as slices are end-to-end deployments (they cover all network segments, access network, core network, transport, service management, etc.), an automatic vertical slice management solution is required in order to maintain the consistency and stability of the entire system.

We argue that a solution based on containers and Kubernetes (K8s) [18] may address this challenge; and this section describes how this can be done.

\subsection{Terminology and global overview}

In the virtualization area, containers (formally software containers) are lightweight, stand-alone, executable packages of software that include everything needed to run it: code, runtime, system tools, etc. Containerized software will always run the same, regardless of the environment. As main advantage, containers isolate software from its surroundings, so effects of software fails are limited to the container. As containers are lightweight, they may be employed intensively in a unique machine and be mixed with other heavier virtualization techniques. Many different mechanisms to create 
containers are nowadays available. In this article we propose to employ the most popular and efficient one nowadays: Docker [19].

The described proposal requires the use of many terms and concepts, which are presented below:

- Over-the-top service (OTTS): It is every end-user service, which employs the communication service provided by the telecommunication operators. It includes traditional voice calls, data services for the IoT, future health applications, etc.

- Communication service (CS): A CS is the collection of functionalities offered by a certain telecommunication operator, to allow the sending of information among a set of users, devices and/or applications. The characteristics of the CSs should be adapted to the OTTS which are going to employ the final users (then CS for IoT scenarios, traditional mobile users... will be defined), but CSs must be neutral and do not interfere with the OTTS operation. Various CSs may be deployed in a same slice, although in this case, the isolation among them must be supported by the telecommunication operator with an adequate design of the logical network (LN).

- Physical node (PN): It is every physical equipment in the core network or in the access network, generic or more specific, employed to support the mobile communications (i.e. the hardware infrastructure)

- $\mathrm{K} 8 \mathrm{~s}$ node $(\mathrm{KN})$ : Each one of the virtual machines which are hosted in the PNs. Each KN may execute network functions belonging to different CSs, each one of them encapsulated in a different container.

- Network operating system (NOS): It is a software layer focused on virtualizing the PNs and creating the required KNs with the requested characteristics and capabilities. This NOS must be location-aware in order to guarantee the CSs covers the area where the final users are going to be located.

- Functional component (FC): A FC it is each one of the network functions that allow supporting a certain CS. A FC usually corresponds with a VNF, which is executed in isolation in a container hosted in a certain $\mathrm{KN}$ (access network functions will be hosted in access network nodes, etc.).

- Logical network (LN): It is the network of FCs (containers or VNFs) that work together in order to support the CS contained in a same slice.

- $\quad \mathrm{K} 8 \mathrm{~s}$ pod (KP): It is each set of FCs belonging to a unique LN (or slice), which are hosted and executed in the same KN (i.e. geographical location). All FCs in a KP share the same IP address, and use different communication ports.

- K8s service (KS): It the set of all KPs belonging to the same slice. It includes the LN and all required control and coordination components (see Section 3.2).

As can be seen, in our proposal each slice is organized as a KS. In order to allow the deployment of KSs, the infrastructure owners implement a NOS and virtualize their generic PN to create dedicated $\mathrm{KN}$ adapted to the CS requirements (e.g. a KN focused on proving a CS that needs a local device register should have enough memory space to maintain these databases). On the other hand, telecommunication operators rent slices, indicating the CS requirements, the CS objective and the support $\mathrm{LN}$ to the infrastructure owners. Using their information, the FC (containers) and KP making up the KS (slice) are generated and deployed. This technique, as we are 
describing below, fulfills all proposed requirements for $5 \mathrm{G}$ architectures and greatly improves network slicing management, through the native Docker and K8s solutions.

\subsection{Design and network architectures}

The 5G-PPP (5G Infrastructure Public Private Partnership), the main standard organization about future $5 \mathrm{G}$ networks in Europe, has proposed various initial architectures in July 2017 for these emerging systems [20].

In particular (see Figure 3(a)) the overall architecture defines three main layers and two important software middleware (the NOS and a CS orchestrator). In this sense, our proposal is totally coherent with this view. With the proposed technique (see Figure 3(b)) network infrastructure may be composed of generic devices (the PNs), which are virtualized by means a NOS. This NOS includes two layers. The first one is a generic hypervisor, focused on create and maintain the KNs (dedicated virtual machines, specialized in the provision of certain CSs) which are hosted into the PNs. The second one is composed by a Guest Operating System (usually Linux) and the Docker layer, which will allow the later deployment of FC in such a way that they are independent one from the others (as required in scenario with network slicing).

This view is coherent with recent proposals (supported by the 5G-PPP) about considering an IaaS solution for the future $5 \mathrm{G}$ infrastructures. Besides, our proposal addresses the problem of infrastructure virtualization with a solution that makes easier the operation of higher layers.

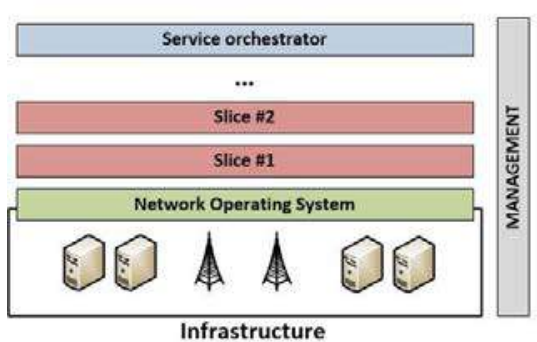

(a)

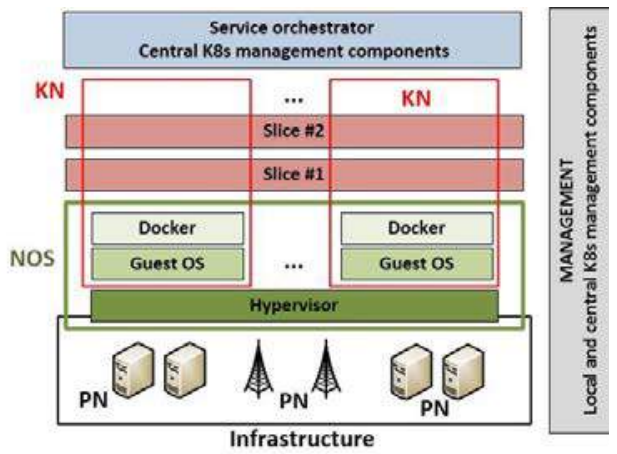

(b)

Fig. 3. (a) 5G-PPP overall architecture (b) Proposed overall architecture

In relation to the second layer, the network layer, our proposal allows the use of all existing NFV and SDN solutions. The telecommunication operators who design the LN should provide the desired network scheme (including control components, dashboards, etc.). FCs may be developed regardless the KNs and /or PNs thanks to the use of the Docker technology. Containers encapsulated each LN into the rented slice(s), isolated from the environment and other LNs.

The third layer is a middleware focused on CS and slice orchestration. These functionalities are totally covered in our proposal by the K8s management functions and components (e.g. the scheduler). Later, more details about this point are provided. 
Finally, a vertical later is also considered: network and service management. In our proposal this layer is also supported by the management functionalities of $\mathrm{K} 8 \mathrm{~s}$ technology. Kubelet, Kube-proxy, controllers, etc. enable the slice, service and nodes management, as components are deployed at all levels, from PN to CS.

In the proposed solution every slice is organized as a KS. The 5G-PPP indicates that every slice must contain six basic functional layers (see Figure 4(a)). With the proposed solution, these layers are divided into two different groups (see Figure 4(b)). On the one hand, telecommunication operators must define the FCs that support their services, including the data plane, the control plane and the management layer. On the other hand, functions related to network slicing, orchestration, service plane and management of these functions are supported by native components of the K8s technology.

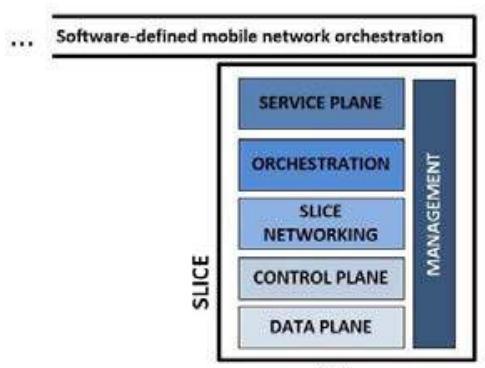

(a)

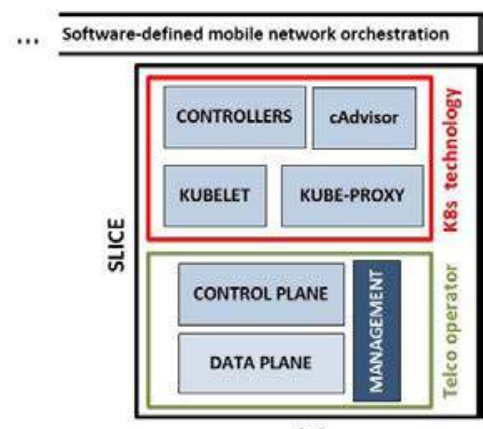

(b)

Fig. 4. (a) 5G-PPP network slice architecture (b) Proposed network slice architecture

In particular, this second group of functions is supported by four components. First, controllers (such as the "DaemonSet Controller" or the "Job Controller") monitor the behavior of KPs and make sure they work as expected. Controllers can also replicate a KP if required, etc. Second, Kubelet is in charge of the start, detention and maintenance of KPs. It notifies to the master node (see Section 3.3) the current state of all KPs and can regenerate a KP if its operation is not as desired. Third, KubeProxy orchestrates the FC and makes sure the appropriate FC receives the data sent to the KN. Finally, cAdvisor monitors the LN, KPs and FCs. Of course, as indicated by the 5G-PPP, inter-slice functions (focused on coordinating the intra-slice domains) will be required. These functions (not defined yet) cannot be supported by K8s components, but they can be supported by one or several new KS (including the FCs that will be defined in the future, such the "inter-slice broker").

\subsection{Improvements in network management}

In the previous subsection, we have remarked various times that using the K8s solutions for slice management, no specific technologies are required. The use of K8s directly addresses and solves these problems. However, in this section we are describing with more details the improvements in network management obtained through the use of this technique. The described functionalities will simplify the 
"Software-defined mobile network orchestration" proposed by the 5G-PPP (see Figure 4).

First, K8s technology defines the concept of "labels" and "selectors". This mechanism allows the identification and grouping of KNs, KPs and KSs, so critical group and management operations may be easily performed. For example, each KS has a unique label, so (if a global unrecoverable error occurs) the entire slice will be automatically regenerated by invoking the adequate selector. This also allows applying operations over slices belonging to the same telecommunication operator (such as updating all its CSs) or making operations over certain network locations (for example modifying faulty software in the KNs of the access network in Napoli).

A unique KP may have several different labels, so it is enabled the definition of different types of slices (as required by the 5G-PPP): from the stand-alone slices (totally independent, with reserved and exclusive resources at all levels) to slices with shared resources (where FCs supporting link and physical level functions are shared among various $\mathrm{KSs}$ ) and resource-unaware slices (where all FCs are shared except those related to the four top layers in the ISO-OSI model). The management of these stations gets also very simplified using the proposed techniques.

These operations are automatically performed by a scheduler. The scheduler runs the pending KPs, selects the appropriate $\mathrm{KN}$ for each $\mathrm{KP}$, manages the QoS and makes sure the PNs does not get overloaded. K8s technology, in order to enable the scheduler operation, also considers a central repository of KPs to be executed, named as etcd. Management operations are based on communications employing JSON (JavaScript Object Notation) objects and the HTTP (Hypertext Transfer Protocol) protocol. In order to validate and secure all communications among KPs, KSs and other external entities, an API (Application Programming Interface) server is also considered. Then, the consistency of operations is guaranteed, so the system cannot enter in an unstable state. At hardware level, all these functions are supported by a special node (the master node) totally isolated from the other KNs.

\section{Experimental validation. Simulations}

As currently there are no reference proposals in the area of implementation solutions for $5 \mathrm{G}$ networks with network slicing, it is very complicated to validate the performance of the proposed technique. However, in order to obtain first evidences of the expected behavior for these future systems, a simulation scenario was designed.

The simulation was performed using the NS3 simulator. This simulator allows the use of TAP bridges and ghost nodes in order to connect real virtual machines with simulated components. In that way, the proposed solution based on Docker and Kubernetes could be implemented, partially, in a real way. The scenario consisted of an access network with five (5) base stations, connected through an IP backbone to a central data center with ten (10) machines. The simulation model of these machines was created to emulate the Dell PowerEdge R330 servers. This infrastructure was virtualized and managed using the proposed technique in this paper.

Using this infrastructure, the number of deployed CS was increased, evaluating the availability of the deployed services. For each situation, twelve simulation were performed, and average values where obtained. Simulated CSs were traditional voice 
calls, where a random number of users between one (1) and 5 (five) was always using the service. The same experiment was performed using a traditional $4 \mathrm{G}$ mobile network as included in the NS3 simulation libraries for LTE technology. Figure 5 presents the results.

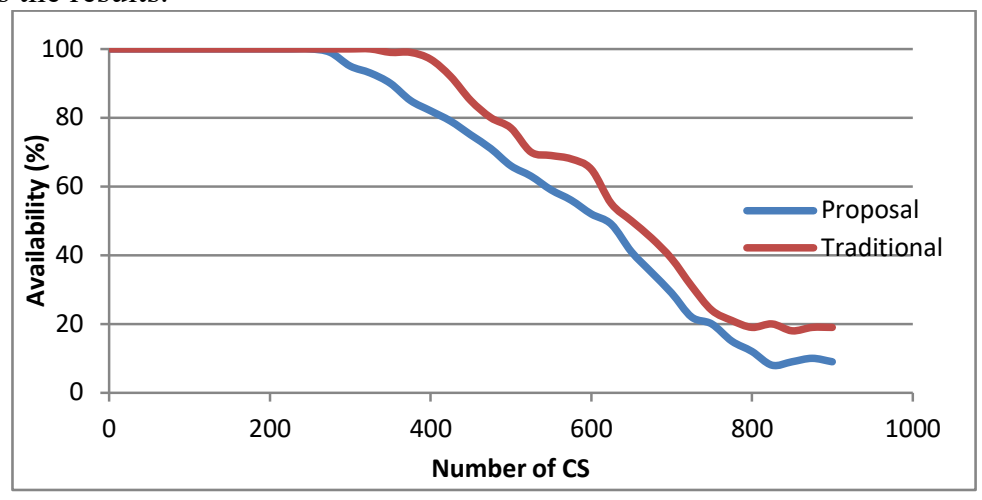

Fig. 5. Results of the proposed simulation

As can be seen, Figure 5 shows the expected exponential evolution for both situations, although the fall in availability when using the proposed technique occurs for a number of CSs 5\% lower. These results are reasonable considering the inefficiencies of a first experiment, so it can be ensured that first evidences show the proposed solution, at least, maintain the same performance of traditional approach (but many advantages in management are obtained).

\section{Conclusions and future works}

In this paper we present a virtualization-based technique for the design, management and implementation of future $5 \mathrm{G}$ systems with network slicing. The proposed technique employs extensively current virtualization technologies such as Docker or Kubernetes in order to create, coordinate and manage slices, services and functional components in future $5 \mathrm{G}$ networks. As a result, many of the current problems on slice management, creation, maintenance or deployment get solved, thanks to the native and existing solutions for containers orchestration.

Future works should consider the proposal of particularized lifecycles for network slices and the definition of specific operations for slice regeneration, update, etc. based on labels and selectors. A prosumer interface for the creation and renting of slices in an automatic way should be also interesting to be investigated in future works.

Acknowledgments. Borja Bordel has received funding from the Ministry of Education through the FPU program (grant number FPU15/03977). Additionally, the research leading to these results has received funding from the Ministry of Economy and Competitiveness through SEMOLA project (TEC2015-68284-R) and from the Autonomous Region of Madrid through MOSI-AGIL-CM project (grant P2013/ICE3019, co-funded by EU Structural Funds FSE and FEDER). 


\section{References}

1. Hasan, M., Hossain, E., \& Niyato, D. (2013). Random access for machine-to-machine communication in LTE-advanced networks: issues and approaches. IEEE communications Magazine, 51(6), 86-93.

2. Bordel, B., Alcarria, R., Robles, T., \& Martín, D. (2017). Cyber-physical systems: Extending pervasive sensing from control theory to the Internet of Things. Pervasive and Mobile Computing, 40, 156-184.

3. de-Rivera, D. S., Martín, D., Alcarria, R., Bordel, B., \& Robles, T. (2017). Towards a Wireless and Low-Power Infrastructure for Representing Information Based on E-Paper Displays. Sustainability, 9(1), 1-12.

4. Robles, T., Bordel, B., Alcarria, R., \& MARTÍN, D. (2017). Mobile Wireless Sensor Networks: Modeling and Analysis of Three-Dimensional Scenarios and Neighbor Discovery in Mobile Data Collection. Adhoc \& Sensor Wireless Networks, 35.

5. Sánchez, B. B., Sánchez-Picot, Á., \& De Rivera, D. S. (2015, July). Using 5G technologies in the Internet of things handovers, problems and challenges. In Innovative Mobile and Internet Services in Ubiquitous Computing (IMIS), 2015 9th International Conference on (pp. 364-369). IEEE.

6. Palattella, M. R., Dohler, M., Grieco, A., Rizzo, G., Torsner, J., Engel, T., \& Ladid, L. (2016). Internet of things in the $5 \mathrm{G}$ era: Enablers, architecture, and business models. IEEE Journal on Selected Areas in Communications, 34(3), 510-527.

7. Foukas, X., Patounas, G., Elmokashfi, A., \& Marina, M. K. (2017). Network Slicing in 5G: Survey and Challenges. IEEE Communications Magazine, 55(5), 94-100.

8. Kirkpatrick, K. (2013). Software-defined networking. Communications of the ACM, 56(9), 16-19.

9. Zhang, H., Liu, N., Chu, X., Long, K., Aghvami, A., \& Leung, V. C. M. (2017). Network Slicing Based 5G and Future Mobile Networks: Mobility, Resource Management, and Challenges. arXiv preprint arXiv:1704.07038.

10. Akyildiz, I. F., Wang, P., \& Lin, S. C. (2015). SoftAir: A software defined networking architecture for $5 \mathrm{G}$ wireless systems. Computer Networks, 85, 1-18.

11. Rost, P., Banchs, A., Berberana, I., Breitbach, M., Doll, M., Droste, H., ... \& Sayadi, B. (2016). Mobile network architecture evolution toward 5G. IEEE Communications Magazine, 54(5), 84-91.

12. Zhou, X., Li, R., Chen, T., \& Zhang, H. (2016). Network slicing as a service: enabling enterprises' own software-defined cellular networks. IEEE Communications Magazine, 54(7), 146-153.

13. Nikaein, N., Schiller, E., Favraud, R., Katsalis, K., Stavropoulos, D., Alyafawi, I., ... \& Korakis, T. (2015, September). Network store: Exploring slicing in future 5g networks. In Proceedings of the 10th International Workshop on Mobility in the Evolving Internet Architecture (pp. 8-13). ACM.

14. Rosen, R. (2014). Linux containers and the future cloud. Linux J, 240(4), 86-95.

15. Banchs, A., Breitbach, M., Costa, X., Doetsch, U., Redana, S., Sartori, C., \& Schotten, H. (2015, May). A NOvel Radio Multiservice adaptive network Architecture for $5 \mathrm{G}$ networks. In Vehicular Technology Conference (VTC Spring), 2015 IEEE 81st (pp. 1-5). IEEE.

16. Yousaf, F. Z., Loureiro, P., Zdarsky, F., Taleb, T., \& Liebsch, M. (2015). Cost analysis of initial deployment strategies for virtualized mobile core network functions. IEEE Communications Magazine, 53(12), 60-66.

17. Samdanis, K., Costa-Perez, X., \& Sciancalepore, V. (2016). From network sharing to multitenancy: The 5G network slice broker. IEEE Communications Magazine, 54(7), 32-39.

18. Kubernetes mainpage. Available online: https://kubernetes.io/ (accessed on 26/11/2017)

19. Docker mainpage. Available online: https://www.docker.com/ (accessed on 26/11/2017) 


\title{
Detecting malicious components in large-scale Internet- of-Things systems and architectures
}

\author{
Borja Bordel $^{1}$, Ramón Alcarria ${ }^{1}$, Diego Sánchez-de-Rivera ${ }^{1}$ \\ ${ }^{1}$ Universidad Politécnica de Madrid, \\ Madrid, España \\ bbordel@dit.upm.es, ramon.alcarria@upm.es,diegosanchez@dit.upm.es
}

\begin{abstract}
Current large-scale Internet-of-Things systems and architectures incorporate many components, such as devices or services, geographic and conceptually very sparse. Thus, for final applications, it is very complicated to deeply know, manage or control the underlying components, which, at the end, generate and process the data they employ. Therefore, new tools to avoid or remove malicious components based only on the available information at high level are required. In this paper we describe a statistical framework for knowledge discovery in order to estimate the uncertainty level associated with the received data by a certain application. Moreover, these results are used as input in a reputation model focused on locating the malicious components. Finally, an experimental validation is provided in order to evaluate the performance of the proposed solution.
\end{abstract}

Keywords: Internet-of-Things, knowledge discovery, security, uncertainty, information systems, pervasive sensing, grid computing

\section{Introduction}

Nowadays, Internet-of-Things (IoT) has matured from its origin as a research concept to commercial products and real deployments, such as the current smart cities [1]. In particular, large-scale IoT pilots are the most interesting and recent topic on IoT innovation [2]. These pilots include very complex systems and architectures which involve a great amount of components (such as devices, services, execution engines, etc.). This complexity facilitates the appearance (deliberate or not) of malicious components; those which provide uncertain data, services or information. In general, IoT architectures try to merge very different devices and other components, which may be (and used to be) geographically sparse and conceptually very distant [3]. Thus, low-level information must be collected, transformed, aggregated and translated various times (using, for example, semantic technologies) before being sent to the high-level final applications. However, none meta-information about the underlying hardware platform (such as sensor sensibility) or other low-level components is provided to the high-level layers.

Therefore, final applications have a very limited knowledge about the system and almost no control over the infrastructure which provides them with the operation data [4]. In this context, extremely important concepts such as the uncertainty level 
associated with the received data or the real quality associated with the offered services cannot be estimated using traditional configuration algorithms (as they require special control information in order to calculate the results) [5]. Instead of that, new solutions based only on the available information at high-level are necessary.

The objective of this paper is to describe a statistical framework for knowledge discovery in order to estimate the uncertainty level associated with the received operation data by a certain application. Additionally, these results are used as input in a reputation model focused on locating the malicious components. Thus, if possible, final applications may discard information from these components.

The rest of the paper is organized as follows: Section 2 describes the state of the art on uncertainty management and reputation models; Section 3 includes the mathematical formalization of the proposed framework and reputation model; Section 4 presents an experimental validation based on a simulated scenario in order to test the performance of the proposed solution; Section 5 contains the experimental results and Section 6 concludes the paper.

\section{Related works}

Uncertainty management is one of the key problems in IoT scenarios; however, little work has treated this topic. Most papers, moreover, are focused on how uncertainties affect control loops and algorithms. In this case, they use to focus the research on Cyber-Physical Systems [6] (sometimes understood as a specific IoT scenario) which may be described using finite difference equations, which are influenced by unknown discrete functions representing the uncertainties [7]. Additionally, some works [8] propose a mathematical framework in order to calculate the optimal reaction in order to cancel the effect of uncertainties in control loops.

On the other hand, typical works on uncertainty management in IoT scenarios try to measure the influence of factors that designers know they do not know (noise, packet losses, etc.). Thus, uncertainty taxonomies [9], modeling [10] and processing [11] are really typical. Nevertheless, factors that designers do not know they do not know are never addressed; and this kind of factors is the most important in large-scale IoT systems. Thus, a more general framework is required.

Papers on uncertainty level estimation in IoT scenarios are strange, and usually research works on this topic talk about trust levels. Any case, there is also little work on trust management for IoT environments. Furthermore, most of these works are based on the concept of reputation.

Some works try to stablish the definition of reputation [12] in IoT systems, and apply the model in a horizontal way (inside the underlying sensor network, in order to calculate the reputation of nodes) [13]. These models, however, only consider Quality-of-Service (QoS) trust metrics like packet forwarding/delivery ratio and energy consumption. More general models have been also proposed [14], including philosophical concepts such as honesty, cooperativeness, or community-interest.

If vertical solutions are considered, works on service reputation are also found [15]. In particular, models based on the evaluation of user's trust in a service and 
service classification [16]; and models considering authentication history and penalty [17] can be found. However, none of these models consider the previously described theoretical formalization, and concepts such as the service honesty are not defined.

In comparison with all the cited works our proposal formalizes the concept of uncertainty level in IoT scenarios using statistical tools. Additionally, definitions initially proposed for sensor networks are extended to services and other IoT components. As a result, malicious components are efficiently located and policies in order to remove or avoid them are enabled.

\section{Formalization of the proposed solution}

Malicious components are those which provide uncertain data, service or information. This behavior may be due to a cyber-attract, a bad programming or a malfunction in hardware (among other reasons). In order to avoid sever damage to entire system, these components must be isolated. With this objective, the concept of "reputation of a IoT entity" is defined.

The reputation of an IoT component $\Sigma, \mathcal{R}_{\Sigma}$, is defined as the global perception of its behavior in the system, in particular, whether transactions including this component present in general positive outcomes. As can be seen, reputation is a global concept, so all components in the system should be involved in its estimation. However, a global definition of reputation may be impractical, thus we also define the concept of local reputation.

The $\Lambda$-local reputation of an IoT component $\Sigma,\left.\mathcal{R}_{\Sigma}\right|_{\Lambda}$, is the local perception of the behavior of the component $\Sigma$ in a certain system's component $\Lambda$, in particular whether transactions including both components present in general positive outcomes.

Then, it is trivial to deduct that the relation between $\mathcal{R}_{\Sigma}$ and $\left.\mathcal{R}_{\Sigma}\right|_{\Lambda}$ is which indicated in (1), where $\mathfrak{C}$ is the set of all components in the system and $\lambda_{\Lambda}$ the relative weight of $\left.\mathcal{R}_{\Sigma}\right|_{\Lambda}$.

$$
\mathcal{R}_{\Sigma}=\left.\sum_{\Lambda \in \mathfrak{C}} \lambda_{\Lambda} \cdot \mathcal{R}_{\Sigma}\right|_{\Lambda}
$$

The $\Lambda$-local reputation of an IoT component $\Sigma$ it is calculated as the weighted sum of three values: the nobleness $\mathcal{N}$, the solidarity $\mathcal{S}$, and the relevance $\mathcal{R} e$ of the component $\Sigma$ perceived by the component $\Lambda$ (2).

$$
\left.\mathcal{R}_{\Sigma}\right|_{\Lambda}=\left[\begin{array}{lll}
\alpha & \beta & \gamma
\end{array}\right] \cdot\left[\begin{array}{c}
\mathcal{N} \\
\mathcal{S} \\
\mathcal{R} e
\end{array}\right]
$$

Thus, a component $\Sigma$ is categorized as malicious by a certain component $\Lambda$ if its $\Lambda$-local reputation falls below a certain threshold $\mu_{\Lambda}$ (a solution which in practical applications should be complemented with, for example, a token-based danger detection technology see Section 5 and 6). On the contrary, it is classified as a regular component. Malicious components should be avoided and, in that way, they would tend to be isolated as time passes.

Although these concepts may be applied to any couple of IoT components in a system or architecture, in this work we are focused on the reputation that final 
applications perceive about their primary data sources (i.e. mainly, services and hardware devices).

In order to evaluate the local reputation, the three parameters which compose it must be defined. For that, four different scenarios may be defined (see Figure 1), depending on two independent criteria.

- Transmission mode: Bidirectional communications generate a more complex model as two data flows have to be considered. In the simplest case, data only flows from the information source to the final application (unidirectional communications).

- Data aggregation: In some occasions, data received by a certain application are obtained by aggregating the flows from various components (various-to-one scheme). In that case, measurements refer a global vision, and estimating the individual reputation is more complicated than in one-to-one schemes (where data are generated only in one component).
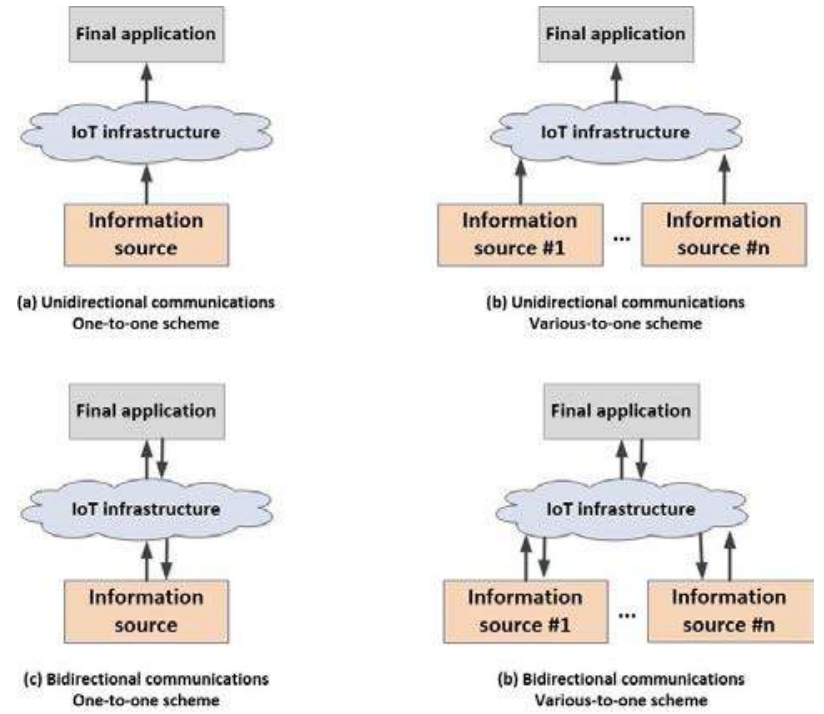

Fig. 1. Possible scenarios for the local reputation calculation

For this first work a reduced model is considered. Thus, only unidirectional communications based on a one-to-one scheme are considered. Additionally, in this first study, it is considered that $\beta=\gamma=0$ and $\alpha=1$, so the value of the local reputation matches the nobleness value. Next subsection describes the calculation of this parameter.

\subsection{Uncertainty level estimation: nobleness calculation}

The nobleness $\mathcal{N}$ of an IoT component $\Sigma$, according to a second component $\Lambda$, is defined as the expectance of $\Lambda$ to obtain correct information from $\Sigma$. This information 
may be production data or meta-information (such as the offered QoS for a certain service).

Nobleness calculation is based on previous experiences, which are weighted in order to limit the effect of events very distant in time. Moreover, it is difficult to stablish the nobleness of a component based on a few interactions. Thus a threshold $N_{t h}$ must be defined in order accumulate the required measurements to start estimating the nobleness value.

Equation (3) represents the mathematical model for nobleness calculation; where $n$ is the number of accumulated nobleness measurements and $h$ is the weighted ratio of the number of times the component behaved nobly (i.e. it sent correct information).

$$
\mathcal{N}=\left\{\begin{array}{c}
1 ; n<N_{t h} \\
\sqrt{2} \frac{h}{\sqrt{1+h^{2}}} ; n>N_{t h}
\end{array}\right.
$$

As can be seen, nobleness follows an algebraic function belonging to the sigmoid class. Thus, $\mathcal{N} \in[0,1] \forall h \in[0,1]$. Moreover, the model includes the presumption of nobleness, as every component is sincere $(\mathcal{N}=1)$ until enough measurements are collected. In order to calculate the weighted ratio $h$, we are considering a geometric sum (4); where the common ratio $r$ can be freely fixed (in order to limit the influence of the past behaviors, as $|r| \rightarrow 0)$. .

$$
h=\sum_{j=0}^{n} u[-j] \cdot r^{j+1}
$$

The sequence $u[\cdot]$ represents the natural ratio of the number of times the component behaved honestly in every time slot. $u[j]$ is defined (5) as the quotient between the times the component provided correct information in the $\mathrm{j}$-th time slot $p_{j}$ and the total number of transactions in that time slot $t_{j}$.

$$
u[j]=\frac{p_{j}}{t_{j}}
$$

In order to calculate whether an IoT component has provided correct information in a certain transaction, we are evaluating the uncertainty level $\theta$ associated with the provided information. If this level remains below a certain threshold $\mu_{h}$ it is considered the component has been honest. In some cases, the detected uncertainty may not be caused by the analyzed component. However, from the final applications' point of view the provided information is uncertain and, in an aggregated vision, the component is not honest.

In order to evaluate the uncertainty level $\theta$ we use the following statistical model. Figure 2 represents the scenario under study. A final application received from an information source (IoT component) a certain information $\overline{\bar{x}}$. In large-scale IoT systems, the uncertainty level associated with $\overline{\bar{x}}$ is the addition of two amounts.

First, the uncertainties $\mathfrak{I}_{I T}$ about the equivalence between the received information $\overline{\bar{x}}$ and the information generated by the information source $\bar{x}$. In this first case, the relation between both data may be described as a surjective stochastic application $T[\cdot]$, as every information $\overline{\bar{x}}$ must be the image of a certain information $\bar{x}$. These uncertainties are caused by the IoT infrastructure, so they are IT uncertainties.

And, second, the uncertainties $\mathfrak{I}_{P H Y}$ about the equivalence between the information generated by the information source $\bar{x}$ and the real information existing in the 
physical world $x$. These uncertainties are caused by physical limitations in the information capture. Therefore, they are physical uncertainties.

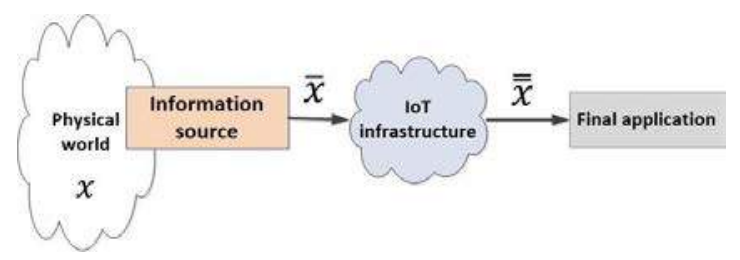

Fig. 2. Scenario under study

Thus, associated with a received information $\overline{\bar{x}}$ there exists an enumerable set of uncertainty sources $\mathfrak{I}=\left\{\mathfrak{I}_{P H Y}, \mathfrak{I}_{I T}\right\}=\left\{i_{k}, k=1, \ldots, K\right\}$ whose cardinality $K$ may reach the cardinality of the natural numbers $\aleph_{0}$. This uncertainty sources transform the process of acquiring a certain information $\overline{\bar{x}}$ in a random experiment $\varepsilon$, which takes values from the discrete sample space $\Omega$.

Each uncertainty source is described as a bi-varied stochastic process (6), being $\Psi$ the sample space of all possible values for the uncertain event.

$$
i_{k}=\mathrm{X}_{k}[m ; \omega, \psi) / \omega \in \Omega, \psi \in \Psi
$$

Stochastic processes are discrete in time as final applications are a cyber component, but the sample space $\Psi$ may be continuous or discrete, depending on the nature of the uncertainty source. For example, the measurement error has a continuous nature; however, the possibility of suffering a cyber-attack is described by a discrete variable. Furthermore, in general, uncertainties' value change pretty slow, so these stochastic processes may be considered stationary during a time slot. As they are unknown effects, stochastic processes are expressed in a parametric way, depending, each one, on a certain parameter $\vartheta_{k}$. Three basic probability density functions or probability distributions may be used to describe uncertainty sources: uniform distributions, triangular distributions and Gaussian distributions (see Figure 3).

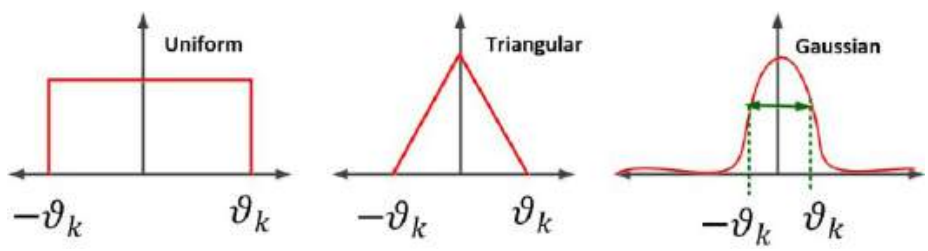

Fig. 3. Basic probability density functions

Uniform distributions are employed to describe unknown effects whose effect is limited to the range $\left[-\vartheta_{k}, \vartheta_{k}\right]$. In data acquisition processes this is most general distribution, as the sample space is bounded. Triangular distributions are employed when, besides the variation range, it is known that the error probability goes down as its value goes up. Finally, if more information is available (for example, if noise is considered) a Gaussian distribution with typical deviation $\vartheta_{k}$ can be employed. 
Considering $\mathcal{F}$ the set of parts of $\Omega$, and a function $P(A) A \in \mathcal{F}$ which fulfills the three Kolmogorov's probability axioms, the experiment $\varepsilon$ is completely characterized with the event algebra $E=\langle\Omega, \mathcal{F}, P(\cdot)\rangle$, which (additionally) is a $\sigma$-algebra.

In this context, it is possible to create a partition $\Pi=\left\{\pi_{1}, \ldots, \pi_{p}\right\}$ of $\Omega$, and select a main value $\delta_{i} \in \pi_{i}$ representing every cluster. Then, the process to estimate the uncertainty level $\theta[j]$ in the $\mathrm{j}$-th time slot is as follows.

When certain information $\overline{\bar{x}}$ is received, it is included in the observations vector $v_{j}$ of the current time slot (7).

$$
v_{j}=\left(\overline{\bar{x}}_{1}, \ldots, \overline{\bar{x}}_{m}\right)
$$

As these observations are independent, it is possible to calculate the value of the $\vartheta_{k}$ parameter for each uncertainty source using the maximum likelihood estimation (MLE) [18] algorithm and the vector $v_{j}$. This method is the most adequate as prior probability distributions are unknown. Then, the event $\pi_{i}$ to which belongs the received information it is located. For each uncertainty source, the probability $\rho_{i}^{j}$ of the received information to really belong to the event $\pi_{i}$ in that time slot is calculated (8).

$$
\rho_{i}^{j}=\int_{\pi_{i}} \mathrm{X}_{k}[j ; \overline{\bar{x}}, \psi) d \psi \quad \text { or } \quad \rho_{i}^{j}=\sum_{\psi \in \pi_{i}} \mathrm{X}_{k}[j ; \overline{\bar{x}}, \psi]
$$

In that way, as uncertainty sources are also independent, the global probability $\rho^{j}$ of $\overline{\bar{x}}$ to belong to $\pi_{i}$ is calculated as a probability multiplication (9).

$$
\rho^{j}=\prod_{k=1}^{K} \rho_{k}^{j}
$$

Finally, the information $\delta_{i}$ is considered to be received with an uncertainty level $\theta[j]$ calculated as indicated in (10).

$$
\theta[j]=1-\rho^{j}
$$

In this transaction, the information source is considered to be honest if meets the condition explained above $\left(\theta[j]>\mu_{h}\right)$.

\section{Experimental validation}

In order to evaluate the proposed solution, an experimental validation was designed. The proposed experiment consisted of a simulation scenario, where a large-scale IoT system was deployed (Figure 4).

The simulated scenario (based on a real European deployment) included four different networks of information sources (public sensors, LoRA devices, etc.) geographically sparse. Each network was composed by one hundred and fifty (150) components. Randomly, the configuration of ten (10) components was created to force them to provide uncertain information (and being considered as malicious). Basically, the precision of the instruments was reduced, the electromagnetic interferences and the packet losses were strengthened and, in one case, it was supposed an intruder controls the component (causing this component to provide erroneous information). One final application was hosted in the FI-WARE ecosystem. 


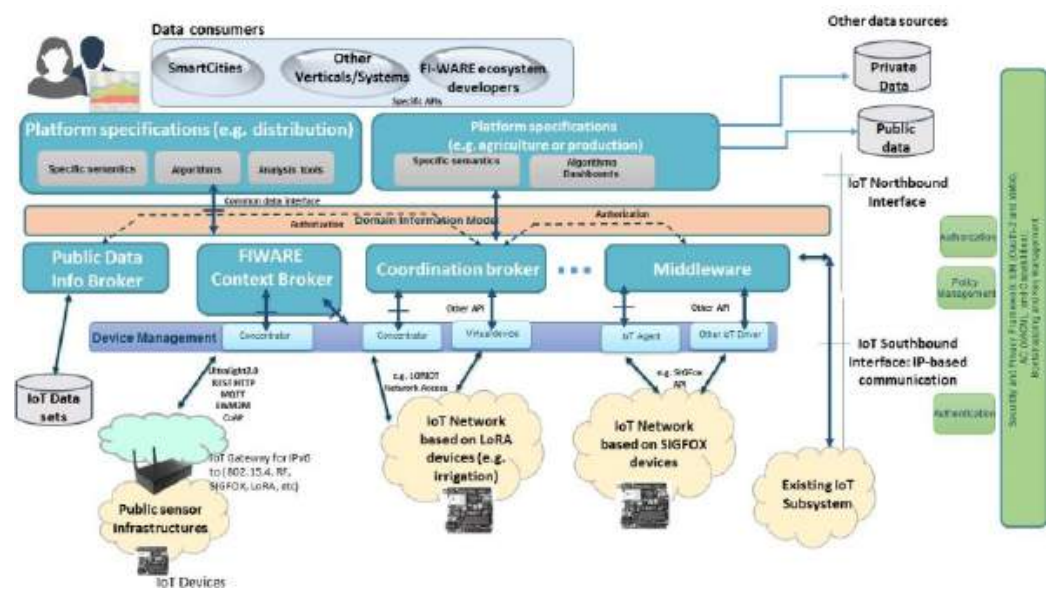

Fig. 4. Simulation scenario

The simulated final application included the proposed uncertainty level calculation algorithm and the described reputation model (expressions from (1) to (10)). The other entities could be measured, but they were not provided with the reputation calculation module.

In order to perform the proposed simulation, the NS3 simulator was employed. NS3 is a network simulator whose scenarios and behavior are controlled and described by means of $\mathrm{C}++$ programs, and which is extensively used in research due to its flexibility. Data about the number of transactions performed by the malicious nodes were collected. Moreover, the evolution of the local reputation of the malicious nodes was monitored.

\section{Results}

Figure 5(a) presents the evolution of the number of transactions performed by the malicious components as times passes. As can be seen, firstly the number of transaction remains constant, but from a time between $t=80$ and $t=200$ minutes, the number of transaction in every malicious node descends slowly but continuously, following an exponential-like law. This exponential tendency is explained by the fact that, once the reputation goes below the stablished threshold, final applications look for other information sources to obtain their data immediately. Depending on the uncertainty of the received data (see Expression 9 and Figure 3), the descent phase can start later or be very abrupt from the beginning. Moreover, components performing a low number of transactions require more time to be isolated, as more time is required to acquire the necessary information about their behavior. Any case, each of the ten malicious components, at the end, got isolated, so the objective of the proposed solution is met. 

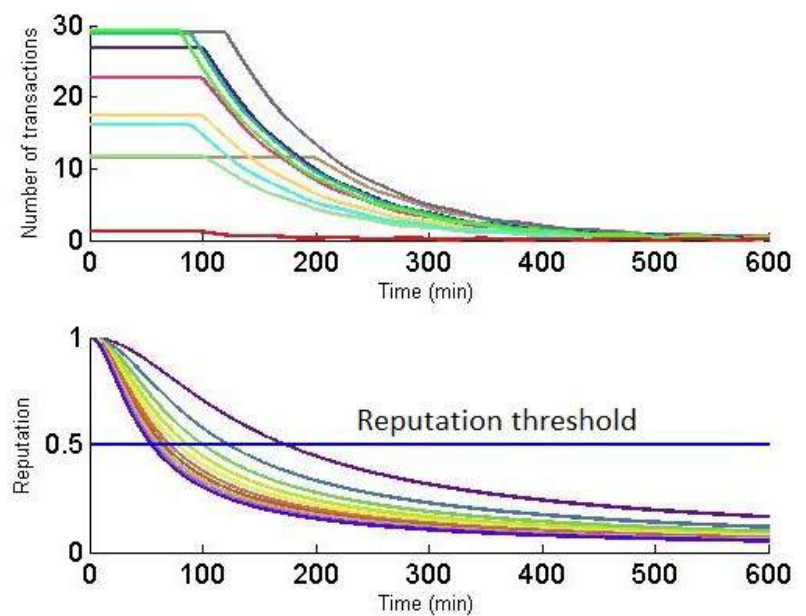

Fig. 5. Simulation results

On the other hand, the correspondence between the amount of performed transactions and the calculated reputation can be seen in Figure 5(b). In this figure a group of sigmoid functions is showed, as the nobleness calculation model is described using these functions. In this case, the reputation level descends constantly since the initial instant, as malicious components behave in a disingenuous way from the beginning. In comparison, the number of performed transaction remains constant for every component, until its reputation goes below the fixed threshold. This fact must be taken into account, as there is a convergence time during which malicious components may affect the entire system. The descent stops when no transactions are performed by the component (see Figure 5(a)) and measurements are not updated.

\section{Conclusions and future works}

In this paper we have described a mathematical framework for the data uncertainty level calculation, which allows final application to estimate the nobleness and the reputation of the information sources and other IoT components in large-scale systems and architectures. Results showed that malicious components get isolated as time passes if implanted the proposed technology.

On the other hand, the proposed reputation model may be enriched with additional measurements (such as the component solidarity) in order to accelerate the isolation process. Future works should consider these ideas (exposed also in Section 3), as well the possibility of including a token-based rapid hazard detection system (in order to avoid severe damage during the convergence time). Finally, both, the formalization of the solution and the simulation scenario, describe the basic problem, so future works should extend the proposed framework to scenarios where bidirectional communications and data aggregation are presented. 
Acknowledgments. Borja Bordel has received funding from the Ministry of Education through the FPU program (grant number FPU15/03977). Additionally, the research leading to these results has received funding from the Ministry of Economy and Competitiveness through SEMOLA project (TEC2015-68284-R) and from the Autonomous Region of Madrid through MOSI-AGIL-CM project (grant P2013/ICE3019, co-funded by EU Structural Funds FSE and FEDER).

\section{References}

1. Sarkar, A. N. Significance of Smart Cities in 21 st Century: An International Business Perspective. FOCUS: Journal of International Business, 2(2), (2016).

2. Hong, K., Lillethun, D., Ramachandran, U. Mobile fog: A programming model for largescale applications on the internet of things. In Proceedings of the second ACM SIGCOMM workshop on Mobile cloud computing, pp. 15-20. ACM. (2013)

3. Lee, H., Jo, S. K., Lee, N., Lee, H. W. A method for co-existing heterogeneous IoT environments based on compressive sensing. In 18th International Conference on Advanced Communication Technology (ICACT) pp. 206-209. IEEE, (2016).

4. Robles, T., Alcarria, R., Martın, D., Navarro, M.: An IoT based reference architecture for smart water management processes. J. Wirel. Mob. Netw. Ubiquit. Comput. Dependable Appl, 6(1), pp. 4-23. (2015)

5. Bordel, B., Alcarria, R., Martín, D., Robles, T. Self-configuration in humanized CyberPhysical Systems. Journal of Ambient Intelligence and Humanized Computing (2016)

6. Stankovic, J. AResearch directions for the internet of things. IEEE Internet of Things Journal, 1(1), pp. 3-9, (2014)

7. Cardenas, A. A., Amin, S., Sastry, S. Secure control: Towards survivable cyber-physical systems. System, (2008)

8. Zhu, Q., Başar, T.: Robust and resilient control design for cyber-physical systems with an application to power systems. In 2011 50th IEEE Conference on Decision and Control and European Control Conference, pp. 4066-4071, IEEE. (2011)

9. Zhang, M., Selic, B., Ali, S., Yue, T., Okariz, O., Norgren, R. Understanding Uncertainty in Cyber-Physical Systems: A Conceptual Model. (2016)

10. Aggarwal, C. C., Ashish, N., Sheth, A. The internet of things: A survey from the datacentric perspective. In Managing and mining sensor data, pp. 383-428. Springer US. (2013).

11. Hasan, S., Curry, E. Approximate semantic matching of events for the internet of things. ACM Transactions on Internet Technology (TOIT), 14(1), 2, (2014).

12. Chen, D., Chang, G., Sun, D.: TRM-IoT: A trust management model based on fuzzy reputation for internet of things. Comput. Sci. Inf. Syst., 8(4), pp. 1207-1228, (2011)

13. Boukercha, A., Xua, L., EL-Khatibb, K.: Trust-based security for wireless ad hoc and sensor networks. Computer Communications Vol. 30, Issues 11-12, 2413-2427. (2007)

14. Bao, F., Chen, R. Trust management for the internet of things and its application to service composition. In World of Wireless, Mobile and Multimedia Networks (WoWMoM),(pp. 16. IEEE, (2012).

15. Chen, L., Yan, Z., Zhang, W.: TruSMS: A trustworthy SMS spam control system based on trust management. Future Generation Computer Systems, 49, pp. 77-93, (2015)

16. Liu, Y., Chen, Z., Xia, F., Lv, X., Bu, F. A trust model based on service classification in mobile services. In Green Computing and Communications (GreenCom), pp. 572-577. IEEE, (2010)

17. Liu, Y., Wang, K. Trust control in heterogeneous networks for internet of things. In International Conference on Computer Application and System Modeling, (2010)

18. Scholz, F. W. Maximum likelihood estimation. Encyclopedia of statistical sciences. (1985). 


\title{
Protecting Industry 4.0 systems against the malicious effects of cyber-physical attacks
}

\author{
Borja Bordel, Ramón Alcarria, Diego Sánchez-de-Rivera, Tomás Robles \\ Universidad Politécnica de Madrid \\ bbordel@dit.upm.es, ramon.alcarria@upm.es, diego.sanchezderiveracordoba@gmail.com, \\ tomas.robles@upm.es
}

\begin{abstract}
Industry 4.0 refers a new industrial paradigm based on CyberPhysical Systems principles. In these new, complex and highly interdependent systems, the traditional definition of "cyber-attack" is not enough to represent all the situations may occur. Furthermore, traditional security policies and defense strategies are not designed to be effective in scenarios mixing cyber and physical elements. In this context, this work presents a new idea about what cyber-physical attacks are, and a technological solution to protect and compensate the malicious effects of these attacks in Industry 4.0 systems. The proposal is based on a specific description language (CP-ADL) for cyber-physical attacks, and a mathematical framework allowing a decision making about the most adequate defense strategy. Finally, and experimental validation is provided, showing with our proposal the impact of cyber-physical attacks is highly reduced.
\end{abstract}

Keywords: Cyber-Physical attacks, Industry 4.0, process execution, CP-ADL, security

\section{Introduction}

The emerging term Industry 4.0 was coined for the first time at the Hannover Fair during the presentation of the "Industry 4.0" initiative in Germany [1]. In general words, Industry 4.0 refers a new industrial scenario where a new type of systems consisting of the intersection of the physical and the cyber world (the so-called CyberPhysical Systems -CPS-) is employed.

Traditional industrial systems are focused on control, so standard security policies consist of feedback loops with the objective of compensating the malicious effects of cyber-attacks [2][3]. However, CPS present some special characteristics (such as the pervasiveness and the hybrid design) which make them vulnerable to a new and more general type of attacks known as cyber-physical attacks [4]. In these attacks, changes (accidental or not) in both (the physical and the cyber space) may appear, but due to the highly interdependency of components in CPS, the effects may influence any other part of the system, and thousands of components could be the final objective of the attack [4]. The genuine approach of cyber-physical attacks is acting on the weakest elements in the system (usually sensors deployed in a public space) in such a way 
that these elements (which have full access to the system) cause a fail in the critical components (as every element in the system is integrated with the others). For example, vandalism, weather damages and, of course, traditional cyber-attacks, may be considered as cyber-physical attacks. CPS, moreover, may be deployed together with critical infrastructures (such as smart grids), so disconnecting the system or waiting until the attack finishes are not valid solutions.

The problem associated with these new attacks is their amplitude: there is an infinite amount of ways to perform a successful cyber-physical attack. Thus, depending on the attacked element, the aggression method and the final objective of the attack, protecting policies must be different. In a simple example, if control components are affected, traditional security policies for control systems should be applied; on the contrary if the data analytics layer is implicated, the attacks could be addressed as a computer virus. In this context, once detected a cyber-physical attack is taken place, it is necessary to infer the type of attack being performed in order to protect the system adequately. However, information about a running attack is obtained as time passes, while the effects in the system are getting worse.

Therefore the objective of this paper is to provide a solution in order to infer the type of cyber-physical attack that an Industry 4.0 system is suffering, as well as a decision making framework to determine if a certain security or protection policy must be applied or if it is more profitable to wait for more information about the attack. Our proposal is based on a specific description language (CP-ADL) for cyberphysical attacks, on the use of XML patterns and templates and on a mathematical framework using the game theory.

The rest of the paper is organized as follows. Section 2 describes the state of the art on Industry 4.0 and CPS security solutions. Section 3 describes the proposed solution. Section 4 explains the simulation scenario used as experimental validation. Finally, Section 5 presents the results of the experimental validation and Section 6 concludes the paper.

\section{State of the art}

Security is one of the most popular research topics nowadays. The use of technological systems to digitalize critical infrastructures has cause an increase in the number of works about that issue. The emerging Industry 4.0 systems are also part of this trend.

Different works may be found. On the one hand, works about security in new generation smart grids have been proposed. Most of them are focused on the design of enhanced control loops [5][6], but cyber-attacks taxonomies have been also described [7][8]. Proposals about protection schemes for CPS applied to smart grids [9] and other systems under cyber-physical attacks [10] have been also described. On the other hand, security solutions for networked control systems and industrial applications have been also investigated [4].

The problem of all these proposals is that they are focused on attacks which introduce perturbations or known malicious signals in the system, so security and protec- 
tion solutions are fixed and rigid. In consequence, these proposals reduce their usability if new or slightly different attacks are performed.

In addition, works about how traditional security solutions (i.e. firewalls, computer shields, etc.) could be applied to CPS and Industry 4.0 scenarios have been recently described [11]. However, this approach only covers partially the problems and vulnerabilities associated with cyber-physical attacks, so more general solutions are required. General reviews about the problems associated with security in these new scenarios have been also reported. Works about critical industrial scenarios [20], Industry 4.0 systems [21] and CPS [22] may be found.

Finally, the concept of cyber-physical attack has been investigated. Abstract taxonomies and description languages have been proposed [12]. These instruments are very useful to classify and infer the use of certain types of cyber-physical attacks.

\section{$3 \quad$ A protection solution for Industry 4.0 systems}

A cyber-physical attack may be described using only six different fields [12] (see Figure 1). Below a brief explanation of each field is provided:

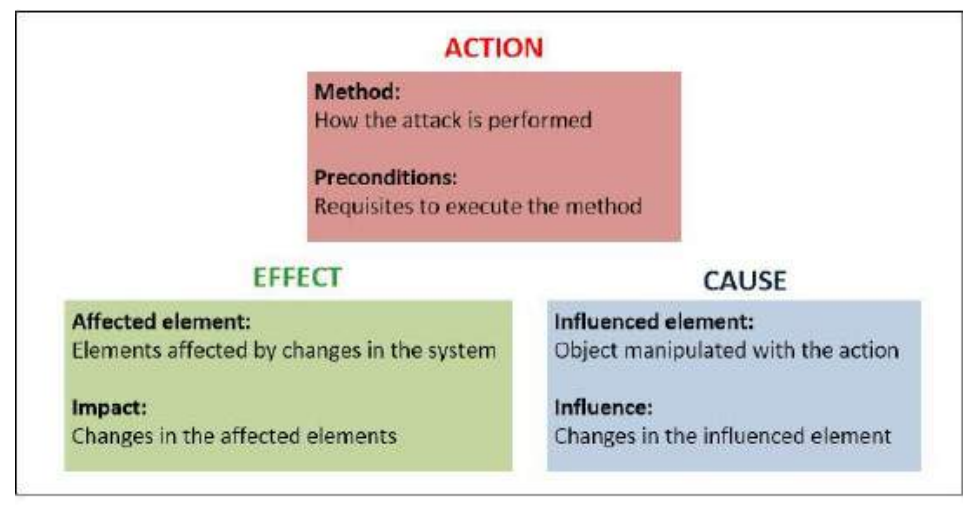

CYBER-PHYSICAL ATTACK

Fig. 1. Description of cyber-physical attacks

- Method: It represents the procedure employed to affect the system

- Preconditions: They list the requirements to be present in the system so the method is a successful way of attacking the system. Together with the "method" this list made up the "action" of the cyber-physical attack.

- Influenced element: It refers the elements which have been manipulated through the described action.

- Influence: The produced changes in the influenced element. Together with the "influenced element", it describes the "cause" of the cyber-physical attack. 
- Affected element: A list of the elements which have been affected by the changes in the system (usually it is the objective of the attack).

- Impact: A description of the changes in the system. Together with the "affected element", it describes the "effect" of the cyber-physical attack.

In this context, a cyber-physical attack may be modeled by means of a XML document, describing the value of each one of the previously identified fields. CP-ADL is a description language [12] focused on this objective (see Figure 2).
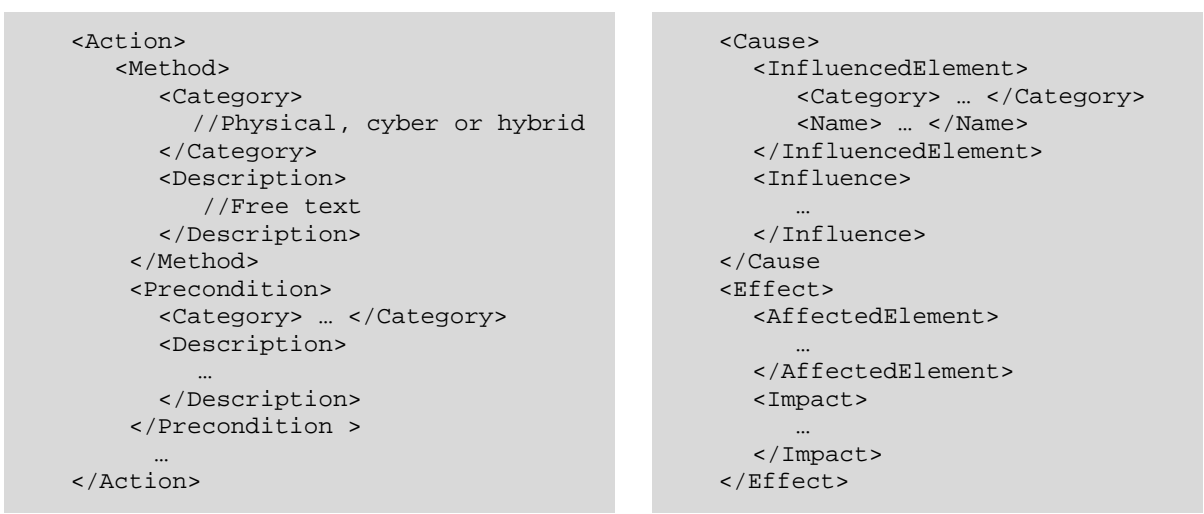

Fig. 2. Example of a generic CP-ADL description

Then, considering this description language, it is proposed a functional architecture in order to support a protection system for Industry 4.0 applications (see Figure 3 ). The proposed architecture is very horizontal and general, so it may be integrated at different levels in Industry 4.o architecture (from the highest to the lowest) and, even, it may be included in different places at the same time, creating a vertical security solution of connected horizontal deployments.

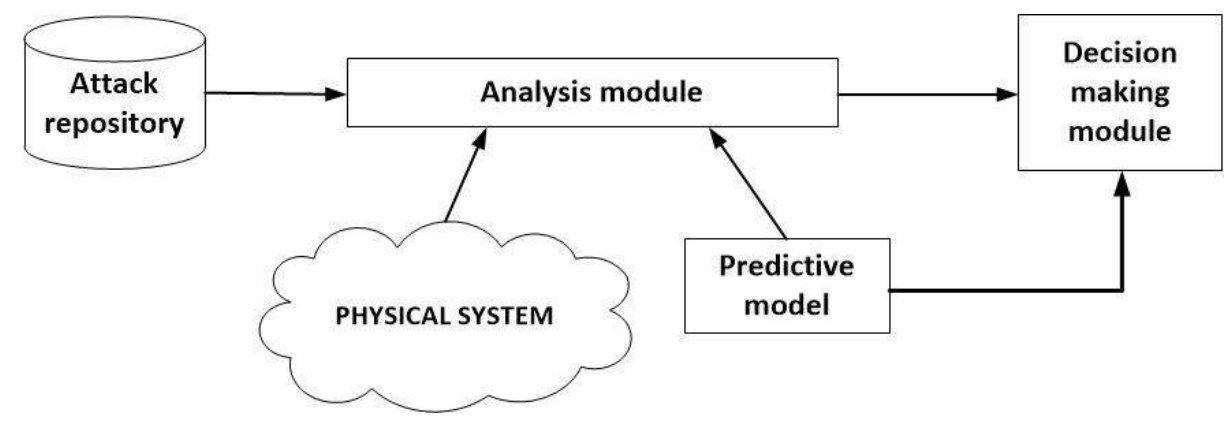

Fig. 3. Functional architecture

In this architecture, different components may be identified. A Predictive model represents the system's state in a certain moment and at some future instants. This model may be updated through a hybrid simulator and interpolation techniques [13]. This information is provided to an Analysis module, where these data are compared to 
the real state of the components in the system. Results of the comparison process are employed to infer if the system is under a cyber-physical attack. In order to do that, a collection of templates describing different cyber-physical attacks (and the security and protection policies associated with these attacks) is included in a Template repository. A distance function is employed to determine the set of templates (i.e. attacks) which are closer to the observations made about the system (a pattern recognition process is, in that way, performed). Finally, this set of patterns is used by a Decision making module to select the protection method to be applied (if decided it is necessary to perform some actions).

Mathematically, the process is described as follows.

An Industry 4.0 system $\mathfrak{I}$ is described in a certain instant $t=T_{0}$ as a collection of $N\left(T_{0}\right)$ components $c_{i}$ whose state is represented through a set of $M_{i}$ parameters $p_{j}$. See (1).

$$
\begin{aligned}
& \mathfrak{T}\left(t=T_{0}\right)=\left\{c_{i}\left(T_{0}\right), \quad i=1, \ldots, N\left(T_{0}\right)\right\}= \\
= & \left\{\left\{p_{j}\left(T_{0}\right), j=1, \ldots, M_{i}\right\}_{i}, \quad i=1, \ldots, N\left(T_{0}\right)\right\}
\end{aligned}
$$

Two different representations of the system are obtained, then, at each moment: on the one hand, the state calculated by the Predictive model $\mathfrak{I}_{p}$ and, on the other hand, the state obtained from the real deployment $\mathfrak{I}_{r}$. The difference between both representations $\varepsilon$ is obtained in order to evaluate whether any component in the system is affected by a cyber-physical attack (2). This difference is calculated for each component $\varepsilon_{c_{i}}$ and for the global system through a deformation estimation process [14]. If the deformation in the state of any component is higher than the maximum allowed threshold $\varepsilon_{t h_{i}}$, then, that component is considered affected (3).

$$
\begin{gathered}
\varepsilon=\left\|F\left(\mathfrak{I}_{p}, \mathfrak{T}_{r}\right)\right\| \\
\varepsilon_{c_{i}}>\varepsilon_{t h_{i}}
\end{gathered}
$$

Where $F(\cdot, \cdot)$ is a strain tensor (i.e. the Jacobian matrix of the application which transforms $\mathfrak{I}_{p}$ into $\mathfrak{I}_{r}$ ) and the operator "norm" $\|\cdot\|$ represents the module of a matrix.

Then, the list of affected components is employed to instantiate a XML template $t$ containing the information about the potential cyber-physical attack the system is suffering (4). The Cyber-Physical Attack Description language (CP-ADL) is employed. Usually, only the fields related to the "effect" of the attack could be described, although depending on the available information sometimes is possible to determine the "cause" as well.

$$
t=X M L\left(\varepsilon_{c_{i}}, \mathfrak{I}_{p}, \mathfrak{I}_{r}\right)
$$

In a standard case, information collected at the first instant $t=T_{0}$ is not enough to determine without any doubt which attack is being taken place or if, in fact, an attack is being performed. Thus, a statistical procedure is needed, so the set of the most probable options have to be calculated. In order to do that, it is considered the collection of templates $\mathbb{T}$ stored in the Template repository. Each one of these templates describes a cyber-physical attack, and it has associated a certain protection policy, adequate for such attack.

The set of most probable attacks $\mathcal{A}$ is then obtained by means of a Dynamic time warping (DTW) pattern recognition process [17], which may be understood as an 
enhanced distance in a generalized space (5). The basic idea is to calculate the proximity of the performed observations to every cyber-attack template.

$$
\mathcal{A}=\operatorname{dtw}(\mathbb{T}, t)
$$

If the DTW algorithm is too heavy to be executed in the scenario under study, a generalized distance could be also employed, such as $d_{1}$ or $d_{\infty}$ (which, at the end, compute the number of coincidences between two templates) [18]. Finally, the set $\mathcal{A}$ may be composed by the attacks whose distance to the instantiated template $t$ is lower than a certain limit; or may be forced to present a specific cardinality.

At this stage, different strategies (one for each cyber-physical attack contained in $\mathcal{A}$ ) may be followed (6) by the system manager (i.e. different protection policies could be applied or no action could be performed), and each one of these strategies produces different benefits/problems (i.e. different payoffs) in the system (7).

$$
\begin{aligned}
S_{\text {admin }} & =\left\{s_{1}^{\text {admin }}, \ldots, s_{q}^{\text {admin }}\right\} \\
U_{\text {admin }} & =\left\{u_{s_{1}^{\text {admin }}}, \ldots, u_{s_{q}^{\text {admin }}}\right\}
\end{aligned}
$$

These payoffs will be directly proportional to the compensation of the malicious effects of the cyber-physical attack produced by the protection policy under study. Moreover, the attacker may be running any of the cyber-physical attacks considered in $\mathcal{A}$, or, even, any other non-considered attack. Sometimes, besides, no attack is running (then, the attacker has one strategy more to choose than the system manager). Thus, the attacker may follow also different strategies (8), each one giving different payoffs (directly proportional to the malicious effects caused in the system) (9).

$$
\begin{aligned}
S_{\text {attack }} & =\left\{s_{1}^{\text {attack }}, \ldots, s_{q+1}^{\text {attack }}\right\} \\
U_{\text {attack }} & =\left\{u_{s_{1}^{\text {attack }}}, \ldots, u_{s_{q+1}^{\text {attack }}}\right\}
\end{aligned}
$$

In that way, a game can be defined, considering the attacker and the system administrator as players of the game (10). The game $G$ is dynamic and the number of considered rounds should be proportional to the observation period. For a standard configuration [19] three rounds should be considered. In this context, the intervention order in the game would be: Attacker-Manager-Attacker. Figure 4 represents the game in an extensive way.

$$
G=[S, U]=\left\{S_{\text {admin }}, S_{\text {attack }} ; U_{\text {admin }}, U_{\text {attack }}\right\}
$$




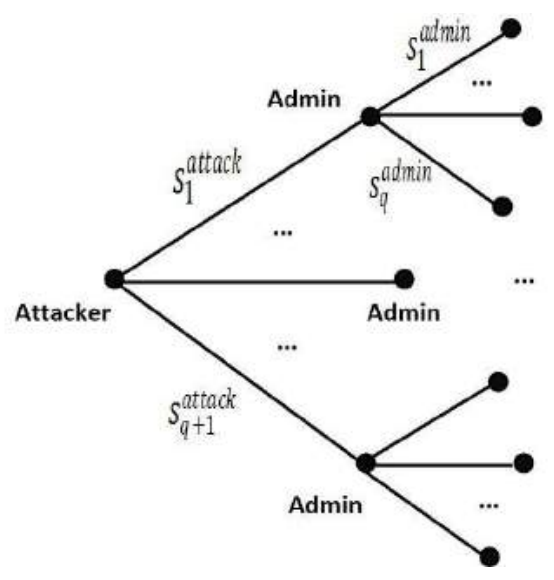

Fig. 4. Proposed dynamic game

Considering the first player who selects first a strategy is the attacker, three different tactics may be followed:

- Minimum effect: In this tactic, the manager selects the strategy which reduces as much as possible the payoff of the attacker. In this approach, the attacker usually tries to change the strategy to obtain a higher payoff.

- Satisfaction and control: In this tactic, it is selected the strategy which gives a highest payoff to the attacker. Thus, its objectives are fulfilled and the manager can be focused on controlling the malicious effects as much as possible. It is a very stable approach but very risky, especially if some effects become uncontrolled.

- Optimum: The system manager selects the strategy that generates a Nash equilibrium [15] (so the game does not advance as no individual incentive is possible). However, this strategy is only successful if the attacker is a rational entity.

Once, following any of the proposed tactics, a protection policy is selected, it is applied (or not, if decided waiting for more information). At this moment, the described process is restarted in order to re-evaluate the system performance and the effects of the selected security solution.

\section{$4 \quad$ Experimental validation}

In this Section we propose a first validation of the proposed solution by means of a simulation environment. The experimental validation consisted of a simulated scenario based on the NS3 simulator.

The proposed Industry 4.0 scenario consisted of different production systems of electronic devices and components. In particular three different types were considered: manufacture of diodes, manufacture of capacitors and manufacture of logic gates. Details about the components which are included in each one of the production systems are provided in the literature [16]. 


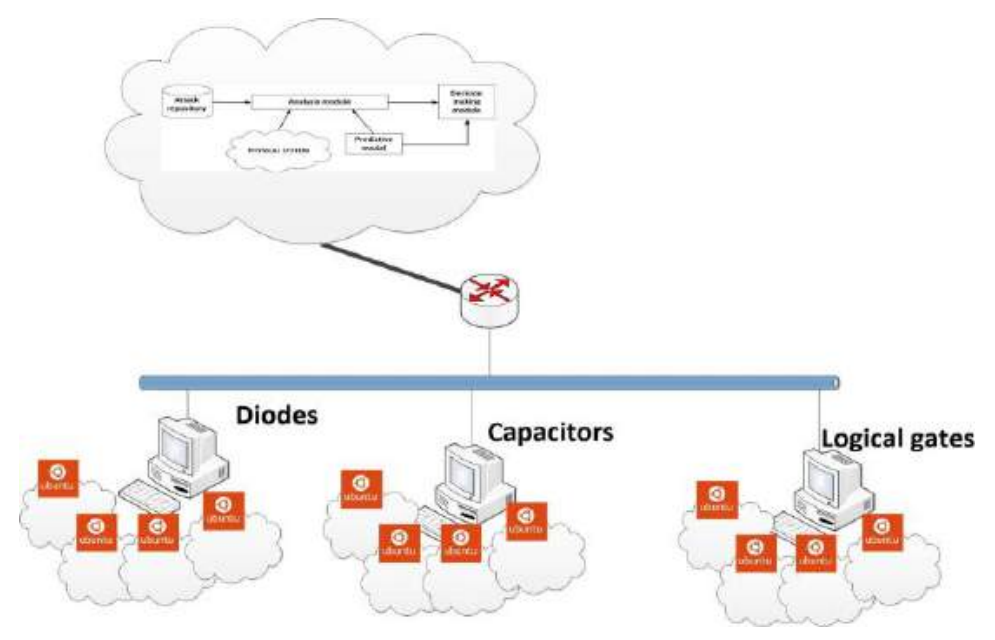

Fig. 5. Simulation scenario

Moreover, the proposed architecture was implemented in the top layer, in order to protect the system against cyber-physical attacks. With this approach, attack detection is slower but the scope of the applied protection policies turns wider. In order to implement the described solution in the components of the simulated scenario, each node in the NS3 simulator was provided with a tap bridge (or ghost node). These bridges are able to connect an internal NS3 component with an external entity, which allows implementing in a very easy way complex algorithms and other proprietary solutions for which there are no $\mathrm{C}++$ libraries (the programming language of the NS3 simulator). In particular, elements in the functional architecture were connected with virtual Linux machines based on the KVM technology (so they may be automatically deployed using the libvirt interface).

The simulation was programmed to introduce a cyber-physical attack in a random way, selecting the type of attack from a previously created catalogue.

Two different experiments were performed in this scenario. During the first experiment it was evaluated the number of attacks which were successfully addressed. Different tactics were considered in order to compare the obtained performance. Moreover, information about the protection procedure was obtained, so the causes of failures were also studied. During the second experiment, the required time to protect the system in an adequate way was measured.

\section{$5 \quad$ Results}

Results of the first experiment are showed on Figure 6. As can be seen, the proposed framework allows protecting successfully the system against cyber-physical attacks in more than $90 \%$ of cases. The tactic which produces the best results is "satis- 
faction and control", as it is the approach which forces the system to employ the most general and ambitious security policy (as the reduction in the malicious effects, in the first step, is considered to be minimum).

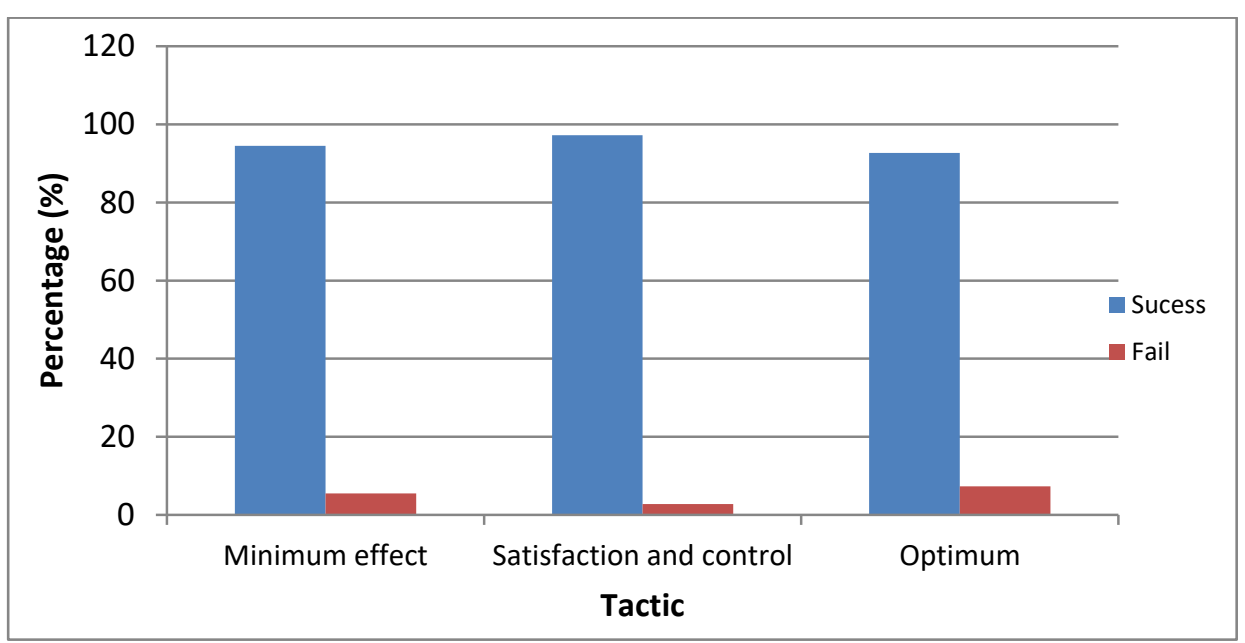

Fig. 6. Results of the first experiment

An study about the causes of failure showed that (in general), optimum tactic fails because the attacker is not a rational entity (for example, it cannot change its behavior depending on the applied security policy). On the other hand, the minimum effect tactic fails as predictions must be very precise to reach that situation and this is quite complicated if a fast response is desired.

Results of the second experiment are showed on Figure 7. As the interesting information is the comparison among the different tactics, but not the particular time values, time scale is normalized using the maximum value.

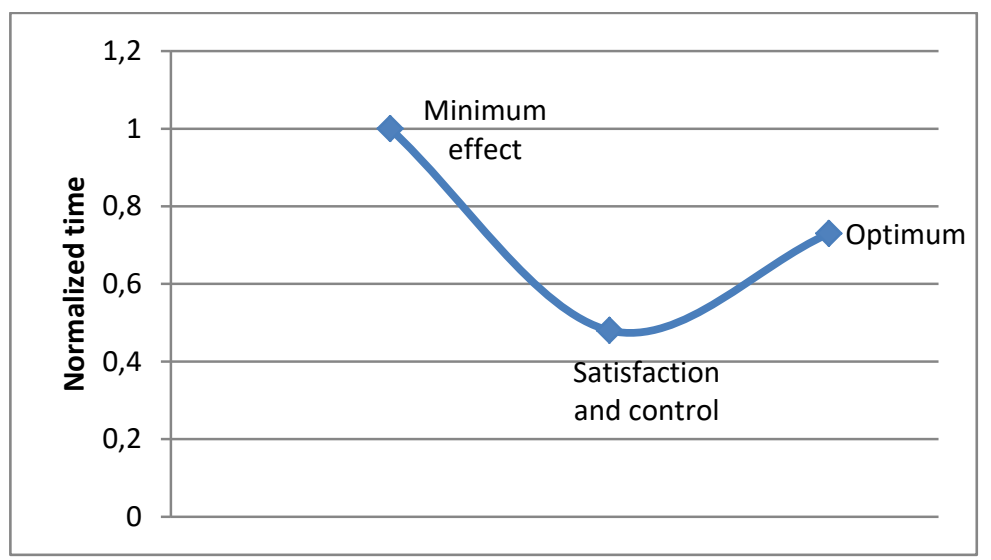

Fig. 7. Results of the second experiment 
As can be seen, the time required to implement and protect the system using the "satisfaction and control" tactic is the lowest. It is a very easy approach to execute, as no effort to control the appearance of new malicious effects is provided. On the contrary, the "minimum effect" approach is a very complicated tactic, as it requires a procedure to compensate the malicious effects of the cyber-physical attack and protection policies to avoid the appearance of new effects.

\section{Conclusions}

Industry 4.0 systems are one the most promising technological paradigms. This new type of solutions may be employed in many different scenarios, including critical infrastructures. In this context, security to be implemented in these systems is a key topic in research.

In this work a new framework to select and apply the most adequate security and protection policy in order to compensate the effects of cyber-physical attacks in Industry 4.0 scenarios is proposed. It consists of a template repository based on CPADL description language, and a decision making process based on the game theory.

Results of the experimental validation showed that in more than $90 \%$ of cases the proposed framework allows protecting Industry 4.0 systems against cyber-physical attacks. "Satisfaction and control" approach is the most successful tactic. Moreover, it is the fastest tactic to implement.

\section{Acknowledgments}

Borja Bordel has received funding from the Ministry of Education through the FPU program (grant number FPU15/03977). Additionally, the research leading to these results has received funding from the Ministry of Economy and Competitiveness through SEMOLA project (TEC2015-68284-R) and from the Autonomous Region of Madrid through MOSI-AGIL-CM project (grant P2013/ICE-3019, co-funded by EU Structural Funds FSE and FEDER).

\section{References}

1. Dujin, A., Geissler, C., Horstkötter, D. Industry 4.0: The new industrial revolution. Roland Berger Strategy Consultants, Munich. (2014).

2. Mo, Y., Kim, T. H. J., Brancik, K., Dickinson, D., Lee, H., Perrig, A., Sinopoli, B. Cyberphysical security of a smart grid infrastructure. Proceedings of the IEEE, 100(1), pp. 195209. (2012).

3. Sridhar, S., Hahn, A., Govindarasu, M. Cyber-physical system security for the electric power grid. Proceedings of the IEEE, 100(1), pp. 210-224. (2012).

4. Pasqualetti, F., Dörfler, F., Bullo, F. Cyber-physical attacks in power networks: Models, fundamental limitations and monitor design. In Decision and Control and European Con- 
trol Conference (CDC-ECC), 2011 50th IEEE Conference on. pp. 2195-2201. (2011, December).

5. Sridhar, S., Hahn, A., Govindarasu, M. Cyber-physical system security for the electric power grid. Proceedings of the IEEE, 100(1), pp. 210-224. (2012).

6. Hahn, A., Ashok, A., Sridhar, S., Govindarasu, M. Cyber-physical security testbeds: Architecture, application, and evaluation for smart grid. IEEE Transactions on Smart Grid, 4(2), pp. 847-855. (2013).

7. Zhu, B., Sastry, S. SCADA-specific intrusion detection/prevention systems: a survey and taxonomy. In Proceedings of the 1st Workshop on Secure Control Systems (SCS) (Vol. 11). (2010, April).

8. Zhu, B., Joseph, A., Sastry, S. A taxonomy of cyber attacks on SCADA systems. In Internet of things (iThings/CPSCom), 2011 international conference on and 4th international conference on cyber, physical and social computing. pp. 380-388 (2011, October)..

9. Mo, Y., Kim, T. H. J., Brancik, K., Dickinson, D., Lee, H., Perrig, A., Sinopoli, B. Cyberphysical security of a smart grid infrastructure. Proceedings of the IEEE, 100(1), pp. 195209. (2012).

10. Clark, R. M., Hakim, S. Cyber-Physical Security. Springer (2017)

11. Genge, B., Fovino, I. N., Siaterlis, C., Masera, M. Analyzing cyber-ph ${ }^{\circ}$ ysical attacks on networked industrial control systems. In International Conference on Critical Infrastructure Protection. pp. 167-183. Springer Berlin Heidelberg. (2011, March).

12. Yampolskiy, M., Horváth, P., Koutsoukos, X. D., Xue, Y., Sztipanovits, J. A language for describing attacks on cyber-physical systems. International Journal of Critical Infrastructure Protection, 8, pp. 40-52. (2015).

13. Bordel Sánchez, B., Alcarria, R., De Rivera, D. S., \& Sánchez-Picot, A. Predictive algorithms for mobility and device lifecycle management in Cyber-Physical Systems. EURASIP Journal on Wireless Communications and Networking, 2016(1), pp. 228. (2016).

14. Bordel, B., Alcarria, R. Jara A. Process execution in humanized Cyber-Physical Systems: soft processes. In Information Systems and Technologies (CISTI), 2017 12th Iberian Conference on. pp. 1-7 (2017, June).

15. Fudenberg, D., Tirole, J. Game theory. (1991).

16. B. Bordel, R. Alcarria, D. Sanchez, A. Sánchez. Enhancing Process Control in Industry 4.0 Scenarios using Cyber-Physical Systems. Journal of Wireless Mobile Networks, Ubiquitous Computing, and Dependable Applications, 7:4. pp. 41-64. (December 2016)

17. Berndt, D. J., Clifford, J. Using dynamic time warping to find patterns in time series. In KDD workshop. Vol. 10, No. 16, pp. 359-370. (1994, July).

18. Munkres, J. R. Topology. Prentice Hall. (2000).

19. Alcarria, R., de Andrés, D. M., Bordel, B., de Rivera, D. S., Sánchez-Picot, Á., Robles, T. A service-oriented monitoring system based on rule evaluation for Home Automation. In Consumer Electronics (ICCE), 2017 IEEE International Conference on. pp. 329-330. IEEE. (2017, January).

20. Uchenna P., Ani D., Hongmei M., Tiwari H. A.. Review of cybersecurity issues in industrial critical infrastructure: manufacturing in perspective, Journal of Cyber Security Technology, 1:1, pp. 32-74. (2017)

21. Waslo, R. Industry 4.0 and cybersecurity - Managing risk in an age of connected production. Deloitte University Press (2017)

22. Cardenas, A., Amin, S., Sinopoli, B., Giani, A., Perrig, A., Sastry, S. Challenges for securing cyber physical systems. In Workshop on future directions in cyber-physical systems security. p. 5. (2009, July). 


\title{
An Intra-Slice Security Solution for Emerging 5G Networks Based on Pseudo-Random Number Generators
}

\author{
BORJA BORDEL ${ }^{\circledR 1}$, AMALIA BEATRIZ ORÚE ${ }^{\circledR 2}$, RAMÓN ALCARRIA', \\ AND DIEGO SÁNCHEZ-DE-RIVERA ${ }^{\oplus 1}$ \\ ${ }^{1}$ Universidad Politécnica de Madrid, 28040 Madrid, Spain \\ ${ }^{2}$ Instituto de Tecnologías Físicas y de la Información, Consejo Superior de Investigaciones Científicas, 28006 Madrid, Spain \\ Corresponding author: Borja Bordel (bbordel@dit.upm.es)
}

This work was supported in part by the Ministry of Economy and Competitiveness through SEMOLA project under Grant TEC2015-68284-R, in part by the Autonomous Region of Madrid through MOSI-AGIL-CM project (co-funded by EU Structural Funds FSE and FEDER) under Grant P2013/ICE-3019, in part by the Ministerio de Economía, Industria y Competitividad, in part by the Agencia Estatal de Investigación, and in part by the Fondo Europeo de Desarrollo Regional through the COPCIS project under Grant TIN2017-84844-C2-1-R. The work of B. Bordel was supported by the Ministry of Education through the FPU Program under Grant FPU15/03977.

\begin{abstract}
Future 5G networks must provide communication services to a great and heterogeneous collection of scenarios: from traditional mobile communications to emerging applications such as Industry 4.0 or the Internet of Things (IoT). In this context, the network slicing technique is defined, where network resources are packaged and assigned in an isolated manner to the sets of users according to their specific requirements. Two different domains are, thus, defined: the intra-slice domain (where dedicated and specific solutions have to be deployed) and the inter-slice domain (including transversal solutions). One of the key topics which should be redefined following this approach is security. Traditionally, some solutions (such as stream ciphers) were not considered in mobile networks. However, 5G systems will be extensively employed in other new and very distinct scenarios, where requirements are different. For example, the use of resource constrained devices with little mobility and real-time data streaming in certain IoT applications suggests the use of stream ciphers (and other similar techniques) as the main security solutions. Therefore, in this paper, we investigate and propose a new security solution for emerging $5 \mathrm{G}$ networks, to be applied in the intra-slice domain. The proposed solutions employ lightweight pseudo-random number generators in order to provide the keystream used in stream ciphers which protect the private information and hide the communication signals in the frequency spectrum using spread spectrum techniques. We also describe and evaluate a first implementation of the proposed solution, using both, a simulation scenario and a real deployment.
\end{abstract}

INDEX TERMS 5G mobile communication, cryptography, Internet of Things, network slicing, random number generation, security.

\section{INTRODUCTION}

Future 5G networks must provide communication services to a great and heterogeneous collection of scenarios. Traditional voice calls or mobile data connections are only two examples among a very wide collection of applications that includes some of the most popular technological paradigms nowadays, such as the Internet-of-Things (IoT) [1], Industry 4.0 [2] or Cyber-Physical Systems (CPS) [3].

A homogeneous portfolio of communication services [4] cannot fulfil the very different requirements needed by all these scenarios. Then, 5G networks must define several operation planes in order to address and meet the needs of all future systems and applications. Each plane should be isolated from the rest of them to, for example, avoid the spreading of malfunctions or cyber-attacks in a public free service (e.g. Internet access in airports).

This design approach is known as network slicing. Network slicing [5] is a technique where network resources are packaged and assigned in an isolated manner to groups of users according to their specific requirements. With this view, the entire network infrastructure is divided into dedicated vertical segments, focused on proving a certain set 


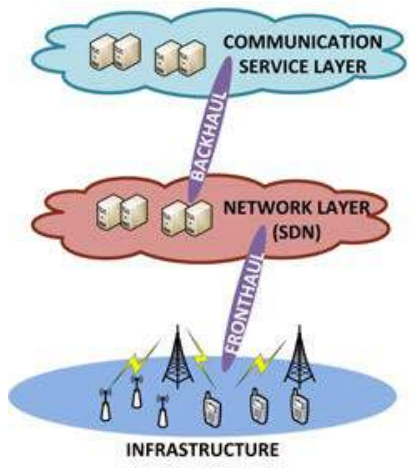

(a)

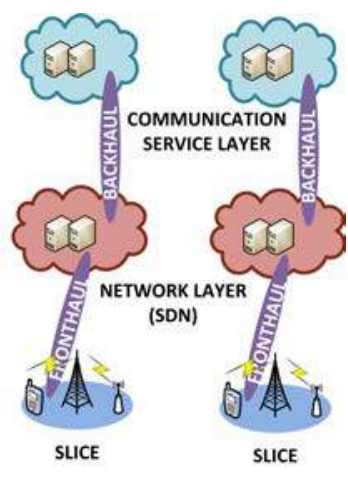

(b)
FIGURE 1. Schematic view of the overall architecture for (a) traditional mobile networks and (b) future $5 \mathrm{G}$ system with network slicing.

of communication services with common requirements, and isolated from the rest of existing slices. Fig. 1 represents in a schematic way the new network architecture in comparison to traditional mobile deployments.

In this new generation of mobile networks, thus, two different domains are defined [6]. The intra-slice domain contains all functions, components and solutions specifically designed to address and solve problems related to the application of mobile communications in specific scenarios (IoT systems, eHealth applications, etc.). On the other hand, inter-slice domain includes all transversal mechanisms that are common to all application scenarios and components focused on slice coordination, service orchestration, management, etc.

This new approach makes it mandatory to redefine most of the existing proposals for mobile networks, in order to ensure they fulfill the requirements of the domain where they are going to be employed. In that way, many intra-slice ad hoc solutions are nowadays needed in order to describe the future functional components that will make up the emerging $5 \mathrm{G}$ networks.

Obviously, this situation also affects one of the key topics in communication engineering nowadays: security.

Traditionally, mobile networks have implemented security solutions adapted to scenarios composed of few powerful devices, which, besides, may present a high level of mobility. Encryption solutions in $4 \mathrm{G}$ networks (for example) include complex algorithms to correct lags and signal drifts in the key sharing process [7]. These techniques are required in traditional scenarios, but they make security solutions very heavy and resource consuming.

On the contrary, new scenarios for $5 \mathrm{G}$ networks, such as IoT applications, present important differences with respect to traditional scenarios. First, the level of mobility is much lower, as usually the associated objects (furniture, appliances, city infrastructures, etc.) are static by default. Later, the use of communication services in these scenarios is not sporadic (as voice calls or connection to the Internet), and usually there is a continuous data flow. Moreover, IoT devices tend to be small and resource constrained, so complex algorithms are not a valid solution. Finally, new cyber-attacks must be considered, as IoT systems (as unattended pervasive wireless systems) are sensitive to interference, spectrum scanning attacks, etc., which are not frequent problems in traditional mobile networks.

Nowadays, most of these applications are supported by other communication technologies such as Bluetooth or ZigBee. Systems using these technologies, however, cannot integrate a great amount of devices or support pervasive deployments. Because of this fact, cryptographic and security solutions are usually based on traditional block ciphers whose computational cost is unaffordable for future embedded devices.

In conclusion, new security solutions for the IoT (and other similar paradigms, like CPS) intra-slice domain are needed. In particular, we argue that stream ciphers, with the appropriate configuration, may address this problem.

Therefore, the objective of this paper is to describe a new stream cipher, specifically designed to be applied in emerging $5 \mathrm{G}$ networks. The cipher will be deployed in order to protect communications between resource constrained devices and base stations in 5G systems. Our proposal is based on a simple lightweight Pseudo Random Number Generator (PRNG), which is the core of an stream cipher that protects the user information, the meta-information of the system (including data size, the encryption scheme, etc.) and hides the communication signals thanks to a spread spectrum technique. The entire solution may be easily implemented in any resource constrained microcontroller or System-on-Chip.

The remainder of this paper is organized as follows. Section II describes the state of the art on stream ciphers for IoT applications. Section III presents the employed PRNG, the proposed initial configuration process and the behavior of the designed cipher. Section IV describes the performed experimental validation. Finally, Section V presents the obtained results and Section VI concludes this work.

\section{STATE OF THE ART}

In the last ten years, many different and novel security solutions for IoT systems have been proposed (including block ciphers [8], hash functions [9] and non-traditional techniques [10]). However, in this section we focus on lightweight stream ciphers, as it is the objective of our work.

Probably, the most important attempt for developing a lightweight stream cipher for emerging technological systems is the ECRYPT II eSTREAM project [11].

In this project, a catalogue of seven totally new functional lightweight stream ciphers was created and released [12], [13]. It includes the HC-128 [14], Rabbit [15], Salsa20/12 [16], SOSEMANUK [17], Grain [18], MICKEY [19] and Trivium [20] ciphers. Some of these algorithms, such as MICKEY, have already been reported to be insecure (a differential fault attack has been reported against MICKEY 2.0 in 2013 [21]), although others are still considered safe (e.g. Trivium) and, they have even been specified as an international standard [22]. 
The only theoretically secure cipher is known as the One Time Pad. It is obtained by combining a truly random key sequence, which is as long as the message, and the message, by means of XOR addition [23], [24]. The problem here is that we need to secretly send that random key sequence to the intended recipient.

The practical alternative is to use a pseudorandom generator: First a pseudorandom keystream is generated from a seed, which plays the role of a real key that is much shorter than the full keystream combined to the message. This seed must be unpredictable and long enough to avoid exhaustive search attacks. In this way the final objective of any PRNG is to generate a cryptographically secure random number sequence, that is, the sequence must have a very long period in which the sequence must be indistinguishable from a perfect random sequence, and must be unpredictable.

In consequence, all stream ciphers (including previously cited ones) are focused on the generation of an adequate key stream, as XOR operation is very simple and supported by all programming languages. As truly random number streams are very difficult to generate nowadays, key streams are generated by means of Pseudo Random Number Generators (PRNGs) [25].

For example, Trivium cipher is based on a PRNG with three shift registers of different lengths. These registers are placed as in a circle, and while samples are moving around, some positions are modified using combinations of other samples in the registers [26]. These PRNGs, based on Linear Feedback Shift Registers (LFSRs), are very common [27], and were the initial phase of PRNGs based on Feedback with Carry Shift Registers (FCSRs) or on combinations of both types of registers [28].

These kinds of proposals, however, are not proved to be secure, as the Linear feedback shift register can be easily cryptanalyzed. Finding a cryptographically secure PRNG is an open problem in cryptography. Some standards (the RC4 algorithm, for example) tried to address this problem and failed [29], [30], and some PRNGs that are considered valid (such as the BBS generator [31]) are very slow to be operated at real-time. On the other hand, in order to improve the randomness of the generated key stream, some proposals consider a chaotic flow [32] as input, although problems in managing chaotic dynamics have reduced the utility of these techniques.

Instead of ad hoc combinations of LFSRs and/or FCSRs, some proposals employ existing pseudorandom generators as initial input. One of these reference proposals is Lagged Fibonacci Pseudorandom Generator (LFG) [33]. Its reasonably good behavior on standard statistical tests makes it perfect to be employed as input to create more complicated PRNGs [34].

Table 1 compares the characteristics of some of the most important lightweight stream ciphers for IoT scenarios nowadays. It can be seen that around $50 \%$ of ciphers are no longer secure, although some of them employ the largest secret keys. On the other hand, usually, faster ciphers are those
TABLE 1. Comparison of the stream ciphers for loT scenarios.

\begin{tabular}{lcccc}
\hline \hline \multicolumn{1}{c}{ Cipher } & $\begin{array}{c}\text { Creation } \\
\text { date }\end{array}$ & $\begin{array}{c}\text { Secure } \\
\text { (Nov. 2017) }\end{array}$ & Key length & Speed \\
\hline HC-128 & 2004 & Yes & 128 & $\uparrow \uparrow$ \\
Rabit & 2003 & Yes & 128 & $\uparrow \uparrow$ \\
Salsa & 2004 & No & 256 & $\uparrow$ \\
SOSEMANUK & 2004 & Yes & 128 & $\mathrm{X}$ \\
Grain & 2004 & No & 80 & $\mathrm{X}$ \\
MICKEY & 2003 & No & 80 & $\mathrm{X}$ \\
Trivium & 2004 & Yes & 80 & $\uparrow \uparrow$ \\
RC4 & 1987 & No & $8-2048$ & $\uparrow$ \\
BBS & 1986 & Yes & It depends & $\downarrow \downarrow$ \\
LFG & 1985 & No & on the target & $\uparrow \uparrow$ \\
Trifork & 2010 & Yes & application & $\uparrow \uparrow$ \\
\hline \hline
\end{tabular}

whose encryption scheme has been broken. There is, in this tendency, an important exception: the Trifork generator.

Current encryption schemes, moreover, not only protect the private user information but also meta-information about the employed cipher. This is very important, as new cyberattacks based only on meta-information have been reported. For example, the length of encrypted samples is sometimes a very valuable knowledge to build a successful attack. Therefore, our proposal considers a solution to protect, also, meta-information.

PRNGs can be also employed in spread spectrum techniques, which can hide the communication signal in the frequency spectrum, making them more robust against interferences and electronic noise, and helping the entire system to get synchronized. In this work we use an LFG-based PRNG as a core of a stream cipher for emerging $5 \mathrm{G}$ network and a spread spectrum technique. The proposed solution also includes this technology.

\section{A STREAM CIPHER FOR 5G NETWORKS}

In this section, the proposed stream cipher is described. The first subsection presents the Trifork PRNG, which is employed in our stream cipher design. Second subsection describes the proposed process for the initial configuration; and in the third subsection the main contribution and its behavior are described in detail.

\section{A. A PRNG FOR REDUCED RESOURCE DEVICES}

One of the lightest PRNG is Tausworthe generator (1), where $c_{i}$ are binary parameters, $b_{i}$ are binary variables and $\oplus$ is an operator representing the exclusive-or addition [35].

$$
b_{n}=c_{q-1} b_{n-1} \oplus c_{q-2} b_{n-1} \oplus \ldots \oplus c_{0} b_{n-q}
$$

It can, besides, generate long random sequences and manybit random numbers (as required by cryptographic applications). In fact, Tausworthe generator produces $k$-bit random numbers, independently from the underlying hardware platform [36]. If $k=1$, the LFSRs cited above are obtained. In its most common implementation, however, only two $c_{i}$ parameters are non-zero (2).

$$
b_{n}=b_{n-r} \oplus b_{n-s}
$$


Tausworthe generators, when implemented as in (2), are understood as a particular case of a more generic collection of PRNGs named as Lagged Fibonacci Generators (LFGs). The general form of LFG [37] is as indicated in (3), where $r>s>0$ are the lags, $\circ$ is an operator indicating a binary operation, $m$ is the base and $\left\{x_{t}, t=0, \ldots, r-1\right\}$ is the $r$-dimensional initialization vector (IV) or seed.

$$
L F\left[r, s, m, \circ ;\left\{x_{t}, t=0, \ldots, r-1\right\}\right]
$$

The logical mapping presented in (4) allows calculating the new elements in the sequence $\left\{x_{n}\right\}$ for $n \geq r$.

$$
x_{n}=x_{n-r} \circ x_{n-s}
$$

The operator $\circ$ usually represents a binary addition, subtraction, a $\bmod -m$ multiplication or a bitwise exclusiveor (XOR). In order to take full advantage of the data representation capacity of electronic devices and computers, usually $m=2^{N}$, being $N$ the word length of the microcontroller executing the LFG.

In order to maximize the repetition period $p$ of this PRNG, a special configuration for the IV, $r$ and $s$ should be selected. In fact, some authors [38] have indicated that LFG need to be initialized at random, and the randomness of the IV is a key factor conditioning the behavior of the entire LFG.

Moreover, LFGs may be expressed as trinomials over the Galois Field of two elements, $G F(2)$, see (5).

$$
x^{r}+x^{s}+1
$$

In general, it is proved that LFGs defined by irreducible, primitive mod -2 trinomials are a good design option [39], as they generate the maximal repetition period $p$ if at least one seed is odd [40] (6).

$$
p=2^{N-1}\left(2^{r}-1\right)
$$

Despite all these advantages, LFGs are not secure, and several faults on their security have been reported [40], [41]. Consequently, Perturbed Lagged Fibonacci Generators (PLFGs) were defined [34].

It is well known that all bits in the random number generated by LFGs have not the same behavior [42]. While the most significant bit (MSB) presents (if the entire PRNG exhibits the maximal repetition period) a period $p_{M S B}=$ $2^{N-1}\left(2^{r}-1\right)$, the least significant bit presents a repetition period $p_{L S B}=\left(2^{r}-1\right)$. Moreover, in general, the $k-t h$ presents a period $p_{k}=2^{k-1}\left(2^{r}-1\right)$. Most randomness problems, thus, are associated to the LSB. In order to address this challenge, it was found that the perturbation of the low and high bits in $x_{n-r}$ and $x_{n-s}$ could improve the performance of the entire PRNG. These perturbations could be introduced by means of any logical operation, but the generation of new additional samples to perform these operations seems to be very costly. Then perturbations are introduced through some of the existing samples generated by the same PLFG.

In its general form (7) a PLFG is defined by a onedimensional map including the same parameters as a standard
LFG and a new control parameter, $d$, to act over the added perturbation.

$$
L F\left[r, s, m, d, \circ ;\left\{x_{t}, t=0, \ldots, r-1\right\}\right]
$$

As said, the induced modifications in the PLFG are perturbations in the least and most significant bits of the samples. Then, the logical mapping that defines the new elements in the sequence $\left\{x_{n}\right\}$ for $n \geq r$ is (8).

$$
\begin{aligned}
x_{n} & =\left(\left(x_{n-r} \oplus x_{n-s}^{\gg}\right)+\left(x_{n-s} \oplus x_{n-r}^{\ll}\right)\right) \bmod m \\
x_{n-s}^{\gg} & =\left(x_{n-s} \gg d\right) \\
x_{n-r}^{\ll} & =\left(x_{n-r} \ll d\right)
\end{aligned}
$$

In (8) $n$ represents time, and $d$ is a constant integer usually selected to fulfill that $2 \leq d \leq 0.7 N$. Operators $\ll$ and $\gg$ represent the left-shift and right-shift operations. In fact, this is a lightweight way of performing multiplications or divisions, when one of the factors is a power of two (9).

$$
\begin{aligned}
& \left(x_{n-s} \gg d\right)=\left\lfloor\frac{x_{n-s}}{2^{d}}\right\rfloor \\
& \left(x_{n-r} \ll d\right)=\left(x_{n-r} \cdot 2^{d}\right) \bmod m
\end{aligned}
$$

As novelty, PLFGs consider three different operation types which highly improve their performance: $m-\bmod$ additions, XOR additions and bit-shift (left-shift and right shift). These operations, together with the introduced perturbations, improve dramatically the randomness and repetition period of the PRNG.

A PLFG is already a good PRNG to be applied in IoT scenarios and future $5 \mathrm{G}$ networks. However, it was reported that statistical attacks could be performed against these systems as the output random numbers are the same employed to obtain the following samples.

A three-branch PLFG was proposed: the Trifork generator [34], which (in fact) has three branches; each one composed of a Perturbed Lagged Fibonacci Generator. In order to obtain the final output of the global PRNG, the outputs of two different PLFGs are added by means of the XOR operation. In this scheme, the third PLFG composing the Trifork will remain totally hidden. This innovative proposal guarantees that the global output sequence is useless to infer neither the internal system parameters, the current or past system state nor the secret keys. This characteristic is very important as it allows us to design a secure meta-information protection algorithm (see Section III.C).

The three PLFGs making up the Trifork generator are interconnected, so they get perturbations in a cyclic manner thanks to a set of three new internal XOR operations. In these operations, the output of a PLFG is combined with the leftshifted output of the precedent PLFG. The left-shift is recommended to be about $\frac{N}{2}$ bits, where $N$ is the bit word size. Using this configuration, the resulting period is much longer than the one obtained from conventional Lagged Fibonacci generators. It has been proved that the global output is unpredictable, as generated sequences pass successfully the most stringent randomness test suites. 


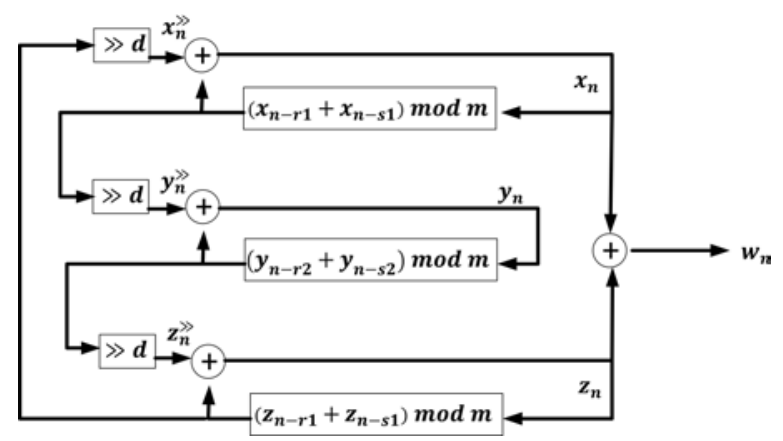

FIGURE 2. Block diagram of the Trifork generator.

The proposed architecture (see Fig. 2) hides the internal random numbers through the XOR combination of three different PLFGs. Moreover, it was found that the combination of three PLFGs allows reducing some operations, so Trifork generator requires less computational power than three separated PLFGs.

With these considerations, the mathematical expression employed to implement the Trifork generator is (10), where $w_{n}$ is the output of the generator.

$$
\begin{aligned}
x_{n} & =\left(\left(x_{n-r 1}+x_{n-s 1}\right) \bmod m\right) \oplus z_{n}^{\gg} \\
y_{n} & =\left(\left(y_{n-r 2}+y_{n-s 2}\right) \bmod m\right) \oplus x_{n}^{\gg} \\
z_{n} & =\left(\left(z_{n-r}+z_{n-s}\right) \bmod m\right) \oplus y_{n}^{\gg} \\
x_{n}^{\gg} & =\left(\left(x_{n-r 1}+x_{n-s 1}\right) \bmod m\right) \gg d \\
y_{n}^{\gg} & =\left(\left(y_{n-r 2}+y_{n-s 2}\right) \bmod m\right) \gg d \\
z_{n}^{\gg} & =\left(\left(z_{n-r 3}+z_{n-s 3}\right) \bmod m\right) \gg d \\
w_{n} & =x_{n} \oplus z_{n}
\end{aligned}
$$

\section{B. INITIAL CONFIGURATION}

As said, for each Trifork generator included in the stream cipher, a key must be shared between the base station and the remote IoT device. It is implicit that all stream ciphers are symmetric cryptographic schemes, so the problem of sharing keys is basic in these scenarios.

In previous works, we have already investigated some algorithms and protocols to share with safety symmetric keys in order to initiate stream ciphers based on PRNGs in 5G networks [43]. In fact, although future $5 \mathrm{G}$ devices to be integrated into the IoT slice (and other similar domains) will be resource constrained, they can also execute in a sporadic way some more complex processes (using, for example, auxiliary co-processors if needed).

In our application scenario, the deployed IoT system and $5 \mathrm{G}$ infrastructure may be represented by a set of two collections (11). $B$ represents the set of $5 \mathrm{G}$ base stations and $D$ the set of IoT devices which are connected to these base stations.

$$
S_{i o t}=\{B, D\}
$$

Fig. 3 represents this scenario, where the number of base stations is fixed to one, without loss of generality.

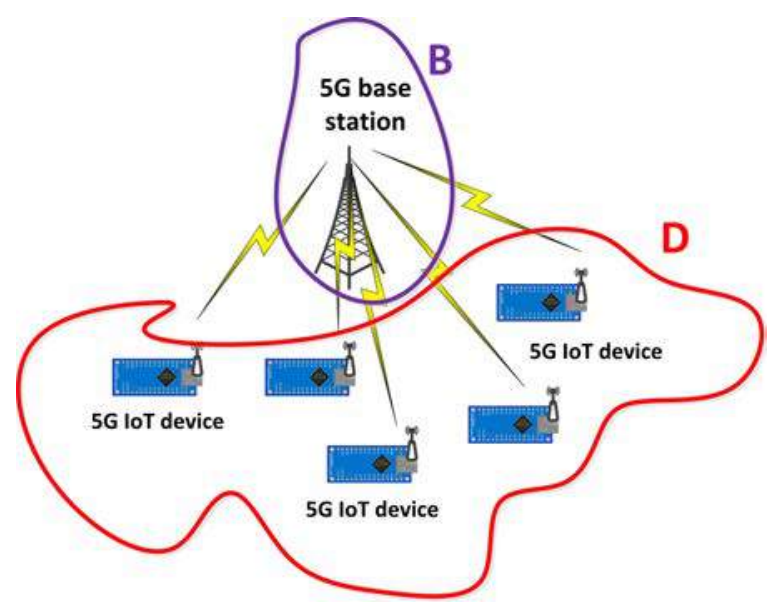

FIGURE 3. Study scenario for this work.

In this scenario, and in almost any other case of symmetric key sharing nowadays [44], a Public Key Infrastructure (PKI) is the most efficient solution to address this problem. In that way, in our system two different cryptographic functions will coexist: $f_{\text {sym }}$ (i.e. the stream symmetric cipher based on a PRNG, see subsection C), and $f_{\text {asym }}$ (i.e. the KPI).

As symmetric cryptographic methods need an asymmetric method to support the symmetric key sharing, in this work we are employing the RSA (Rivest, Shamir and Adleman) algorithm [45], as it can be employed to encrypt and to digitally sign documents. Besides, there are efficient implementations of this algorithm in almost any existing programming language [46].

The designed protocol to share the symmetric keys based on the RSA cryptographic algorithm is very simple. It consists of a triple handshaking procedure (the most recommended process to establish a communication link, secure or not), where mechanisms to address and solve the eventually problematic situations (packet loss, unexpected delays in the communications channel, etc.) have been considered.

Fig. 4 presents a message sequence chart describing the basic use case and behavior of the proposed protocol.

Basically, the base station, which has a powerful computational infrastructure, must calculate and broadcast its RSA public key (so, the costliest procedures are not performed by the IoT devices). This key is also signed, so the identity of the owner is certified.

Each 5G IoT device receives the public key and, when it wants to stablish a secure communication link, employs it to encrypt and send to the base station the private symmetric keys which are going to be used to initialize the stream cipher.

In order to guarantee the key sharing process has been performed successfully, the base station and the 5G IoT device confirm each other the transaction using the initialized stream cipher. If shared symmetric keys contain any error, the acknowledgment (ACK) message will not be recovered (either in the base station or in the IoT device), and the key sharing process could be repeated. 


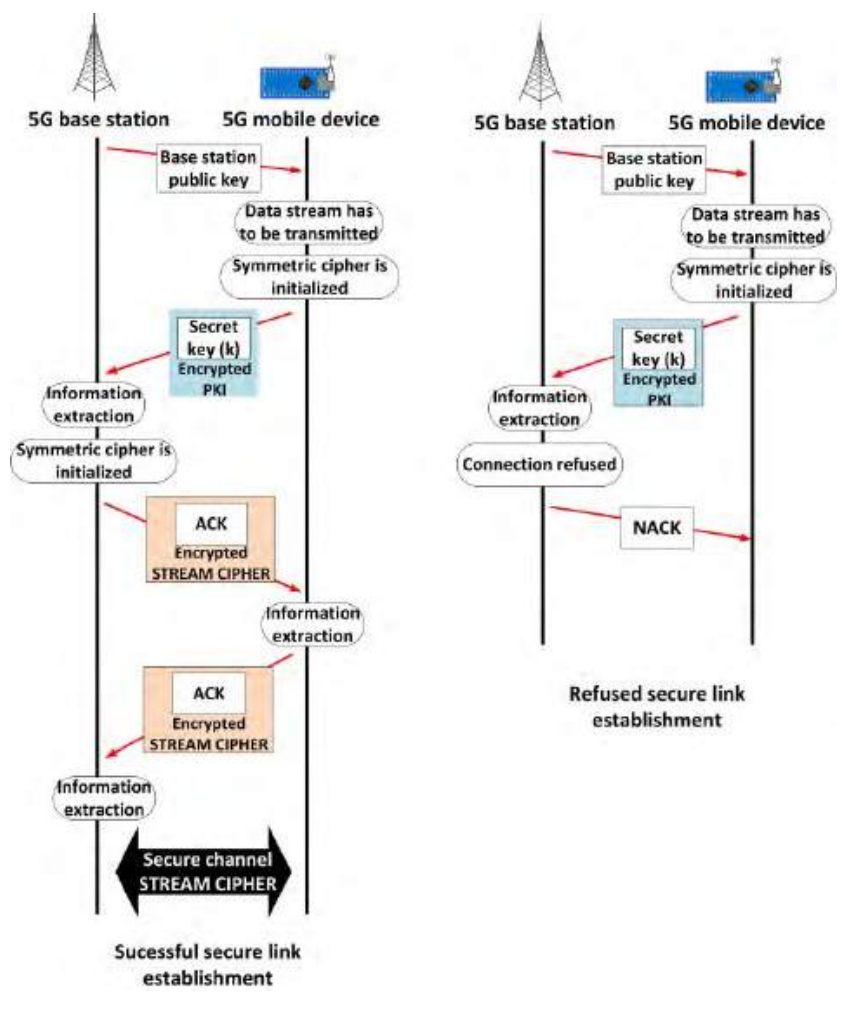

FIGURE 4. Message sequence chart of the proposed initialization protocol: successful secure link establishment and refused secure link establishment.

This triple handshaking process allows checking that a bidirectional secure link is open and, besides (see Section III.C), enables the symmetric ciphers (both in the base station and the IoT device) to get synchronized.

On the other hand, if the base station is congested, the IoT device has not contracted any communication service, etc., the 5G network may refuse the link establishment and a nonencrypted NACK message is sent.

The proposed protocol also includes timers and forwarding techniques in order to get a secure link in aggressive environments; i.e. in presence of high delays, bursts of errors, interference, etc. A detailed analysis of all possible cases is not the objective of this section, as previous works have addressed this problem [43]. Only, as an example, Fig 5 presents two different situations (those which have been proved to be the most probable [43]).

In the first one (Fig. 5, on the left), it is represented the proposed solution for situations where packet sending suffers a great delay. As can be seen, basically, a timer is triggered every time a packet is sent during the initiation process. If no answer is received before this timer expires, then, the packet is sent again. Eventually, a response to the first request could be received. Thanks to a transaction number included in every packet, this response may be discarded, waiting for the answer to the last request.

In the second one (Fig. 5, on the right) it is represented the protocol reaction if an error occurs during the key sharing process and any of the sent messages (the keys or the

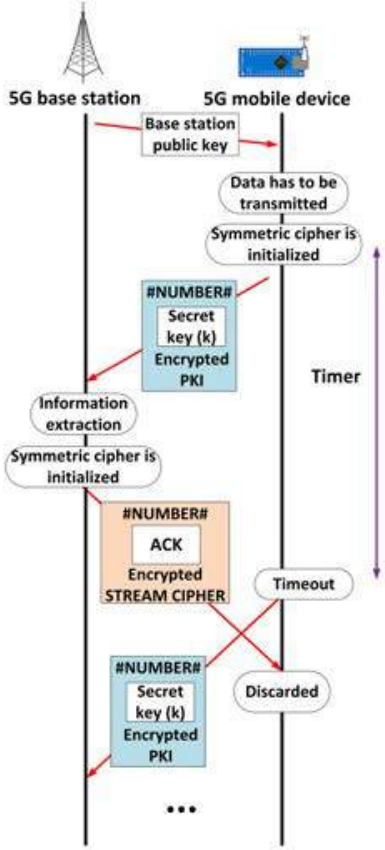

Secure link establishment using a channel with a high packet loss rate

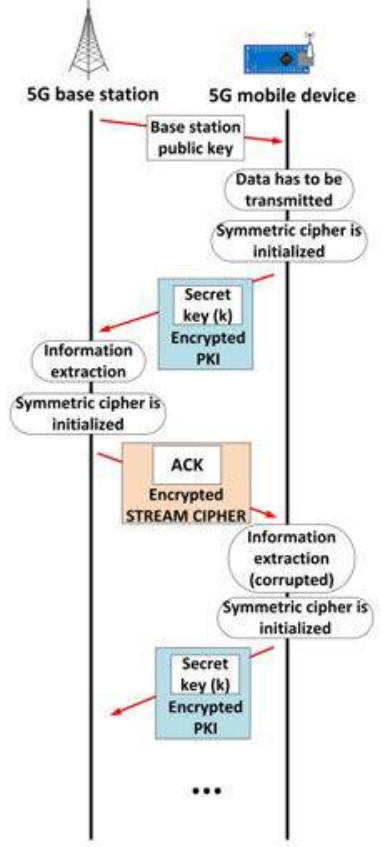

Secure link establishment with corrupted packets
FIGURE 5. Message sequence chart of the proposed initialization protocol: secure link establishment in radio channels with delays and packet loss rate; and secure link establishment with corrupted packets.

acknowledgments) cannot be recovered adequately. In particular, once the IoT device detects an error has occurred, it reinitiates the process.

In order to avoid the system to enter in an infinite loop, if any problem (delays, communication errors, etc.) persists beyond a certain number of attempts, the procedures is cancelled, and user applications are informed that the communication link could not be established.

Considering the private keys have been correctly shared, both remote stream ciphers may be initialized, and the encryption process can start.

\section{SYSTEM OPERATION}

The proposed stream cipher consists of a transmitter and a receptor, which are slightly different. The stream cipher receives a constant sequence of samples which are obtained each $T_{S}$ seconds (the sampling period) from an analog information source (with a maximum bandwidth $f_{B}$ ). Each sample has a length of $N$ bits, according to the word length in the underlying hardware architecture. The stream cipher encrypts the information, randomizes the length of the original samples (so no meta-information about the core of the cipher is transmitted) and generates a pseudorandom code (synchronized with the encryption code) employed in a spread spectrum system which hides the communication signals in the frequency spectrum below the noise level.

Fig. 6 presents a block diagram of the transmitter. The scheme has three main parts: the encryption core, the meta-information protection module, and the synchronized 


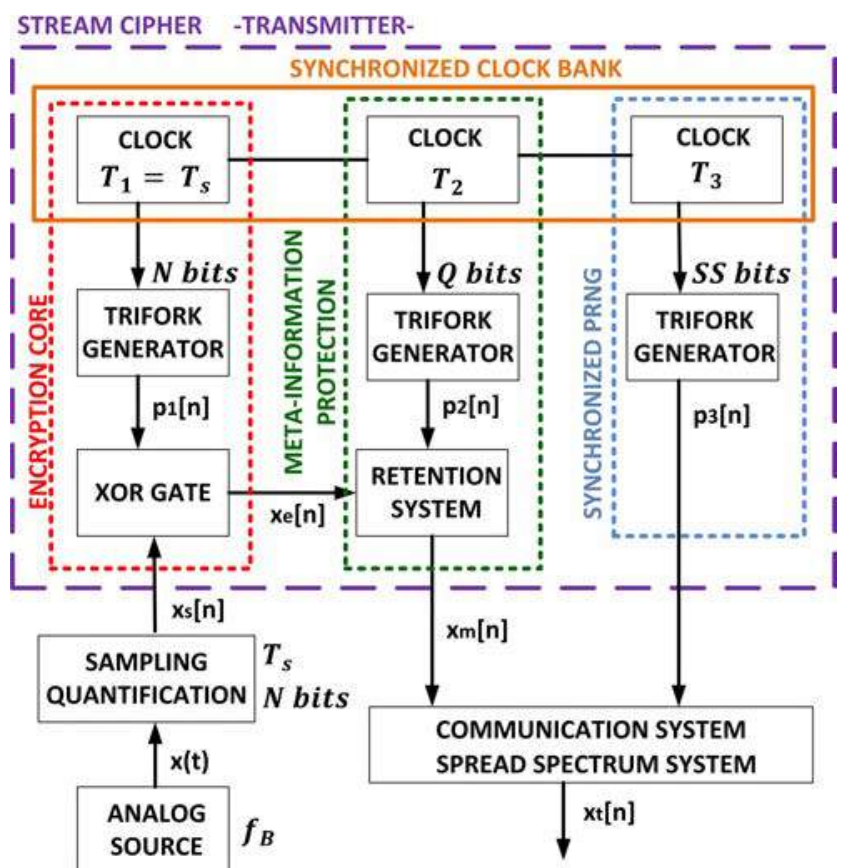

FIGURE 6. Block diagram of the transmitter of the proposed stream cipher.

PRNG to feed the spread spectrum system (not included in the cipher's design as it belongs to the radio communication system).

In the next paragraphs we are explaining each one of these three main parts in detail.

Before describing the encryption core, it is important to note that three different Trifork generators are considered in the proposed cipher. In consequence, the global private key of our system has a length around $9 N$ bits, where $N$ the length of the original samples being encrypted (the precise value depends on the specific configuration of the cipher). Nowadays, any hardware platform may support samples with, at least, 10 bits (including the simplest microcontrollers, such as the well-known Arduino project [47]). Thus, the proposed scheme employs key lengths similar to most standard stream ciphers (see Table 1). In this way, the proposed scheme, at least, has the same security level of existing solutions (or even higher).

As can be seen, the encryption core of the proposed cipher is made of a Trifork generator and a (software) XOR gate. The XOR gate receives two data sequences to be operated. Signal $x_{s}[n]$ is composed of $N$ bit samples, with a sample period of $T_{S}$ seconds. In order to guarantee the digital signal $x_{S}[n]$ correctly represents the original analog signal $x(t)$, it must be guaranteed that the Nyquist theorem is fulfilled (12).

$$
T_{s} \leq \frac{1}{2 \cdot f_{B}}
$$

In that way, $x_{S}[n]$ takes values in the range $\left\{0, \ldots, 2^{N}-1\right\}$, and it has a symbol rate as indicated in (14), and a binary rate as indicated in (14).

$$
\begin{aligned}
& \lambda_{s}=\frac{1}{T_{s}} \\
& \lambda_{b}=\frac{N}{T_{s}}
\end{aligned}
$$

The second injected data flow in the XOR gate $p_{1}[n]$ is a pseudorandom number sequence generated by a Trifork PRNG. This generator is configured to produce a signal with the same characteristics (sample length, and symbol and binary rate) than the information signal. That requirement is necessary to apply the proposed encryption mechanism.

As said, as a main encryption mechanism, a XOR gate is included in our proposal (15).

$$
x_{e}[n]=x_{s}[n] \oplus p_{1}[n]
$$

XOR encryption may be highly strong or very weak, depending on the use of this technology. If signal $p_{1}[n]$ is a sequence of random numbers, then, all possible values have the same probability, and this characteristic is transferred to the encrypted signal (16).

$$
P\left(p_{1}[n]=\xi_{i}\right)=P\left(x_{e}[n]=\xi_{i}\right)=\varphi_{1}=\frac{1}{2^{N}} \forall \xi_{i}
$$

On the other hand, because of the structure of XOR operation, given a sample of the encrypted signal $x_{e}[n]$, all possible values in the range of the original signal $x_{s}[n]$ have the same probability of having produced that sample (17).

$$
P\left(x_{s}[n]=\xi_{j} \mid x_{e}[n]=\xi_{i}\right)=\varphi_{2}=\frac{1}{2^{N}} \forall \xi_{i}, \xi_{j}
$$

Then, considering the Shannon's information theory, the mutual information between the original and the encrypted signal $I\left(x_{s} ; x_{e}\right)$ represents the residual information that remains in the encrypted signal about the original one (18). A simple calculation proves that this quantity is zero. Thus, the encrypted signal does not contain information coming from the original signal.

$$
\begin{aligned}
I\left(x_{s} ; x_{e}\right)=\sum_{i=0}^{2^{N}-1} \sum_{j=0}^{2^{N}-1} P\left(x_{s}=\xi_{j}, x_{e}=\xi_{i}\right) \\
\cdot \log \left(\frac{P\left(x_{s}=\xi_{j} \mid x_{e}=\xi_{i}\right)}{P\left(x_{e}=\xi_{i}\right)}\right)=0
\end{aligned}
$$

This statistical demonstration is not valid if signal $p_{1}[n]$ has a short period (i.e. it cannot be considered random during the entire data transmission and the PRNG is not secure) or if the same pseudorandom sequence is employed to encrypt various messages. In both cases a simple cryptanalysis may break the encryption. In order to address the first problem, the Trifork generator has been proved to be secure with a very long period and a good random behavior. In order to address the second problem, each time a transmission starts a new configuration is generated for the stream cipher (as described in the previous section). 


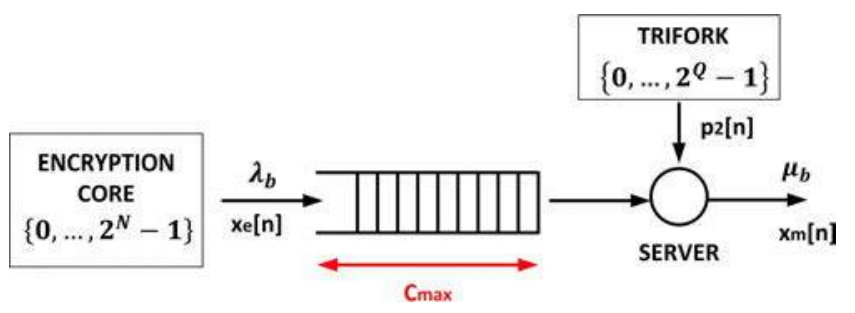

FIGURE 7. Block diagram of the retention system.

In that way, an encrypted signal $x_{e}[n]$ protecting the original private information is obtained. This signal, however, still contains some meta-information about the system.

In fact, recently, it has been proved that meta-information may be also successfully employed to break encryptions considered secure [48]. Trifork generator partially addresses this problem as the random signal $p_{1}[n]$ is not the direct output of the PLFGs, but a XOR combination of them. However, samples in $x_{e}[n]$ still contain an important meta-information about the system: the sample length.

Although attacks against the proposed encryption core are complicated to perform, a good quality input information for them is that the signals $p_{1}[n]$ and $x_{s}[n]$ take values in the range $\left\{0, \ldots, 2^{N}-1\right\}$. This information could be perturbed if additional unused bits are included into each sample (for example), but then a percentage of the available bit rate is wasted. Instead of that, our proposal considers a metainformation protection phase, focused on hiding the information about the sample length.

The proposed meta-information protection phase consists of a Trifork generator and a retention system.

Trifork generator is configured to produce $Q$ bit random numbers with a period of $T_{2}$ seconds. The clock that controls the operation of this Trifork generator is synchronized with the clock that controls the operation of the encryption core. This is important in order to enable the information recovering in the receptor. In conclusion, the generated random signal $p_{2}[n]$ has a symbol rate as indicated in (19), and a binary rate as indicated in (20).

$$
\begin{aligned}
\gamma_{s} & =\frac{1}{T_{2}} \\
\gamma_{b} & =\frac{Q}{T_{2}}
\end{aligned}
$$

The retention system is showed on Fig. 7. It consists of a FIFO (First In First Out) binary queue, where output samples from the encryption core are stored as sequences of bit. This queue is served by a unique server which each $T_{2}$ seconds sends a new sample made of the first $M$ bits in the queue. If there are not enough bits in the queue to create a $M$ bit sample, the server waits until they are received. In this context, $M$ is a random number generated by the Trifork PRNG which takes values in the range $\left\{0, \ldots, 2^{Q}-1\right\}$.

With the proposed scheme, the sample length of the original system is hidden. Besides, the word length of the Trifork generator in the meta-information protection phase is also hidden. Although by collecting enough samples and analyzing their length it is probable to find the value of $Q$, there is no guarantee to obtain the real value.

On the other hand, with the proposed scheme, the random signal $p_{2}[n]$ may be easily recovered by a cyber attacker. However, that circumstance does not reduce the security level of the proposed system as, thanks to the XOR operation, it is impossible to find out the internal state of the Trifork generator using only the output sequence. Besides, although the original sample length was deducted, that does not break the encryption. Therefore, the proposed scheme is a valid meta-information protection solution.

The length of the output samples from the metainformation protection module follows a uniform distribution in the range $\left\{0, \ldots, 2^{Q}-1\right\}$, as the Trifork generator that controls the server.

As the described retention system includes a queue, it is necessary to study this scheme using the queue theory, in order to configure all design parameters properly.

The first consideration we must do is to guarantee that the retention system is not congested. In this case, as only one server is considered, the congestion level $\rho$ is equal to the traffic volume $A$ supported by the retention system. In order to guarantee the system is not congested (so a percentage of the samples will be lost), the traffic volume cannot be greater than 1 Erlang. The Erlang's theory defines the traffic volume as the quotient of average input traffic rate divided by the average output traffic rate. As the proposed queue is a binary queue, this parameter is properly estimated using binary rates (21).

$$
\rho=A=\frac{\lambda_{b}}{\mu_{b}} \leq 1
$$

The input binary rate in the retention system is constant (15), and equal to the binary rate of signal $x_{e}[n], \lambda_{b}$. The output binary rate $\mu_{b}$, however, depends on the output sample length, which follows a uniform distribution.

The average value of the output sample length may be easily obtained (22). And, as a sample is extracted from the queue each $T_{2}$ seconds, the average output binary rate is directly obtained (23)

$$
\begin{aligned}
\eta_{t} & =\sum_{i=0}^{2^{Q}-1} \frac{i}{2^{Q}}=\frac{2^{Q}\left(0+2^{Q}-1\right)}{2^{Q} \cdot 2}=\frac{2^{Q}-1}{2} \\
\mu_{b} & =\frac{2^{Q}-1}{2 \cdot T_{2}}
\end{aligned}
$$

Using this information, we obtain a relation between parameters in the encryption core and parameters in the metainformation protection step that is necessary to fulfill (24).

$$
A=\frac{\frac{N}{T_{s}}}{\frac{2 Q-1}{2 \cdot T_{2}}}=\frac{2 \cdot N}{2^{Q}-1} \cdot \frac{T_{2}}{T_{s}} \leq 1
$$

If it is guaranteed that $A \leq 1$, there are not structural sample losses in the retention system, but there is a certain 


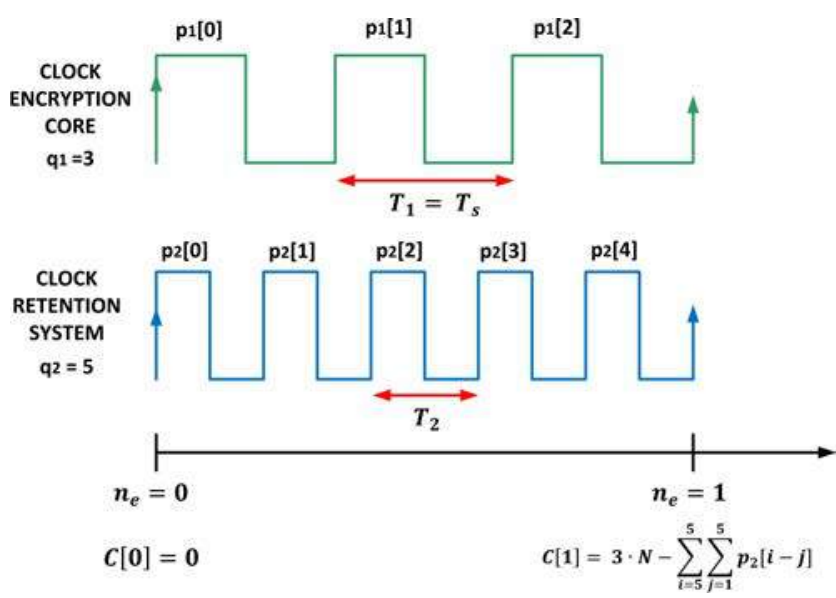

FIGURE 8. Temporal diagram of signals $\mathrm{p}_{1}[\mathrm{n}]$ and $\mathrm{p}_{2}[\mathrm{n}]$.

loss probability $P_{L}$ because of the limited capacity of the queue.

In general, to operate correctly, the queue must be able to store at least one input sample (i.e. $N$ bits). Nevertheless, if $Q>N$, then, the queue must be able to store (at least) as much samples as needed to accumulate $2^{Q}-1$ bits (the maximum sample length in the retention system). Thus, there is a minimum capacity $C_{\min }$ the queue must have (25).

$$
C_{\min }=N \cdot\left\lceil\frac{2^{Q}-1}{N}\right\rceil
$$

The queue capacity $C$ may be higher than $C_{\min }$ depending on the loss probability $P_{L}$ the system can tolerate. Traditional mathematical analyses in queue theory are based on Markovian traffic, so they are not applicable in our case [49]. On the other hand, proposals for general traffic models (such as the Kingman's formula [50]) are designed for pure waiting systems (where queues are infinite), and they are not valid in this context either. Thus, in order to evaluate the loss probability in our scenario, we are analyzing the evolution of the queue capacity in time.

As we have said, parameters $T_{1}$ and $T_{2}$ may be freely chosen, considering the previously mentioned limits (13) (25). However, in order to guarantee a good synchronization among the clocks in the clock bank, enabling an analysis of the queue capacity; we are adding a new requirement: $T_{1}$ and $T_{2}$ must have a rational factor of proportionality (26).

$$
\frac{T_{2}}{T_{s}}=\frac{q_{1}}{q_{2}} q_{1}, q_{2} \in \mathbb{N}
$$

This requirement means that, every $q_{2} T_{2}=q_{1} T_{1}$ seconds, signals $p_{1}[n]$ and $p_{2}[n]$ present a common edge (a rising edge or a falling edge); a characteristic that allows them to be synchronized. Temporal instants when both signals present a common edge are noted as $n_{e}$. See Fig. 8

In (27) it is described the queue capacity in these temporal instants. Besides, if we take as initial condition that $C[0]=0$ (the queue is empty when the system starts operating), the queue capacity may be calculated as in (28)

$$
\begin{aligned}
& C\left[n_{e}\right]=C\left[n_{e}-1\right]+q_{1} \cdot N-\sum_{j=1}^{q_{2}} p_{2}\left[q_{2} \cdot n_{e}-j\right] \\
& C\left[n_{e}\right]=n_{e} \cdot q_{1} \cdot N-\sum_{i=q_{2}}^{n_{e} \cdot q_{2}} \sum_{j=1}^{q_{2}} p_{2}[i-j]
\end{aligned}
$$

Considering the maximum queue capacity is $C_{\max }$, samples are lost at $n_{e}$ if this maximum capacity is overcome (29). Operating, and taking probabilistic values for random terms, an expression for the loss probability at $n=n_{e}$ is obtained (30).

$$
\begin{aligned}
C\left[n_{e}\right] & =n_{e} \cdot q_{1} \cdot N-\sum_{i=q_{2}}^{n_{e} \cdot q_{2}} \sum_{j=1}^{q_{2}} p_{2}[i-j]>C_{\max } \\
P_{L}\left[n_{e}\right] & =P\left(\sum_{i=q_{2}}^{n_{e} \cdot q_{2}} \sum_{j=1}^{q_{2}} p_{2}[i-j]<n_{e} \cdot q_{1} \cdot N-C_{\max }\right)
\end{aligned}
$$

In order to obtain the global loss probability, it must be considered the aggregated result of the entire temporal series (31).

$$
P_{L}=\sum_{n_{e}=1}^{\infty} P_{L}\left[n_{e}\right]
$$

The proposed expression for the loss probability depends explicitly on four parameters: $N, C_{\max }, q_{1}$ and $q_{2}$. Besides, it depends implicitly on the parameter $Q$. However, before considering (31) as a valid expression to obtain the loss probability, it must be guaranteed that the series presents a convergent sum. In order to guarantee that, the distribution function of the summation in (31) is analyzed.

Each individual realization $p_{2}[n]$ in that summation is described by a uniform distribution $U[u]$ in the range $\left\{0, \ldots, 2^{Q}-1\right\}$. This distribution is characteristic of the Trifork generator and of all samples it generates. As each realization (random number) is independent from the previous and the later ones, the probability density function of the summation of $k$ realizations may be calculated as the convolution of the probability density function of the individual realizations (32). In this case, the convolution of $U[u]$ with itself $k$ times (33) is represented by the operator $\operatorname{conv}_{k}(\cdot)$.

$$
\begin{aligned}
p d f\left(X_{1}+\ldots+X_{k}\right) & =p d f\left(X_{1}\right) * \ldots * p d f\left(X_{k}\right) \\
p d f\left(\sum_{j=1}^{k} p_{2}[j]\right) & =\operatorname{conv}_{k}(U[u])
\end{aligned}
$$

The central limit theorem establishes that for large values of $k$ (in practice for $k>6$ ), the probability density function $(p d f)$ described by $\operatorname{conv}_{k}(U[u])$ is a Gaussian distribution. The mean and the variance of this distribution may be easily calculated from the mean and variance of the uniform 


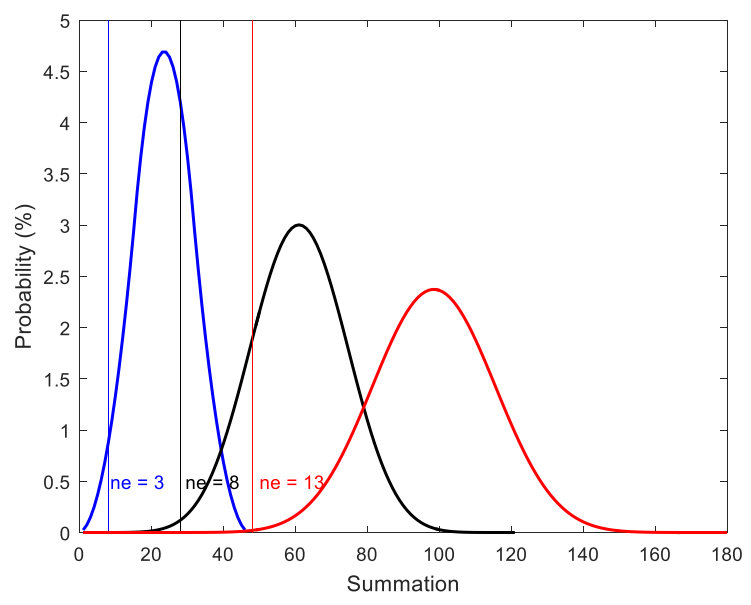

FIGURE 9. Representation of the evolution in the pdf as the number of samples in the summation goes up. The area below the limit (vertical lines) is smaller as $\mathbf{n}_{\mathbf{e}}$ grows up. Configuration parameters: $\mathbf{N}=\mathbf{Q}=\mathbf{4}$. $C_{\max }=4 . q_{1}=q_{2}=1$. Blue graphic $\left(n_{e}=3\right)$. Black graphic $\left(n_{e}=8\right)$. Red graphic $\left(n_{e}=13\right)$.

distribution (34).

$$
\begin{aligned}
\operatorname{conv}_{k}(U[u]) & =\Phi_{\eta_{g}, \sigma_{g}}[u] \\
\eta_{g} & =k \cdot \eta_{t} \\
\sigma_{g}^{2} & =k \cdot \sigma_{t}^{2}=k \cdot \frac{2^{Q}-1}{12}
\end{aligned}
$$

Therefore, as $k$ goes up, the mean value of the corresponding probability density function $(p d f)$ moves to higher values in the abscissa axis. The increasing speed of the mean value is $\eta_{t}$ units per convolution. The resulting Gaussian function also widens as $k$ goes up; however, its widening speed is lower, only $\frac{\eta_{t}}{\sqrt{3}}$ units per convolution.

Finally, in (30), the limit value for which the probability $\left(n_{e} \cdot q_{1} \cdot N-C_{\max }\right)$ is calculated also increases as $n_{e}$ grows up. However, its increasing speed is much lower, $q_{1} \cdot N$ units per convolution.

As a result of all this information, as $n_{e}$ grows up, the area of the corresponding Gausian $p d f$ that remains below the calculation limit is smaller. Then, its contribution to the sum of the series in (31) is also smaller and, thus, this sum is guaranteed to be convergent. Fig. 9 shows this situation.

Therefore, it is possible to numerically obtain graphics and tables that help people to choose the proper values for the design parameters $Q, N, C_{\max }, q_{1}$ and $q_{2}$, given the maximum allowed loss probability $P_{L}$ or vice versa. Fig. 10 and Fig. 11 are examples of these graphics. Fig. 10 represents the loss probability depending on the capacity of the queue $C_{\text {max }}$ for different values of $Q$. Fig. 11 represents the loss probability depending on the capacity the parameter $q_{1}$ for different values of $q_{2}$. As can be seen, for current standard values and sample lengths, the loss probability is very low; as the traffic volume supported by the system is much lower than 1 Erlang (congestion situations only appear for small values such as $N=Q=2$ ).

The output signal from the meta-information protection phase $x_{m}[n]$ protects both, the original private information

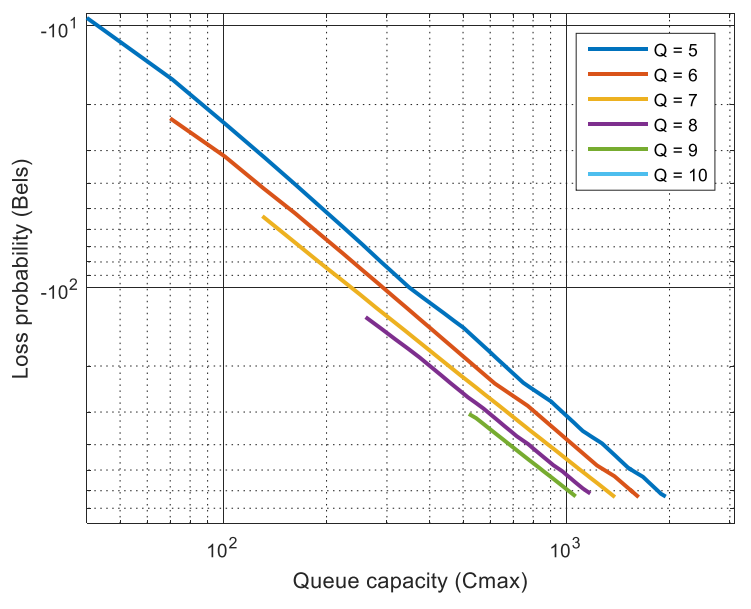

FIGURE 10. Loss probability depending on the queue capacity $\mathbf{C}_{\max }$ for different values of $\mathbf{Q}$ parameter. Loss probability is represented in bels. The employed numerical algorithm has a precision of $0.1 \%$ Configuration parameters: $\mathbf{N}=10 . q_{1}=q_{2}=1$.

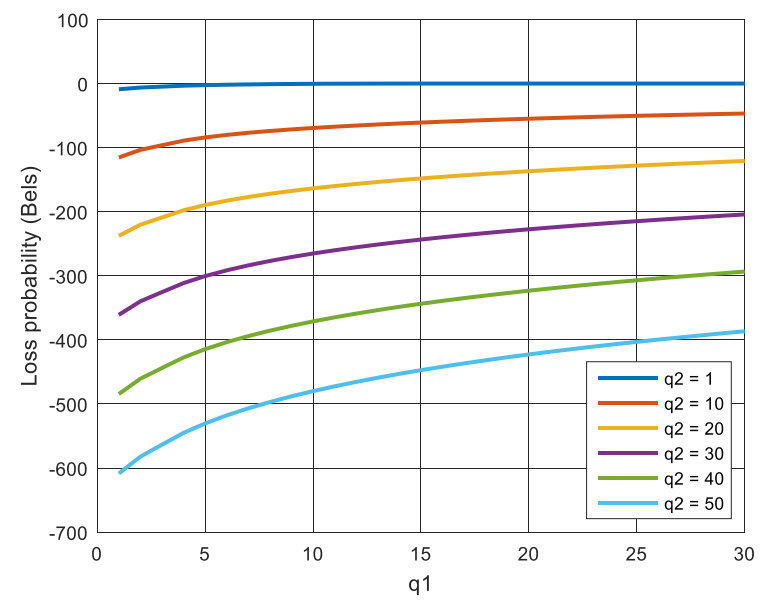

FIGURE 11. Loss probability depending on the $q_{1}$ parameter for different values of $q_{2}$ parameter. Loss probability is represented in bels. The employed numerical algorithm has a precision of $0.1 \%$ Configuration parameters: $\mathbf{N}=Q=4$. $C_{\max }=4$.

and the meta-information. However, it may be still detected in the frequency spectrum, so any attacker could easily capture the communication signals, although he cannot access to the protected information. Besides, attackers could try to generate interferences or deny the communication services by producing electronic noise or placing any conductive object to reflect the radio signals.

In order to address all these problems, a spread spectrum (SS) technique may be considered. The application of these techniques belongs to the communication system, and is outside the objective of this paper. Nevertheless, in the proposed system, the necessary pseudorandom number sequence to support these solutions can be generated apart from the communication module, so this third PRNG may be employed to synchronize the remote transmitter and receptor.

The proposed synchronized PNRG consists of a third Trifork generator which is controlled by a third clock, 


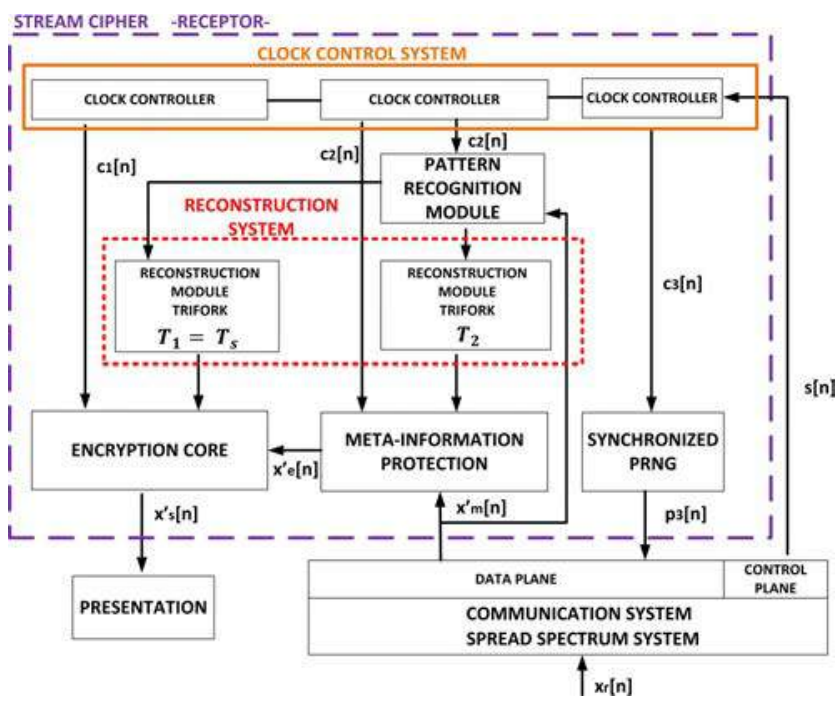

FIGURE 12. Block diagram of the receptor of the proposed stream cipher.

synchronized with the two previous ones. In particular it must be guaranteed that the period of this clock presents a rational factor of proportionality with $T_{1}$ and $T_{2}$ (35). It must be taken into account that, in order to feed a SS system, the period $T_{3}$ must be much shorter than $T_{1}$ and $T_{2}$.

$$
\frac{T_{3}}{T_{s}}=\frac{q_{3}}{q_{1}} ; \quad \frac{T_{3}}{T_{2}}=\frac{q_{3}}{q_{2}} q_{1}, q_{2}, q_{3} \in \mathbb{N}
$$

Using the secure signal $x_{m}[n]$ and the random signal generated by this synchronized PNRG, $p_{3}[n]$, the communication module sends the private information to the receptor.

In the receptor, an equivalent stream cipher to the one deployed in the transmitter is included. Fig. 12 presents the corresponding block diagram. Some details are not included, as components making up each one of the three main parts of the stream cipher (the encryption core, the meta-information protection module and the synchronized PRNG) are equal to the ones previously described for the transmitter. As can be seen, however, there are some important new modules. These new modules perform complex algorithms, so the receptor or the proposed stream cipher must be always placed in the base station. The proposed initiation protocol (see Section III.B) is designed by following this requirement.

The receptor of the proposed stream cipher includes three new functionalities: the clock control system, the pattern recognition module and the reconstruction system.

When the communication and the SS systems receive an information signal $x_{r}^{\prime}[n]$ with a transmission power below the noise level, they employ the random signal $p_{3}^{\prime}[n]$ to recover a signal $x_{m}^{\prime}[n]$ with an adequate Signal to Noise Ratio (SNR). In order to do that, it is necessary to adequately synchronize the pseudorandom code in the transmitter and the receptor.

Every SS system has the ability of getting automatically synchronized through the calculation of the autocorrelation (36). Moving in time the pseudorandom sequence, the synchronization between the transmitter and the receptor will be reached when the autocorrelation is maximal.

$$
\begin{aligned}
\theta\left(n_{0}\right) & =\sum_{i=n_{i}}^{n_{i}+k} p_{3}^{\prime}[i] p_{3}[i]=\sum_{i=n_{i}}^{n_{i}+k} p_{3}\left[i+n_{0}\right] p_{3}[i] \\
& = \begin{cases}k+1 & \text { if } n_{0}=0 \\
\downarrow \downarrow \downarrow & \text { if } n_{0} \neq 0\end{cases}
\end{aligned}
$$

These movements in time of the pseudorandom sequence $p_{3}^{\prime}[n]$ are obtained through a synchronization signal $s[n]$, being able to stop and run the master clock by means of a clock controller. This clock controller is connected to two additional controllers, which stop and run the other two master clocks in the cipher, so the entire clock bank is synchronized and controlled by a clock control system.

Nevertheless, getting the master clocks synchronized does not guarantee the PRNGs in the receptor are totally synchronized too. In fact, SS systems have the possibility of storing or removing some samples of the pseudorandom sequences. In practice, this means that when clocks are synchronized, PRNGs (i.e. Trifork generators in the encryption core and the meta-information protection module) are "almost-synchronized", there are no guarantees that they are completely synchronized.

In order to guarantee a total synchronization of the PRNG, a pattern recognition module is included in the cipher. This module receives the signal $x_{m}^{\prime}[n]$ whose sample length represents the sequence of generated pseudorandom numbers by the Trifork generator in the meta-information protection phase in the transmitter. This sequence is extracted and analyzed in order to infer the particular time instant $n=n_{0}$ for which the PRNG must be configured so it generates the same numerical sequence as described by the sample length in $x_{m}^{\prime}[n]$.

As the receptor has the symmetric keys, and the clock control system has placed the PRNG in an "almostsynchronized" state, it is not complicated to deduct this specific instant using a simple program and a Trifork generator configured as the PNRG in the meta-information protection module.

The information about this instant, $n=n_{0}$, is transmitted to a reconstruction module, consisting of a fast evolution Trifork generator which is able to recover the internal state of the PNRG for the indicated time instant. This module, finally, places the PRNG of the meta-information protection system in a total synchronization state with the corresponding PRNG in the transmitter.

It is important to note that, in the receptor, the meta-information protection module is not necessary, as the original length may be directly reconstructed knowing that samples have a length of $N$ bits. However, this module in receptor allows the cipher to detect problems, cyber-attacks, false transmissions and fails in the synchronization. As in the transmitter, if no enough bits are received to create a sample of $N$ bits, then, the encryption core (in this case) waits until more bits are received. Thus, a flow of $N$-bit samples $x_{e}^{\prime}[n]$ 
is obtained as output of this module. This signal contains the encrypted private information to be recovered.

At this point, it must be considered that clocks in the clock bank have a rational factor of proportionality. Thus, every so often, both clocks have a common edge, and both associated PRNG produce a new sample at the same time. In particular (see Fig. 8) the first common edge corresponds to the transmission beginning. Therefore, once deduced the time instant $n=n_{0}$, the Trifork generator in the encryption core may be also synchronized with the corresponding one in the transmitter by means of a second reconstruction module. As a result, a new pseudorandom number sequence $p_{1}^{\prime}[n]$ is generated.

Using the generated sequence of random numbers $p_{1}^{\prime}[n]$ and the signal $x_{e}^{\prime}[n]$, the private information may be deciphered and recovered using the same XOR operation employed in the transmitter (37). The obtained signal $x_{s}^{\prime}[n]$ will be equal to the original private information $x_{s}[n]$ if both PRNG (in the transmitter and in the receptor) are totally synchronized.

$$
\begin{aligned}
x_{s}^{\prime}[n] & =x_{e}^{\prime}[n] \oplus p_{1}^{\prime}[n] \\
& =x_{s}[n] \oplus p_{1}[n] \oplus p_{1}[n] \\
& =x_{s}[n]
\end{aligned}
$$

The synchronization process may be complex and time consuming, so (in our proposal) it is performed by the base stations. In traditional mobile networks, where devices present a high mobility level, these techniques are useless as the synchronization state is easily lost due to mobility. However, as we said, in the studied intra-slice domain, devices have a very low and limited mobility level, so there is a very low probability of the synchronization state to get affected due to this fact.

\section{EXPERIMENTAL VALIDATION}

In relation to the proposed stream cipher, evaluating the security level of the proposed encryption scheme has no sense, as it is directly equivalent to the randomness of the Trifork generator, which has been deeply evaluated in the literature [34]. Besides, important characteristics such as the meta-information protection are not correctly considered in these analyses. Therefore, in this case, we have designed an evaluation method based on key performance indicators (KPI).

Four different experiments were designed in order to evaluate (i) the synchronization time depending on the number of active communication links, (ii) the synchronization time depending on the communication links being synchronized at the same time, (iii) the binary loss probability depending on the number of active communication links and (iv) the amount of resources the proposed scheme requires from a resource constrained microcontroller. The first three experiments were based on a simulation scenario. The final experiment was performed using a first practical implementation of the proposed solution.
The designed simulation scenario was based on the NS3 simulator, a network simulator very flexible and configurable. It is an open source tool where scenarios are represented as $\mathrm{C}++$ programs. This tool was deployed in a Linux (Ubuntu 16.04) machine with 8GB of RAM memory and an Intel i7 processor.

The simulation scenario consisted of a unique base station and a variable population of IoT devices (see Fig. 3). Models for radio channels, interferences, network protocols, etc. were taken from the NS3 libraries for mobile networks. The simulation model for the base station was configured according to the characteristics of current real base stations [51]. In particular, if the Digital Signal Processors (which belong to the communication system) are not considered, most modern base station nowadays [51] are composed of a relatively small amount of hardware: a general purpose embedded processor, 15 Kilobits of SRAM and 32 Mbyte of DRAM.

In order to pack and assign these resources to the base station, a virtual machine was deployed. It was configured using container technologies; specifically, a Kernel-based Virtual Machine (KVM) was created. It was deployed by means of the libVirt Application Programming Interface (API), so the machine can be managed directly from the $\mathrm{C}++$ program describing the simulation scenario.

With the objective of connecting the virtual machine and the simulated scenario represented inside the NS3 simulator, the base station in the simulation was provided with a TAP bridge. These bridges forward the input traffic in the simulated base station to the external virtual machine, so real algorithms, solutions and protocols may be easily tested.

As in this first three experiments, the impact of the proposed solution in the IoT devices is not evaluated (we are considering they can easily support the stream cipher), these IoT components are represented only as simulated nodes (which are also programmable in the NS3 simulator).

In the first experiment, a set of simulations was planned. With each new simulation, the number of IoT devices is incremented in one unit. These devices try to stablish a secure communication link and get synchronized with the base station sequentially. For each simulation, the synchronization time required by the last device to be synchronized is measured. New simulations were performed until the last IoT device could not get synchronized.

During the second experiment, the same scenario of first experiment was designed. However, in this case, IoT devices try to get synchronized at the same time, instead of sequentially. The total required time to synchronize all IoT devices is measured. New simulations were performed until IoT devices cannot get synchronized.

In these two first experiments, simulations were configured for different values of $Q$ parameter. Other relevant configuration parameters were fixed to represent a real situation nowadays: $N=10$

Finally, in the third experiment, a slightly different scenario was created. In this new simulation, IoT devices were supposed to be already synchronized. All IoT devices in the 
scenario are using an active communication link to stream a binary data flow. With each new simulation, the number of IoT devices increments in one unit. The binary loss probability is evaluated in each simulation, until it is close to $100 \%$. In order to evaluate the binary loss probability only from to the proposed stream cipher, we reduce the impact of the communication channel considering it ideal (without packet losses, interferences, the binary rate may be as high as desired, etc.). Simulations in this experiment were configured for different values of $Q$ parameter. The other configuration parameters were fixed to represent a real situation nowadays: $N=10, q_{1}=q_{2}=1$

The last, and fourth, experiment was very different from the previous ones.

In order to evaluate the resource consumption of the stream cipher when implemented in resource constrained microcontrollers, we developed an Arduino program describing the cipher proposed for transmission. We evaluate the additional use in RAM memory, program space (Flash memory) and computational time due to the use of our proposal. As a practical consequence we are analyzing the limits of the proposal to be used in the application scenario.

The experiment was repeated for different values of $Q$ and $C$ parameters. The other configuration parameters were fixed to represent a real situation nowadays: $N=10, q_{1}=q_{2}=1$

As a hardware platform, we employed an Arduino Nano board. It includes an AVR microcontroller, the ATmega328 microcontroller. It also has $32 \mathrm{~KB}$ of Flash memory, $2 \mathrm{~KB}$ of SRAM memory and $1 \mathrm{~KB}$ of EEPROM (which is rarely employed).

\section{RESULTS}

In this section results of the described experiments in the previous section are presented and discussed.

In order to remove from the results of the first experiment (as much as possible) variations in the execution process of the simulations due to exogenous variables (e.g. delays operations performed by the Operating Systems), for each case twelve different simulations were performed. The average of all these measures was obtained in order to calculate the final results. Fig. 13 shows the obtained data.

As can be seen, the maximum synchronization time for the proposed stream cipher is around $T_{\text {syn }}=30$ seconds. This maximum time may vary around $15 \%$ depending on exogenous variables, which do not directly depend on the proposed solution.

Evolution curves present two different areas. In the first zone, the synchronization time remains constant, around $T_{\text {syn }}=0.9$ seconds, showing small fluctuations, which are not related to the proposed cipher. This value is equal for all values of $Q$ parameters, as operation to be performed (such as memory allocation) are almost independent of these parameters. Then (in the second zone), once a certain number of active connections is overcome, the synchronization time starts growing exponentially, until the maximum synchronization is reached.

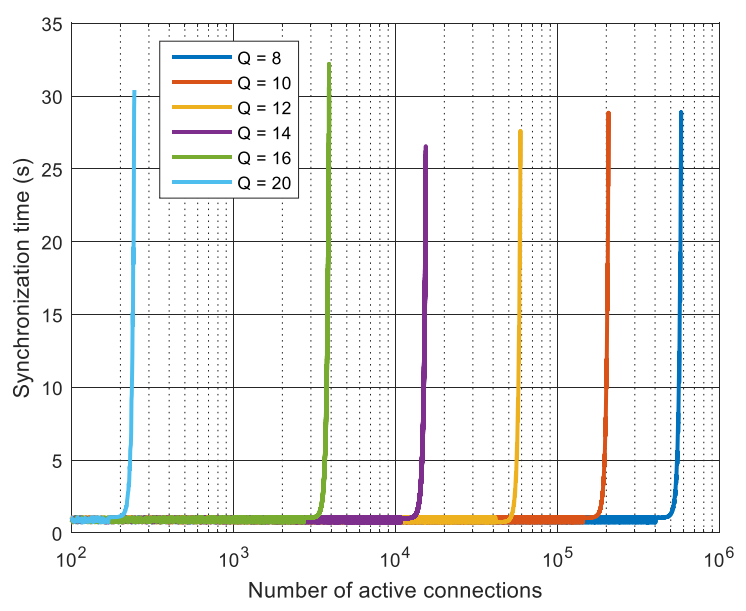

FIGURE 13. Results of the first experiment: synchronization time depending on the number of active communication links.

Both the number of active connection for which the maximum synchronization time is reached, and the growth rate in the exponential zone strongly depend on the $Q$ parameter.

As can be seen, as $Q$ goes up, the growth rate in the exponential zone and the number of active connection for which the maximum synchronization time is reached increase. In particular, for $Q=20$, the maximum number of active connections is $N_{\text {conne }}=244$, whereas for $Q=8$, the maximum number of active connections is $N_{\text {conne }}=5.8 \cdot 10^{5}$. In fact, as $Q$ parameter grows, the length of keys, the maximum capacity of the queue, etc., must be higher, and a lower number of connections can be maintained at the same time. Any case, if we consider that nowadays a base station can only maintain around one hundred simultaneous connections (due to limits in the use of the spectrum, data rate, etc.), the proposed solution does not limit the performance of the base station in any aspect.

In scenarios composed of pervasive infrastructures (such as IoT scenarios), when the system is powered up, many devices will try to get synchronized at the same time. In order to evaluate the response of the base station in this situation the second experiment was carried out. Fig 14 shows the obtained results.

As can be seen, once more, results strongly depend on $Q$ parameter. Curves in Fig. 14 presents, also, two areas. In the first area, the global evolution of the synchronization time is linear with the number of devices that are trying to get synchronized. The slope of this line is higher as $Q$ goes up (it must be remarked that axes are logarithmic). This situation may be explained by the small-time differences in the execution of the synchronization operations (such as memory allocation). Although considering only one realization these differences are not appreciable (see Fig. 13), they have an important effect when analyzing the accumulation of several realizations.

Fast variations in this slope may also appear when the base station starts being congested. The maximum number of devices that may be synchronized at the same time is 


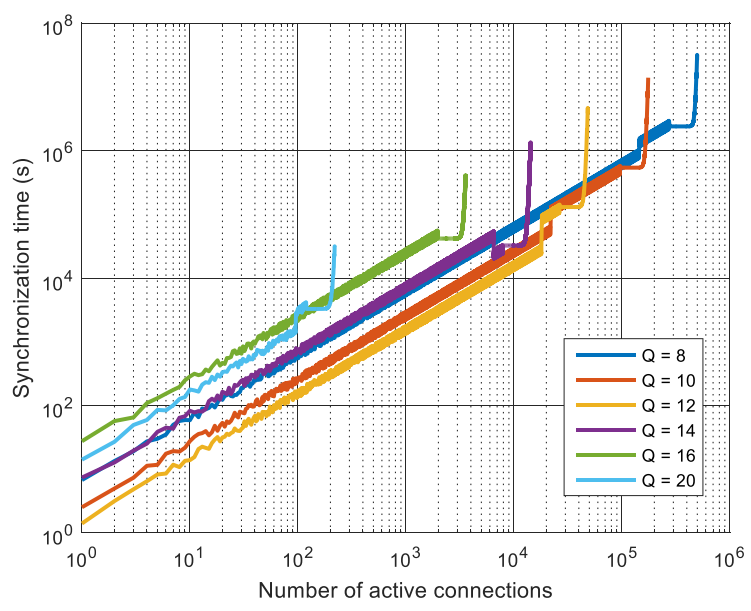

FIGURE 14. Results of the second experiment: synchronization time depending on the communication links being synchronized at the same time.

similar to the maximum number of active connections which still allow new synchronizations. A difference around $10 \%$ between both values may be seen (the maximum number of devices that may be synchronized at the same time is lower, as it could be expected).

The obtained curves also present a second area. In this area the synchronization time goes up exponentially, indicating that the base station is in a congested state. The growth rate in this case also depends on the value of $Q$ parameter. As the number of devices that can be synchronized is lower as $Q$ parameter grows (see Fig. 13), the maximum accumulated synchronization time is also lower if $Q$ is higher.

In conclusion, we may guarantee that the proposed cipher does not affect the synchronization of the IoT deployments in 5G mobile networks, as the imposed limits are above the real operation limits of base stations.

With previous experiments, the maximum number of devices that may be connected at the same time to a unique base station is evaluated. However, we have no information about the quality of the communication links between the base station and these devices. Experiment three aims to obtain that information. Fig. 15 shows the obtained results.

As can be seen, obtained curves are sigmoid-like functions. The binary loss probability remains between $0 \%$ and $5 \%$ if the number of active connections is below the maximum number of simultaneous connections a unique base station can handle (see Fig. 13). In particular for $Q=20$ the binary loss probability is below $2 \%$ for $N_{\text {conne }} \lesssim 250$, whereas for $Q=8$ this situation is maintained for $N_{\text {conne }} \lesssim 6 \cdot 10^{5}$.

Once the maximum number of connections is overcome, the binary loss probability starts growing, as no memory is available to handle the extra input flows. This probability has an asymptote for $p=100 \%$, since, as the quantity of data that are rejected goes up, the probability gets closer to $100 \%$. Nevertheless, there is always a certain percentage of data that are accepted, so probability must be lower than $100 \%$ (although as close to this value as desired).

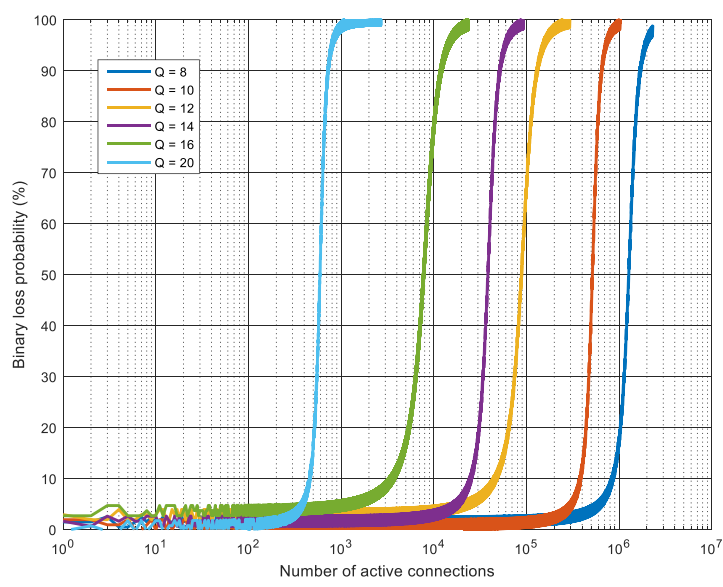

FIGURE 15. Results of the third experiment: binary loss probability depending on the number of active communication links.

Small fluctuations may appear in the curves because of standard problems, such as the probability of queue to be overflowed.

The three described experiments are focused on base stations. However, IoT devices are (at least) as important as these components. In particular, we must guarantee that the proposed cipher is light enough to be implemented in resource constrained microcontrollers. Table 2 presents the results of the last experiment, where an implementation of the proposed cipher in a small microcontroller is evaluted.

As can be seen, the implementation of the proposed cipher is very lightweight, and it does not almost consume resources in the microcontroller. The use of the program space remains equal, and the use of RAM has grown only between $1 \%$ (for $C=C_{\min }$ ) and $2 \%$ (for $C=2 \cdot C_{\min }$ ). As we are using an Arduino board, which is not prepared to work with bits at low level, the designed program must employ standard data types and the obtained program is almost independent from the value of $Q$ parameter (in practice, for small values of $Q$ we are allocating extra space for bits that are not going to be used).

As a consequence of a program that is independent from the value of $Q$, the sample processing time is also independent from this parameter. In this case, since microcontrollers only admit sequential programming (not parallel programming), the required time to update the three modules in the cipher is not negligible. In particular, the required time to process one sample has been multiplied by 10 . In practice this implies that the maximum admissible bandwidth for input signals has been reduced in the same factor. Traditionally, Arduino board may consider analog signal with a maximum bandwidth up to $4.5 \mathrm{MHz}$. When using our cipher, this quantity is reduced to $450 \mathrm{KHz}$.

Despite this fact, standard analog signals in IoT scenarios have a much smaller bandwidth; usually no more than $100 \mathrm{KHz}$ (almost three times the required throughput for voice transmissions). Therefore, the proposed cipher will not affect the wide majority of IoT applications, although they are based on resource constrained devices. 
TABLE 2. Resource consumption of the proposed cipher in microcontrollers.

\begin{tabular}{|c|c|c|c|c|}
\hline & Situation & $\begin{array}{l}\text { Use of } \\
\text { RAM }\end{array}$ & $\begin{array}{c}\text { Use of } \\
\text { program } \\
\text { space }\end{array}$ & $\begin{array}{l}\text { Processing time of } \\
\text { one sample }\end{array}$ \\
\hline \multirow{4}{*}{ 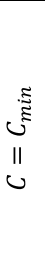 } & Without cipher & $9 \%$ & $8 \%$ & $112 \mu \mathrm{s}$ \\
\hline & Cipher $Q=8$ & $10 \%$ & $8 \%$ & $1019 \mu \mathrm{s}$ \\
\hline & Cipher $Q=16$ & $10 \%$ & $8 \%$ & $1020 \mu \mathrm{s}$ \\
\hline & Cipher $Q=32$ & $10 \%$ & $8 \%$ & $1020 \mu \mathrm{s}$ \\
\hline \multirow{4}{*}{$\begin{array}{l}\stackrel{\tilde{\Xi}}{\tilde{E}} \\
\dot{N} \\
\| \\
ن\end{array}$} & Without cipher & $9 \%$ & $8 \%$ & $112 \mu \mathrm{s}$ \\
\hline & Cipher $\mathrm{Q}=8$ & $11 \%$ & $8 \%$ & $1019 \mu \mathrm{s}$ \\
\hline & Cipher $Q=16$ & $11 \%$ & $8 \%$ & $1020 \mu \mathrm{s}$ \\
\hline & Cipher $\mathrm{Q}=32$ & $11 \%$ & $8 \%$ & $1020 \mu \mathrm{s}$ \\
\hline
\end{tabular}

\section{CONCLUSIONS AND FUTURE WORKS}

In this paper we have investigated and proposed a new security solution for emerging $5 \mathrm{G}$ networks, to be applied in the intra-slice domain.

The proposed solution consists of a stream cipher based on lightweight pseudo-random number generators which protect the private information, the meta-information and hide the communication signals in the frequency spectrum using spread spectrum techniques.

We have also described and evaluated a first implementation of the proposed solution, using both, a simulation scenario and a real deployment. Results showed that the proposed cipher may be implemented in both, resource constrained devices and future $5 \mathrm{G}$ base stations.

Although the sample processing time in resource constrained IoT devices is multiplied by ten if using the proposed cipher, it has been proved that the performance of none of $5 \mathrm{G}$ network components (both IoT devices and base stations) will be penalized if they include the proposed solution.

Future works will evaluate the performance of the proposed solution in microelectronic devices, such as Systemon-chip, as these hardware components are envisioned to be the basis of future engineered solutions. On the other hand, an efficient resource-constraint hardware implementation of the proposed technology would be an interesting topic to be investigated nowadays.

Considering more technical aspects, problems and possible solutions when using the presented proposal in noncontinuous transmissions should be evaluated (effects of bursts of information, synchronization problems, etc.).

Besides, and finally, the combination of the proposed cryptographic solution with other security methods should be investigated. Frameworks including cryptographic techniques, trust management technologies and other similar solutions should be integrated to design a totally secure future Internet-of-Things.

\section{REFERENCES}

[1] J. Gubbi, R. Buyya, S. Marusic, and M. Palaniswami, "Internet of Things (IoT): A vision, architectural elements, and future directions," Future Generat. Comput. Syst., vol. 29, no. 7, pp. 1645-1660, 2013.

[2] B. B. Sánchez, R. Alcarria, D. S. de Rivera, and Á. S. Picot, "Enhancing process control in industry 4.0 scenarios using cyber-physical systems," $J$. Wireless Mobile Netw., vol. 7, no. 4, pp. 41-64, 2016.

[3] B. Bordel, R. Alcarria, T. Robles, and D. Martín, "Cyber-physical systems: Extending pervasive sensing from control theory to the Internet of Things," Pervasive Mobile Comput., vol. 40, pp. 156-184, Sep. 2017.

[4] Y.-B. Lin, Y.-W. Lin, J.-C. Wu, and Y.-C. Wang, "An investigation of telecom mobile data billing plans," J. Internet Serv. Inf. Secur, vol. 6, no. 3, pp. 1-26, 2016.

[5] X. Foukas, G. Patounas, A. Elmokashfi, and M. K. Marina, "Network slicing in 5G: Survey and challenges," IEEE Commun. Mag., vol. 55, no. 5, pp. 94-100, May 2017.

[6] View on 5G Architecture, 5G PPP Architecture Working Group, White Paper Version 2.0, Dec. 2017. [Online]. Available: https://5gppp.eu/wp-content/uploads/2018/01/5G-PPP-5G-Architecture-WhitePaper-Jan-2018-v2.0.pdf

[7] G. Orhanou and S. El-Hajji, "The new LTE cryptographic algorithms EEA3 and EIA3," Appl. Math, vol. 7, no. 6, pp. 2385-2390, 2013.

[8] T. Shirai, K. Shibutani, T. Akishita, S. Moriai, and T. Iwata, "The 128-bit blockcipher CLEFIA," in Fast Software Encryption, vol. 4593. Berlin, Germany: Springer, 2007, pp. 181-195.

[9] J. P. Aumasson, L. Henzen, W. Meier, and M. Naya-Plasencia, "Quark: A lightweight hash," in Proc. Int. Workshop Cryptograph. Hardw. Embedded Syst. (CHES), vol. 6225, Aug. 2010, pp. 1-15.

[10] M. S. Rahman, A. Basu, and S. Kiyomoto, "Decentralized ciphertextpolicy attribute-based encryption: A post-quantum construction," J. Internet Serv. Inf. Secur., vol. 7, no. 3, pp. 1-16, 2017.

[11] M. Katagi and S. Moriai, "Lightweight cryptography for the Internet of Things," Sony Corp., Tokyo, Japan, Tech. Rep. 03-2011, 2008, pp. 7-10.

[12] eSTREAM: The ECRYPT Stream Cipher Project. Accessed: Mar. 17, 2018. [Online]. Available: http://www.ecrypt.eu.org/stream/

[13] M. Robshaw and O. Billet, New Stream Cipher Designs: The eSTREAM Finalists, vol. 4986. Berlin, Germany: Springer-Verlag, 2008.

[14] H. Wu, The Stream Cipher HC-128 (Lecture Notes in Computer Science), vol. 4986. Berlin, Germany: Springer-Verlag, 2008, pp. 39-47.

[15] M. Boesgaard, M. Vesterager, T. Pedersen, J. Christiansen, and O. Scavenius, "Rabbit: A new high-performance stream cipher," in Fast Software Encryption. Berlin, Germany: Springer-Verlag, 2003, pp. 307-329.

[16] D. J. Bernstein, "The Salsa20 family of stream ciphers," in New Stream Cipher Designs (Lecture Notes in Computer Science), vol. 4986. Berlin, Germany: Springer, 2008, pp. 84-97.

[17] C. Berbain et al., "SOSEMANUK, a fast software-oriented stream cipher," in New Stream Cipher Designs (Lecture Notes in Computer Science), vol. 4986. 2008, pp. 98-118.

[18] M. Hell, T. Johansson, and W. Meier, "Grain: A stream cipher for constrained environments," Int. J. Wireless Mobile Comput., vol. 2, no. 1, pp. 86-93, 2007.

[19] S. Babbage and M. Dodd, "The MICKEY stream ciphers," in New Stream Cipher Designs. Berlin, Germany: Springer, 2008, pp. 191-209.

[20] C. de Canniere and B. Preneel, "Trivium," in New Stream Cipher Designs. Berlin, Germany: Springer-Verlag, 2008, pp. 244-266.

[21] S. Banik and S. Maitra, "A differential fault attack on MICKEY 2.0," in Proc. Int. Workshop Cryptograph. Hardw. Embedded Syst., Aug. 2013, pp. 215-232.

[22] Information Technology-Security Techniques-Lightweight Cryptography-Part 3: Stream Ciphers, document ISO/IEC 291923:2012, 2012. [Online]. Available: https://www.iso.org/standard/56426. html

[23] R. Beaulieu, D. Shors, J. Smith, S. Treatman-Clark, B. Weeks, and L. Wingers, "SIMON and SPECK: Block ciphers for the Internet of Things," Nat. Secur. Agency, Fort Meade, MD, USA, Tech. Rep., 2015, p. 585 .

[24] E. Biham and O. Dunkelman, "Cryptanalysis of the A5/1 GSM stream cipher," in Progress in Cryptology-INDOCRYPT. Berlin, Germany: Springer, 2000, pp. 43-51.

[25] A. B. Orúe, L. H. Encinas, V. Fernández, and F. Montoya, "A review of cryptographically secure PRNGs in constrained devices for the IoT," in Proc. Int. Joint Conf. (SOCO), Sep. 2017, pp. 672-682. 
[26] Y. Tian, G. Chen, and J. Li, "On the design of trivium," School Inf. Secur Eng., Shanghai Jiaotong Univ., Shanghai, China Tech. Rep. 431-2009, 2009, p. 431

[27] Y. Zhang, D. Xiao, W. Wen, H. Nan, and M. Su, "Secure binary arithmetic coding based on digitalized modified logistic map and linear feedback shift register," Commun. Nonlinear Sci. Numer. Simul., vol. 27, nos. 1-3, pp. 22-29, 2015

[28] M. B. Shemaili, C. Y. Yeun, K. Mubarak, and M. J. Zemerly, "A new lightweight hybrid cryptographic algorithm for the Internet of Things," in Proc. Int. Conf. Internet Technol. Secur. Trans., Dec. 2012, pp. 87-92.

[29] A. Klein, "Attacks on the RC4 stream cipher," Des., Codes Cryptogr. vol. 48, no. 3, pp. 269-286, 2008.

[30] L. Dorrendorf, Z. Gutterman, and B. Pinkas, "Cryptanalysis of the random number generator of the Windows Operating System," ACM Trans. Inf. Syst. Secur., vol. 13, no. 1, 2009, Art. no. 10 .

[31] L. Blum, M. Blum, and M. Shub, "A simple unpredictable pseudo-random number generator," SIAM J. Comput., vol. 15, no. 2, pp. 364-383, 1986.

[32] A. B. O. López, G. Marañon, A. G. Estévez, G. P. Dégano, M. R. García, and F. M. Vitini, "Trident, a new pseudo random number generator based on coupled chaotic maps," in Proc. 3rd Int. Workshop Comput. Intell. Secur. Inf. Syst. (CISIS), Oct. 2010, pp. 183-190.

[33] G. Marsaglia and L.-H. Tsay, "Matrices and the structure of random number sequences," Linear Algebra Appl., vol. 67, pp. 147-156, Jun. 1985.

[34] A. B. Orue, F. Montoya, and L. H. Encinas, "Trifork, a new pseudorandom number generator based on lagged fibonacci maps," J. Comput. Sci. Eng., vol. 2, no. 2, pp. 1-6, Aug. 2010.

[35] W. H. Payne, "FORTRAN Tausworthe pseudorandom number generator," Commun. ACM, vol. 13, no. 1, 1970, Art. no. 57.

[36] S. Tezuka and P. L'Ecuyer, "Efficient and portable combined Tausworthe random number generators," ACM Trans. Model. Comput. Simul., vol. 1, no. 2, pp. 99-112, 1991.

[37] Y. Bi, "A reconfigurable supercomputing library for accelerated parallel lagged-Fibonacci pseudorandom number generation," M.S. thesis, University of Tennessee, Knoxville, TN, USA, 2006.

[38] N. S. Altman, "Bit-wise behavior of random number generators," SIAM J. Sci. Statist. Comput., vol. 9, no. 5, pp. 941-949, 1988.

[39] D. E. Knuth, The Art of Computer Programming, Seminumerical Algorithms, vol. 2, 3rd ed. London, U.K.: Pearson Education, 1997.

[40] R. Anderson, "On Fibonacci keystream generators," in Proc. Int. Workshop Fast Softw. Encryption, Dec. 1994, pp. 346-352.

[41] W. W. Tsang et al., "Development of cryptographic random number generators,” Dept. Comput. Sci. Inf. Syst., Univ. Hong Kong, Hong Kong, HKU CSIS Tech. Rep. TR-2003-07, 2003.

[42] M. K. Chetry and W. V. Kandaswamy, "A note on self-shrinking lagged fibonacci generator," IJ Netw. Secur, vol. 10, no. 3, pp. 185-187, 2010

[43] B. B. Bordel and R. Alcarria, "Secure sensor data transmission in 5G networks using pseudorandom number generators," in Proc. Res. Briefs Inf. Commun. Technol. Evol. (ReBICTE), vol. 3. 2017, pp. 1-11.

[44] T. Heer, O. Garcia-Morchon, R. Hummen, S. L. Keoh, S. S. Kumar, and K. Wehrle, "Security challenges in the IP-based Internet of Things," Wireless Pers. Commun., vol. 61, no. 3, pp. 527-542, 2011.

[45] R. L. Rivest, A. Shamir, and L. Adleman, "A method for obtaining digita signatures and public-key cryptosystems," Commun. ACM, vol. 21, no. 2, pp. 120-126, Feb. 1978.

[46] H. Seo, Z. Liu, J. Großschädl, and H. Kim, "Efficient arithmetic on ARM-NEON and its application for high-speed RSA implementation,' Secur. Commun. Netw., vol. 9, no. 18, pp. 5401-5411, 2016.

[47] S. F. Barrett, "Arduino microcontroller processing for everyone!" Synthesis Lectures Digit. Circuits Syst., vol. 8, no. 4, pp. 1-513, 2013.

[48] T. Jager and J. Somorovsky, "How to break XML encryption," in Proc. 18th ACM Conf. Comput. Commun. Secur., Oct. 2011, pp. 413-422.

[49] A. Braverman, J. G. Dai, and J. Feng, "Stein's method for steady-state diffusion approximations: An introduction through the Erlang-A and ErlangC models," Stochastic Syst., vol. 6, no. 2, pp. 301-366, 2017.

[50] J. F. C. Kingman and M. F. Atiyah, "The single server queue in heavy traffic," Oper. Manage., Critical Perspect. Bus. Manage., vol. 57. 2003, p. 40 .

[51] A. Hemani et al., "Network on chip: An architecture for billion transistor era," in Proc. IEEE NorChip Conf., vol. 31, Nov. 2002, p. 11.

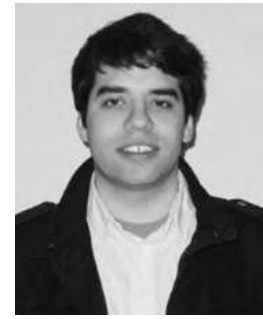

BORJA BORDEL received the B.S. and M.S degrees in telecommunication engineering from the Technical University of Madrid, in 2012 and 2014, respectively. He is currently pursuing the Ph.D. degree in telematics engineering with the Telecommunication Engineering School, UPM. His current research interests include cyberphysical systems, wireless sensor networks, radio access technologies, communication protocols, and complex systems.

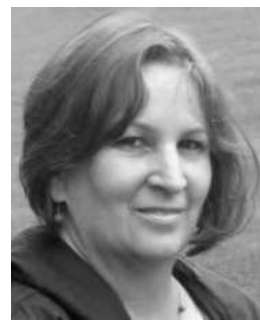

AMALIA BEATRIZ ORÚE received the B.E. and M.S. degrees in telecommunications systems from the University of Oriente, Cuba, in 1984 and 1998 respectively, and the $\mathrm{Ph} . \mathrm{D}$. degree from the Polytechnic University of Madrid, Spain, in 2013. She is currently a Researcher with the Department of Information and Communications Technologies, Institute of Physical and Information Technologies, Spanish National Research Council, Madrid Spain. She has served as a referee for different SCI journals and international conferences. She has published several research papers in international scientific journals, peer-reviewed workshops, and conferences. Her current research interests include cryptography, Internet of Things security, information security, teaching methods of cryptography, and educational innovation. She is a member of the IEEE Education Society and the IEEE Council on RFID.

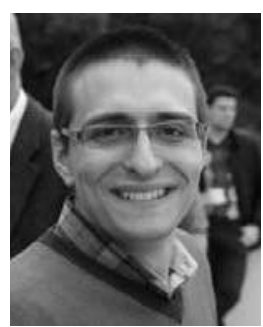

RAMÓN ALCARRIA received the M.S. and Ph.D. degrees in telecommunication engineering from the Technical University of Madrid, in 2008 and 2013, respectively. He is currently an Assistant Professor with the E.T.S.I Topography, Technica University of Madrid. He has been involved in several research and development European and National projects related to future internet, Internet of Things, and service composition. His current research interests include service architectures, sensor networks, human-computer interaction, and prosumer environments.

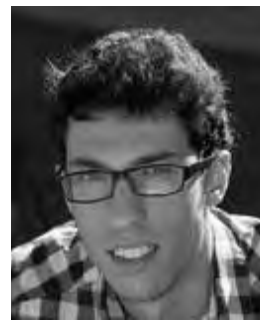

DIEGO SÁNCHEZ-DE-RIVERA received the B.Sc. and M.S. degrees in telecommunication engineering from the Technical University of Madrid. He is currently pursuing the Ph.D. degree with the E.T.S.I Telecommunications, Technical University of Madrid. His current research interests include Internet of Things, sensor networks, and Web development. 


\section{Discussion, Conclusions and Future Works}

In this section, and considering the selected papers presented in the previous section, it is described a general overview of the proposed solutions and the innovative technologies developed. Results of the performed experiments are also analyzed together, so a general discussion about the developed investigation may be done. From this analysis some relevant and significant conclusion are extracted and presented. Finally, considering the performed analysis discussion and conclusion extraction, some pending challenges, future research opportunities and future works are proposed.

\subsection{General overview of the proposed solution}

After analyzing the existing technologies in the context of Cyber-Physical systems (by studying works in the state-of-the-art) in respect to functional architectures, application scenarios and global capabilities (among other important topics); the most interesting proposals for process execution application are identified (with special interest on the NIST's proposals). The paper "Cyber-physical systems: Extending pervasive sensing from control theory to the Internet of Things" by Bordel et al. (Section 3.2) reports the obtained results.

With the obtained information from paper "Cyber-physical systems: Extending pervasive sensing from control theory to the Internet of Things" by Bordel et al., it is proposed a first scheme to execute processes in humanized CPS.

The global solution proposed in this PhD Thesis work starts with the design of a reference architecture reported in the paper "Physical processes control in Industry 4.0based systems: a focus on Cyber-Physical Systems" by Bordel et al. In this paper it is described an eight-level reference architecture, based on the NIST's reference architecture from which transversal components have been removed and two additional interconnection layers (middleware) have been added to adapt the proposal to process execution systems. 
Considering the reference architecture proposed in the paper "Physical processes control in Industry 4.0-based systems: a focus on Cyber-Physical Systems" by Bordel et al., it is proposed an implementation architecture describing the components that make up humanized CPS in the paper "Building smart adaptable Cyber-Physical Systems: definitions, classification and elements" by Bordel et al. It is identified in which level each component is placed, if they are hardware or software; and, overall, which components are already available, which ones must be adapted from existing products and which elements must be designed.

Considering the reference architecture previously defined, and the implementation architecture and the components identified in the paper "Building smart adaptable Cyber-Physical Systems: definitions, classification and elements" by Bordel et al.; in the paper "Building enhanced environmental traceability solutions: From Thing-to-Thing communications to Generalized Cyber-Physical Systems" by Bordel et al. it is proposed a high-level functional architecture, using general components that cover the essential functions required to execute processes in humanized CPS. In this work a more detailed functional architecture and specific models where all components that make up humanized CPS (including humans acting as service providers), their relations and their main characteristics are also described. These models will be later employed in predictive management solutions based on simulators, and in orchestrated management schemes, where a virtual representation of the underlying physical platform is proposed.

This detailed functional architecture proposed in the previous paper is employed for first time to execute processes in the paper "TF4SM: a framework for developing traceability solutions in small manufacturing companies" by Bordel et al. (see Section 3.3). This paper proposes, besides a functional architecture, a real system consisting of three main components performs mentioned functionalities in a simplified manner. In this work, besides, it is implemented for first time a cyber-physical device (a smart glove and a smart table) that can operate into humanized CPS. These devices are employed to monitor the activities being performed by people and to explicitly notify information about them to other components in the system. This information is employed to make the process advance and trigger other activities.

In particular, in this paper, processes previously identified as "event-driven" in the paper "Physical processes control in Industry 4.0-based systems: a focus on CyberPhysical Systems" by Bordel et al. are executed. In these processes the process control is in charge of low-level devices hardcoded programming describing processes (independently from the definitions at high-level). In this scenario, nevertheless, some interfaces to connect and send explicit information about low-level events (present and or future) to high-level components are also included. As it is described in the paper "Enhancing Process Control in Industry 4.0 Scenarios using Cyber-Physical Systems" by Bordel et al., where a stricter theoretical formalization of the event-driven process execution is proposed, these interfaces enable high-level components to control the activities performed by hardware devices in an independent manner. Furthermore, it is 
also described in this paper that these event-driven processes are modeled by means of state machines.

State-machines for these processes are defined at high level, and are transformed using service catalogues (see Section 3.4) and transformation laws to create various service definitions at various levels. The state machine remains in the same state until an explicit event notification from the physical platform is received. Then, and depending on the particular process, the machine advances towards the next state executing both the input activities in the target state and the output activities in the source state. Activities may consist of control actions to maintain the process within a certain limit, new processes that are triggered, etc.

This bottom-up approach is complemented with a top-down view proposed in the paper "Process execution in Cyber-Physical Systems using cloud and Cyber-Physical Internet services" by Bordel et al. In this paper it is described in a very detailed manner the functional architecture required to execute process in humanized Cyber-Physical Systems, emphasizing the components that make up the infrastructure in each one of the proposed abstraction levels. Besides it is described an algorithm to select the best location to execute a service among the local platform, virtual entities or external cloud services, presenting the required interfaces to read the service catalogue created at each level. Moreover, special components (such statistical service invocators) are employed regarding the specific characteristics of processes related to the physical world.

In this approach it is proposed as best technology to represent and execute processes a high-level executable processes description language known as YAWL (Van Der Aalst et al., 2005). This language permits describing processes both at business level and production level, and all process configurations can be described, although, before that, a domain-specific language (for example BPMN (White, 2008)) is used to create the process by domain-experts, employing a transformation step to change this domain definition to an executable definition in YAWL.

In respect to the described process execution systems in these previous works, a solution is missed that creates and maintains the services collection in each one of the abstraction layers, to later being able to transform processes and involve participants (devices, humans or entities in charge of the execution).

A general overview of the proposed service configuration solution, all the proposed abstraction layers, the main functionalities required from a service self-configuration solution, and first discussions about how to create heterogeneous services by the coordination of entities with very different behavior and capabilities (in the context of Humanized Cyber-Physical systems) are proposed in the paper "Low-Level Service Management in Cyber-Physical Systems" by Bordel et al. (see Section 3.5). In this paper it is also explained how to define costs and quality estimations about services, considering how distinct entities execute the activities that compose services. This information is stored with the execution scheme of each service, as it is essential to 
make a decision about the best location to execute each service with the previously described algorithms.

In general, three different layers are identified in service self-configuration technologies for humanized Cyber-Physical systems (see paper "Low-Level Service Management in Cyber-Physical Systems" by Bordel et al.).

First, it is described the self-configuration at physical level. It is described how transducers are connected to computation nodes and other physical devices, and how these nodes detect available capabilities and send this information to the management component where these functionalities and grouped to define low-level services and manage the platform in an easier manner. Paper "Plug-and-play transducers in cyberphysical systems for device-driven applications" by Bordel et al. (Section 3.5) describes this physical technology.

Considering the information obtained by computation nodes using the technologies proposed in the paper "Plug-and-play transducers in cyber-physical systems for device-driven applications" by Bordel et al., in the paper "Fast self-configuration in service-oriented Smart Environments for real-time applications" by Bordel et al. it is proposed a fast solution to compose heterogeneous services by coordinating different physical capacities notified by different hardware nodes. Considering some previously defined templates, the proposed technology creates heterogeneous production services coordinating different physical capabilities. By composing several production levels, business level services are defined to create the corresponding catalogue. Finally, a description of the composition of each high-level service is obtained together with a description of its costs and initial QoS.

On the other hand, it is also investigated a solution to, considering the global state of people at real-time, make a decision about the services that humans in the CyberPhysical Systems can execute. The solution includes a decision algorithm to create the corresponding service catalogue, a mechanism to assign a certain QoS to these services and a technique to enable the composition of thee services among them, and with services provided by hardware elements, in order to increase the heterogeneity of services and the wealth of the service catalogue. All these solutions are based on the Maslow's works (Maslow, 1943) and require knowing the motivational state of people at real-time. Depending on the motivational state required to execute each service and the motivational state of humans in the systems, both the service catalogue and the corresponding QoS are calculated. This solution is described in the paper "Selfconfiguration in humanized cyber-physical systems" by Bordel et al. (Section 3.5).

In both papers, the one where the execution of event-driven processes was described ("TF4SM: a framework for developing traceability solutions in small manufacturing companies" by Bordel et al.) and the paper where the self-configuration solution for humanized Cyber-Physical Systems as presented ("Self-configuration in humanized cyber-physical systems" by Bordel et al.), it is indicated that new tools to monitor people in an efficient and precise manner are required, as well as new 
technologies to adapt the hard execution of process performed by physical devices to the flexible, uncontrollable and variable behavior of people. Therefore, some papers describing these required solutions were also reported.

In that way, in the paper "Assessment of human motivation through analysis of physiological and emotional signals in Industry 4.0 scenarios" by Bordel and Alcarria it is described some algorithms and mechanisms to monitor the motivational state of people, which is employed in the self-configuration process for services provided by humans in the paper "Self-configuration in humanized cyber-physical systems" by Bordel et al.. The monitoring and calculation solutions are based on the acquisition of physiological and emotional signals which are processed together considering the Maslow's ideas in order to obtain an estimation of the motivational state.

Also, in Section 3.6, in the paper "T4AI: A system for monitoring people based on improved wearable devices" by Pérez, Bordel et al., how monitoring devices for people are designed and constructed is described in detail at hardware level. These devices are employed to obtain signals in the paper "Assessment of human motivation through analysis of physiological and emotional signals in Industry 4.0 scenarios" by Bordel and Alcarria, and to monitor the activities being performed by people in process execution systems as explained in the paper focused on event-driven processes, "TF4SM: a framework for developing traceability solutions in small manufacturing companies" by Bordel et al. (see Section 3.4).

In the previously referred paper, it is proposed a very simple mechanism to recognize the activity being performed by people, and then use this information to control the process execution. In order to improve the people's freedom to execute activities and allow the creation of a much larger catalogue of permitted activities (practically unlimited), it is proposed an advanced pattern recognition technique based on neural and Bayesian networks in the paper "Recognition of activities of daily living in Enhanced Living". In that way, humans can perform almost any activity, without requiring any previous hard configuration (as needed in the previous work "TF4SM: a framework for developing traceability solutions in small manufacturing companies" by Bordel et al.).

Also, in order to flex and enable a freer execution of processes by humans, in the previous works it was also identified the need of defining a new process model adapted to human behavior. In this new approach processes are not validated considering a maximum permitted error in measurements or tasks, but more complex techniques are employed. In the paper "Process execution in humanized Cyberphysical systems: soft processes" by Bordel et al. (Section 3.6) three different process model types are identified depending on their adaptation to the human behavior (hard, rigid and soft); being defined for first time the idea of soft process in the context of humanized CPS. Basically, these processes are validated using deformation functions, which are applied to the entire process or activities. 
As specific technologies are required to integrate humans in Cyber-Physical Systems and manage their flexible behavior, it is also needed special mechanisms to manage hardware devices and their lifecycle, in order to maintain services in an available state. In particular, as CPS are distributed by definition, solutions to manage task executions in dynamic platforms are required; as well as techniques to enable device to selfmanage. In order to enable the resource self-management by hardware devices, and to allow pervasive physical platform to accept and execute tasks in a choreographed and coordinated manner among the different entities that support a certain heterogeneous service, in the paper "A Hardware-Supported Algorithm for Self-Managed and Choreographed Task Execution in Sensor Networks" by Bordel et al. in Section 3.7, a hardware-supported algorithm is proposed. The proposed algorithm can be implemented in all types of devices, and s based on the idea of "gas" which represents the available resources of devices at a certain moment and it is consumed as operations are executed. This solution is complemented with a filter (implement in a powerful control gateway, for example) that prevents the physical platform to get congested, which is based on the traditional Erlang's queue theory.

Despite the use of choreography solutions, hardware device lifecycle presents other important aspects (such as mobility) which must be also considered. Thus, in the paper "Predictive algorithms for mobility and device lifecycle management in Cyber-Physical Systems" by Bordel et al. it is proposed a management solution based on predictions about the future state of the systems and the composing devices, so no operation gets cancelled, pending or blocked. The proposed solution is based on simulation and interpolation techniques to calculate the future state of a humanized Cyber-Physical System, and information which is employed to manage operations in the physical platform if an important change is expected to happen.

Referred simulation techniques are supported by a specific humanized CPS simulator, where it is necessary to coordinate both the social subsystem as the communication and physical subsystems (representing humans, cyber components and the physical world). Thus, in the paper "A Methodology for the Design of Application-Specific Cyber-Physical Social Sensing Co-Simulators" by Bordel et al. (see Section 3.7) it is proposed a methodology to build simulators adequate to predict the future state of CPS and being integrated into predictive management solutions, as the one previously described. These simulators are configured using the models reported in the paper "Building enhanced environmental traceability solutions: From Thing-toThing communications to Generalized Cyber-Physical Systems" by Bordel et al. (see Section 3.3)

In all previously described works, it is said that communication among the different components that make up a humanized CPS is a critical issue to be considered. Although this is essential among any set of components within the proposed architecture, it is especially important in relation to physical devices as they may be deployed in a sparse way in an area without communication infrastructure, which can strongly affect the performance of the process execution system. In order to evaluate 
the impact of the communication technologies in humanized CPS a new wireless channel model is proposed in the paper "Mobile Wireless Sensor Networks: Modeling and Analysis of Three-Dimensional Scenarios and Neighbor Discovery in Mobile Data Collection" by Robles, Bordel et al. (in Section 3.8). In this new channel model, important characteristics of devices in Humanized CPS are considered, such as the node mobility, the connection procedures of wireless technologies (such as WiFi or Bluetooth) or the possibility of performing handovers. The proposed model is based on the addition of some harmonic functions to regular trajectories and the introduction of stochastic terms in propagation expressions.

The situation where handovers must be performed is analyzed in detail in the paper "Using 5G technologies in the Internet of things handovers, problems and challenges" by Bordel et al. The conclusion of this works if the need of designing a new network scheme for future scenarios, what is known as fifth generation communications. Thus, in the paper "Virtualization-Based Techniques for the Design, Management and Implementation of Future 5G Systems with Network Slicing" by Bordel et al. (see Section 3.8) it is proposed a new network design and implementation for humanized Cyber-Physical Systems to increase their flexibility with a much more adequate communication scheme. The proposed solution is based on virtualization techniques such as Dockers and existing white papers of international organizations such as the 5GPPP (5GPPP, 2018).

Finally, an important and independent aspect the proposed solution is how to secure humanized CPS focused on process execution.

First, in the paper "Protecting Industry 4.0 Systems Against the Malicious Effects of Cyber-Physical Attacks" by Bordel et al. (see Section 3.9), they are investigated the types of attacks that may suffer a humanized CPS; being identified a new class of attack named as "cyber-physical attack". These attacks may be described using a specific language and present some special characteristics that can be classified using six different categories. Using these descriptions, it is proposed a security technology for humanized CPS where an intelligent module using the game theory is able to make a decision about if a cyber-attack is being produced and perform the smartest strategy at each moment.

On the other hand, two additional proposals are proposed in order to protect humanized CPS against two specific possible attacks. In the paper "Detecting Malicious Components in Large-Scale Internet-of-Things Systems and Architectures" by Bordel et al. it is proposed an algorithm being able to detect malicious, infected or untrustworthy nodes in the system, isolate them and prevent them to affect the entire system. Moreover, in the paper "An intra-slice security solution for emerging 5G networks based on pseudo-random number generators" by Bordel et al. a cryptographic solution based on symmetric key techniques is proposed, which allows protecting the private information about users (for example the physiological signals employed in the paper "Assessment of human motivation through analysis of 
physiological and emotional signals in Industry 4.0 scenarios" by Bordel and Alcarria). The proposed solution is lightweight so it can be implemented in resource constraint devices as which employed to monitor people in humanized CPS.

The final solutions include an architecture valid to execute all process types considered in humanized CPS, a list of all possible process models in these systems and the service self-configuration and self-adaptation technologies employed to generate and maintain the service catalogues employed to transform and execute processes. Monitoring techniques to evaluate and manage humans and their behavior, as well as management techniques to control hardware devices and their lifecycle are also included in the proposed solution. Finally, new communication technologies to improve the flexibility of the proposed solution, and security proposals to protect the users' private information and the system against malicious nodes are designed and evaluated as well.

Table 2 summarizes the contribution of each paper solution in Section 3 to the global.

Table 2. Overview of the proposed solution

\begin{tabular}{|c|c|c|}
\hline Paper & Section & Main contribution \\
\hline $\begin{array}{l}\text { Cyber-physical systems: Extending } \\
\text { pervasive sensing from control theory to } \\
\text { the Internet of Things [JCR- Q2] }\end{array}$ & 3.2 & $\begin{array}{l}\text { An analysis of the state-of-the-art in Cyber-Physical Systems, } \\
\text { considering all elements that may be part of CPS, the different } \\
\text { proposed architectures and the main standardization initiatives }\end{array}$ \\
\hline $\begin{array}{l}\text { Building smart adaptable Cyber- } \\
\text { Physical Systems: definitions, } \\
\text { classification and elements }\end{array}$ & 3.3 & $\begin{array}{l}\text { An implementation architecture describing all physical elements and } \\
\text { software components that make up a humanized CPS, including the } \\
\text { main required functionalities such as intelligent management }\end{array}$ \\
\hline $\begin{array}{l}\text { Physical processes control in Industry } \\
\text { 4.0-based systems: a focus on Cyber- } \\
\text { Physical Systems }\end{array}$ & 3.3 & $\begin{array}{l}\text { The different processes to be executed in humanized CPS, a reference } \\
\text { architecture to support process execution systems using humanized } \\
\text { CPS. }\end{array}$ \\
\hline $\begin{array}{l}\text { Building enhanced environmental } \\
\text { traceability solutions: From Thing-to- } \\
\text { Thing communications to Generalized } \\
\text { Cyber-Physical }\end{array}$ & 3.3 & $\begin{array}{l}\text { An exhaustive model to describe humanized Cyber-Physical Systems, } \\
\text { its components, their relations and their main characteristics. Also it } \\
\text { is included a flexible functional architecture to build process } \\
\text { execution systems using cyber-physical technologies }\end{array}$ \\
\hline $\begin{array}{l}\text { Process execution in Cyber-Physical } \\
\text { Systems using cloud and Cyber- } \\
\text { Physical Internet services [JCR- Q2] }\end{array}$ & 3.4 & $\begin{array}{l}\text { A detailed functional architecture and execution algorithms to execute } \\
\text { computational processes with a top-down approach. }\end{array}$ \\
\hline $\begin{array}{l}\text { TF4SM: a framework for developing } \\
\text { traceability solutions in small } \\
\text { manufacturing companies [JCR- Q1] }\end{array}$ & 3.4 & $\begin{array}{l}\text { A detailed functional architecture and hardware devices to execute } \\
\text { low-level defined processes controlled by state machines. }\end{array}$ \\
\hline $\begin{array}{l}\text { Enhancing Process Control in Industry } \\
\text { 4.0 Scenarios using Cyber-Physical } \\
\text { Systems [SJR-Q2] }\end{array}$ & 3.4 & $\begin{array}{l}\text { A complete formalization of the execution of event-driven processes, } \\
\text { considering state machines and specific description languages. }\end{array}$ \\
\hline $\begin{array}{l}\text { Low-Level Service Management in } \\
\text { Cyber-Physical Systems }\end{array}$ & 3.5 & $\begin{array}{l}\text { A global overview of the required service self-configuration processes } \\
\text { in humanized CPS, including algorithms to evaluate the QoS }\end{array}$ \\
\hline $\begin{array}{l}\text { Self-configuration in humanized cyber- } \\
\text { physical systems [JCR- Q3] }\end{array}$ & 3.5 & $\begin{array}{l}\text { A self-configuration algorithm based on Malow's theories to define } \\
\text { heterogeneous services provided by people }\end{array}$ \\
\hline Fast self-configuration in service- & 3.5 & A mechanism to compose heterogeneous services at different levels \\
\hline
\end{tabular}




\begin{tabular}{|c|c|c|}
\hline $\begin{array}{l}\text { oriented Smart Environments for real- } \\
\text { time applications [JCR- Q4] }\end{array}$ & & $\begin{array}{l}\text { by coordinating capabilities from different hardware devices and, } \\
\text { even, people. }\end{array}$ \\
\hline $\begin{array}{l}\text { Plug-and-play transducers in cyber- } \\
\text { physical systems for device-driven } \\
\text { applications }\end{array}$ & 3.5 & $\begin{array}{l}\text { Low-level self-configuration algorithms to enable computing nodes to } \\
\text { learn about their own capabilities and use this information to be } \\
\text { integrated into humanized CPS. }\end{array}$ \\
\hline $\begin{array}{l}\text { Process execution in humanized Cyber- } \\
\text { physical systems: soft processes }\end{array}$ & 3.6 & $\begin{array}{l}\text { A new process type based on the idea of "deformation" which is valid } \\
\text { to execute processes based on human provided services. }\end{array}$ \\
\hline $\begin{array}{l}\text { Assessment of human motivation } \\
\text { through analysis of physiological and } \\
\text { emotional signals in Industry } 4.0 \\
\text { scenarios [JCR- Q3] }\end{array}$ & 3.6 & $\begin{array}{l}\text { A monitoring algorithm and system to obtain information about the } \\
\text { motivational state of humans, from the analysis of psychological and } \\
\text { emotional signals. Devices to monitor these signals are designed. }\end{array}$ \\
\hline $\begin{array}{l}\text { Recognition of activities of daily living } \\
\text { in Enhanced Living Environments }\end{array}$ & 3.6 & $\begin{array}{l}\text { A pattern recognition algorithm to infer the activities being } \\
\text { performed by people at each moment, from the analysis of their } \\
\text { action }\end{array}$ \\
\hline $\begin{array}{l}\text { T4AI: A system for monitoring people } \\
\text { based on improved wearable devices }\end{array}$ & 3.6 & $\begin{array}{l}\text { Hardware devices to monitor human actions in an unobtrusive } \\
\text { manner and enable the recognition of activities they perform }\end{array}$ \\
\hline $\begin{array}{l}\text { Predictive algorithms for mobility and } \\
\text { device lifecycle management in Cyber- } \\
\text { Physical Systems [JCR- Q3] }\end{array}$ & 3.7 & $\begin{array}{l}\text { A management algorithm for hardware devices (and probably } \\
\text { people) based on the prediction of the future state of the system using } \\
\text { simulators and interpolation techniques }\end{array}$ \\
\hline $\begin{array}{l}\text { A Methodology for the Design of } \\
\text { Application-Specific Cyber-Physical } \\
\text { Social Sensing Co-Simulators [JCR- Q1] }\end{array}$ & 3.7 & $\begin{array}{l}\text { A methodology to build simulators for humanized CPS, by } \\
\text { coordinating domain specific simulators for social, communication } \\
\text { (network) and physical simulations. }\end{array}$ \\
\hline $\begin{array}{l}\text { A Hardware-Supported Algorithm for } \\
\text { Self-Managed and Choreographed Task } \\
\text { Execution in Sensor Networks [JCR- Q1] }\end{array}$ & 3.7 & $\begin{array}{l}\text { A hardware-supported algorithm to enable the self-management of } \\
\text { resources by computing nodes, and the choreographed task } \\
\text { execution. }\end{array}$ \\
\hline $\begin{array}{l}\text { Mobile Wireless Sensor Networks: } \\
\text { Modeling and Analysis of Three- } \\
\text { Dimensional Scenarios and Neighbor } \\
\text { Discovery in Mobile Data Collection } \\
\text { [JCR- Q4] }\end{array}$ & 3.8 & $\begin{array}{l}\text { A model for scenarios based on geographically sparse sensor } \\
\text { networks, where data are extracted by mobile data mules. The model } \\
\text { is based on the addition of harmonic functions to regular trajectories, } \\
\text { and the inclusion of the connection procedures of main commercial } \\
\text { wireless communication technologies }\end{array}$ \\
\hline $\begin{array}{l}\text { Using } 5 \mathrm{G} \text { technologies in the Internet of } \\
\text { things handovers, problems and } \\
\text { challenges }\end{array}$ & 3.8 & $\begin{array}{l}\text { A deep analysis of handovers in the context of Internet of Things } \\
\text { systems and future } 5 \mathrm{G} \text { is provided using simulation techniques }\end{array}$ \\
\hline $\begin{array}{l}\text { Virtualization-Based Techniques for the } \\
\text { Design, Management and } \\
\text { Implementation of Future 5G Systems } \\
\text { with Network Slicing }\end{array}$ & 3.8 & $\begin{array}{l}\text { An innovative approach to build future networks using commercial } \\
\text { virtualization technologies, such as kubernetes, and enable the } \\
\text { creation of different slices for future fourth industrial revolution } \\
\text { scenarios }\end{array}$ \\
\hline $\begin{array}{l}\text { Detecting Malicious Components in } \\
\text { Large-Scale Internet-of-Things Systems } \\
\text { and Architectures }\end{array}$ & 3.9 & $\begin{array}{l}\text { A mechanism and algorithm to detect malicious components (those } \\
\text { which provide false information) in the context of humanized Cyber- } \\
\text { Physical systems, and isolate them to protect the system }\end{array}$ \\
\hline $\begin{array}{l}\text { Protecting Industry } 4.0 \text { Systems Against } \\
\text { the Malicious Effects of Cyber-Physical } \\
\text { Attacks }\end{array}$ & 3.9 & $\begin{array}{l}\text { An intelligent security solution to detect cyber-physical attacks using } \\
\text { a set of predefined templates, and select the mort appropriate } \\
\text { protection strategy using the game theory }\end{array}$ \\
\hline $\begin{array}{l}\text { An intra-slice security solution for } \\
\text { emerging } 5 G \text { networks based on } \\
\text { pseudo-random number generators } \\
\text { [JCR- Q1] }\end{array}$ & 3.9 & $\begin{array}{l}\text { A cryptographic solution based on symmetric key techniques to } \\
\text { protect user private information, specifically those data that are } \\
\text { transmitted as a continuous flow such as physiological signals. }\end{array}$ \\
\hline
\end{tabular}




\subsection{Result analysis and discussion}

All papers included in the collection presented in Section 3 provide a validation study where both technical and global characteristics in a relevant scenario are analyzed. In each paper the main properties, limits and the usability of the particular technical proposals are evaluated, but also the adequacy and the performance of the described solutions in some relevant cases according to the described methodology that guides this PhD Thesis work.

In particular, papers included in Section 3.4, related to the process execution and management in the context of humanized Cyber-Physical Systems, describe validations in holistic cases and scenarios and, therefore, from these papers are extracted most part of the results that proves Hypothesis\#1. On the other hand, paper in the next thematic units, in Subsection 3.5, Subsection 3.6 and Subsection 3.7, include experimental validation in embedded cases, which provide partial and complementary information about the proposed solutions, technologies and components in those works.

To prove the validity of Hypothesis $\# \mathbf{1}$ it is not performed a statistical analysis of the aggregated data from all cases is not performed. Instead, specific statistical processing methods are considered to study (within each case) the humanization and flexibility level during process execution in humanized CPS-based systems, and to compare the results to traditional solutions. .

Specifically, the most employed procedure is the Mann-Whitney U test. The MannWhitney $U$ test is a nonparametric test of the null hypothesis that two samples come from the same population against an alternative hypothesis, comparing the mean values of the two samples. It is used to evaluate if two different data populations are similar or different (higher or lower). The $p$ value indicates the significance level of Mann-Whitney U test.

In both papers "Process execution in Cyber-Physical Systems using cloud and Cyber-Physical Internet services" by Bordel et al. and "Building enhanced environmental traceability solutions: From Thing-to-Thing communications to Generalized Cyber-Physical Systems" by Bordel et al., they are reported success rates in process execution with humanized CPS above the ones that are obtained using existing solutions. In all cases and scenarios, the success rate is above 95\%. In these same papers it is also reported an improvement in the execution time and better scalability, especially when event-driven processes are executed, when the amount of information traffic through communication networks grows up as more devices are working and executing operations in parallel.

In the same manner, in the paper "TF4SM: a framework for developing traceability solutions in small manufacturing companies" by Bordel et al. it is reported a relevant flexibility during process execution in cases where people performs an essential role as service providers. Besides, it is showed an important decreasing in the number of made errors by workers integrated into humanized Cyber-Physical systems. 
It must be taken into account that selected scenarios are relevant for this $\mathrm{PhD}$ Thesis works, but obtained results could be different if the proposed solutions are deployed in a real industrial environment including only domain workers without technological skills. In order to evaluate somehow the impact that the consideration of these real scenarios could have in the obtained results, in the paper "Building enhanced environmental traceability solutions: From Thing-to-Thing communications to Generalized Cyber-Physical Systems" by Bordel et al. (also in Section 3.4) it is proposed an experiment where industrial experts inform about their experience using humanized CPS. Results report an important improvement in all KPIs (Key Performance Indicators) for executions systems based on humanized CPS: from the usability and ease to create and define processes, to the flexibility during the execution and the correctness and success applying control policies.

Solutions described in papers in Section 3.4, especially in the paper "Process execution in Cyber-Physical Systems using cloud and Cyber-Physical Internet services" by Bordel et al., already considers the effects of technologies proposed in other papers, such as those related to self-configuration or the human integration into CPS. Therefore, described results validate Hypothesis \#1.

It must be noted, however, that in the employed case study methodology, general conclusions are extracted from repeated observations in some relevant scenarios. Nonetheless, future application scenarios may include some cases, which nowadays are not relevant and, then, have not been considered in the experimental validation. In this case, Hypothesis $\mathbb{1}$ would not be valid. In that way, it is important before selecting an application scenario to guarantee that it is clearly and totally represented by those relevant cases selected for experiments in this $\mathrm{PhD}$ Thesis work.

Moreover, and finally, obtained results in the paper "Fast self-configuration in service-oriented Smart Environments for real-time applications" by Bordel et al. (Section 3.5) proves that it is possible to build real-time applications using humanized CPS, as it is possible to calculate and maintain the catalogues of heterogeneous services, which support the process execution, also at real time.

In respect to Hypothesis\#2, results reported in the paper “Mobile Wireless Sensor Networks: Modeling and Analysis of Three-Dimensional Scenarios and Neighbor Discovery in Mobile Data Collection" by Robles, Bordel et al. and in the paper "Using 5G technologies in the Internet of things handovers, problems and challenges" by Bordel et al. must be considered. They prove that depending on the communication system configuration, how geographically sparse the physical platform is, if there is or there is not a reliable communication infrastructure, etc., it is possible the flexibility (and probably, humanization) in process execution using humanized CPS to strongly decrease. Even, it is possible the system to stop and get blocked, as communications are not available enough time to define a valid service catalogue and (while being valid) order the process execution to the low-level components. In fact, scenarios focused on ad-hoc connections cannot support process execution systems based on 
humanized CPS, although some types of CPS could be deployed in those scenarios (for example CPS for Smart Agriculture). In general, connections must be permanent and stable during a relatively long time to make viable the deployment of a process execution system based on humanized Cyber-Physical systems (and, then, a flexible execution of processes). Hypothesis\#2 is then validated, as the influence of the communication networks in the flexibility (overall) and humanization (in a lower level) during process execution gets proved.

It is important to note that results related to Hypothesis\#2 do not stablish new design laws or imply the need of redefining process execution systems based on humanized CPS. They stablish limits to the selectable application scenarios where these systems may be successfully deployed. In particular, scenarios where the physical platform is geographically sparse cannot be considered.

The last group of results contained in this PhD Thesis work, which must be analyzed, is related to security schemes. In this PhD Thesis work, three different security solutions for humanized CPS have been proposed, each one associated to an experimental validation where the impact of these technologies in the process execution is evaluated.

As can be seen in the paper "An intra-slice security solution for emerging 5G networks based on pseudo-random number generators" by Bordel et al. the required time to execute the encryption procedure is negligible, and no relevant impact on the process execution system efficiency, flexibility or humanization is detected. On the contrary, it is reported a significant increase in the memory usage, but this do not affect most important characteristics of process execution systems. Then, it can be ensured that security techniques do not affect the flexibility or humanization of humanized CPS, as the execution speed, scalability, effectiveness and success rate remain invariant.

The same conclusions may be extracted for proposals contained in papers "Detecting Malicious Components in Large-Scale Internet-of-Things Systems and Architectures" by Bordel et al. and "Protecting Industry 4.0 Systems Against the Malicious Effects of Cyber-Physical Attacks" by Bordel et al. (see Section 3.9). As can be seen in both cases (especially in the paper "Protecting Industry 4.0 Systems Against the Malicious Effects of Cyber-Physical Attacks" by Bordel et al.) the security solution executes in parallel to the process execution algorithms, and the required time to detect an attack and trigger a solution might be considered minimum in comparison to the required time to execute a high-level process. In that way, Hypothesis \#3 is also validated.

Any case, it must be noted that security schemes are very dependent on the number of entities that are executing operations in parallel at a certain moment. Thus, the use of pervasive and very dense physical platform will probably impose new requirements, and security technologies will need a higher amount of resources to be effective. However, the scalability evaluations provided in the paper "Detecting 
Malicious Components in Large-Scale Internet-of-Things Systems and Architectures" by Bordel et al. shows that the increase in the number of physical components must be very strong to seriously affect the performance of the system. Nonetheless, as future applications or scenarios are unknown, these limits must be considered.

\subsection{Conclusions}

This $\mathrm{PhD}$ Thesis work is motivated by the need to explore new process execution systems adapted to the requirements and necessities that will be born from the fourth industrial revolution also known as the era of Cyber-Physical Systems.

The need includes the definition of new process models, execution algorithms, as well as service self-configuration technologies to coordinate the heterogeneous capacities available in different entities. Services are composed by coordinating both hardware devices and humans (which in future technological systems could act as service providers), integrated into process execution systems. It is also required to talk about security and communication networks.

This need and its associated proposals arise after performing an exhaustive analysis of the state-of-the-art, identifying the nowadays existing technologies, their properties, the different approaches to CPS, the different architectures that have been reported, the components that might be part of CPS and the main standardization initiatives reported until now.

Nonetheless, it has been noted that it does not exist a valid proposal for all future scenarios associated to the fourth industrial revolution of Industry 4.0. And, overall, there is no proposal adapted to scenarios where people intervene as active agents and their participation is essential; as for example in manufacturing companies.

- To address this first problem, it is defined a reference architecture especially designed for process execution systems based on humanized Cyber-Physical systems (defined as those CPS where people have an active role). It is also proposed an implementation architecture where the independent physical and software components that make up these systems are identified. Moreover, and finally, a detailed functional architecture describing all functionalities that must be included in humanized CPS to be effective as process execution systems is also proposed.

In a more and more automated world, the process definition techniques in such a way that processes could be later executed by a technological solution (in an independent, flexible and automated way), and tools for domain experts without engineering or programming skills to define processes in an easy manner, are topic receiving a great attention, especially if related to Industry 4.0 .

- Therefore, in this PhD Thesis work, mechanisms to enable the flexible, humanized and automatic execution of processes using the previously described 
architectures are proposed. These contributions include decision algorithms to enable the local execution of tasks or to externalize them to virtual entities, cloud services or third-party production systems. The referred mechanisms also include the definition of processes in an executable manner, as well as technologies to transform these description languages into different abstraction layers, as well as into domain-specific languages employed by domain experts to define processes they desire to execute in an easy manner. They have been also considered other process types, not firstly defined at high-level, as those which are controlled by people or devices at low-level, or those which are eventdriven (for which techniques to define some control laws or states the process must meet or pass through are also described).

The necessity to provide services at a very high-level in process execution systems, some services which are usually created by joining smaller activities executed at lower levels; as well as the use of a service-oriented architecture in almost all future engineered systems because of its flexibility, causes the need of defining service configuration mechanisms. These mechanisms must be self-configuration technologies to define and maintain heterogeneous services created by coordinating entities with a very different behavior and capabilities.

- Contributions relative to this problem that are included in this $\mathrm{PhD}$ Thesis work allow the creation and definition of a service catalogue at all abstraction layers where it is required, from a minimum template; and enable associating to each service a QoS indicator which is employed to make decisions about the locations where particular tasks and processes are executed. Solutions adequate to device supported services and human provided services (these solutions based on the Maslow's works) are reported, as well as technologies to configure hybrid services.

The integration of humans in Cyber-Physical systems is a pending research challenge that must be addressed, as well as an opportunity to discover new application scenarios and businesses. However, several technological issues must be solved in relation to this challenge.

- In order to address this challenge, in this PhD Thesis work it is proposed a new process model named as "soft process", which is validated using the concept of deformation (instead of the traditional maximum permitted error), as it is an idea much flexible and closer to human behavior. Mechanisms to recognize the activities being performed by people and, then, being able to control the evolution of processes executed by them, are also described. Finally, they are proposed devices and algorithms to monitor the state of humans, especially physiological and emotional signals. This information is employed to obtain an estimation of the human motivational state which is used to decide which services and with which quality might be executed by people in the CPS. 
Device management techniques in distributed computing platforms, as humanized CPS, are one of the most popular and studied research challenges in Telecommunication engineering. Very different proposal related to this topic have been reported, but humanized CPS present some special characteristics: the inclusion of resource constraint devices with a very heterogeneous behavior, which are unable to execute tasks making up a process (so tasks must be decomposed into smaller operations), etc.

- Contributions presented in this $\mathrm{PhD}$ Thesis work about this topic include hardware supported mechanisms that enable the device self-management, in order to select if they can accept the execution of a certain operation; algorithms to prevent the entire system congestion and solutions to enable the choreographed execution of tasks among entities in charge of executing operations composing those tasks. Besides, predictive management policies are also presented in order to simulate and learn about the future system states and make decision about event related to the device lifecycle before they occur.

As all telecommunication systems, the basic technology in humanized CyberPhysical Systems is communication networks. Research related to communications and future applications and scenarios such as Cyber-Physical Systems is a very popular research line nowadays.

- In order to solve this problem, in this PhD Thesis work, it is proposed a new model for wireless channels, created by adding some harmonic functions to the main and essential trajectories created by nodes while moving. The connection process for the most popular commercial technologies nowadays is also considered in the model. It is also proposed a network architecture for future systems (fifth generation systems) based on virtualization technologies. This proposal is based on a detailed study about handovers in mobile networks and Cyber-Physical systems, where devices have a small coverage range and are deployed in a very dense manner.

Finally, in order to enable the flexible process execution and the service selfconfiguration (where private information about people and their physiological and emotional state is employed), security policies must be considered.

- In order to provide security to humanized CPS, in this PhD Thesis work they are proposed three different mechanisms. On the one hand, an encryption scheme based on symmetric key technologies in order to protect private personal information is described. On the other hand, a solution and algorithm to detect malicious components based on the ideas of trust and reputation is described, which allows detecting and isolating infected components. Finally, an intelligent algorithm to protect humanized CPS against general attacks, based on game theory and a specific description language, is also reported. 
In this PhD Thesis work, and according to the proposed case study methodology, is has been developed a set of experiments and validation in real and simulation scenarios that allow extending the proposed work from the theoretical discussion to the real engineering. Namely:

- The process management techniques and solutions are evaluated through statistical techniques and survey research. Relevant scenarios where these solutions have been validated include production systems, manufacturing companies and assembling lines.

- Self-configuration technologies, including those referred to humans and those focused on devices, are evaluated in scenarios related to Smart Environments and Ambient Intelligence deployments.

- Scenarios related to Ambient Intelligence deployments are also employed to validate proposals focused on integrating humans into CPS. Besides, Industry 4.0 applications are selected as relevant cases to experiment with monitoring techniques applied to know the motivational state of people.

- Cases where physical device management technologies are validated are exclusively focused on IoT systems and Smart Cities. Large IoT deployments are, besides, the relevant scenarios where security technologies are evaluated (using simulation techniques). Smart Cities are also referred in works related to fifth generation communication networks.

- Finally, Smart agriculture applications are the main scenario where the proposed wireless communication channel model is evaluated using simulation techniques. The described analysis about handovers and CPS is also focused on this study case.

Considering all previous analyses and discussions, it can be ensured that the proposed research objectives (see Section 1) have been fulfilled, and the established initial hypotheses (see also Section 1) validated.

\subsection{Future works}

This section describes the planned future works, related to the contributions described in this PhD Thesis work.

The main pending challenge which must be developed in future works, as it is not addressed in this $\mathrm{PhD}$ Thesis work, in the integration of time references into humanized Cyber-Physical Systems. In fact, timing is one of the main research challenges related to Cyber-Physical systems, which in this PhD Thesis works has been only partially addressed.

In particular, some process description languages, such as YAWL, may include time references to be considered during execution, as for example the maximum timeout for a task. However, there is not any vertical solution in the proposed architectures to add time information and feed process execution with the desired time requirements. 
Besides, no solution to adapt the time understanding of people and hardware devices is proposed.

Therefore, future works should address this challenge and design mechanism to integrate timing in process definition, to enable algorithms and functional components to understand time information, and finally to include a learning algorithms or smart system being able to harmonize the different understanding of time that are present in humanized Cyber-Physical Systems.

Another pending challenge is the proposal of a management system to control emergencies related to services provided by people. In services provided by hardware devices, it is always possible (if necessary) to cancel all operations sending the appropriate message, removing the power supply, etc. Nevertheless, it is very difficult, and it is a problem not addressed in PhD Thesis work, how to cancel executions performed by people. This is especially critical when actions performed by people may cause a general fault in the system, affect other people or even might be a danger for the service provider.

Although proposed solutions include some information displays and other warning systems (as described in some paper in Section 3.4), it is assumed that people obey every moment the information showed in those devices. This assumption, however, might not be true, so proposed displays showing emergency information must be complemented with additional solutions to avoid dangerous situations and, then, totally stop activities being performed, suppress dangerous behaviors somehow and/or evacuate people if necessary (solutions known as "industrial security systems").

Data processing techniques to be applied in humanized CPS are also a challenge to be addressed in future works. These techniques should be employed to transform information in a certain abstraction layer to valid information for a new target abstraction level. This includes transformation techniques for process definitions, for data and metadata. Paradigms such as Big Data may help to solve some aspects of this challenge.

Finally, and apart from aspects related to security or communication networks which are works that are moving away from the main focus of this PhD Thesis work, the last main challenge that should be addressed in future works is the definition of management algorithms based on orchestration techniques.

In this PhD Thesis work it has been proposed a self-management algorithm based on a choreographed execution of tasks, so hardware devices are in charge of operations depending on their available resources. However, all devices may execute a certain operation a priori, as it is assumed that all of them execute operations with a homogeneous quality (apart from congestion situation which have been already considered). In real deployments this assumption may not be true, so this PhD Thesis work should be extended to situations where an orchestration module is desirable. This new module would decide which device(s) can or must execute a certain 
operation depending on the offered by each one QoS through the self-configuration procedures. 


\section{Bibliography}

5GPPP Whitepapers. Available online: https://5g-ppp.eu/white-papers/ (accessed 02 June 2018)

Ackoff, R. L. (1962). Scientific method: Optimizing applied research decisions.

Ackoff, R. L., \& Sasinieni, M. W. (1968). Fundamentals of operations research.

Akyildiz, I. F., Su, W., Sankarasubramaniam, Y., \& Cayirci, E. (2002). Wireless sensor networks: a survey. Computer networks, 38(4), 393-422.

Baeg, S. H., Park, J. H., Koh, J., Park, K. W., \& Baeg, M. H. (2007, October). Building a smart home environment for service robots based on RFID and sensor networks. In Control, Automation and Systems, 2007. ICCAS'07. International Conference on (pp. 1078-1082). IEEE.

Churchman, C. W. (1961). Prediction and optimal decision (pp. 51-56). Englewood Cliffs, NJ: Prentice-Hall.

Cohen, D. I., \& Cohen, D. I. (1991). Introduction to computer theory (Vol. 2). New York: Wiley.

Cook, D., \& Das, S. K. (2004). Smart environments: Technology, protocols and applications (Vol. 43). John Wiley \& Sons.

Coughlan, P., \& Coghlan, D. (2002). Action research for operations management. International journal of operations \& production management, 22(2), 220-240.

Daneels, A., \& Salter, W. (1999). What is SCADA?.

Das, S. K., \& Cook, D. J. (2005, December). Designing smart environments: A paradigm based on learning and prediction. In International Conference on Pattern Recognition and Machine Intelligence (pp. 80-90). Springer, Berlin, Heidelberg.

De Brito, M. P., \& Dekker, R. (2004). A framework for reverse logistics. In Reverse logistics (pp. 3-27). Springer Berlin Heidelberg. 
Eisenhardt, K. M. (1989). Building theories from case study research. Academy of management review, 14(4), 532-550.

Emery, F., \& Thorsrud, E. (1976). Democracy at work: The report of the Norwegian industrial democracy program (Vol. 2). Springer.

Forza, C. (2010). Surveys. In Researching operations management (pp. 98-175). Routledge.

Frohlich, M. T. (2002). Techniques for improving response rates in OM survey research. Journal of Operations Management, 20(1), 53-62..

Glaser, B. G., \& Strauss, A. L. (2017). Discovery of grounded theory: Strategies for qualitative research. Routledge.

Gross, N. C., Giacquinta, J. B., \& Bernstein, M. (1971). Implementing organizational innovations: A sociological analysis of planned educational change. Basic Books.

Jazdi, N. (2014, May). Cyber physical systems in the context of Industry 4.0. In Automation, Quality and Testing, Robotics, 2014 IEEE International Conference on (pp. 1-4). IEEE.

La, H. J., \& Kim, S. D. (2010, August). A service-based approach to designing cyber physical systems. In Computer and Information Science (ICIS), 2010 IEEE/ACIS 9th International Conference on (pp. 895-900). IEEE.

Lee, E. A. (2006, October). Cyber-physical systems-are computing foundations adequate. In Position Paper for NSF Workshop On Cyber-Physical Systems: Research Motivation, Techniques and Roadmap (Vol. 2, pp. 1-9).

Lee, E. A. (2015). The past, present and future of cyber-physical systems: A focus on models. Sensors, 15(3), 4837-4869.

Lewin, K. (1946). Action research and minority problems. Journal of social issues, 2(4), 34-46.

Lukkien, J. (2016). Cyber Physical Systems: a perspective. Available online: http://www.win.tue.nl/ johanl/docs/CPS\%20v2\%20presented.pdf (accessed on 26 May 16, 2018).

Ma, H. D. (2011). Internet of things: Objectives and scientific challenges. Journal of Computer science and Technology, 26(6), 919-924.

Malhotra, M. K., \& Grover, V. (1998). An assessment of survey research in POM: from constructs to theory. Journal of operations management, 16(4), 407-425.

Maslow, A. H. (1943). A theory of human motivation. Psychological review, 50(4), 370.

Miles, M. B., \& Huberman, A. M. (1984). Qualitative data analysis: A sourcebook of new methods. In Qualitative data analysis: a sourcebook of new methods. Sage publications 
National Institute of Standards and Technology. CPS Public Working Group presentation. $\quad$ http://www.nist.gov/el/upload/CPS-PWG-Kickoff-WebinarPresentation-FINAL.pdf (accessed on 26 May 2018).

Nixon, P., Dobson, S., \& Lacey, G. (2000, June). Managing smart environments. In Proceedings of the Workshop on Software Engineering for Wearable and Pervasive Computing.

Popperp, K. R. (1959). The Logic of Scientific Discovery. University Press.

Ramos, C., Augusto, J. C., \& Shapiro, D. (2008). Ambient intelligence-the next step for artificial intelligence. IEEE Intelligent Systems, 23(2), 15-18.

Rivett, P. (1972). Principles of model building.

Schmidt, A. (2000). Implicit human computer interaction through context. Personal technologies, 4(2-3), 191-199.

Susman, G. I., \& Evered, R. D. (1978). An assessment of the scientific merits of action research. Administrative science quarterly, 582-603.

Tarraf, D. C. (2013). Control of cyber-physical systems. Proc. of Lecture Notes in Control and Information Sciences, 449.

Van Der Aalst, W. M., \& Ter Hofstede, A. H. (2005). YAWL: yet another workflow language. Information systems, 30(4), 245-275.

Voss, C., Tsikriktsis, N., \& Frohlich, M. (2002). Case research in operations management. International journal of operations \& production management, 22(2), 195-219.

Wan, J., Chen, M., Xia, F., Di, L., \& Zhou, K. (2013). From machine-to-machine communications towards cyber-physical systems. Computer Science and Information Systems, 10(3), 1105-1128.

Weiser, M. (1991). The Computer for the 21 st Century. Scientific american, 265(3), 94105.

White, S. A. (2008). BPMN modeling and reference guide: understanding and using BPMN. Future Strategies Inc..

Wu, F. J., Kao, Y. F., \& Tseng, Y. C. (2011). From wireless sensor networks towards cyber physical systems. Pervasive and Mobile computing, 7(4), 397-413.

Yin, R. K. (2017). Case study research and applications: Design and methods. Sage publications. 


\section{Annex A. Complete collection of publications. Additional results}

During the course of this $\mathrm{PhD}$ Thesis work, the author has made a number of publications, some of them directly related to the contributions and some indirectly related, but that, somehow, contributed to validate the ideas and proposed solutions.

\section{A.1 Publications directly related to the research objectives}

Publications directly related to the objectives of this PhD Thesis work can be classified into the following groups:

\section{Publications in international journals listed in JCR ranking}

1. Bordel Sánchez, B., Alcarria, R., Martín, D., \& Robles, T. (2015). TF4SM: a framework for developing traceability solutions in small manufacturing companies. Sensors, 15(11), 29478-29510. [IF: 2.033 - Q1 - JCR 2015]

2. Bordel, B., Alcarria, R., Robles, T., \& Martín, D. (2017). Cyber-physical systems: Extending pervasive sensing from control theory to the Internet of Things. Pervasive and Mobile Computing, 40, 156-184. [IF: 2.349 - Q2 - JCR 2016]

3. Sánchez, B. B., Alcarria, R., De Rivera, D. S., \& Sánchez-Picot, A. (2016). Predictive algorithms for mobility and device lifecycle management in CyberPhysical Systems. EURASIP Journal on Wireless Communications and Networking, 2016(1), 228. [IF: 1.529 - Q3 - JCR 2016]

4. Bordel, B., Alcarria, R., Martín, D., Robles, T., \& de Rivera, D. S. (2017). Selfconfiguration in humanized cyber-physical systems. Journal of Ambient Intelligence and Humanized Computing, 8(4), 485-496. [IF: 1.588 - Q3 - JCR 2016]

5. Robles, T., Bordel, B., Alcarria, R., \& Martin, D. (2017). Mobile Wireless Sensor Networks: Modeling and Analysis of Three-Dimensional Scenarios and Neighbor Discovery in Mobile Data Collection. Adhoc \& Sensor Wireless Networks, 35. [IF: 1.034 - Q4 - JCR 2016] 
6. Sánchez, B. B., Alcarria, R., Sánchez-Picot, Á., \& Sánchez-de-Rivera, D. (2017). A Methodology for the Design of Application-Specific Cyber-Physical Social Sensing Co-Simulators. Sensors, 17(10), 2177. [IF: 2.677 - Q1 - JCR 2016]

7. Bordel, B., Miguel, C., Alcarria, R., \& Robles, T. (2018). A Hardware-Supported Algorithm for Self-Managed and Choreographed Task Execution in Sensor Networks. Sensors, 18(3), 812. [IF: 2.677 - Q1 - JCR 2016]

8. Bordel, B., Alcarria, R., de Rivera, D. S., \& Robles, T. (2018). Process execution in Cyber-Physical Systems using cloud and Cyber-Physical Internet services. The Journal of Supercomputing, 1-43. [IF: 1.929 - Q2 - JCR 2016]

9. Bordel, B., Alcarria, R., Sánchez de Rivera, D., Martín, D., \& Robles, T. (2018). Fast self-configuration in service-oriented Smart Environments for real-time applications. Journal of Ambient Intelligence and Smart Environments, 10(2), 143-167. [IF: 0.809 - Q4 - JCR 2016]

10. Bordel, B., Orúe, A. B., Alcarria, R., \& Sánchez-de-Rivera, D. (2018). An intraslice security solution for emerging $5 \mathrm{G}$ networks based on pseudo-random number generators. IEEE Access, 6, 16149-16164. [IF: 3.244 - Q1 - JCR 2016]

11. Bordel, B., \& Alcarria, R. (2017). Assessment of human motivation through analysis of physiological and emotional signals in Industry 4.0 scenarios. Journal of Ambient Intelligence and Humanized Computing, 1-21. [IF: 1.588 Q3 - JCR 2016]

\section{Publications in international journals listed in SJR ranking}

12. Sánchez, B. B., Alcarria, R., de Rivera, D. S., \& Sánchez-Picot, Á. (2016). Enhancing Process Control in Industry 4.0 Scenarios using Cyber-Physical Systems. JoWUA, 7(4), 41-64. [IF: 0.337 - Q2 -SJR 2016]

\section{Other publications in international journals}

13. Pérez-Jiménez, M., Sánchez, B. B., \& Alcarria, R. (2016). T4AI: A system for monitoring people based on improved wearable devices. Research Briefs on Information \& Communication Technology Evolution (ReBICTE), 2, 1-16.

14. Bordel, B., Alcarria, R., Manso, M. A., \& Jara, A. (2017). Building enhanced environmental traceability solutions: From Thing-to-Thing communications to Generalized Cyber-Physical Systems. Journal of Internet Services and Information Security (JISIS), 7(3), 17-33.

15. Bordel, B., Pérez-Jiménez, M., \& Sánchez-de-Rivera, D. Recognition of activities of daily living in Enhanced Living Environments. IT CoNvergence PRActice (INPRA), 2, 1-16 


\section{Publications in international conferences listed in the CORE ranking}

16. Bordel, B., Alcarria, R., Sánchez-de-Rivera, D., \& Robles, T. (2017, November). Protecting Industry 4.0 Systems Against the Malicious Effects of Cyber-Physical Attacks. In International Conference on Ubiquitous Computing and Ambient Intelligence (pp. 161-171). Springer, Cham. [CORE ranking - C]

17. Bordel, B., Alcarria, R., \& Jara, A. (2017, June). Process execution in humanized Cyber-physical systems: soft processes. In Information Systems and Technologies (CISTI), 2017 12th Iberian Conference on (pp. 1-7). IEEE. [CORE ranking - C Regional]

18. Bordel, B., de Rivera, D. S., \& Alcarria, R. (2018, March). Virtualization-Based Techniques for the Design, Management and Implementation of Future 5G Systems with Network Slicing. In World Conference on Information Systems and Technologies (pp. 133-143). Springer, Cham. [CORE ranking - C]

19. Bordel, B., Alcarria, R., \& Sánchez-de-Rivera, D. (2017, April). Detecting Malicious Components in Large-Scale Internet-of-Things Systems and Architectures. In World Conference on Information Systems and Technologies (pp. 155-165). Springer, Cham. [CORE ranking - C Regional]

\section{Publications in other international conferences}

20. Bordel, B., Alcarria, R., Pérez-Jiménez, M., Robles, T., Martín, D., \& de Rivera, D. S. (2015, December). Building smart adaptable Cyber-Physical Systems: definitions, classification and elements. In International Conference on Ubiquitous Computing and Ambient Intelligence (pp. 144-149). Springer, Cham.

21. Bordel, B., de Rivera, D. S., \& Alcarria, R. (2016, July). Plug-and-play transducers in cyber-physical systems for device-driven applications. In Innovative Mobile and Internet Services in Ubiquitous Computing (IMIS), 2016 10th International Conference on (pp. 316-321). IEEE.

22. Bordel, B., Alcarria, R., Sánchez-de-Rivera, D., \& Jara, A. (2017, July). Low-Level Service Management in Cyber-Physical Systems. In International Conference on Innovative Mobile and Internet Services in Ubiquitous Computing (pp. 860872). Springer, Cham.

23. Bordel, B., Alcarria, R., Sánchez-de-Rivera, D., \& Robles, T. (2017, November). Protecting Industry 4.0 Systems Against the Malicious Effects of Cyber-Physical Attacks. In International Conference on Ubiquitous Computing and Ambient Intelligence (pp. 161-171). Springer, Cham. 


\section{A.2 Other publications by the author, indirectly related to this PhD Thesis}

Publications indirectly related to the objectives of this $\mathrm{PhD}$ Thesis work can be classified into the following groups:

\section{Publications in international journals listed in JCR ranking}

1. Mareca, M. P., \& Bordel, B. (2017). Improving the complexity of the Lorenz dynamics. Complexity, 2017. [IF: 4.621 - Q1 - JCR 2016]

2. Sánchez-de-Rivera, D., Martín, D., Alcarria, R., Bordel, B., \& Robles, T. (2017). Towards a Wireless and Low-Power Infrastructure for Representing Information Based on E-Paper Displays. Sustainability, 9(1), 76. [IF: 4.88 - Q2 - JCR 2016]

3. Alcarria, R., Bordel, B., Martín, D., \& De Rivera, D. S. (2017). Rule-based monitoring and coordination of resource consumption in smart communities. IEEE Transactions on Consumer Electronics, 63(2), 191-199. [IF: 4.347 - Q2 - JCR 2016]

4. Bordel, B., Alcarria, R., Morales, A., \& Castillo, I. (2018). A framework for enhancing mobile workflow execution through injection of flexible security controls. Analog Integrated Circuits and Signal Processing, 1-14. [IF: 1.347 - Q4 - JCR 2016]

5. Martín, Diego, Bordel, Borja, Alcarria, Ramón \& Castro, Yone (2018). Improving Learning Tasks for Mentally Handicapped People Using AmI Environments Based on Cyber-Physical Systems. Mobile Information Systems, 2018, 1-12. [IF: 0.849 - Q4 - JCR 2016]

6. Sánchez-Picot, A., Martín, D., Bordel, B., \& Sánchez de Rivera, D. AmI environments simulations approach integrating social and network aspects: A case study. Journal of Ambient Intelligence and Smart Environments, (accepted for publication) [IF: 0.809 - Q4 - JCR 2016]

7. Bordel Sánchez, B., Alcarria, R., Martín, D., \& Sánchez-Picot, A. Trust provision in the Internet of Things using transversal blockchain networks. Intelligent Automation \& Soft Computing, (accepted for publication)

[IF: $0.644-$ Q4 - JCR 2016]

\section{Publications in international journals listed in SJR ranking}

8. Alcarria, R., Bordel, B., de Andrs, D. M., \& Robles, T. (2018). Enhanced Peer Assessment in MOOC Evaluation Through Assignment and Review Analysis. International Journal of Emerging Technologies in Learning (iJET), 13(1), 206219. [IF: 0.218 - Q3 -SJR 2017] 


\section{Other publications in international journals}

9. Sánchez, B. B., de Rivera, D. S., \& Sánchez-Picot, A. (2016). Building unobtrusive wearable devices: an ergonomic cybernetic glove. J. Internet Serv. Inf. Secur., 6(2), 37-52.

\section{Publications in international conferences listed in the CORE ranking}

10. Sánchez-Picot, Á., Martín, D., de Rivera, D. S., Bordel, B., \& Robles, T. (2016, March). Modeling and simulation of interactions among people and devices in ambient intelligence environments. In Advanced Information Networking and Applications Workshops (WAINA), 2016 30th International Conference on (pp. 784-789). IEEE. [CORE ranking - B]

11. Alcarria, R., Bordel, B., Manso, M. Á., Iturrioz, T., \& Pérez, M. (2018, January). Analyzing UAV-Based Remote Sensing and WSN Support for Data Fusion. In International Conference on Information Theoretic Security (pp. 756-766). Springer, Cham [CORE ranking - C]

12. Mareca, P., \& Bordel, B. (2018, March). An Intra-slice Chaotic-Based Security Solution for Privacy Preservation in Future 5G Systems. In World Conference on Information Systems and Technologies (pp. 144-154). Springer, Cham. [CORE ranking - C]

13. Bordel, B., Alcarria, R., Rizzo, G., \& Jara, A. (2018, January). Creating Predictive Models for Forecasting the Accident Rate in Mountain Roads Using VANETs. In International Conference on Information Theoretic Security (pp. 319-329). Springer, Cham. [CORE ranking - C]

14. Bordel, B., \& Mareca, M. P. (2017, June). Using wikis in the higher education: The case of Wikipedia. In Information Systems and Technologies (CISTI), 2017 12th Iberian Conference on (pp. 1-6). IEEE. [CORE ranking - C Regional]

15. Mareca, P., \& Bordel, B. (2017, April). A Robust Implementation of a Chaotic Cryptosystem for Streaming Communications in Wireless Sensor Networks. In World Conference on Information Systems and Technologies (pp. 95-104). Springer, Cham. [CORE ranking - C]

16. Mareca, P., \& Bordel, B. (2016, June). CAOS SUITE: A software tool for the analysis and simulation of chaotic Dynamical Systems. From chaos to hyperchaos. In Information Systems and Technologies (CISTI), 2016 11th Iberian Conference on (pp. 1-7). IEEE. [CORE ranking - C Regional]

17. Bordel, B., Mareca, P., \& Alcarria, R. (2016, June). Bioengineering learning models to explain nonlinarearity and chaos. In Information Systems and Technologies (CISTI), 2016 11th Iberian Conference on (pp. 1-7). IEEE. [CORE ranking - C Regional]

18. de Rivera, D. S., Alcarria, R., Martín, D., Sánchez-Picot, Á., Bordel, B., \& Robles, T. (2016, March). Distributed Query Results and IoT Data in a Publish-Subscribe 
Network Implementing User Notifications. In Advanced Information Networking and Applications Workshops (WAINA), 2016 30th International Conference on (pp. 778-783). IEEE. [CORE ranking - B]

\section{Publications in other international conferences}

19. Alcarria, R., de Andrés, D. M., Bordel, B., de Rivera, D. S., Sánchez-Picot, Á., \& Robles, T. (2017, January). A service-oriented monitoring system based on rule evaluation for Home Automation. In Consumer Electronics (ICCE), 2017 IEEE International Conference on (pp. 329-330). IEEE.

20. de Rivera, D. S., Alcarria, R., de Andres, D. M., Bordel, B., \& Robles, T. (2016, January). An autonomous information device with e-paper display for personal environments. In Consumer Electronics (ICCE), 2016 IEEE International Conference on (pp. 139-140). IEEE.

21. Alcarria, R., Bordel, B., \& Jara, A. (2017, July). Flexible Service Provision in Context-Aware Cyber-Physical Systems. In International Conference on Innovative Mobile and Internet Services in Ubiquitous Computing (pp. 873883). Springer, Cham.

22. Bordel, B., Alcarria, R., Martín, D., \& Sánchez-de-Rivera, D. (2017, May). Improving MOOC Student Learning Through Enhanced Peer-to-Peer Tasks. In European Conference on Massive Open Online Courses (pp. 140-149). Springer, Cham.

23. Martín, D., Bordel, B., Alcarria, R., Sánchez-Picot, Á., de Rivera, D. S., \& Robles, T. (2016, November). Improving Learning Tasks for Mentally Handicapped People Using AmI Environments Based on Cyber-Physical Systems. In International Conference on Ubiquitous Computing and Ambient Intelligence (pp. 166-177). Springer, Cham.

24. Martín, D., Bordel, B., Alcarria, R., Sánchez-Picot, Á., de Rivera, D. S., \& Robles, T. (2017, November). Prosumerization Approach to Semantic Ambient Intelligence Platforms. In International Conference on Ubiquitous Computing and Ambient Intelligence (pp. 109-120). Springer, Cham.

25. Morales, A., Castillo-Velazquez, J. I., Bordel, B., \& Hernandez, M. (2017, September). Enhancing mobile workflow execution by modeling flexible security controls. In Sensors Networks Smart and Emerging Technologies (SENSET), 2017 (pp. 1-4).

26. Sánchez-Picot, Á., de Andrés, D. M., Sánchez, B. B., Garrido, R. P. A., de Rivera, D. S., \& Robles, T. (2016). Towards a Simulation of AmI Environments Integrating Social and Network Simulations. In AmILP@ ECAI.

27. Bordel, B., \& Alcarria, R. (2017). Secure sensor data transmission in 5G networks using pseudorandom number generators. In 2nd International Symposium on Mobile Internet Security, 2017 (pp. 1-11) 
28. Alcarria, R; Bordel, B; Manso, MA; de Andres, DM, PROPOSAL OF AN EXPERIENTIAL LEARNING ENVIRONMENT IN THE CLOUD FOR THE CAPTURE AND PROCESSING OF GEOSPATIAL INFORMATION BY HIGHER EDUCATION STUDENTS10TH INTERNATIONAL CONFERENCE OF EDUCATION, RESEARCH AND INNOVATION (ICERI2017) ICERI Proceedings 10th Annual International Conference of Education, Research and Innovation (ICERI) NOV 16-18, $2017 \quad$ Seville, SPAIN 2340-1095

29. Bordel, B; Alcarria, R; Martin, D; Manso, MA IMPACT EVALUATION OF THE TUTORING METHODOLOGY IN THE COMPETENCE ACQUISITION BY STUDENTS DURING THE ELABORATION OF THE FINAL PROJECT 10TH INTERNATIONAL CONFERENCE OF EDUCATION, RESEARCH AND INNOVATION (ICERI2017) ICERI Proceedings10th Annual International Conference of Education, Research and Innovation (ICERI) NOV 16-18, 2017 Seville, SPAIN 2340-1095 
